NUREG/CR-5787

EGG-2657

Vol. 2

\title{
Timing Analysis of PWR Fuel Pin Failures
}

Final Report

Appendices $\mathbf{K}-\mathbf{L}$

Prepared by

K. R. Jones, N. I. Wade, K. R. Katsma,

L. J. Siefken, M. Straka

Idaho National Engineering Laboratory EG\&G Idaho, Inc.

Prepared for

U.S. Nuclear Regulatory Commission 


\section{AVAILABILITY NOTICE}

Availabilly of Reference Materials Cited in NRC Publications

Most documents cited in NRC publications will be avallable from one of the following sources:

1. The NRC Public Document Room, 2120 L Street, NW., Lower Level, Washington, DC 20555

2. The Superintendent of Documents. U.S. Goverment Printing Ofice, P.O. Box 37082, Washington, DC 20013-7082

3. The National Technical InIormation Service, Springfield, VA 22161

Although the listing that follows represents the majority of documents cited In NRC publications, it is not intended to be exhaustive.

Referenced documents avallable for inspection and copying for a fee from the NPC Public Document Room include NRC correspondence and internal NRC memoranda; NRC bulletins, clrculars, information notices. inspection and investigation notices: licensee event reports; vendor reports and correspondence: Commission papers: and applicant and licensee documents and correspondence.

The following documents in the NUREG series are avallable for purchase from the GPO Sales Program: formal NRC statt and contractor reports, NRC-sponsored conterenco proceedings, International agreement reports, grant publications, and NRC booklets and brochures. Also avallable are regulatory quides, NRC regulations in the Code of Federal Regulations. and Nuclear Regulatory Commission Issuances.

Documents avallable from the National Tochnical information Service include NUREQ-series reports and technical reports prepared by other Federal agencies and reports prepared by the Atomic Energy Commission. Foreruner agency to the Nuclear Regulatory Commission.

Documents avaliable from public and special technical libraries include all open hiterature thems, such as books, journal articles, and transactions. Federal Register notices. Federal and State legislatlon. and congressional reports can usually be obtained from these libraries.

Documents such as theses, dissertations, foreign reports and translations, and non-NRC conference proceedings are avaliable for purchase from the organization sponsoring the publication cited.

Single copies of NRC draft reports are avallable free, to the extent of supply, upon written request to the Office of Administration. Distribution and Mall Services Section. U.S. Nuclear Regulatory Commission. Washington. DC 20555.

Copies of industry codes and standards used in a substantive manner in the NRC regulatory process are maintained at the NRC Library, 7920 Norfolk Avenue. Bethesda, Maryland, for use by the public. Codes and standards are usually copyrighted and may be purchased from the originating organization or, If they are American National Standards, from the American National Standards Institute, 1430 Broadway, New York. NY 10018.

\section{DISCLAIMER NOTICE}

This report was prepared as an account of work sponsored by an agency of the United States Govemment. Neither the United States Government nor any agency thereof, or any of their employees, makes any warranty, expressed or implied, or assumes any legal liability of responsibility for any third party's use, or the results of such use, of any information, apparatus, product or process disclosed in this report, or represents that its use by such third party would not infringe privately owned rights. 


\section{DISCLAIMER}

This report was prepared as an account of work sponsored by an agency of the United States Government. Neither the United States Government nor any agency Thereof, nor any of their employees, makes any warranty, express or implied, or assumes any legal liability or responsibility for the accuracy, completeness, or usefulness of any information, apparatus, product, or process disclosed, or represents that its use would not infringe privately owned rights. Reference herein to any specific commercial product, process, or service by trade name, trademark, manufacturer, or otherwise does not necessarily constitute or imply its endorsement, recommendation, or favoring by the United States Government or any agency thereof. The views and opinions of authors expressed herein do not necessarily state or reflect those of the United States Government or any agency thereof. 


\section{DISCLAIMER}

Portions of this document may be illegible in electronic image products. Images are produced from the best available original document. 


\section{Timing Analysis of PWR Fuel Pin Failures}

Final Report

Appendices $\mathrm{K}-\mathrm{L}$

Manuscript Completed: August 1992

Date Published: September 1992

Prepared by

K. R. Jones, N. I. Wade, K. R. Katsma,

I. J. Siefken, M. Straka*

Idaho National Engineering I aboratory

Managed by the U.S. Department of Energy

EG\&G Idaho, Inc.

Idaho Ialls, ID 83415

Prepared for

Division of Safety Issue Resolution

Office of Nuclear Regulatory Research

U.S. Nuclear Regulatory Commission

Washington, DC 20555

NRC FIN L1611

Under DOE Contract No. DE-AC07-76ID01570

*Halliburton NUS

1301 E. 17 th

Idaho Falls, ID 83404

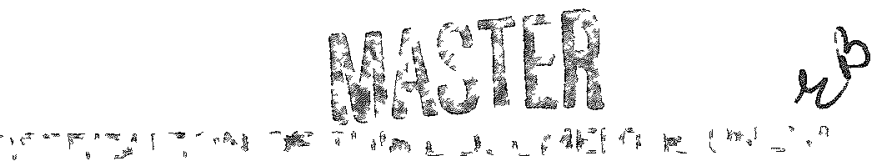





\begin{abstract}
Research has been conducted to develop and demonstrate a methodology for calculation of the time interval between receipt of the containment isolation signals and the first fuel pin failure for loss-of-coolant accidents (LOCAs). Demonstration calculations were performed for a Babcock and Wilcox (B\&W) design (Oconee) and a Westinghouse (W) four-loop design (Seabrook). Sensitivity studies were performed to assess the impacts of fuel pin burnup, axial peaking factor, break size. emergency core cooling system availability, and main coolant pump trip on these times. The analysis was performed using the following codes: FRAPCON-2, for the calculation of steady-state fuel behavior; SCDAP/RELAP5/MOD3 and TRACPF1/MODI, for the calculation of the transient thermal-hydraulic conditions in the reactor system: and FRAP-T6, for the calculation of transient fuel behavior. In addition to the calculation of fuel pin failure timing. this analysis provides a comparison of the predicted results of SCDAP/RELAP5/MOD3 and TRAC-PF1/MOD1 for large-break LOCA analysis.
\end{abstract}

Using SCDAP/RELAP5/MOD3 thermal-hydraulic data. the shortest time intervals calculated between initiation of containment isolation and fuel pin failure are 10.4 seconds and 19.1 seconds for the $B \& W$ and $W$ plants, respectively. Using data generated by TRAC-PFI/MOD1, the shortest intervals are 10.3 seconds and 29.1 seconds for the $B \& W$ and $W$ plants, respectively. These intervals are for a double-ended, offset-shear. cold leg break, using the technical specification maximum peaking factor and applied to fuel with maximum design burnup. Using peaking factors commensurate with actual burnups would result in longer intervals for both reactor designs.

FIN L1611-LOCA Pin Failure Source Term 


\section{CONTENTS \\ VOLUME 1}

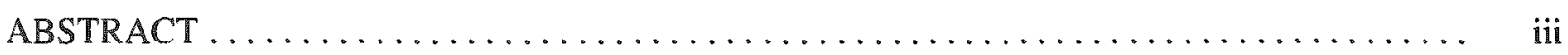

LIST OF TABLES $\ldots \ldots \ldots \ldots \ldots \ldots \ldots \ldots \ldots \ldots \ldots \ldots \ldots \ldots \ldots \ldots \ldots$

LIST OF FIGURES $\ldots \ldots \ldots \ldots \ldots \ldots \ldots \ldots \ldots \ldots \ldots \ldots \ldots \ldots \ldots \ldots \ldots \ldots \ldots \ldots$

EXECUTIVE SUMMARY $\ldots \ldots \ldots \ldots \ldots \ldots \ldots \ldots \ldots \ldots \ldots \ldots \ldots \ldots \ldots \ldots \ldots \ldots \ldots$

FOREWORD $\ldots \ldots \ldots \ldots \ldots \ldots \ldots \ldots \ldots \ldots \ldots \ldots \ldots \ldots \ldots \ldots \ldots \ldots \ldots \ldots \ldots \ldots \ldots \ldots$

ACKNOWLEDGMENTS $\ldots \ldots \ldots \ldots \ldots \ldots \ldots \ldots \ldots \ldots \ldots \ldots \ldots \ldots \ldots \ldots \ldots \ldots$

ACRONYMS $\ldots \ldots \ldots \ldots \ldots \ldots \ldots \ldots \ldots \ldots \ldots \ldots \ldots \ldots \ldots \ldots \ldots \ldots \ldots \ldots \ldots \ldots \ldots \ldots$

1. INTRODUCTION $\ldots \ldots \ldots \ldots \ldots \ldots \ldots \ldots \ldots \ldots \ldots \ldots \ldots \ldots \ldots \ldots \ldots$

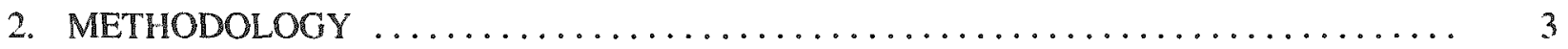

3. SOFTWARE DEVELOPMENT $\ldots \ldots \ldots \ldots \ldots \ldots \ldots \ldots \ldots \ldots \ldots \ldots \ldots \ldots \ldots \ldots \ldots$

4. THERMAL-HYDRAULIC MODEL DEVELOPMENT $\ldots \ldots \ldots \ldots \ldots \ldots \ldots \ldots$

4.1 Oconee SCDAP/RELAP5/MOD3 Model Development $\ldots \ldots \ldots \ldots \ldots \ldots \ldots \ldots \ldots$

4.2 Seabrook SCDAP/RELAP5/MOD3 Model Development $\ldots \ldots \ldots \ldots \ldots \ldots \ldots \ldots \ldots$

4.3 TRAC-PF1/MOD1 Model Development $\ldots \ldots \ldots \ldots \ldots \ldots \ldots \ldots \ldots \ldots \ldots \ldots \ldots \ldots \ldots$

5. FUEL PERFORMANCE MODEL DEVELOPMENT $\ldots \ldots \ldots \ldots \ldots \ldots \ldots \ldots \ldots \ldots$

5.1 FRAPCON-2 and FRAP-T6 Fuel Performance Model Development .......... 23

5.2 SCDAP Fuel Performance Model Development $\ldots \ldots \ldots \ldots \ldots \ldots \ldots \ldots \ldots \ldots \ldots \ldots \ldots$

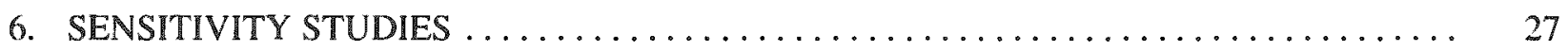

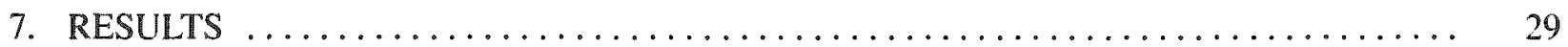

7.1 Results Generated Using SCDAP/RELAP5/MOD $3 \ldots \ldots \ldots \ldots \ldots \ldots \ldots \ldots$

7.1.1 SCDAP/RELAP5/MOD3 Calculations ....................... 29

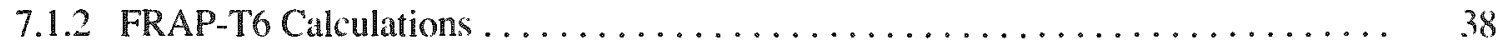

7.1.3 Comparison of SCDAP/RELAP5/MOD3 and FRAP-T6 Fuel Pin

7.2 Results Generated Using TRAC-PFI/MOD $1 \ldots \ldots \ldots \ldots \ldots \ldots \ldots \ldots \ldots$

7.2.1 Oconee Model ....................................... 49

7.2 .2 Seabrook Model ...................................... 50 


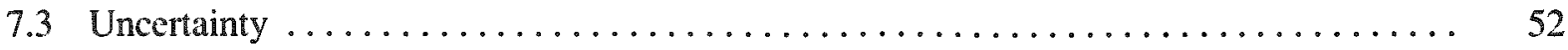

8. TECHNICAL FINDINGS $\ldots \ldots \ldots \ldots \ldots \ldots \ldots \ldots \ldots \ldots \ldots \ldots \ldots \ldots \ldots \ldots \ldots$

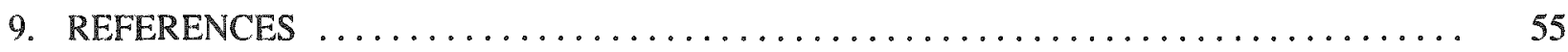

Appendix A-Quality Control $\ldots \ldots \ldots \ldots \ldots \ldots \ldots \ldots \ldots \ldots \ldots \ldots \ldots \ldots \ldots \ldots \ldots \ldots$

Appendix B-Preliminary LOCA PIN Failure Analysis Results Using the Surry Plant

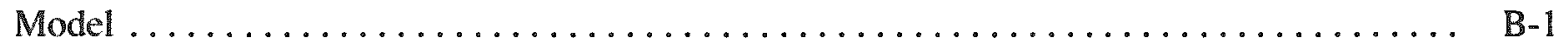

Appendix $\mathrm{C}-$ Conversion of FRAPCON-2 and FRAP-T6 to Fortran-77 ...........

Appendix D-Development of the FRAPR5 and TRAC2FRAP Interface Codes $\ldots \ldots \ldots \ldots$ D-1

Appendix E-SCDAP/RELAP5/MOD3 Input Deck Preparation and Results $\ldots \ldots \ldots \ldots \ldots$ E-1

Appendix $\mathrm{F}$-Core Power Distribution Calculation for Oconee $\ldots \ldots \ldots \ldots \ldots \ldots \ldots \ldots$ F-I

Appendix $\mathrm{G}-$ Core Power Distribution Calculation for Seabrook $\ldots \ldots \ldots \ldots \ldots \ldots \ldots$

Appendix H-TRAC-PF1/MOD1 Input Deck Preparation and Results $\ldots \ldots \ldots \ldots \ldots \ldots \ldots$

Appendix I-FRAPCON-2 Input Deck Preparation and Results $\ldots \ldots \ldots \ldots \ldots \ldots \ldots \ldots$

Appendix $\mathrm{J}-$ FRAP-T6 Input Deck Preparation and Results $\ldots \ldots \ldots \ldots \ldots \ldots \ldots \ldots \ldots$

\section{VOLUME 2}

Appendix $\mathrm{K}$-Plots for the Timing Analysis of PWR Fuel Pin Failures for Oconee $\ldots \ldots \ldots \ldots$

Appendix L-Plots for the Timing Analysis of PWR Fuel Pin Failures for Seabrook ....... L-1 


\section{LIST OF TABLES}

ES-1. FRAP-T6-calculated hot fuel pin failure time ( $s$ ) and location as a function of burnup and peaking factor for a complete, double-ended, offset-shear LOCA for

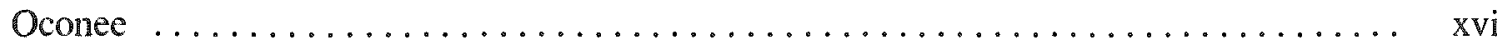

ES-2. FRAP-T6-calculated hot fuel pin failure time ( $\$$ ) and location as a function of burnup and peaking factor for a complete, double-ended. offset-shear LOCA for Seabrook

ES-3. FRAP-T6-calculated fuel pin failure time (s) for the Oconee 100\% DBA case using thermal-hydraulic conditions generated by TRAC-PFI/MOD $1 \ldots \ldots \ldots \ldots \ldots$ xvii

ES-4. FRAP-T6-calculated fuel pin failure time (s) for the Seabrook 100\% DBA case using thermal-hydraulic conditions generated by TRAC-PFI/MOD $1 \ldots \ldots \ldots \ldots \ldots$. $\ldots$ ii

ES-5. Timing summary for worst-case LOCA runs using highest burnup and peaking

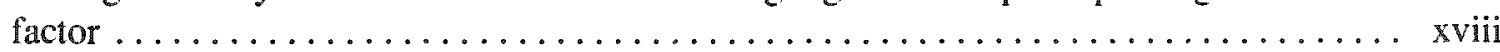

1. Containment isolation trip setpoints for Oconee $\ldots \ldots \ldots \ldots \ldots \ldots \ldots \ldots \ldots$

2. Containment isolation trip setpoints for Seabrook $\ldots \ldots \ldots \ldots \ldots \ldots \ldots \ldots \ldots \ldots$

3. Summary of fuel design characteristics $\ldots \ldots \ldots \ldots \ldots \ldots \ldots \ldots \ldots \ldots \ldots \ldots \ldots \ldots$

4. SCDAP/RELAP5/MOD3 results (in seconds) for Oconee and Seabrook . . . . . . . . . 29

5. $\quad$ FRAP-T6-calculated hot fuel pin failure time ( $s$ ) and location as a function of burnup and peaking factor for the Oconee $100 \%$ DBA without ECCS (Case 1) ........

6. FRAP-T6-calculated hot fuel pin failure time (s) and location as a function of burnup and peaking factor for the Oconee $90 \%$ DBA without ECCS (Case 2) ........

7. FRAP-T6-calculated hot fuel pin failure time ( $(\mathrm{s})$ and location as a function of burnup and peaking factor for the Oconee $75 \%$ DBA without ECCS (Case 3) ........

8. FRAP-T6-calculated hot fuel pin failure time $(\$)$ and location as a function of burnup and peaking factor for the Oconee $50 \%$ DBA without ECCS (Case 4) .........

9. FRAP-T6-calculated hot fuel pin failure time ( $s$ ) and location as a function of burnup and peaking factor for the Oconee $100 \%$ DBA without ECCS and with

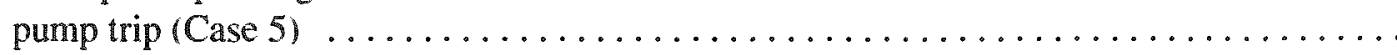

10. FRAP-T6-calculated hot fuel pin failure time (s) and location as a function of burnup and peaking factor for the Oconee $100 \%$ DBA with ECCS on (Case 7) ........

11. FRAP-T6-calculated hot fuel pin failure time (s) and location as a function of burnup and peaking factor for the Oconee $100 \%$ DBA with pump trip and ECCS

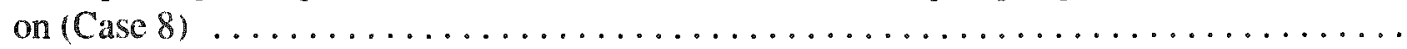

12. FRAP-T6-calculated hot fuel pin failure time ( $s$ ) and location as a function of burnup and peaking factor for the Oconee 6 in. break without ECCS (Case 6 ) 
13. FRAP-T6-calculated hot fuel pin failure time ( $\mathrm{s}$ ) and location as a function of burnup and peaking factor for the Oconee 6 in. break with ECCS (Case 9) .........

14. FRAP-T6-calculated hot fuel pin failure time (s) and location as a function of burnup and peaking factor for the Seabrook 100\% DBA without ECCS (Case 1) .......

15. FRAP-T6-calculated hot fuel pin failure time (s) and location as a function of burnup and peaking factor for the Seabrook 90\% DBA without ECCS (Case 2) .......

16. FRAP-T6-calculated hot fuel pin failure time (s) and location as a function of burnup and peaking factor for the Seabrook $75 \%$ DBA without ECCS (Case 3) .......

17. FRAP-T6-calculated hot fuel pin failure time (s) and location as a function of burnup and peaking factor for the Seabrook 50\% DBA without ECCS (Case 4) .......

18. FRAP-T6-calculated hot fuel pin failure time (s) and location as a function of burnup and peaking factor for the Seabrook 100\% DBA without ECCS and with

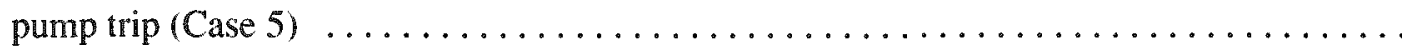

19. FRAP-T6-calculated hot fuel pin failure time (s) and location as a function of burnup and peaking factor for the Seabrook 100\% DBA with ECCS on (Case 7) ......

20. FRAP-T6-calculated hot fuel pin failure time $(\mathrm{s})$ and location as a function of burnup and peaking factor for the Seabrook $100 \%$ DBA with pump trip and ECCS

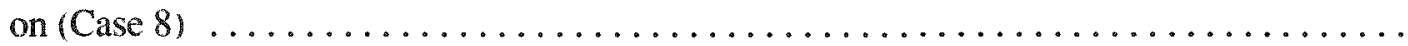

21. FRAP-T6-calculated hot fuel pin failure time (s) and location as a function of burnup and peaking factor for the Seabrook 6-in. break without ECCS (Case 6) .......

22. FRAP-T6-calculated hol fuel pin failure time ( $s$ ) and location as a function of burnup and peaking factor for the Seabrook 6-in. break with ECCS (Case 9) ..........

23. FRAP-T6-calculated hot fuel pin failure lime (s) and location as a function of burnup and peaking factor for the Oconee $100 \%$ DBA without ECCS and with

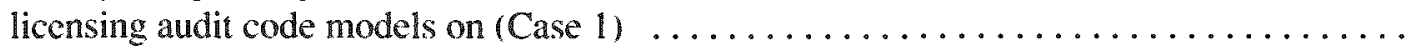

24. FRAP-T6-calculated hot fuel pin failure time (s) and location as a function of bumup and peaking factor for the Seabrook $100 \%$ DBA without ECCS and with

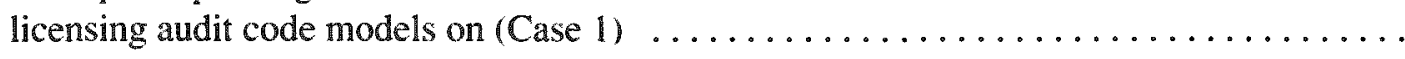

25. A comparison of FRAP-T6 fuel pin failure time (s) using TRAC-PF1/MOD1 and SCDAP/RELAP5/MOD3 thermal-hydraulic data for the $100 \%$ DBA case for Seabrook

26. A comparison of FRAP-T6 fuel pin failure time (s) using TRAC-PF1/MOD1 and SCDAP/RELAP5/MOD3 thermal-hydraulic data for the 100\% DBA case for

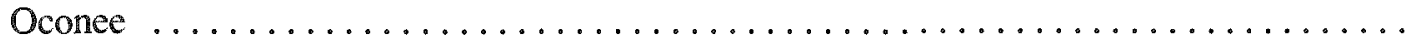

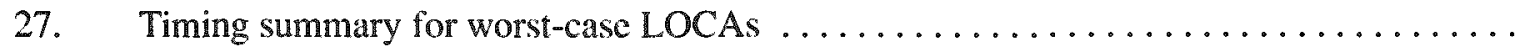




\section{LIST OF FIGURES}

ES-1. Flow chart of methodology using SCDAP/RELAP5/MOD3 thermal-hydraulic data ..... xii

ES-2. Flow chart of methodology using TRAC-PFI/MOD1 thermal-hydraulic data $\ldots \ldots \ldots$ xiii

1. Flow chart of methodology using SCDAP/RELAP5/MOD3 thermal-hydraulic

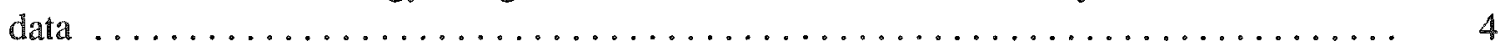

2. Flow chart of methodology using TRAC-PF1/MOD1 thermal-hydraulic data $\ldots \ldots \ldots \ldots$

3. SCDAP/RELAP5/MOD3 nodalization diagram of the Oconee reactor ........... 9

4. SCDAP/RELAP5/MOD3 nodalization diagram of the Oconee primary loop A $\ldots \ldots \ldots 10$

5. SCDAP/RELAP5/MOD3 nodalization diagram of the Oconee primary loop B ..... 11

6. SCDAP/RELAP5/MOD3 nodalization diagram of the Oconee pressurizer $\ldots \ldots \ldots \ldots$

7. SCDAP/RELAP5/MOD3 nodalization diagram of the Seabrook plant $\ldots \ldots \ldots \ldots$

8. SCDAP/RELAP5/MOD3 nodalization diagram of the Seabrook reactor $\ldots \ldots \ldots \ldots$

9. TRAC-PF1/MOD1 nodalization diagram of the Oconee system model $\ldots \ldots \ldots \ldots$

10. TRAC-PF1/MOD1 vessel axial nodalization for the Oconee model $\ldots \ldots \ldots \ldots \ldots$

11. TRAC-PF1/MOD1 nodalization diagram of the Seabrook system model ......... 20

12. TRAC-PF1/MODI vessel axial nodalization for the Seabrook model $\ldots \ldots \ldots \ldots \ldots$

13. TRAC-PF1/MOD1 loop nodalization for the Seabrook model $\ldots \ldots \ldots \ldots \ldots \ldots \ldots$

14. Steady-state radial temperature profile for the Oconee 5-GWd/MTU fuel pin at the

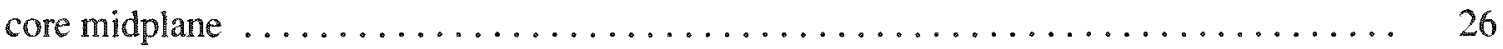

15. Steady-state radial temperature profile for the Oconee $55-\mathrm{GWd} / \mathrm{MTU}$ fuel pin at

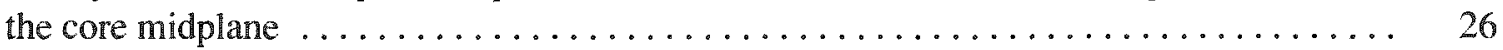

16. SCDAP/RELAP5/MOD3-calculated core thermal power for the Oconee

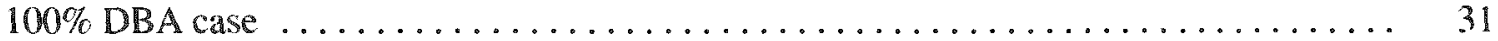

17. SCDAP/RELAP5/MOD3-calculated collapsed reactor water level for the Oconee

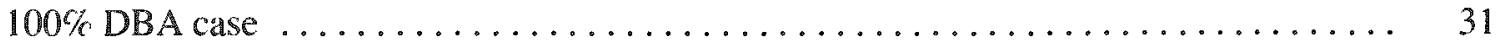

18. SCDAP/RELAP5/MOD3-calculated reactor upper head and pressurizer pressure

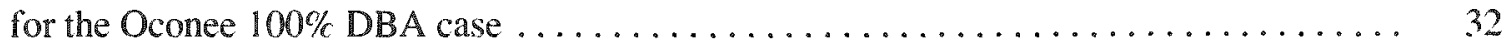

19. SCDAP/RELAP5/MOD3-calculated containment pressure for the Oconee

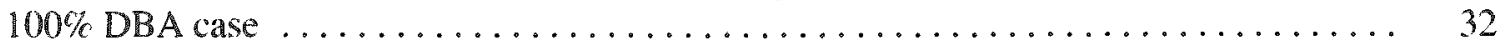

20. SCDAP/RELAP5/MOD3-calculated total break flow for the Oconee $100 \%$ DBA

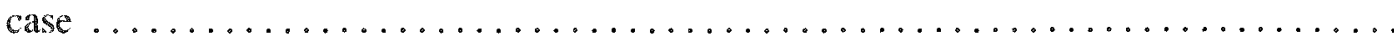


21. SCDAP/RELAP5/MOD3-calculated accumulator liquid volume for the Oconee

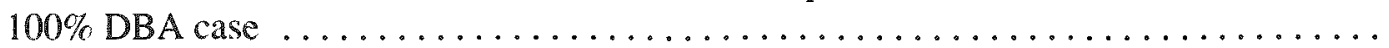

22. SCDAP/RELAP5/MOD3-calculated hot leg flow for the Oconee $100 \%$ DBA case ..... 34

23. SCDAP/RELAP5/MOD3-calculated cold leg flow for the Oconee $100 \%$ DBA

24. SCDAP/RELAP5/MOD3-calculated hot channel core flow for the Oconee

25. SCDAP/RELAP5/MOD3-calculated internal pin pressure for the Oconee $100 \%$ DBA case

26. SCDAP/RELAP5/MOD3-calculated fuel centerline temperature for the $55-\mathrm{GWd} / \mathrm{MTU}$ fuel pin for the Oconee $100 \%$ DBA case $\ldots . . \ldots \ldots \ldots \ldots \ldots$

27. SCDAP/RELAP5/MOD3-calculated cladding surface temperature for the $55-\mathrm{GWd}$ /MTU fuel pin for the Oconee $100 \%$ DBA case $\ldots \ldots \ldots \ldots \ldots \ldots \ldots$

28. SCDAP/RELAP5/MOD3-calculated cladding hoop strains for the 55-GWd/MTU

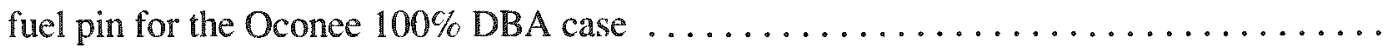

29. FRAP-T6-calculated internal pin pressure for the 55-GWd,MTU pin, peaking factor of 2.63 , Oconee $100 \%$ DBA case . . . . . . . . . . . . . . . . . . . .

30. FRAP-T6-calculated failure probability for the 55-GWd/MTU pin, peaking factor

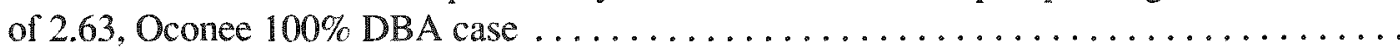

31. FRAP-T6-calculated cladding hoop strain for the 55-GWd/MTU pin, peaking factor of 2.63 , Oconee $100 \%$ DBA case . . . . . . . . . . . . . . . . . . . .

32. FRAP-T6-calculated cladding surface temperature for the 55-GWd/MTU pin,

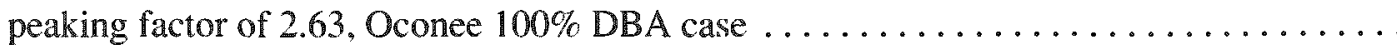

33. FRAP-T6-calculated fuel centerline temperature for the 55-GWd/MTU pin, peaking factor of 2.63, Oconee $100 \%$ DBA case $\ldots \ldots \ldots \ldots \ldots \ldots \ldots \ldots \ldots \ldots \ldots$

34. FRAP-T6-calculated oxide thickness for the 55-GWd/MTU pin, peaking factor of 2.63 , Oconee $100 \%$ DBA case . . . . . . . . . . . . . . . . . . . . . . . 
Appendix K

\section{Plots for the Timing Analysis of PWR} Fuel Pin Failures for Oconee 



\section{APPENDIX K \\ Plots FOR the Timing ANAlysis of PWR \\ Fuel Pin failures for OconeE}

Appendix $K$ contains the plotted results for the timing anlaysis of PWR fuel $p$ in failures for the Oconee reactor. Section $K-1$ contains the SCDAP/RELAP5/MOD3 plots of total core power, collapsed reactor water level, reactor upper head and pressurizer dome pressures, containment pressure, fission product release, internal pin pressures, fuel centerline temperatures, cladding surface temperatures, hoop strains, total break flow, accumulator flow, accumulator liquid volume, hot leg flows, cold leg flows, hot channel core flow, downcomer void fractions, mass error, time step size, and cpu time for the nine accident scenarios. Section K-2 contains the FRAP-T6 plots of failure probability, internal pin pressure, cladding hoop strain, cladding surface temperature, fuel centerline temperature, and oxide thickness for the nine accident scenarios. Section $K-3$ contains plots comparing TRAC-PF1/MODI and SCDAP/RELAP5/MOD3 results for the 100\% DBA case for the following variables: core thermal power, collapsed reactor water level, presurizer dome and reactor upper head pressures, total break flow, accumulator flow, accumulator liquid volume, hot leg flow, cold leg flow, hot channel core flow, downcomer void fractions, and time step size. Tables $k-1, k-2$, and $k-3$ provide a listing of the plot variables. 


\section{K-1. SCDAP/RELAP5/MOD3 PLOTTEd Results FOR OCONEE}

Table K-1. Description of SCDAP/RELAP5/MOD3 plot variables for Oconee.

\begin{tabular}{|c|c|}
\hline Variable & Description \\
\hline 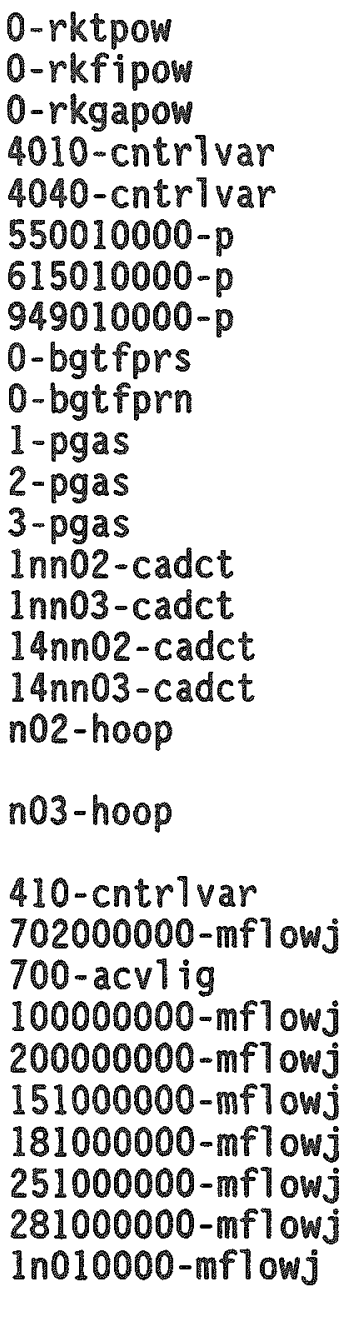 & 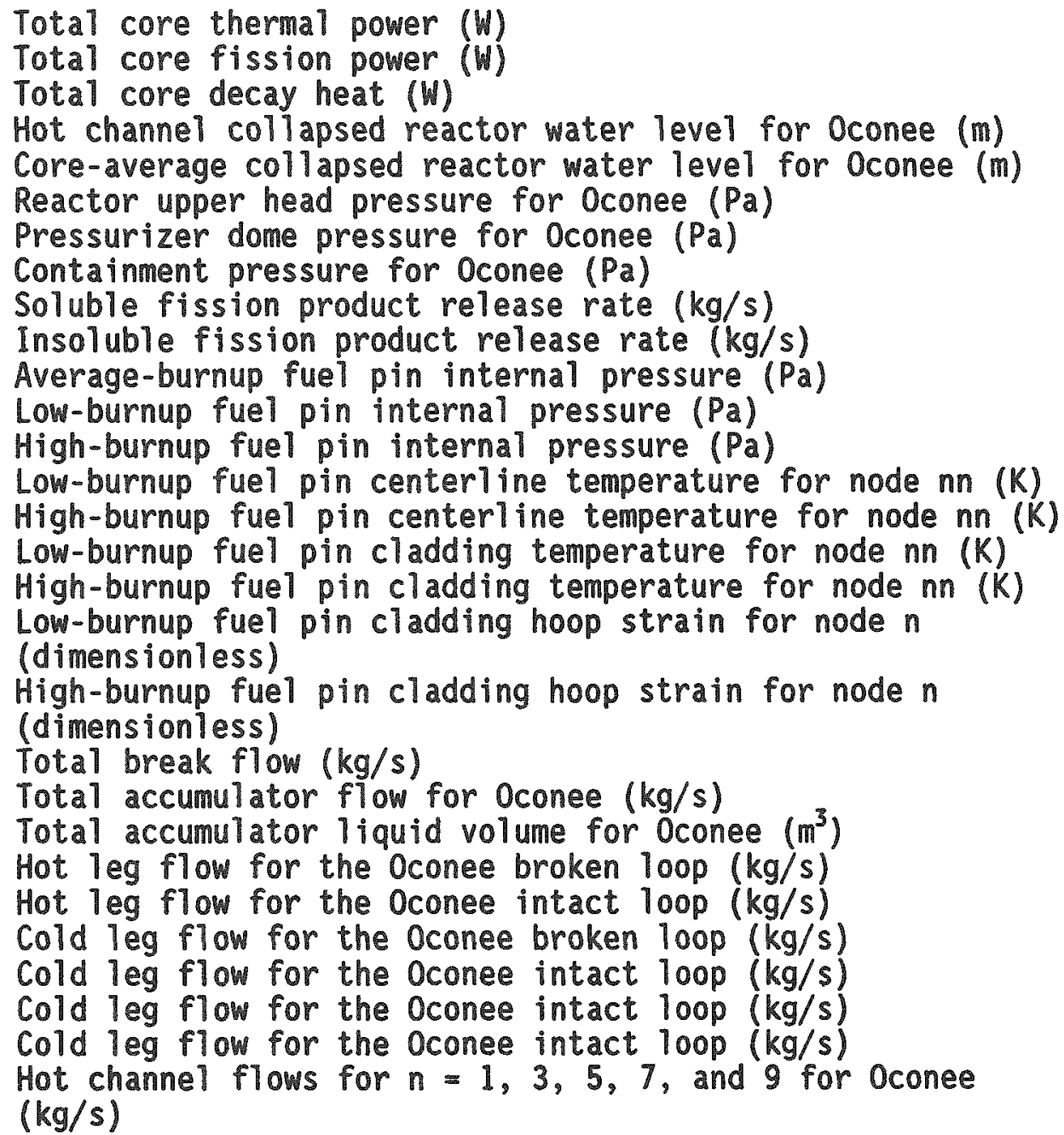 \\
\hline
\end{tabular}


B\&W (OCONEE) 100\% DBA LOCA PIN FAILLRE

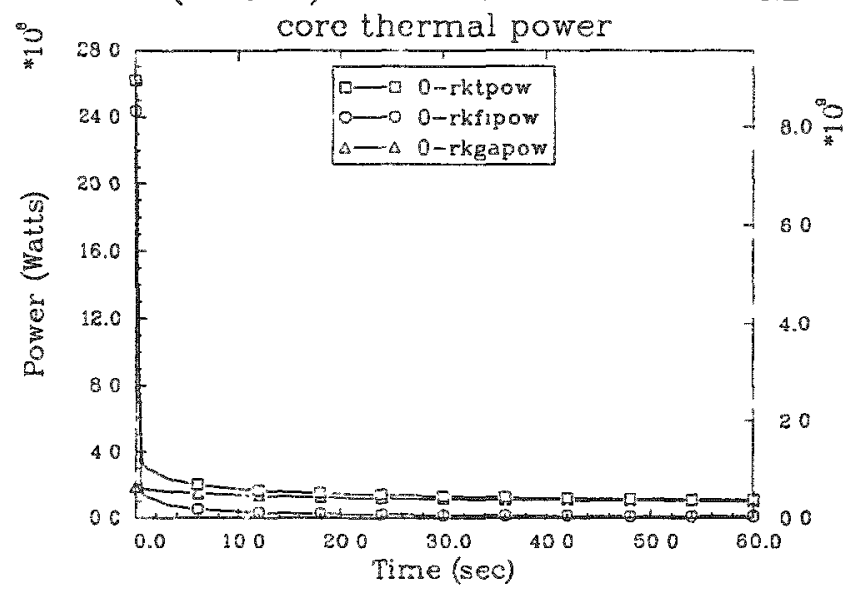

B\&W (OCONEE) 100\% DBA LOCA PIN FAILURE

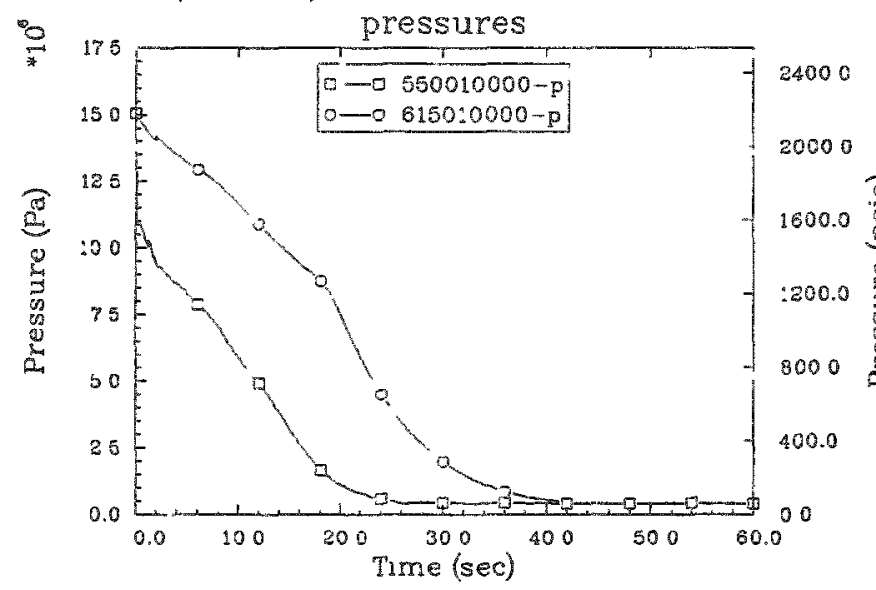

B\&W (OCONEE) 100\% DBA LOCA PIN FAILURE

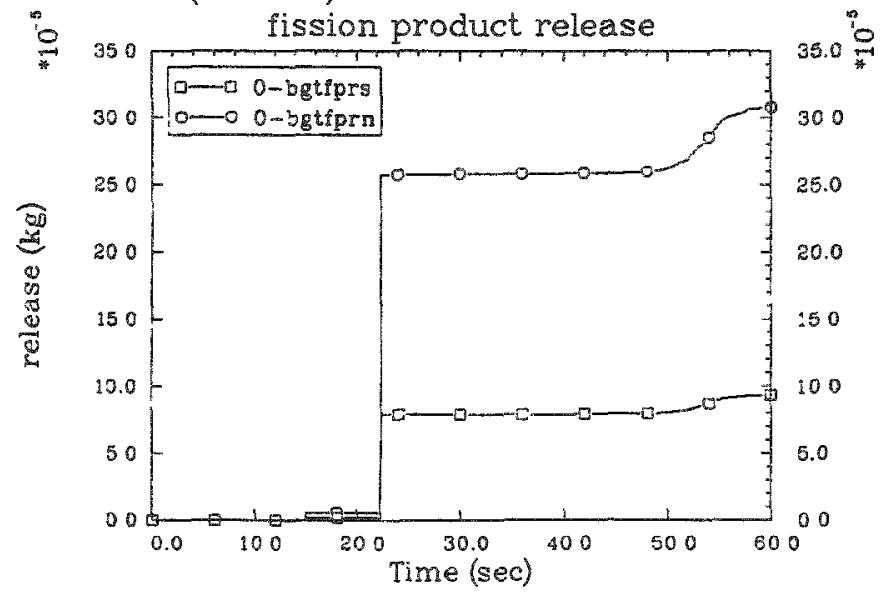

BSEW (OCONEE) 100\% DBA LOCA PIN FAILURE'

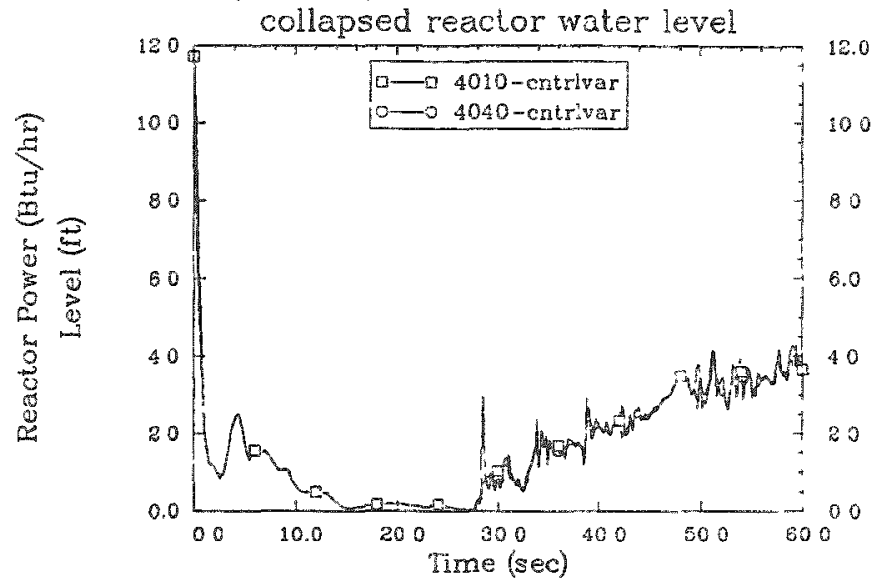

B\&W (OCONEE) 100\% DBA LOCA PIN FAILURE

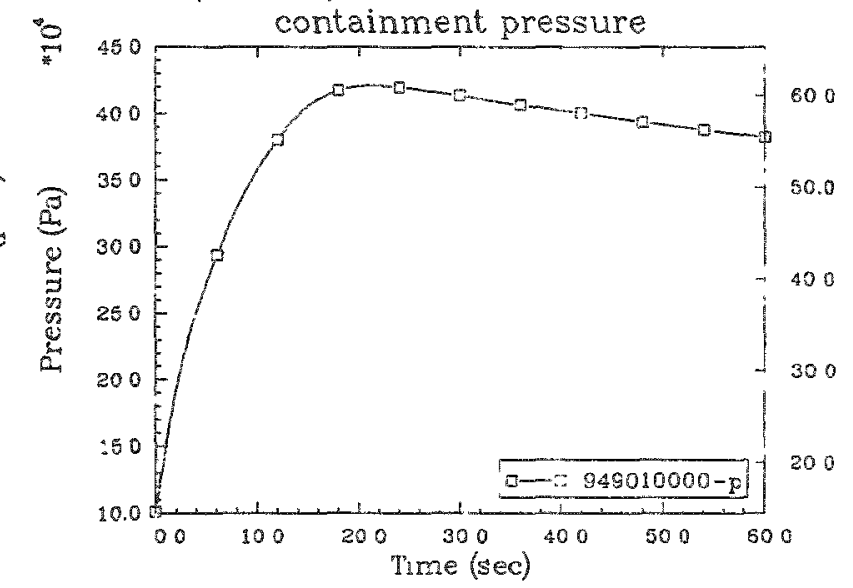

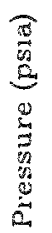

B\&W (OCONEE) 100\% DBA LOCA PIN FAILURE

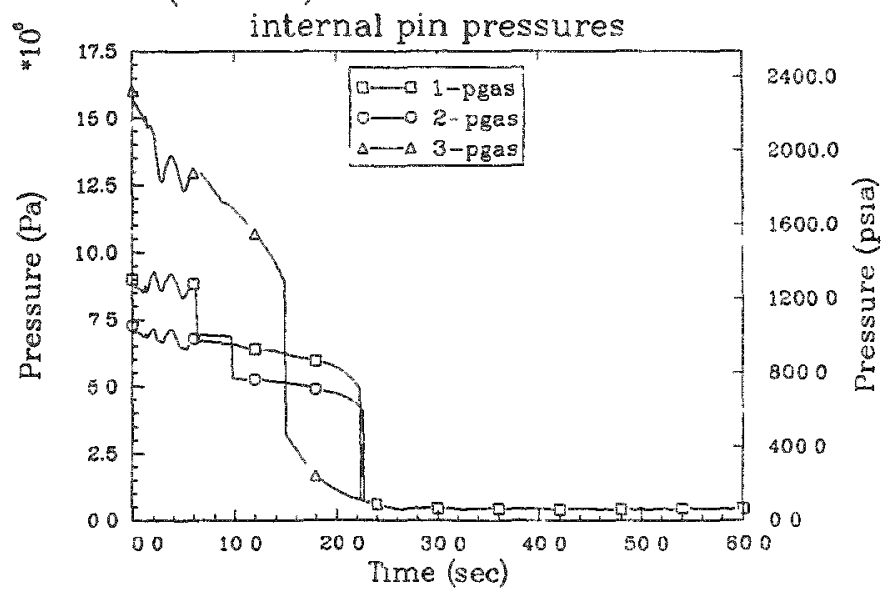


B\&W (OCONEE) 100\% DBA LOCA PIN FAILCRE

fuel centerline temperatures for 5 GWD/MTU pin

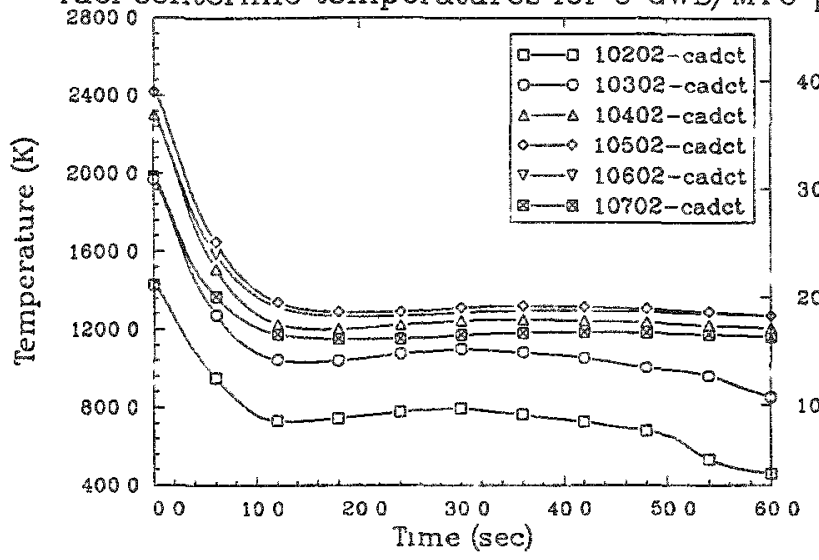

B\&W (OCONEE) 100\% DBA LOCA PIN FAILURE cladding surface temperatures for $5 \mathrm{GWD} / \mathrm{MTU}$ pin

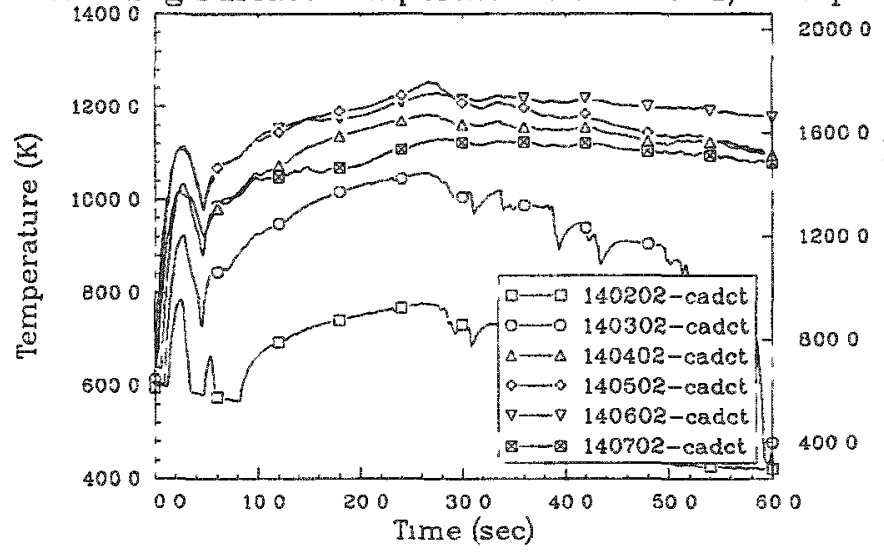

B\&W (OCONEE) 100\% DBA LOCA PIN FAILURE

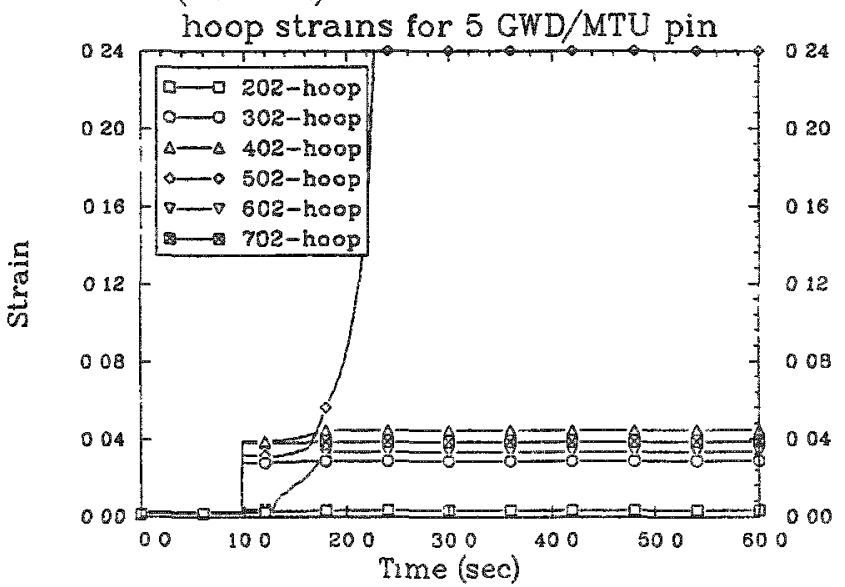

B\&W (OCONEE) 100\% DBA LOCA PIN FALUURE fuel centerhne temperatures for 55 GWD/MTU pin

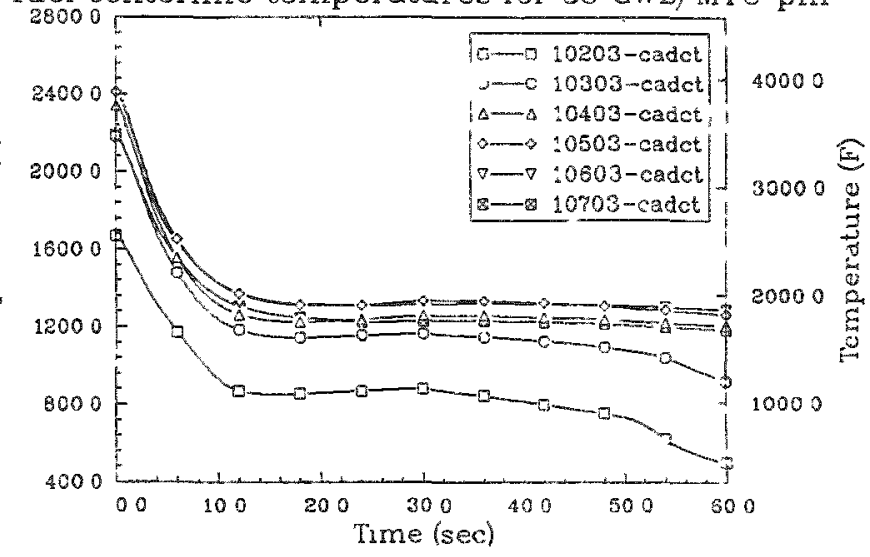

B8W (OCONEE) 100\% DBA LOCA PIN FAILURE cladding surface temperatures for $55 \mathrm{GWD} / \mathrm{MTU}$ pin $14000 \longrightarrow 20000$

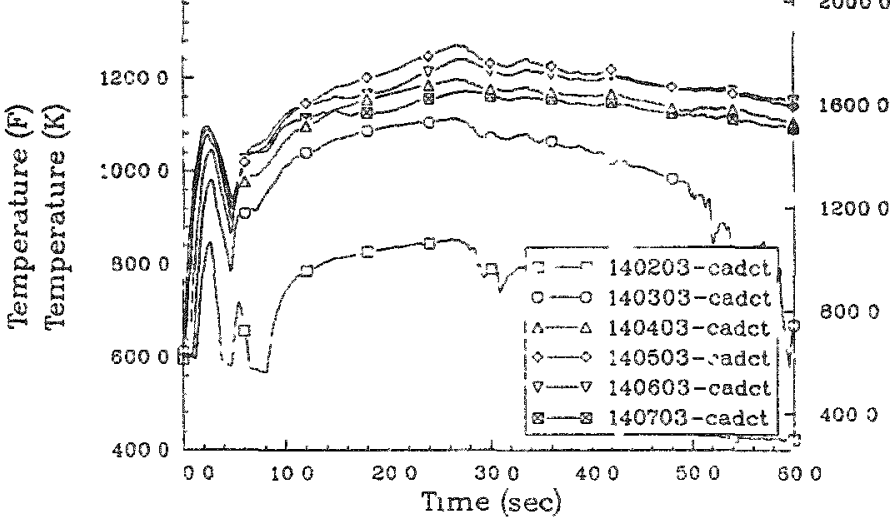

B\&W (OCONEE) 100\% DBA LOCA PIN FAILURE

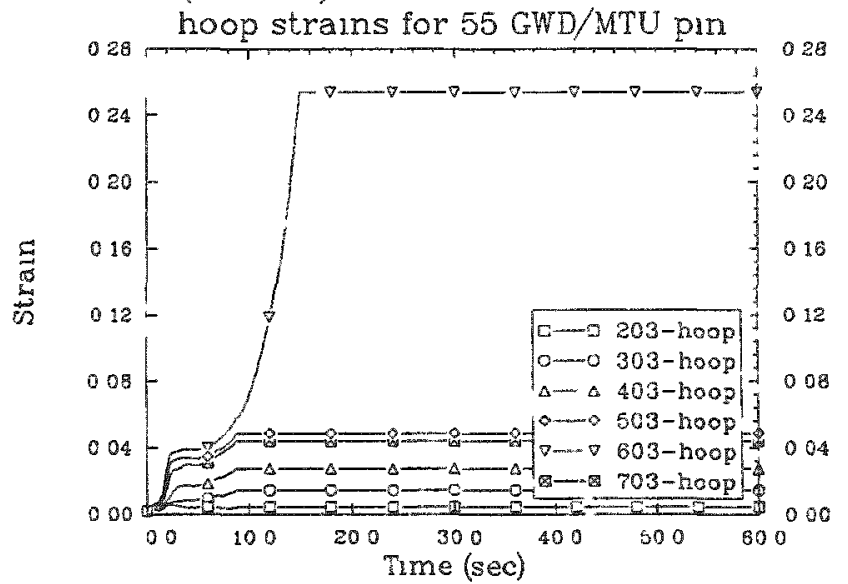


8\&W (OCONEE) 100\% DBA LOCA PIN FAILURE

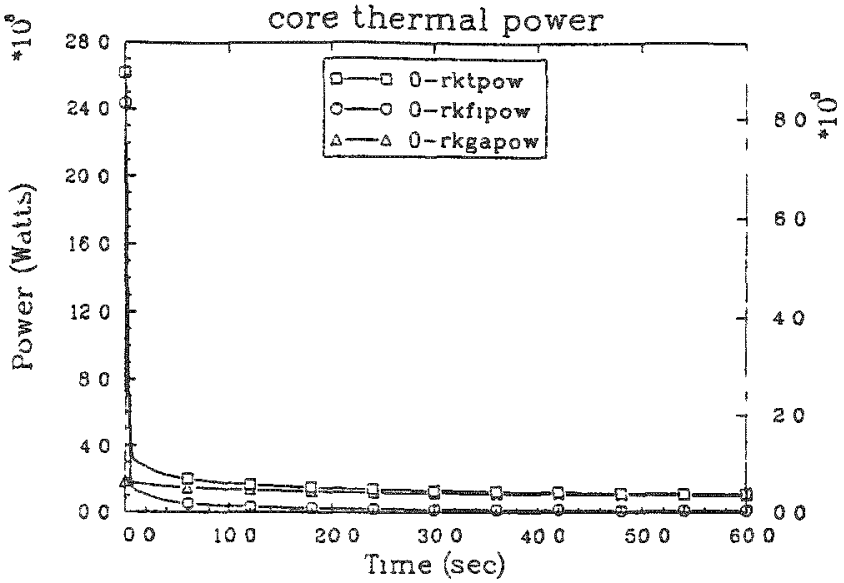

B\&W (OCONEE) 100\% DBA LOCA PIN FAILURE

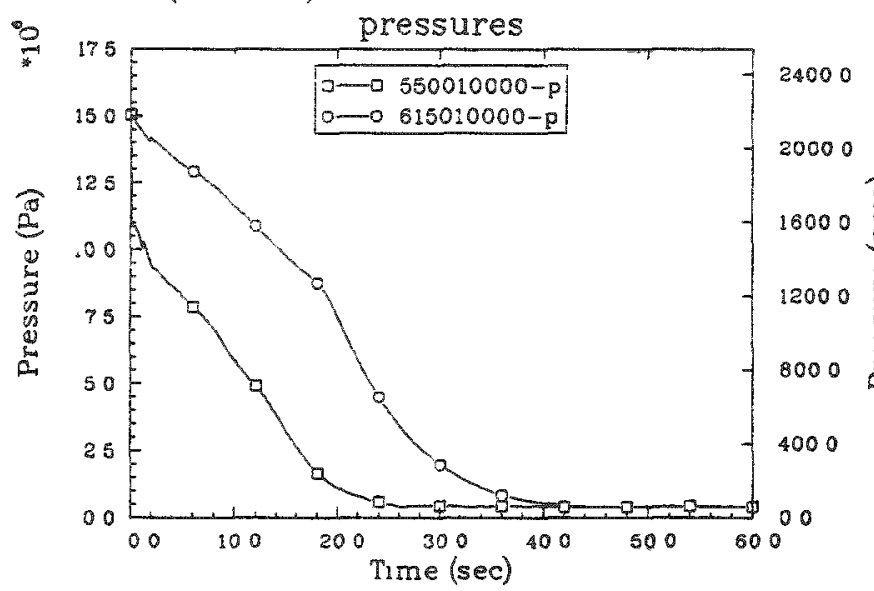

B\&W (OCONEE) 100\% DBA LOCA PIN FAILURE

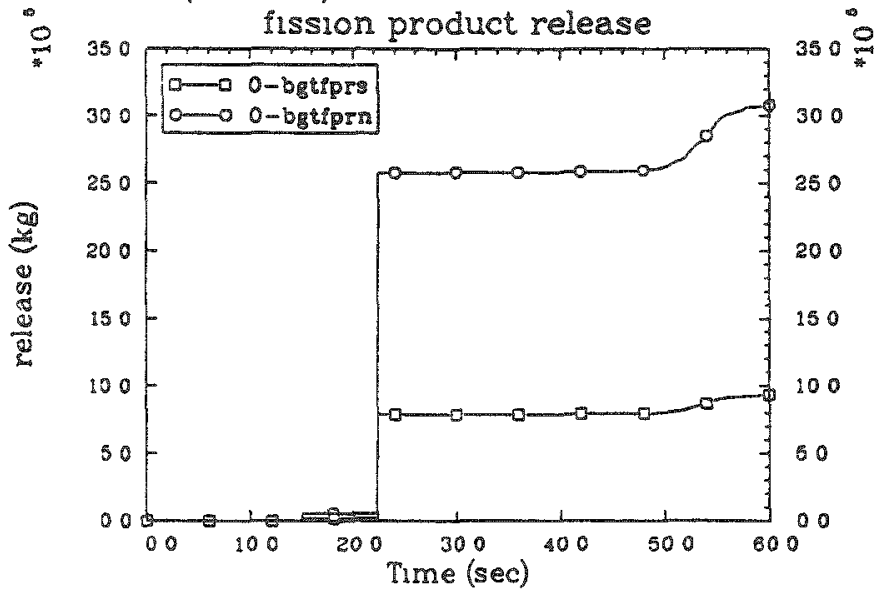

B\&W (OCONEE) 100\% DBA LOCA PIN FAILURE collapsed reactor water level

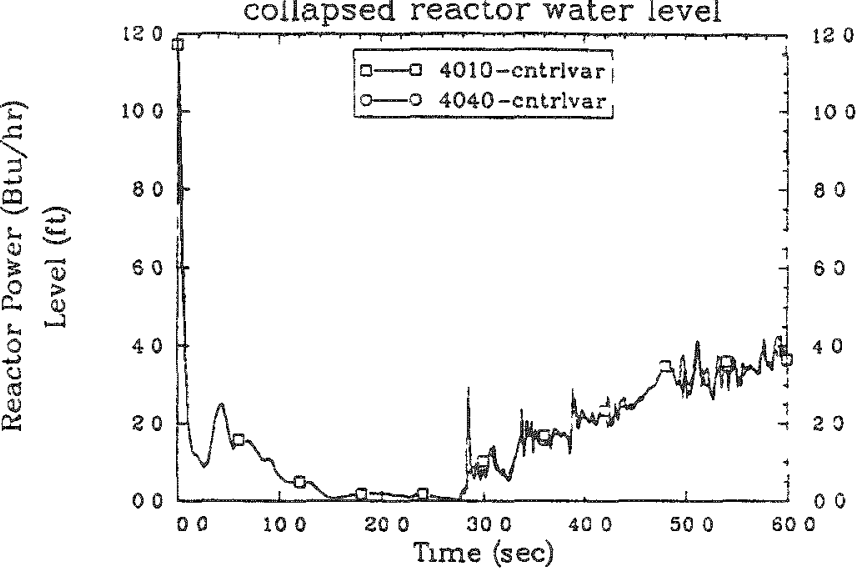

B\&W (OCONEE) 100\% DBA LOCA PIN FAILURE

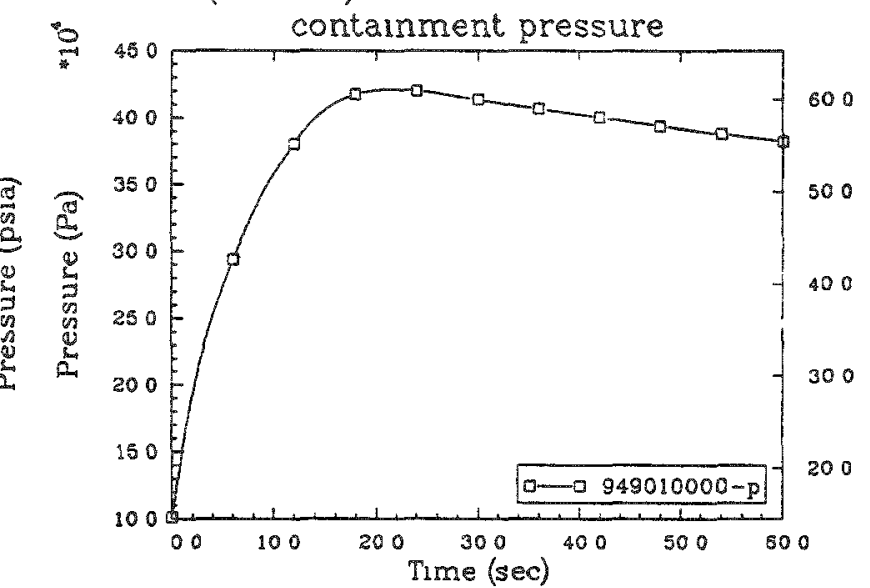

B\&W (OCONEE) 100\% DBA LOCA PIN FAILURE

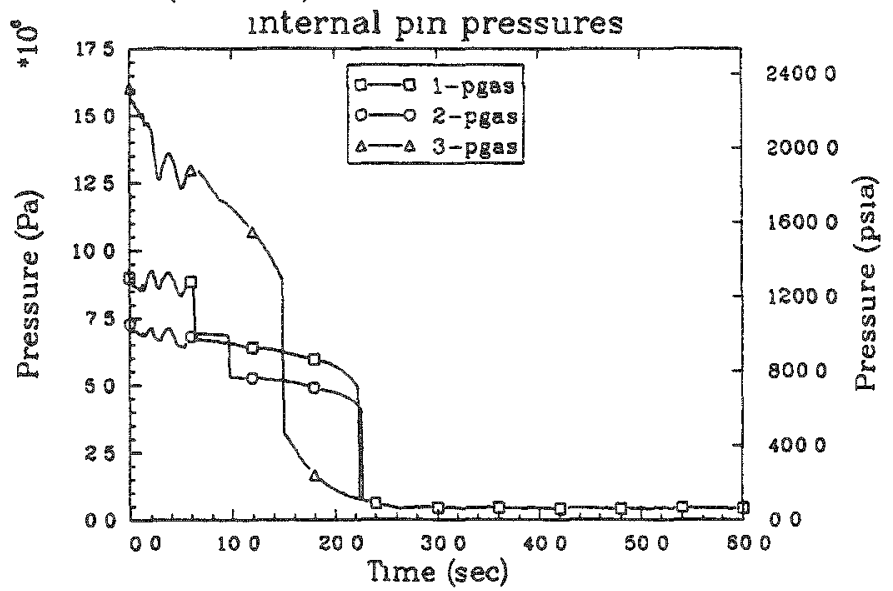


B\&W (OCONEE) 100\% DBA LOCA PIN FAILURE

fuel centerline temperatures for 5 GWD/MTU pin

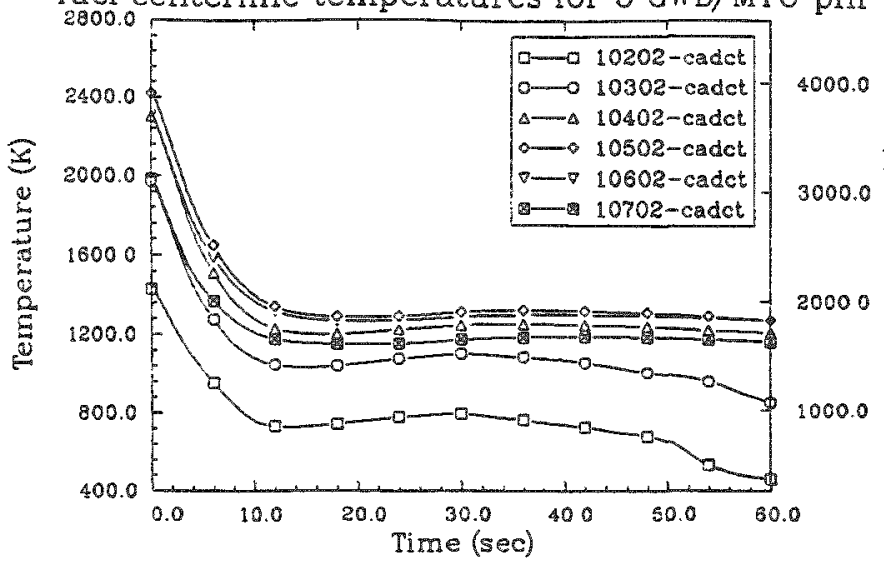

B8W (OCONEE) 100\% DBA LOCA PIN FAILURE

cladding surface temperatures for 5 GWD/MTU pin

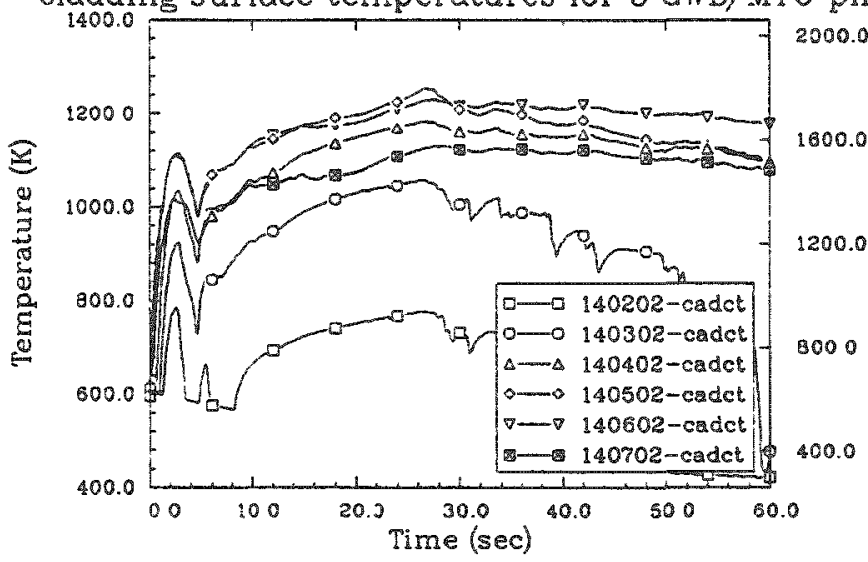

B\&W (OCONEE) 100\% DBA LOCA PIN FAILURE

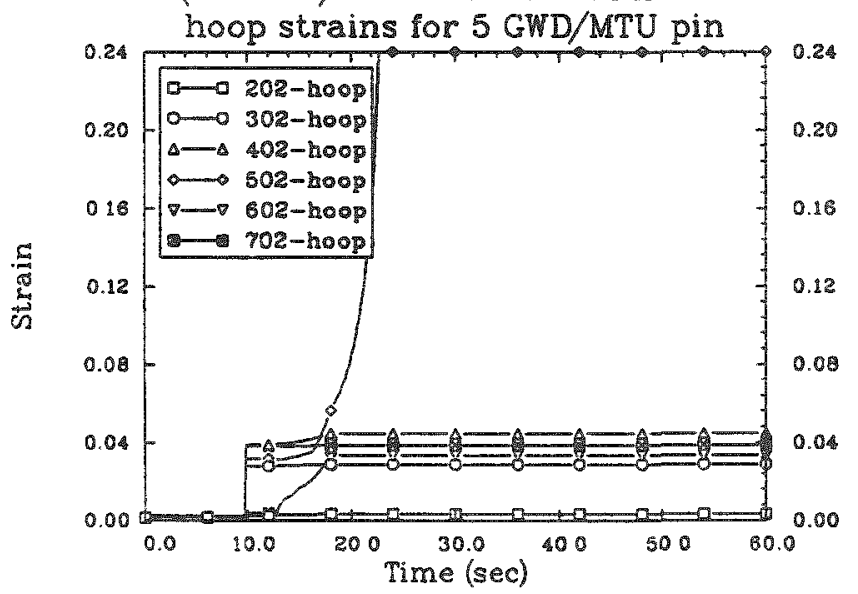

B\&W (OCONEE) 100\% DBA LOCA PIN FAILURE fuel centerline temperatures for 55 GWD/MTU pin

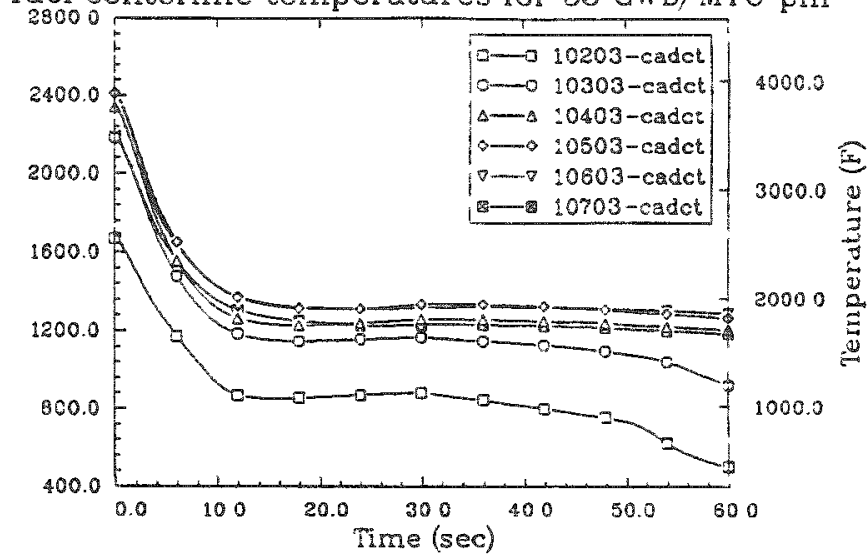

B\&W (OCONEE) 100\% DBA LOCA PIN FAILURE cladding surface temperatures for $55 \mathrm{GWD} / \mathrm{MTU}$ pin

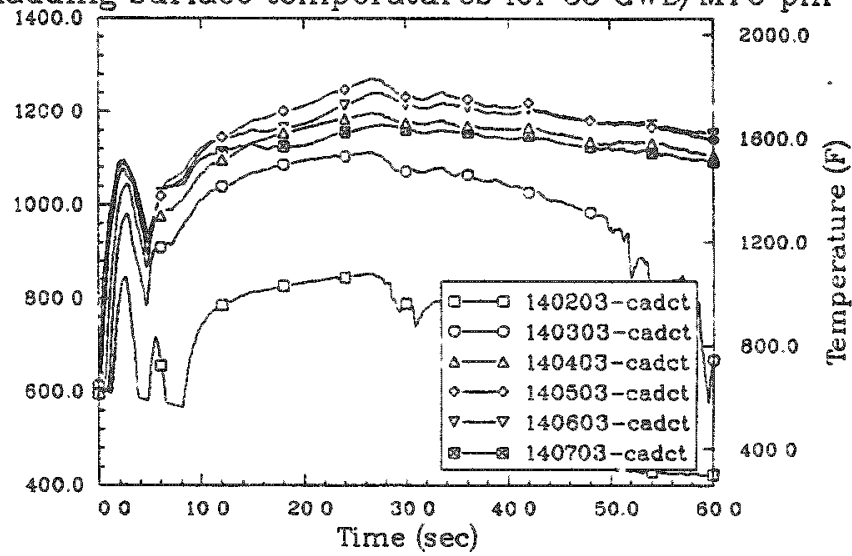

B\&W (OCONEE) 100\% DBA LOCA PIN FAILURE

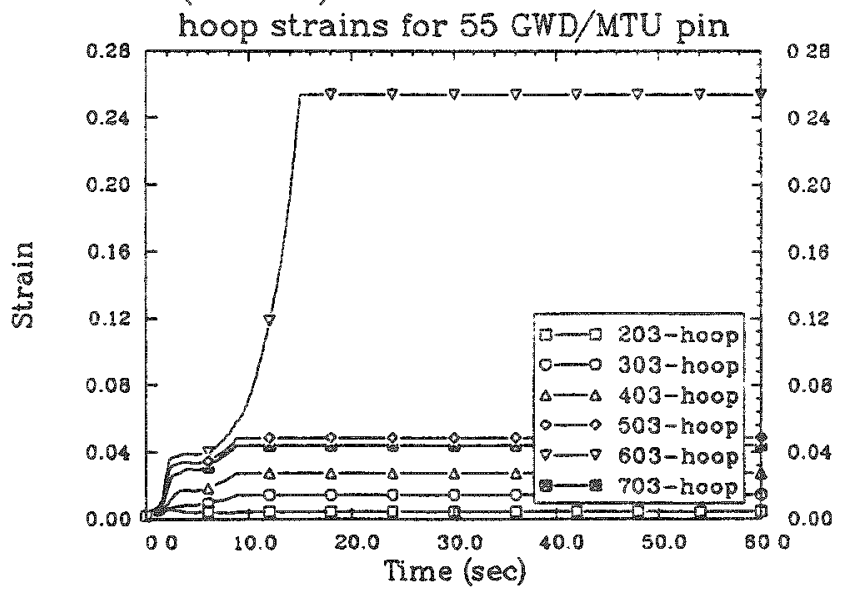


B\&I (OCONIT) 100\% DBA LOCA PIN FAILURE

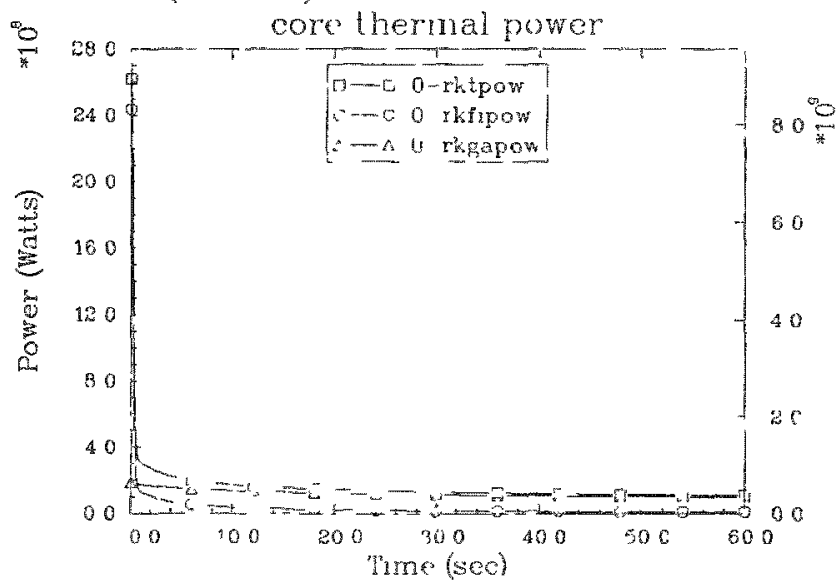

B\&W (OCON PF) 100\% DBA LOCA IIN FAILURE

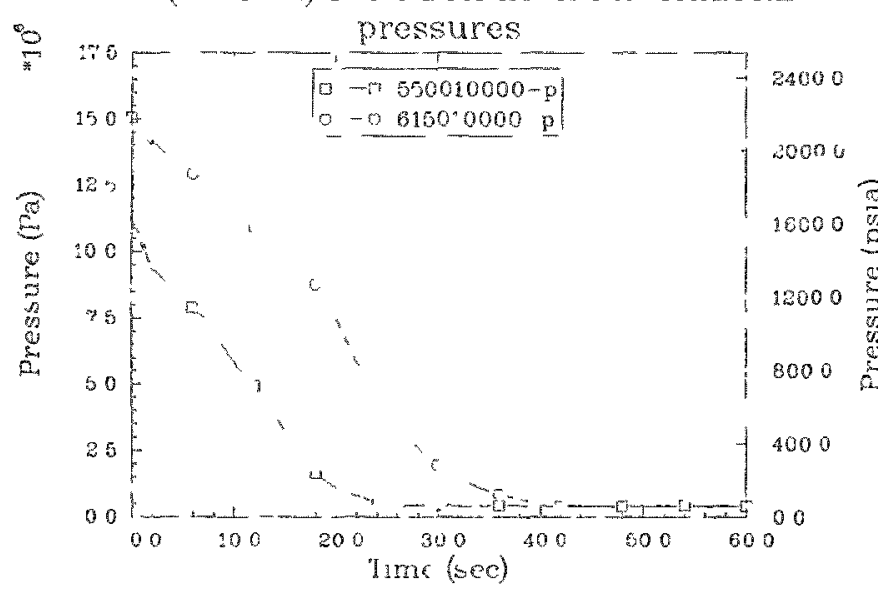

B\&W (OCONLE) 100\% DHA LUCA PIN FAILURE

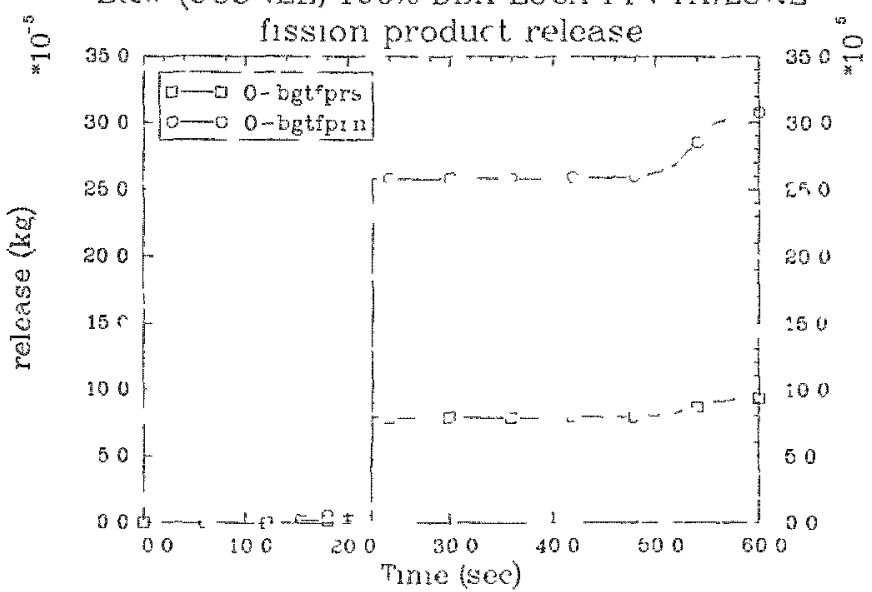

B\&W (OCONEE) 100\% DBA LOCA PIN FAILURE' collapsed reactor water level

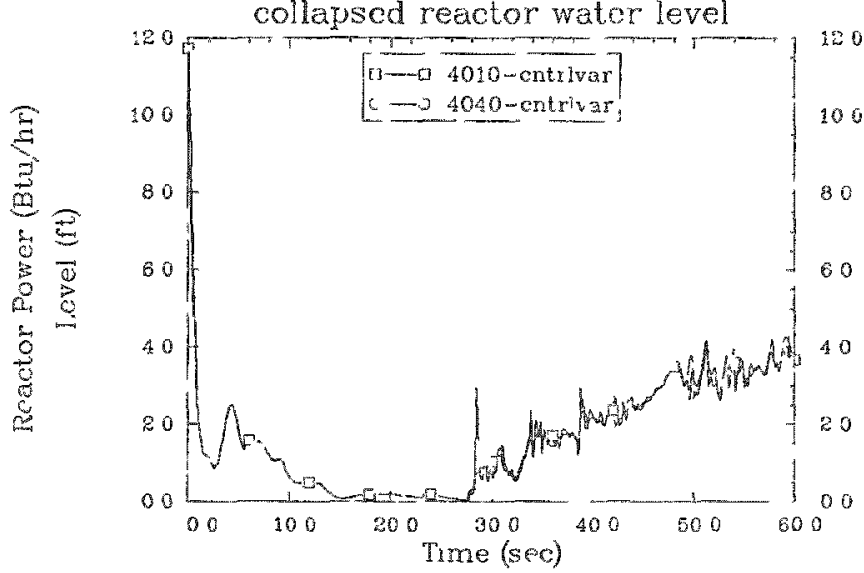

B\&W (OCONEE) 100\% DIBA LOCA PIN FAILURE

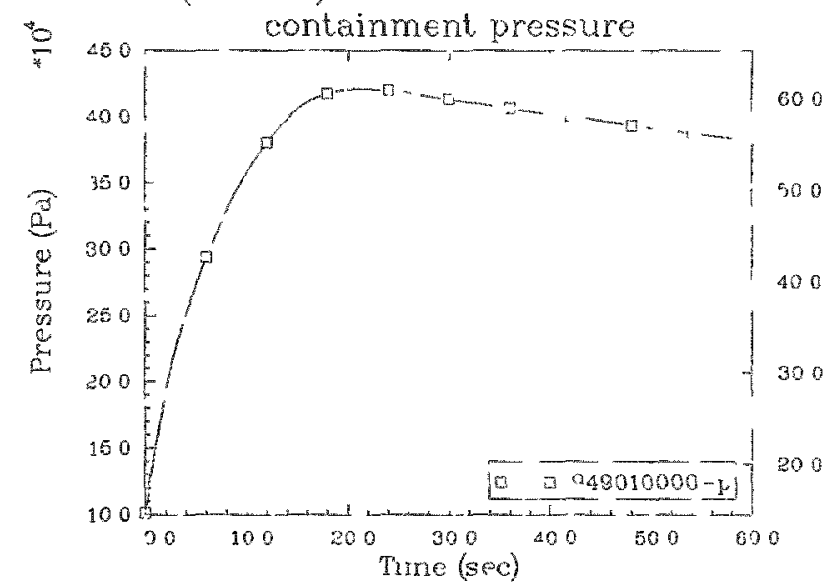

B\&W (OCONCE) 100\% DBA LOCA PIN IALLURE

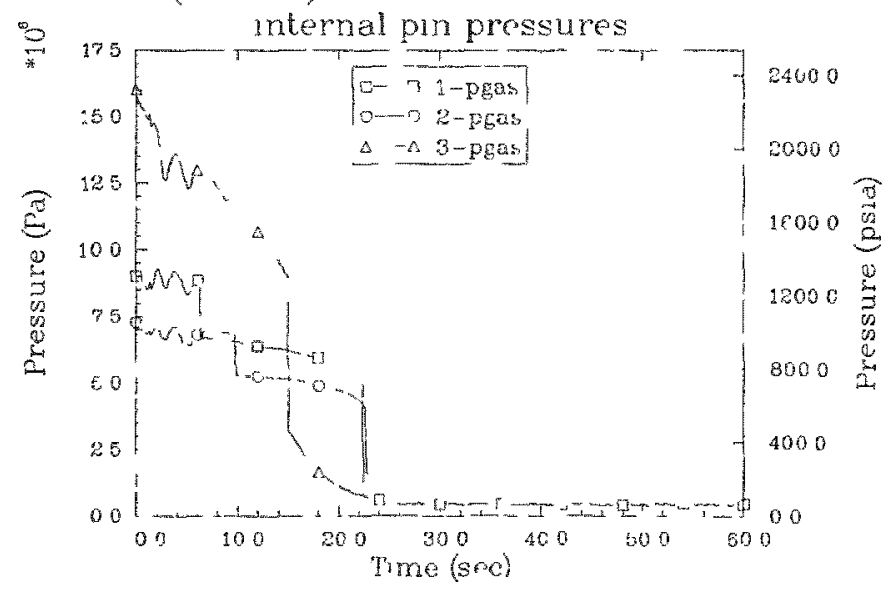


B\&W (OCONEE) 100\% DBA LOCA PIN FAIIURE

fuel conterline temperatures for 5 GWD/MTU pin

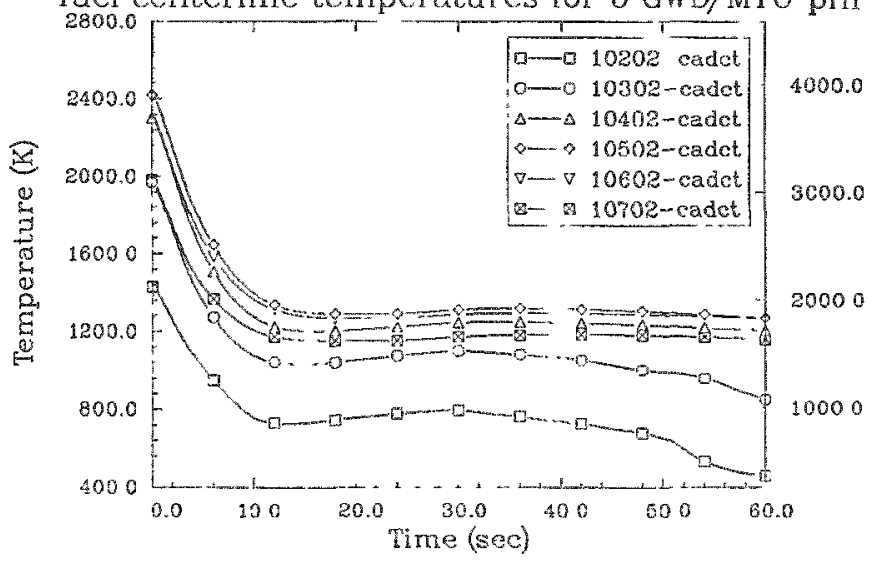

B\&W (OCONFF) 100\% DBA LOCA PIN FALLURF,

cladding surface temperatures for 5 GWD/MTU pin

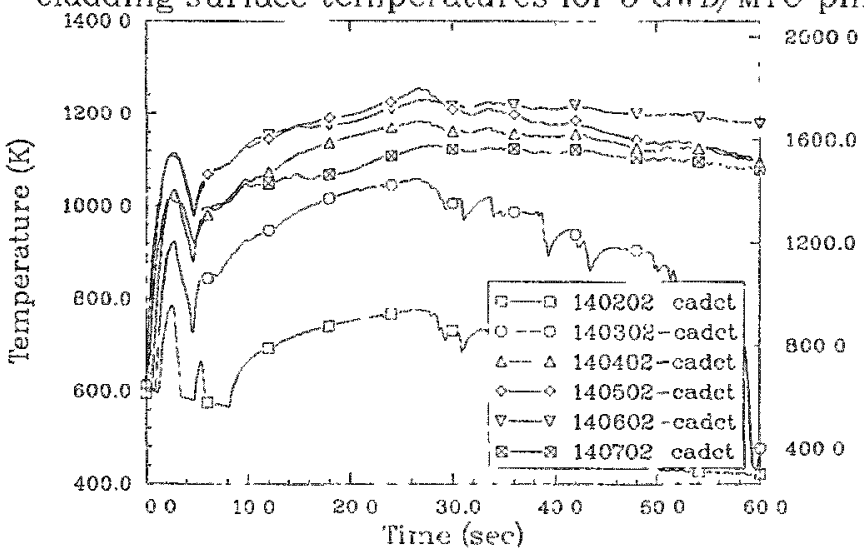

B\&W (OCONEE) 100\% DBA LOCA PIN FAIIUPE:

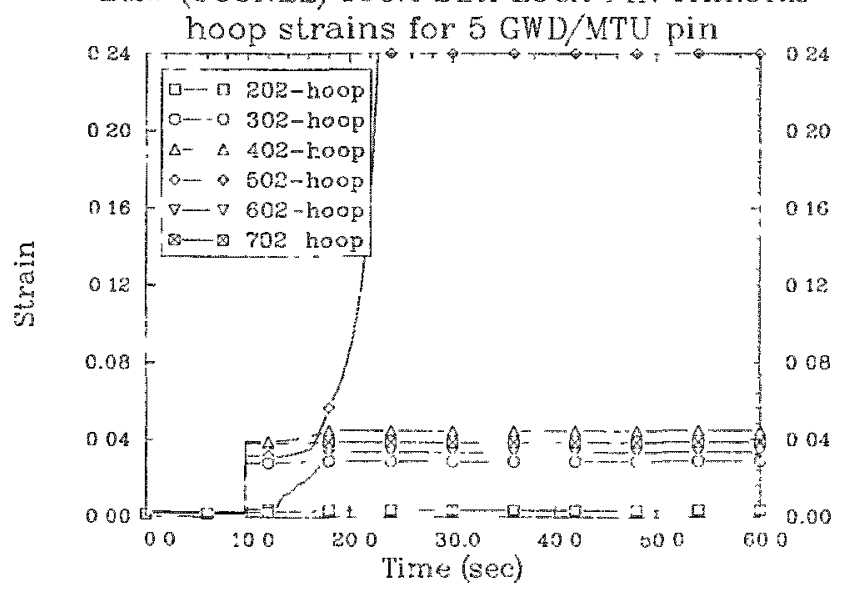

B\&W (OCONEE) 100\% DBA LOCA PIN FAILURE fuel centerline temperatures for $55 \mathrm{GWD} / \mathrm{MTU}$ pin

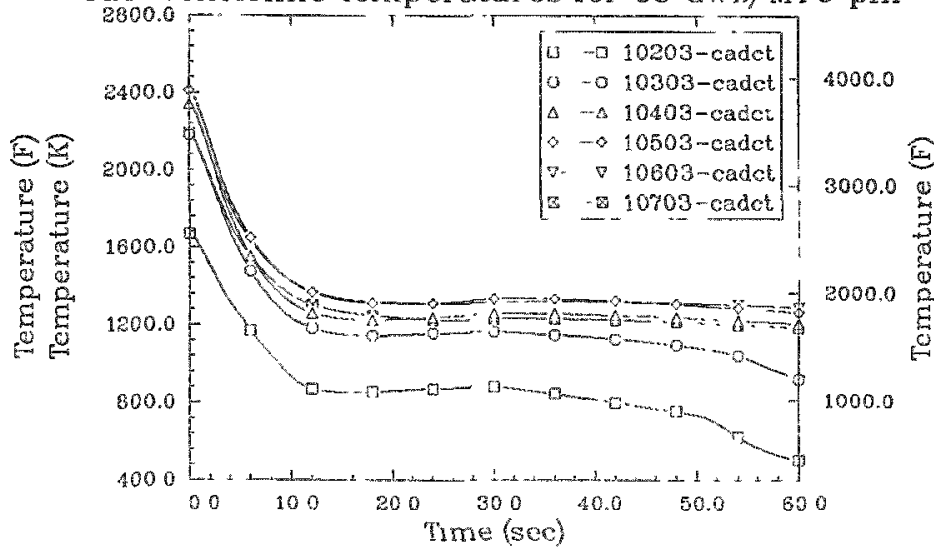

B\&W (OCONEE) 100\% DBA IOCA PIN FAILURE cladding surface tomperatures for 55 GWD/MTU pin

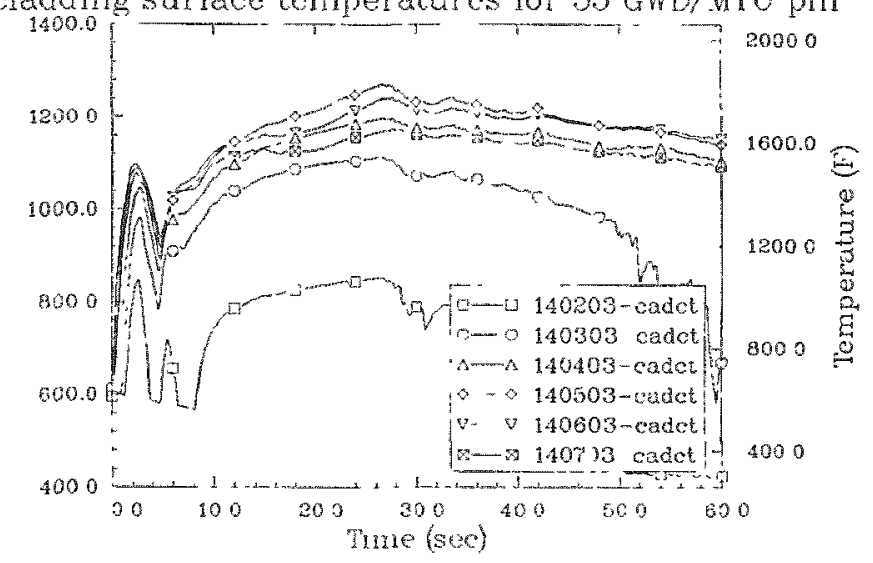

B\&W (OCONEE) 100\% DBA IOCA PIN FALUURE

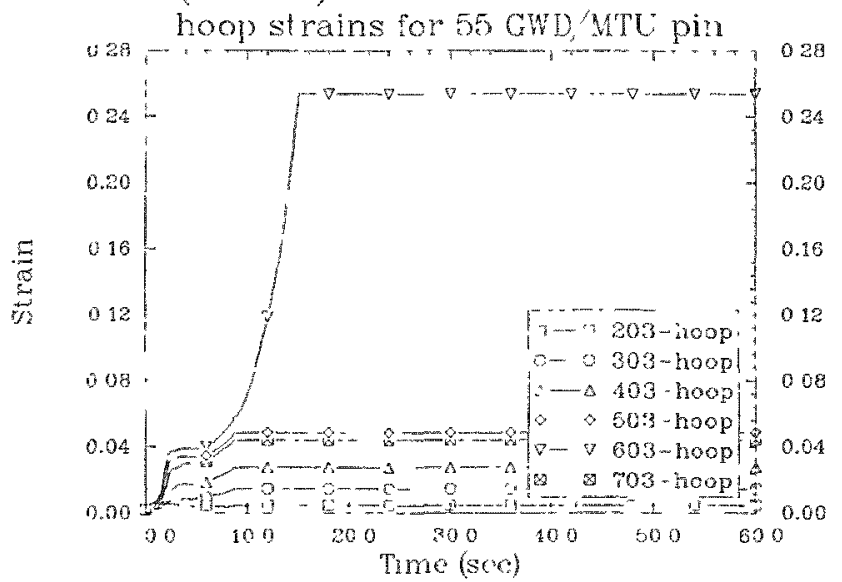



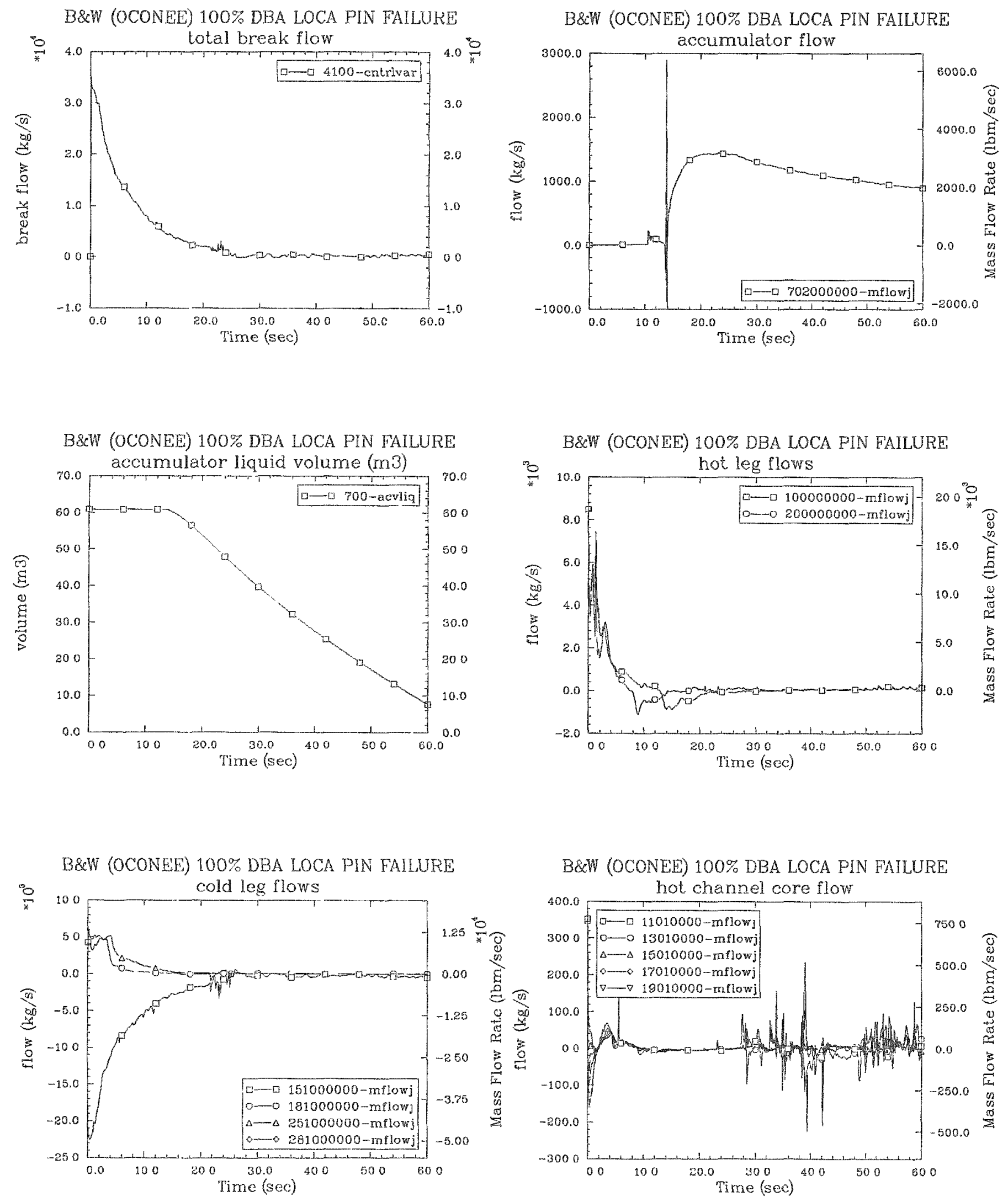
B\&W (OCONEE) 100\% DBA LOCA PIN FAILURE

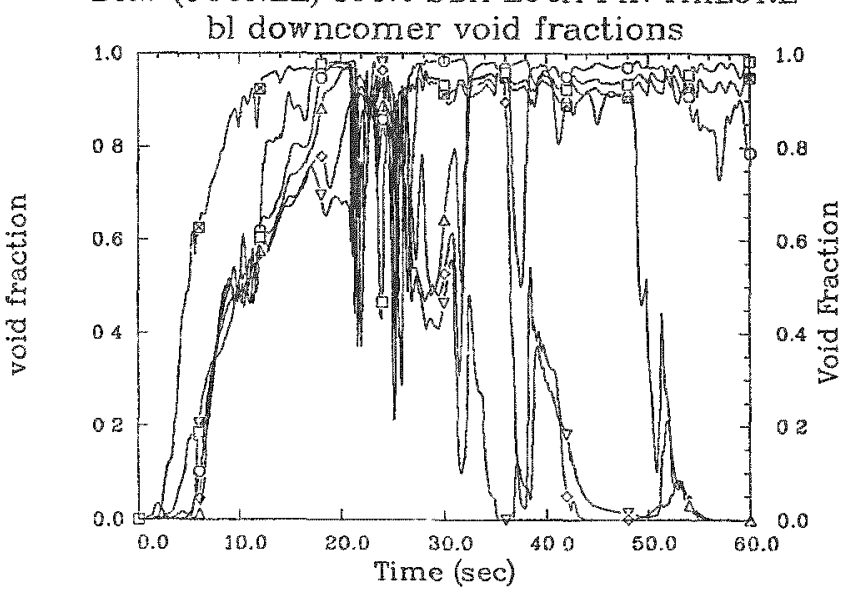

B\&W (OCONEE) 100\% DBA LOCA PIN FAILURE

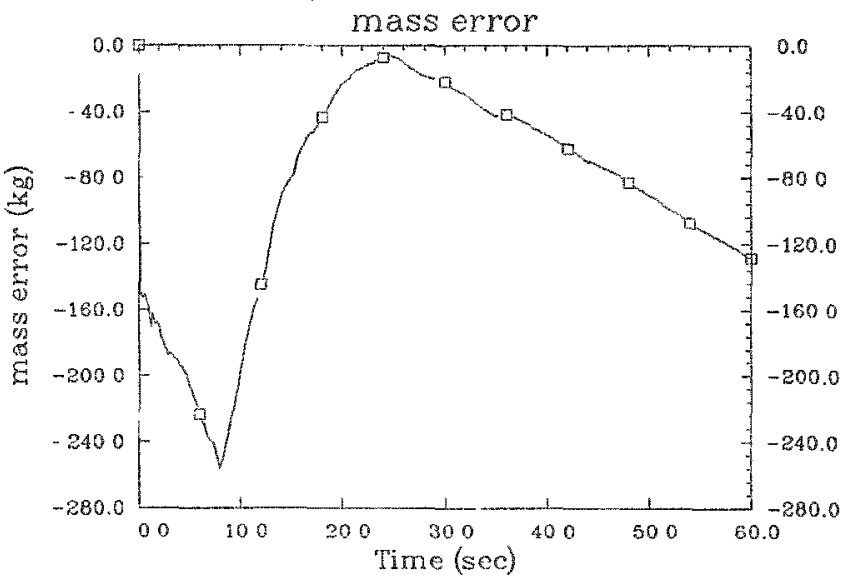

B\&W (OCONEE) 100\% DBA LOCA PIN FAILURE

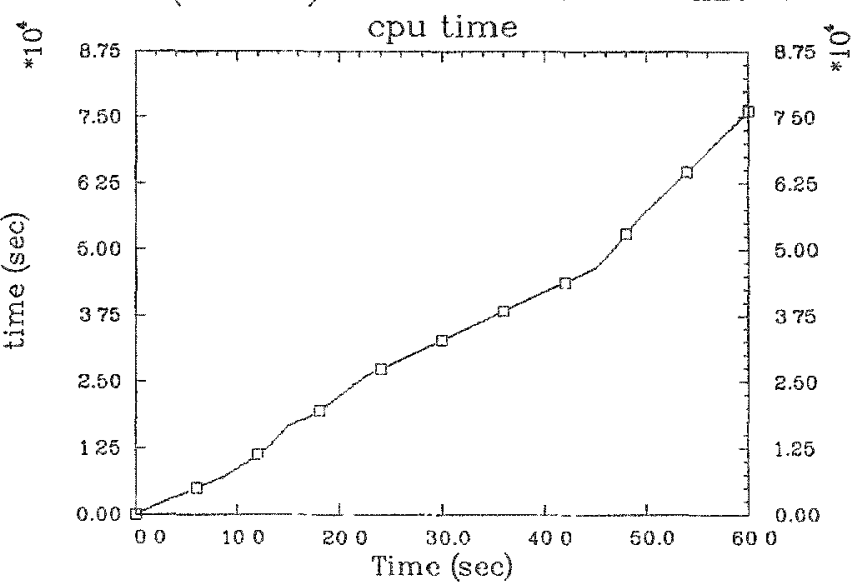

B\&W (OCONEE) 100\% DBA LOCA PIN FAILURE

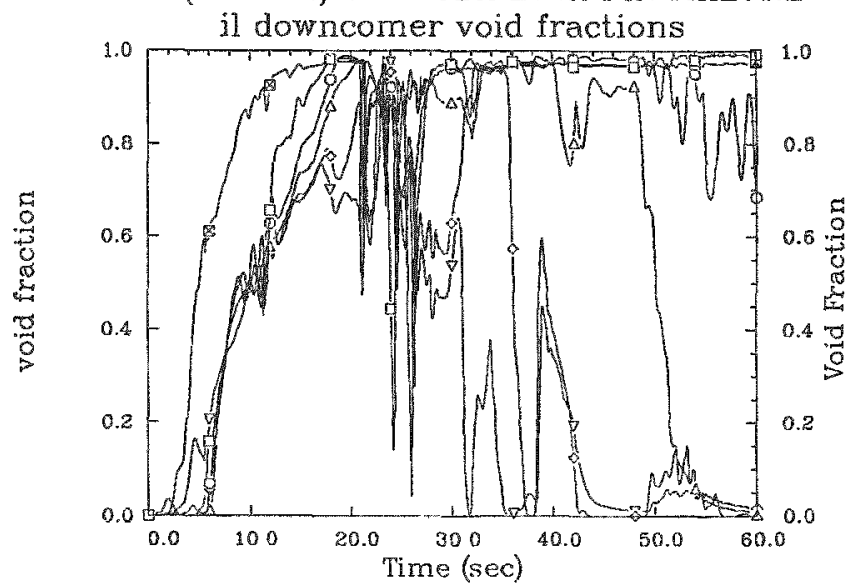

B\&W (OCONEE) 100\% DBA LOCA PIN FAILURE

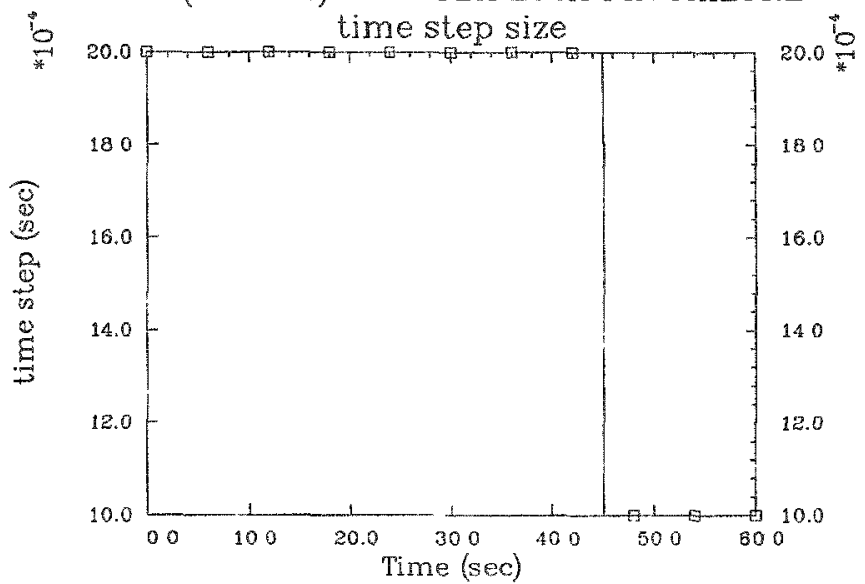


B\&W (OCONEE) 90\% DBA LOCA PIN FALUURE

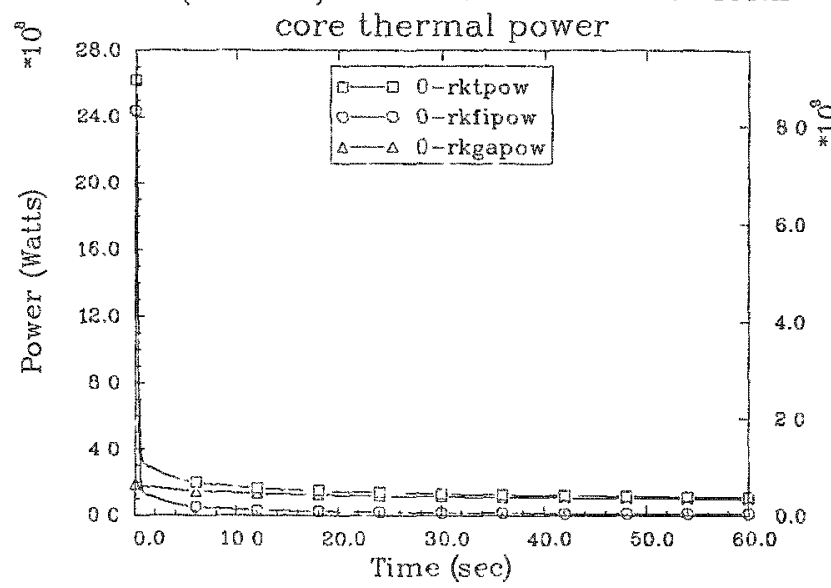

B\&W (OCONEE) 90\% DBA LOCA PIN FAILURE

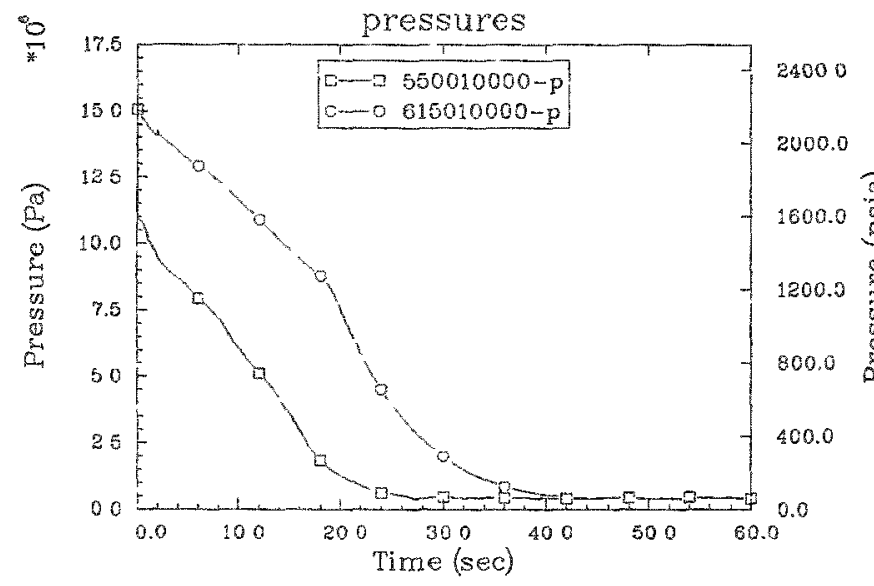

B\&W (OCONEE) 90\% DBA LOCA PIN FAILURE collapsed reactor water level

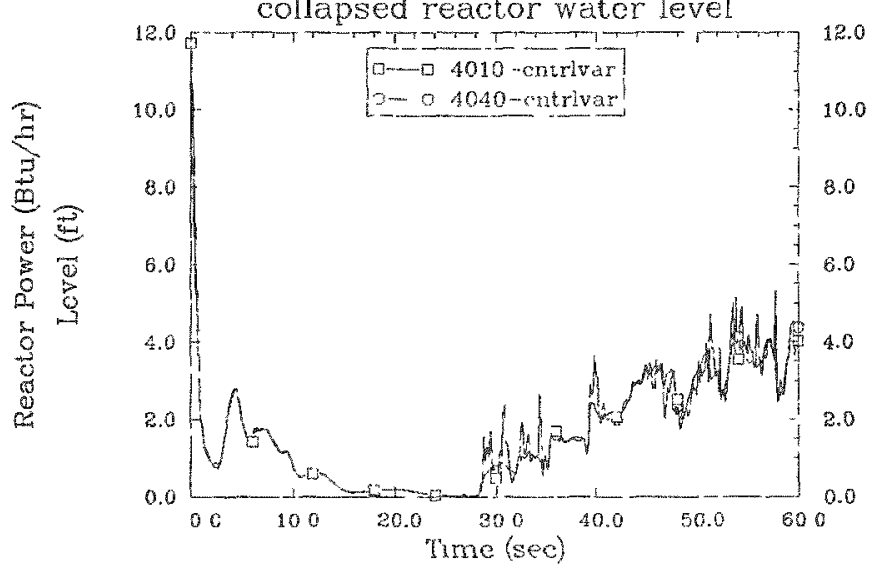

B\&W (OCONLE) 90\% DBA LOCA PIN FALUURE

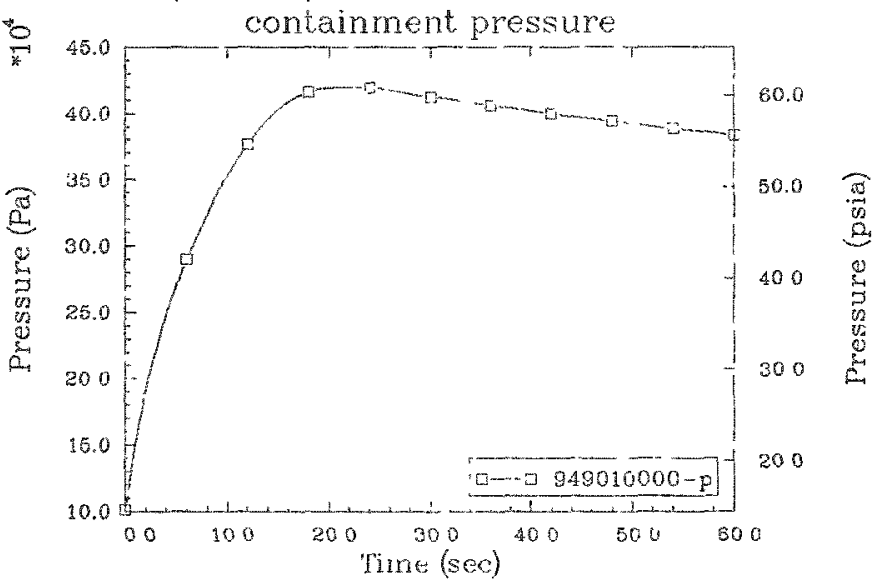

B\&W (OCONEE) 90\% DBA LOCA PIN FAILURE

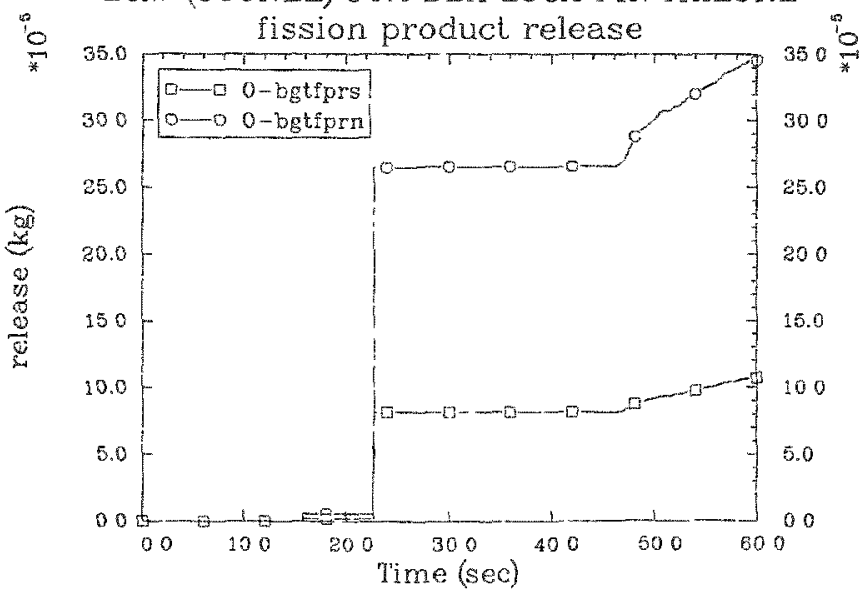

B\&W (OCONEE) 90\% DBA LOCA PIN FALUURE

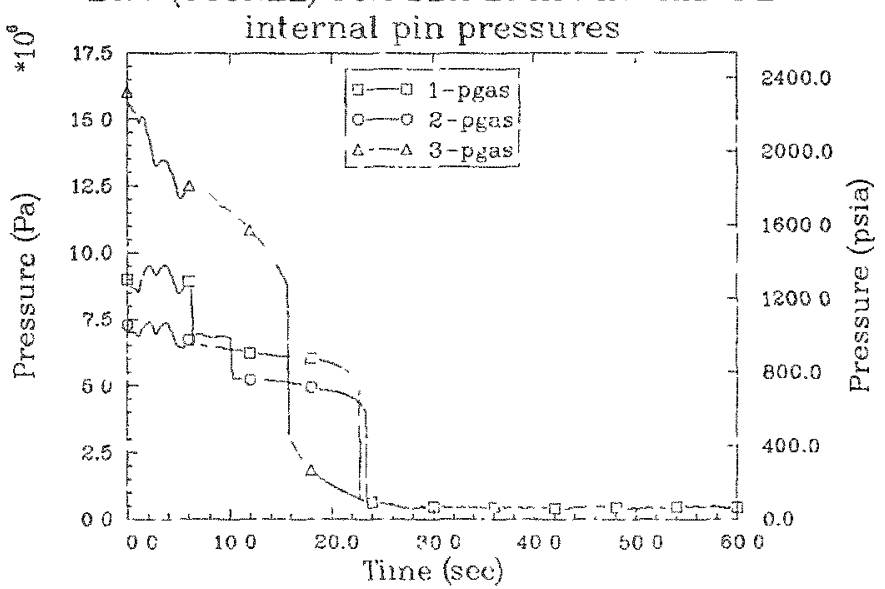


B\&W (OCONEE) 90\% DBA LOCA PIN FAILURE fuel centerline temperatures for 5 GWD/MTU pin

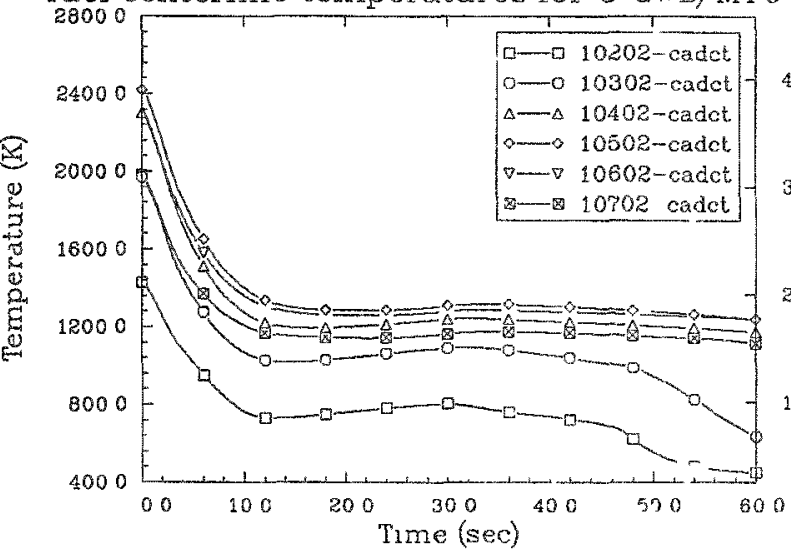

B\&W (OCONEE) 90\% DBA LOCA PIN FAILURE cladding surface temperatures for $5 \mathrm{GWD} / \mathrm{MIU}$ pin

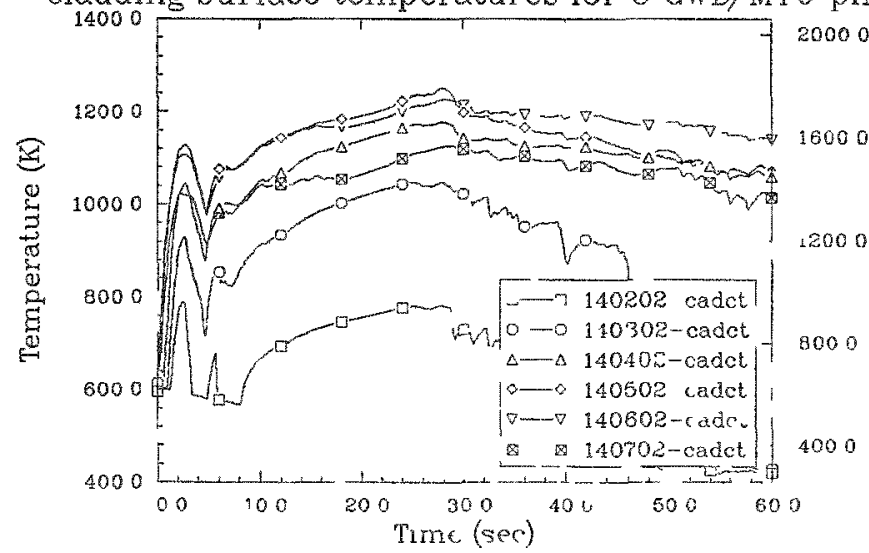

B\&W (OCONEE) 90\% DBA LOCA PIN FAILURE

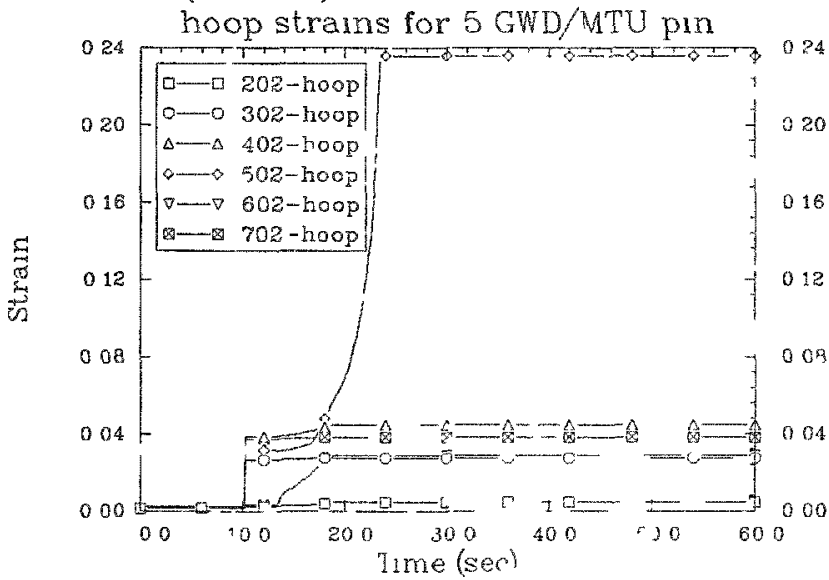

B\&W (OCONEE) 90\% DBA LOCA PIN FAILURE fuel centerline temperatures for 55 GWD/MTU pin

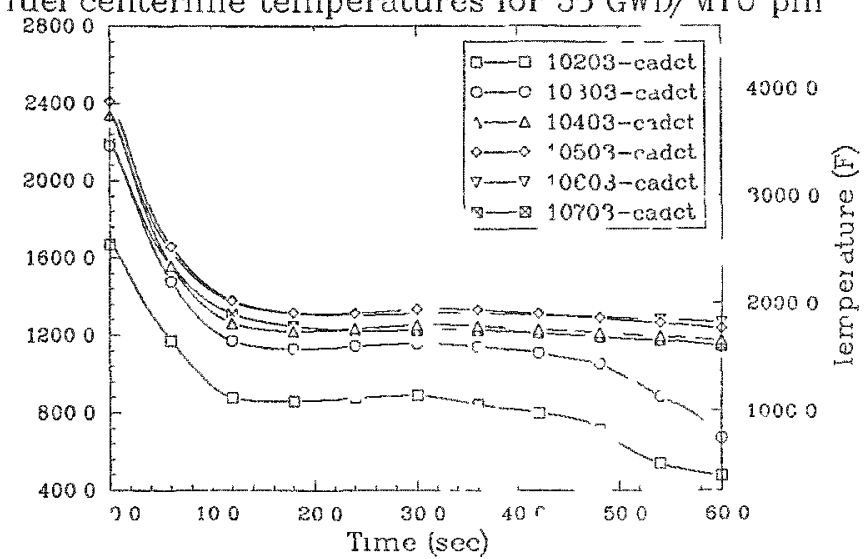

B\&W (OCONEE) 90\% DBA LOCA PI TAIL C'RE cladding surface temperatures for 55 GWD/MTU pm

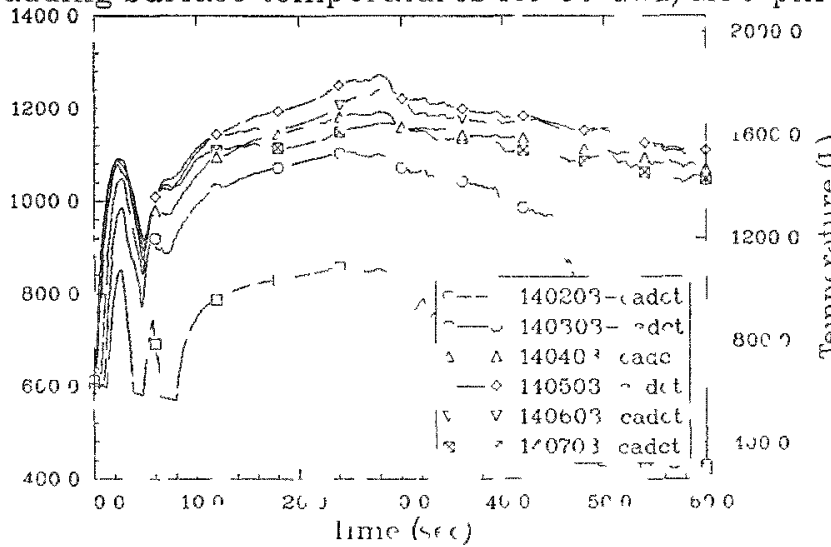

B\&W (OCONEE) 90\% DBA IOCA PI FAII URT

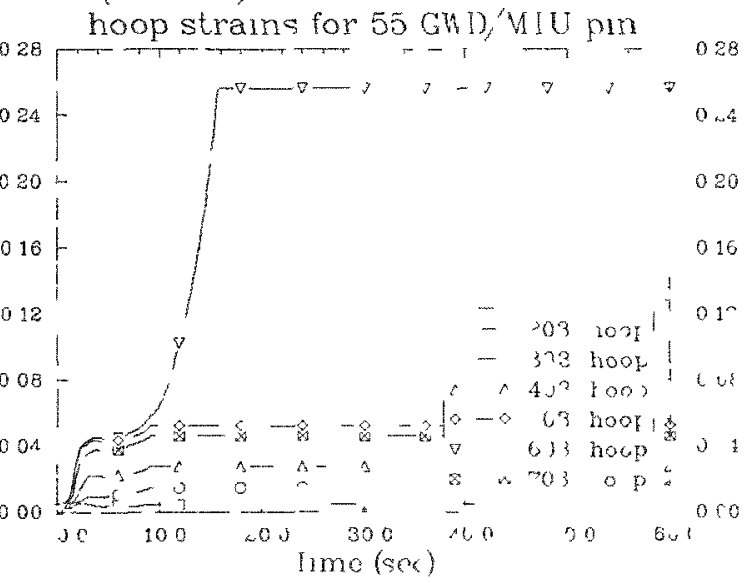


B\&W (OCONEE) 90\% DHA LOCA PIN FALL RE

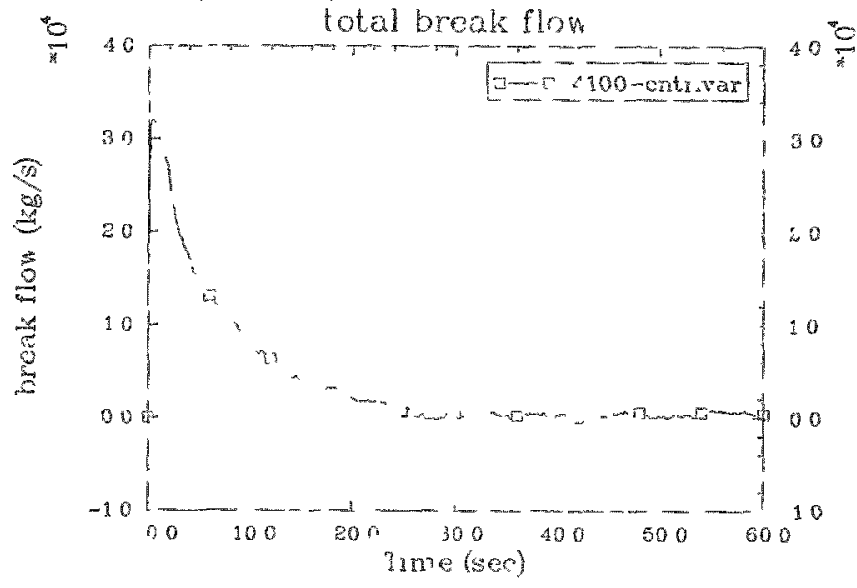

B\&W (OCONET) 90:\% DBA IOCA PJN MALURT,

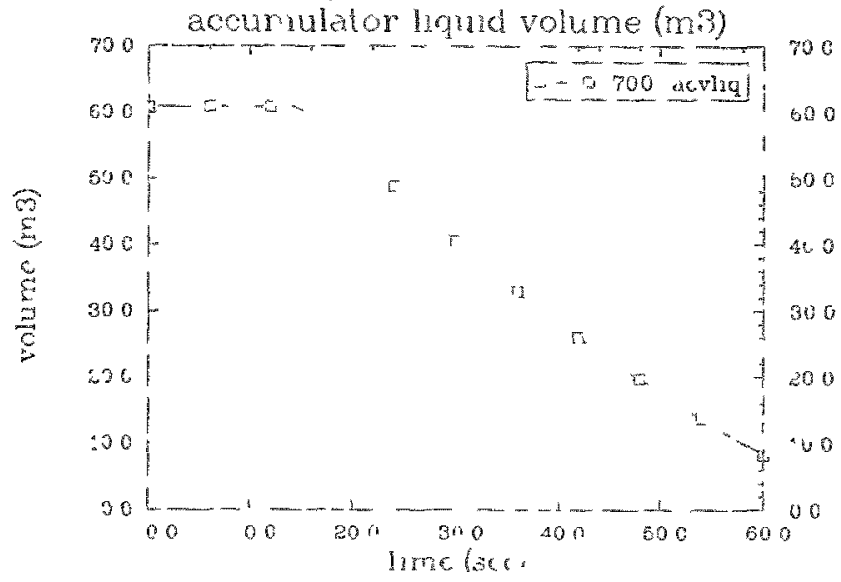

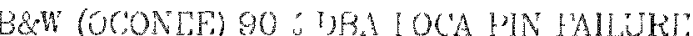

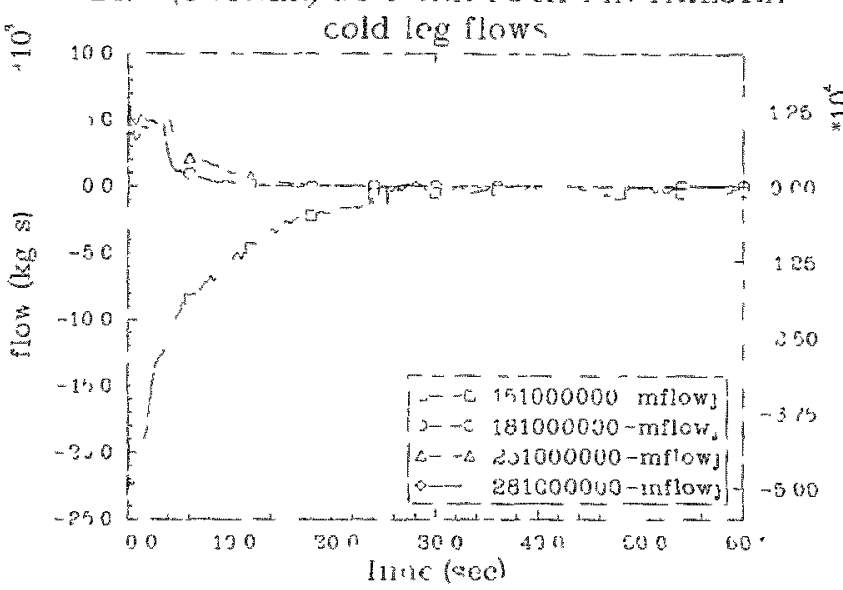

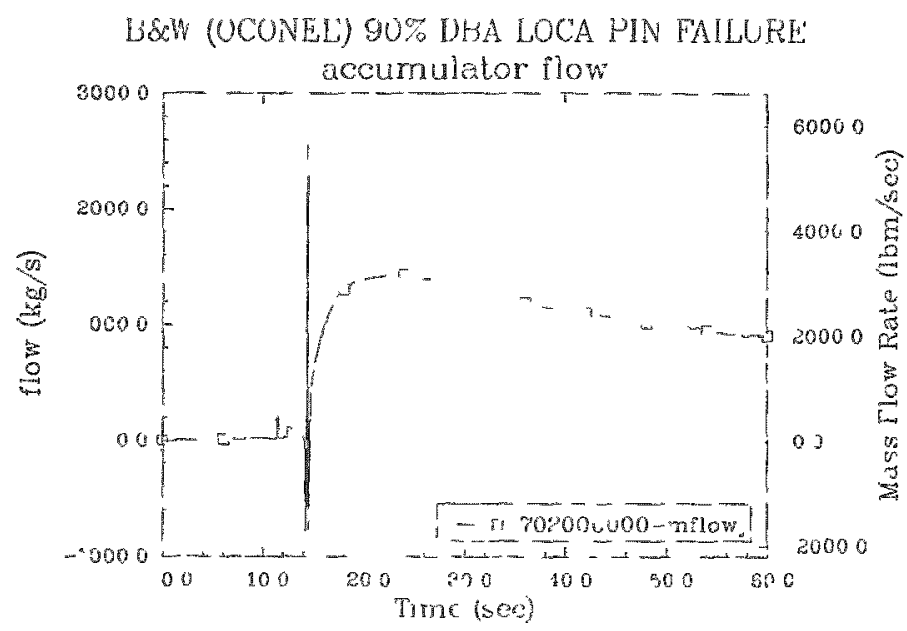

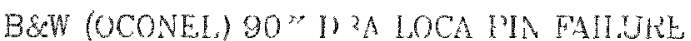

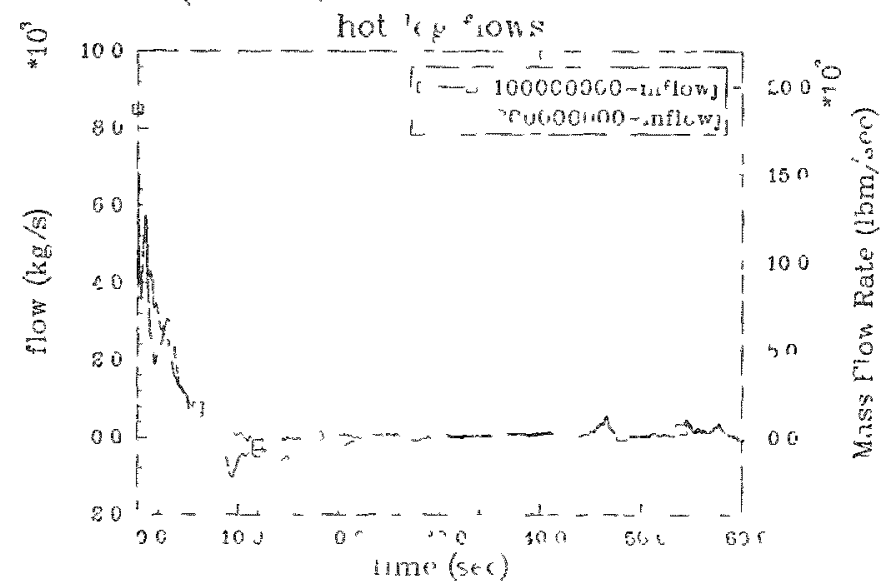

Bow (OCONDL) 10\% DHA, UCA H MAR

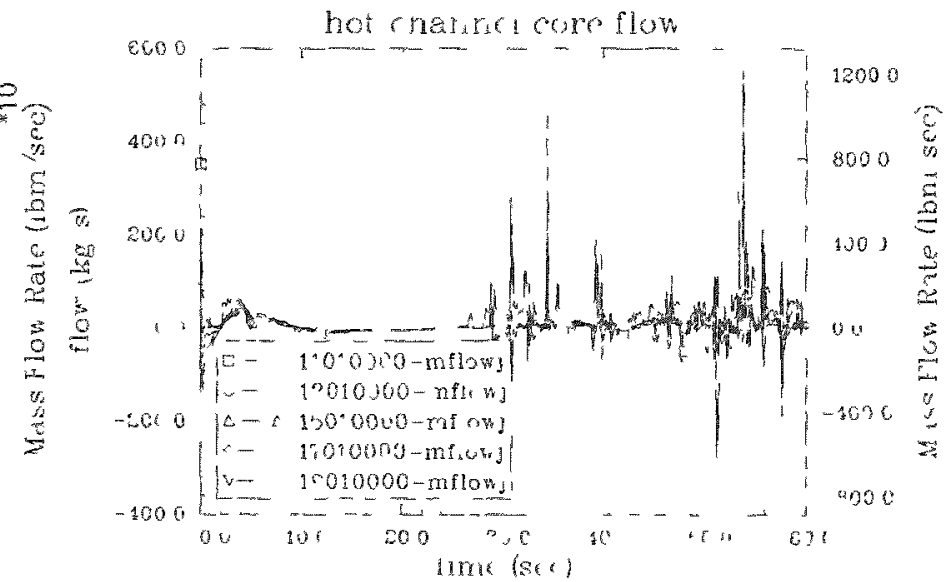




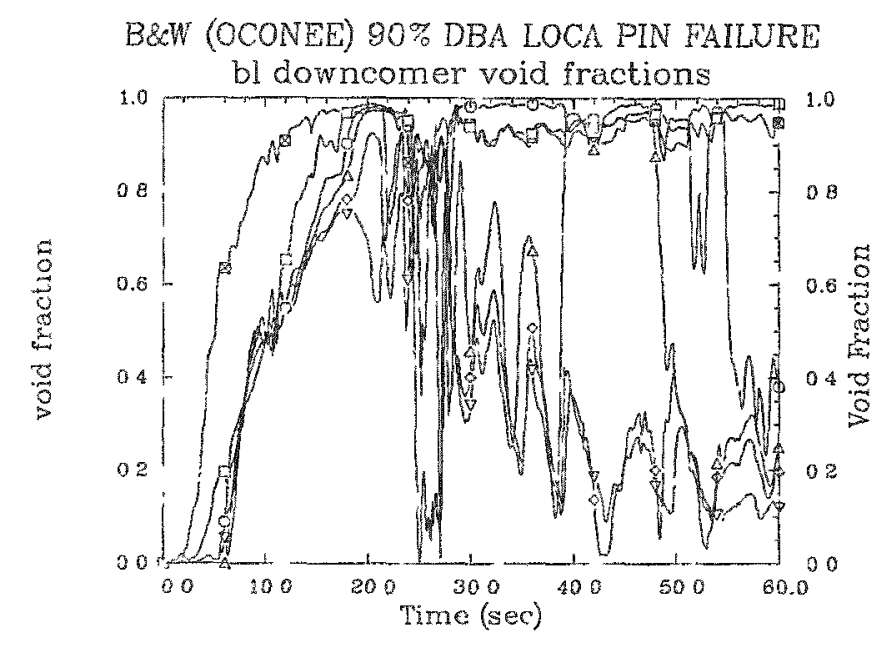

B\&W (OCONEE) 90\% DBA I.OCA FIN FAILURE

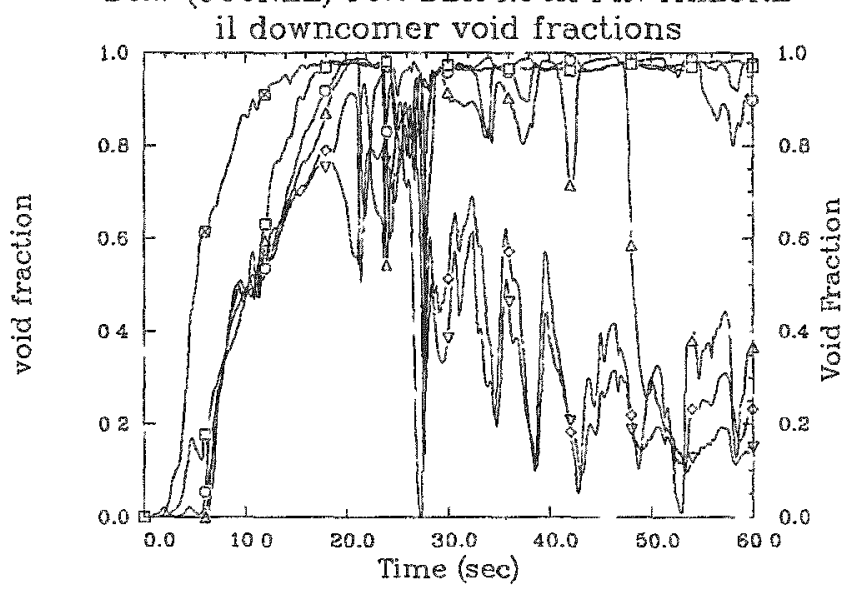

\section{B\&W (OCONEE) 90\% DBA LOCA PIN FAILURE}
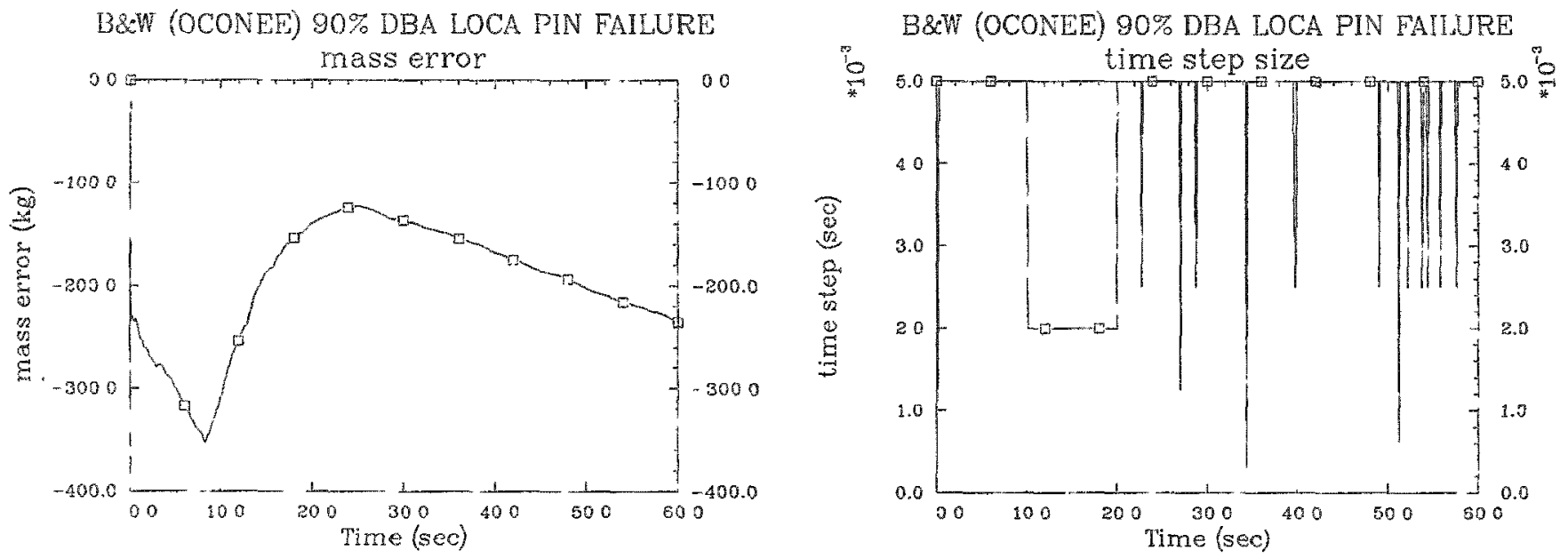

B\&W (OCONEE) 90\% DBA LOCA PIN FAILURF,

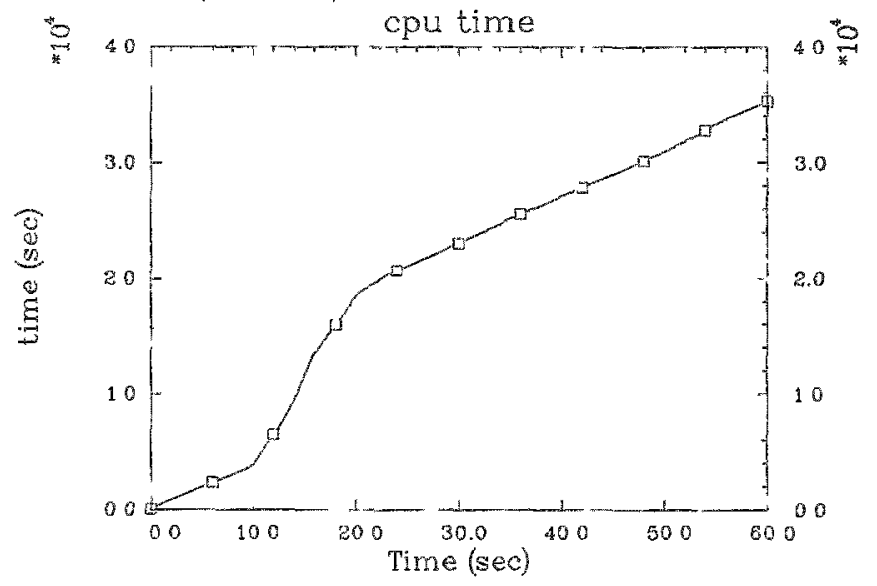


B\&W (OCONEE) 75\% DBA LOCA PIN FAILURE

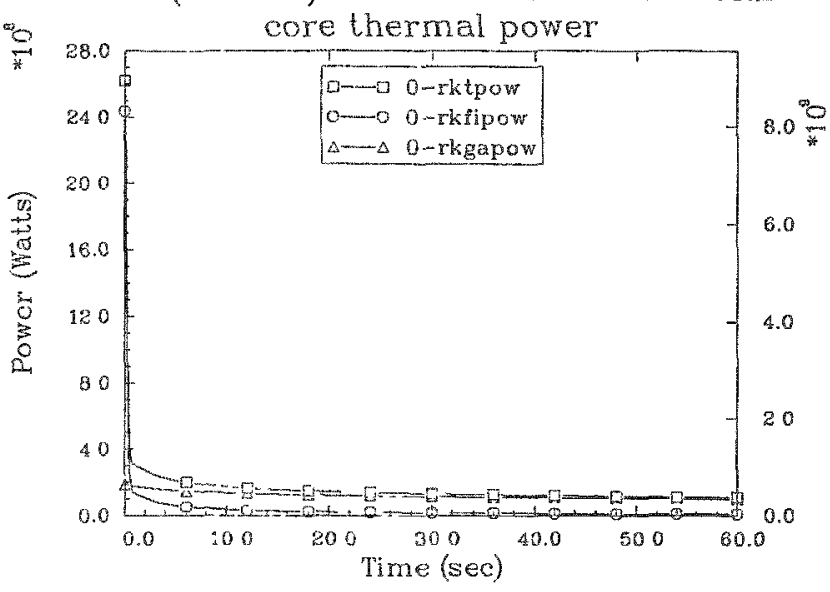

B\&W (OCONEF) 75\% DBA LOCA PIN FAILURE

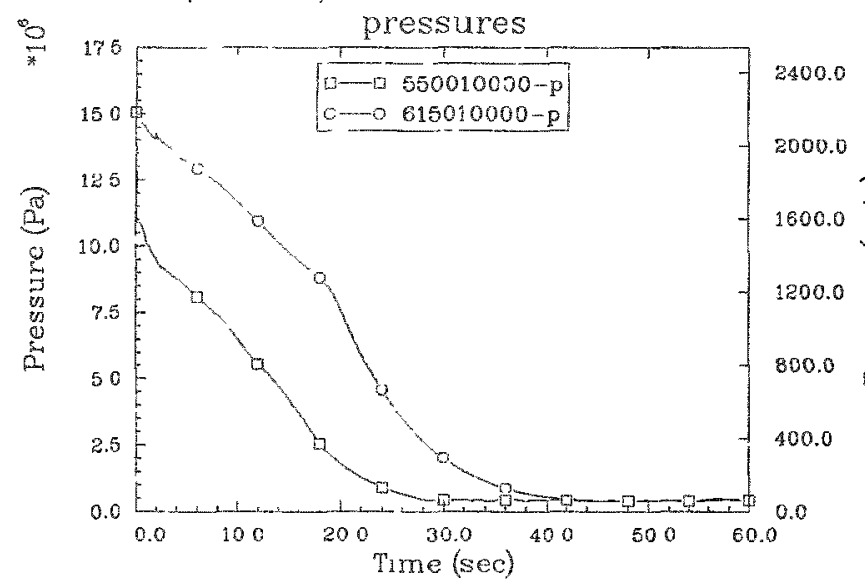

B\&W (OCONEE) $75 \%$ DBA LOCA PIN FAILURE

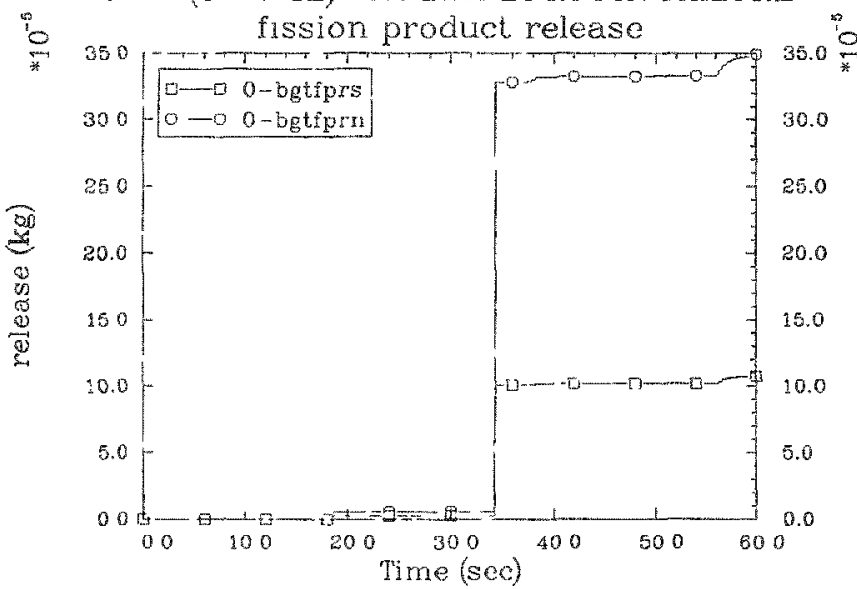

BEW (OCONEE) 75\% DBA LOCA PIN FAILURE

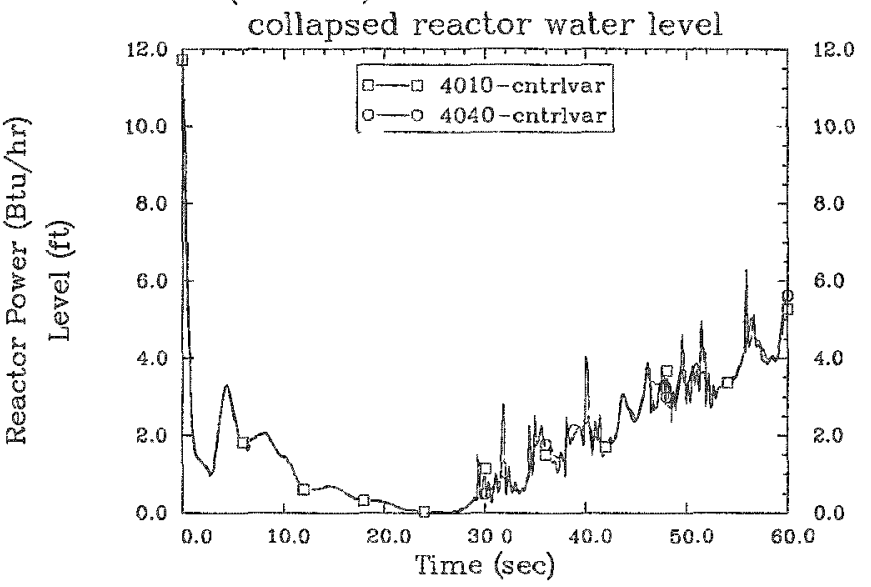

B\&W (OCONEE) 75\% DBA LOCA PIN FAILURE

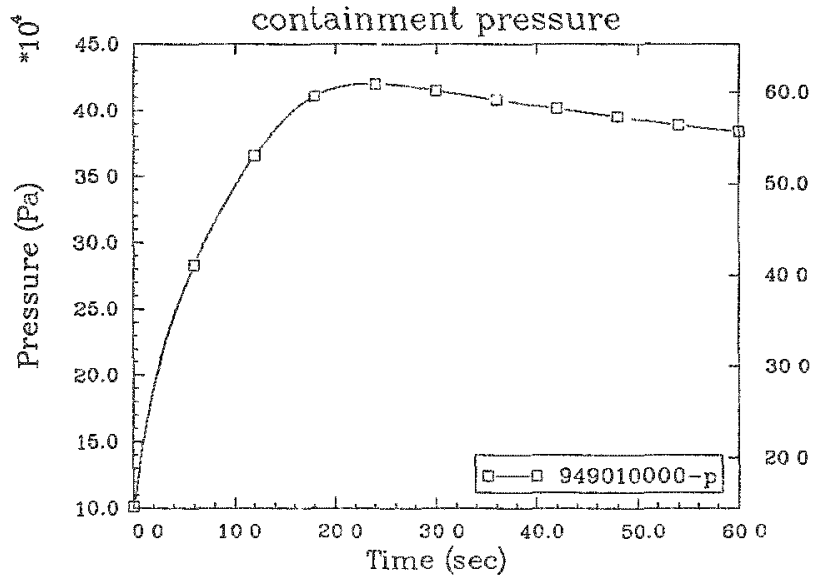

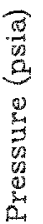

B\&W (OCONEE) $75 \%$ DBA LOCA PIN FAILURE

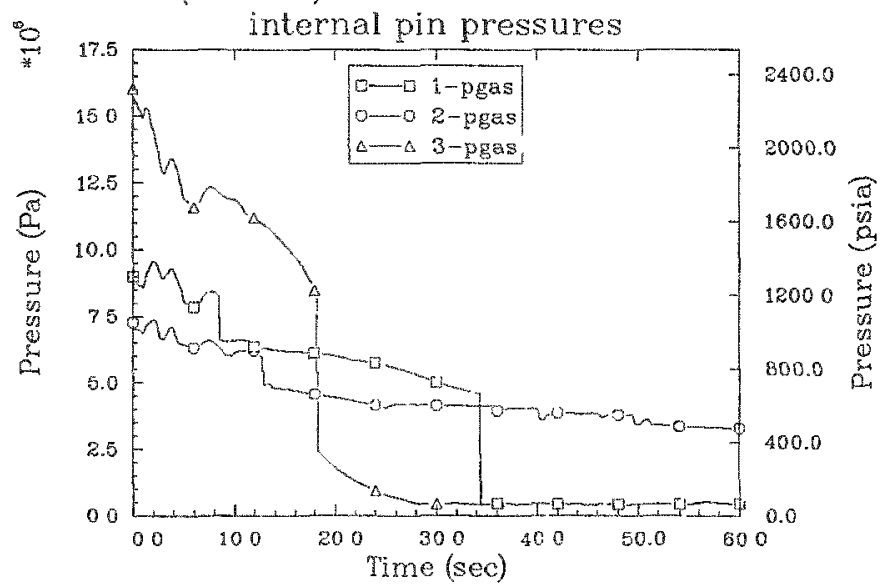


B\&W (OCONEE) 75\% DBA LOCA PIN FAILURE fuel centerline temperatures for $5 \mathrm{GWD} / \mathrm{MTU}$ pin

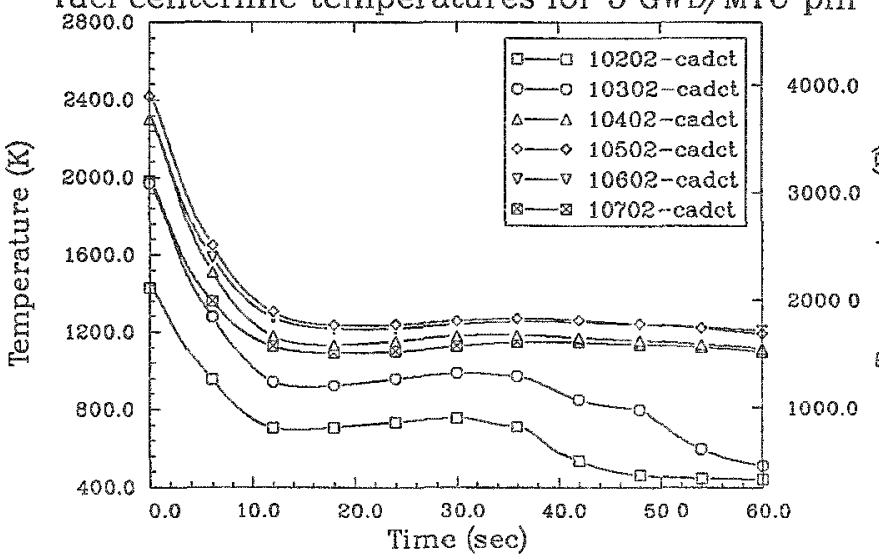

B8.W (OCONEE) 75\% DBA LOCA PIN FAILURE cladding surface temperatures for 5 GWD/MTU pin

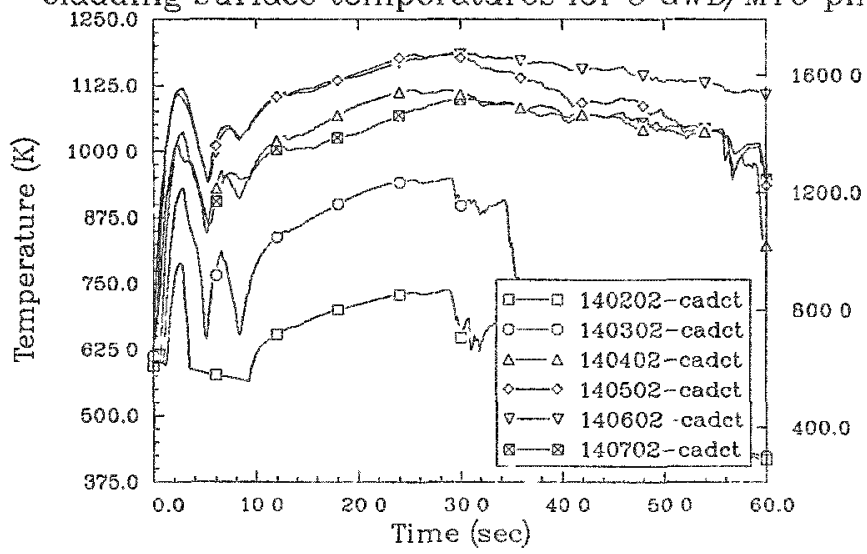

B\&W (OCONEE) 75\% DBA LOCA PIN FAILURE hoop strains for 5 GWD/MTU pin

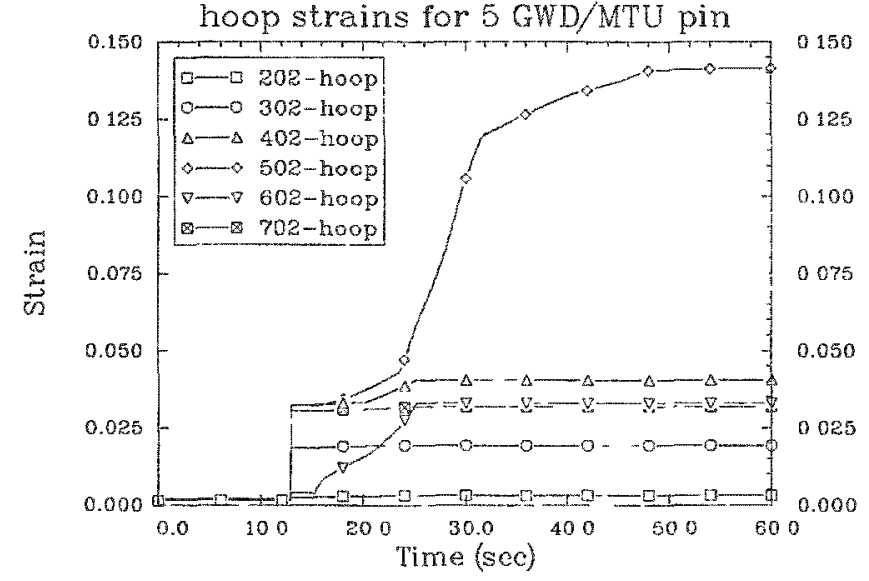

B\&W (OCONEE) 75\% DBA LOCA PIN FAILURE fuel centerline temperatures for $55 \mathrm{GWD} / \mathrm{MTU}$ pin

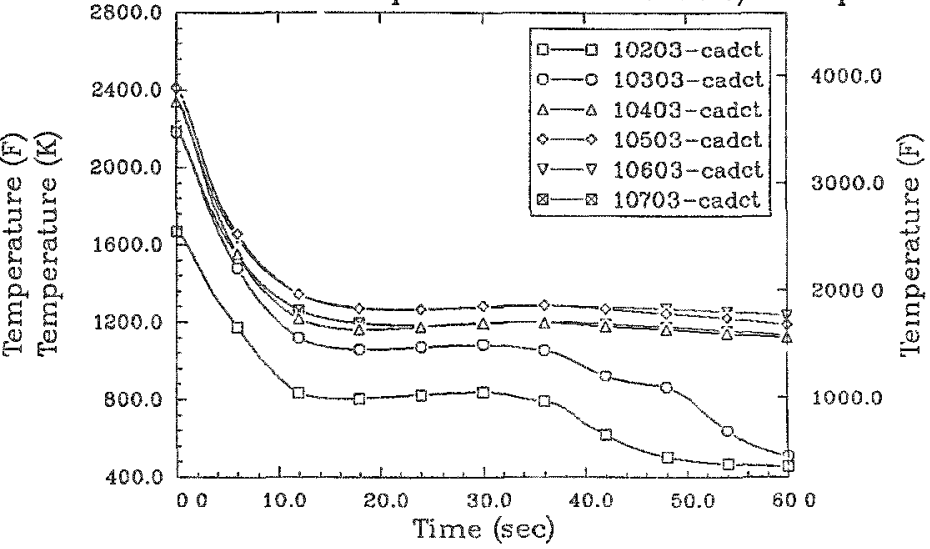

B\&W (OCONEE) 75\% DBA LOCA PIN FAILURE cladding surface temperatures for 55 GWD/MTU pin

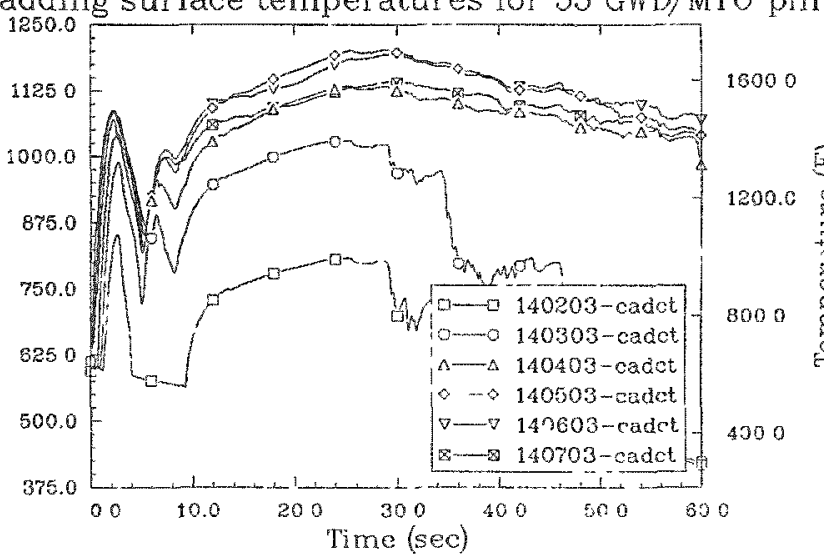

B\&W (OCONEE) 75\% DBA LOCA PIN FAILURE

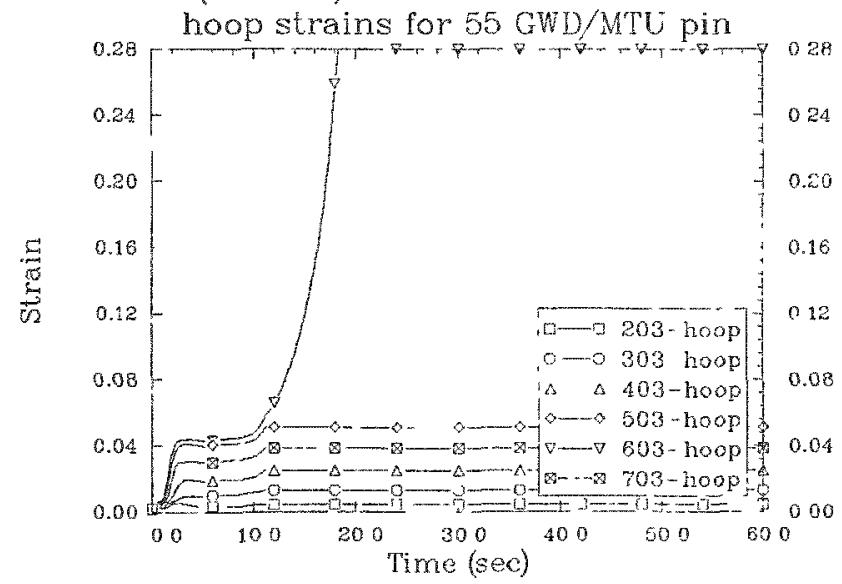



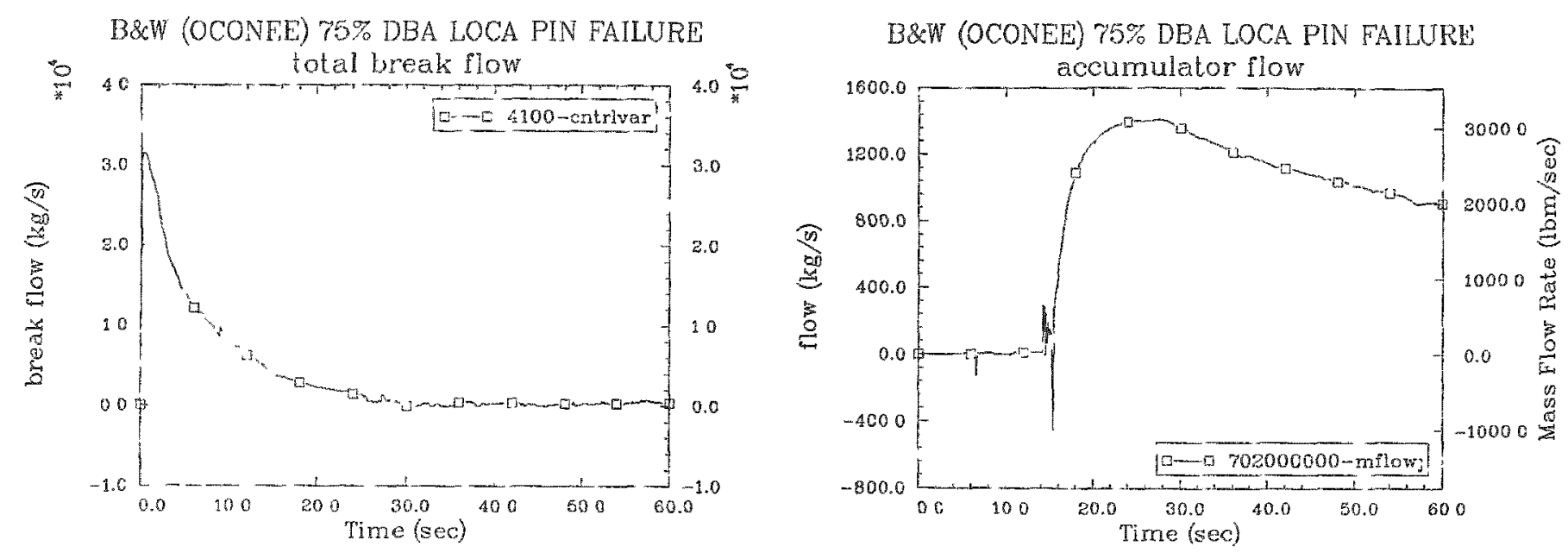

B\&W 10CONEE) 75\% DBA IOOCA PIN FAILURE

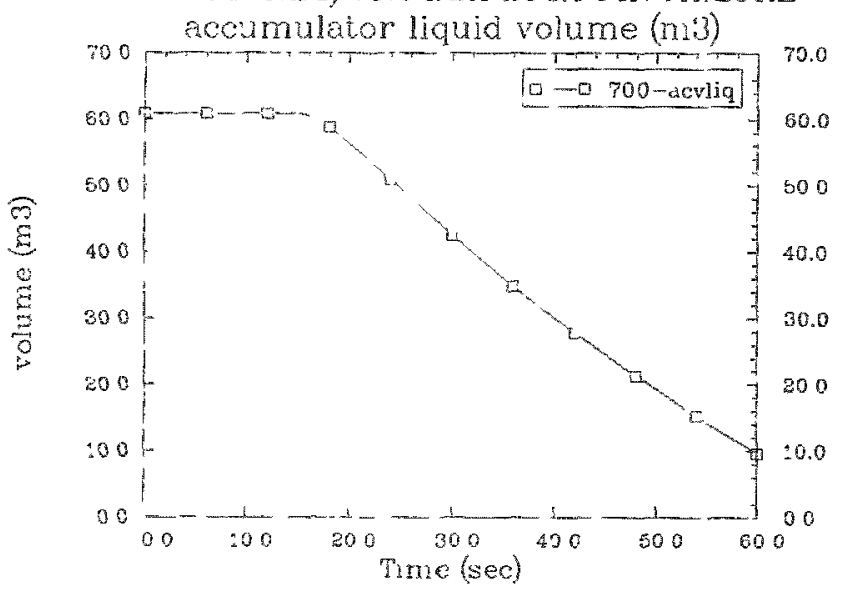

B\&W (OCONEE) 75:: DBA LOCA PIN FALUURE

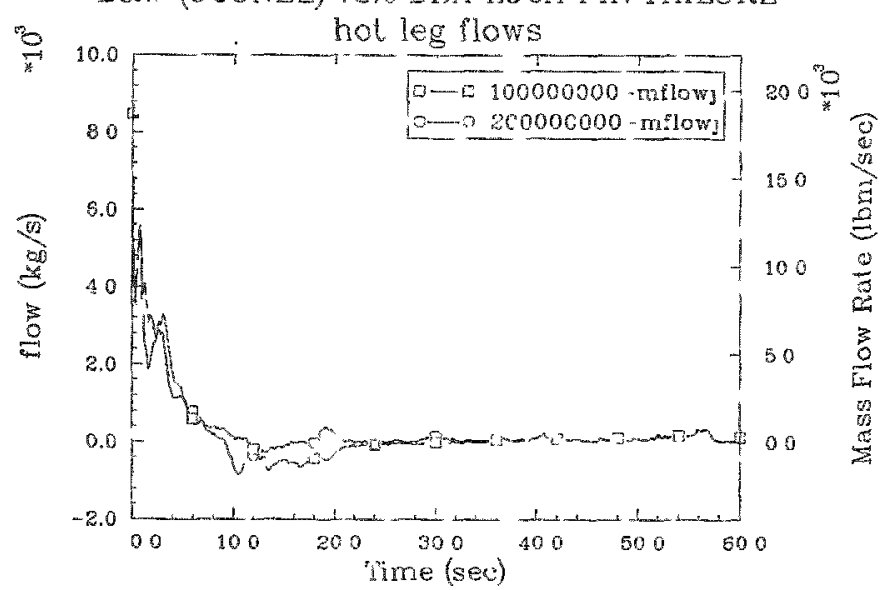

BCW (OCONEL) 75\% DBA LOCA PIN FAILURE

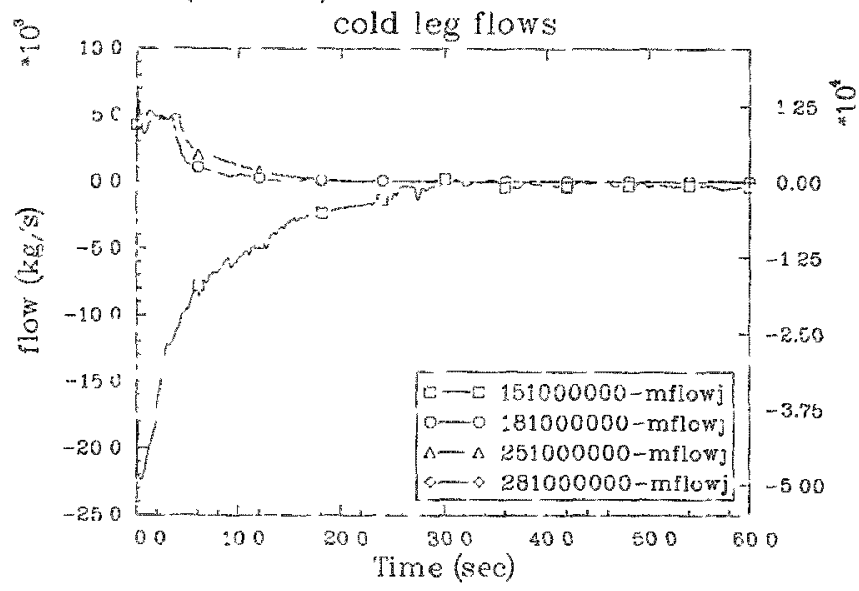

B\&W (OCONEE) 75\% DBA LOCA PIN YAILURE

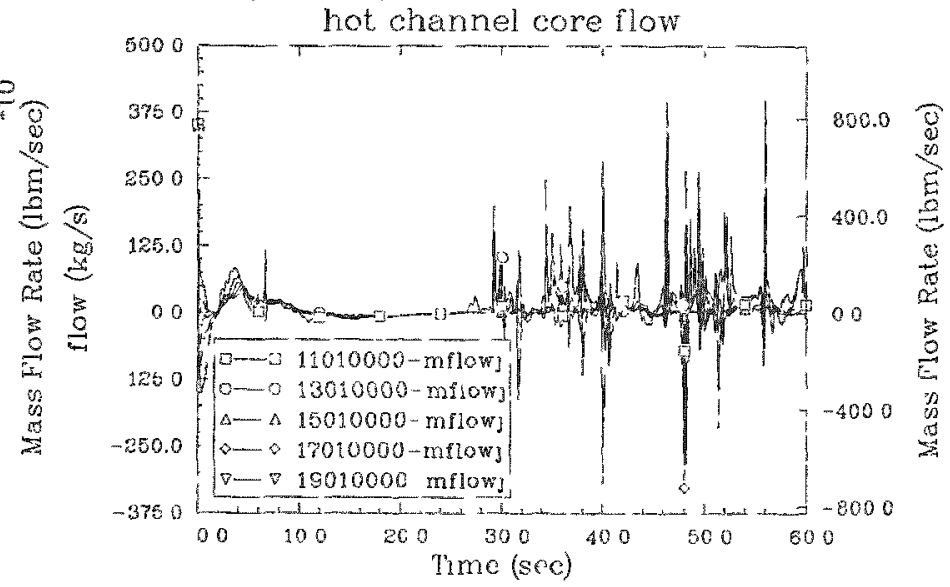



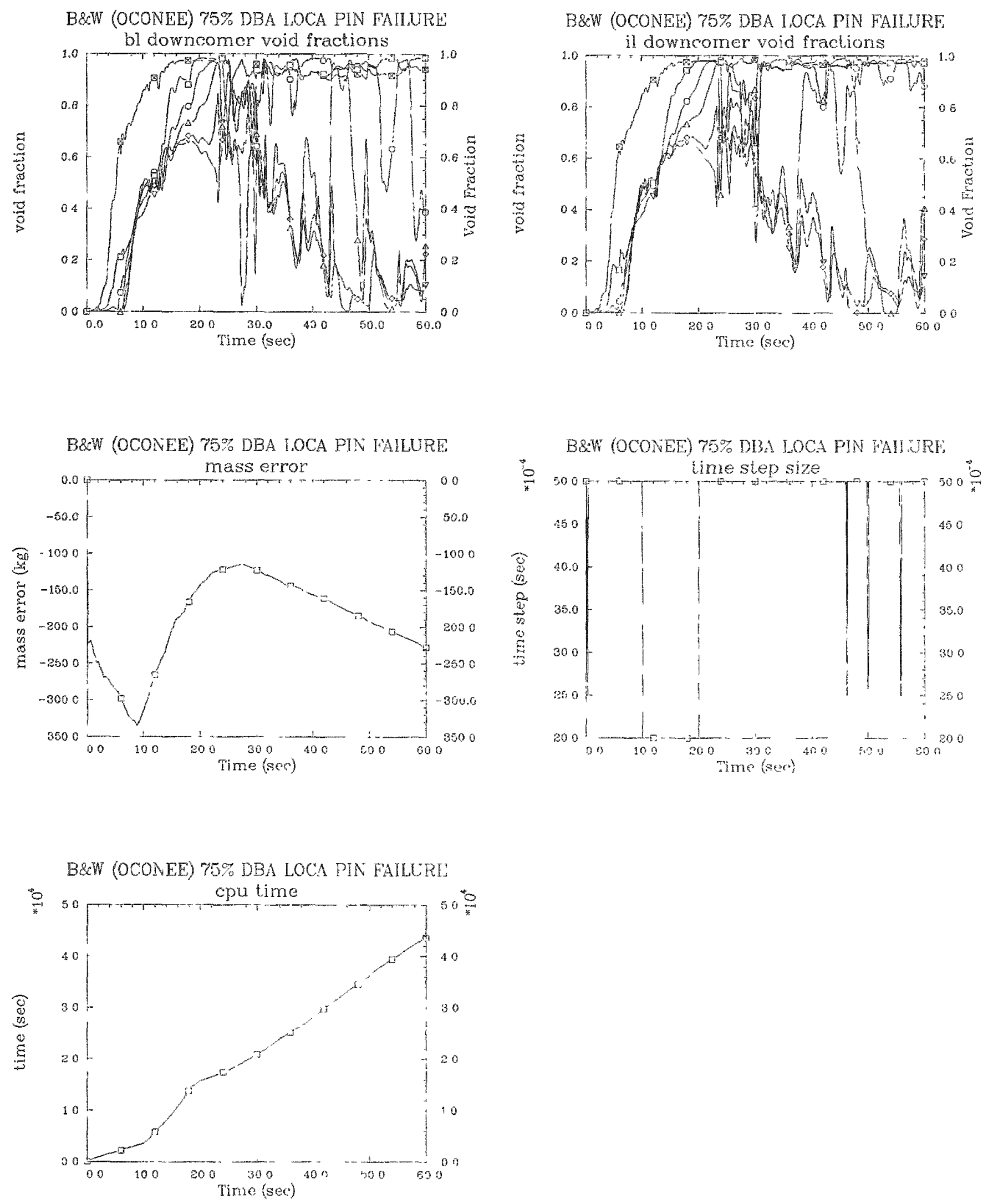
B\&W (OCONEE) 50\% DBA LOCA PIN FAILURE core thermal power

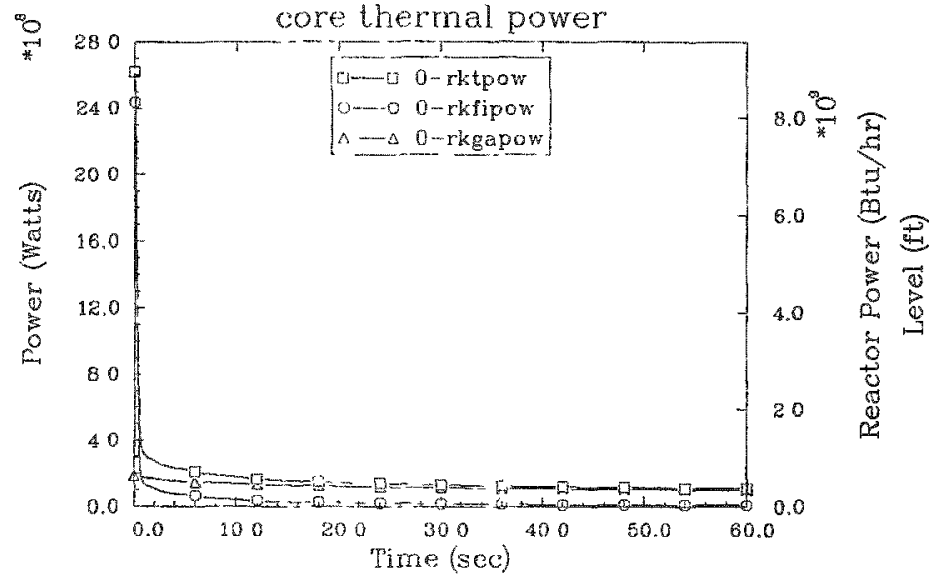

B\&W (OCONEE) 50\% DBA LOCA PIN FALURE collapsed reactor water level

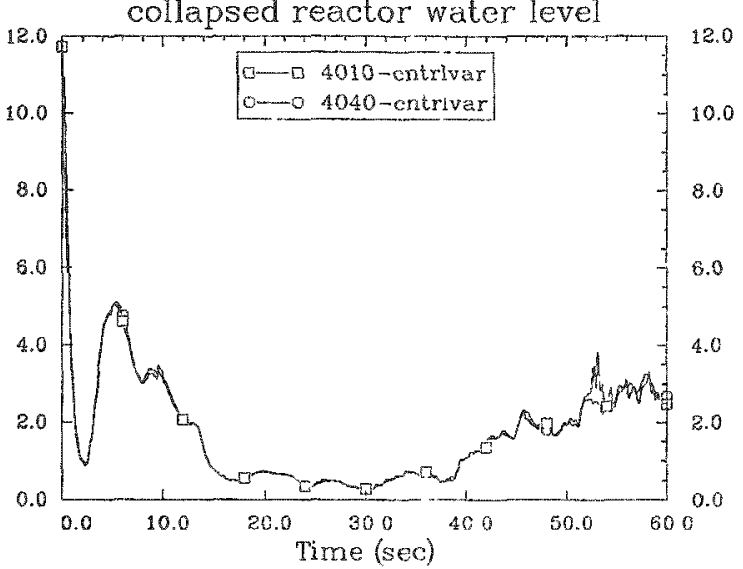

B\&W (OCONEL) 50\% DBA LOCA PIN FAILURE

$$
\stackrel{8}{7}
$$

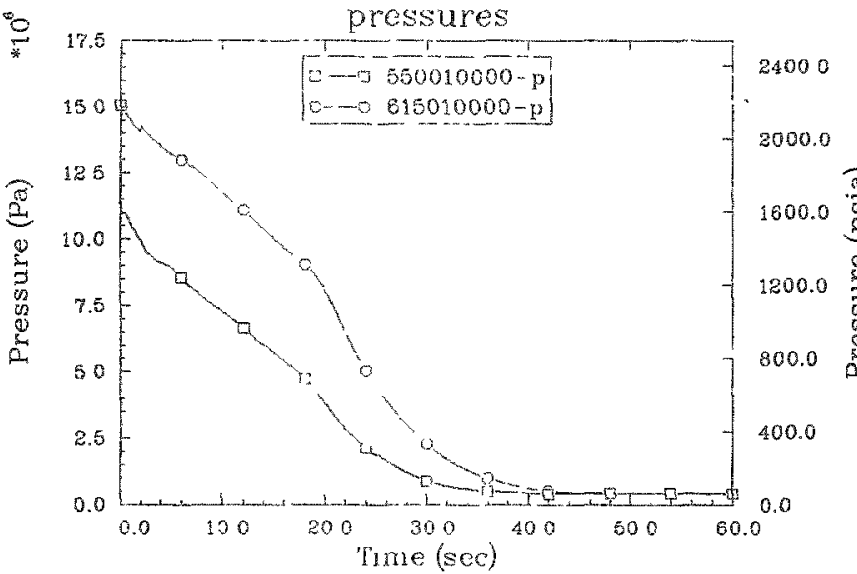

B\&W (OCONLE) 50\% DBA LOCA PIN FAILURE

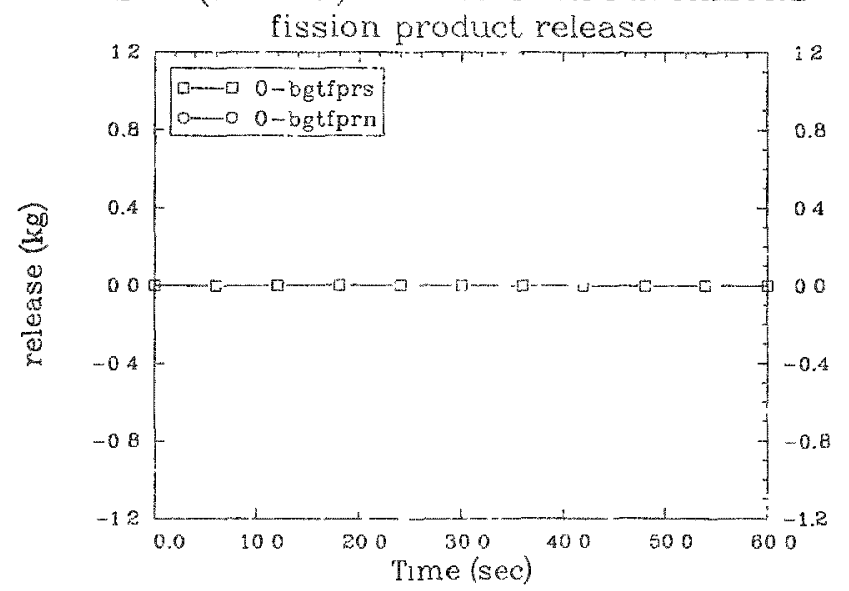

B\&W (OCONEE) 50\% DBA LOCA PIN FAILURE

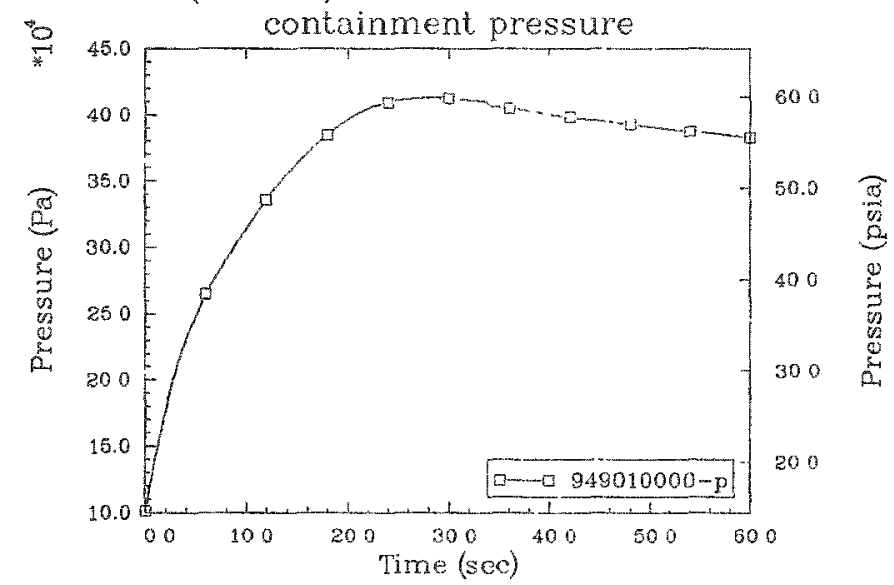

B\&W (OCONEE) 50\% DBA LOCA PIN FAILURE

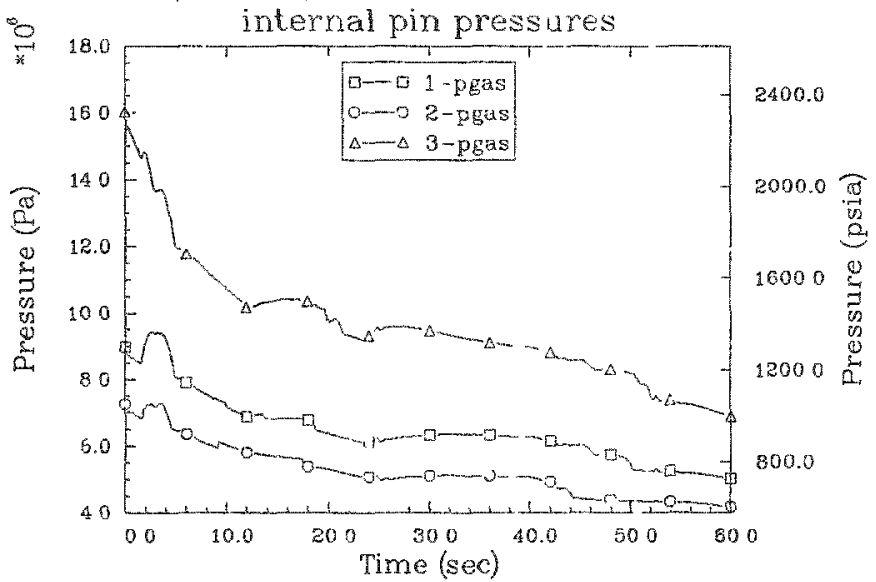


B8W (OCONEE) 50\% DBA LOCA PIN FAILURE fuel centerline temperatures for 5 GWD/MTU pin

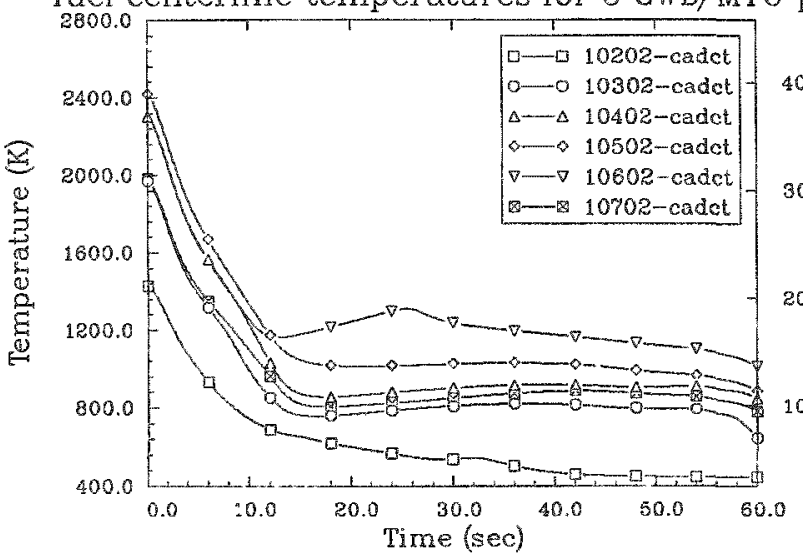

B\&W (OCONEE) 50\% DBA LOCA PIN FAILURE cladding surface temperatures for 5 GWD/MTU pin

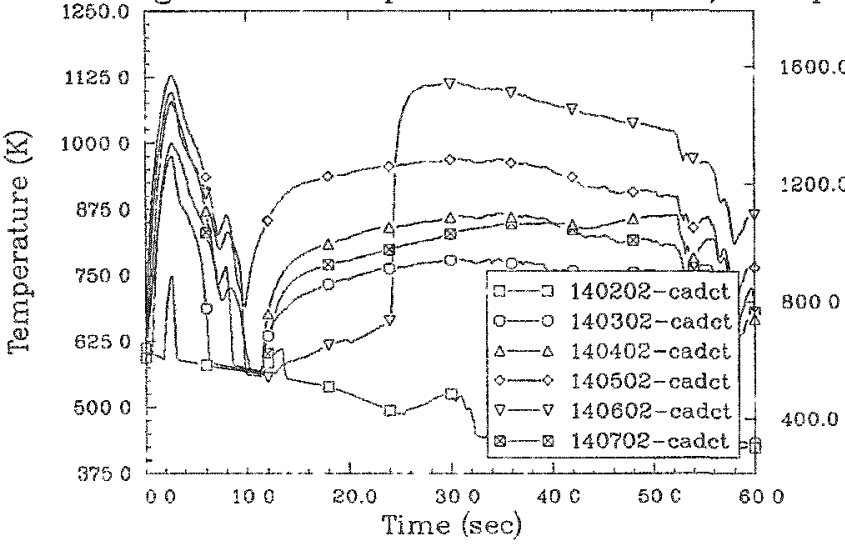

B\&W (OCONEE) 50\% DBA LOCA PIN FALURE

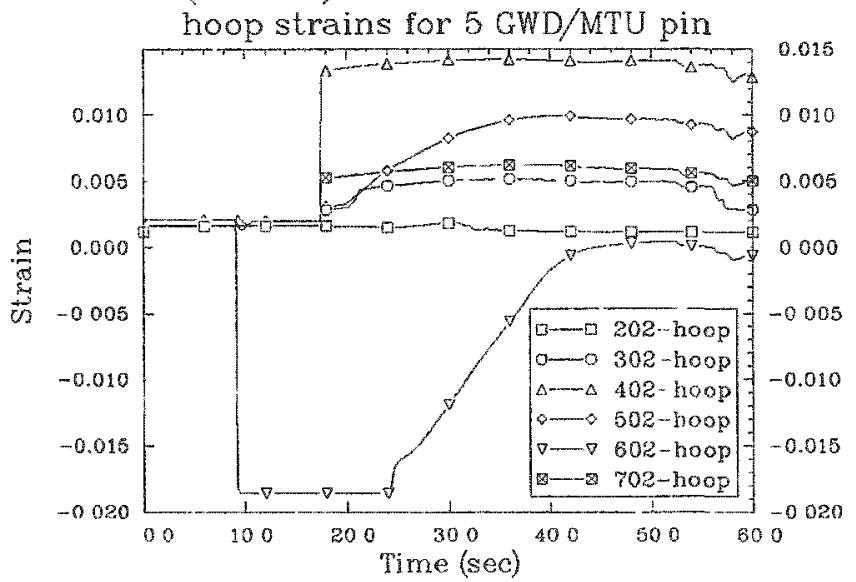

B\&W (OCONEE) 50\% DBA LOCA PIN FAILURE fuel centerline temperatures for 55 GWD/MTU pin

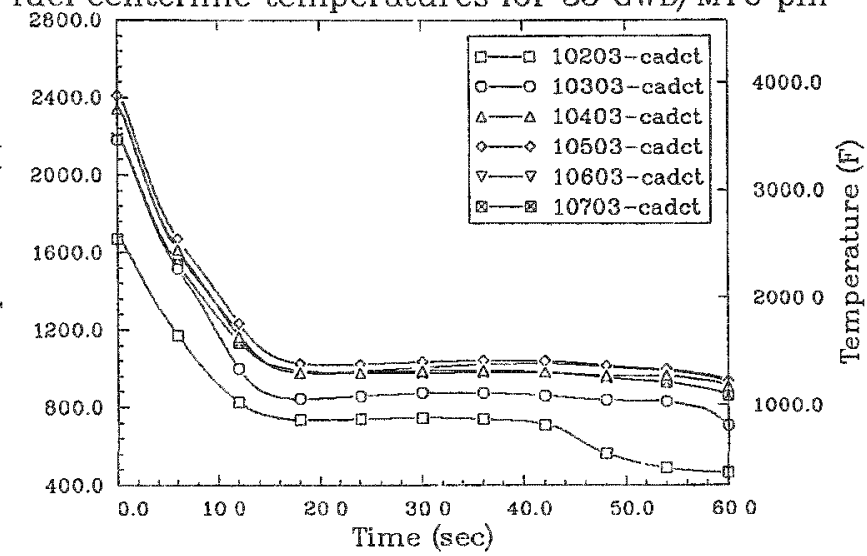

B\&W (OCONEE) 50\% DBA LOCA PIN FALLURE cladding surface temperatures for 55 GWD/MTU pin

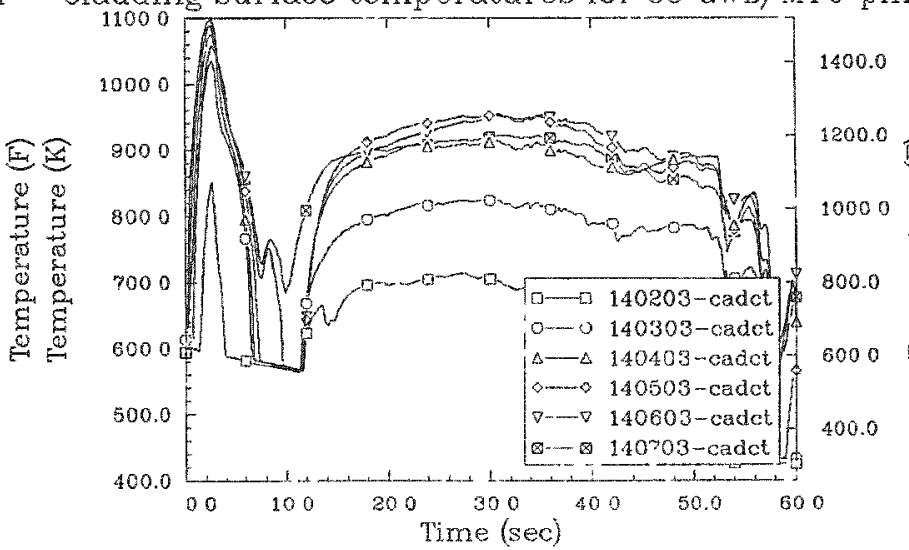

B\&W (OCONEE) 50\% DBA LOCA PIN FAILURE

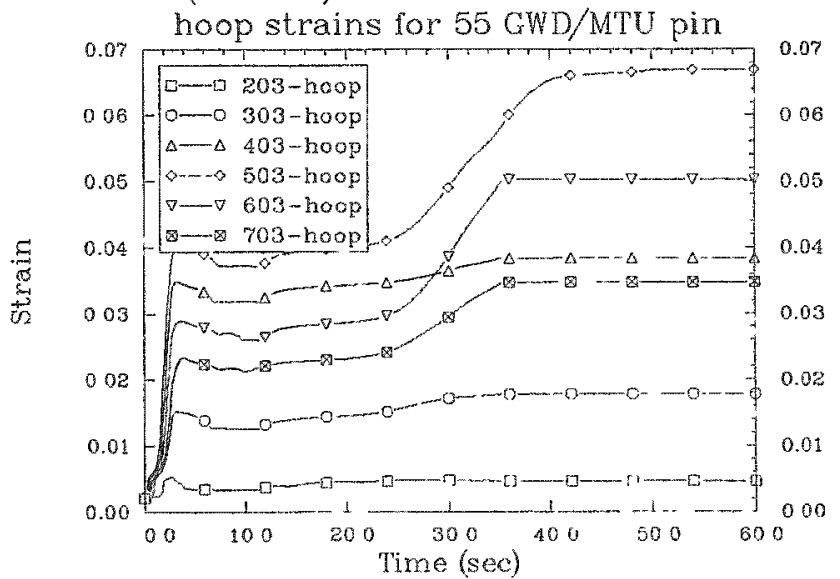


B\&W (OCONEE) $50 \%$ DBA IOCA PIN IAILURE

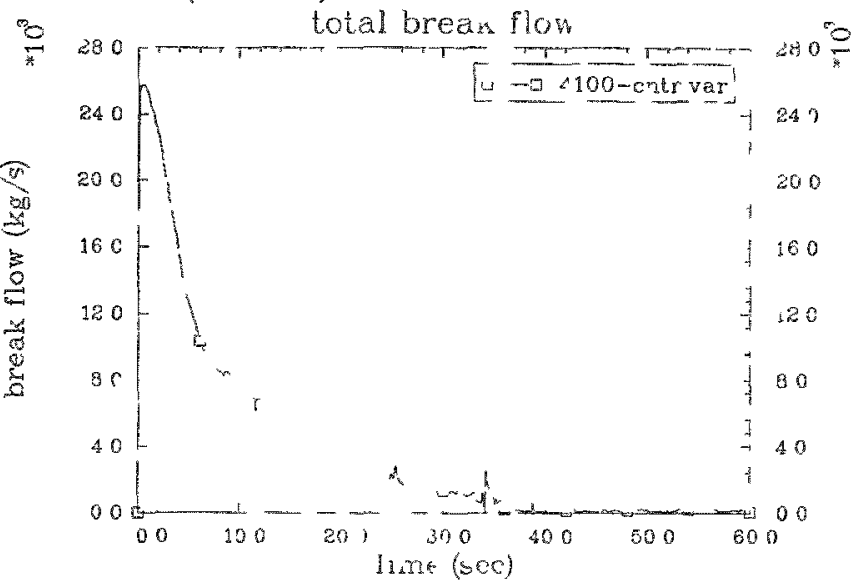

B\&W (OCONEL) 50\% DBA IOCA PIN FALLRE

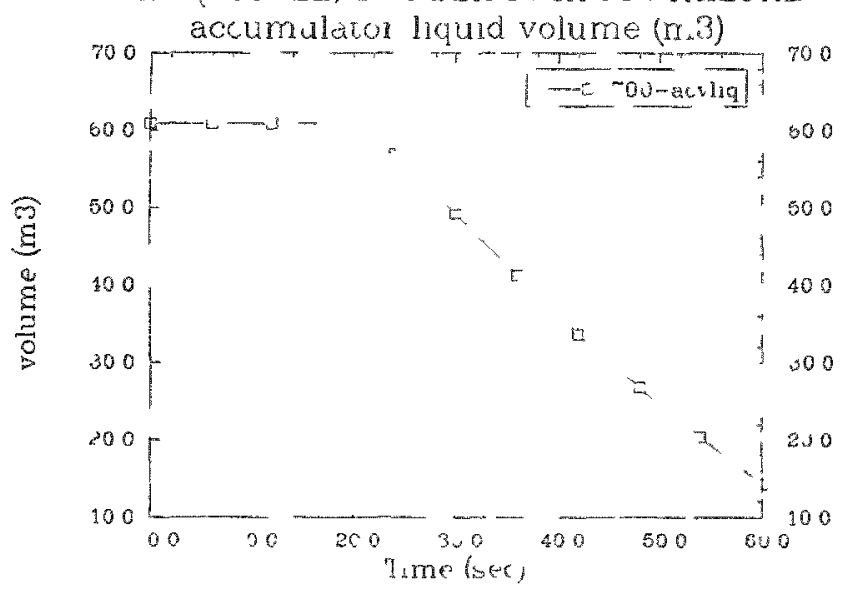

B\&W (OCONEF) 50॰ DBA IOCA PIN FAILURE

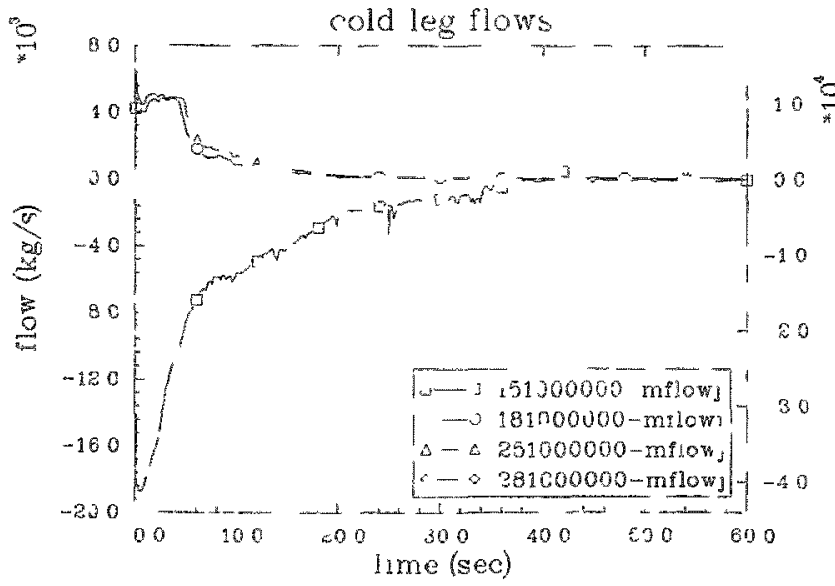

B\&W (OCONEE) 50\% DBA IOCA HIN : ILURF

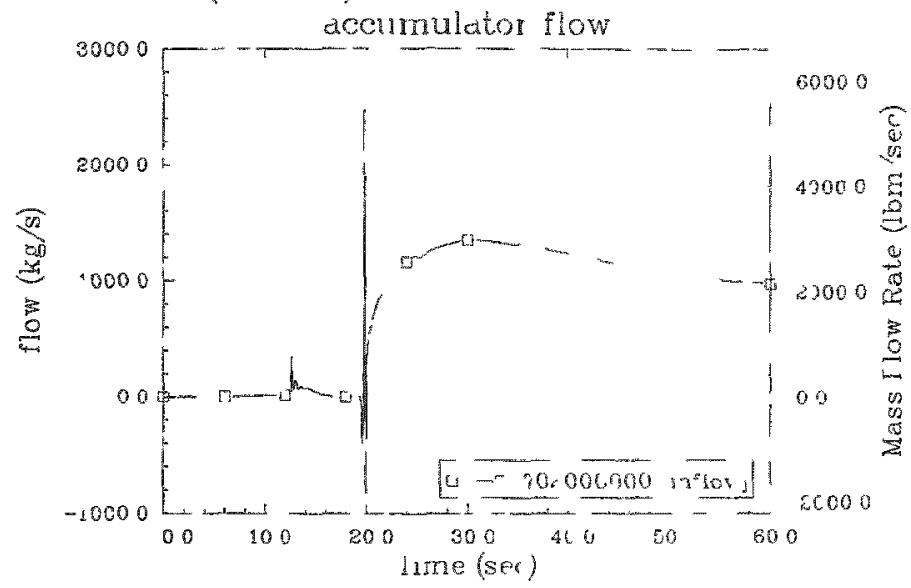

B\&W (OCONLE) $50 \%$ DBA I $\times$ 'A PIV A AILLL

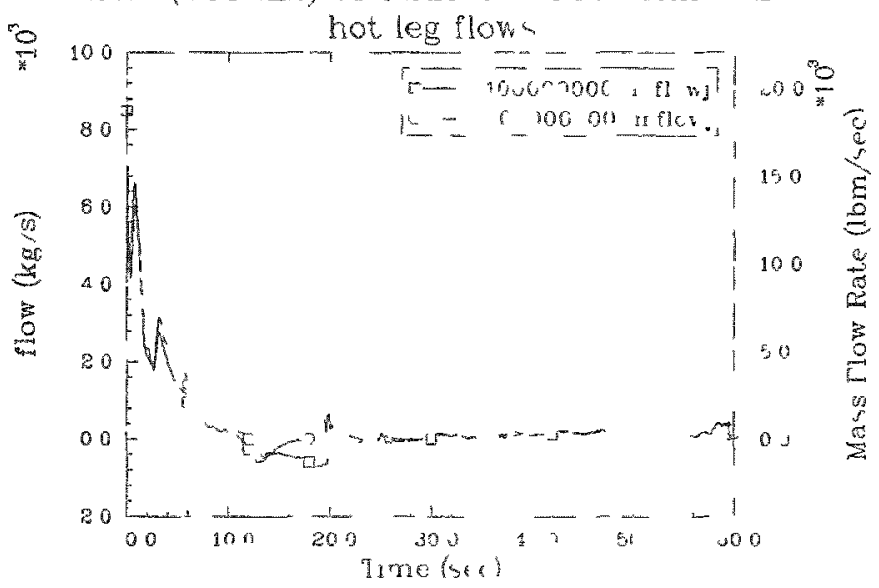

B\&W IOCONEE) 50 DHA WOL A PIN IALLIRI

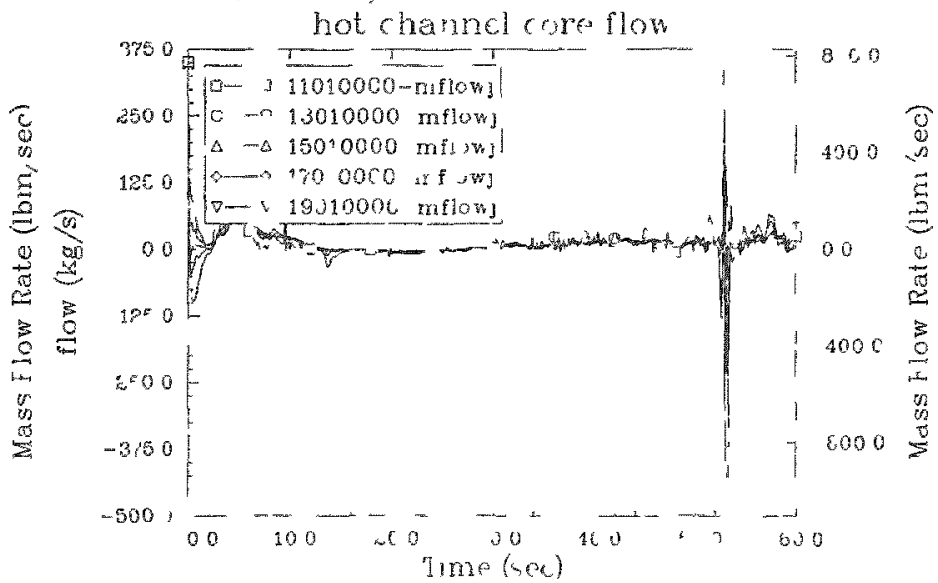




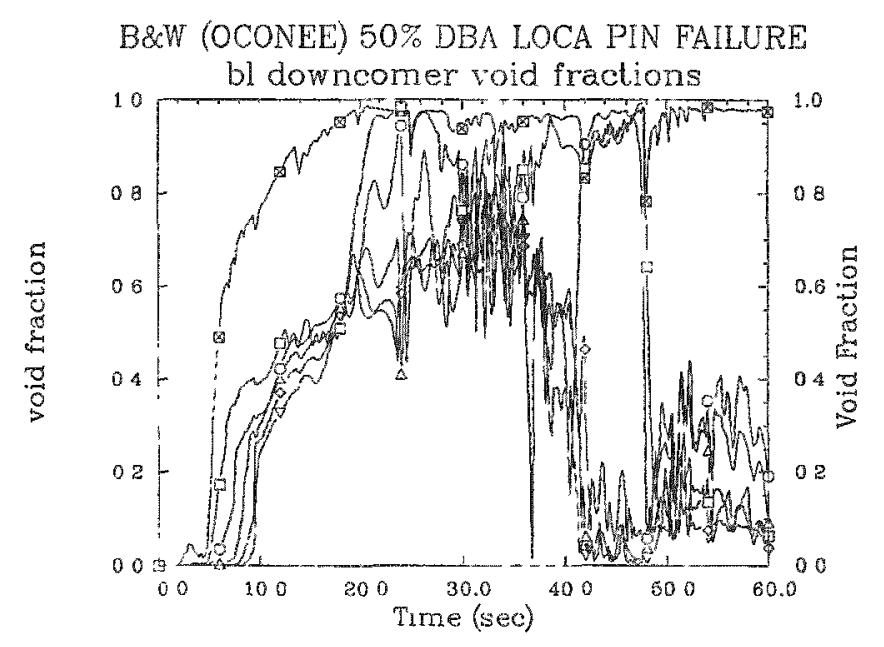

B\&W (OCONFE) 50\% DBA LOCA PIN FAILURE

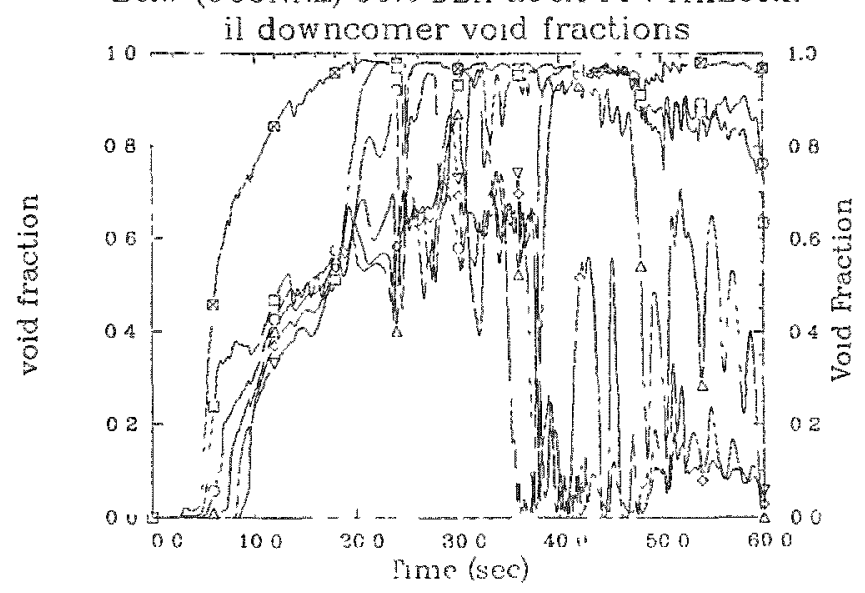

B\&W (OCONEE) 50\% DBA LOCA PIN FAILURE

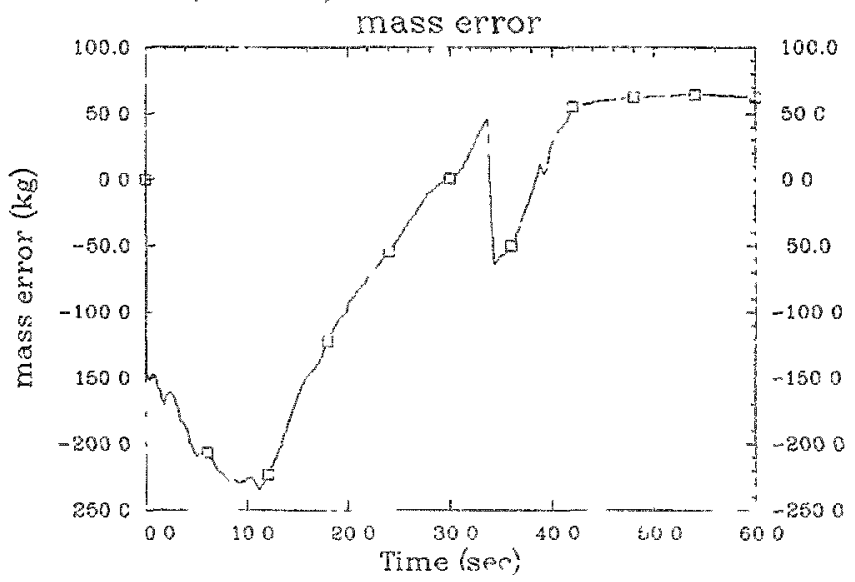

18\&W (OCONLT) 50\% DBA JOC PIV FUIITKF

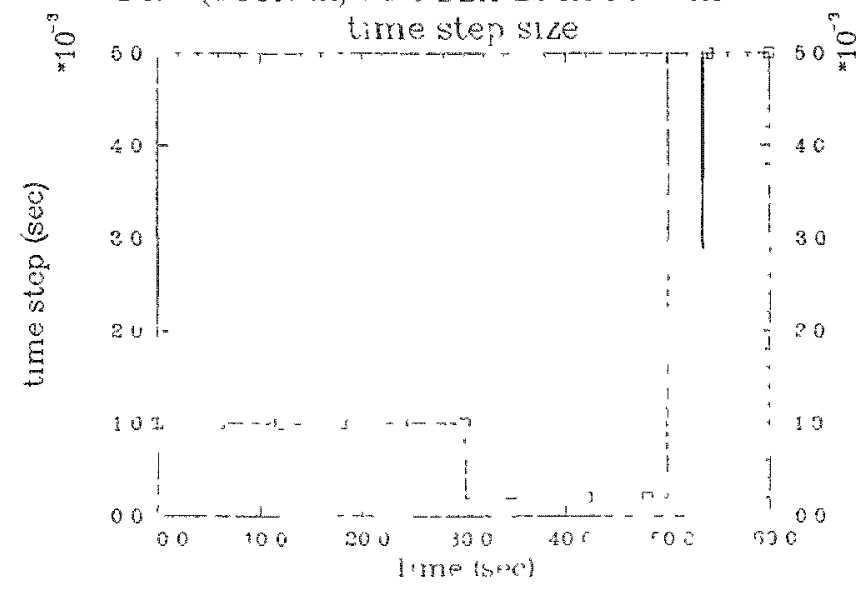

B\&W (OCONEE) 50\% DBA LOCA PIN FAILURE

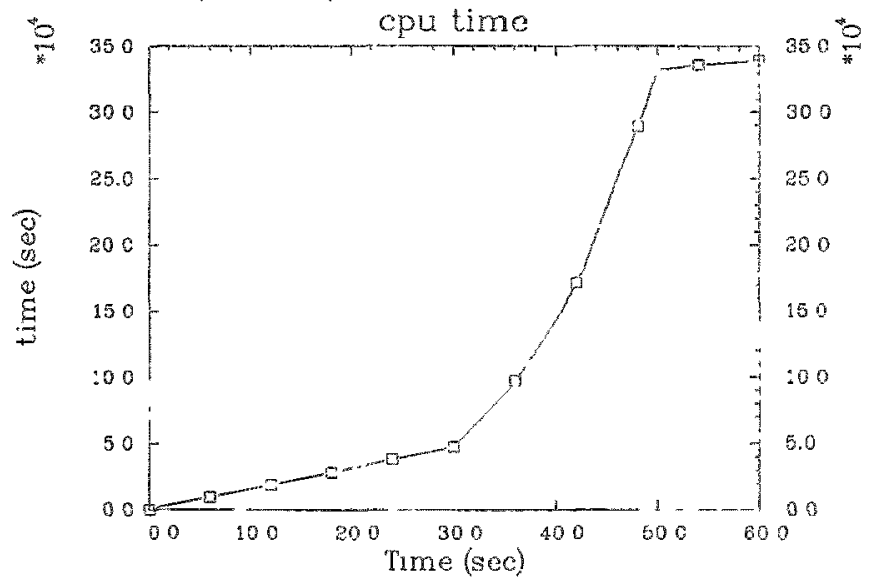


B\&W (OCONEE) SMALL BREAK LOCA PIN FAILURE

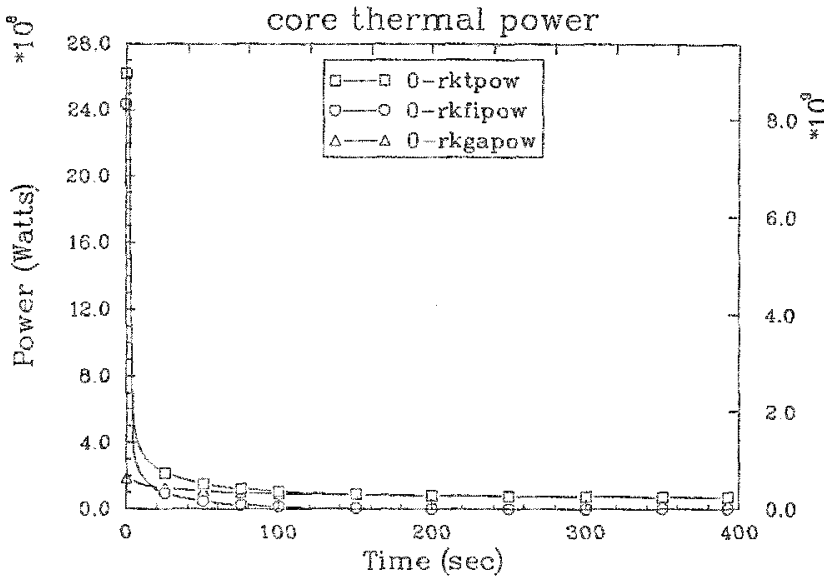

B\&W (OCONEE) SMALL BREAK LOCA PIN FALLURE

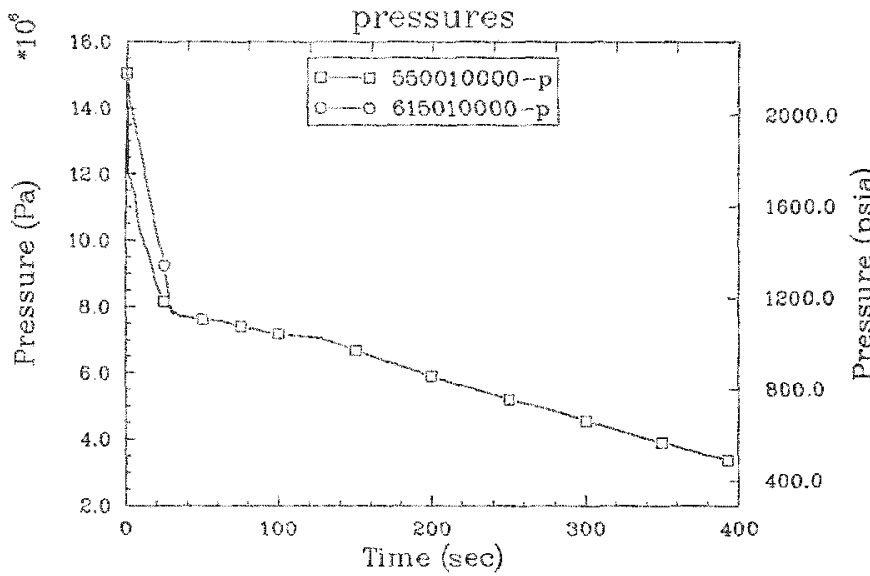

B\&W (OCONEE) SMALL BREAK LOCA PIN FAILURE

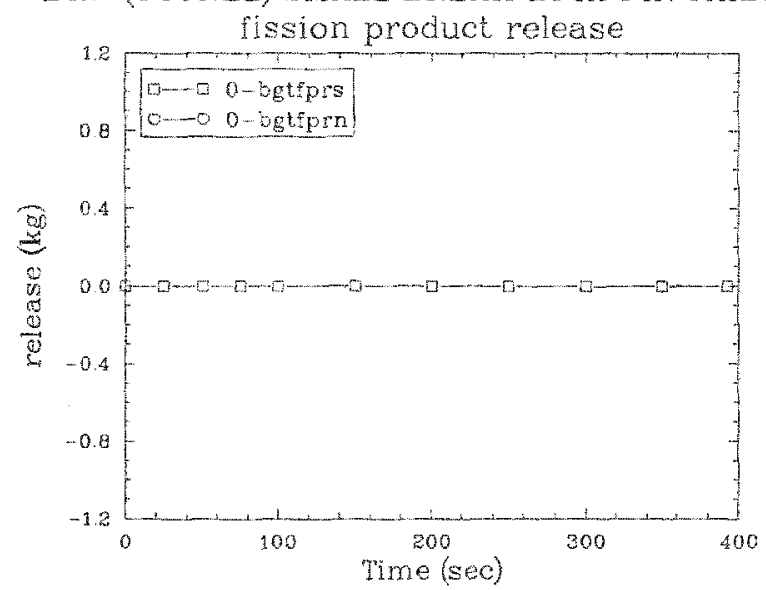

B\&W (OCONEE) SMALL BREAK LOCA PIN FAILURE

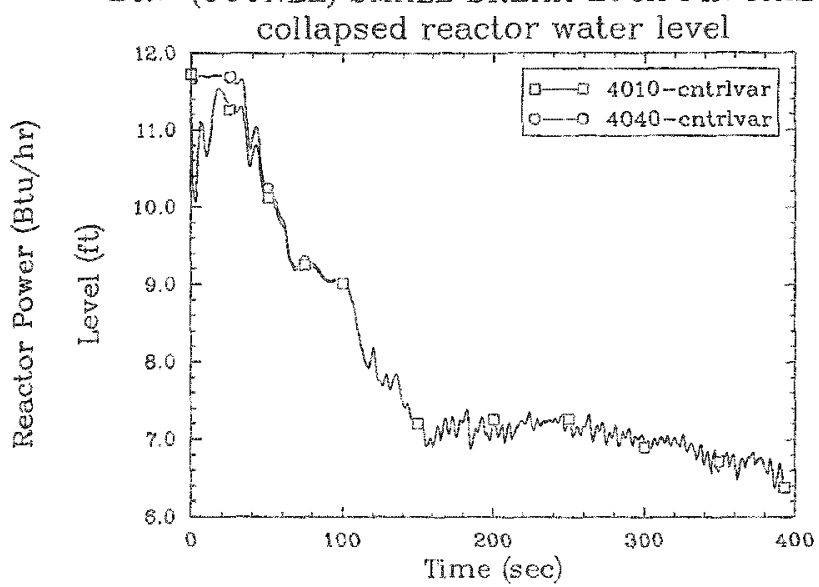

B\&W (OCONEE) SMALI, BREAK LOCA PIN FAILURE

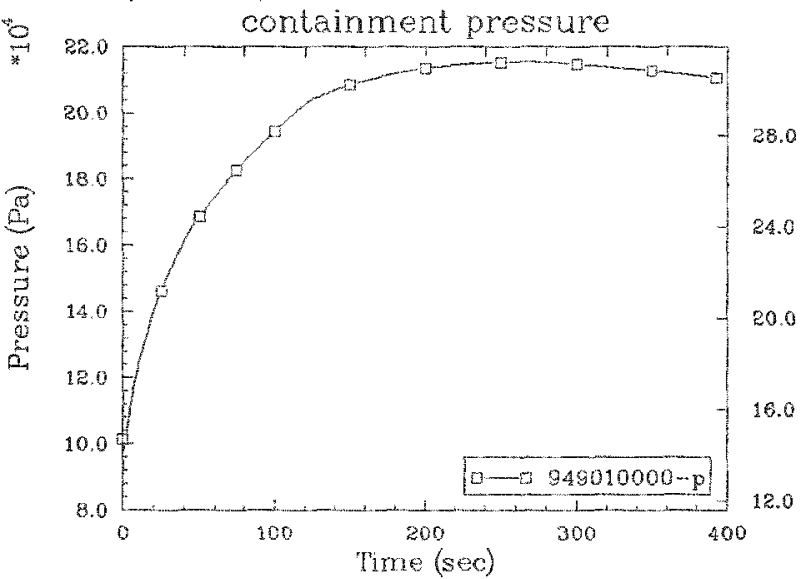

B\&W (OCONEE) SMALL, BREAK LOCA PIN FAIUURE

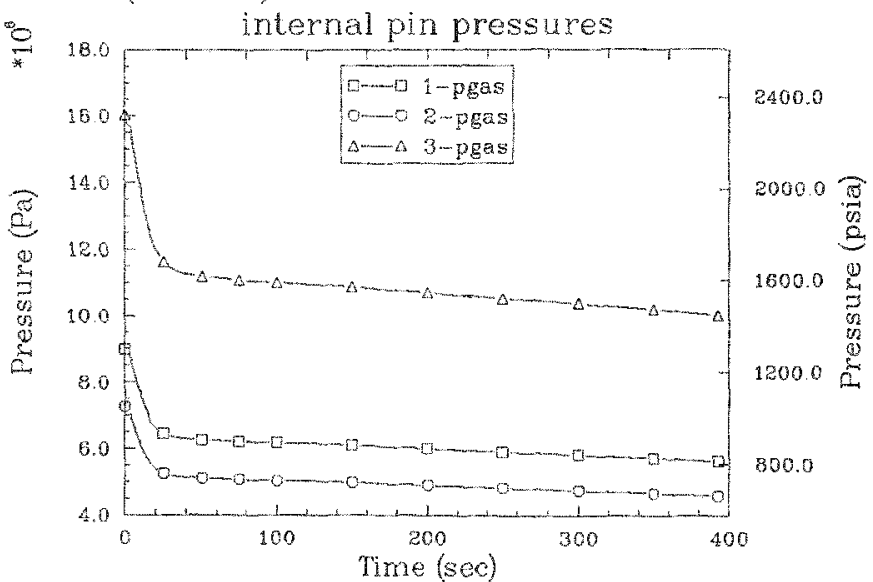


B\&W (OCONEE) SMALIL BREAK LOCA PIN FAILURE

fuel centerline temperatures for 5 GWD/MTU pin

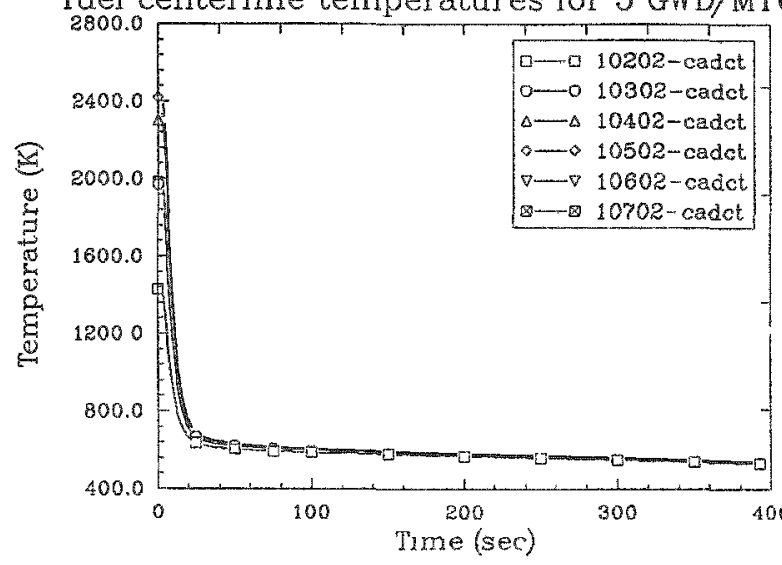

B\&W (OCONEE) SMALL BREAK LOCA PIN FAILURE cladding surface temperatures for 5 GWD/MTU pin

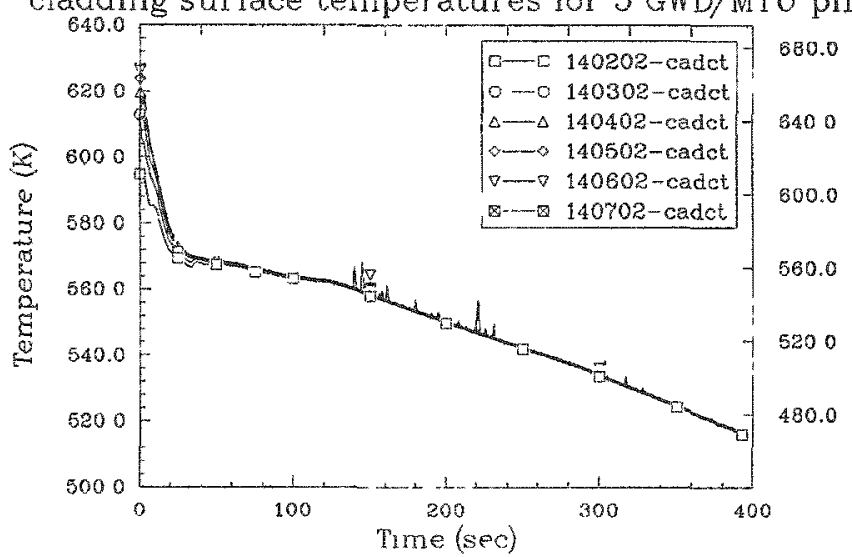

B\&W (OCONEE) SMALL BREAK LOCA PIN FAILURE

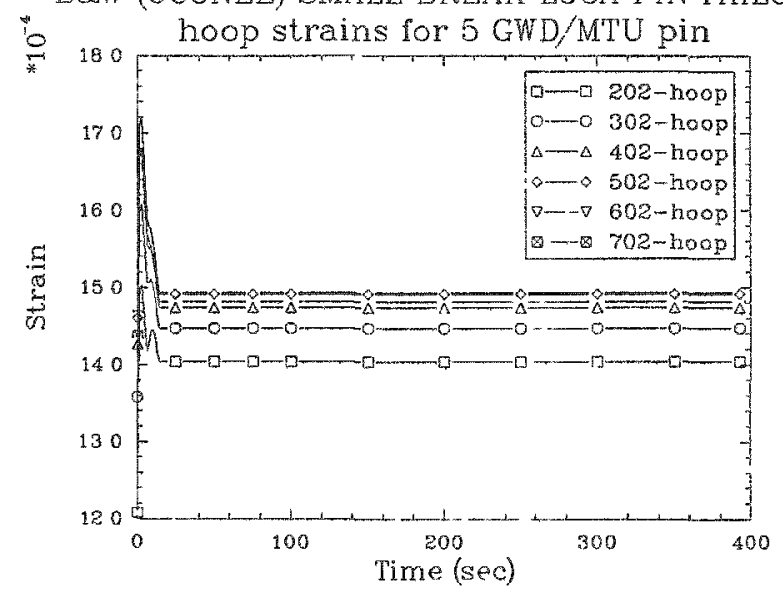
4000.0
B\&W (OCONEE) SMALI BREAK LOCA PIN FALLURE fuel centerline temperatures for 55 GWD/MTU pin

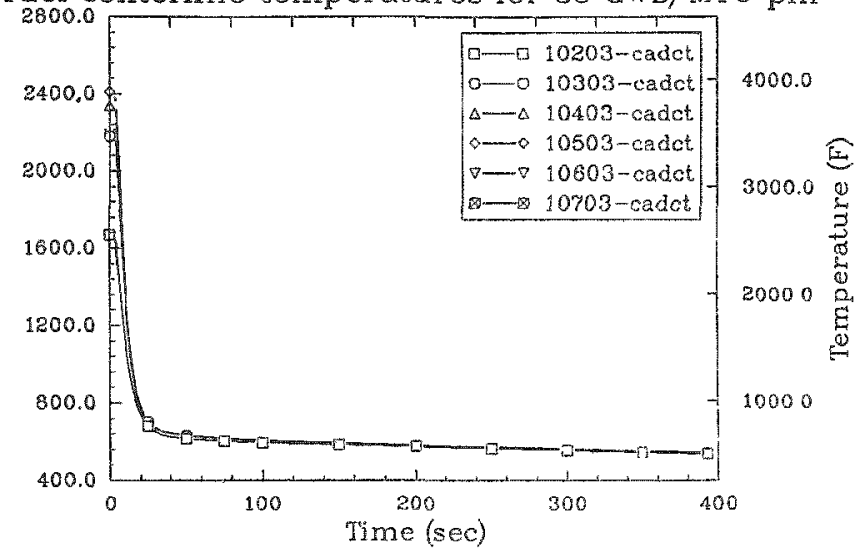

B\&W (OCONEE) SMALL BREAK IOOCA PIN FALUURE cladding surface temperatures for $55 \mathrm{GWD} / \mathrm{MTU}$ pin

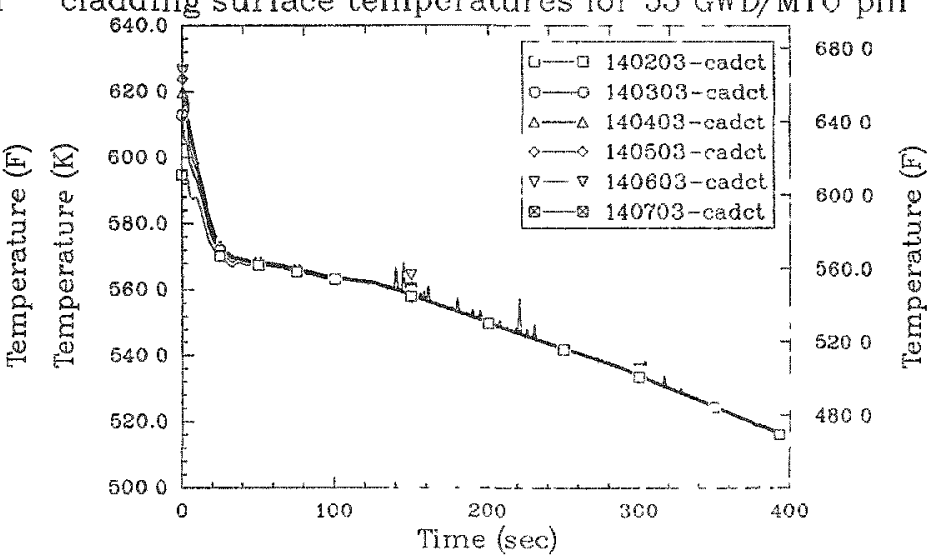

B\&W (OCONEE) SMALL, BREAK LOCA PIN PAILURE

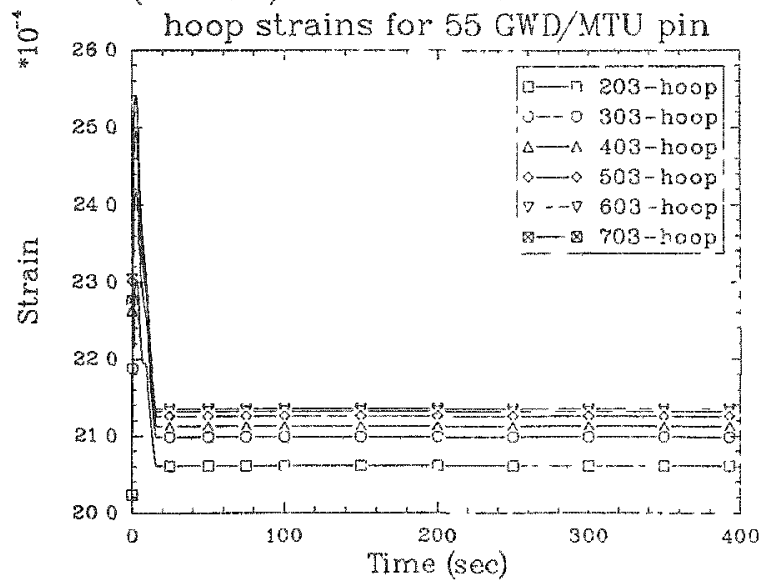



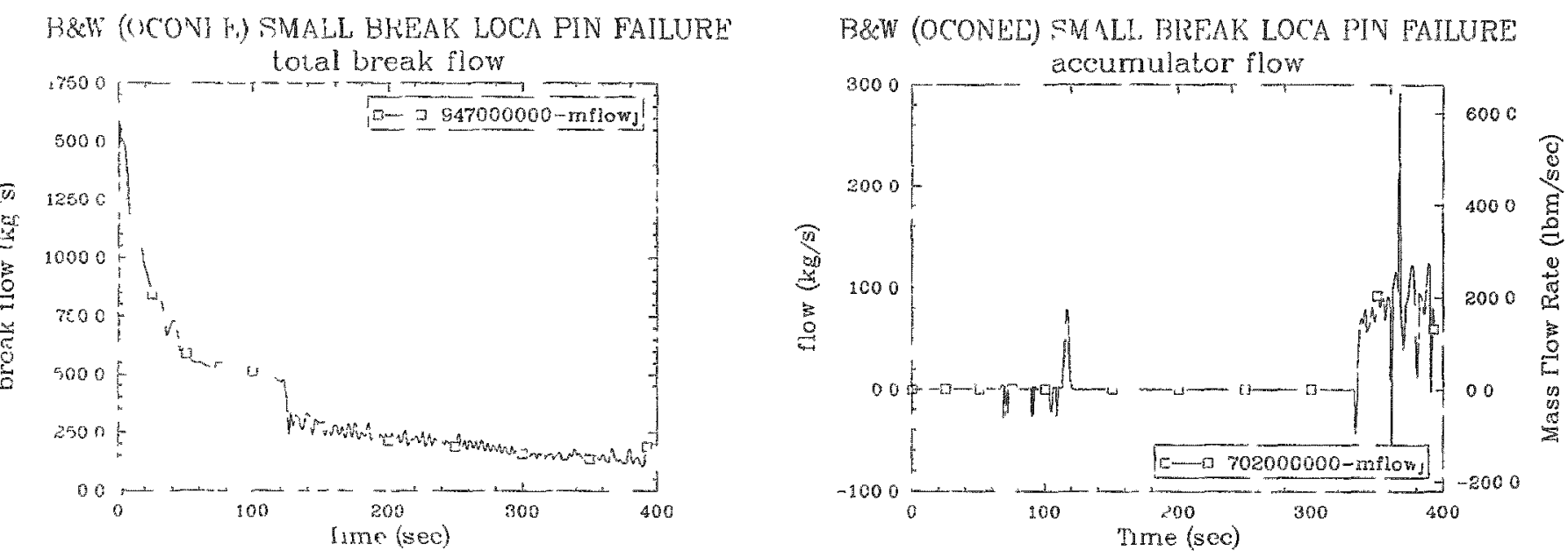

13R (OCONEL) :MALL BREAK LO'A PIN FAILURE

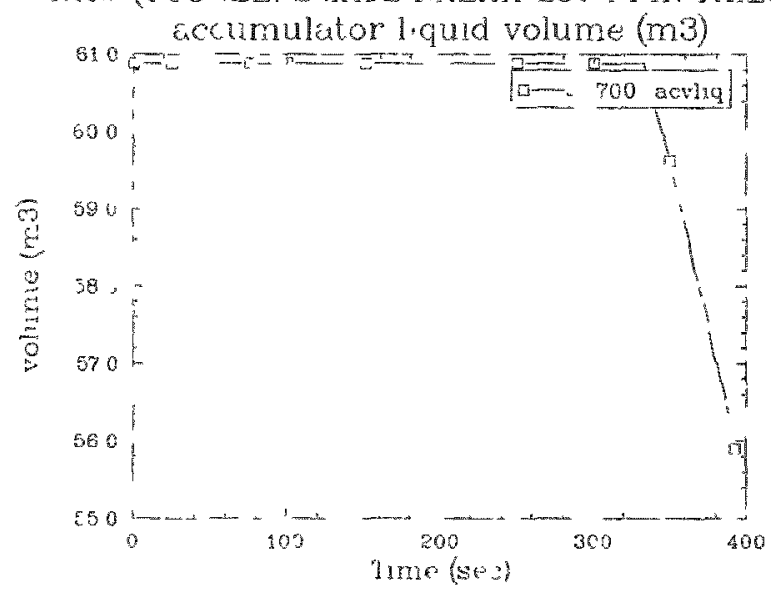

B\&WT (OCONEI) SMALI, IREAR LOCA PIN FAILURE
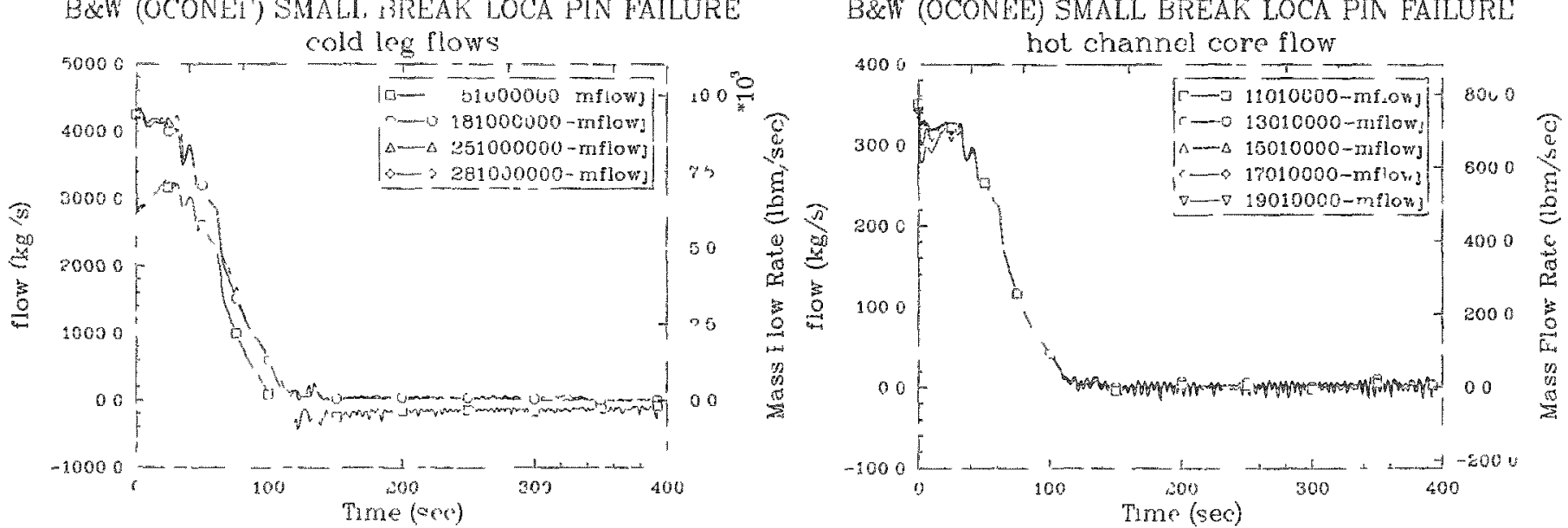
B\&W (OCONEE') SMALI, BRLAK LOCA PIN FAILURE

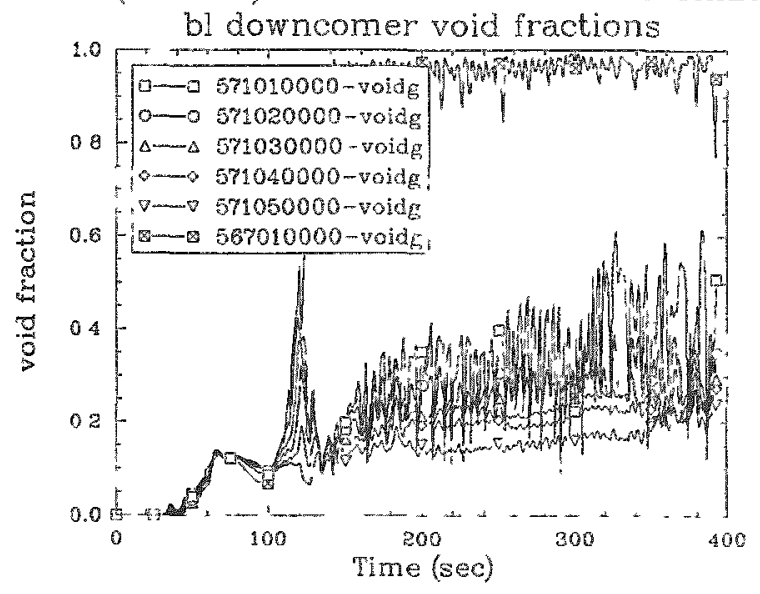

B\&W (OCONEE) SMALL BREAK LOCA PIN FALLIREL

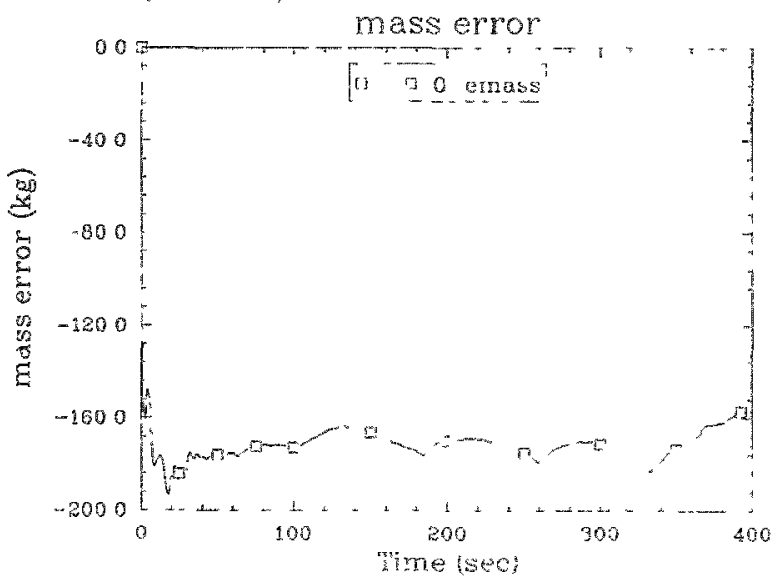

B\&W (OCONEE) SMALL BREAR LOCA P.N FAILLRE

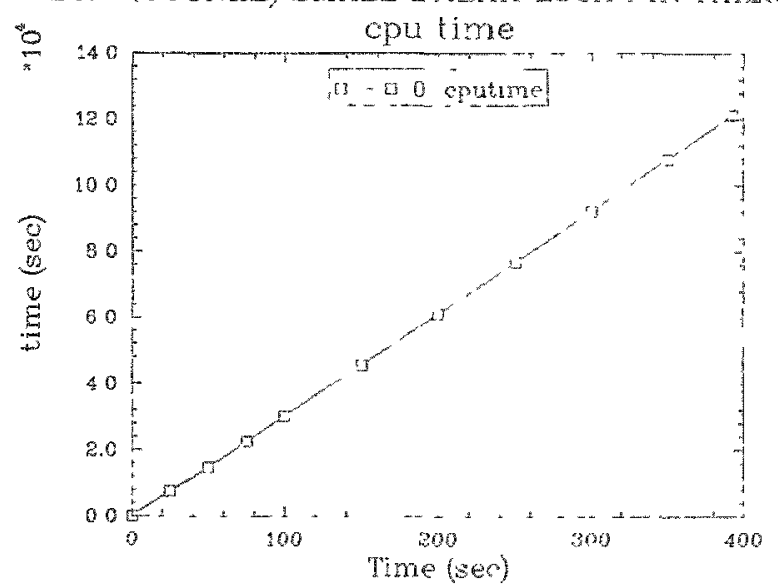

B\&W (OCONEE) SMALL BREAK IOCA PIN FAILURE il downcomer void fractions

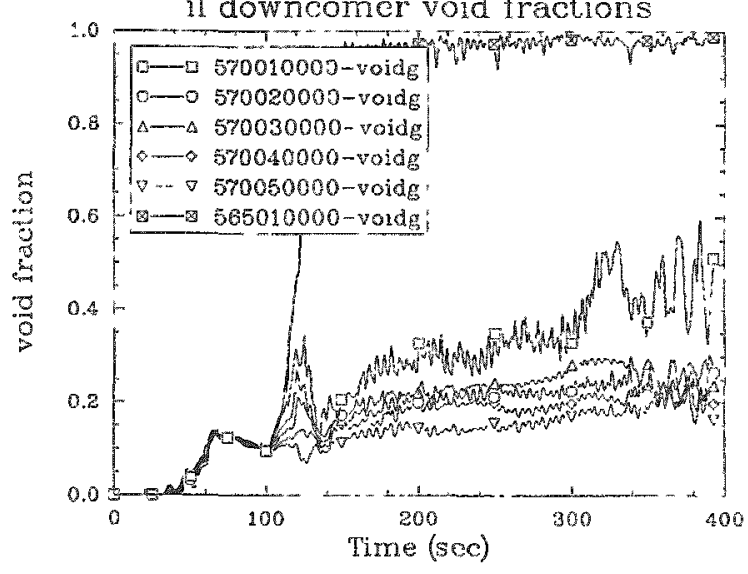

B\&W (OCONEE) SMALL BREAK LOCA PIN FAIILRL

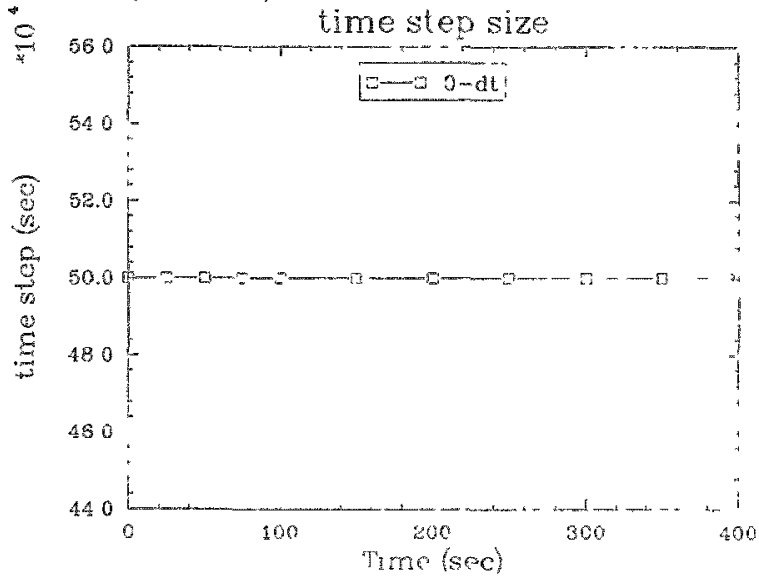


B\&W (OCONLE) 100\% DBA LPF, PUMP TRTP

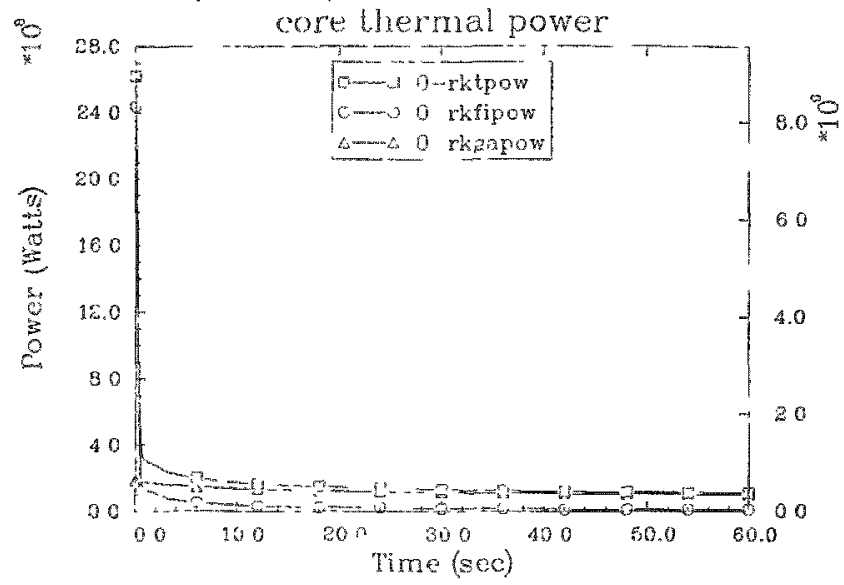

B\&W (OCONHE) 100\% IDBA LPF, PUMP TRIP

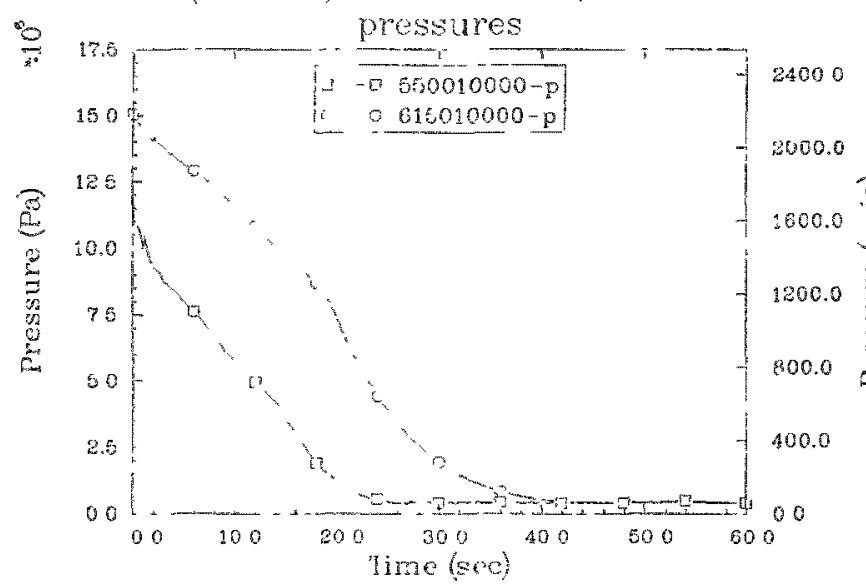

BSW (OCONEE) 100\% DBA LPF, PUMP TRIP

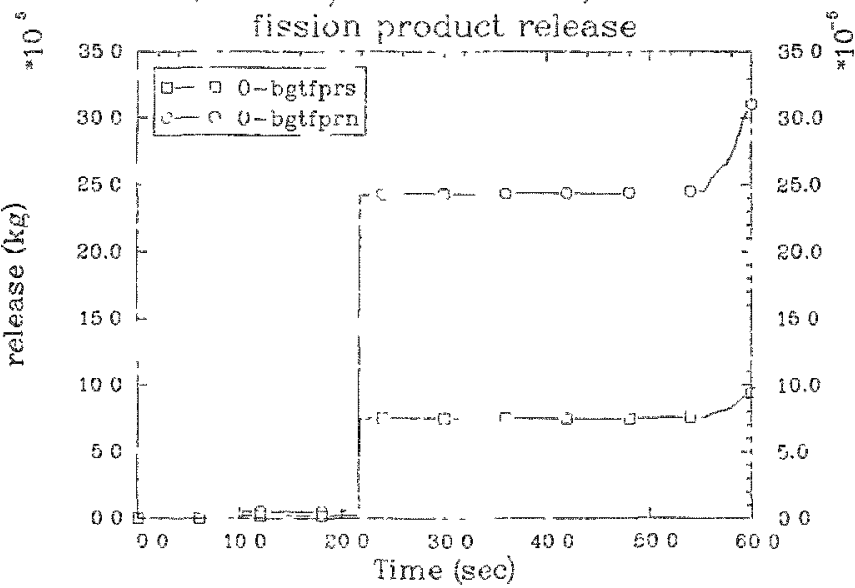

BSW (OCONEE) 100\% DBA LPF, PUMP TRIP

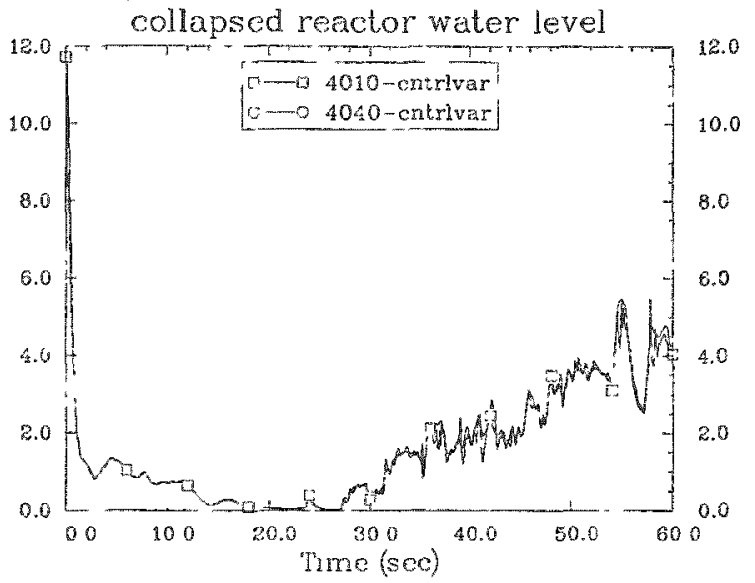

BÂW (OCONEF) 100\% DBA LPF, PUMP TRIP

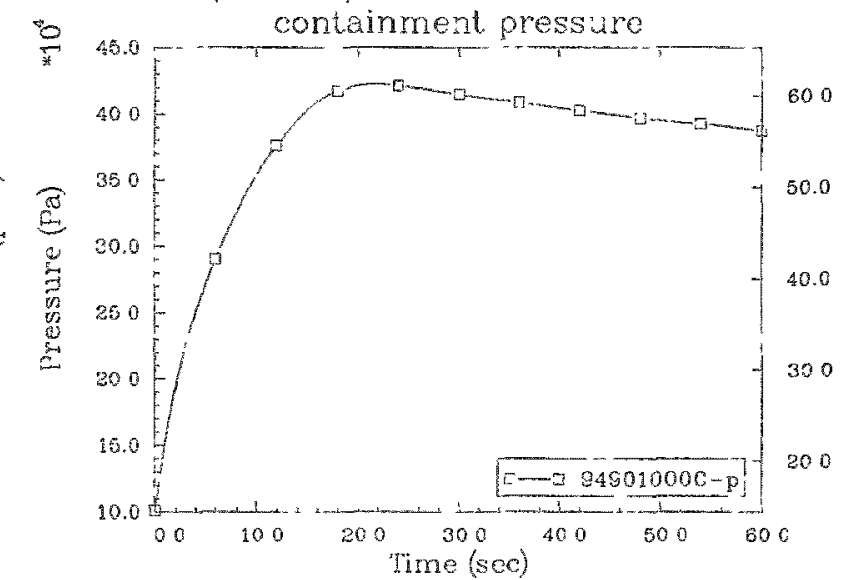

B\&W (OCONEE) 100\% DBA LPF, PUMP TRIP

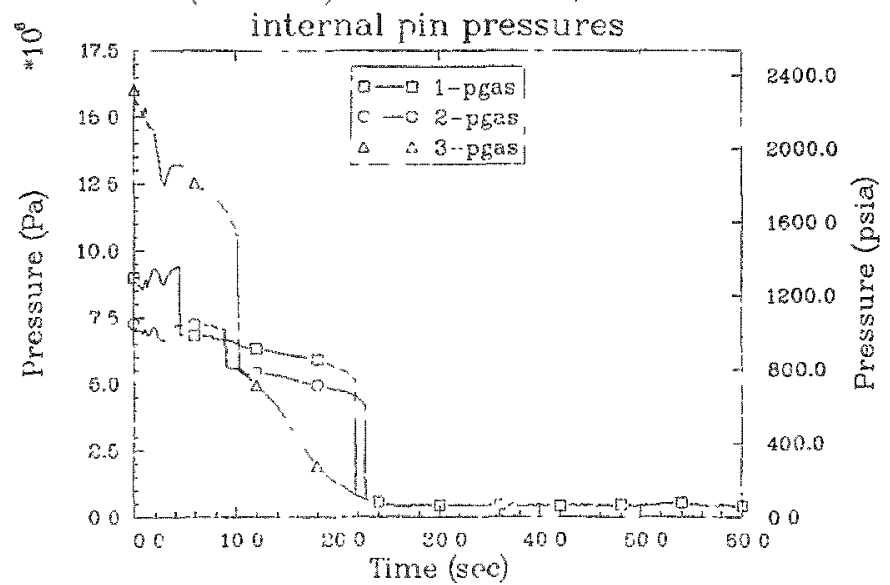


B\&W (OCONEE) 100\% DBA LPH, PUMP TRIP

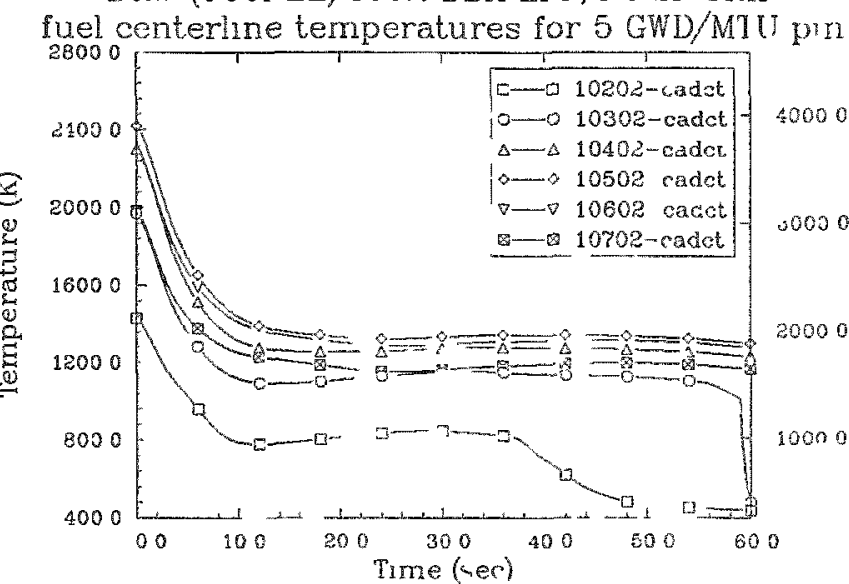

B\&F (OCONEE) 100\% DBA LPF, PUMP TRIP cladding surface temperatures for $5 \mathrm{CWD} / \mathrm{MTU}$ pin

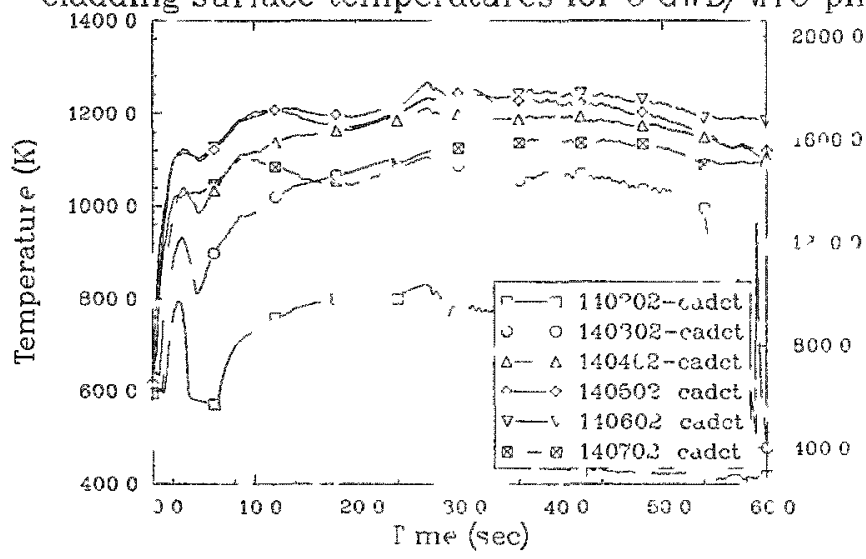

B\&W (OCONEE) 100\% DBA I PF, PUMP PRIP hoop strams for 5 GWD/MTU pin

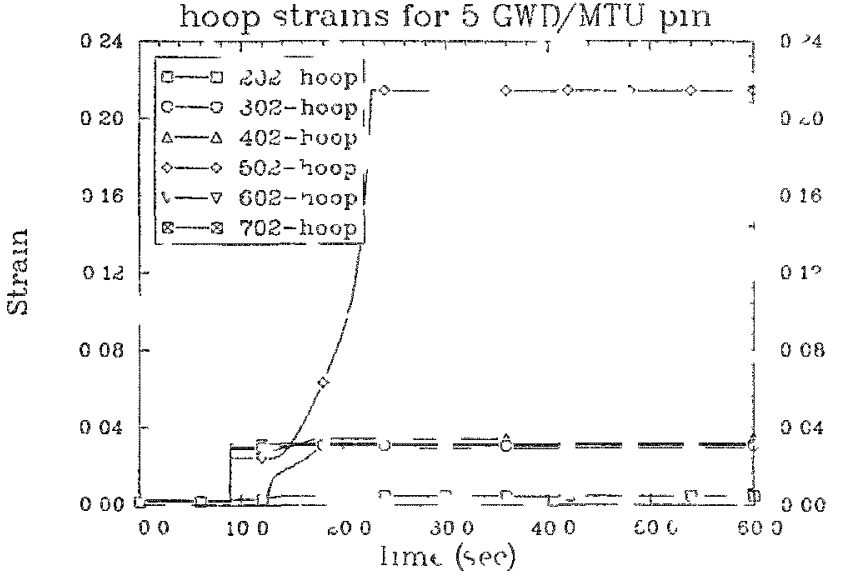

B\&W (OCONL $\mathrm{L}$ ) 100\% DBA IPF, PUMP TRIP fuel centerine tomperatures for 55 GWD/MTU pin

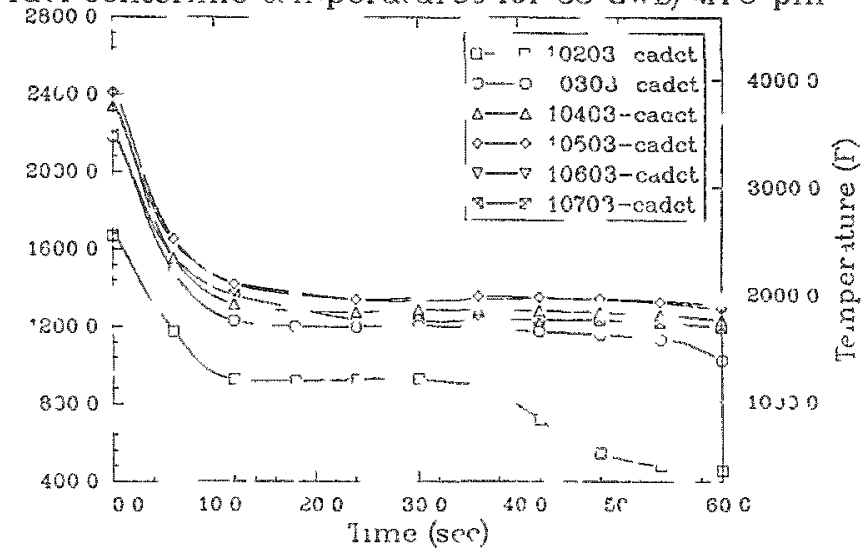

B\&W (OCONCL) 100\% DBA I PF PUMF TRIP cladding surface temperatures for 55 GWD MTU pin

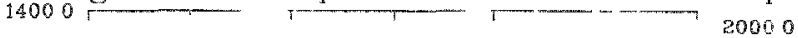

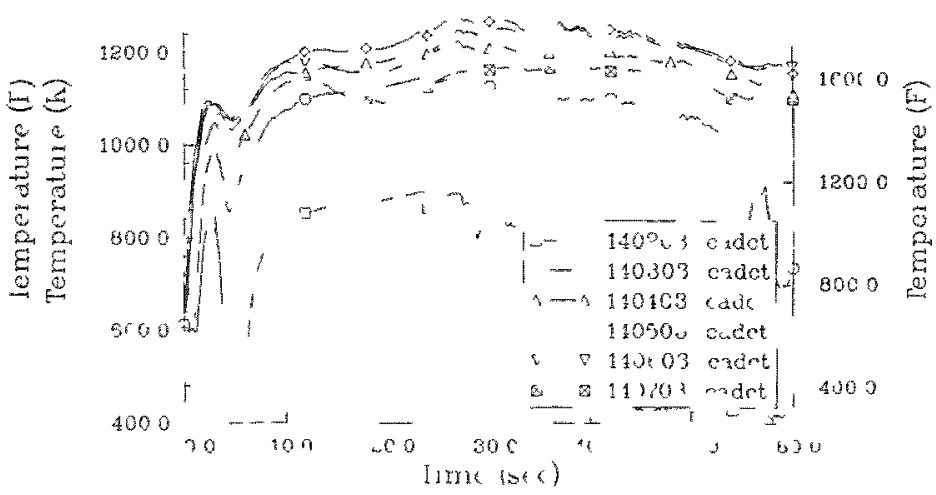

B\&W (OCONL) 100. DB I 'T 'Th TEL'

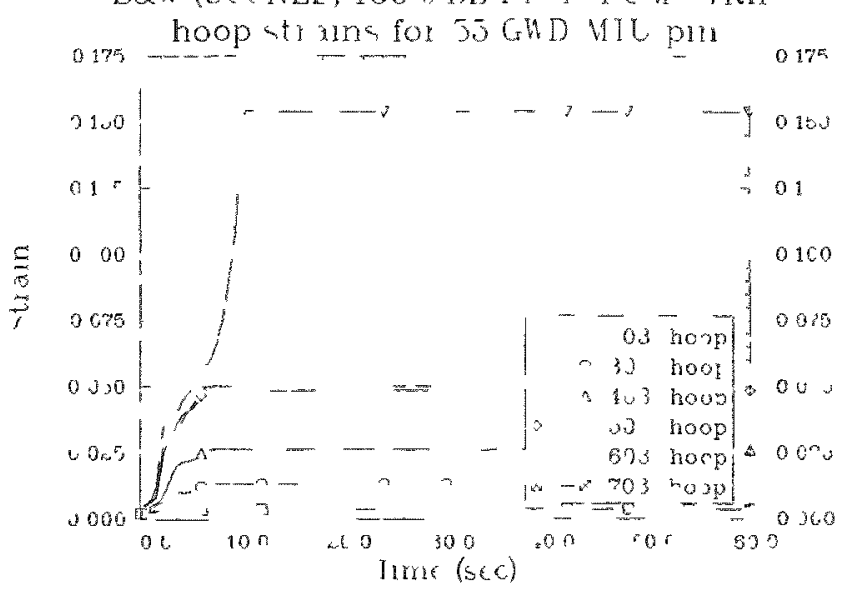



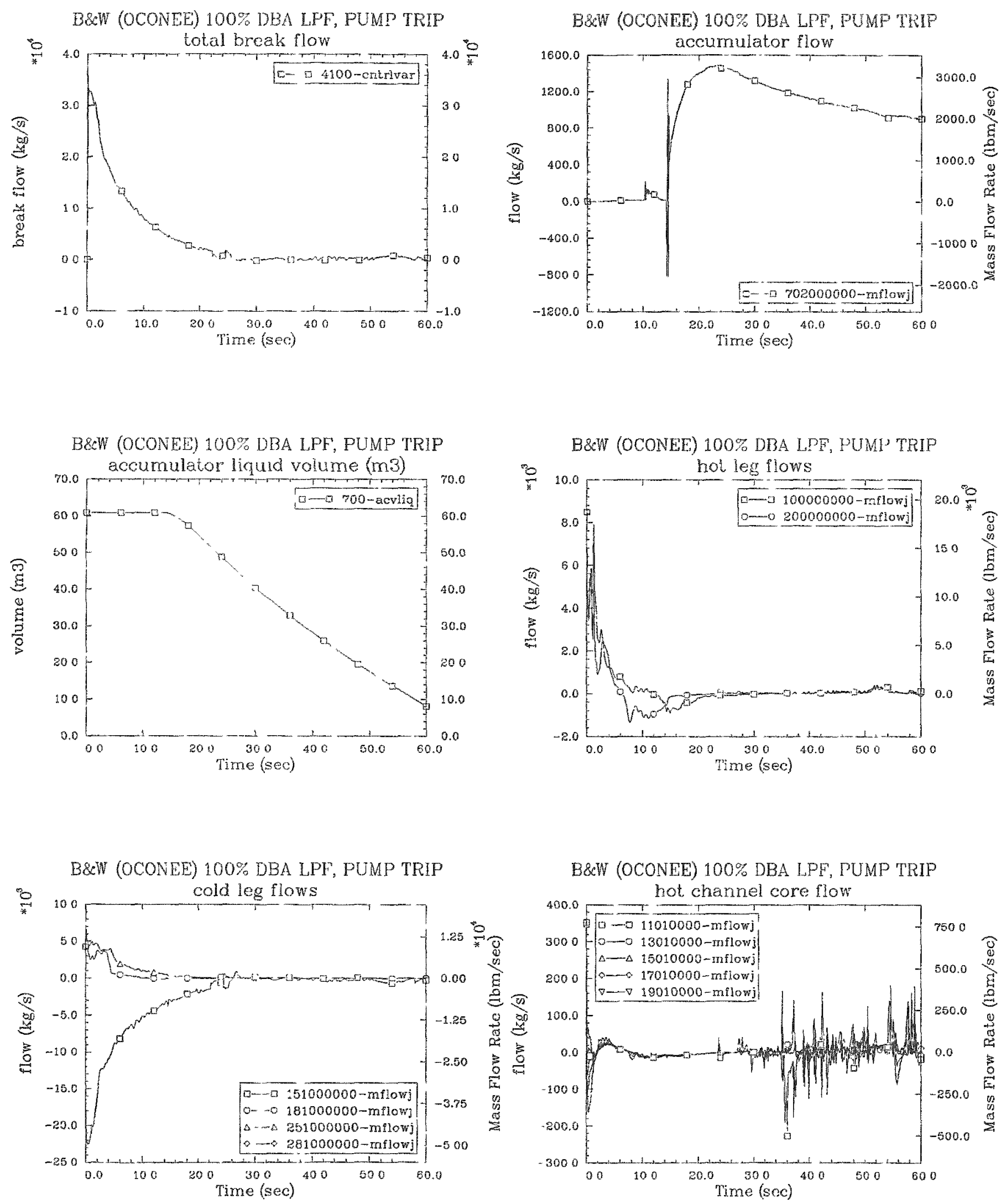

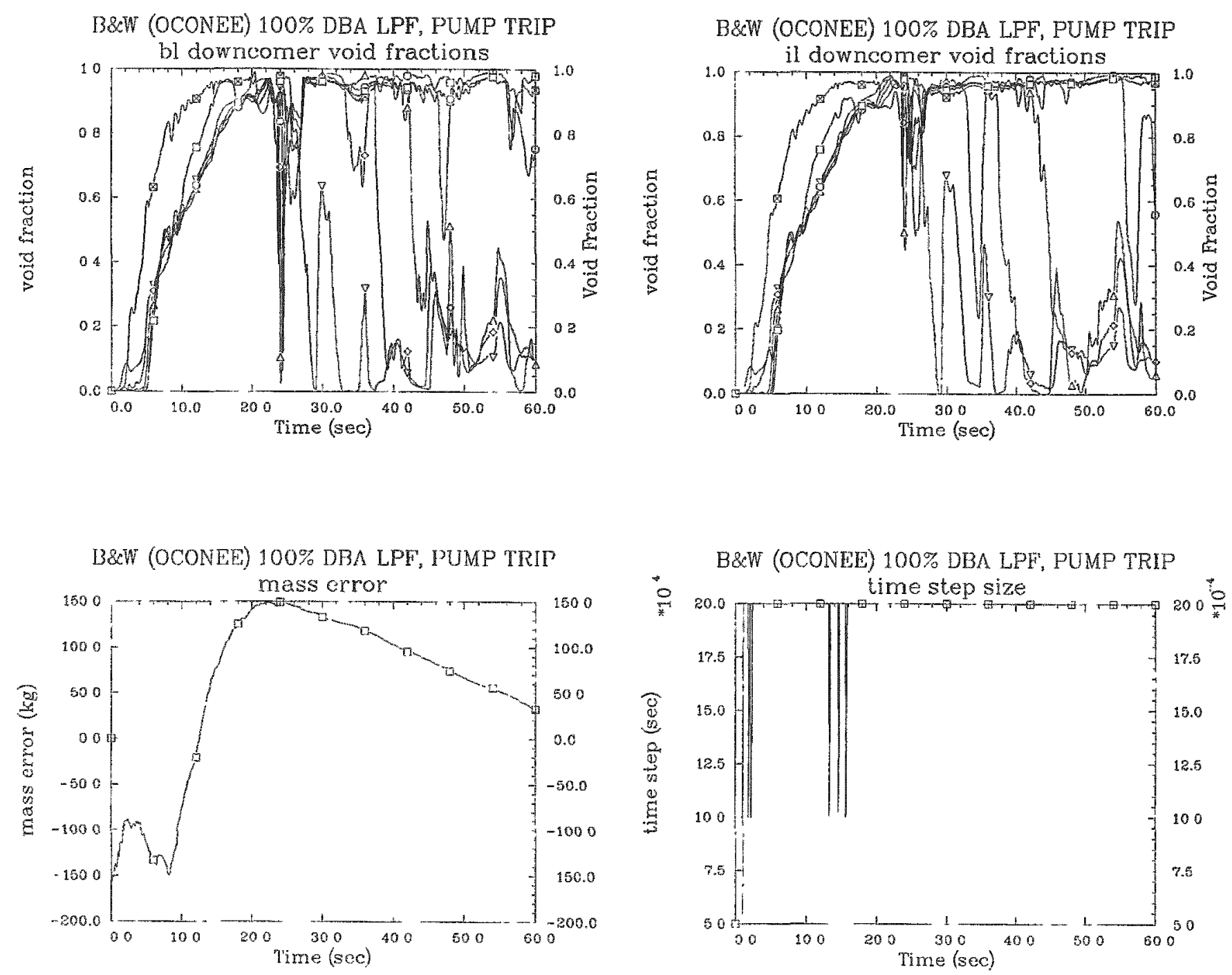

B\&W (OCONEF) 100\% DBA LPF, PUMP TRIP

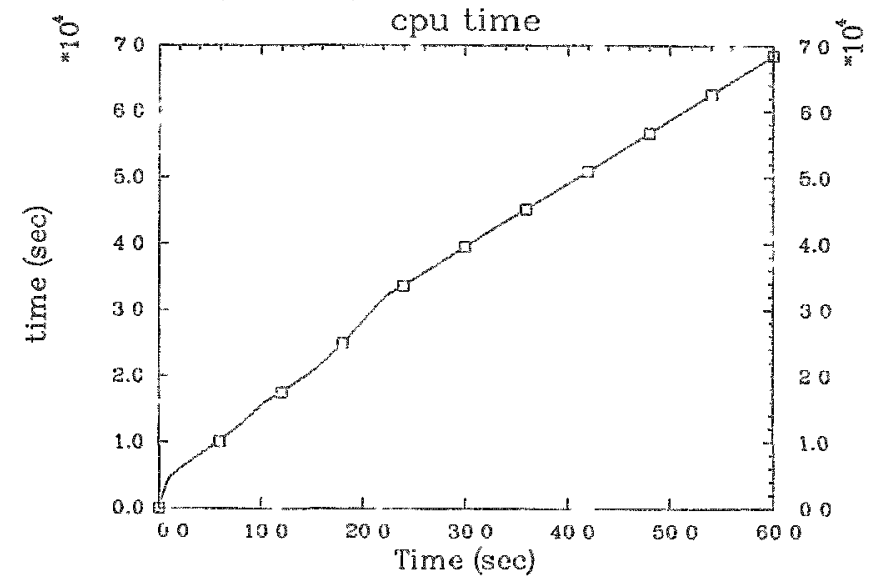


B\&W (OCONEE) 100\% DBA LPF W/ ECCS core thermal power

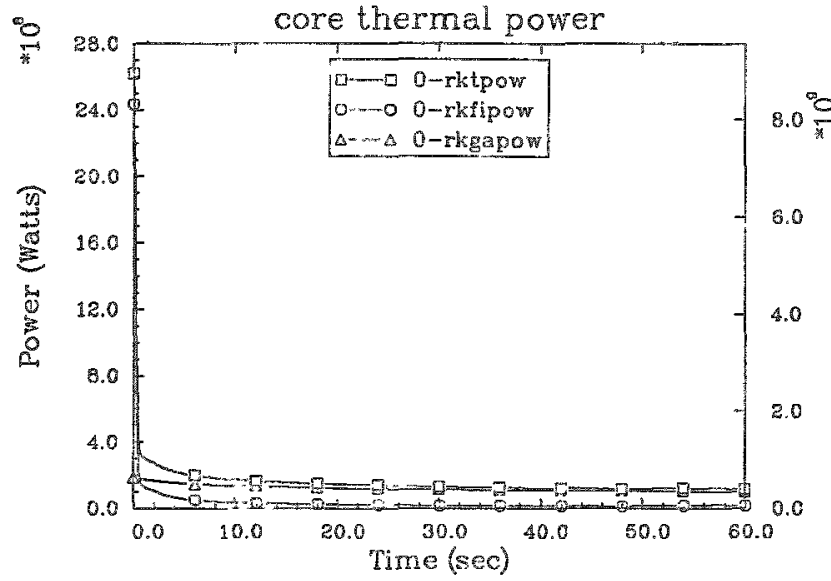

B\&W (OCONEE) 100\% DBA LPF W/ ECCS

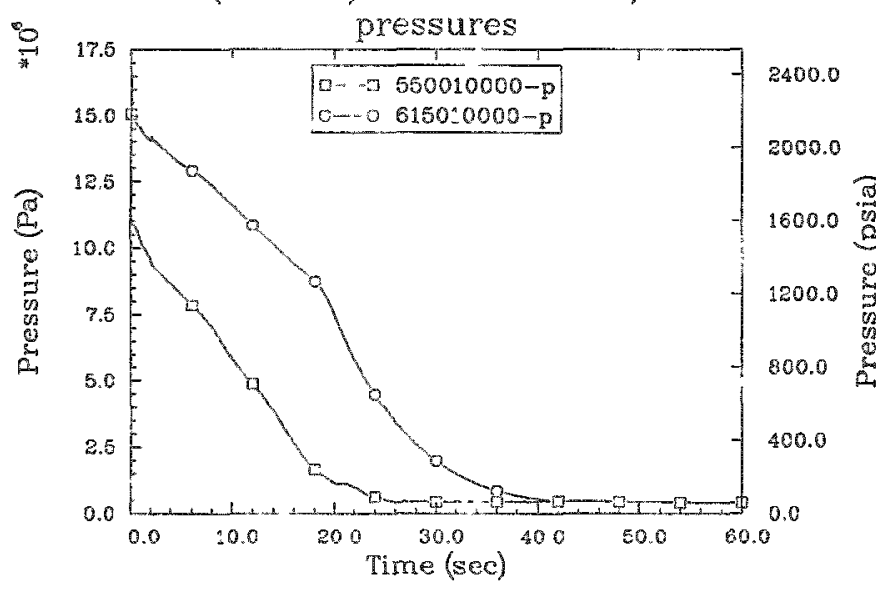

B\&W (OCONEE) 100\% DBA LPF W/ ECCS

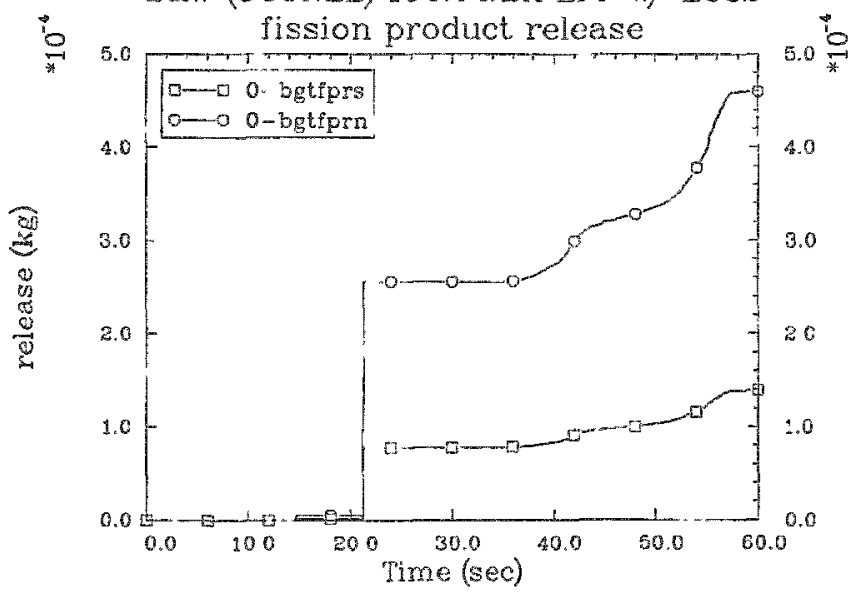

BEW (OCONEE) 100\% DBA LPF W/ ECCS collapsed reactor water level

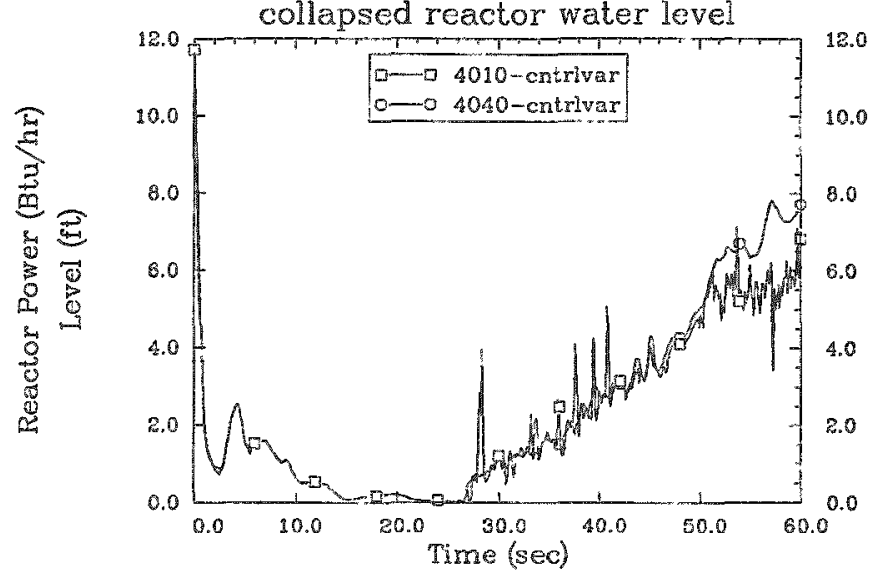

B\&W (OCONEE) 100\% DBA LPF W/ ECCS

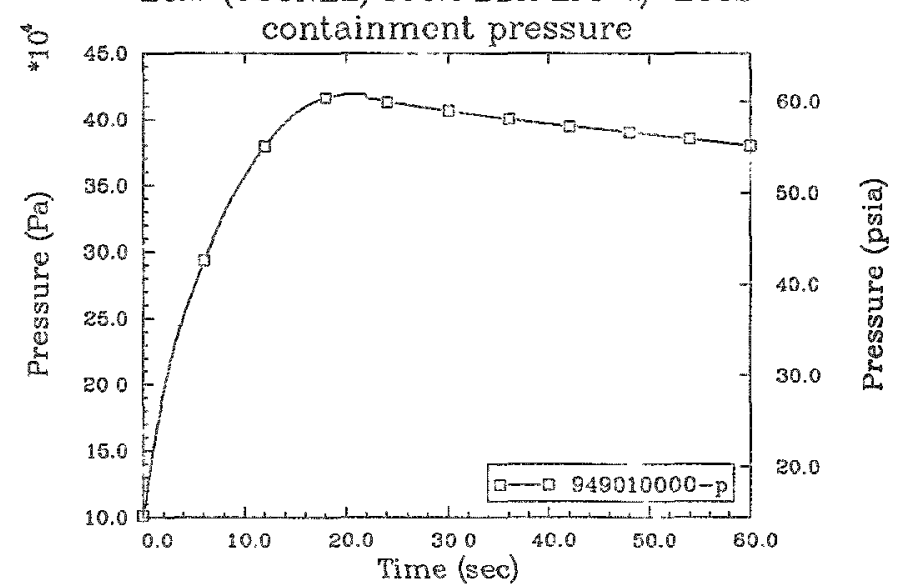

B\&W (OCONEE) 100\% DBA I.PF W/ ECCS

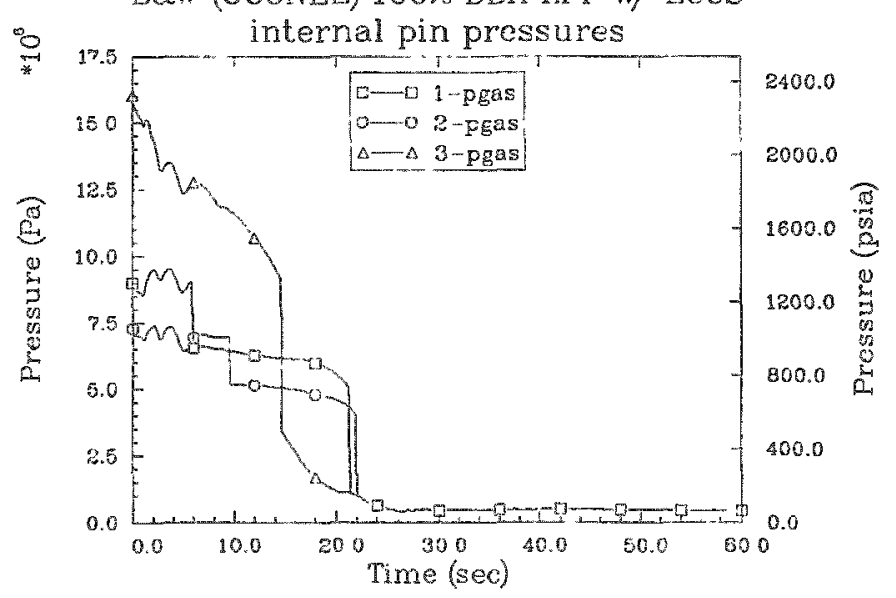


B\&W (OCONEE) 100\% DBA LPF W/ ECCS

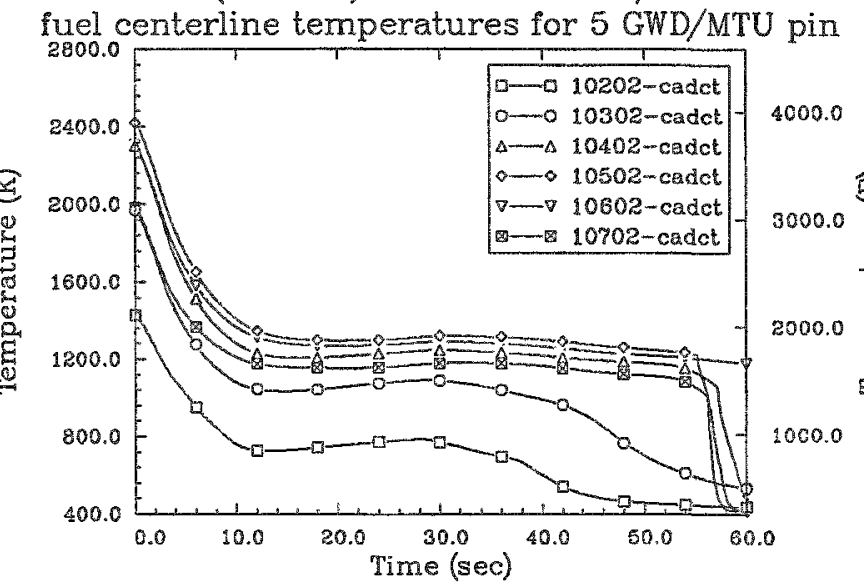

B\&W (OCONEE) 100\% DBA LPF W/ ECCS cladding surface temperatures for $5 \mathrm{GWD} / \mathrm{MTU}$ pin

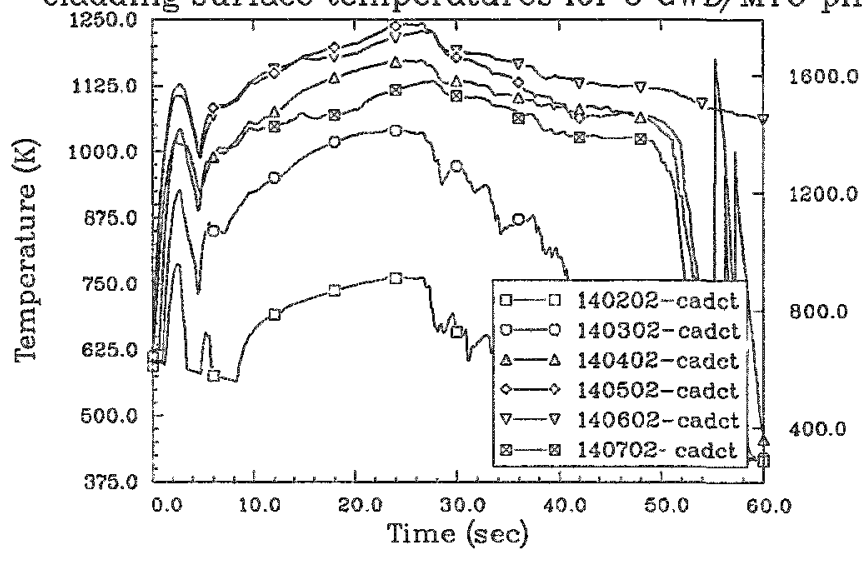

B\&W (OCONEE) 100\% DBA LPF W/ ECCS

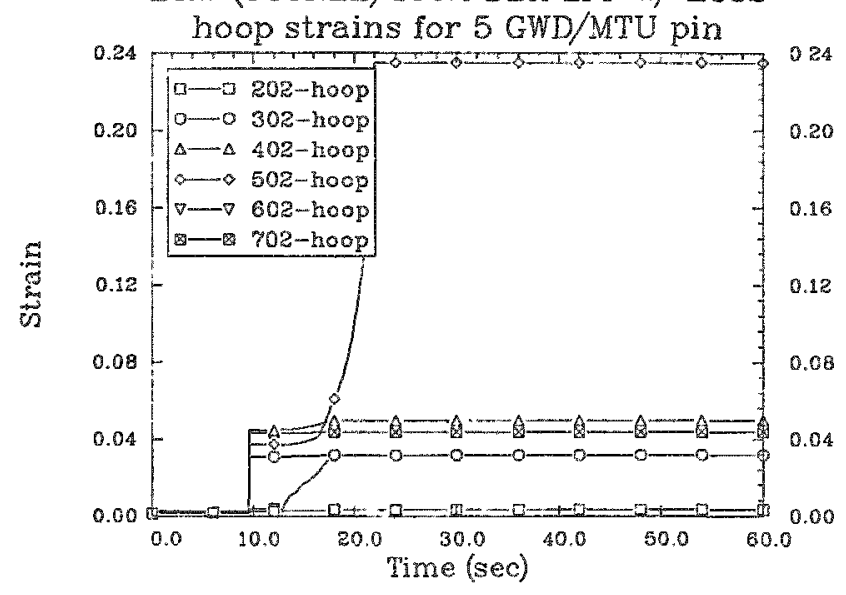

B\&W (OCONEE) 100\% DBA LPF W/ ECCS

fuel centerline temperatures for $55 \mathrm{GWD} / \mathrm{MTU}$ pin

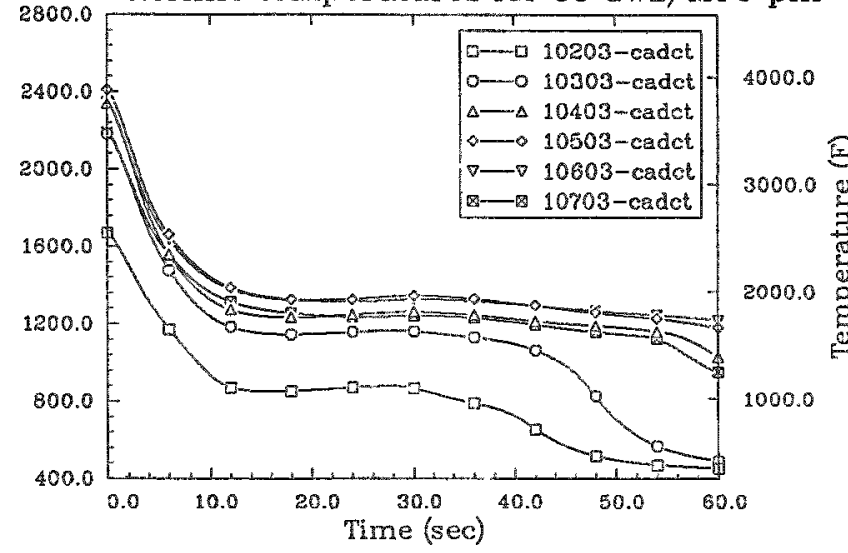

B\&W (OCONEE) 100\% DBA LPF W/ ECCS

cladding surface temperatures for $55 \mathrm{GWD} / \mathrm{MTU}$ pin
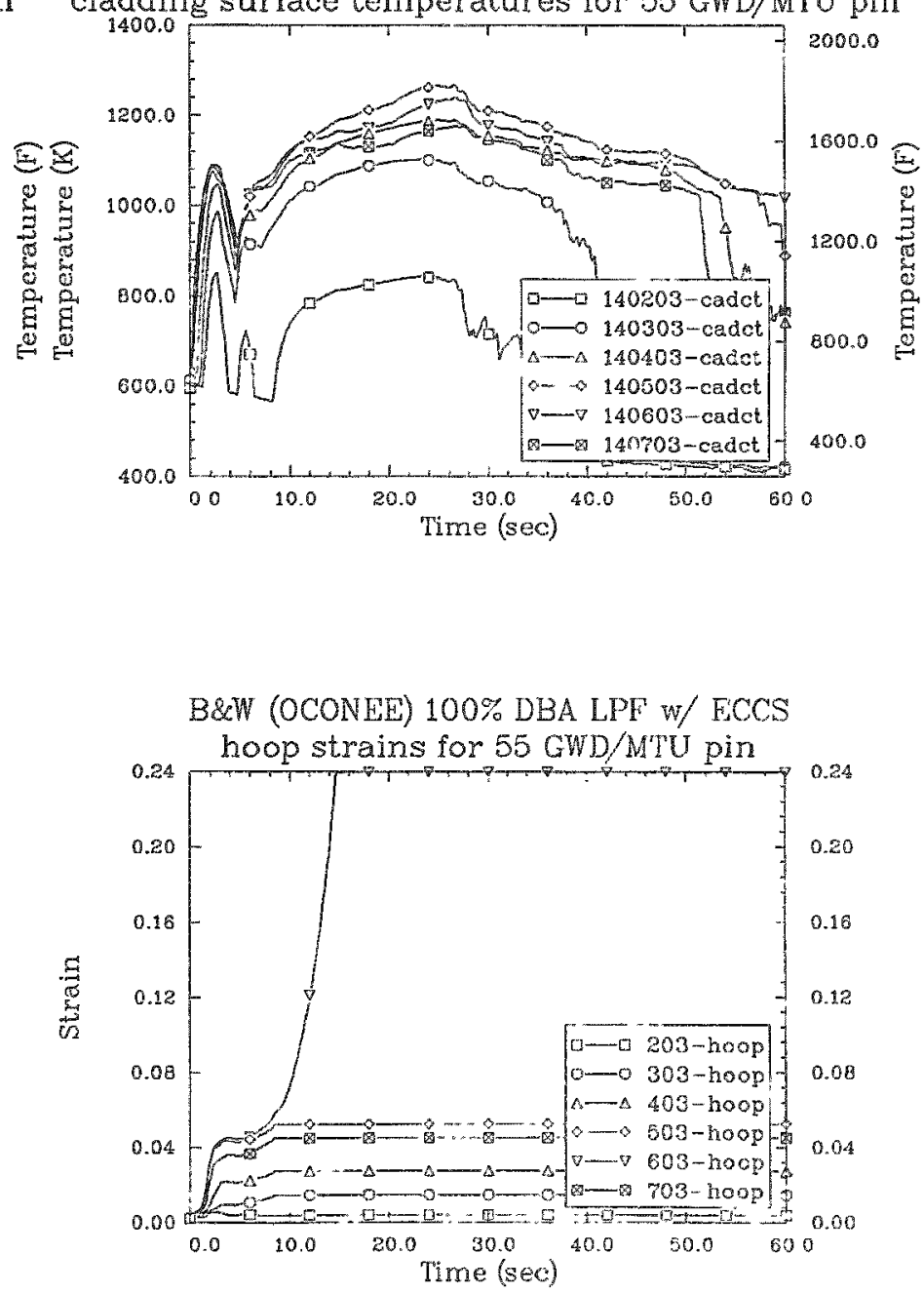

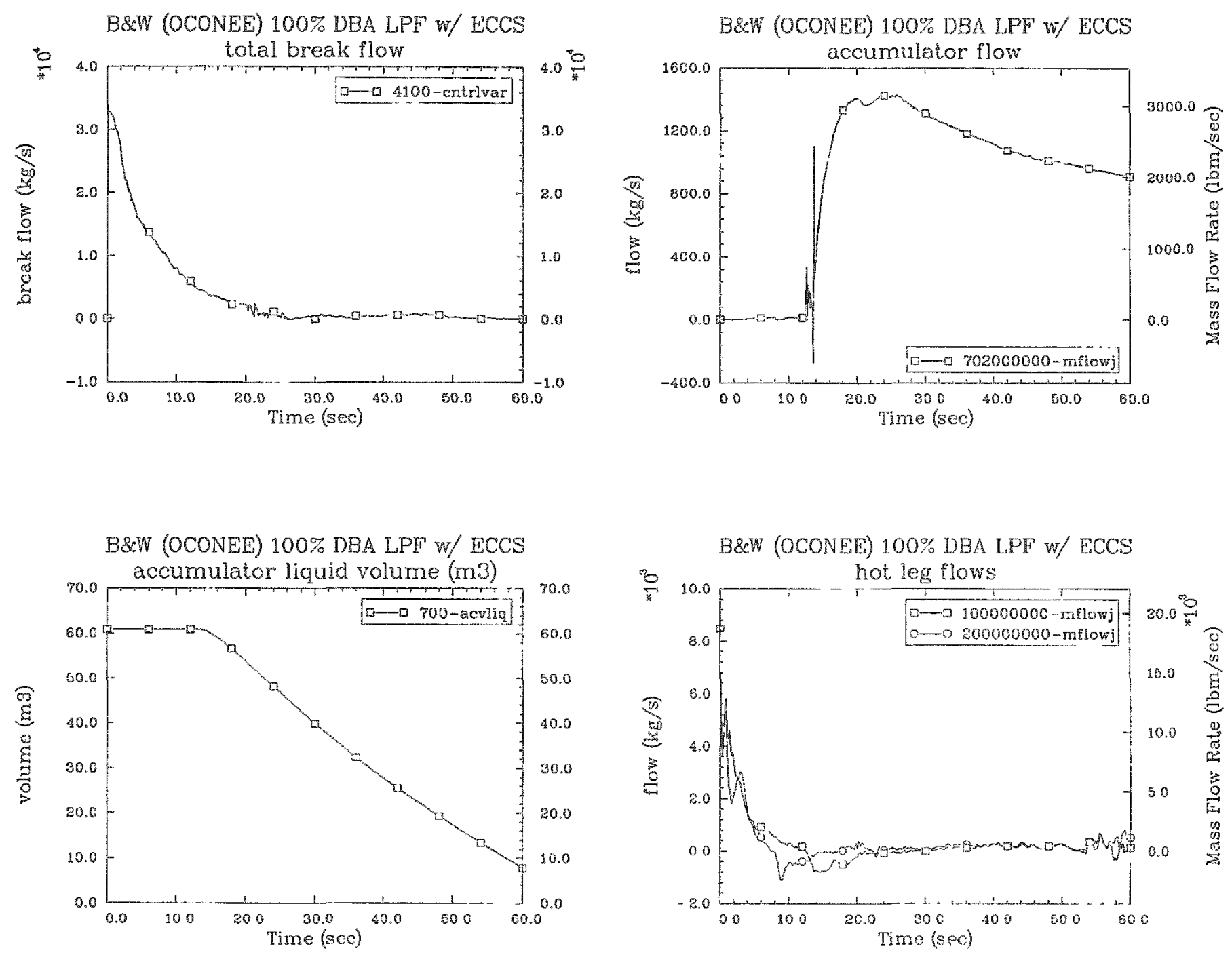

B\&W (OCONEE) 100\% DBA L.PF W/ ECCS

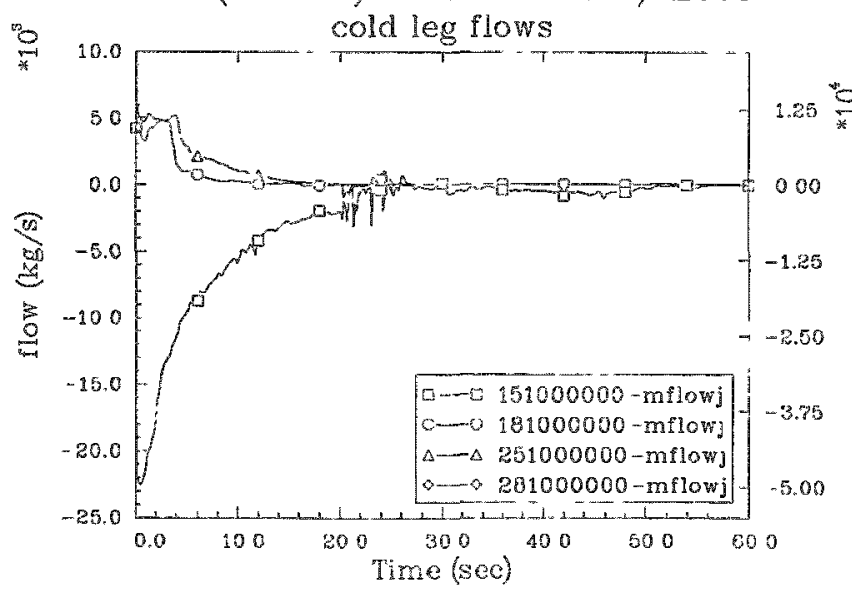

B\&W (OCONEE) 100\% DBA IPF W/ LCCS

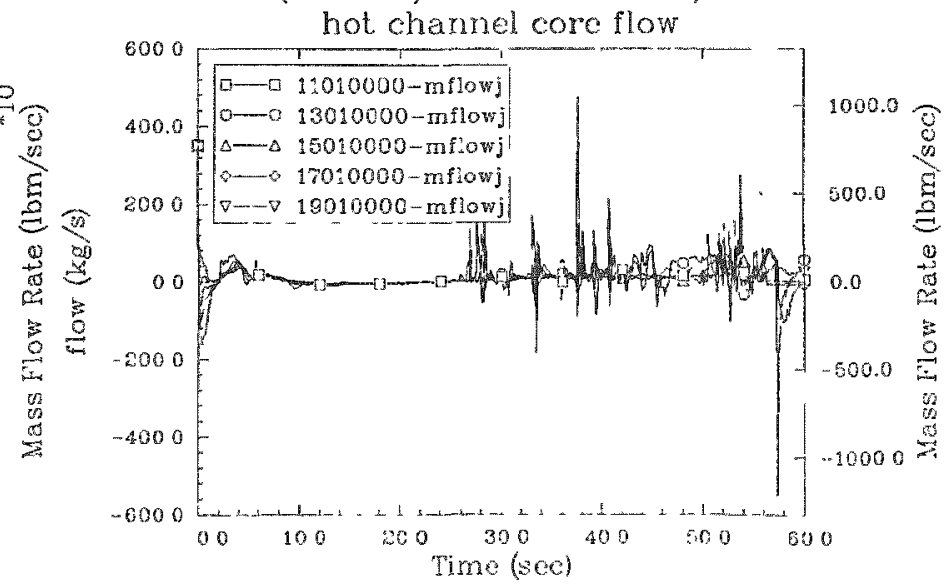


B\&W (OCONEE) 100\% DBA LPI' W/ ECCS

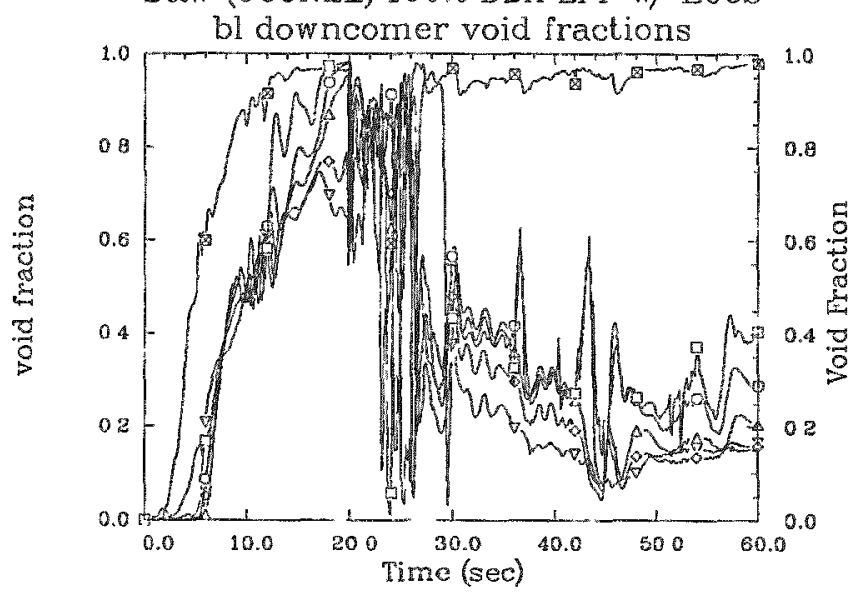

B\&W (OCONEE) 100\% DBA LPF' W/ ECCS

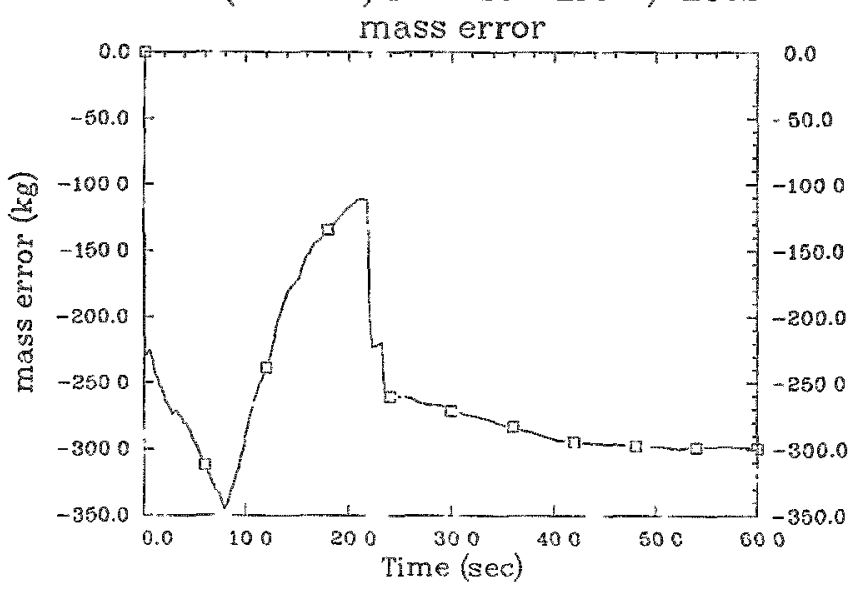

B\&W (OCONEF) 100\% DBA LPF W/ ECCS

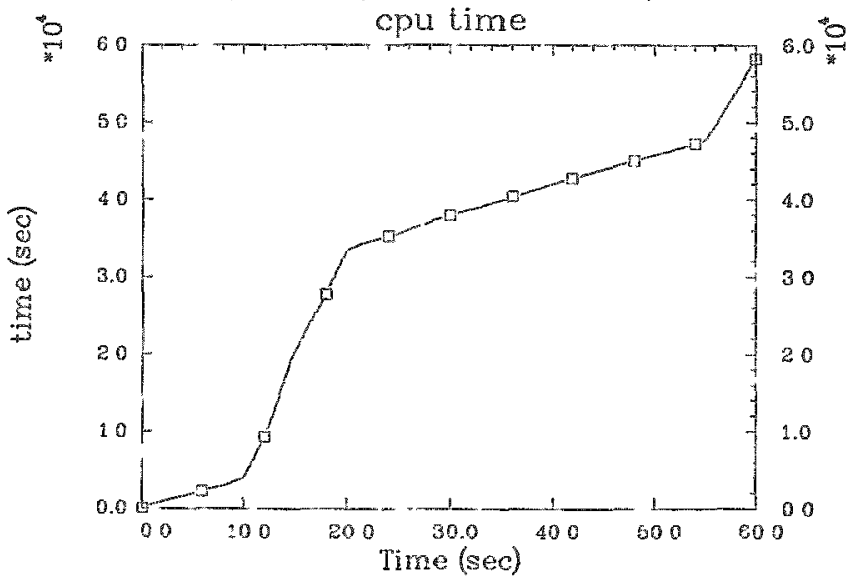

B\&W (OCONEE) 100\% DBA LPF W/ ECCS

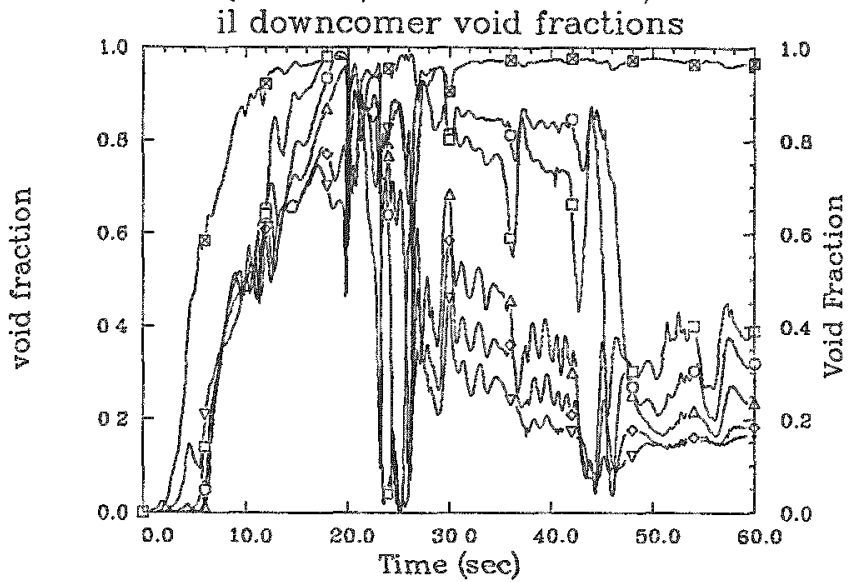

B\&W (OCONEE) 100\% DBA L.PF W/ ECCS

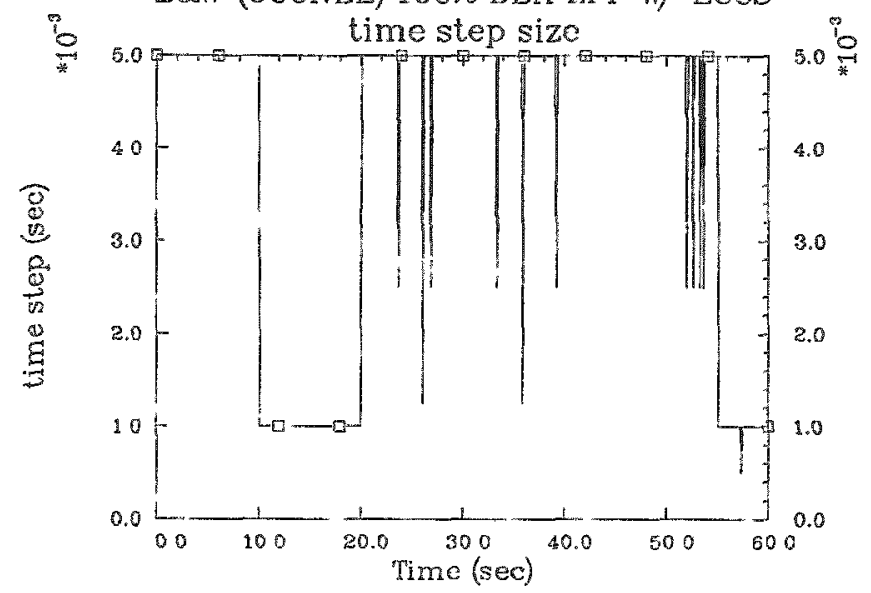

B\&W (OCONEE) 100\% DBA L.PF W/ ECCS

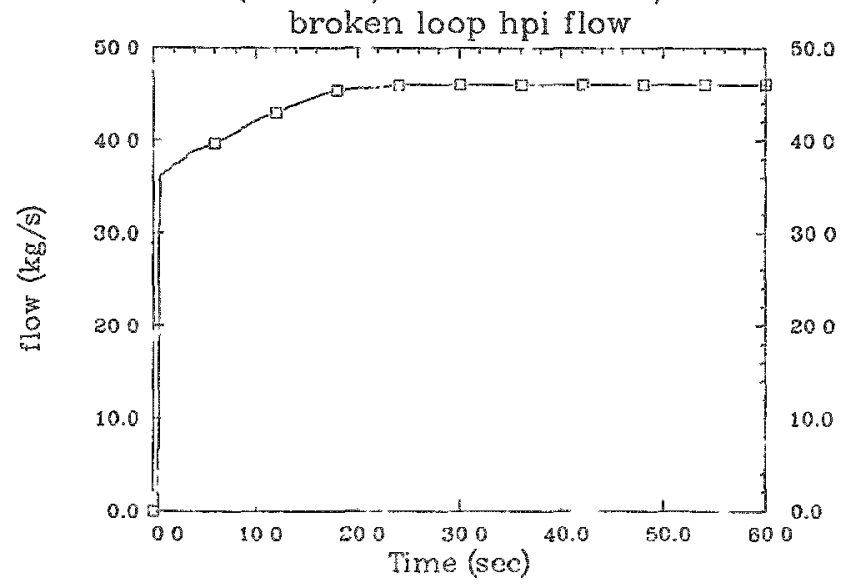



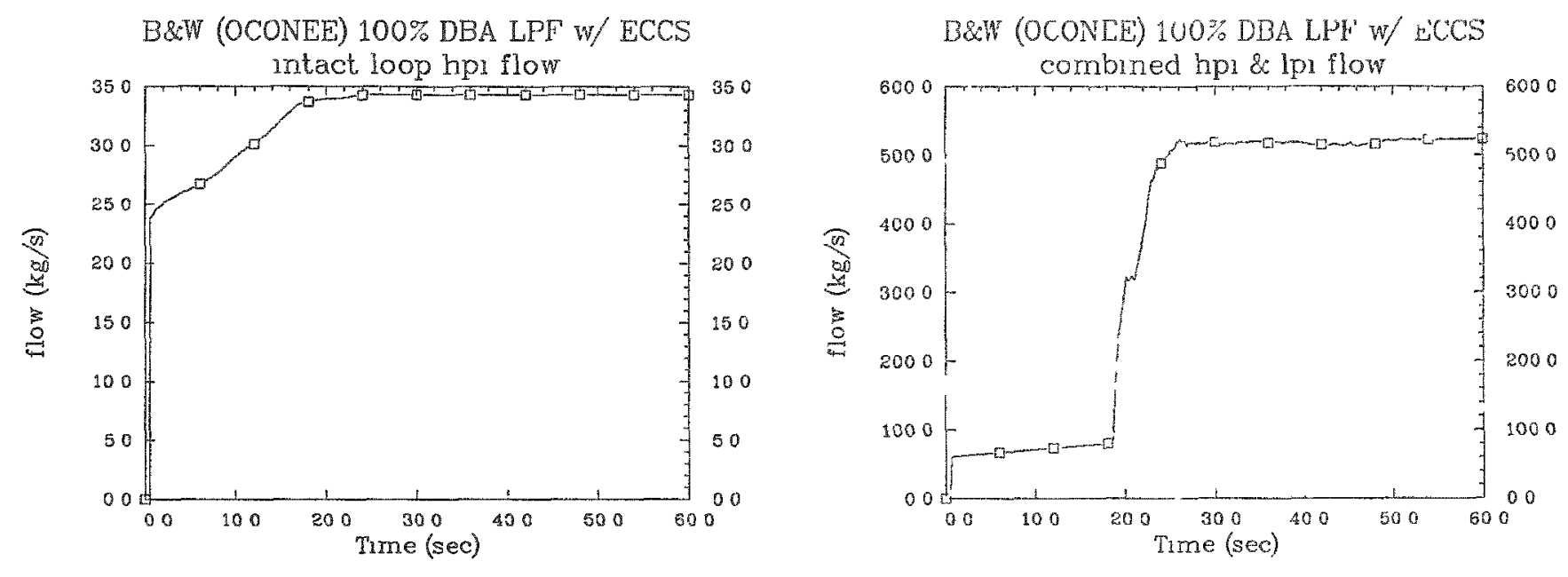
B\&W (OCONEE) 100\% DBA LPF, PUMP TRIP \& ECCS

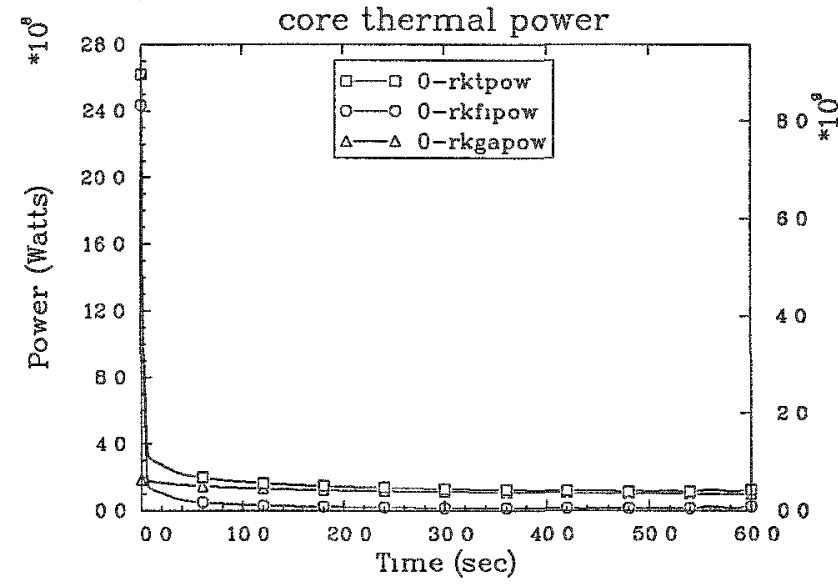

B\&W (OCONEE) 100\% DBA LPF, PUMP TRIP \& ECCS

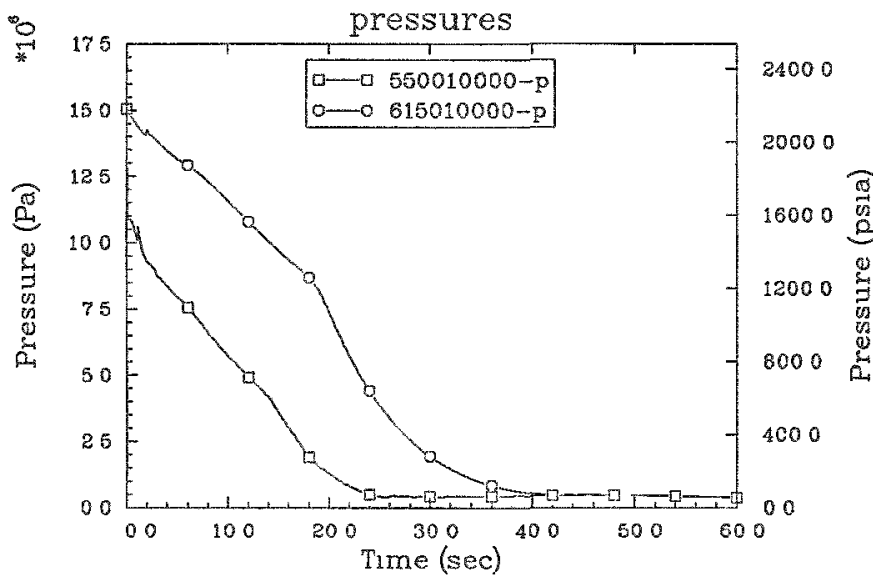

B\&W (OCONEE) 100\% DBA LPF, PUMP TRIP \& ECCS

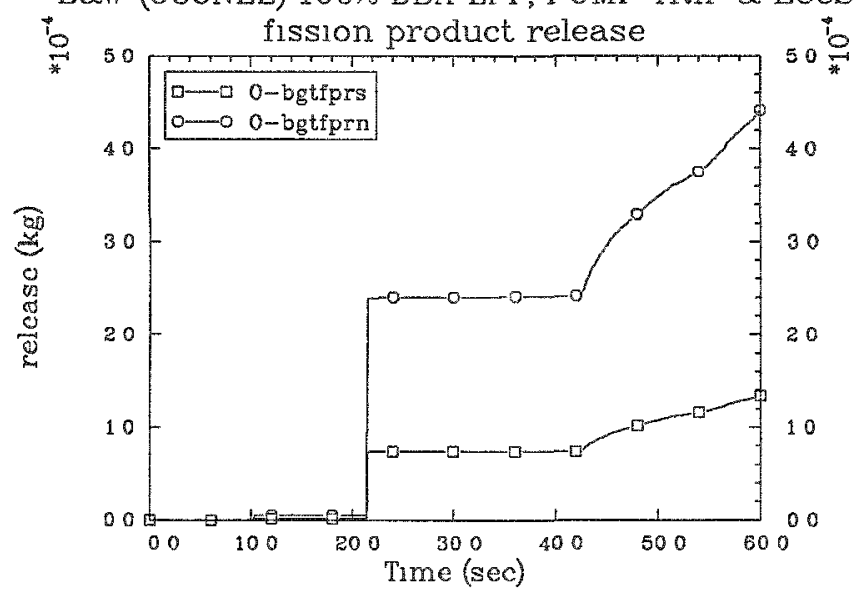

B\&W (OCONEE) 100\% DBA LPF, PUMP TRIP \& ECCS

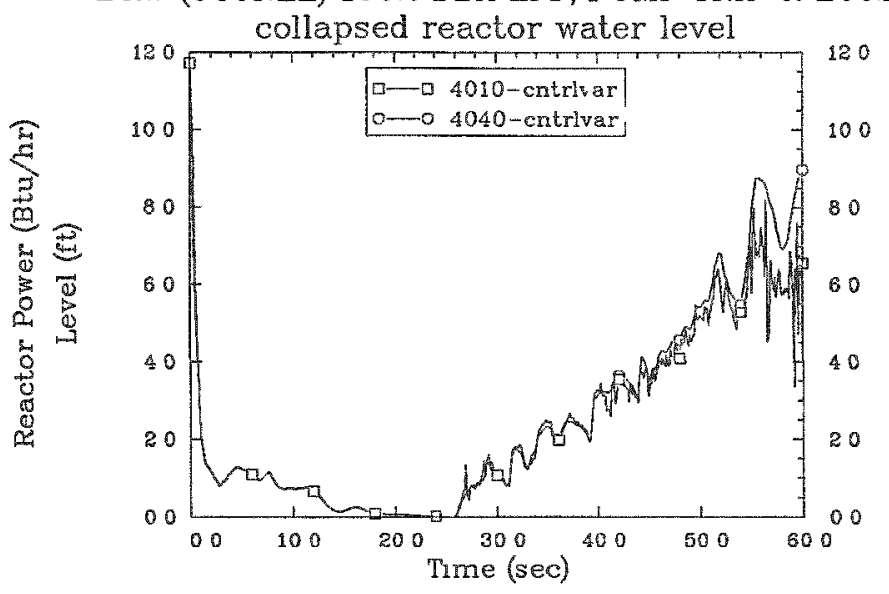

B\&W (OCONEE) 100\% DBA LPF, PUMP TRIP \& ECCS

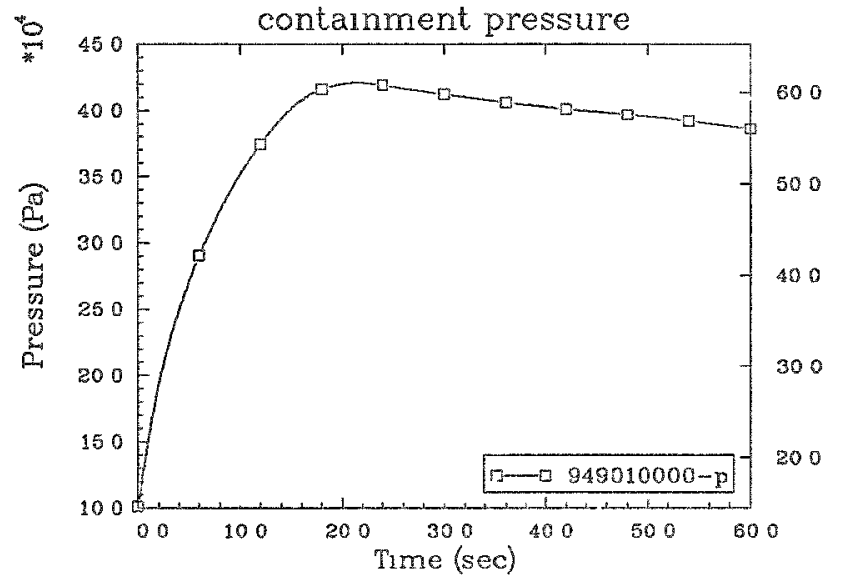

B\&W (OCONEE) 100\% DBA LPF, PUMP TRIP \& ECCS

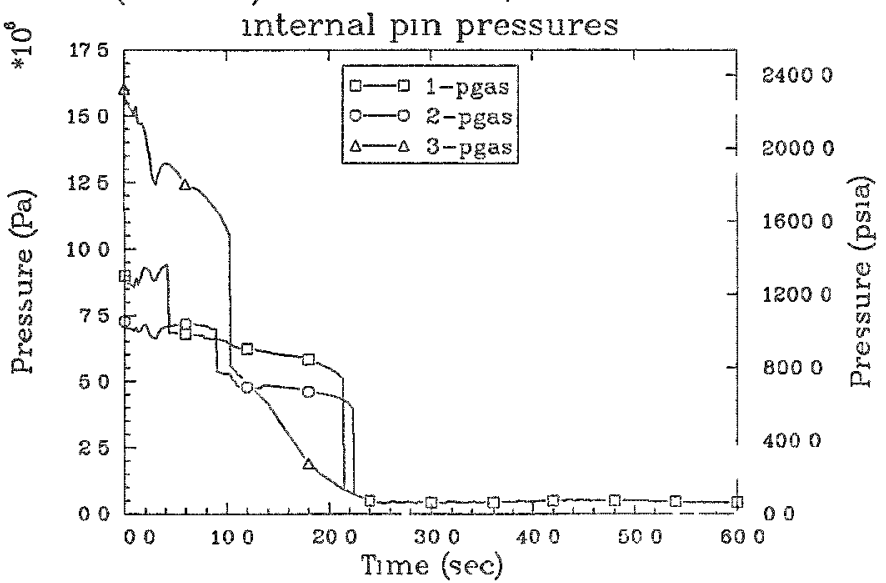


B\&W (OCONEE) 100\% DHA LPF, PUMP TRIP \& ECCS fuel centerline temporatures for 5 GWD/MTU pin

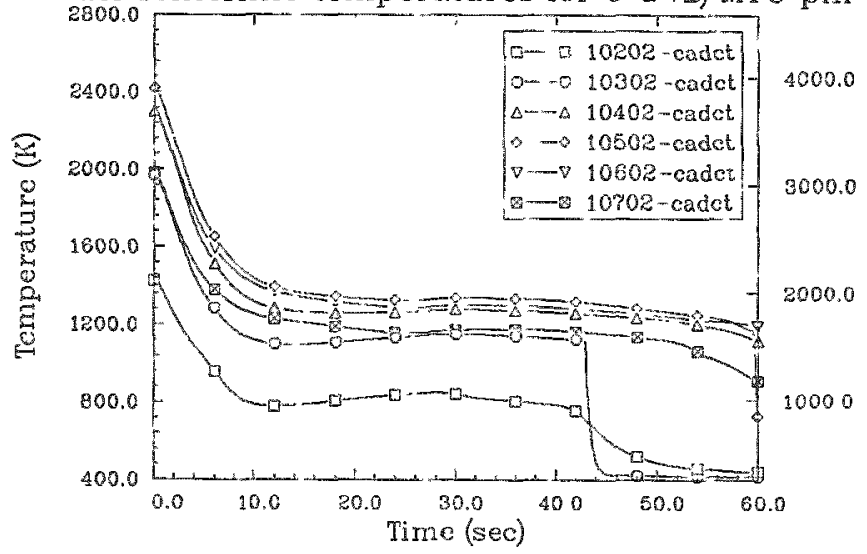

B\&W (OCONEE) 100\% DBA LPF, PUMP TRIP \& ECCS cladding surface temperatures for 5 GWD/MTU pin

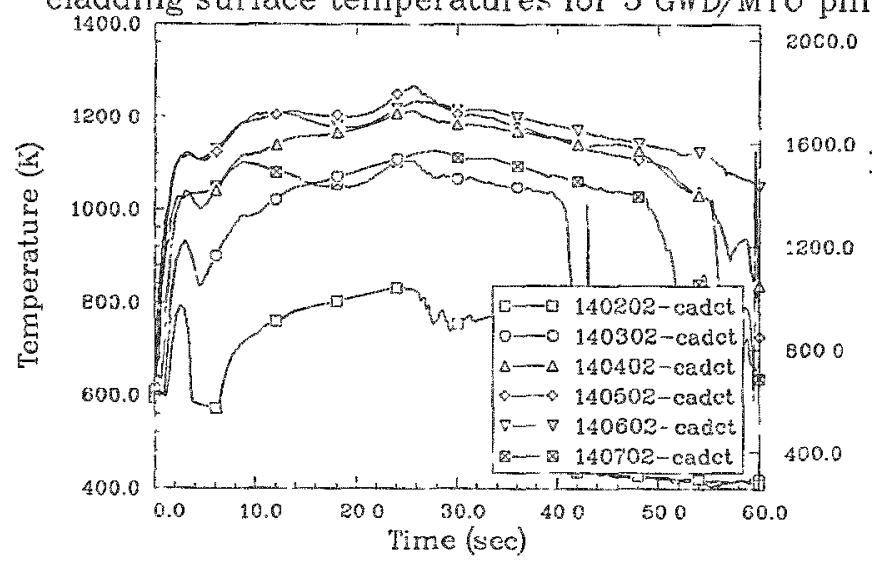

B8W (OCONEE) 100\% DBA LPF, PUMP TRIP \& ECCS

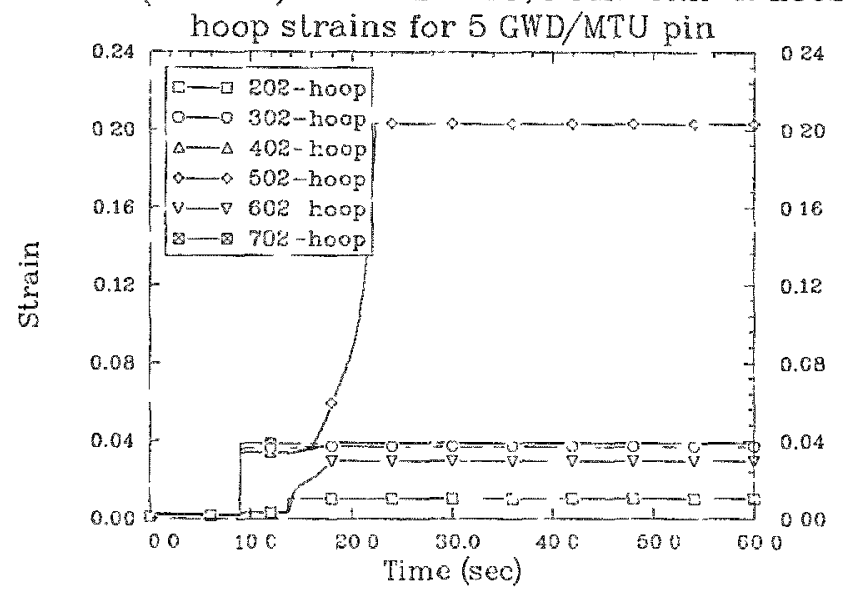

B\&W (OCONEE) 100\% DBA LPF, PUMP TRIP \& ECCS fuel centerline temperatures for 55 GWD/MTU pin

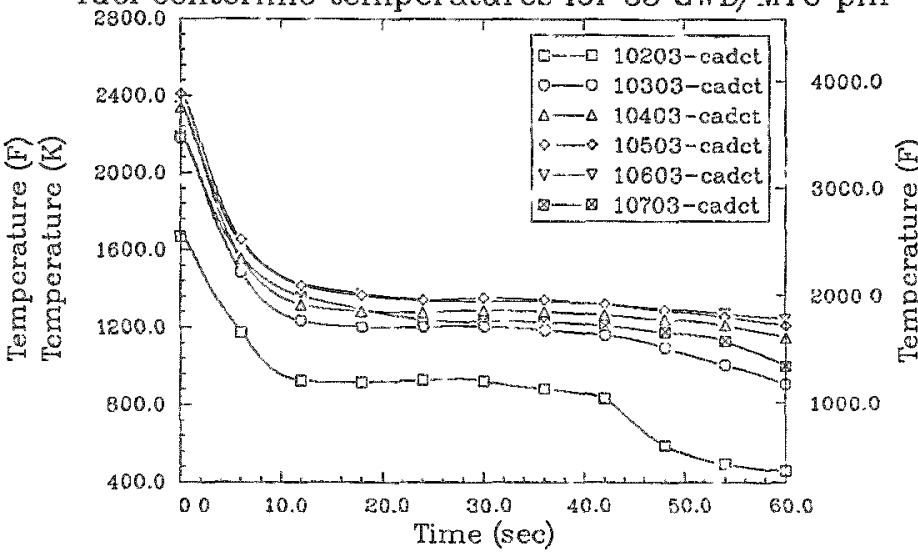

B\&W (OCONEE) 100\% DBA LPF, PUMP TRIP \& ECCS cladding surface temperatures for $55 \mathrm{GWD} / \mathrm{MTU}$ pin

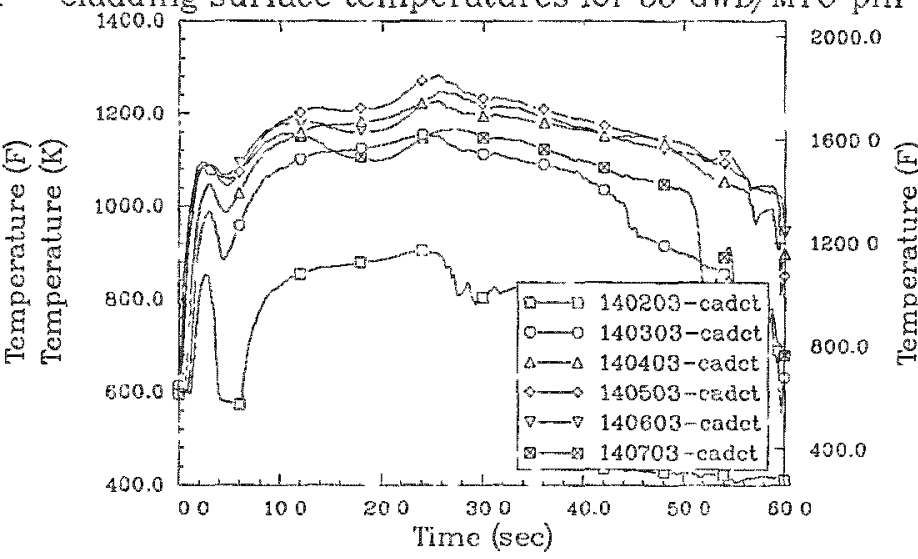

B\&W (OCONEE) 100\% DBA LPF, PUMP TRIP \& ECCS

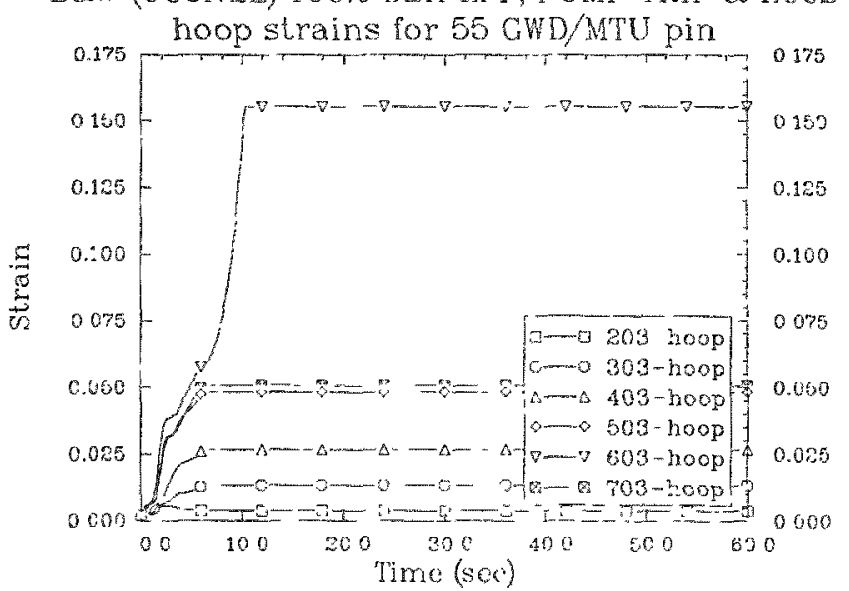


B\&W (OCONEE) 100\% DBA LPF, PUMP TRIP \& ECCS

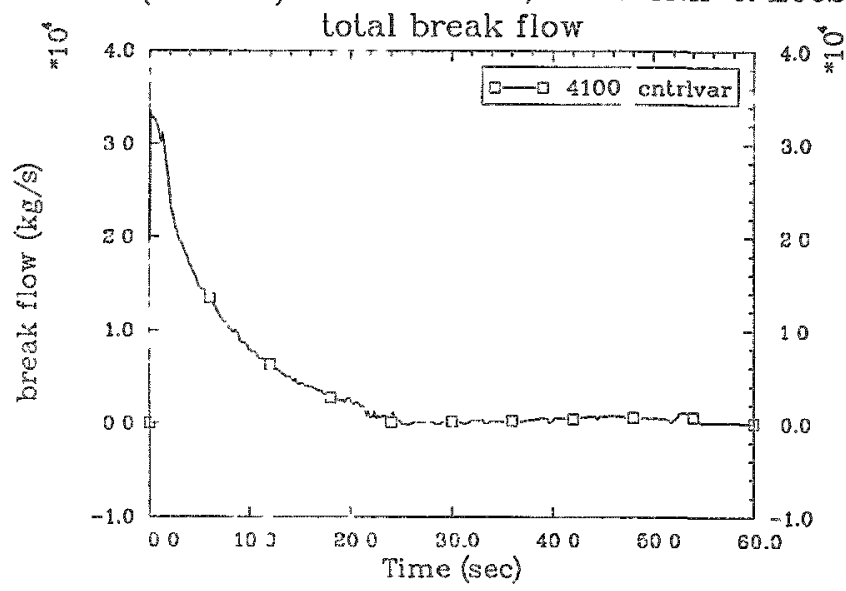

B\&W (OCONEE) 100\% DBA LPF, PUMP TRIP \& ECCS

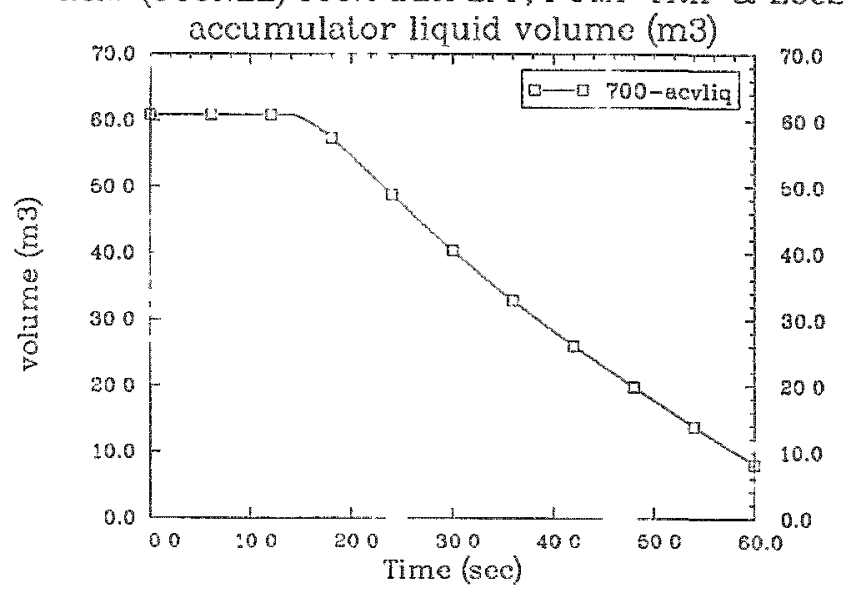

B\&W (OCONFE) 100\% DBA LIPF, PUMP TRIP \& ECCS

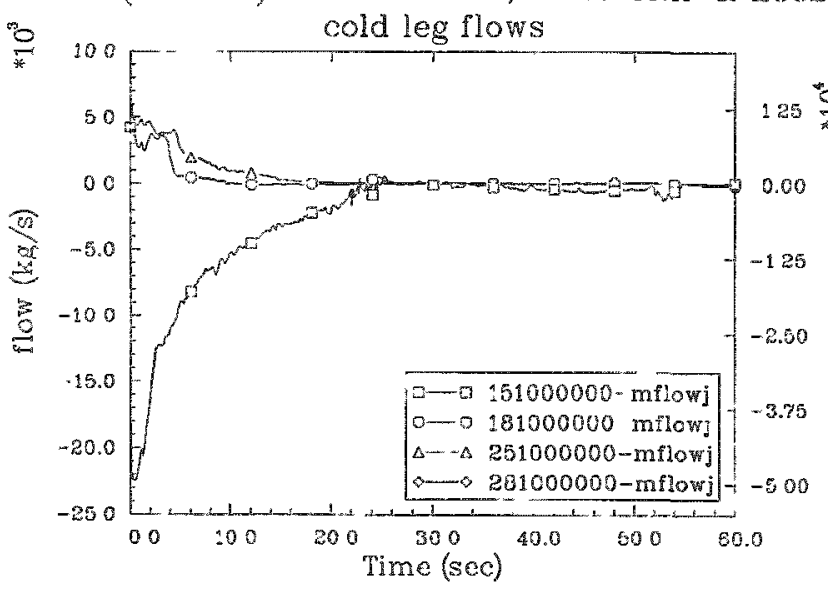

B\&W (OCONEE) 100\% DBA LPF, PUMP TRIP \& ECCS

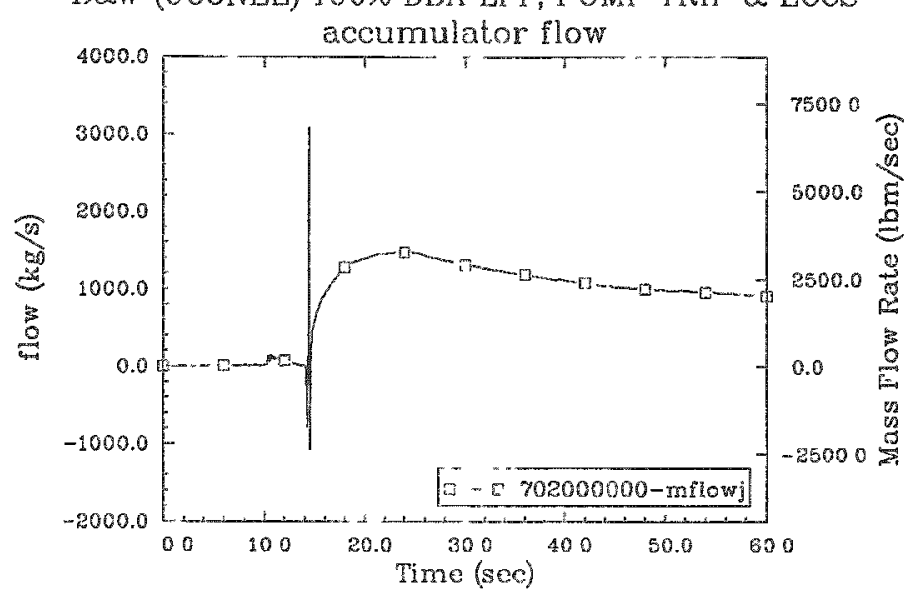

B\&W (OCONEE) 100\% DBA LIPF, PUMP TRIP \& ECCS

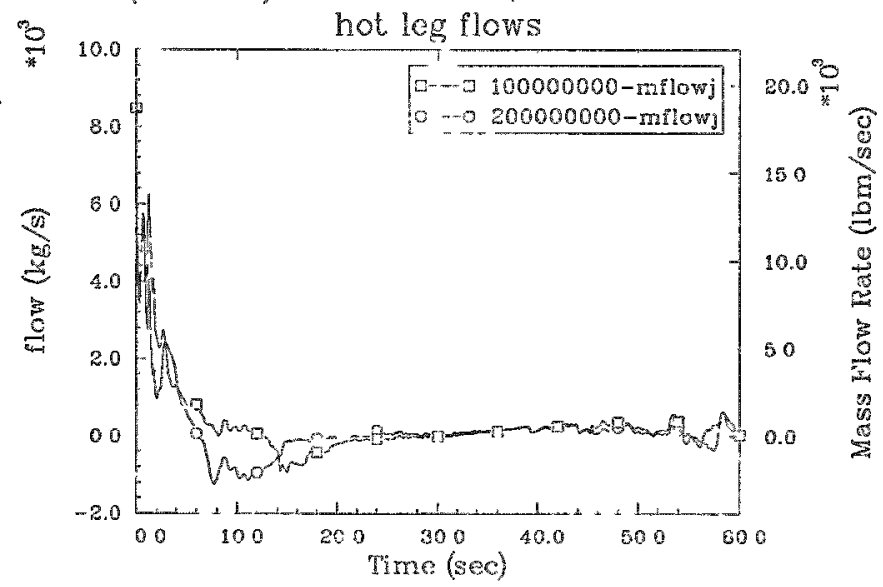

B\&W (OCONEE) 100\% DBA I.PF, PUMP TRIP \& ECCS

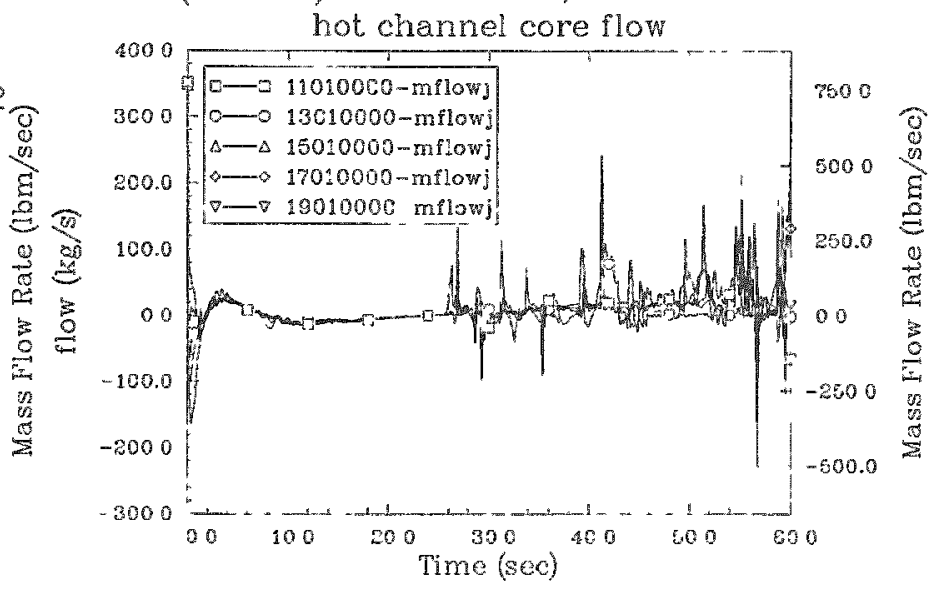


B\&W (OCONEE) 100\% DBA LPF, PUMP TRIP \& ECCS

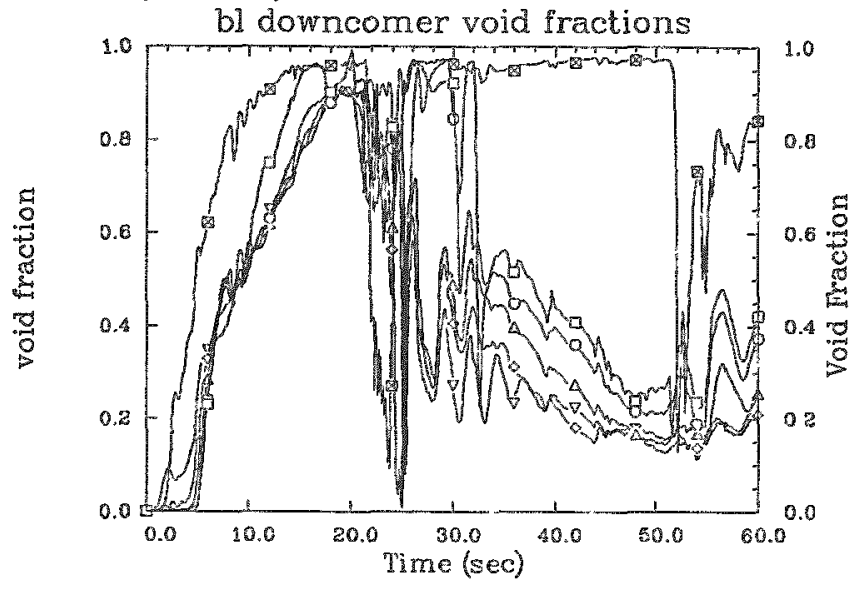

B\&W (OCONEE) 100\% DBA LPF, PUMP TRIP \& ECCS

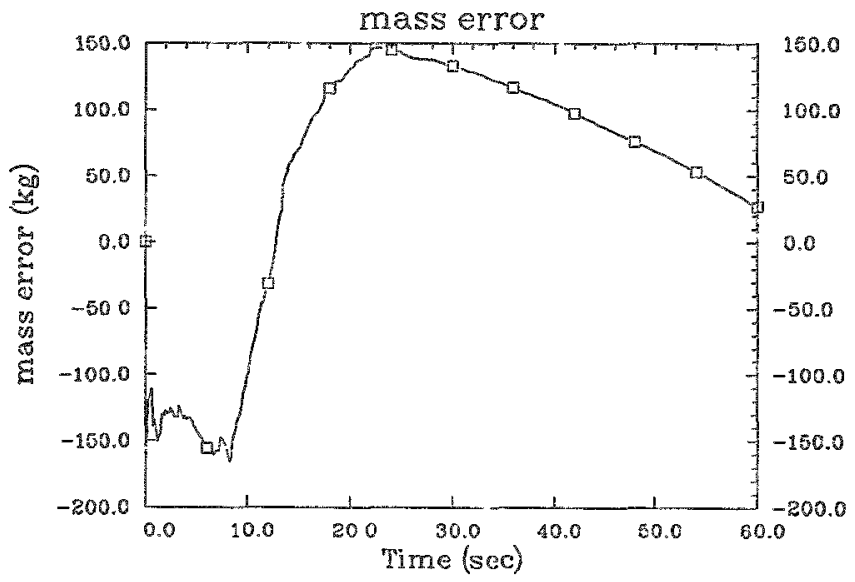

B\&W (OCONEE) 100\% DBA LPF, PUMP TRIP \& ECCS

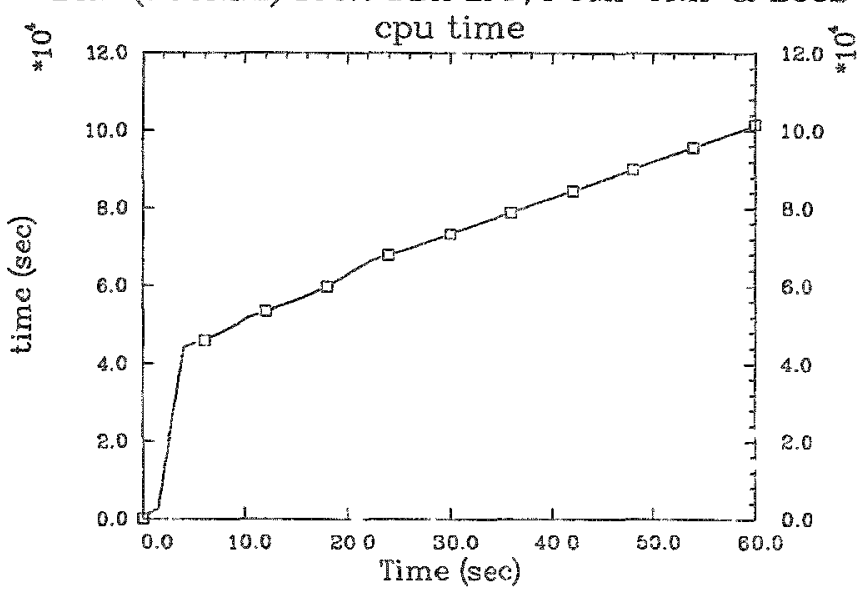

B\&W (OCONEE) 100\% DBA LPF, PUMP TRIP \& ECCS

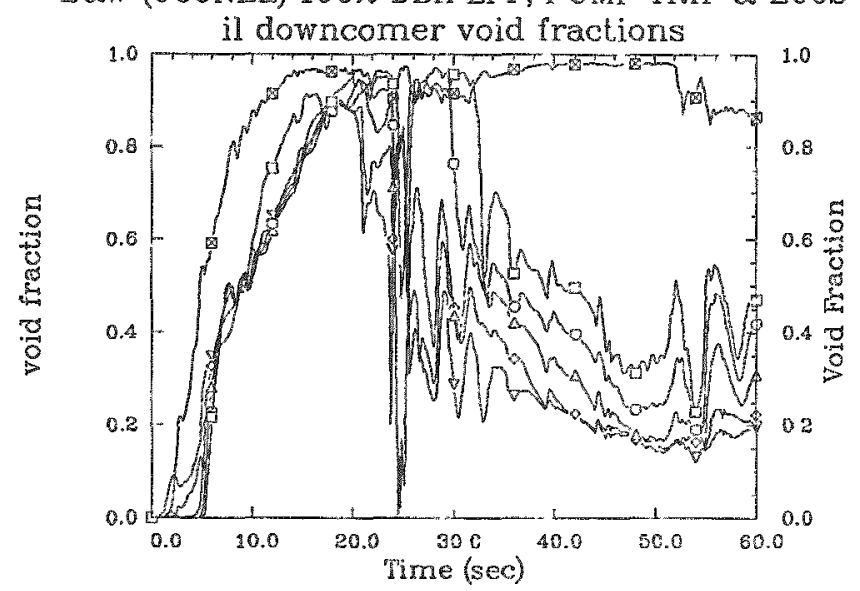

B\&W (OCONEE) 100\% DBA LPF, PUMP TRIP \& ECCS

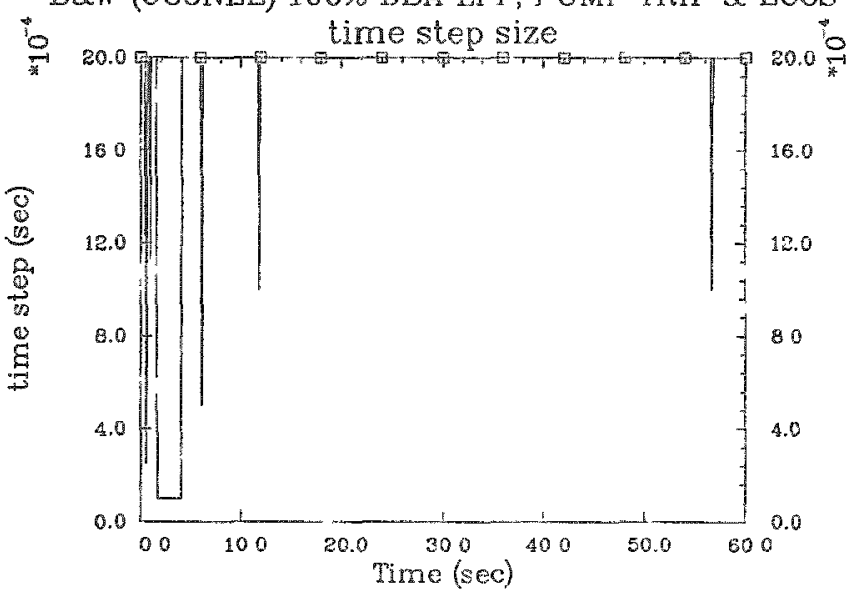

B\&W (OCONEE) 100\% DBAA LPI, PUMP TRIP \& ECCS

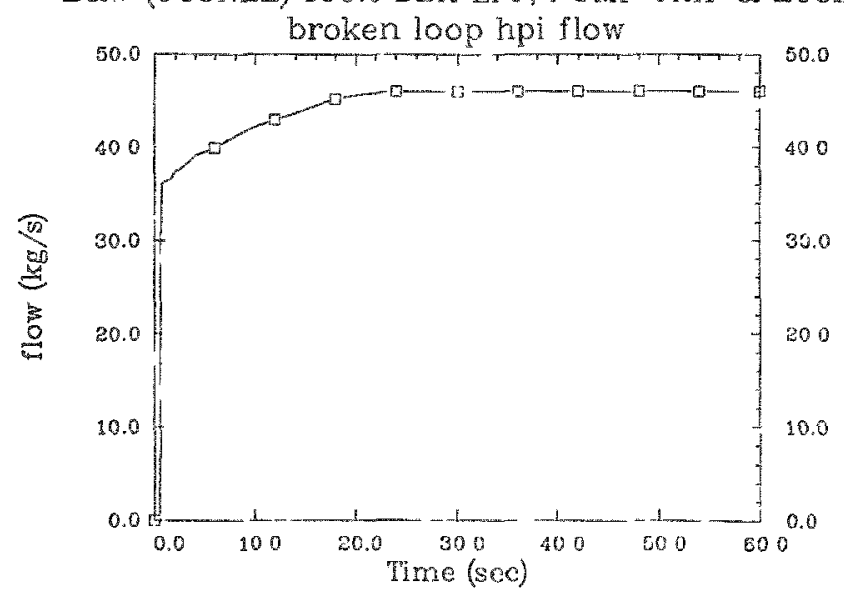


B\&W (OCONEE) 100\% DBA LPF, PUMP TRIP \& ECCS

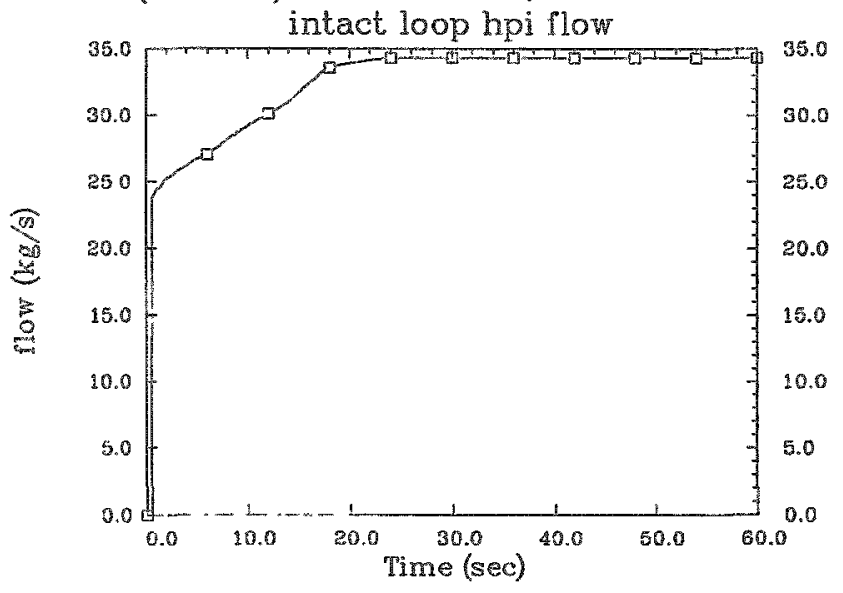

B\&W (OCONEE) 100\% DBA LPF, PUMP TRIP \& ECCS

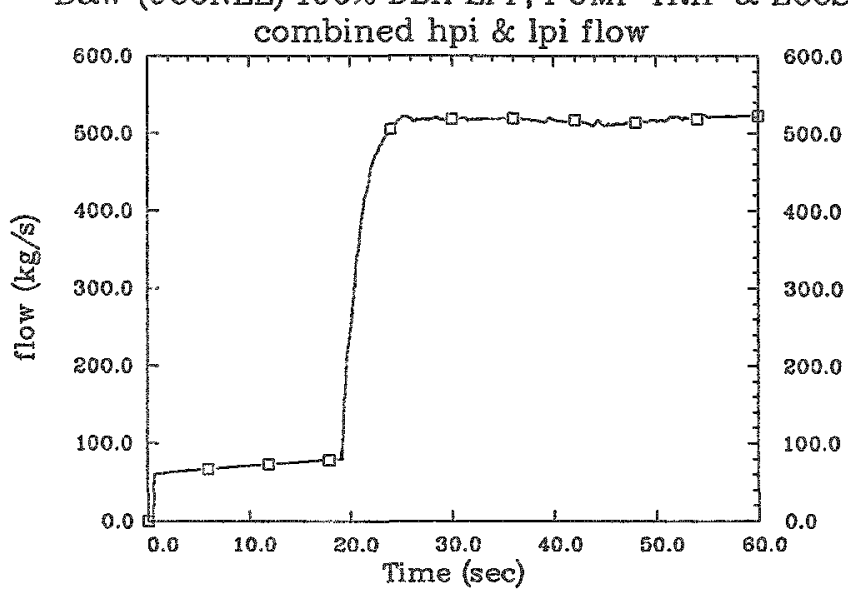



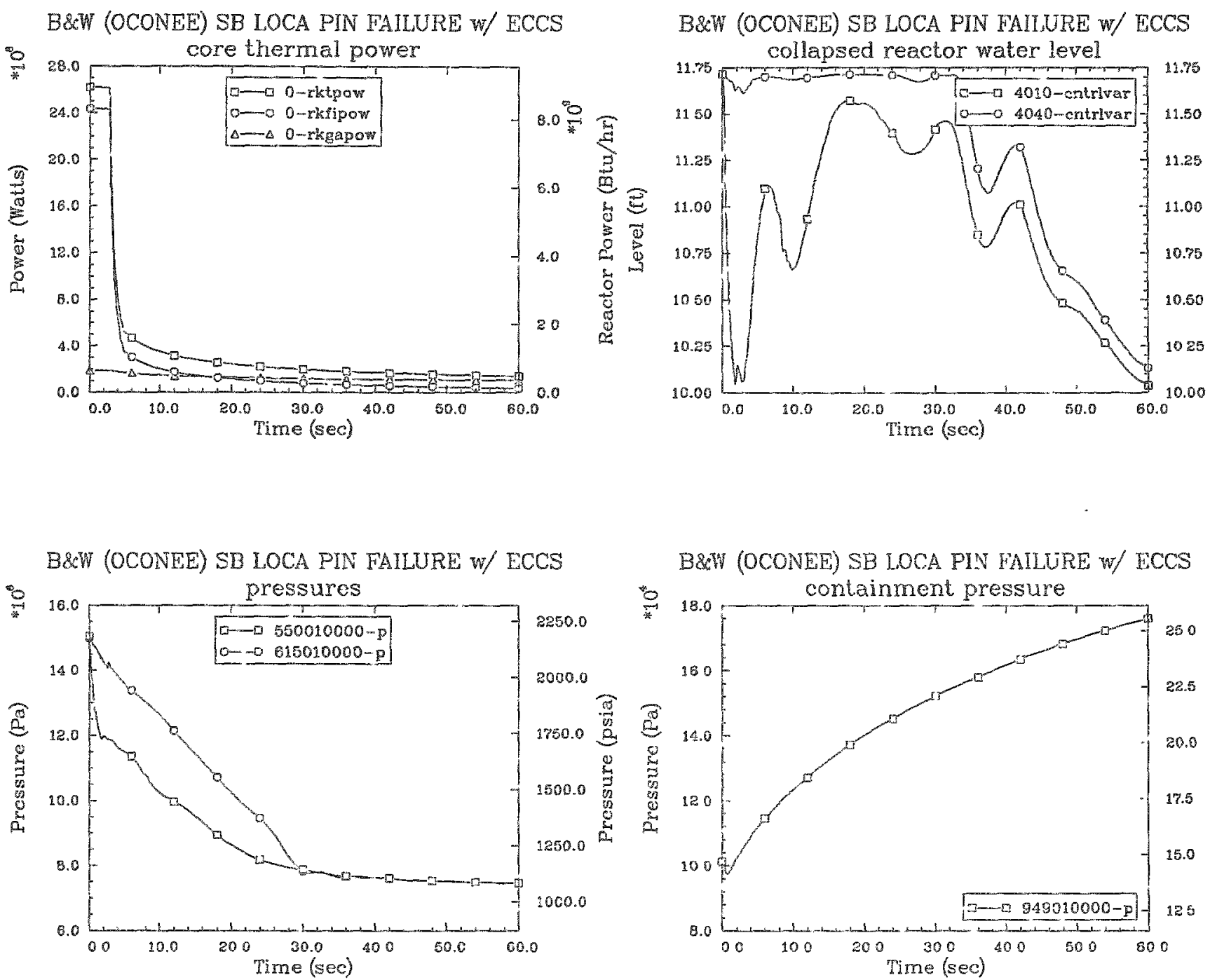

B\&W (OCONEE) SB LOCA PIN FALURE W/ ECCS

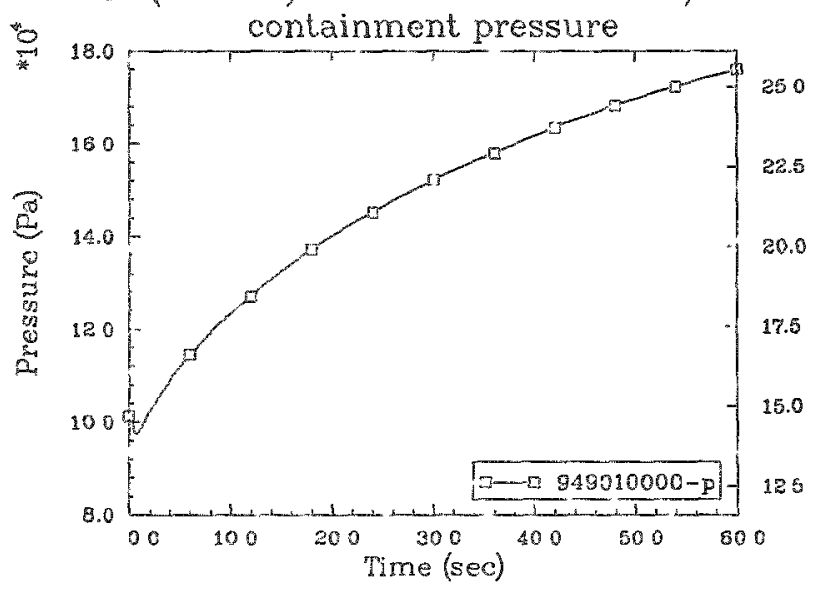

B\&W (OCONEE) SB LOCA PIN FAILURE W/ ECCS
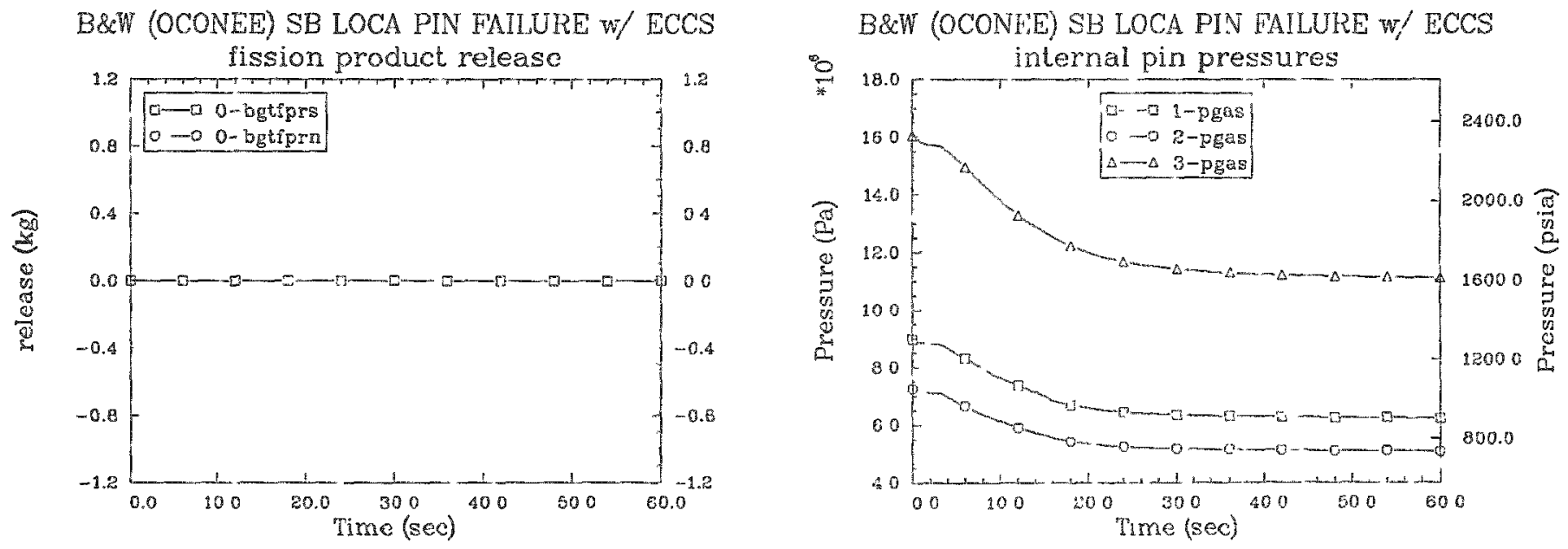
B\&W (OCONEE) SB LOCA PIN FAILURE W/ ECCS

fuel centerline temperatures for $5 \mathrm{GWD} / \mathrm{MTU}$ pin

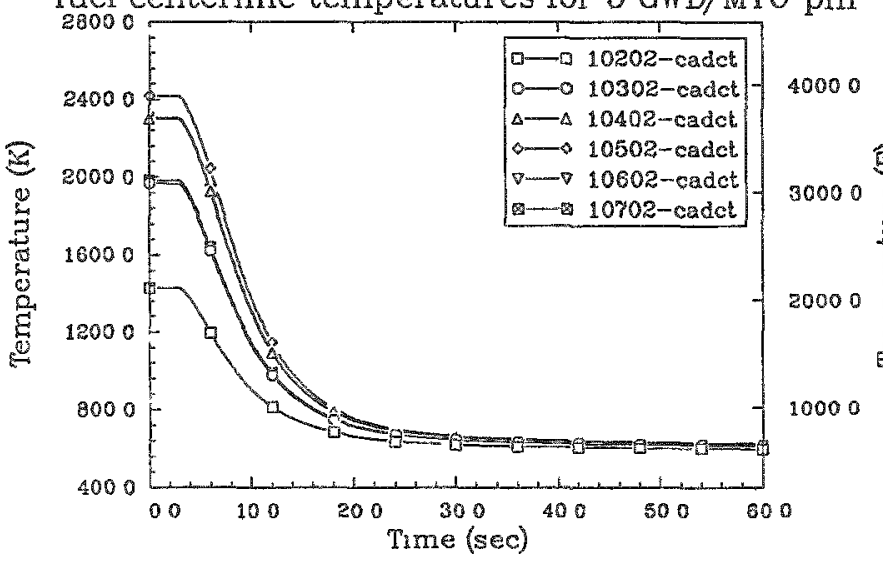

B\&W (OCONEE) SB LOCA PIN FAILURE W/ ECCS cladding surface temperatures for $5 \mathrm{GWD} / \mathrm{MTU}$ pin

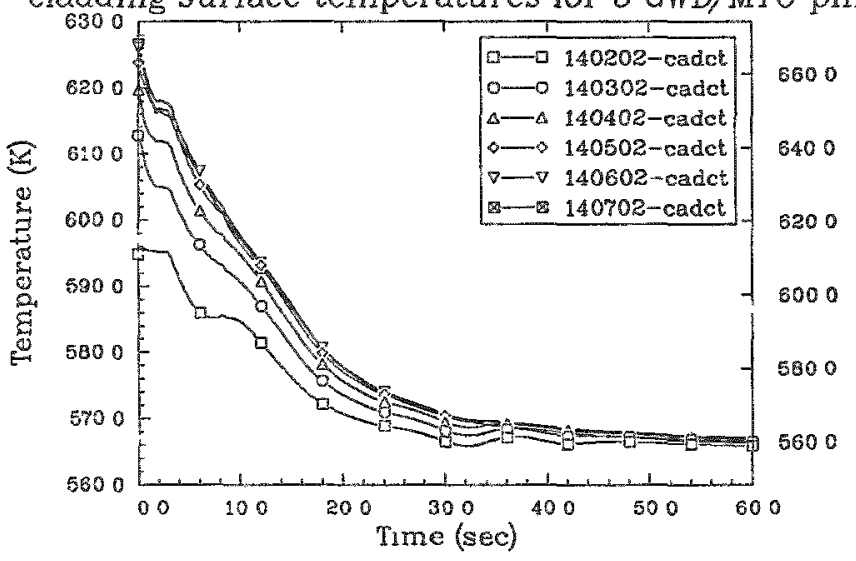

B\&W (OCONEE) SB LOCA PIN FAILURE w/ ECCS

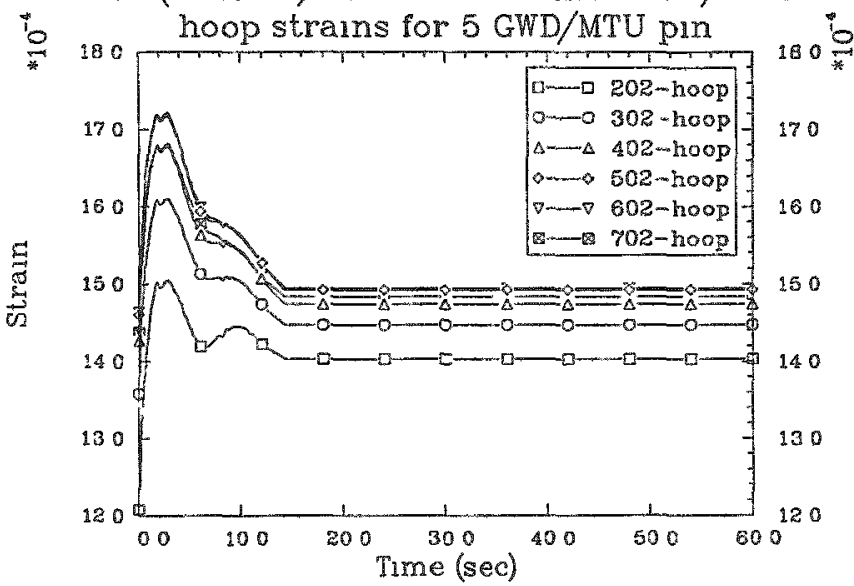

B\&W (OCONEE) SB LOCA PIN FAILURE W/ ECCS fuel centerline temperatures for 55 GWD/MTU pin

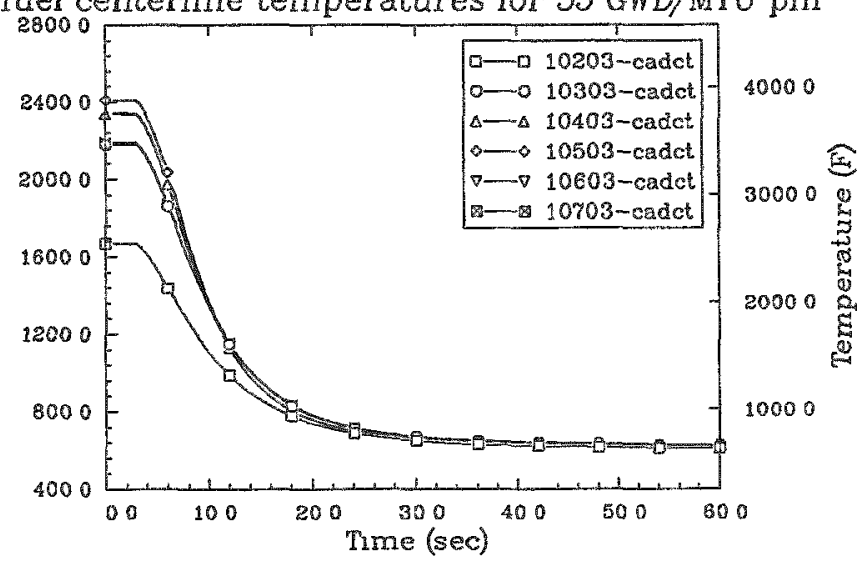

B\&W (OCONEE) SB LOCA PIN FAILURE W/ ECCS cladding surface temperatures for 55 GWD/MTU pin

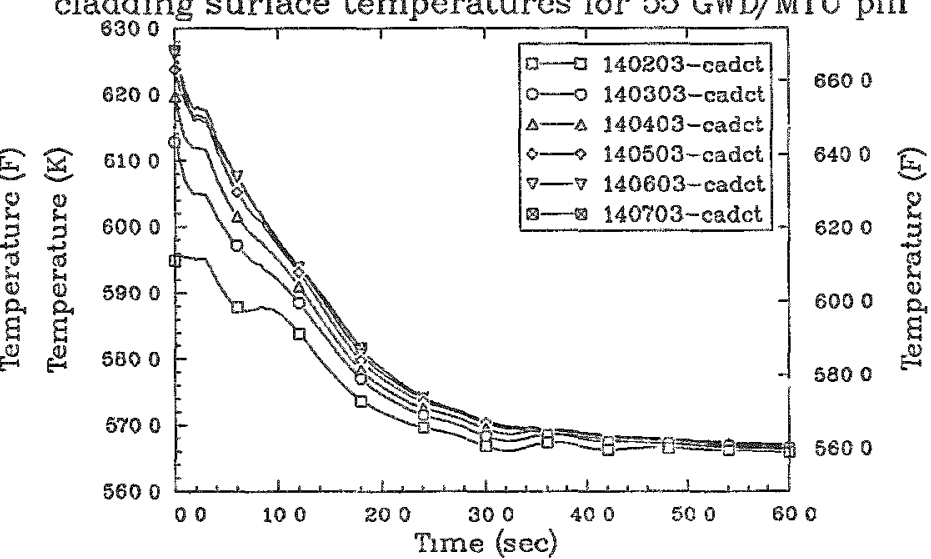

B\&W (OCONEE) SB LOCA PIN FAILURE w/ ECCS

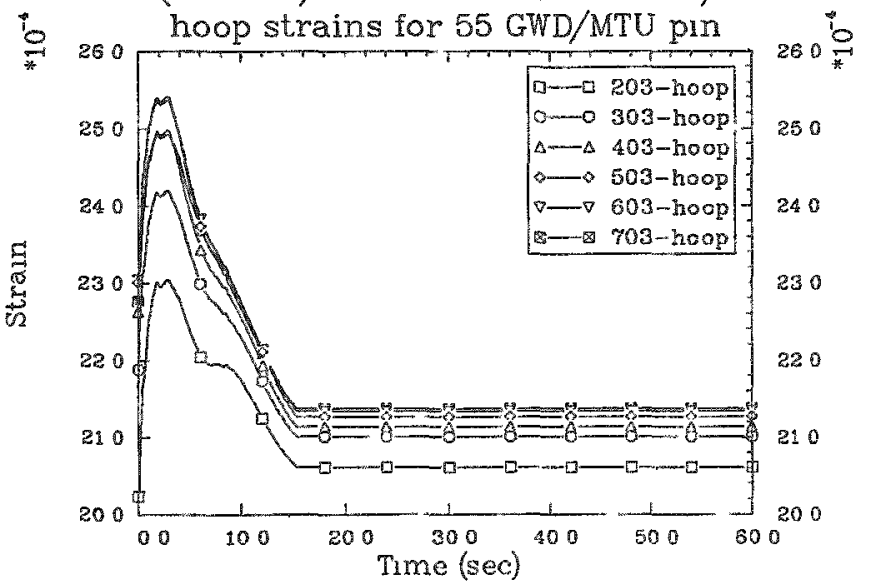




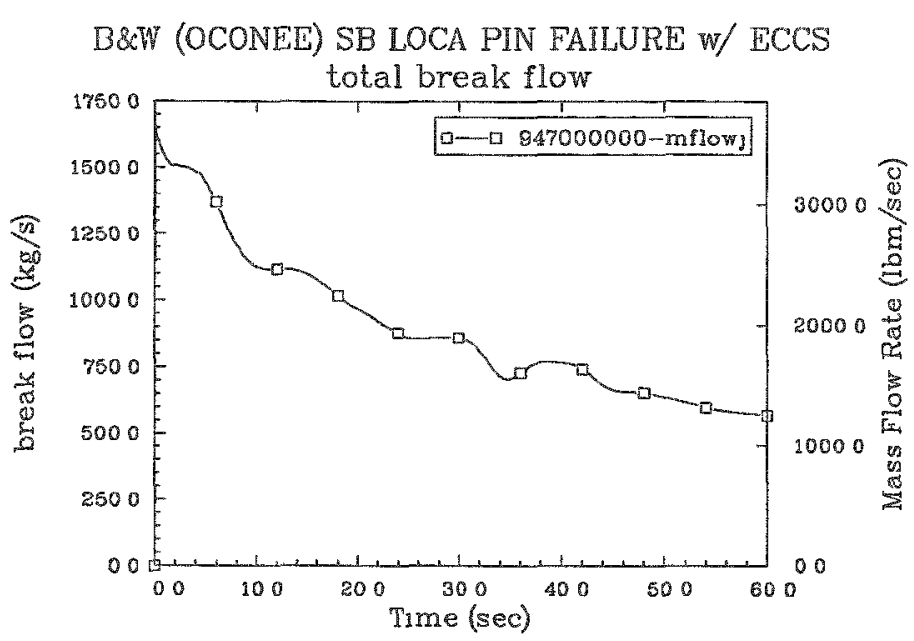

B\&W (OCONEE) SB LOCA PIN FAILURE W/ ECCS
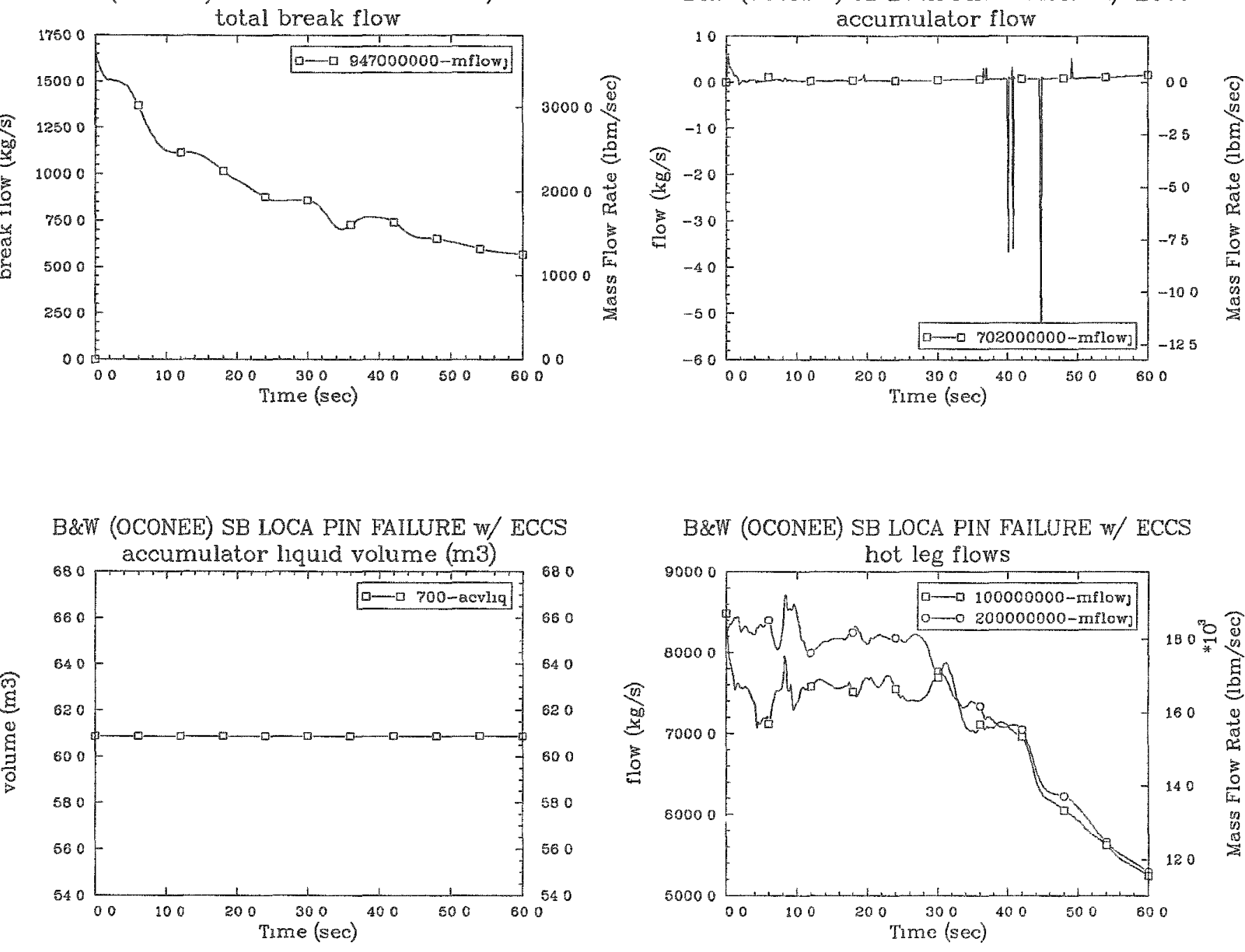

B\&W (OCONEE) SB LOCA PIN FAILURE W/ ECCS

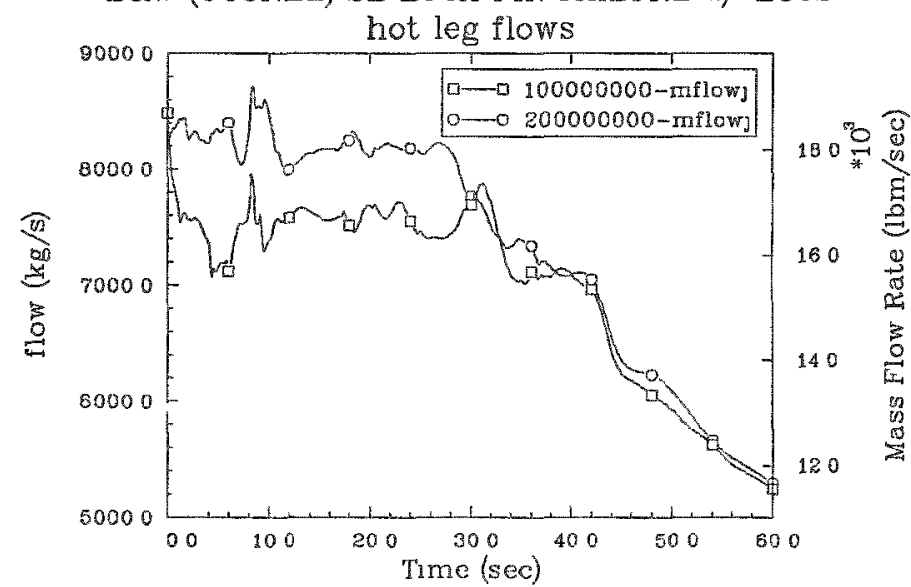

B\&W (OCONEE) SB LOCA PIN FAILURE: w/ ECCS

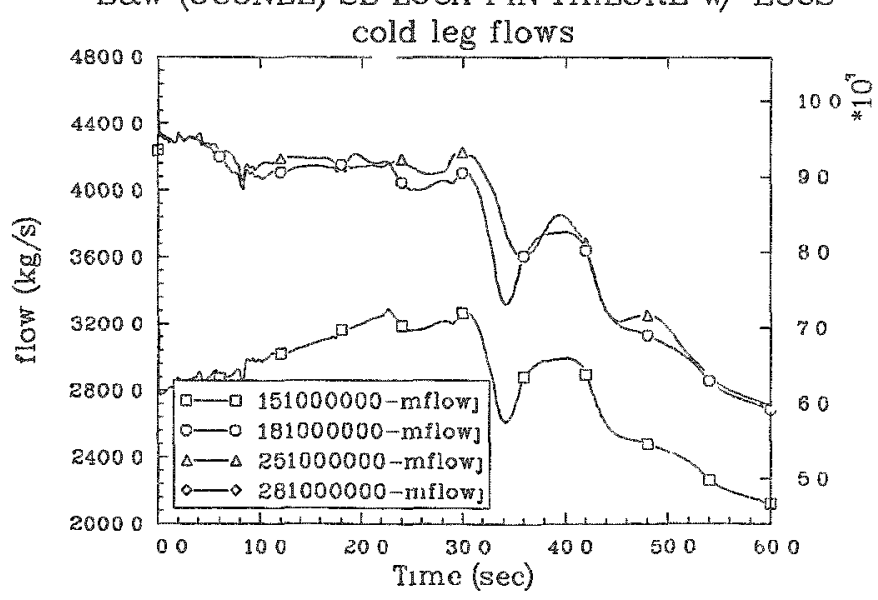

B\&W (OCONEE) SB LOCA PIN FAILURE w/ ECCS

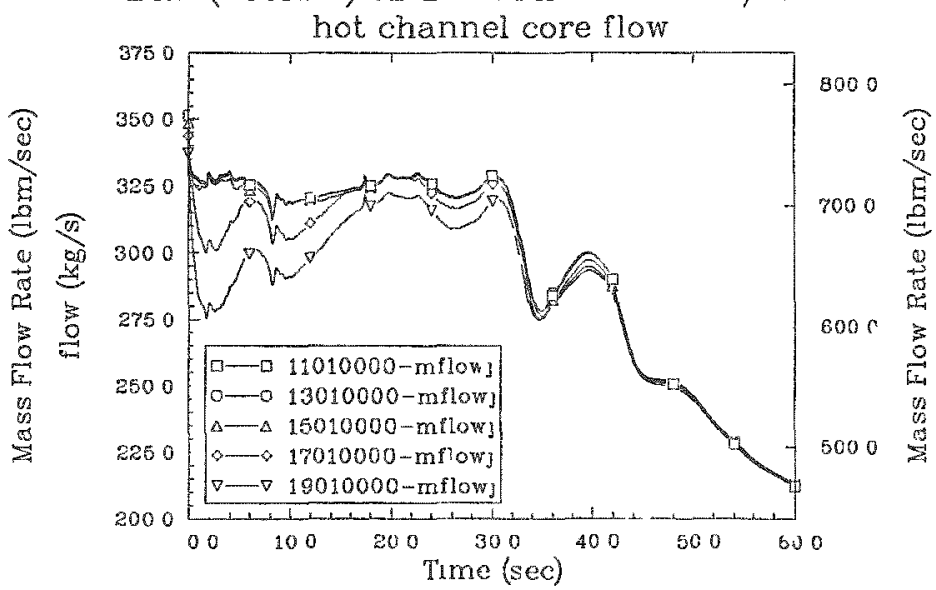




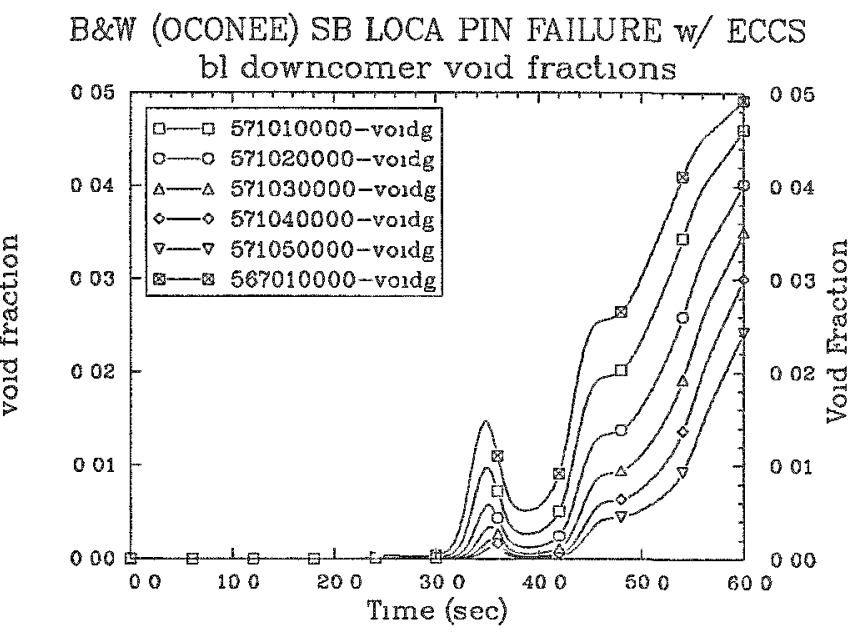

B\&W (OCONEE) SB LOCA PIN FAILURE W/ ECCS

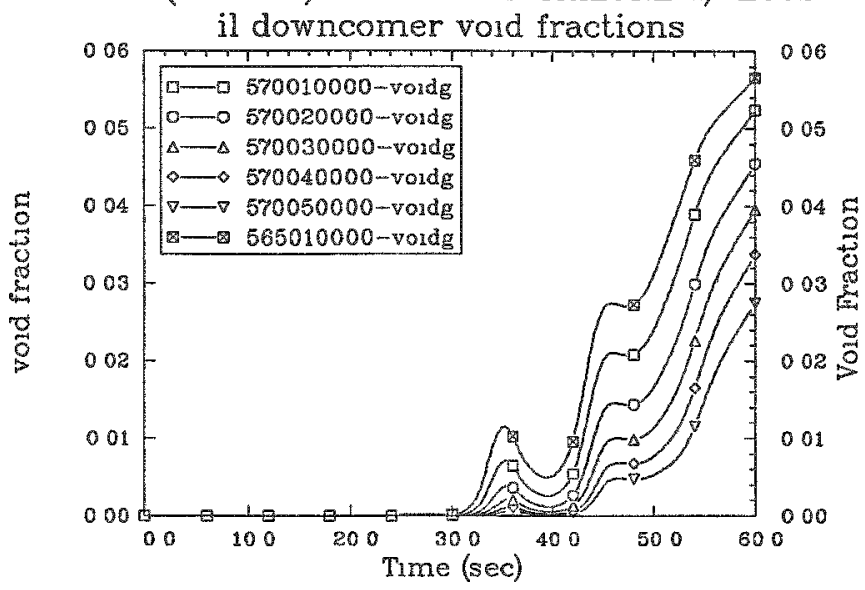

B\&W (OCONEE) SB LOCA PIN FAIIURE W/ ECCS
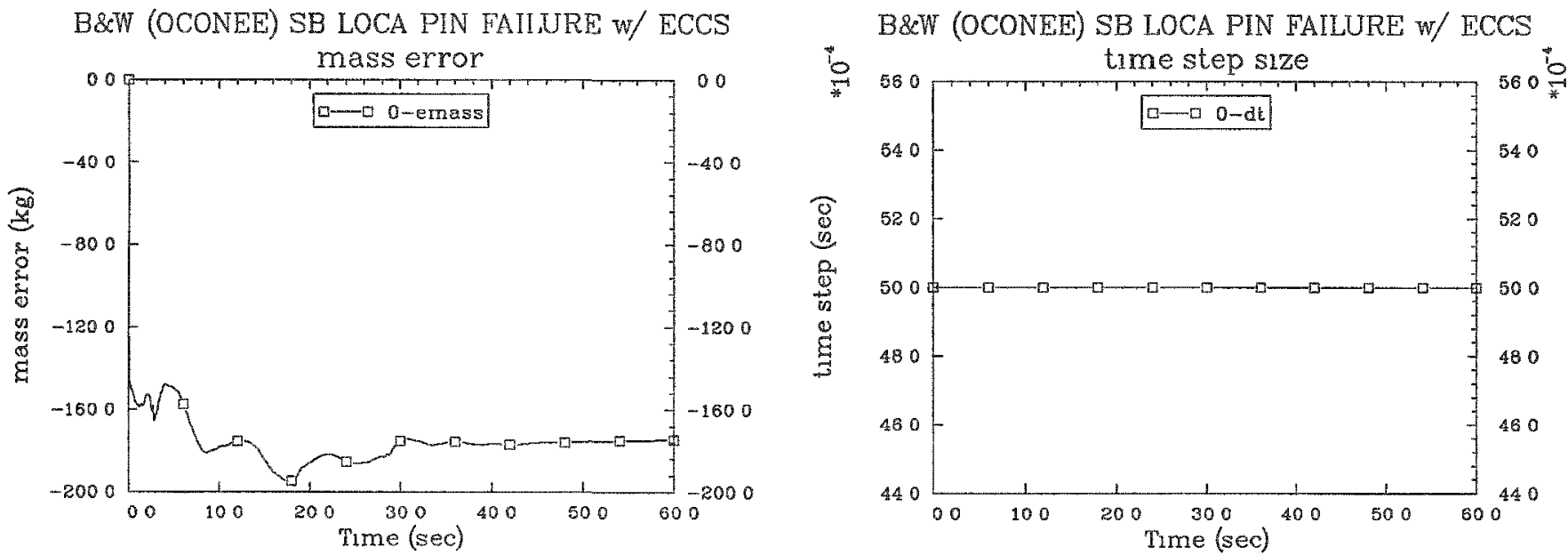

B\&W (OCONEE) SB LOCA PIN FAILURE W/ ECCS

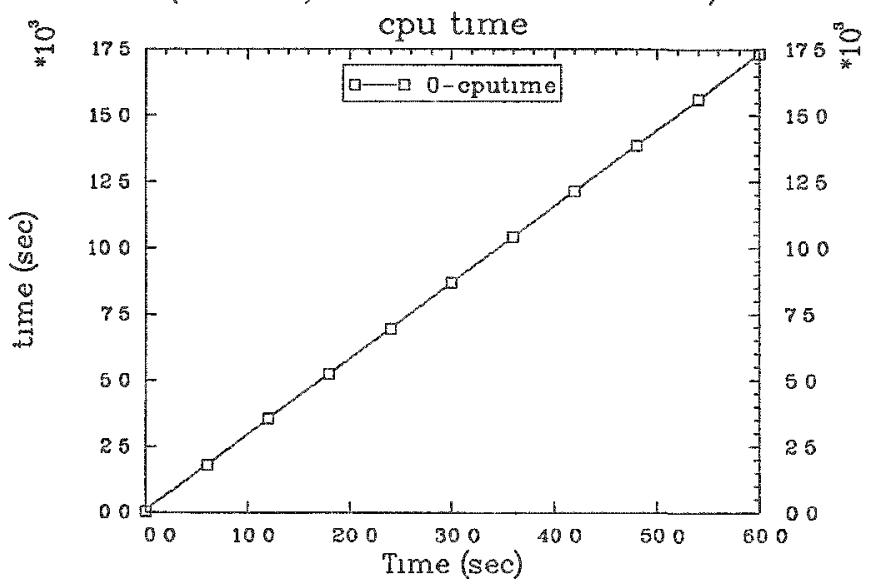

B\&W (OCONEE) SB LOCA PIN FAILURE W/ ECCS

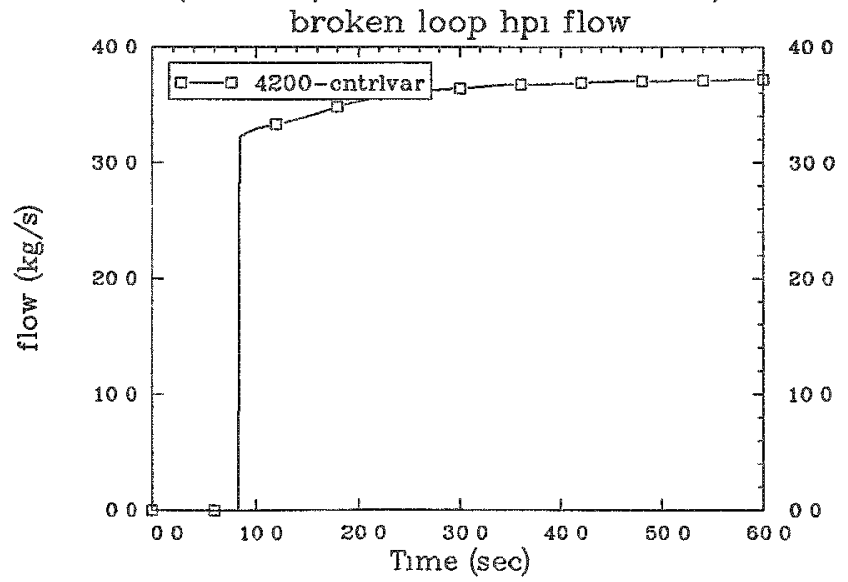



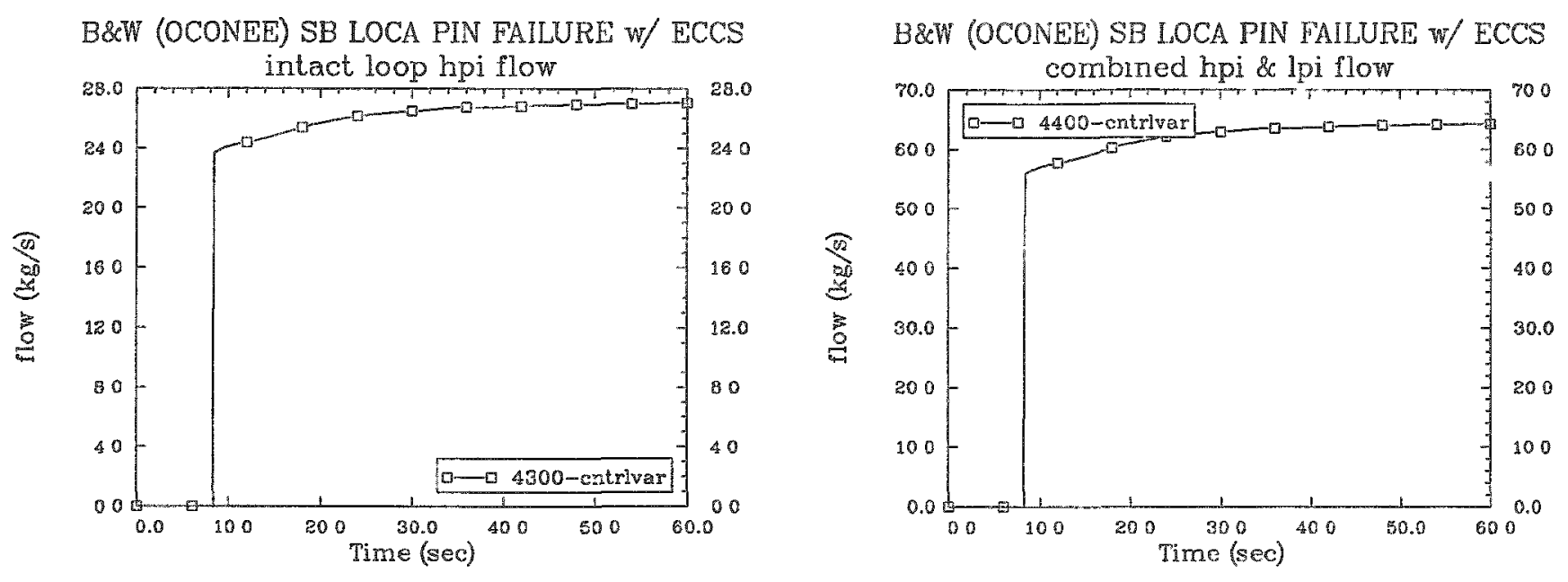


\section{K-2. FRAP-T6 PLOTTEd Results fOR OCONEE}

Table K-2. Description of FRAP-T6 plot variables for Oconee.

\begin{tabular}{ll}
\hline Variable & \multicolumn{1}{c}{ Description } \\
\hline 0-ffr & Failure probability \\
o-prspln & Internal pin pressure (psia, $\mathrm{Pa}$ ) \\
$n$-cladhsn & Cladding hoop strain at axial node $n$ \\
$n$-cladote & Cladding surface temperature at axial node $n\left({ }^{\circ} \mathrm{F}, \mathrm{K}\right)$ \\
$n$-ctemp & Fuel centerline temperature at axial node $n\left({ }^{\circ} \mathrm{F}, \mathrm{K}\right)$ \\
$\mathrm{n}$-ooxtn & Oxide thickness at axial node $\mathrm{n}(\mathrm{in} ., \mathrm{mm})$ \\
\hline
\end{tabular}



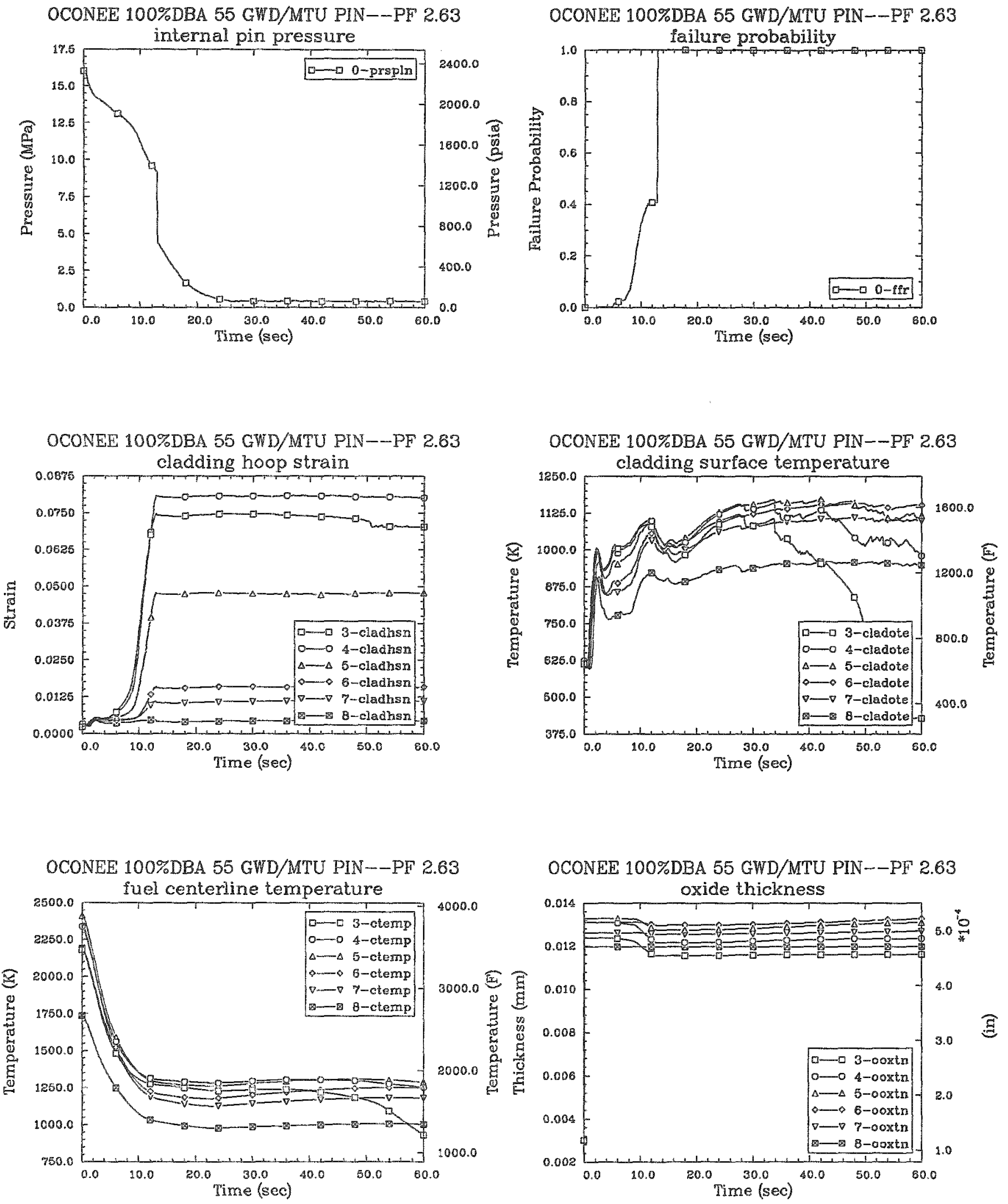

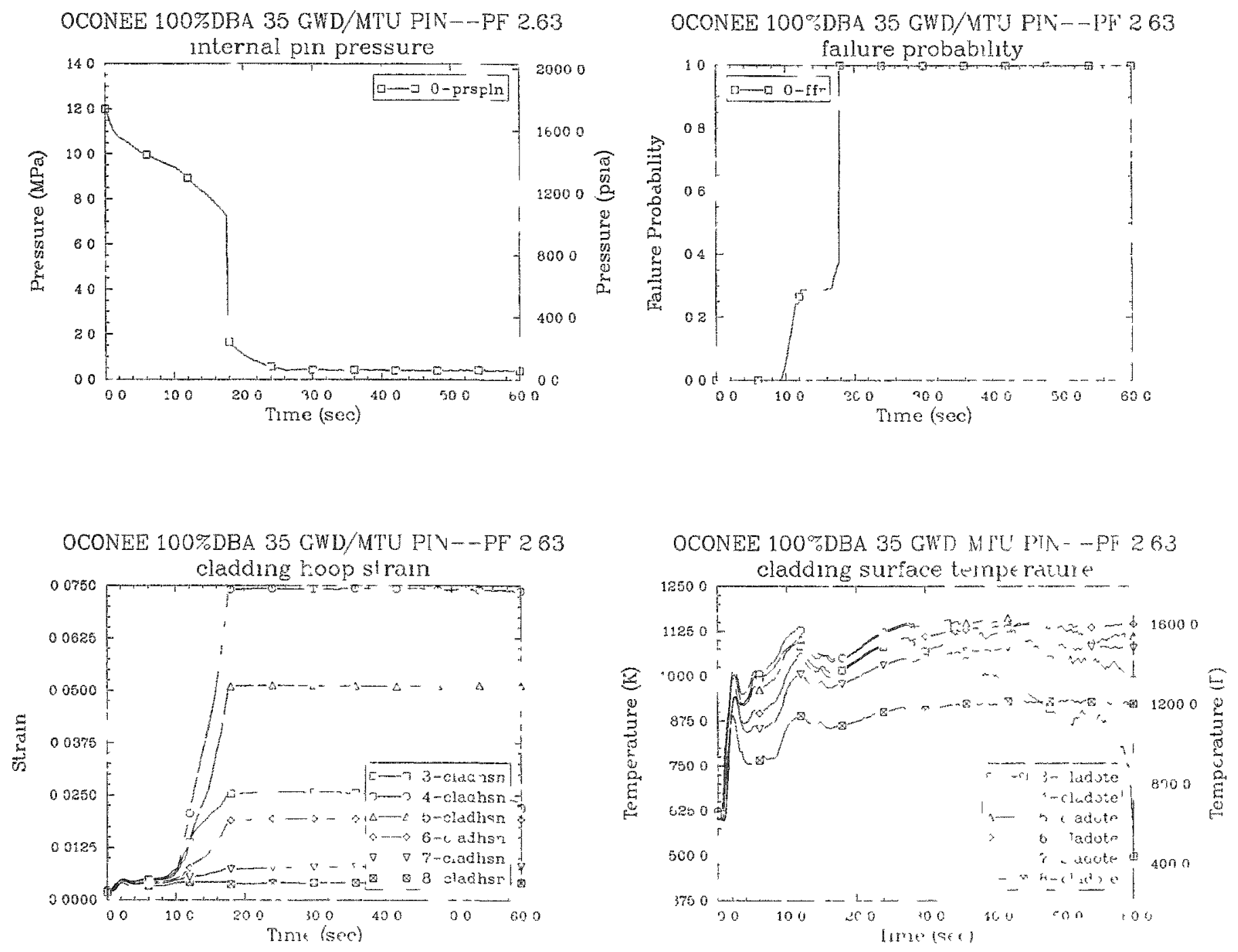

OCONEE 100\%DBA 35 GWD, VT: PIN PI'263

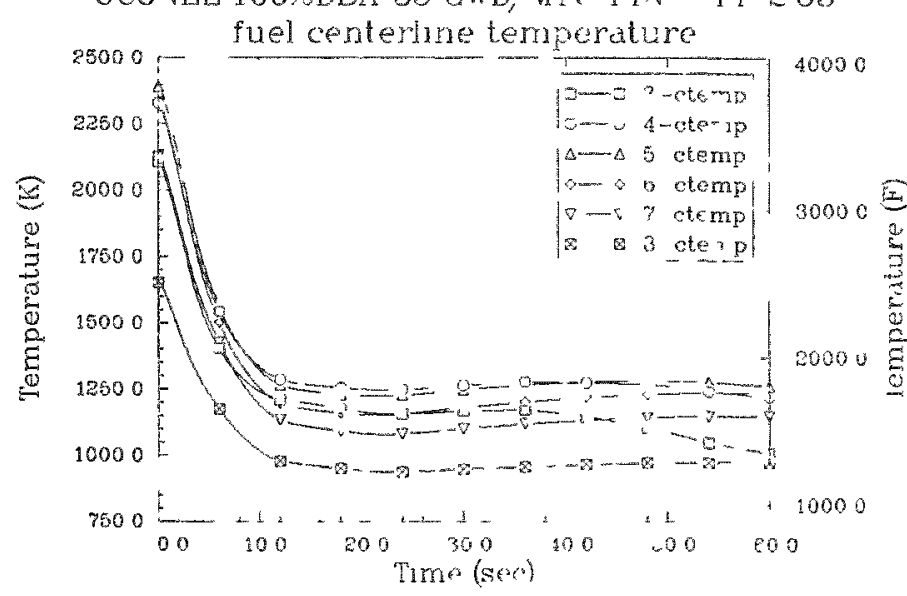

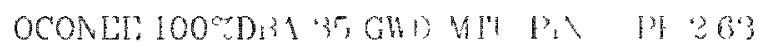

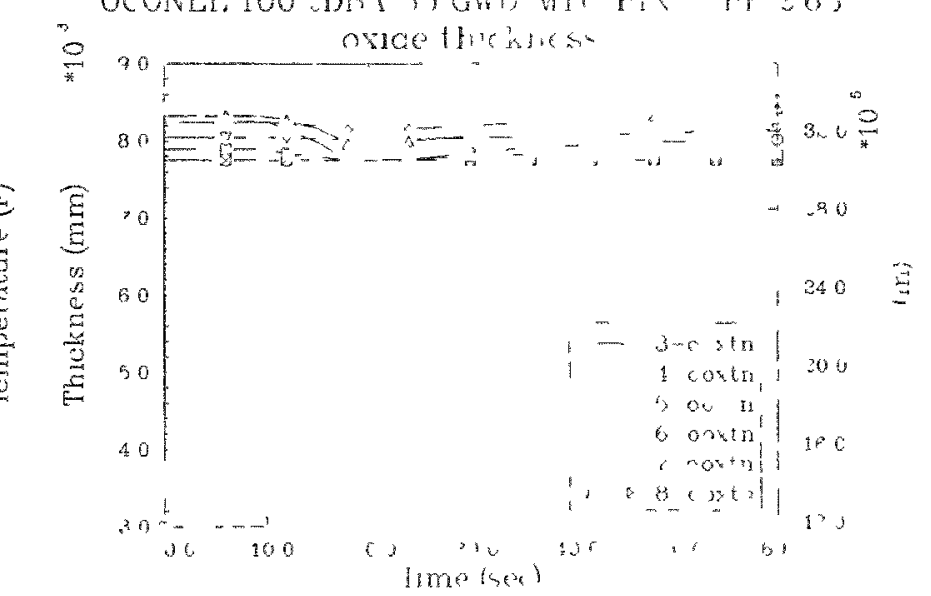



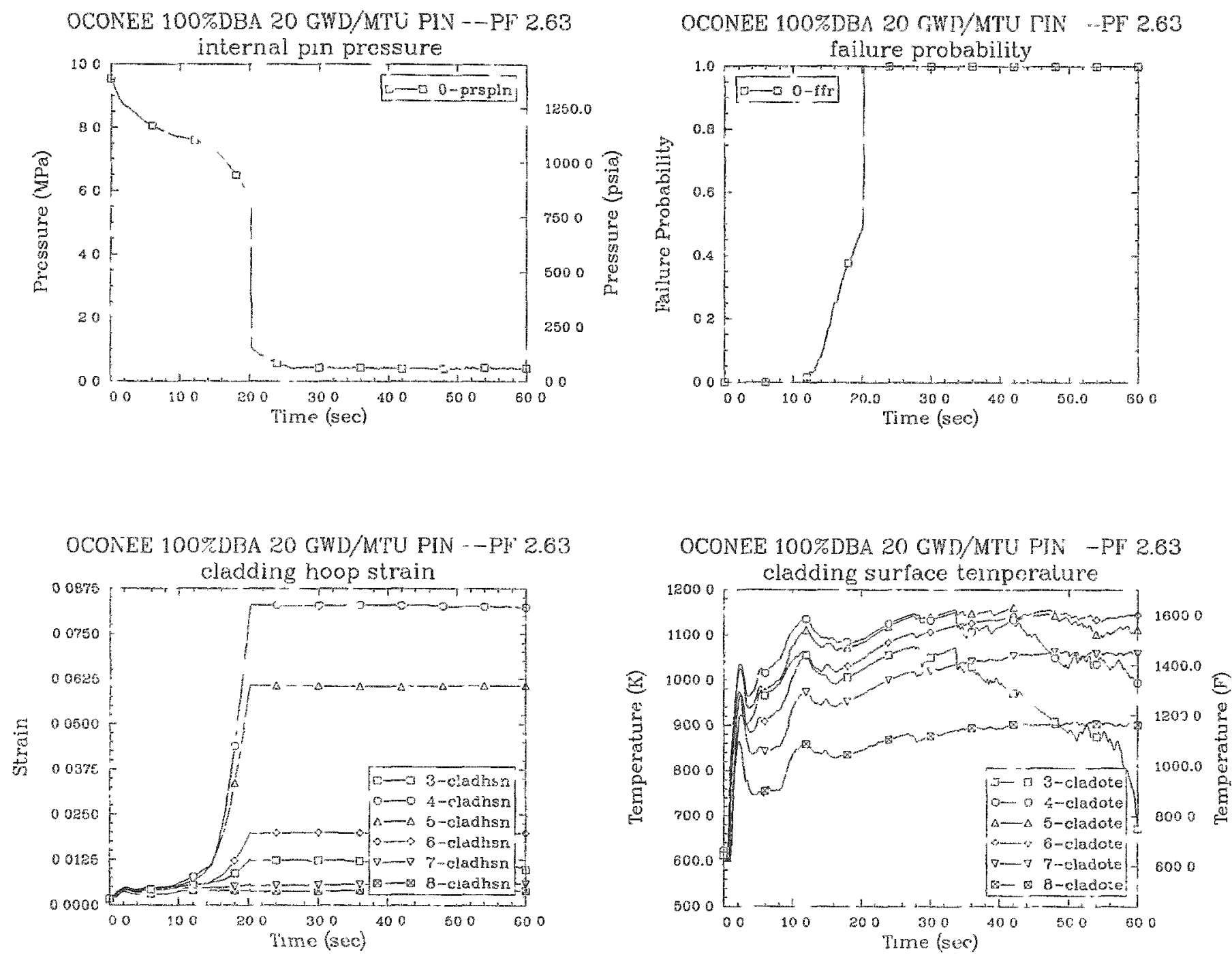

OCONEE 100\%DBA 20 GWD/MTU PIN-DF 2.63

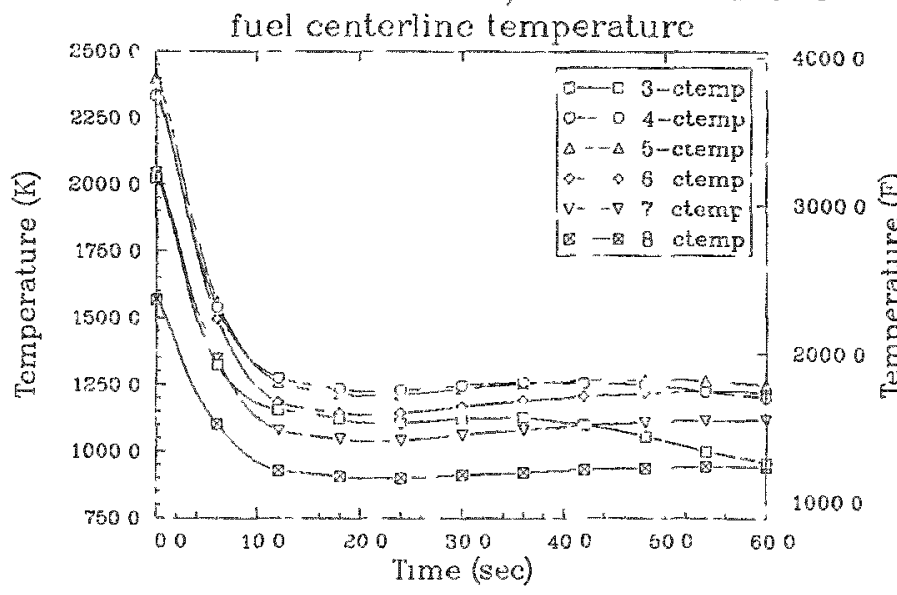

OCONEE 100\%DBA 20 CWD/MTU PIN-DPF 2.63

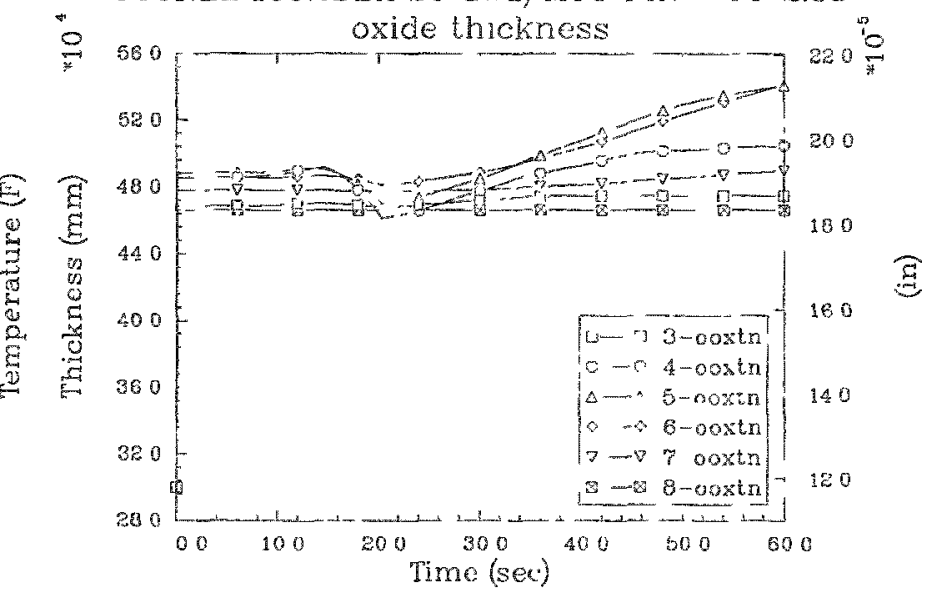



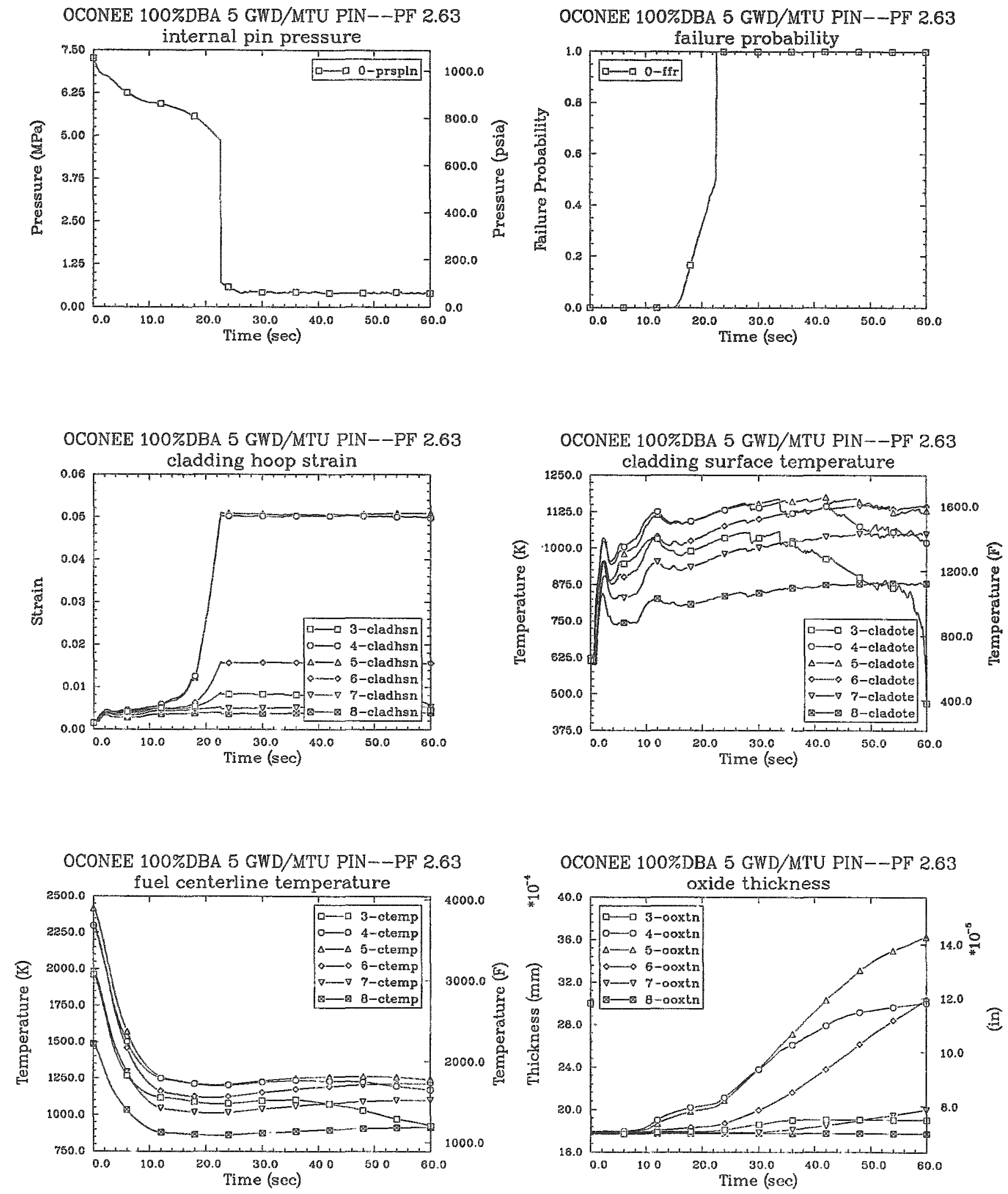
OCONEE 100\%DBA 55 GWD/TU PIN--PF 2.4 internal pin pressure
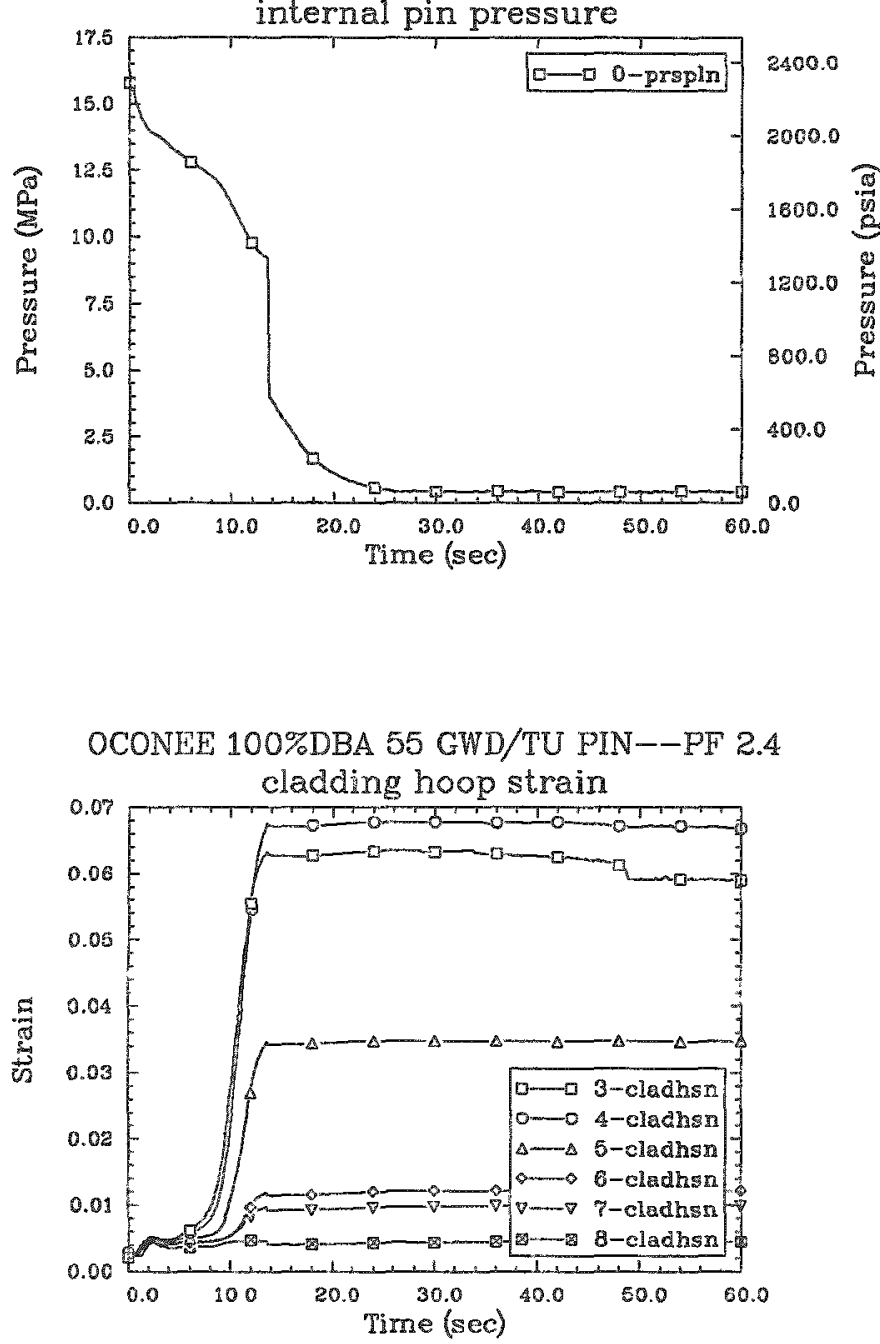

OCONEE 100\%DBA 55 GWD/TU PIN--PF 2.4

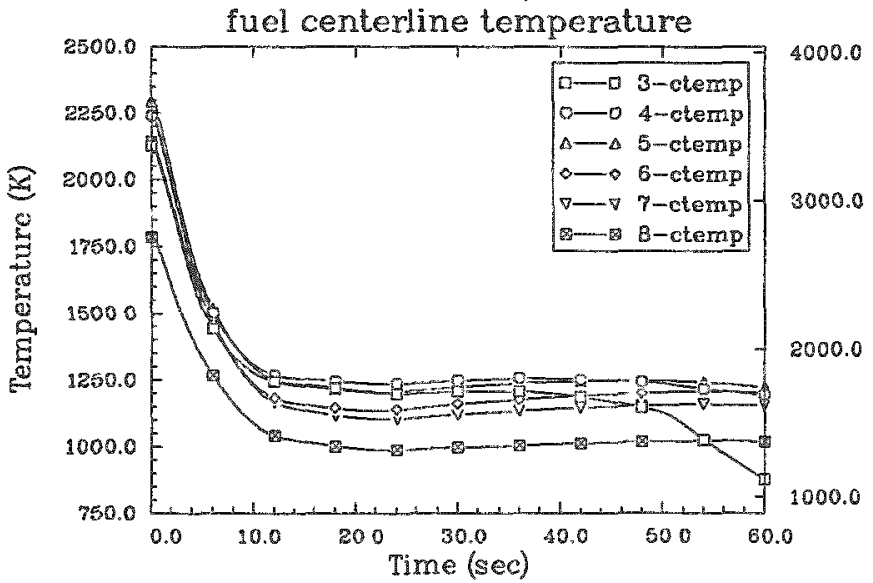

OCONEE 100\%DBA $55 \mathrm{GWD} / \mathrm{TU}$ PIN-DF 2.4 failure probability
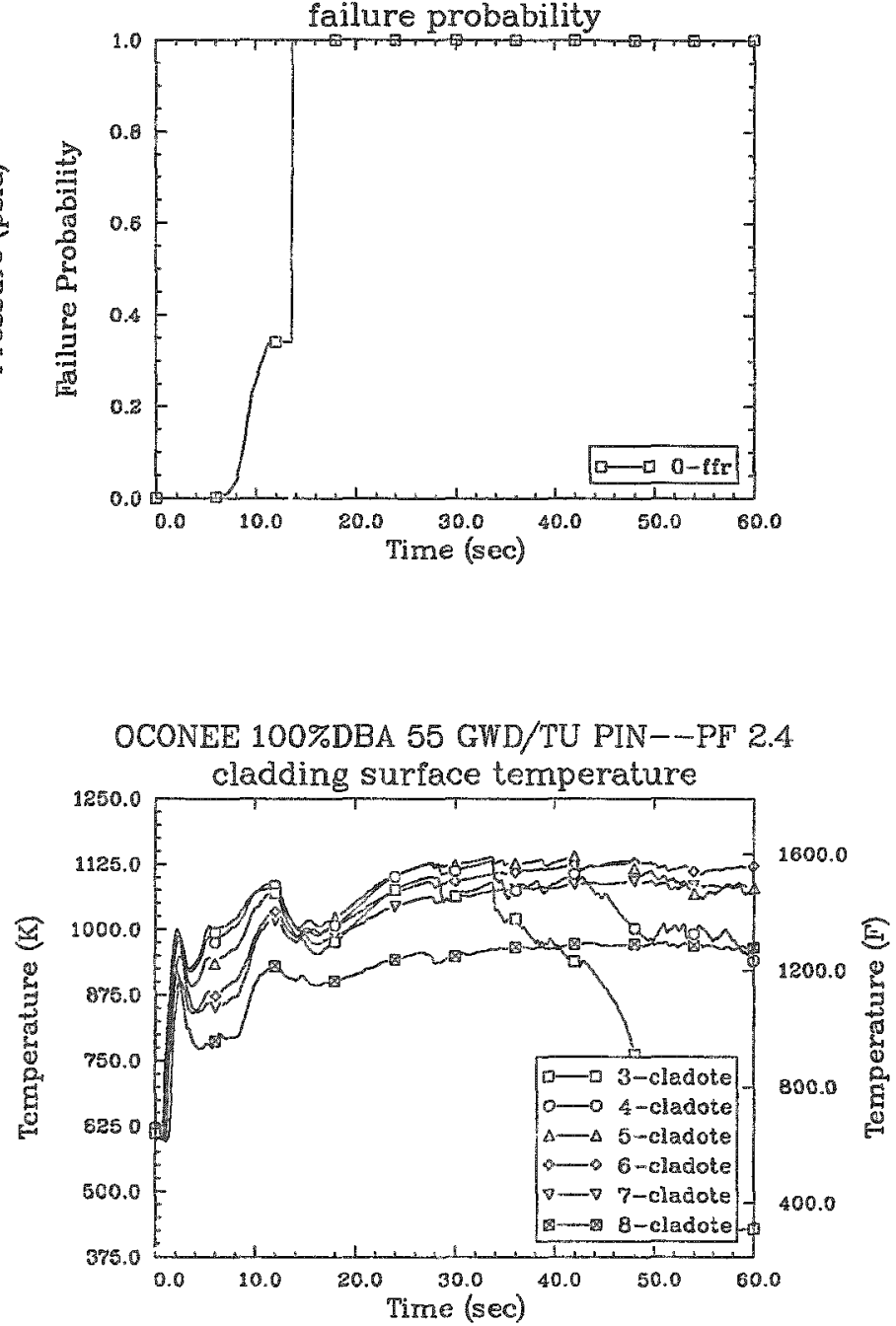

OCONEE 100\%DBA 55 GWD/TU PIN--PF 2.4

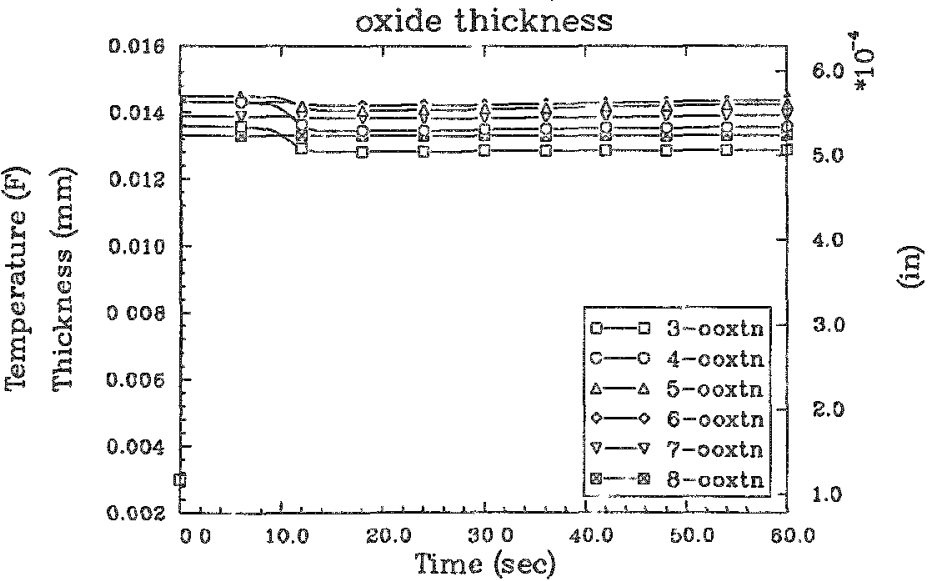



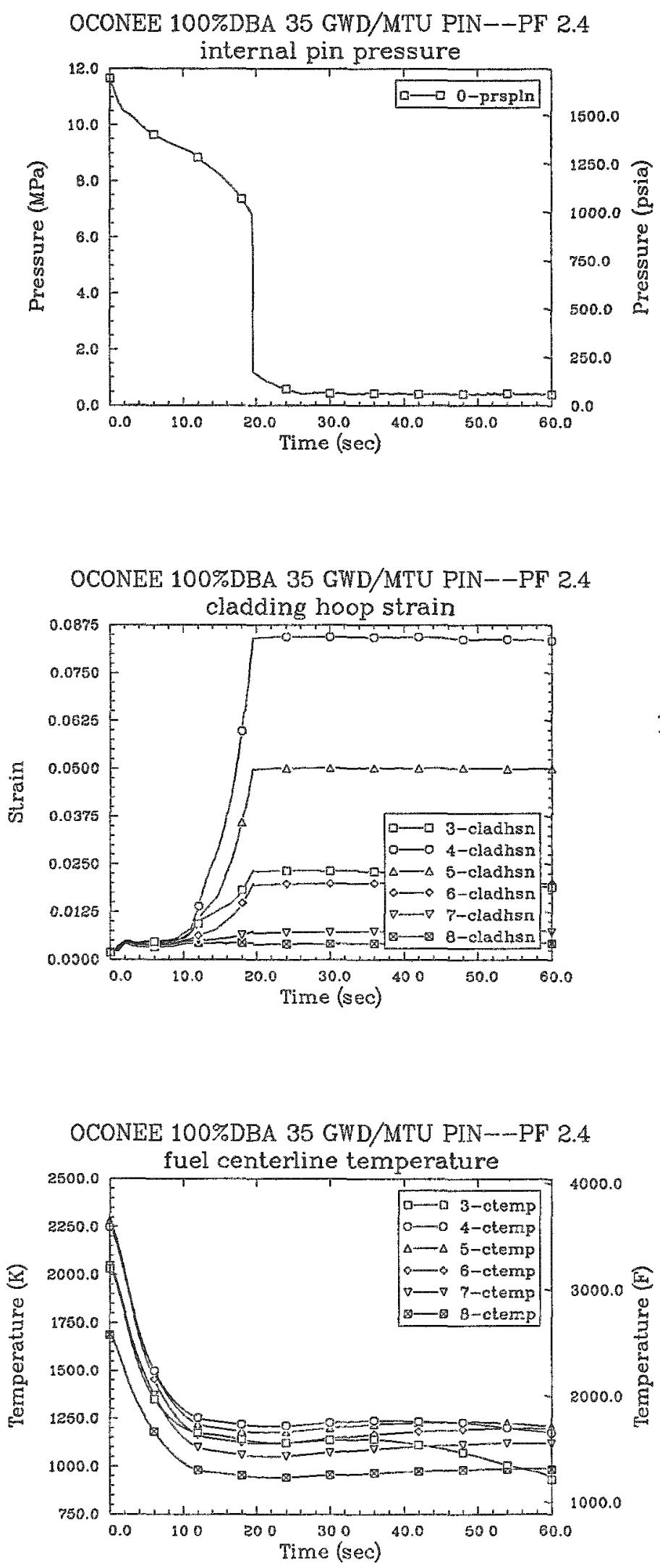
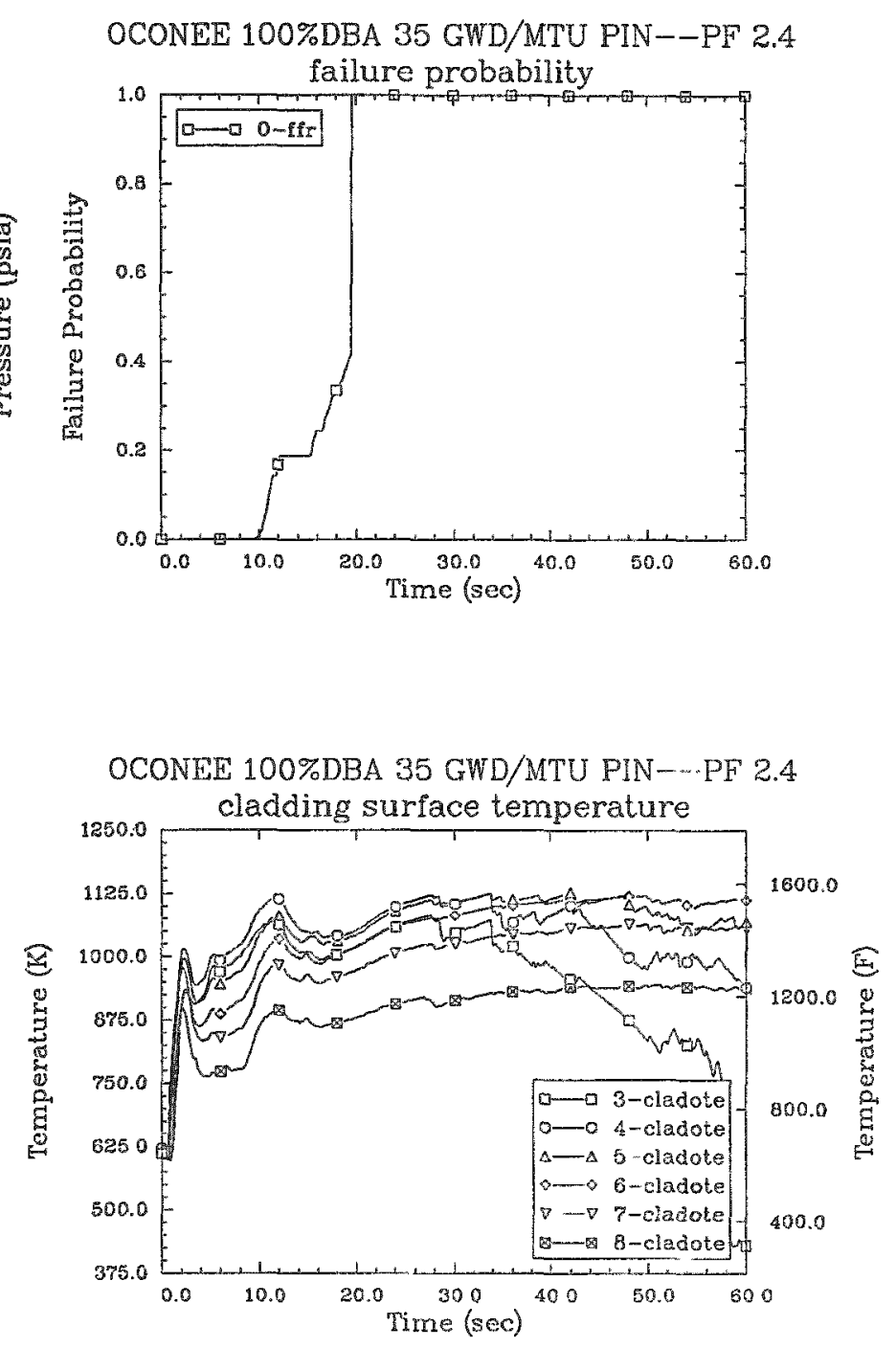

OCONEE 100\%DBA 35 GWD/MTU PIN--PF 2.4

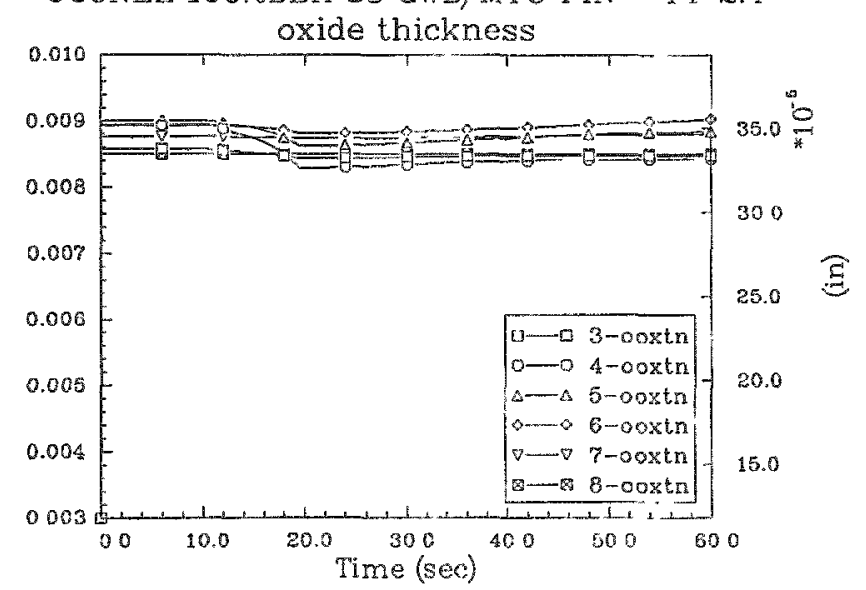




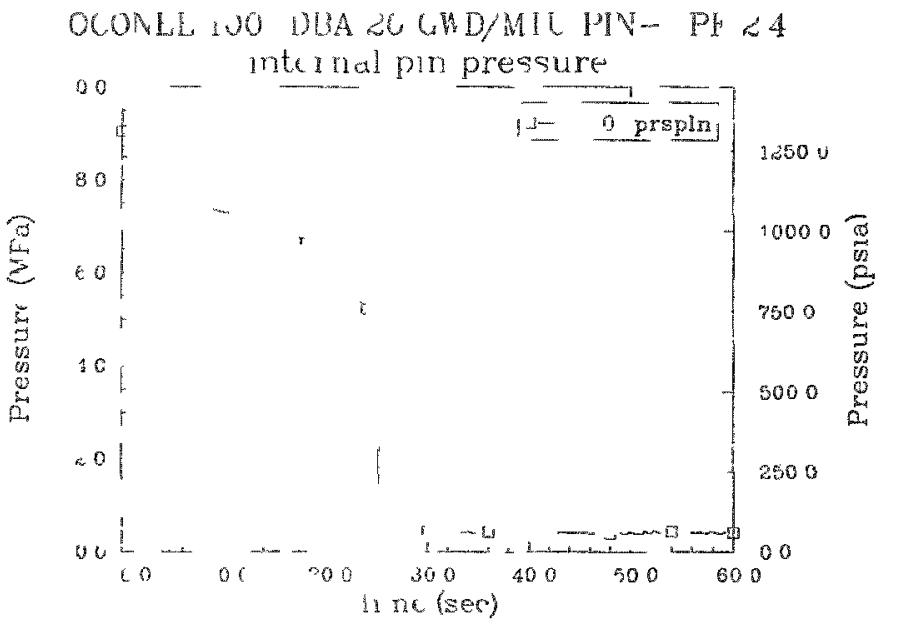

OCONEI 100\%OUBA 2UGMU MTU IN IN 24
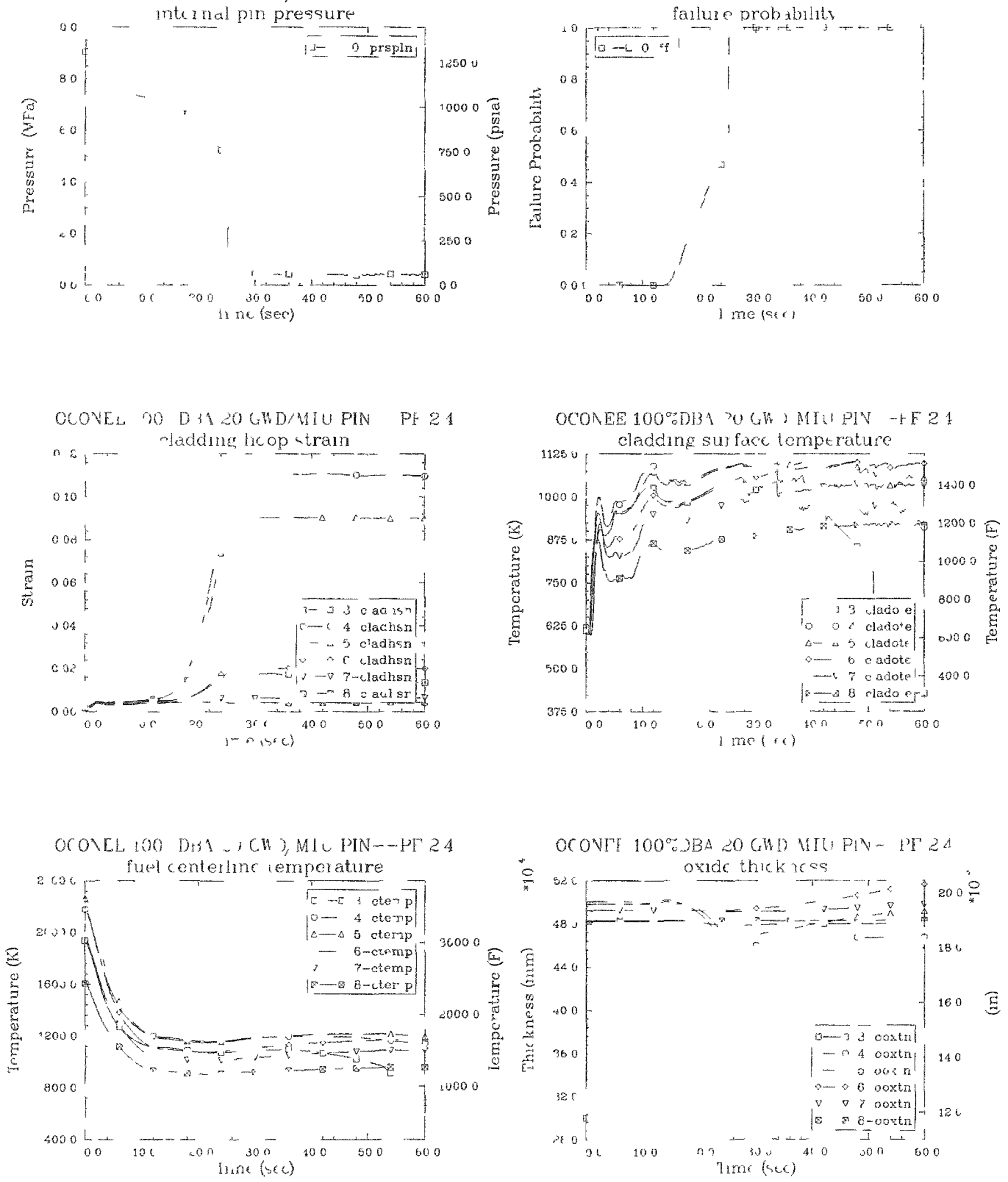

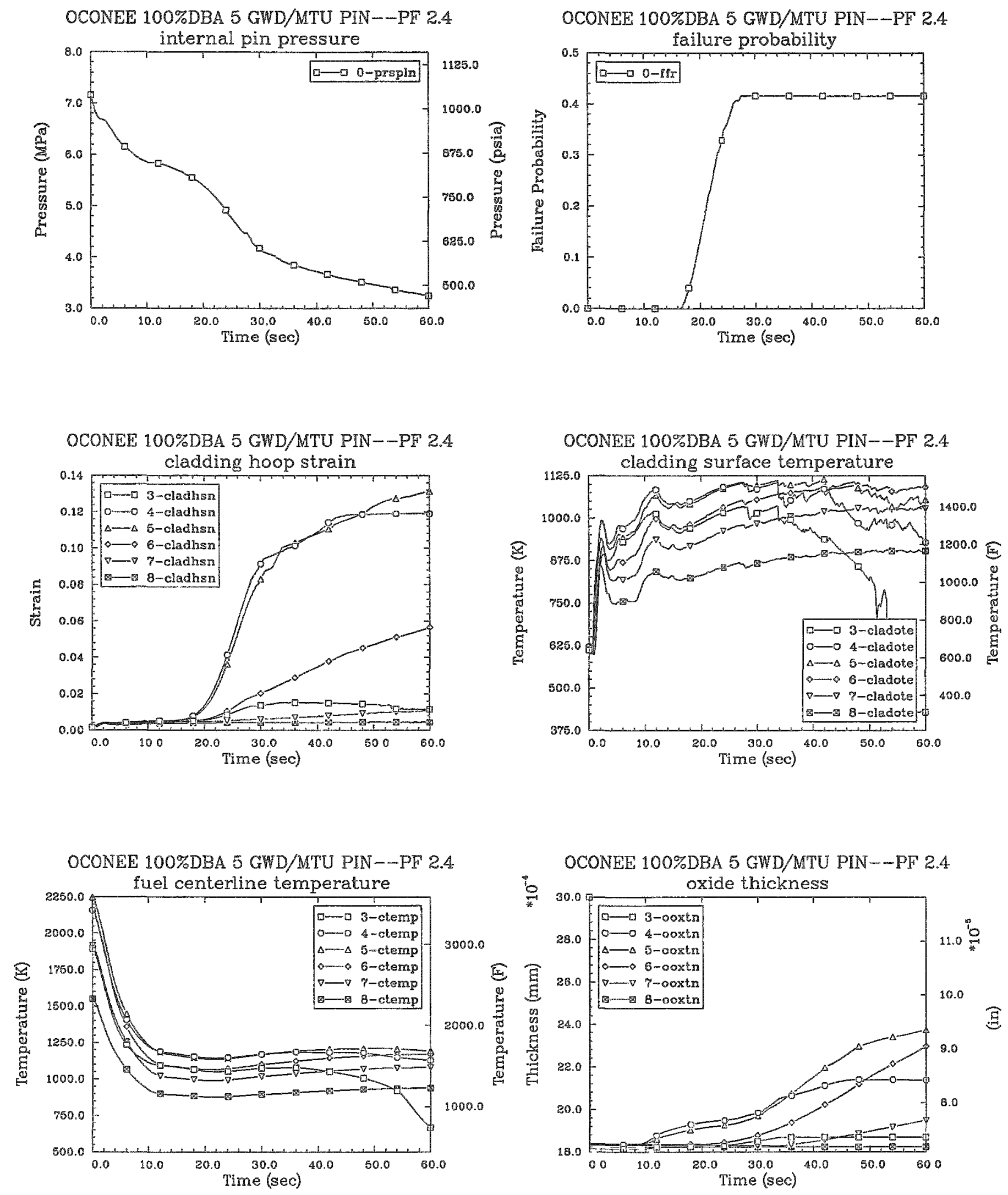

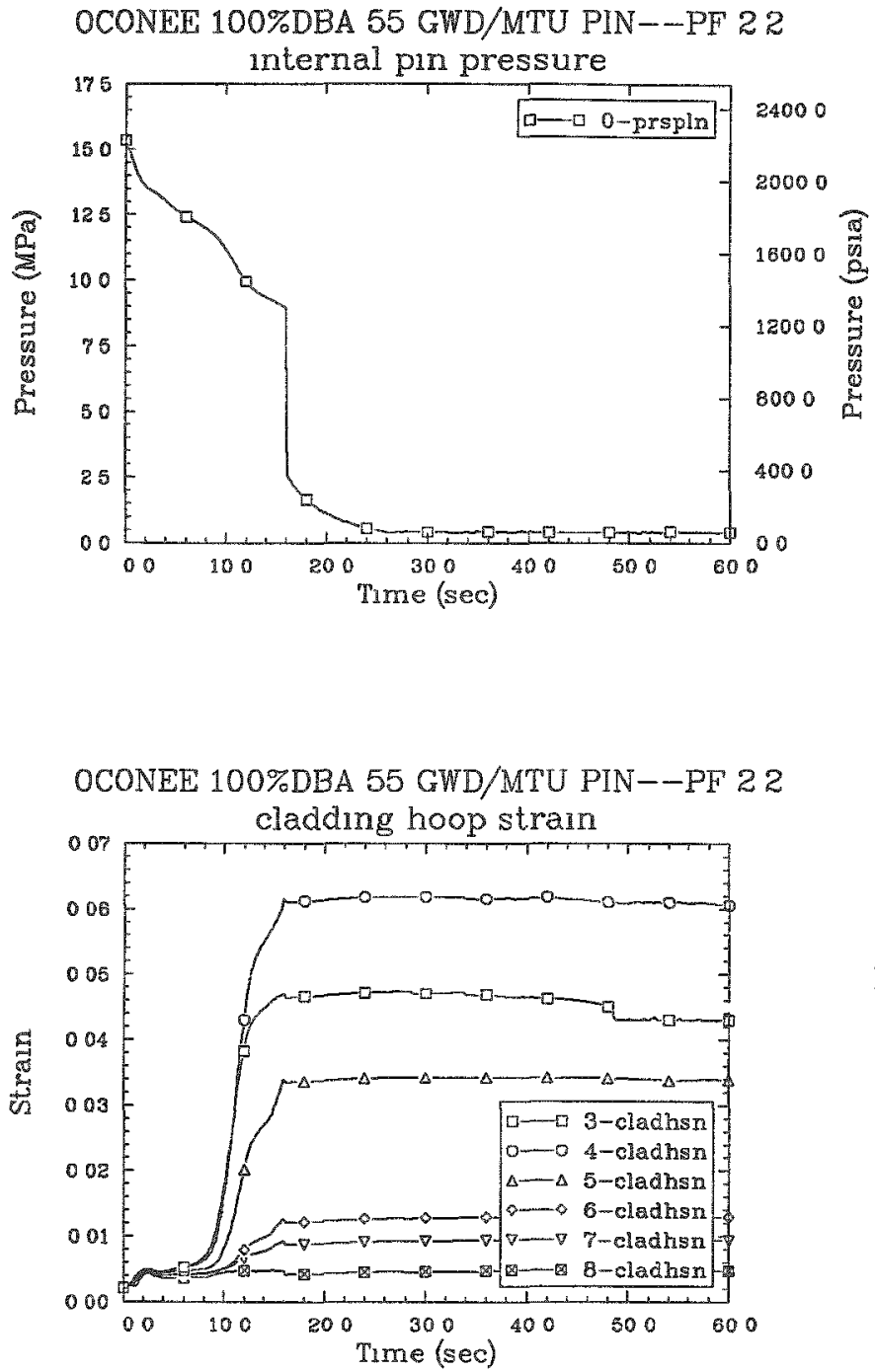

OCONEE 100\%DBA 55 GWD/MTU PIN--PF 22

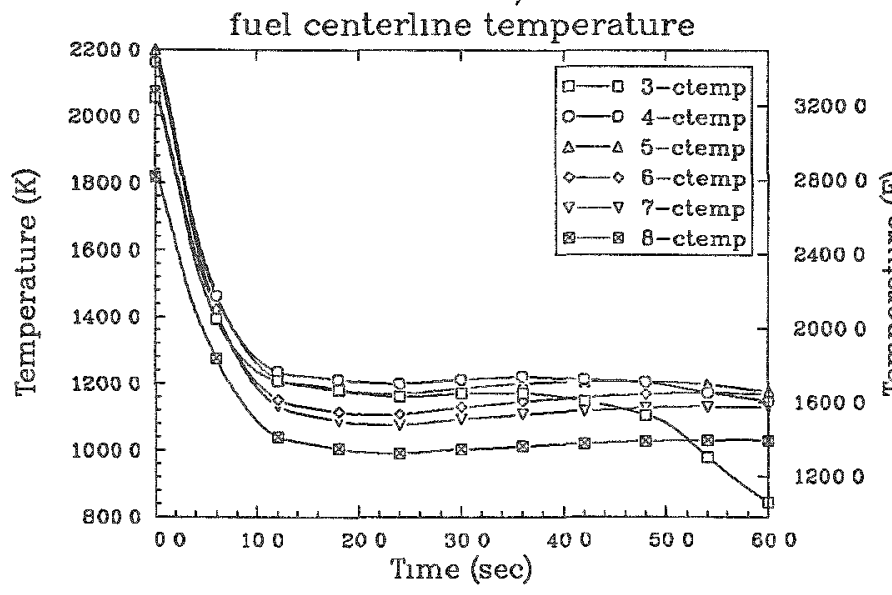

OCONEE 100\%DBA 55 GWD/MTU PIN--PF 22

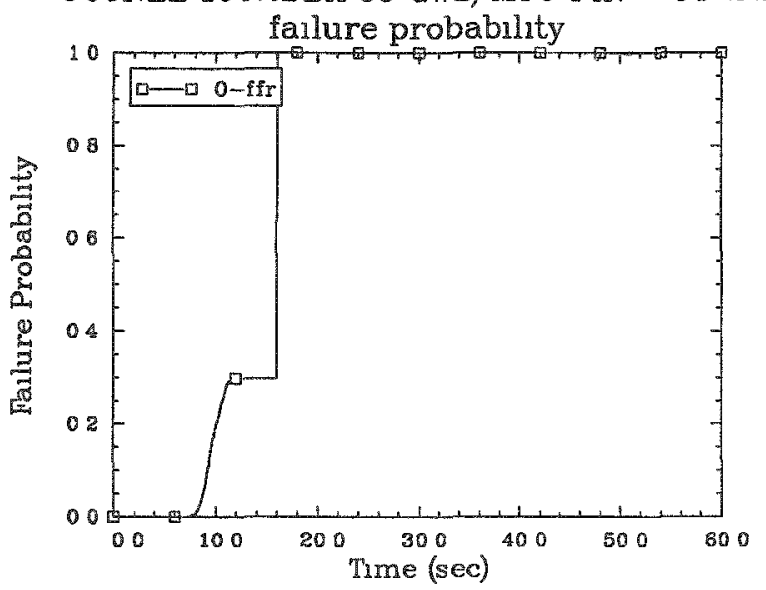

OCONEE $100 \%$ DBA 55 GWD/MTU PIN--PF 22

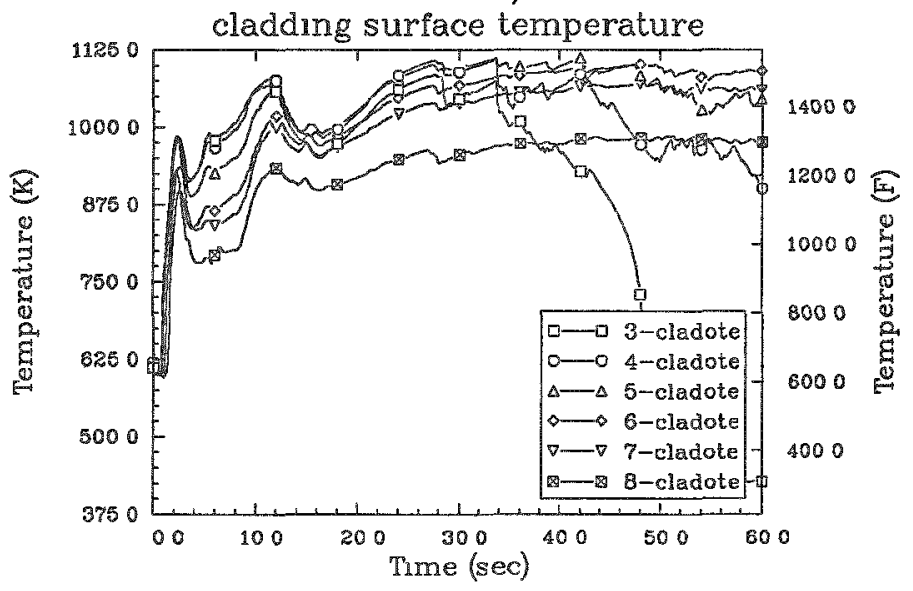

OCONEE 100\%DBA 55 GWD/MTU PIN-DF 22

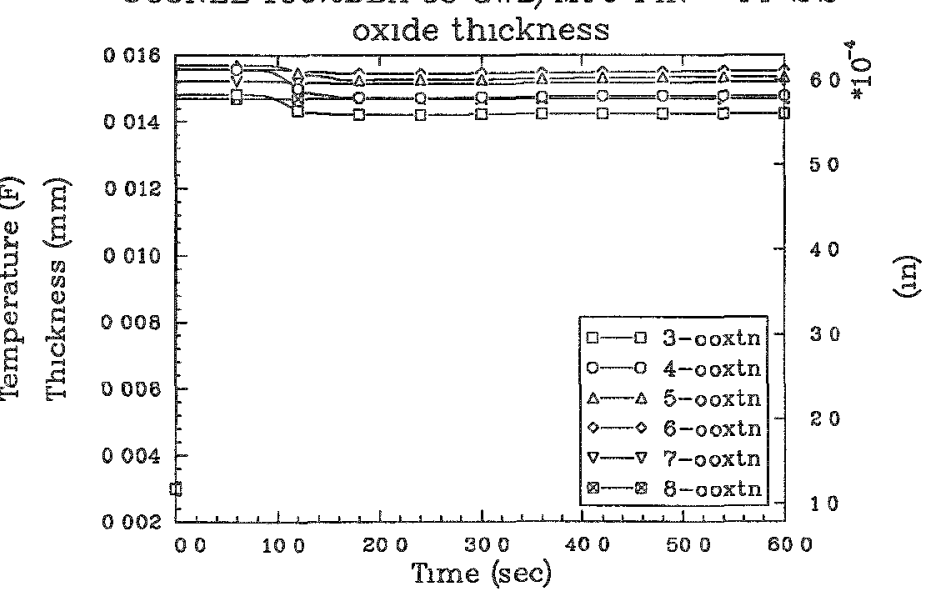



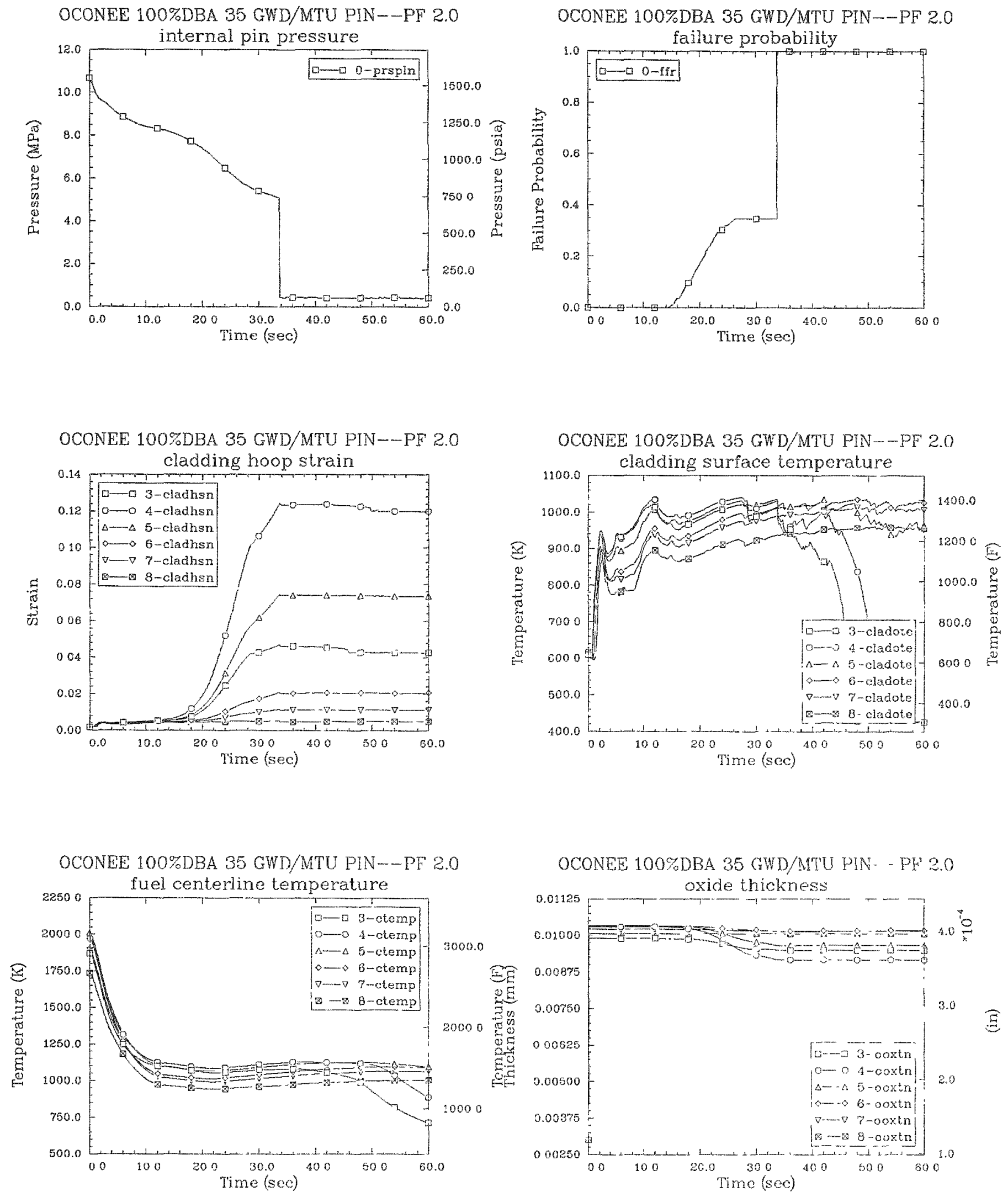

OCONEE 100\%DBA 35 GWD/MTU PIN - PF 2.0

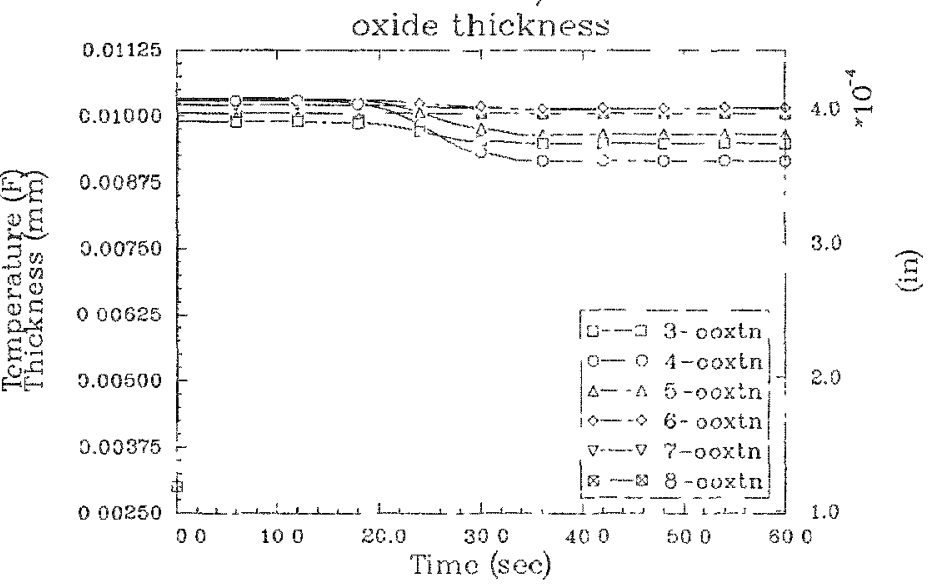



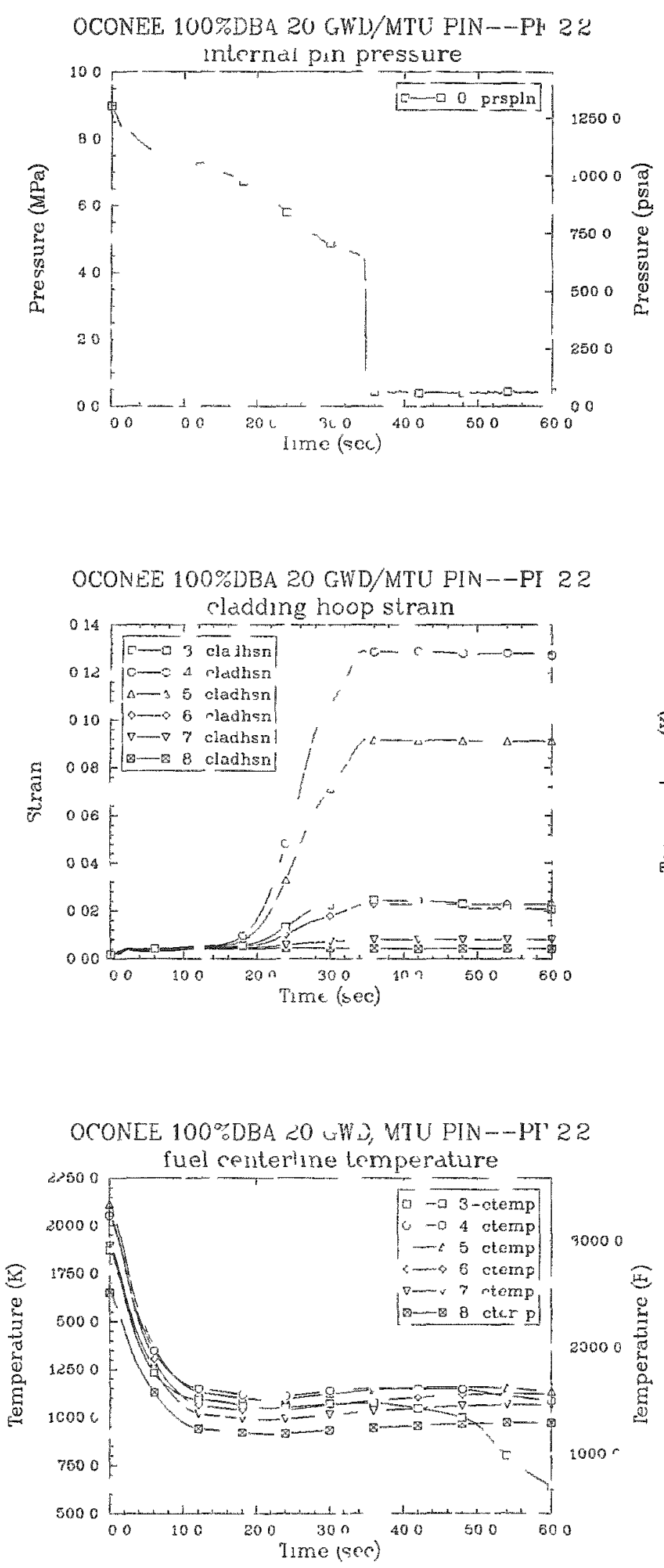

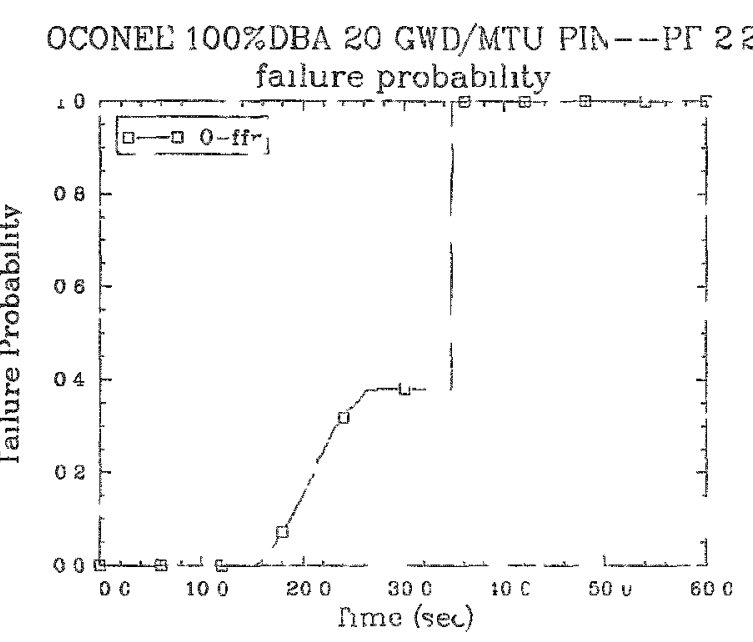

OCONEL 100\%DBA 20 GWD/MTU PIN-DP 22
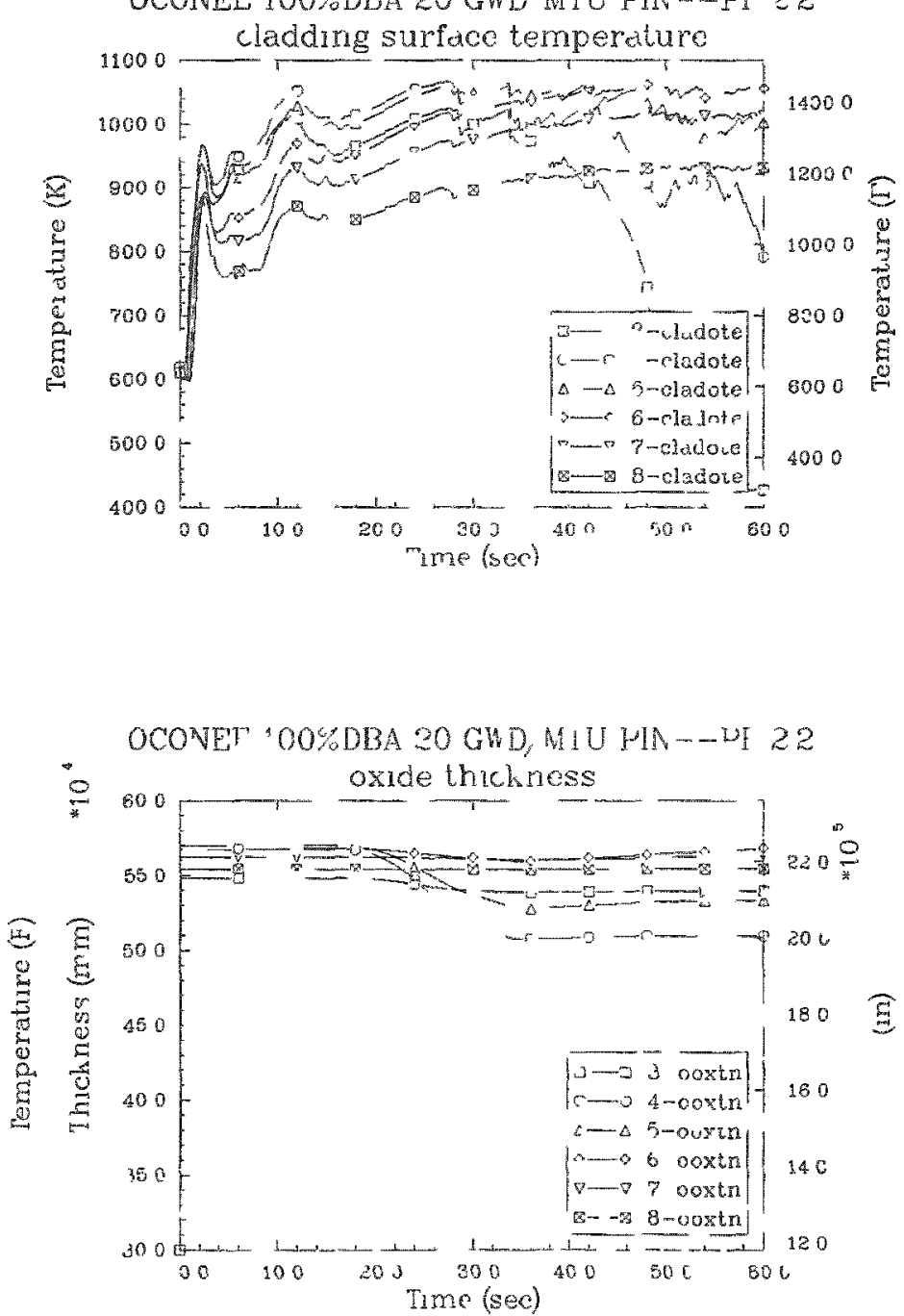

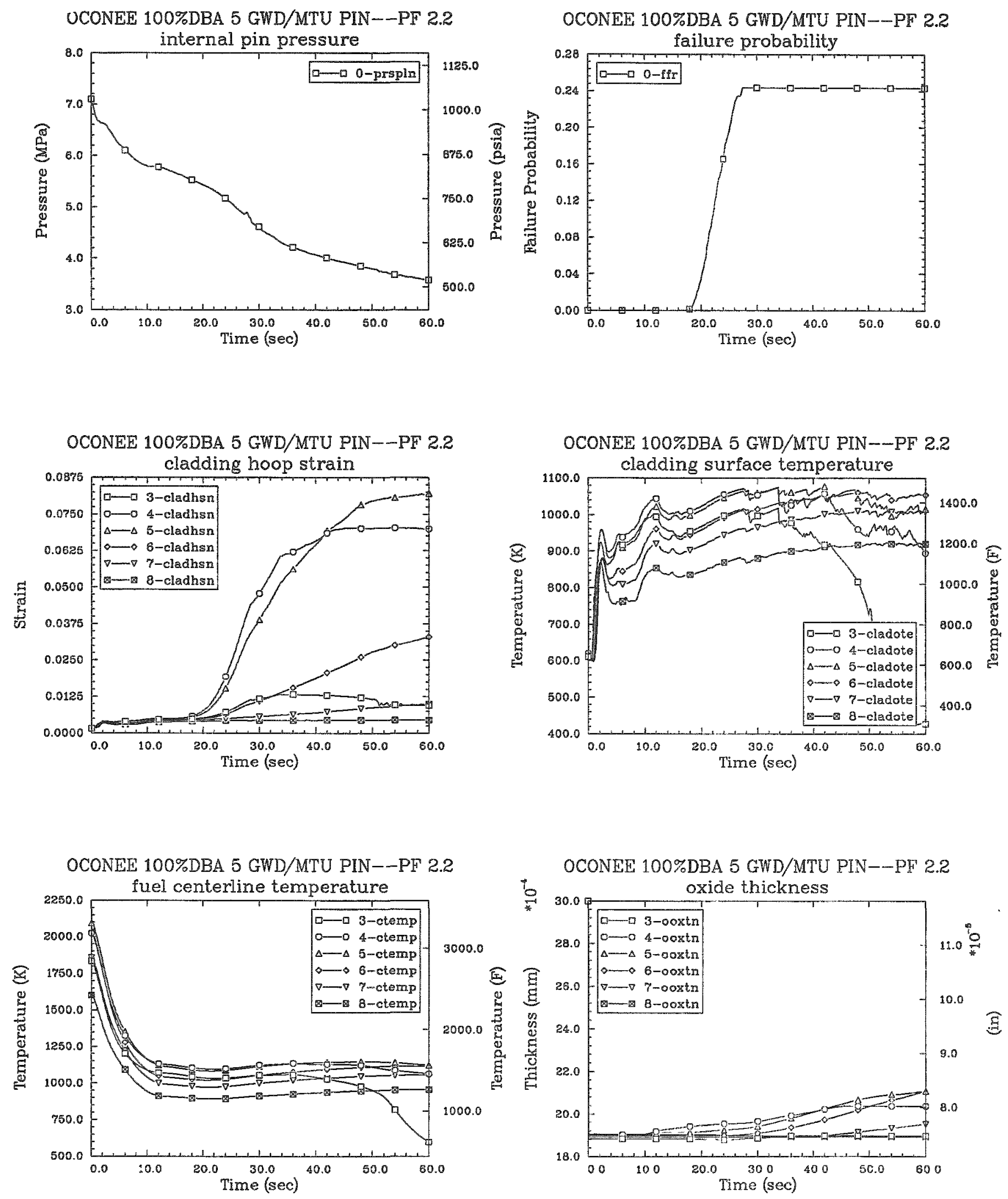

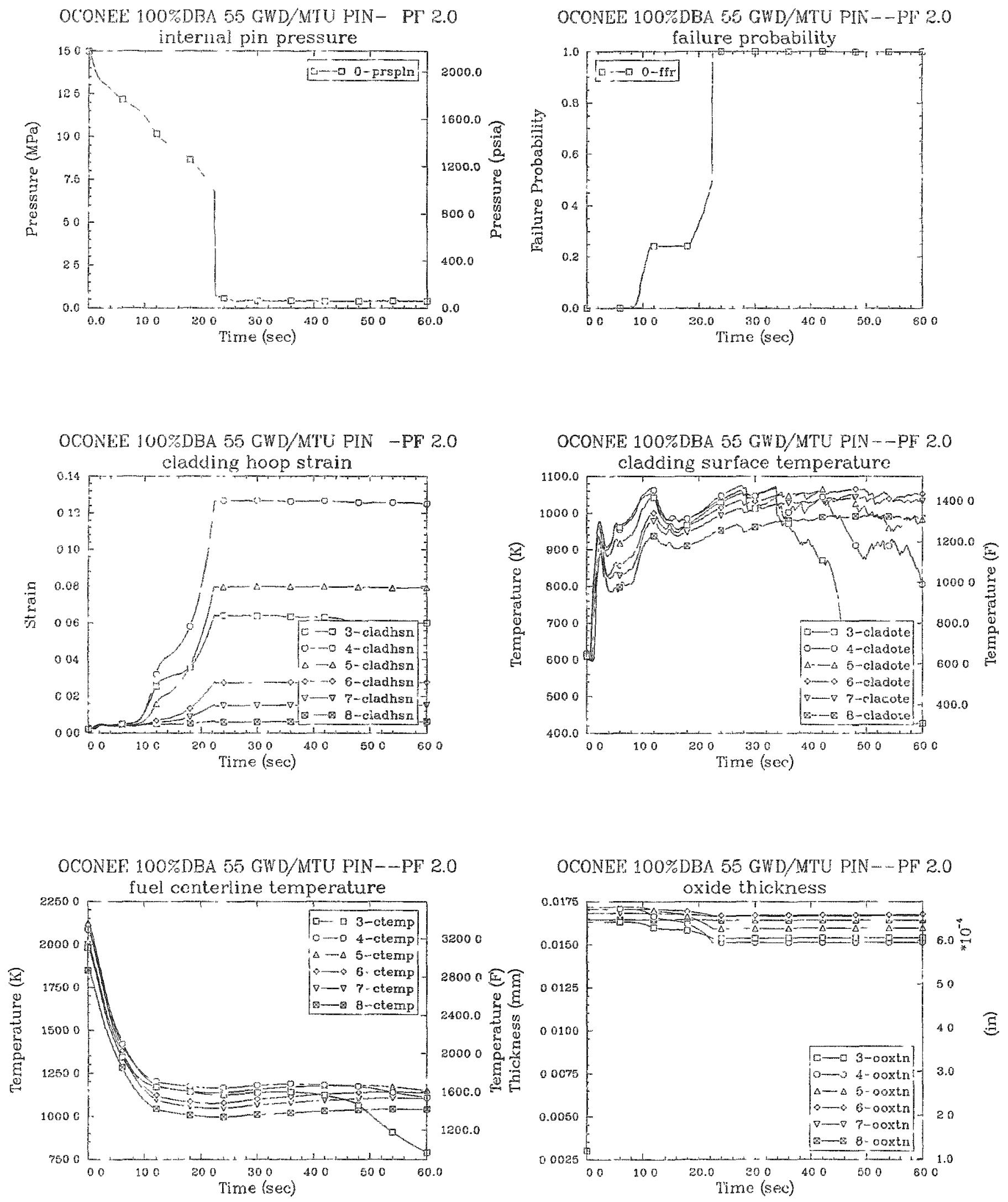

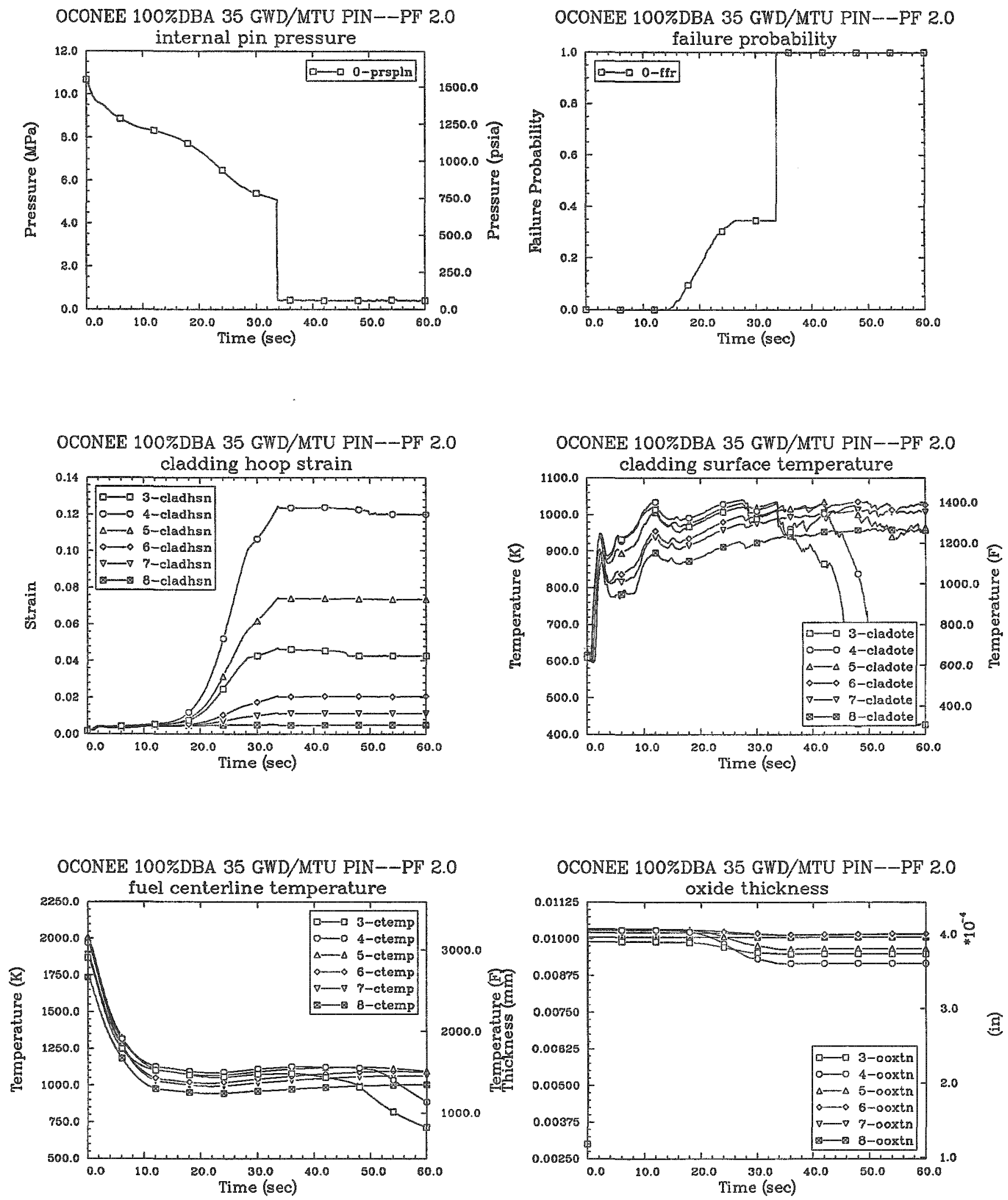
OCONEE 100\%DBA 20 GWD/MTU PIN--.PF 2.0
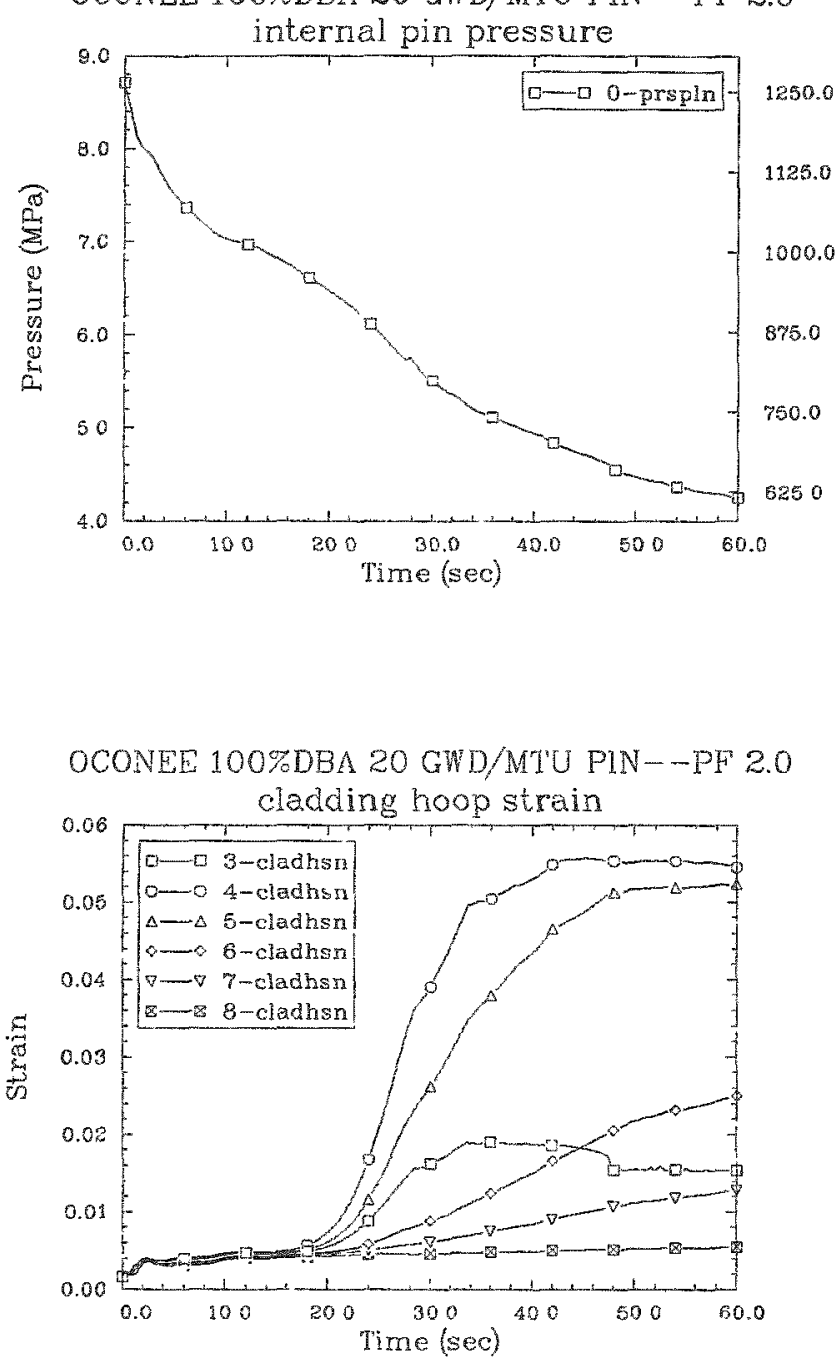

OCONEE 100\%DBA 20 GWD/MTU PIN--PF 2.0

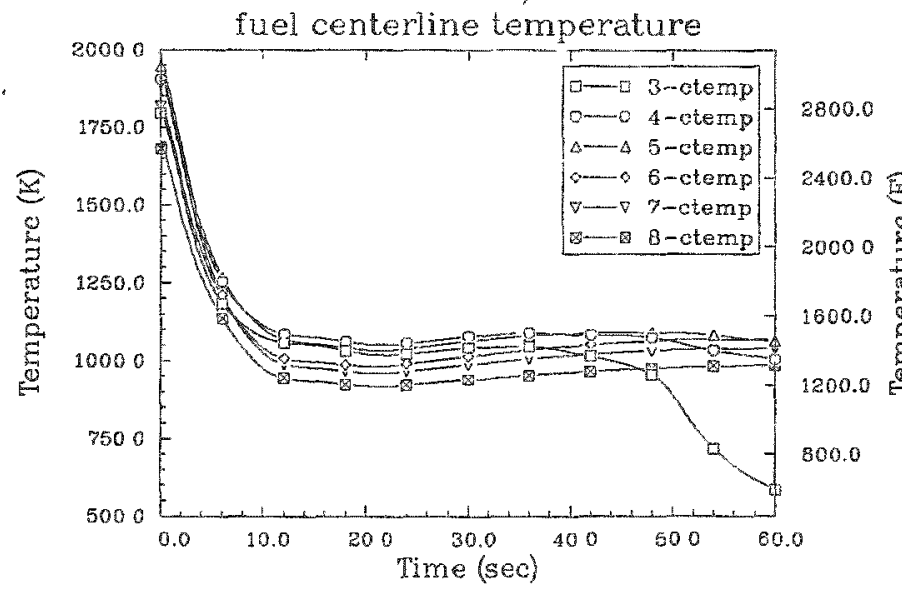

OCONEE 100\%DBA 20 GWD/MTU PIN_-PF 2.0

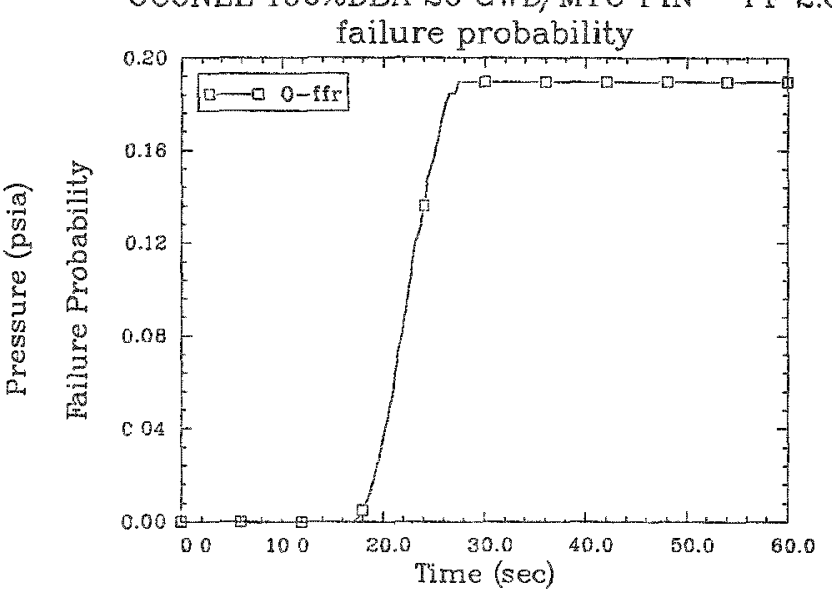

OCONEE 100\%DBA 20 GWD/MTU PIN--PF 2.0

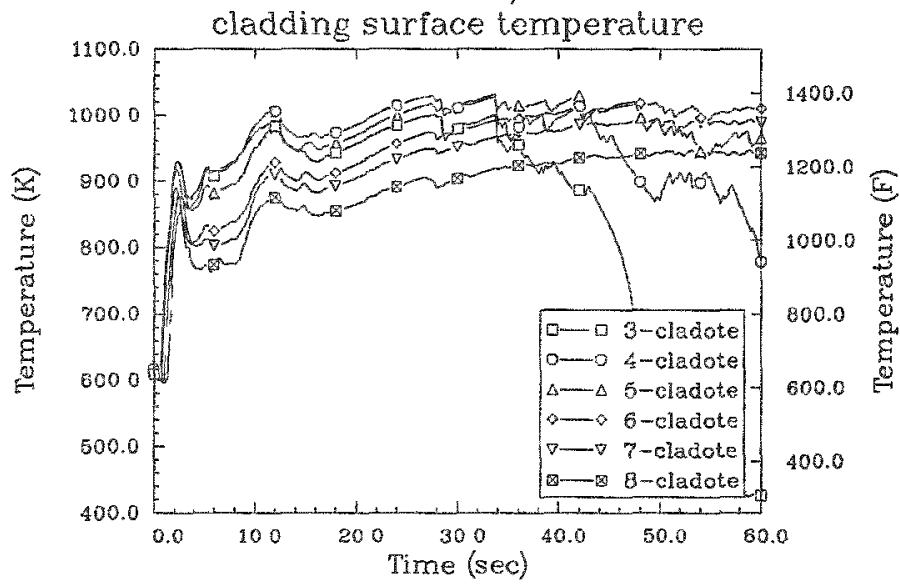

OCONEE 100\%DBA 20 GWD/MTU PIN--PF 2.0

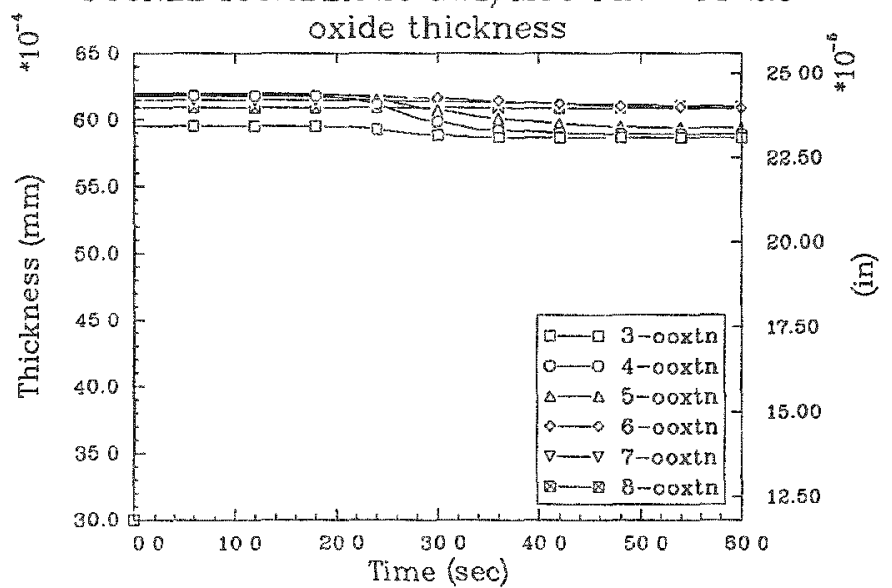



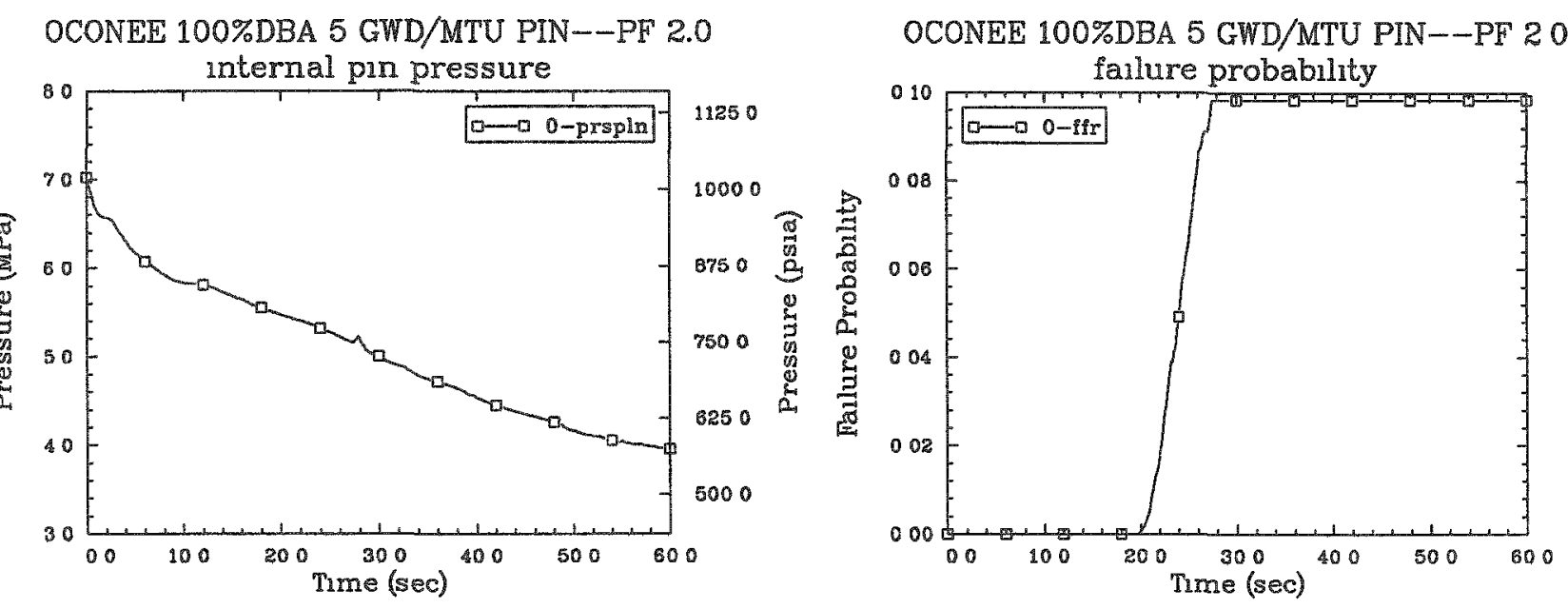

OCONEE 100\%DBA 5 GWD/MTU PIN--PF 20
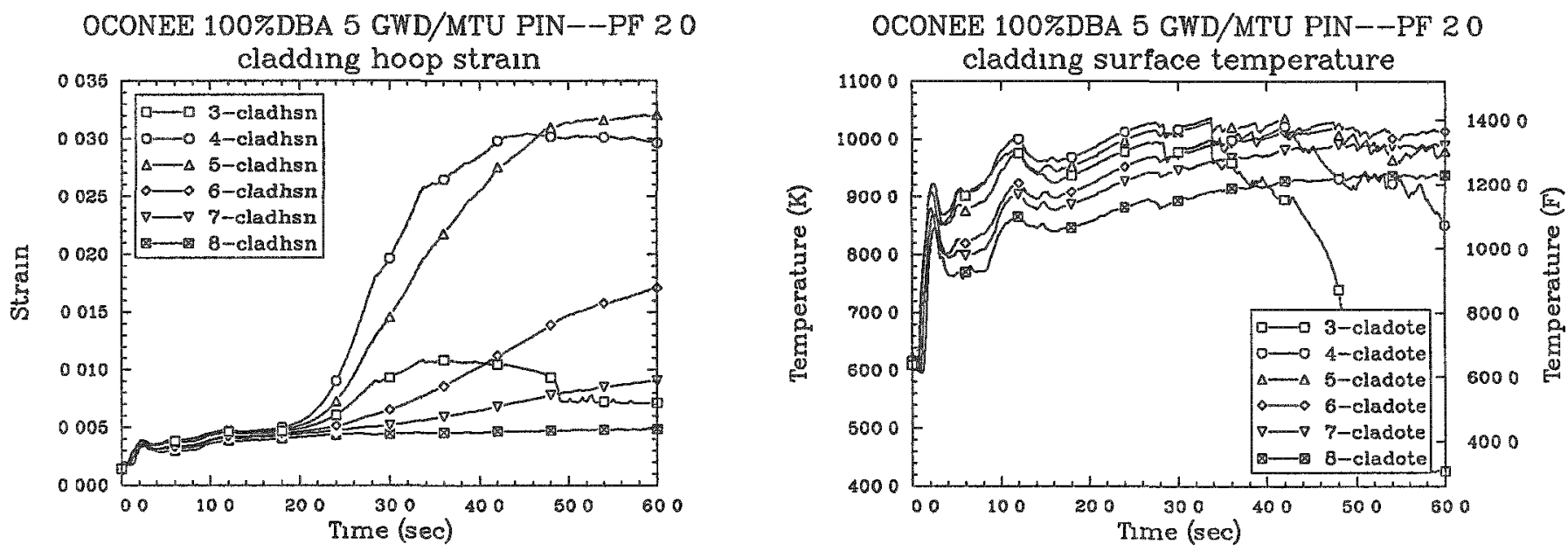

OCONEE 100\%DBA 5 GWD/MTU PIN--PF 20

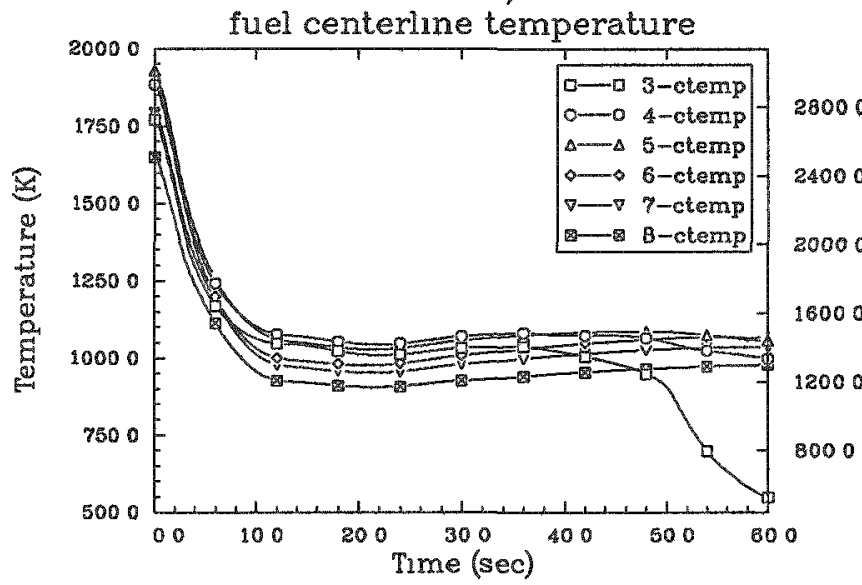

OCONEE 100\%DBA 5 GWD/MTU PIN--PF 20

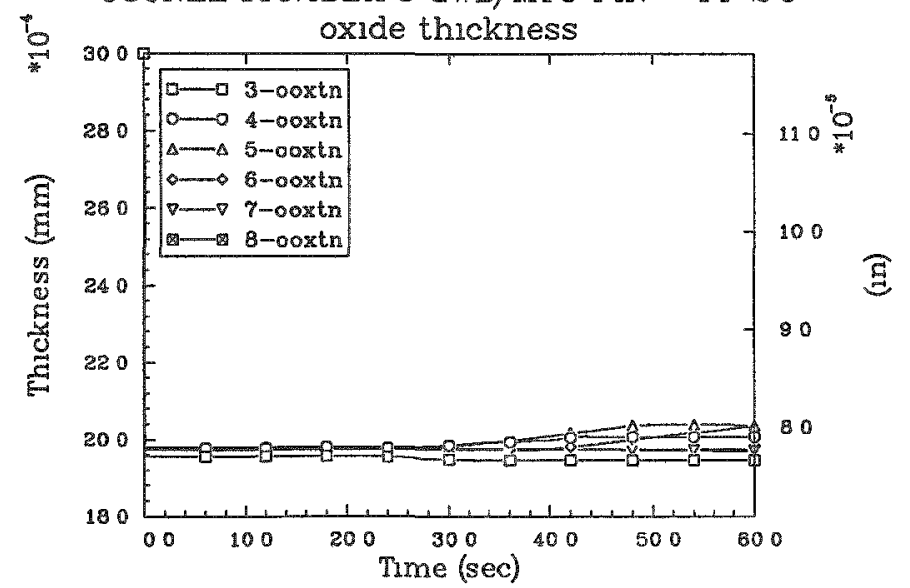



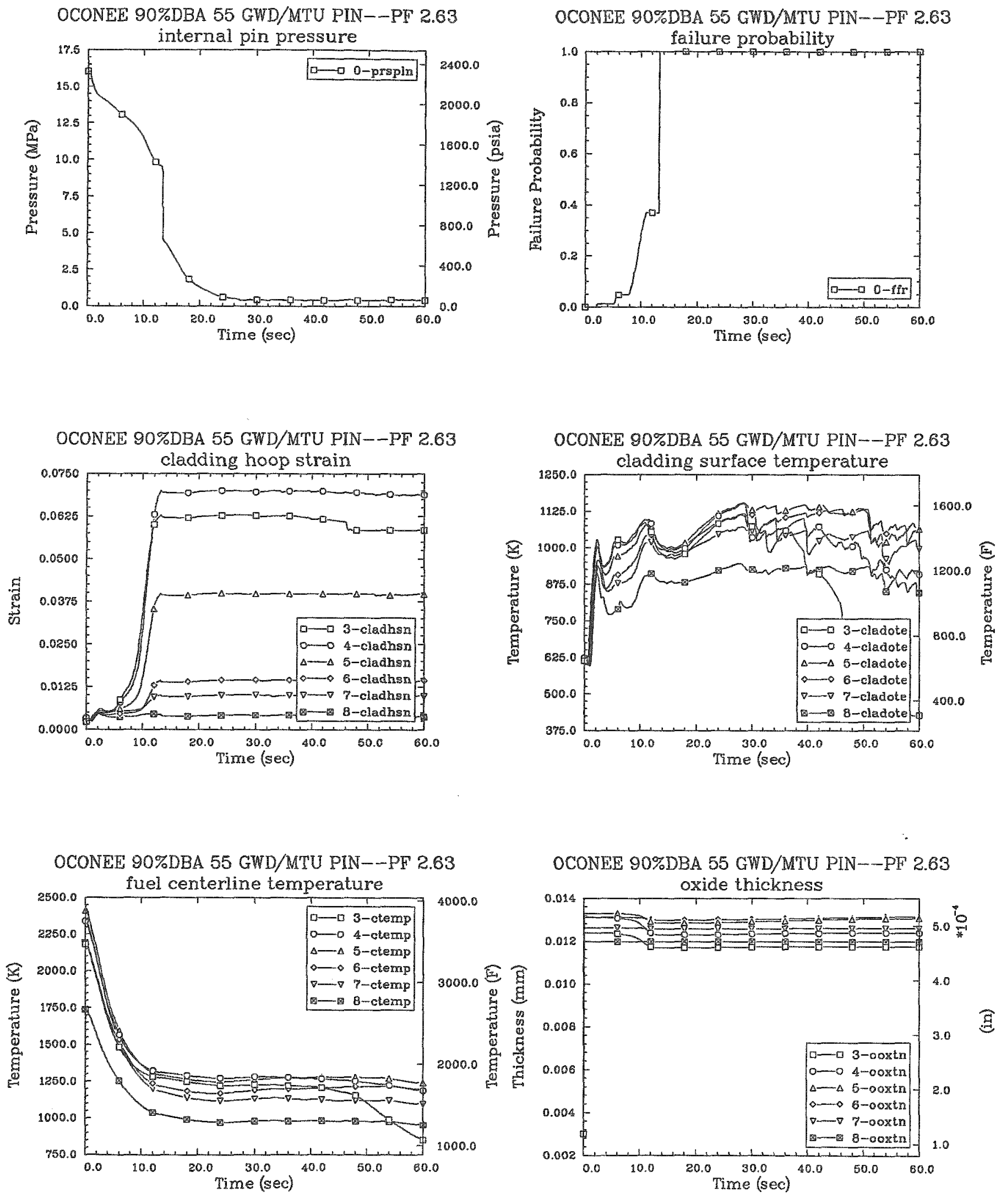

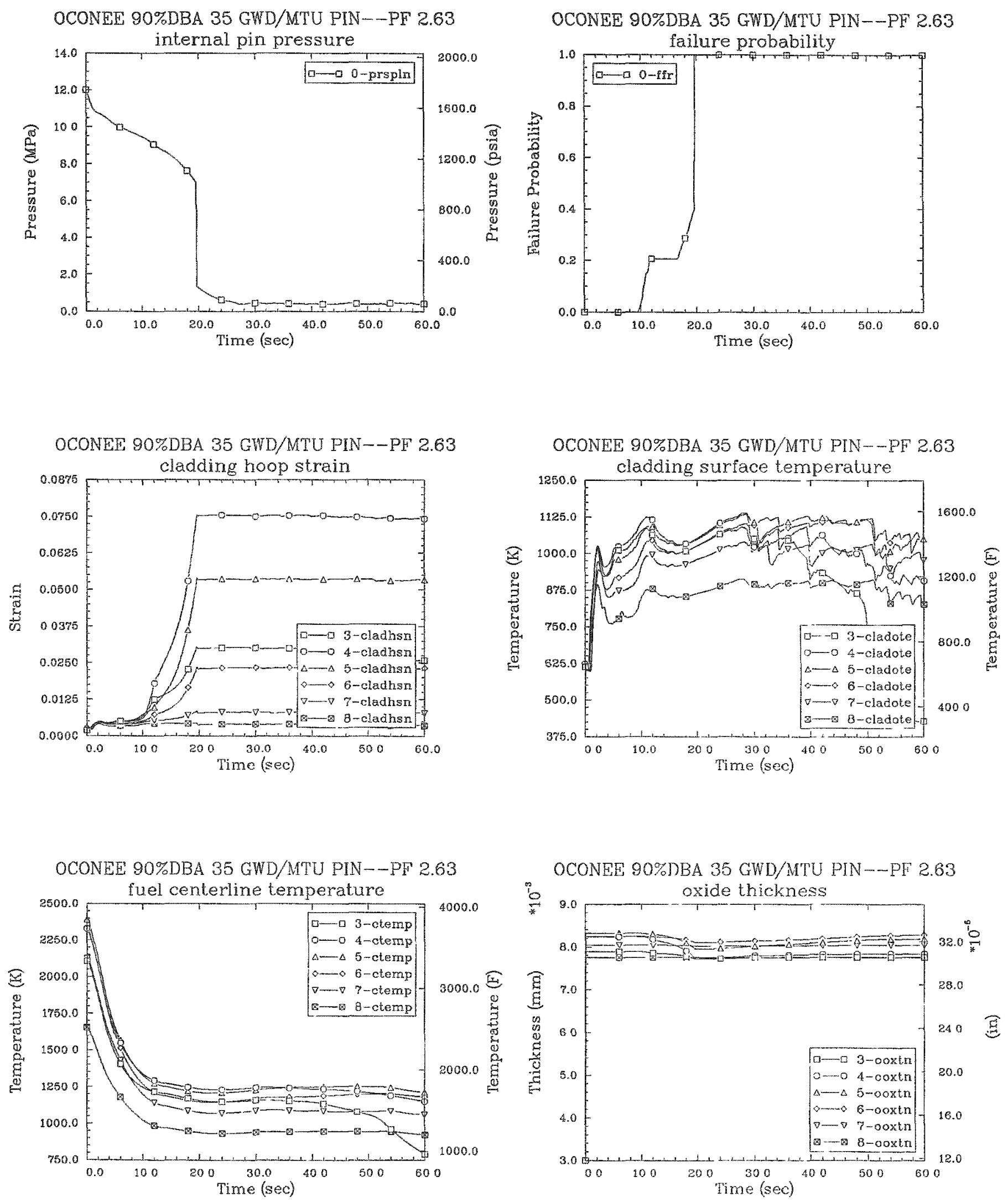

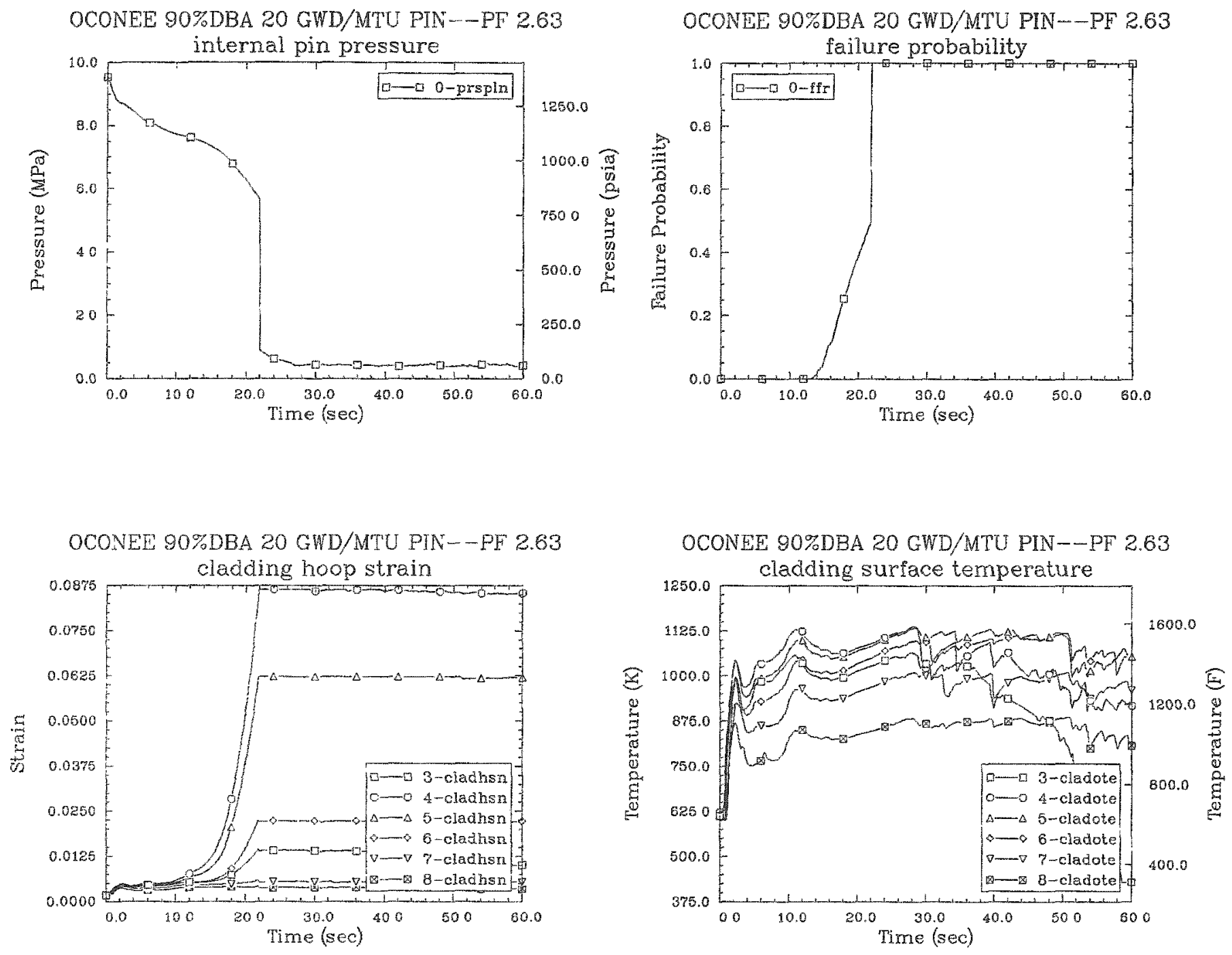

OCONEE 90\%DBA 20 GWD/MTU PIN-DPF 2.63

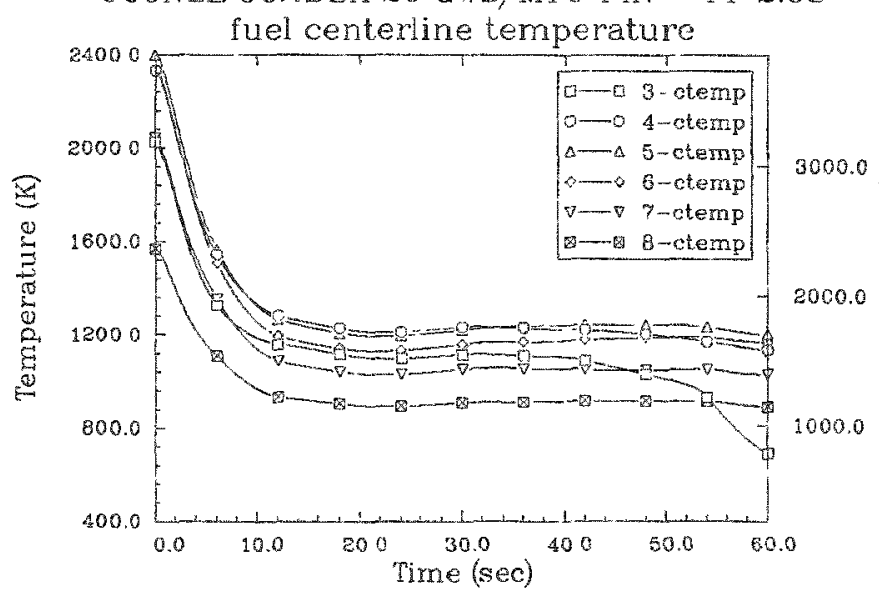

OCONEE 90\%DBA $20 \mathrm{GWD} / \mathrm{MTU}$ PIN-DF 2.63

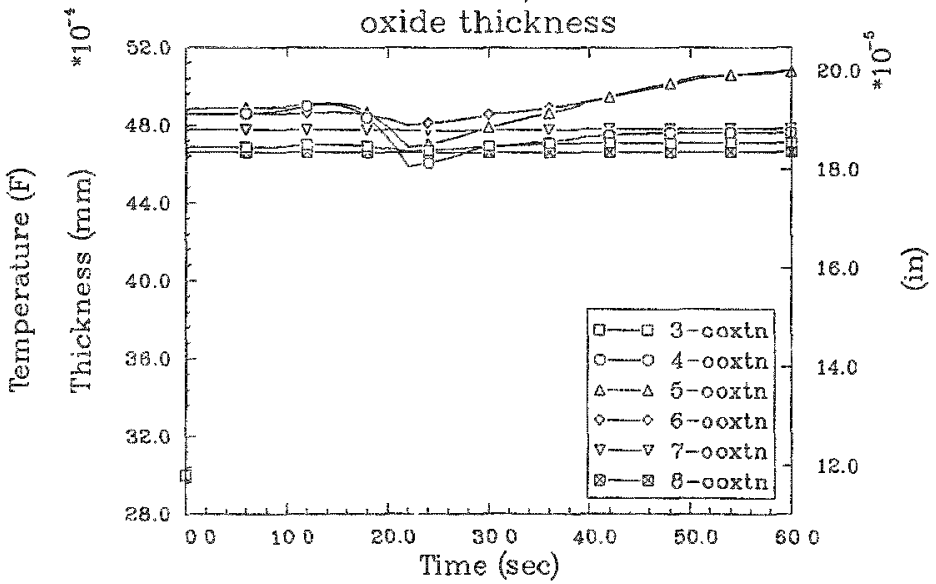



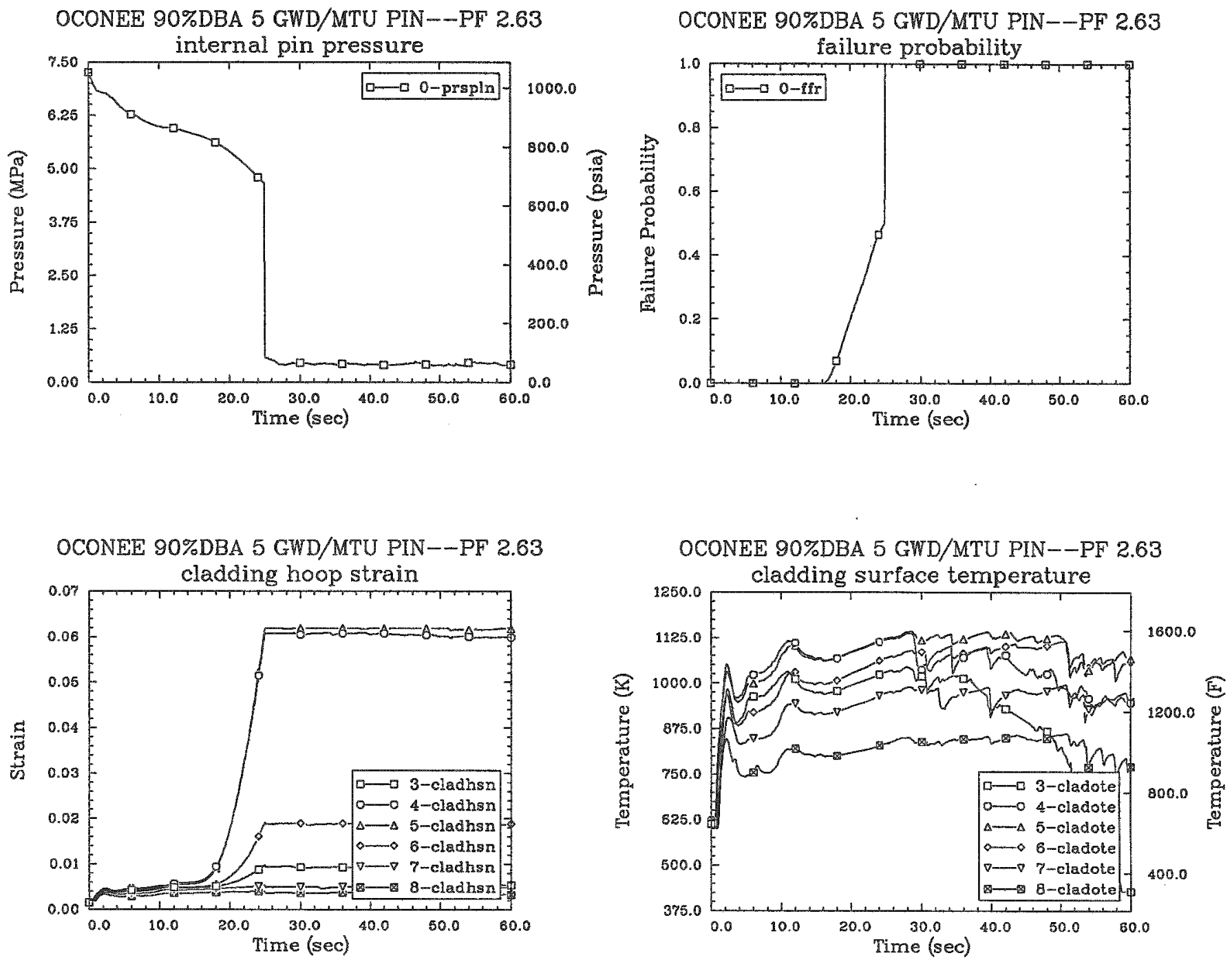

OCONEE 90\%DBA 5 GWD/MTU PIN--PF 2.63
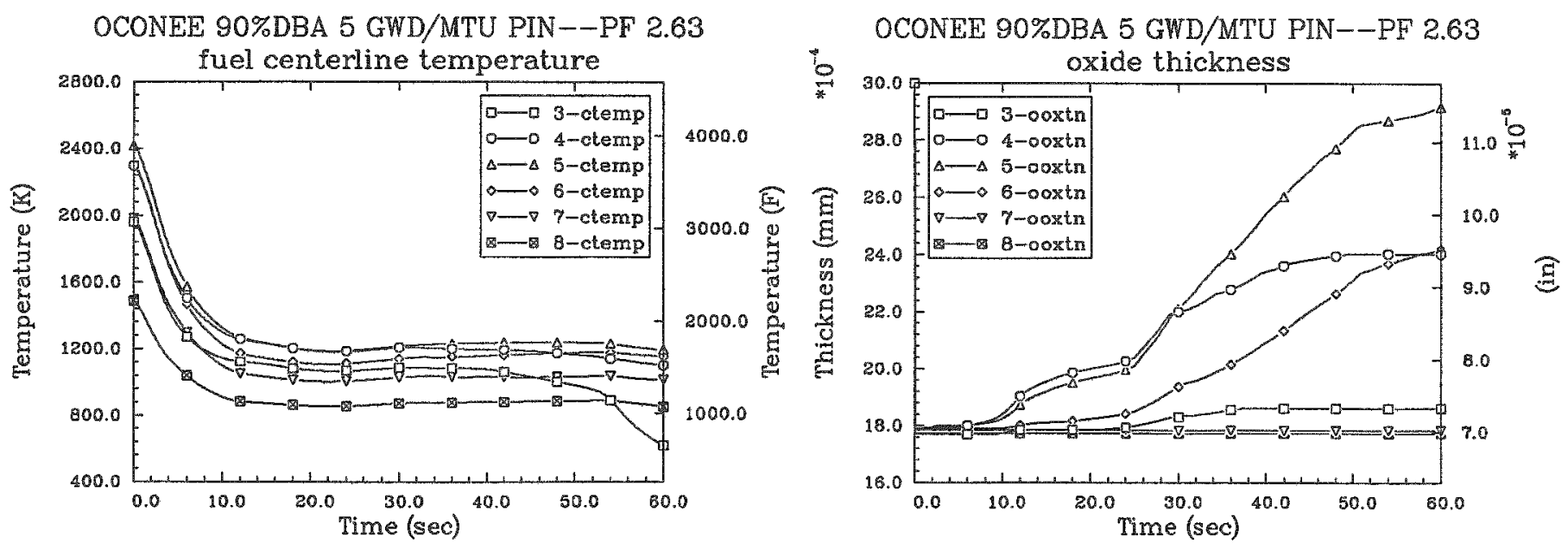

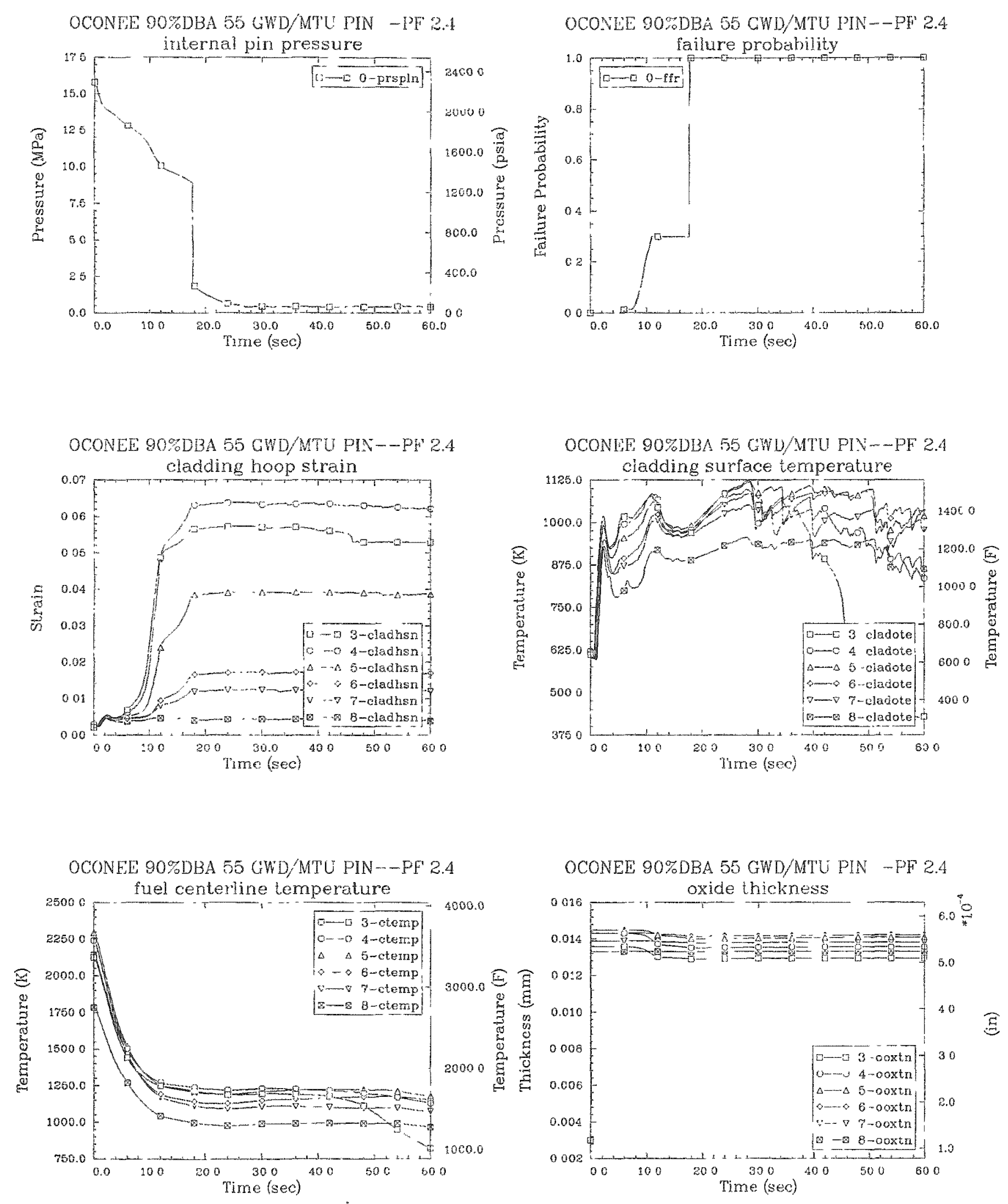

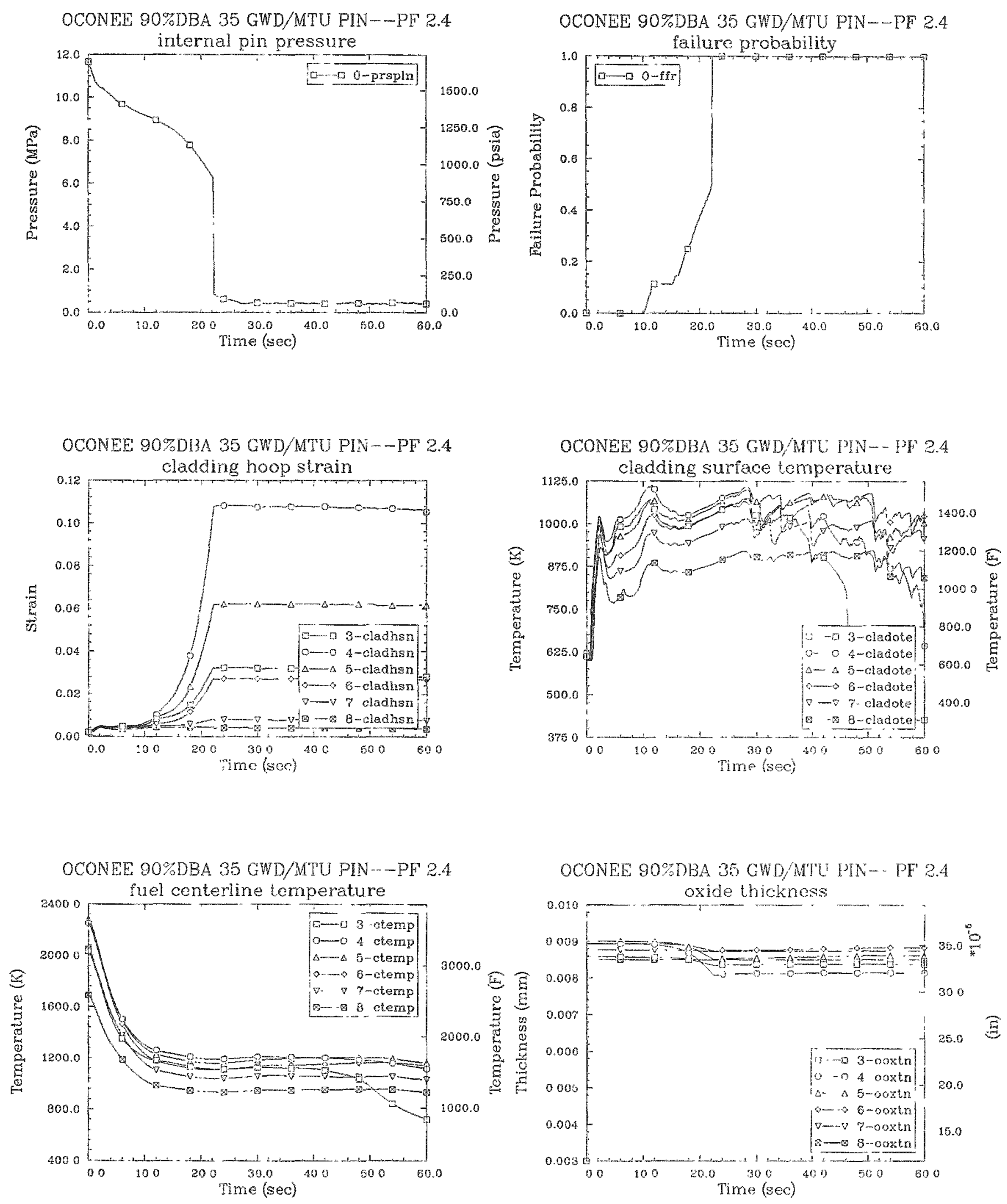
OCONEE 90\%DBA $20 \mathrm{GWD} / \mathrm{MTU}$ PIN-DF 2.4

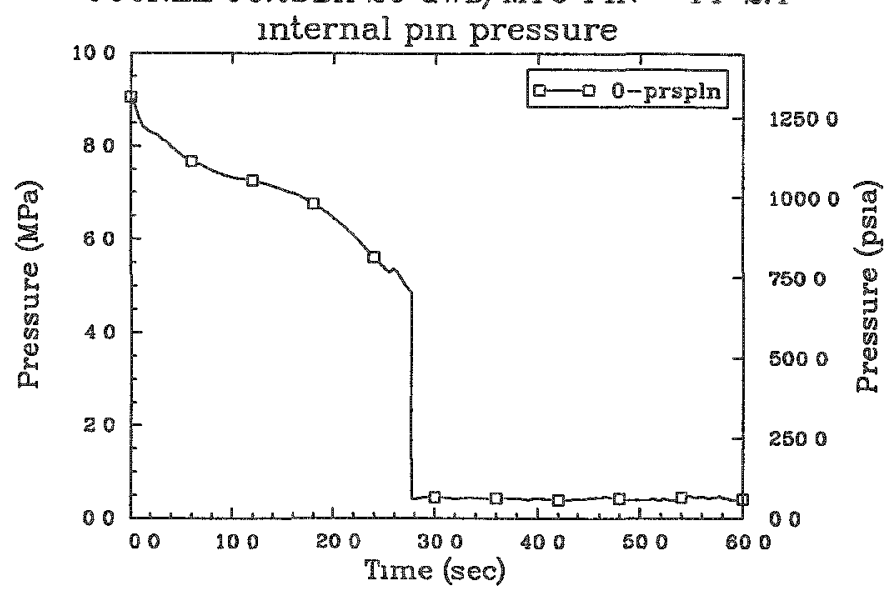

OCONEE 90\%DBA $20 \mathrm{GWD} / \mathrm{MTU}$ PIN--PF 2.4 cladding hoop strain

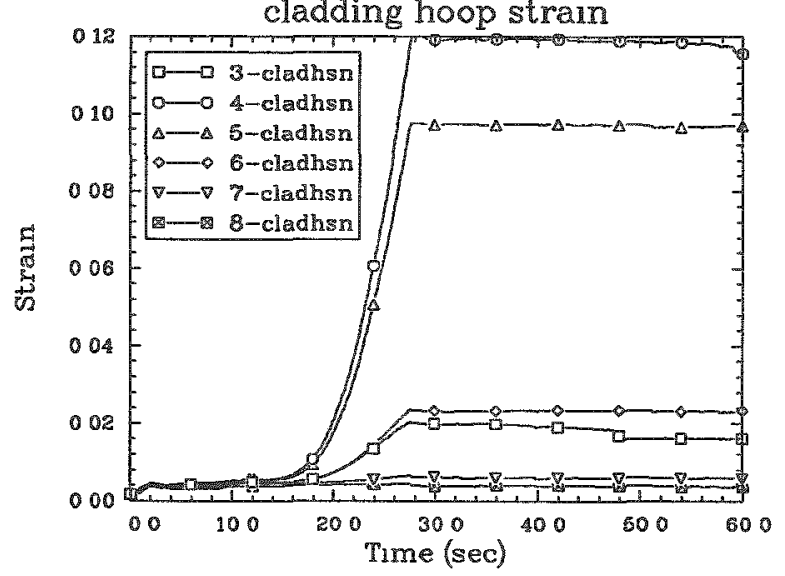

OCONEE 90\%DBA $20 \mathrm{GWD} / \mathrm{MTU}$ PIN--PF 24

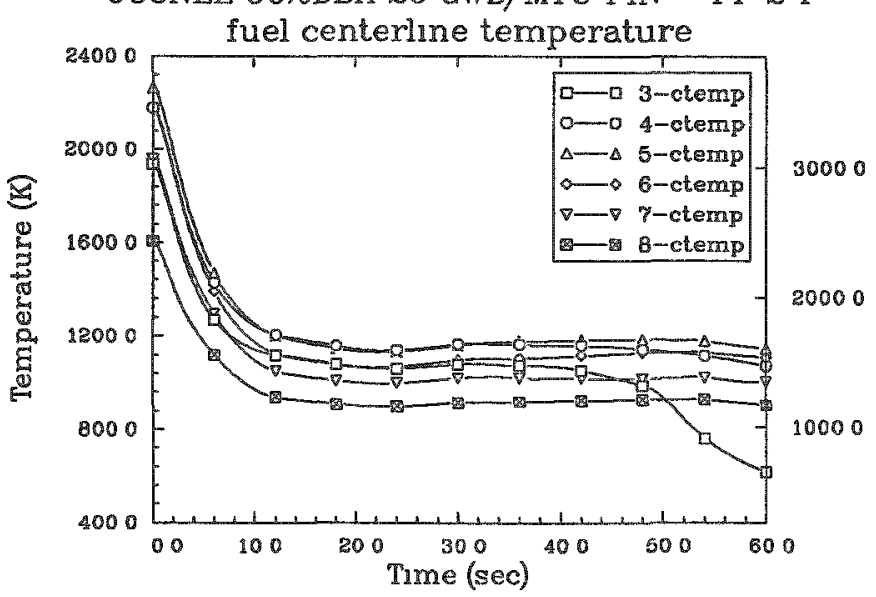

OCONEE 90\%DBA 20 GWD/MTU PIN--PF 2.4

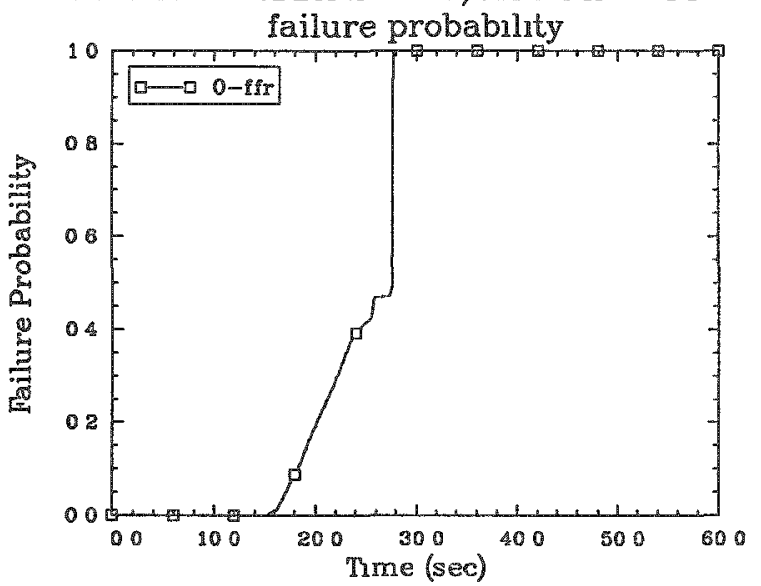

OCONEE 90\%DBA 20 GWD/MTU PIN--PF 24 cladding surface temperature
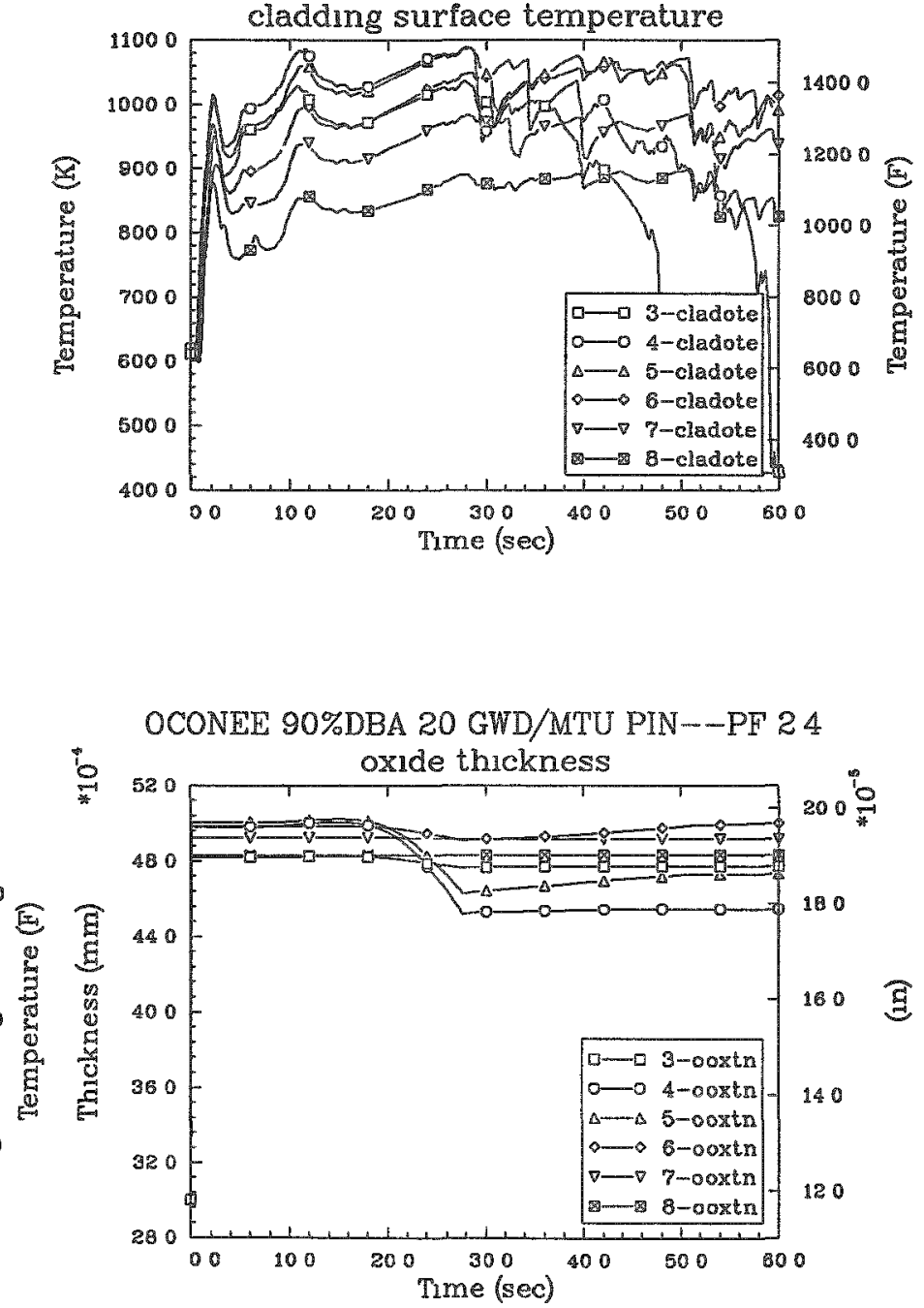
OCONEE 90\%DBA 5 GWD/MTU PIN--PF 2.4

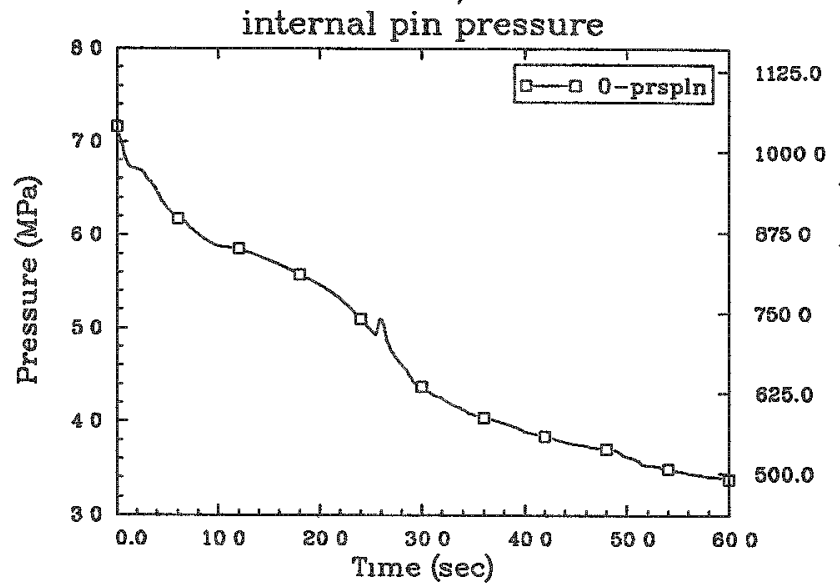

OCONEE 90\%DBA 5 GWD/MTU PIN--PF 2.4 cladding hoop strain

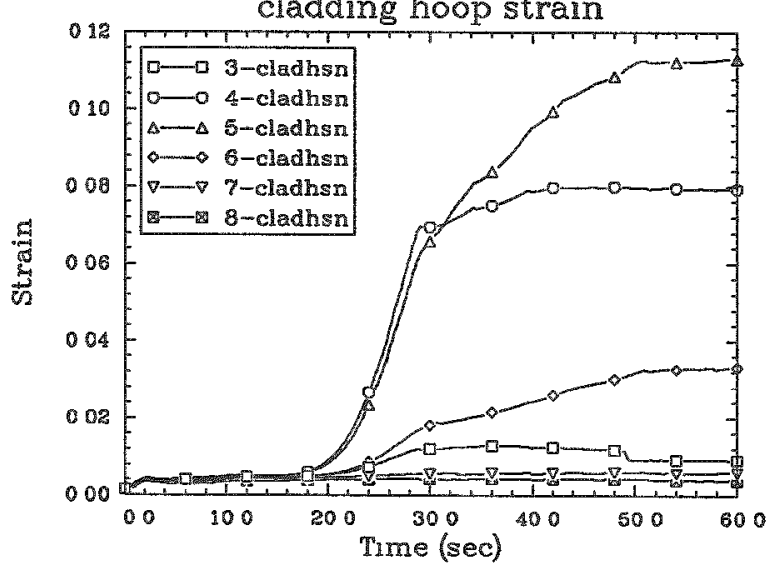

OCONEE 90\%DBA 5 GWD/MTU PIN--PF 24 fuel centerline temperature

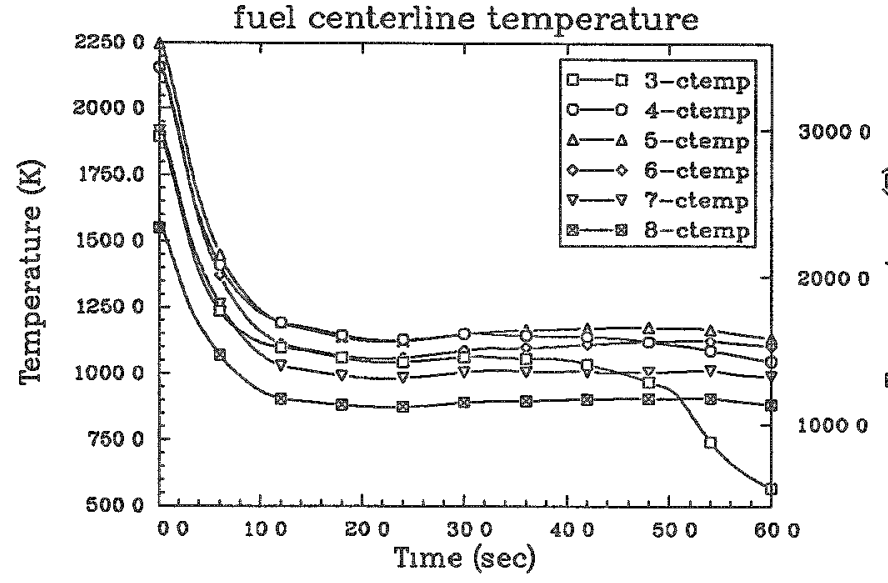

OCONEE 90\%DBA 5 GWD/MTU PIN--PF 2.4 failure probability

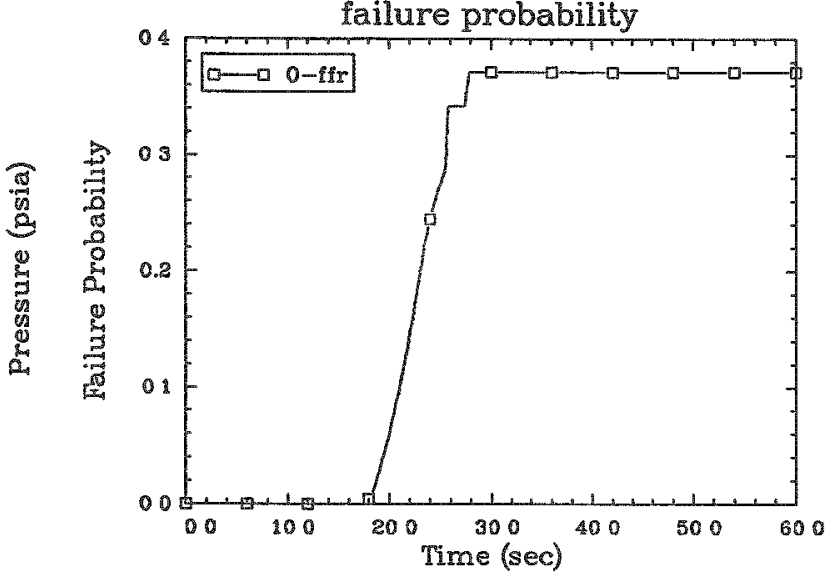

OCONEE 90\%DBA 5 GWD/MTU PIN--PF 2.4

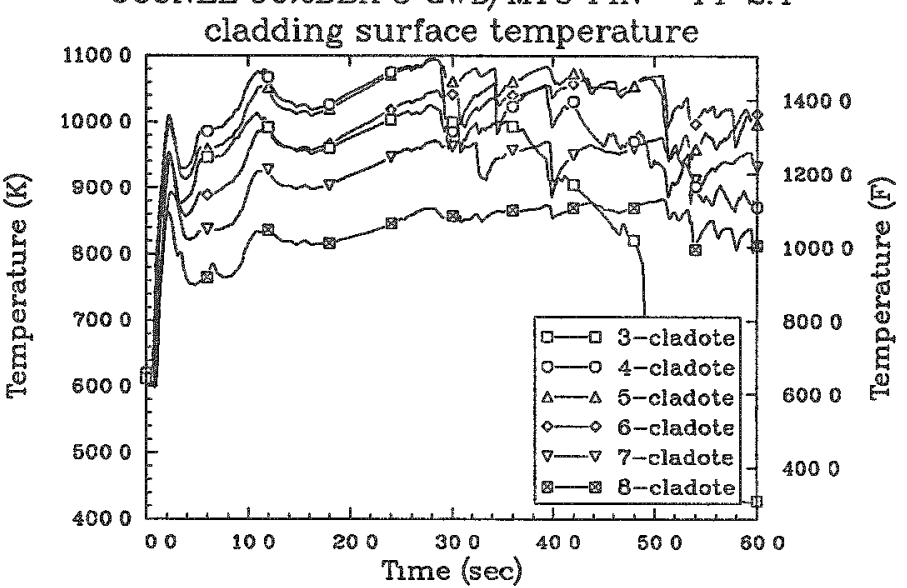

OCONEE 90\%DBA 5 GWD/MTU PIN--PF 2.4

i움 oxide thickness

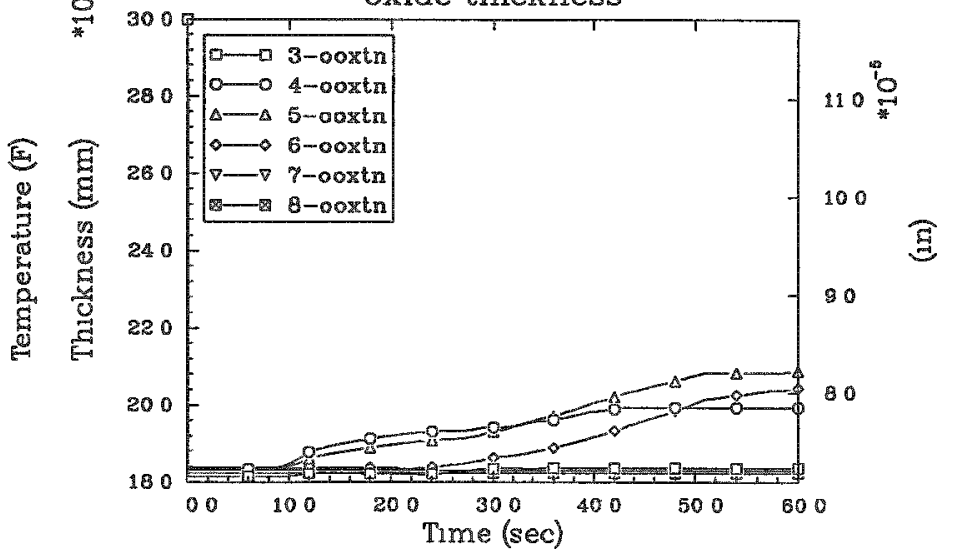



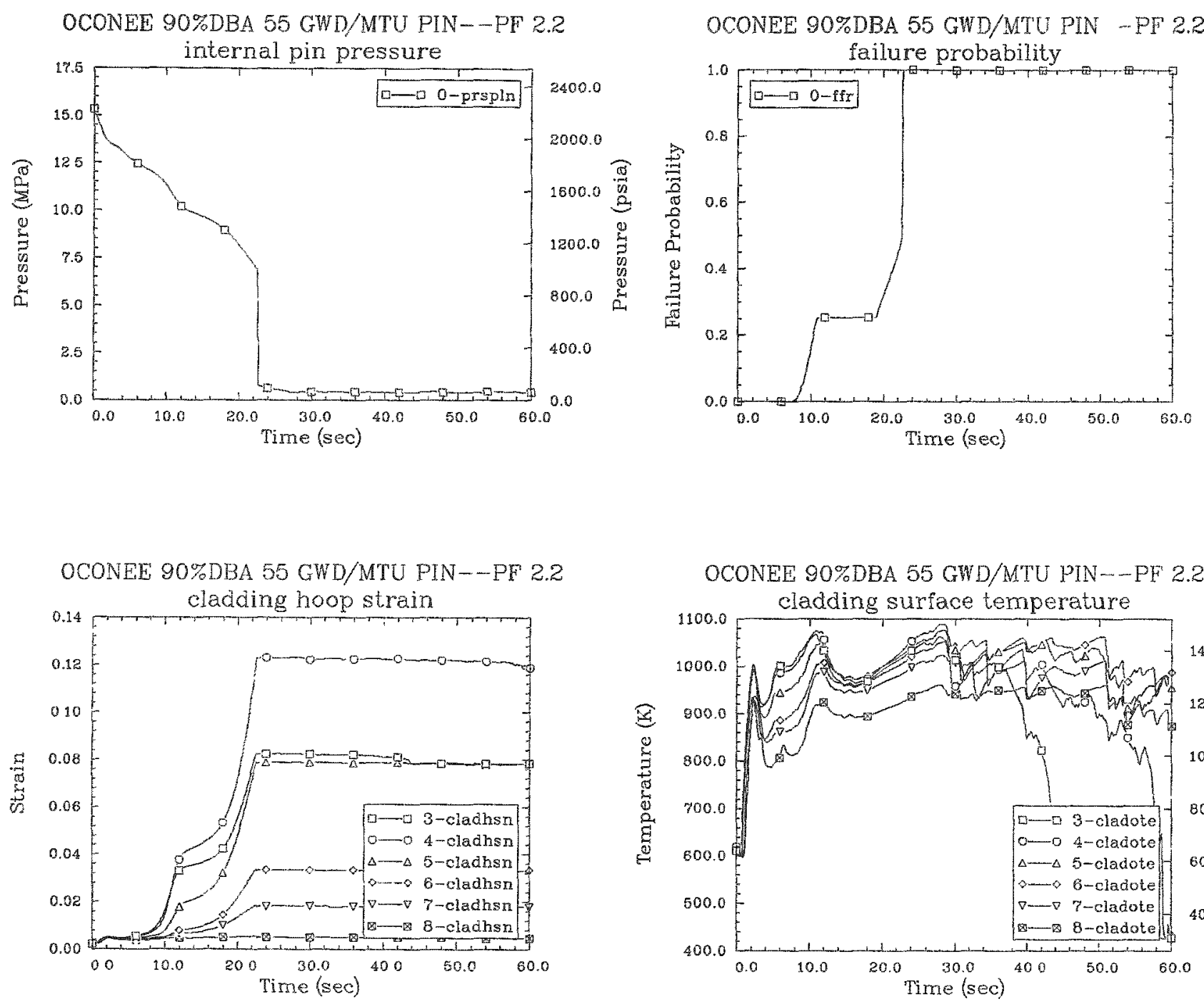

OCONEE 90\%DBA 55 GWD/MTU PIN-D-PF 2.2

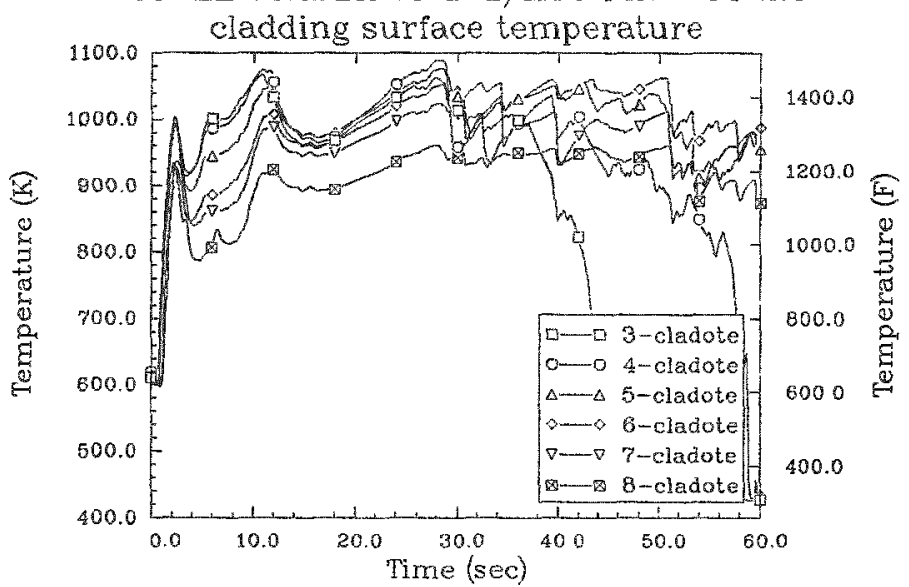

OCONEE 90\%DBA 55 GWD/MTU PIN--PF 2.2

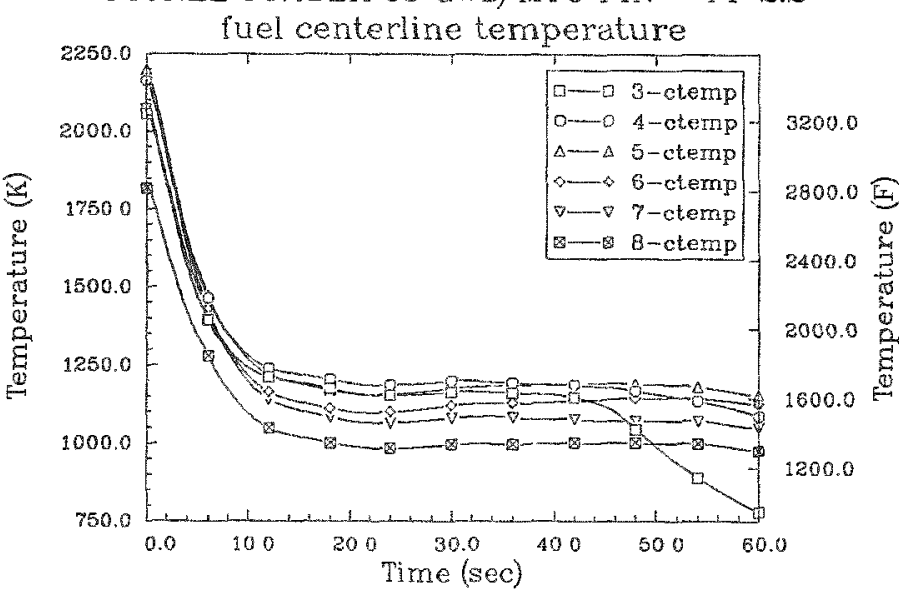

OCONEE 90\%DBA 55 GWD/MTU PIN--PF 2.2

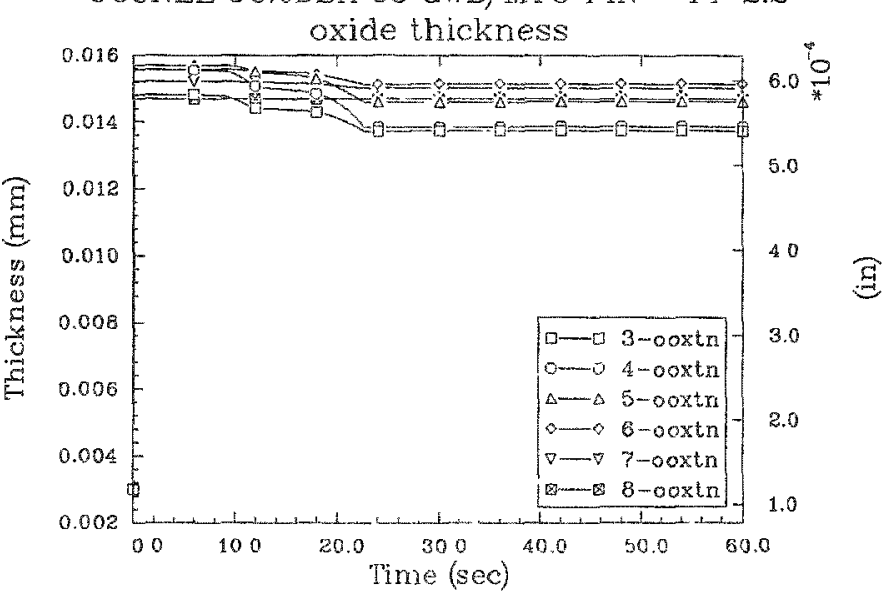




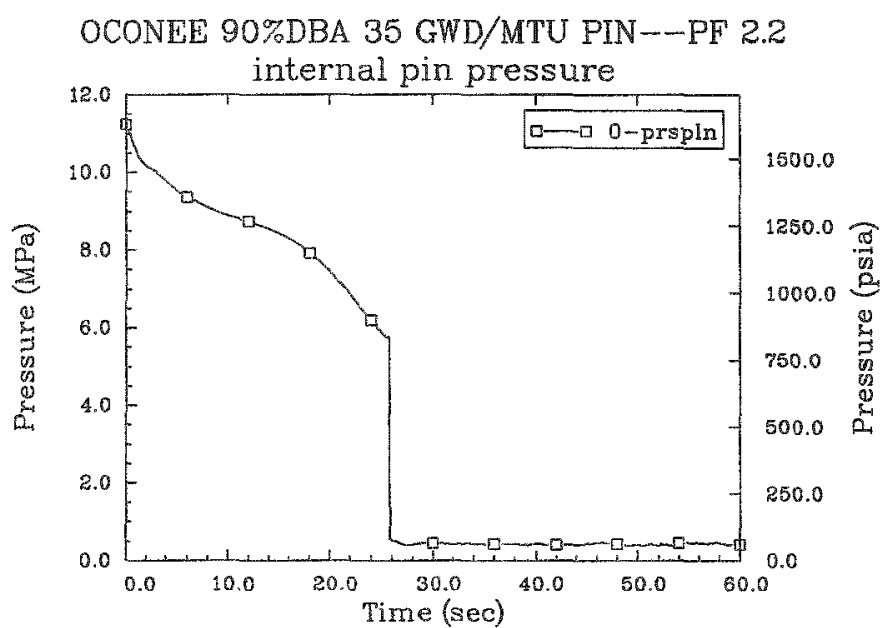

OCONEE 90\%DBA 35 GWD/MTU PIN--PF 2.2
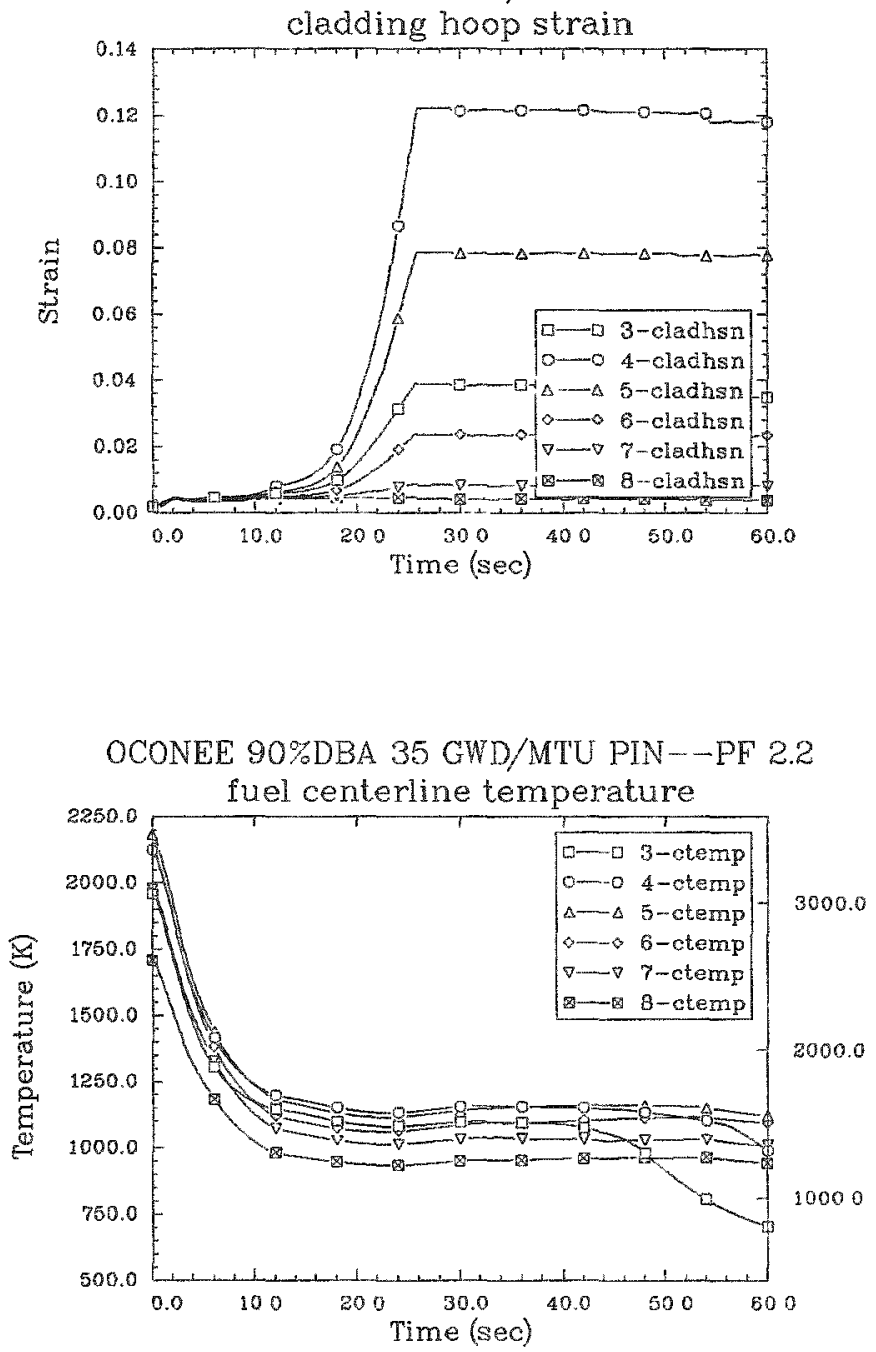

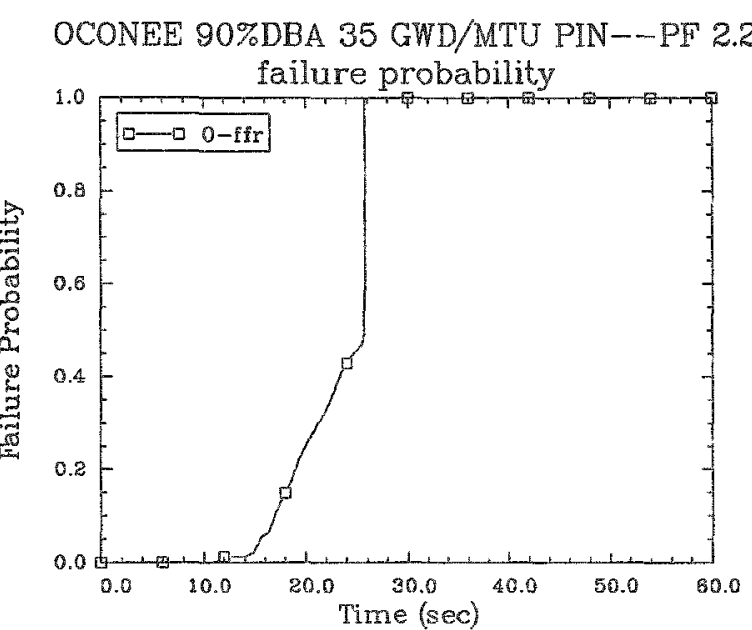

OCONEE 90\%DBA 35 GWD/MTU PIN--PF 2.2

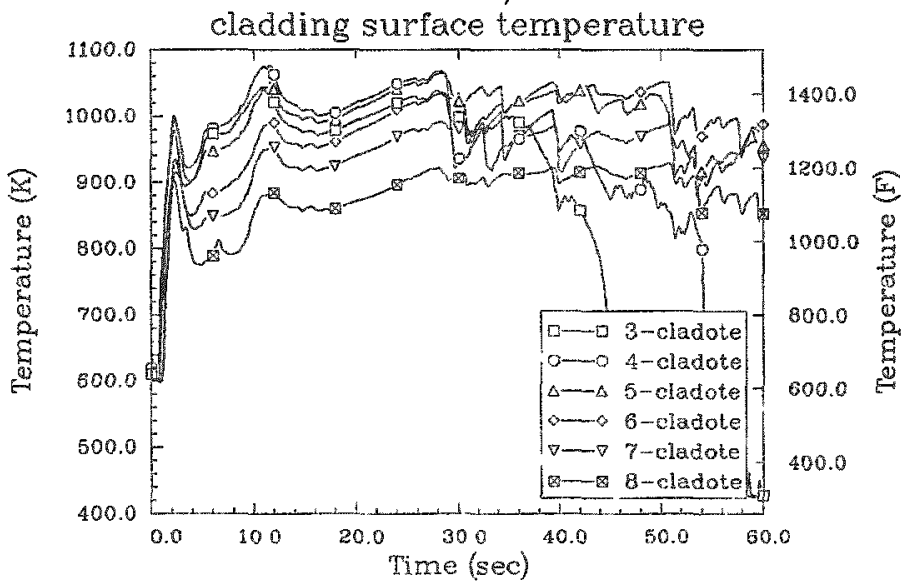

OCONEE 90\%DBA 35 GWD/MTU PIN--PF 2.2

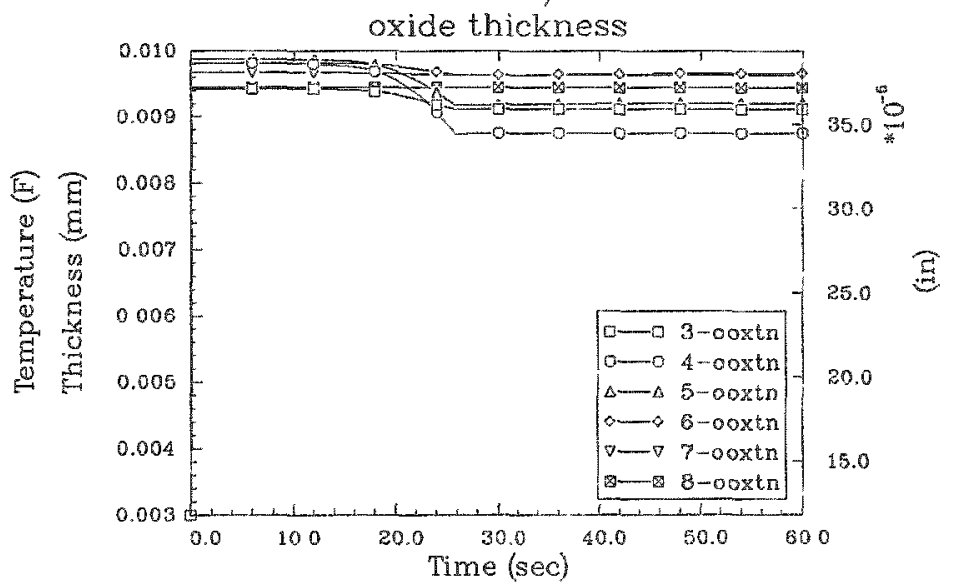


OCONEE 90\%DBA 20 GWD/MTU PIN--PF 2.2

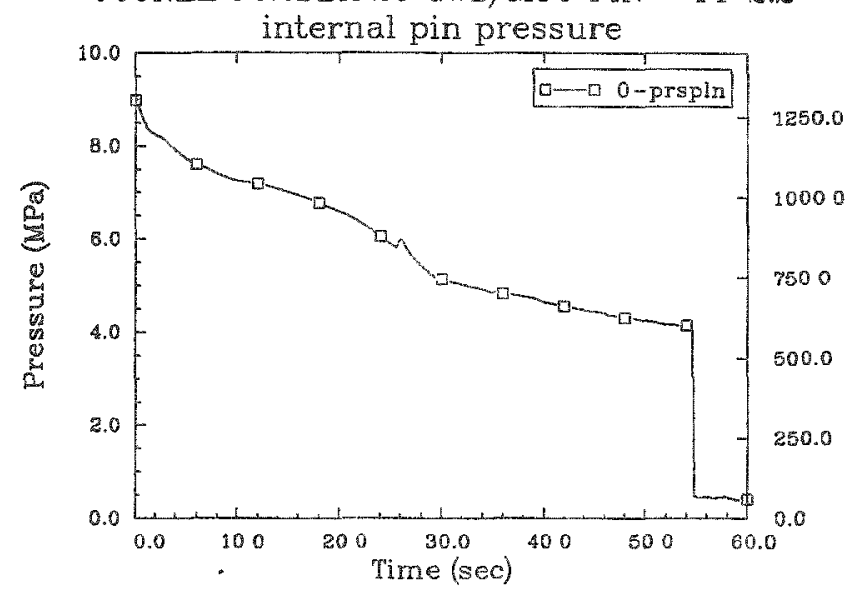

OCONEE 90\%DBA $20 \mathrm{GWD} / \mathrm{MTU}$ PIN--PF 2.2

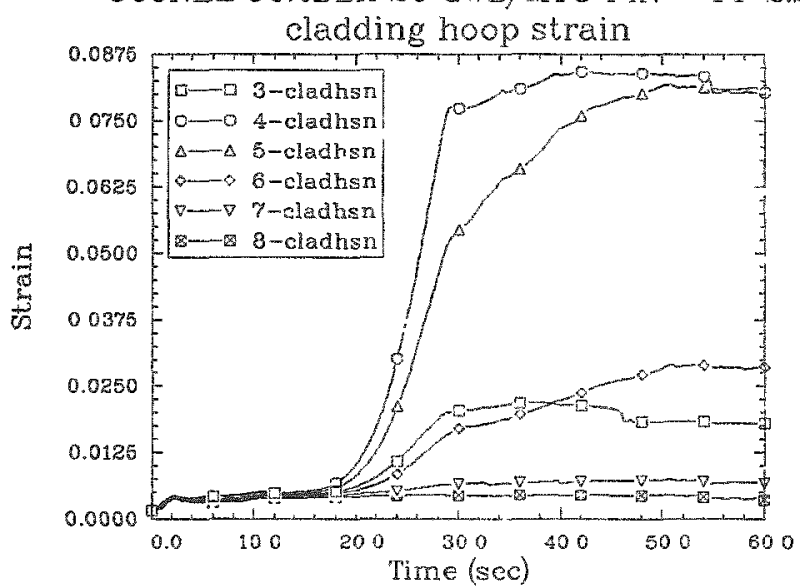

OCONEE 90\%DBA 20 GWD/MTU PIN-DF 2.2

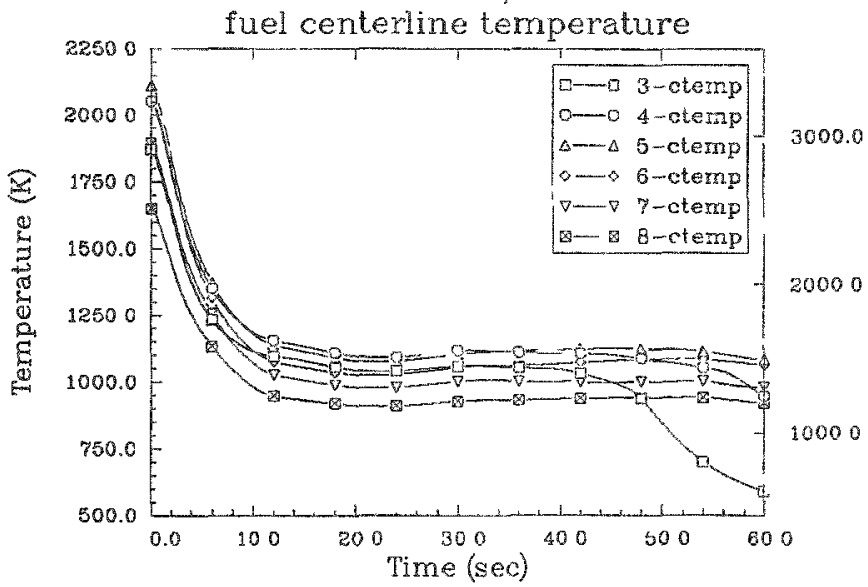

OCONEE 90\%DBA 20 GWD/MTU PIN--PF 2.2

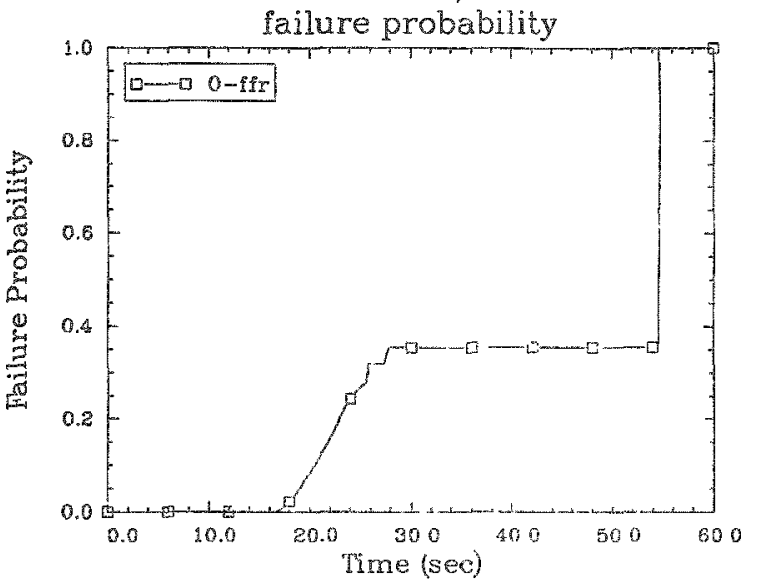

OCONEE 90\%DBA $20 \mathrm{GWD} / \mathrm{MTU}$ PIN--PF 2.2
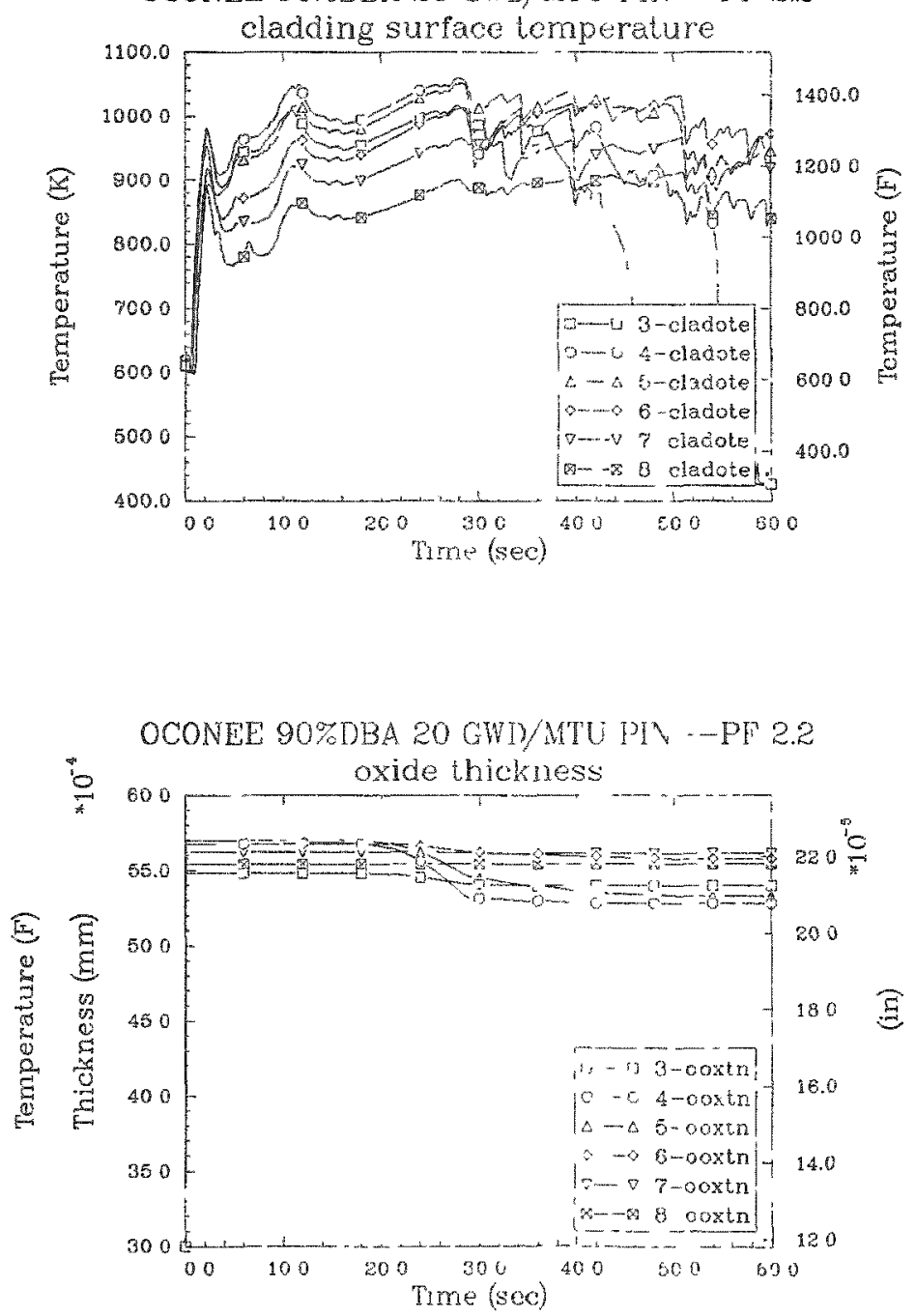
OCONEE 90\%DBA 5 GWD/MTU PIN--PF 2.2
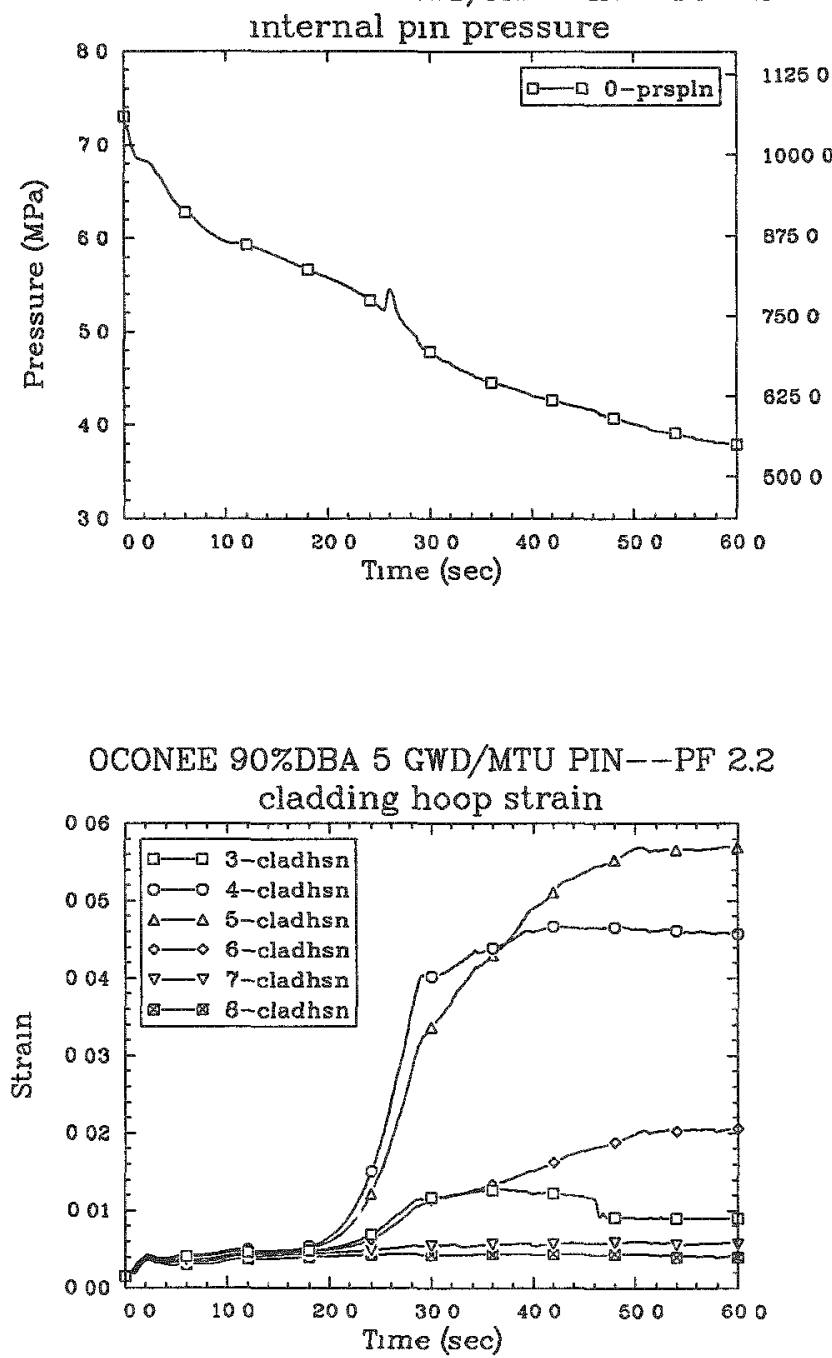

OCONEE 90\%DBA 5 GWD/MTU PIN--PF 22

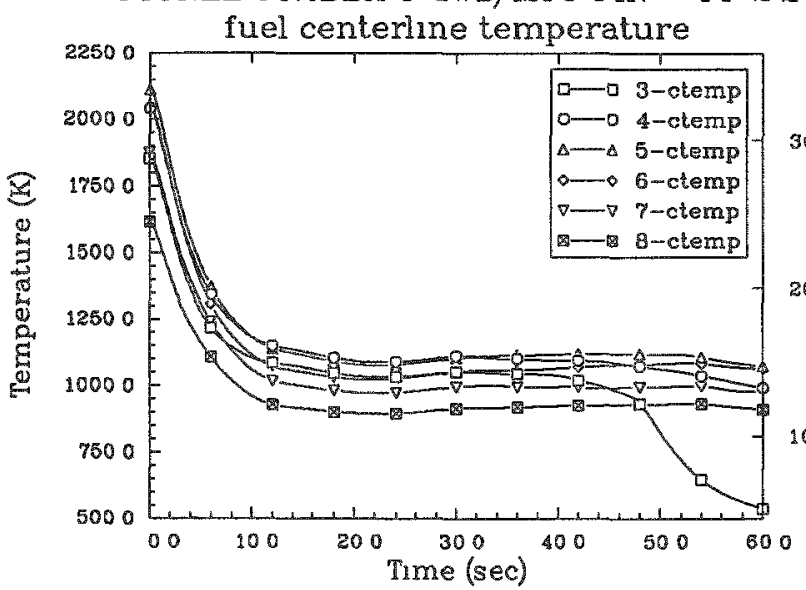

OCONEE 90\%DBA 5 GWD/MTU PIN--PF 22 failure probability

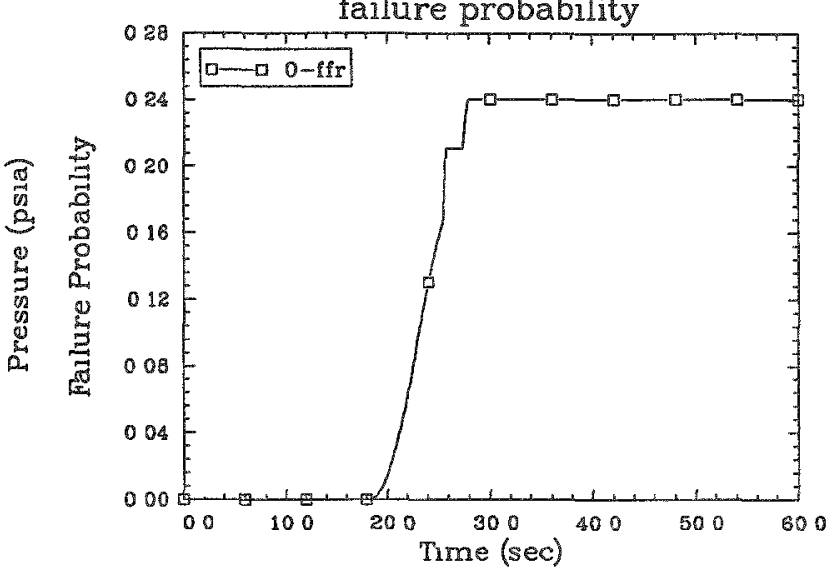

OCONEE 90\%DBA 5 GWD/MTU PIN--PF 22 cladding surface temperature

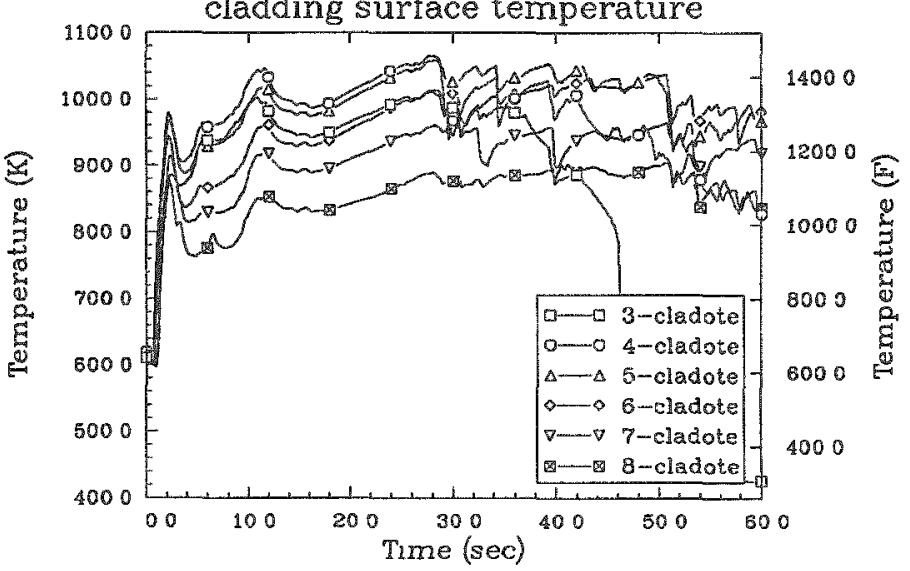

OCONEE 90\%DBA 5 GWD/MTU PIN--PF 22

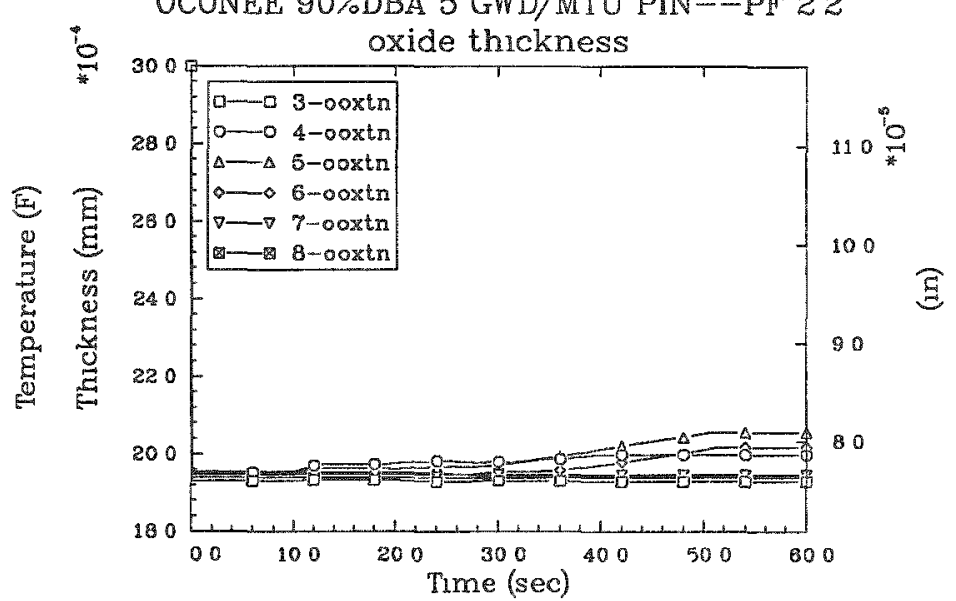




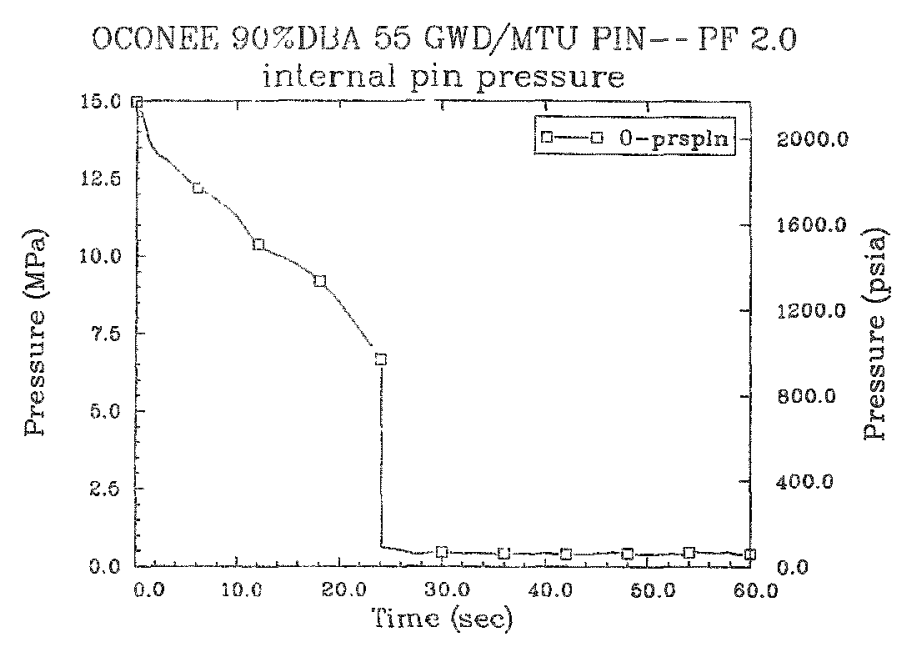

OCONEE 90\%DBA 55 GWD/MTU PIN--PF 2.0

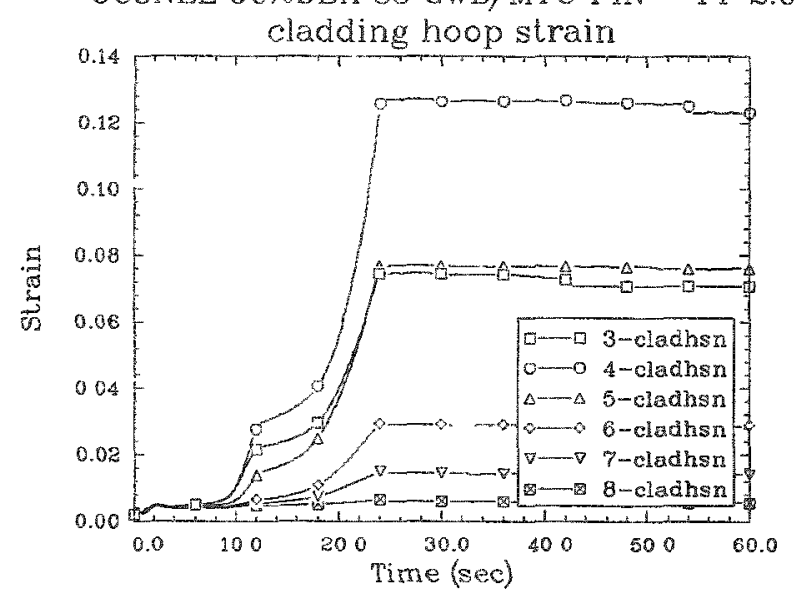

OCONEE 90\%DBA 55 GWD/MTU PIN--PF 2.0

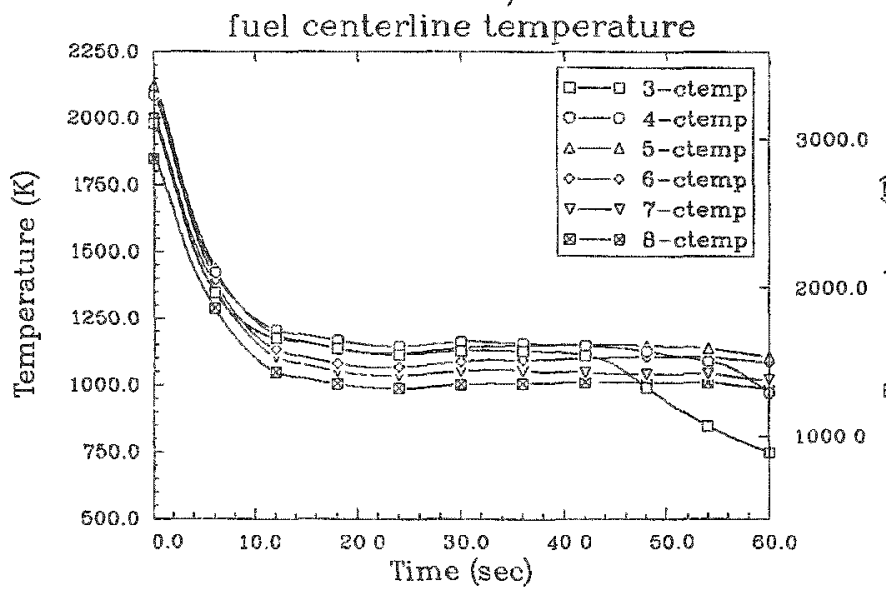

OCONEE 90\%DBA 55 GWD/MTU PIN- -. PF 2.0

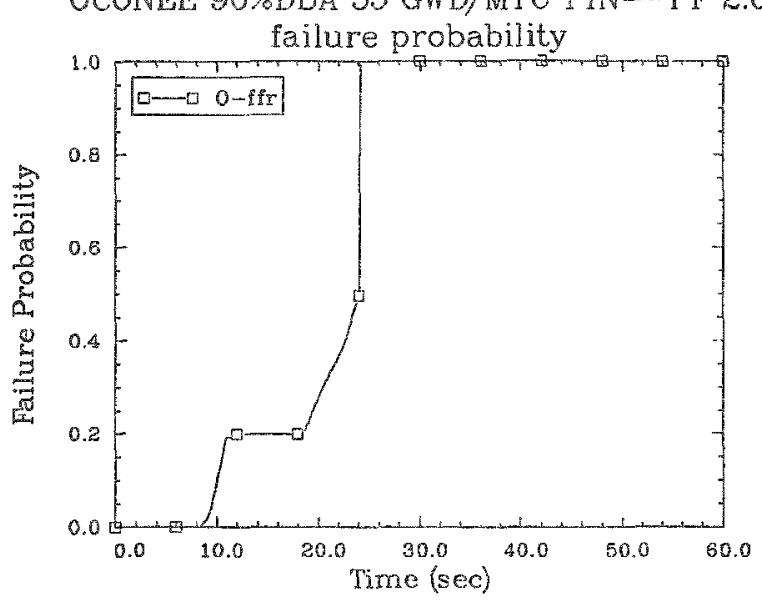

OCONEE 90\%DBA 55 GWD/MTU PIN--PF 2.0

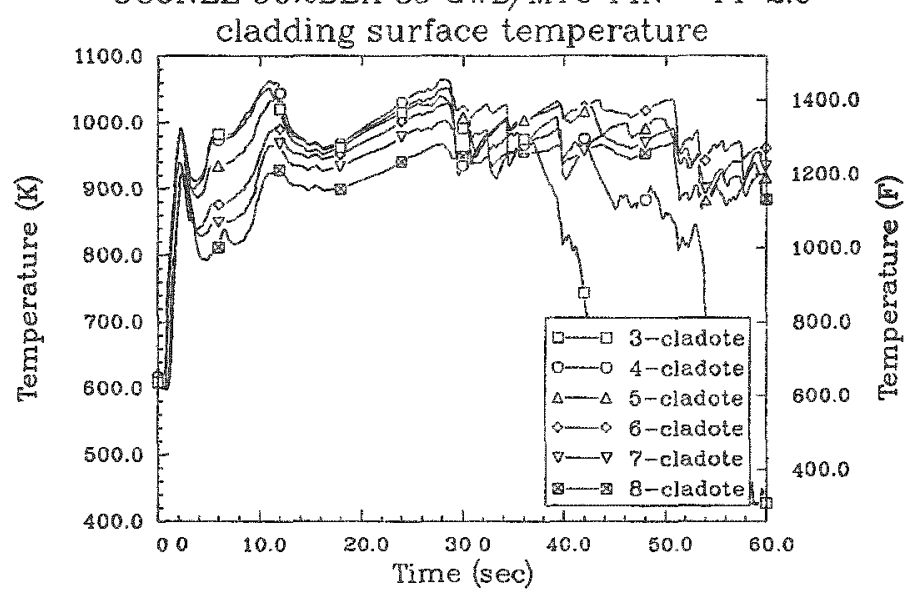

OCONEE 90\%DBA 55 GWD/MTU PIN--PF 2.0

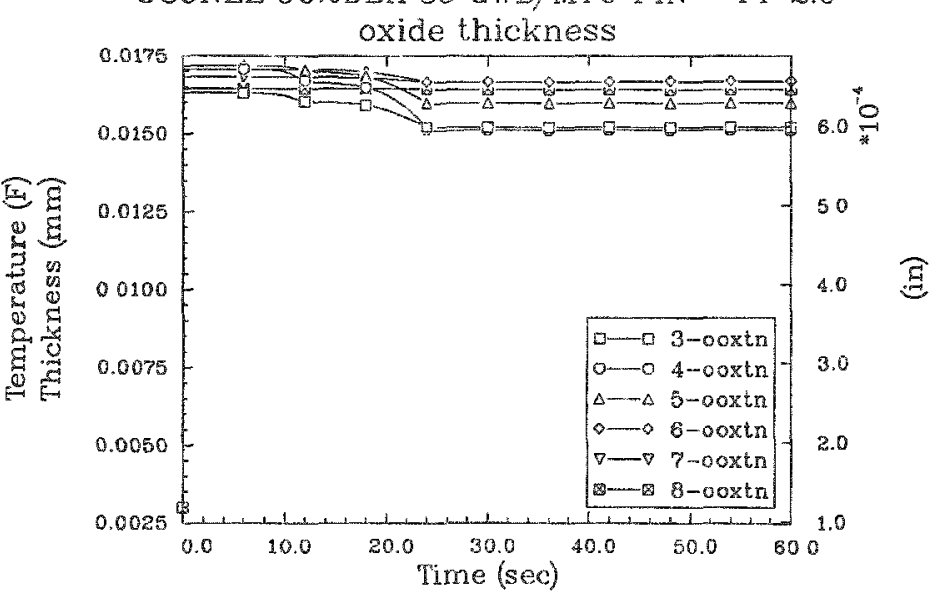



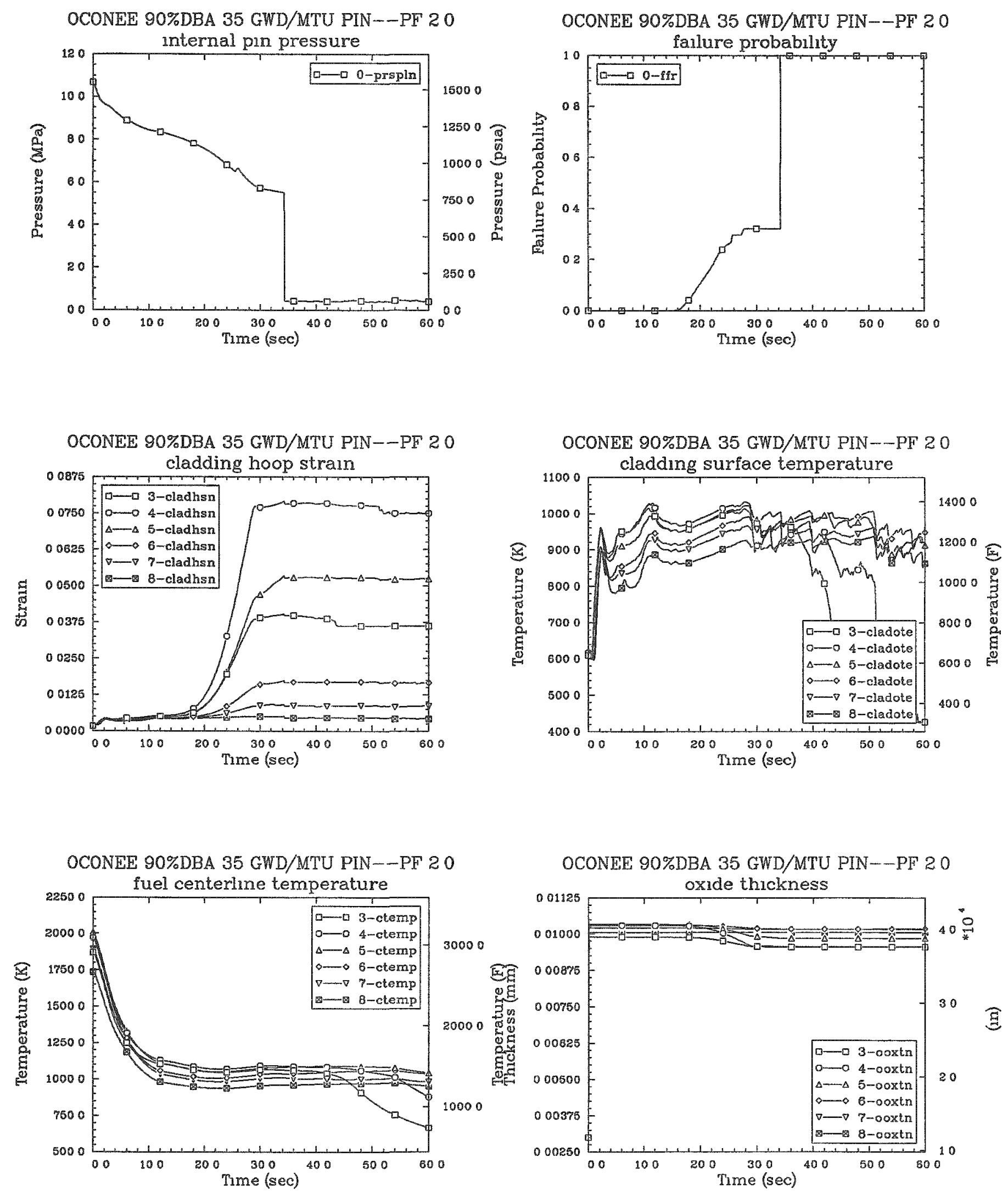

OCONEE 90\%DBA 35 GWD/MTU PIN--PF 20

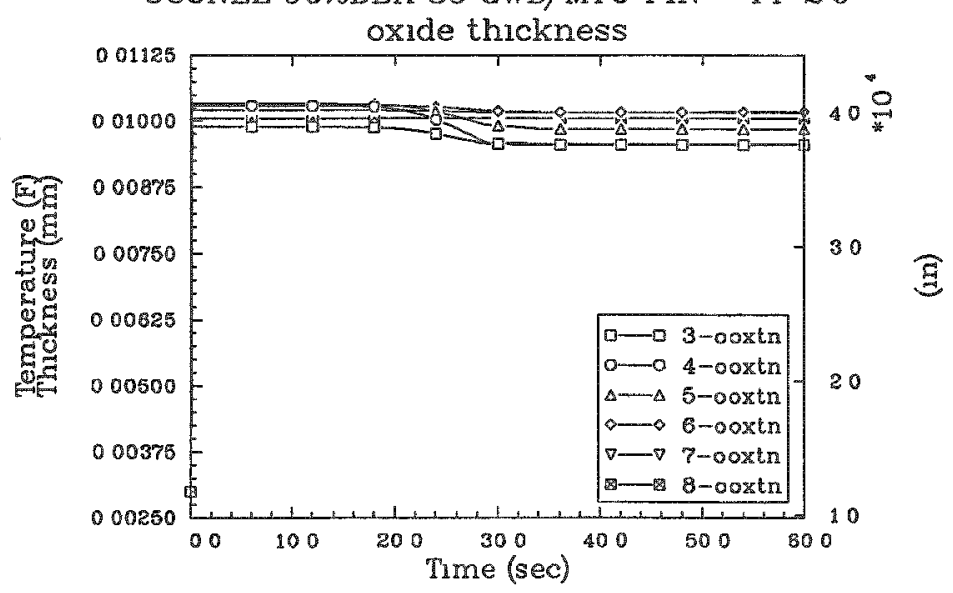


OCONEE 90\%DBA $20 \mathrm{GWD} / \mathrm{MTU}$ PIN--PF 2.0

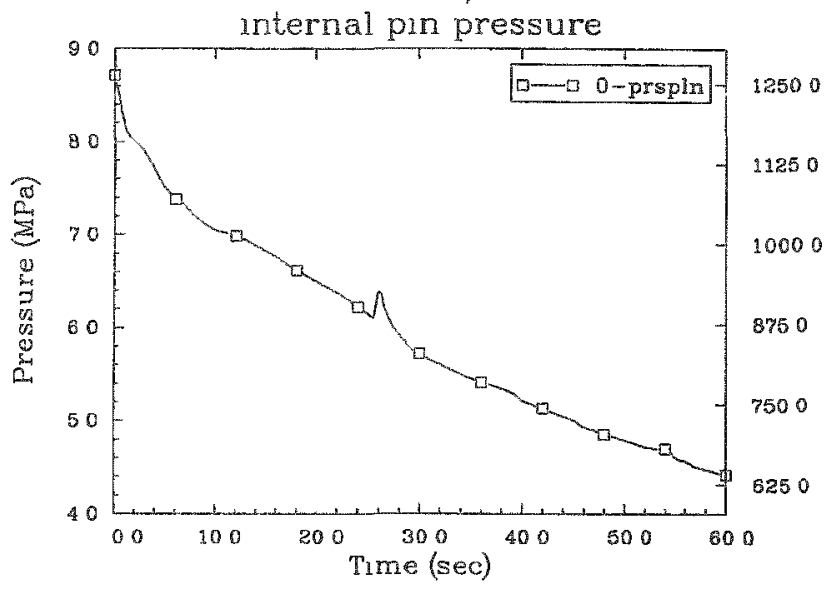

OCONEE 90\%DBA $20 \mathrm{GWD} / \mathrm{MTU}$ PIN--PF 20

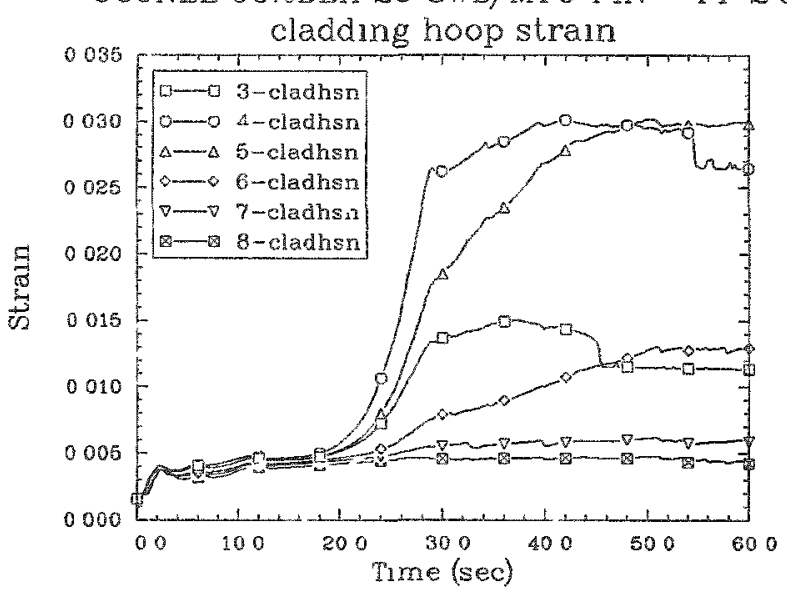

OCONEE 90\%DBA 20 GWD/MTU PIN--PF 20

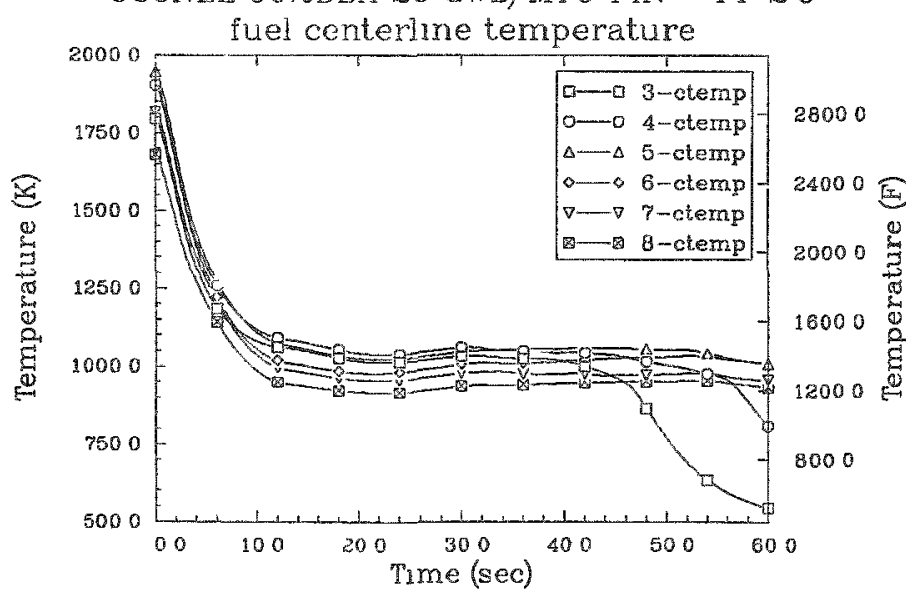

OCONEE 90\%DBA $20 \mathrm{GWD} / \mathrm{MTU}$ PIN--PF 2.0 fallure probability

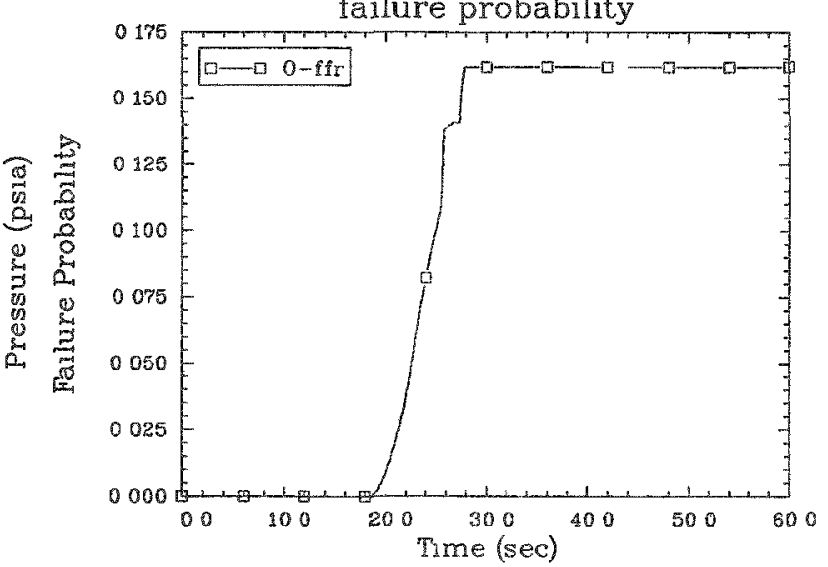

OCONEE 90\%DBA $20 \mathrm{GWD} / \mathrm{MTU}$ PIN--PF 20

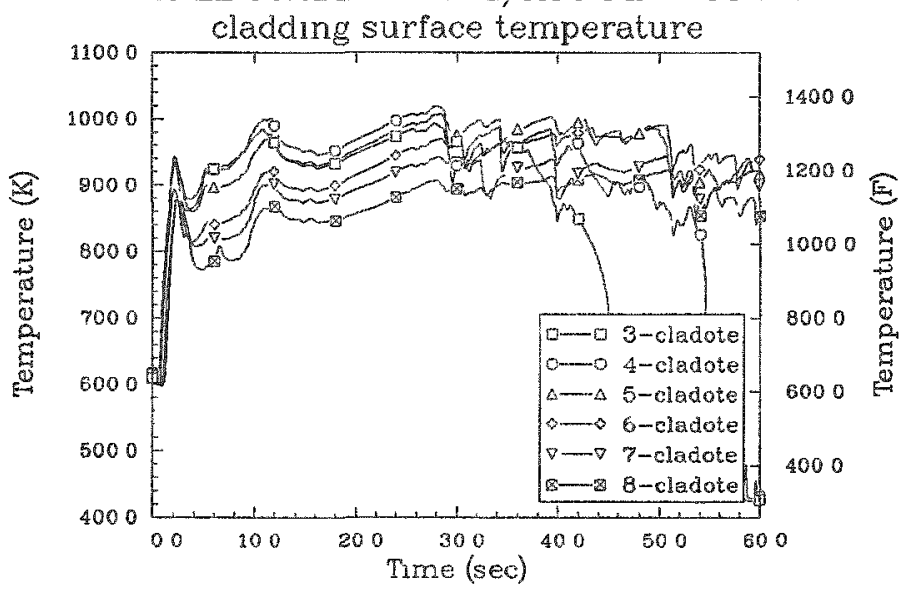

OCONEE 90\%DBA $20 \mathrm{GWD} / \mathrm{MTU}$ PIN--PF 20

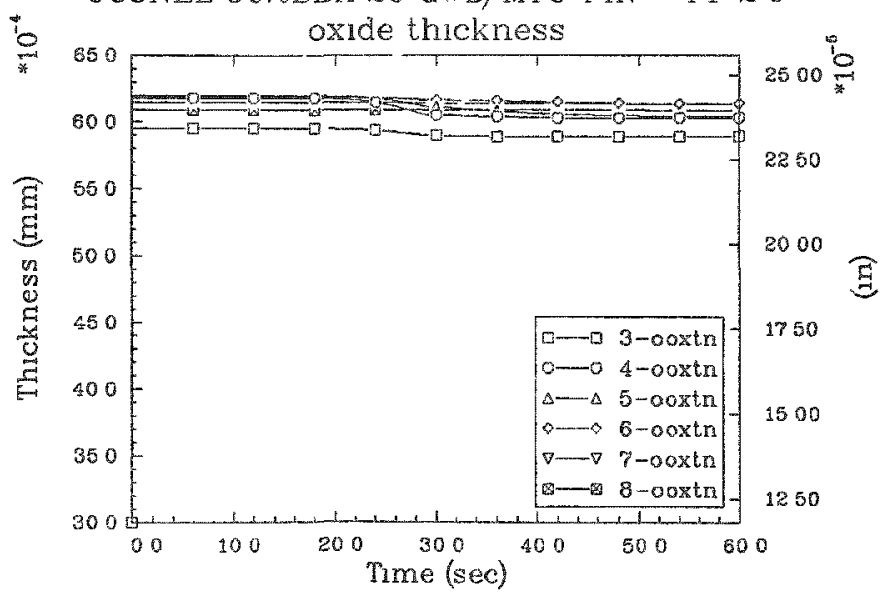


OCONEE 90\%DBA 5 GWD/MTU PIN--PF 2.0

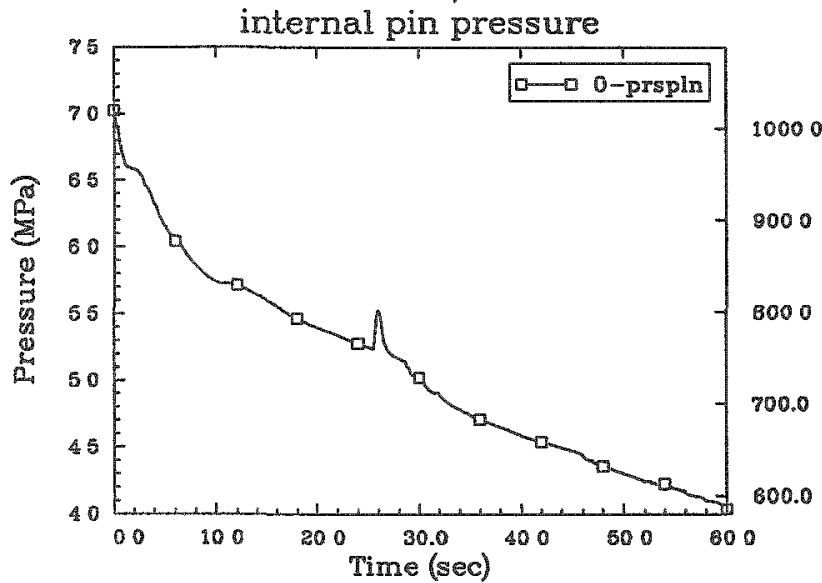

OCONEE 90\%DBA 5 GWD/MTU PIN--PF 2.0 cladding hoop strain

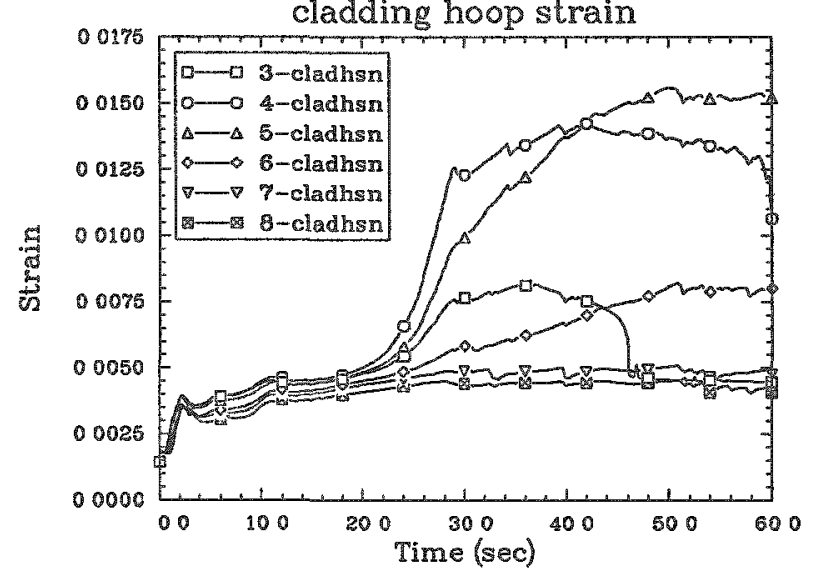

OCONEE 90\%DBA 5 GWD/MTU PIN--PF 2.0

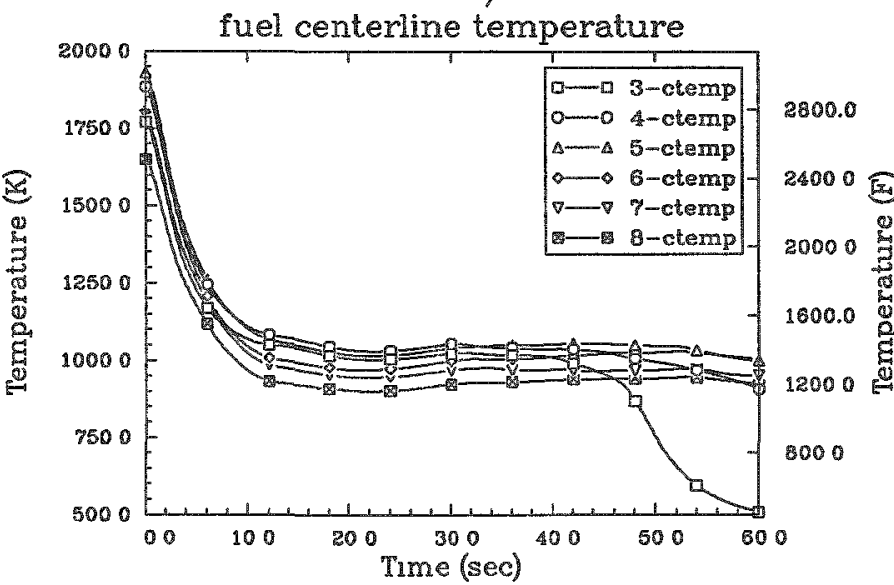

OCONEE 90\%DBA 5 GWD/MTU PIN--PF 2.0 failure probability

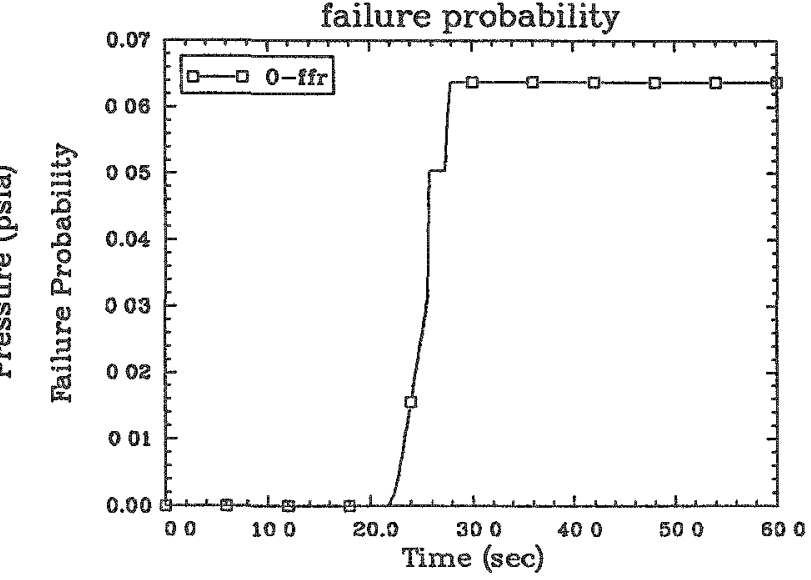

OCONEE 90\%DBA 5 GWD/MTU PIN--PF 2.0 cladding surface temperature

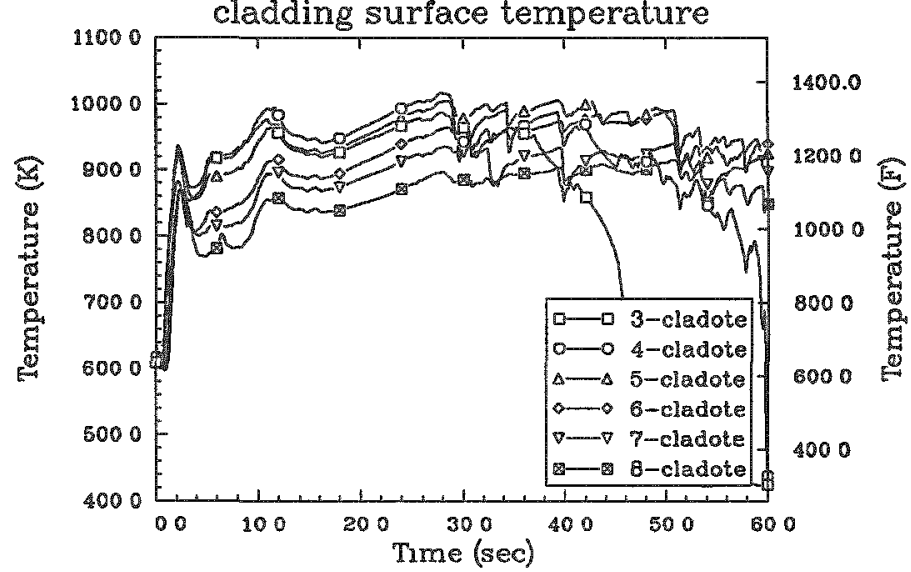

OCONEE 90\%DBA 5 GWD/MTU PIN--PF 2.0

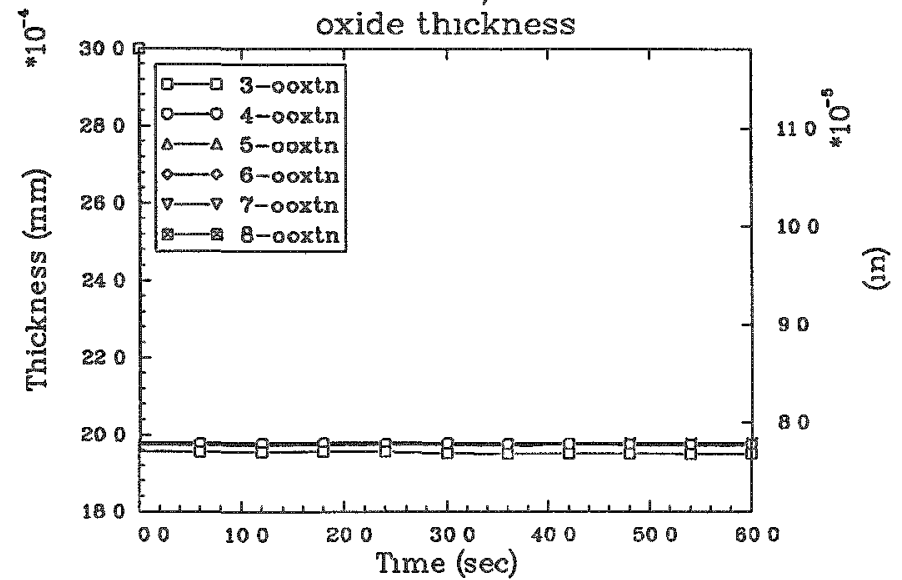




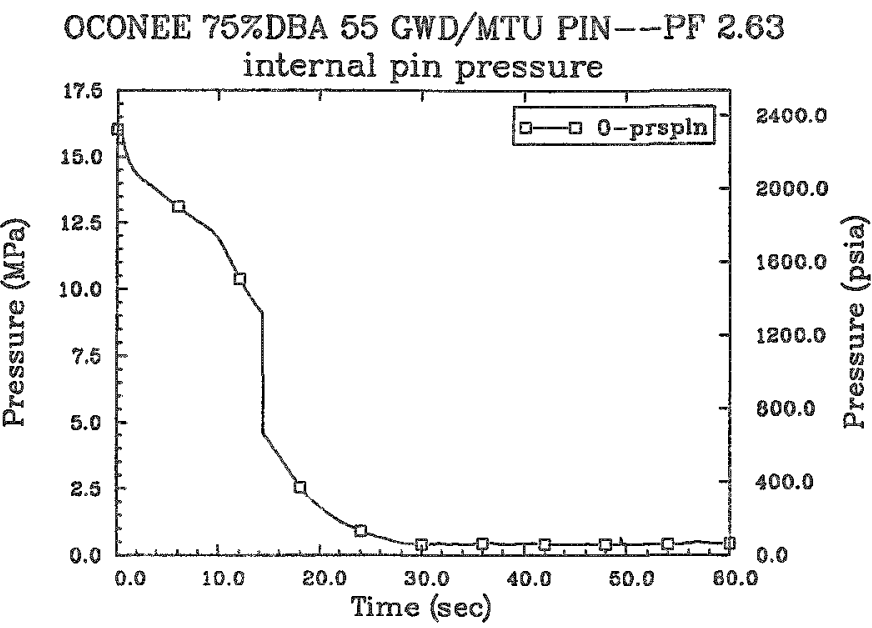

OCONEE 75\%DBA 55 GWD/MTU PIN--PF 2.63 cladding hoop strain

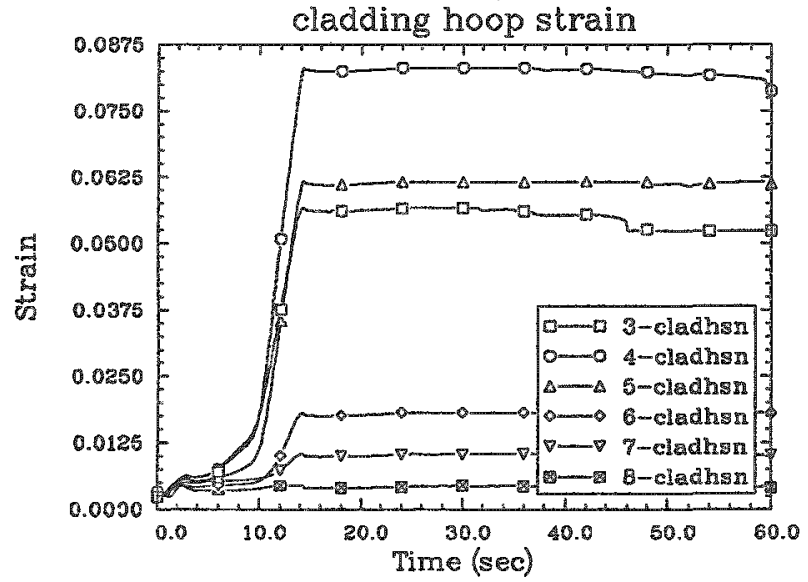

OCONEE 75\%DBA 55 GWD/MTU PIN---PF 2.63

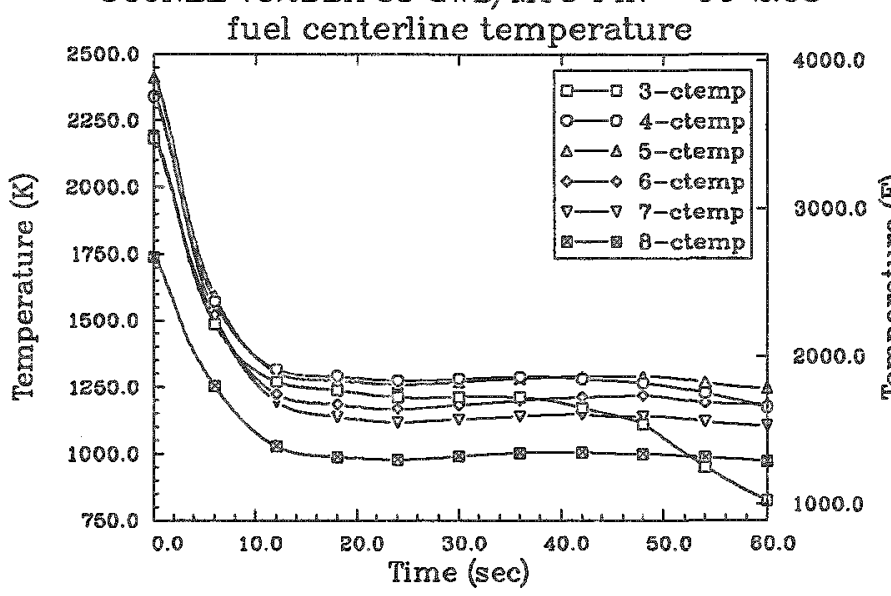

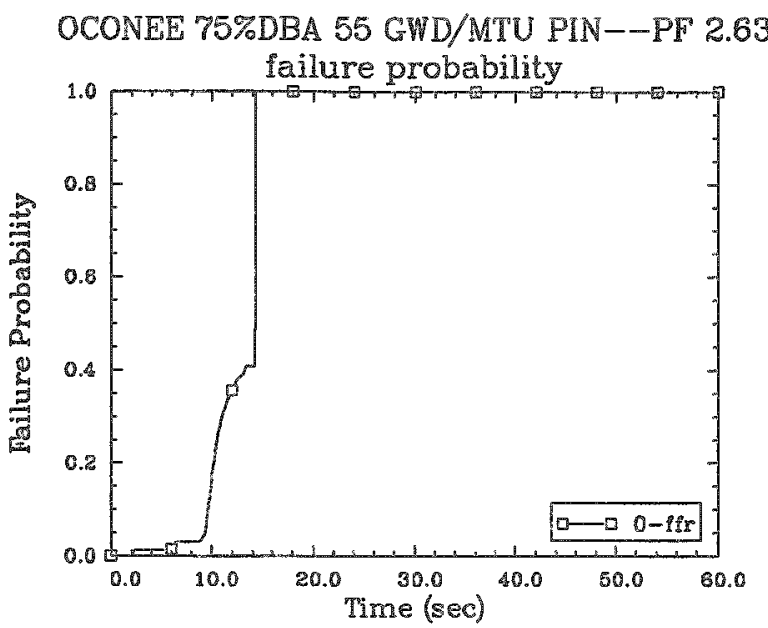

OCONEE 75\%DBA 55 GWD/MTU PIN--PF 2.63

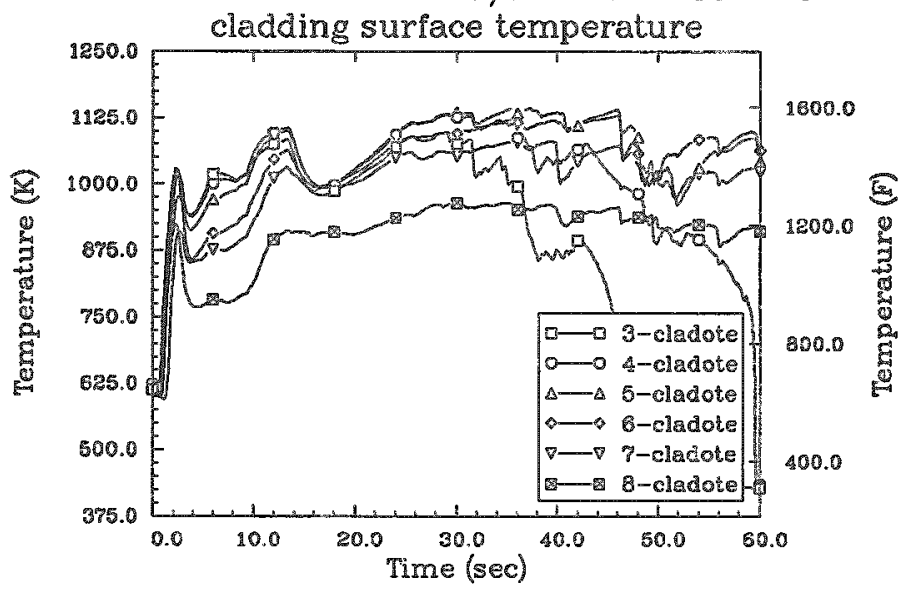

OCONEE 75\%DBA 55 GWD/MTU PIN-DF 2.63 oxide thickness

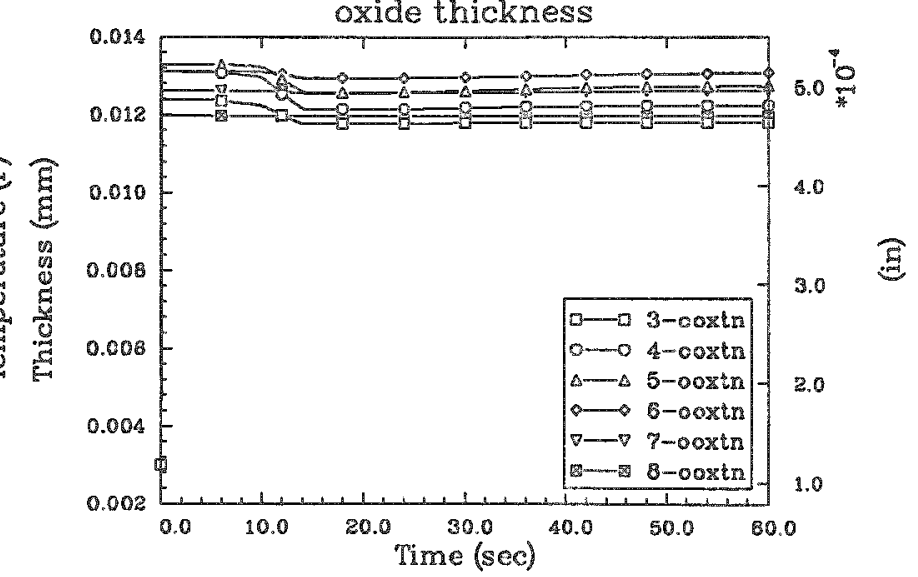



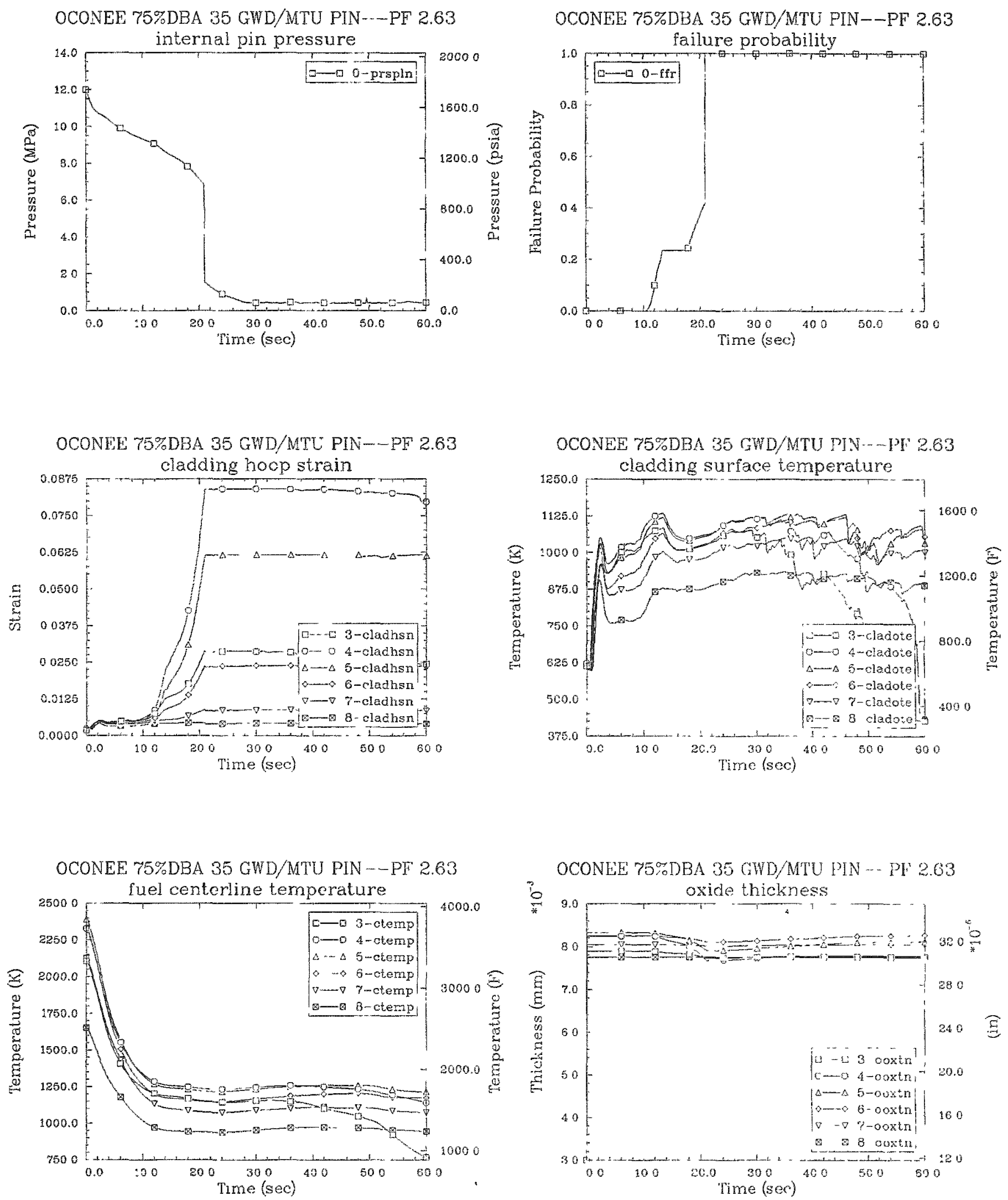

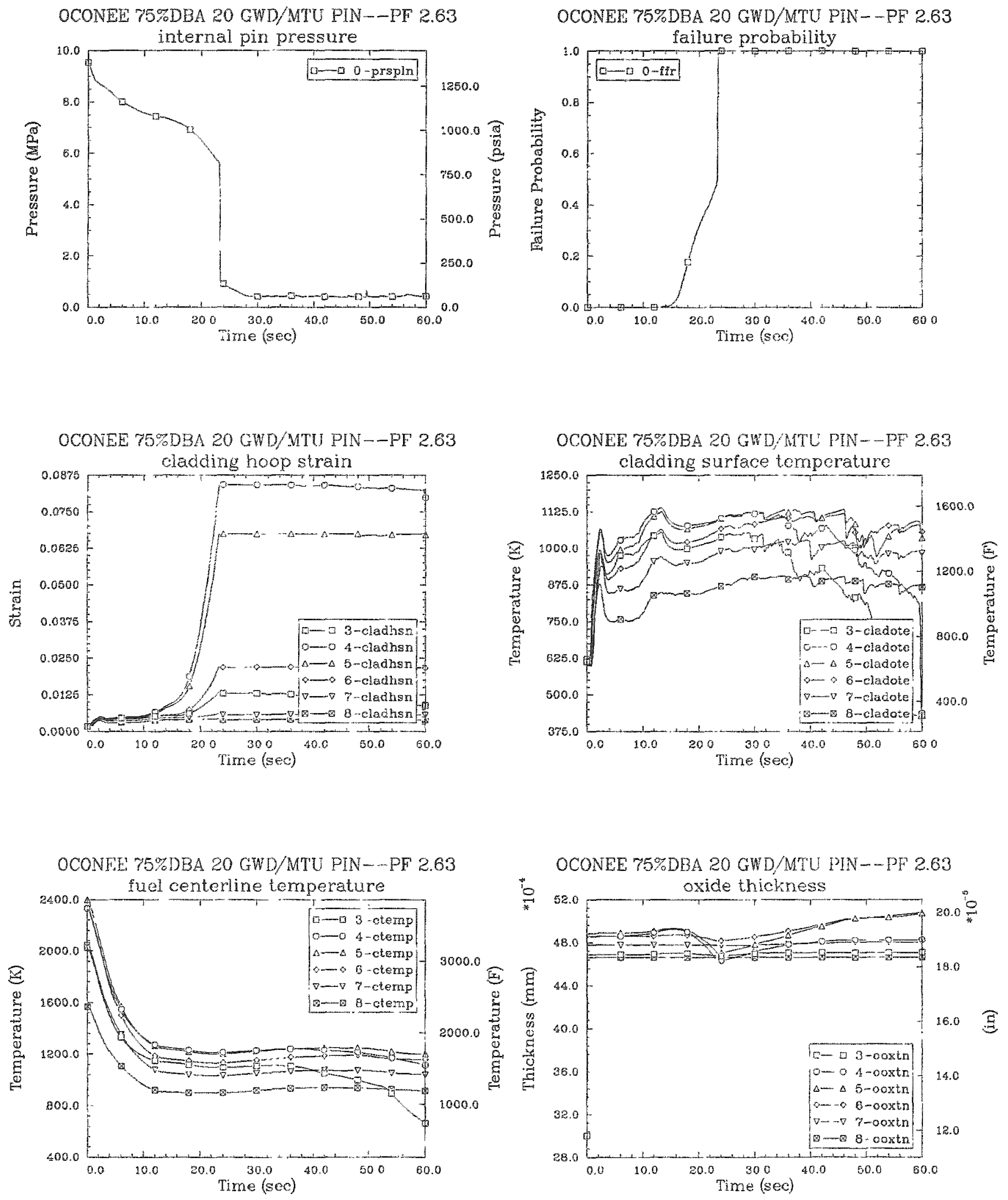
OCONEE 75\%DBA 5 GWD/MTU PIN--PF 2.63

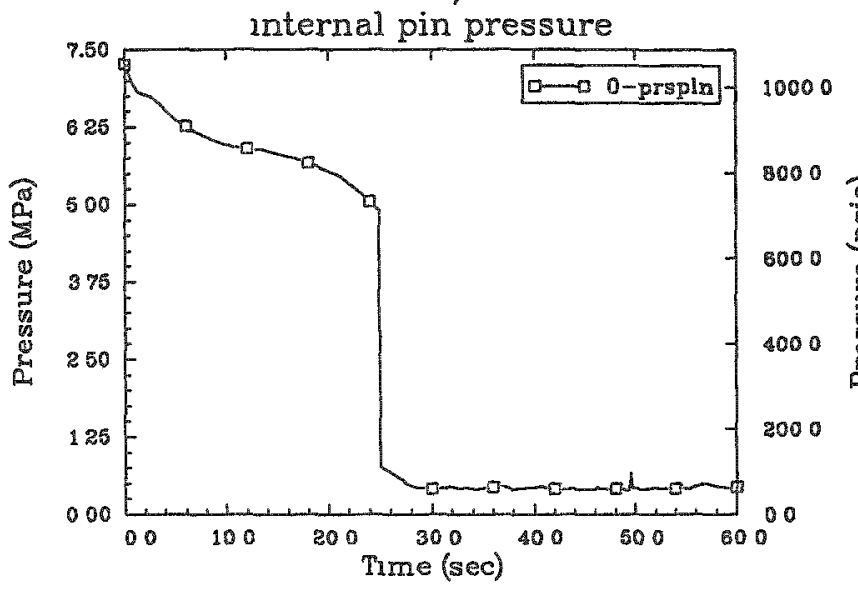

OCONEE 75\%DBA 5 GWD/MTU PIN--PF 263

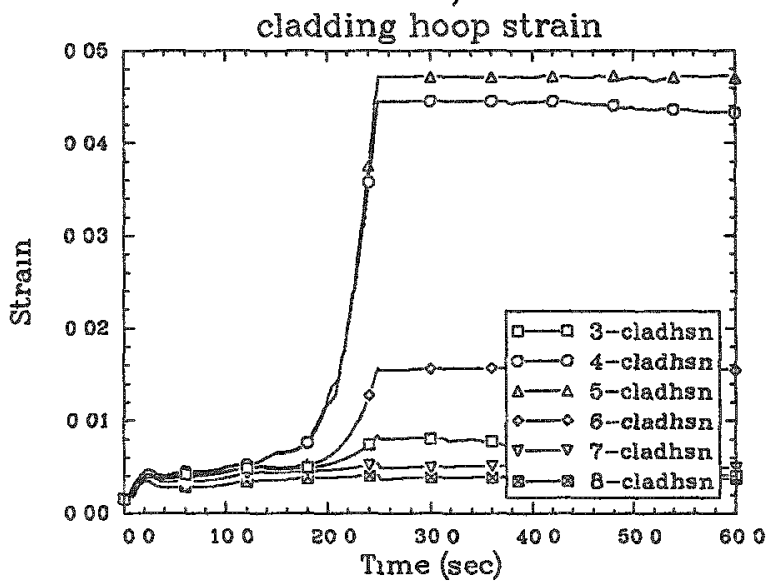

OCONEE 75\%DBA 5 GWD/MTU PIN--PF 263

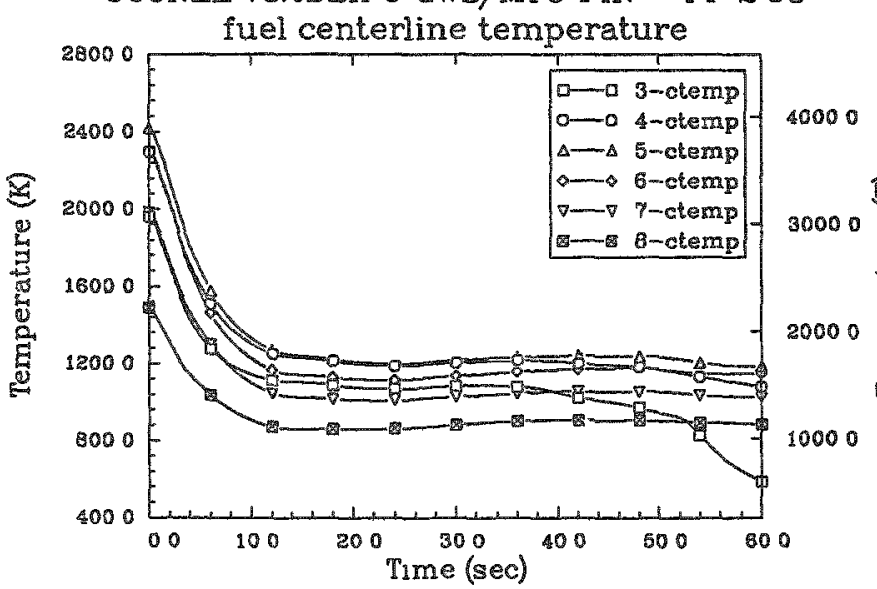

OCONEE 75\%DBA 5 GWD/MTU PIN--PF 2.63

fallure probability

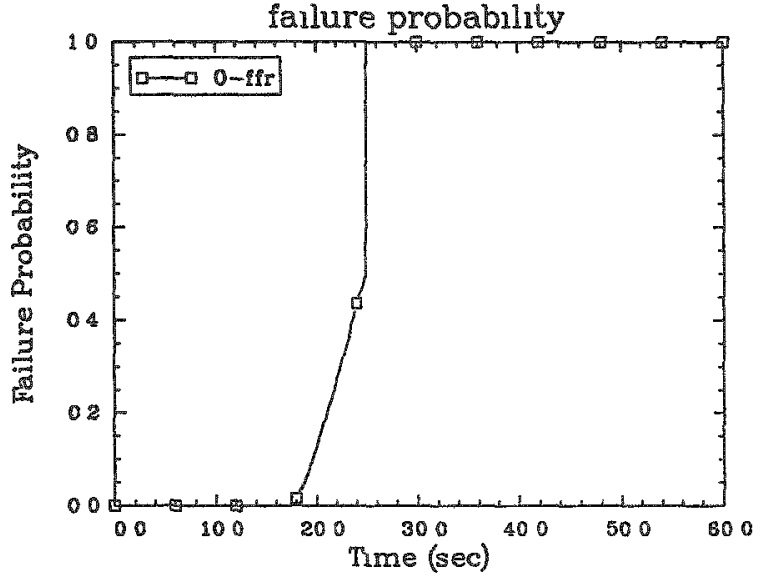

OCONEE 75\%DBA 5 GWD/MTU PIN--PF 2.63

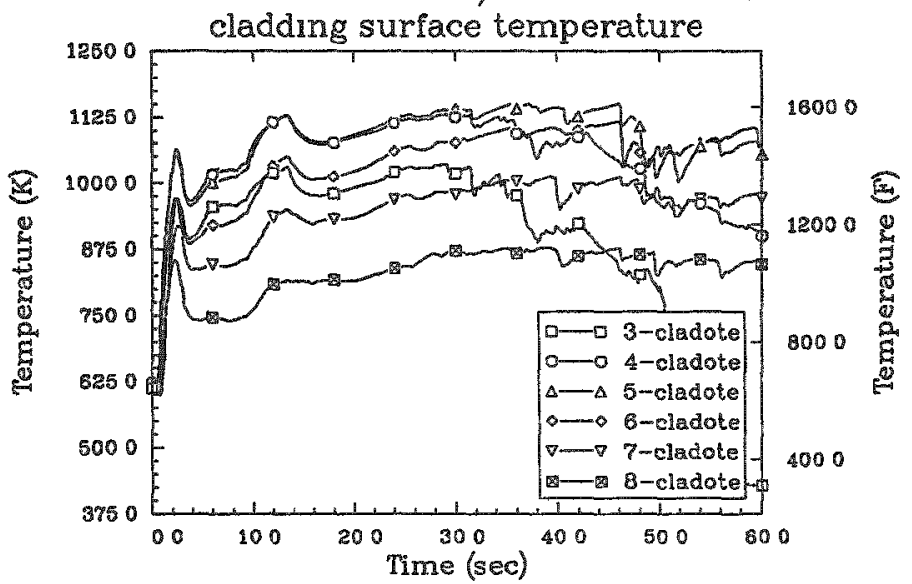

OCONEE 75\%DBA 5 GWD/MTU PIN-PF 263

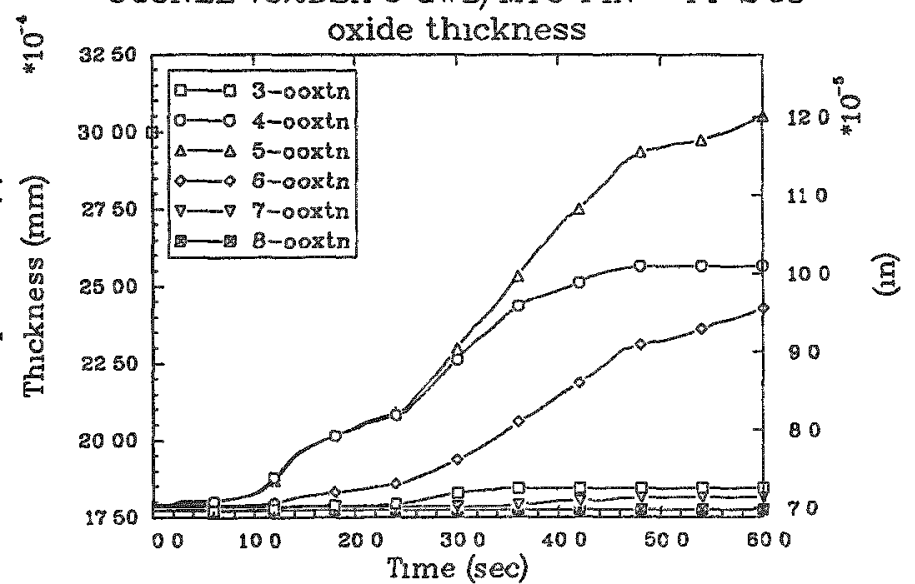



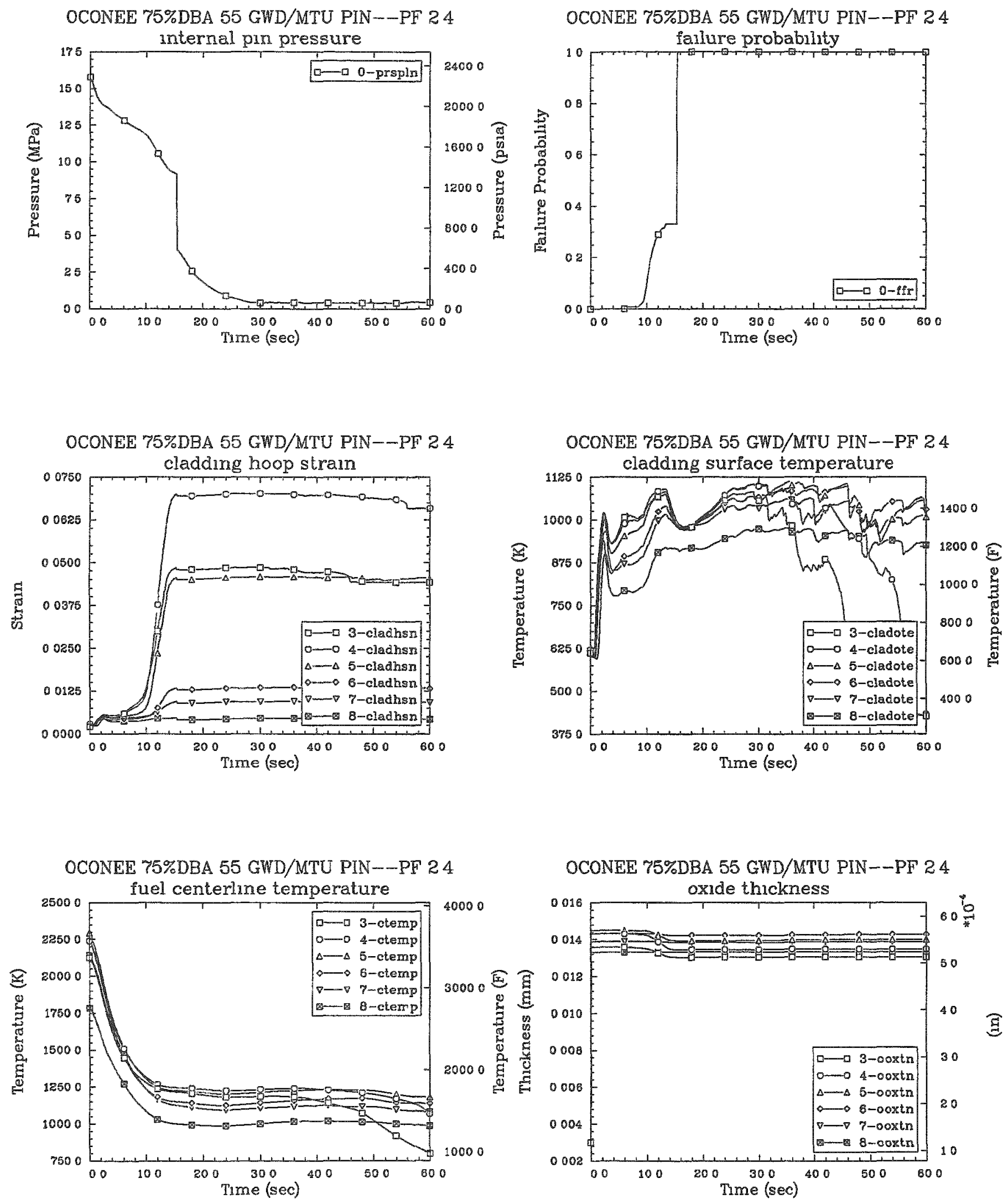

OCONEE 75\%DBA 55 GWD/MTU PIN--PF 24

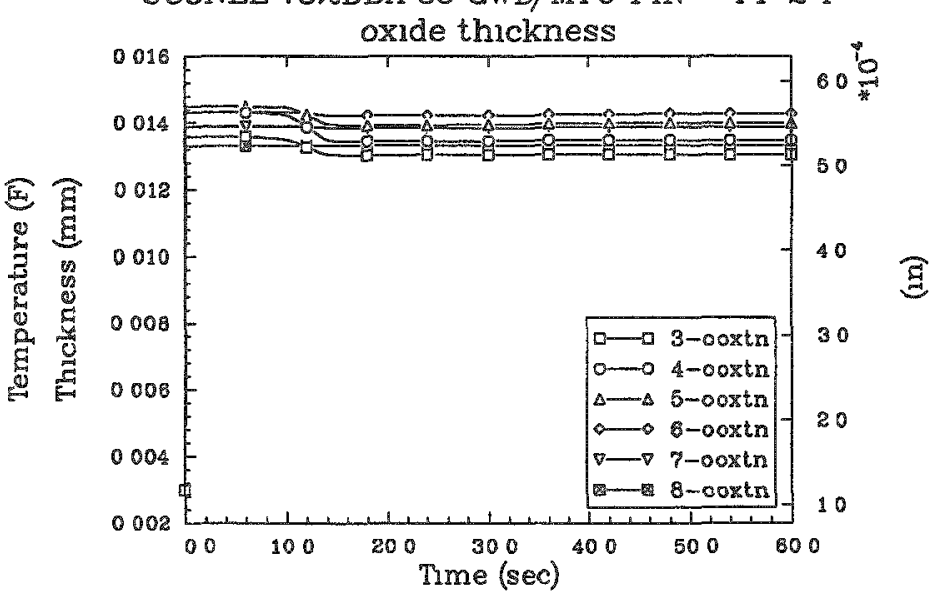


OCONEE 75\%DBA 35 GWD/MTU PIN--PF 2.4
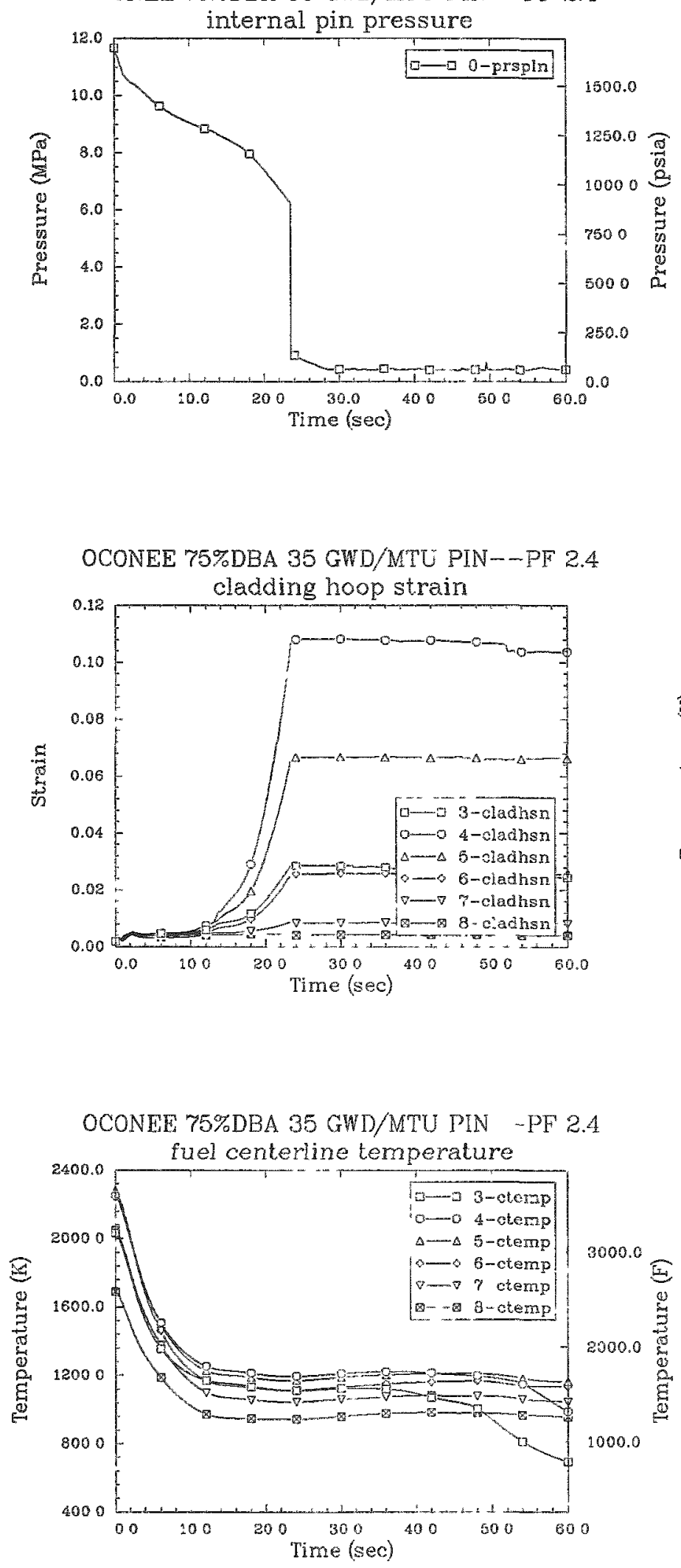

OCONEE 75\%DBA 35 GWD/MTU PIN--PF 2.4

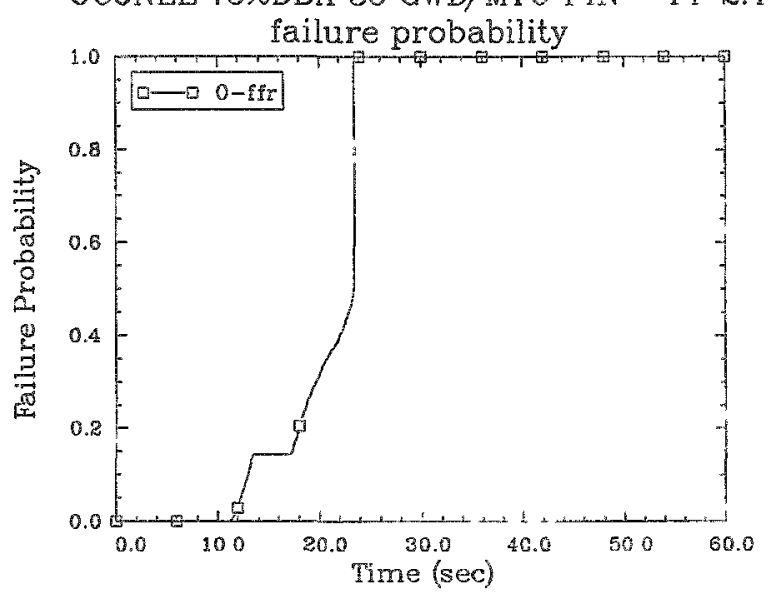

OCONEE 75\%DBA 35 GWD/MTU PIN--PF 24
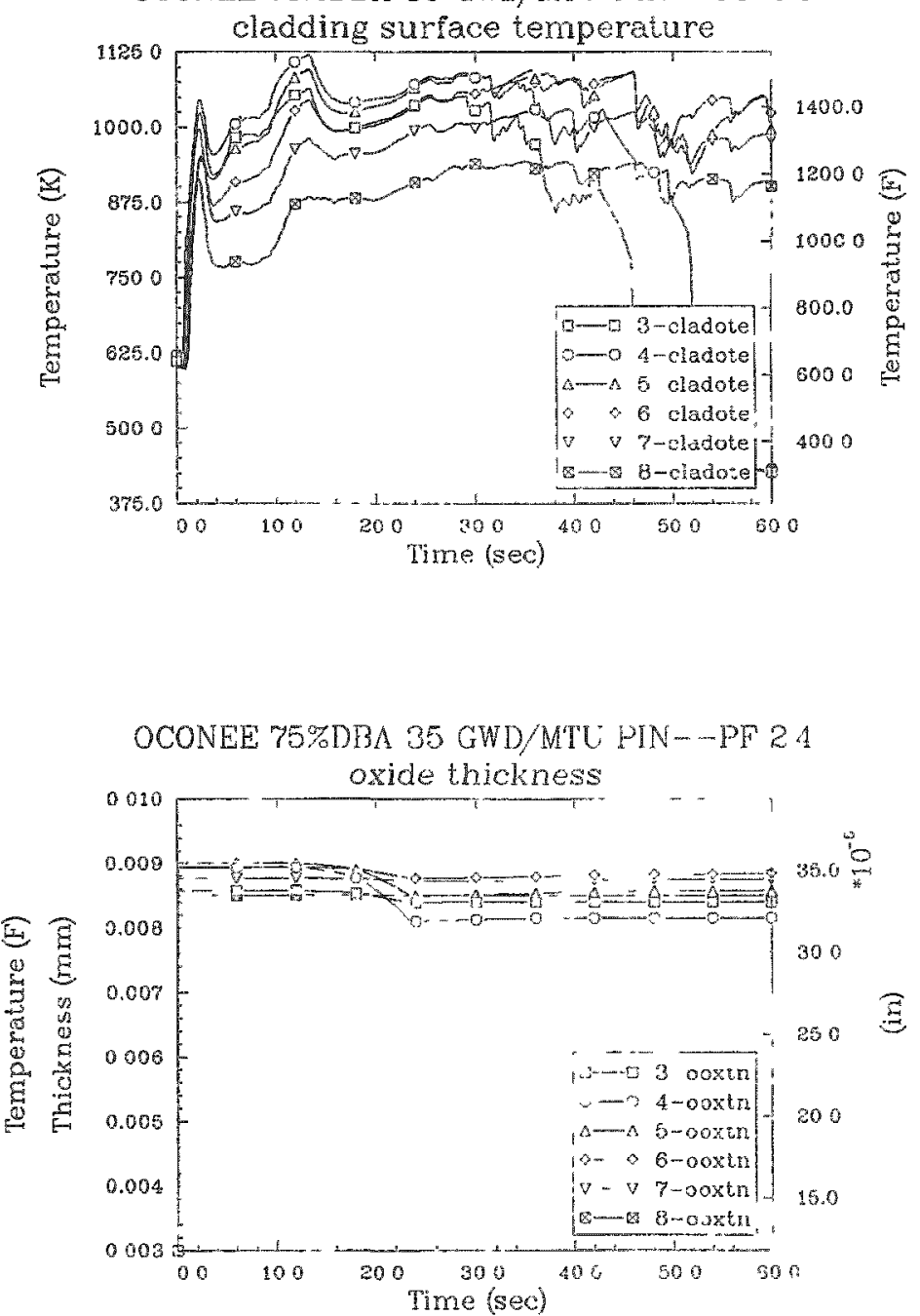

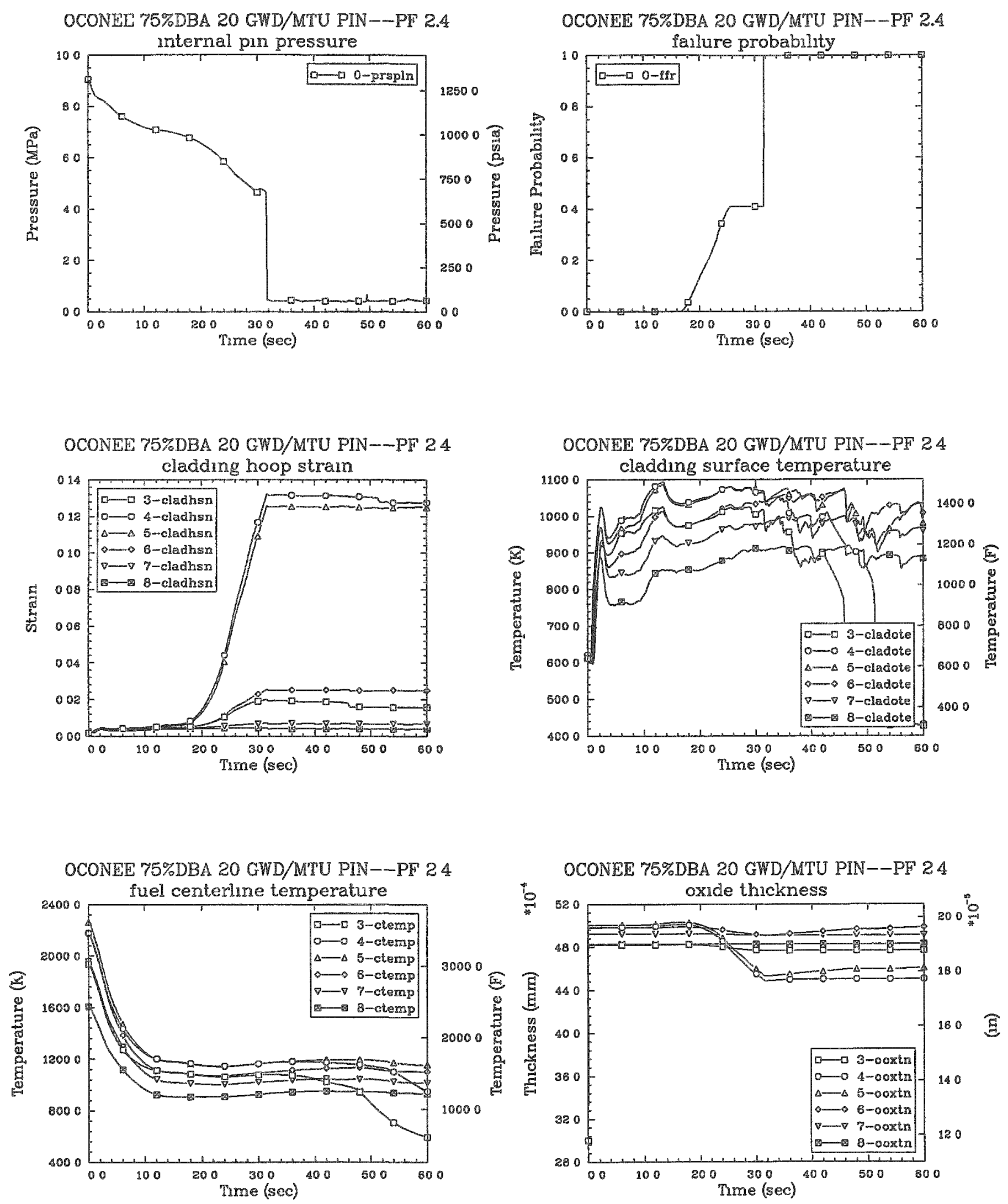

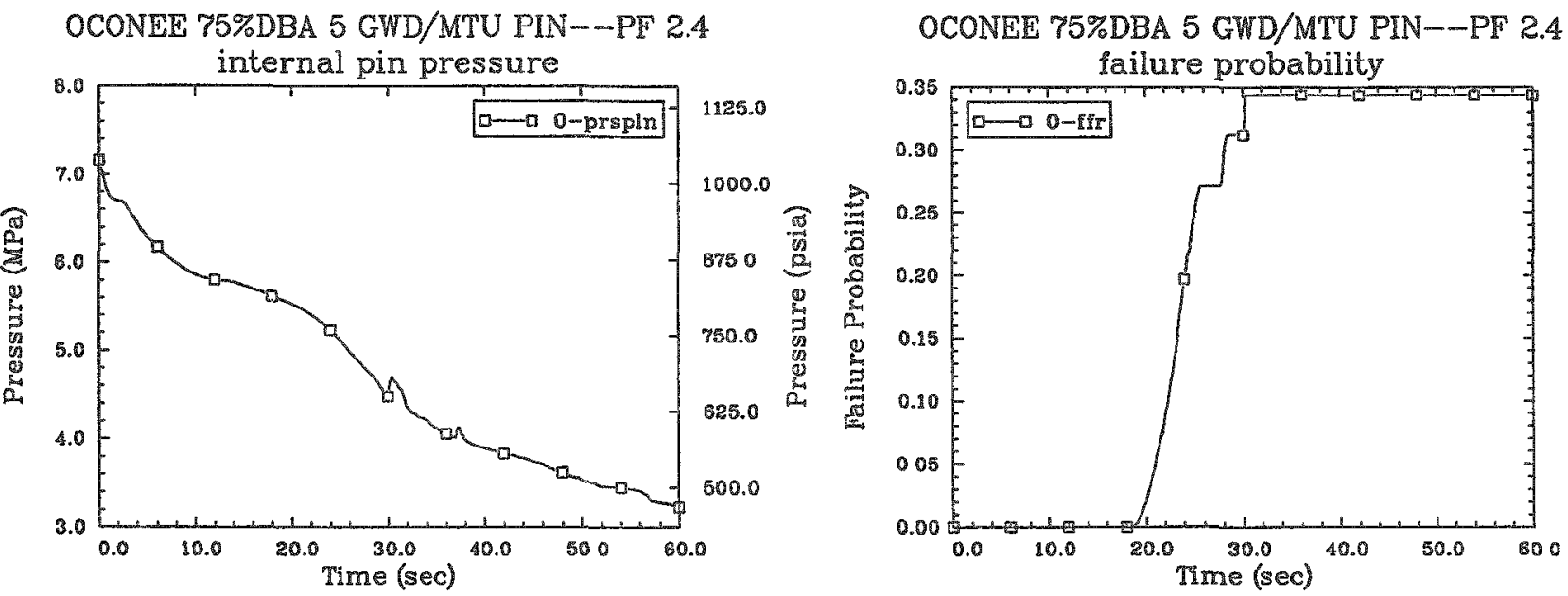

OCONEE 75\%DBA 5 GWD/MTU PIN--PF 2.4 cladding hoop strain

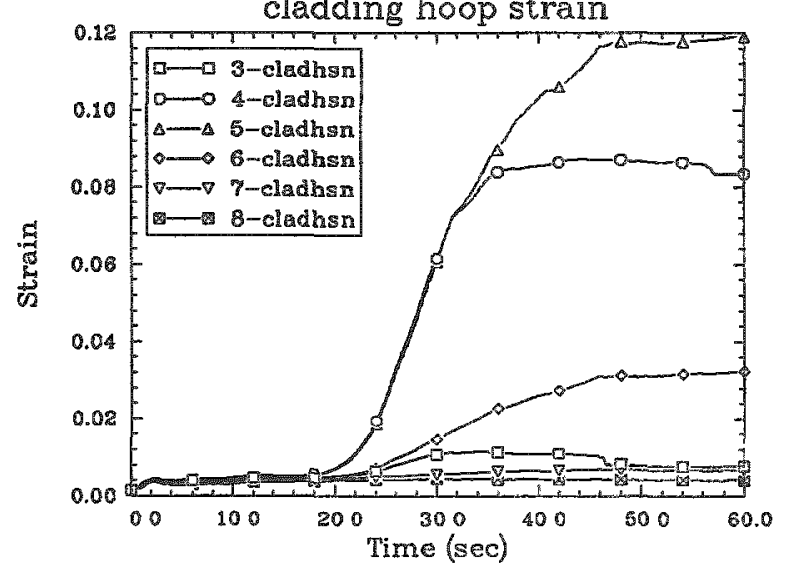

OCONEE 75\%DBA 5 GWD/MTU PIN-DF 2.4
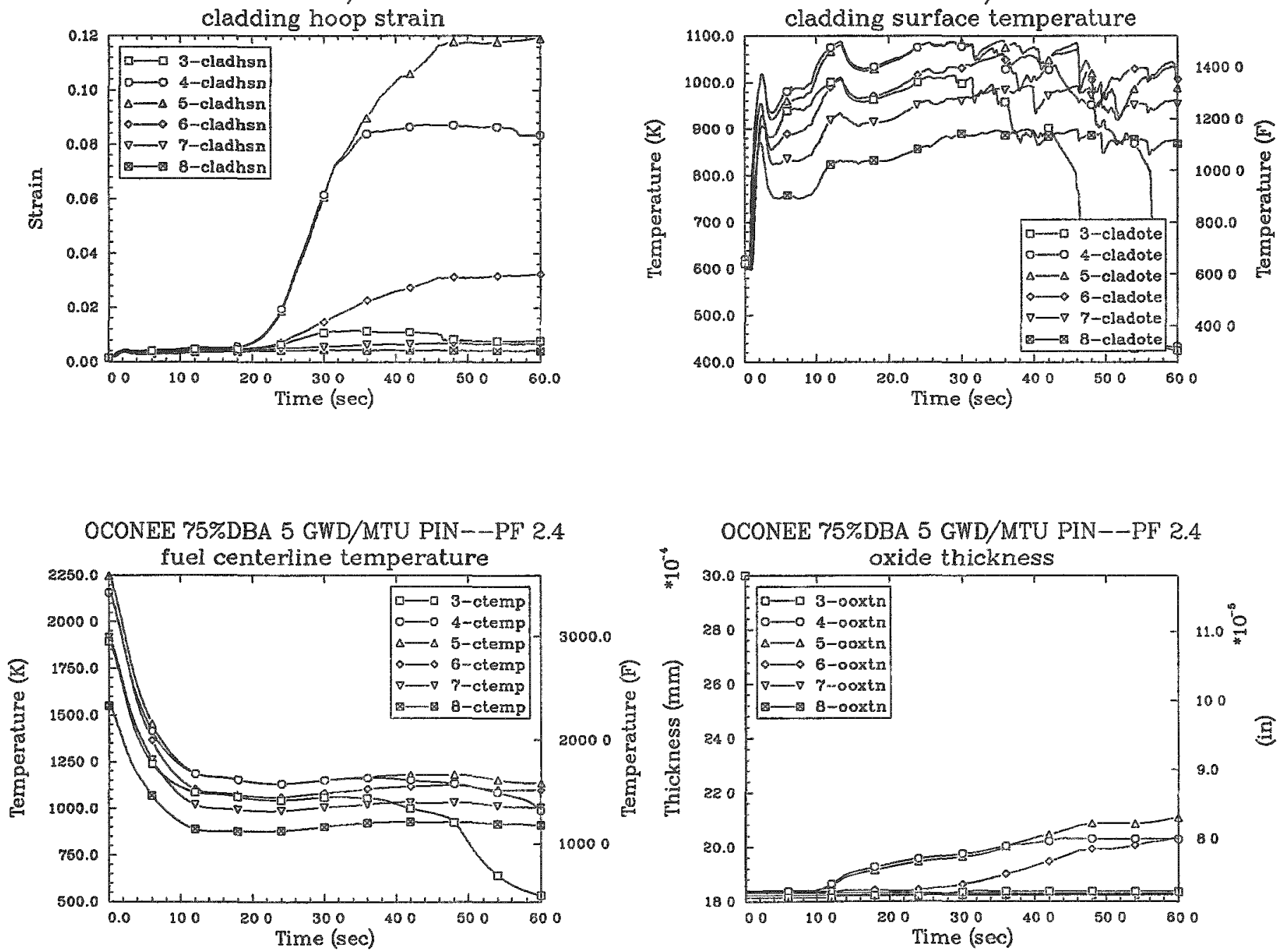

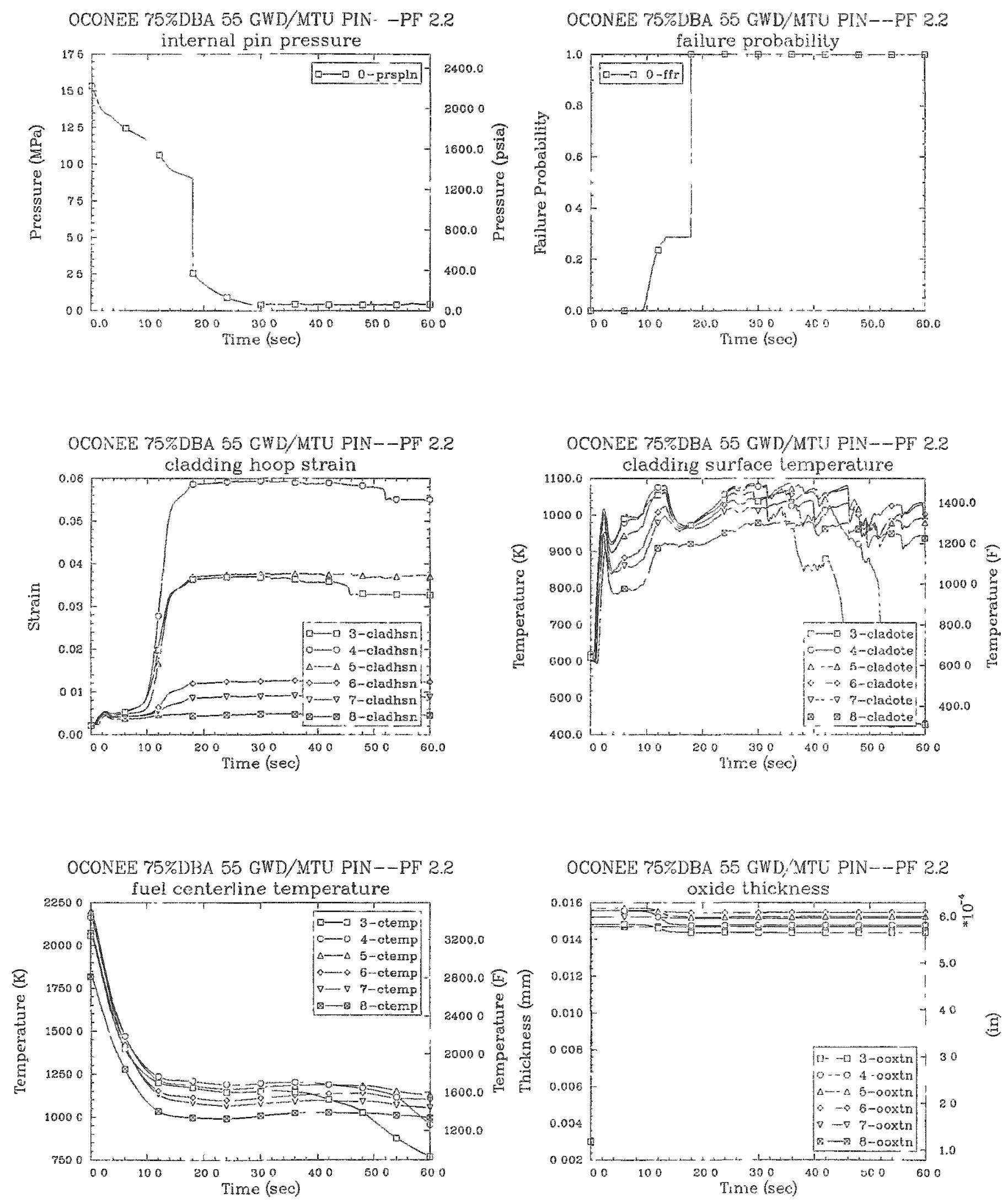

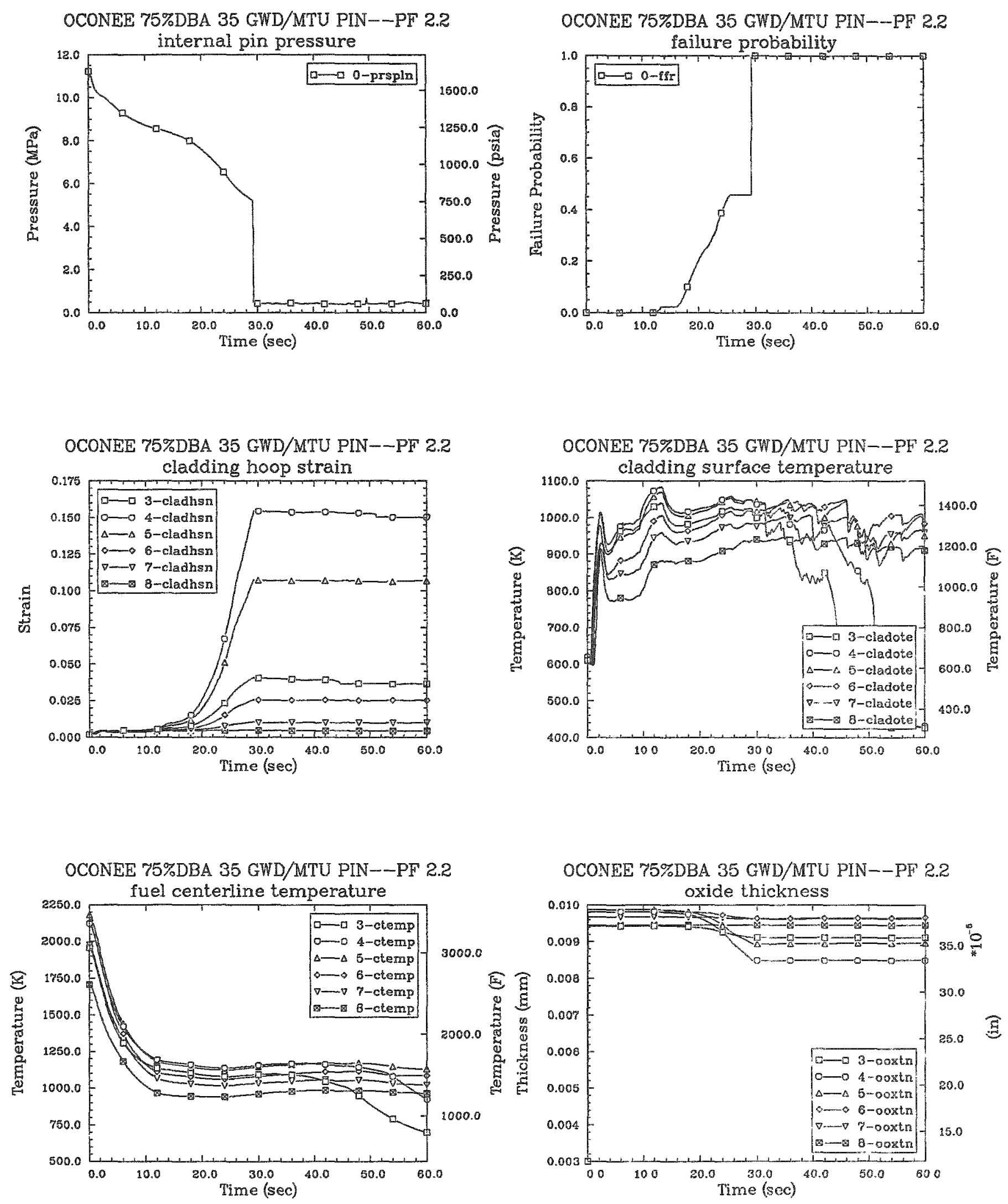

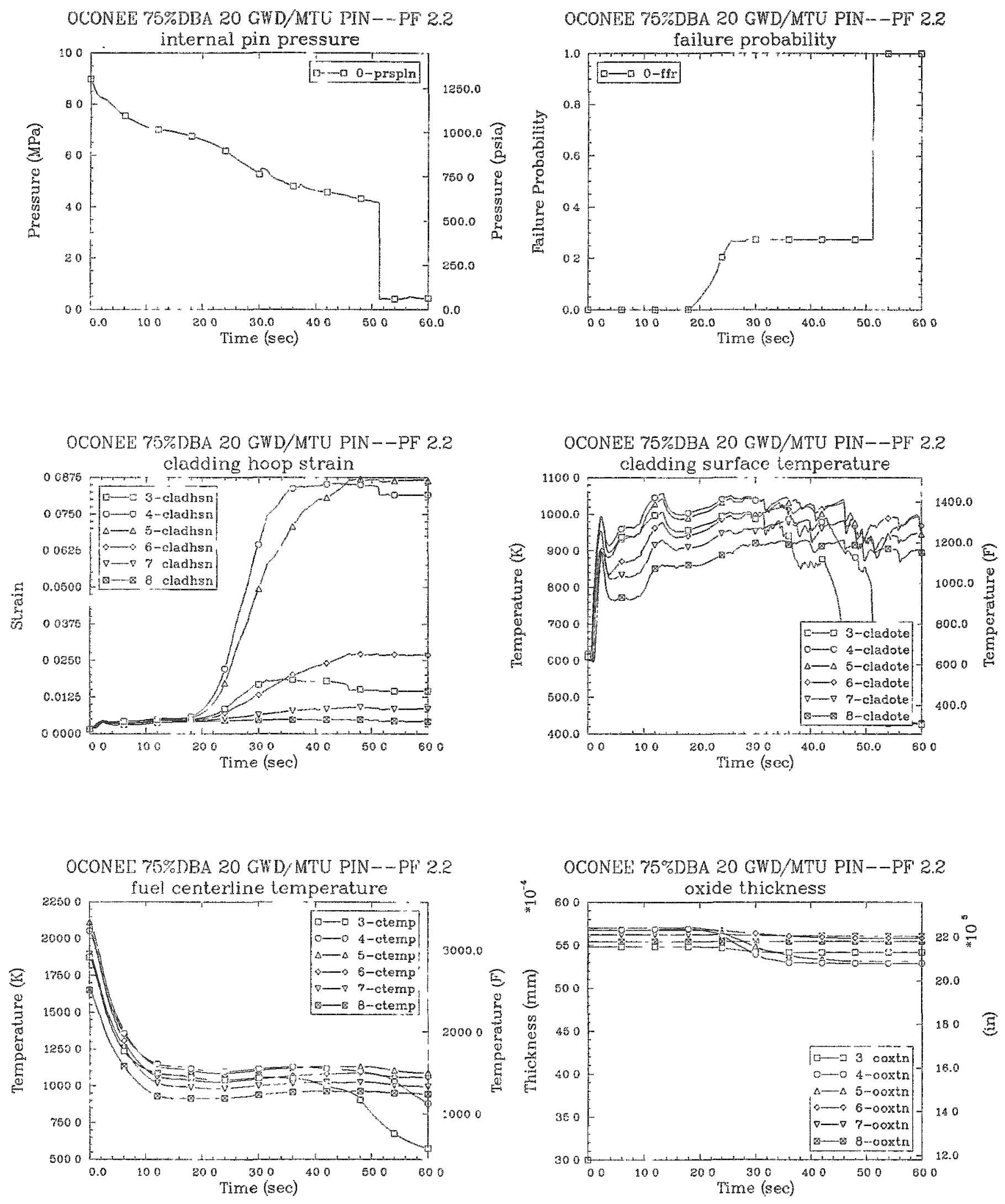

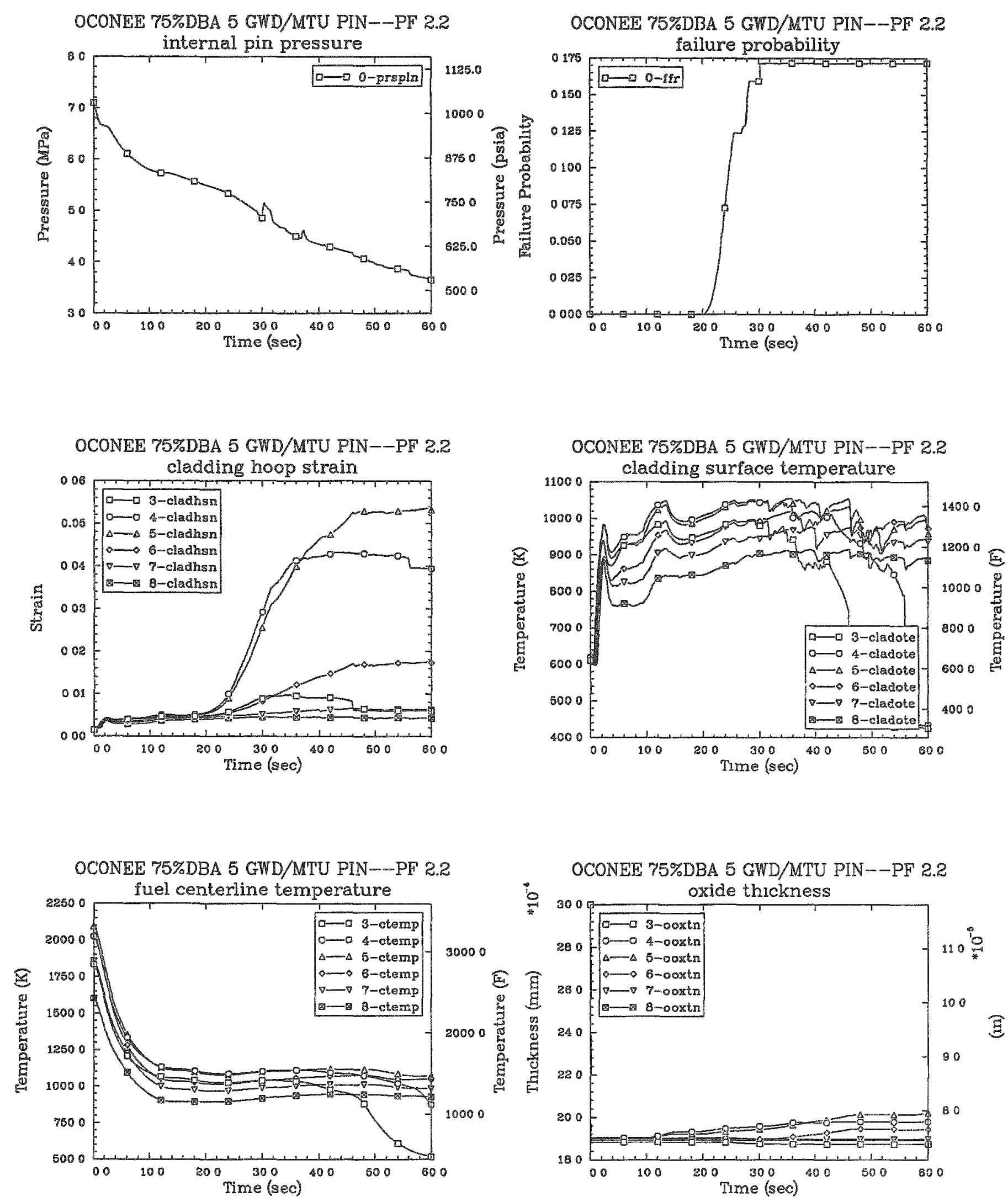

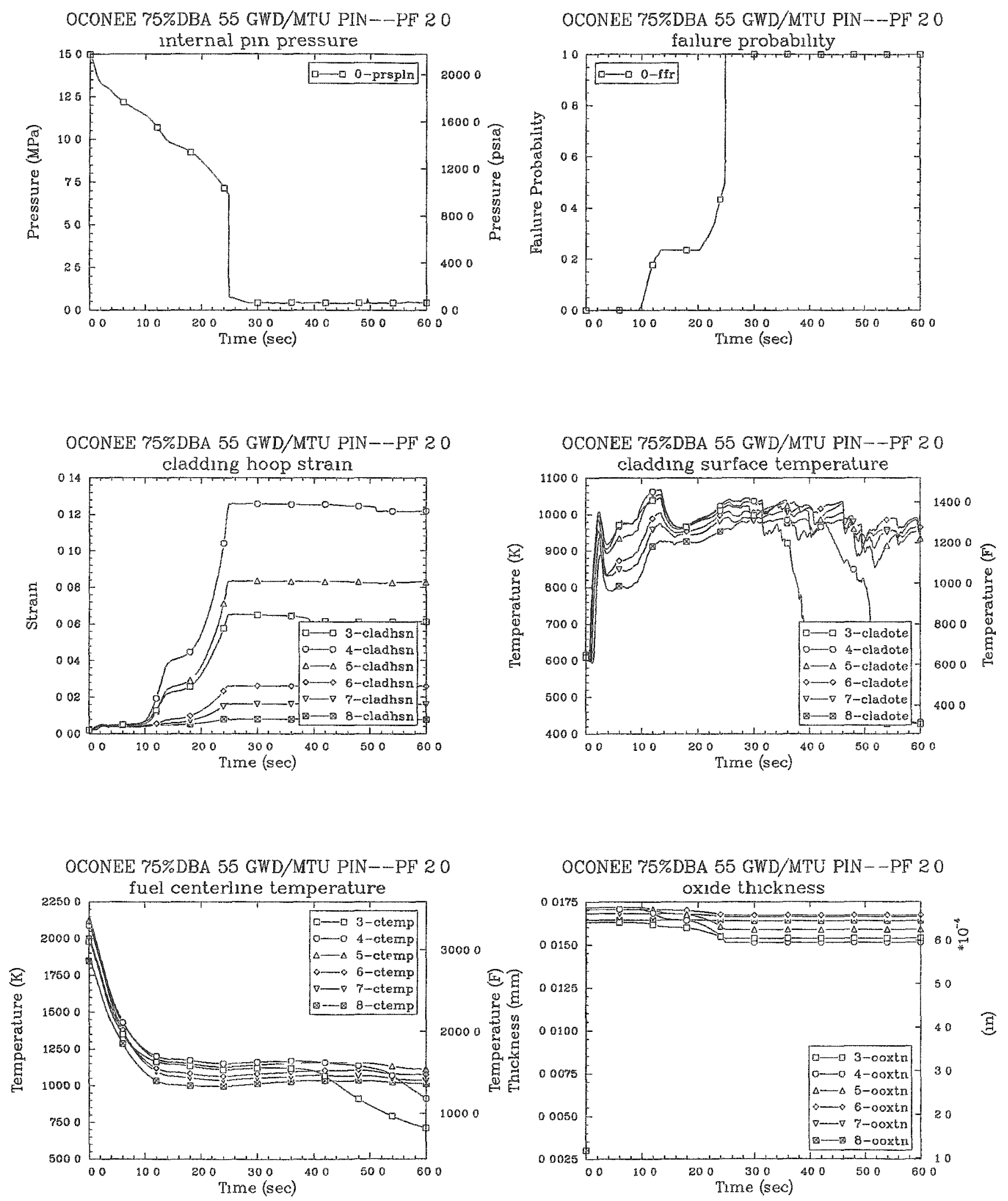

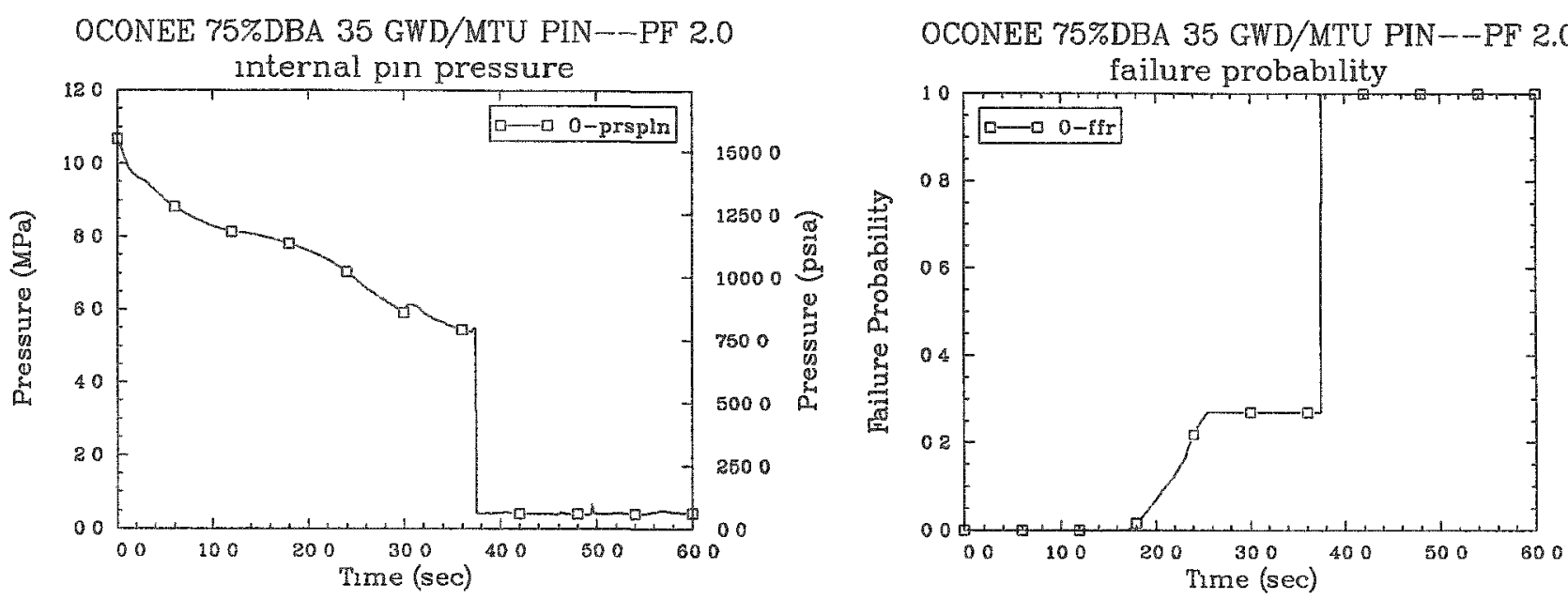

OCONEE 75\%DBA $35 \mathrm{GWD} / \mathrm{MTU}$ PIN--PF 20

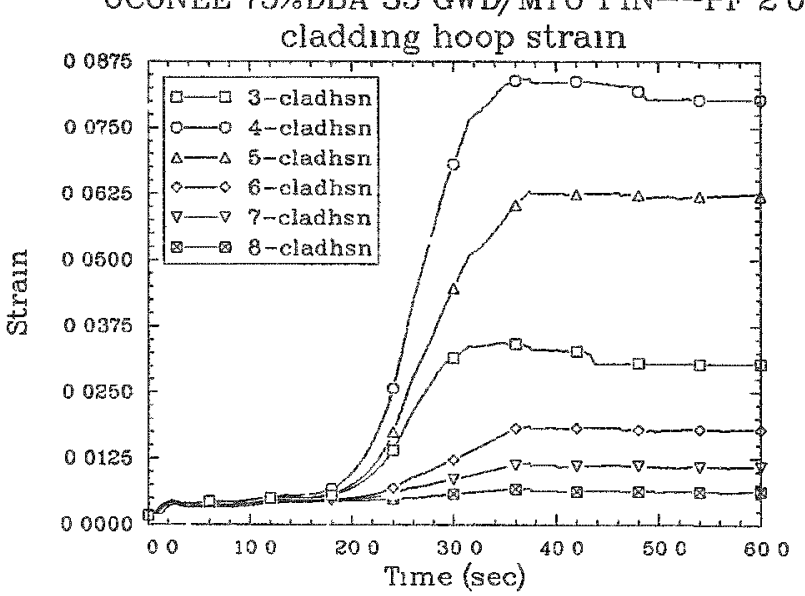

OCONEE 75\%DBA 35 GWD/MTU PIN--PF 20

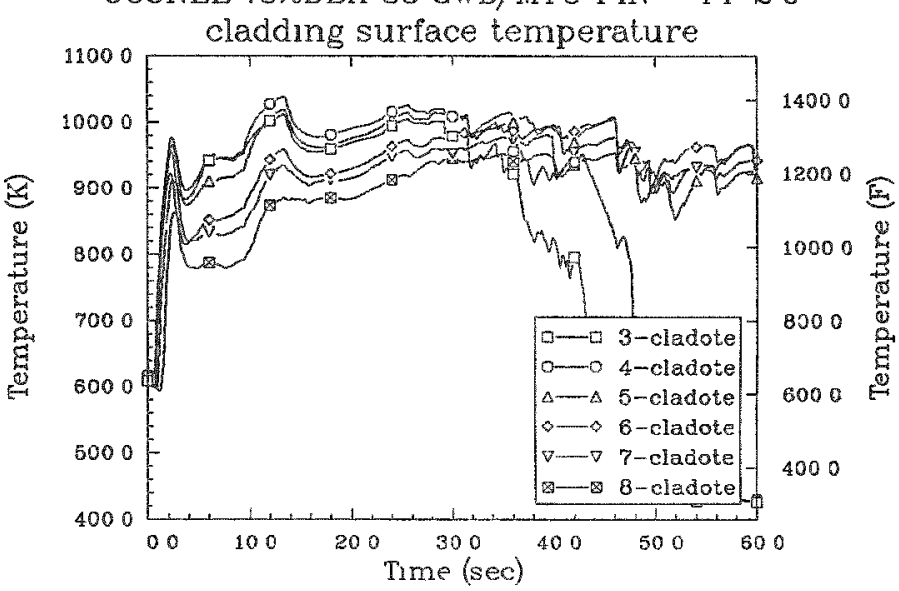

OCONEE 75\%DBA 35 GWD/MTU PIN--Pr̈ 20

OCONEE 75\%DBA 35 GWD/MTU PIN-DP 20
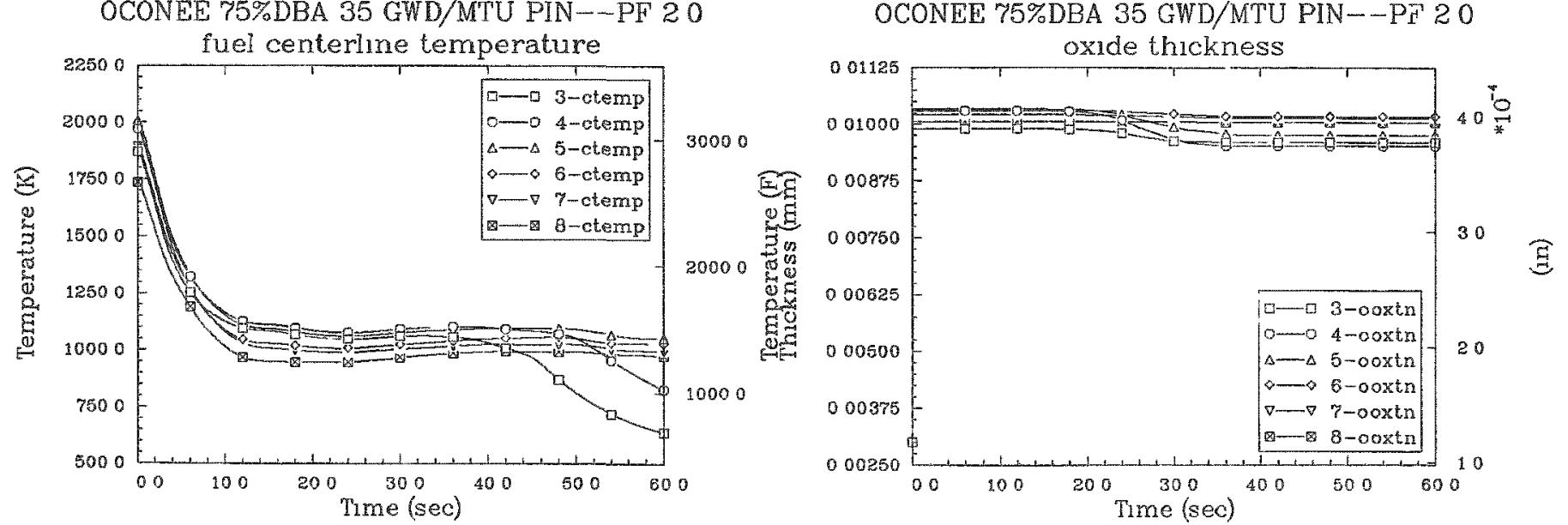
OCONEE 75\%DBA 20 GWD/MTU PIN--PF 20

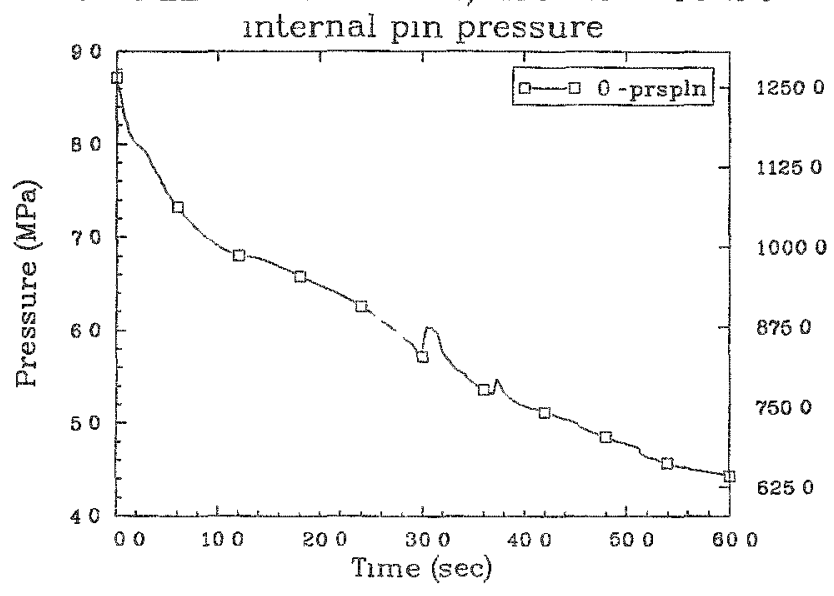

OCONEE 75\%DBA 20 GWD/MTU PIN--PF 20

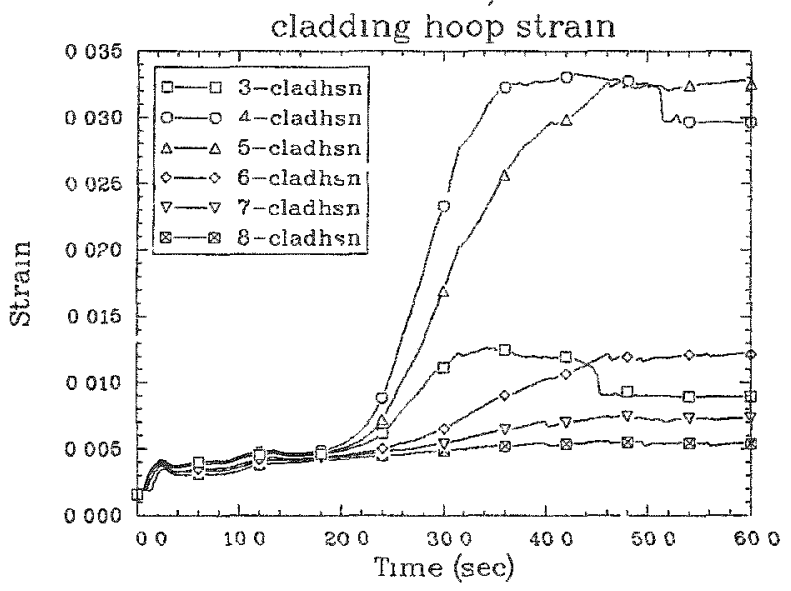

OCONEE 75\%DBA 20 GWD/MTU PIN- -PF 20

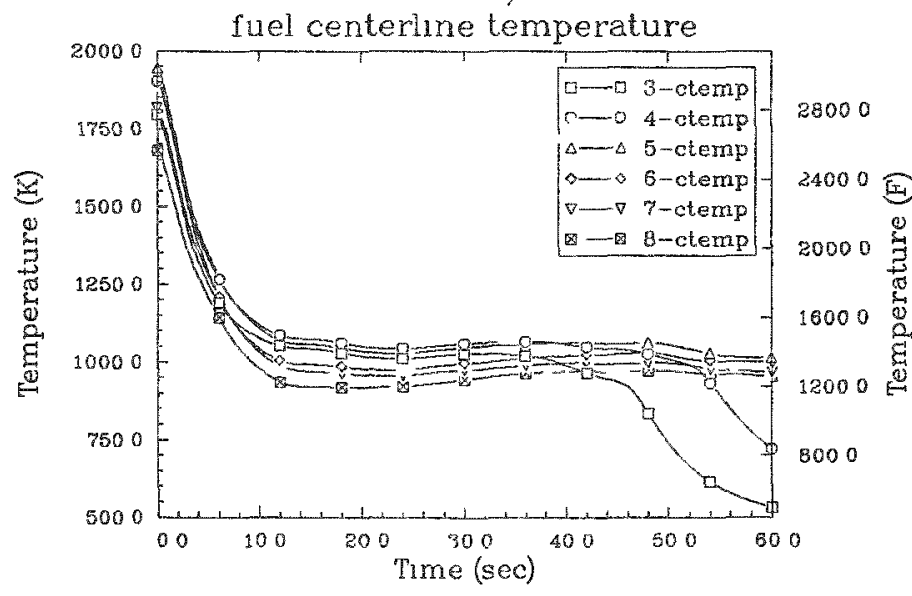

OCONFE 75\%DBA $20 \mathrm{CWD} / \mathrm{MTU}$ PIN--PF 20 fallure probability

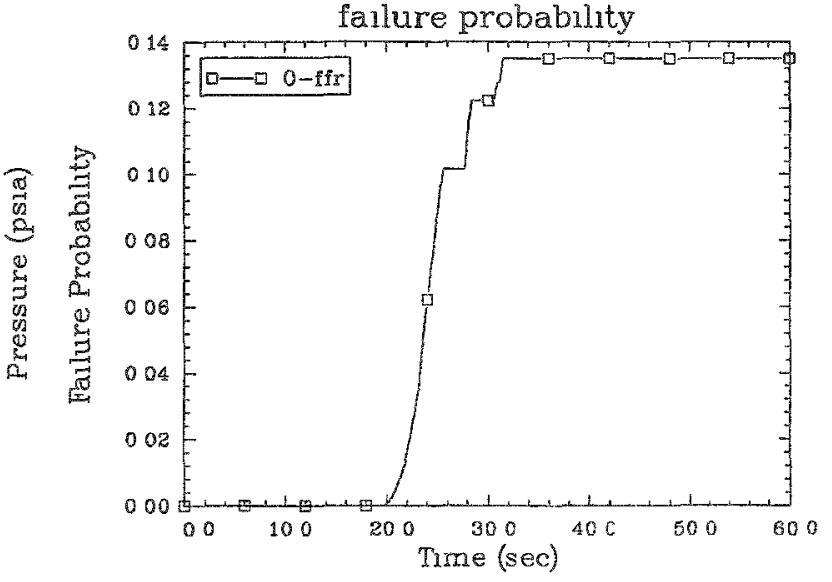

OCONEE 75\%DBA 20 GWD/MTU PIN--PF 20

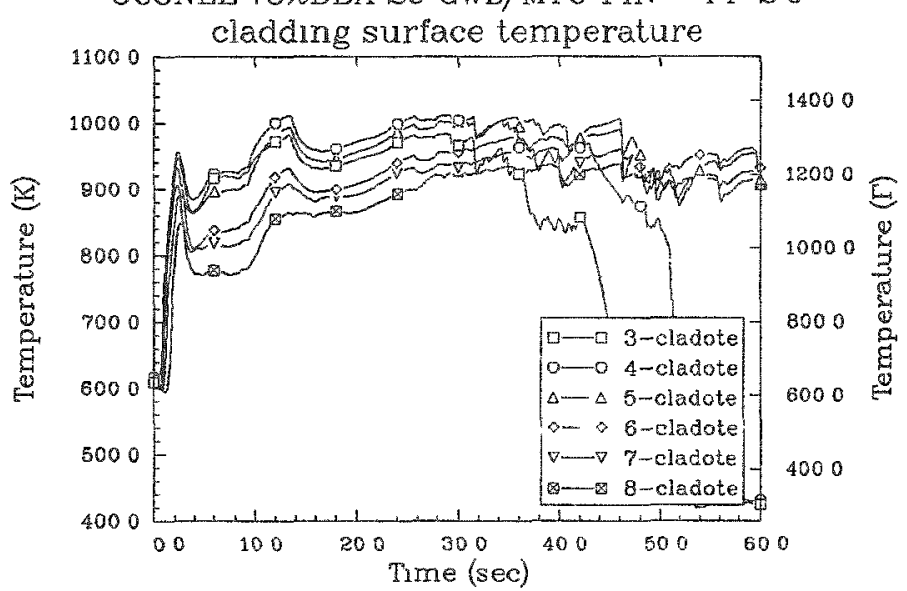

OCONEE 75\%DBA 20 GWD/MTU PIN--PF 20

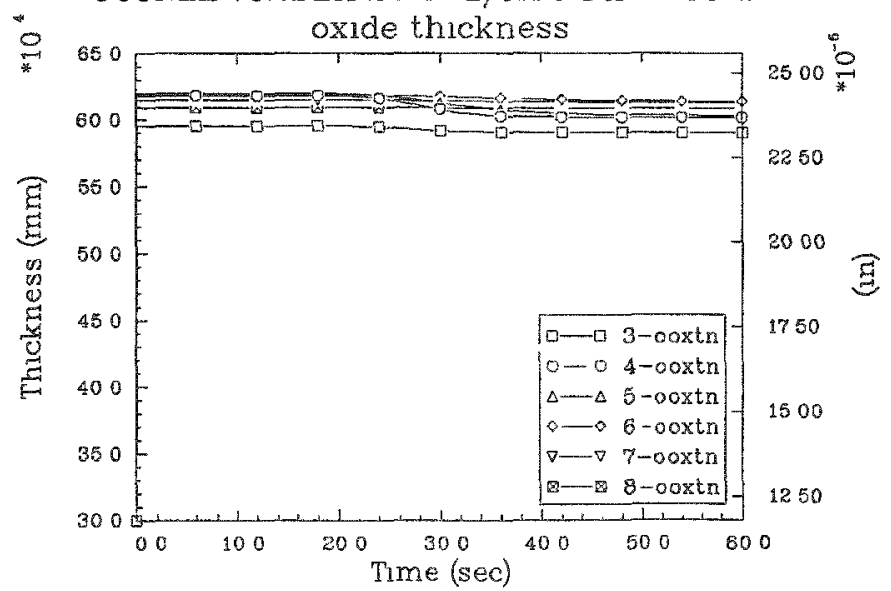


OCONEE 75\%DBA 5 GWD/MTU PIN--PF 2.0
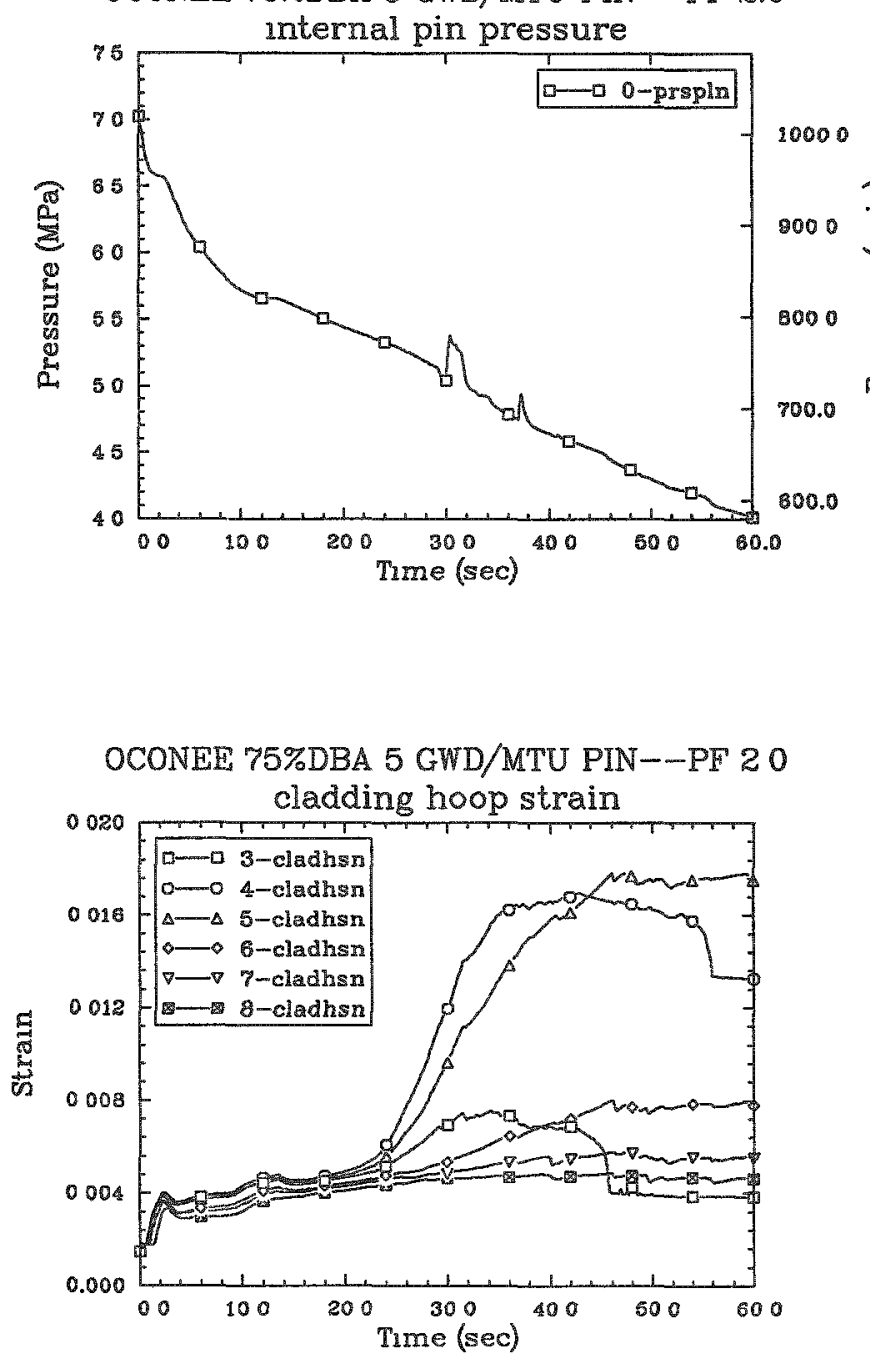

OCONEE 75\%DBA 5 GWD/MTU PIN--PF 20

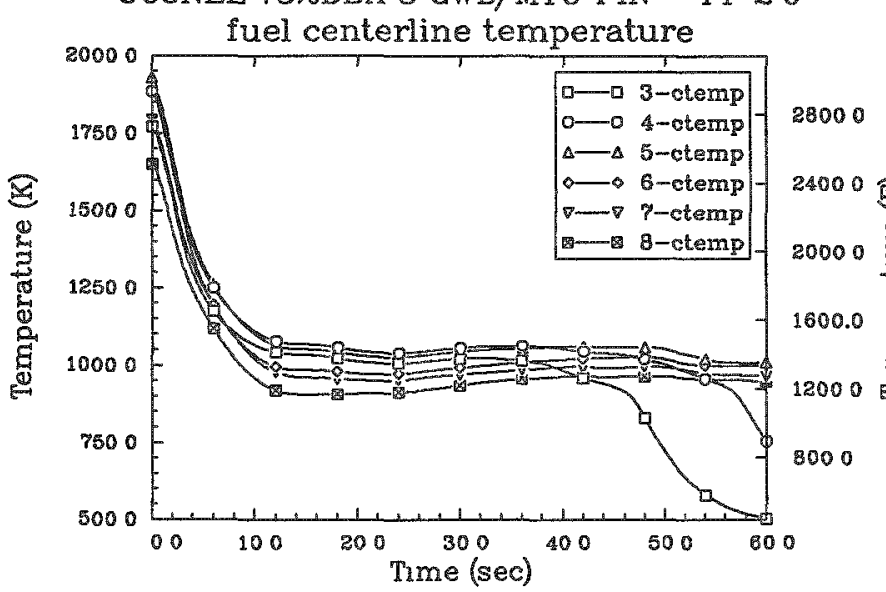

OCONEE 75\%DBA 5 GWD/MTU PIN--PF 2.0

failure probability

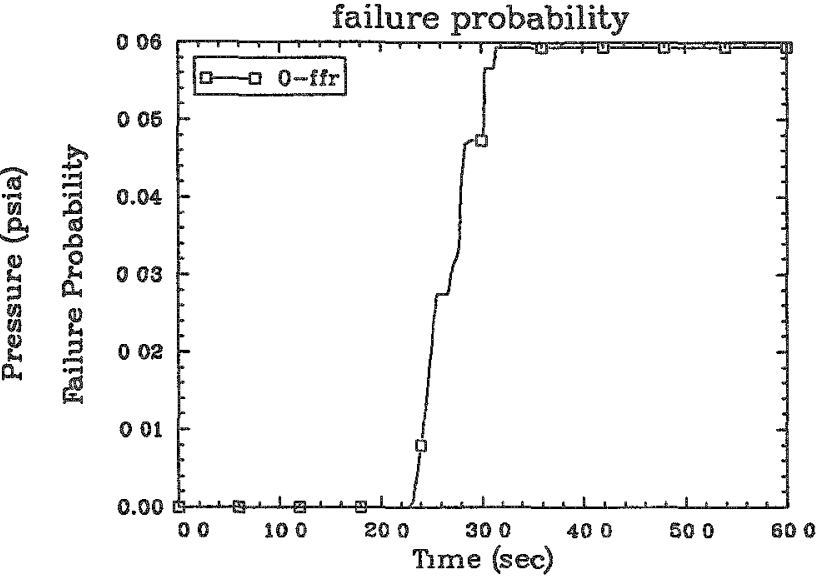

OCONEE 75\%DBA 5 GWD/MTU PIN-DF 2.0 cladding surface temperature

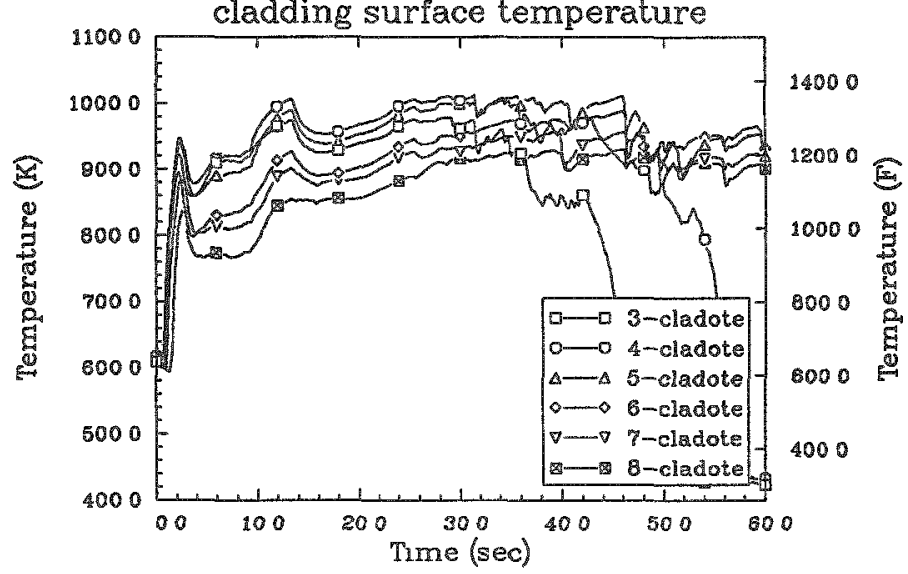

OCONEE 75\%DBA 5 GWD/MTU PIN-PF 20

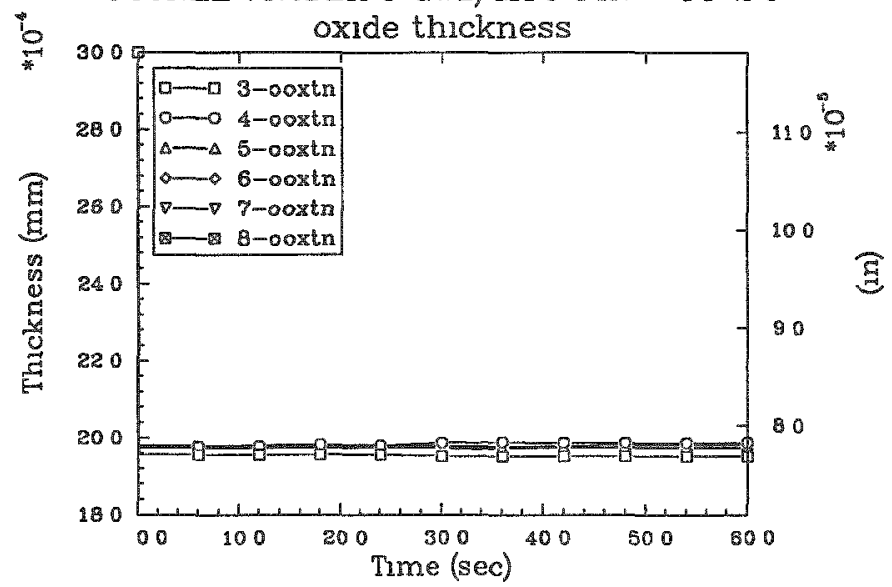




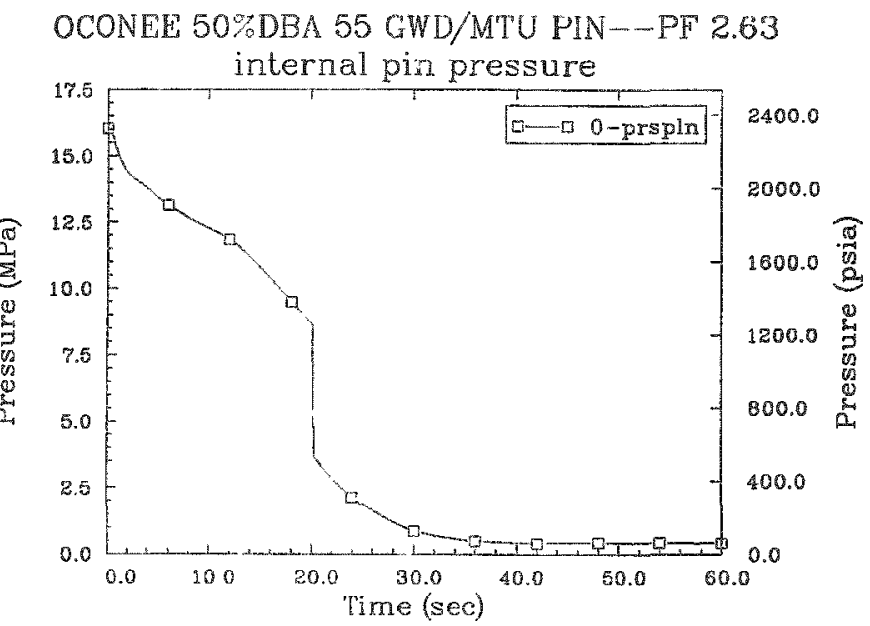

OCONEE 50\%DBA 55 GWD/MTU PIN--PF 2.63

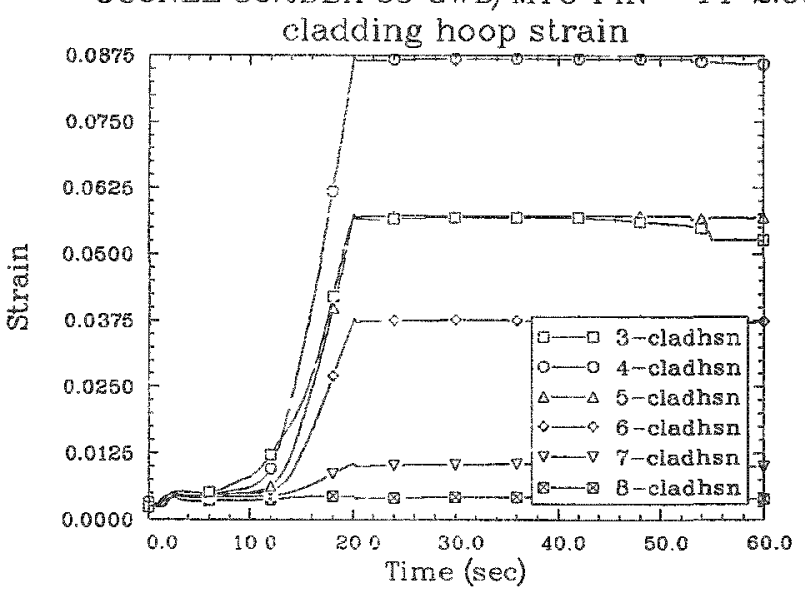

OCONEE 50\%DBA 55 GWD/MTU PIN--PF 2.63

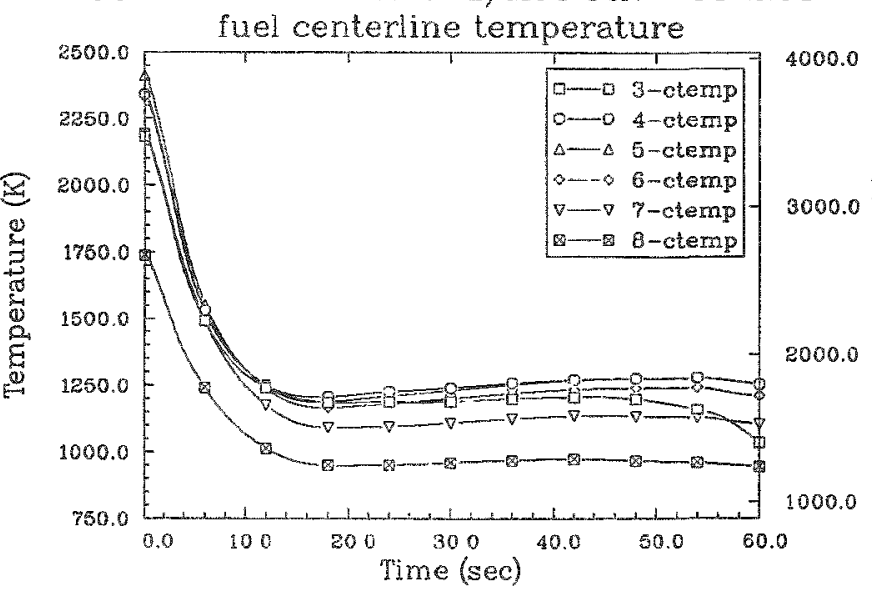

OCONEE 50\%DBA 55 GWD/MTU PIN--PF 2.63

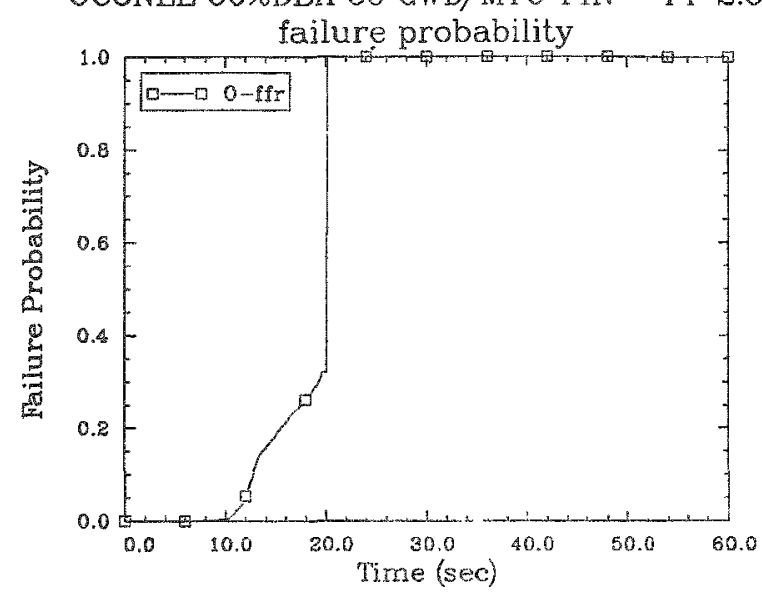

OCONEE 50\%DBA 55 GWD/MTU PIN--PF 2.63

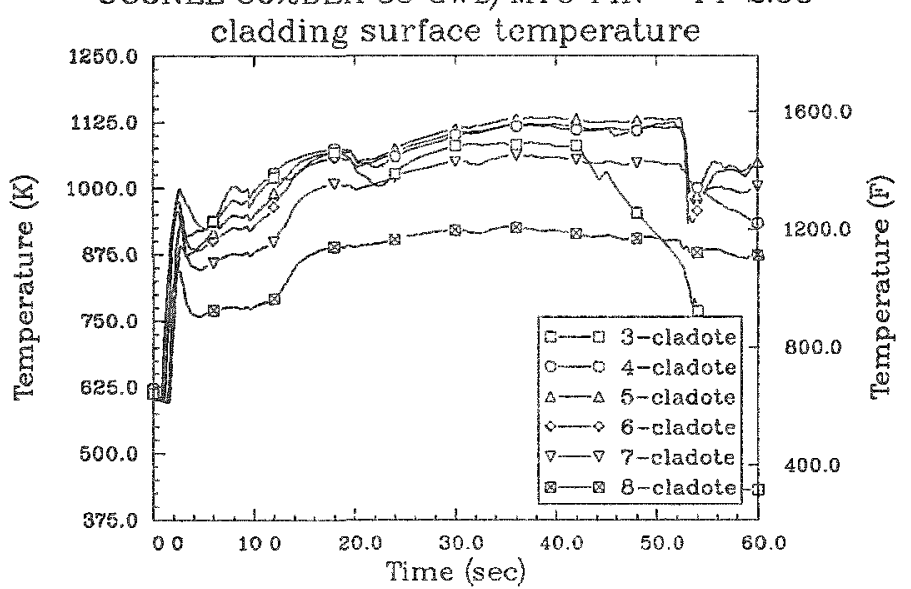

OCONEE 50\%DBA 55 GWD/MTU PIN--PF 2.63 oxide thickness

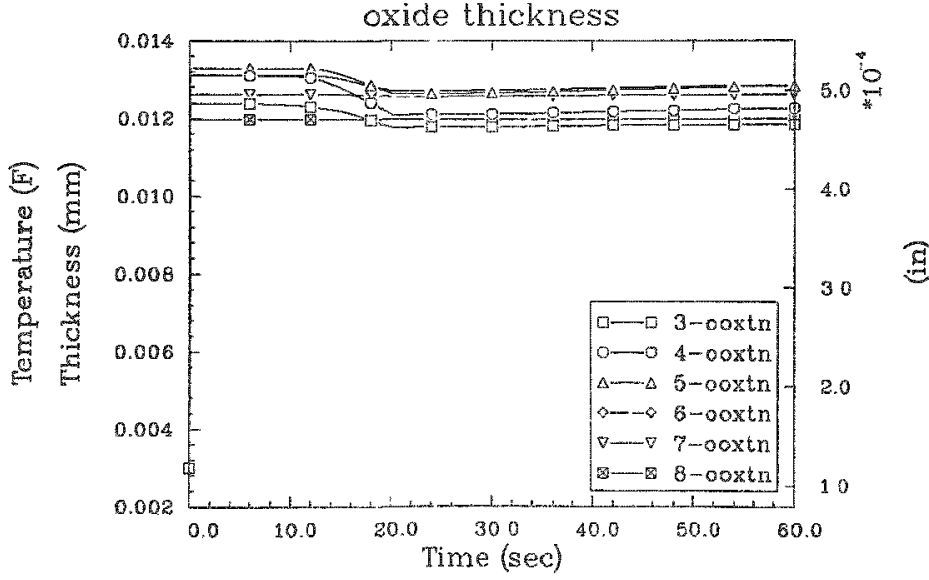



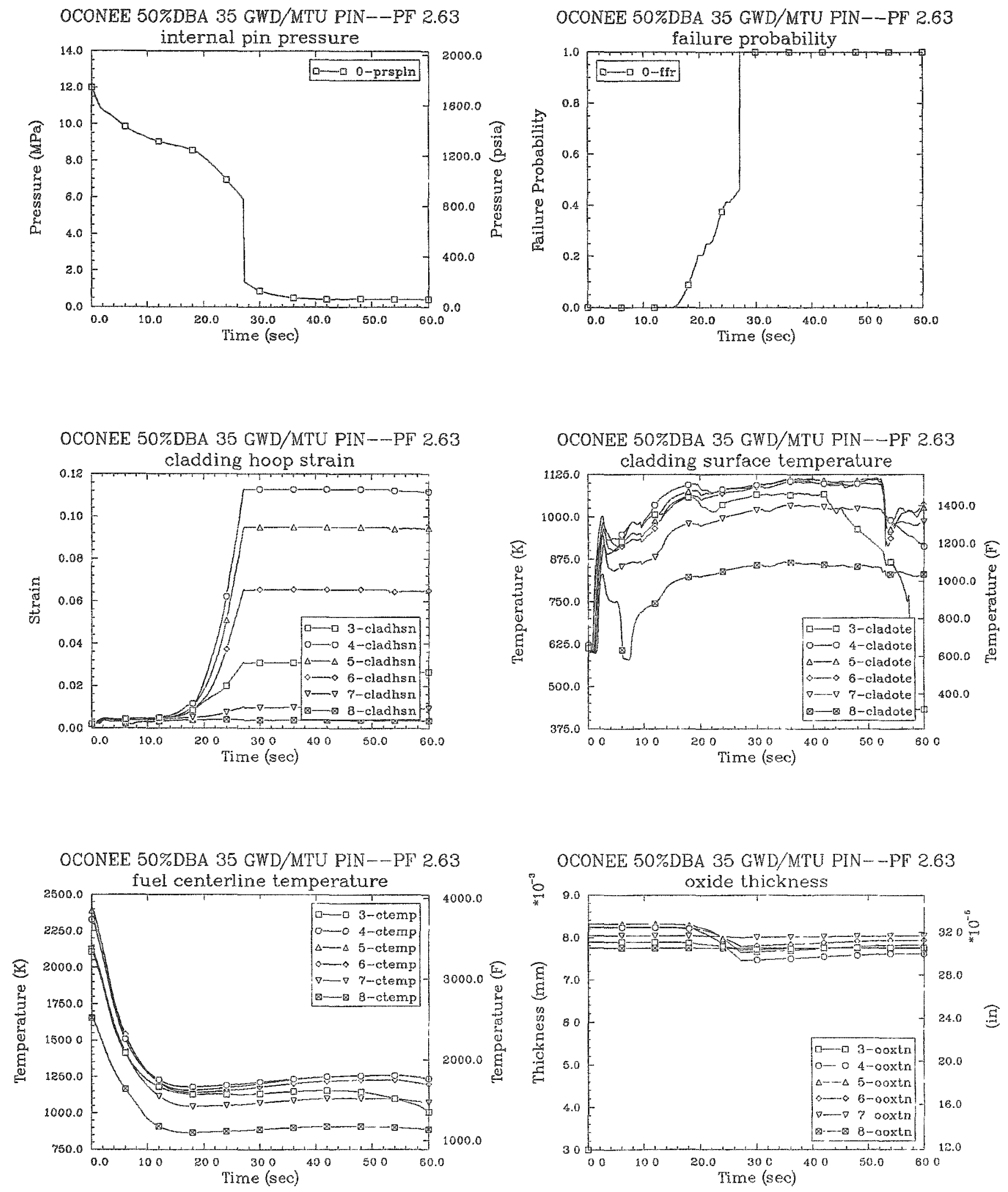

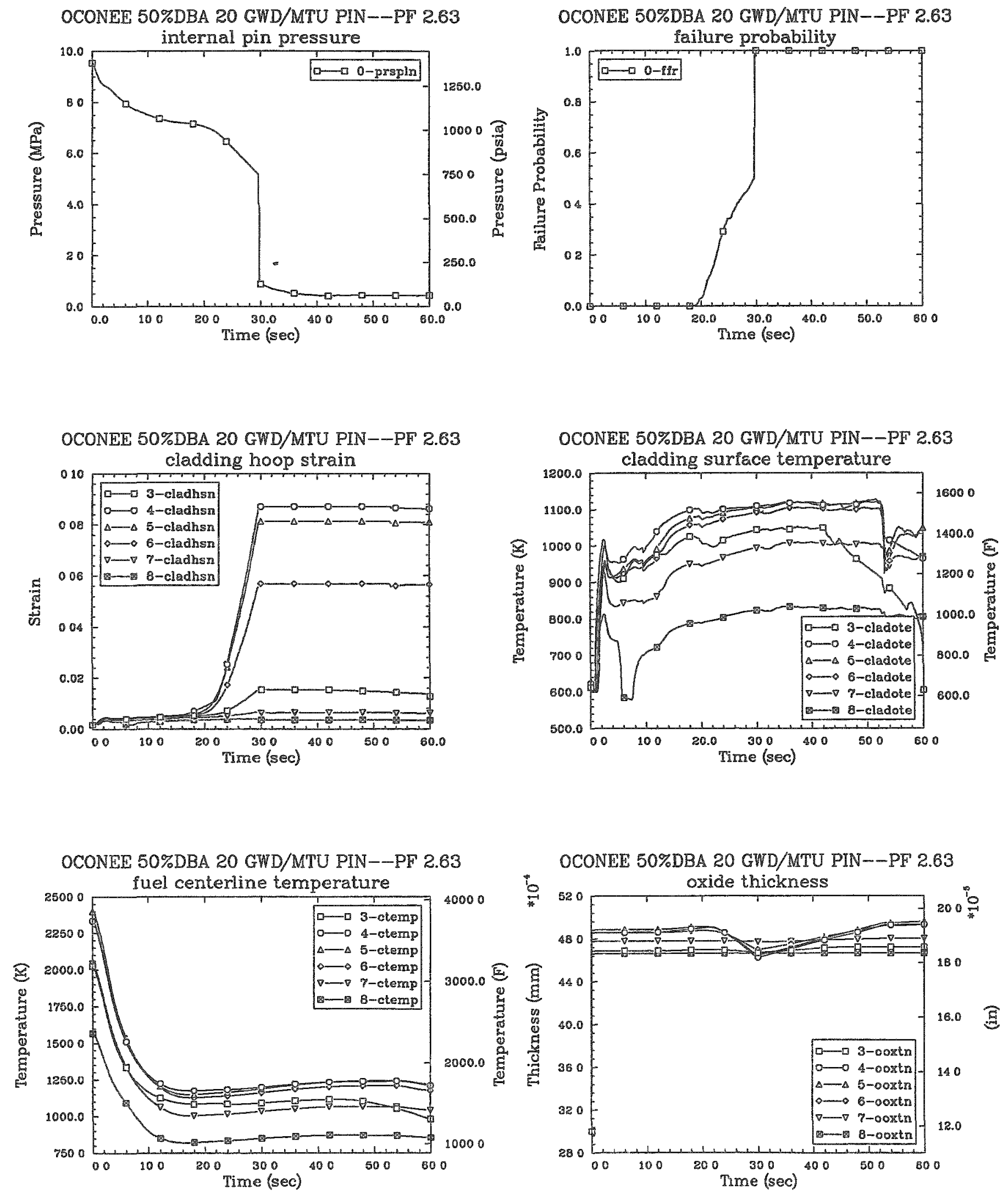

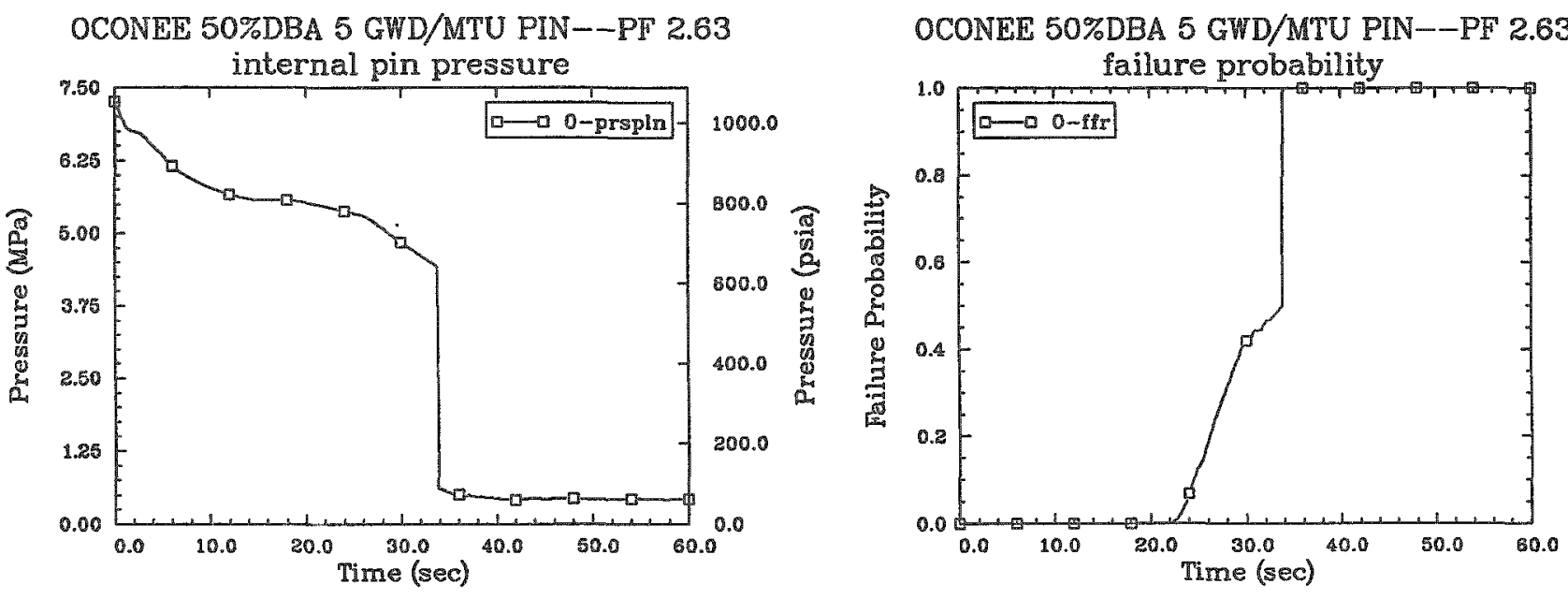

\section{OCONEE 50\%DBA 5 GWD/MTU PIN-DF 2.63}

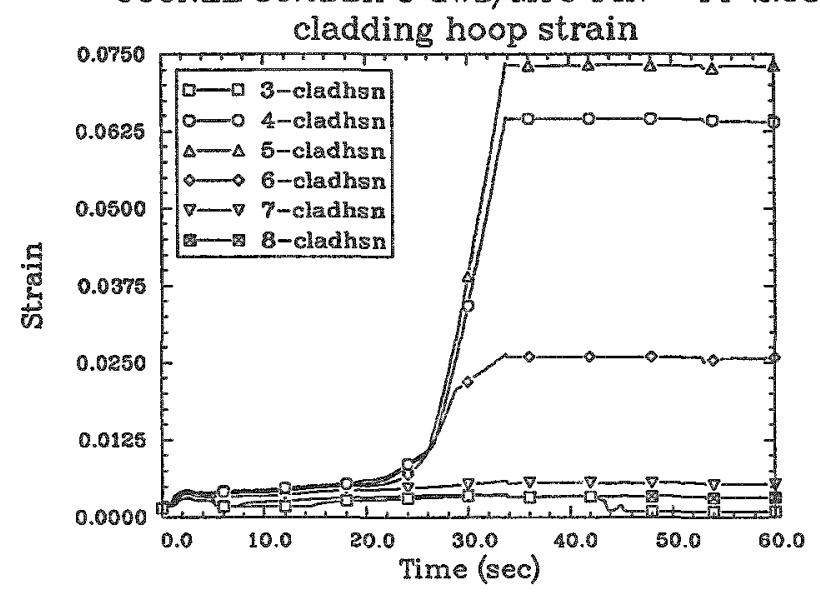

OCONEE 50\%DBA 5 GWD/MTU PIN-DF 2.63

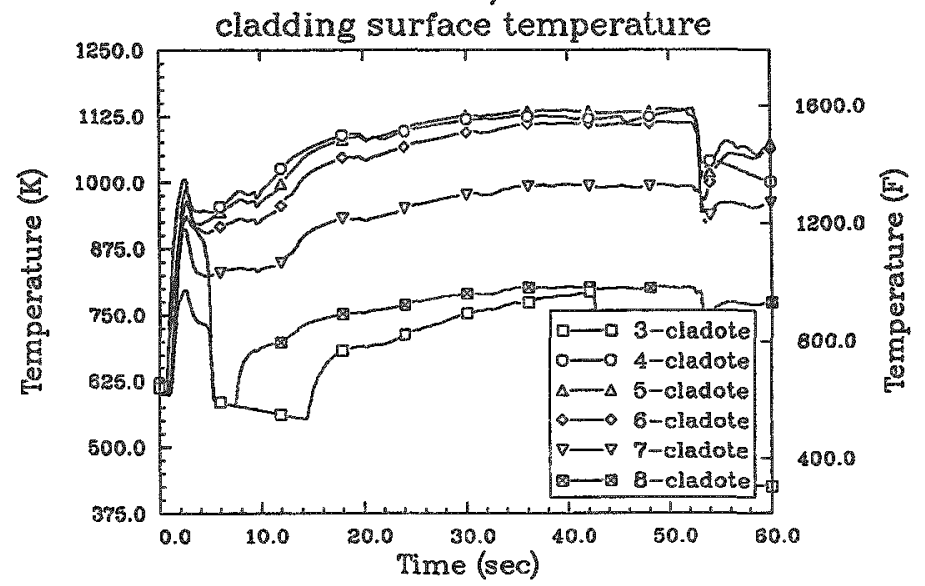

CONEE 50\%DBA 5 GWD/MTU PIN--PF 2.63
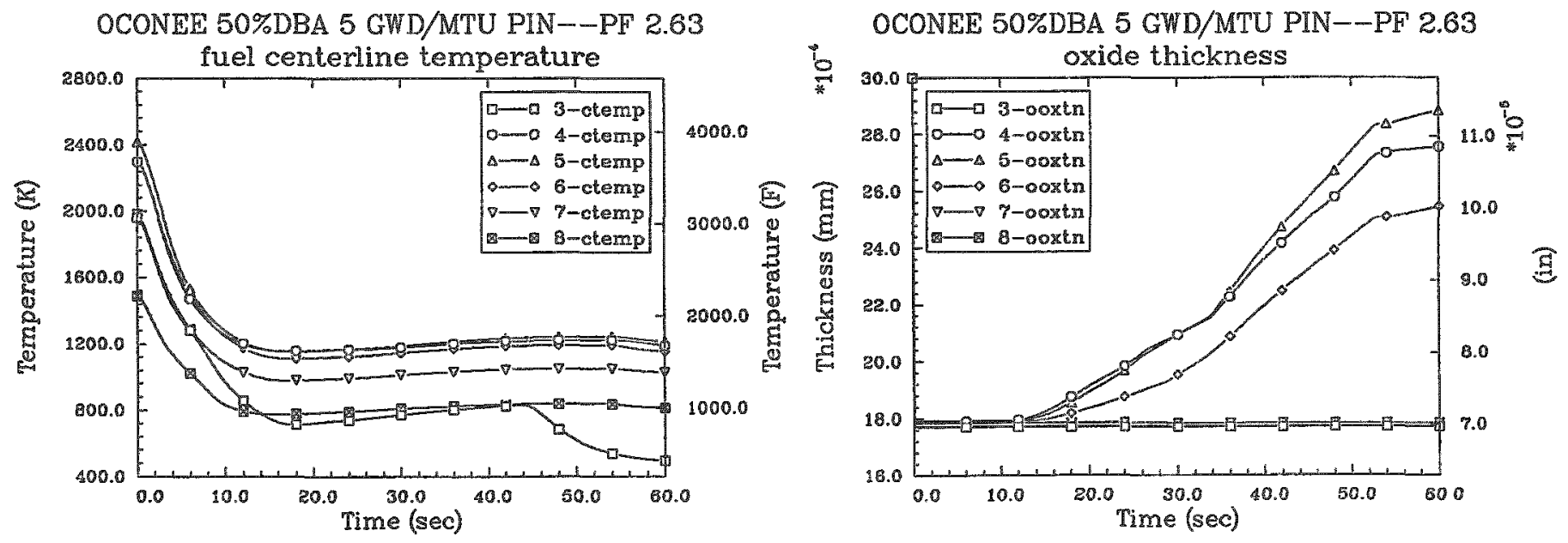
OCONEE 50\%DBA 55 GWD/MTU PIN-DF 24

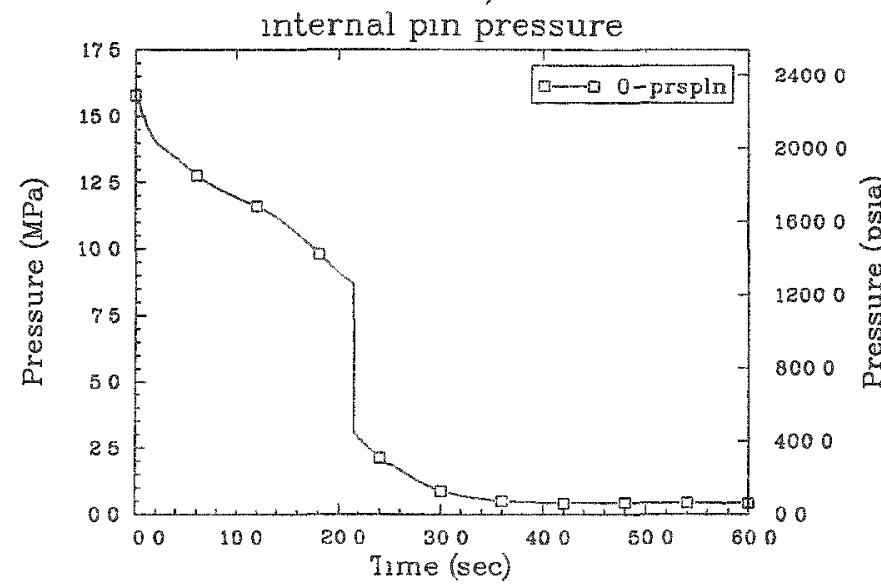

OCONEE 50\%DBA 55 GWD/MTU PIN--PF 24

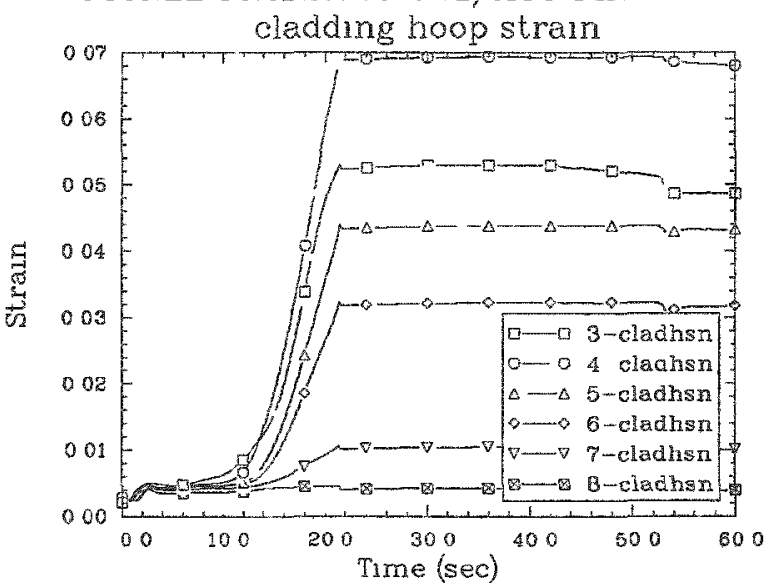

OCONEL 50\%DBA 55 GWD/MTU PIN--PF 24

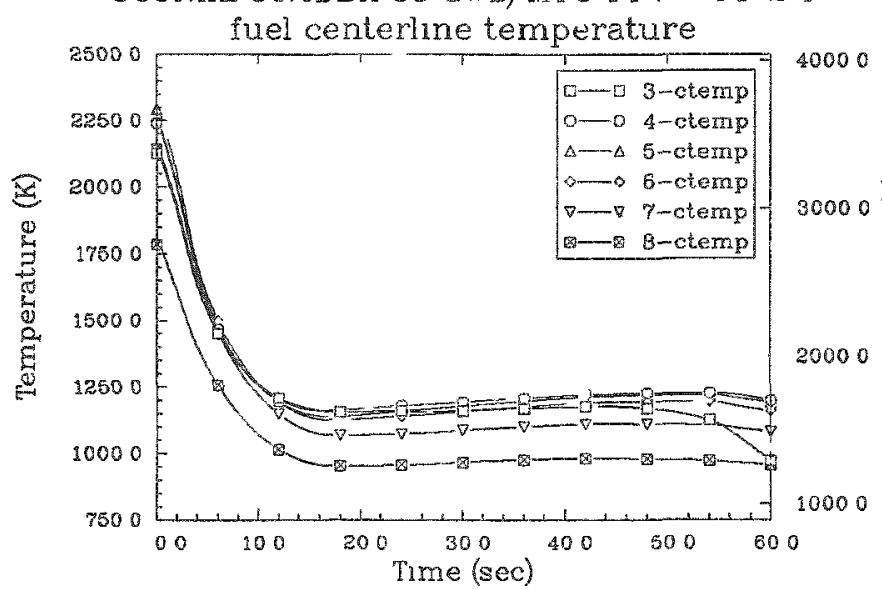

OCONEE 50\%DBA 55 GWD/MTU PIN--PF 24

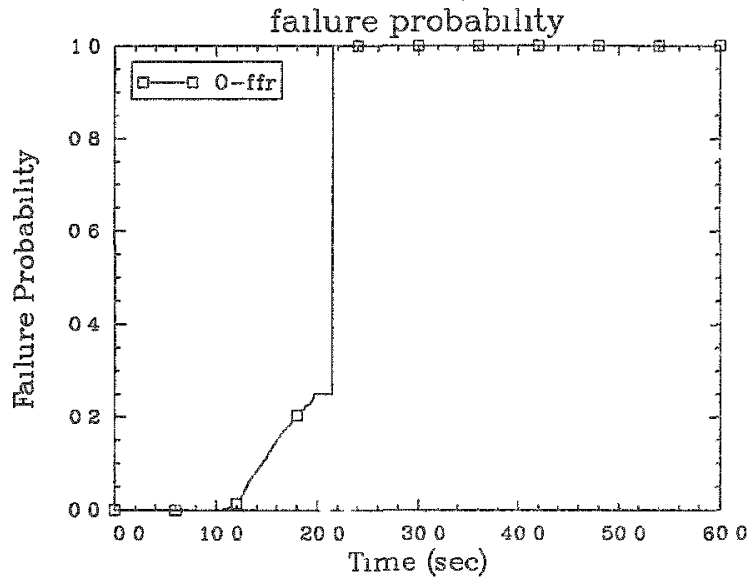

OCONEE 50\%DBA 55 GWD/MTU PIN--PF 24

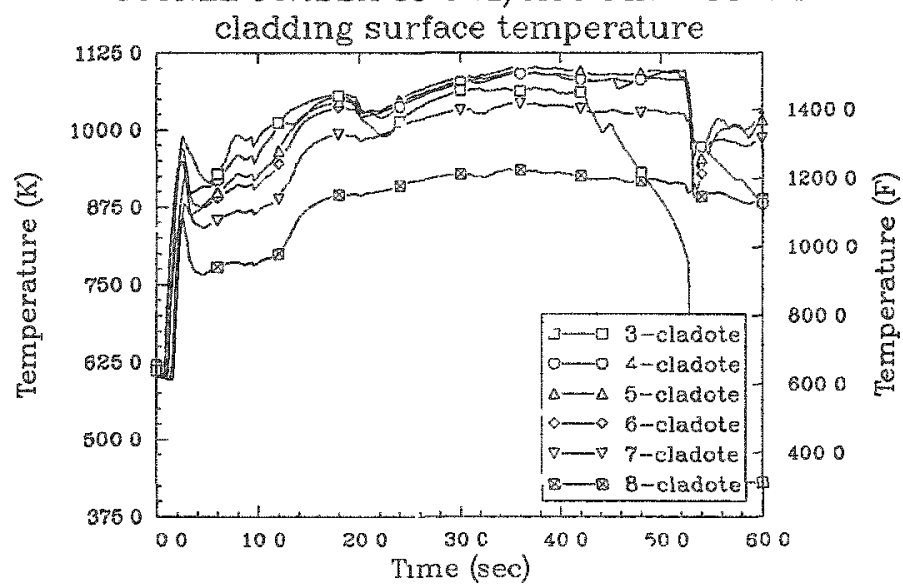

OCONEE 50\%DBA 55 GWD/MTU PIN--PF 24

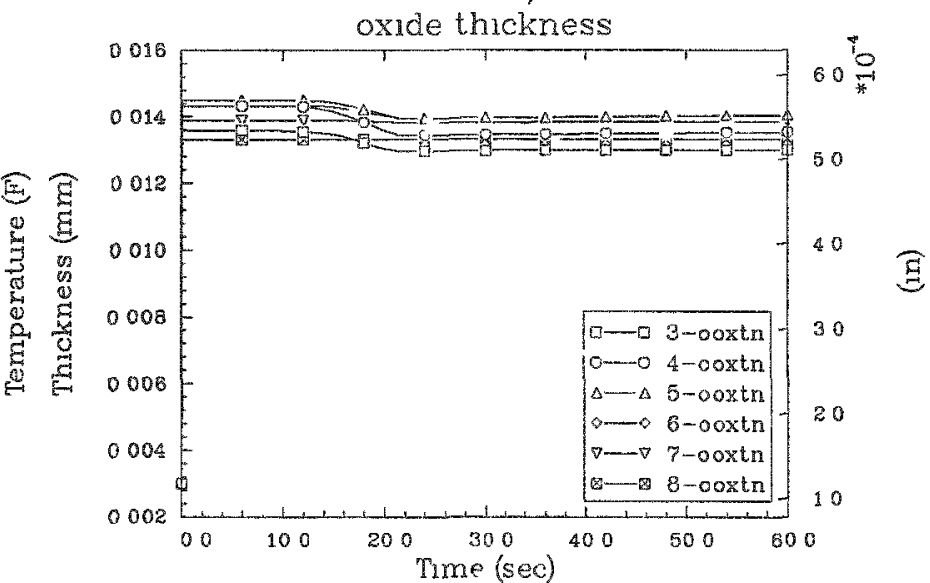



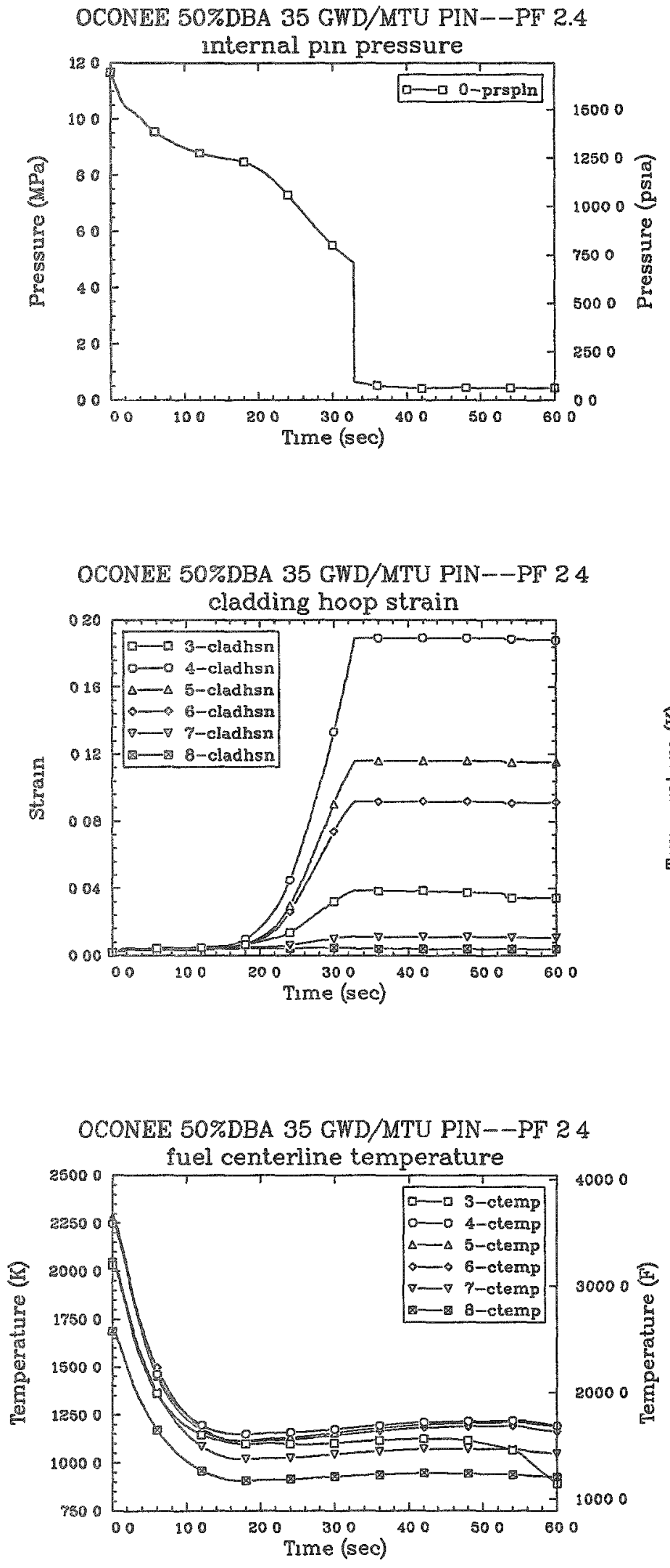

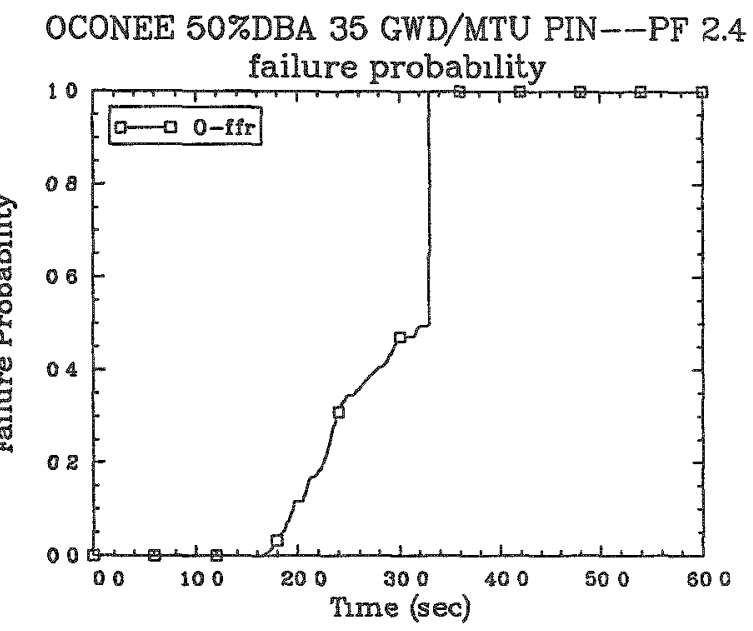

OCONEE 50\%DBA 35 GWD/MTU PIN--PF 24

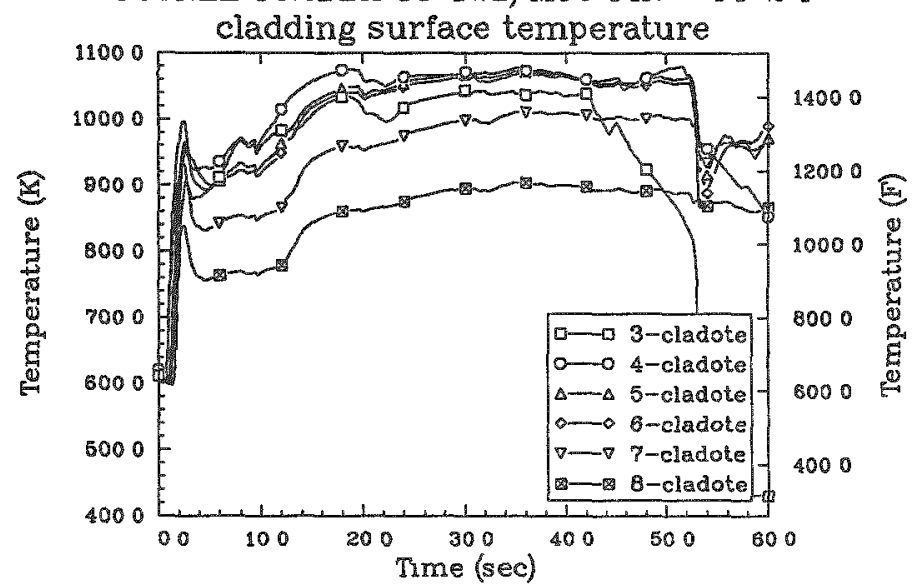

OCONEE 50\%DBA 35 GWD/MTU PIN--PF 24

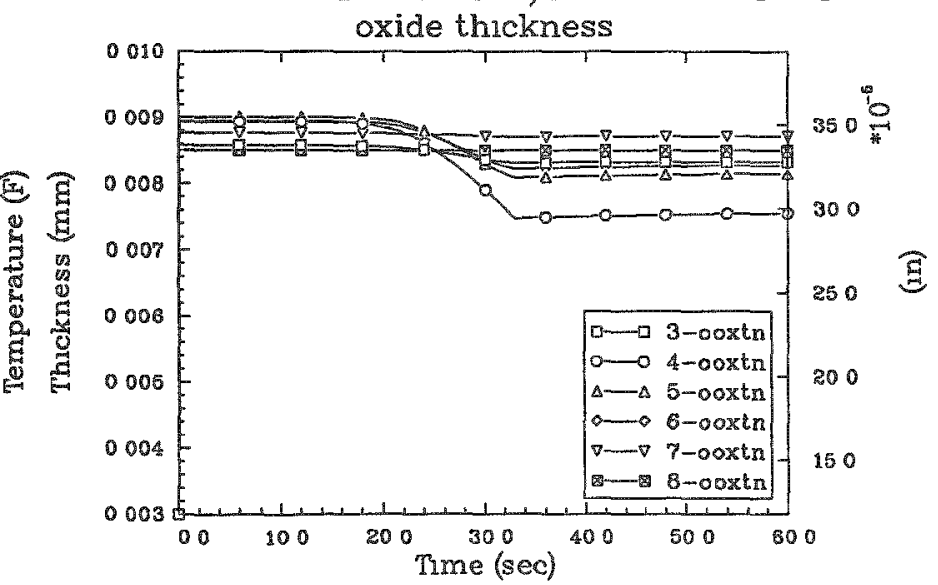



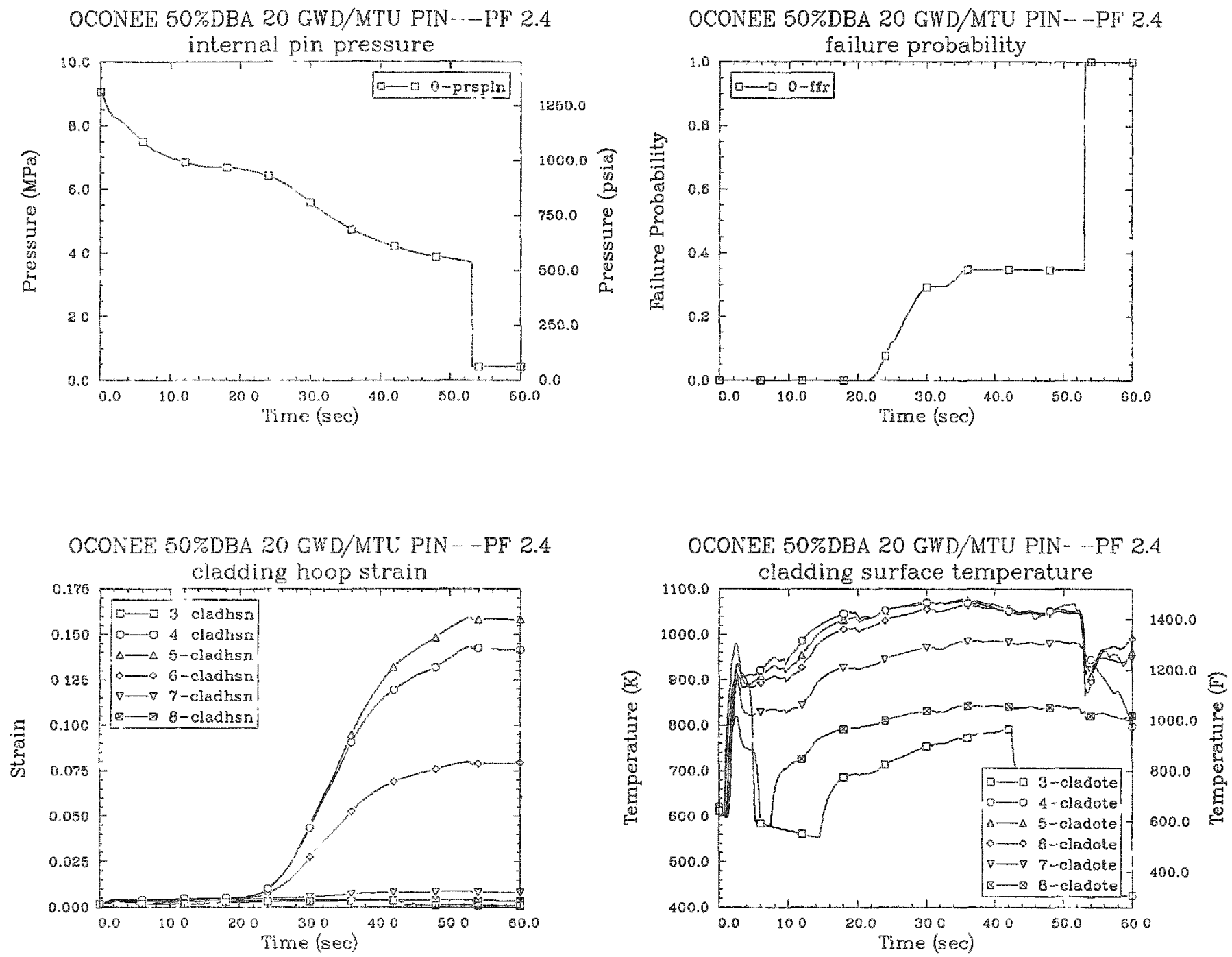

OCONEE 50\%DEA $20 \mathrm{GWD} / \mathrm{MTU}$ PIN--PF 2.4

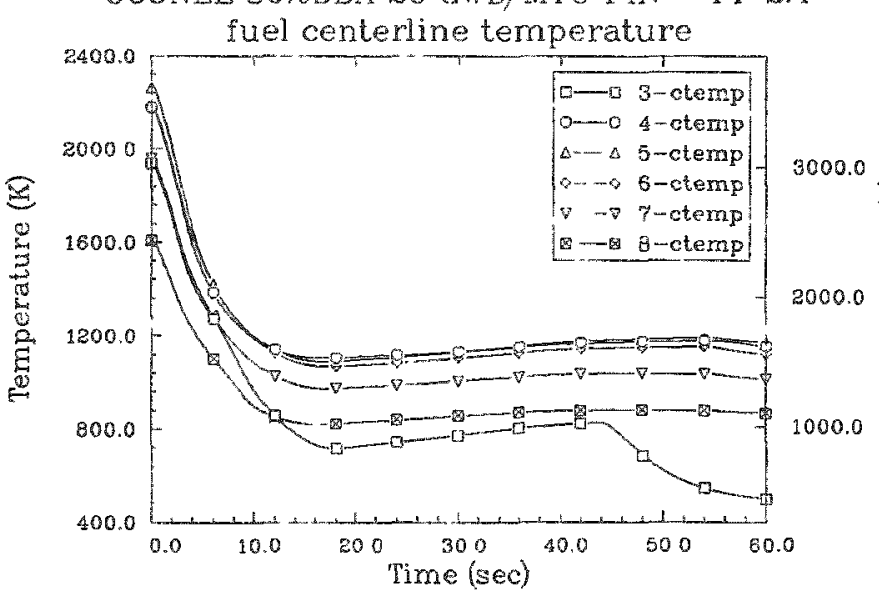

OCONEE 50\%DBA 20 GWD/MTU PIN--PF 2.4

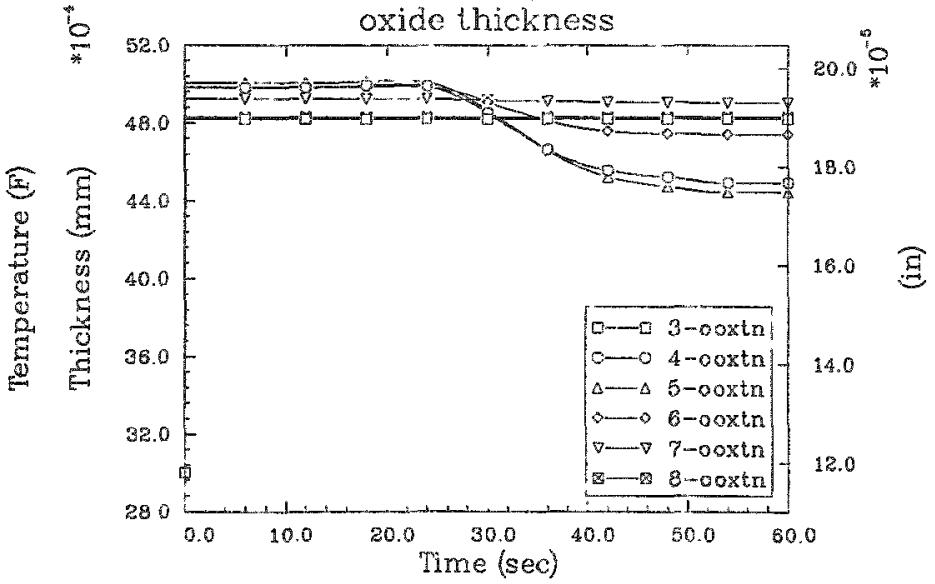



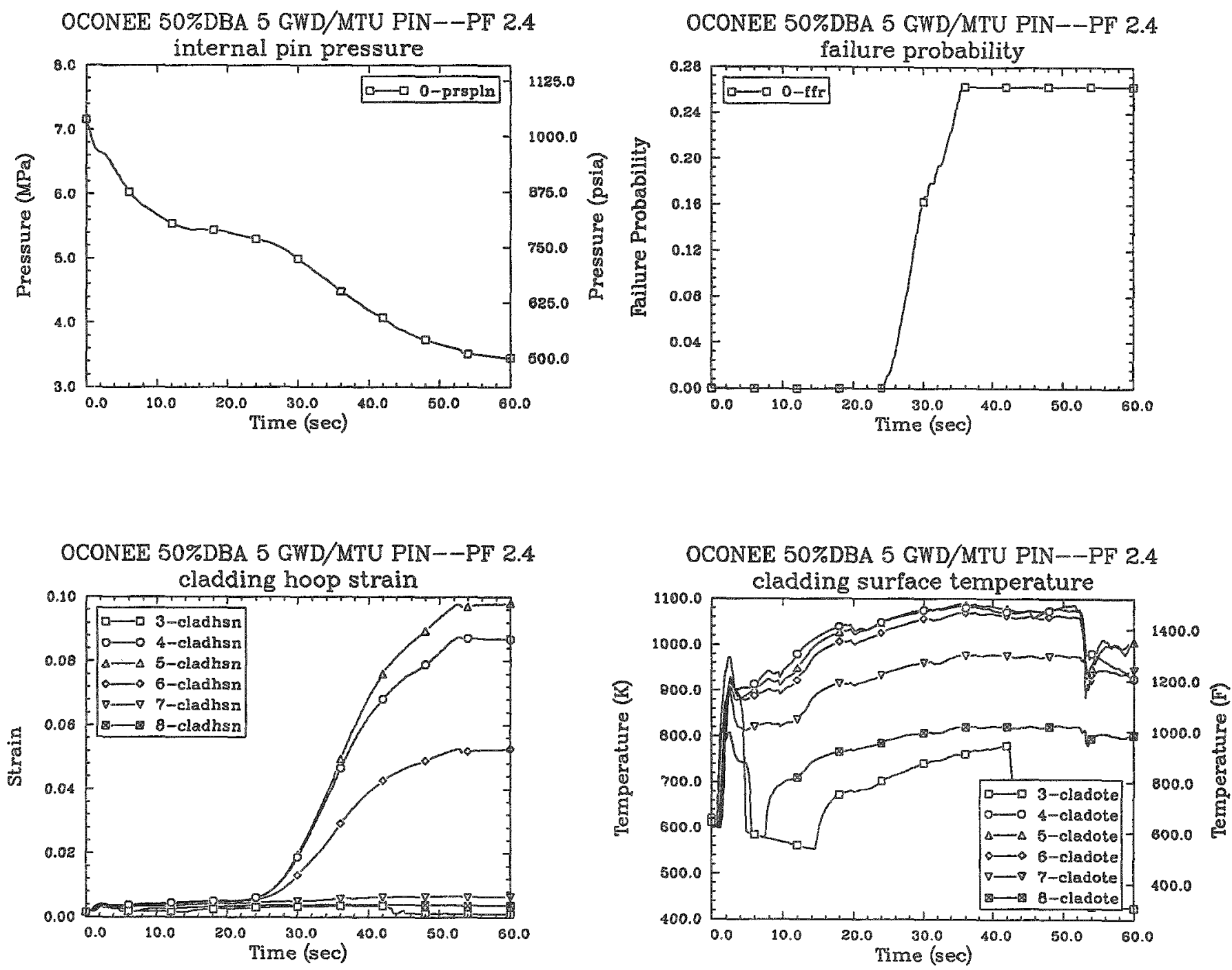

OCONEE 50\%DBA 5 GWD/MTU PIN--PF 2.4
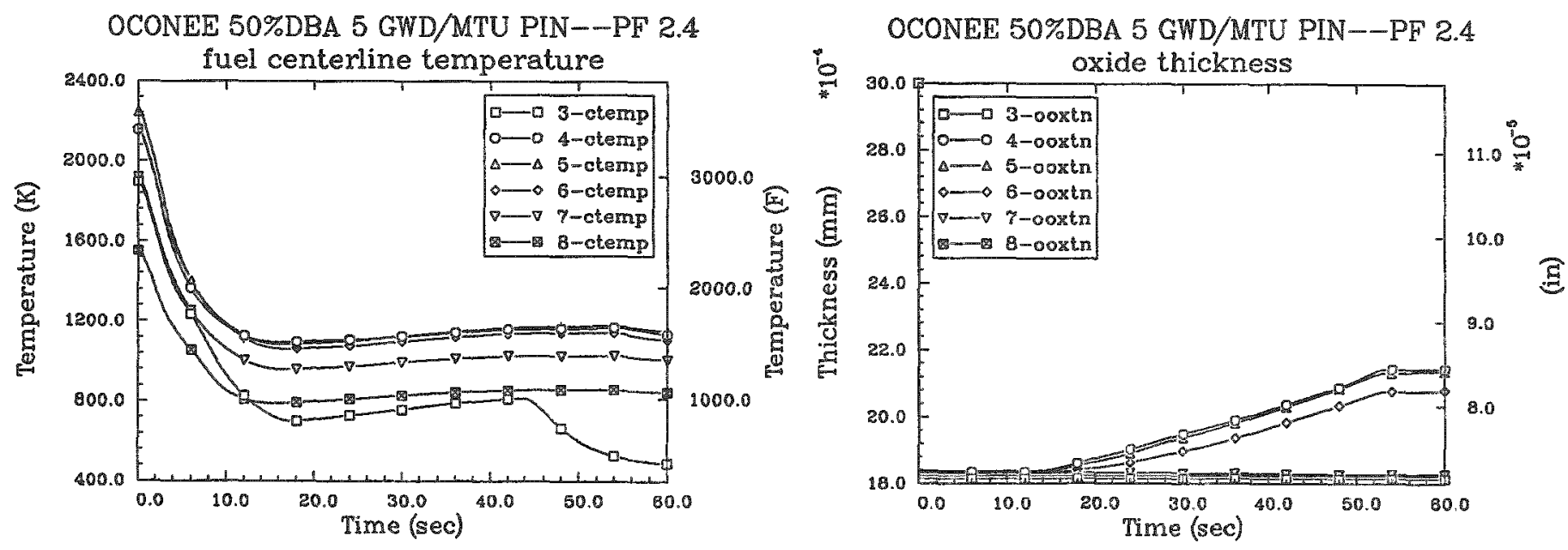
OCONEE 50\%DBA 55 GWD/MTU PIN-PF 2.2

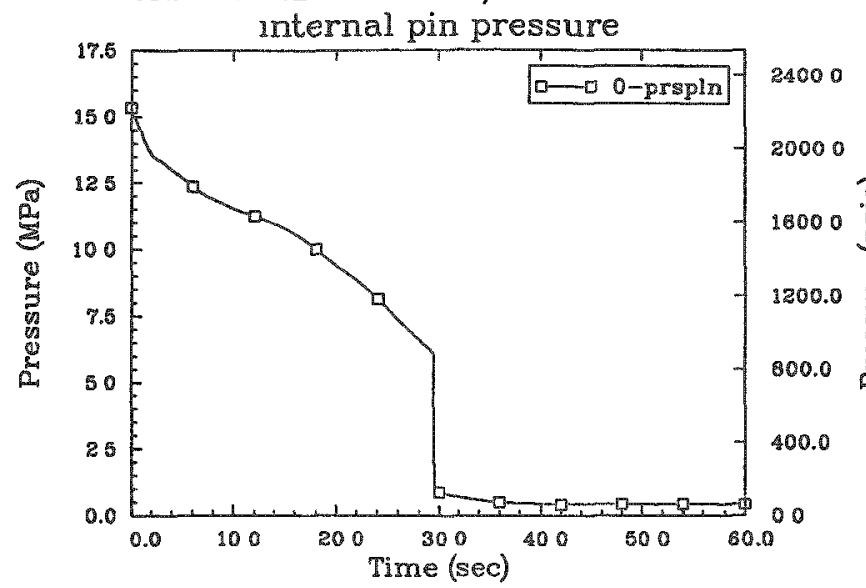

OCONEE 50\%DBA 55 GWD/MTU PIN--PF 2.2

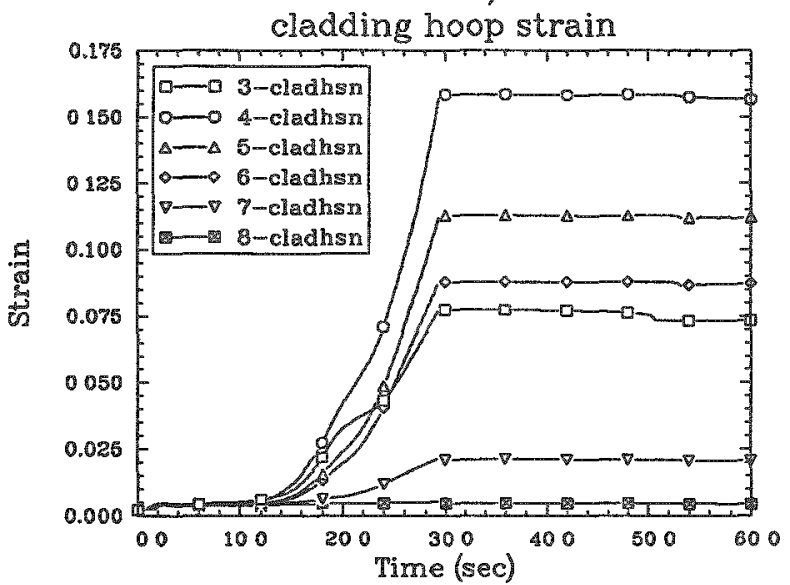

OCONEE 50\%DBA 55 GWD/MTU PIN--PF 2.2

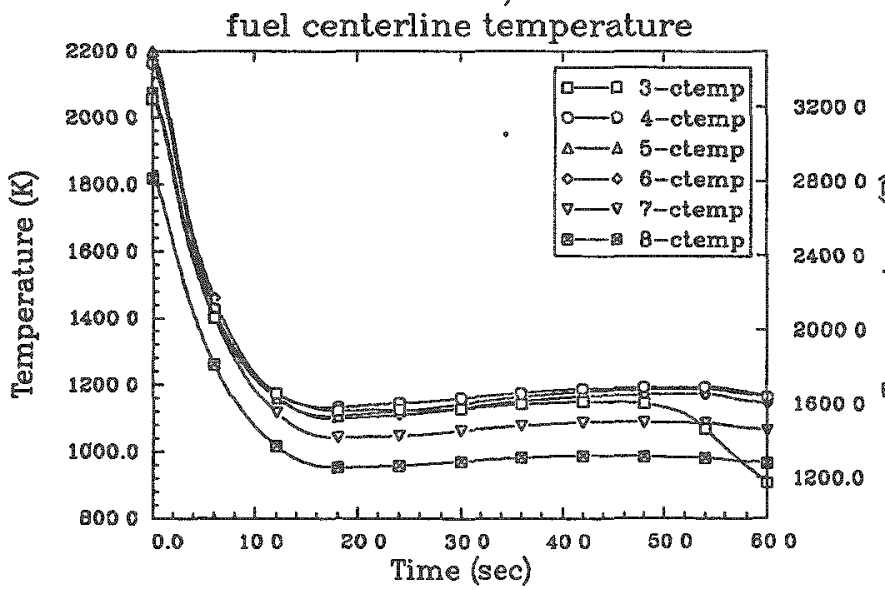

OCONEE 50\%DBA 55 GWD/MTU PIN--PF 2.2 failure probability

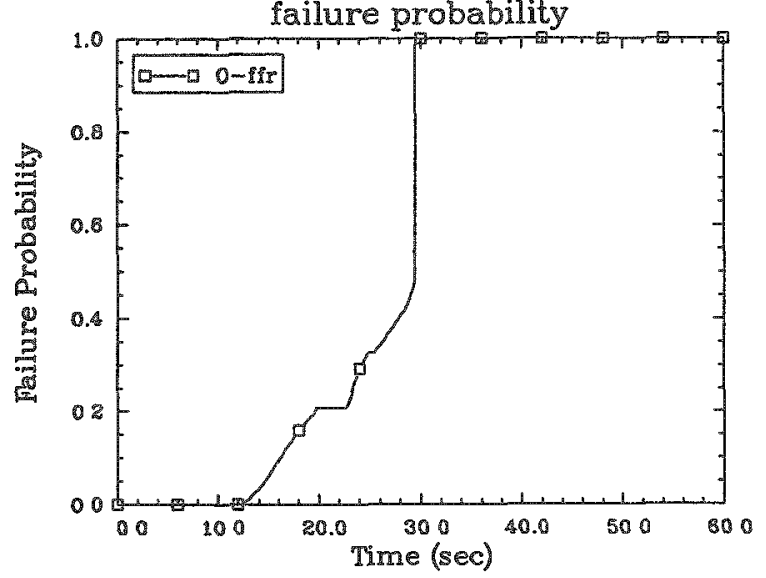

OCONEE 50\%DBA 55 GWD/MTU PIN--PF 2.2

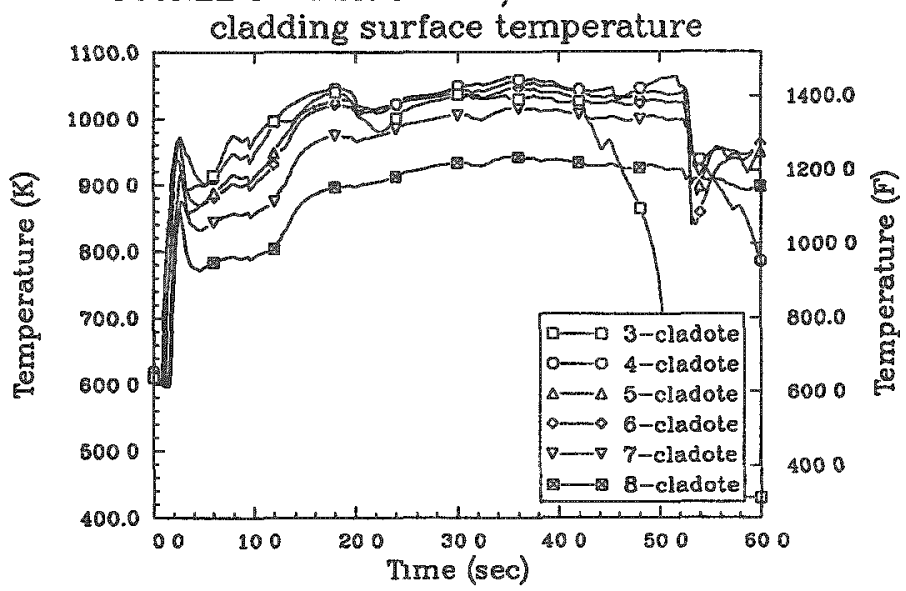

OCONEE 50\%DBA 55 GWD/MTU PIN--PF 2.2

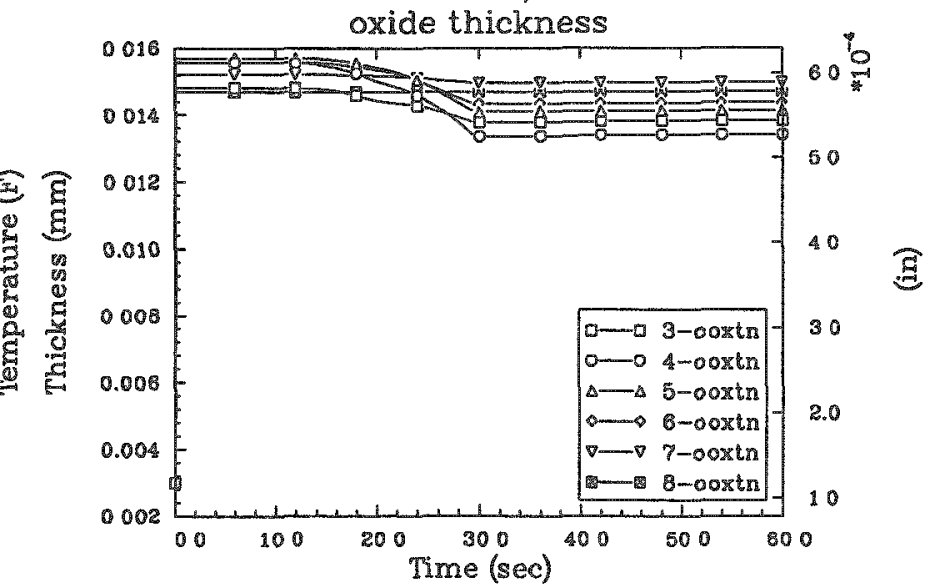



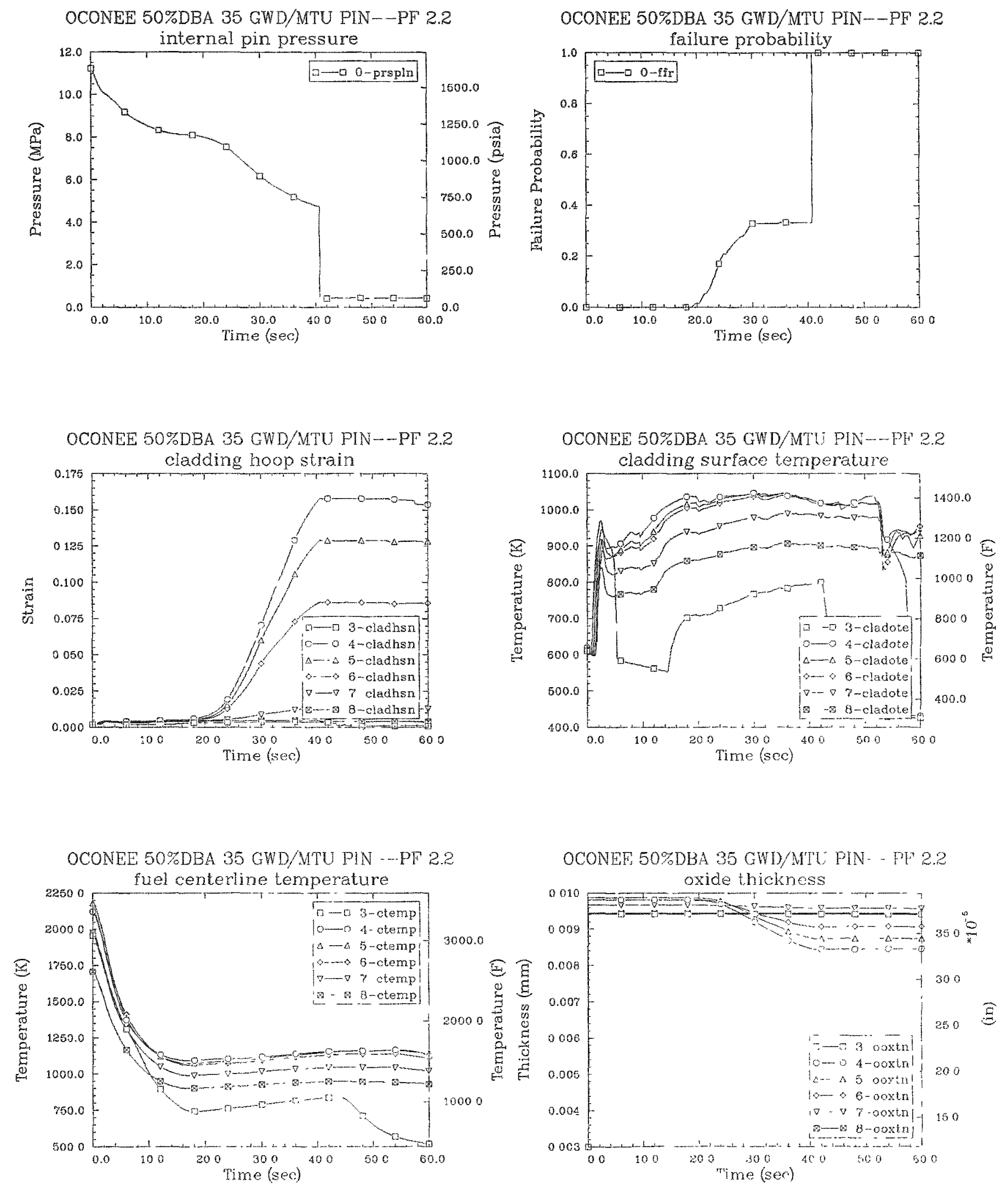

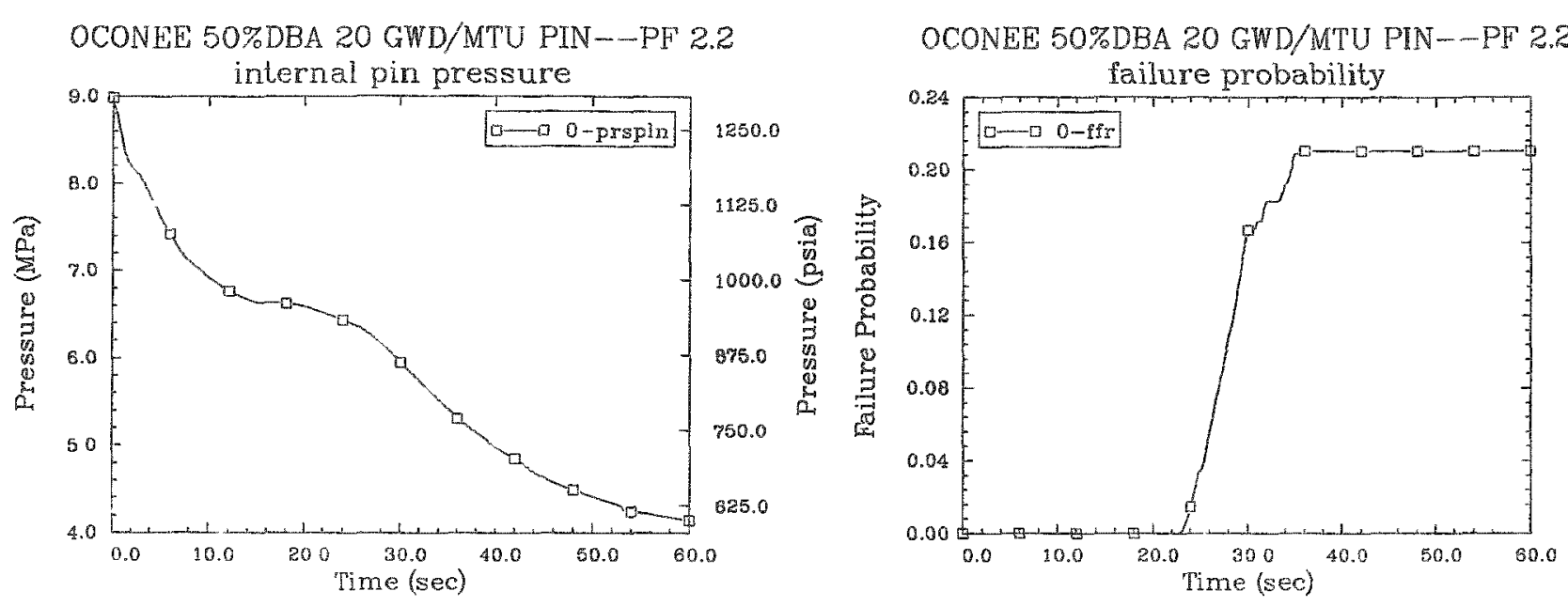

OCONEE 50\%DBA 20 GWD/MTU PIN--PF 2.2

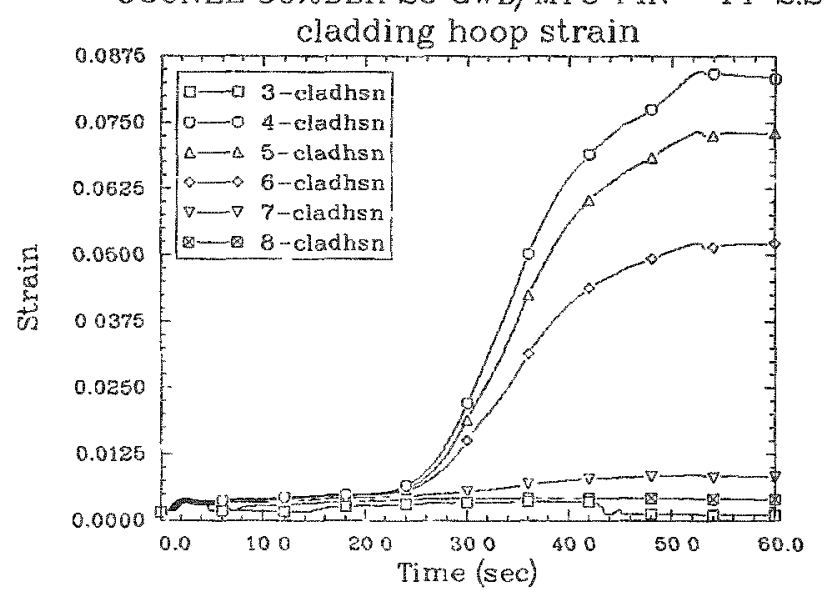

OCONEE 50\%DBA 20 GWD/MTU PIN--PF 2.2

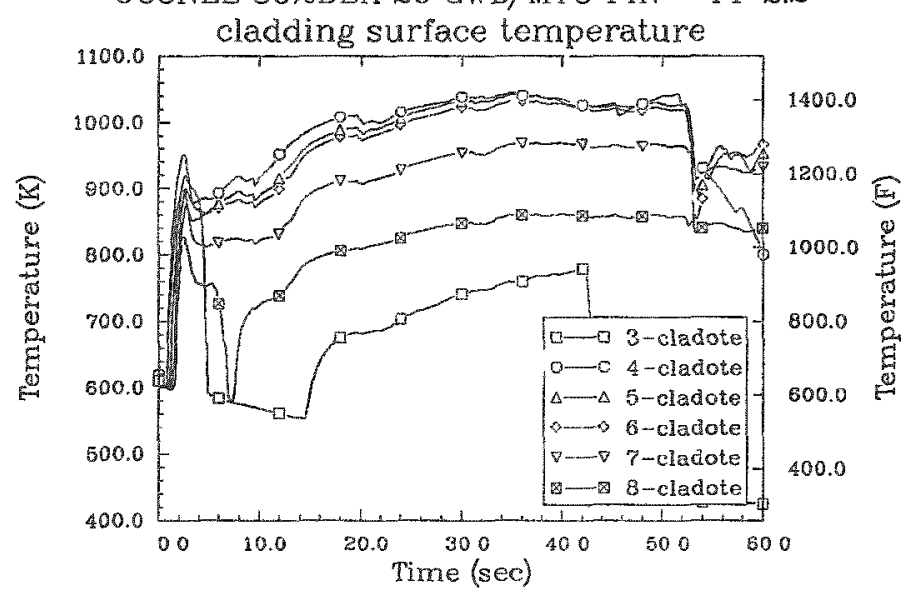

OCONEE 50\%DBA $20 \mathrm{GWD} / \mathrm{MTU}$ PIN--PF 2.2
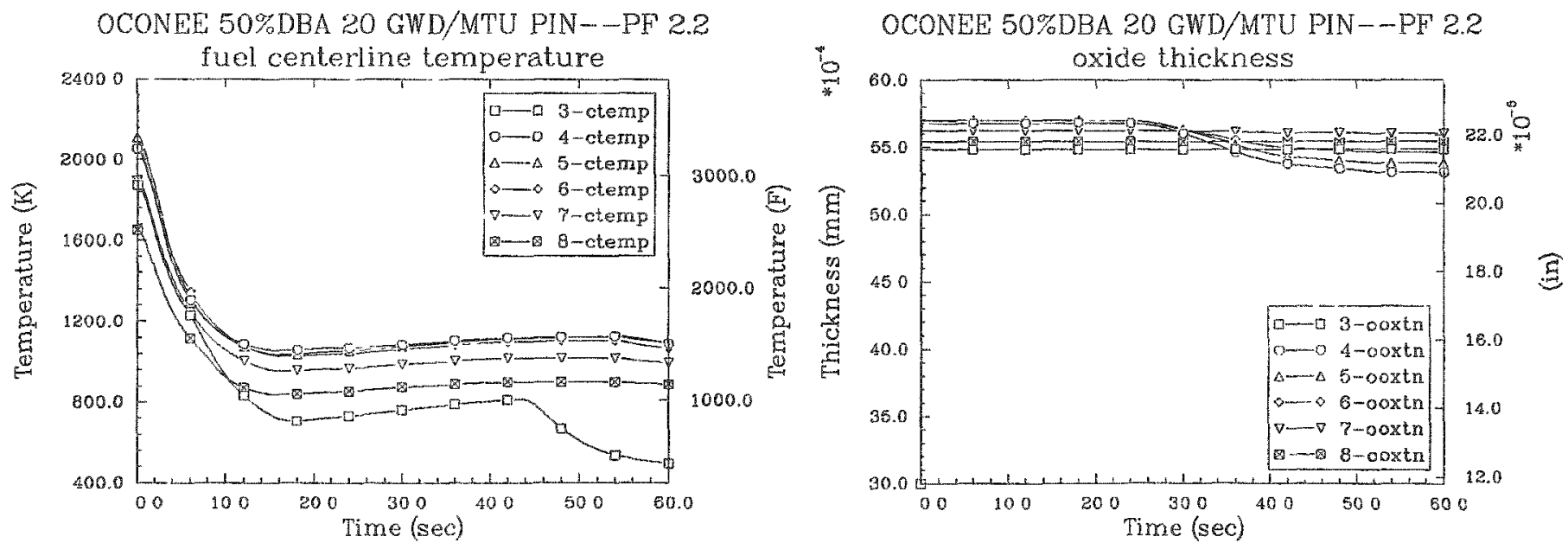

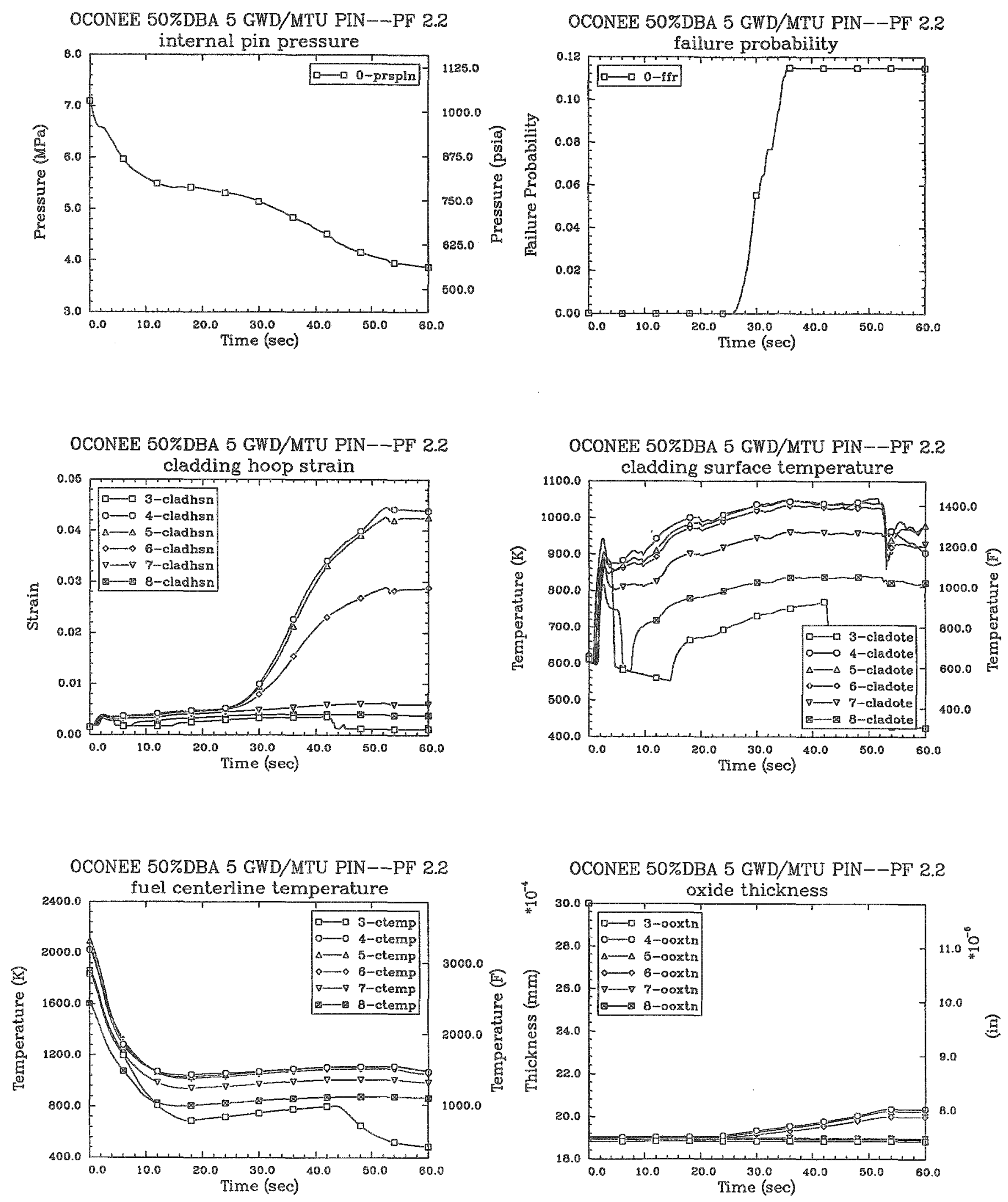
OCONEE 50\%DBA 55 GWD/MTU PIN--PF 2.0
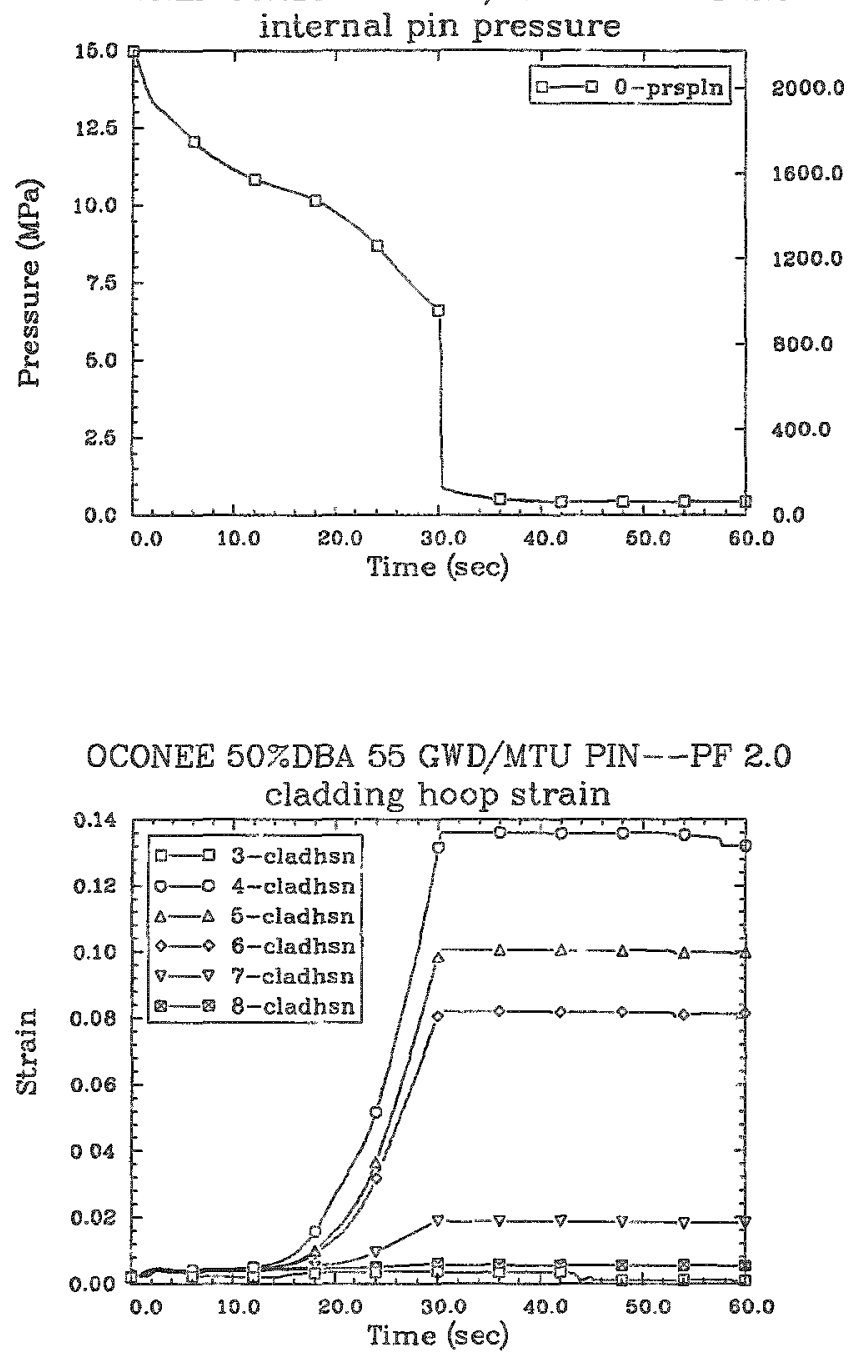

OCONEE 50\%DBA 55 GWD/MTU PIN--PF' 2.0

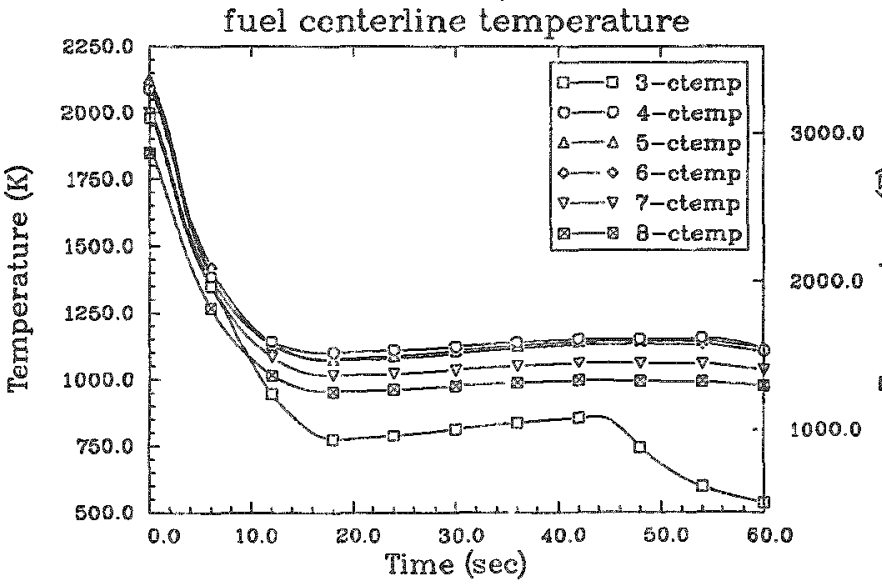

OCONEE 50\%DBA 55 GWD/MTU PIN--PF 2.0 failure probability

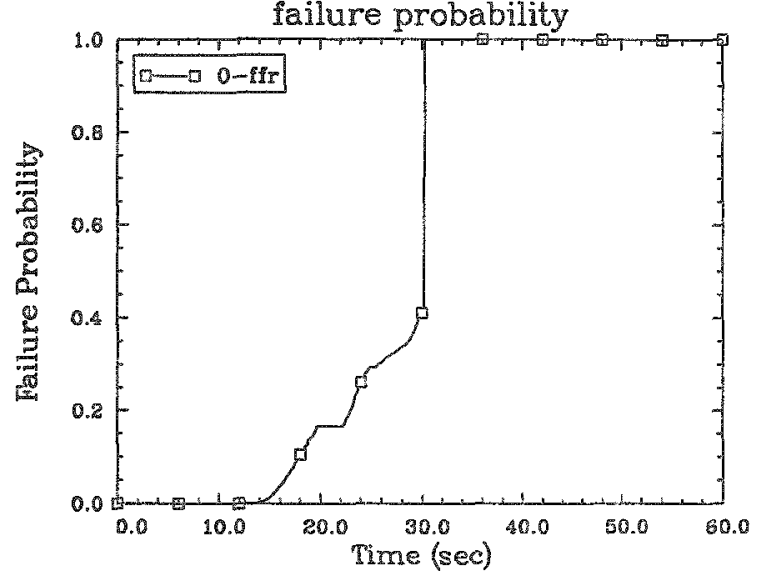

OCONEE 50\%DBA 55 GWD/MTU PIN-PF 2.0

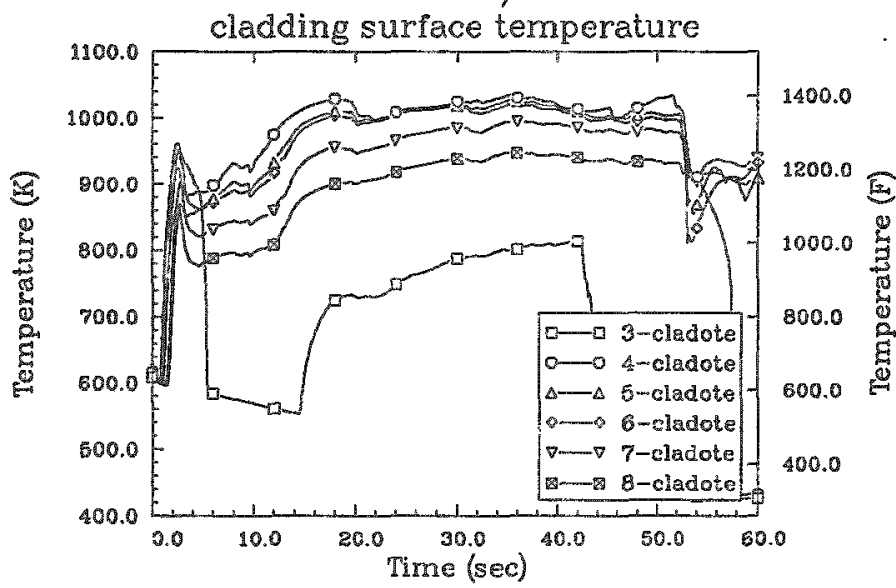

OCONEE 50\%DBA 55 GWD/MTU PIN--PF 2.0

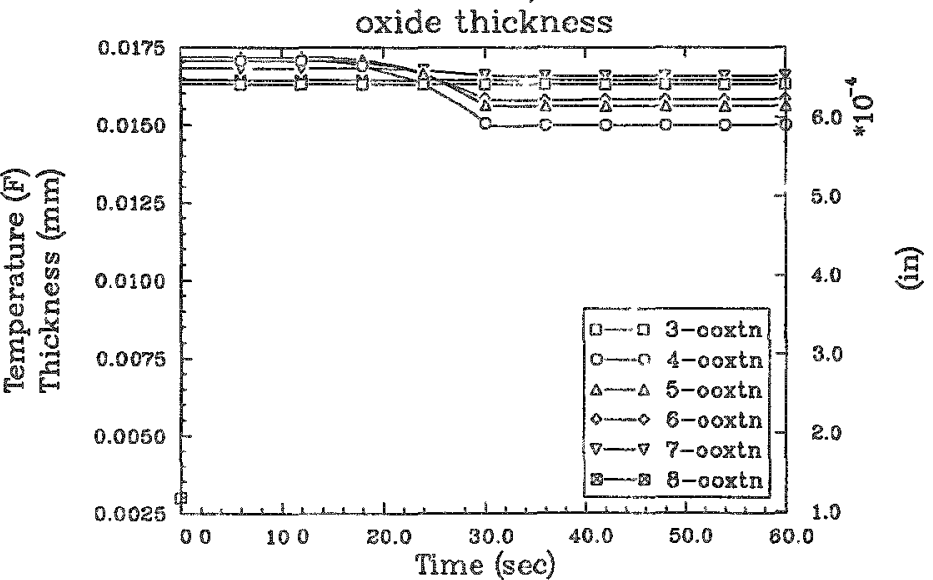



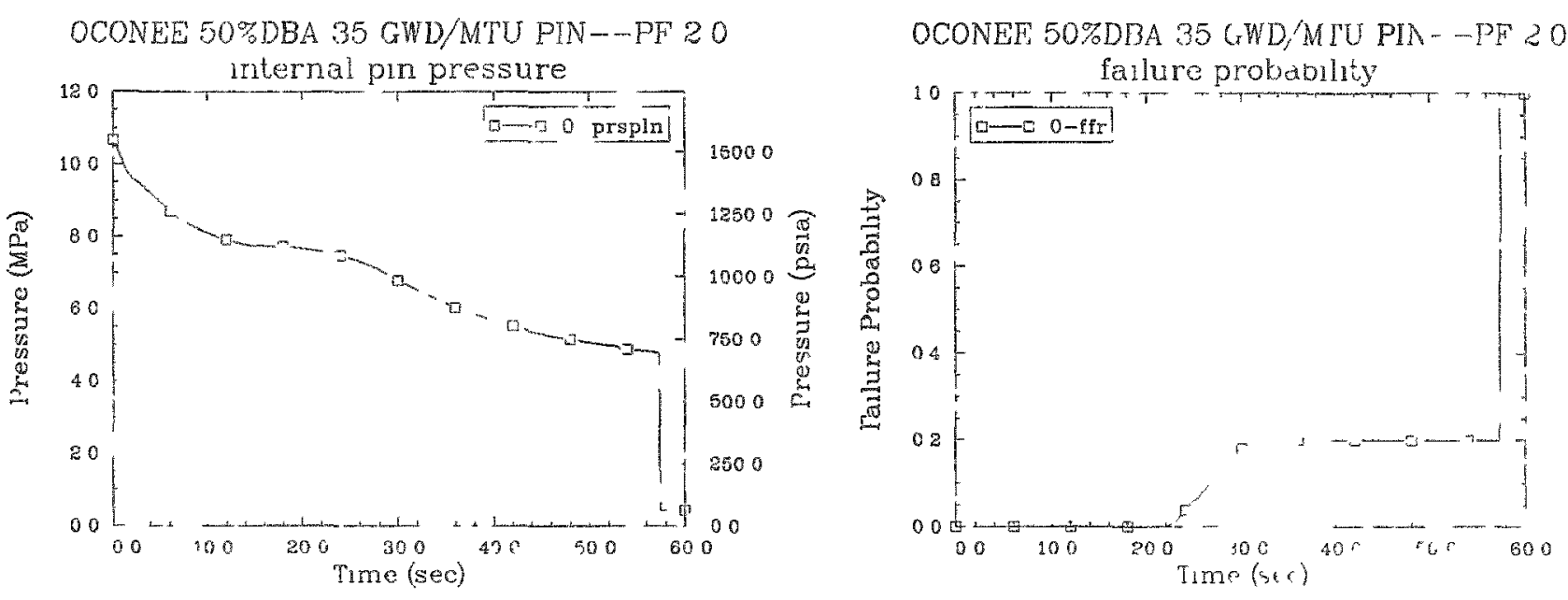

OCONEF 50\%DBA 3', GWD,AOL PIN--PF 20

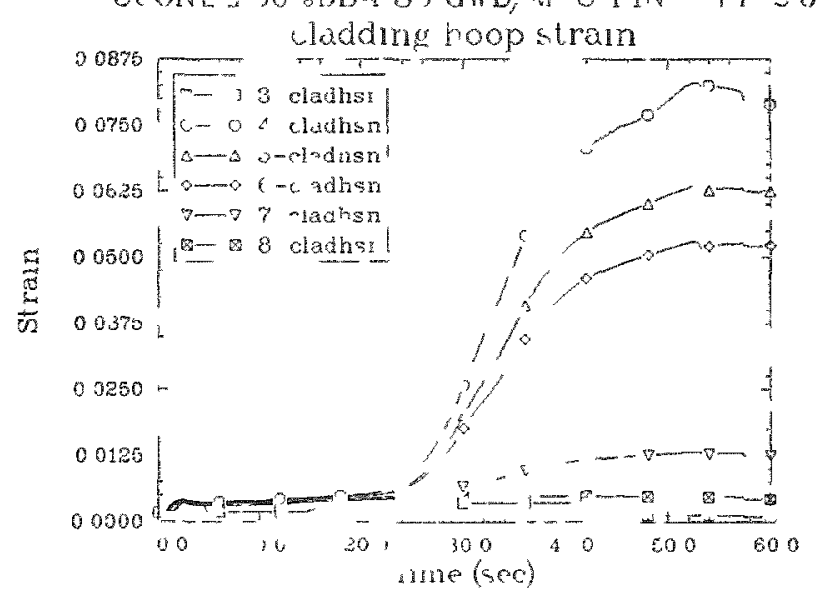

OCONEF. 50\%DEI 35 (MI) \& I PIV - P 20

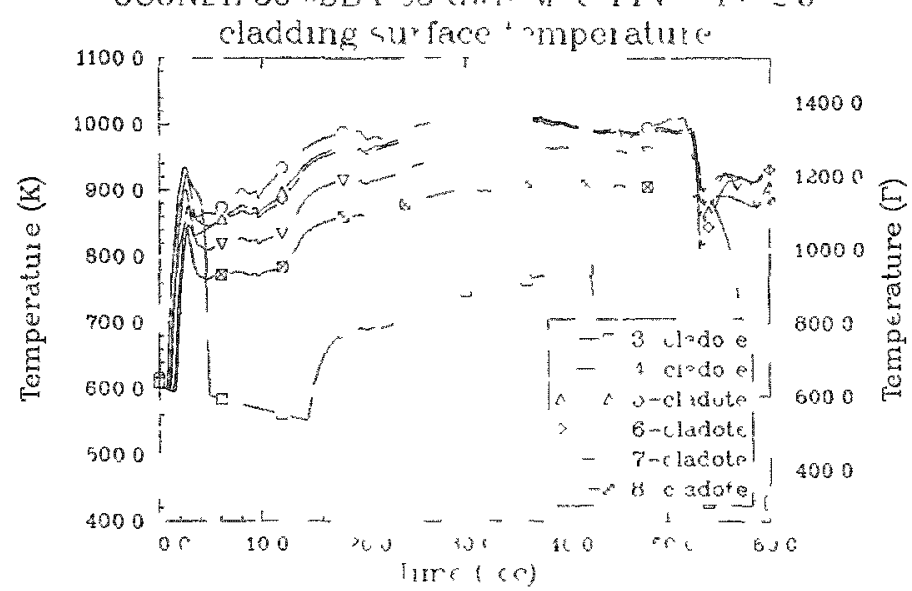

OCONEL 50\%DHA $35 \mathrm{GWD} / \mathrm{MUU}$ PIN--PF 20

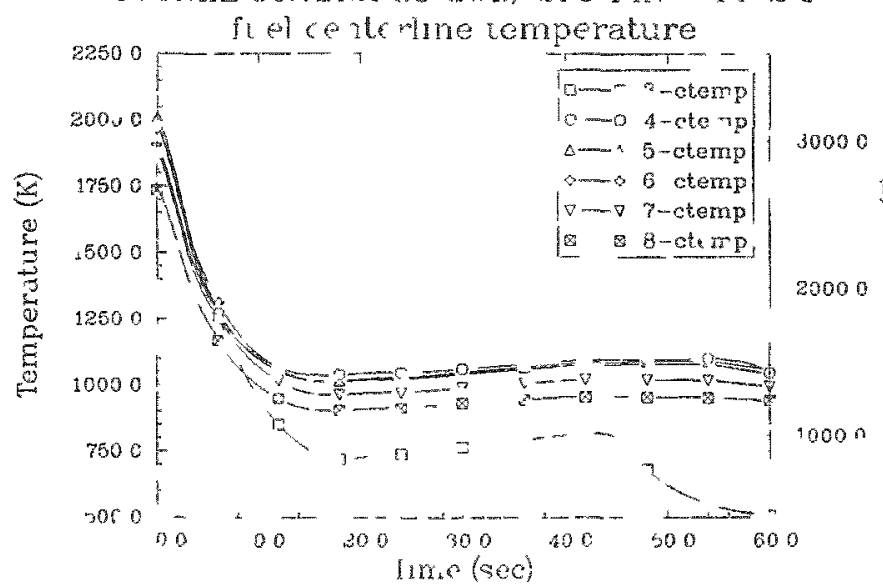

OCONFF 50\%DBA $35 \mathrm{CHD} M$, PIV PP 20

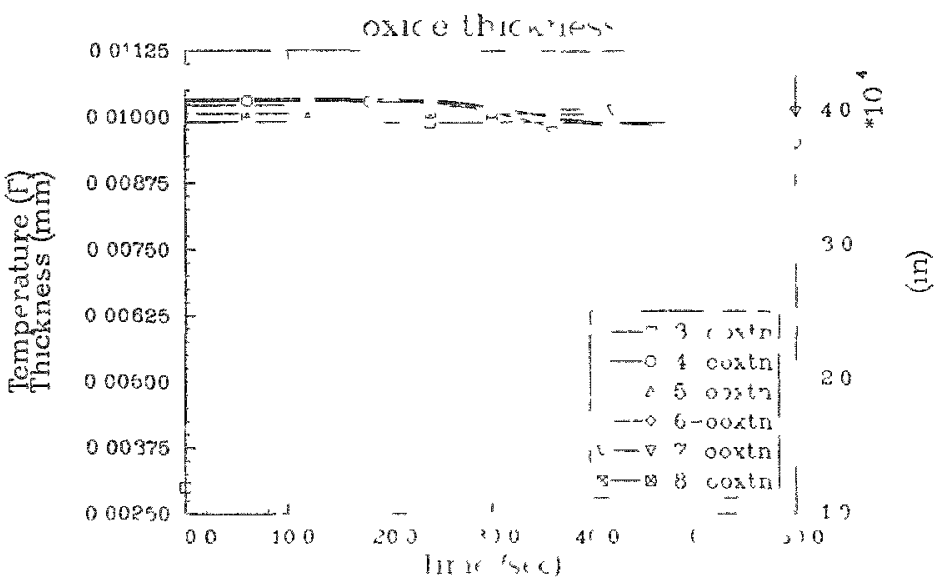



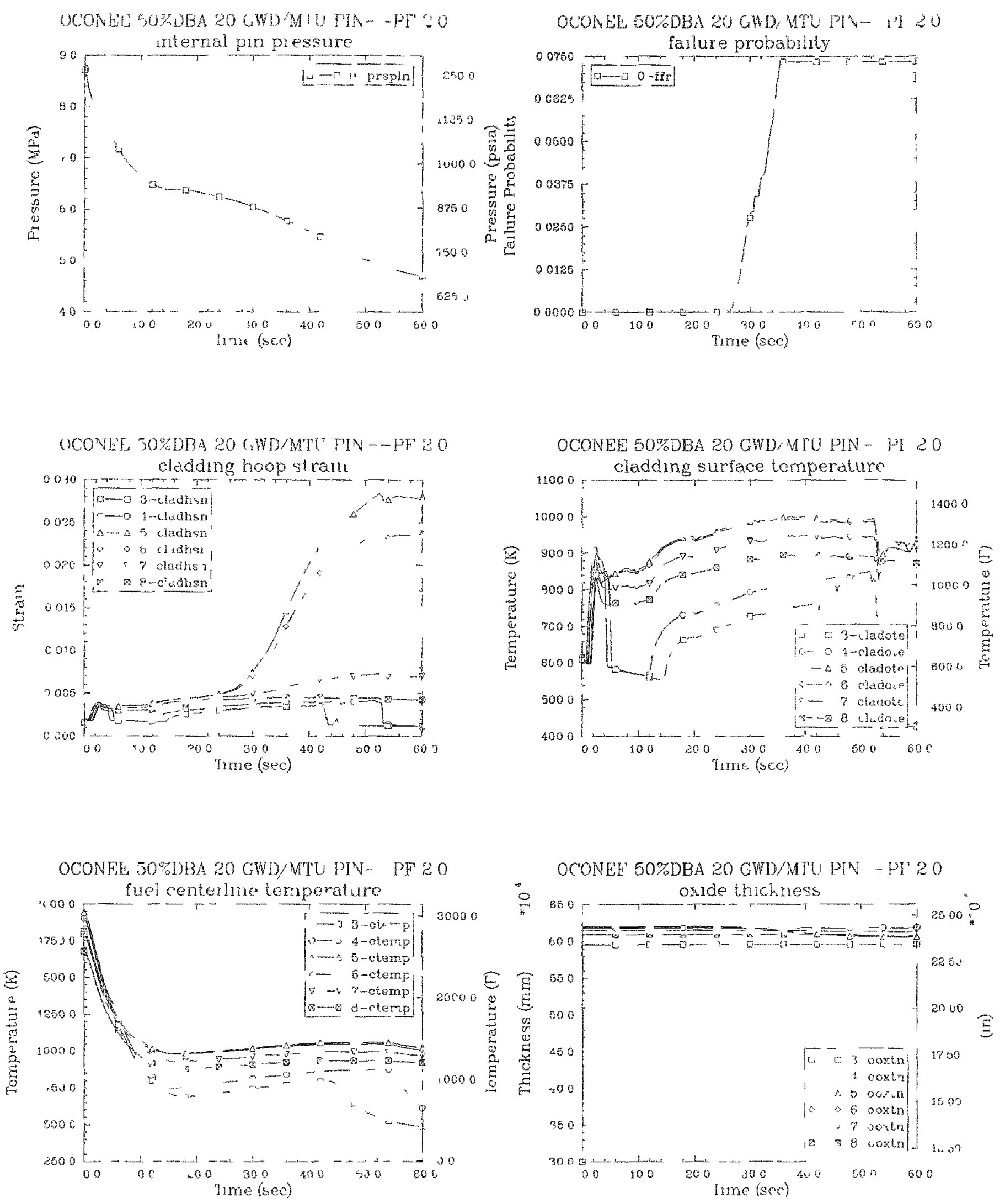
OCONEE 50\%DBA 5 GWD/MTU PIN--PF 2.0

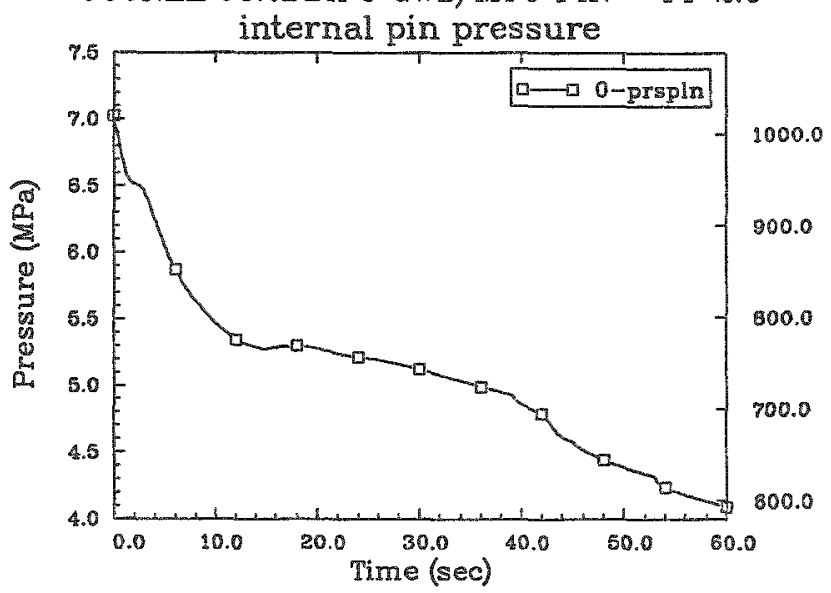

OCONEE 50\%DBA 5 GWD/MTU PIN--PF 2.0

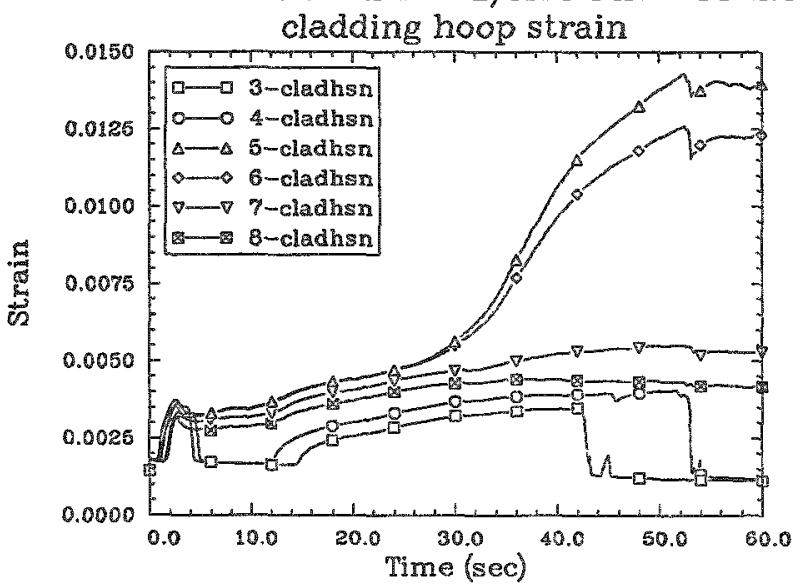

OCONEE 50\%DBA 5 GWD/MTU PIN--PF 2.0

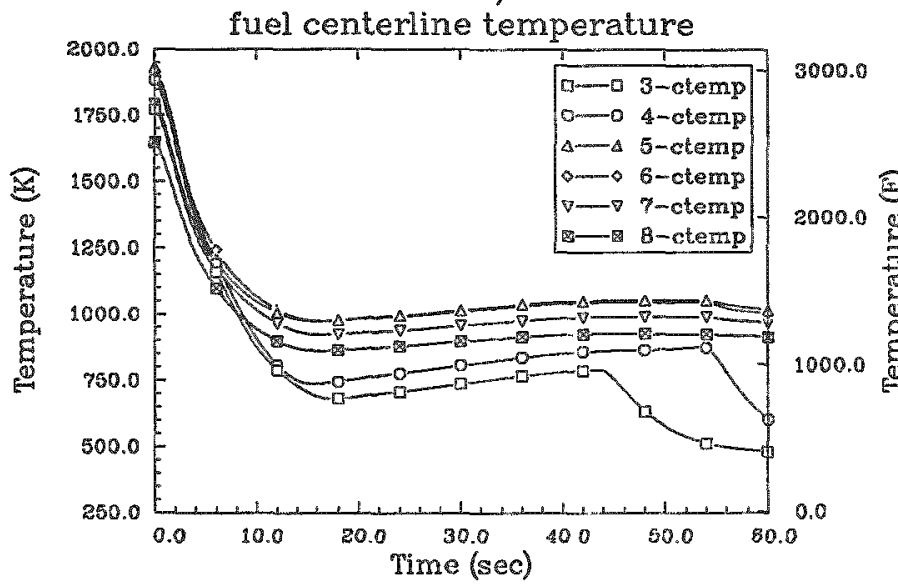

OCONEE 50\%DBA 5 GWD/MTU PIN--PF 2.0 failure probability

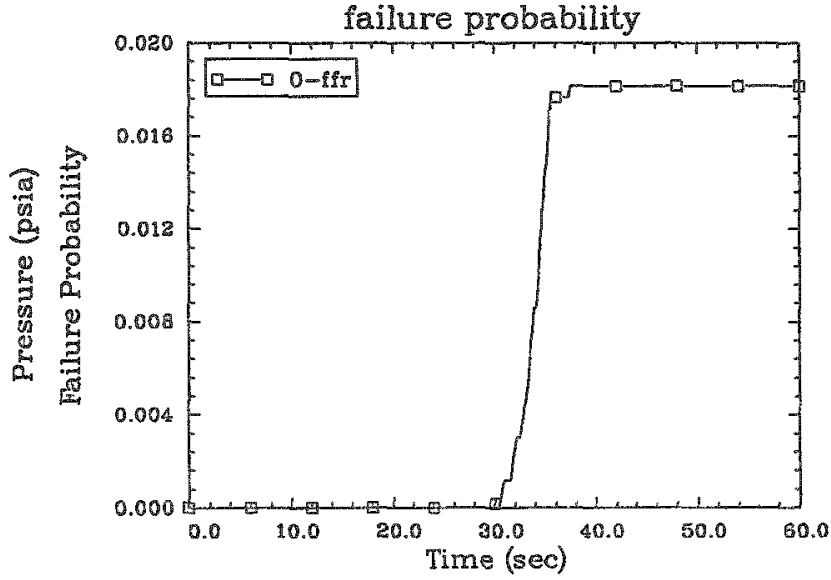

OCONEE 50\%DBA 5 GWD/MTU PIN--PF 2.0 cladding surface temperature

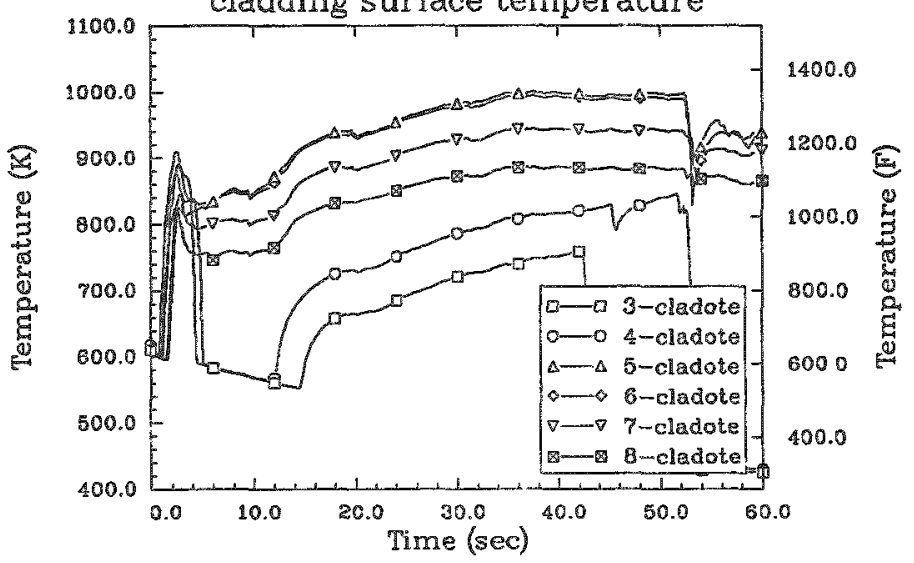

OCONEE 50\%DBA 5 GWD/MTU PIN--PF 2.0

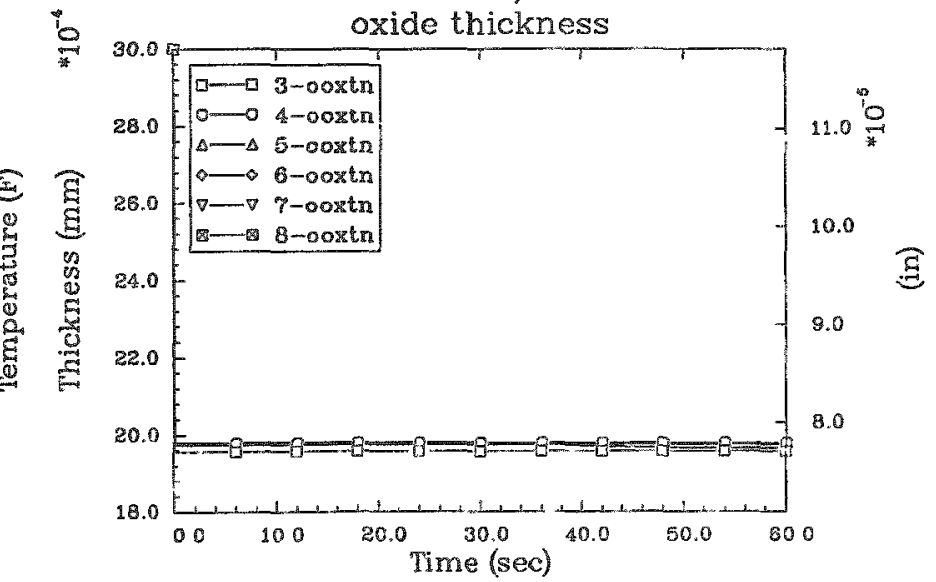




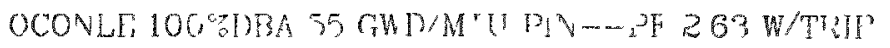

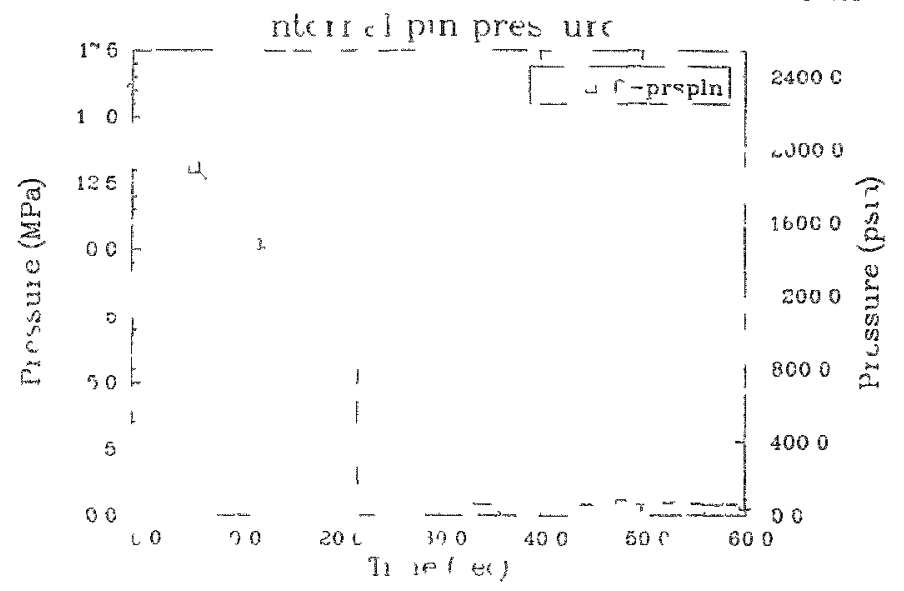

OCONELA $100 \%$ DBA 55 GWD/MIL PN - PH $203 \mathrm{~W} /$ TRIP

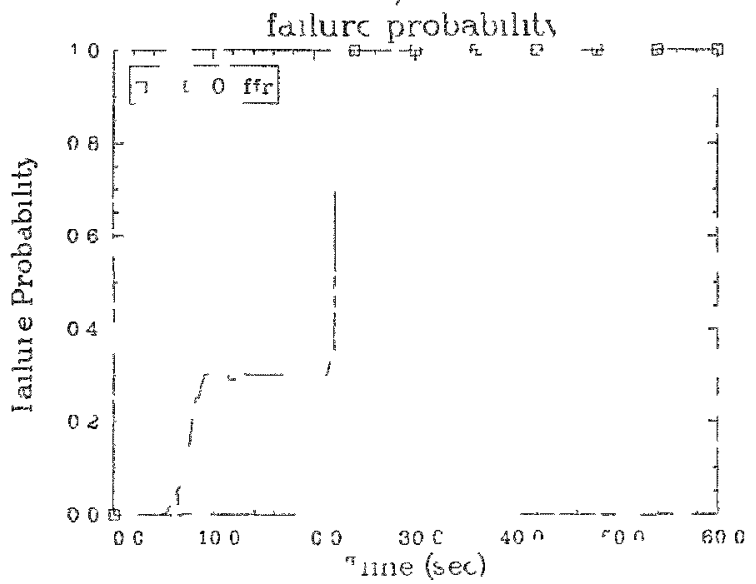

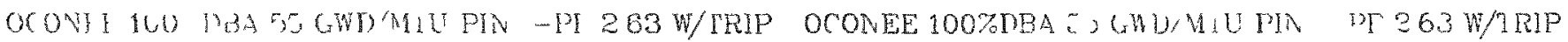
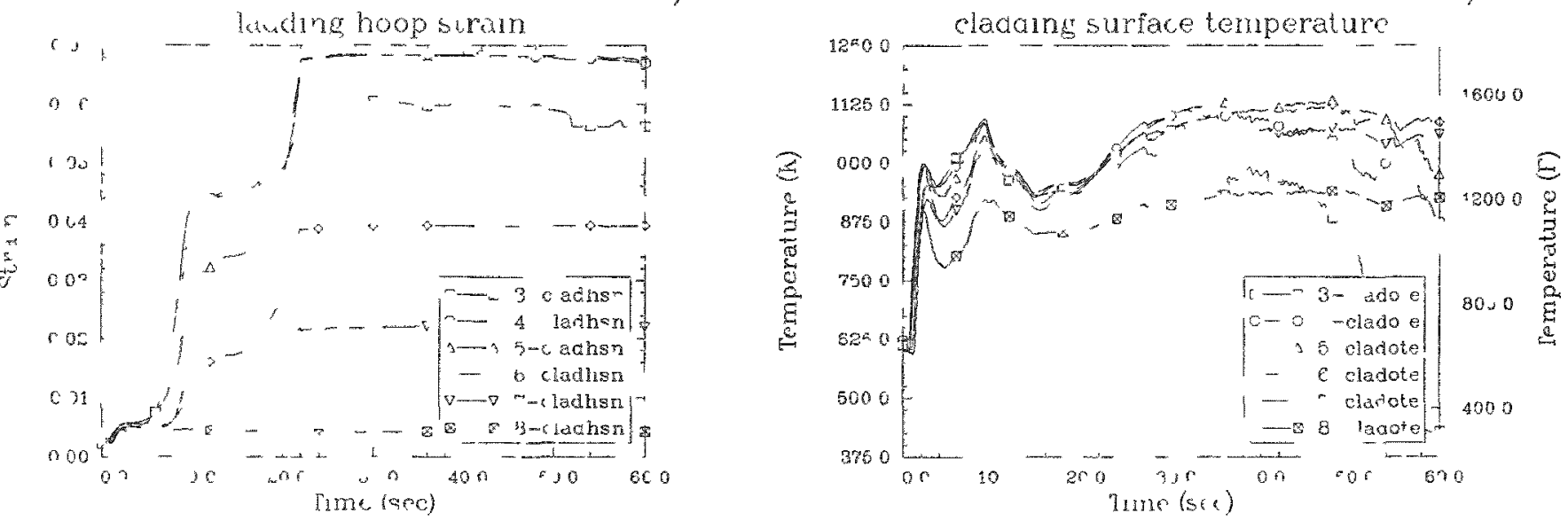

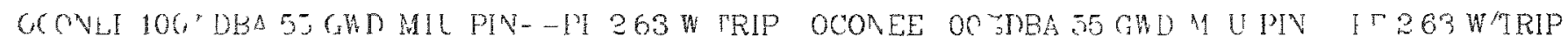
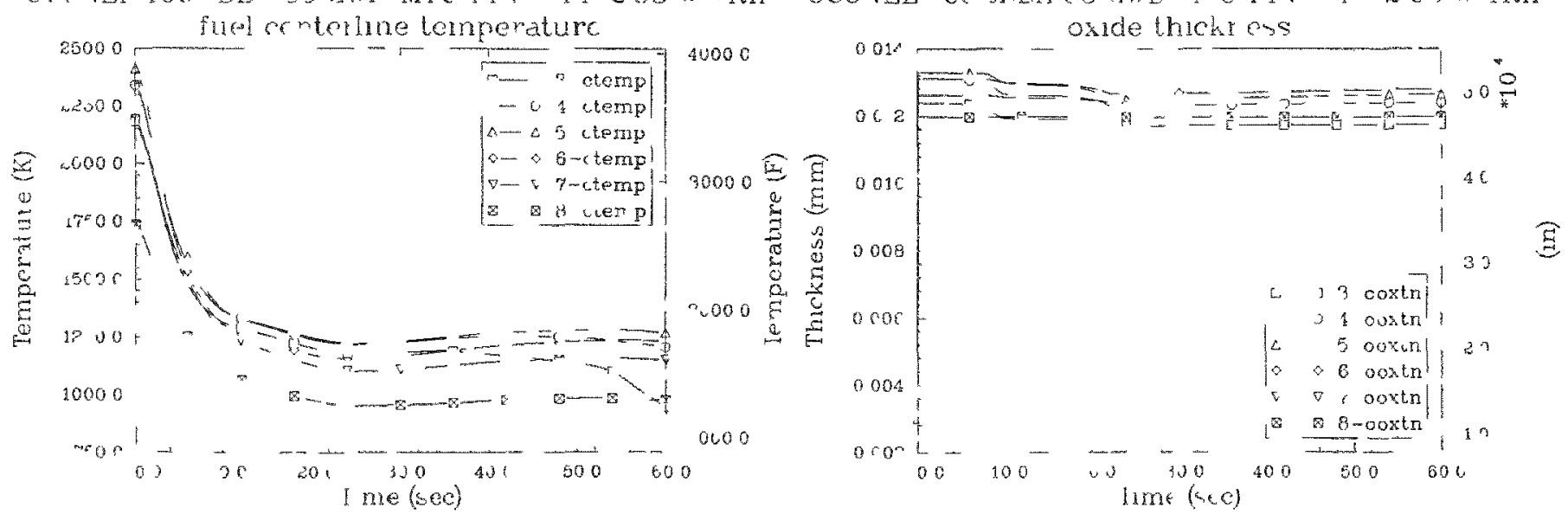

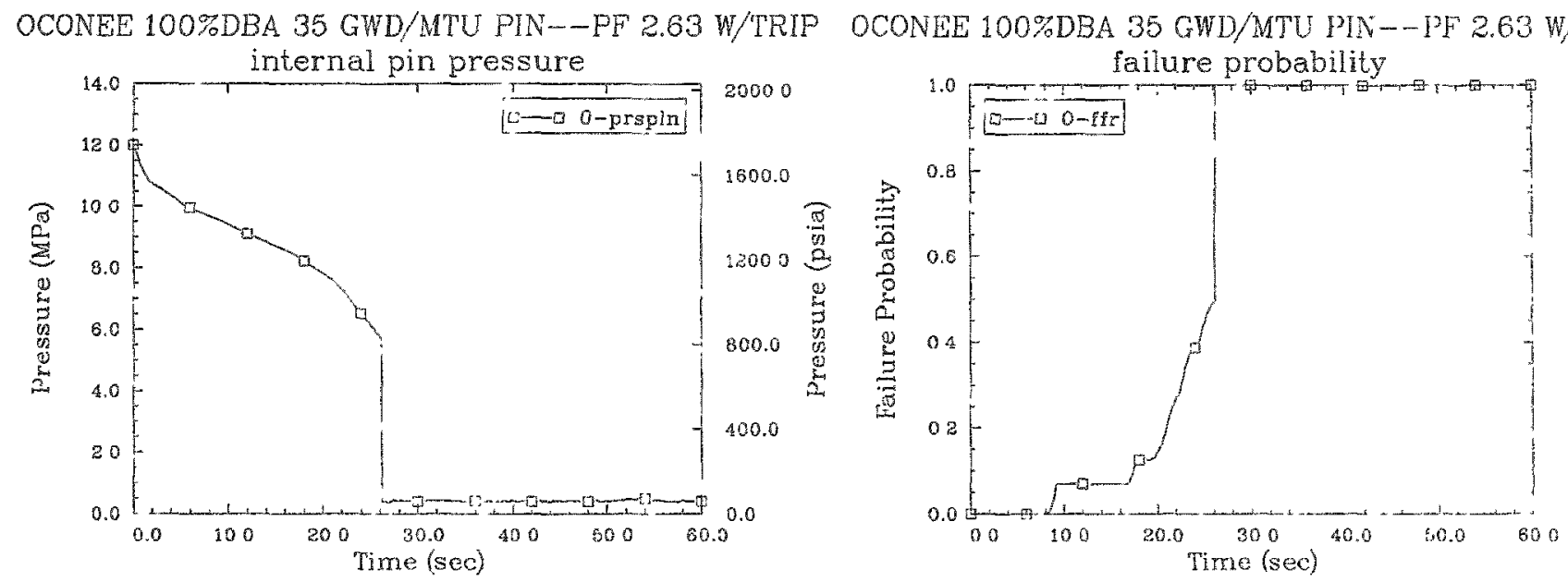

OCONEE 100\%DBA 35 GWD/MTU PIN--PF 2.63 W/TRIP cladding hoop strain
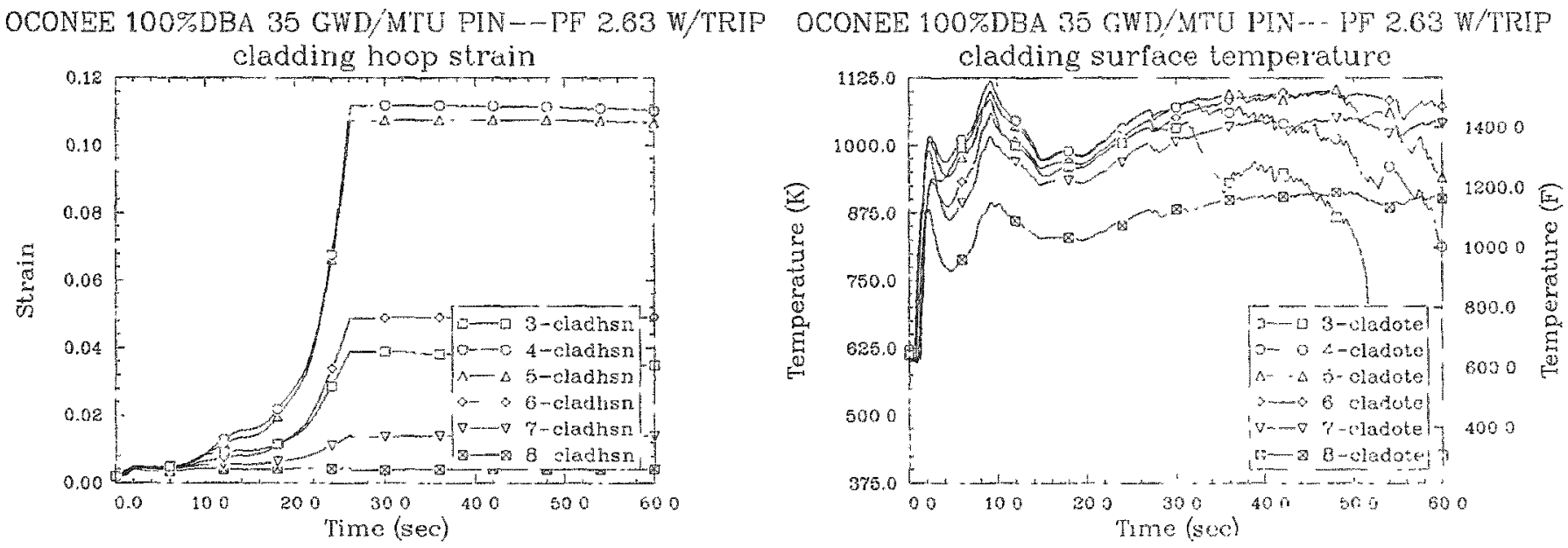

OCONEE 100\%DBA 35 GWD/MTU PIN--PF 2.63 W/TRIP

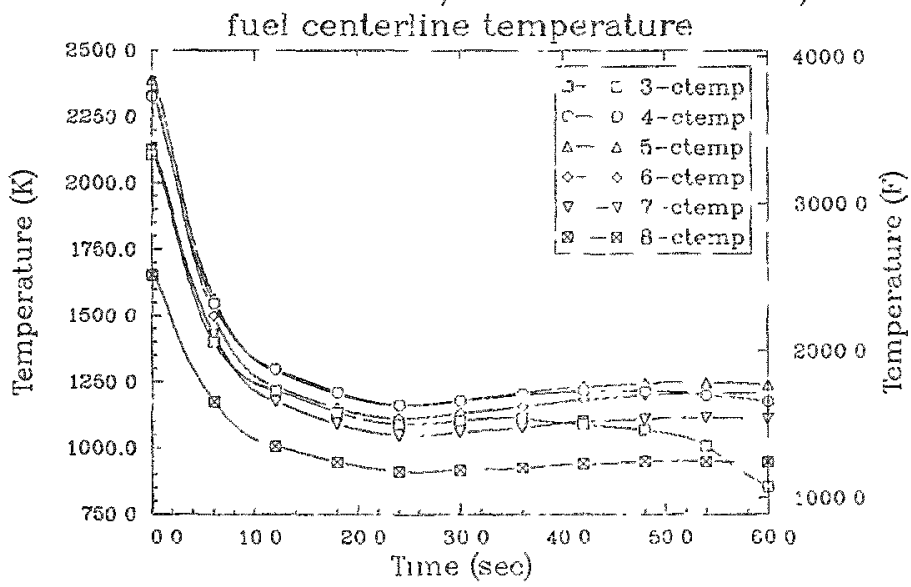

OCONEE 100\%DBA 35 GWD/MTU PIN I2 263 WTRLP

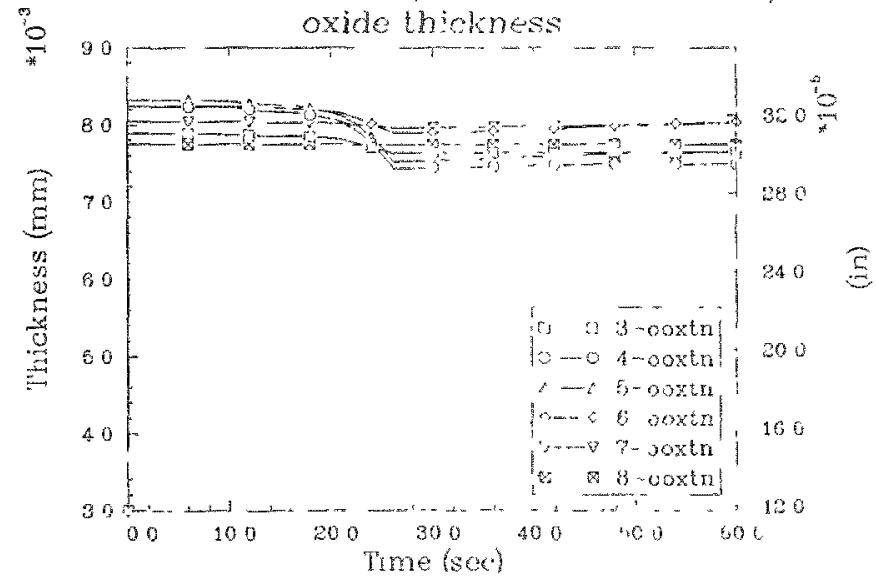



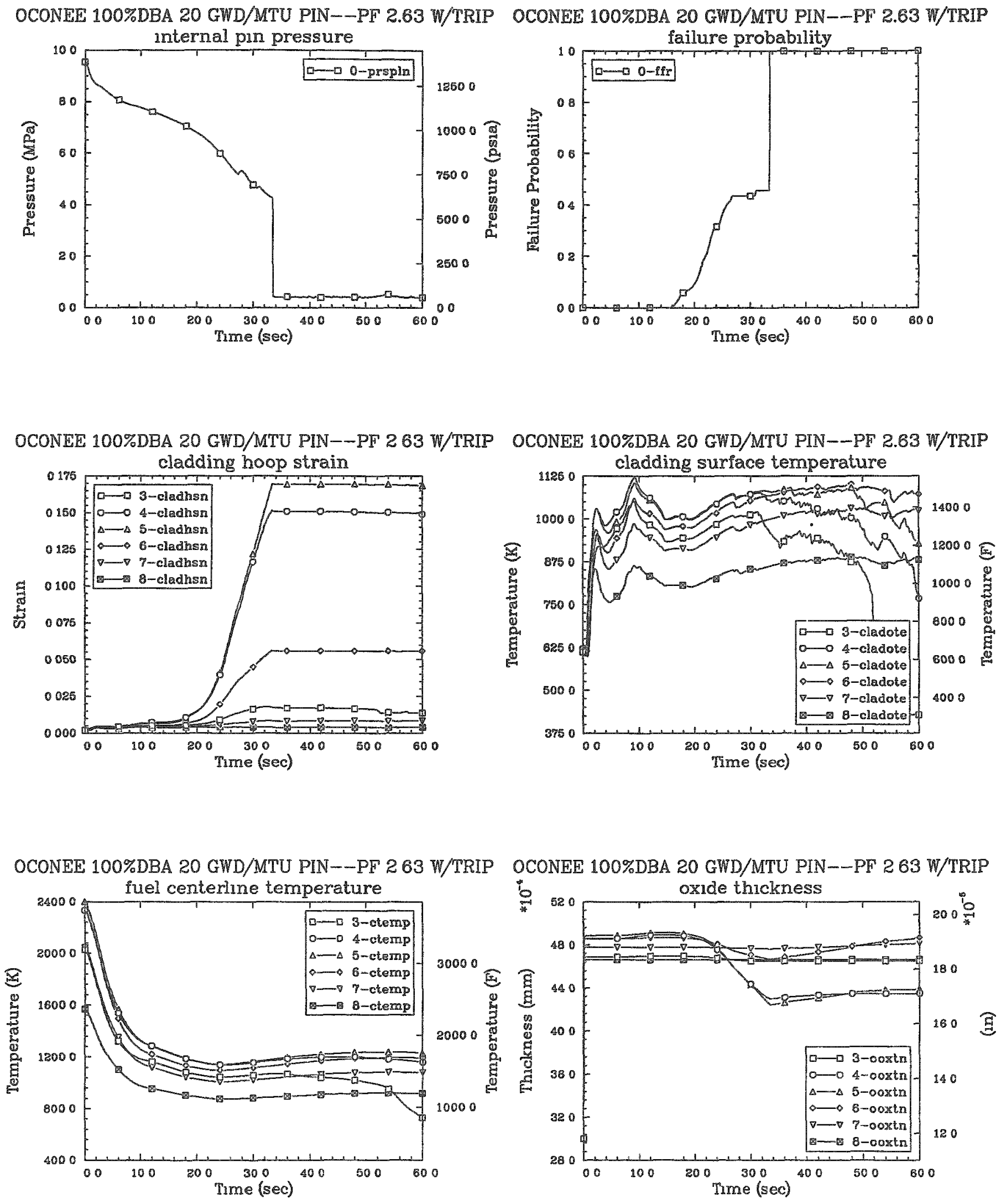

OCONEE 100\%DBA $20 \mathrm{GWD} / \mathrm{MTU}$ PIN--PF $263 \mathrm{~W} / \mathrm{TRIP}$

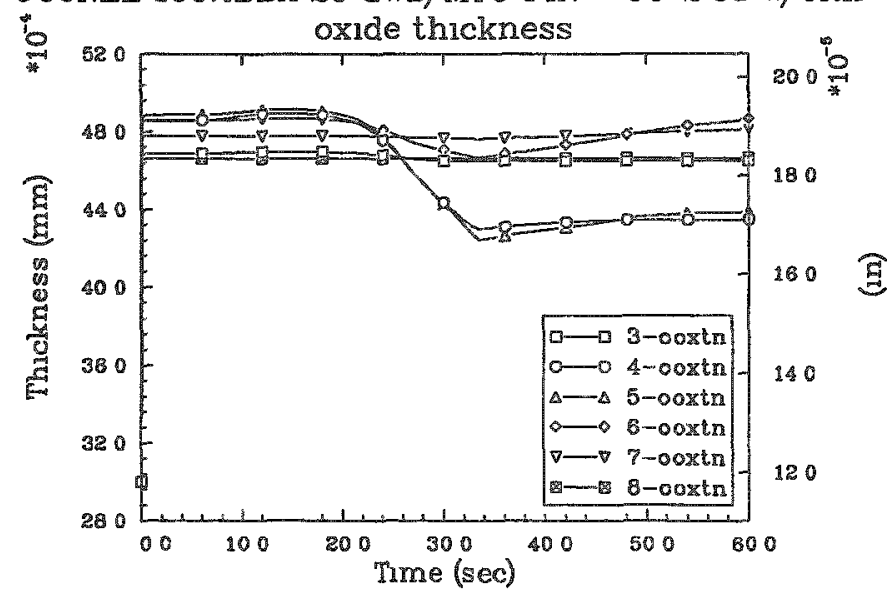


OCONEE 100\%DBA 5 GWD/MTU PIN--PF 2.63 W/TRIP
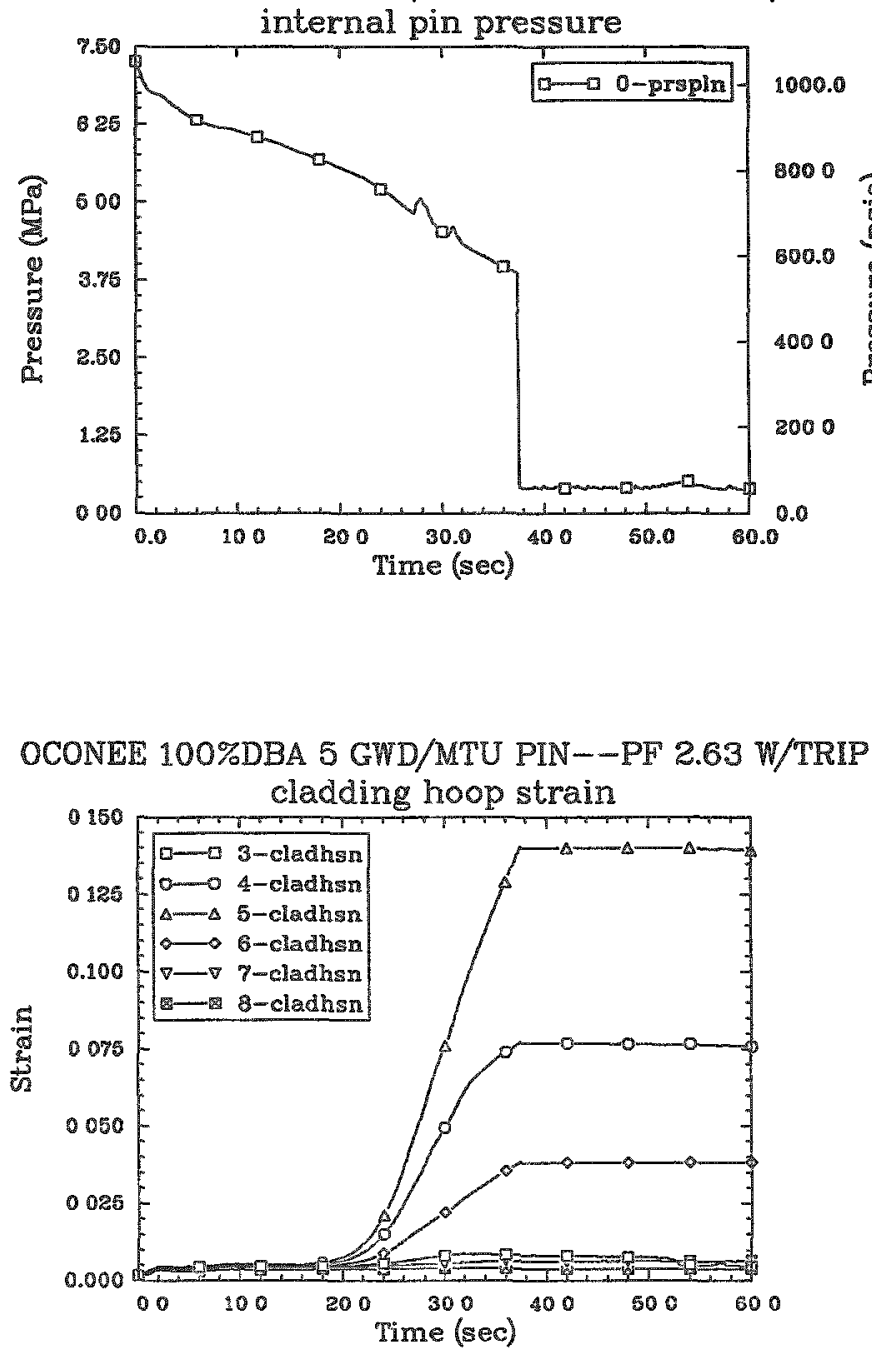

OCONEE 100\%DBA 5 GWD/MTU PIN_-.PF 2.63 W/TRIP

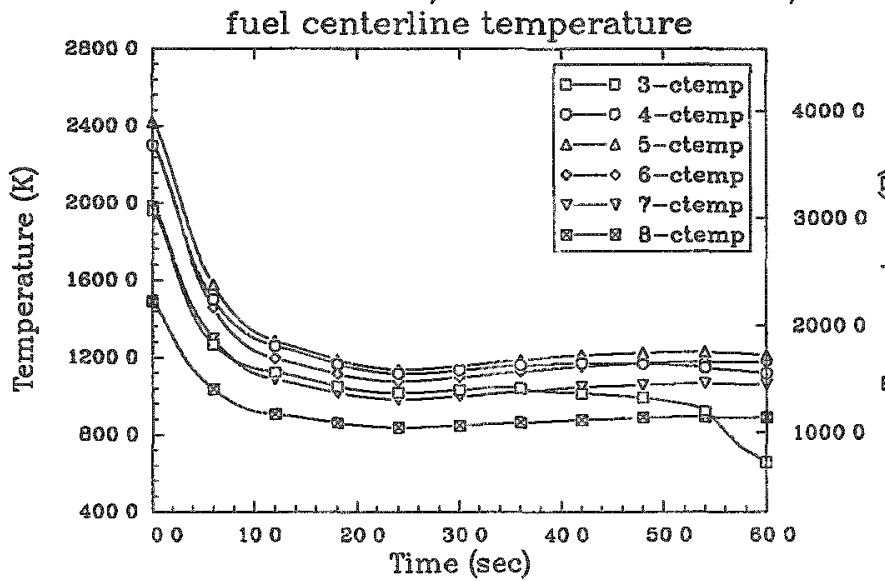

OCONEE 100\%DBA 5 GWD/MTU PIN--PF 2.63 W/TRIP failure probability

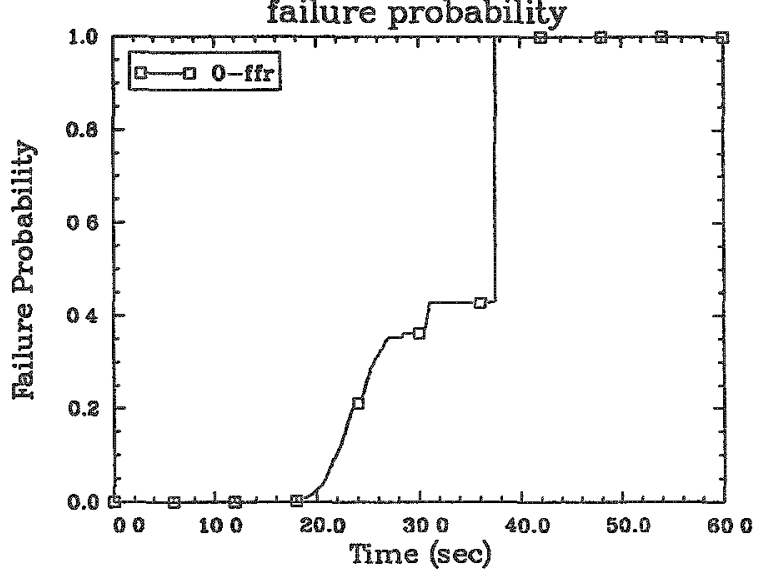

OCONEE 100\%DBA 5 GWD/MTU PIN--PF 2.63 W/TRIP oxide thickness

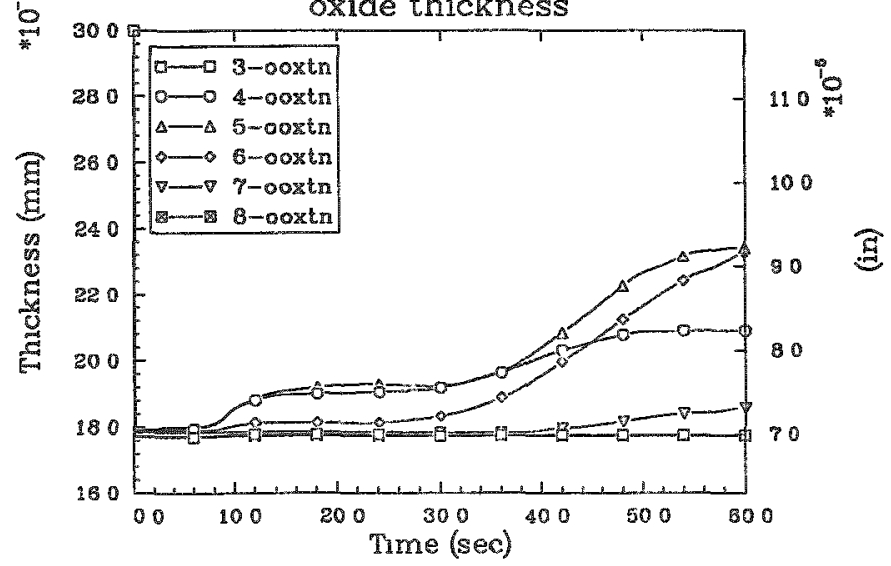




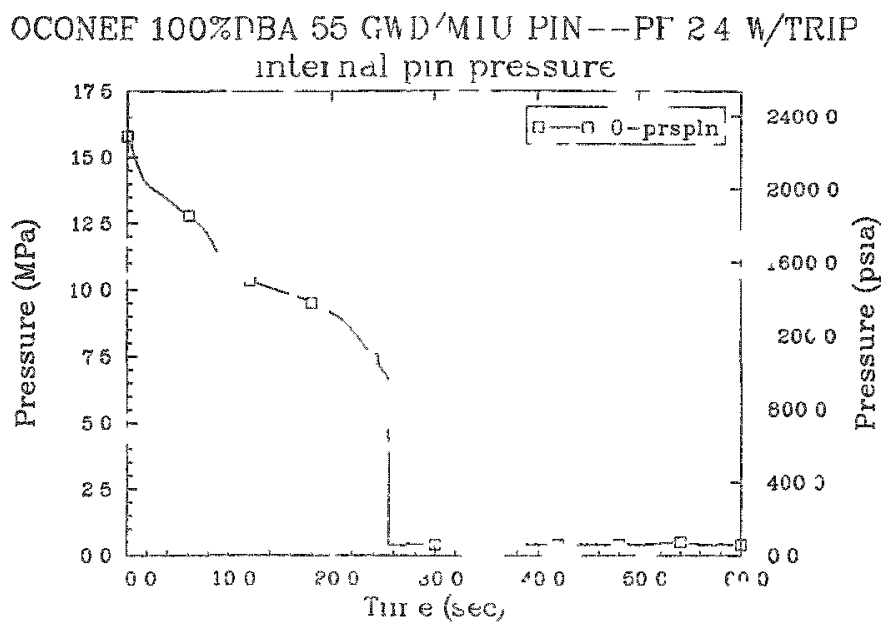

OCONEE 100\%DBA 55 GWD/MIU PIV--PF 24 W/TRIP

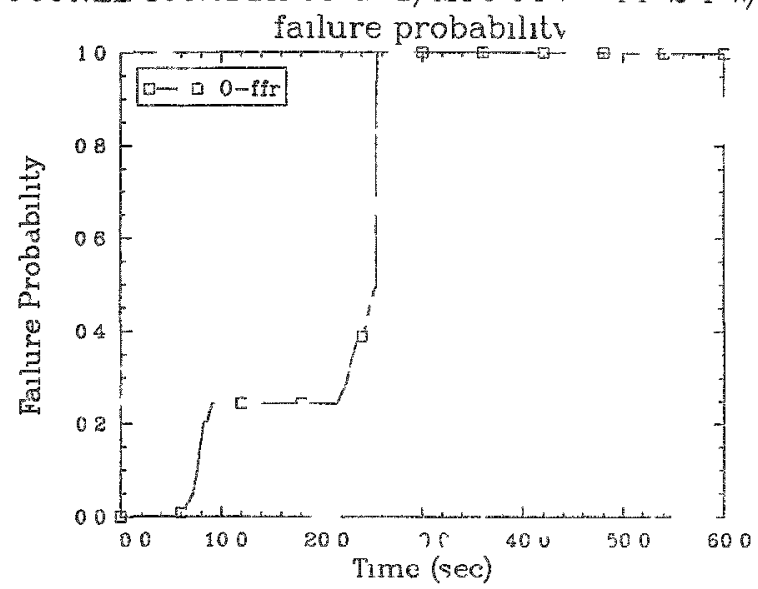

OCONEI 100\%DBA 5 GWD MIU PIV--PI 24 W/ IRIP

OCONEE 100\%DBA 55 GWD/MTU PIV-DP 24 W/IRIP
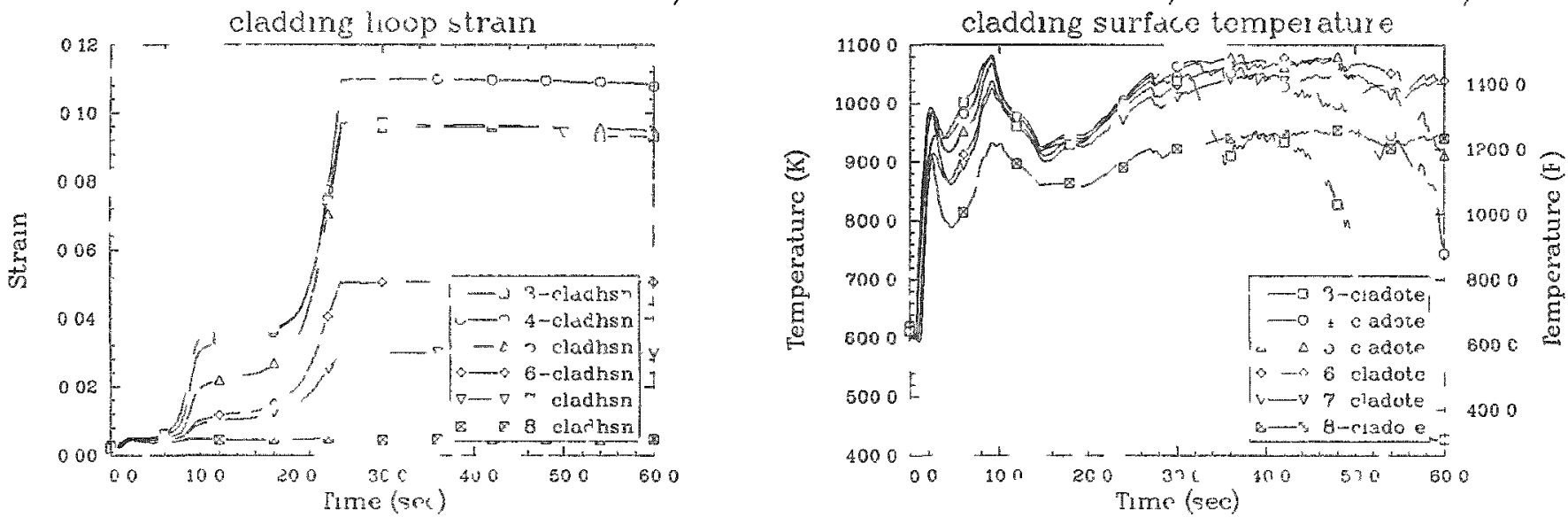

OCONEL 100 ${ }^{\circ}$ DBA 55 GWD, M U PIV-CHH 24 W/IRIP

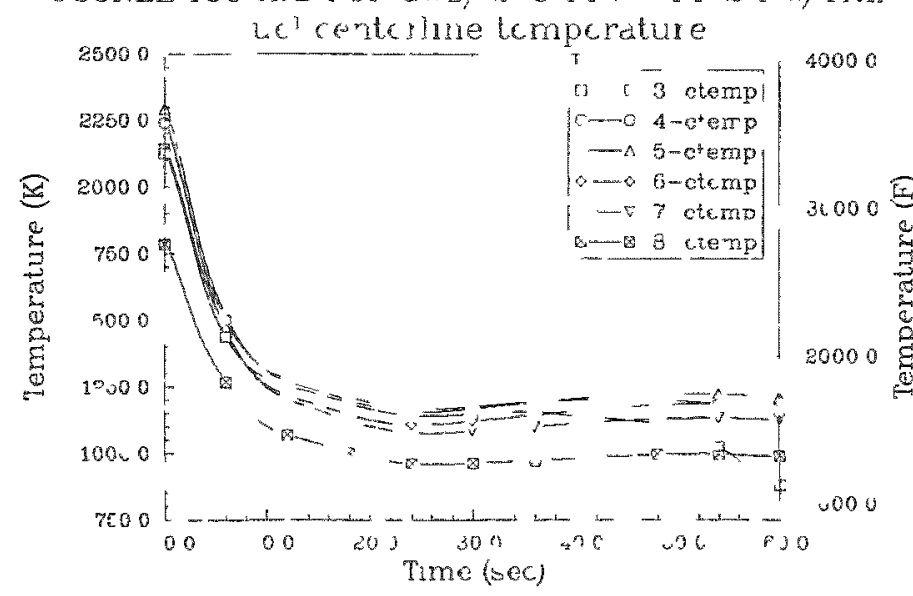

OCONEL 100\%DBA $55 \mathrm{GWD}$ /MTU PIV -PH $24 \mathrm{~W} / \mathrm{RIP}$

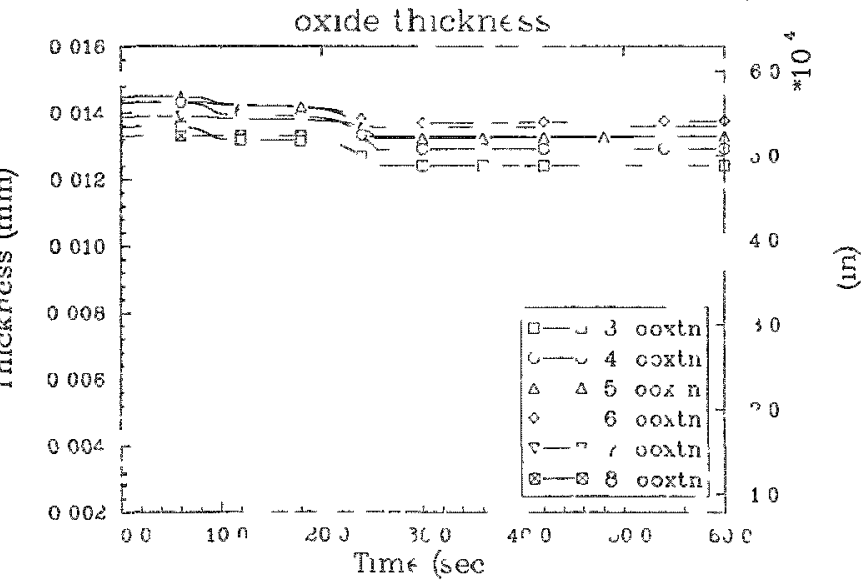


OCONEE 100\%DBA 35 GWD/MTU PIN--PF 2.4 W/TRIP internal pin pressure

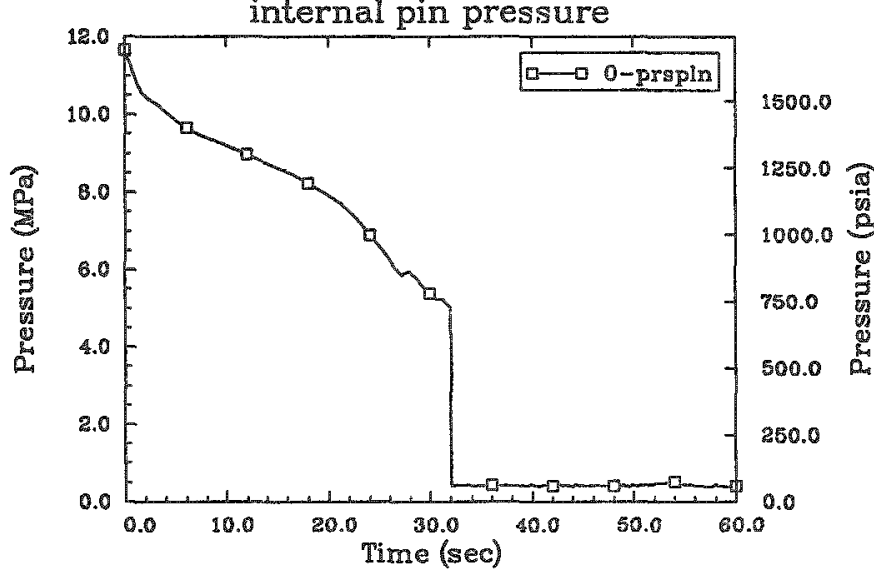

OCONEE 100\%DBA 35 GWD/MTU PIN--PF 2.4 W/TRIP failure probability

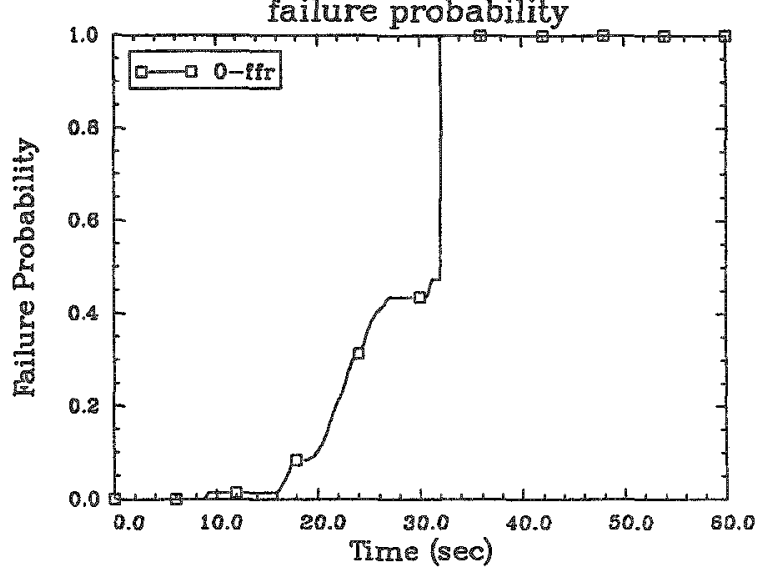

OCONEE 100\%DBA 35 GWD/MTU PIN--PF 2.4 W/TRIP cladding hoop strain

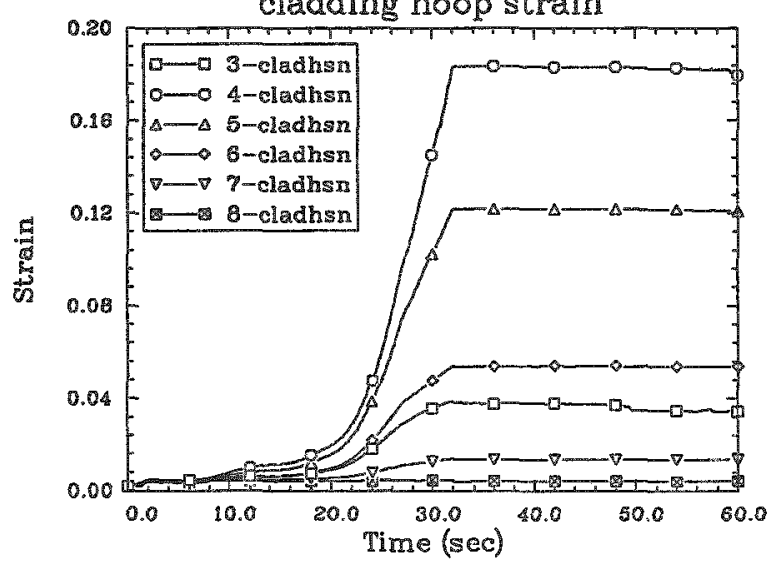

OCONEE 100\%DBA 35 GWD/MTU PIN--PF 2.4 W/TRIP

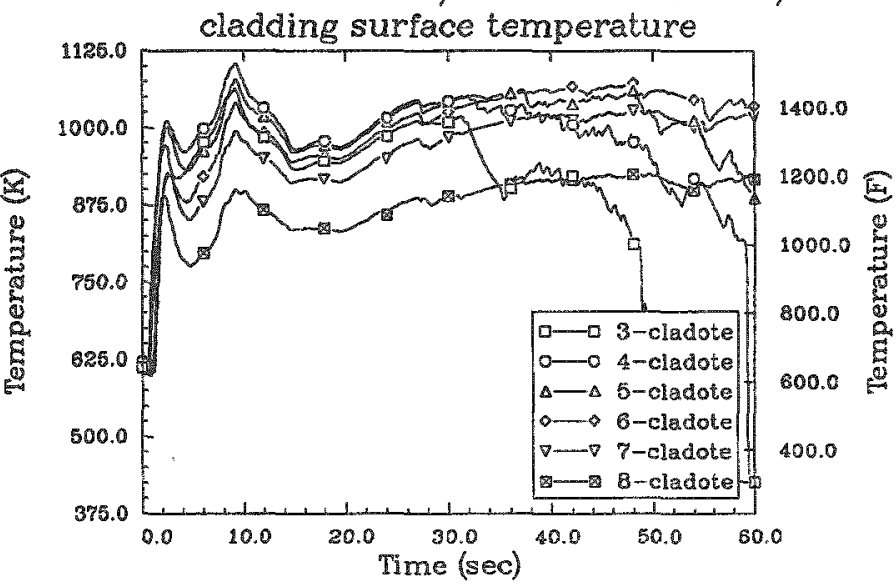

OCONEE 100\%DBA 35 GWD/MTU PIN--PF 2.4 W/TRIP

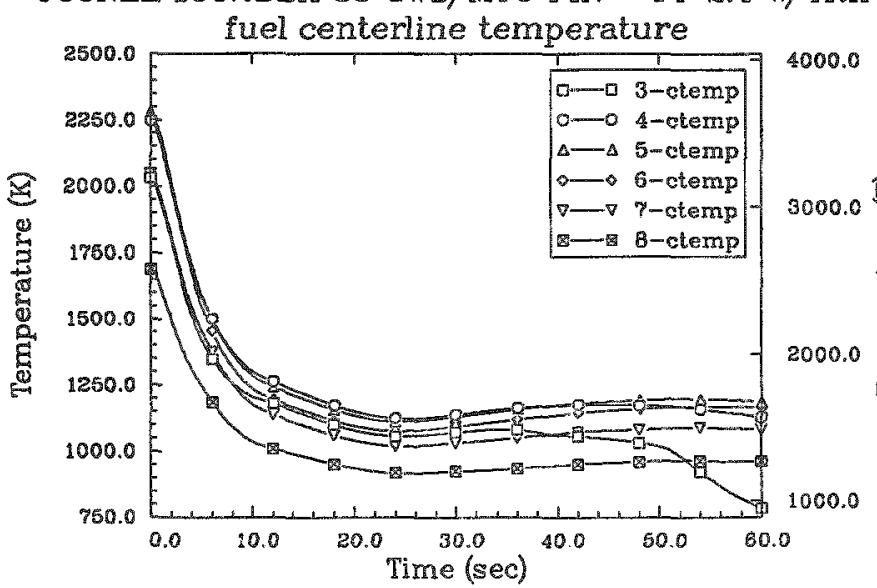

OCONEE 100\%DBA 35 GWD/MTU PIN--PF 2.4 W/TRIP oxide thickness

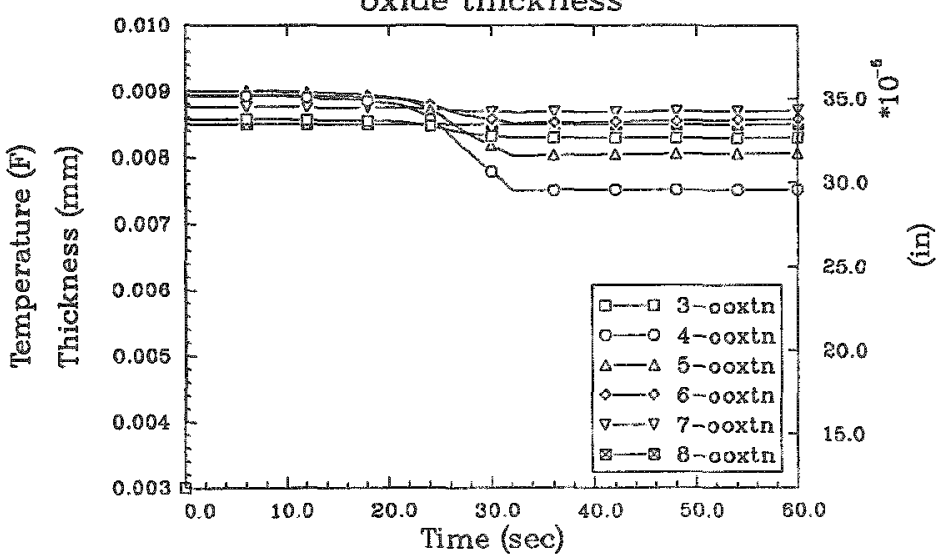


OCONEE 100\%DBA 20 GWD/MTU PIN--PF 2.4 W/TRIP internal pin pressure

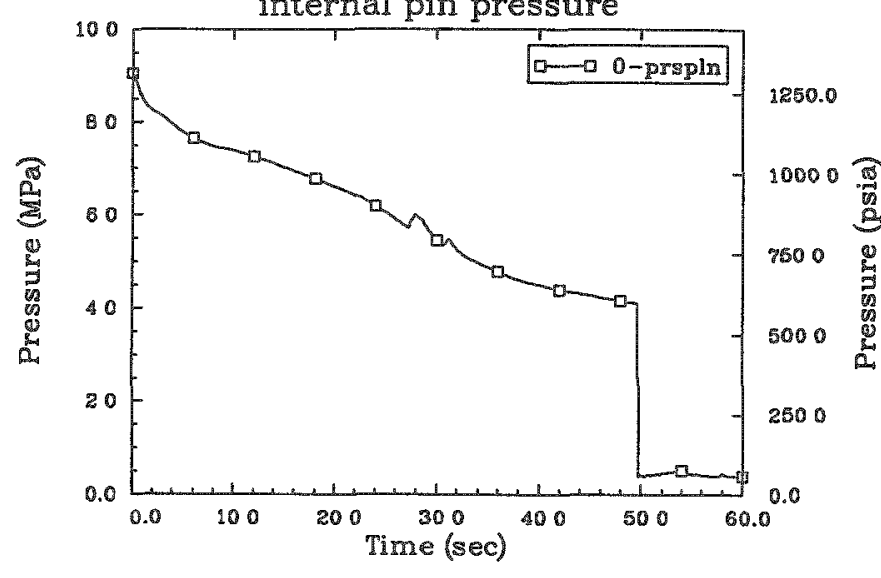

OCONEE 100\%DBA 20 GWD/MTU PIN--PF 2.4 W/TRIP failure probability

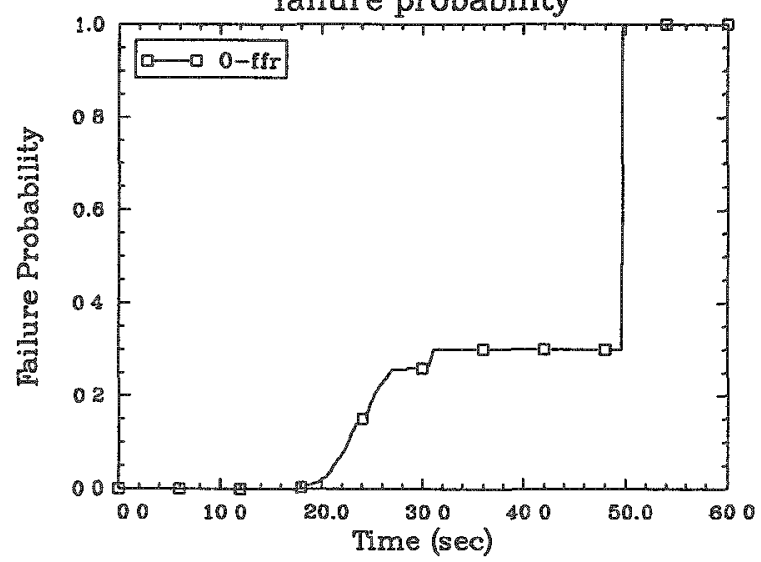

OCONEE 100\%DBA 20 GWD/MTU PIN--PF 2.4 W/TRIP

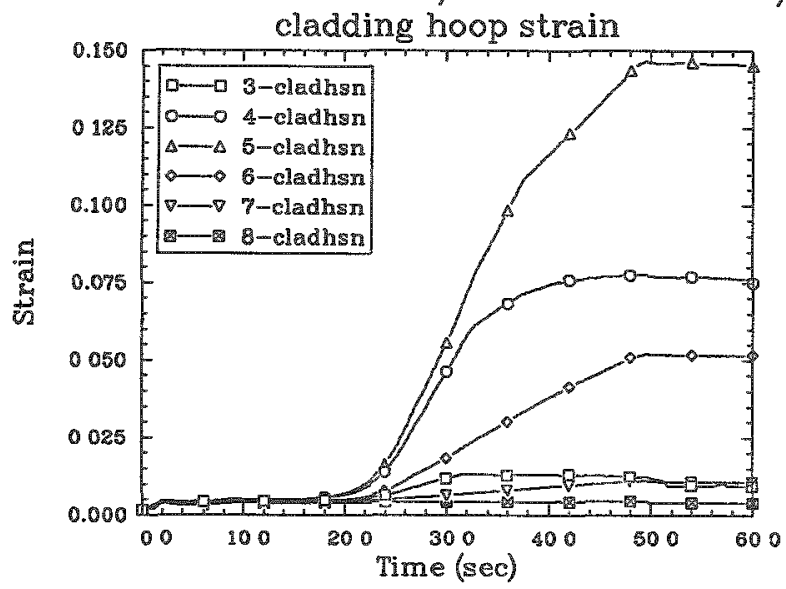

OCONEE 100\%DBA 20 GWD/MTU PIN--PF 2.4 W/TRIP

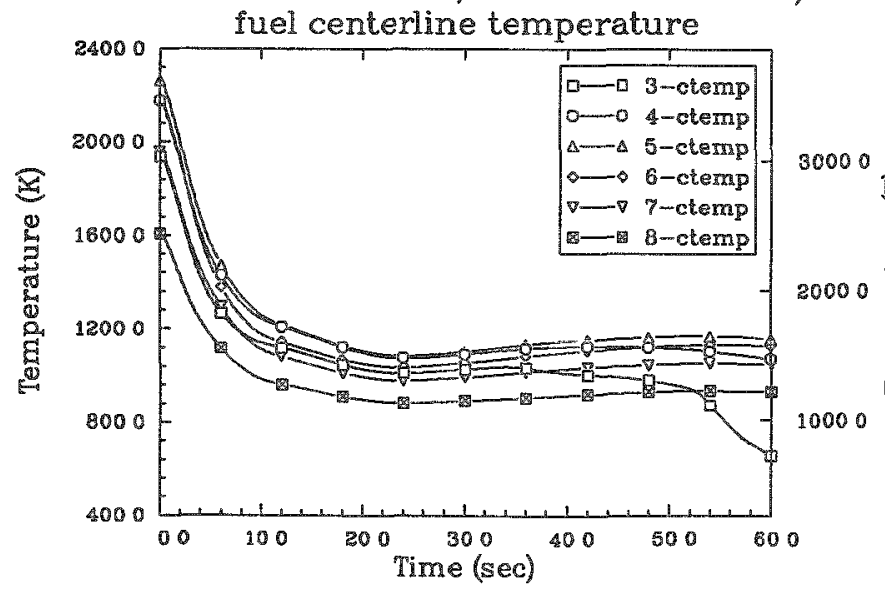

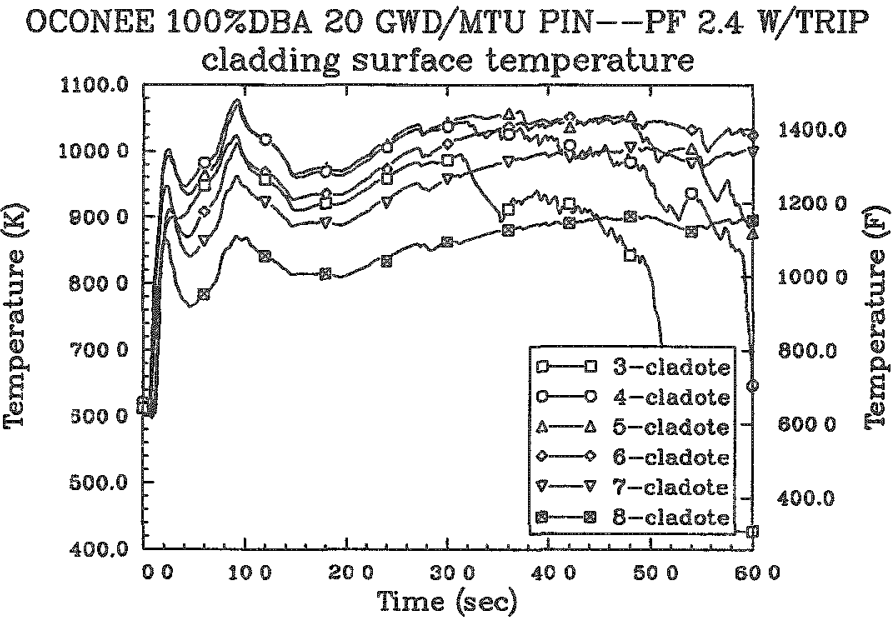

OCONEE 100\%DBA 20 GWD/MTU PIN--PF 2.4 W/TRIP

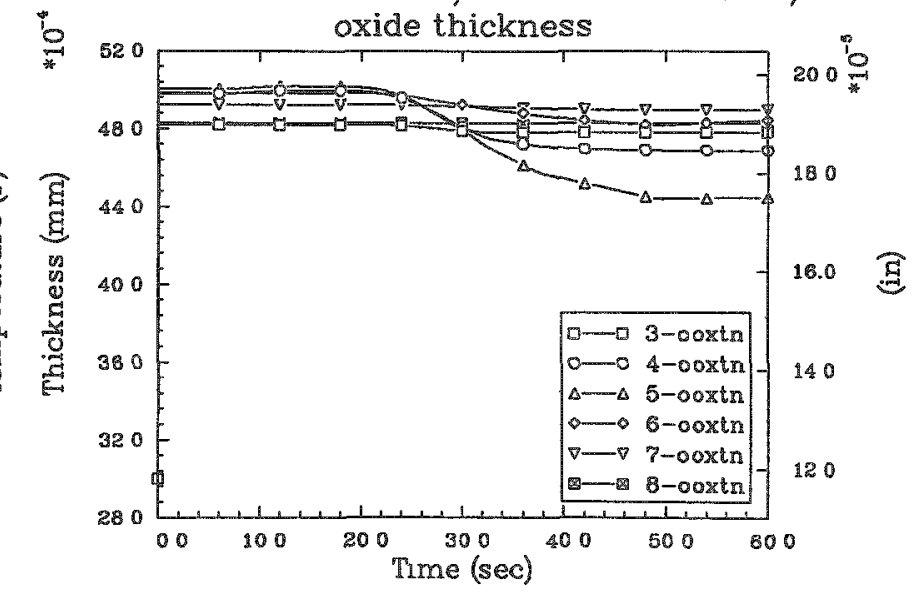


OCONEE 100\%DBA 5 GWD/MTU PIN--PF 2.4 W/TRIP internal pin pressure
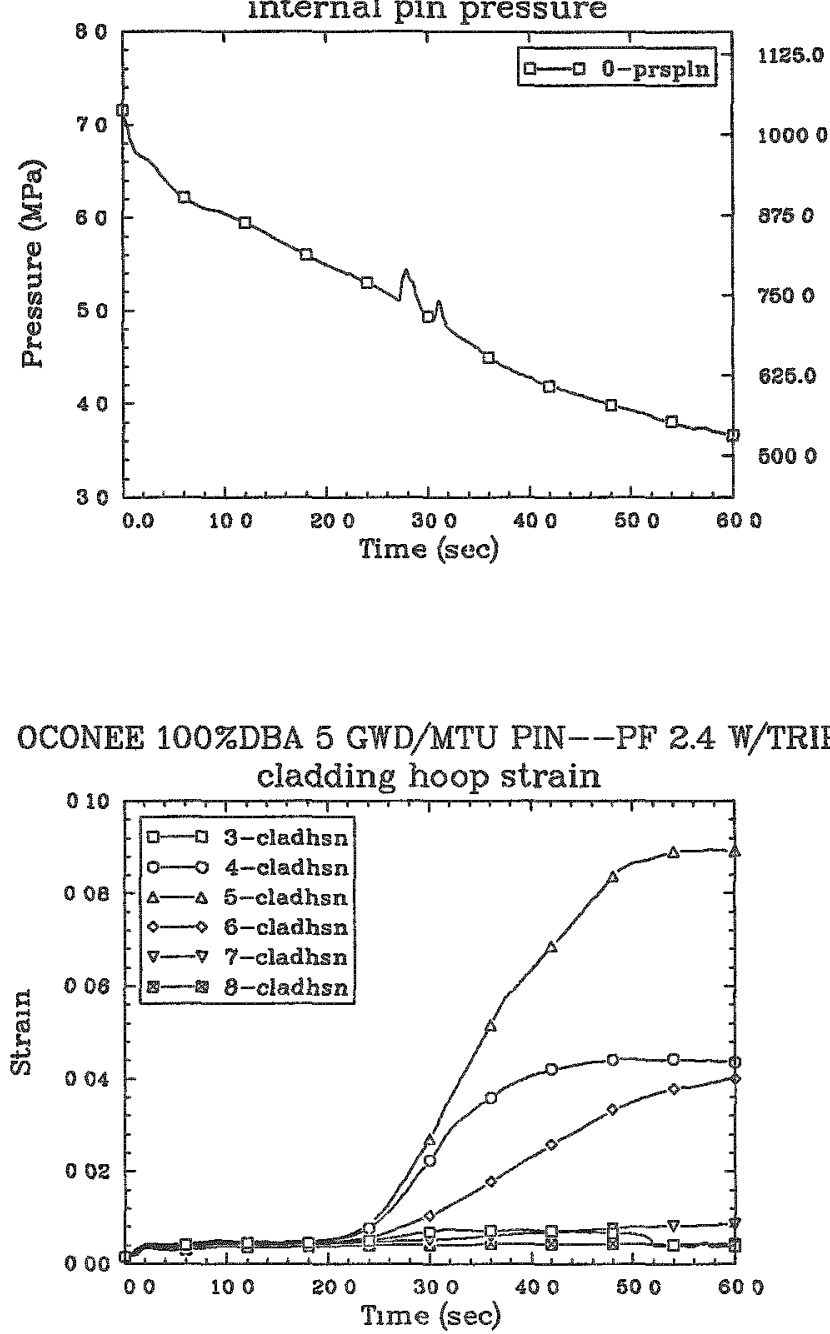

OCONEE 100\%DBA 5 GWD/MTU PIN--PF 24 W/TRIP

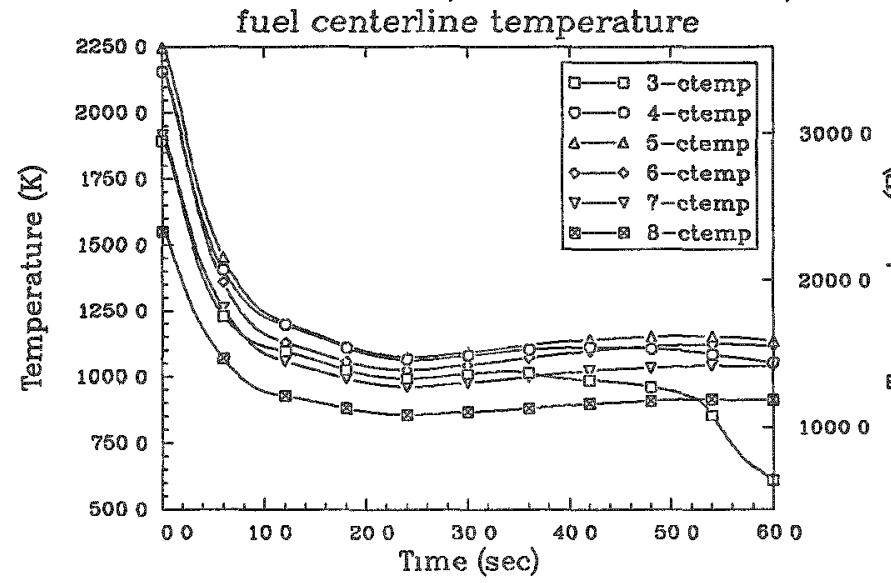

OCONEE 100\%DBA 5 GWD/MTU PIN--PF 2.4 W/TRIP failure probability
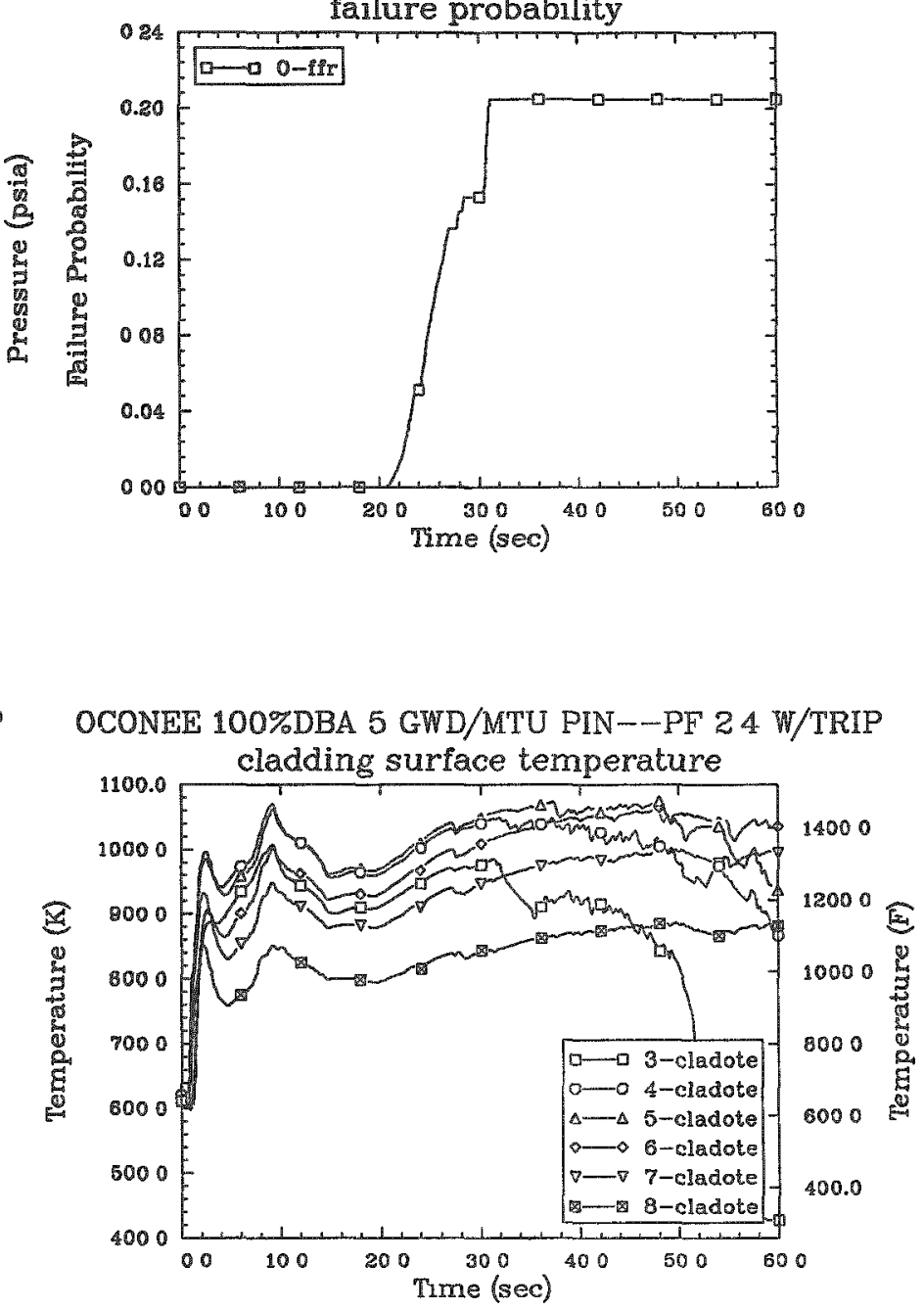

OCONEE 100\%DBA 5 GWD/MTU PIN--PF 24 W/TRIP

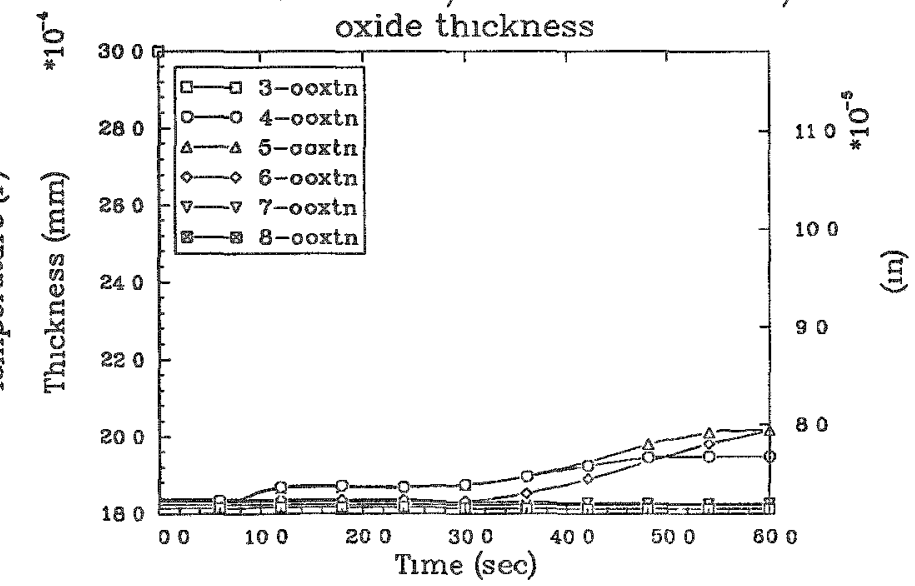


OCONEE 100\%DBA 55 GWD/MTU PIN--PF 2.2 W/TRIP internal pin pressure

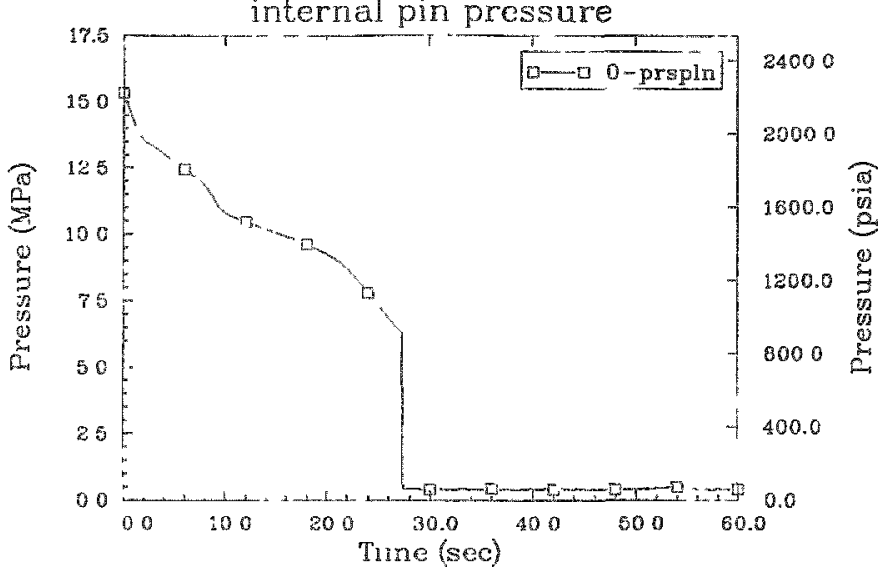

OCONEE 100\%DBA 55 GWD/MTU PIN - PF 2.2 W/TRIP

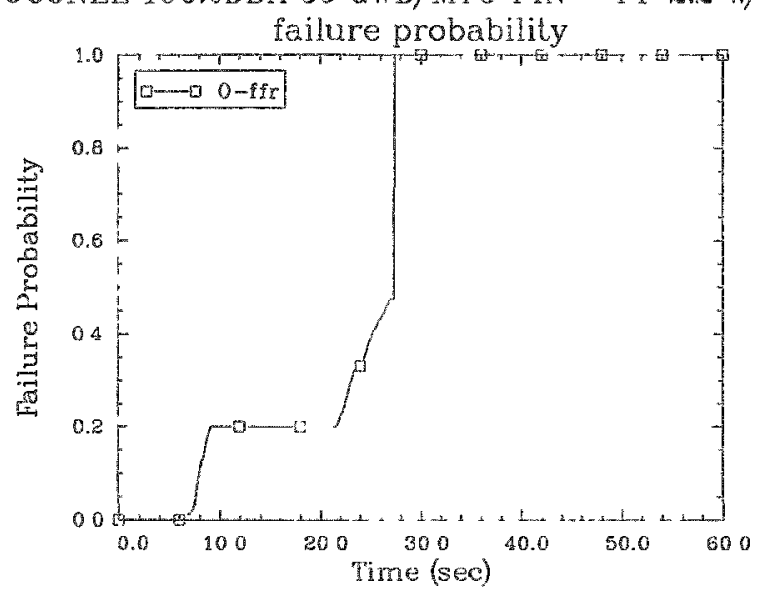

OCONEE 100\%DBA 55 GWD/MTU PIN--PF 2.2 W/TRIP cludding hoop strain

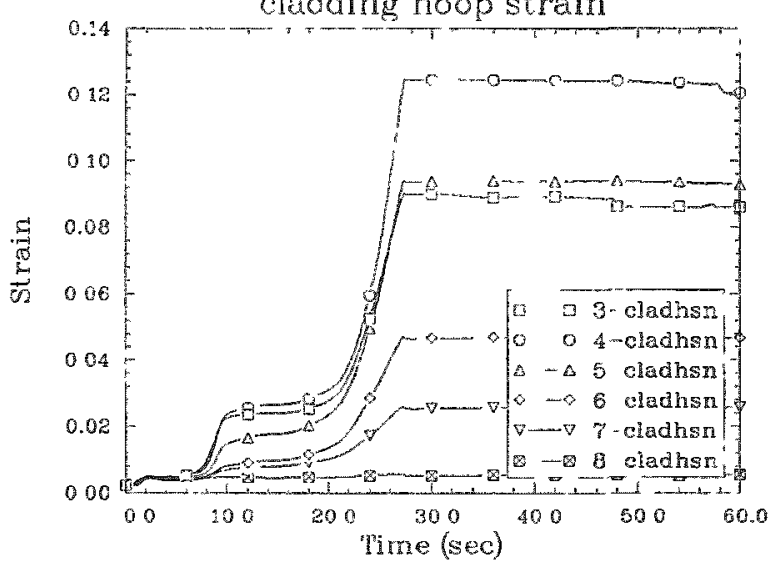

OCONEE 100\%DBA 55 GWD/MTU PIN-- PH 2.2 W/TRIP

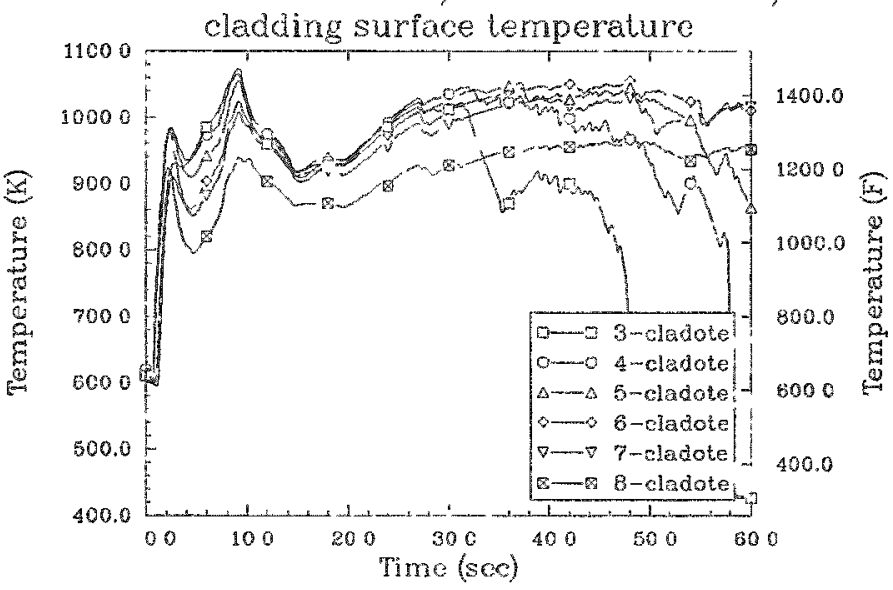

OCONEl' 100\%DBA 55 GWD,MTU PIN--PF 2.2 W/TRIP

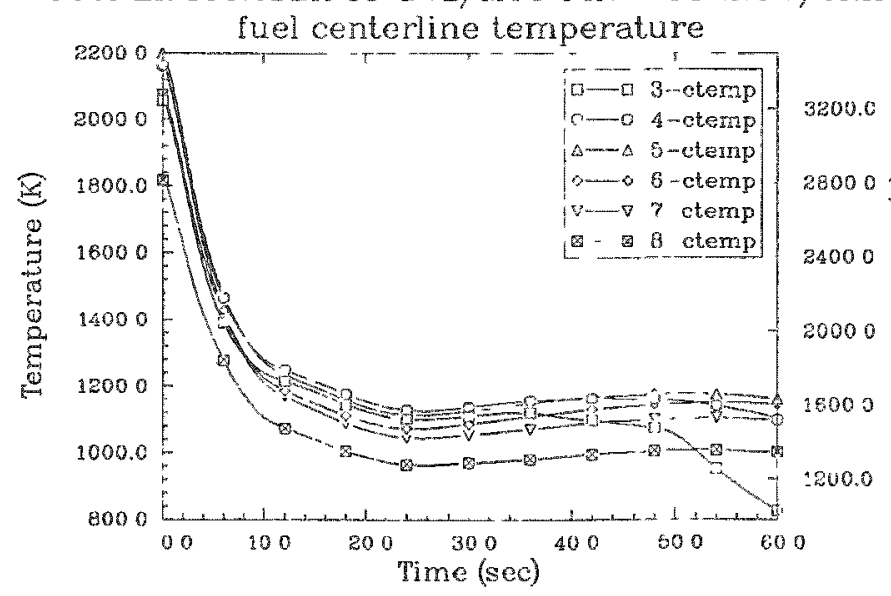

OCONEE 100\%DBA 55 GWD/MTU PIN-..PF 2.2 W/TRIP

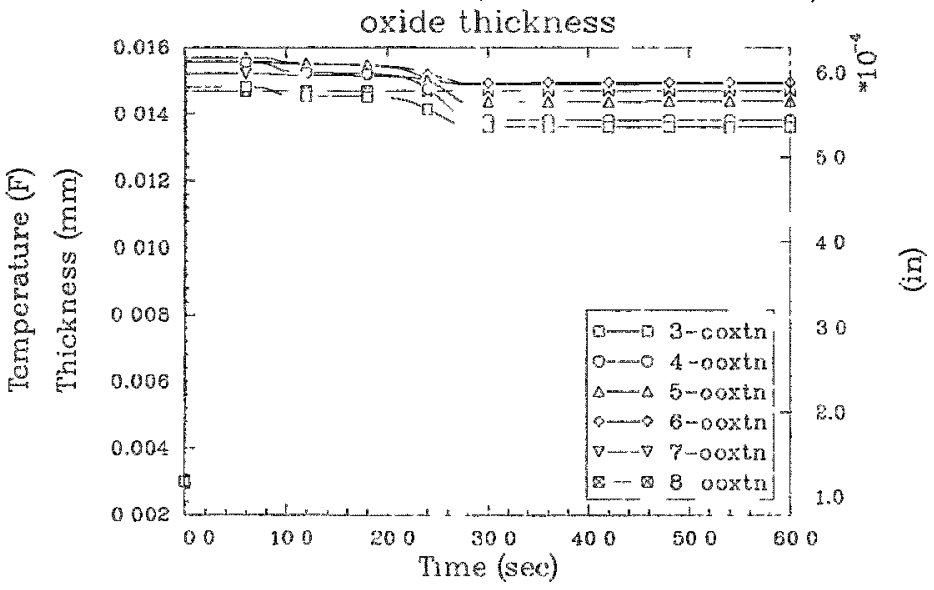


OCONEE 100\%DBA $35 \mathrm{GWD} / \mathrm{MTU}$ PIN--PF $2.2 \mathrm{~W} / \mathrm{TRIP}$

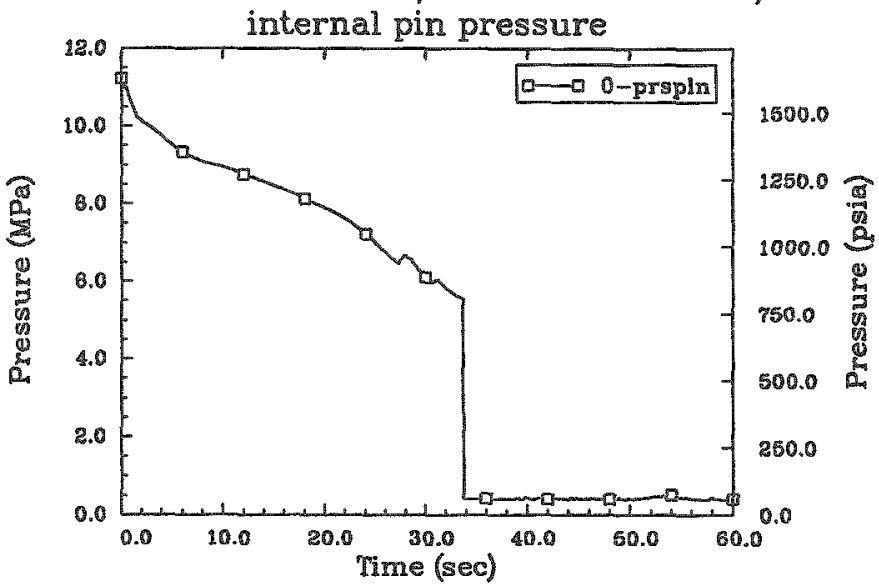

OCONEE 100\%DBA 35 GWD/MTU PIN--PF 2.2 W/TRIP failure probability

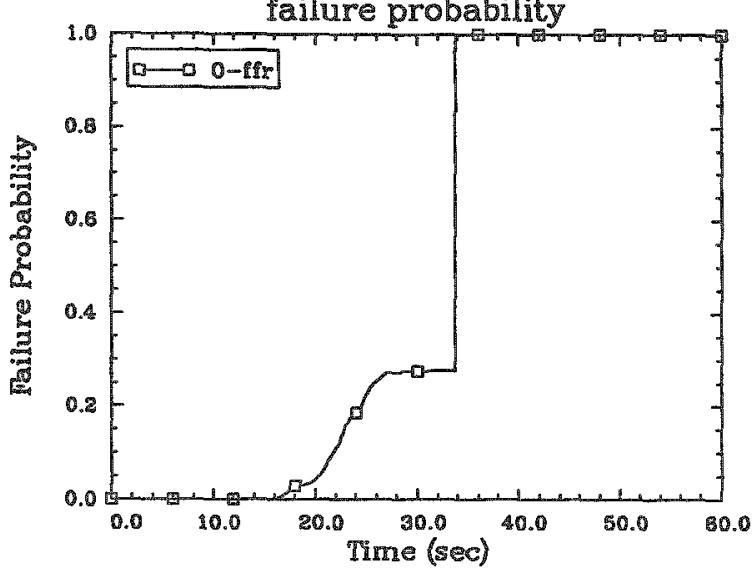

OCONEE 100\%DBA 35 GWD/MTU PIN--PF 2.2 W/TRIP cladding hoop strain

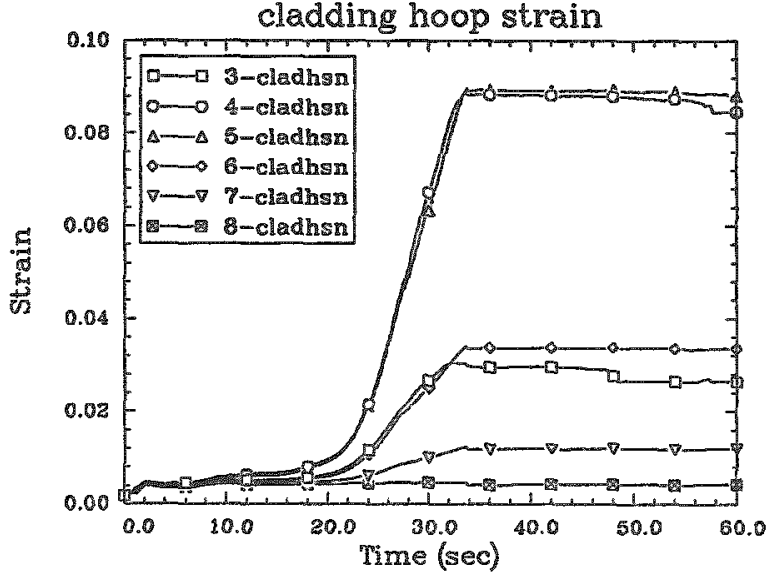

OCONEE 100\%DBA 35 GWD/MTU PIN--PF 2.2 W/TRIP

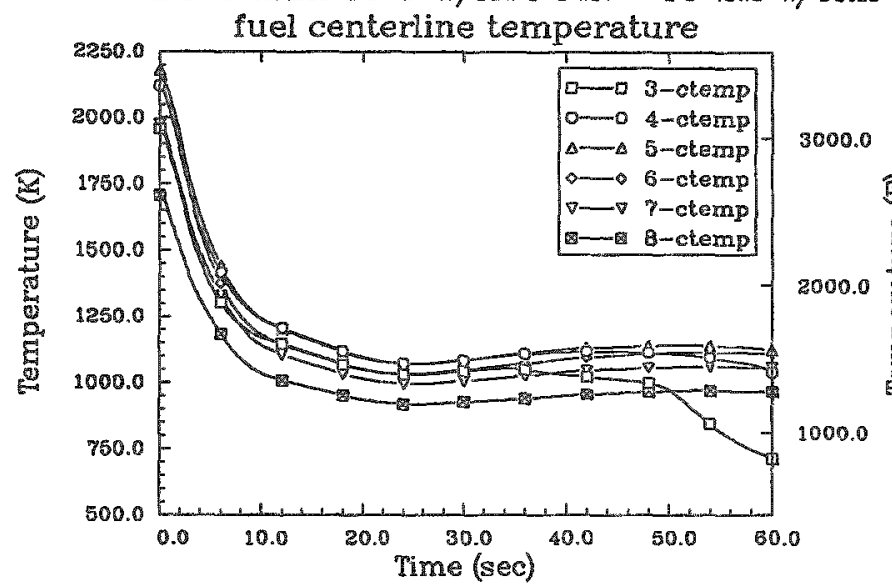

OCONEE 100\%DBA 35 GWD/MTU PIN--PF 2.2 W/TRIP

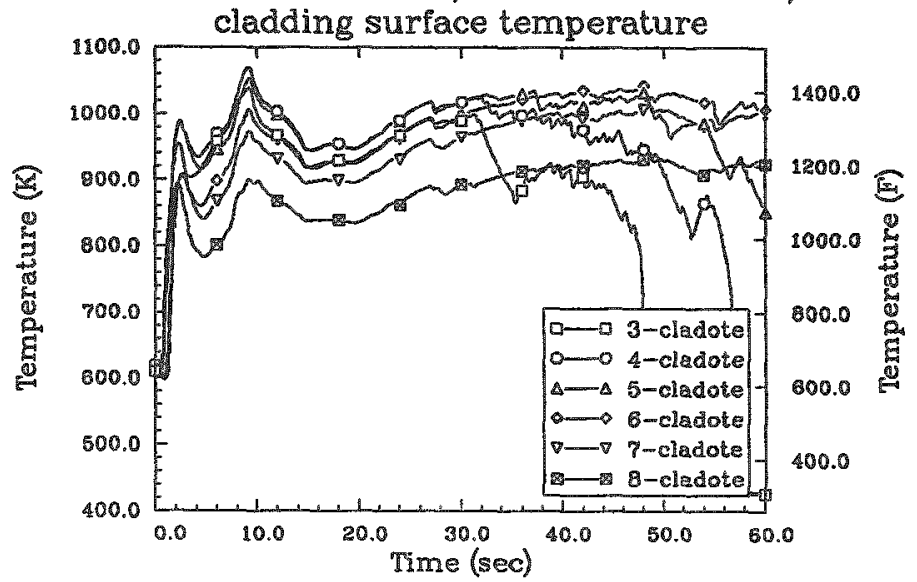

OCONEE 100\%DBA 35 GWD/MTU PIN-PF 2.2 W/TRIP

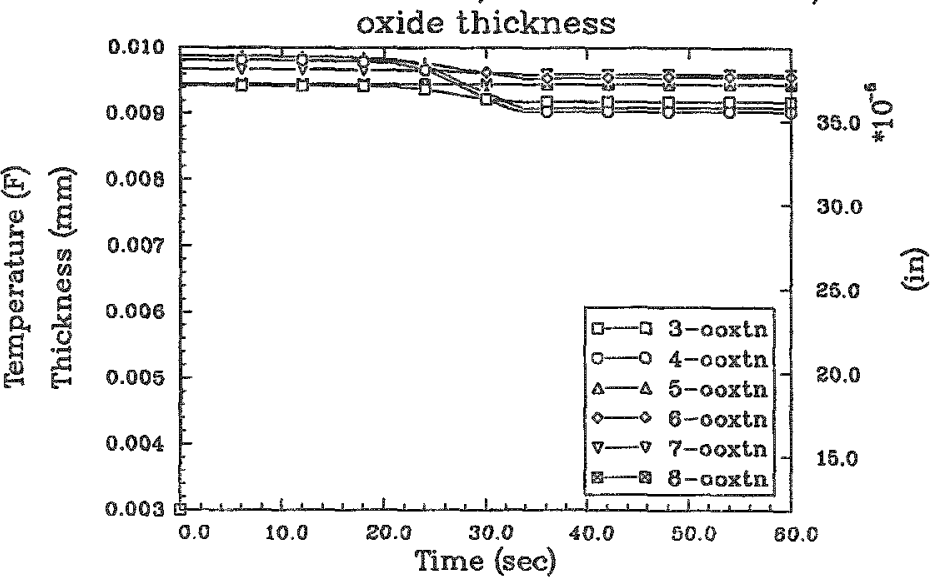


OCONEE $100 \%$ DBA 20 GWD/MTU PIN-PF 2.2 W/TRIP

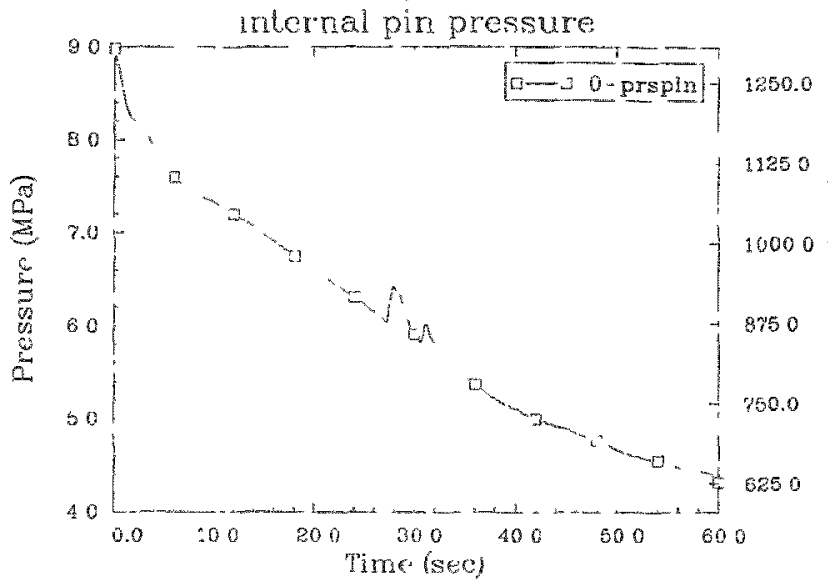

OCONEF 100\%DBA 20 GWD/MTU PIN - -PF $2.2 \mathrm{~W} / \mathrm{TRIP}$

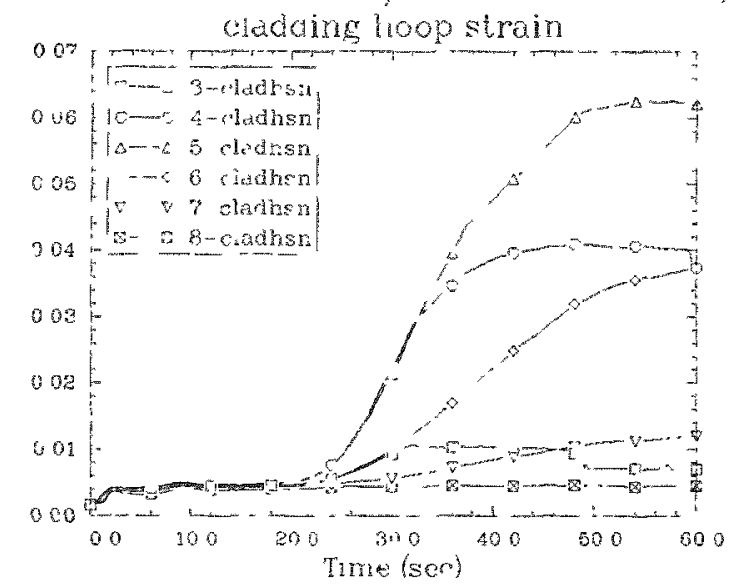

OCONEE 100\%DBA 20 GWD/MTL PIN - -PF 2.2 W/TRIP

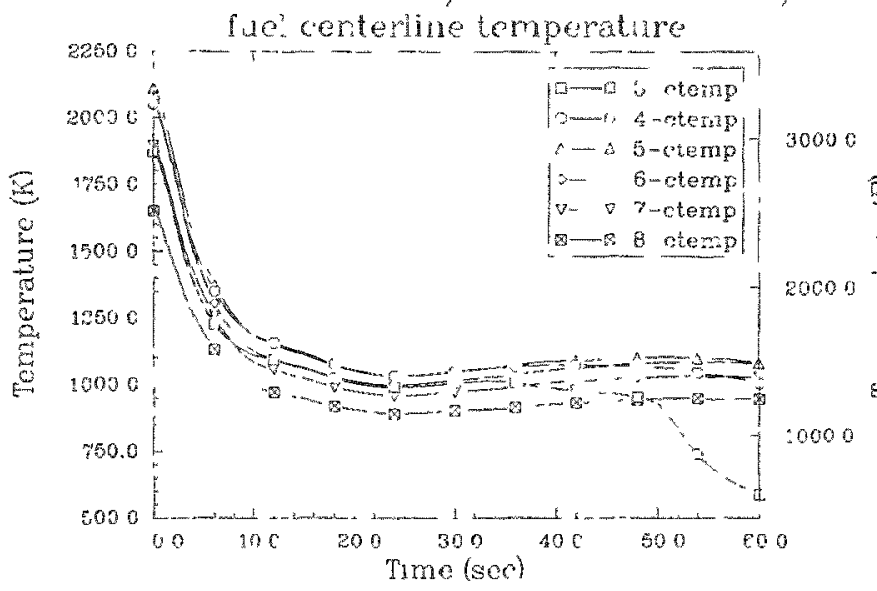

OCONES $100 \%$ DBA 20 GWD/MTU PIN-PI $22 \mathrm{~W} / \mathrm{CRIP}$ failure probability

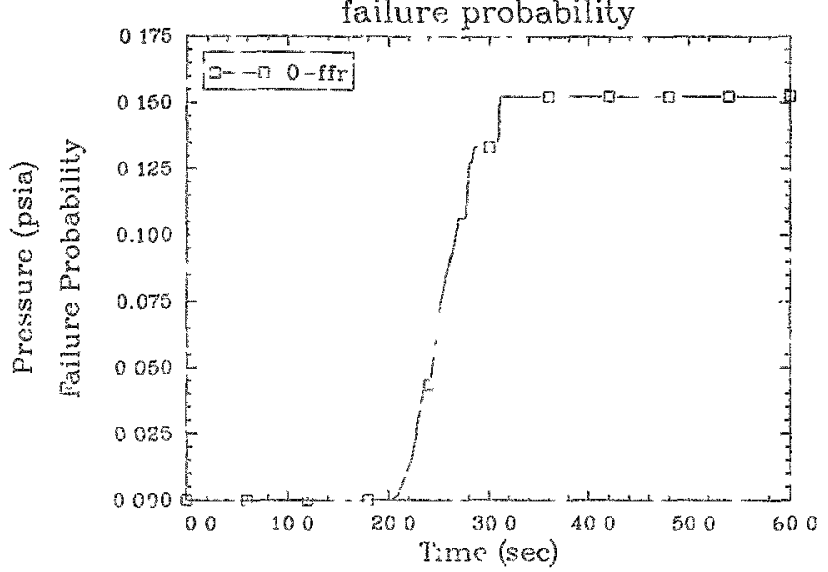

OCONEE 100\%DBA 20 CWD/MTH PIN-PF $2.2 \mathrm{~W} / \mathrm{TRIP}$

io

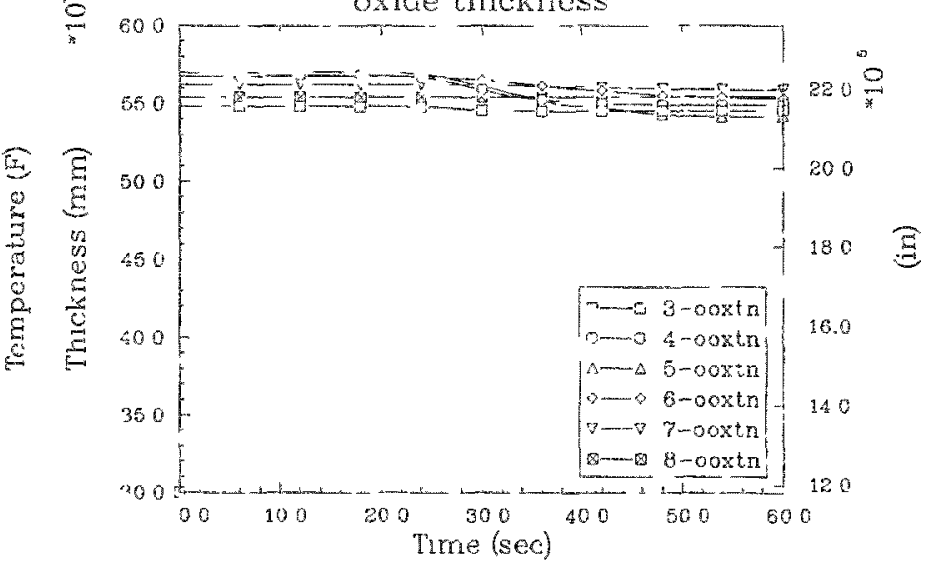


OCONEE 100\%DBA 5 GWD/MTU PIN--PF 2.2 W/TRIP

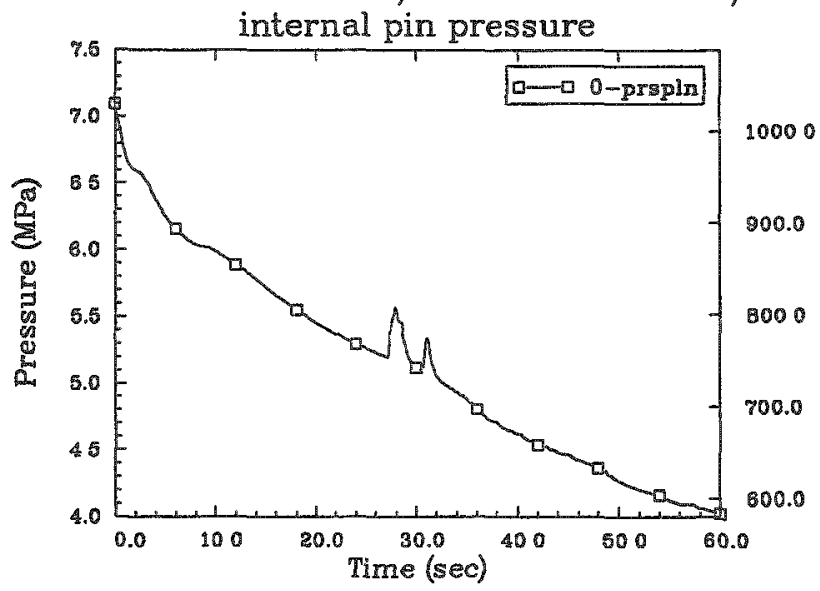

OCONEE 100\%DBA 5 GWD/MTU PIN--PF $2.2 \mathrm{~W} / \mathrm{TRIP}$ cladding hoop strain

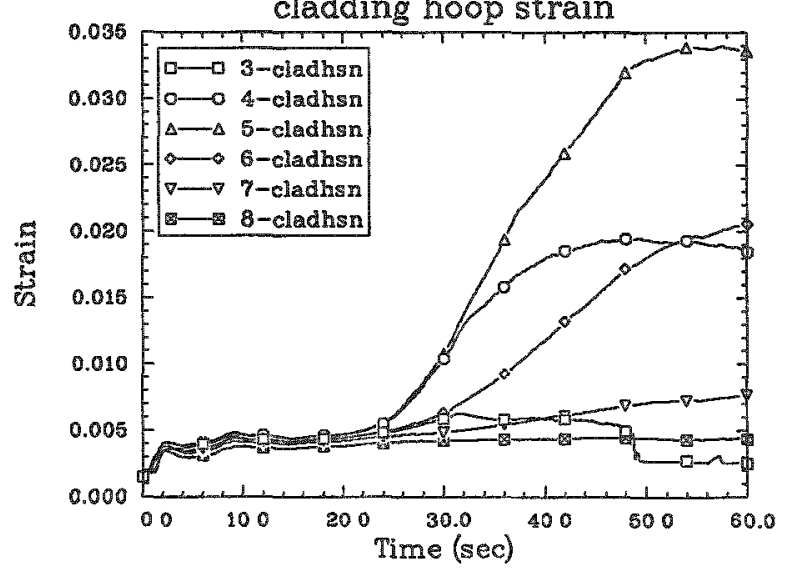

OCONEE 100\%DBA 5 GWD/MTU PIN--PF 2.2 W/TRIP

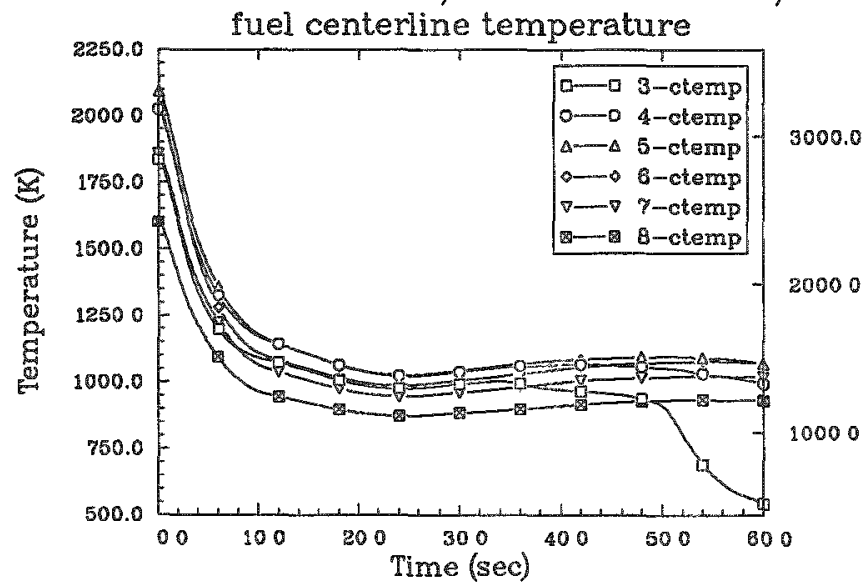

OCONEE 100\%DBA 5 GWD/MTU PIN-DF 2.2 W/TRIP failure probability

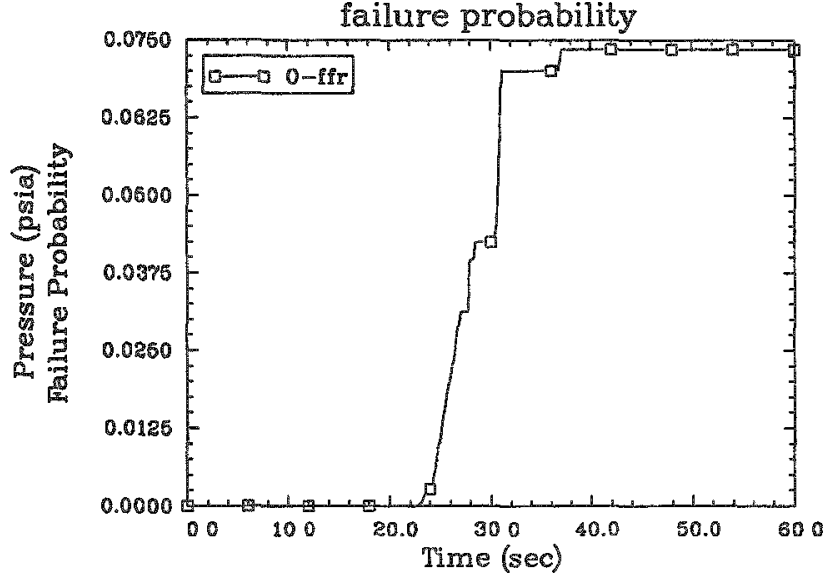

OCONEE 100\%DBA 5 GWD/MTU PIN--PF 2.2 W/TRIP

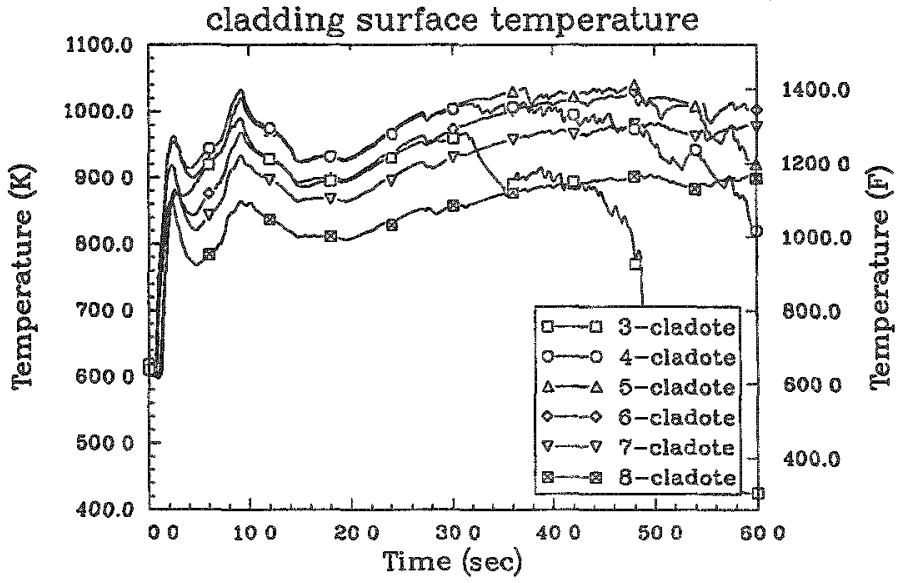

OCONEE 100\%DBA 5 GWD/MTU PIN--.PF 2.2 W/TRIP

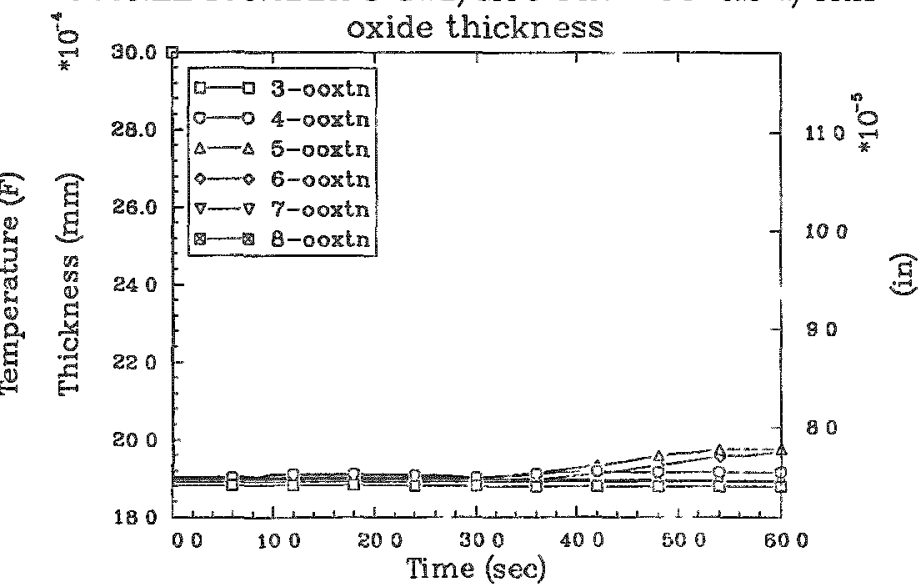


OCONEE 100\%DBA 55 GWD/MTU PIN--PF 20 W/TRIP

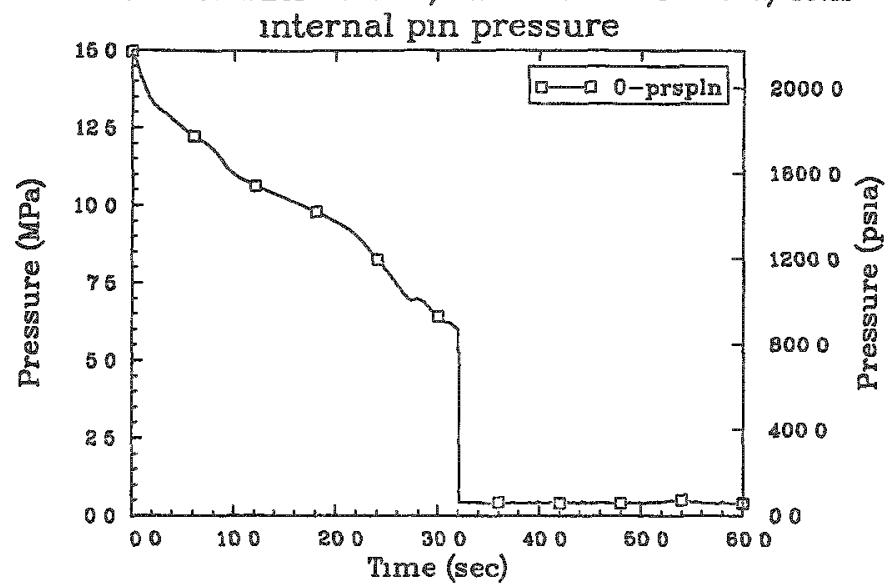

OCONEE 100\%DBA 55 GWD/MTU PIN--PF 20 W/TRIP fallure probability

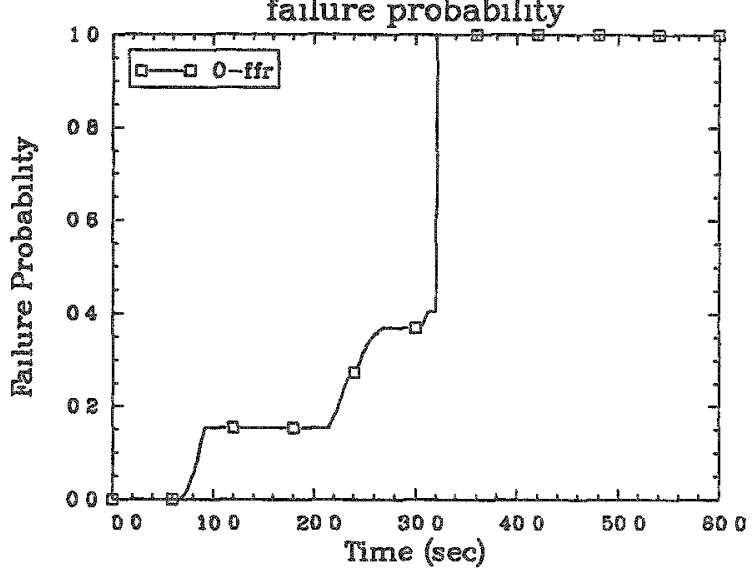

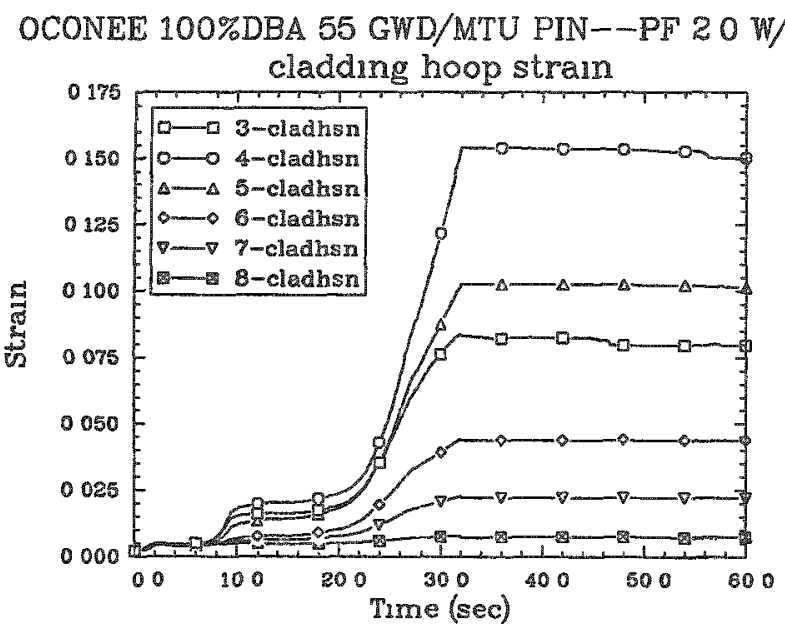

OCONEE 100\%DBA 55 GWD/MTU PIN--PF 20 W/TRIP

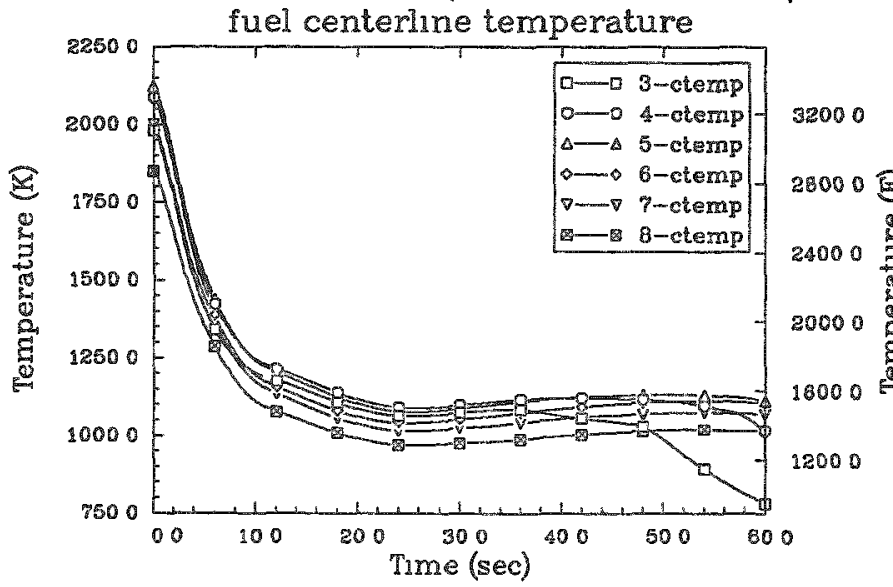

OCONEE 100\%DBA 55 GWD/MTU PIN--PF 20 W/TRIP

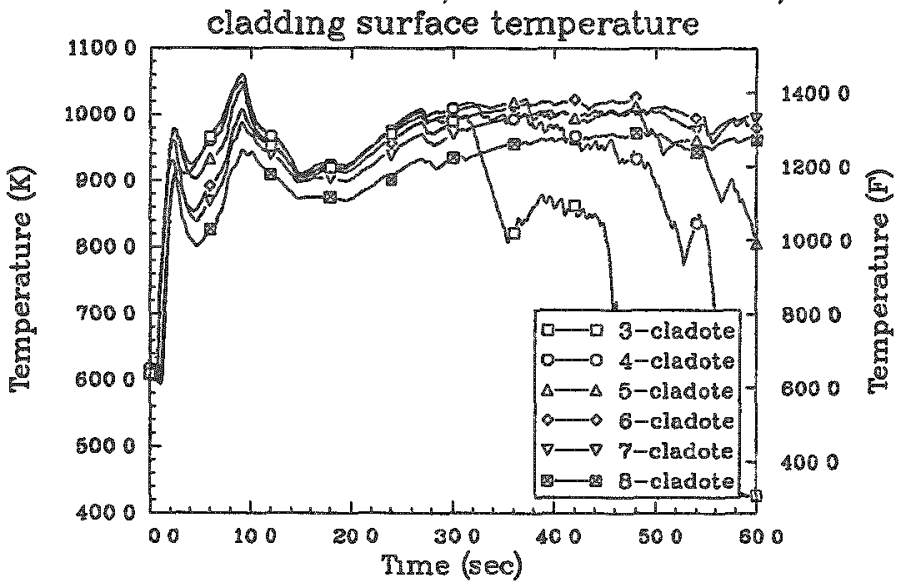

OCONEE 100\%DBA 55 GWD/MTU PIN--PF 20 W/TRIP

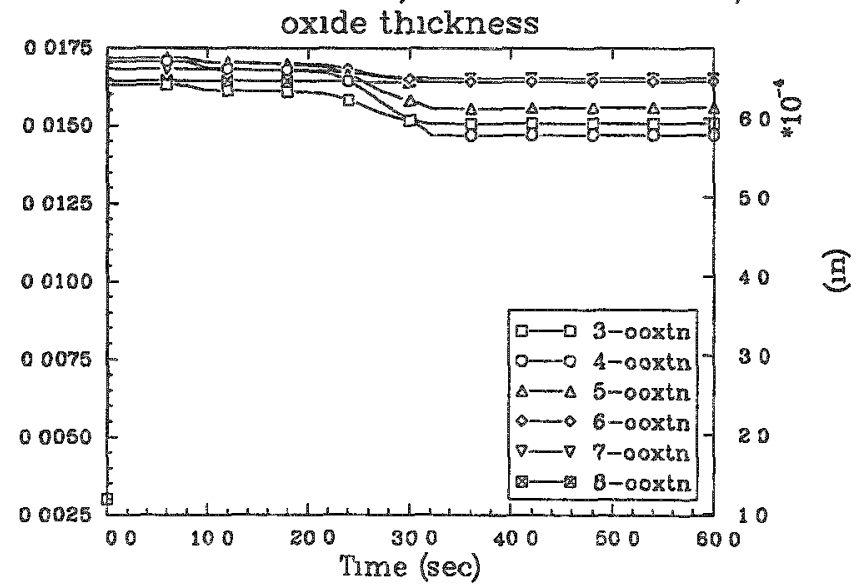


OCONEE 100\%DBA 35 GWD/MTU PIN--PF 2.0 W/TRIP

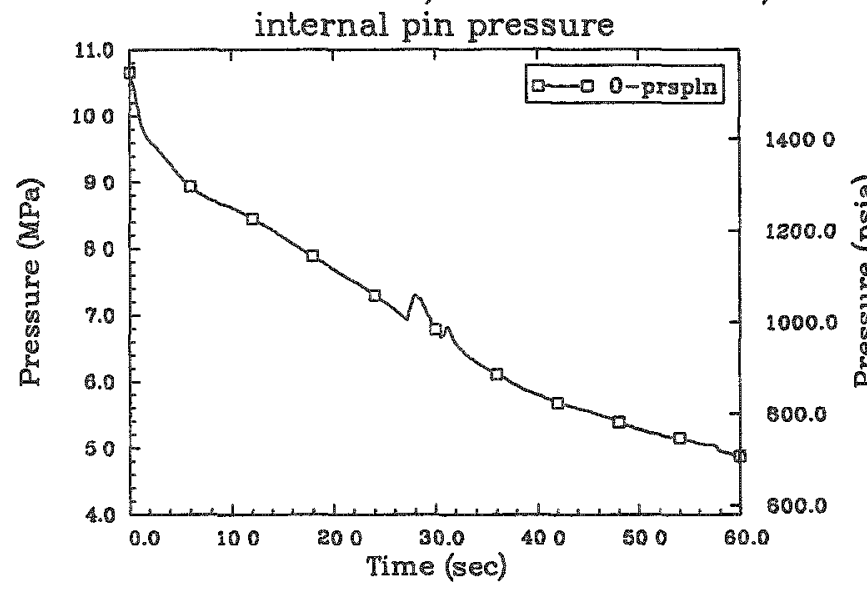

OCONEE 100\%DBA 35 GWD/MTU PIN--PF $2.0 \mathrm{~W} / \mathrm{TRIP}$ failure probability

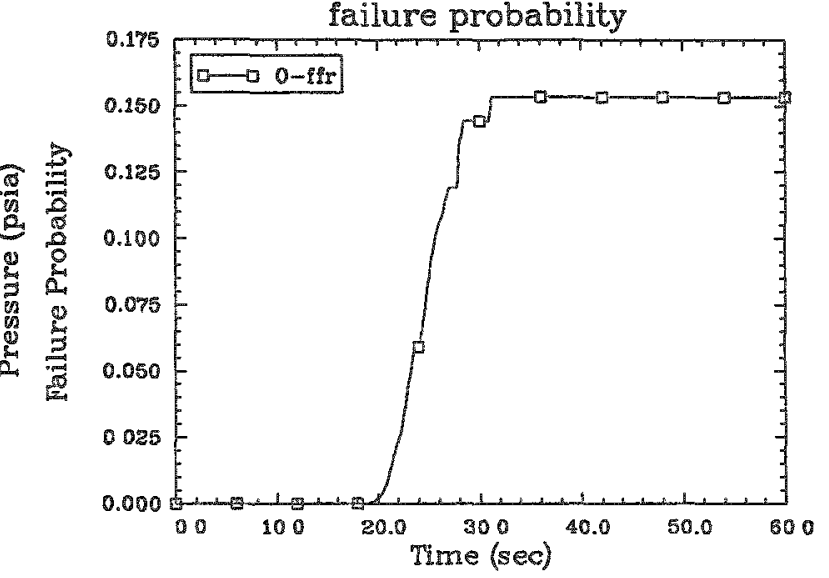

OCONEE 100\%DBA 35 GWD/MTU PIN--PF 2.0 W/TRIP

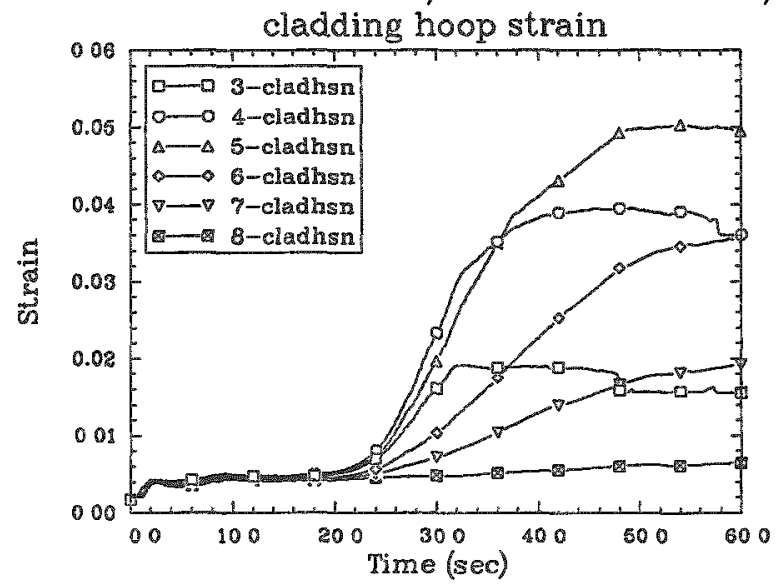

OCONEE 100\%DBA 35 GWD/MTU PIN--PF $2.0 \mathrm{~W} / \mathrm{TRIP}$

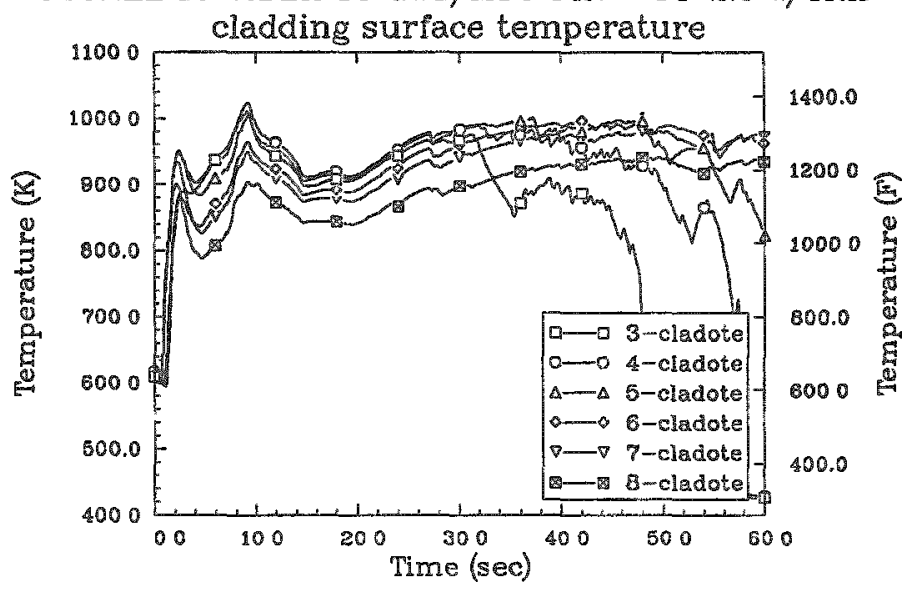

OCONEE 100\%DBA 35 GWD/MTU PIN--PF 2.0 W/TRIP

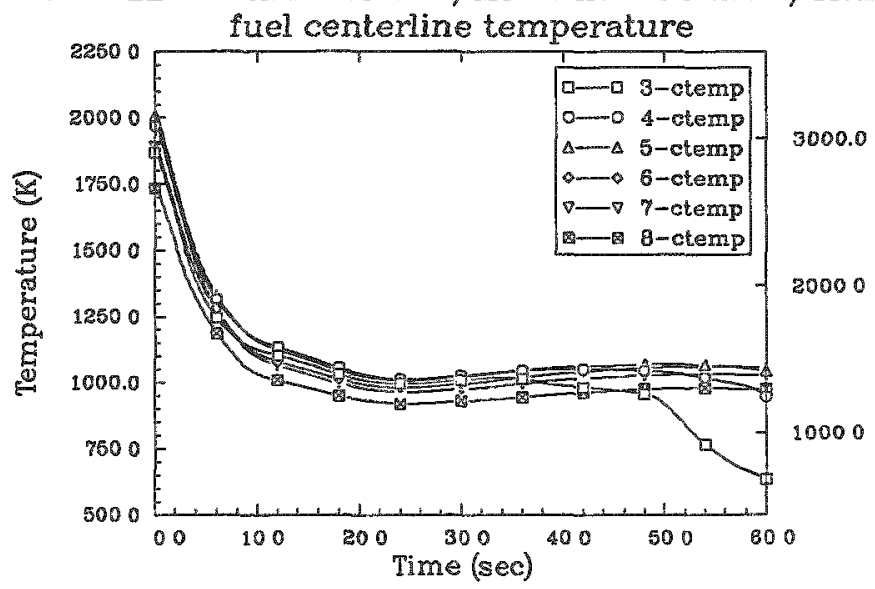

OCONEE 100\%DBA 35 GWD/MTU PIN--PF 2.0 W/TRIP oxide thickness

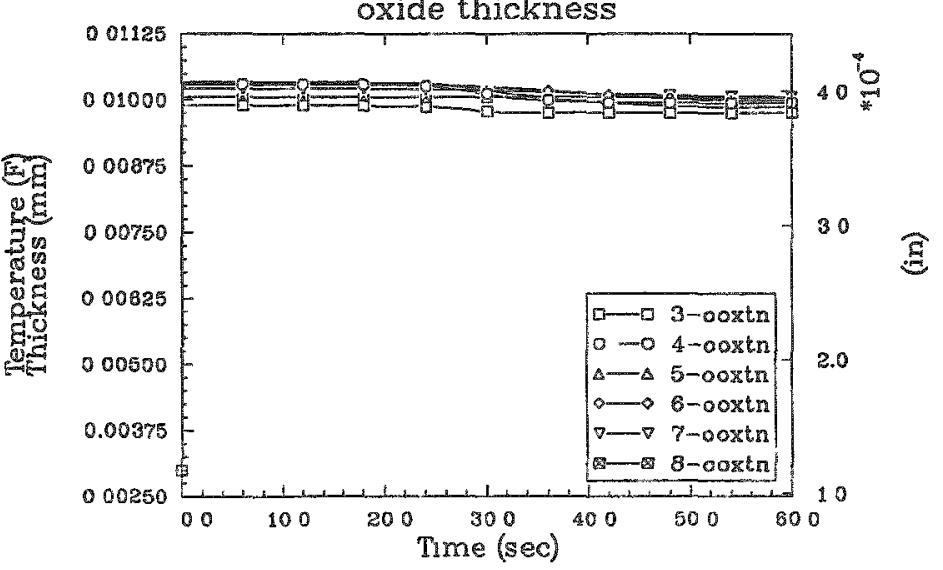


OCONEE 100\%DBA $20 \mathrm{GWD} / \mathrm{MTU}$ PIN -...PF 2.0 W/TRIP internal pin pressure

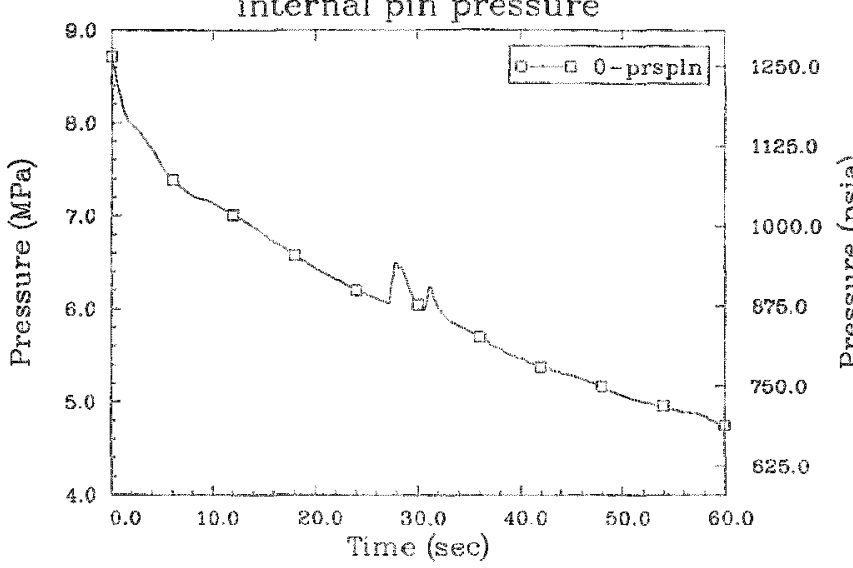

OCONEE 100\%DBA $20 \mathrm{GWD} / \mathrm{MTU}$ PIN-DP $2.0 \mathrm{~W} / \mathrm{TRIP}$ cladding hoop strain

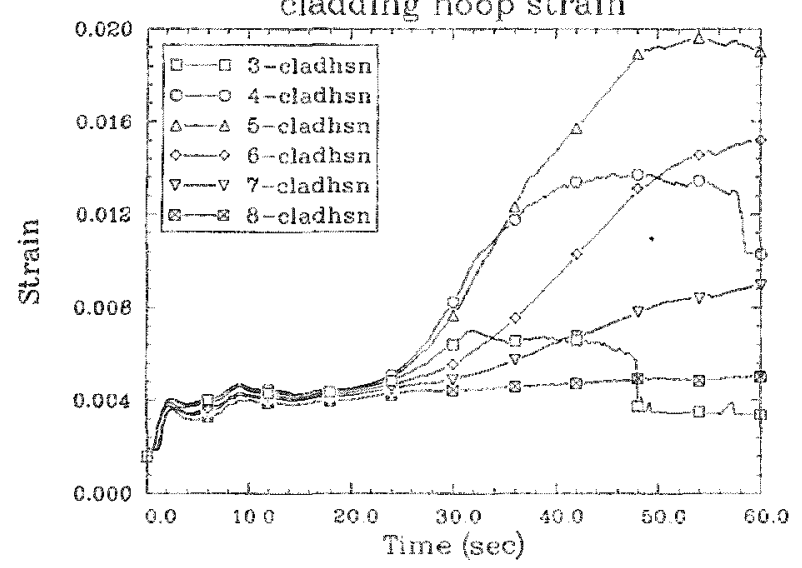

OCONEE 100\%DBA $20 \mathrm{GWD} / \mathrm{MTU} \mathrm{PIN}-\mathrm{PF} 2.0 \mathrm{~W} / \mathrm{TRIP}$

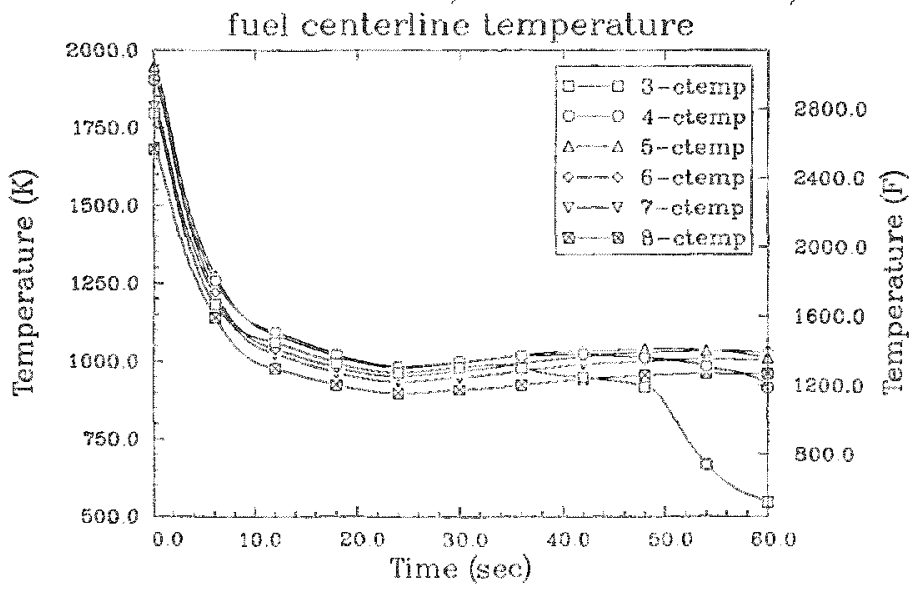

OCONEE 100\%DBA 20 GWD/MTU PIN-DF $2.0 \mathrm{~W} / \mathrm{TRIP}$ failure probability

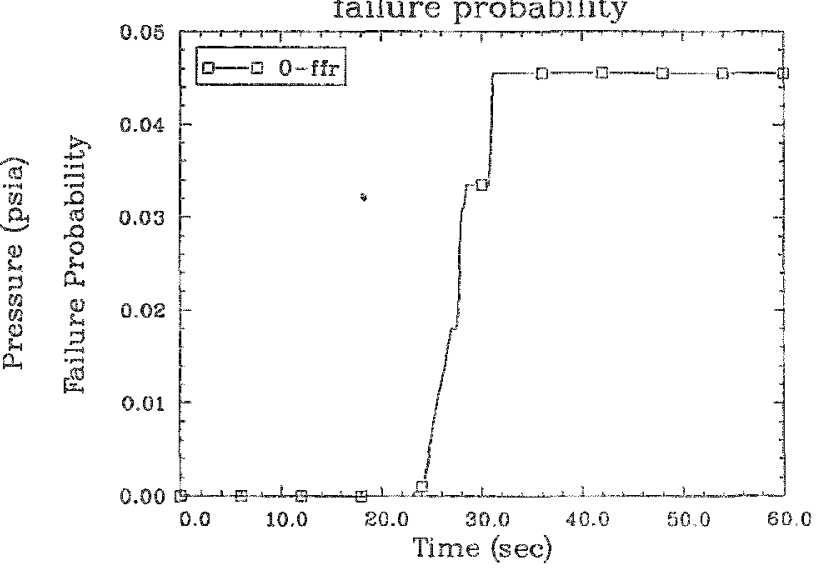

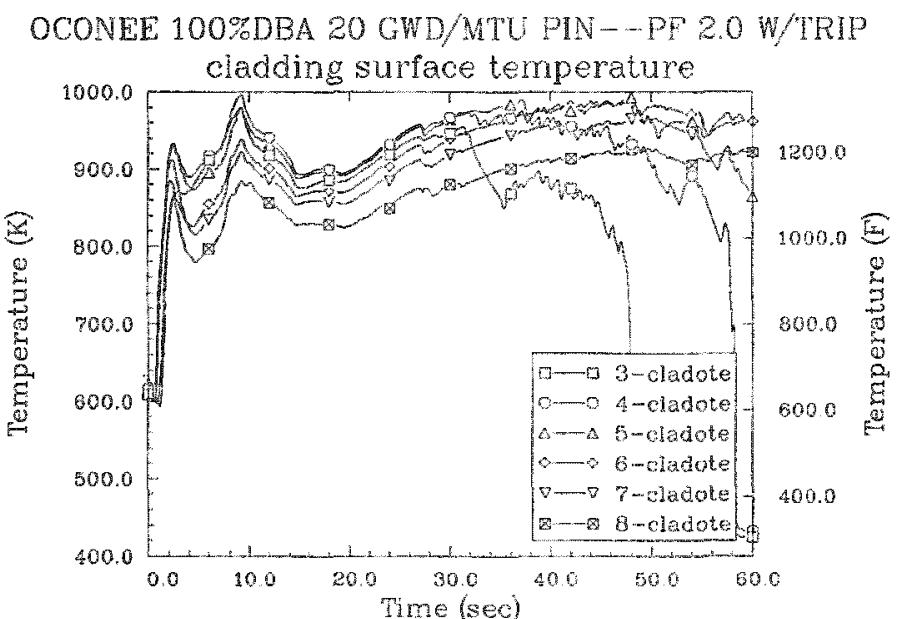

OCONEE 100\%DBA 20 GWD/MTU PIN-.PF 20 W/TRIP

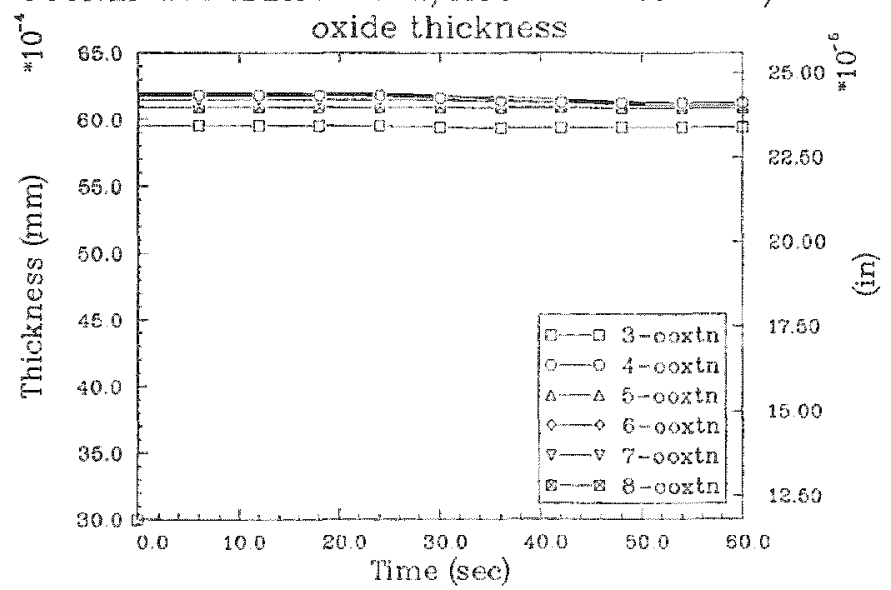


OCONEE 100\%DBA 5 GWD/MTU PIN-DF 2.0 W/TRIP

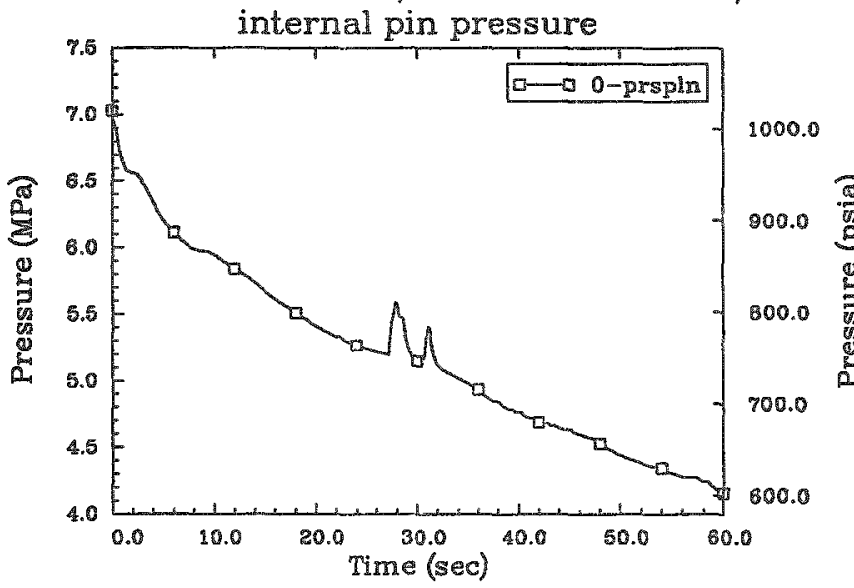

OCONEE 100\%DBA 5 GWD/MTU PIN--PF $2.0 \mathrm{~W} / \mathrm{TRIP}$ cladding hoop strain

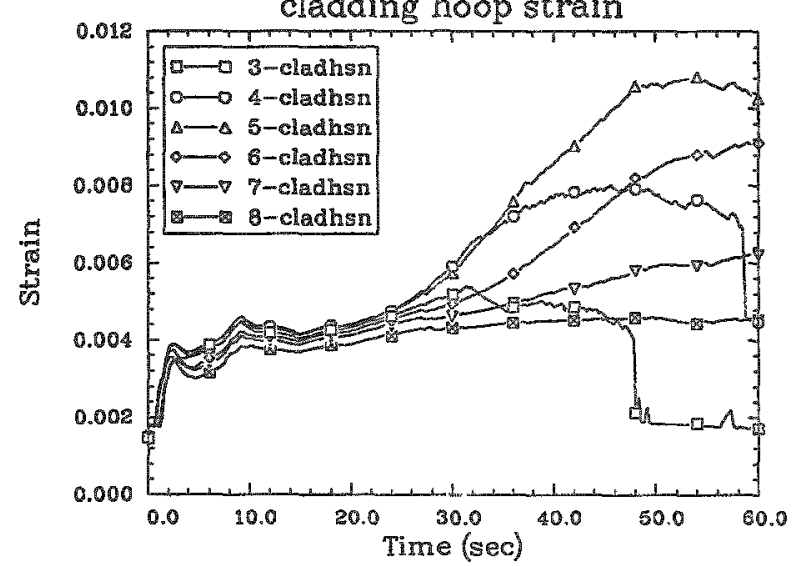

OCONEE 100\%DBA 5 GWD/MTU PIN--PF 2.0 W/TRIP

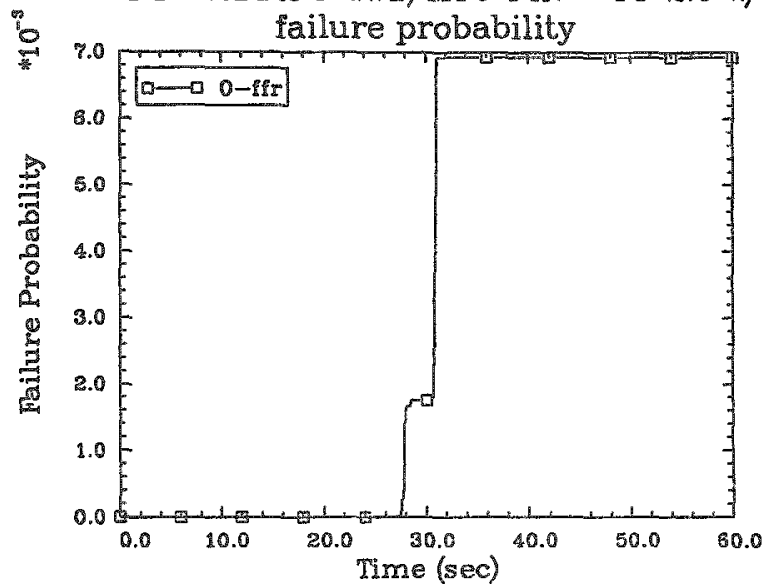

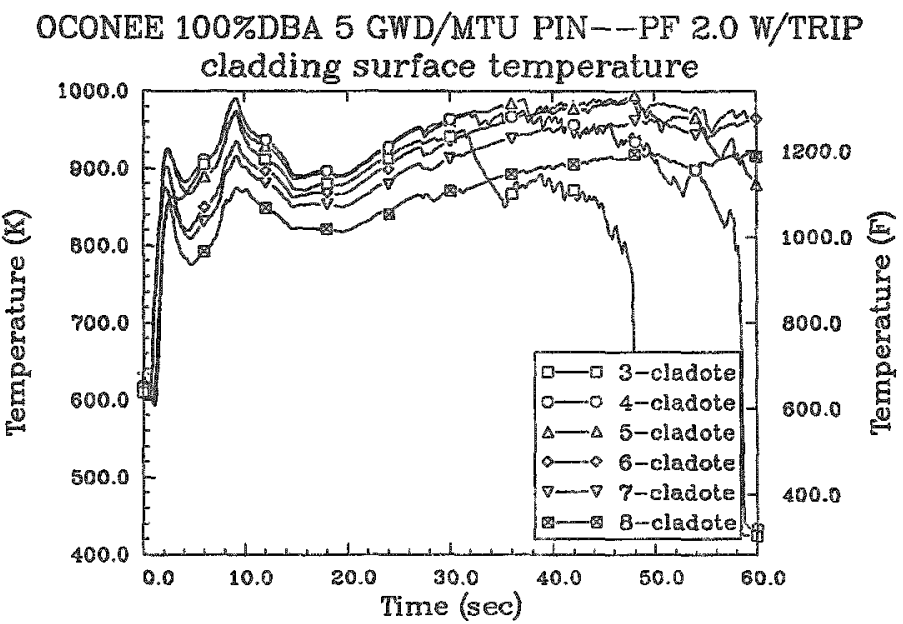

OCONEE 100\%DBA 5 GWD/MTU PIN--PF 2.0 W/TRIP

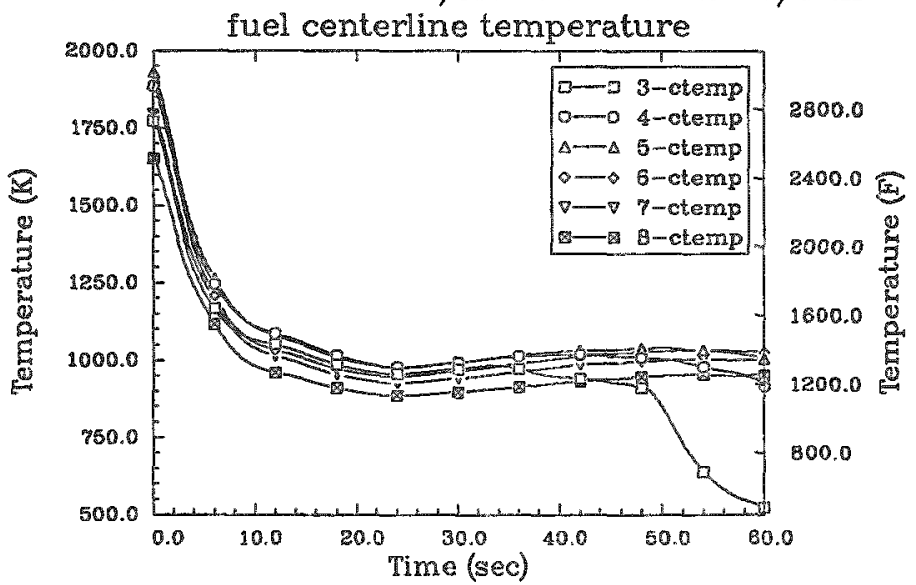

OCONEE 100\%DBA 5 GWD/MTU PIN--PF 2.0 W/TRIP

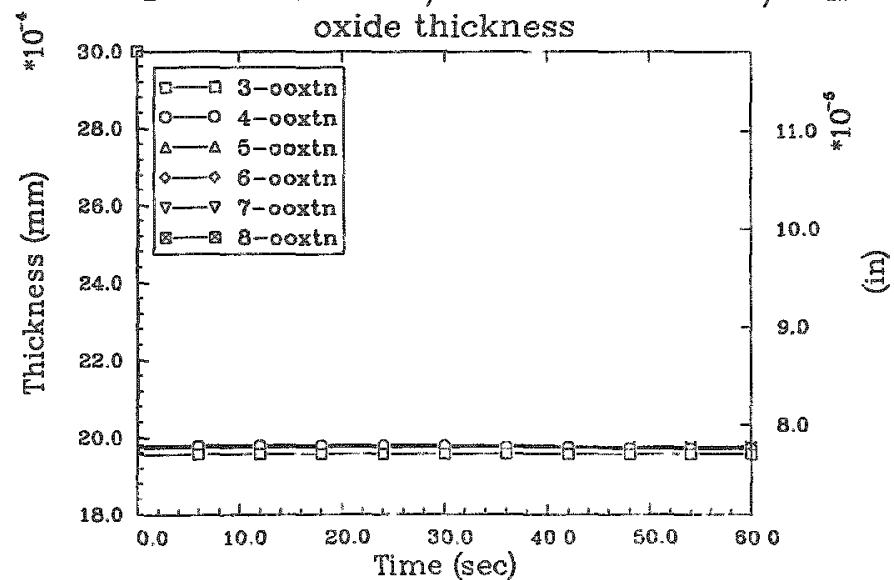



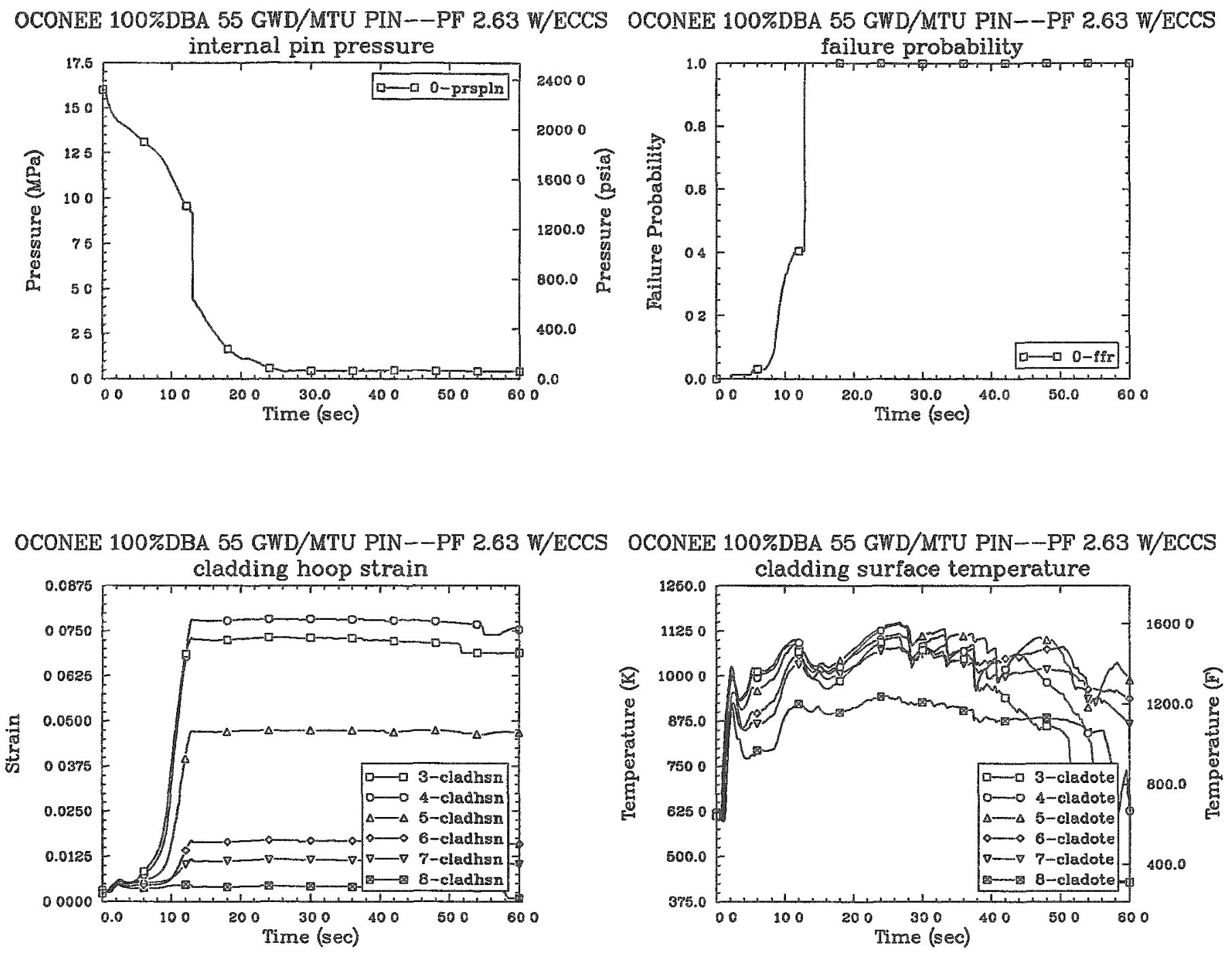

OCONEE 100\%DBA 55 GWD/MTU PIN--PF 2.63 W/ECCS

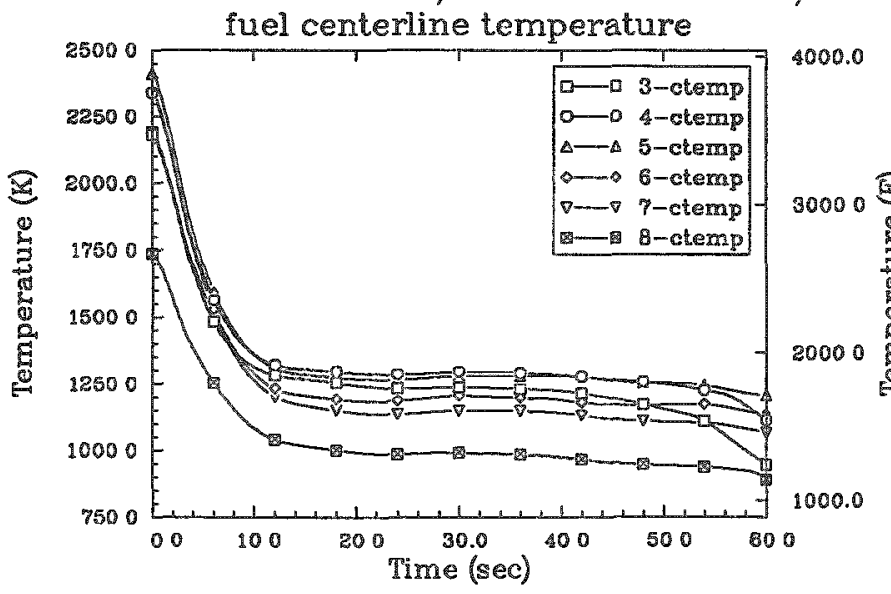

OCONEE 100\%DBA 55 GWD/MTU PIN--PF 2.63 W/ECCS

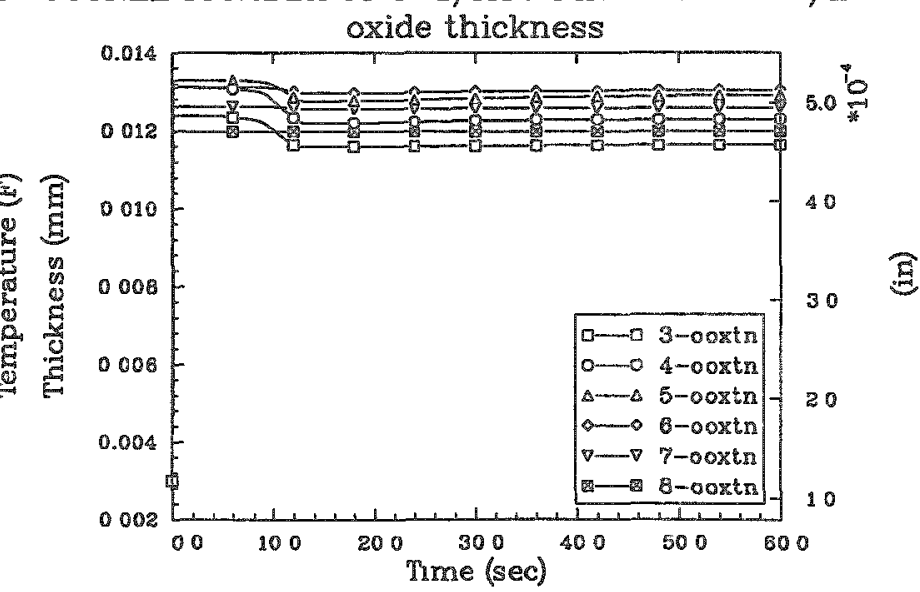




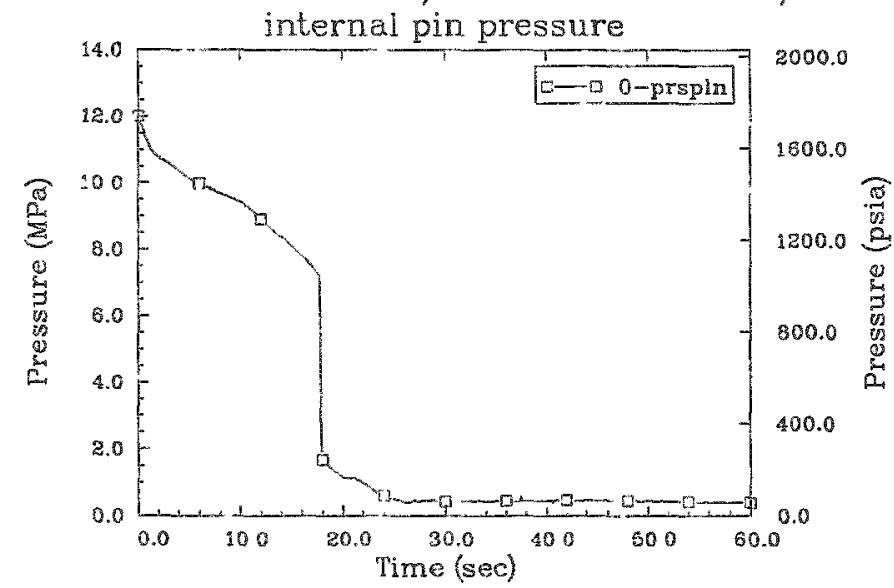

OCONEE 100\%DBA 35 GWD/MTU PIN--PF 2.63 W/ECCS

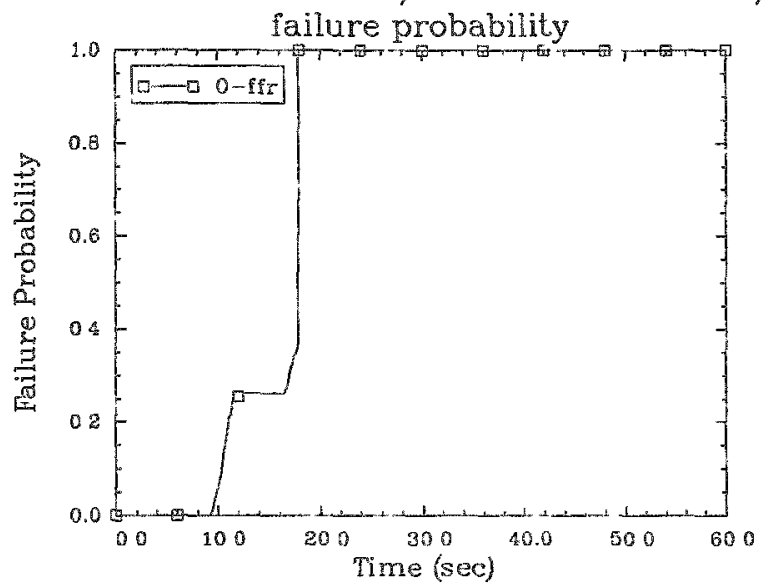

OCONEE 100\%DBA 35 GWD/MTU PIN -.-PF 2.63 W/ECCS

OCONEE 100\%DBA 35 GWD/MTU PIN - PF 2.63 W/ECCS
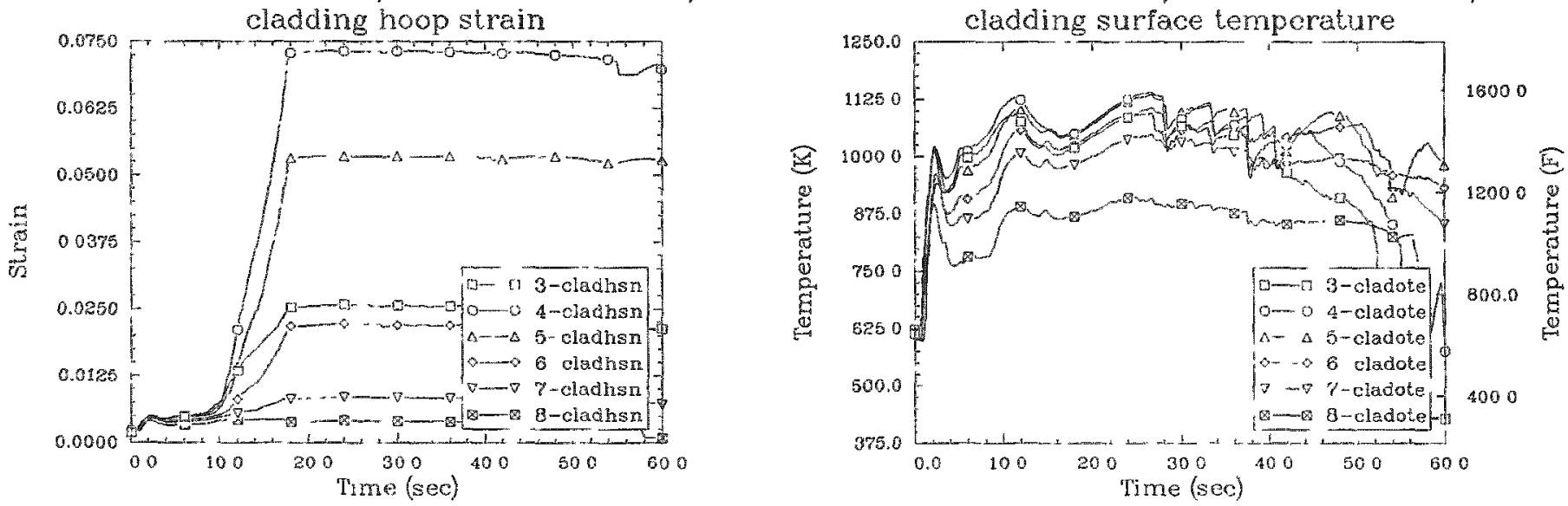

OCONEE 100\%DBA 35 GWD/MTU PIN--PF 2.63 W/FCCS

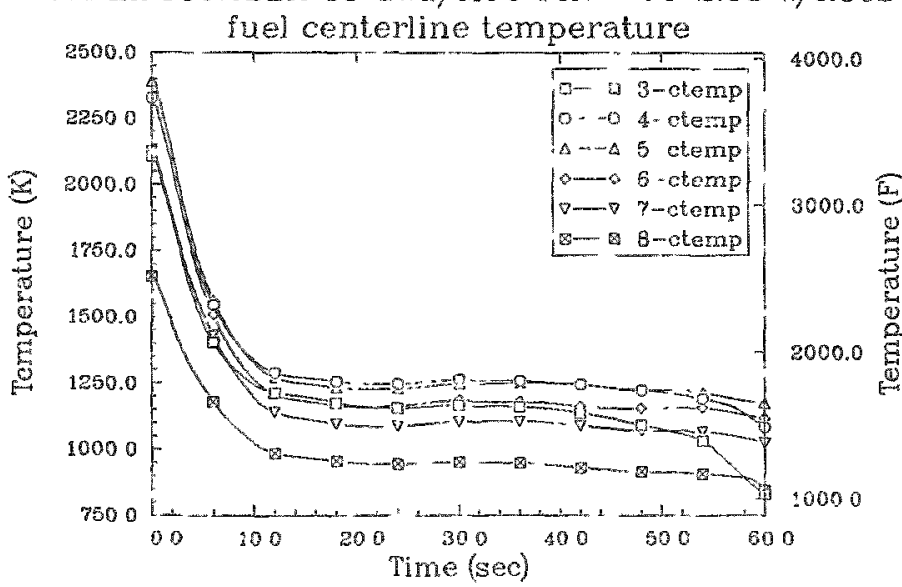

OCONEF 100\%DBA 35 GWD/MTU PIN - PF 263 W/ECCS

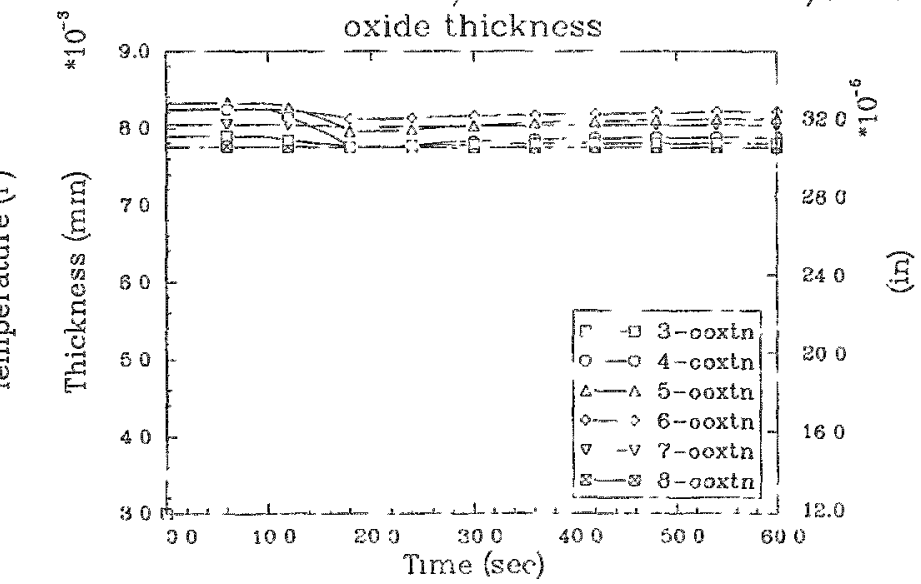


OCONEF 100\%DBA 20 GWD/MTU HA- - PB 2.63 W/ECCS

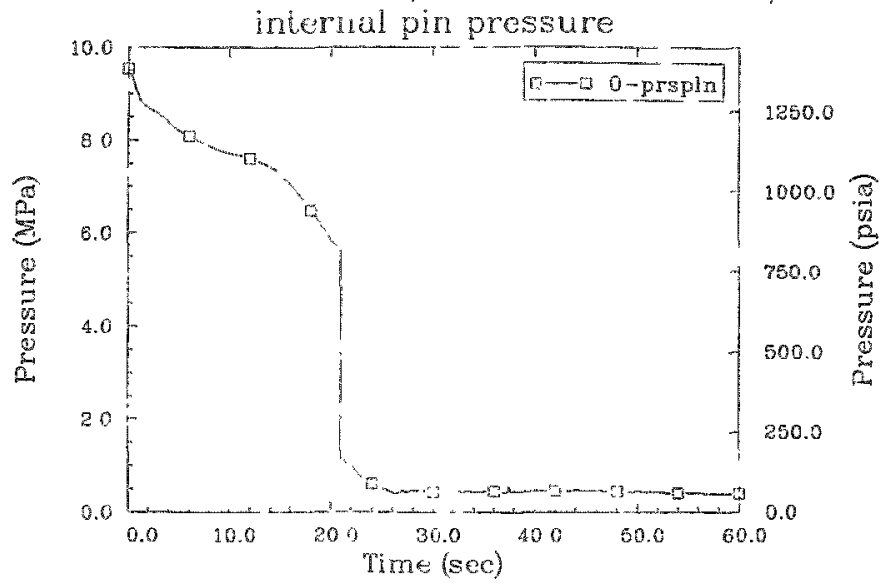

OCONEE 100\%DBA 20 GWD/MTU PIN--DF 2.63 W/ECCS failure probability

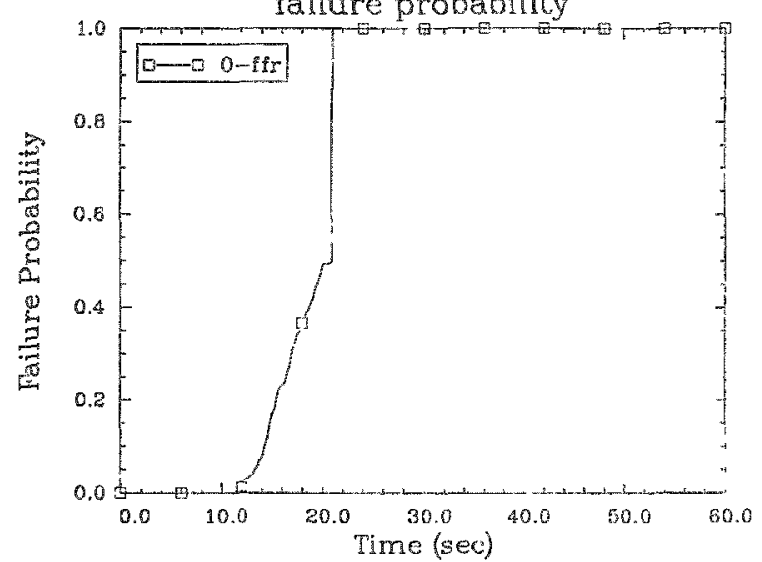

OCONEF $100 \%$ DBA 20 GWD/MTU PIN-IPF 2.63 W/ECCS

OCONEE 100\%DBA $20 \mathrm{GWD} / \mathrm{MTU}$ PIN - PF 2.63 W/ECCS
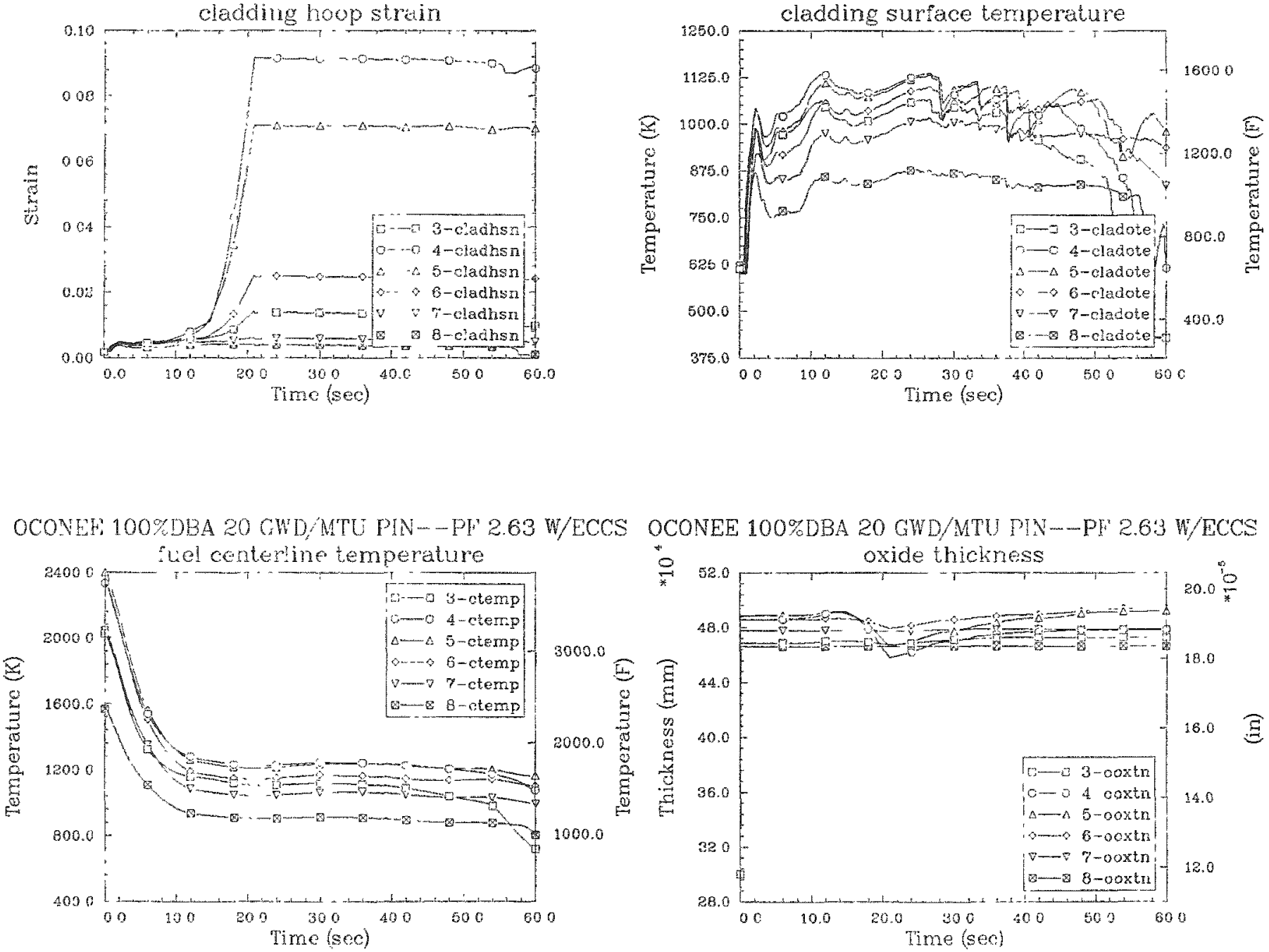
OCONEE 100\%DBA 5 GWD/MTU PIN--PF 2.63 W/ECCS

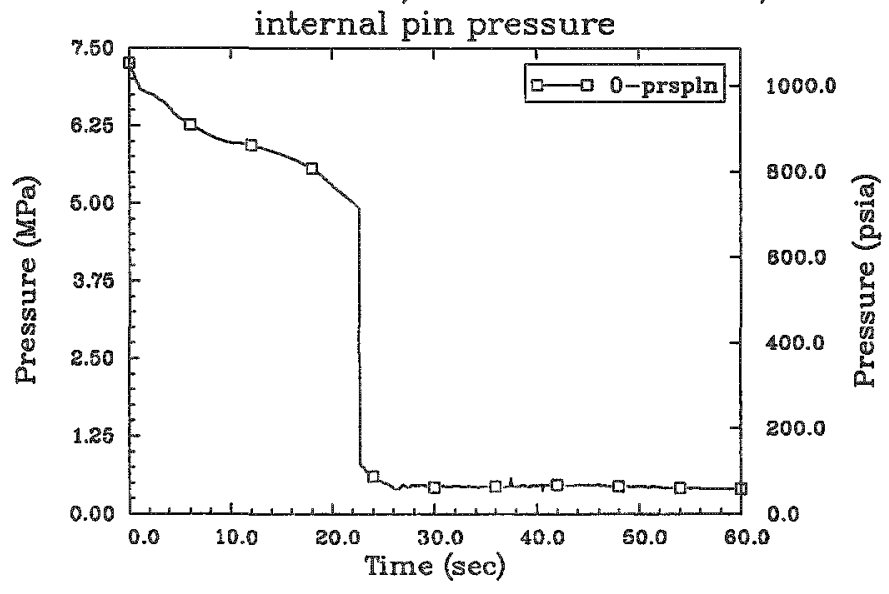

OCONEE 100\%DBA 5 GWD/MTU PIN-DPF 2.63 W/ECCS

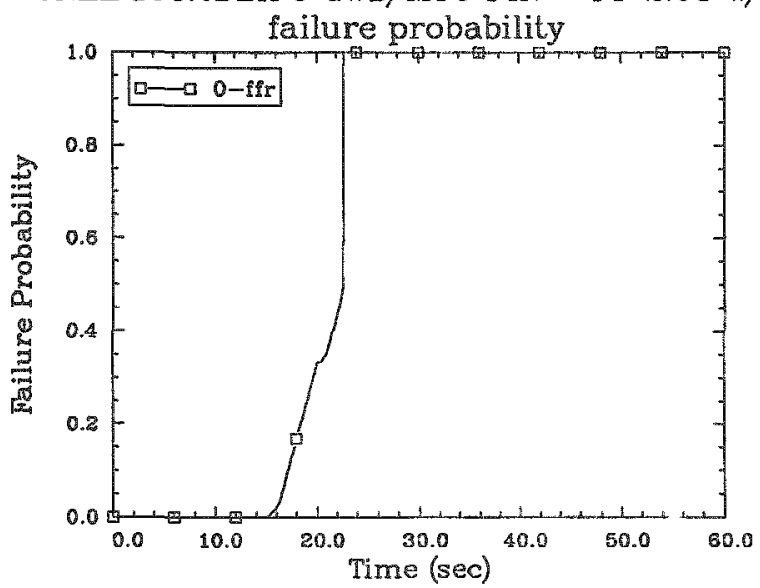

OCONEE 100\%DBA 5 GWD/MTU PIN--PF 2.63 W/ECCS

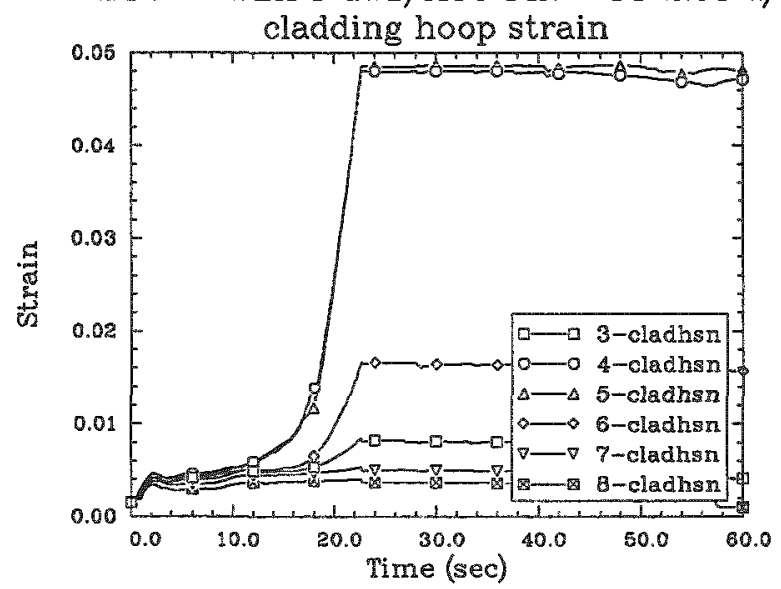

OCONEE 100\%DBA 5 GWD/MTU PIN--PF 2.63 W/ECCS

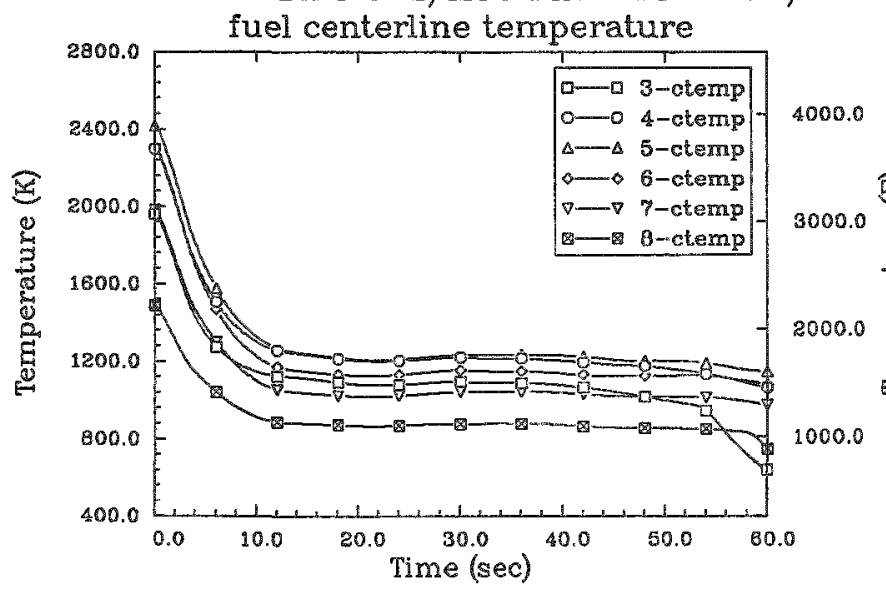

OCONEE 100\%DBA 5 GWD/MTU PIN--PF 2.63 W/ECCS

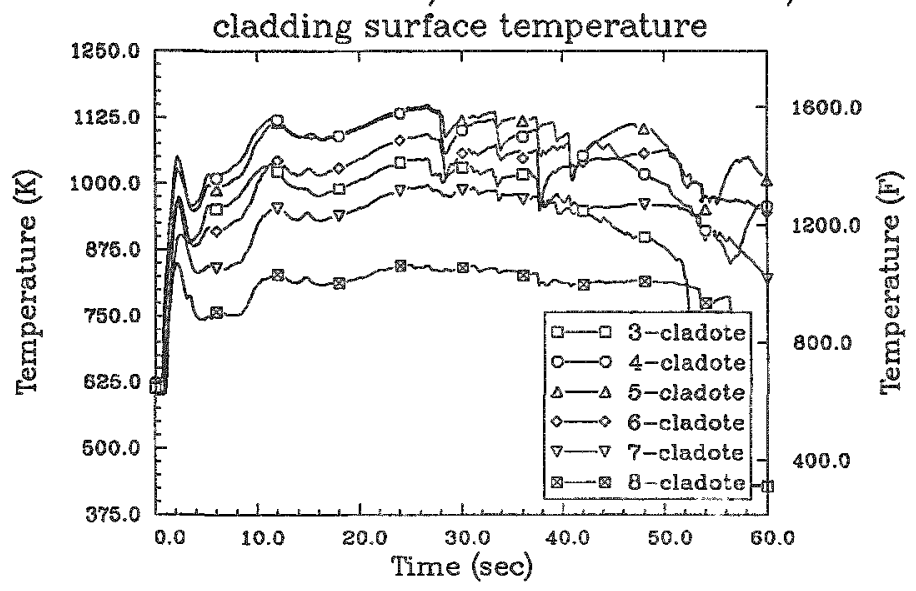

OCONEE 100\%DBA 5 GWD/MTU PIN--PF 2.63 W/ECCS

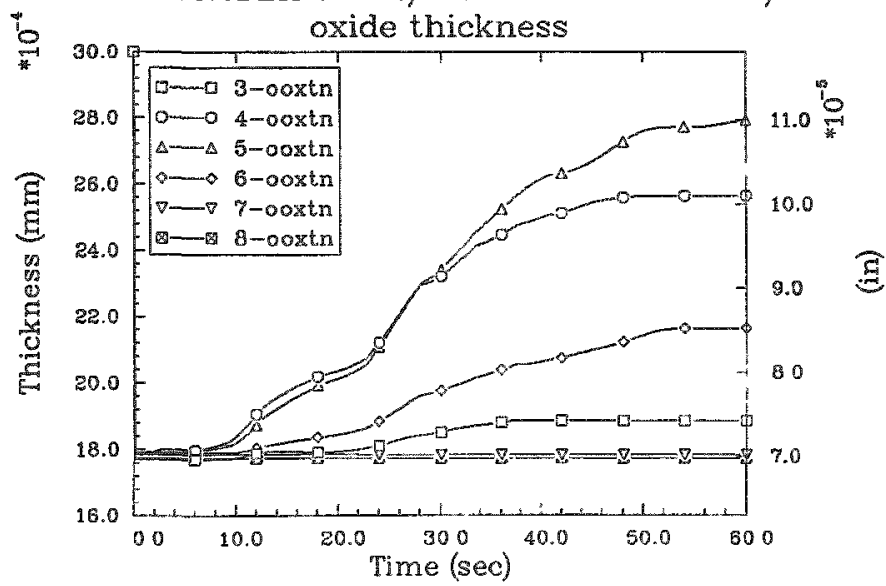


OCONEE 100\%DBA 55 GWD/MTU PIN--PF 2.4 W/ECCS

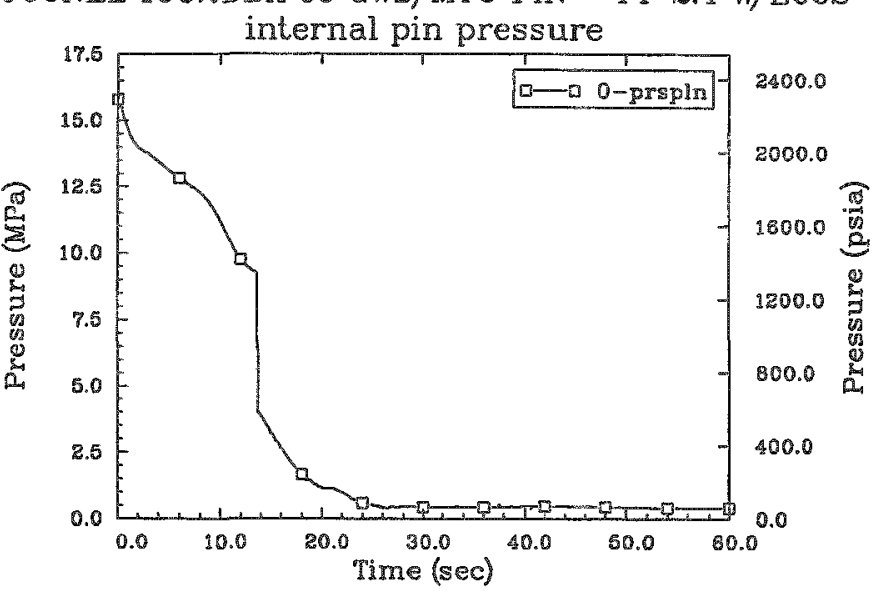

OCONEE 100\%DBA 55 GWD/MTU PIN--PF 2.4 W/ECCS failure probability

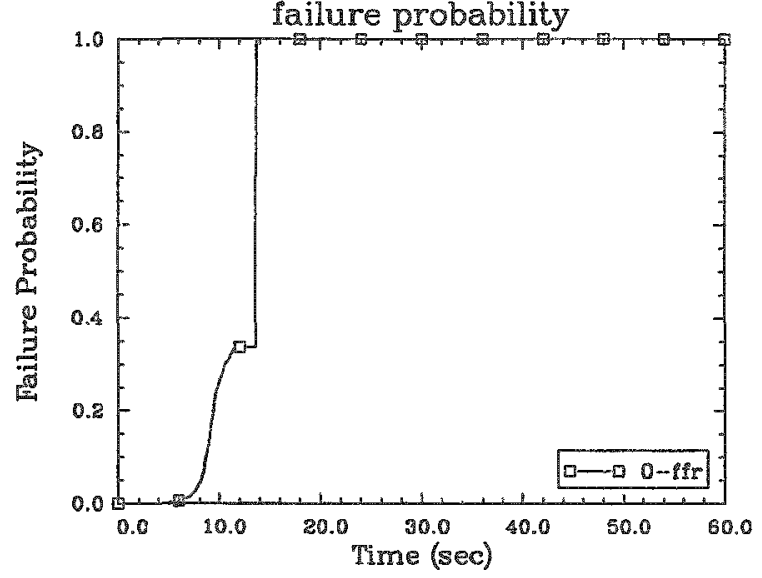

OCONEE 100\%DBA 55 GWD/MTU PIN--PF 2.4 W/ECCS cladding hoop strain

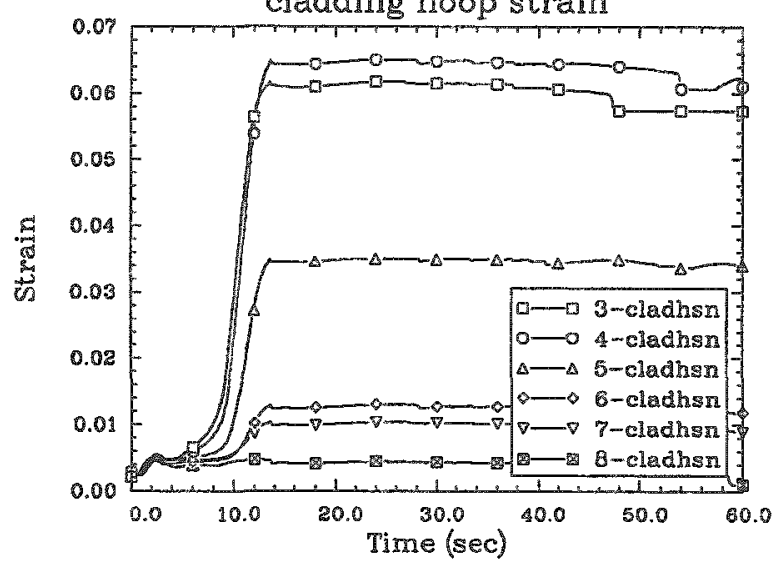

OCONEE 100\%DBA 55 GWD/MTU PIN--PF 2.4 W/ECCS

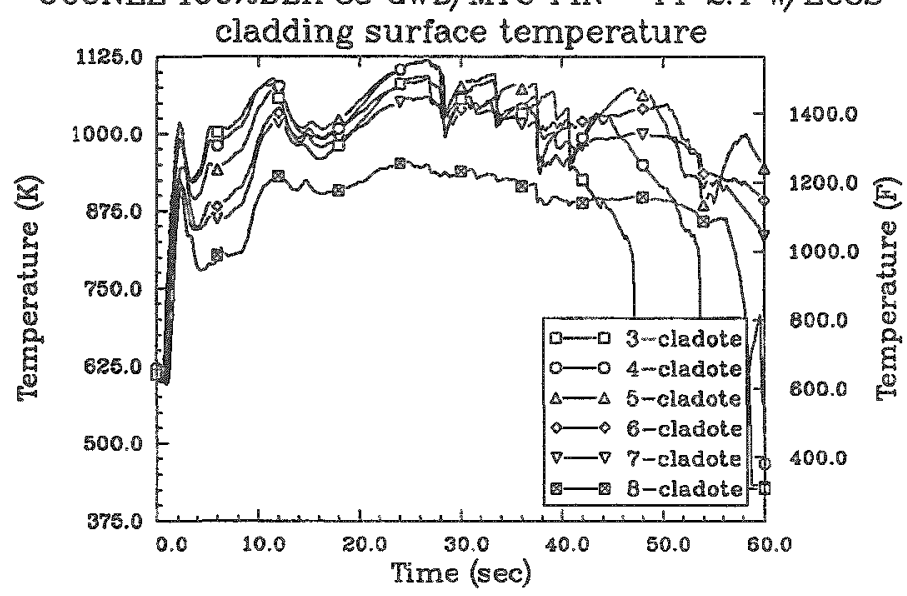

OCONEE 100\%DBA 55 GWD/MTU PIN--PF 2.4 W/ECCS

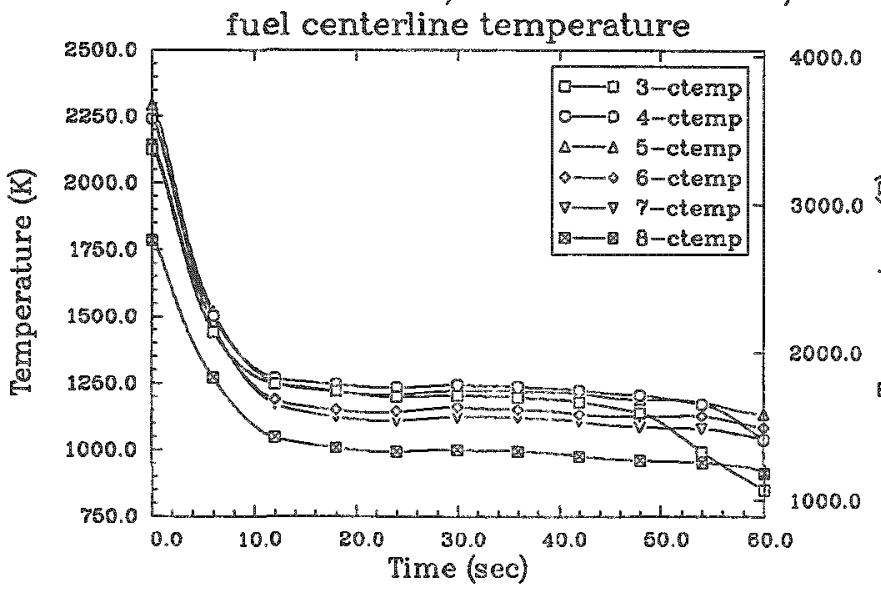

OCONEE 100\%DBA 55 GWD/MTU PIN--PF 2.4 W/ECCS

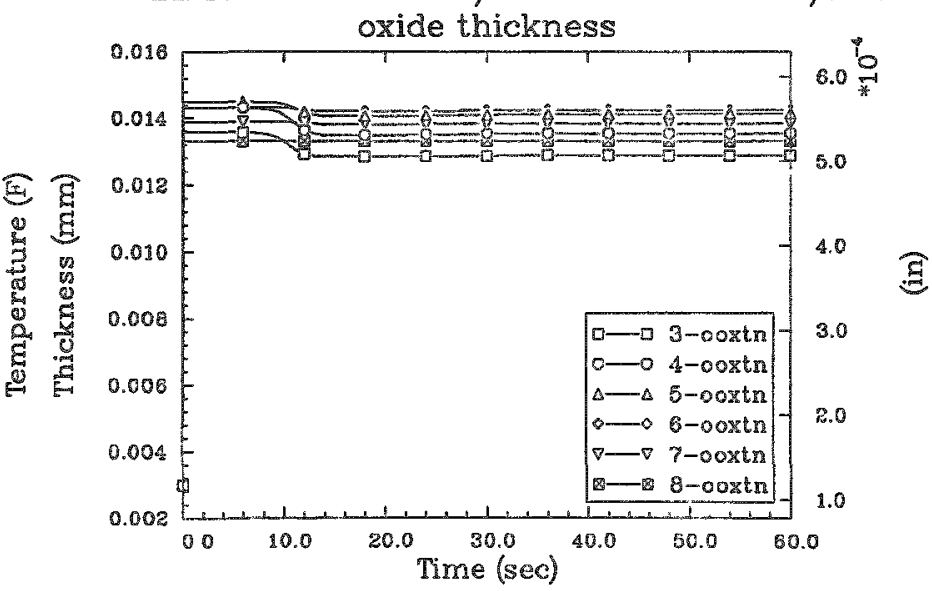



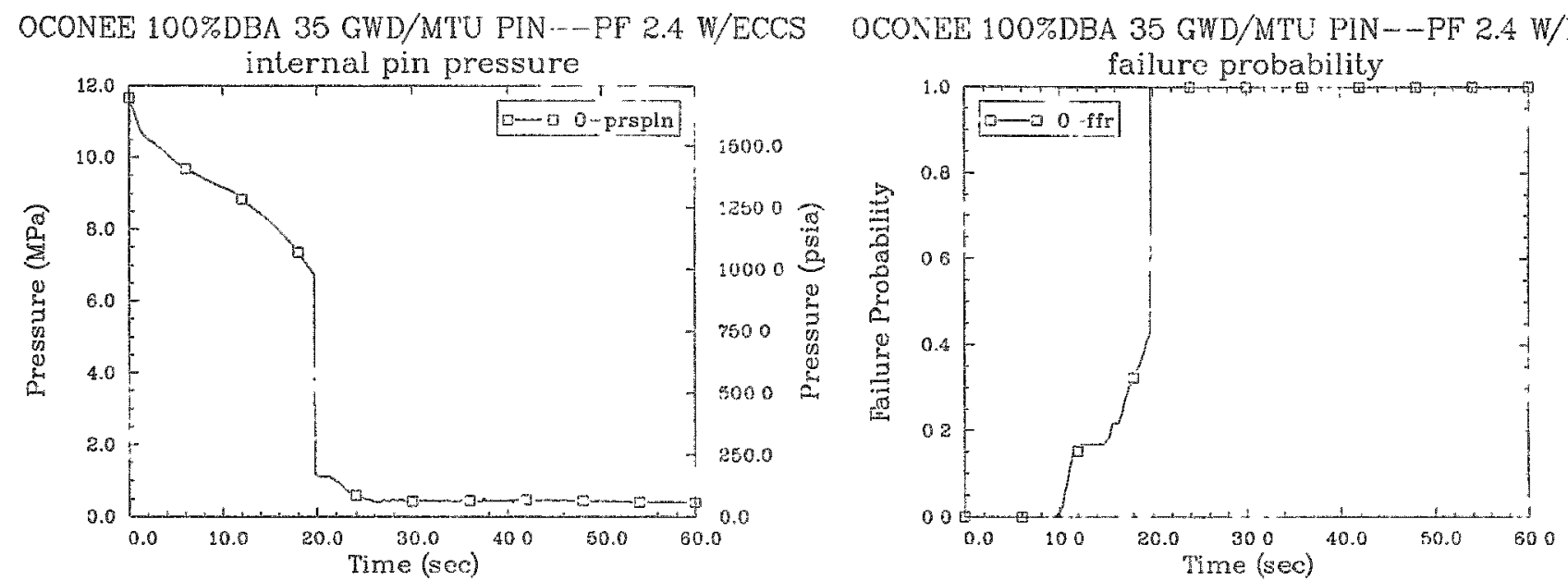

OCONEE 100\%DBA 35 GWD/MTU PIN-- PF 2.4 W/FCCS

OCONEE 100\%DBA 35 GWD/MTU PIN--PF 2.4 W/ECCS
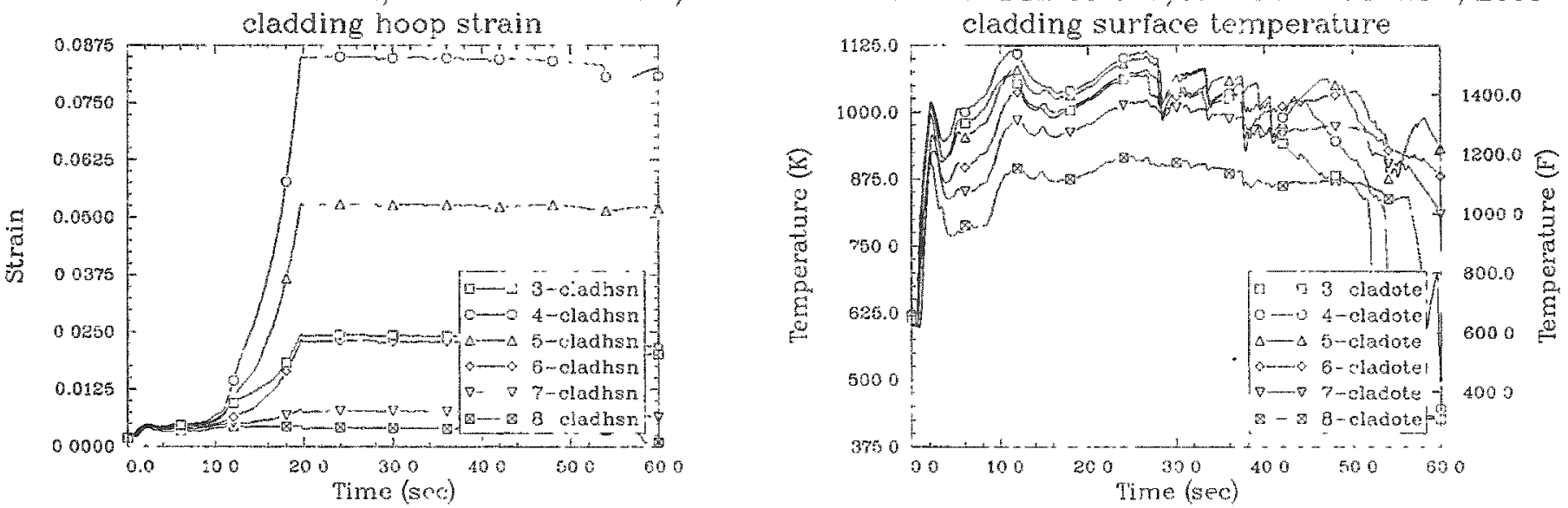

OCONEE 100\%DEA 35 GWD/MTU PIN--IF 2.4 W/LCCS

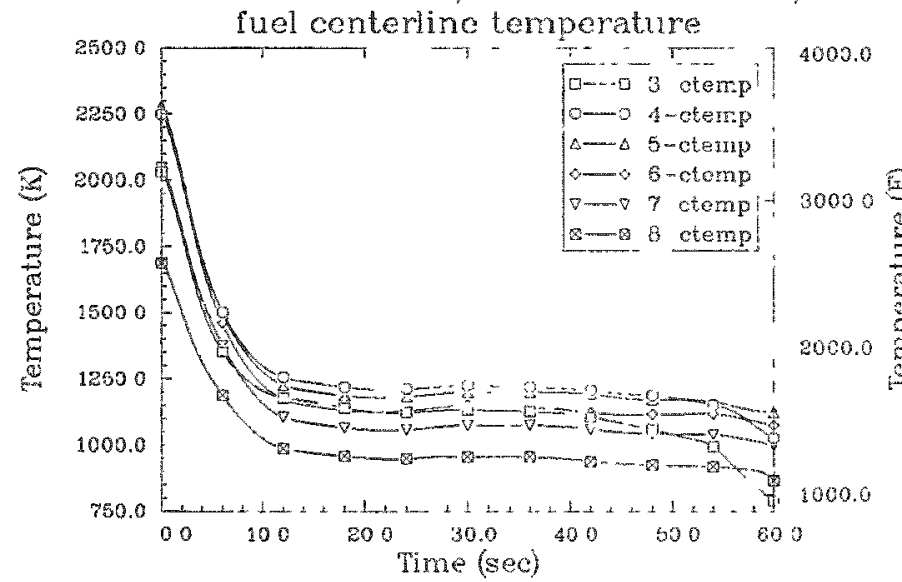

DCONEE $100 \%$ DBA 35 GWD/MTU PIN-FF 2.4 W/FCCS

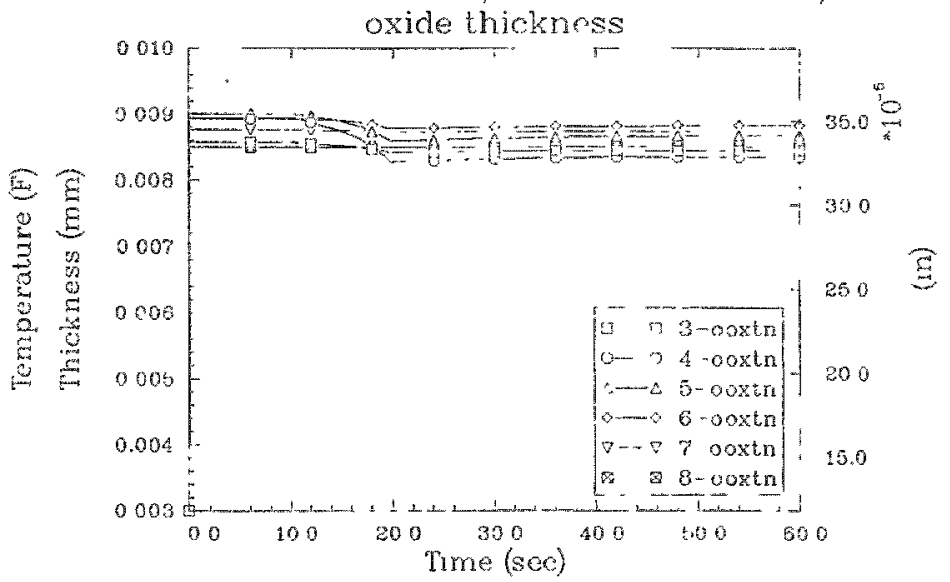


OCONEE 100\%DBA 20 GWD/MTU PIN-DPF 2.4 W/ECCS

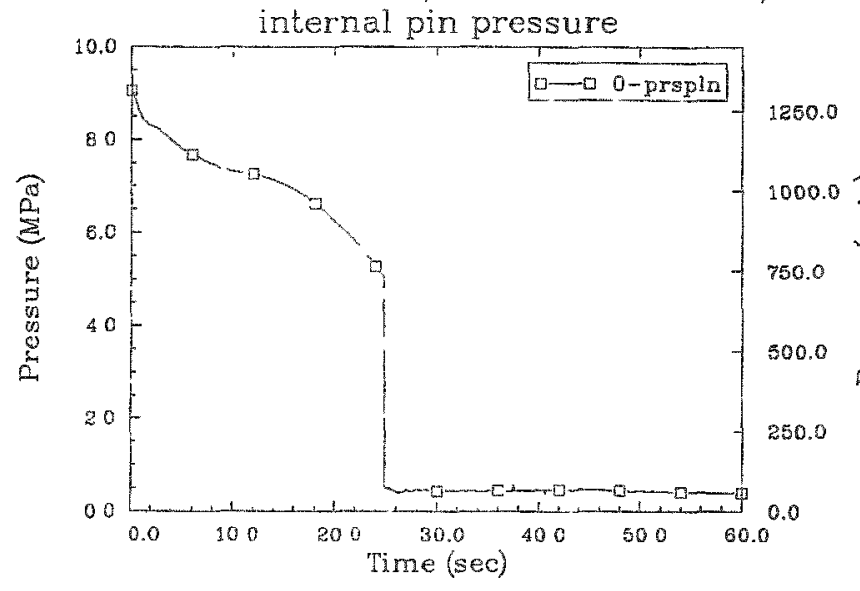

OCONEE 100\%DBA 20 GWD/MTU PIN-PF 2.4 W/ECCS

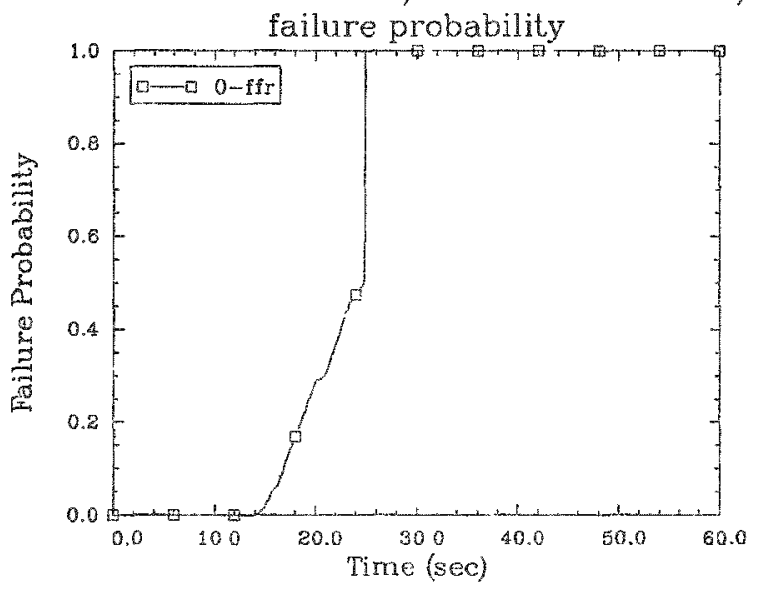

OCONEE 100\%DBA 20 GWD,MTU PIN--PF $2.4 \mathrm{~W} / \mathrm{ECCS}$

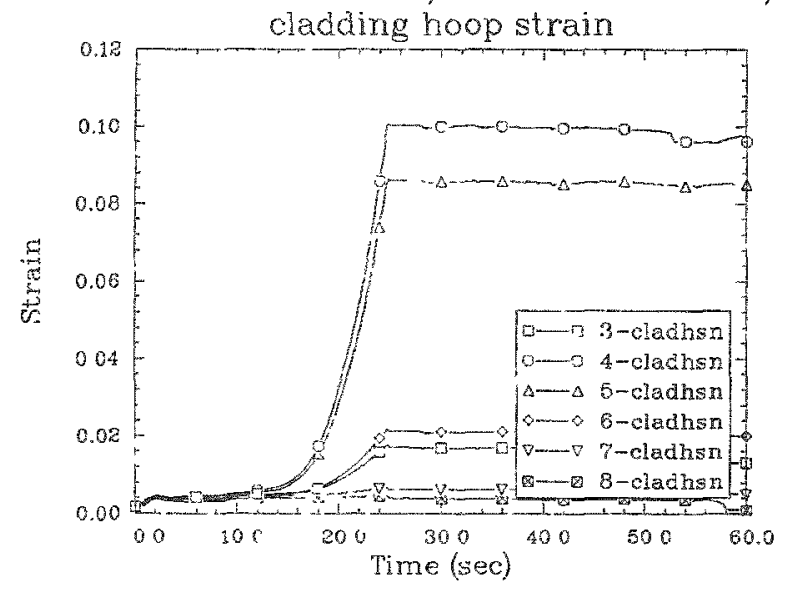

OCONEE 100\%DBA 20 GWD/MTU PIN--PF 2.4 W/ECCS

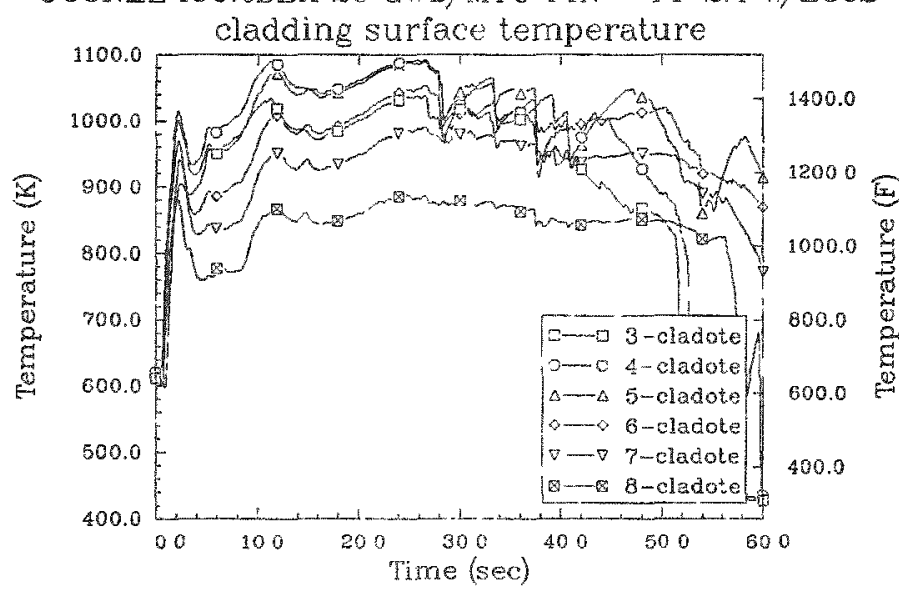

OCONEE 100\%DBA 20 GWD/MTU PIN--PF 2.4 W/ECCS

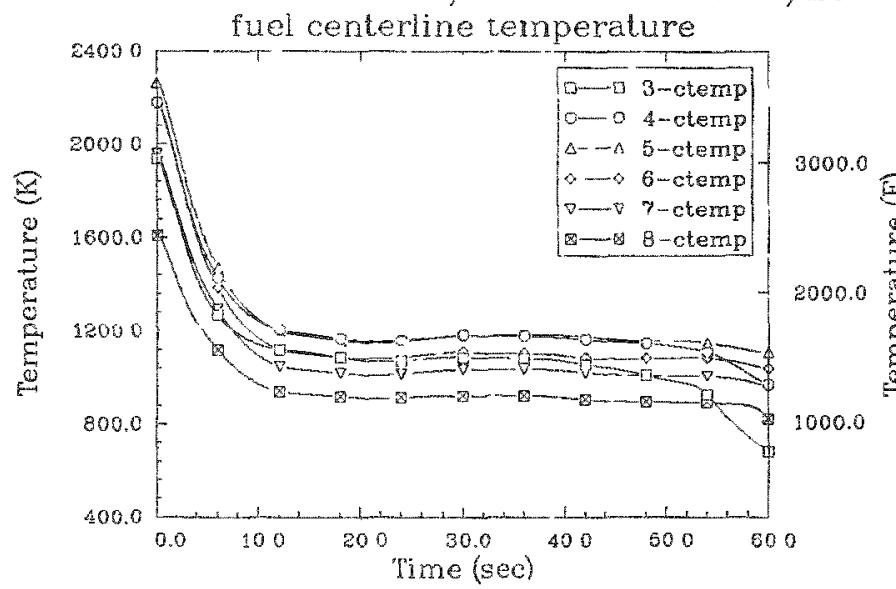

OCONEE $100 \%$ DBA 20 GWD/MIU PIN -..PF 2.4 W/ECCS

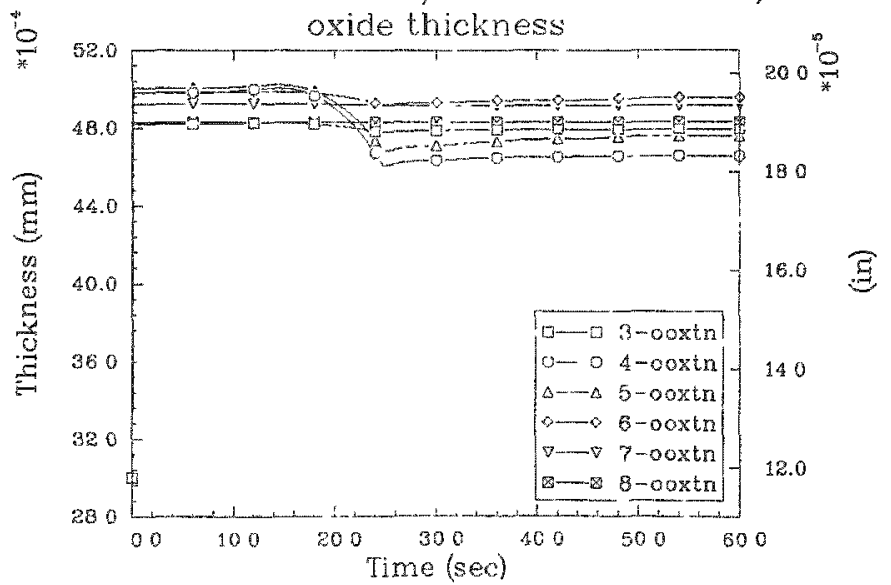


OCONEE 100\%DBA 5 GWD/MTU PIN--PF 2.4 W/ECCS internal pin pressure

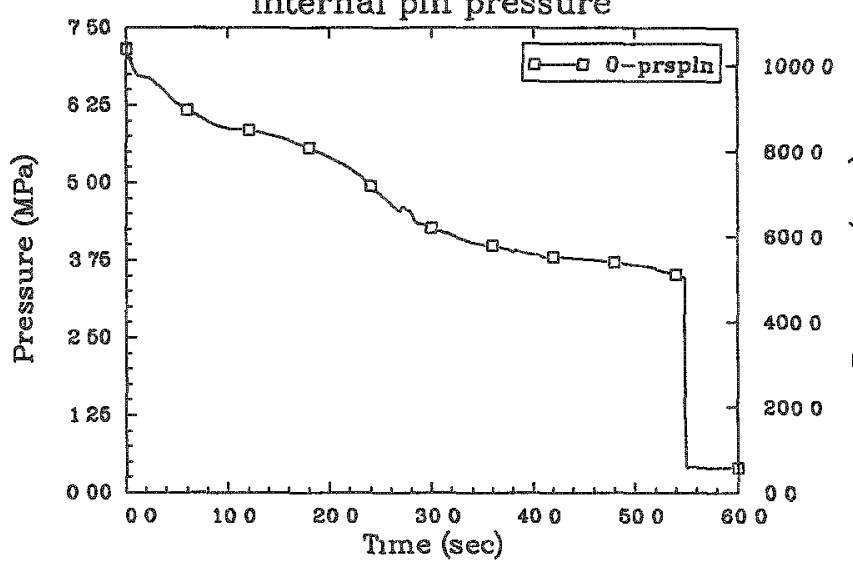

OCONEE 100\%DBA 5 GWD/MTU PIN--PF 24 W/ECCS cladding hoop stram

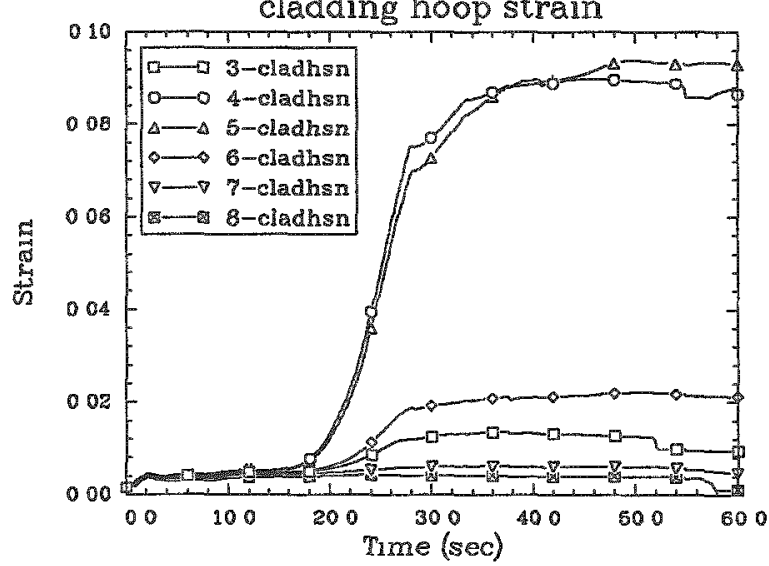

OCONEE 100\%DBA 5 GWD/MTU PIN--PF 24 W/ECCS

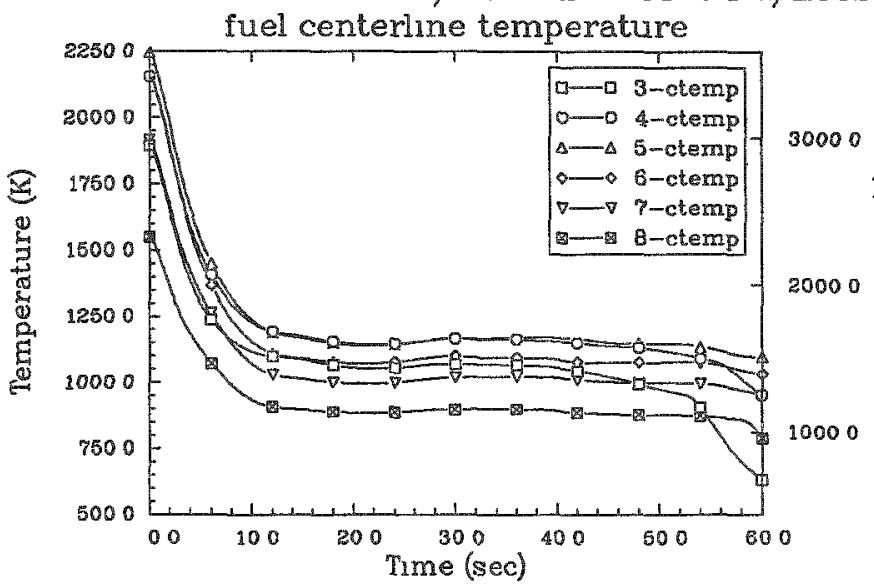

OCONEE 100\%DBA 5 GWD/MTU PIN--PF 2.4 W/ECCS failure probability

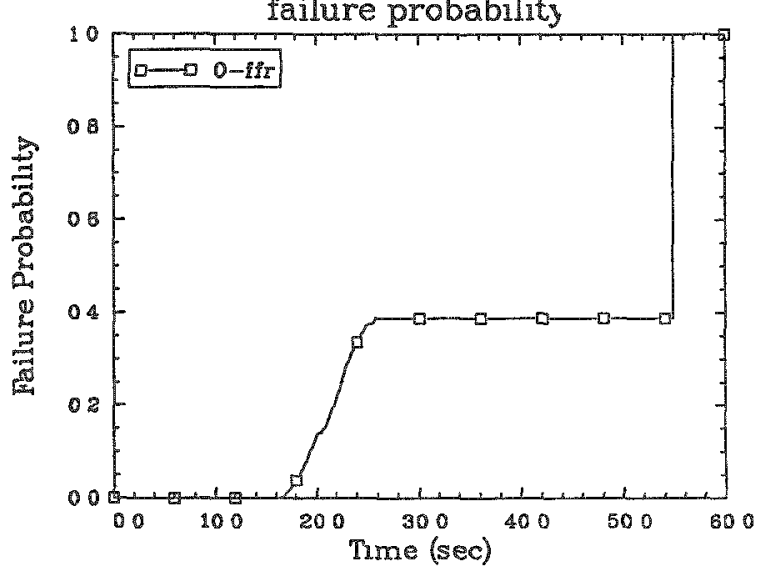

OCONEE 100\%DBA 5 GWD/MTU PIN--PF 24 W/ECCS

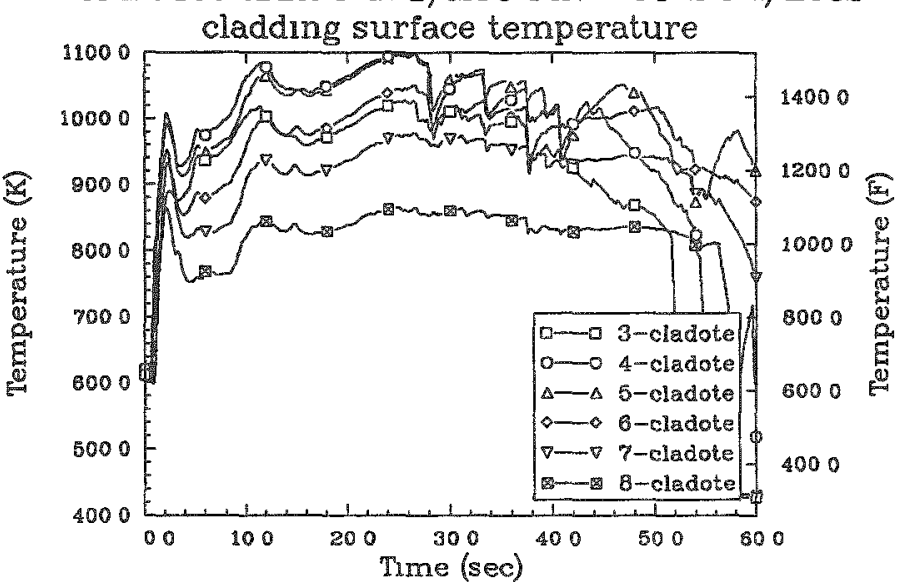

OCONEE 100\%DBA 5 GWD/MTU PIN--PF 24 W/ECCS i。

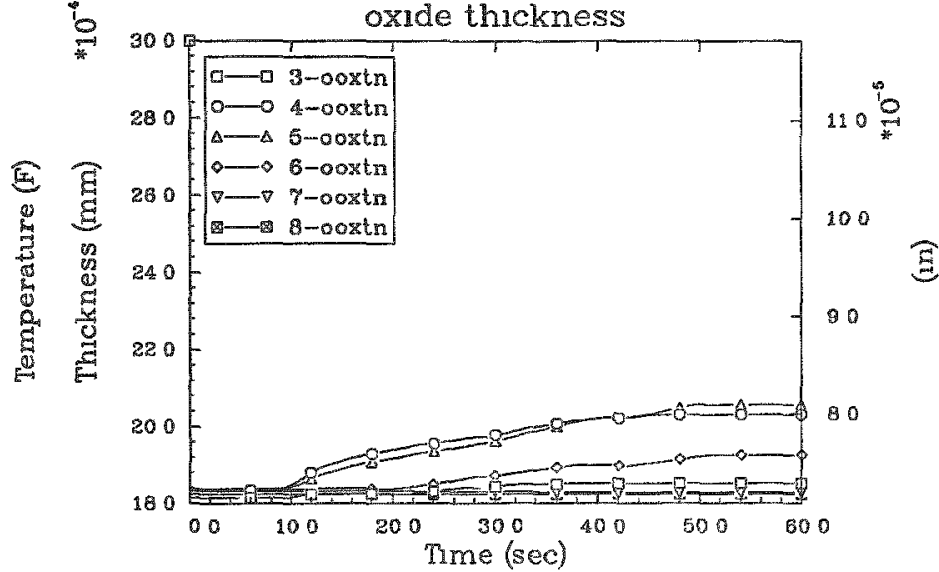


OCONFF 100\%DBA 55 GWD/MIU PIN--PF 22 W/ECCS

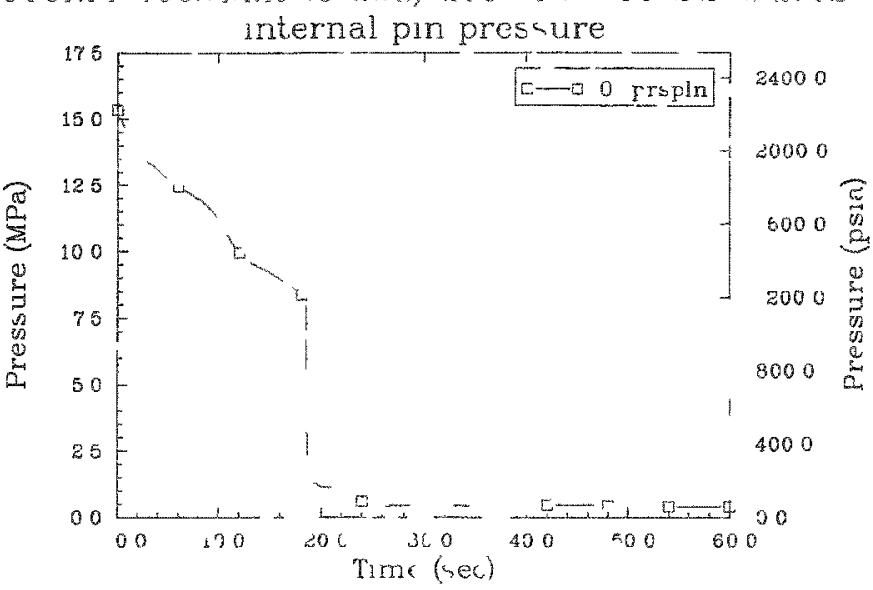

OCONFF $100 \%$ DBA 55 GWD/MUU PI $-\mathrm{P}_{1} 23 \mathrm{~W} / \mathrm{LCCS}$ fallur probabilitv

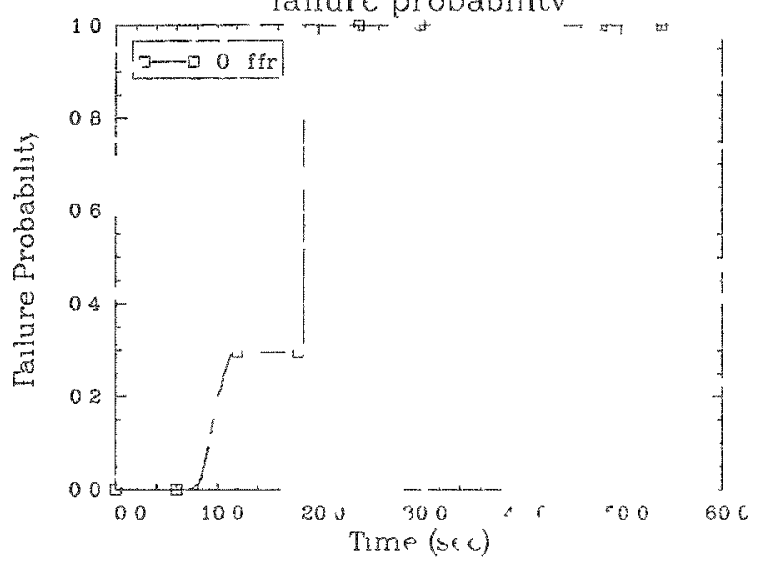

OCONLL 100\%DBA 53 GWD, MIL PIN--PI $22 \mathrm{~W} / \mathrm{ECCS}$

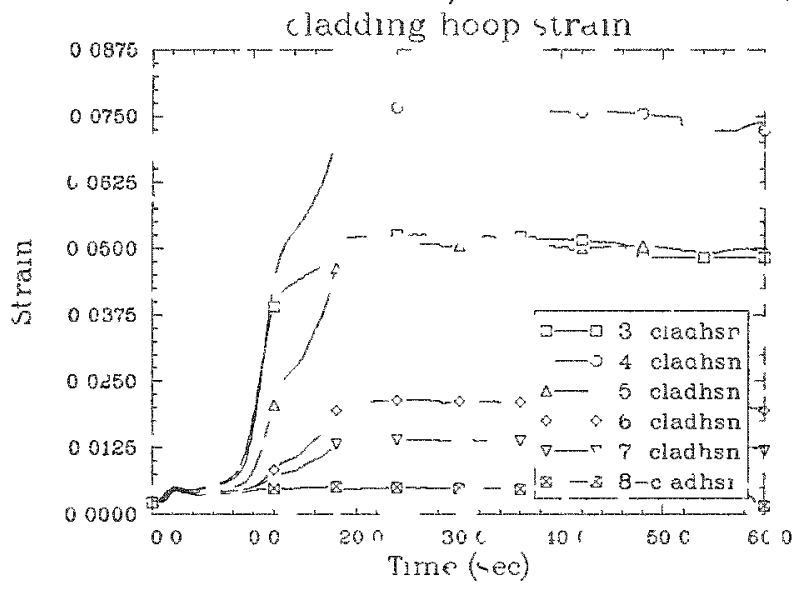

OCONEL 100\%DBA 55 GWD MIL PIV -PT 22 W/ECCS

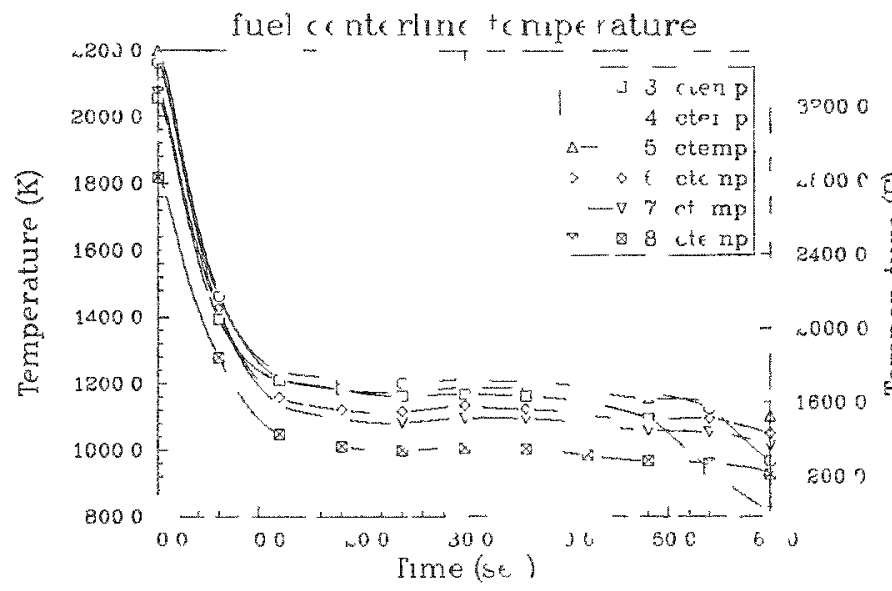

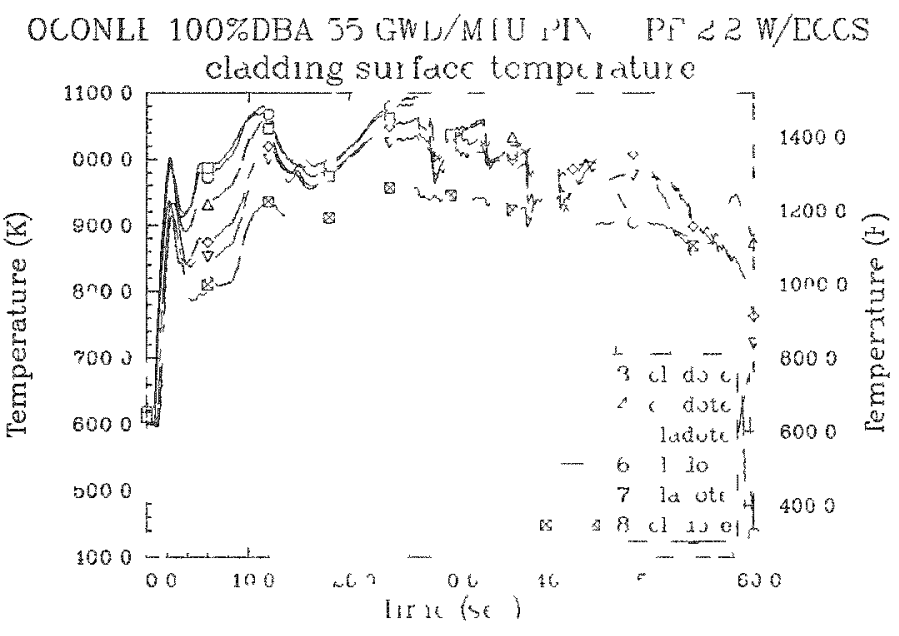

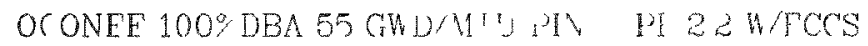

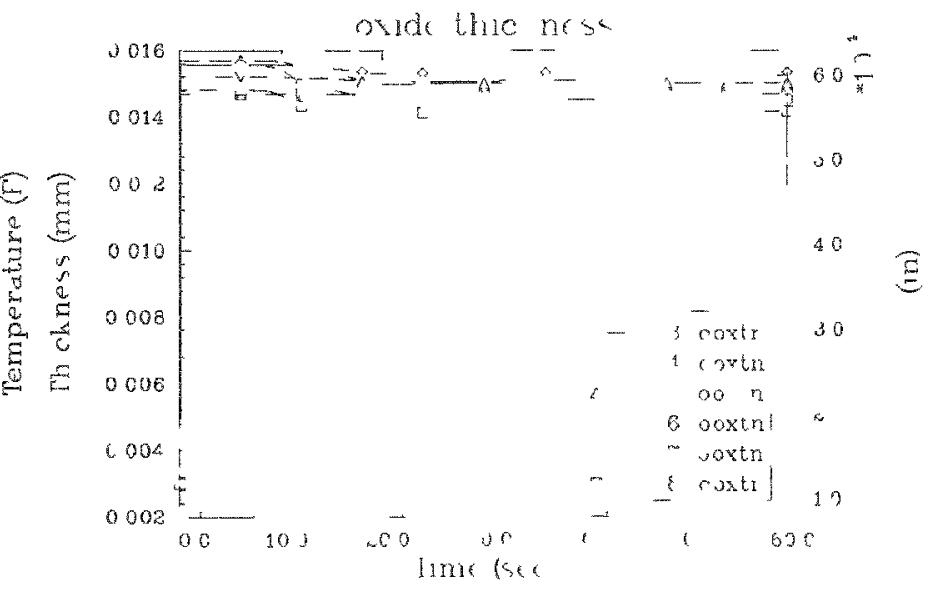


OCONEE 100\%DBA $35 \mathrm{GWD} / \mathrm{MTU}$ PIN--PF $22 \mathrm{~W} / \mathrm{ECCS}$ internal pin pressure

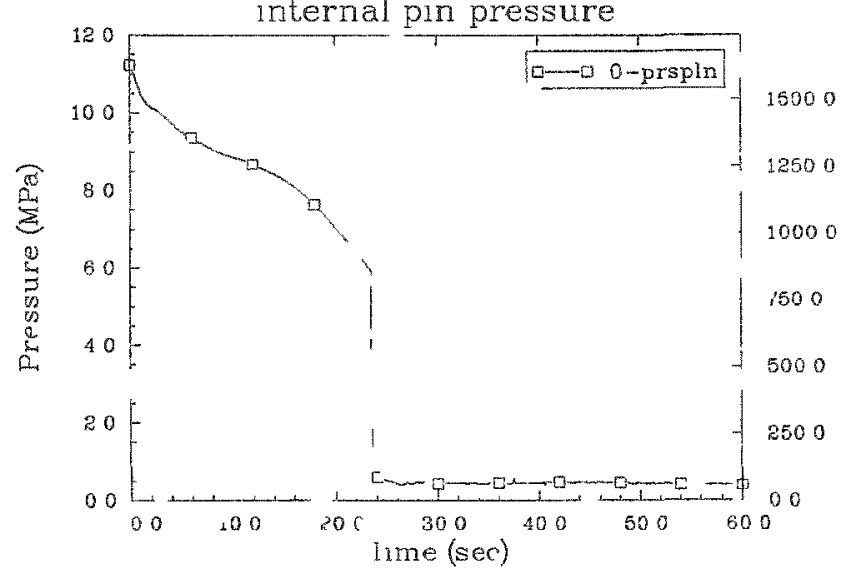

OCONLE 100\%DBA 35 GWD/MIU PIN-PT $22 \mathrm{~W} / \mathrm{ECCS}$ cladding hoop stran

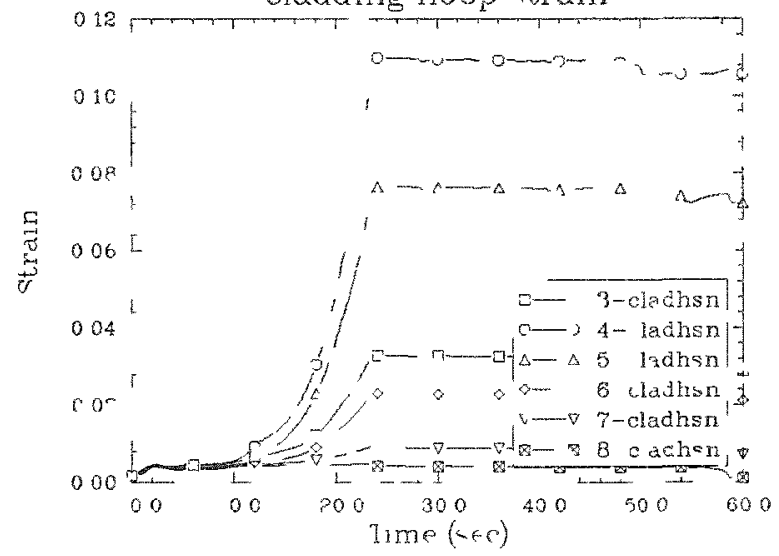

OCONEE 100\%DBA 35 GWD/MTU PIN - PI $22 \mathrm{~W} / \mathrm{CCS}$

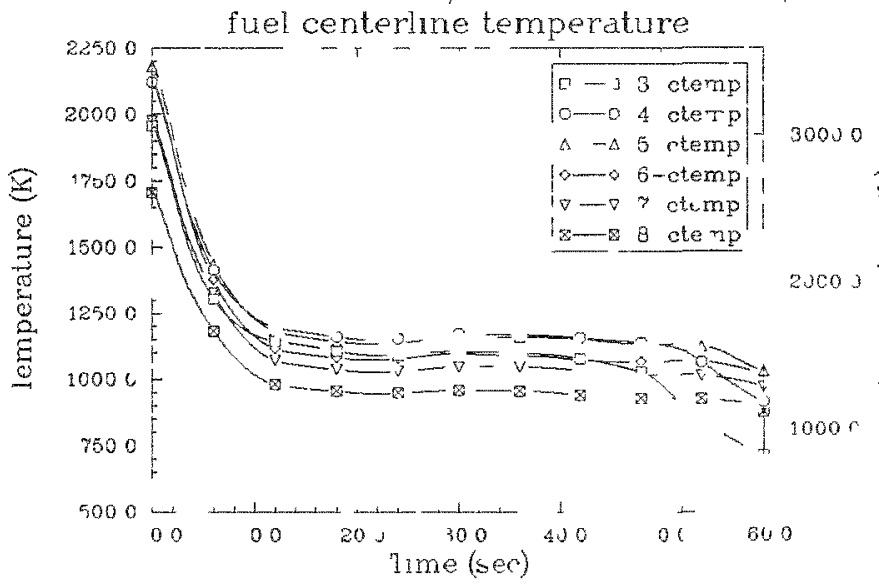

OCONEF 100\%DBA 35 GWD/MTU PIN--PI $22 \mathrm{~W} / \mathrm{FCCS}$

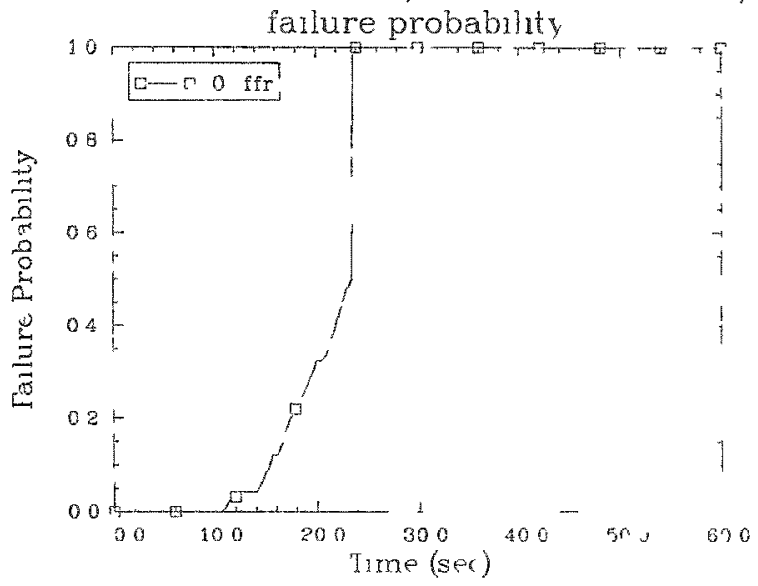


OCONCE 100\%DBA 20 CWD/MTU PIN-PI 2.2 W/ECCS internal pin pressure

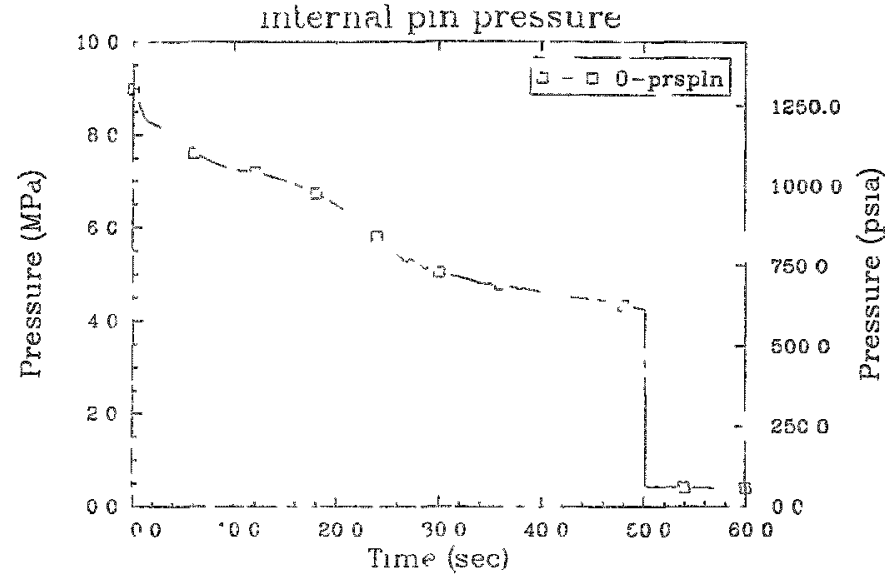

OCONEE 100\%DBA 20 GWD/MTU PIN-- PF 22 W/ECCS falure probabinty

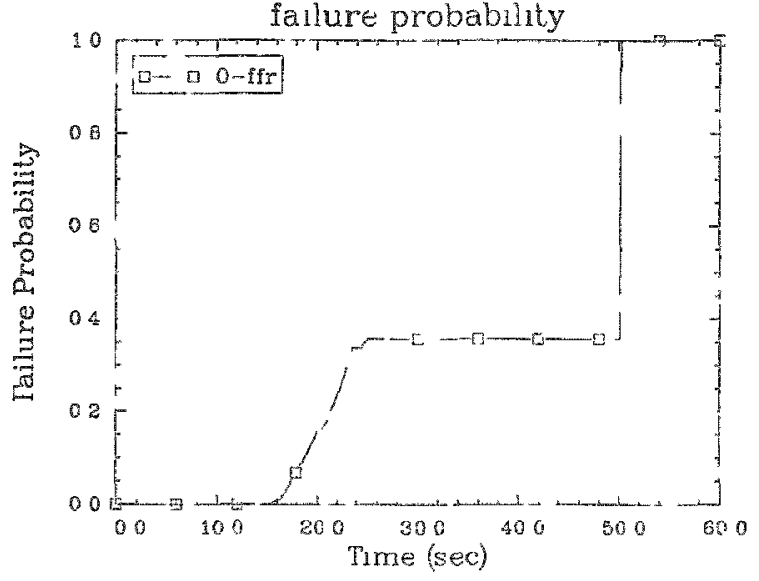

OCONEE 100\%DBA $20 \mathrm{GWL}$ MTL PIN--PF 22 W/ECCS cladding loop stram

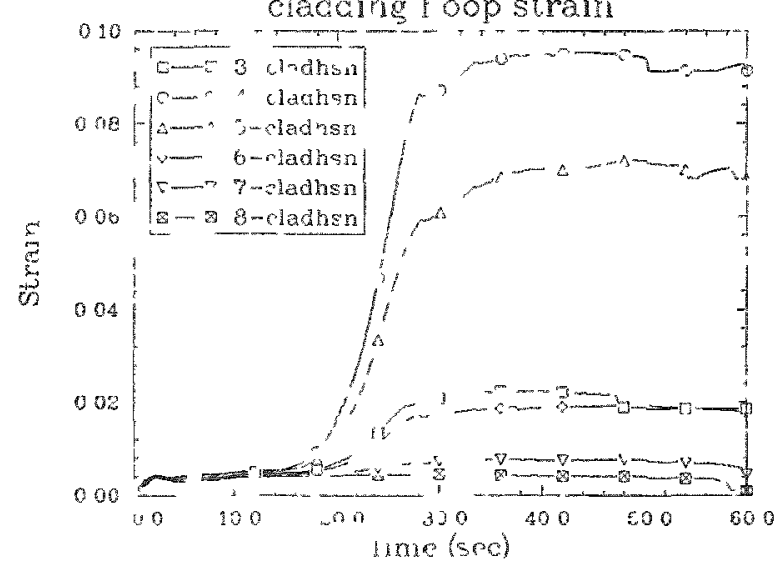

OCONEE 100\%DBA 20 GWD/MTU PIN--HF 2 Z W/ECCS cladding surface temporature

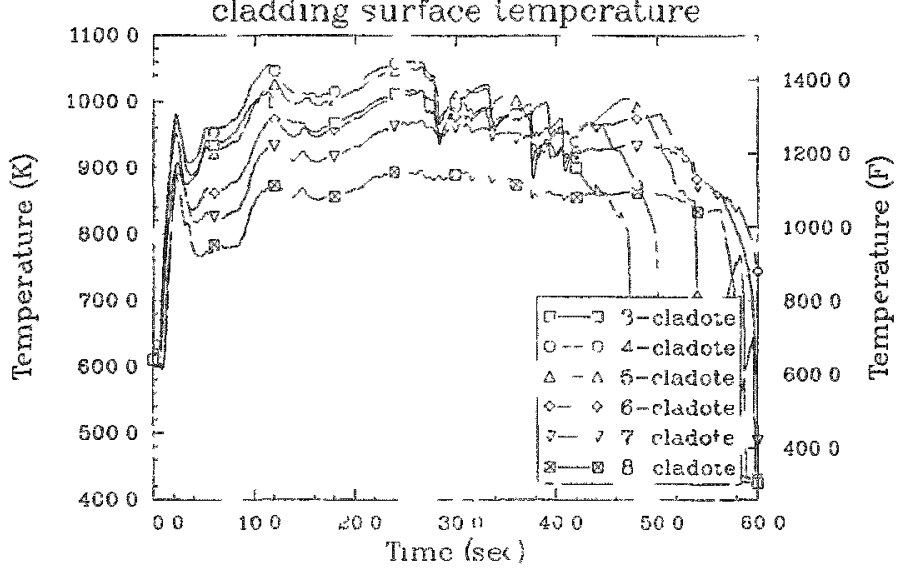

OCONEI 100 STHA 20 GWI MT HIN PF 22 W/LCCS

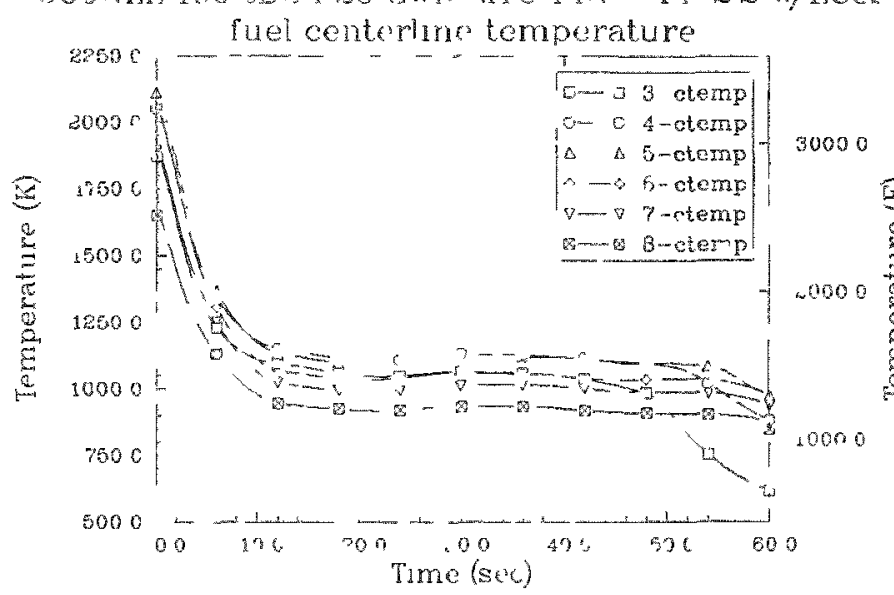

OCONEE 100\%DBA $20 \mathrm{GWD}$ MIL PI -- PF 22 W/FCCS

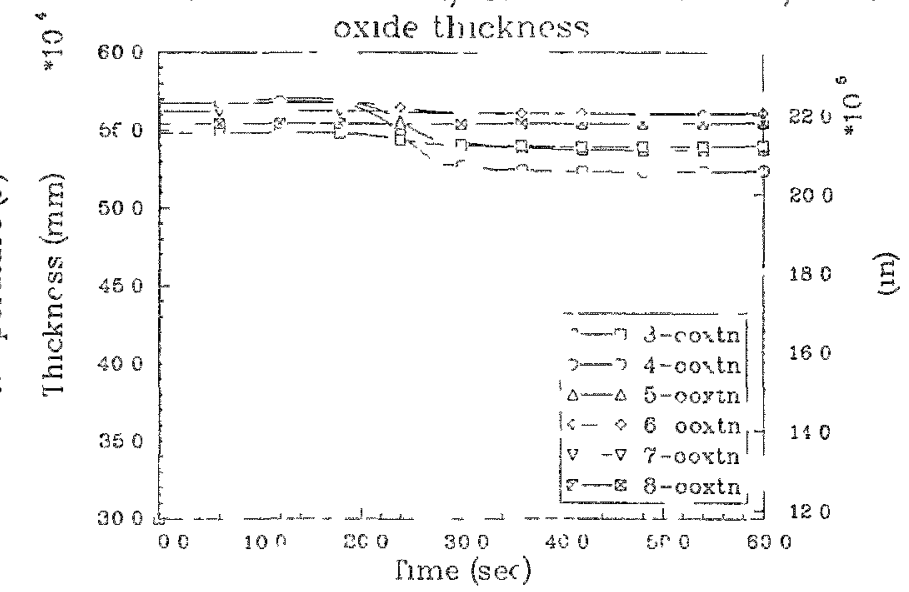


OCONEE 100\%DBA 5 GWD/MTU PIN--PF 2.2 W/ECCS

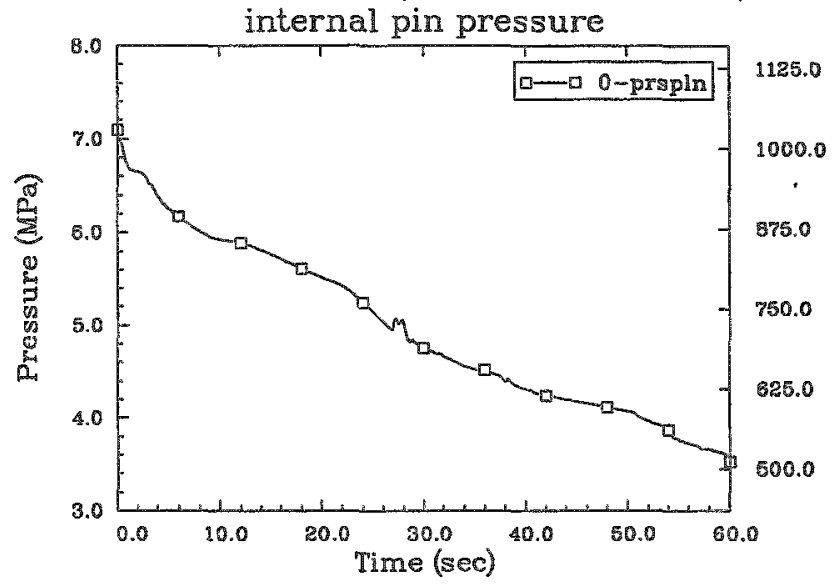

OCONEE 100\%DBA 5 GWD/MTU PIN--PF 2.2 W/ECCS cladding hoop strain

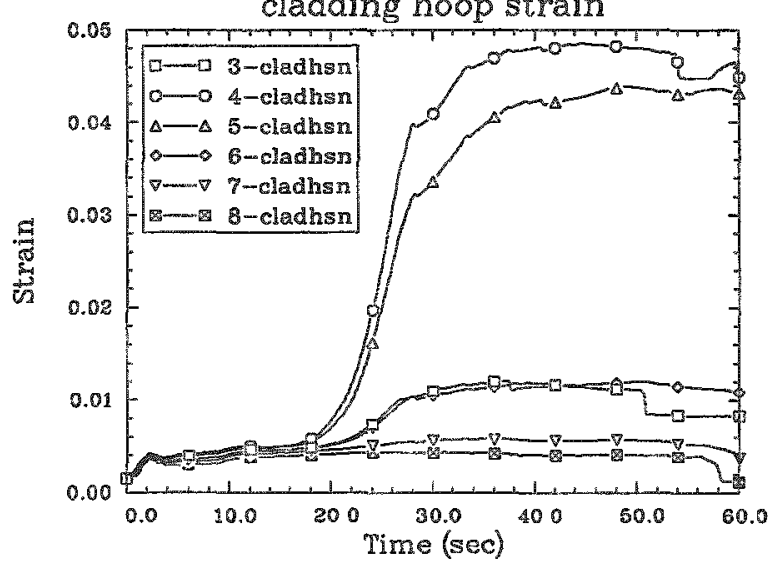

OCONEE 100\%DBA 5 GWD/MTU PIN--PF 2.2 W/ECCS

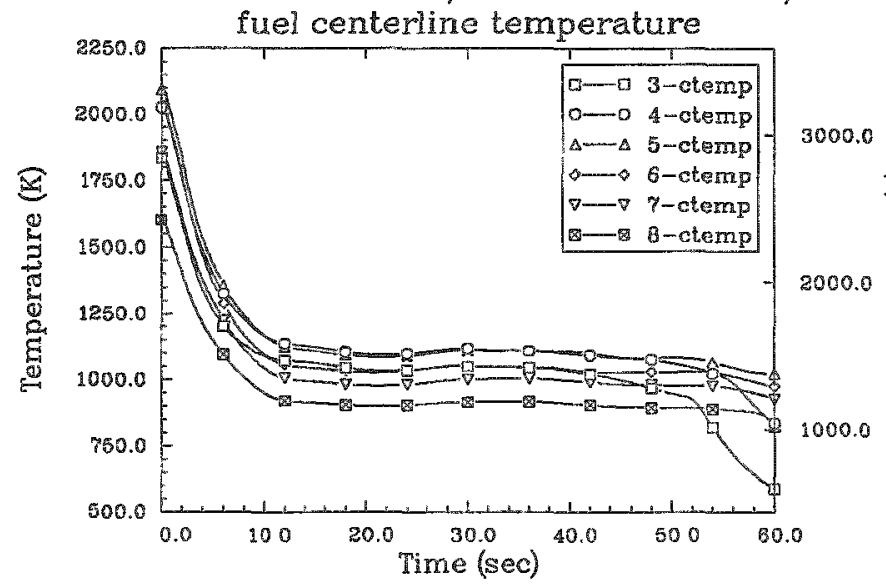

OCONEE 100\%DBA 5 GWD/MTU PIN--PF 2.2 W/ECCS

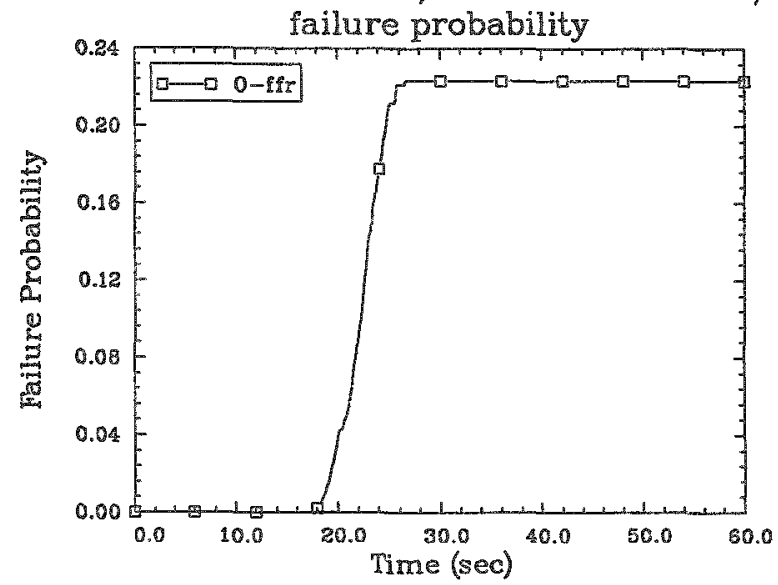

OCONEE 100\%DBA 5 GWD/MTU PIN--PF 2.2 W/ECCS

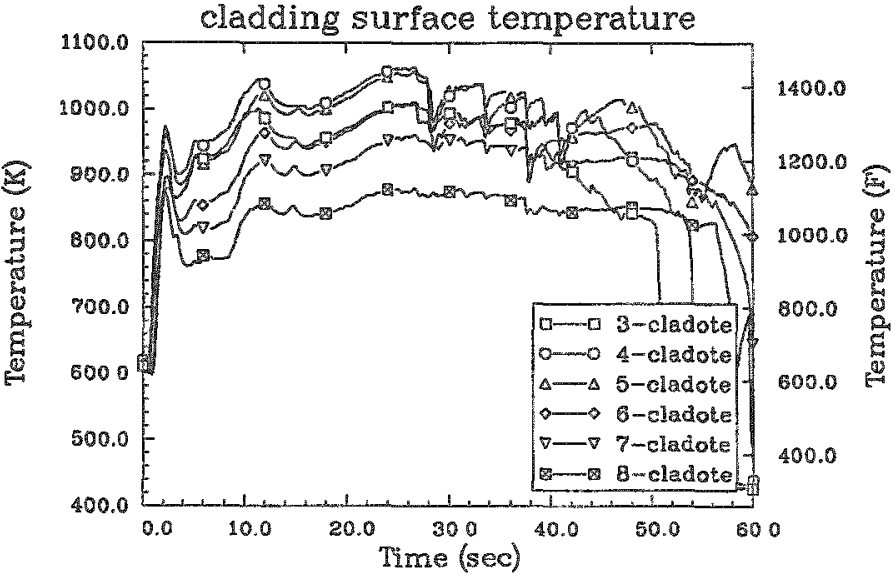

OCONEE 100\%DBA 5 GWD/MTU PIN--PF 2.2 W/ECCS

io oxide thickness

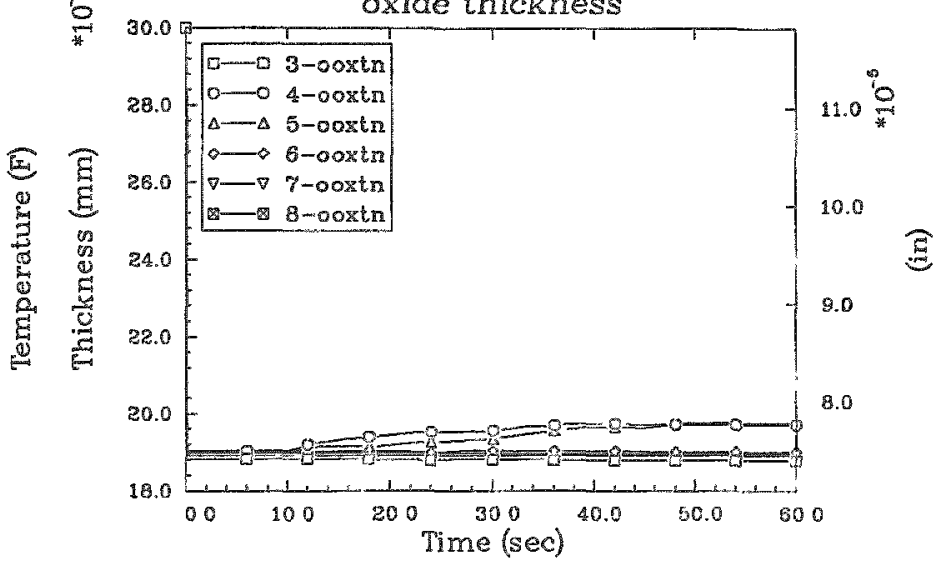


OCONEL 100\%DB $\$ 55$ GWD/MTL PIN-PT $20 \mathrm{~W} / \mathrm{LCCS}$ mbernal pin pressure

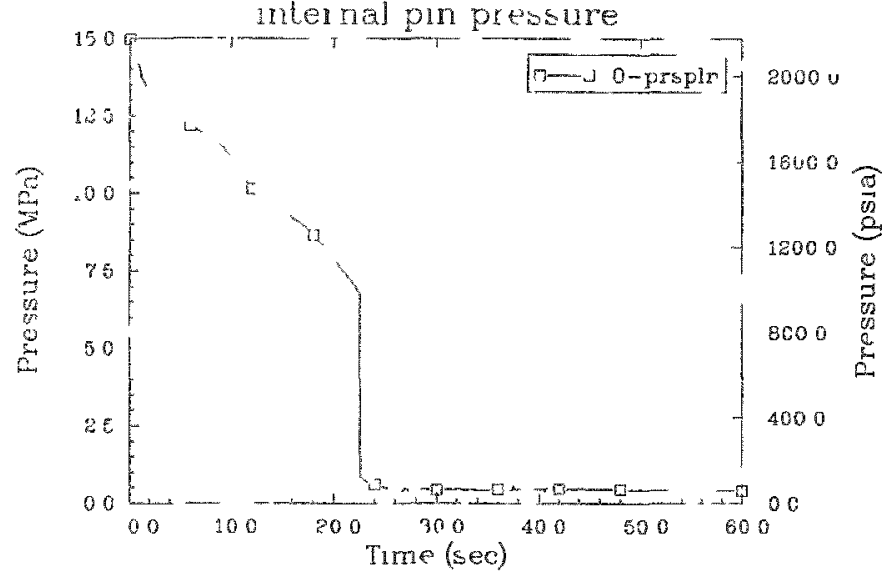

OCONLL 100 7DBA 55 GWD/MTU PIN-PF 20 W/CCCS

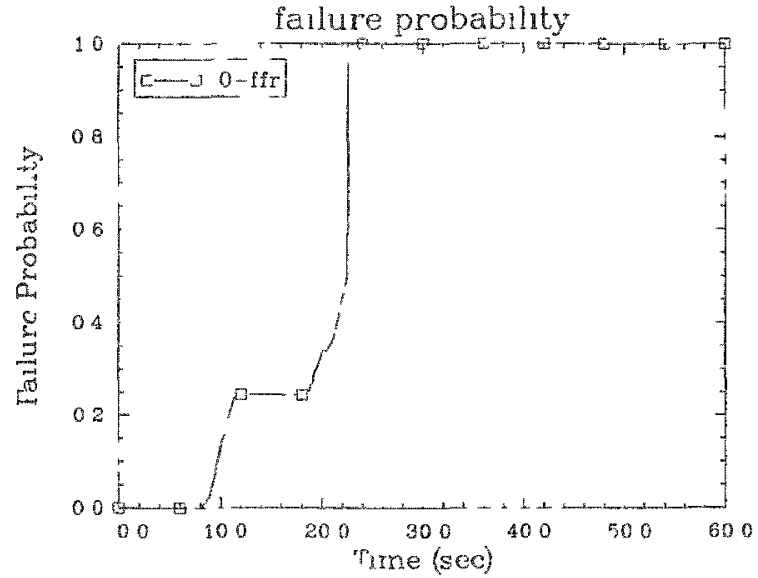

OCO\EE 100 DRA 55 SWD/MTU PIN--PF 20 W/ECCS

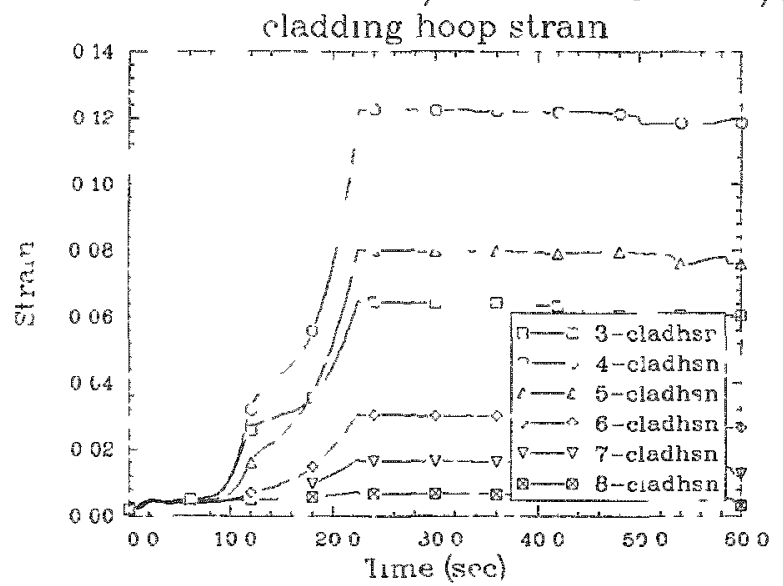

OCONEE 100\%DBA 55 GWD/MIL PIN--IPF 20 W/LCCS

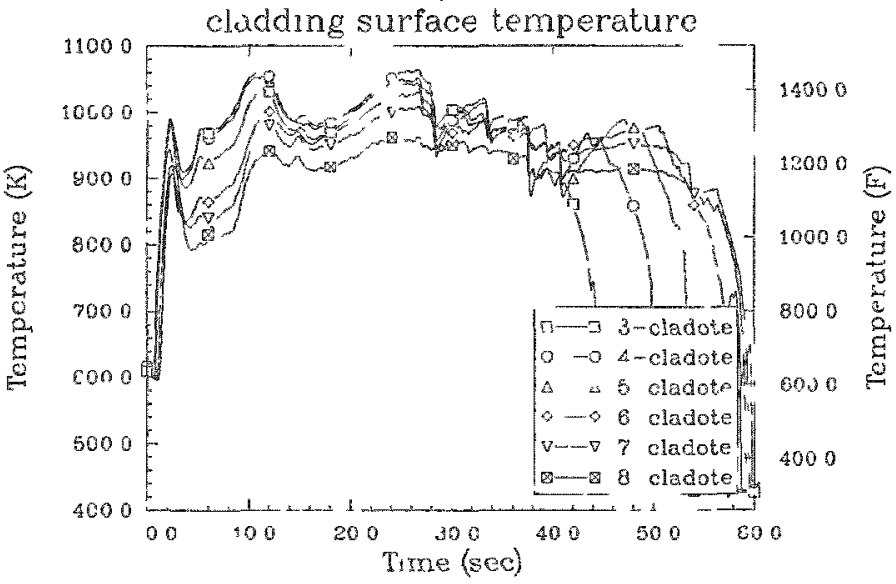

OCONEE 100\%DBA 55 GWD/MTU PIN-PF 20 W/ECCS

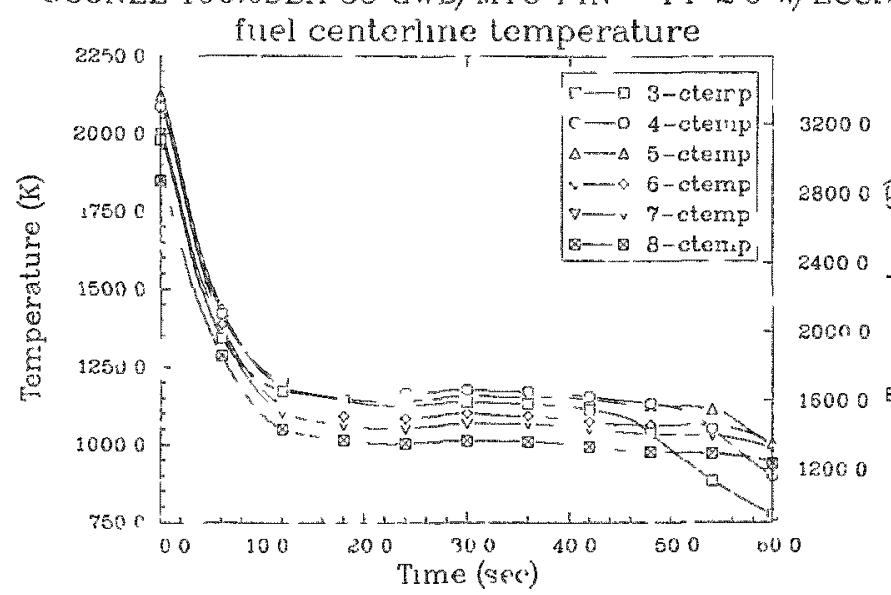

OCONEE 100\%DBA 55 GWD/MTU PIN--PF 20 W/CCCS

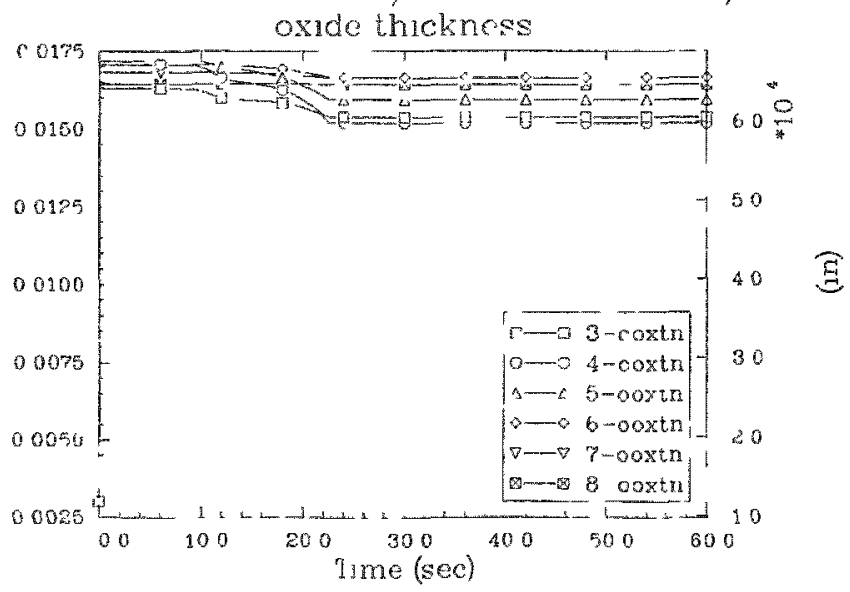


OCONEE 100\%DBA 35 GWD/MTU PIN--PF 2.0 W/ECCS

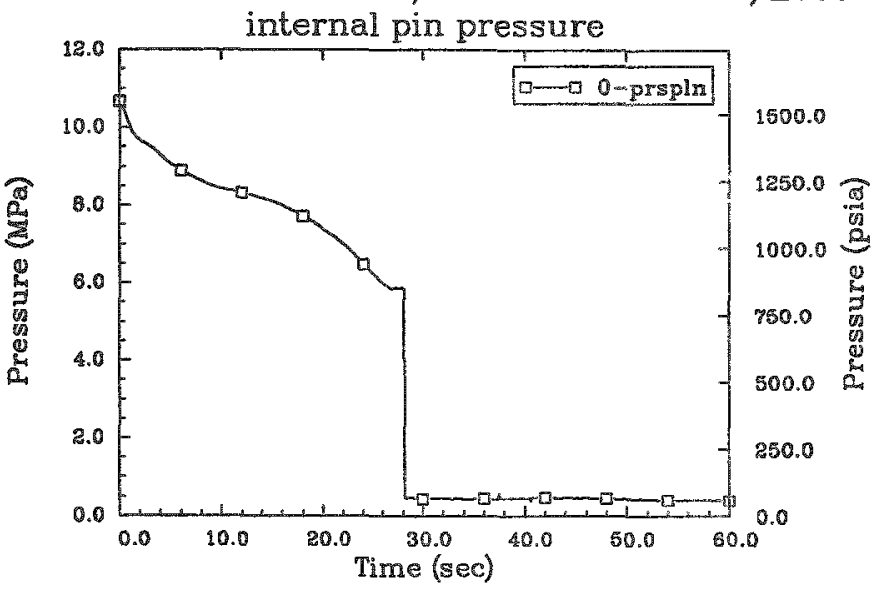

OCONEE 100\%DBA 35 GWD/MTU PIN--PF $2.0 \mathrm{~W} / \mathrm{ECCS}$ failure probability

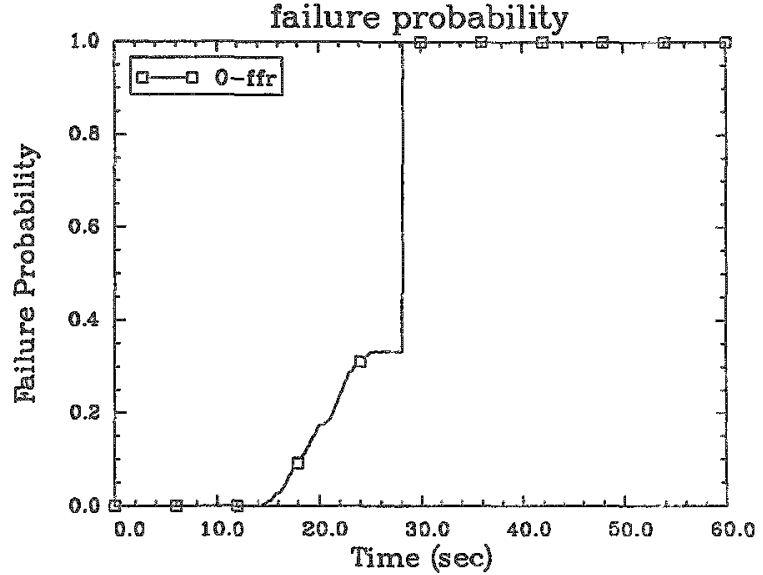

OCONEE 100\%DBA 35 GWD/MTU PIN--PF 2.0 W/ECCS cladding hoop strain

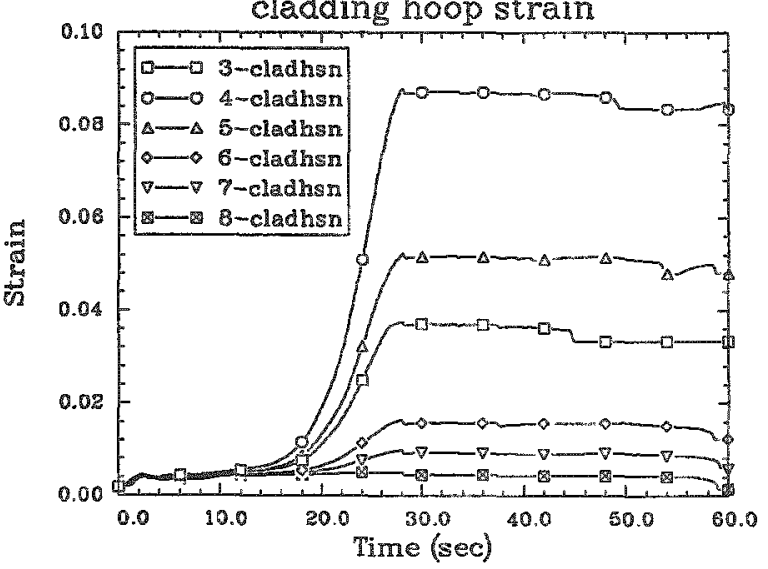

OCONEE 100\%DBA 35 GWD/MTU PIN--PF $2.0 \mathrm{~W} / \mathrm{ECCS}$

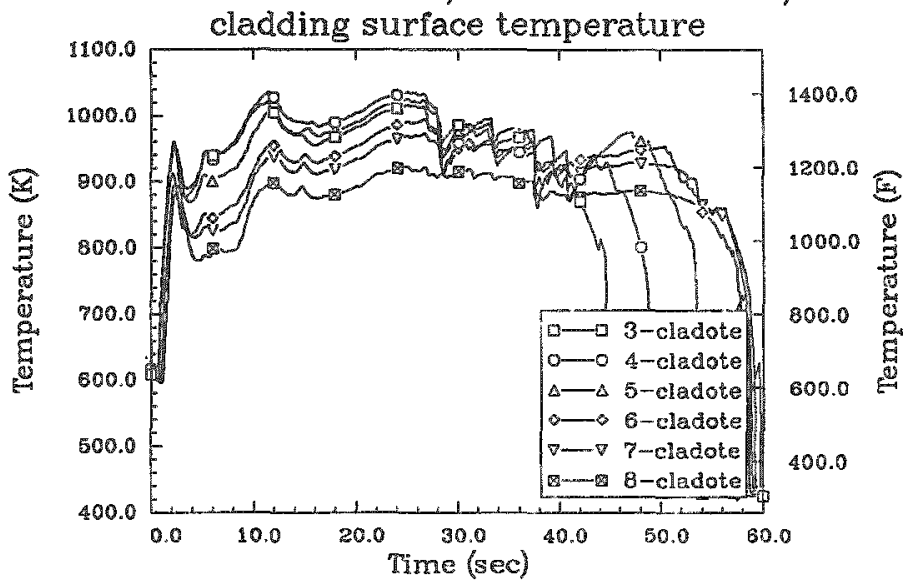

OCONEE 100\%DBA 35 GWD/MTU PIN--PF 2.0 W/ECCS

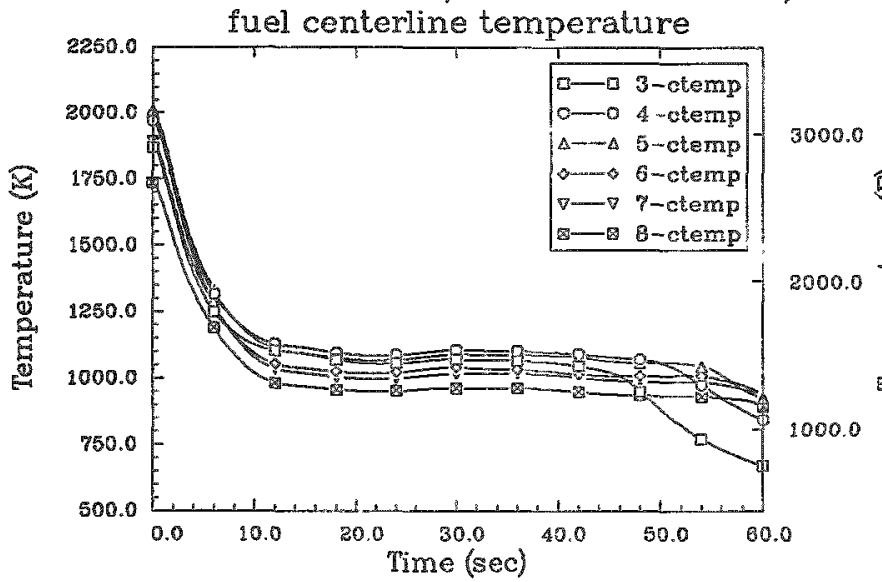

OCONEE 100\%DBA 35 GWD/MTU PIN--PF 2.0 W/ECCS

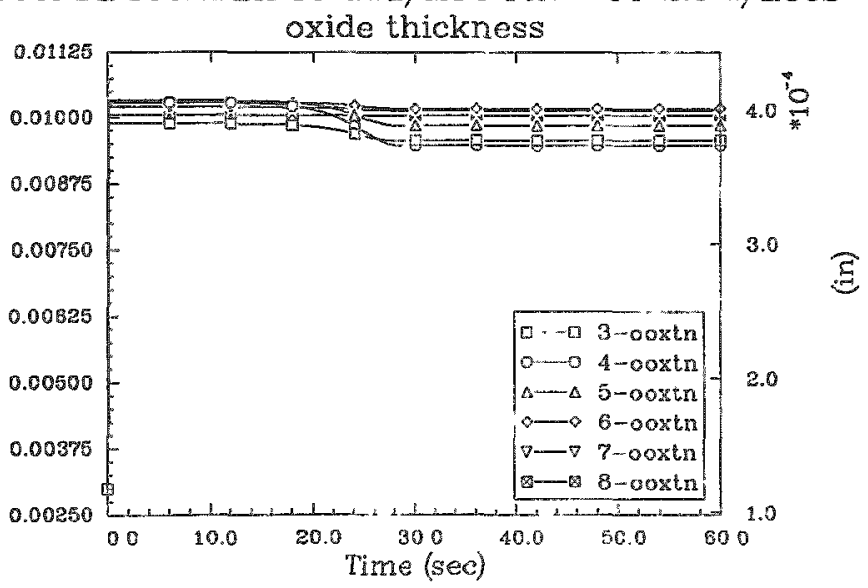


OCONEE 100\%DBA $20 \mathrm{GWD} / \mathrm{MTU}$ PIN-PF $2.0 \mathrm{~W} / \mathrm{BCCS}$ internal pin pressure

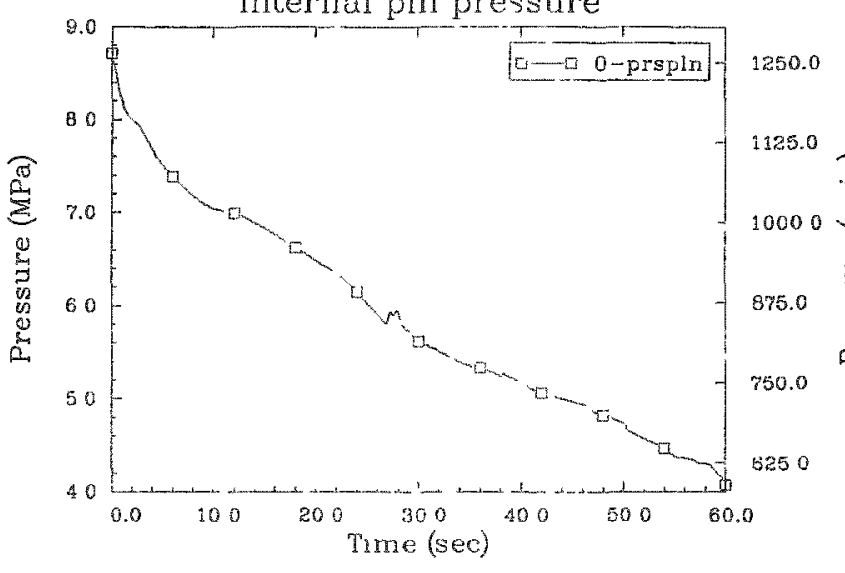

OCONEE $100 \%$ DBA 20 GWD/MIL PIN--PF $2.0 \mathrm{~W} / \mathrm{ECCS}$

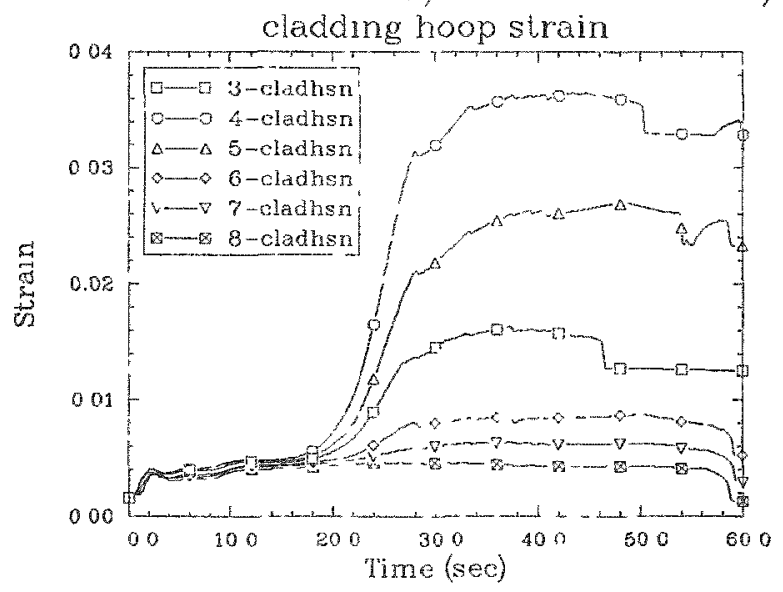

OCONEE 100\%DBA 20 GWD/MJU PIN-..PH $2.0 \mathrm{~W} / \mathrm{ECCS}$

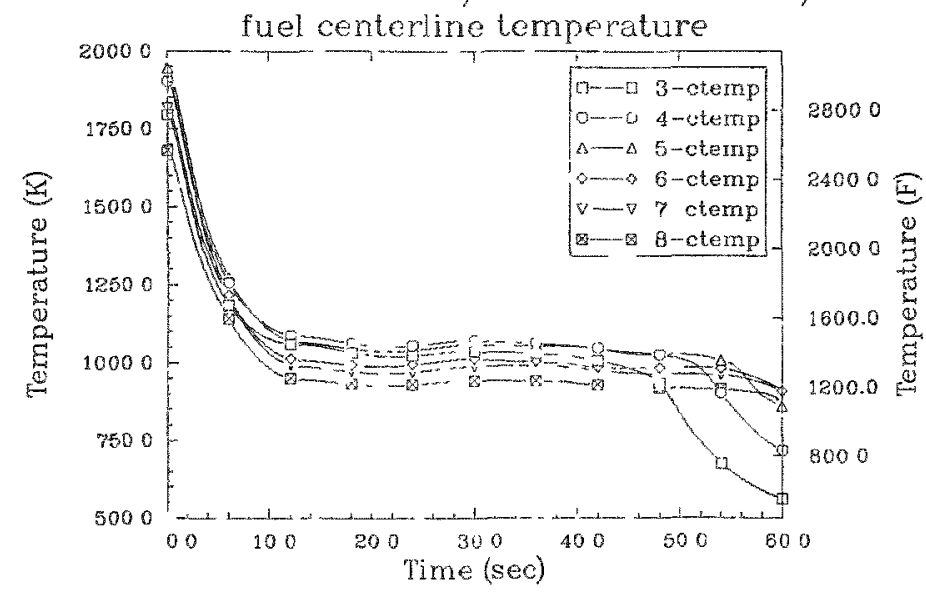

OCONEE 100\%DBA 20 GWD/MTU PIN PF 20 W/ECCS failure probability

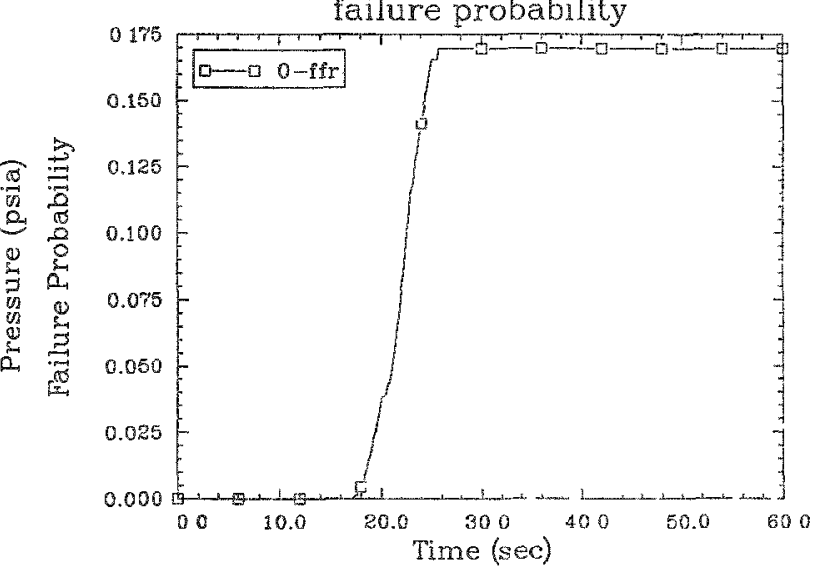

OCONEE 100\%DBA 20 GWD/MTU PIN-PF $20 \mathrm{~W} / \mathrm{ECCS}$

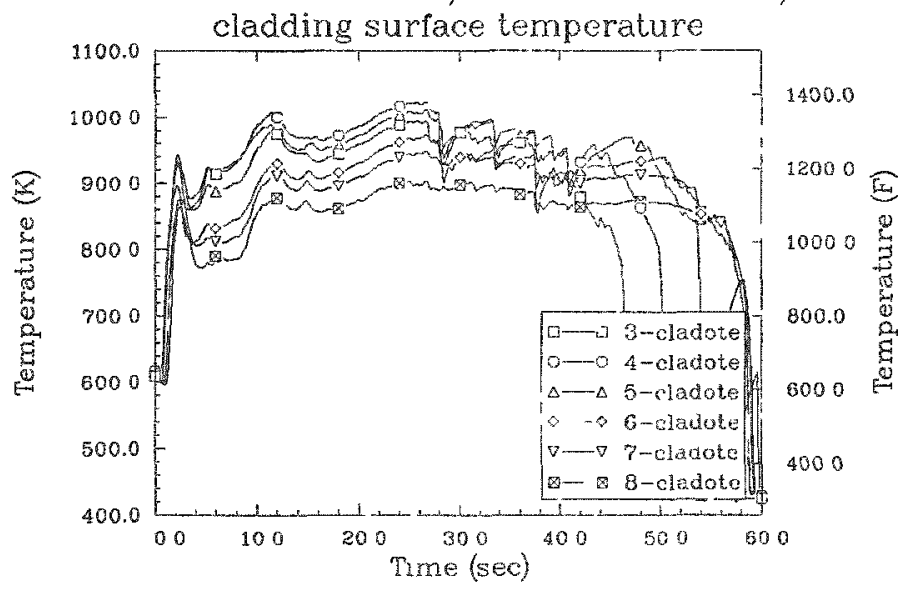

OCONEE 100\%DBA 20 GWD/MTL PIN-PF 20 W/ECCS

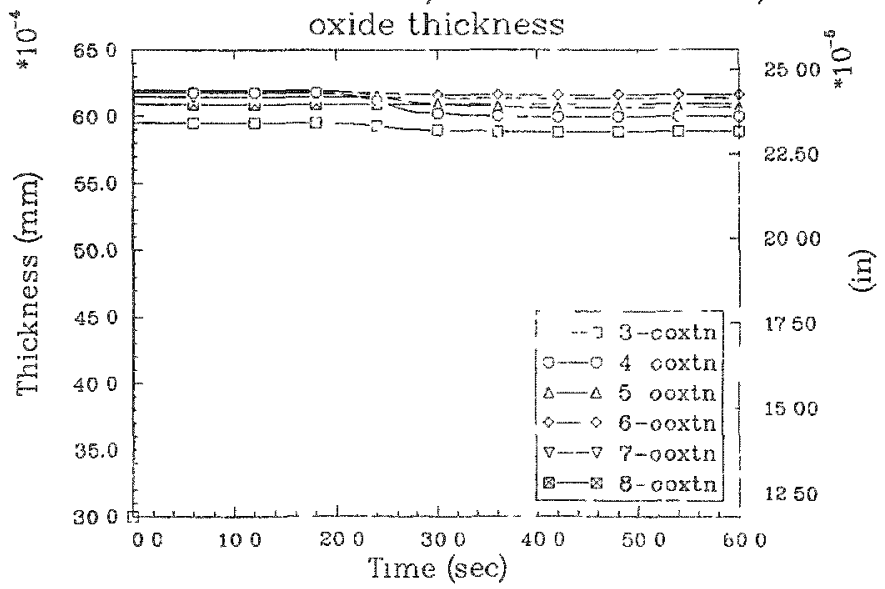


OCONEE 100\%DBA 5 GWD/MTU PIN--PF $2.0 \mathrm{~W} / \mathrm{ECCS}$ internal pin pressure

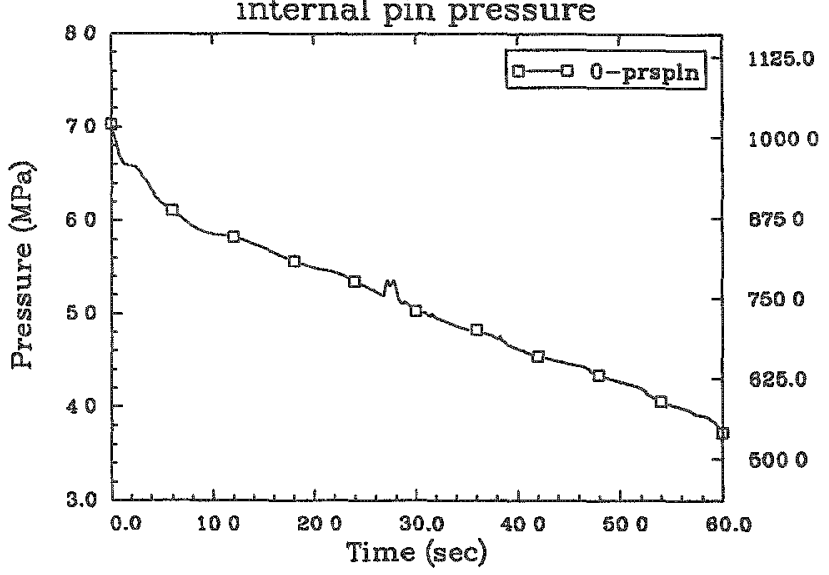

OCONEE 100\%DBA 5 GWD/MTU PIN--PF 2.0 W/ECCS cladding hoop strain

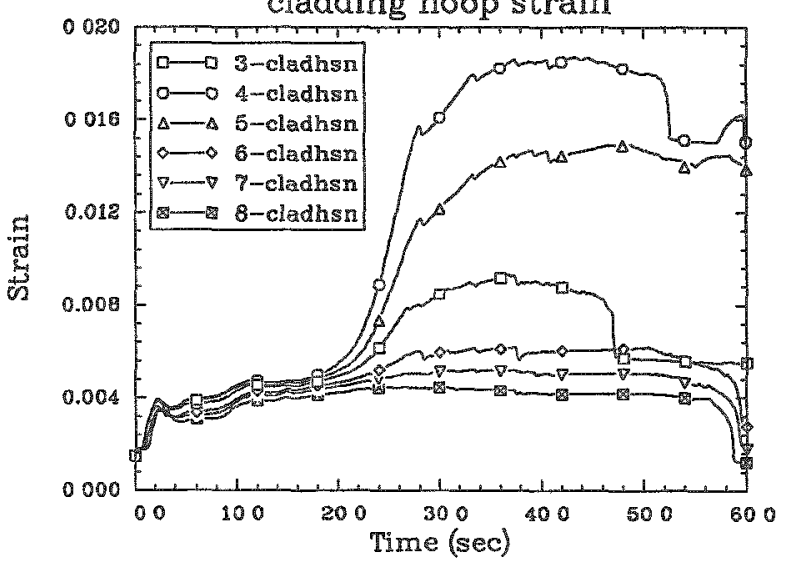

OCONEE 100\%DBA 5 GWD/MTU PIN--PF 2.0 W/ECCS

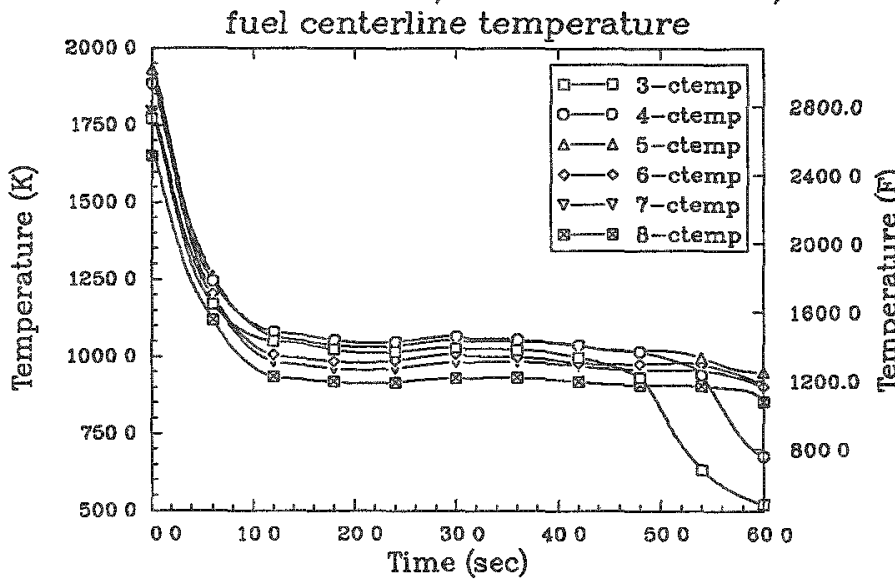

OCONEE 100\%DBA 5 GWD/MTU PIN--PF 2.0 W/ECCS failure probability

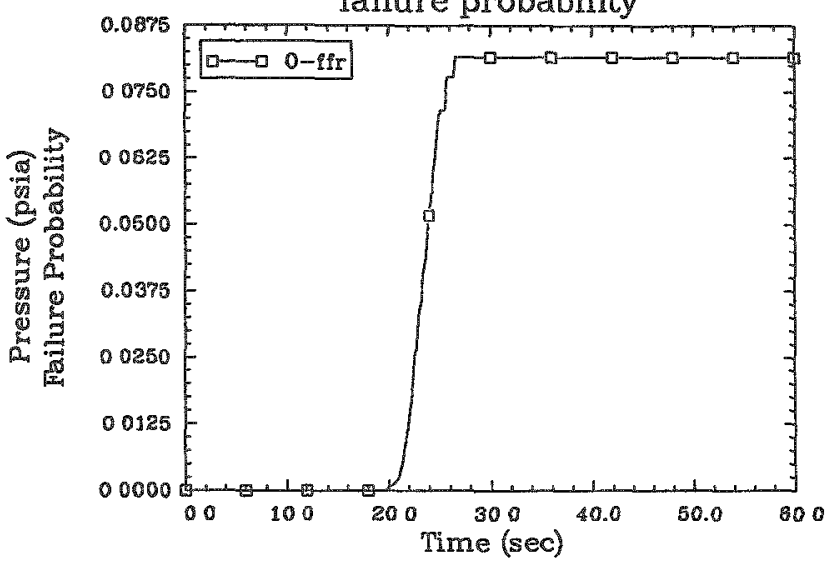

OCONEE 100\%DBA 5 GWD/MTU PIN--PF $2.0 \mathrm{~W} / \mathrm{ECCS}$

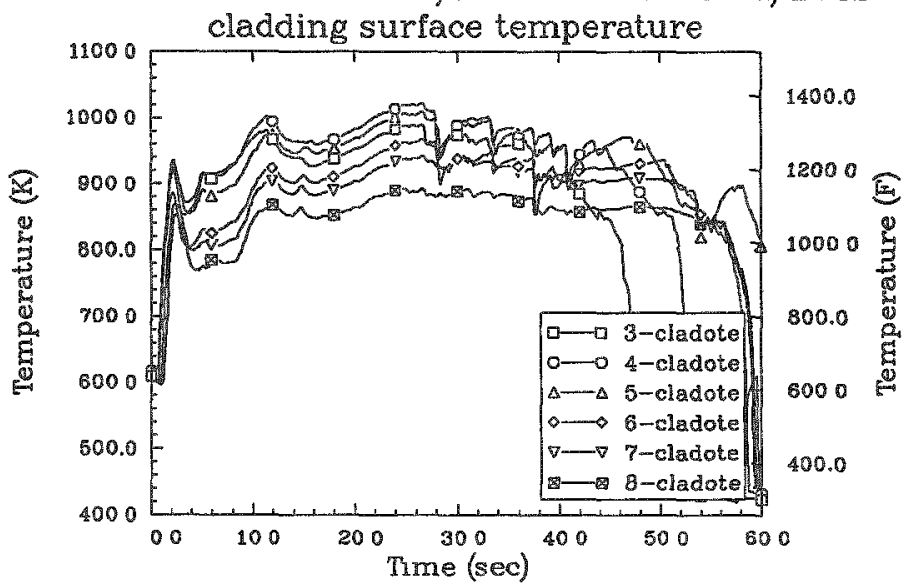

OCONEE 100\%DBA 5 GWD/MTU PIN--PF 2.0 W/ECCS

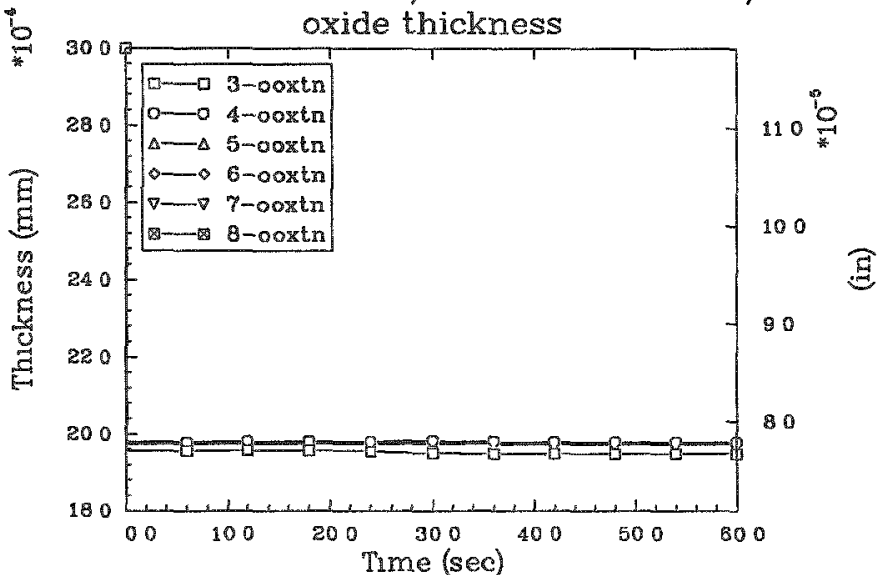


OCONEE 100\%DBA 55 GWD/MTU PIN--PF 2.63 W/E\&PT OCONEE 100\%DBA 55 GWD/MTU PIN--PF 2.63 W/E\&PT
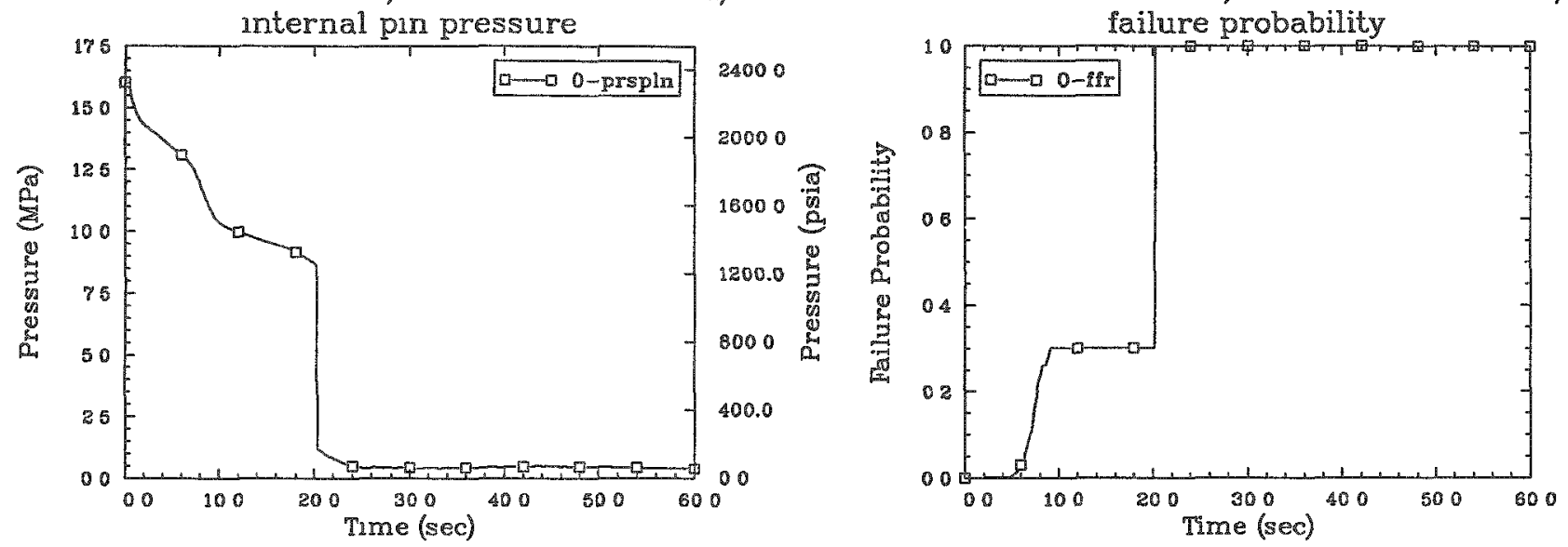

OCONEE 100\%DBA $55 \mathrm{GWD} / \mathrm{MTU}$ PIN--PF $2.63 \mathrm{~W} / \mathrm{E} \& \mathrm{PT}$ OCONEE 100\%DBA $55 \mathrm{GWD} / \mathrm{MTU}$ PIN--PF 2.63 W/E\&PT
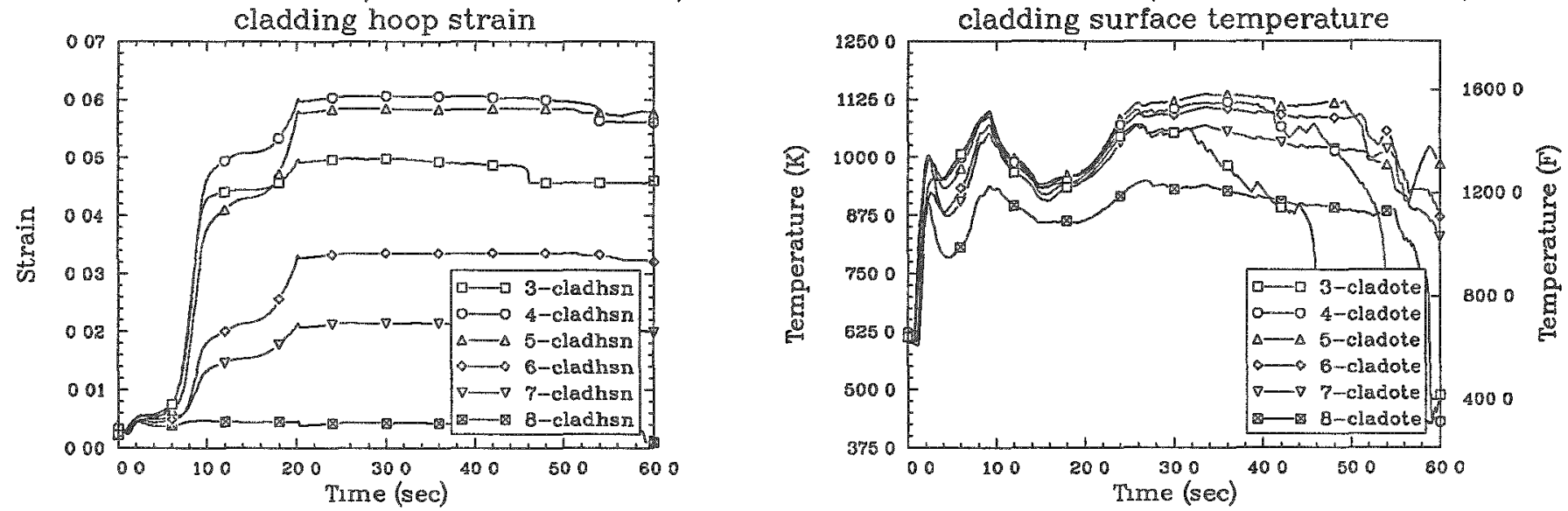

OCONEE 100\%DBA 55 GWD/MTU PIN--PF 2.63 W/E\&PT OCONEE 100\%DBA 55 GWD/MTU PIN--PF 263 W/E\&PT
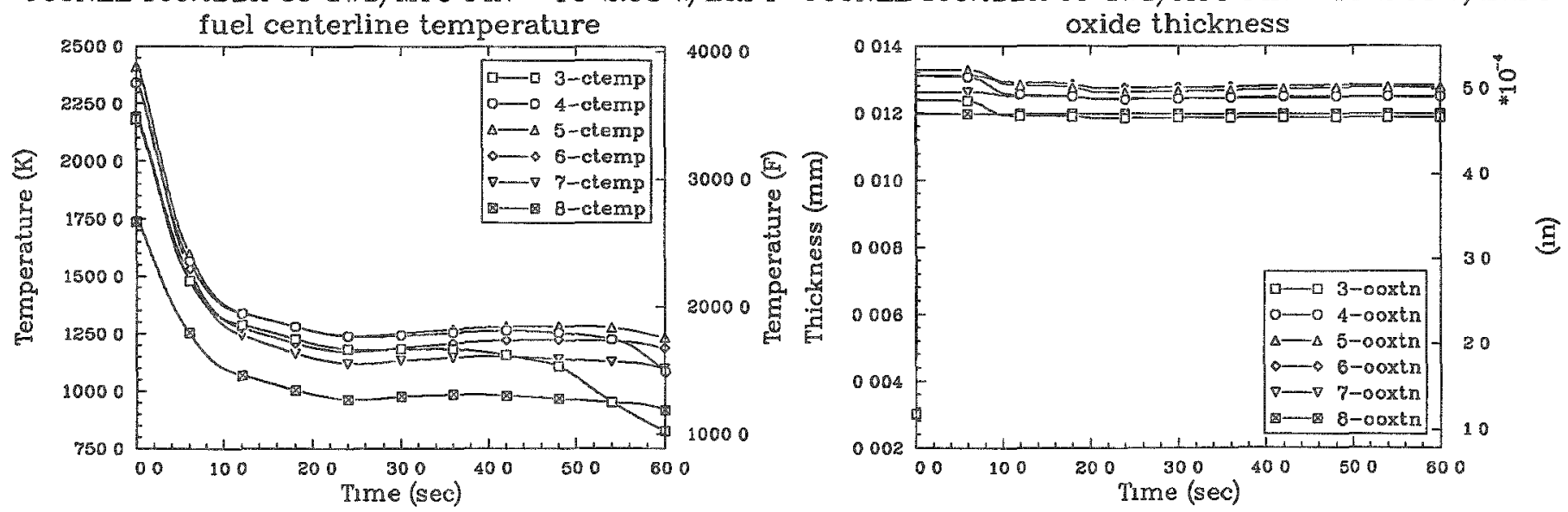
OCONEE 100\%DBA 35 GWD/MTU PIN--PF 2.63 W/E\&PT OCONEE 100\%DBA 35 GWD/MTU PIN--PF 2.63 W/E\&PT
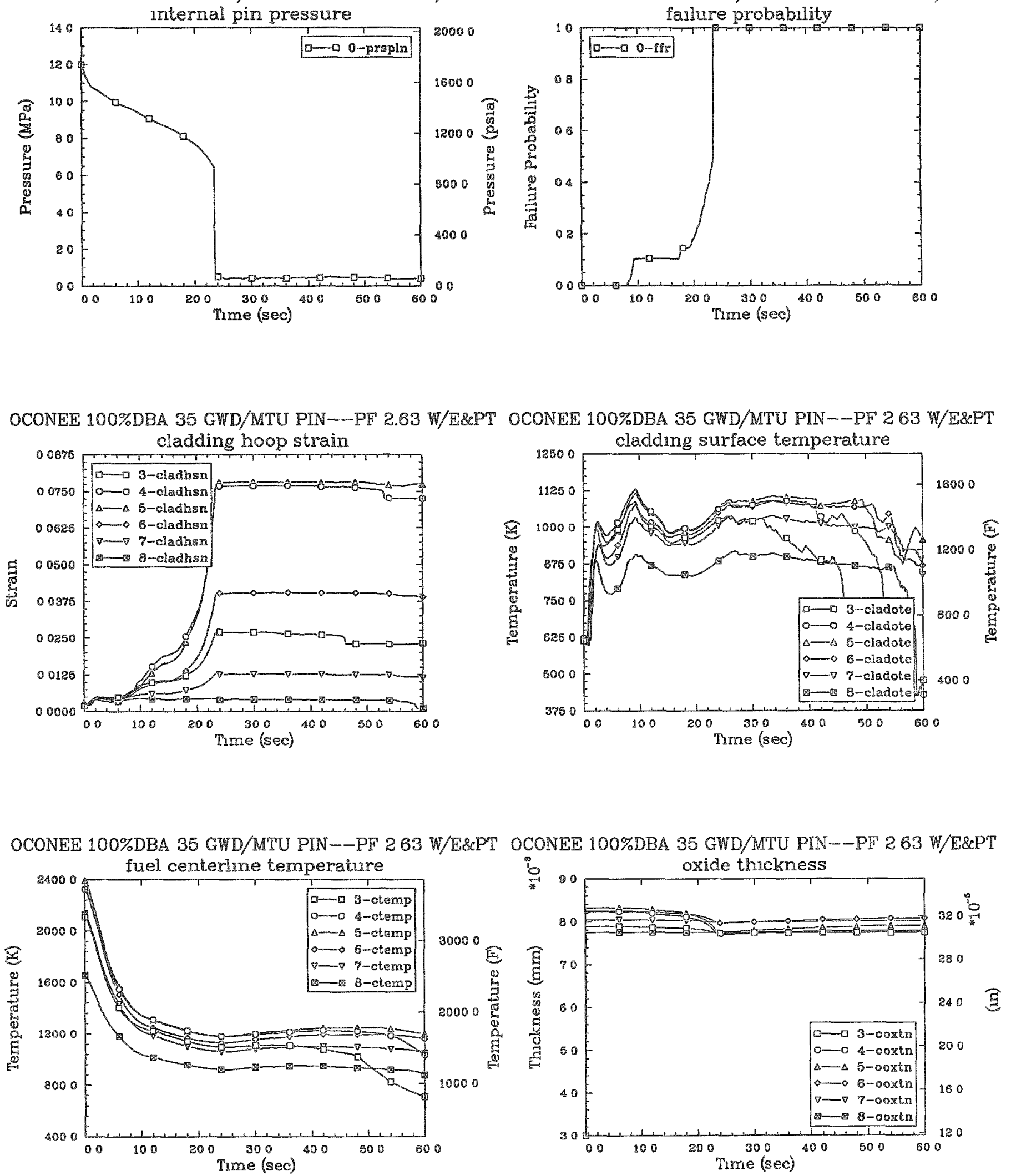


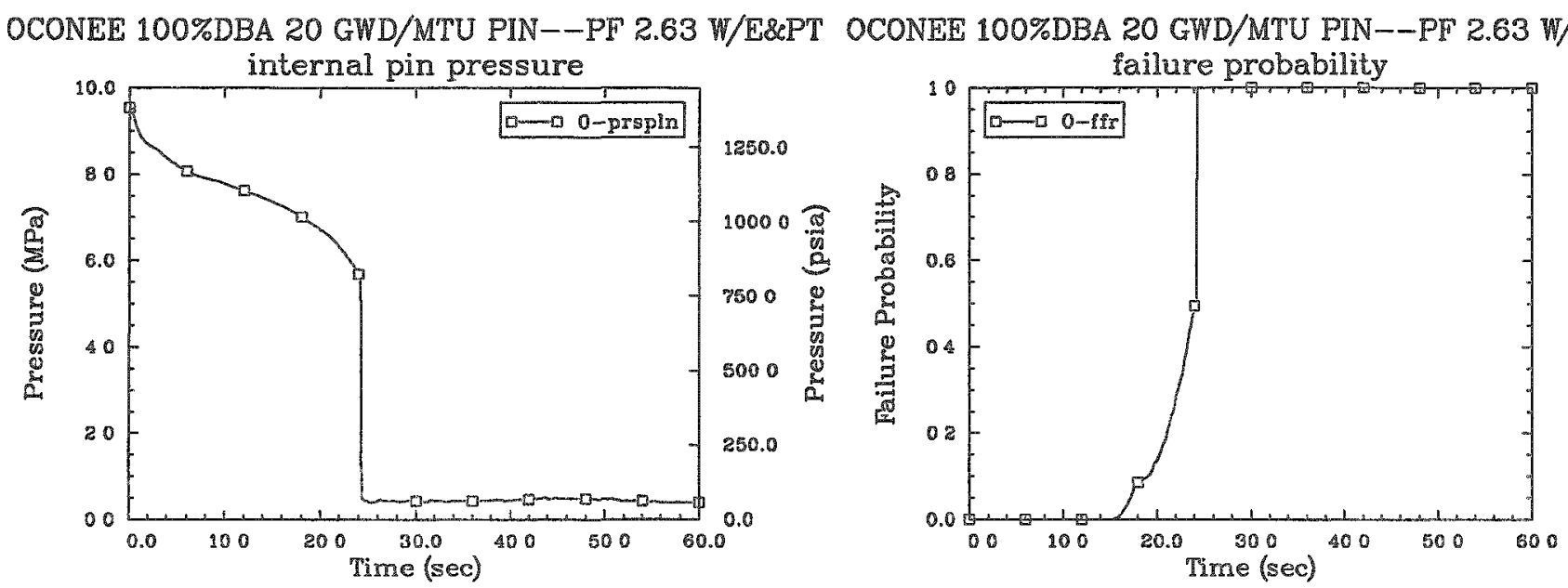

OCONEE 100\%DBA $20 \mathrm{GWD} / \mathrm{MTU}$ PIN--PF 2.63 W/E\&PT OCONEE 100\%DBA $20 \mathrm{GWD} / \mathrm{MTU}$ PIN-PF 2.63 W/E\&PT
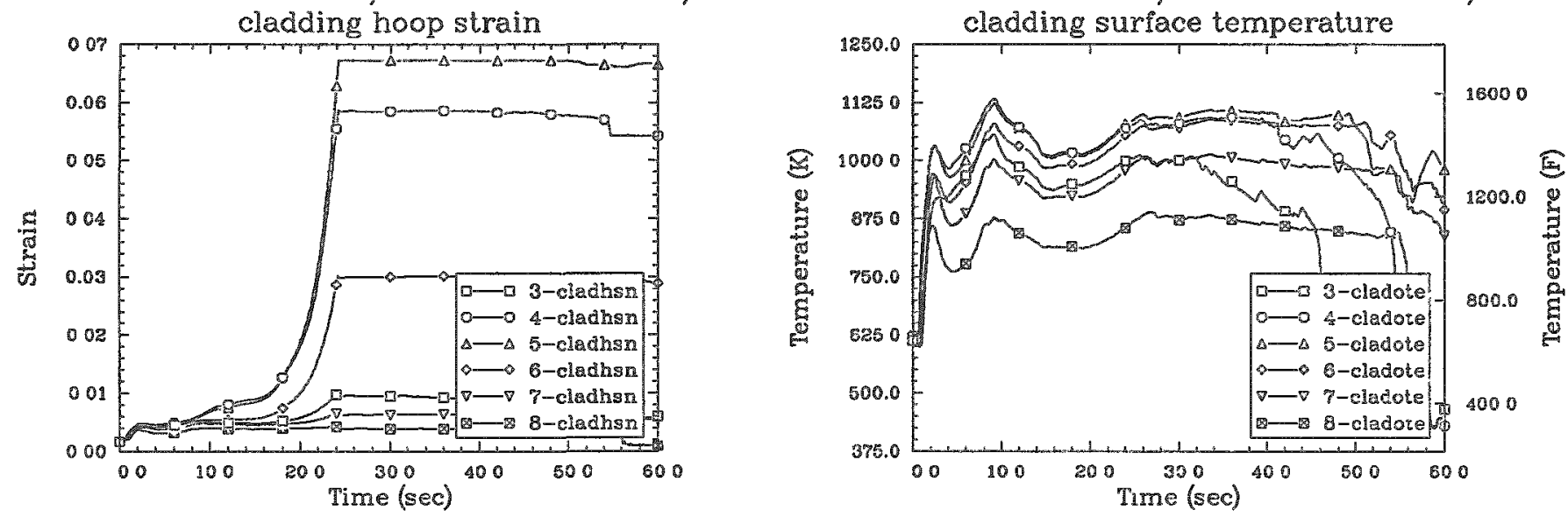

OCONEE 100\%DBA 20 GWD/MTU PIN--PF 2.63 W/E\&PT

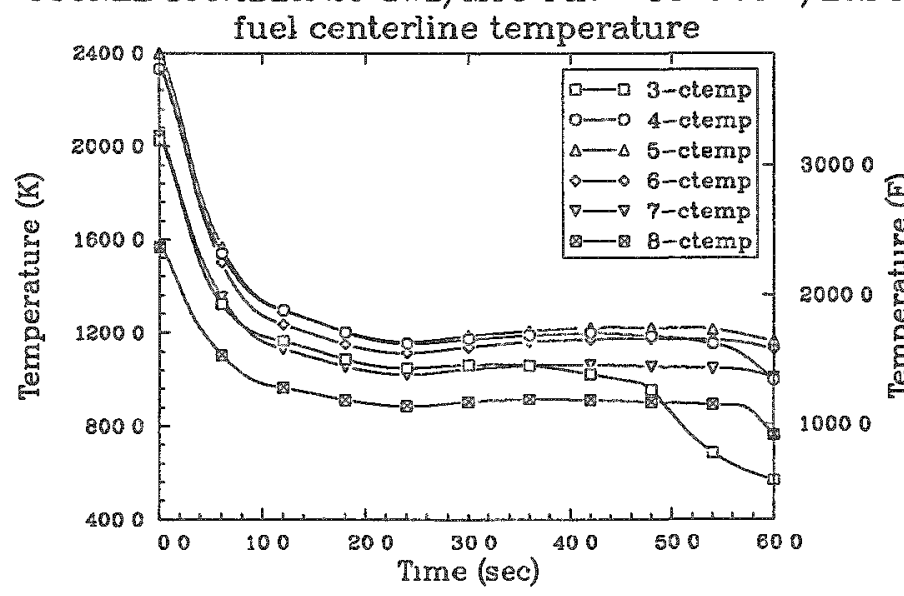

OCONEE 100\%DBA $20 \mathrm{GWD} / \mathrm{MTU}$ PIN--PF 2.63 W/E\&PT
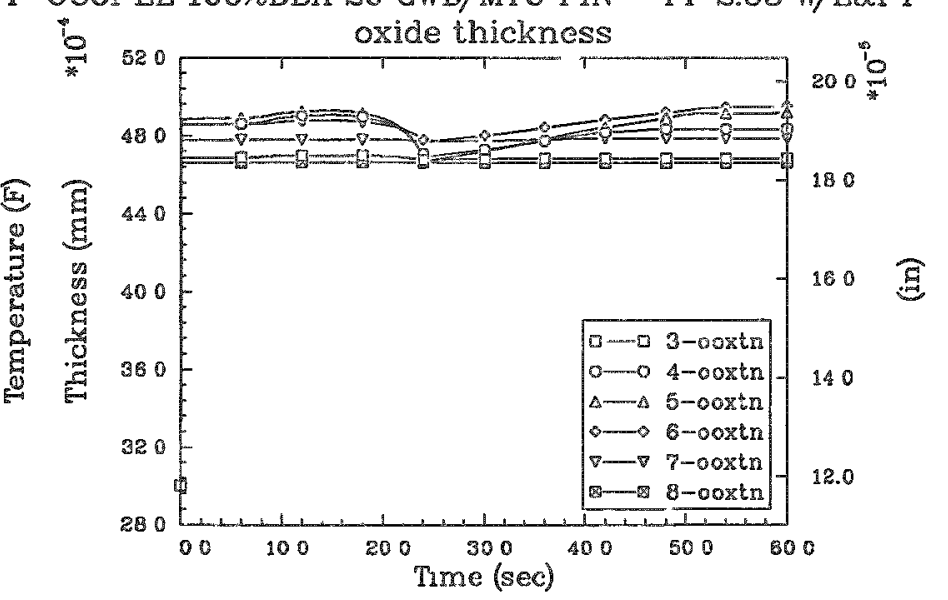
OCONEE 100\%DBA 5 GWD/MTU PIN--PF 2.63 W/E\&PT

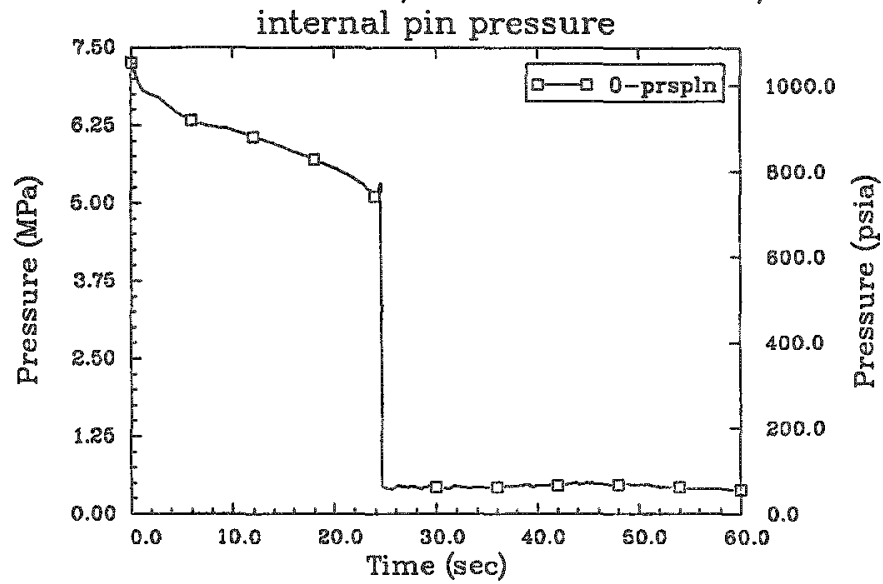

OCONEE 100\%DBA 5 GWD/MTU PIN--PF 2.63 W/E\&PT

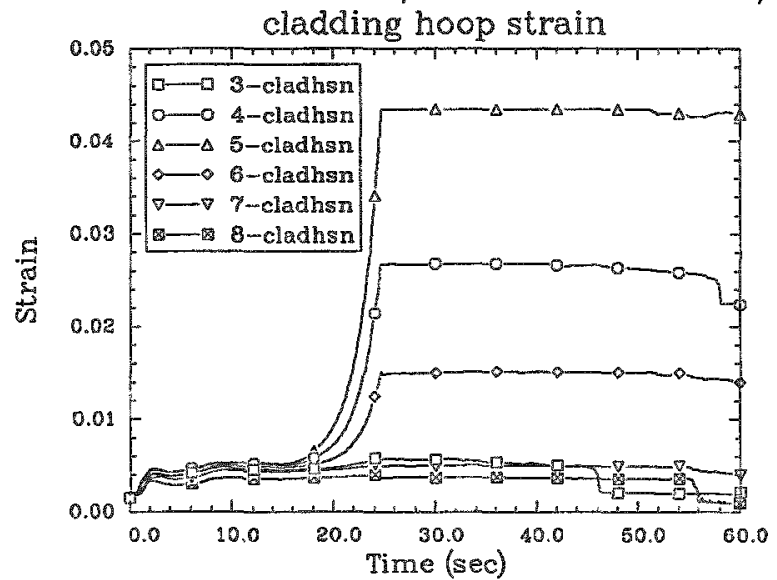

OCONEE 100\%DBA 5 GWD/MTU PIN--PF 2.63 W/E\&PT

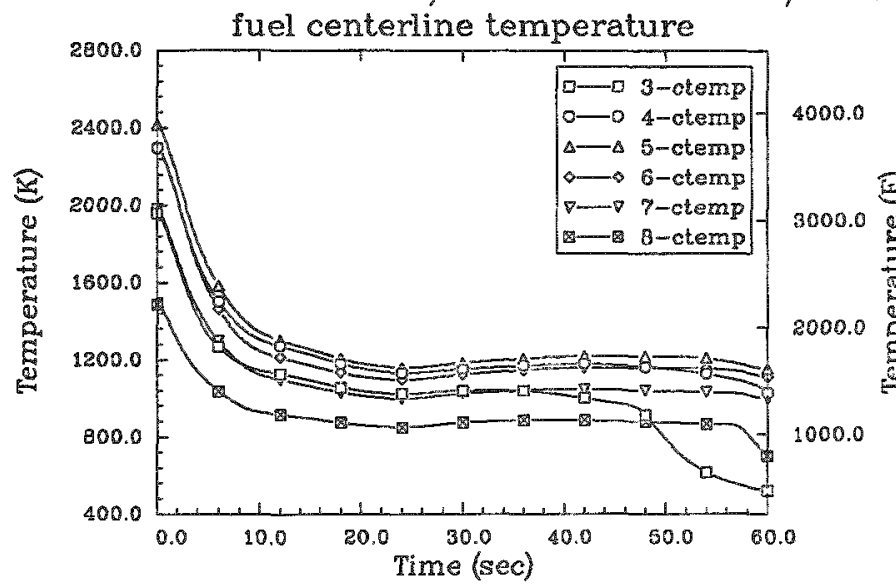

OCONEE 100\%DBA 5 GWD/MTU PIN--PF 2.63 W/E\&PT

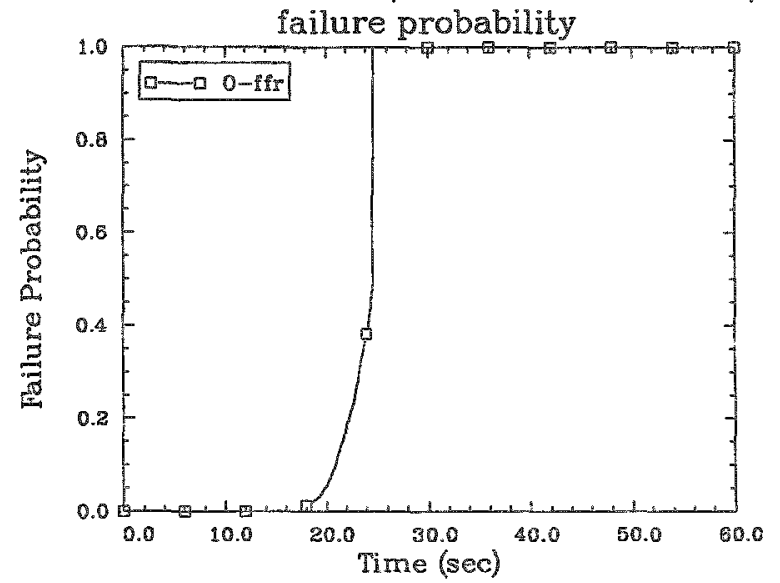

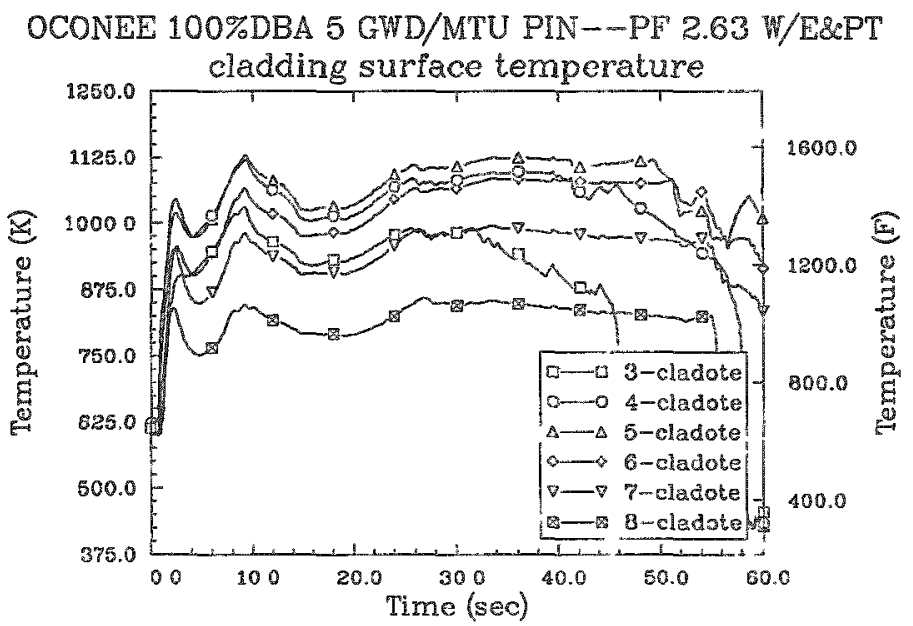

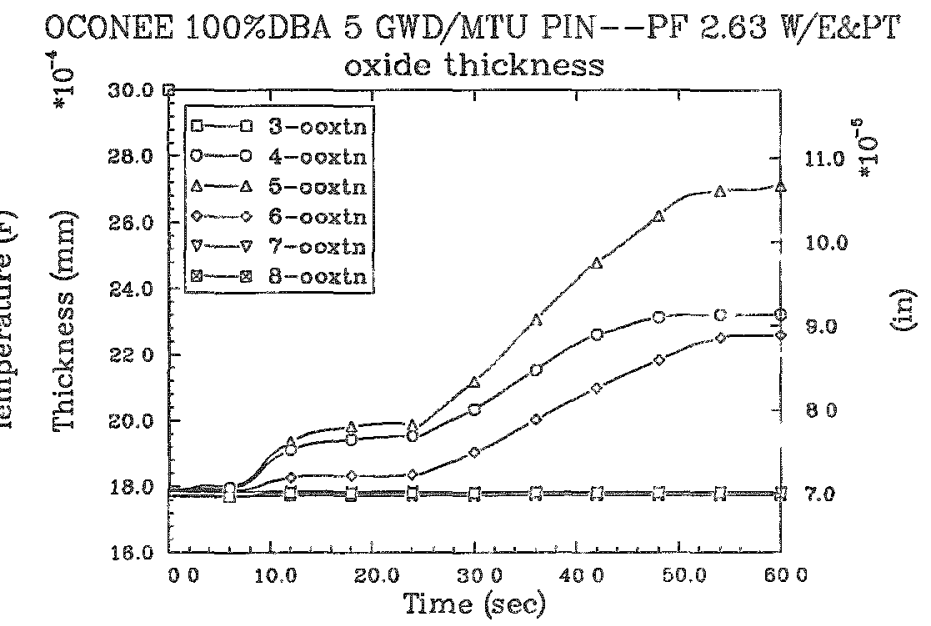



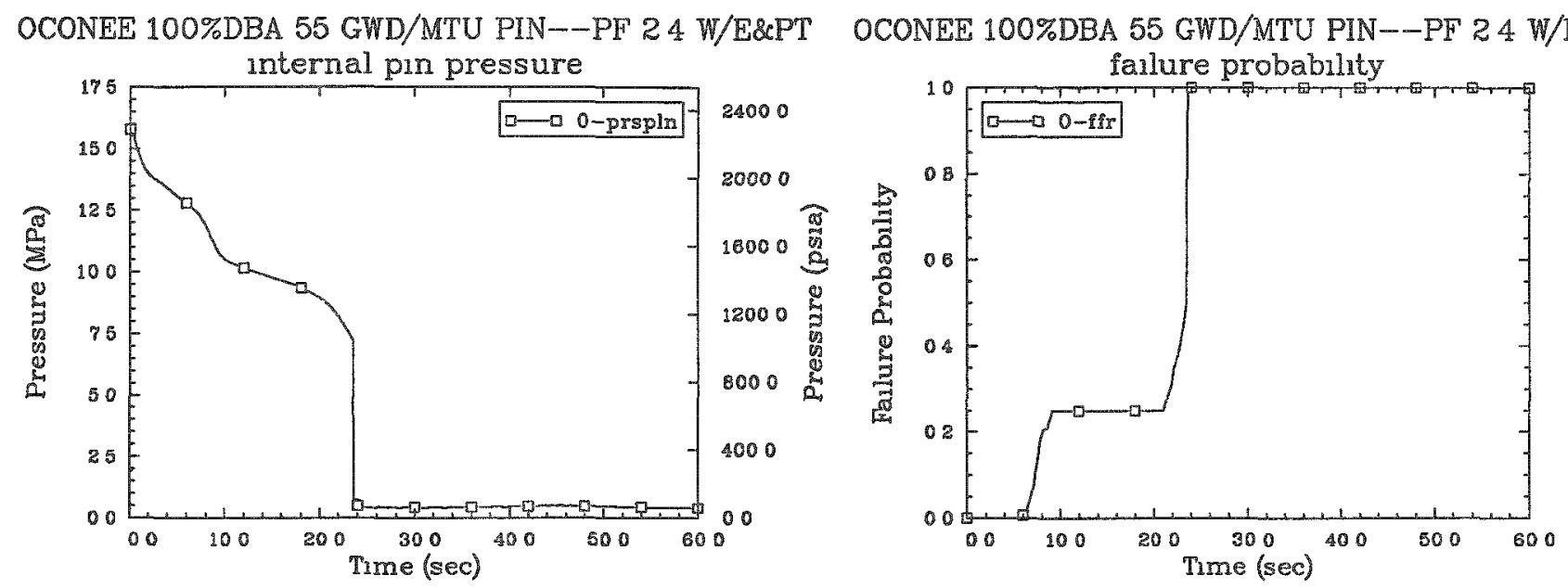

OCONEE 100\%DBA $55 \mathrm{GWD} / \mathrm{MTU}$ PIN--PF 24 W/E\&PT
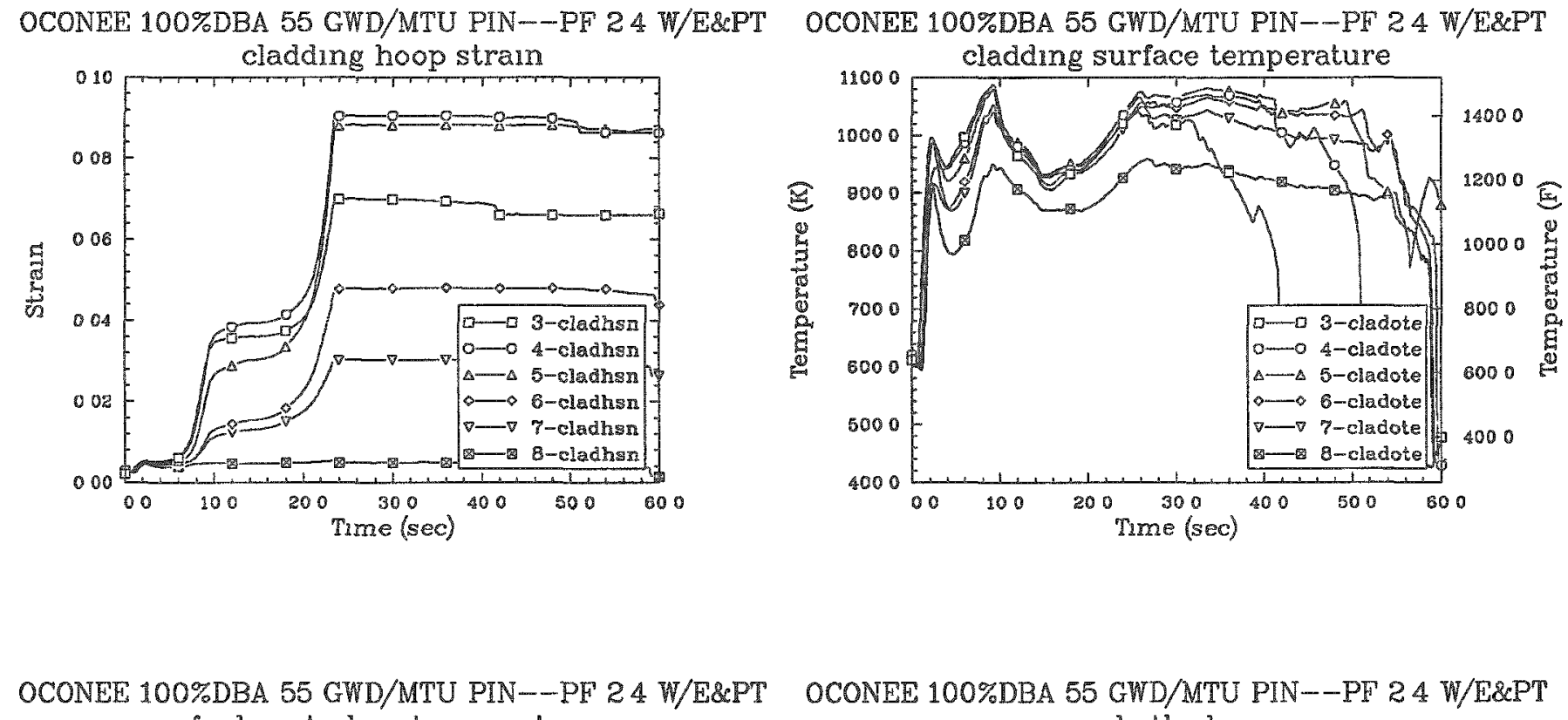

OCONEE 100\%DBA 55 GWD/MTU PIN--PF 24 W/E\&PT
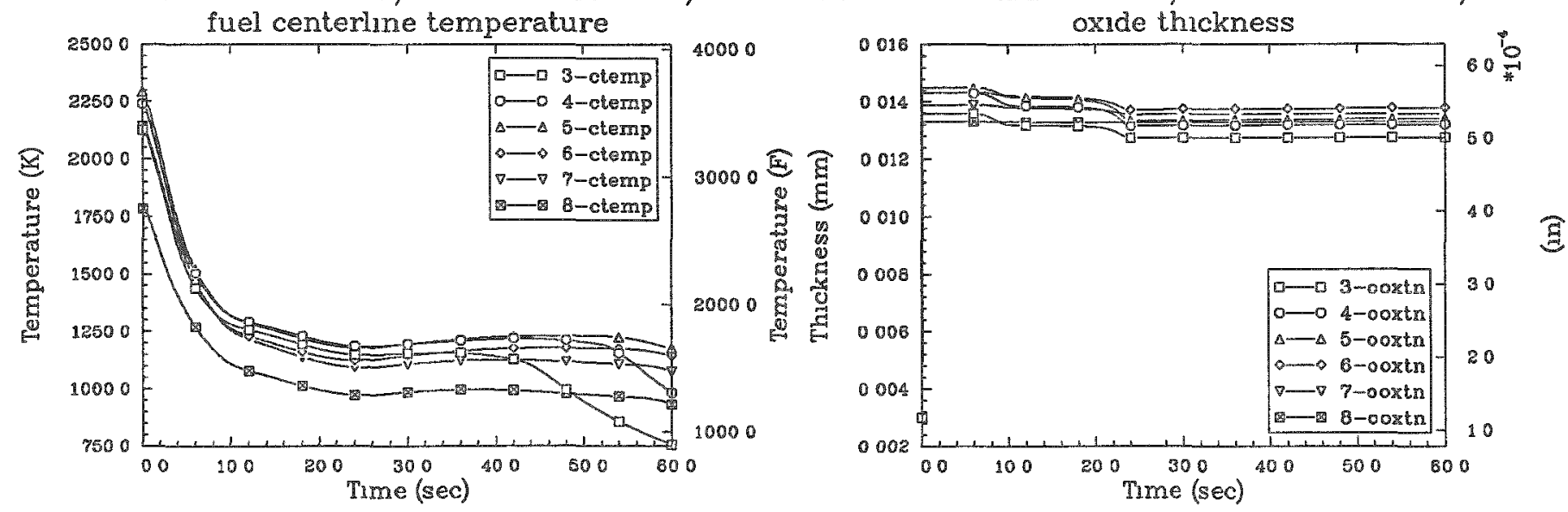
OCONEE 100\%DBA $35 \mathrm{GWD} / \mathrm{MTU}$ PIN--PF $2.4 \mathrm{~W} / \mathrm{E} \& \mathrm{PT}$

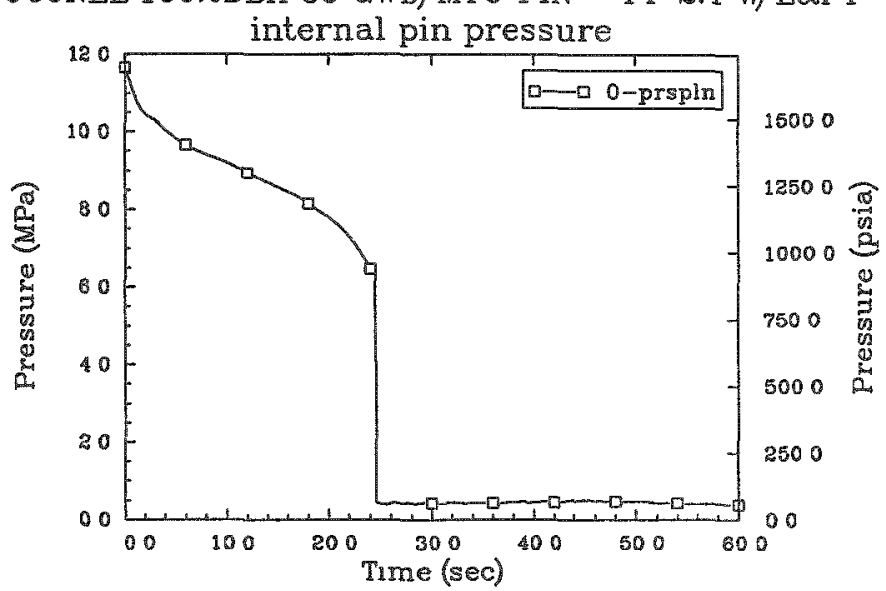

OCONEE 100\%DBA 35 GWD/MTU PIN-DF 2.4 W/E\&PT failure probability

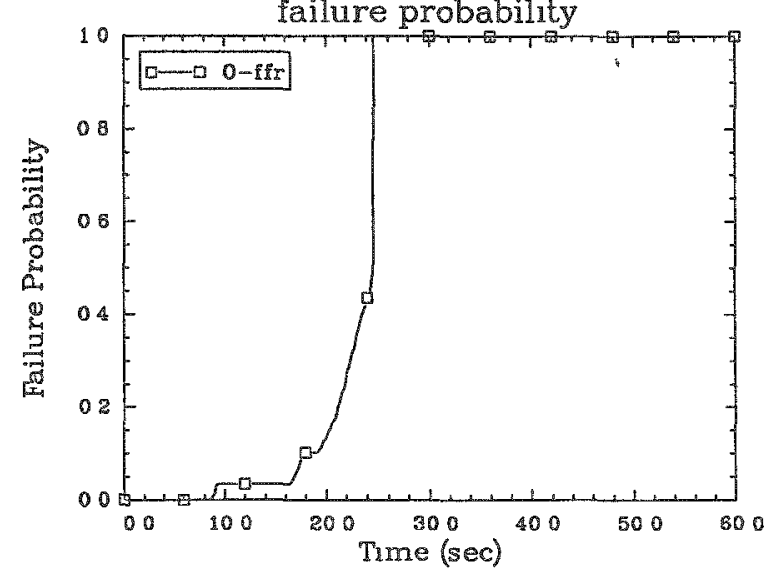

OCONEE 100\%DBA 35 GWD/MTU PIN--PF 2.4 W/E\&PT

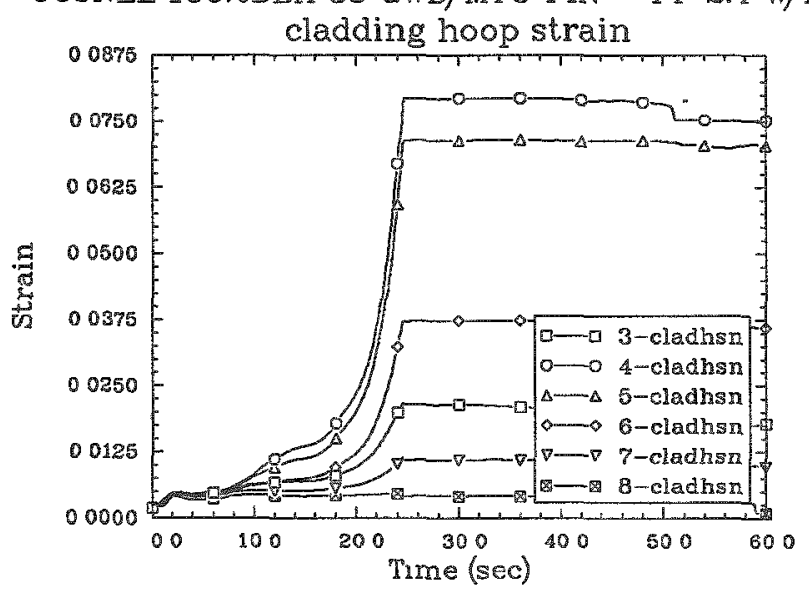

OCONEE 100\%DBA 35 GWD/MTU PIN--PF 24 W/E\&PT

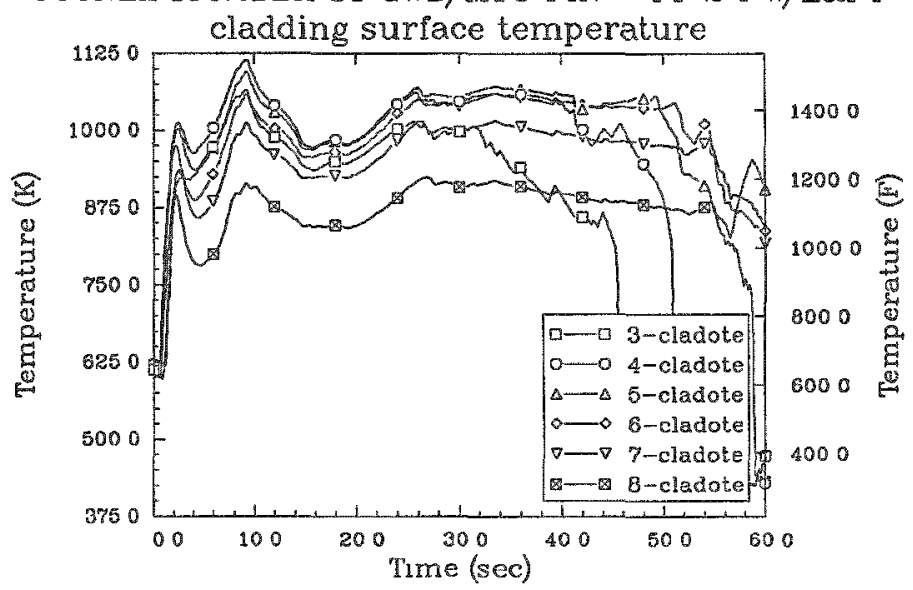

OCONEE 100\%DBA 35 GWD/MTU PIN--PF 2.4 W/E\&PT

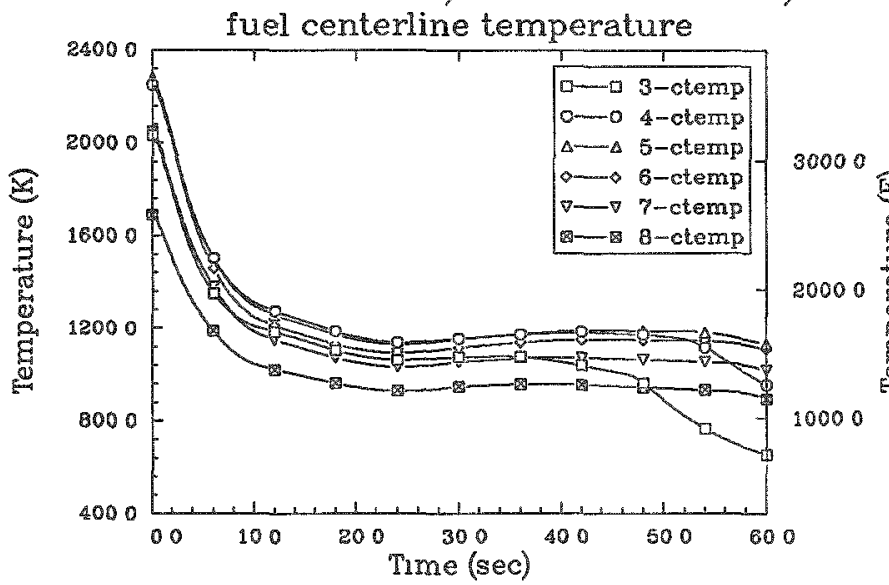

OCONEE 100\%DBA 35 GWD/MTU PIN--PF 24 W/E\&PT

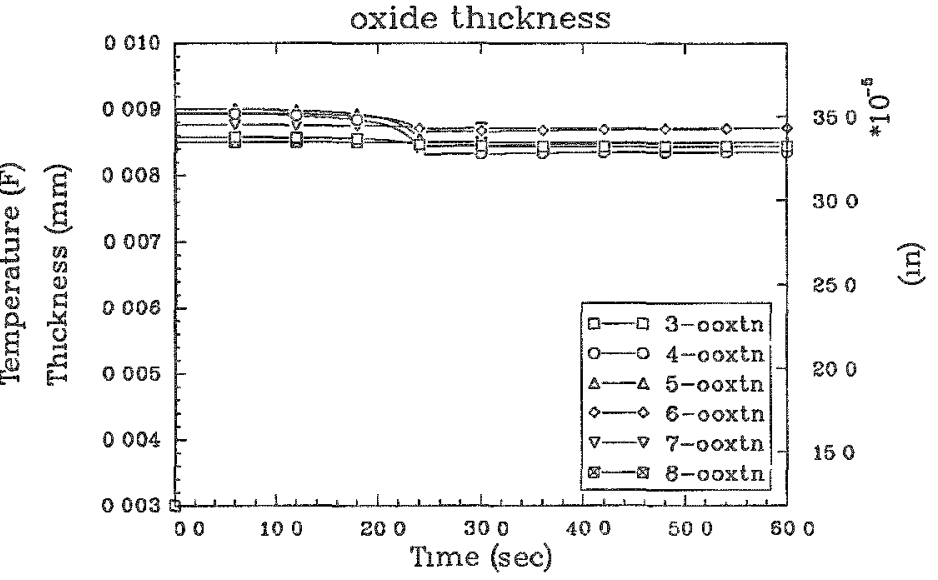


OCONEE 100\%DBA $20 \mathrm{GWD} / \mathrm{MTU} \mathrm{PIN}--\mathrm{PF} 24 \mathrm{~W} / \mathrm{E} \& \mathrm{PT}$

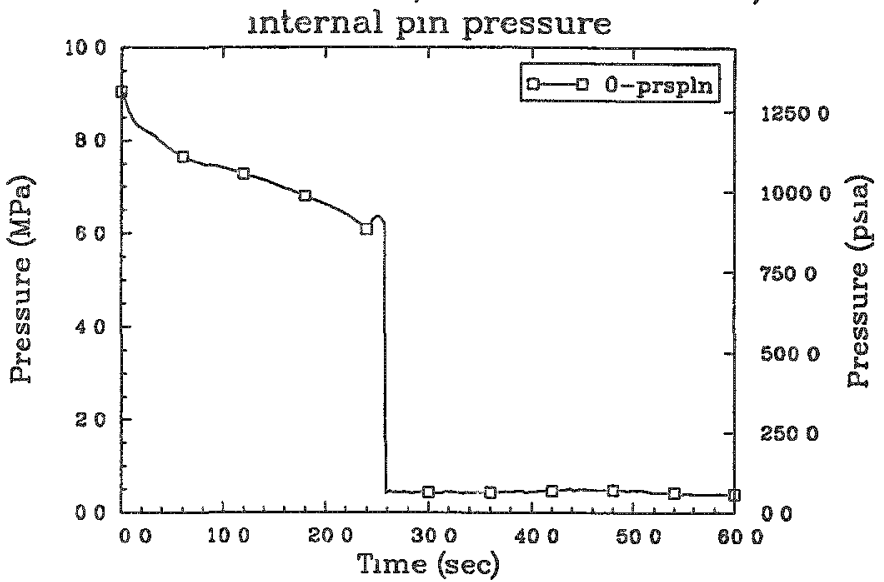

OCONEE 100\%DBA 20 GWD/MTU PIN--PF 24 W/E\&PT

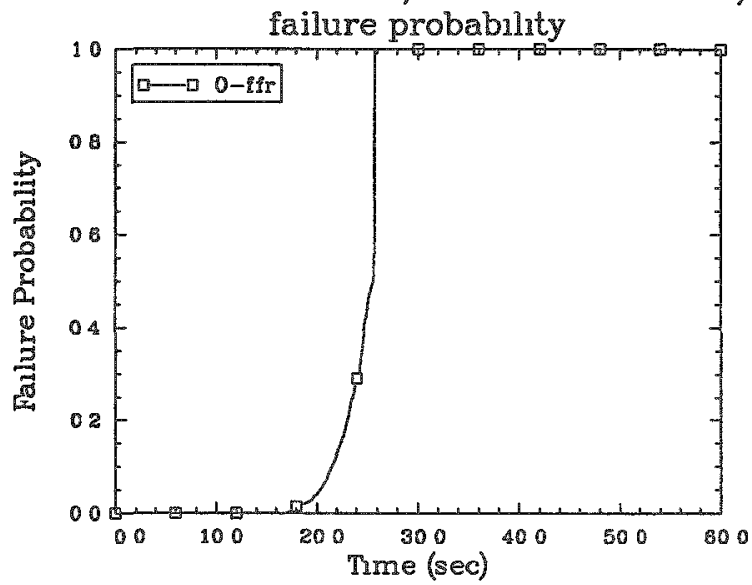

OCONEE 100\%DBA $20 \mathrm{GWD} / \mathrm{MTU}$ PIN--PF $24 \mathrm{~W} / \mathrm{E} \& \mathrm{PT}$ cladding hoop stram

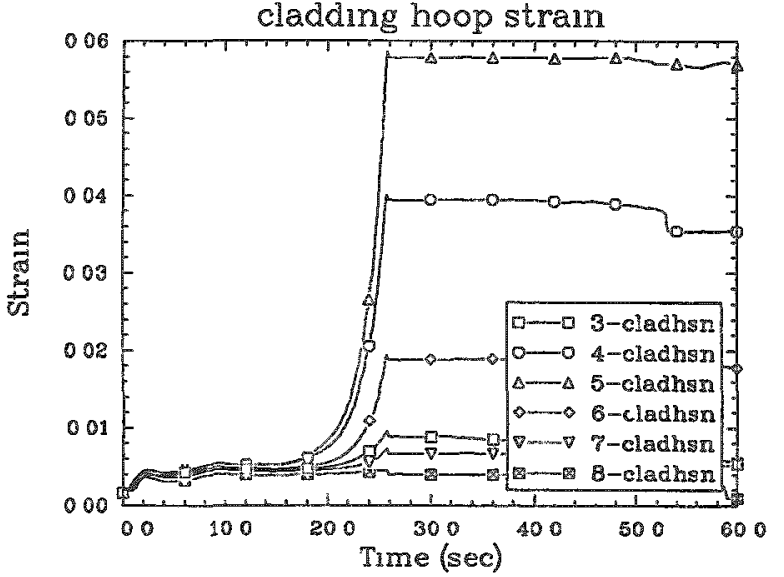

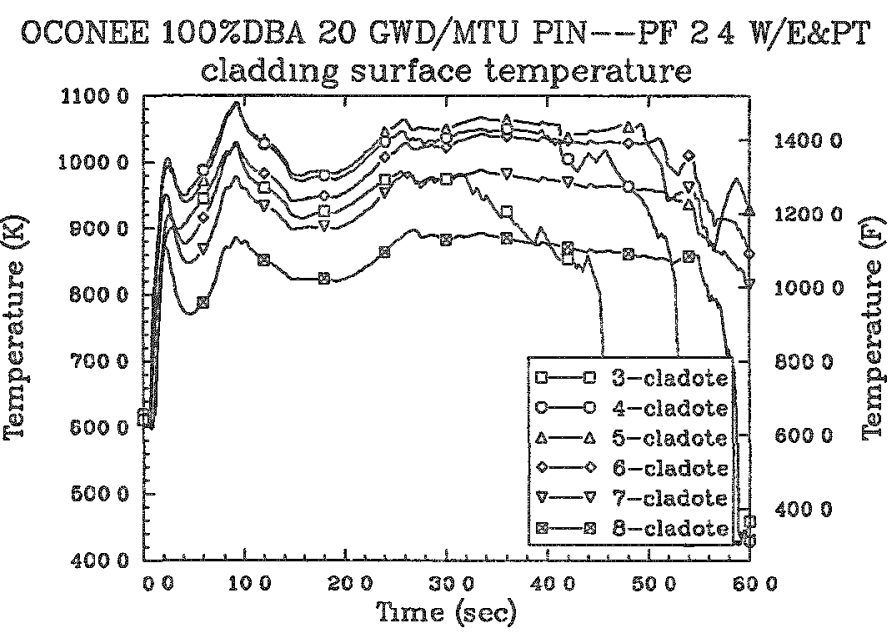

OCONEE 100\%DBA $20 \mathrm{GWD} / \mathrm{MTU} \mathrm{PIN}-\mathrm{PF} 24 \mathrm{~W} / \mathrm{E} \& \mathrm{PT}$

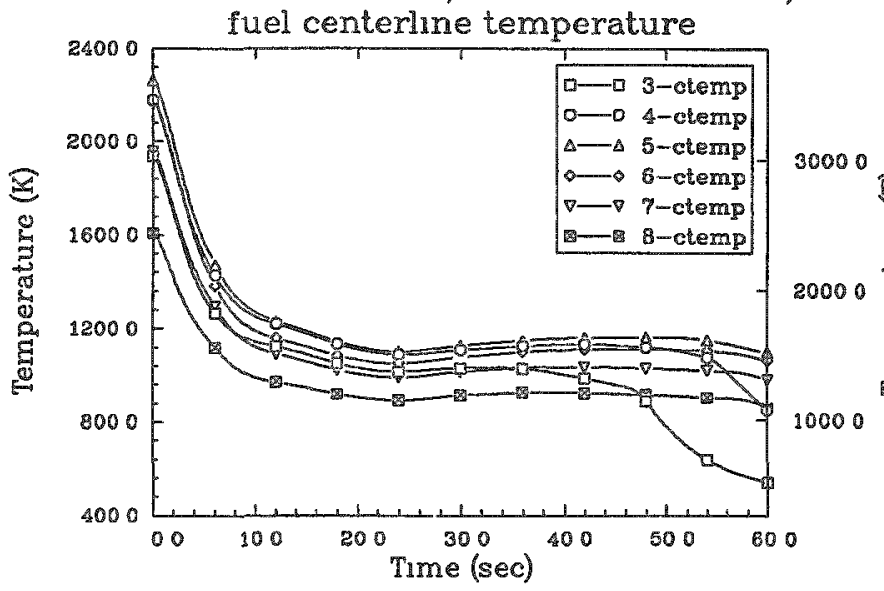

OCONEE 100\%DBA $20 \mathrm{GWD} / \mathrm{MTU}$ PIN--PF 24 W/E\&PT

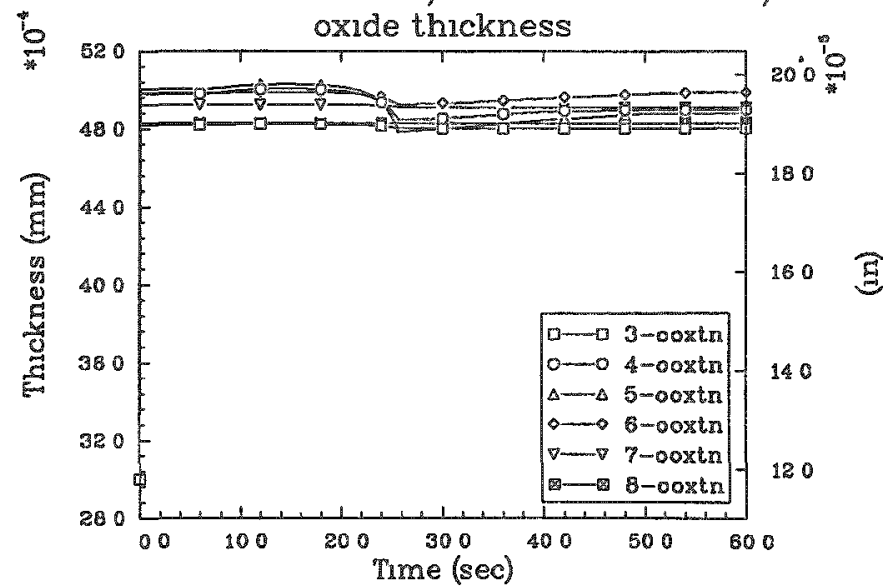




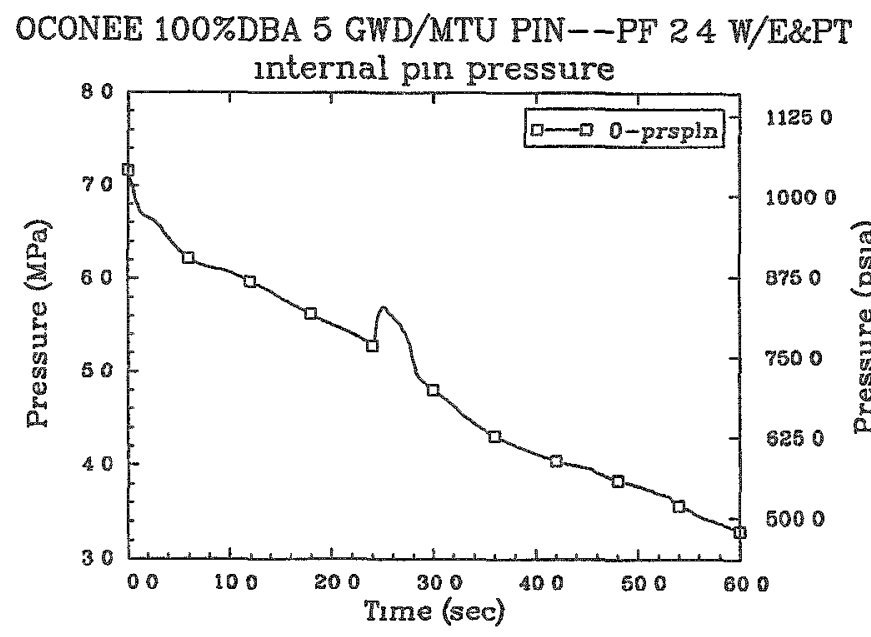

OCONEE 100\%DBA 5 GWD/MTU PIN--PF 2.4 W/E\&PT

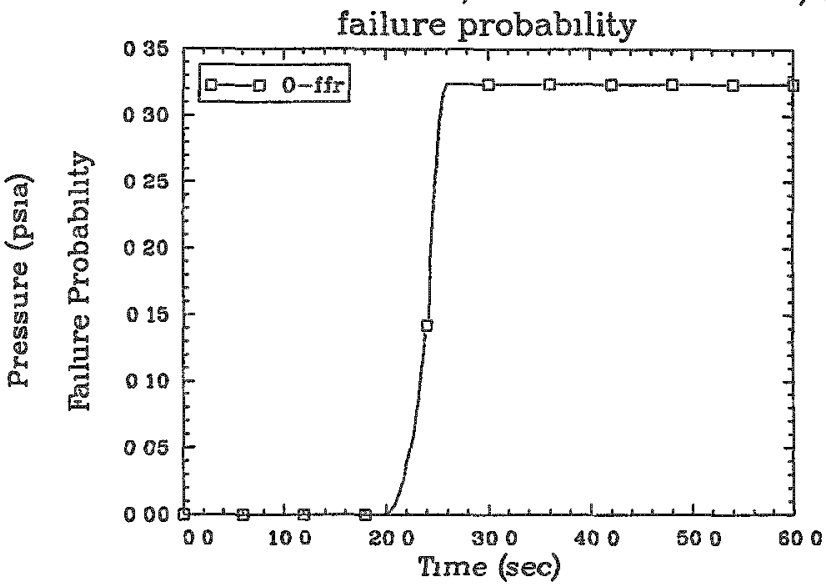

OCONEE 100\%DBA 5 GWD/MTU PIN--PF 24 W/E\&PT cladding hoop strain

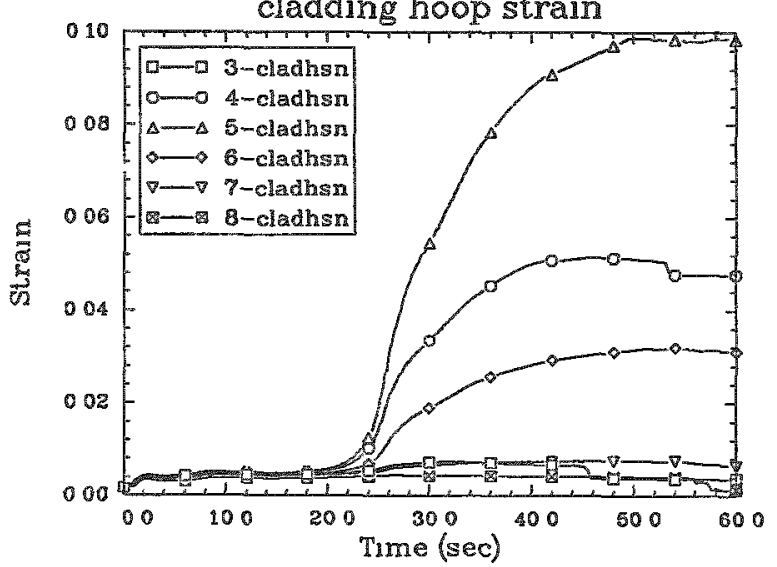

OCONEE 100\%DBA 5 GWD/MTU PIN--PF 24 W/E\&PT

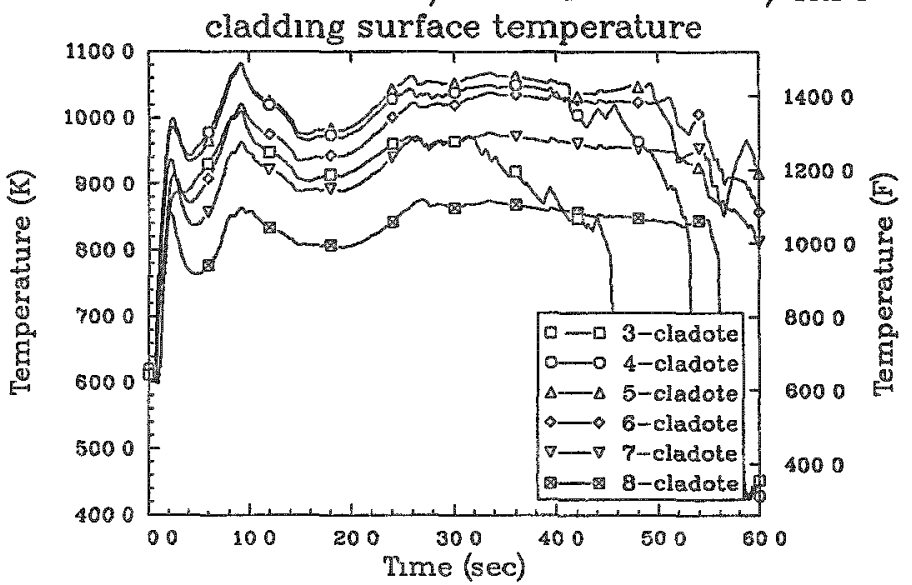

OCONEC 100\%DBA 5 GWD/MTU PIN--PF 24 W/E\&PT

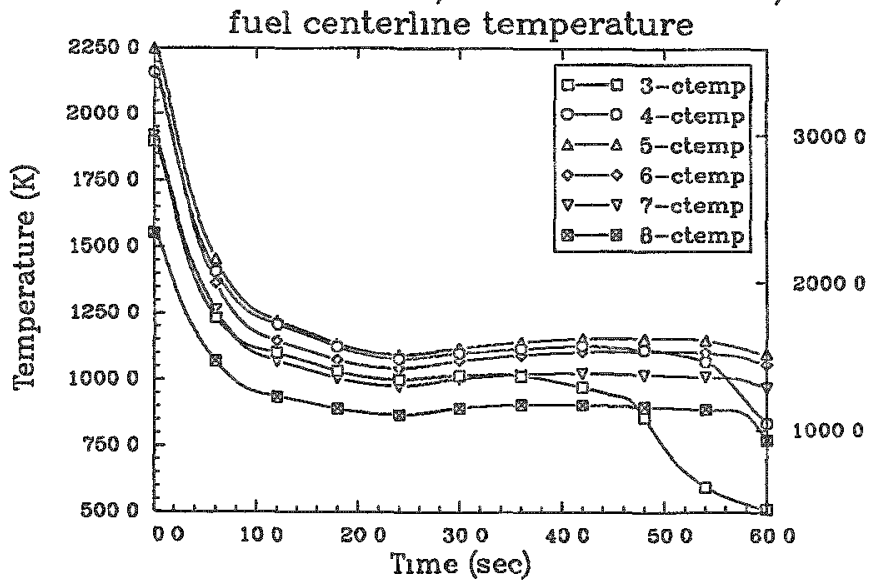

OCONEE 100\%DBA 5 GWD/MTU PIN--PF 24 W/E\&PT

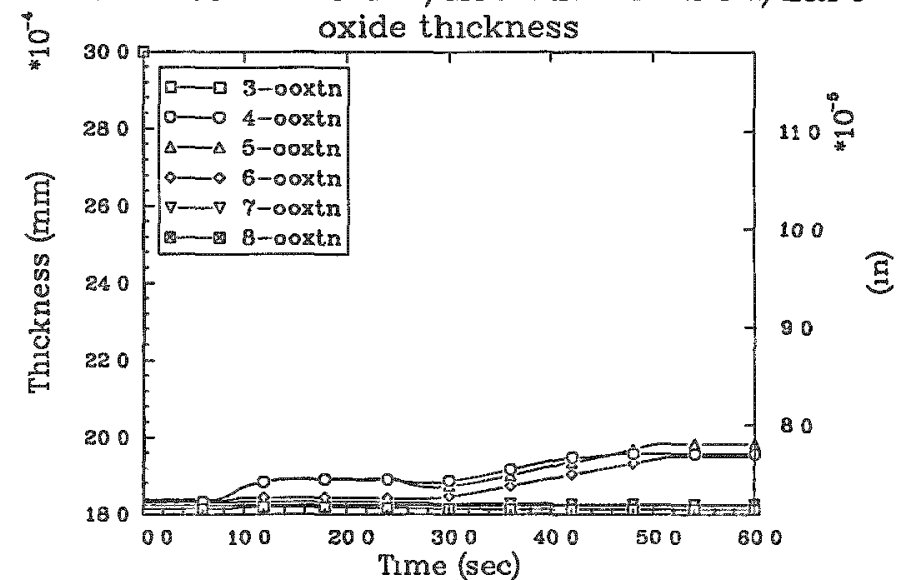


OCONEE 100\%DBA 55 GWD/MTU PIN--PF 22 W/E\&PT

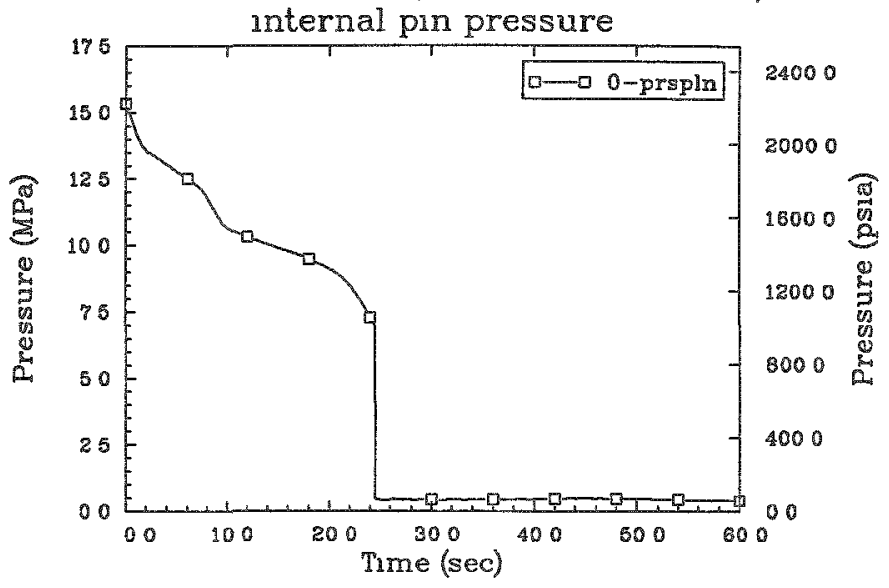

OCONEE 100\%DBA 55 GWD/MTU PIN--PF 22 W/L\&PT

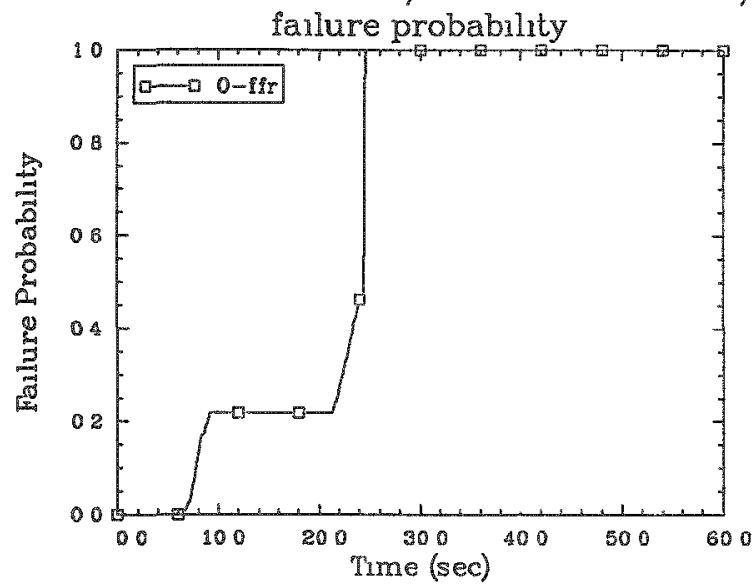

OCONEE 100\%DBA 55 GWD/MTU PIN--PF 22 W/E\&PT cladding hoop strain

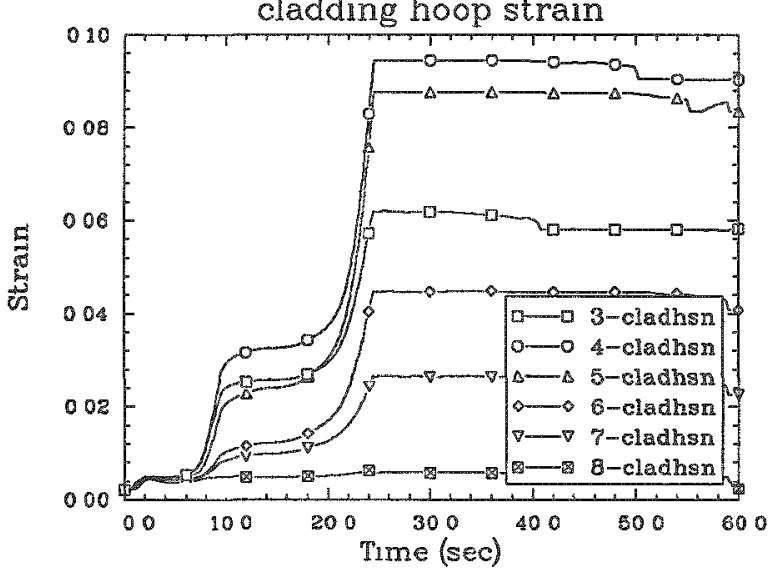

OCONEE 100\%DBA 55 GWD/MTU PIN--PF 22 W/E\&PT

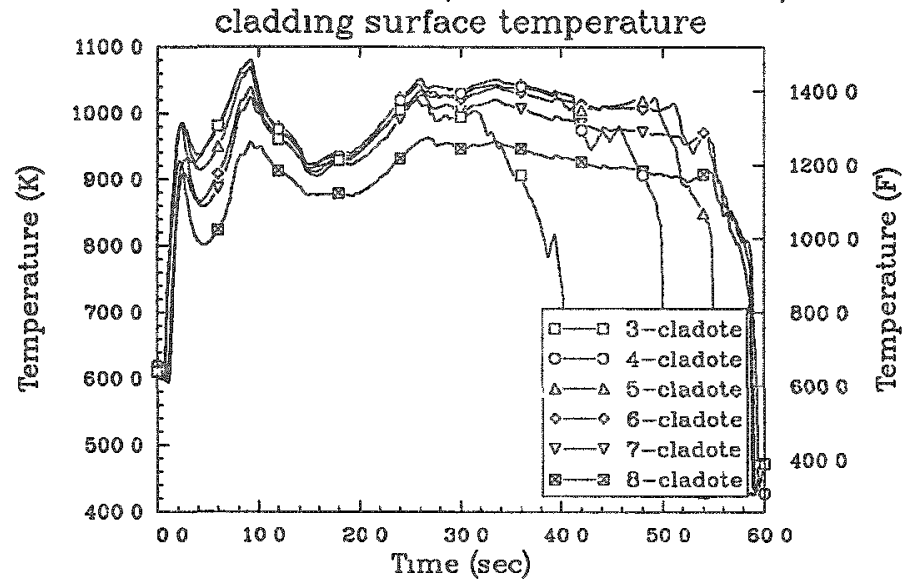

OCONEE 100\%DBA 55 GWD/MTU PIN--PF 22 W/E\&PT

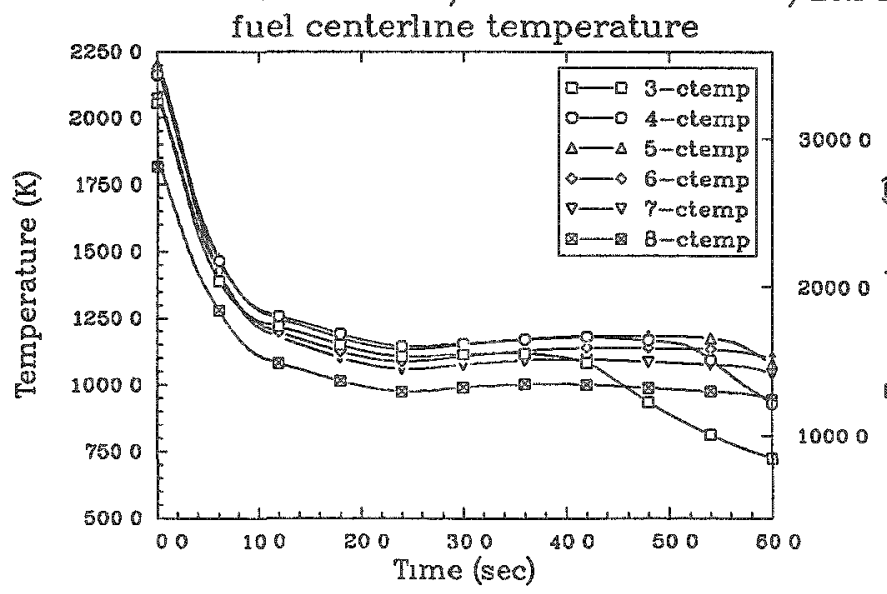

OCONEE $100 \%$ DBA 55 GWD/MTU PIN--PF 22 W/E\&PT

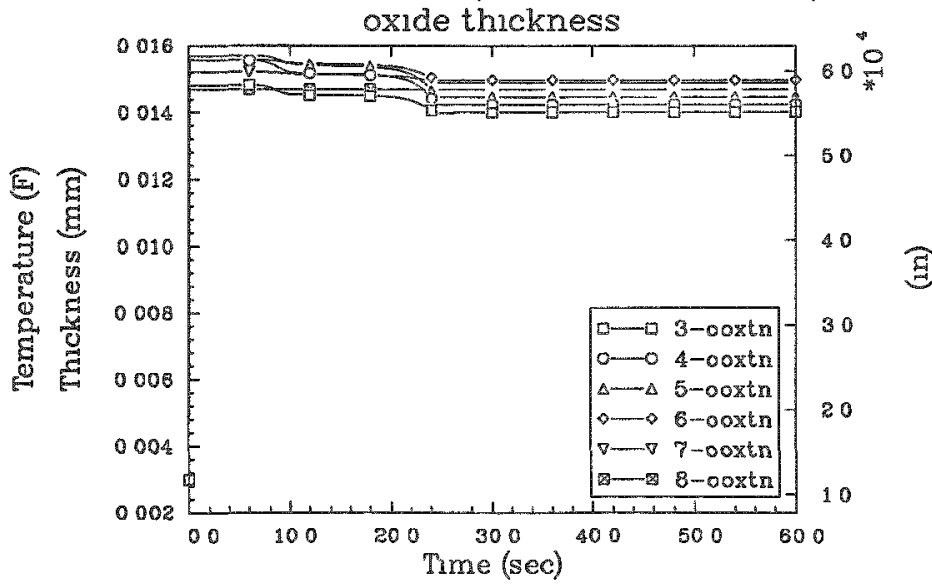


OCONEE 100\%DBA 35 GWD/MTU PIN--PF 22 W/E\&PT unternal pin pressure

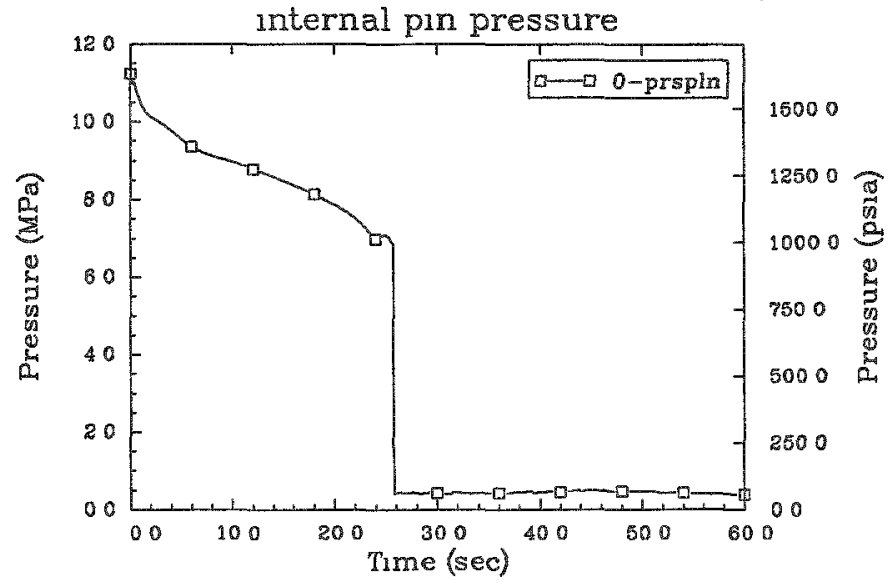

OCONEE 100\%DBA 35 GWD/MTU PIN--PF 22 W/E\&PT

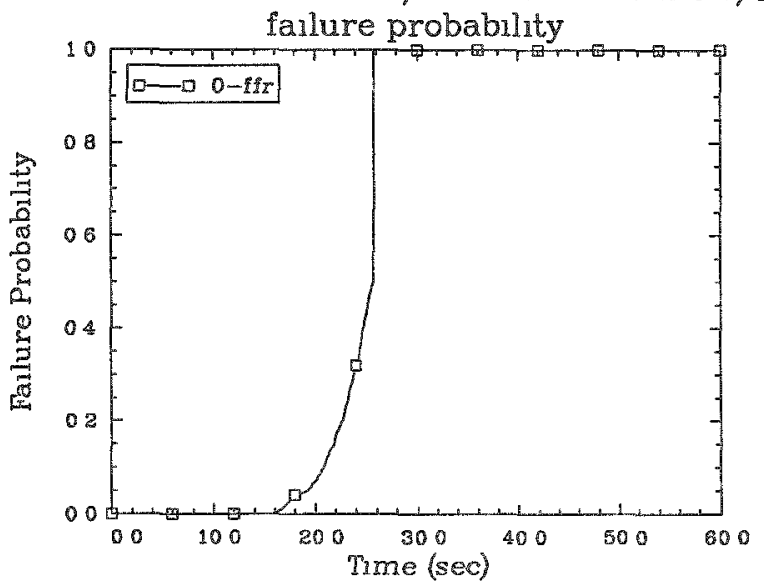

OCONEE 100\%DBA 35 GWD/MTU PIN--PF 22 W/E\&PT

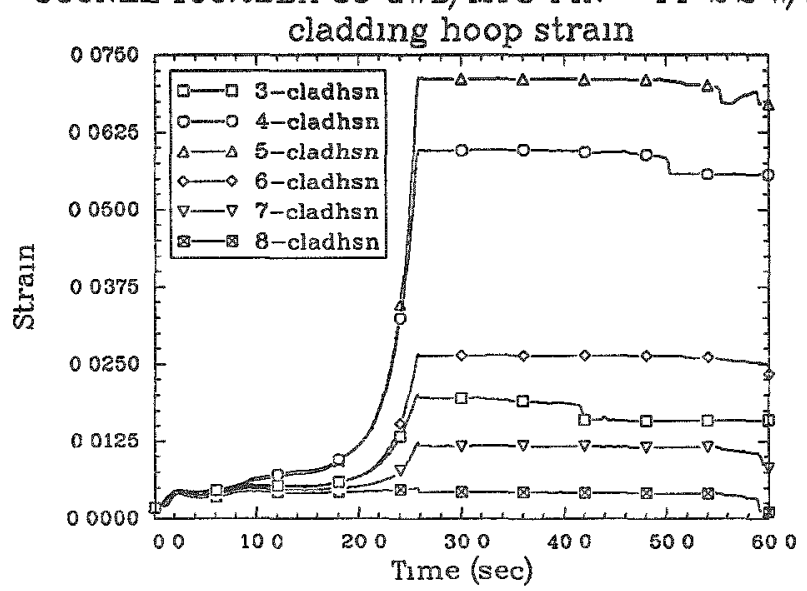

OCONEE 100\%DBA 35 GWD/MTU PIN--PF 22 W/E\&PT

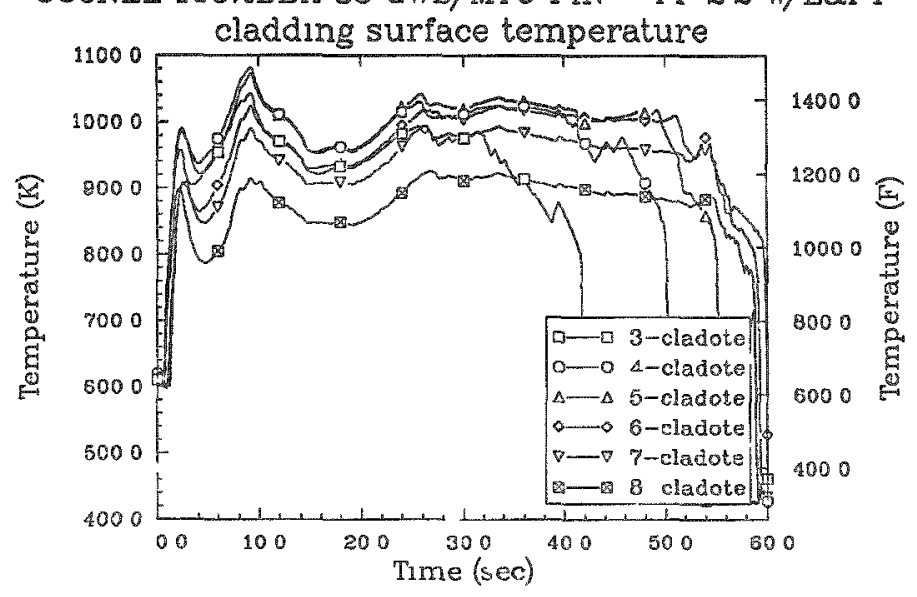

OCONEE 100\%DBA 35 GWD/MTU PIN--PF 22 W/E\&PT

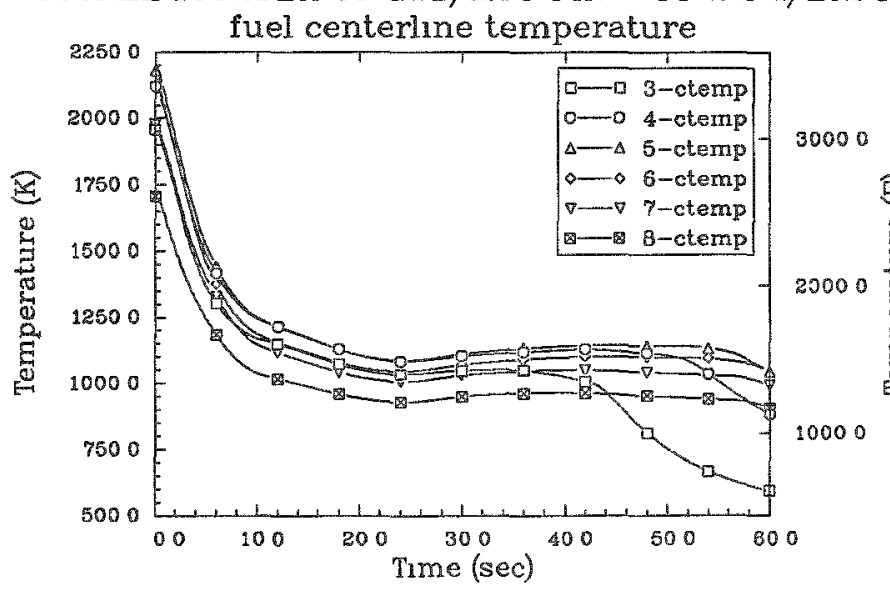

OCONEE 100\%DBA 35 GWD/MTU PIN--PF 22 W/E\&PT

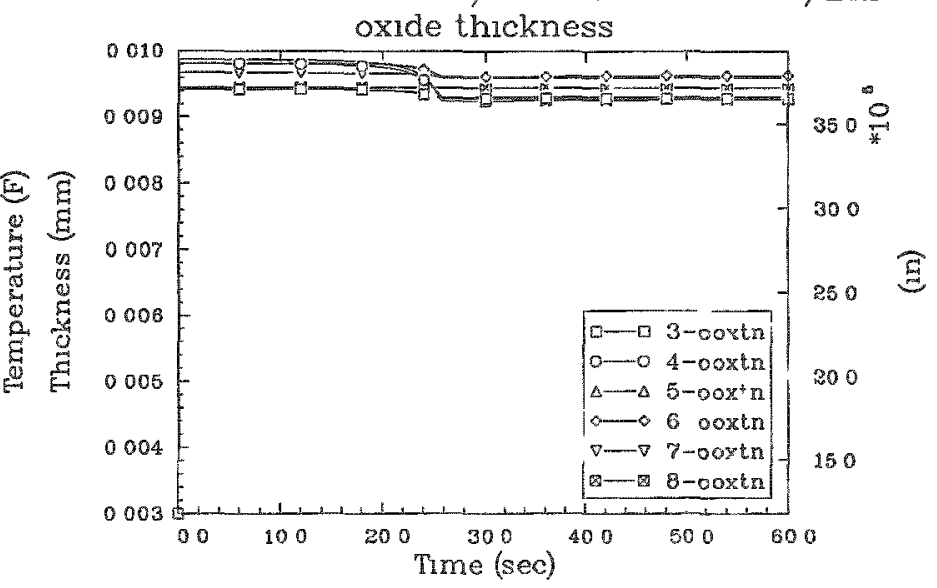


OCONEE 100\%DBA $20 \mathrm{GWD} / \mathrm{MTU}$ PIN--PF $2.2 \mathrm{~W} / \mathrm{E} \& \mathrm{PT}$

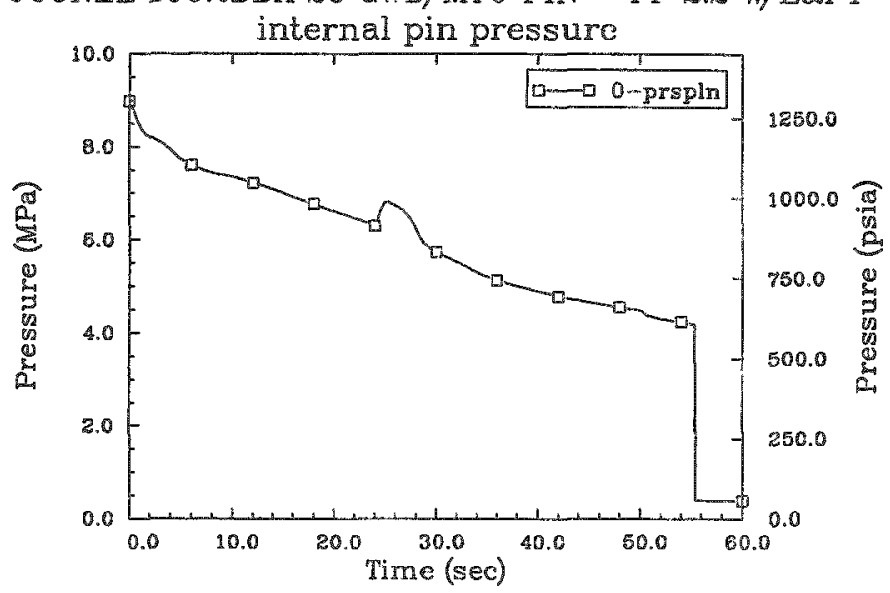

OCONEE 100\%DBA $20 \mathrm{GWD} / \mathrm{MTU}$ PIN-DF $2.2 \mathrm{~W} / \mathrm{E} \& \mathrm{PT}$

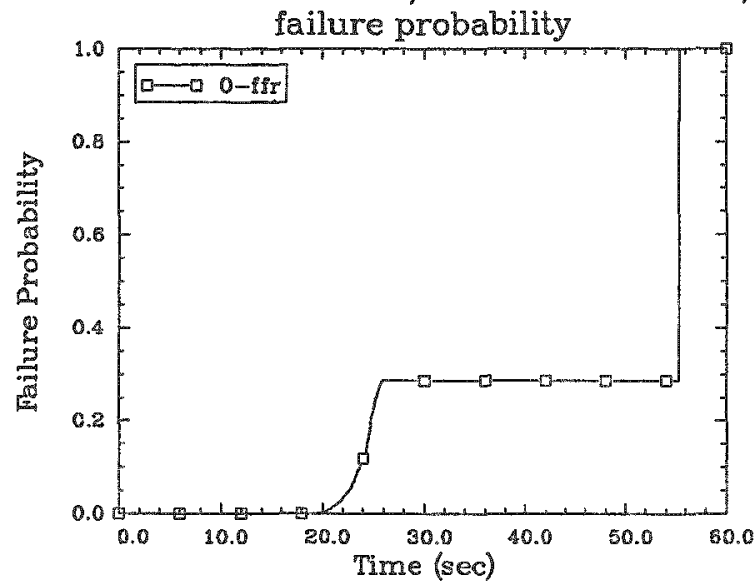

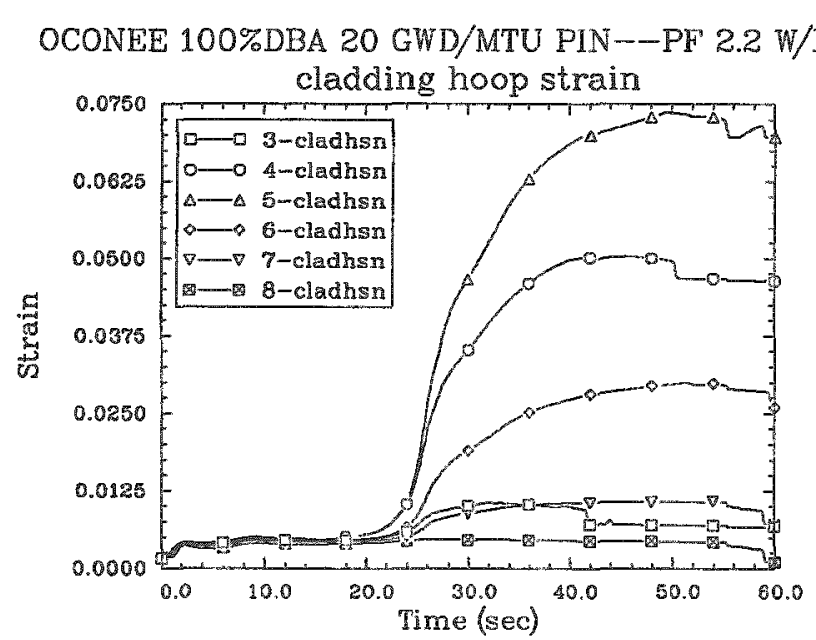

OCONEE 100\%DBA $20 \mathrm{GWD} / \mathrm{MTU}$ PIN--PF 2.2 W/E\&PT

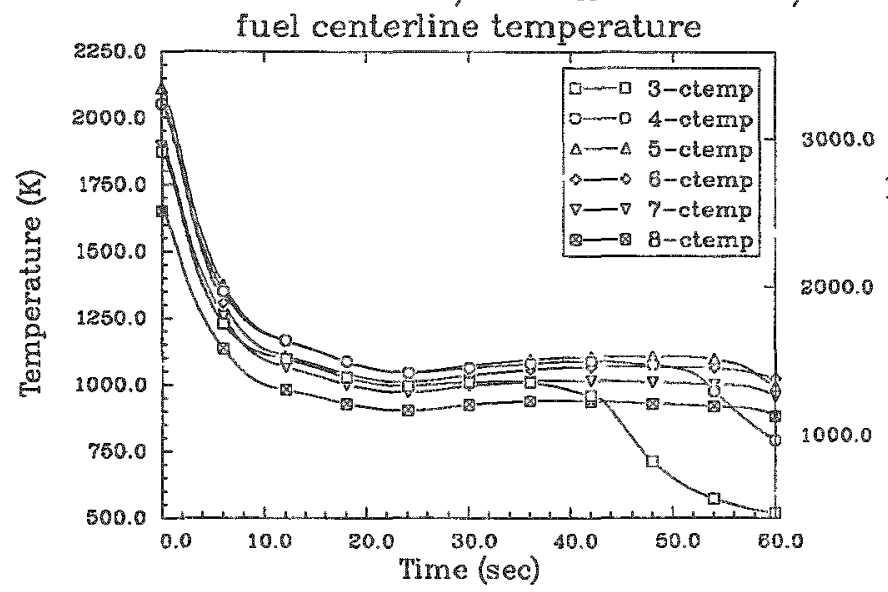

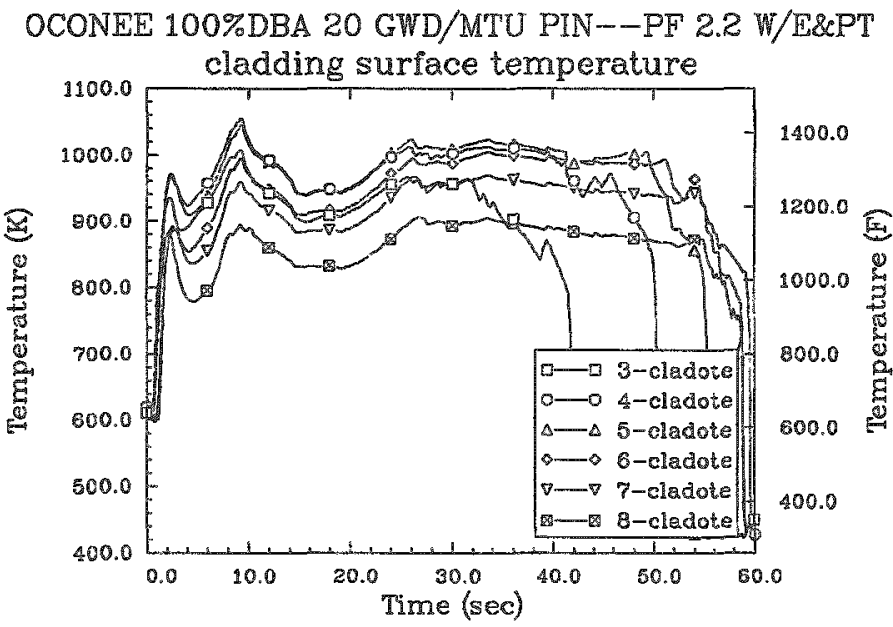

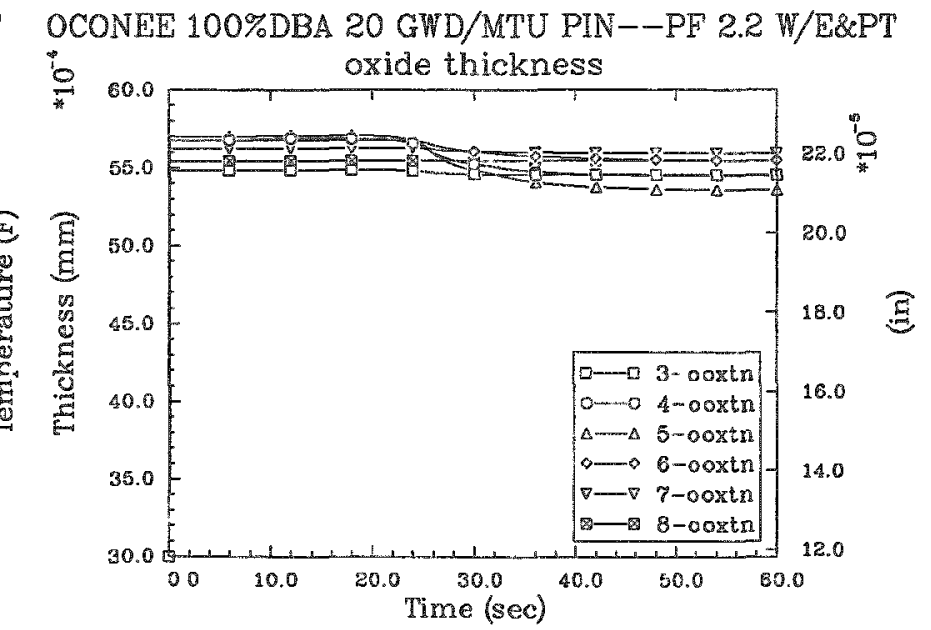


OCONEE 100\%DBA 5 GWD/MTU PIN--PF $2.2 \mathrm{~W} / \mathrm{E} \& \mathrm{PT}$

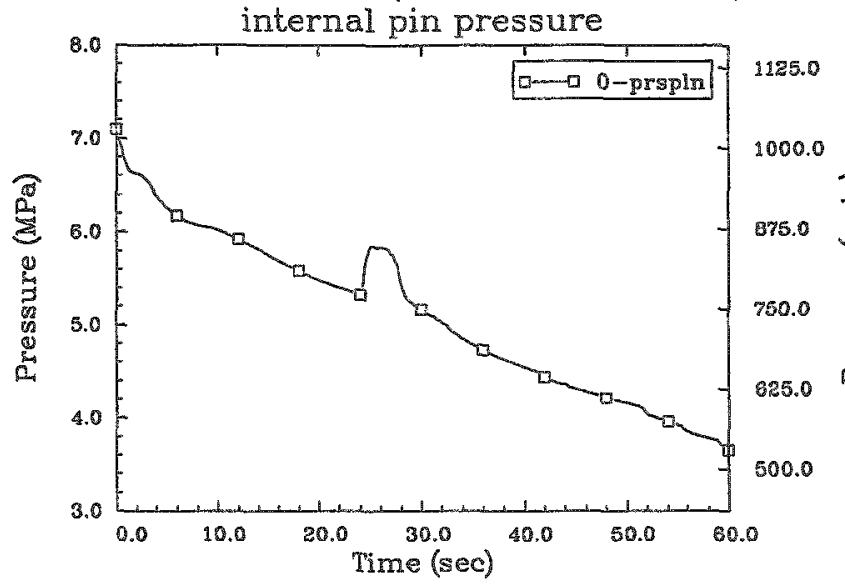

OCONEE 100\%DBA 5 GWD/MTU PIN--PF 2.2 W/E\&PT cladding hoop strain

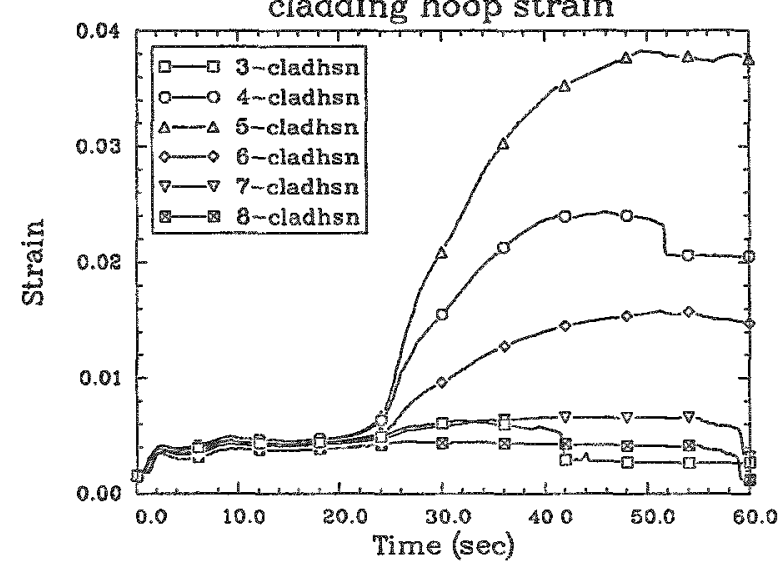

OCONEE 100\%DBA 5 GWD/MTU PIN--PF 2.2 W/E\&PT

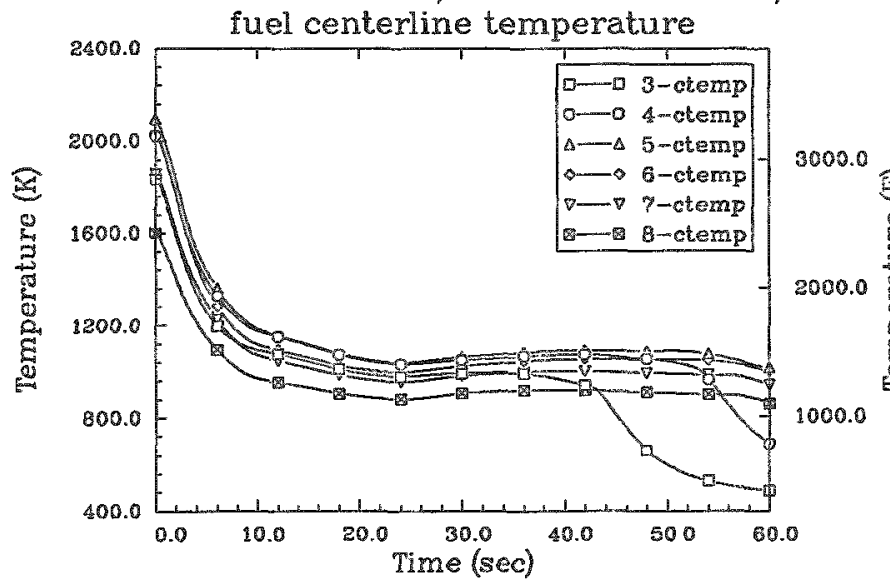

OCONEE 100\%DBA 5 GWD/MTU PIN--PF 2.2 W/E\&PT failure probability

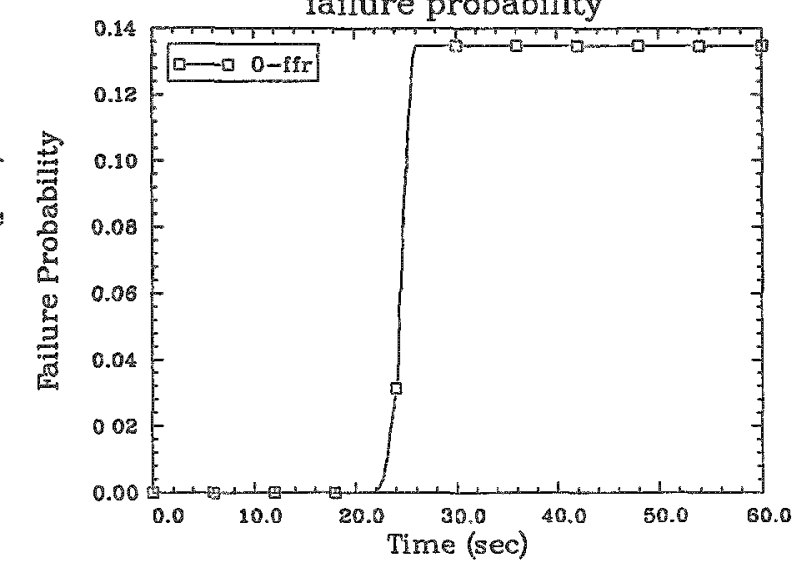

OCONEE 100\%DBA 5 GWD/MTU PIN--PP 2.2 W/E\&PT cladding surface temperature

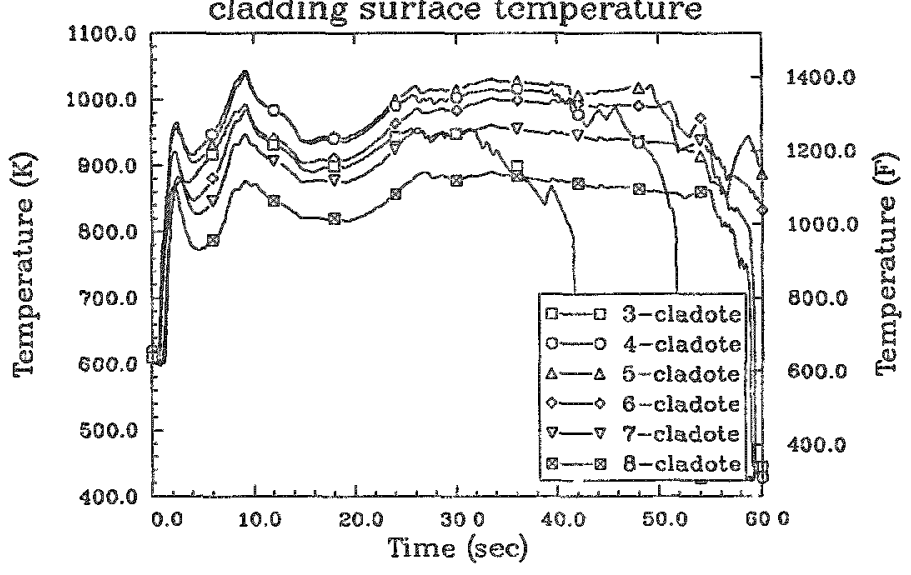

OCONEE 100\%DBA 5 GWD/MTU PIN--PF 2.2 W/E\&PT

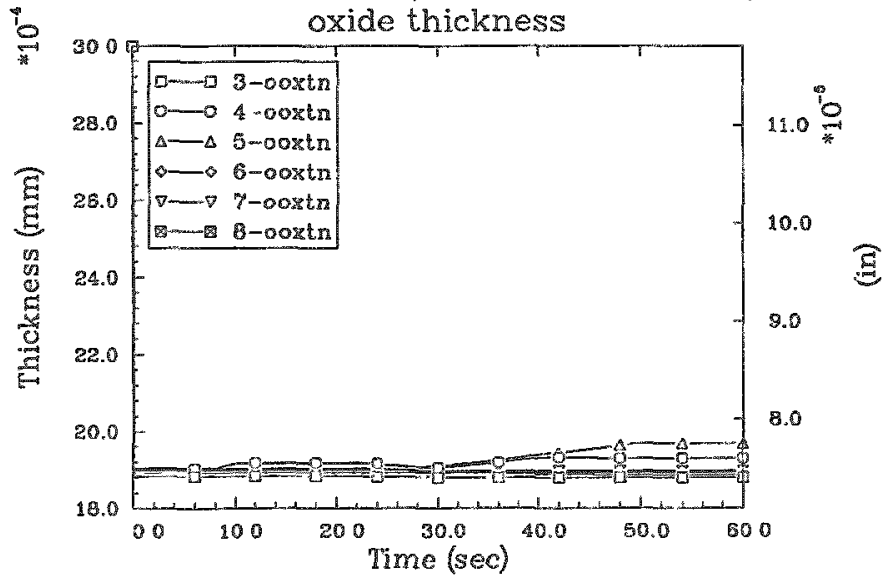


OCONEE 100\%DBA 55 GWD/MTU PIN--PF 20 W/E\&PT

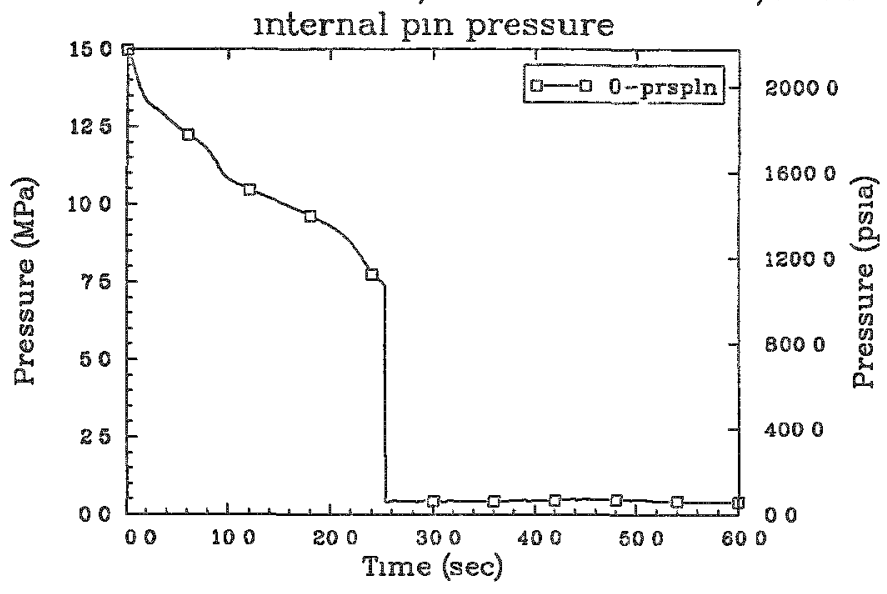

OCONEE 100\%DBA 55 GWD/MTU PIN--PF 20 W/E\&PT

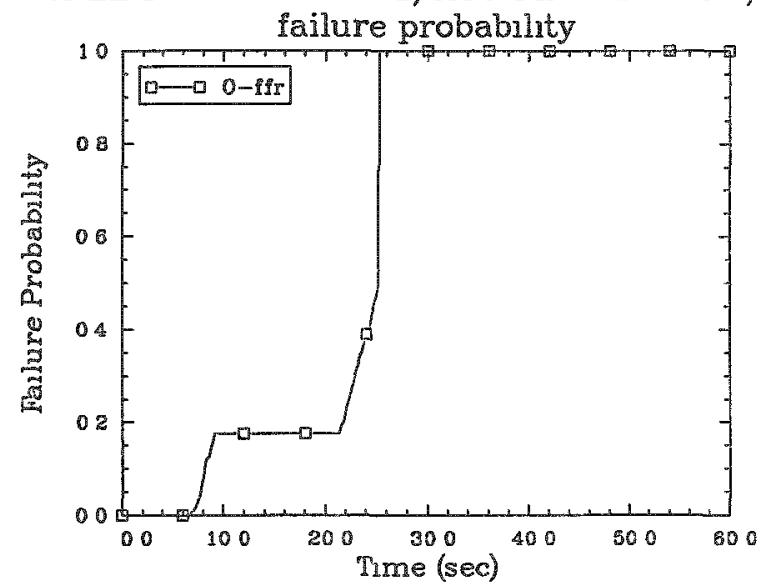

OCONEE 100\%DBA 55 GWD/MTU PIN--PF 20 W/E\&PT cladding hoop strain

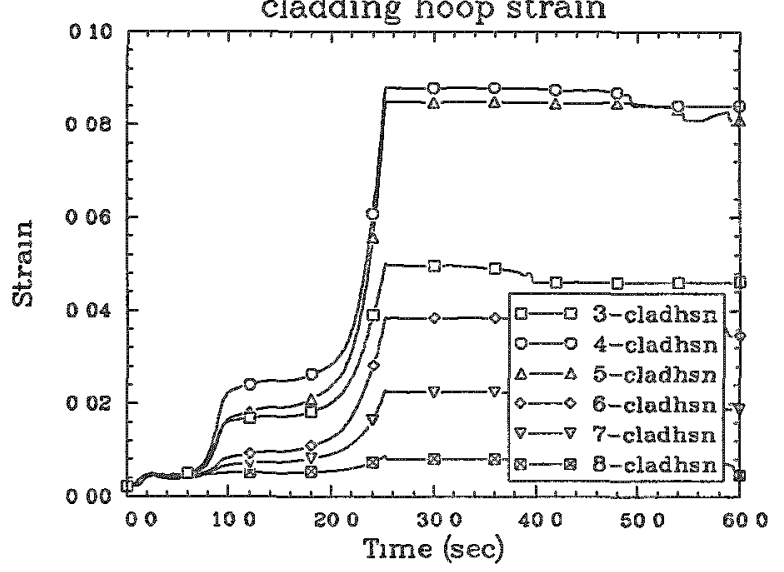

OCONEE 100\%DBA 55 GWD/MTU PIN--PF 20 W/E\&PT

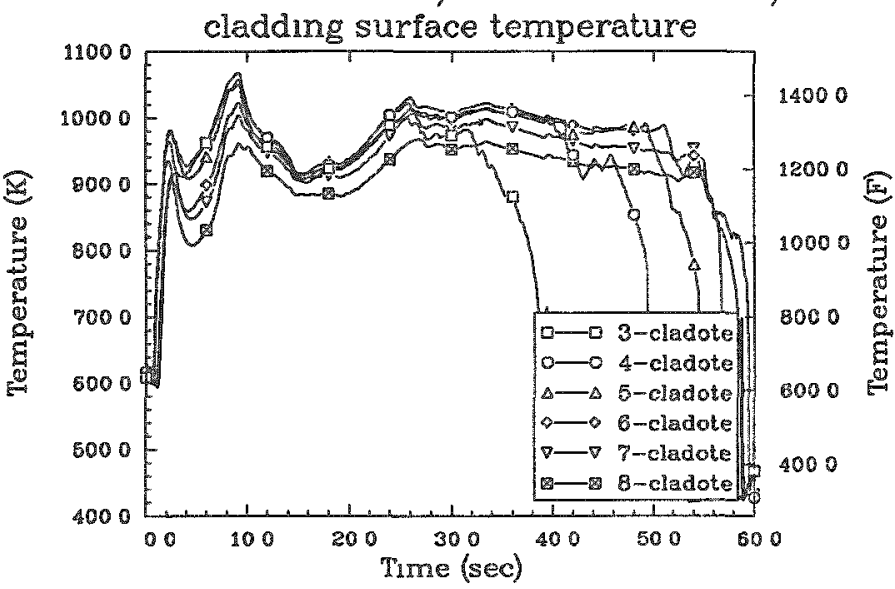

\section{OCONEE 100\%DBA 55 GWD/MTU PIN- PF 20 W/E\&PT}

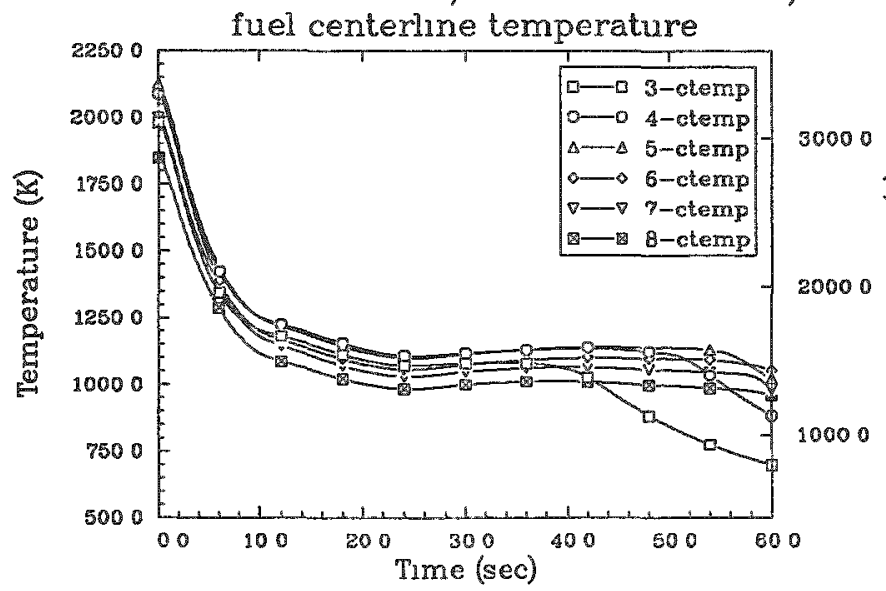

OCONEE 100\%DBA 55 GWD/MTU PIN--PF 20 W/E\&PT

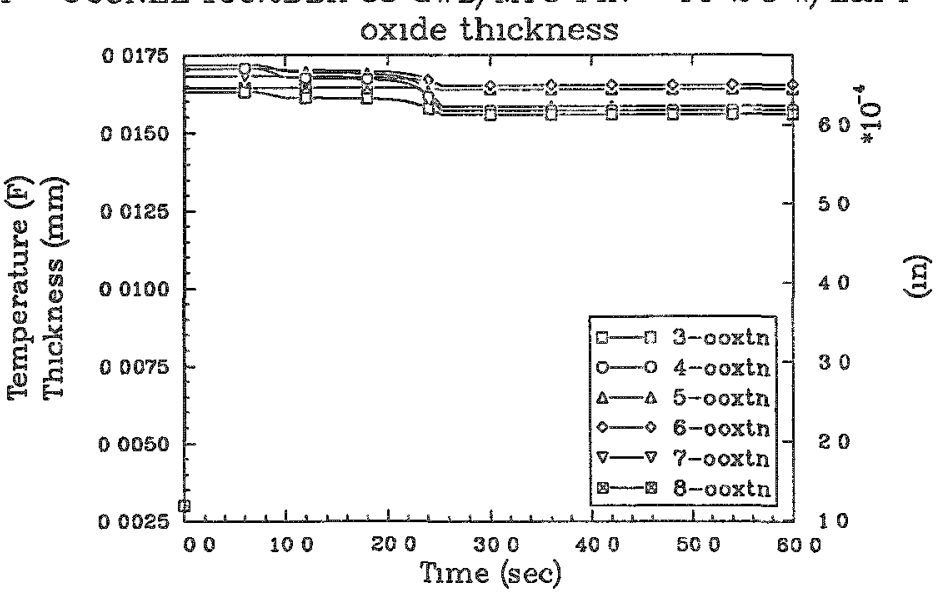


OCONEE 100\%DBA 35 GWD/MTU PIN--PF 2.0 W/E\&PT

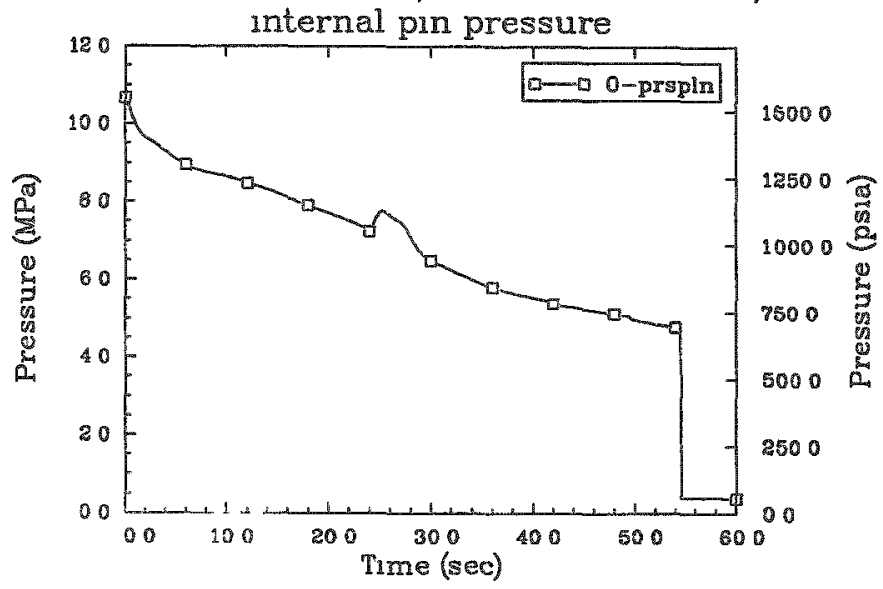

OCONEE 100\%DBA $35 \mathrm{GWD} / \mathrm{MTU}$ PIN--PF $2.0 \mathrm{~W} / \mathrm{E} \& \mathrm{PT}$

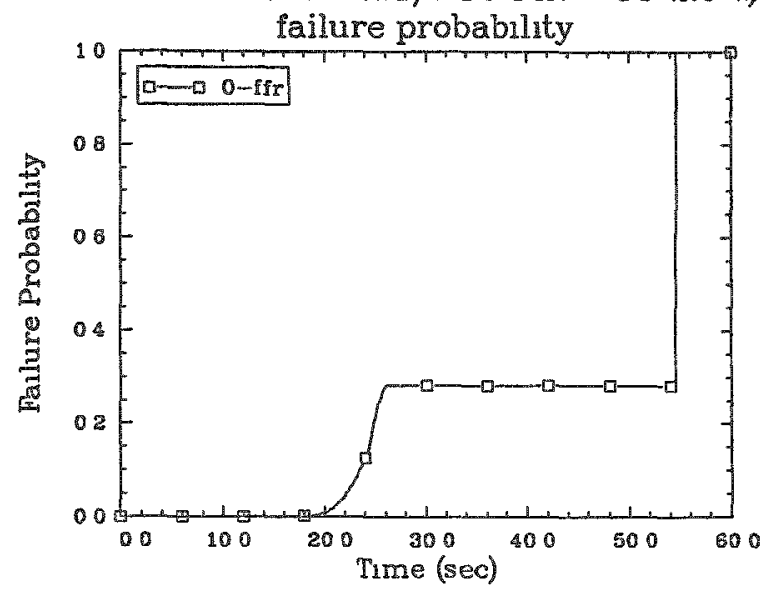

OCONEE 100\%DBA 35 GWD/MTU PIN-PF 20 W/E\&PT

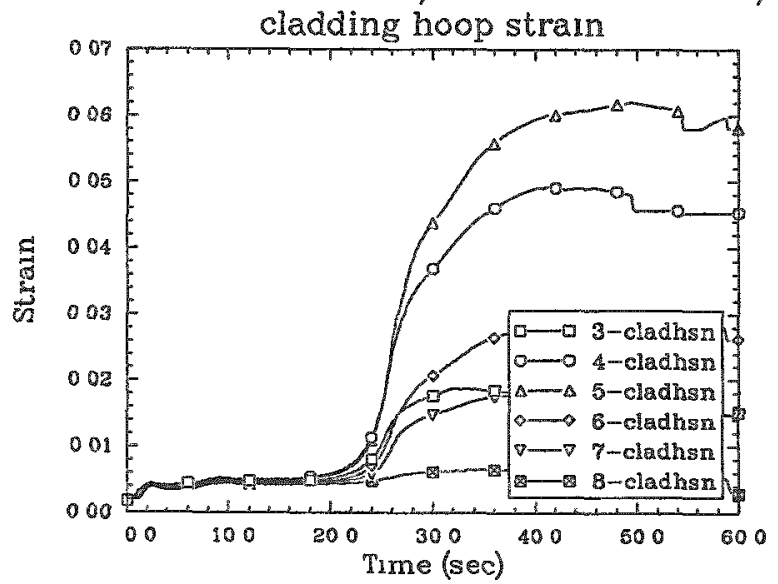

OCONEE 100\%DBA 35 GWD/MTU PIN--PF 20 W/E\&PT

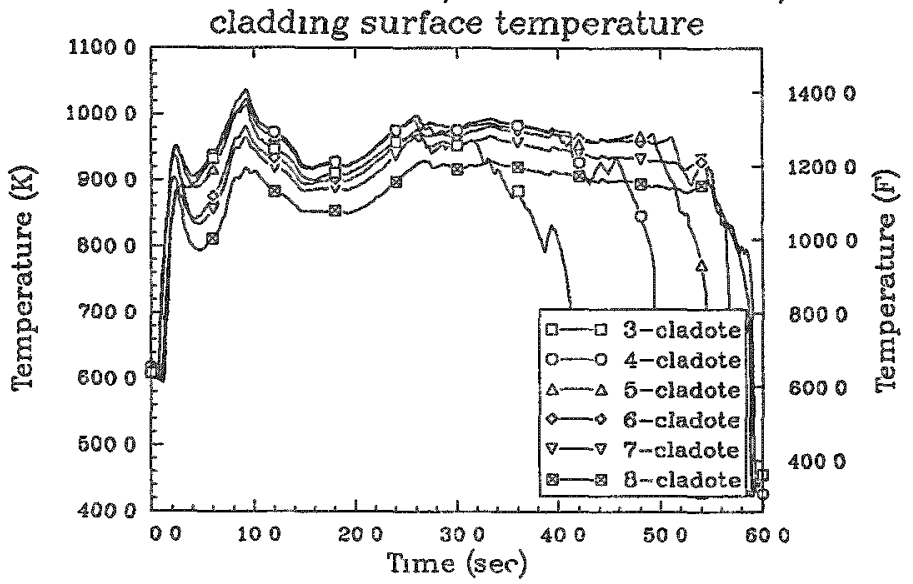

OCONEE 100\%DBA 35 GWD/MTU PIN-DF 20 W/E\&PT

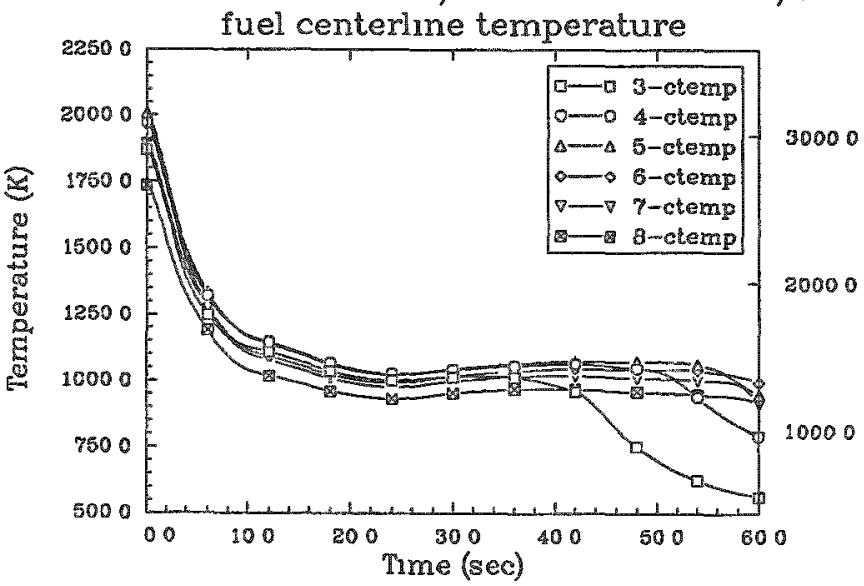

OCONEE 100\%DBA 35 GWD/MTU PIN--PF 20 W/E\&PT

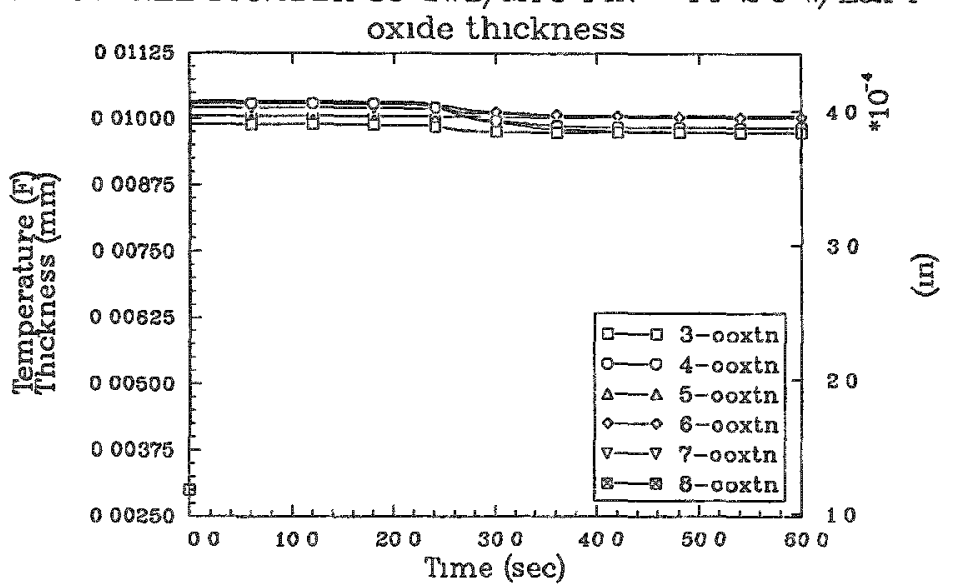




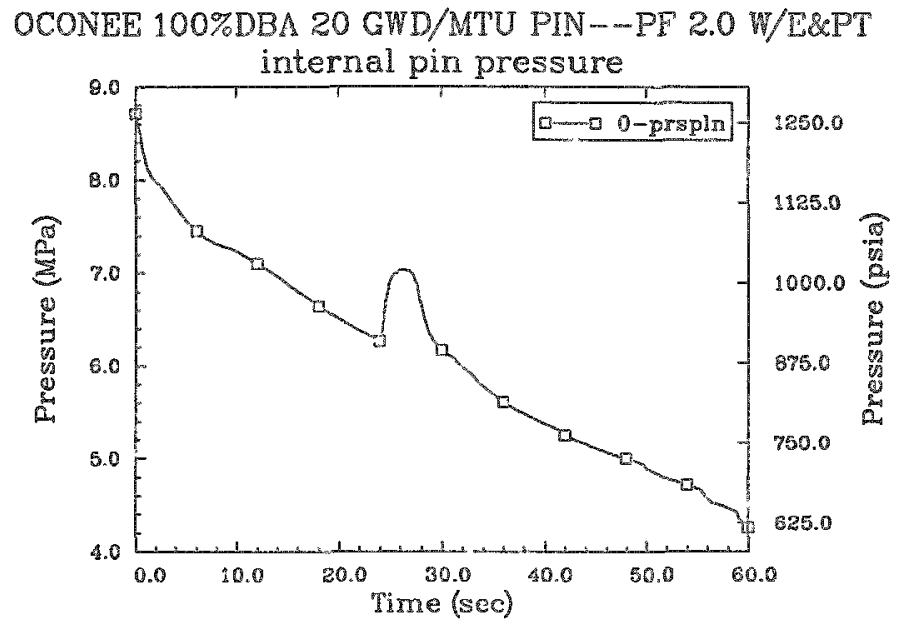

OCONEE 100\%DBA $20 \mathrm{GWD} / \mathrm{MTU}$ PIN--PF $2.0 \mathrm{~W} / \mathrm{E} \& \mathrm{PT}$
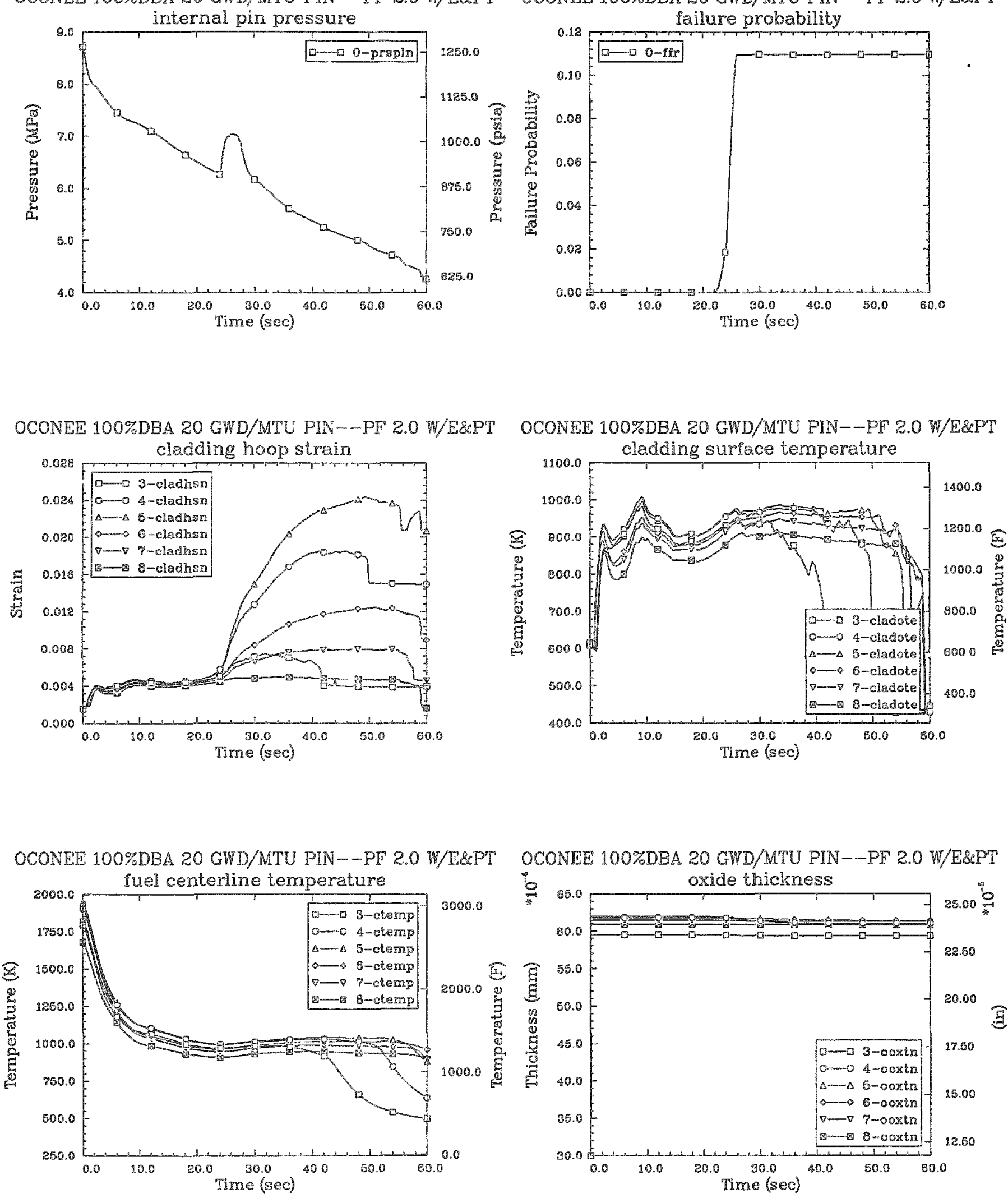

OCONEE 100\%DBA 20 GWD/MTU PIN--PF $2.0 \mathrm{~W} / \mathrm{E} \& \mathrm{PT}$

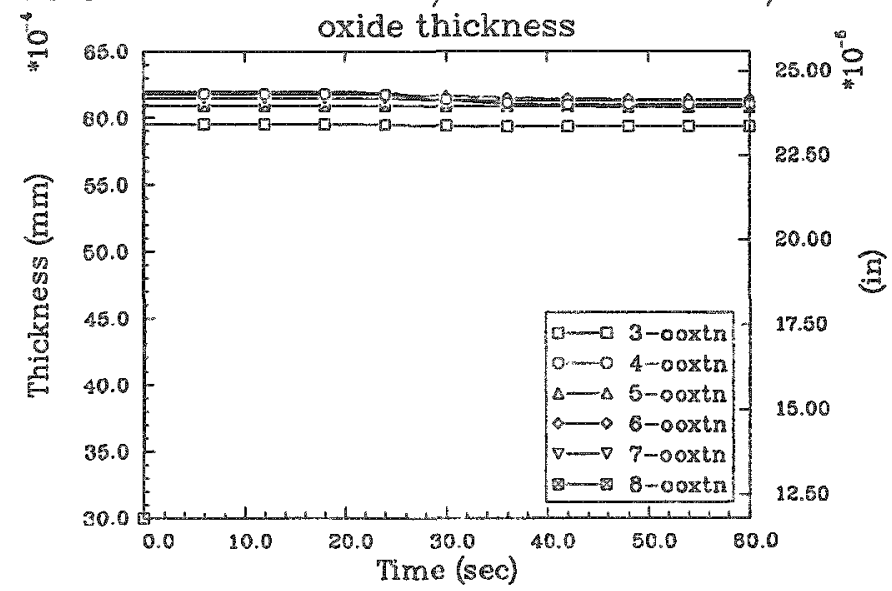


OCONEE 100\%DBA 5 GWD/MTU PIN--PF 2.0 W/E\&PT

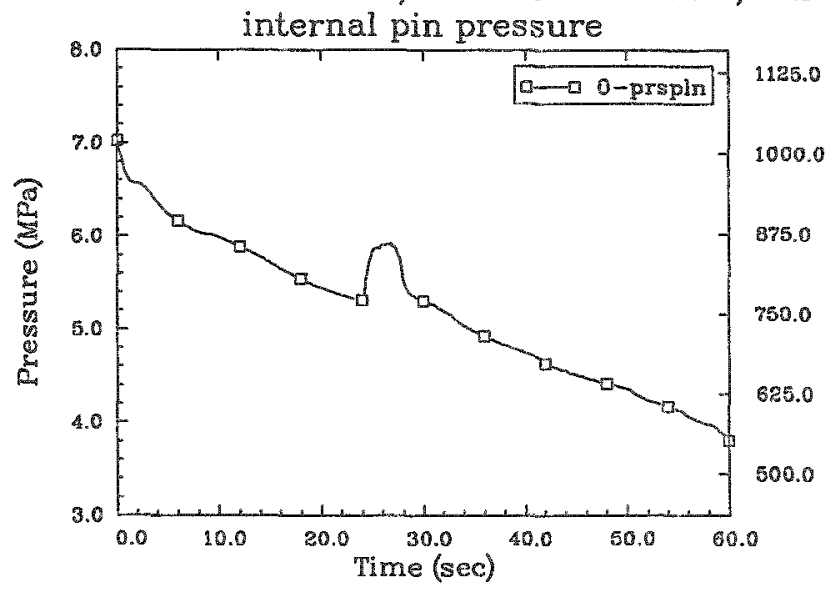

OCONEE 100\%DBA 5 GWD/MTU PIN--PF 2.0 W/E\&PT cladding hoop strain
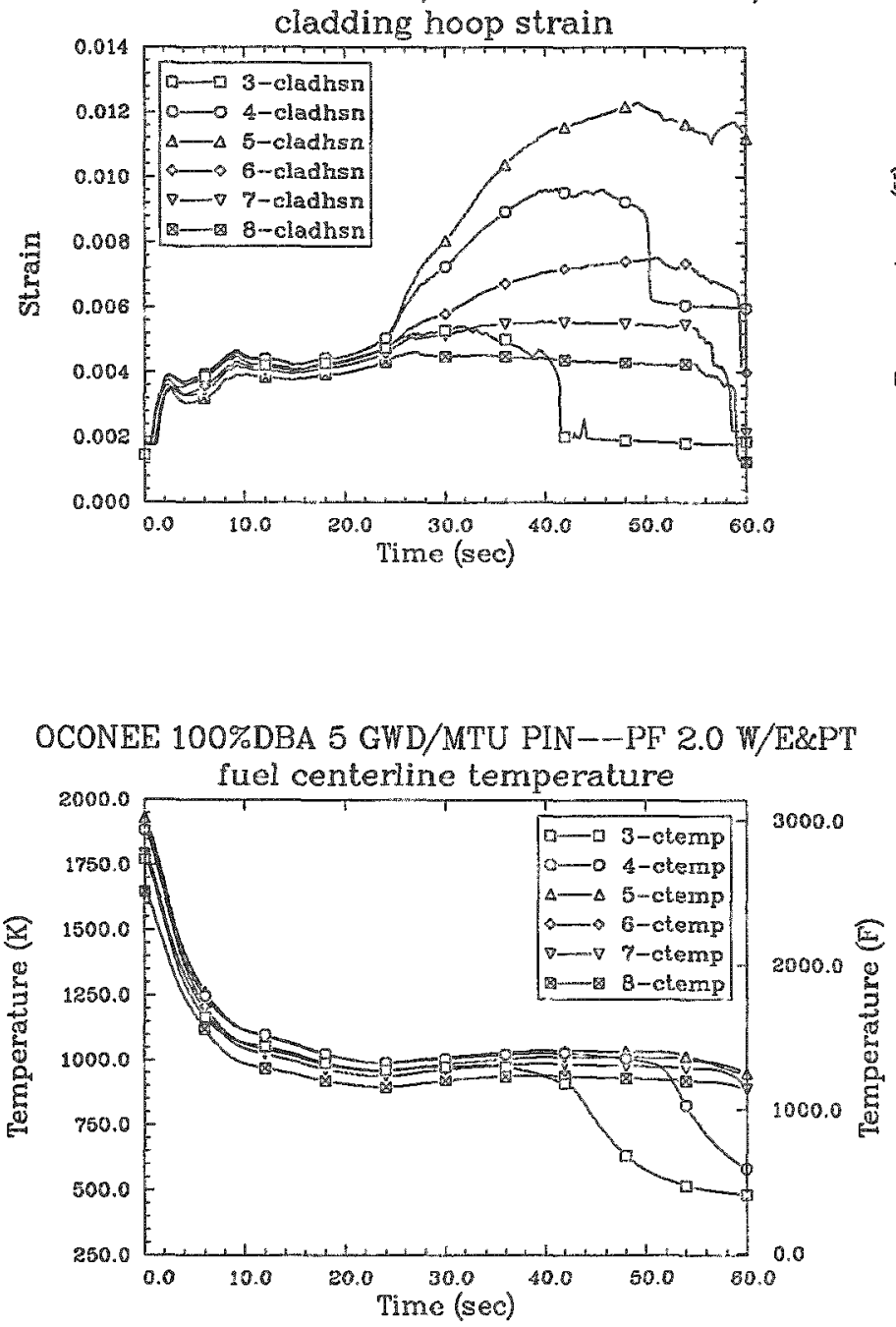

OCONEE 100\%DBA 5 GWD/MTU PIN--PF 2.0 W/E\&PT

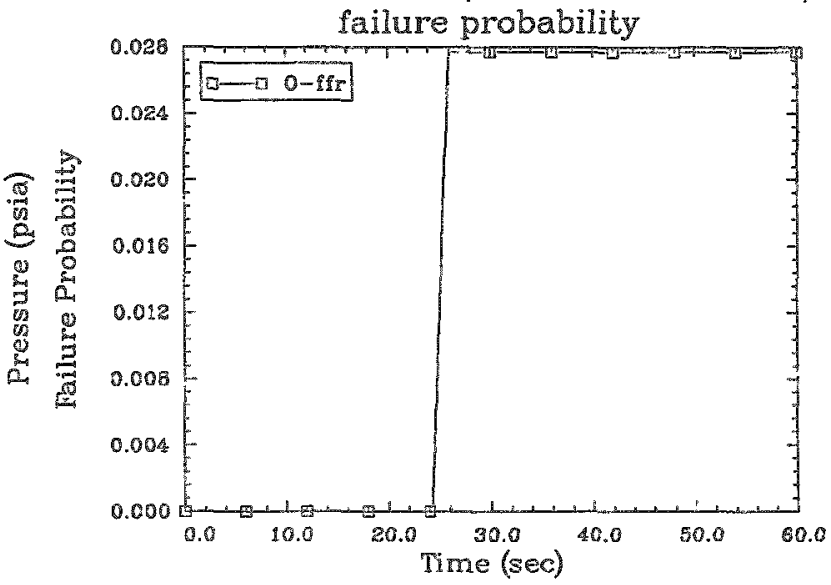

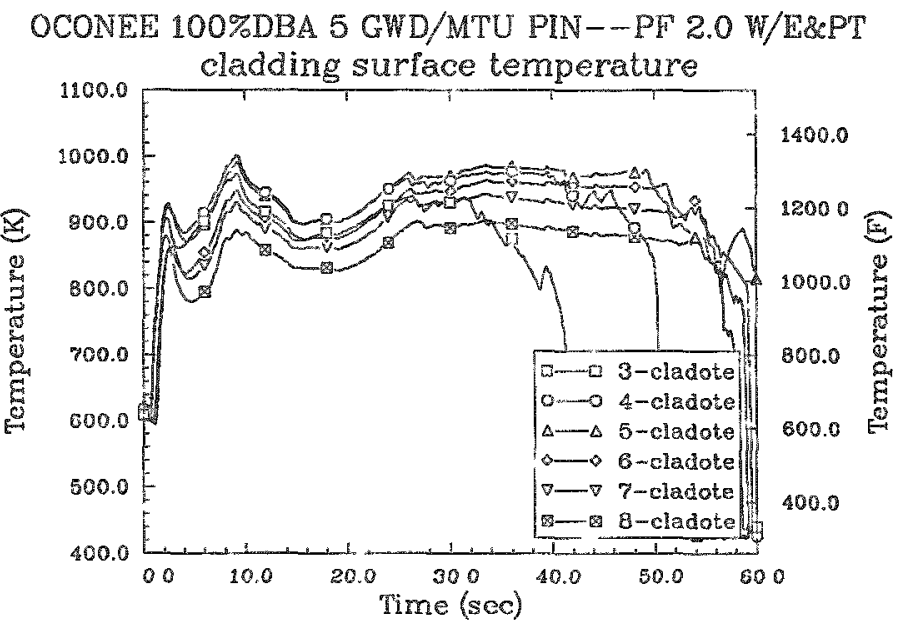

OCONEE 100\%DBA 5 GWD/MTU PIN-DF 2.0 W/E\&PT

oxide thickness

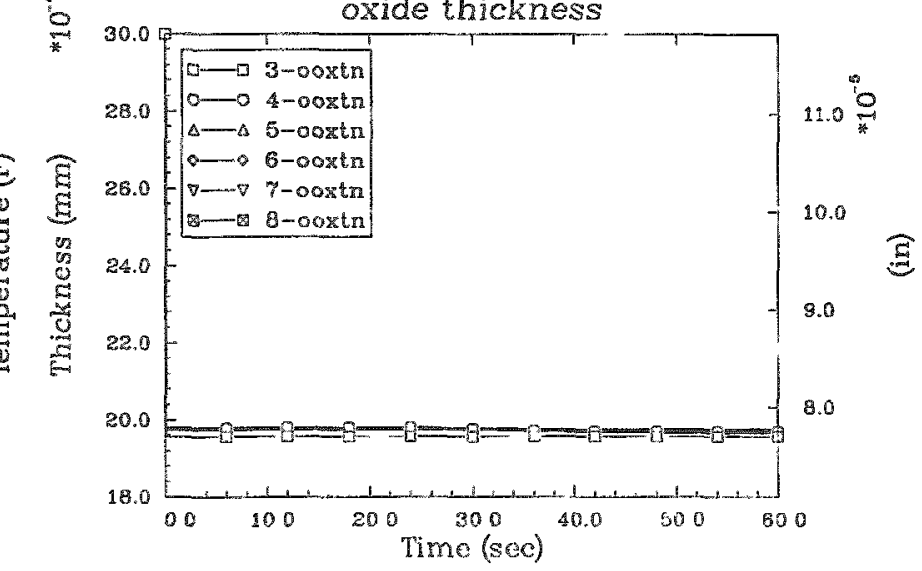




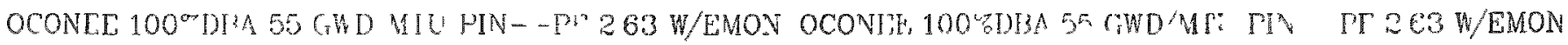
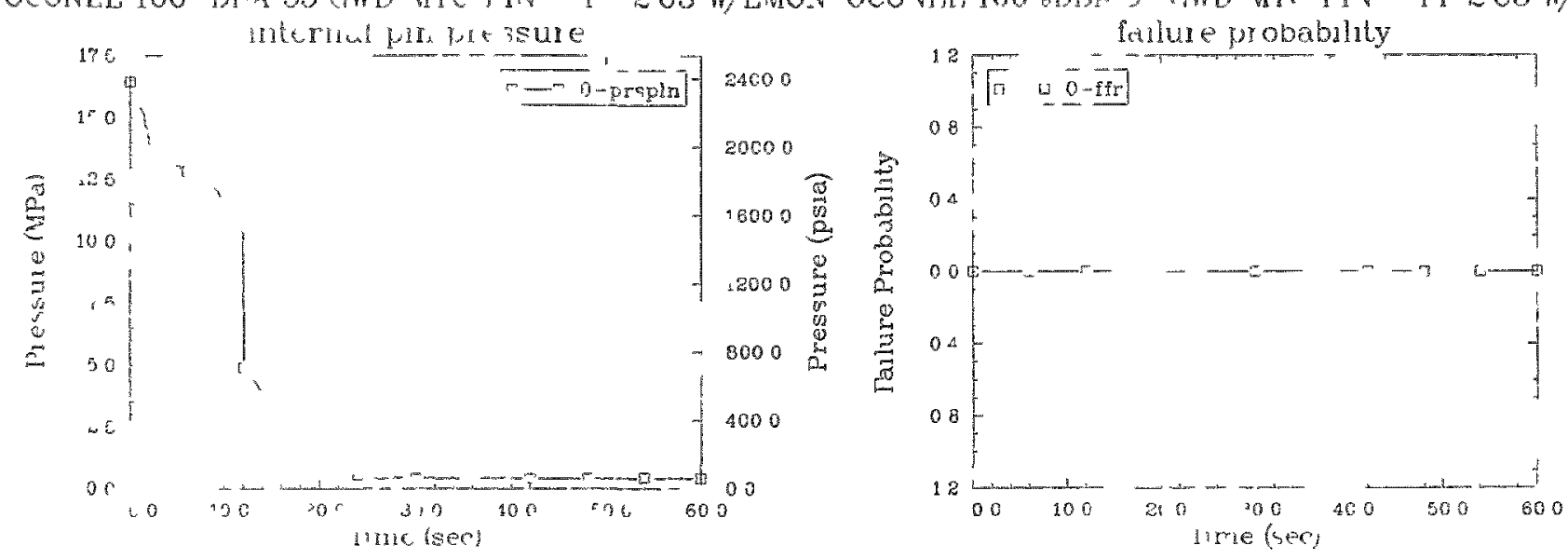

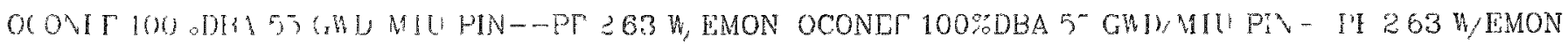
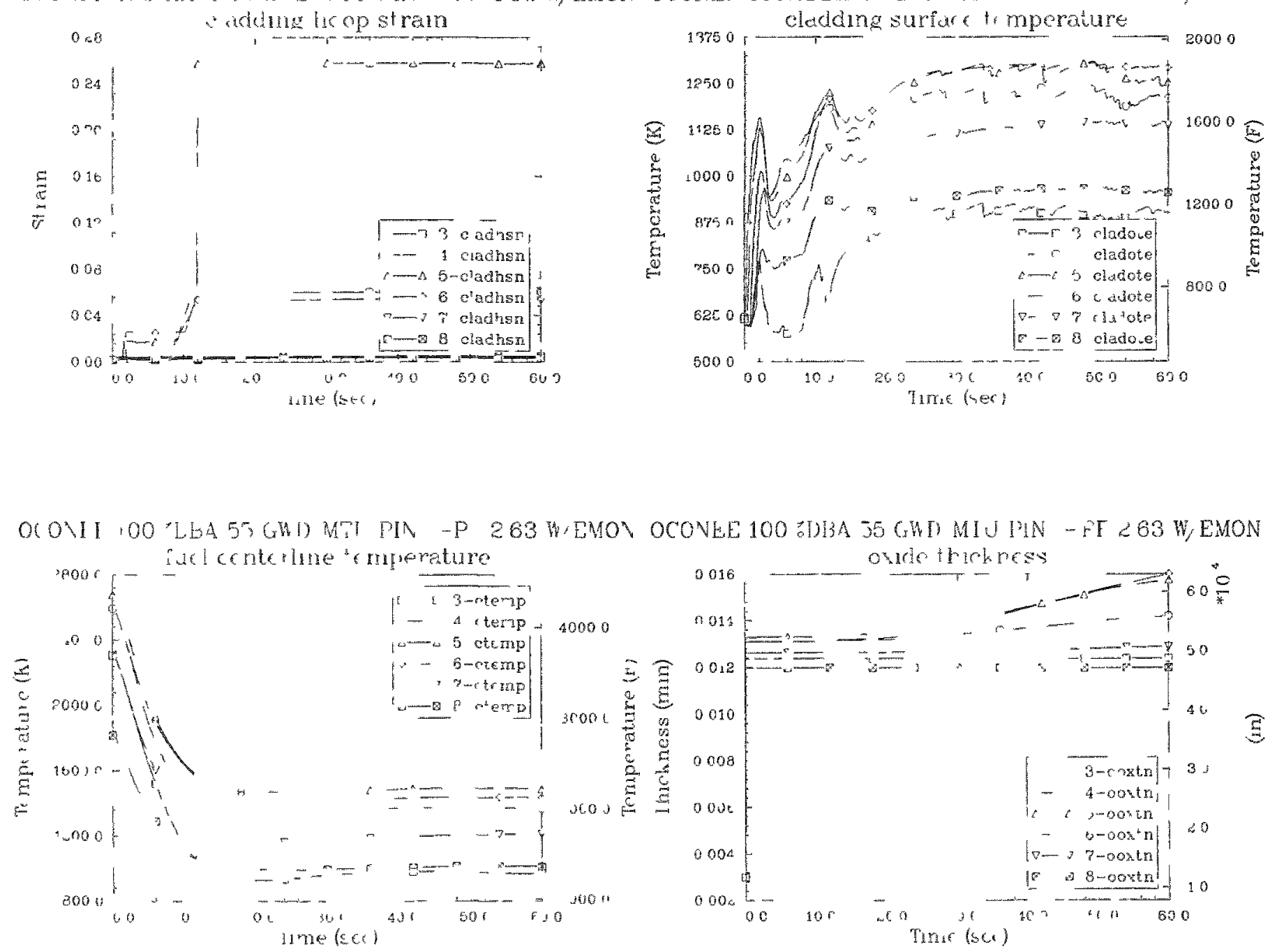
OCONEE 100\%DBA 35 GWD/MTU HIN--PF 263 W/LMON CCONRE 100\%DIBA 35 GWD MTU PIN--HF 263 W/ThON

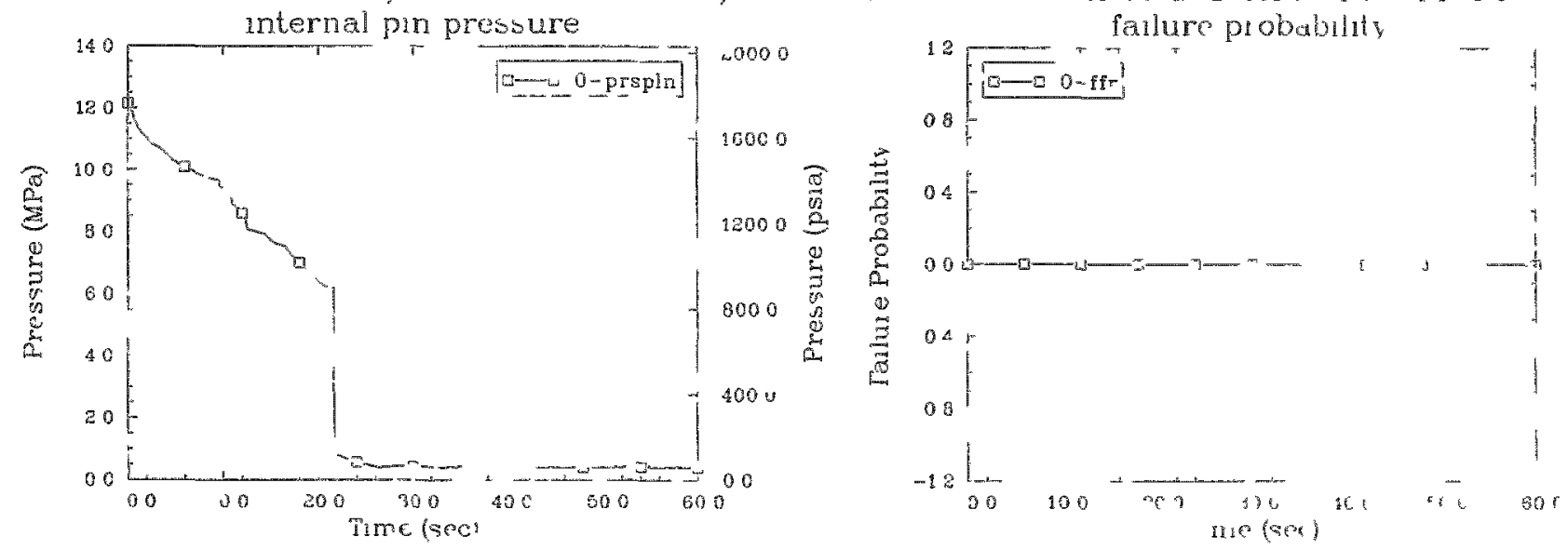

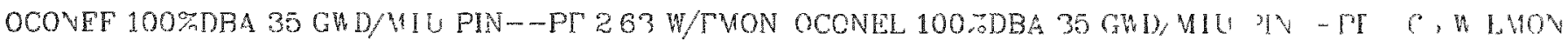
cladding hoop strein
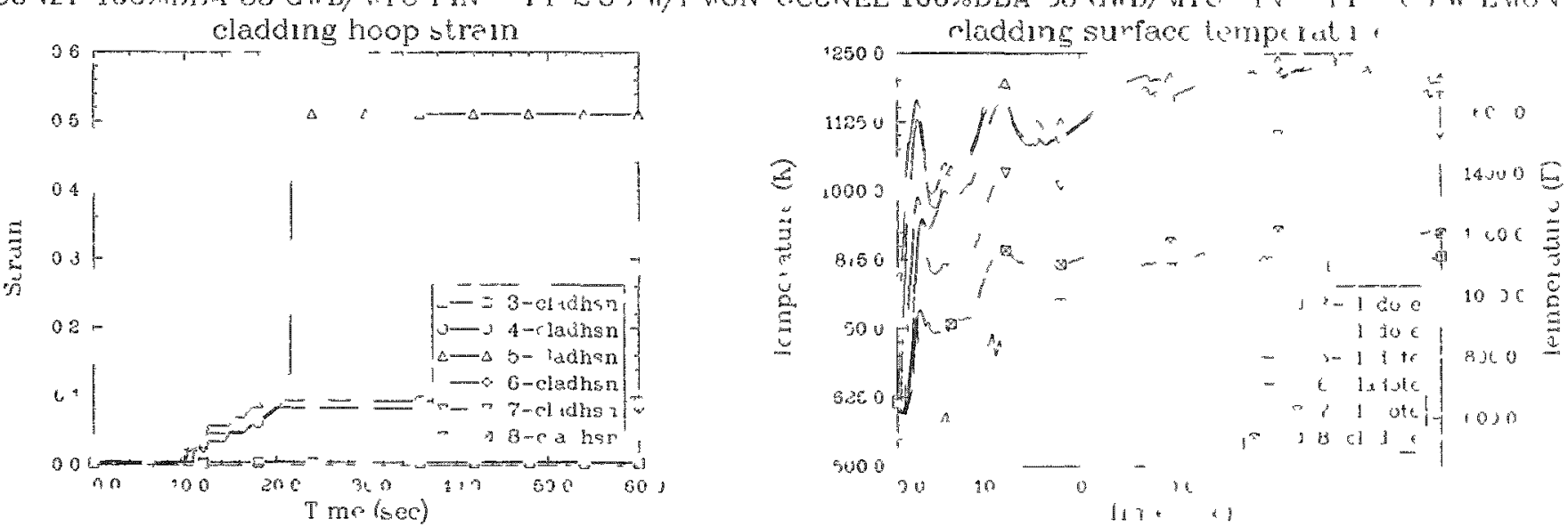

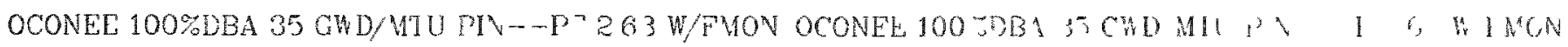

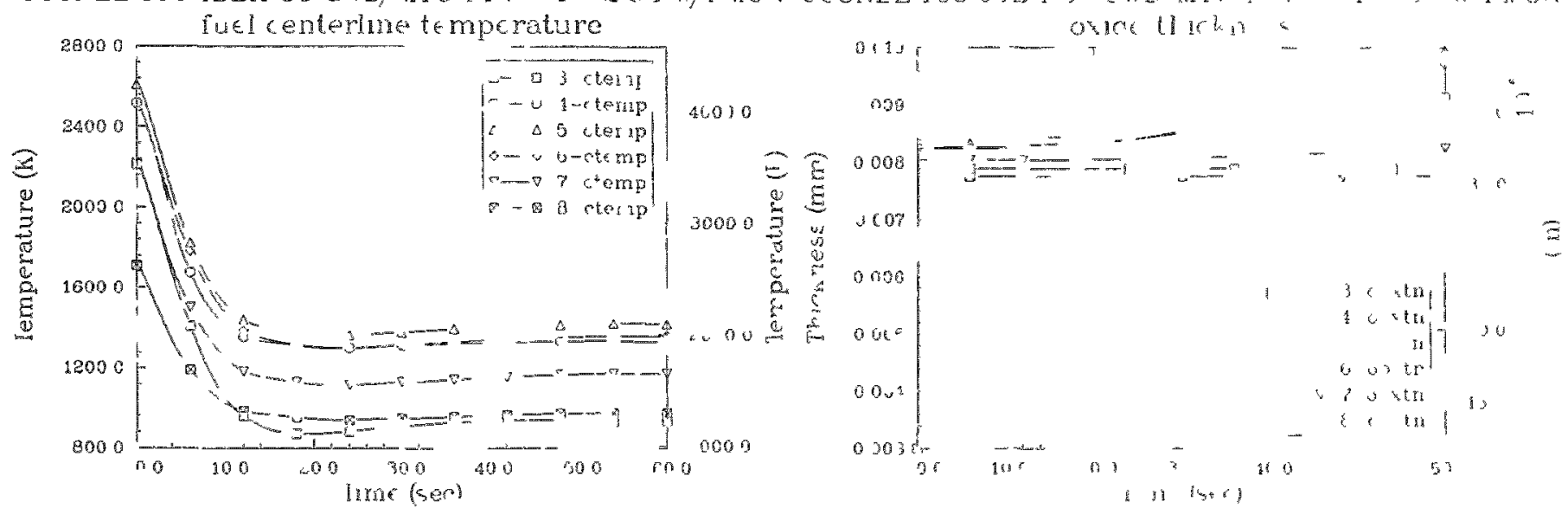


OCONEE 100\%DBA 20 GWD/MTU PIN--PF 2.63 W/EMON OCONEE 100\%DBA 20 GWD/MTU PIN--PF 2.63 W/EMON

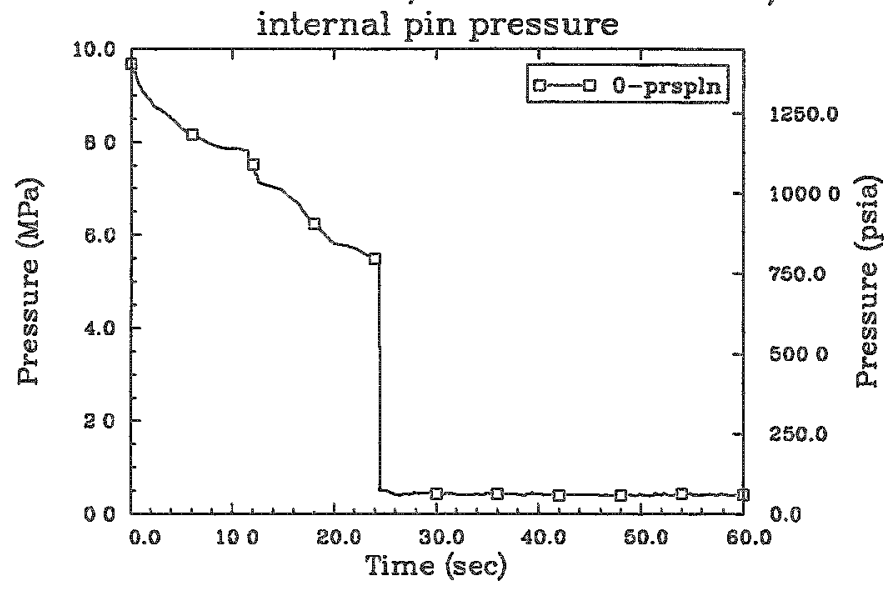
failure probability

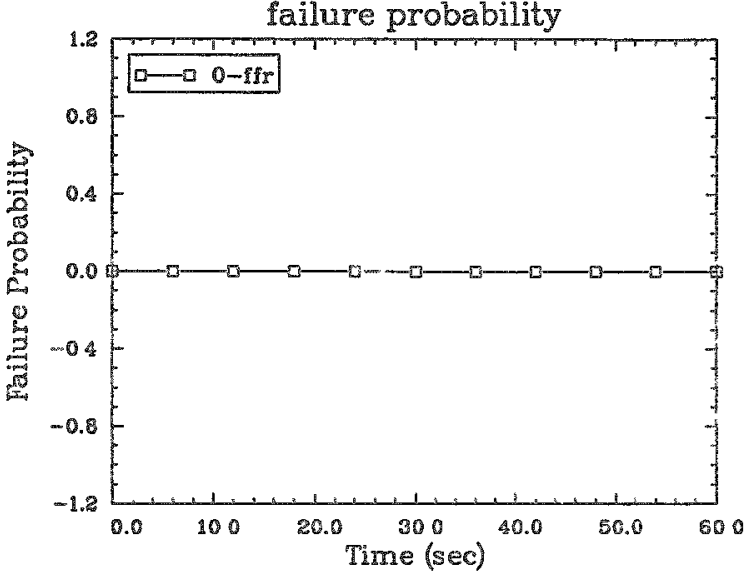

OCONEE 100\%DBA 20 GWD/MTU PIN--PF 2.63 W/EMON OCONEE 100\%DBA 20 GWD/MTU PIN--PF 2.63 W/EMON
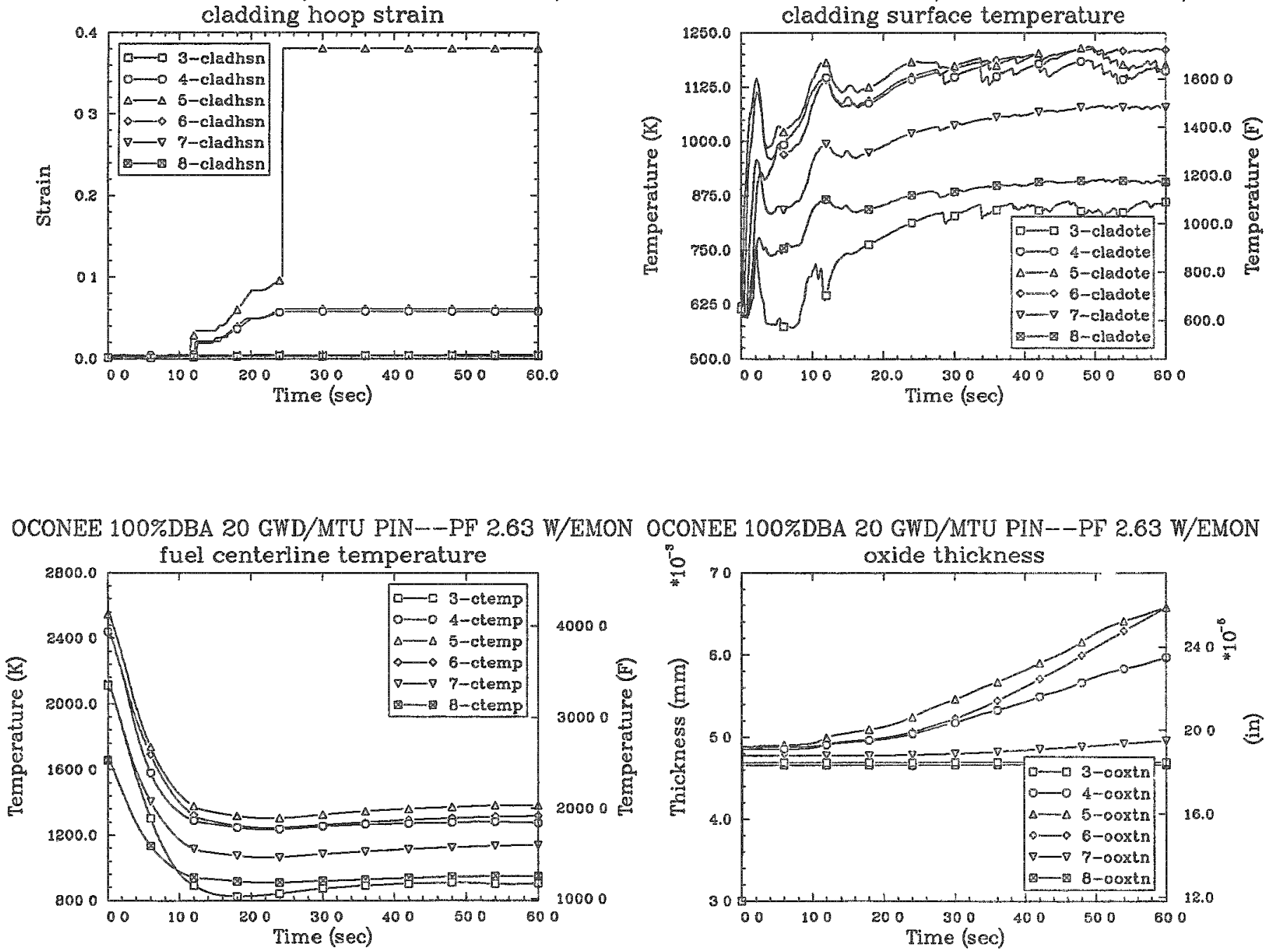
OCONEE 100\%DBA 5 GWD/MTU PIN--PF 2.63 W/EMON internal pin pressure

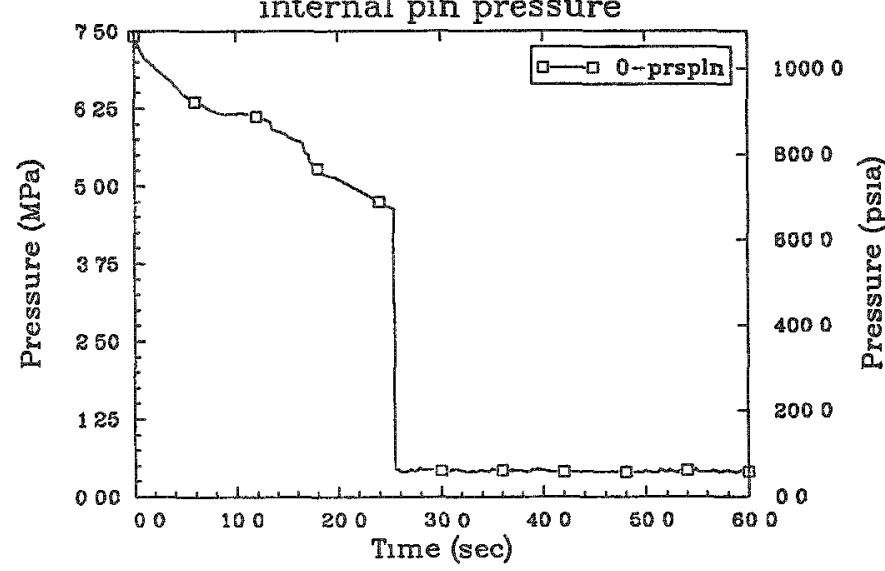

OCONEE 100\%DBA 5 GWD/MTU PIN--PF 2.63 W/EMON cladding hoop strain

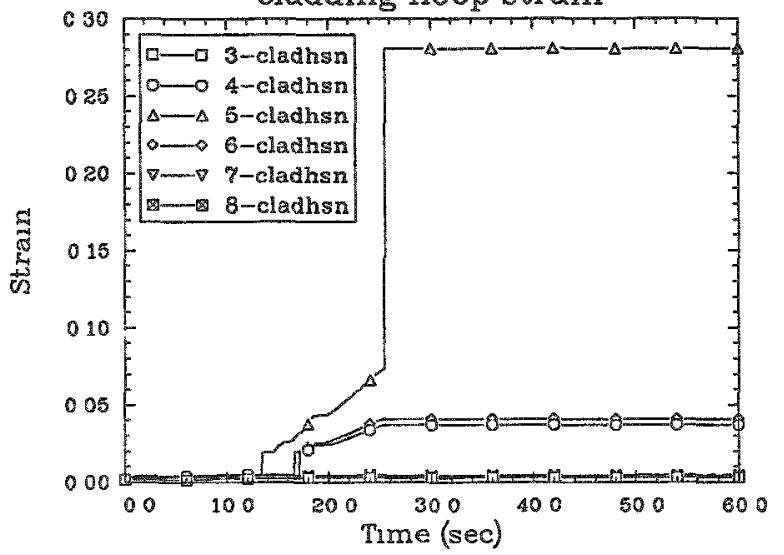

OCONEE 100\%DBA 5 GWD/MTU PIN--PF 2.63 W/EMON failure probability

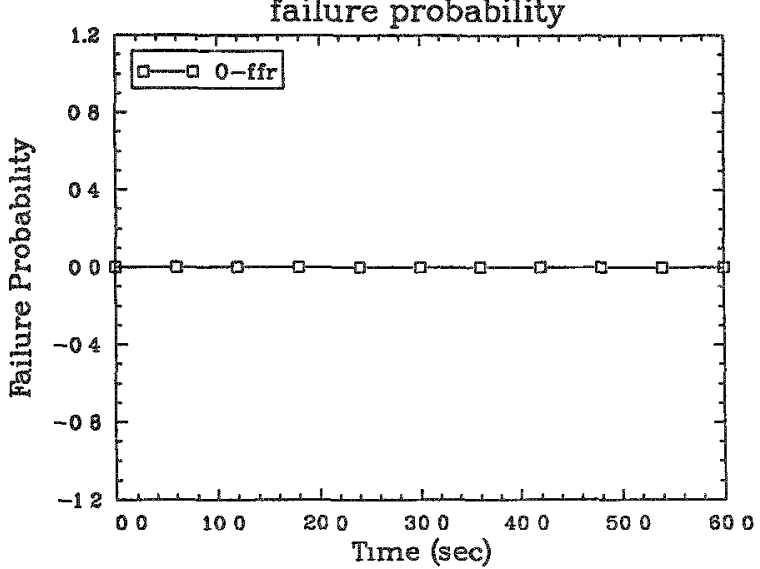

OCONEE 100\%DBA 5 GWD/MTU PIN--PF 2.63 W/EMON

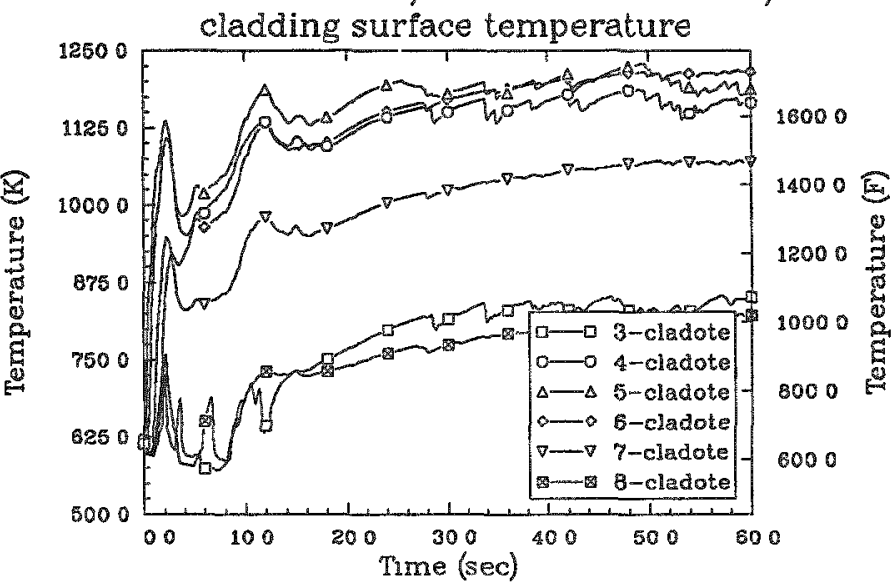

OCONEE 100\%DBA 5 GWD/MTU PIN--PF 263 W/EMON

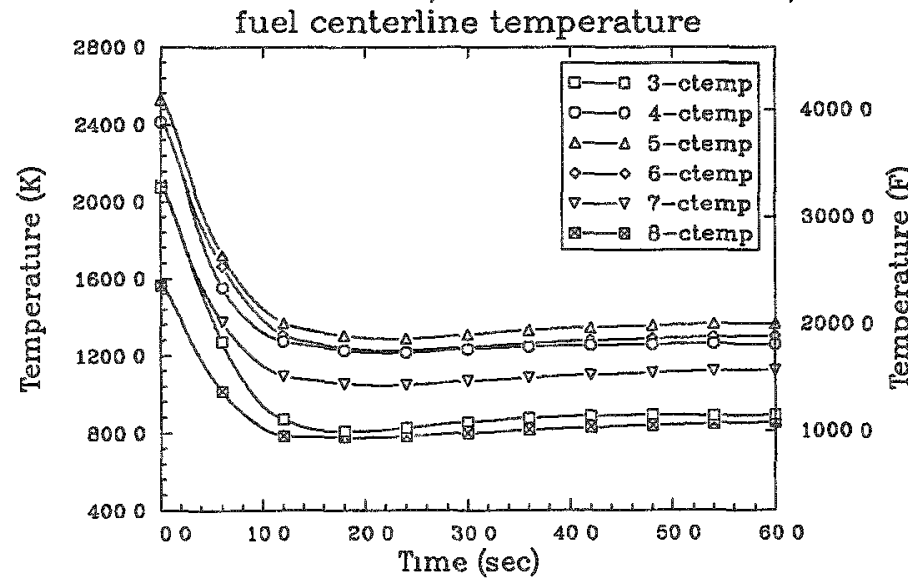

OCONEE 100\%DBA 5 GWD/MTU PIN--PF 263 W/EMON

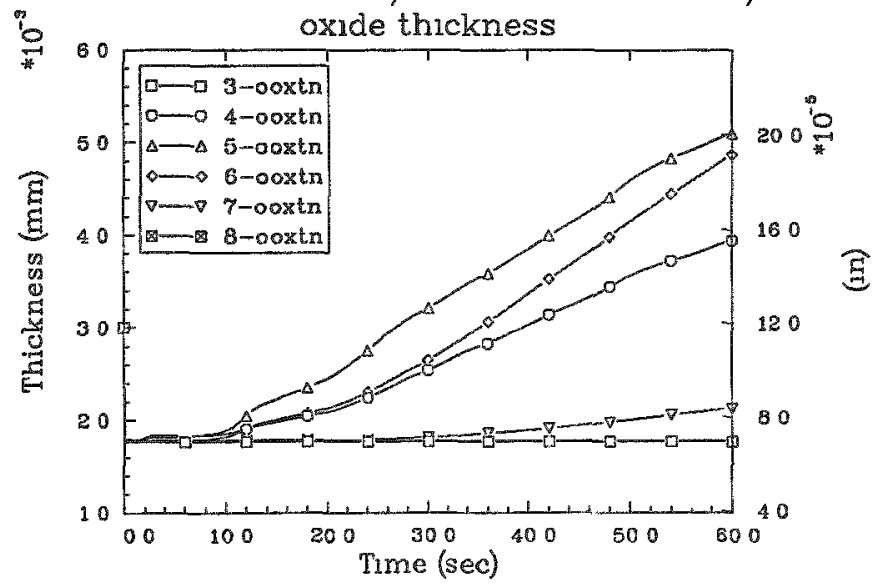


OCUNEH 100\%DH155 GHD/MTU PIN-PF 2.4 W/EMON

OCONEE 100\%DBA 55 GWD/MTI PIN-DI 24 W/EMON
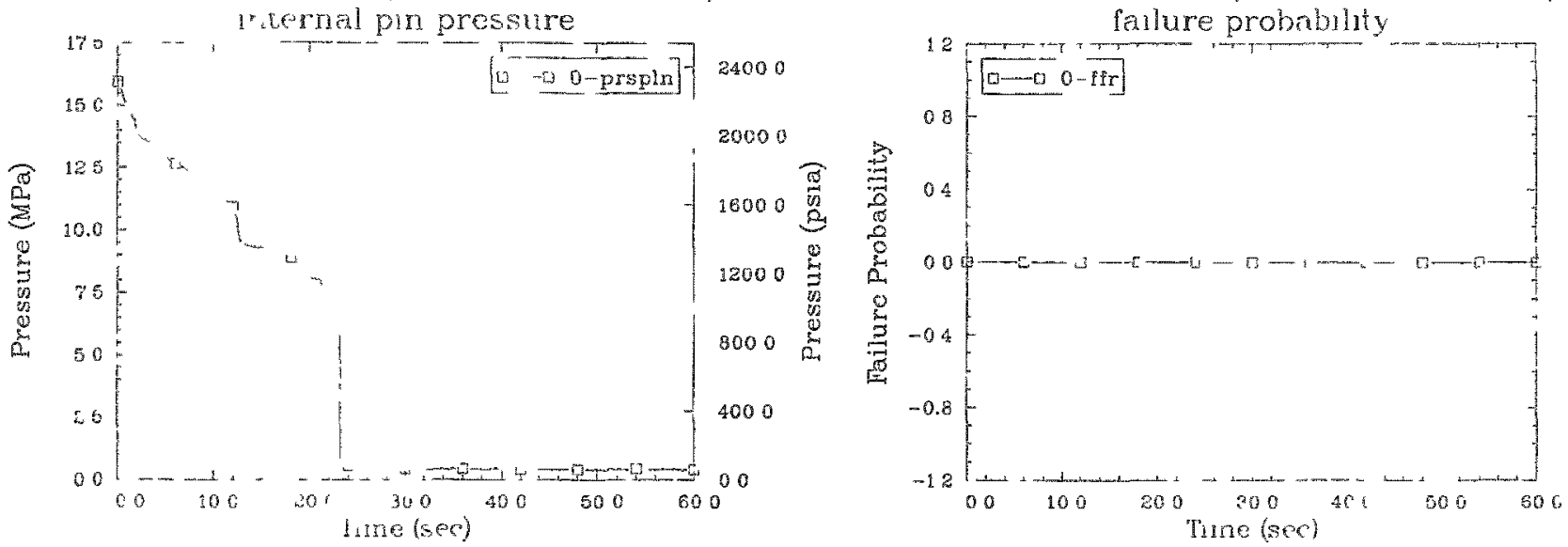

OCONHE $100 \%$ DHA DO GWD/MTL PIN--PF $24 \% / E M O N$

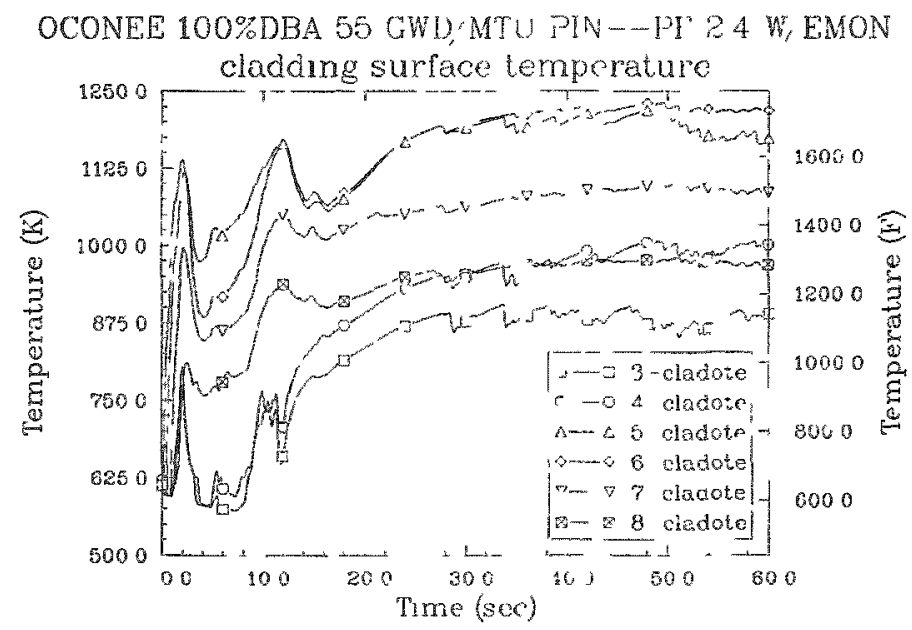

OCONEL 100\%DBA 55 GWD MUU PI - PF 24 W/EMON

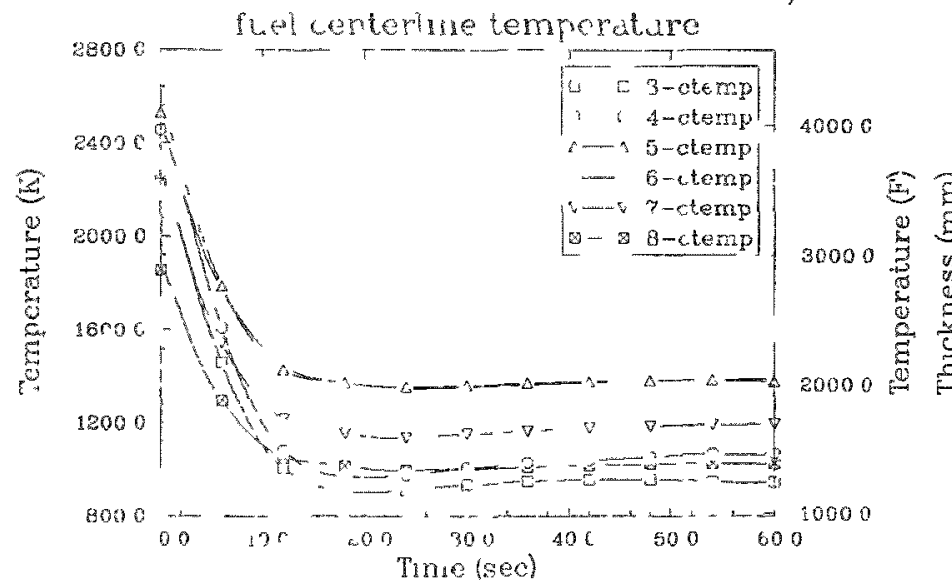

OCONEE 100\%DBA 55 GWU/MUU PIN--1\% 2.4 W/LMON

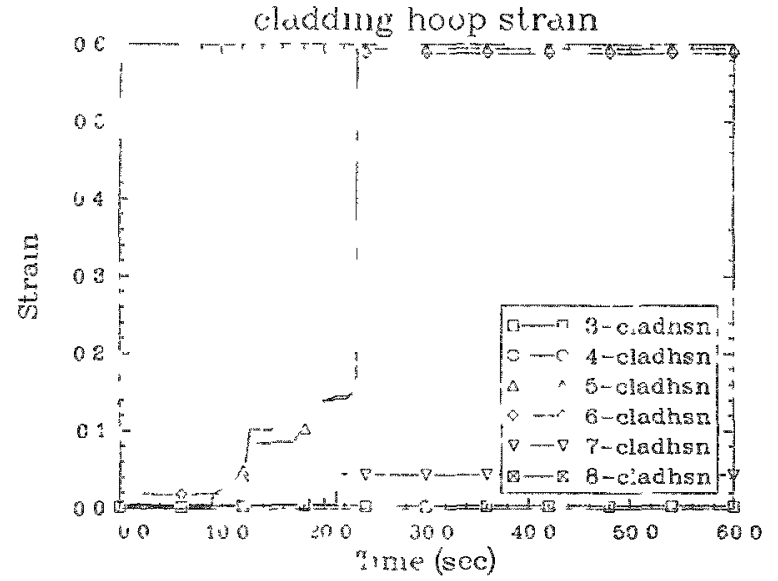


OCONEE 100\%DBA 35 GWD/MTU PIN--PF 2.4 W/EMON

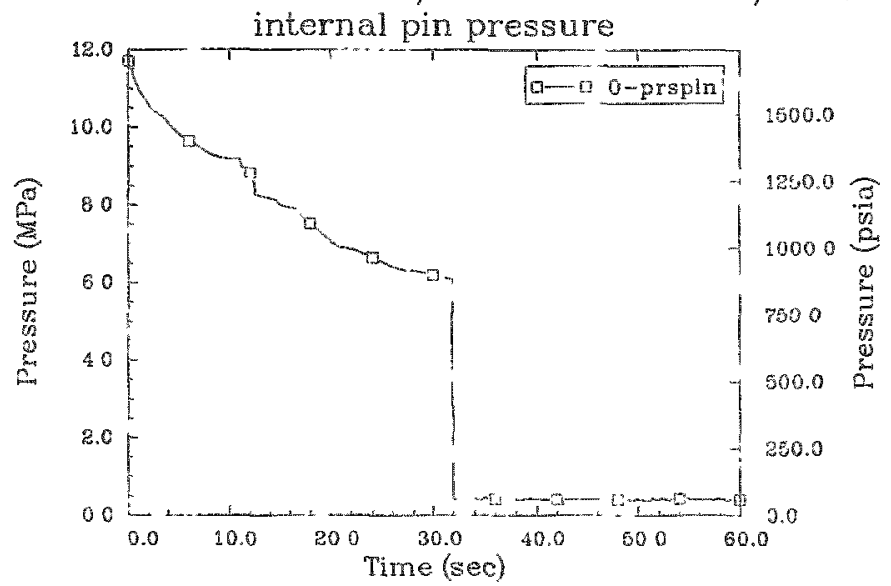

OCONEE 100\%DBA 35 GWD/MTU PIN--PF 2.4 W/EMON

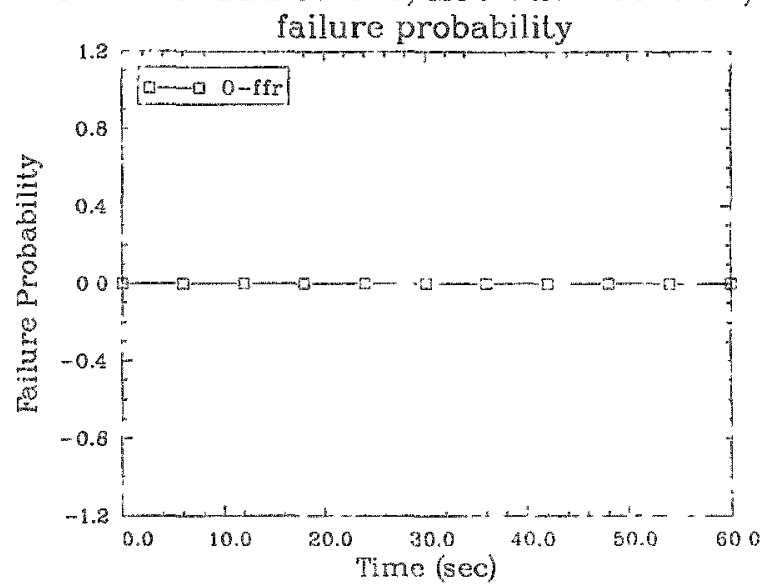

OCONEE $200 \%$ DBA 35 GWD/MTU PIN-PI 24 W/EMON

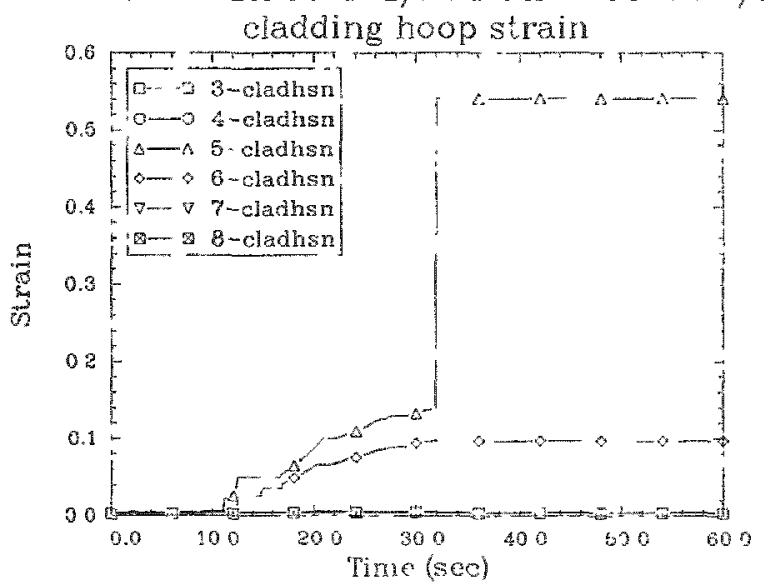

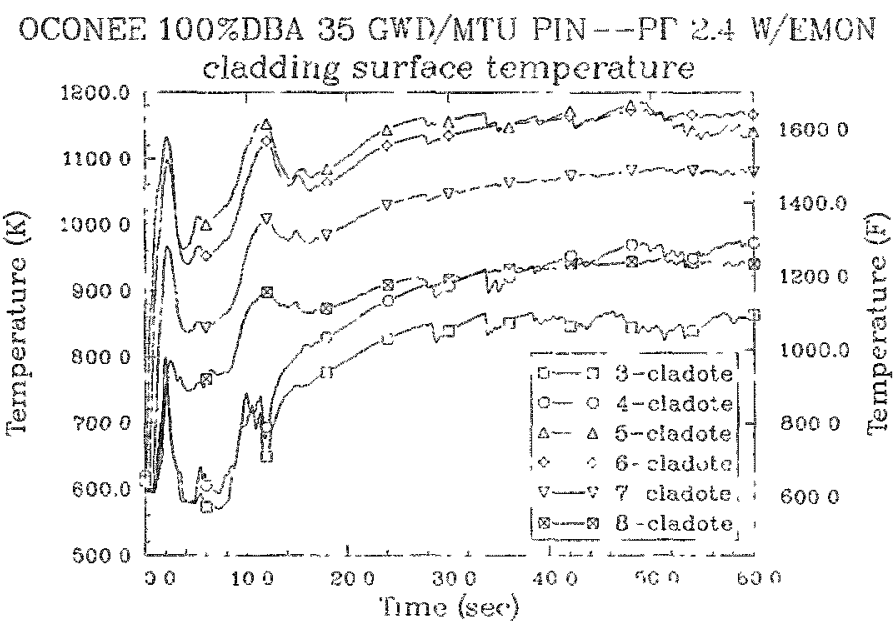

OCONEE 100\%DBA 35 GWD/MTU PIN- -PF 2.4 W/EMON

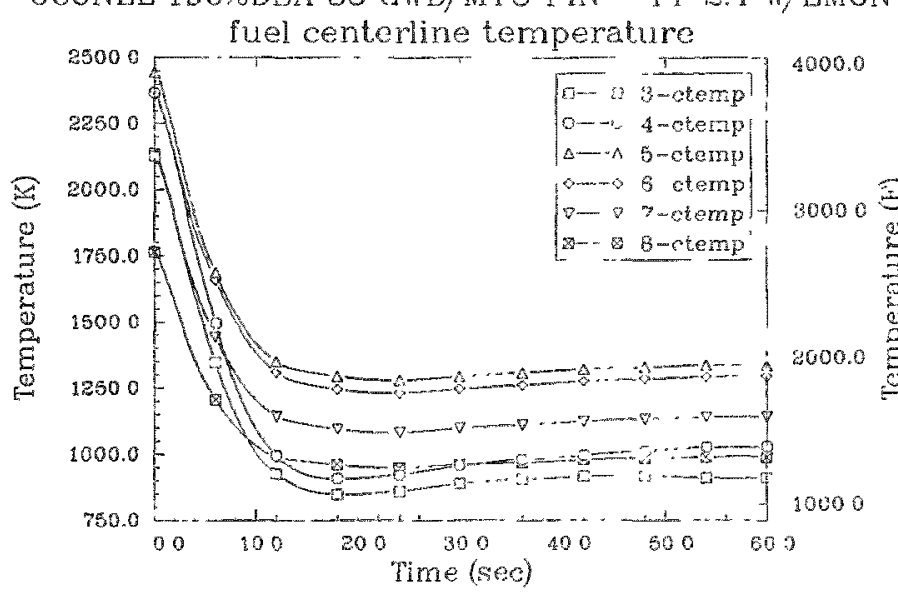

OCONEL 100\%DBA 35 CWD/MTU PIN--PF 2.4 W/FMON oxide thickness

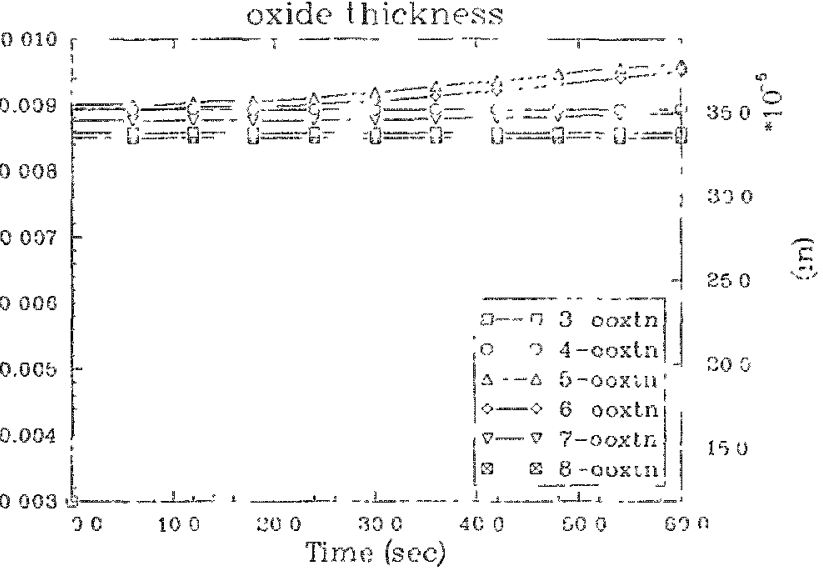


OCONEE 100\%DBA 2U GWD, MIU PIN -PL 24 W/EMON

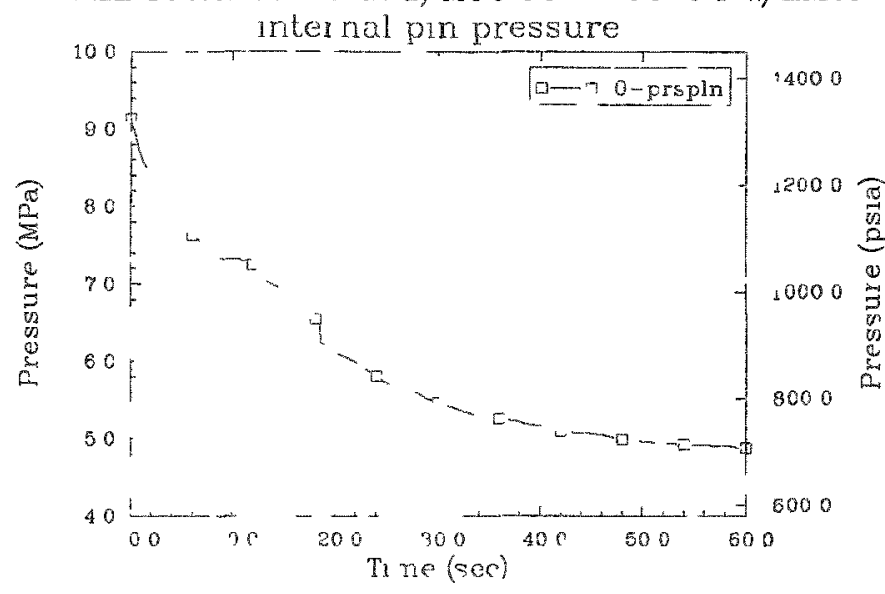

OCONLE 100\%DBA 20 GWD/MTU PIN--PF 24 W/LMON

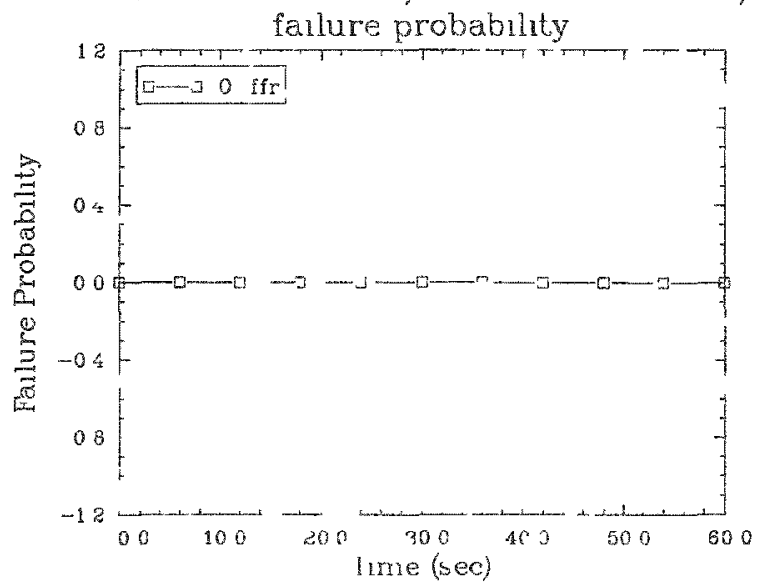

OCONL1 $100 \% \mathrm{DB} 120 \mathrm{GWD} / \mathrm{M}$ CU PIV -PT 24 W/LMON

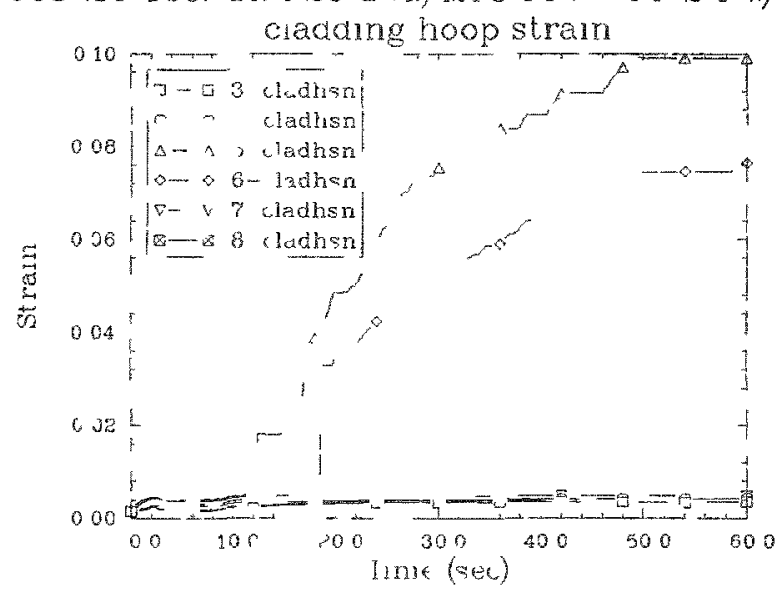

OCONEE 100\%DHA $20 \mathrm{CHD} / \mathrm{MTU}$ PIN--PT $24 \mathrm{~W} / \mathrm{EMON}$

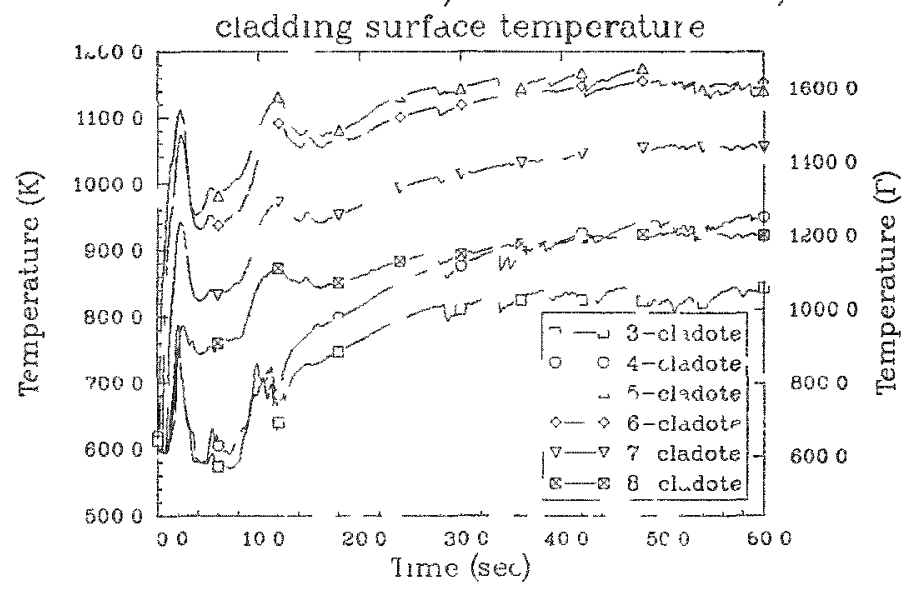

OCONEE 100\%DBA 20 GWD, MTU PIN PГ 24 W/EMON

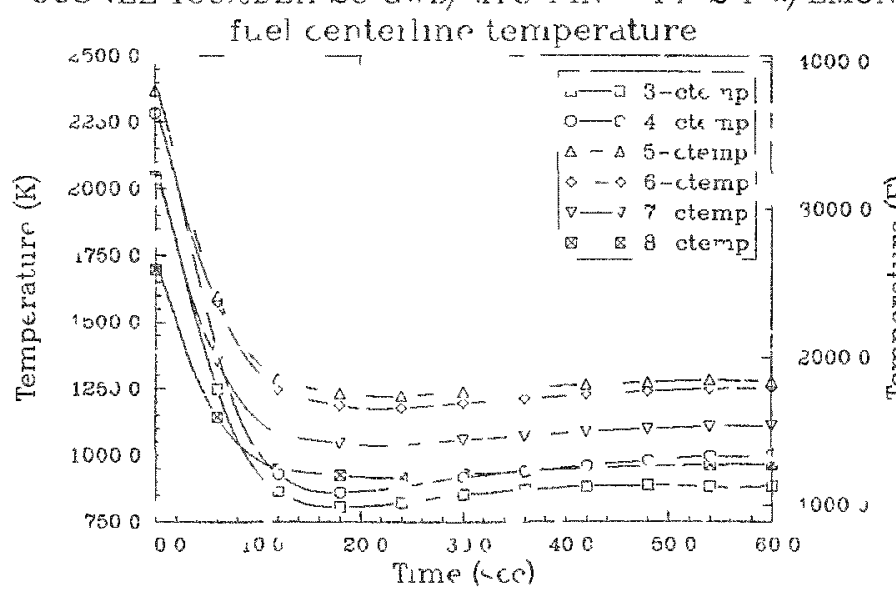

OCONET 100\%DBA $20 \mathrm{GHD} / \mathrm{MLL}$ PIN - PГ $24 \mathrm{~W} / \mathrm{LMON}$ ¿ $600 \ldots \ldots$ oxide thickness

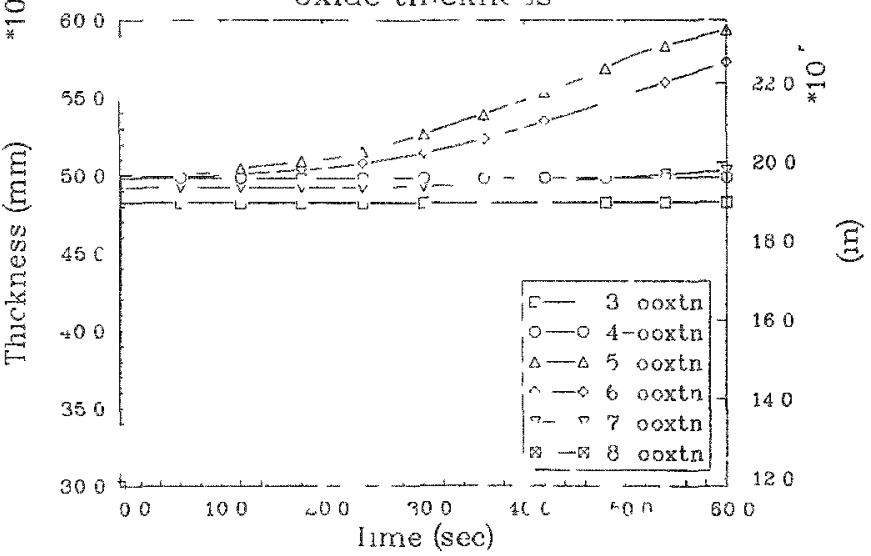


OCONEE 100\%DBA 5 GWD/MTU PIN--PF 2.4 W/EMON internal pun pressure

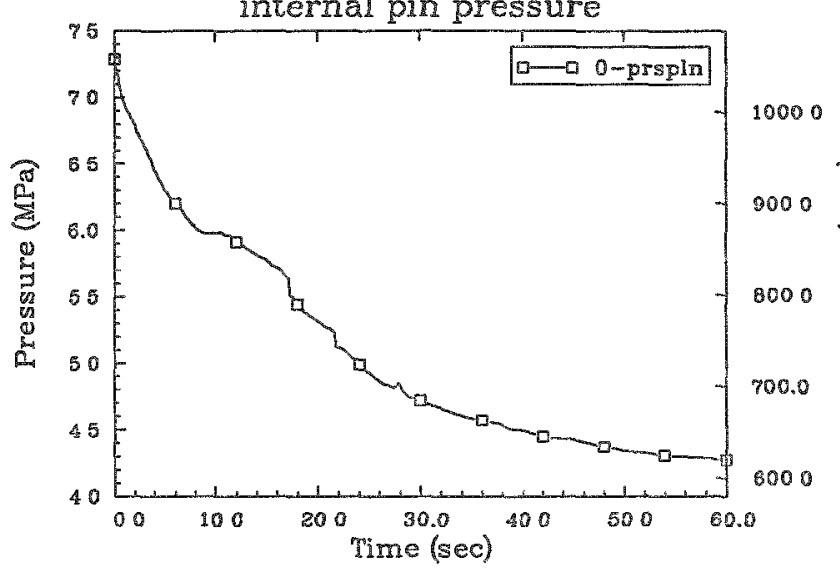

OCONEE 100\%DBA 5 GWD/MTU PIN--PF 2.4 W/EMON cladding hoop strain

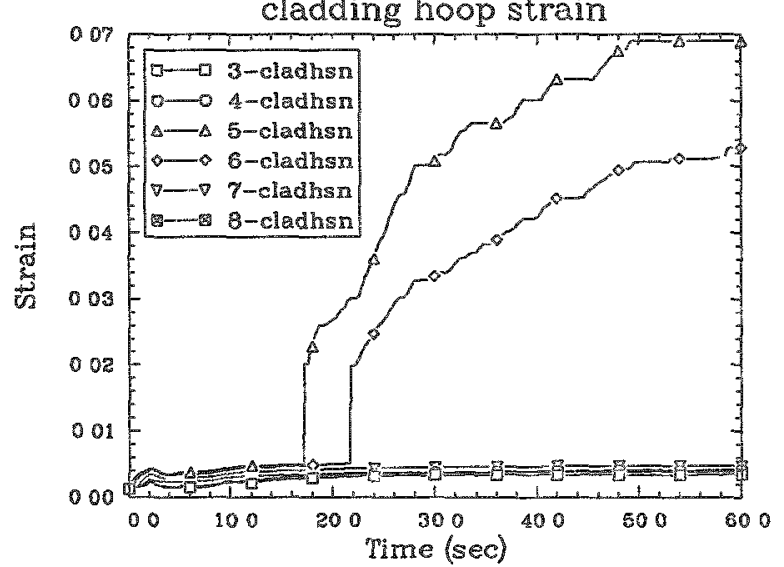

OCONEE 100\%DBA 5 GWD/MTU PIN--PF 2.4 W/EMON

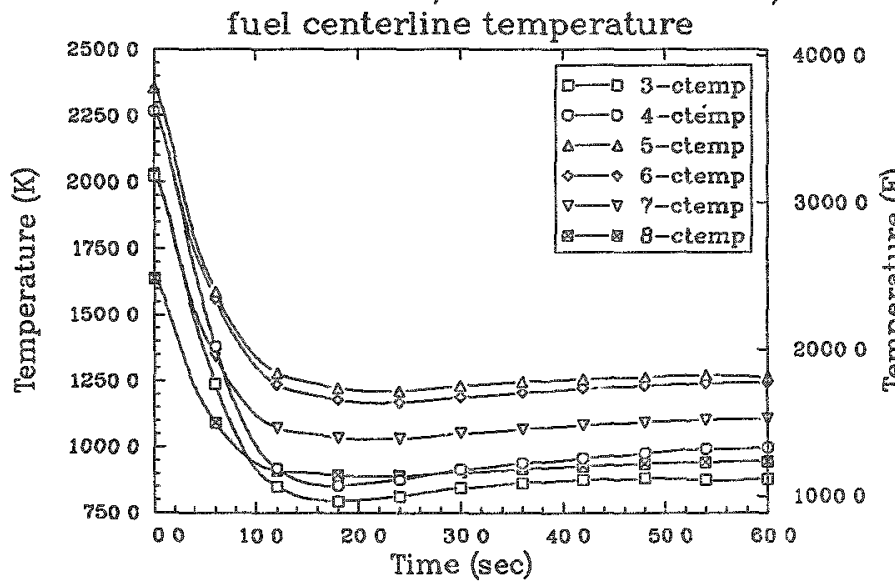

OCONEE 100\%DBA 5 GWD/MTU PIN--PF 2.4 W/EMON failure probability

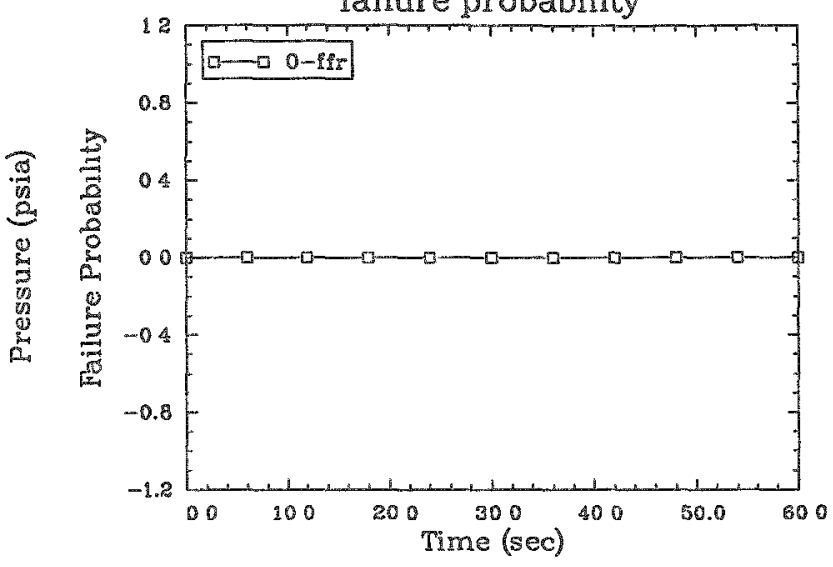

OCONEE 100\%DBA 5 GWD/MTU PIN-DF 2.4 W/EMON

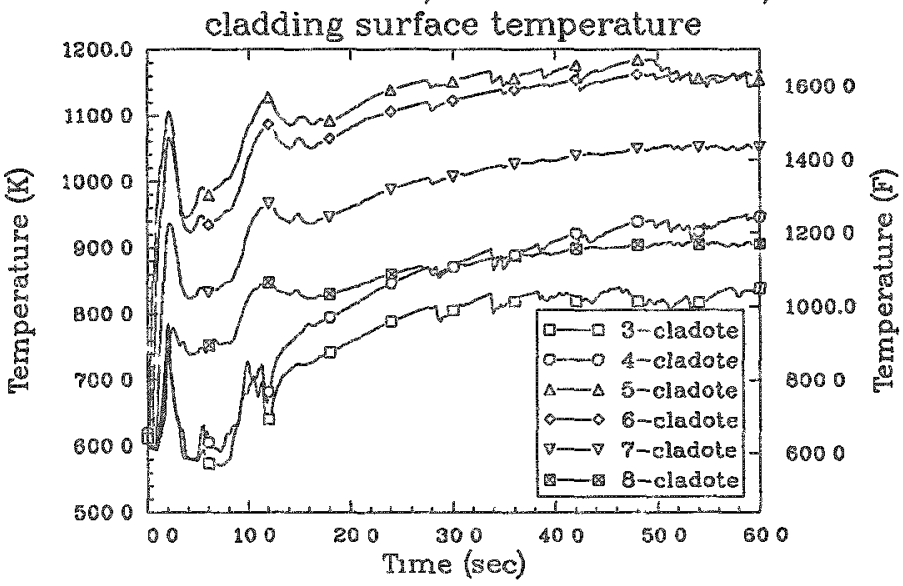

OCONEE 100\%DBA 5 GWD/MTU PIN--PF 2.4 W/EMON

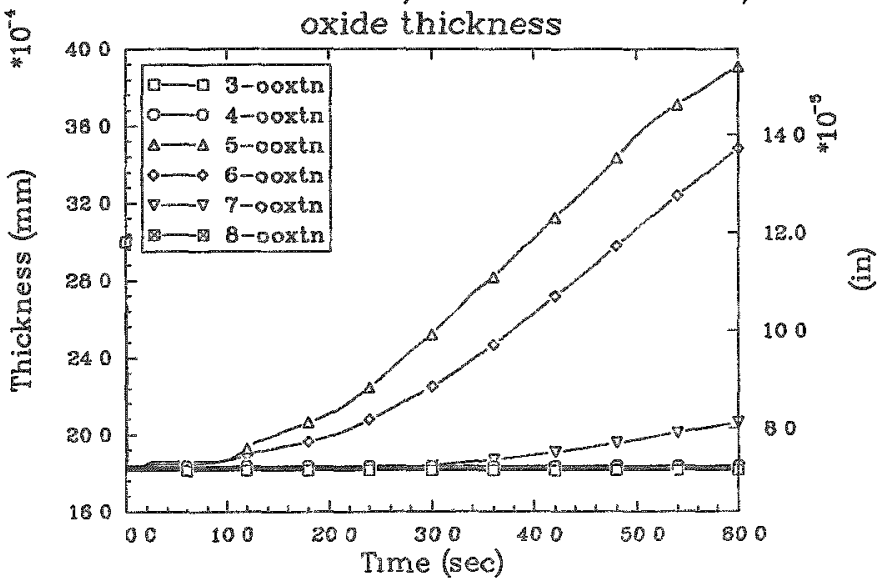


OCONEE 100\%DBA 55 GWD/MTU PIN--PF 2.2 W/EMON

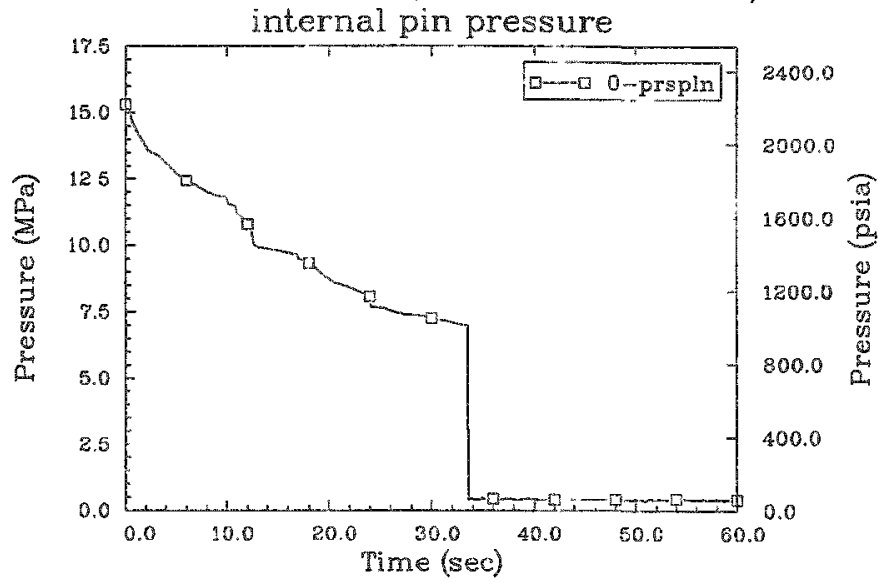

OCONEE 100\%DBA 55 GWD/MTU PIN--PF 2.2 W/EMON failure probability

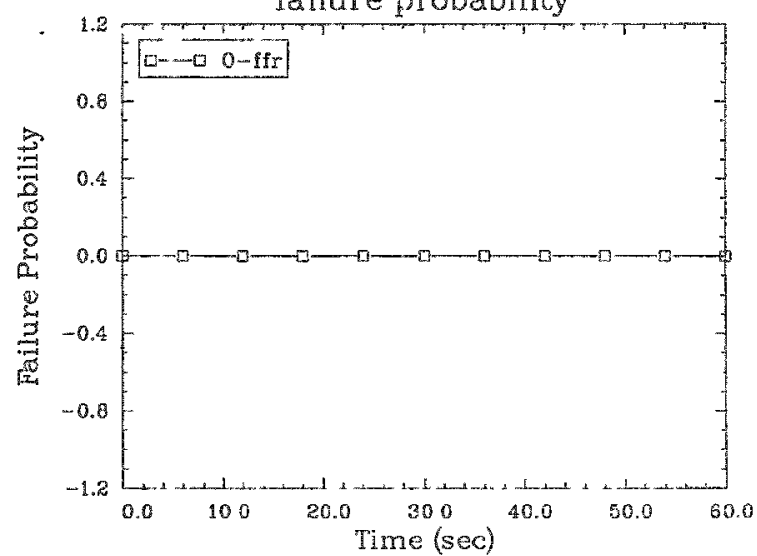

OCONEE 100\%DBA 55 GWD/MTU PIN--PF 2.2 W/EMON cladding hoop strain

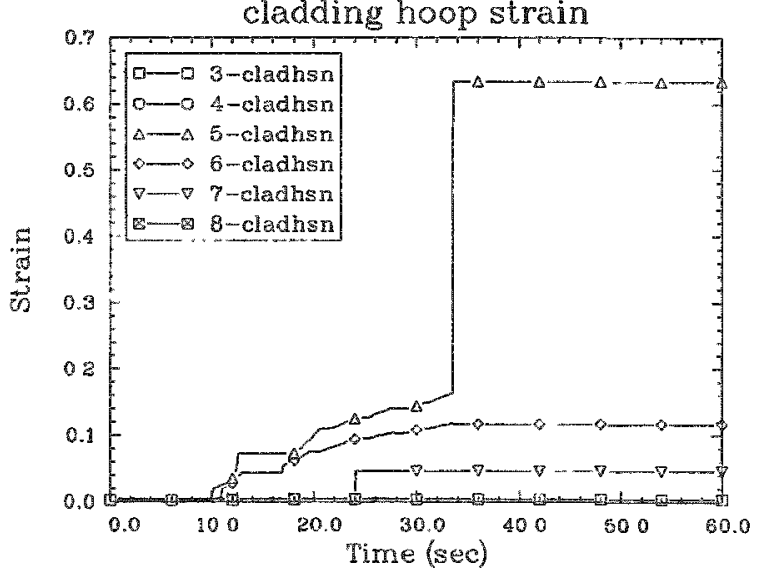

OCONEF 100\%DBA 55 GWD/MTU PIN--PF 2.2 W/EMON

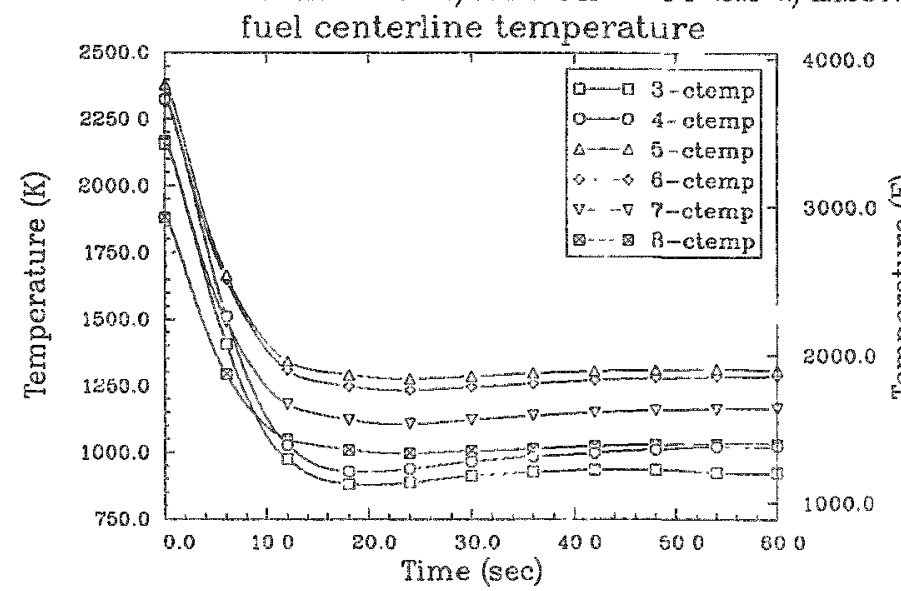

OCONEE 100\%DBA 55 GWD/MTU PIN--PF 2.2 W/EMON

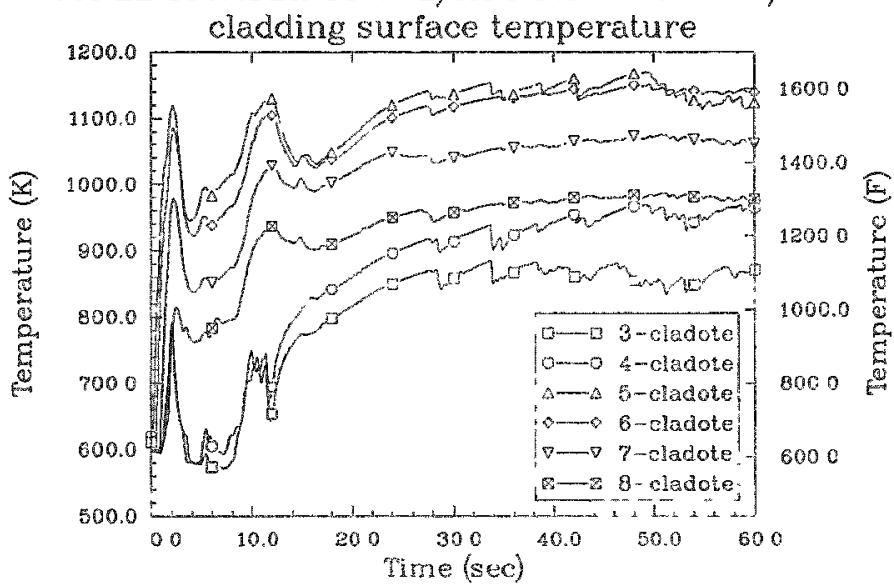

OCONEE 100\%DBA 55 GWD/MTU PIN- PF 2.2 W/EMON oxide thickness

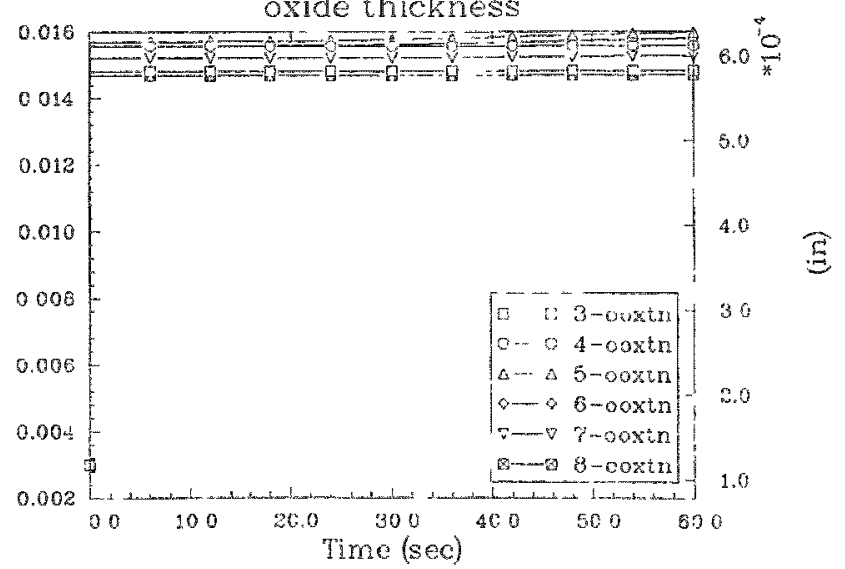


OCONEE 100\%DBA 35 GWD/MTU PIN--PF 2.2 W/EMON

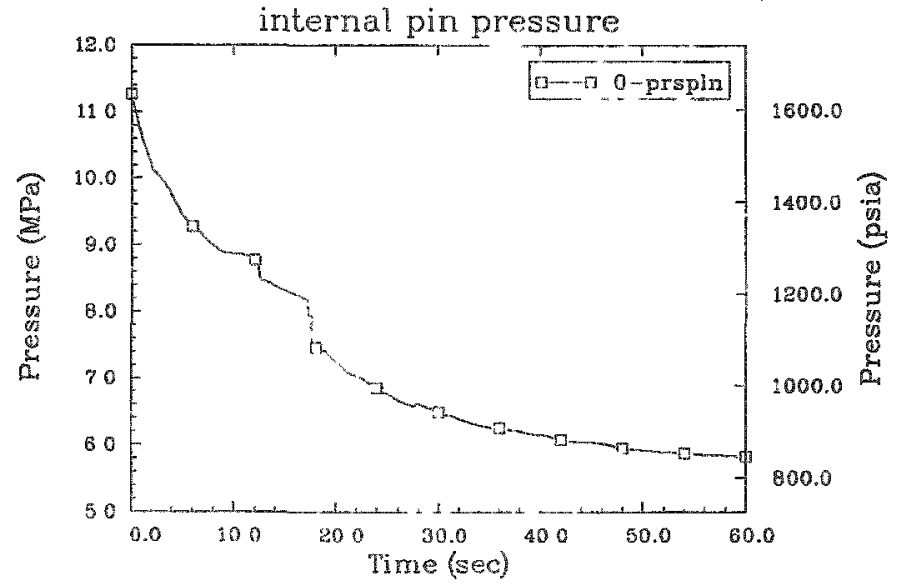

OCONEE 100\%DBA 35 GWD/MTU PIN--PF 2.2 W/EMON failure probability

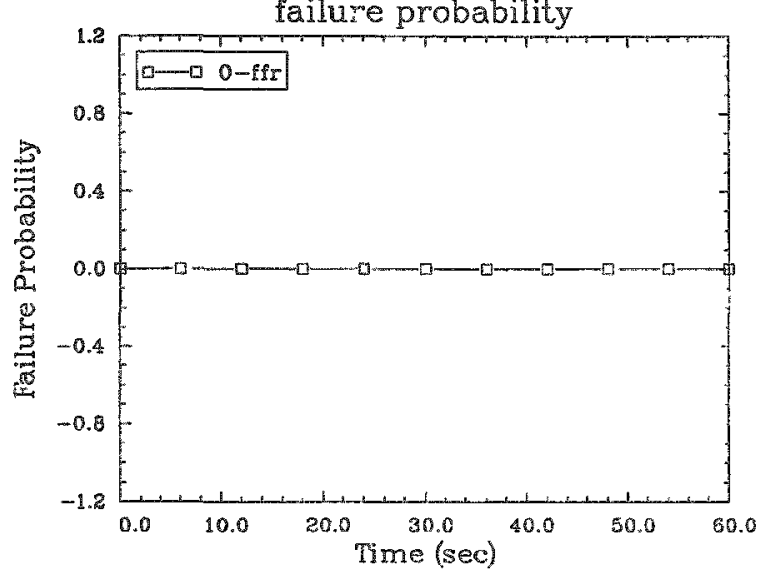

OCONEE 100\%DBA 35 GWD/MTU PIN--PF $2.2 \mathrm{~W} / \mathrm{EMON}$

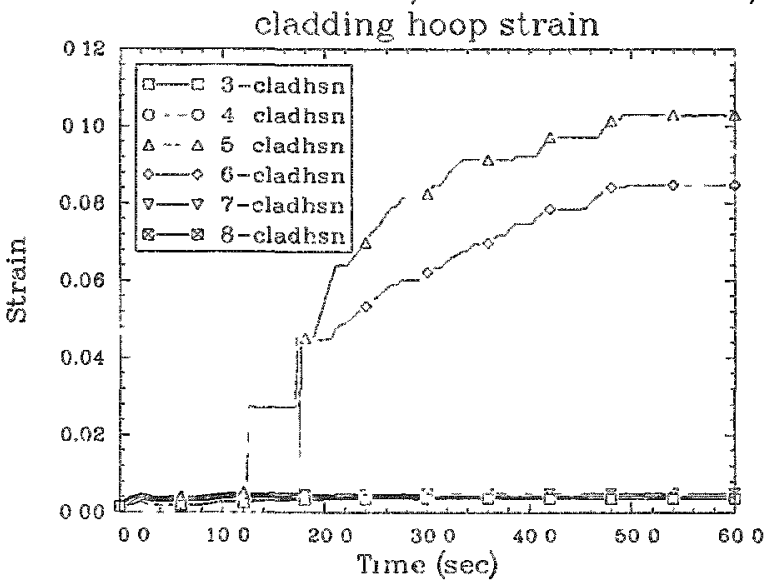

OCONEE 100\%DBA 35 GWD/MTU PIN_-PF 2.2 W/TMON

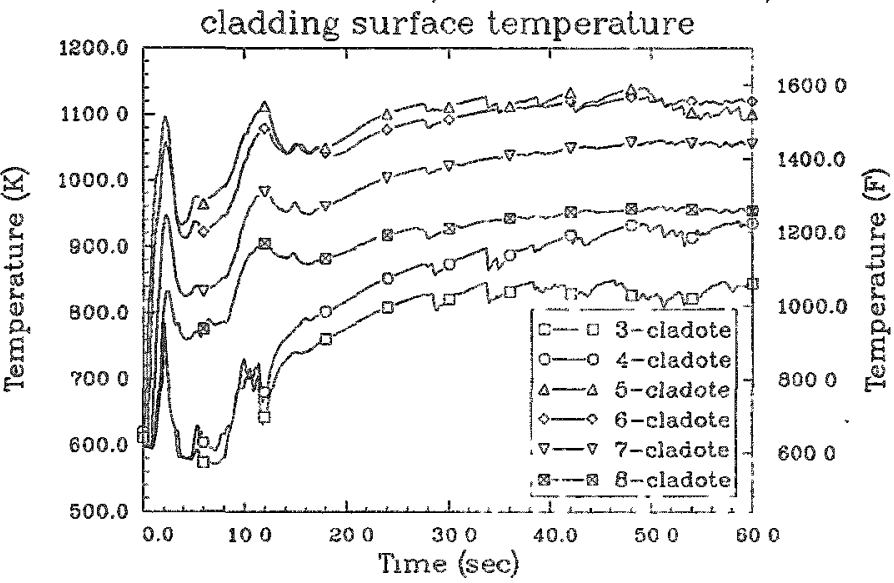

OCONEE 100\%DBA 35 GWD/MTU PIN--PF 2.2 W/EMON

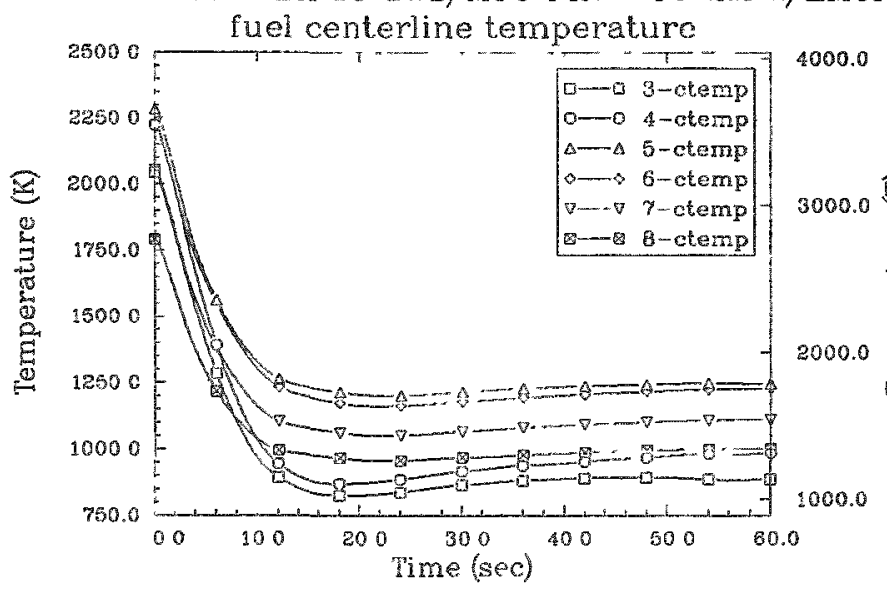

OCONEE 100\%DBA 35 GWD/MTU PIN-. PF 2.2 W/EMON oxide thickness

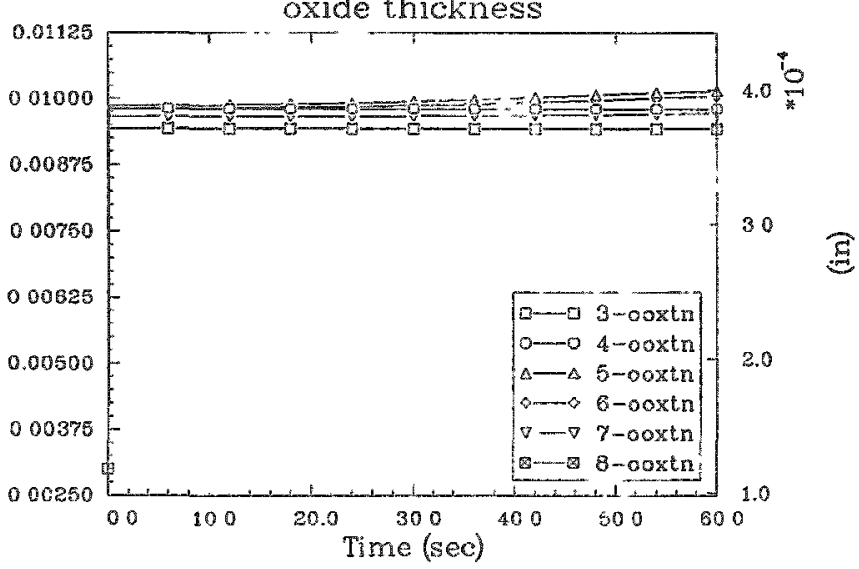



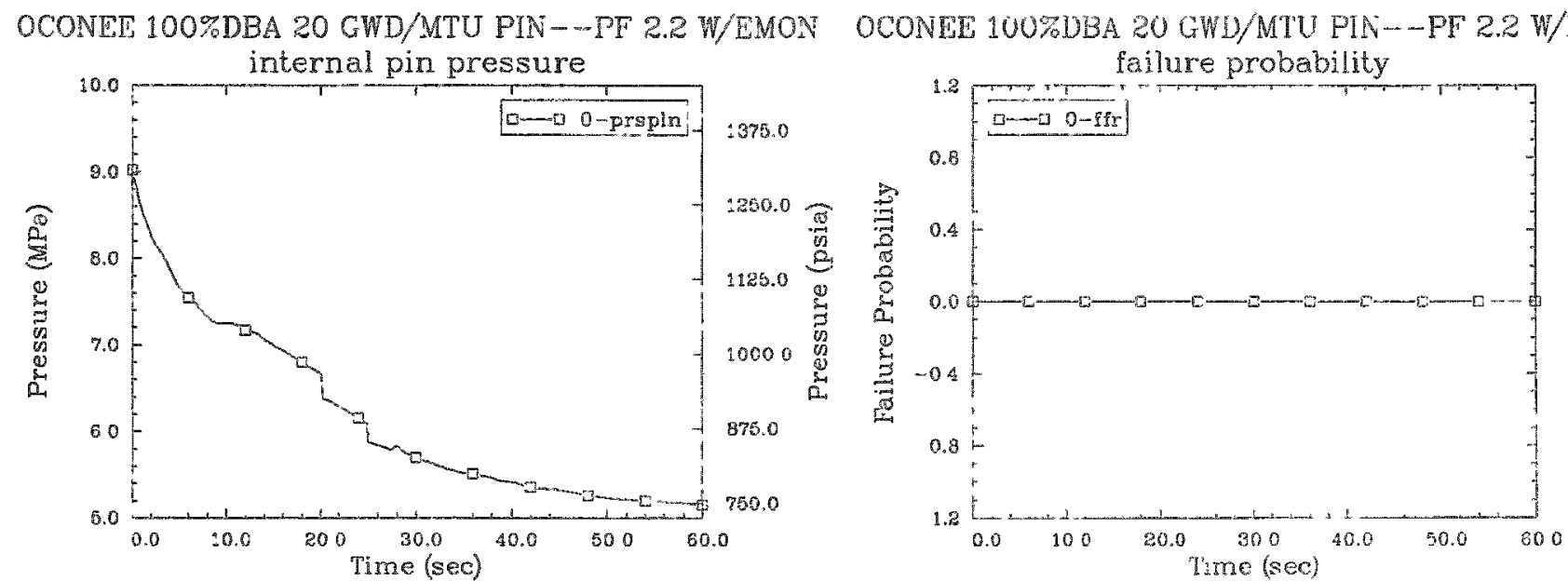

OCONEE 100\%DBA 20 GWD/MTU PIN-PF' 2.2 W/LMON

OCONEE 100\%DBA 20 GWD/MTU PIN- - PF 2.2 W/EMON
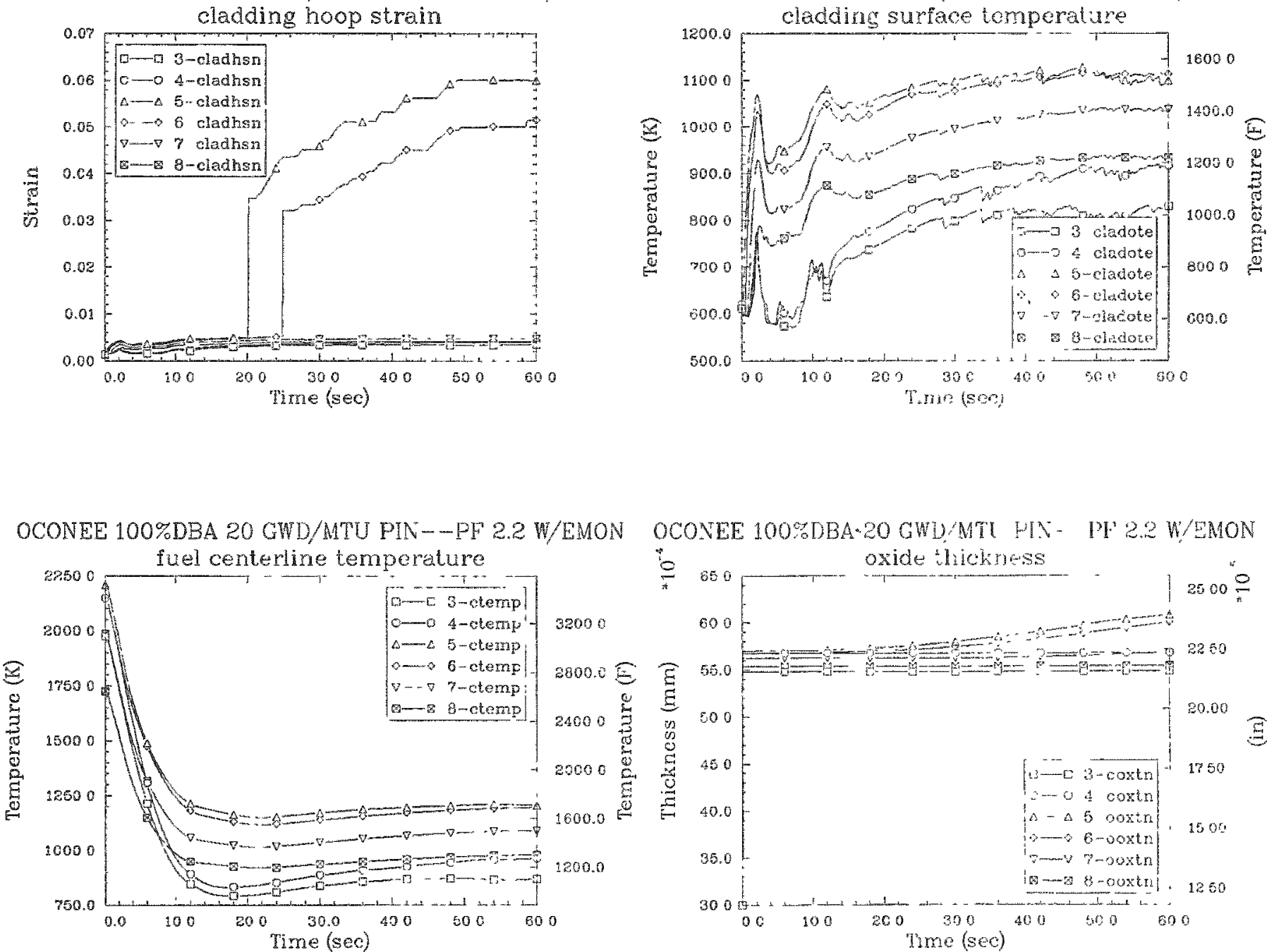

OCONEE 100\%DBA-20 GWD MTT HIN- PF 2.2 W/EMON

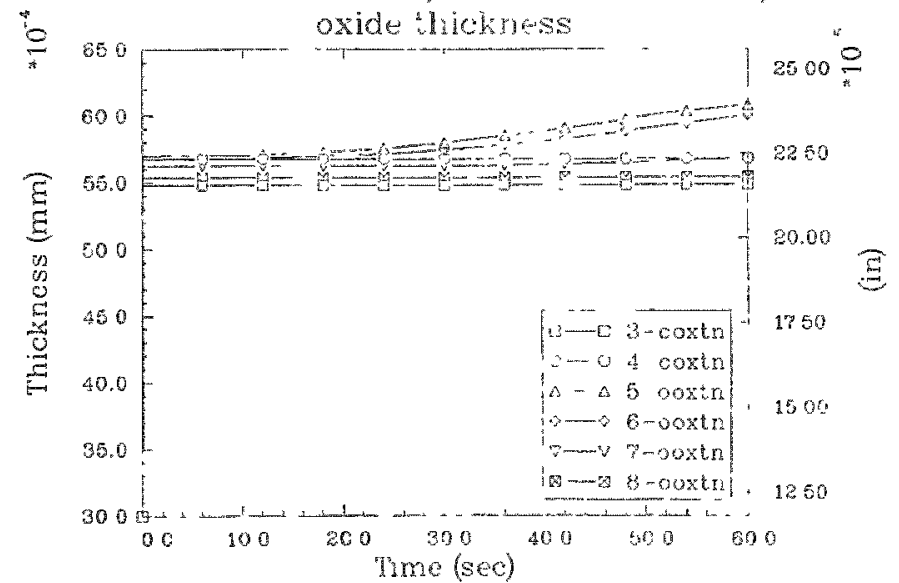




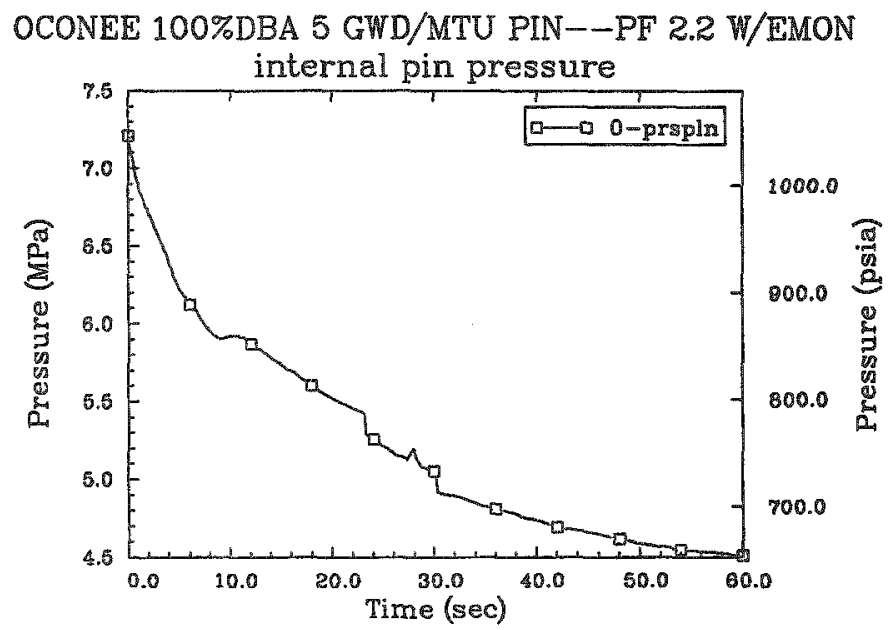

OCONEE 100\%DBA 5 GWD/MTU PIN--PF 2.2 W/EMON

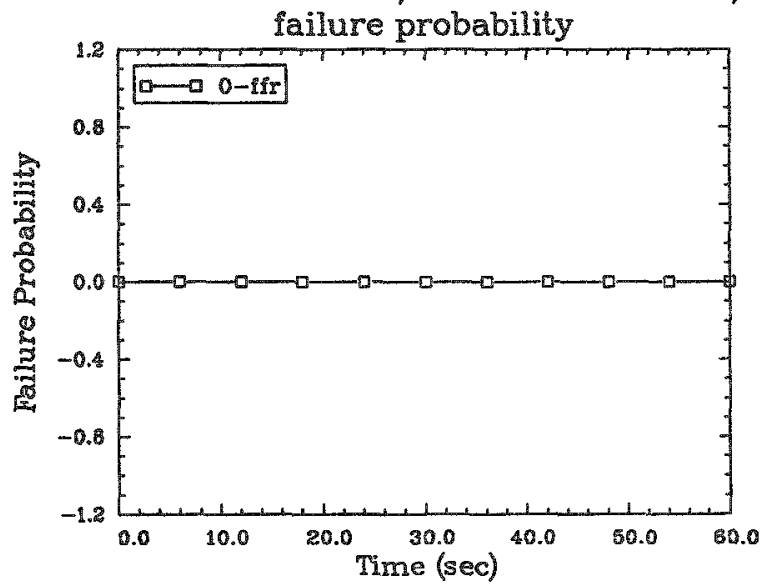

OCONEE 100\%DBA 5 GWD/MTU PIN--PF 2.2 W/EMON

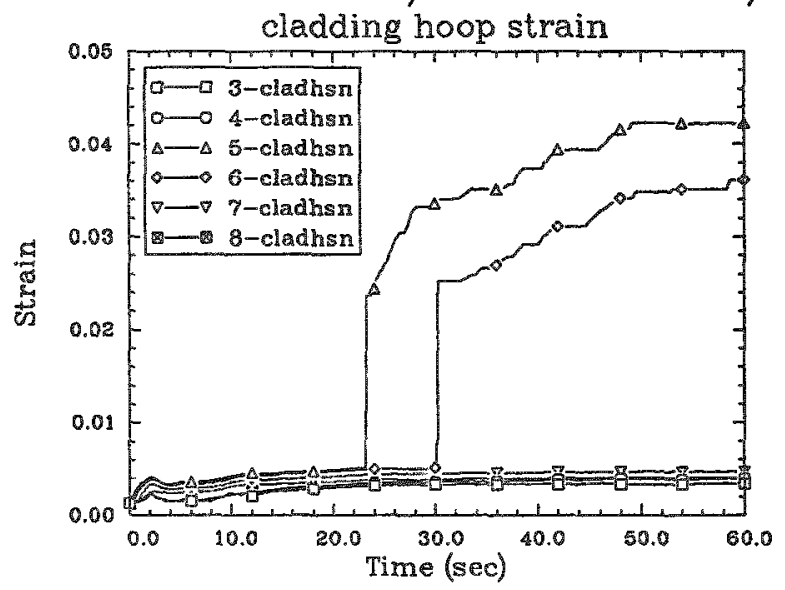

OCONEE 100\%DBA 5 GWD/MTU PIN--PF 2.2 W/EMON

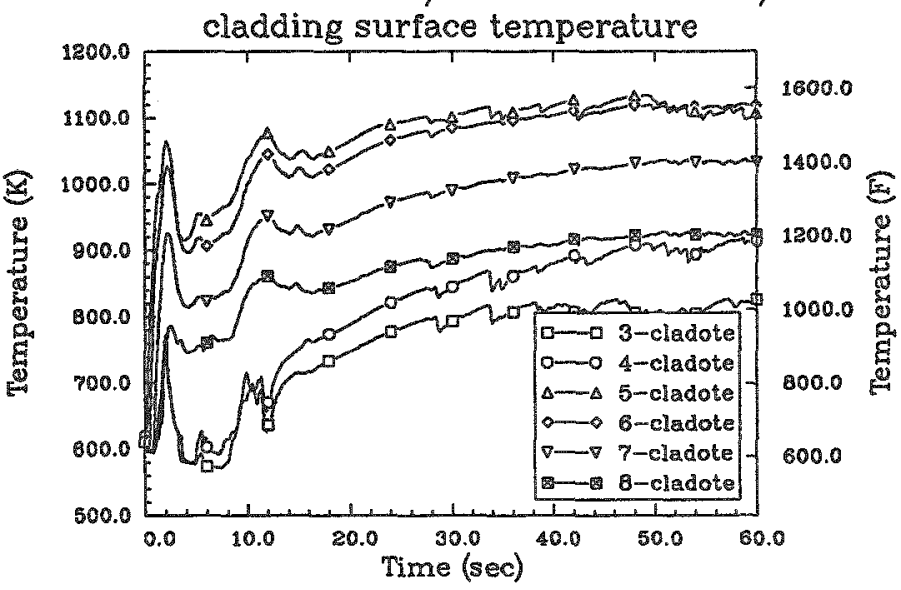

OCONEE 100\%DBA 5 GWD/MTU PIN--PF 2.2 W/EMON

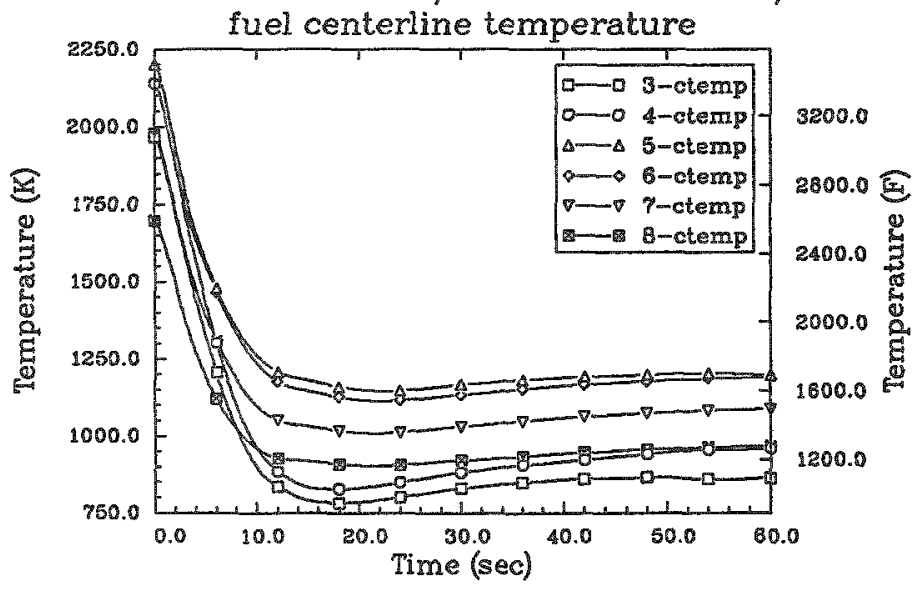

OCONEE 100\%DBA 5 GWD/MTU PIN--PF 2.2 W/EMON

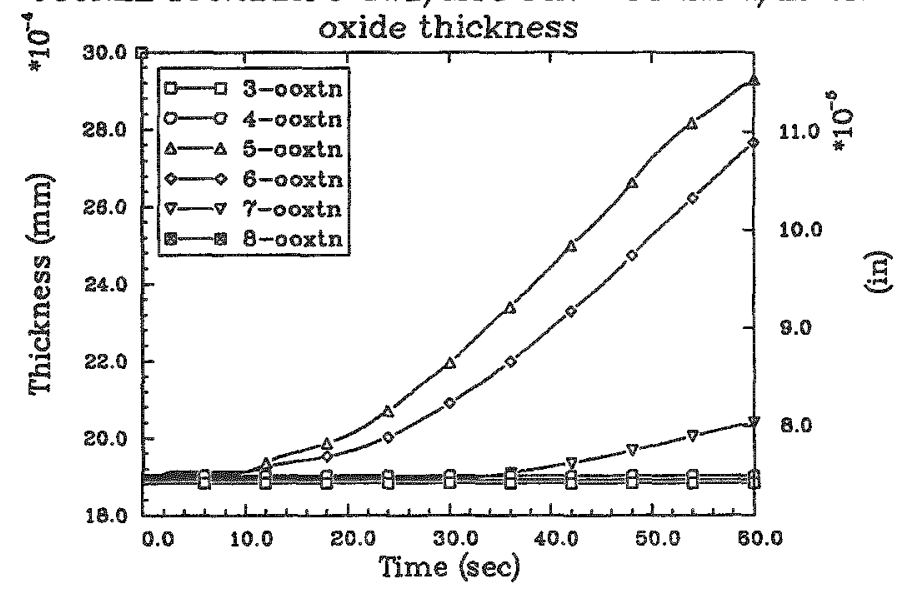


OCONEE 100\%DBA 55 GWD/MTU PIN--PF 2.0 W/EMON

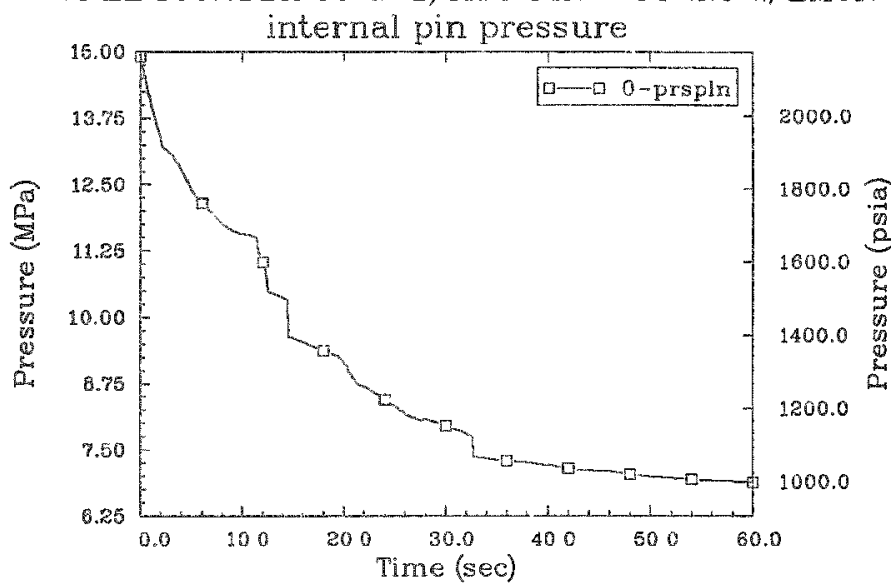

OCONEE 100\%DBA 55 GWD/MTU PIN-- -PF 2.0 W/EMON

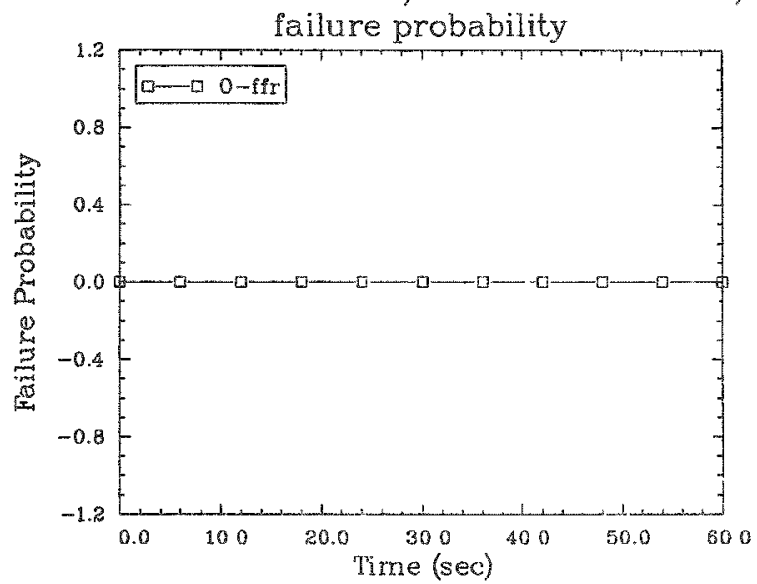

OCONEE 100\%DBA 55 GWD/MTU PIN--PF 2.0 W/EMON cladding hoop strain

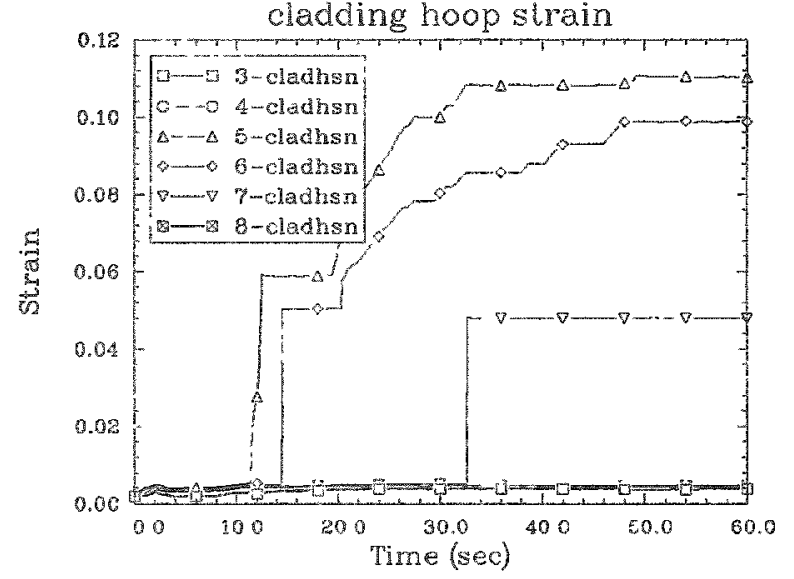

OCONEE 100\%DBA 55 GWD/MTU PIN--PF 2.0 W/EMON

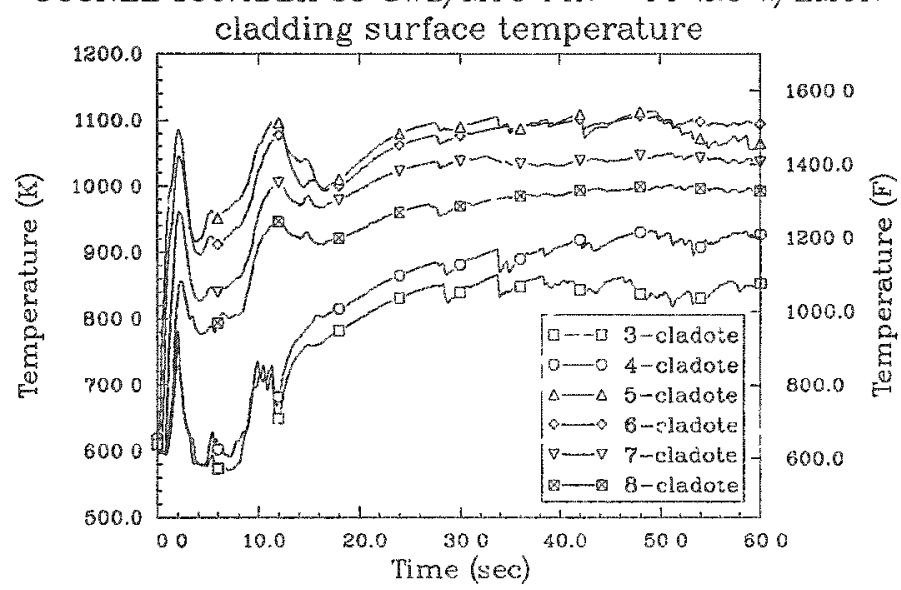

OCONEE 100\%NBA 55 GWD/MTU PIN--PF 2.0 W/EMON

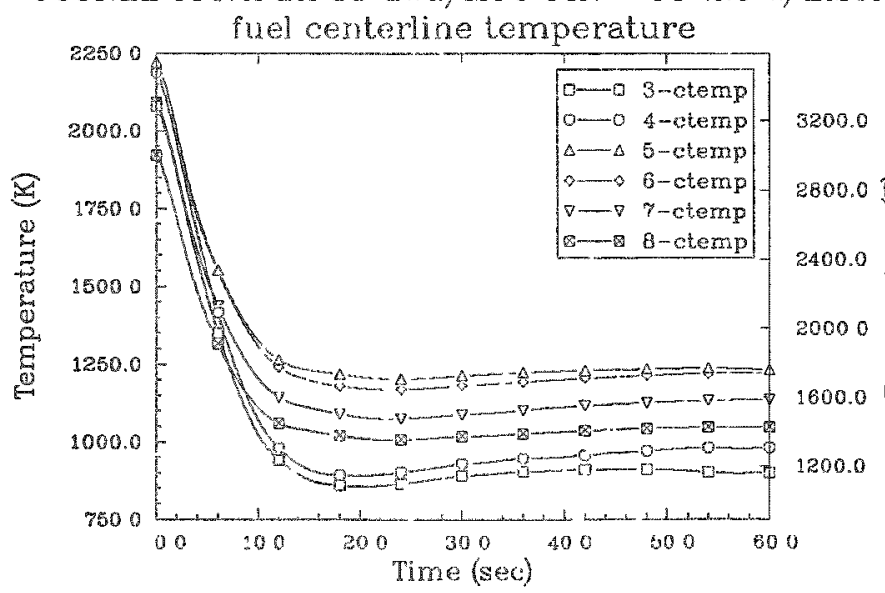

OCONEE 100\%DBA 55 GWD/MTU PIN--PF 2.0 W/EMON oxide thickness

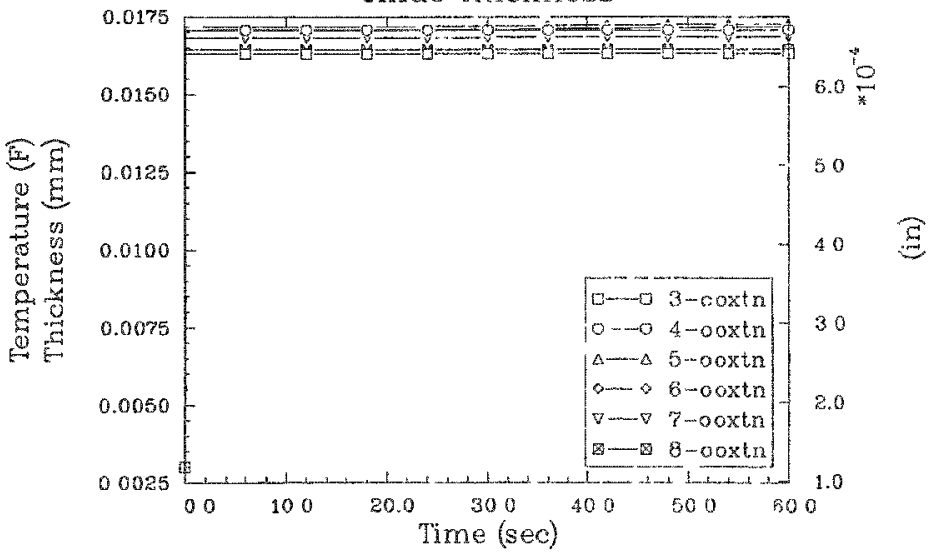


OCONEE 100\%DBA 35 GWD/MTU PIN--PF 2.0 W/EMON

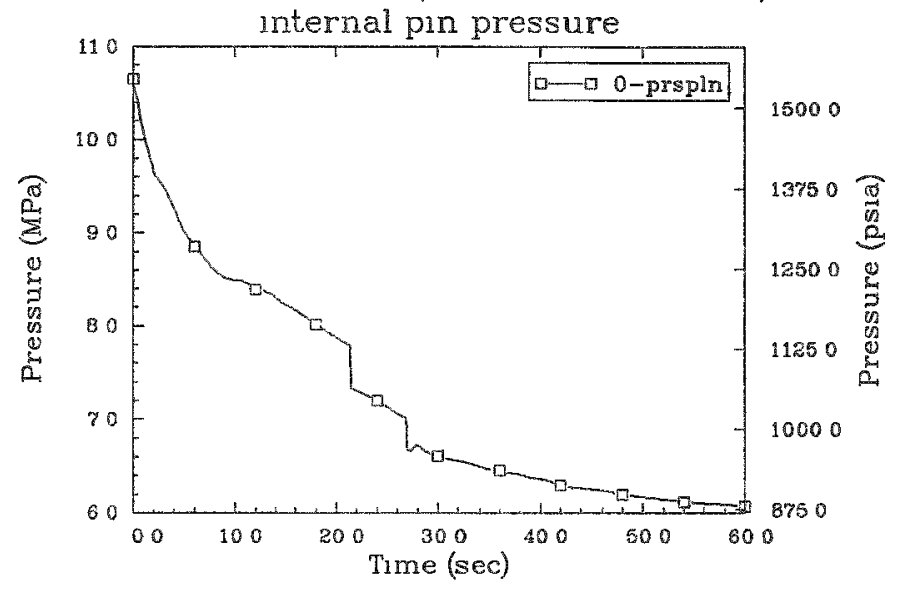

OCONEE 100\%DBA 35 GWD/MTU PIN--PF 20 W/EMON failure probability

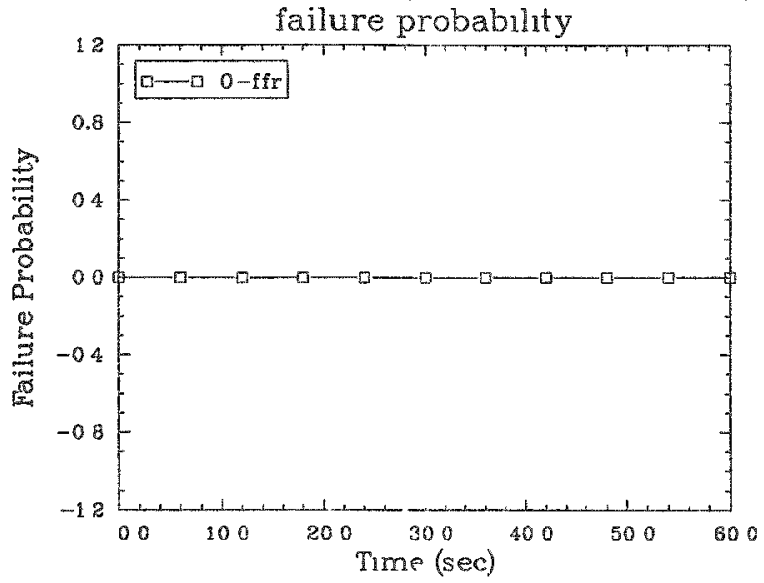

OCONEE 100\%DBA 35 GWD/MTU PIN--PF 2.0 W/EMON

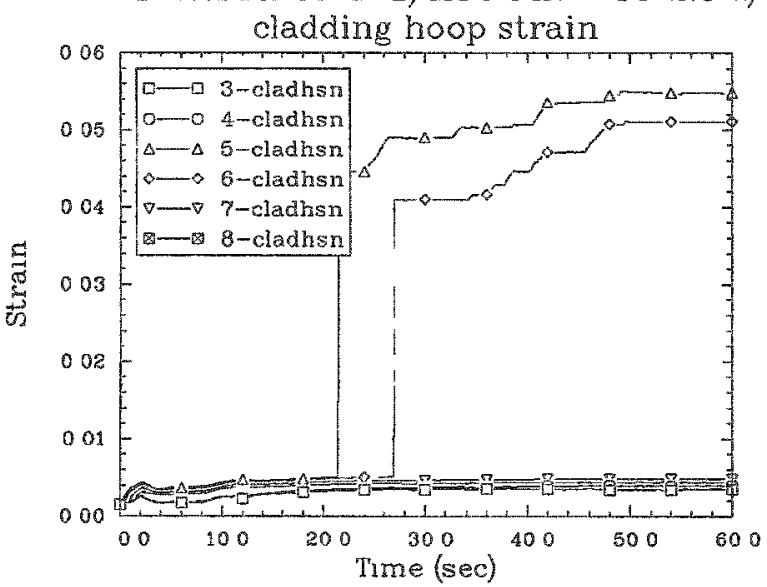

OCONEE 100\%DBA 35 GWD/MTU PIN--PF 20 W/EMON

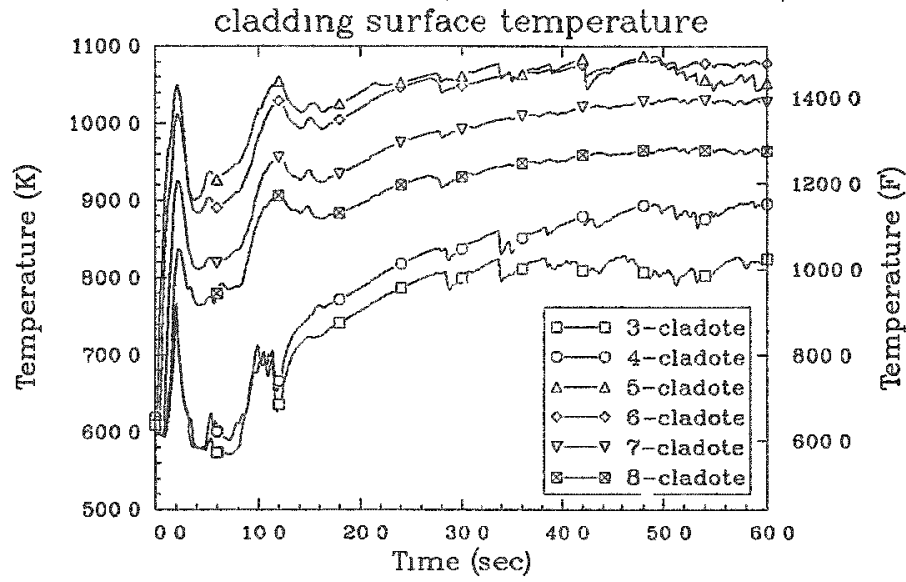

OCONEE 100\%DBA 35 GWD/MTU PIN--PF 20 W/EMON

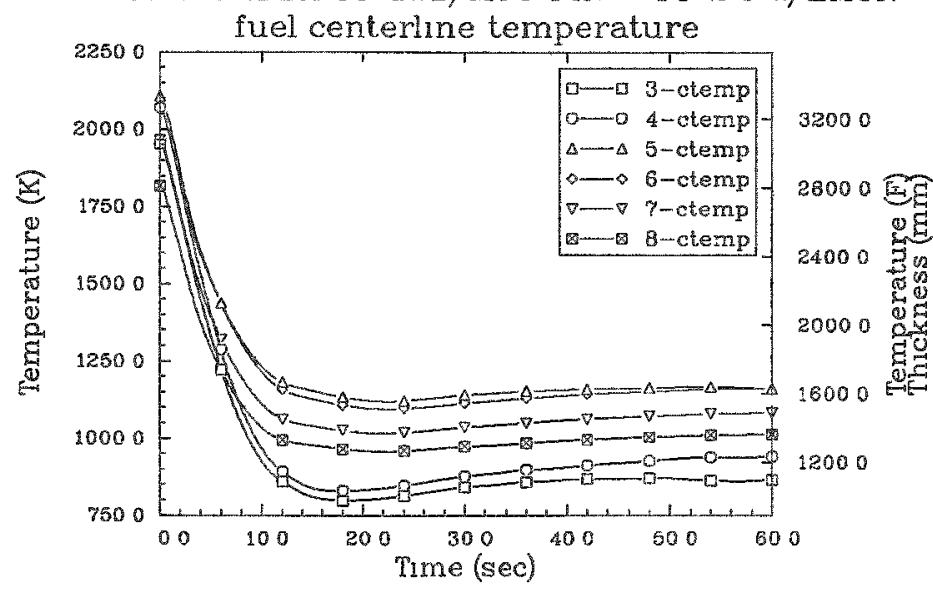

OCONEE 100\%DBA 35 GWD/MTU PIN--PF 20 W/EMON

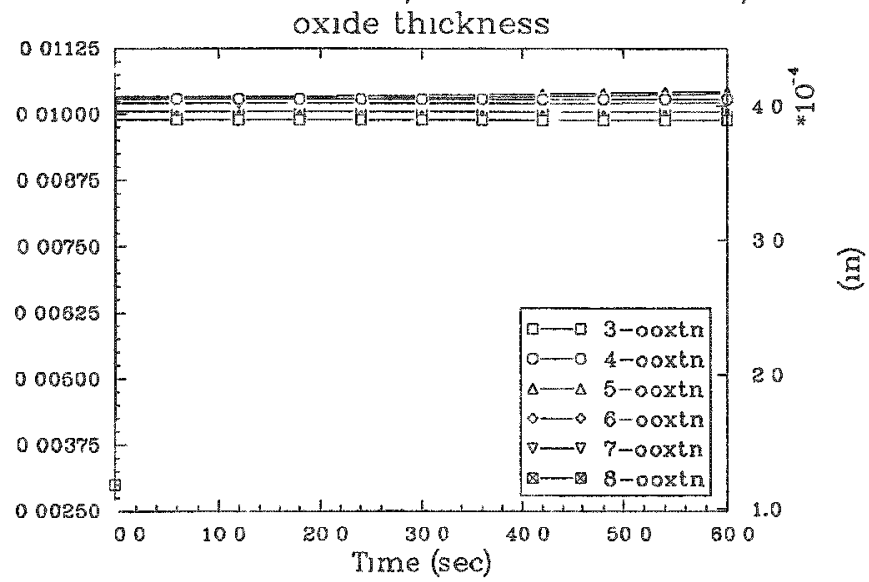


OCONEE 100\%DBA 20 GWD/MTU PIN--PF 2.0 W/EMON

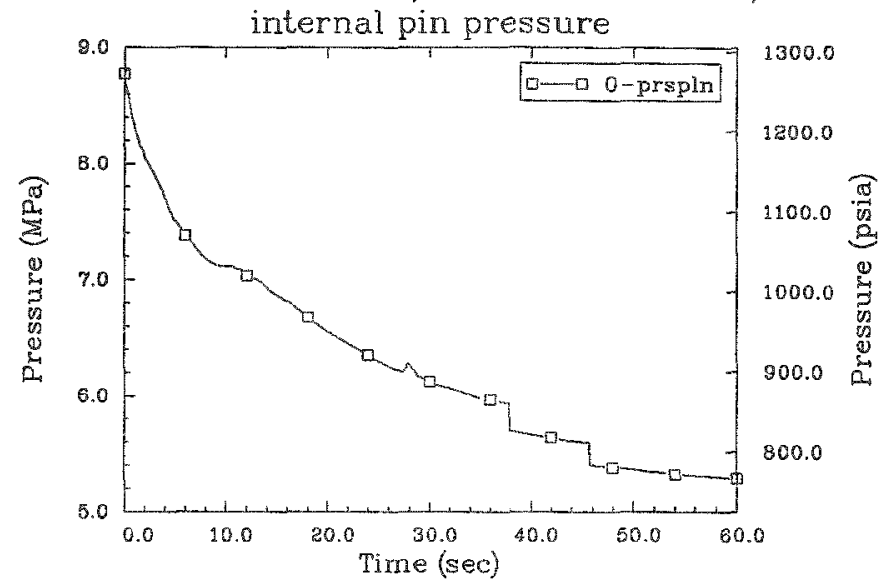

OCONEE 100\%DBA 20 GWD/MTU PIN--PF 2.0 W/EMON

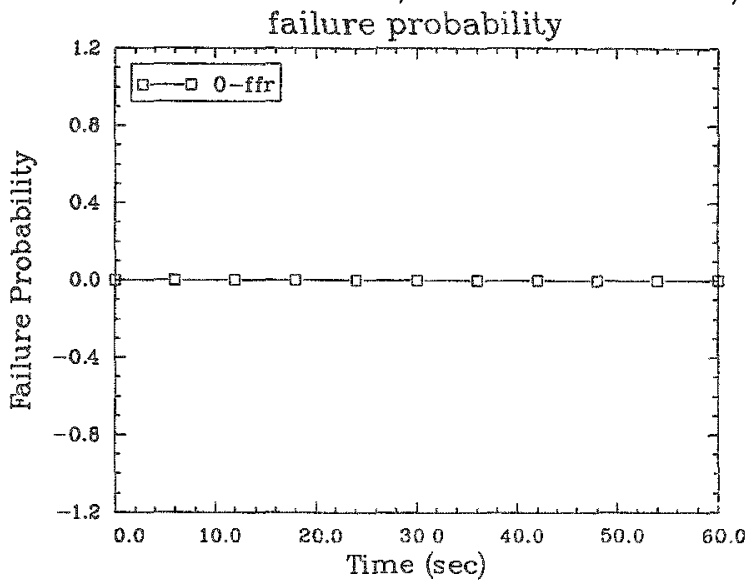

OCONEE 100\%DBA $20 \mathrm{GWD} / \mathrm{MTU}$ PIN--PF $2.0 \mathrm{~W} / \mathrm{EMON}$ cladding hoop strain

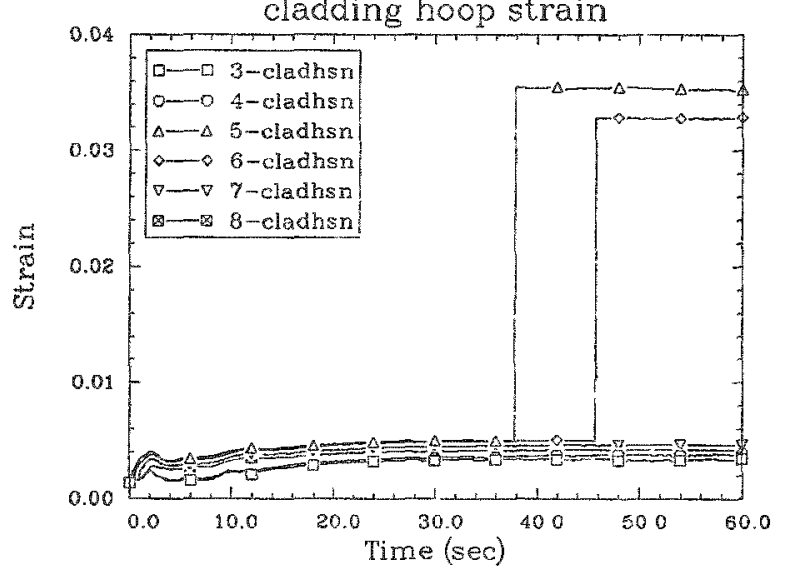

OCONEE 100\%DBA $20 \mathrm{GWD} / \mathrm{MTU}$ PIN---PF $2.0 \mathrm{~W} / \mathrm{EMON}$

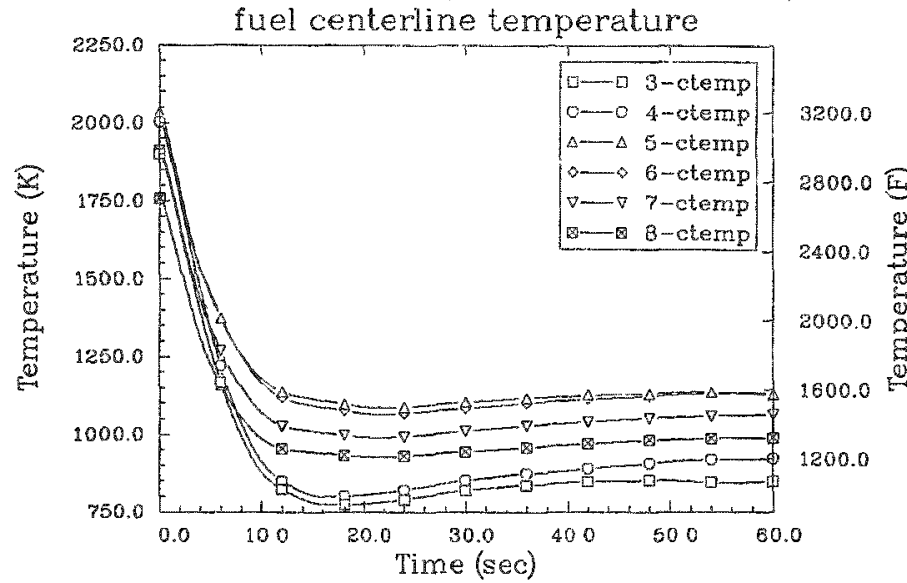

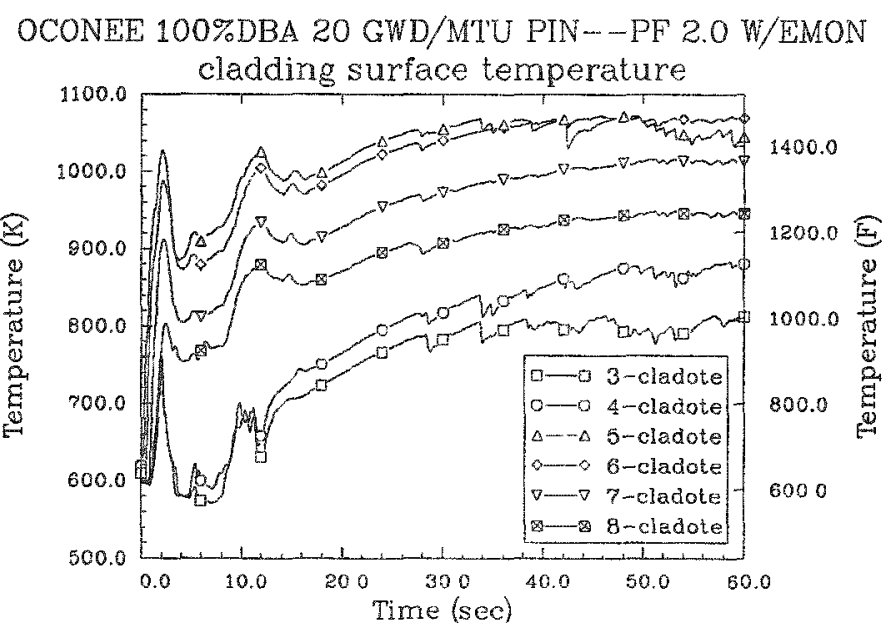

OCONEE 100\%DBA $20 \mathrm{GWD} / \mathrm{MTU}$ PIN -..PF $2.0 \mathrm{~W} / \mathrm{EMON}$

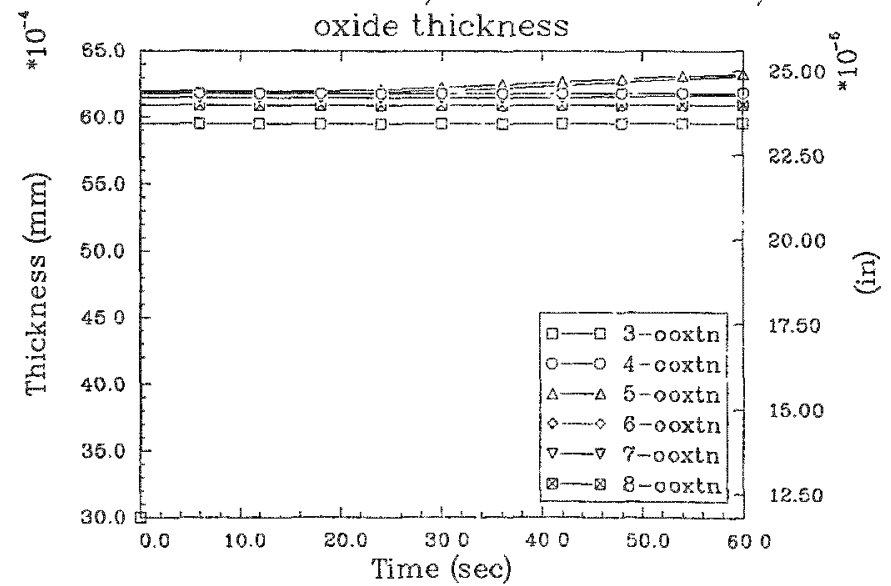


OCONEE 100\%DBA 5 GWD/MTU PIN--PF 2.0 W/EMON

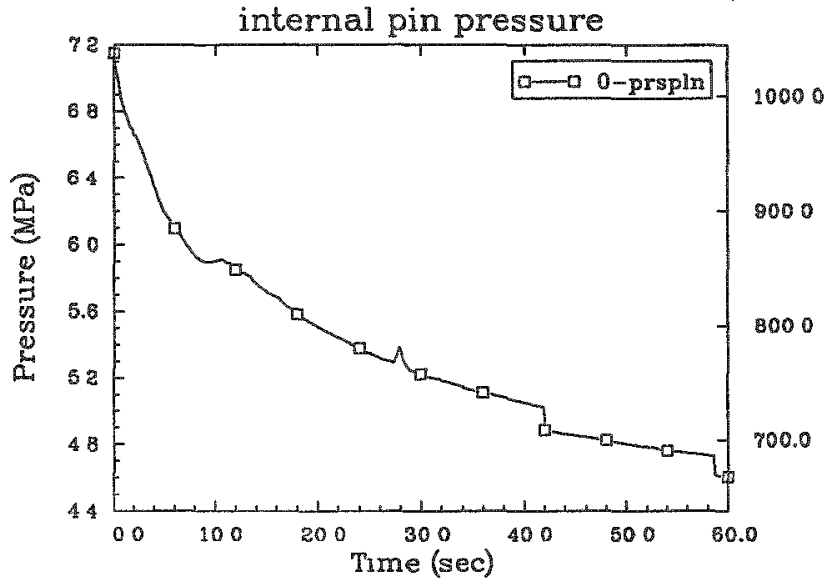

OCONEE 100\%DBA 5 GWD/MTU PIN--PF $2.0 \mathrm{~W} / \mathrm{EMON}$

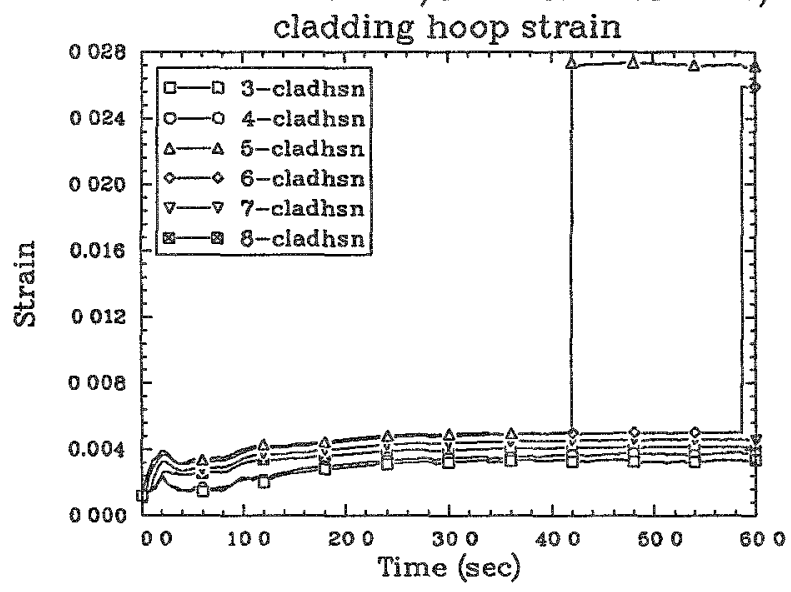

OCONEE 100\%DBA 5 GWD/MTU PIN--PF 2.0 W/EMON

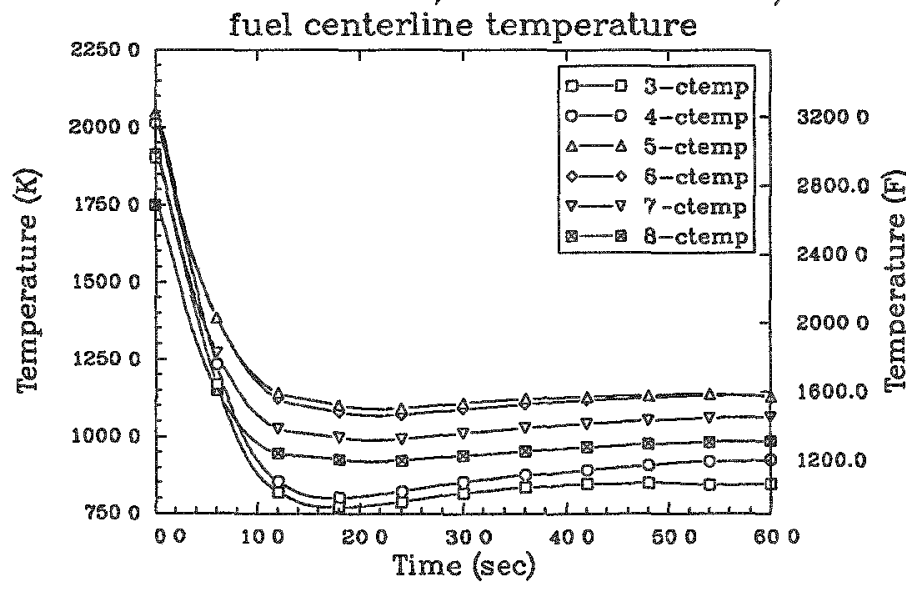

OCONEE 100\%DBA 5 GWD/MTU PIN--PF 2.0 W/EMON failure probability
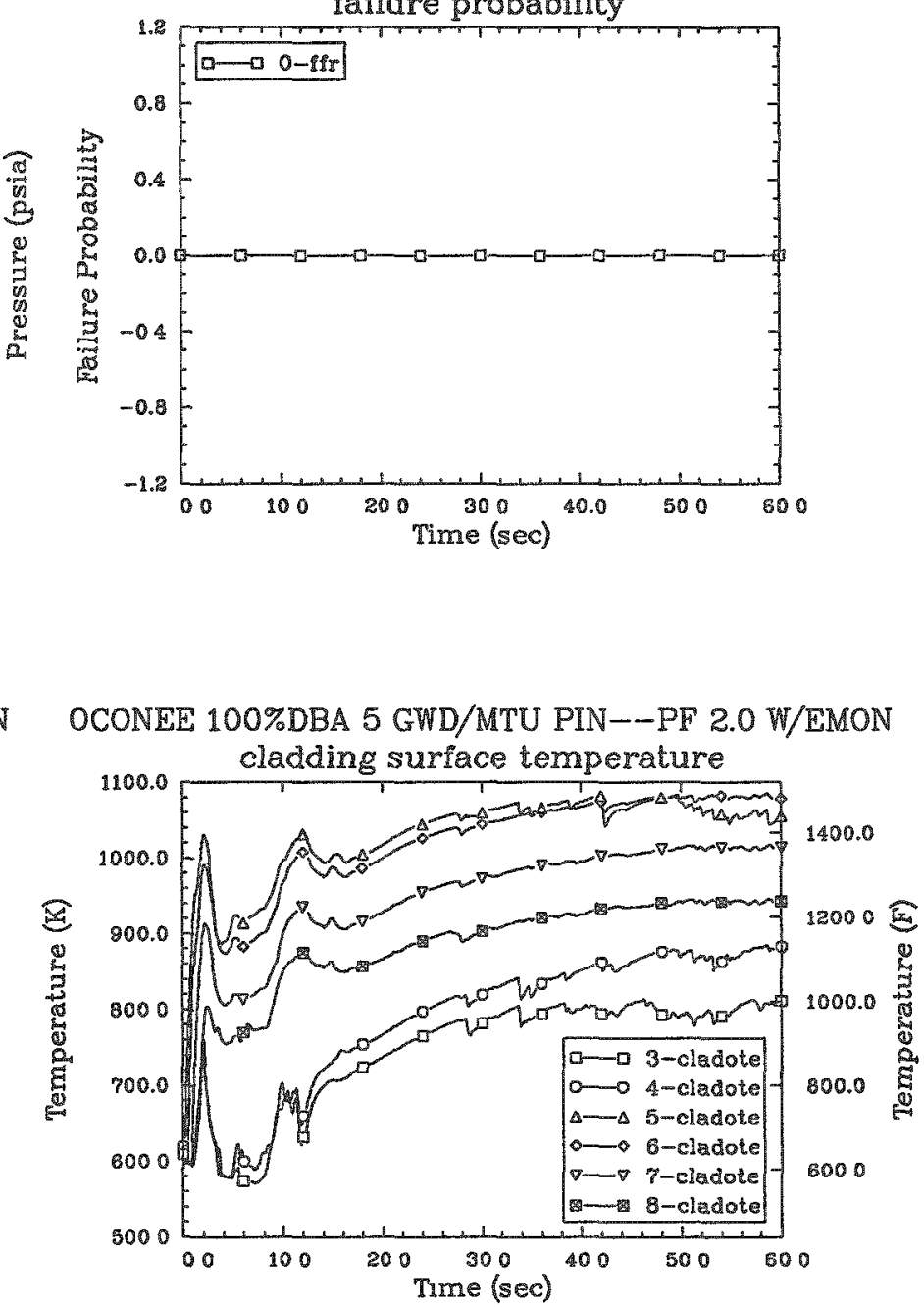

OCONEE 100\%DBA 5 GWD/MTU PIN--PF 2.0 W/EMON

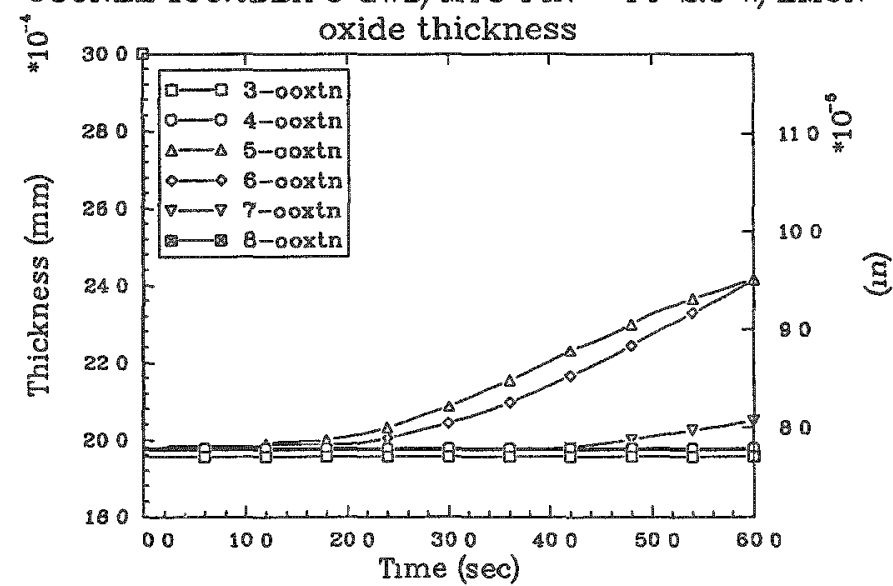


OCONEE SMBRK 55 GWD/MTU PIN--PF 2.63
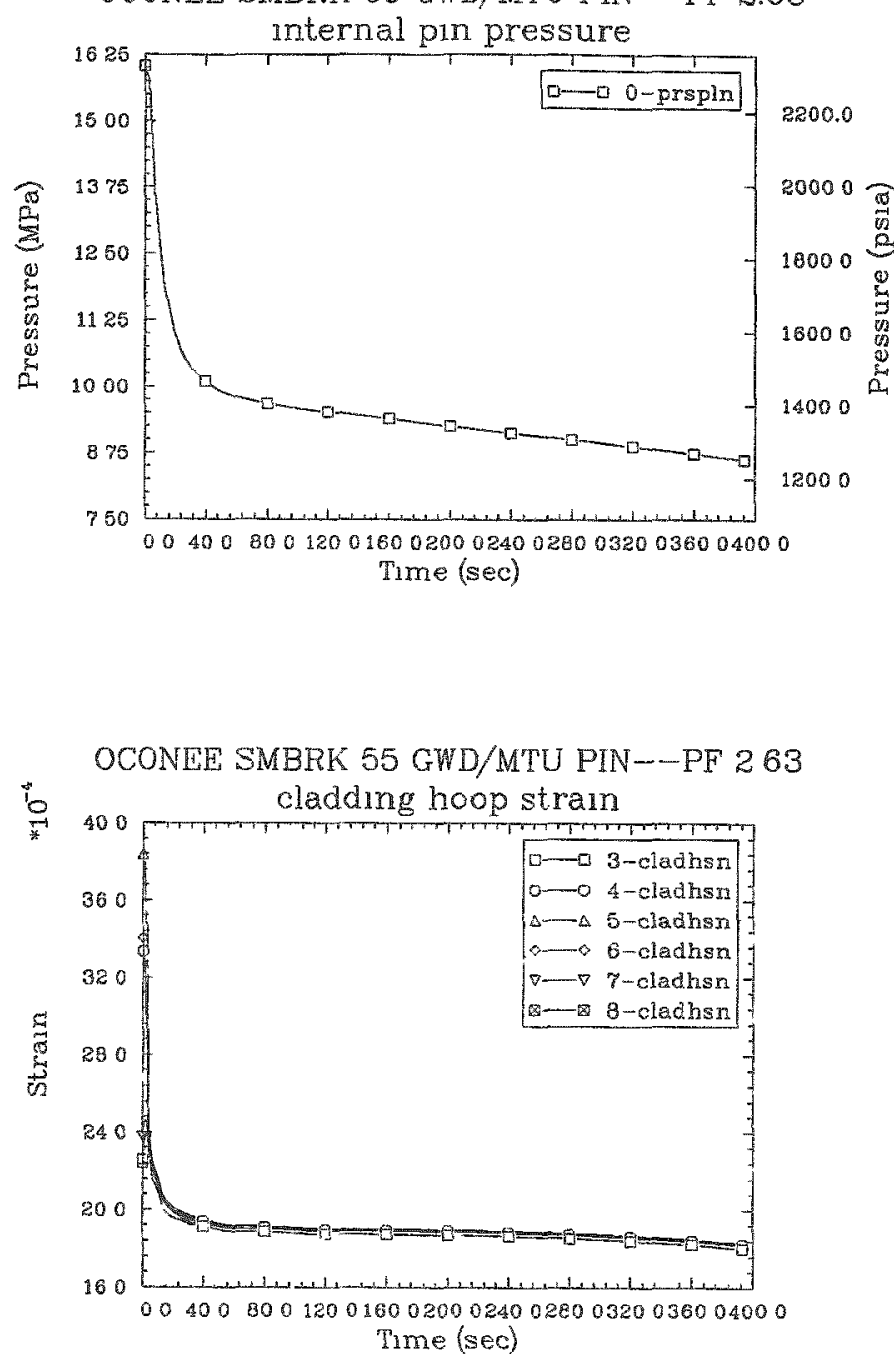

OCONEE SMBRK 55 GWD/MTU PIN - -PF 263

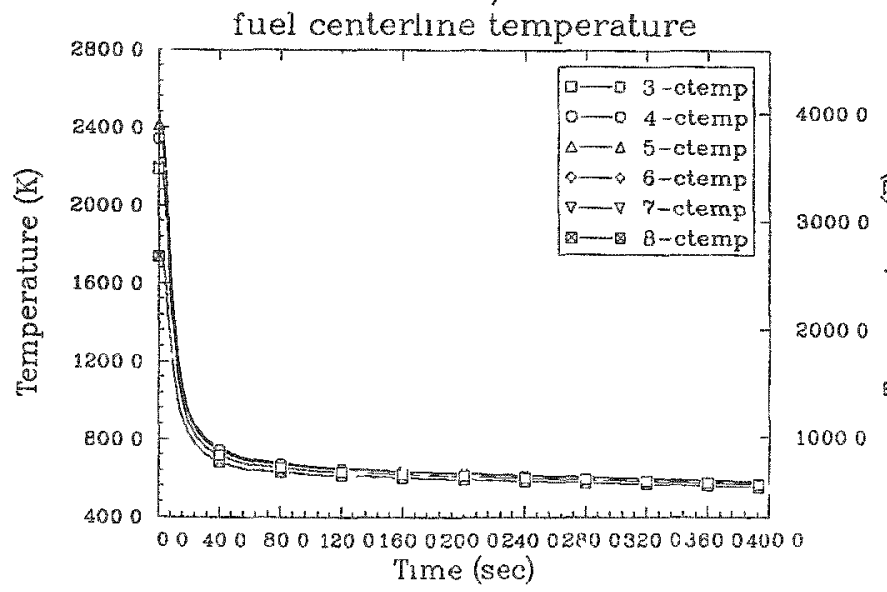

OCONEE SMBRK 55 GWD/MTU PIN-DF 263 faslure probability

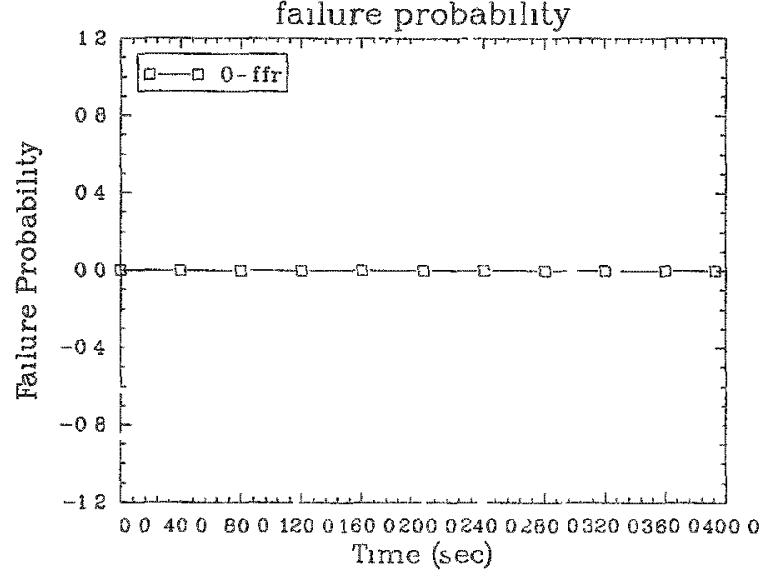

OCONEE SMBRK 55 GWD/MTU PIN-- PF 263

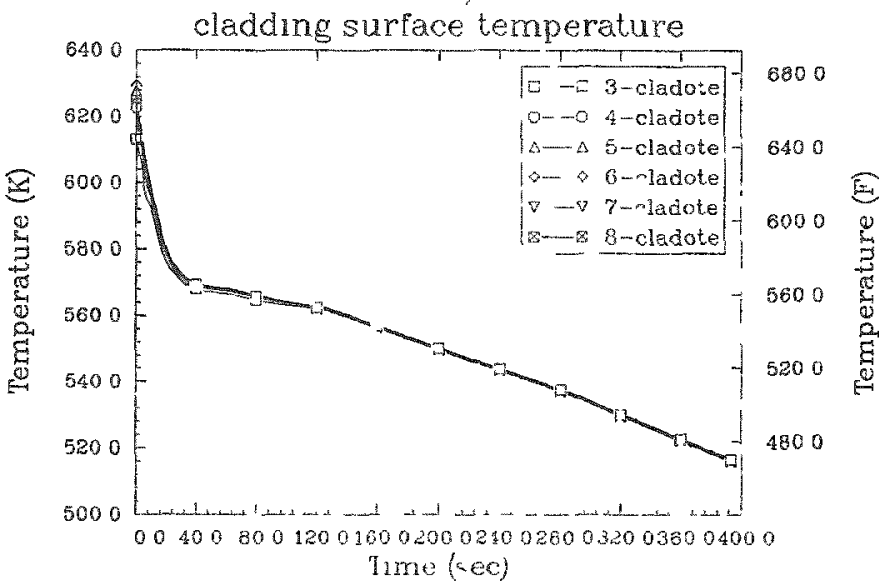

OCONEE SMBRK 55 GWD/MTL PIN -FI 263 oxide thickness

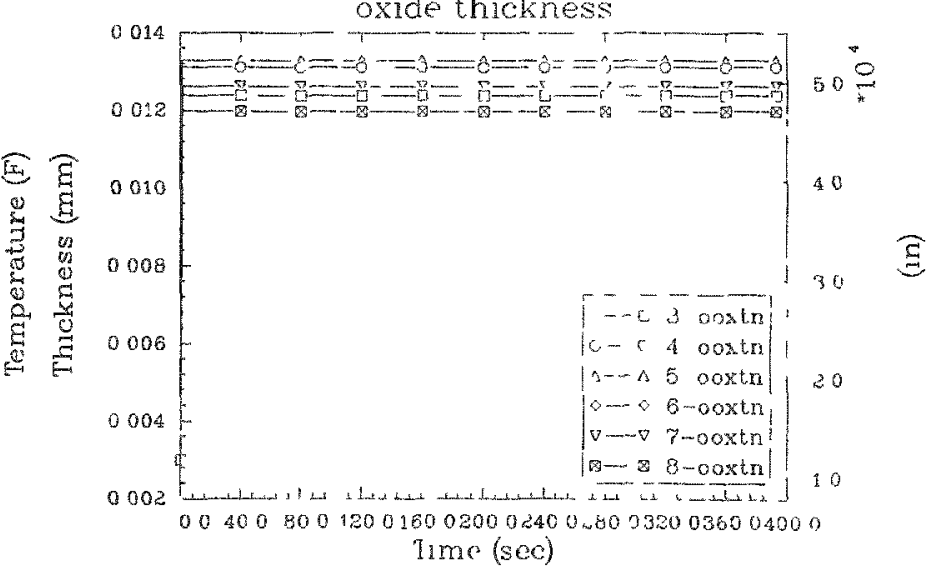



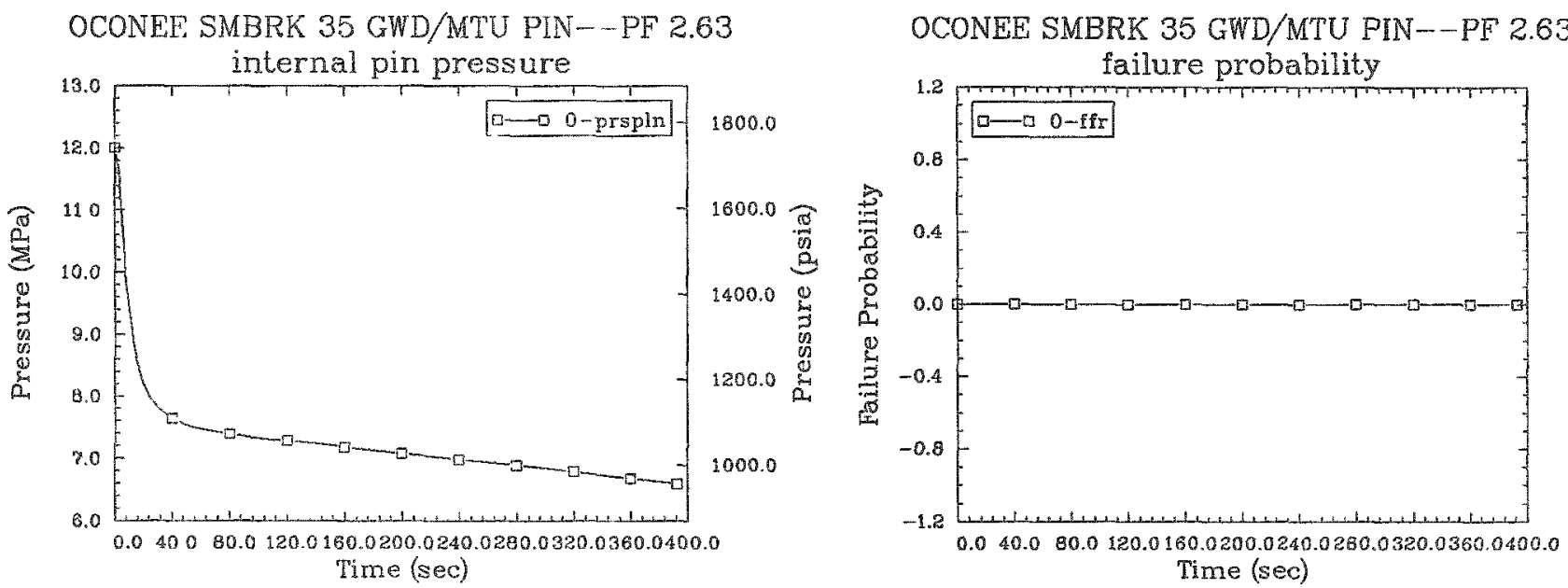

OCONEE SMBRK 35 GWD/MTU PIN--PF 2.63

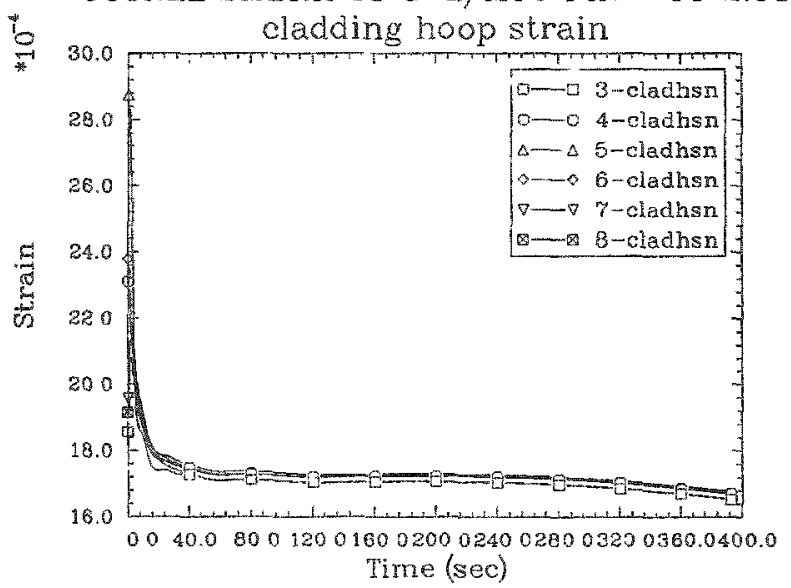

OCONEE SMBRK 35 GWD/MTU PIN--PF 2.63

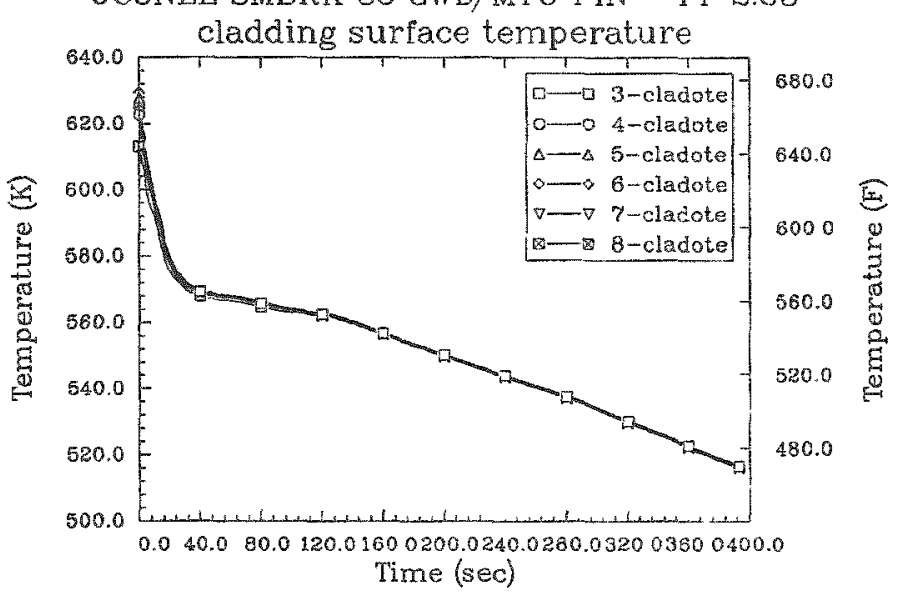

OCONEE SMBRK 35 GWD/MTU PIN--PF 2.63

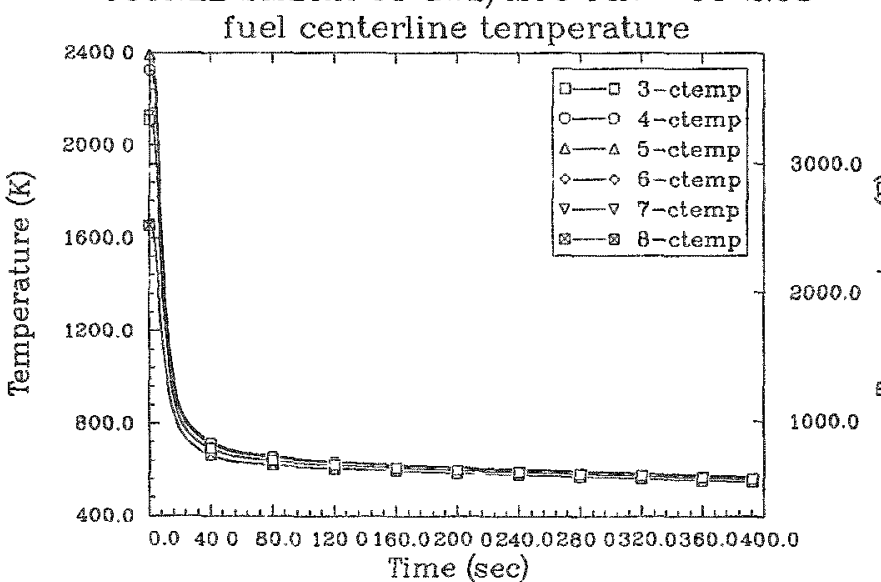

OCONEE SMBRK 35 GWD/MTU PIN--PF 2.63

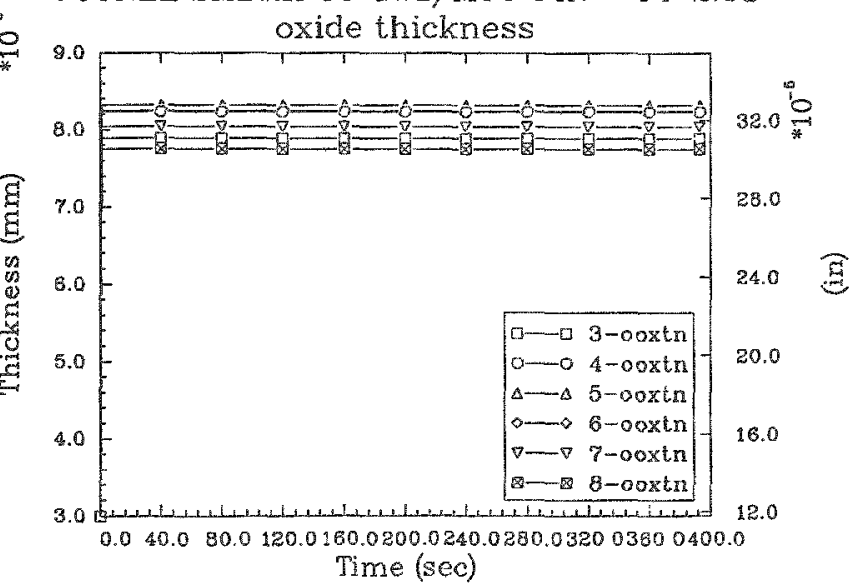



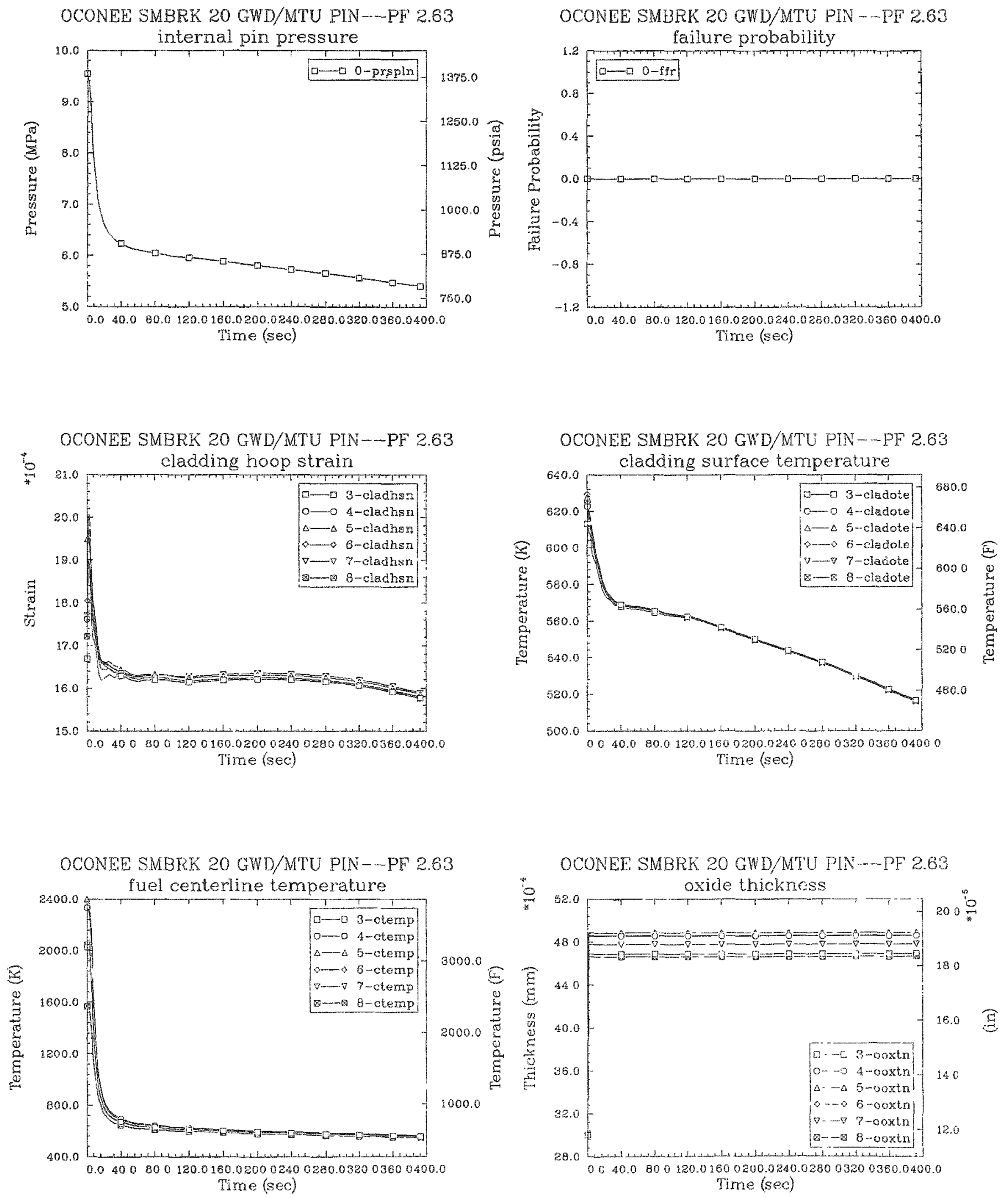

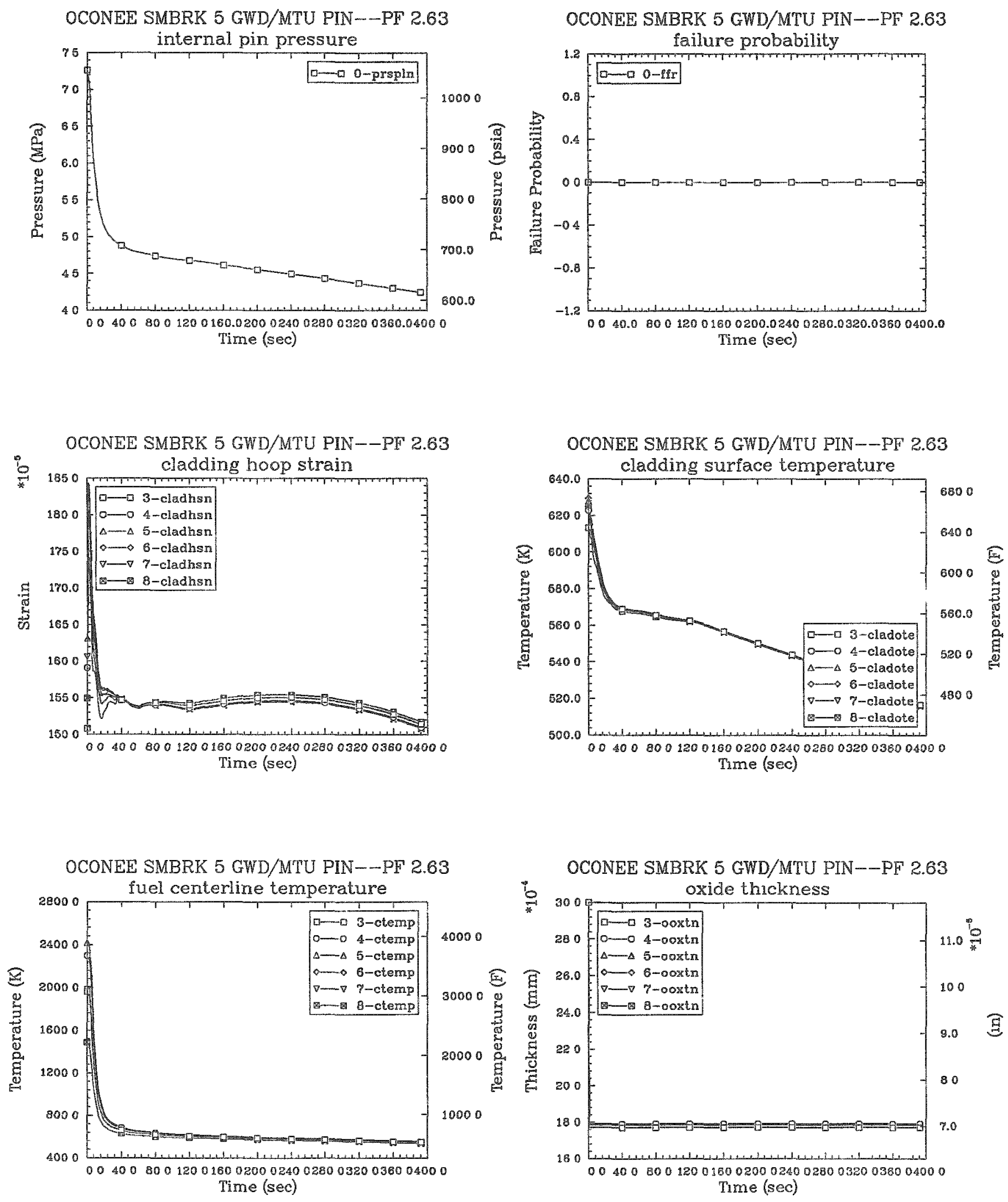


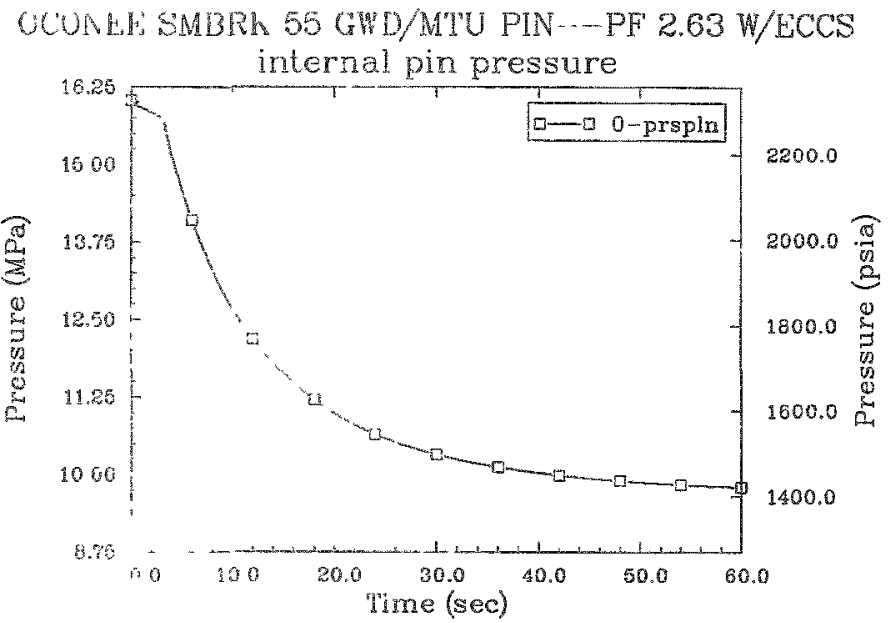

OCONEE SMBRK 55 GWD/MTU PIN--PH' 2.63 W/ECCS

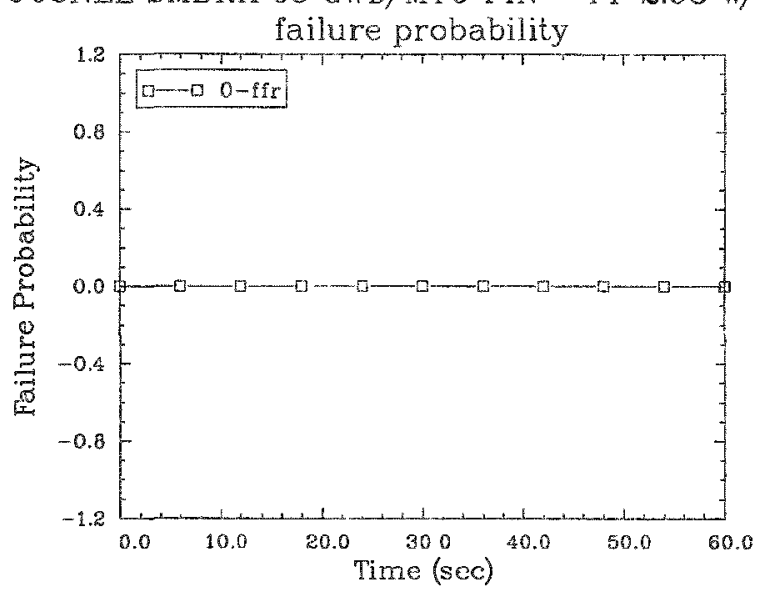

DCONEE SMBRK 55 GWD/MTU PIN--PF 2.63 W/ECCS

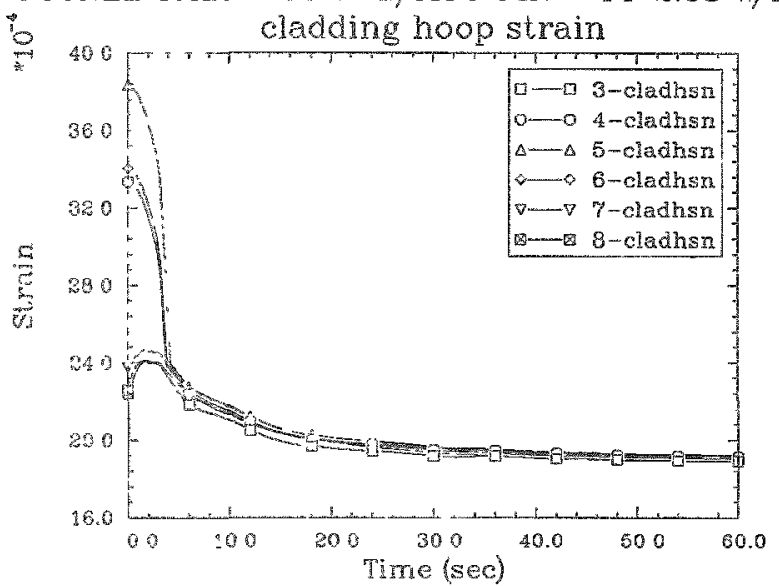

OCONEE SMBRK 55 GWD/MTU PIN - -PF 2.63 W/ECCS
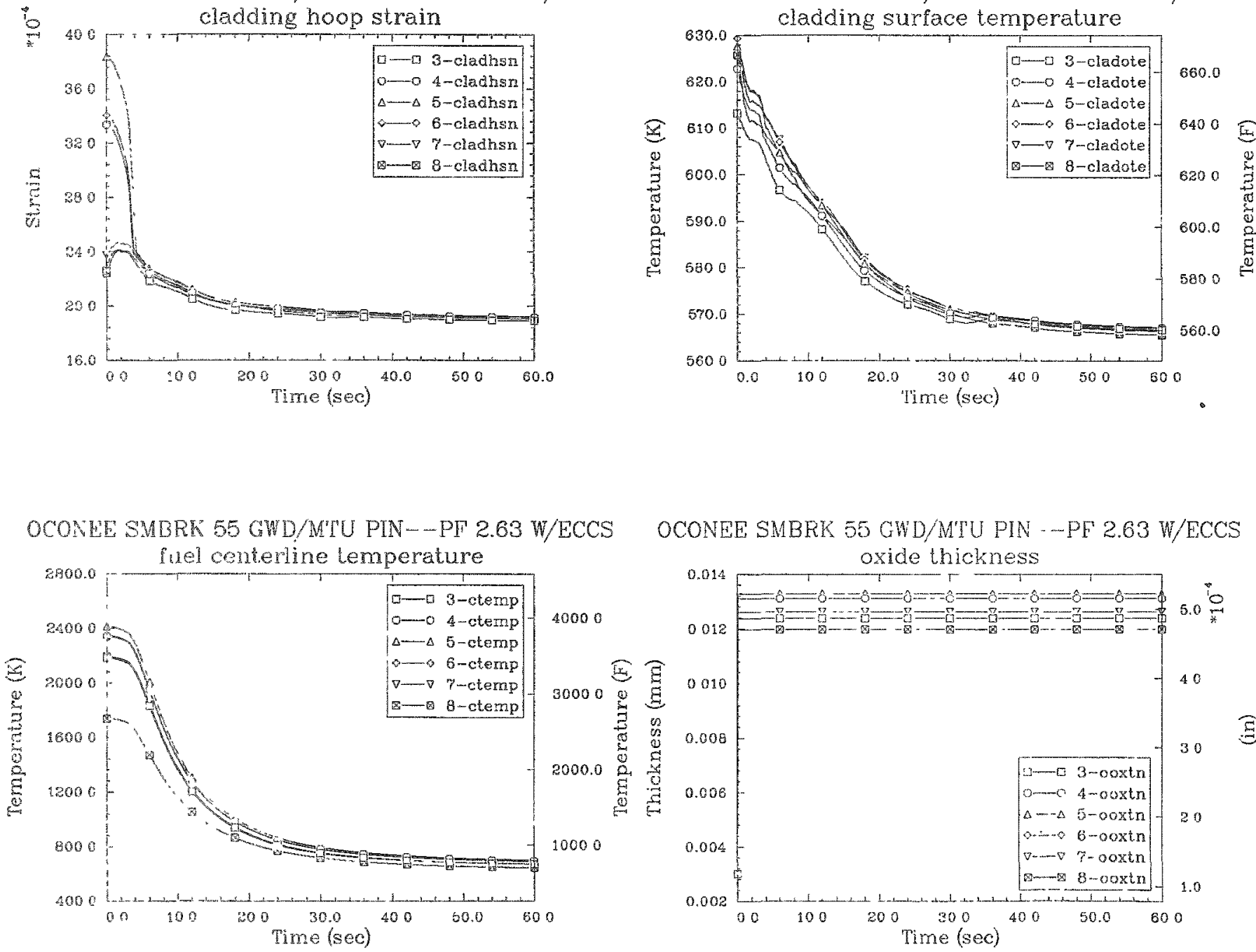

OCONEE SMBRK 55 GWD/MTU PIN -.-PF $2.63 \mathrm{~W} / \mathrm{ECCS}$

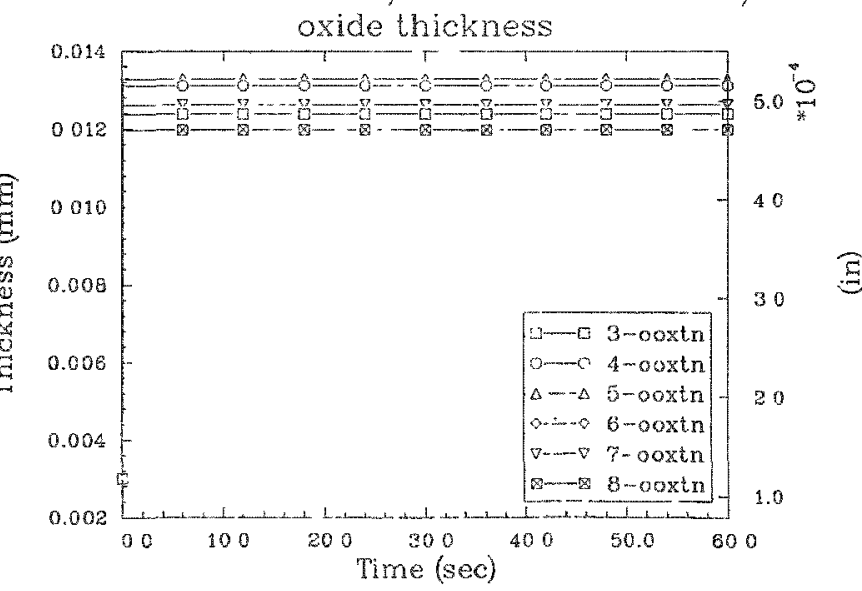


OCONEE SMBRK 35 GWD/MTU PIN--PF 263 W/ECCS

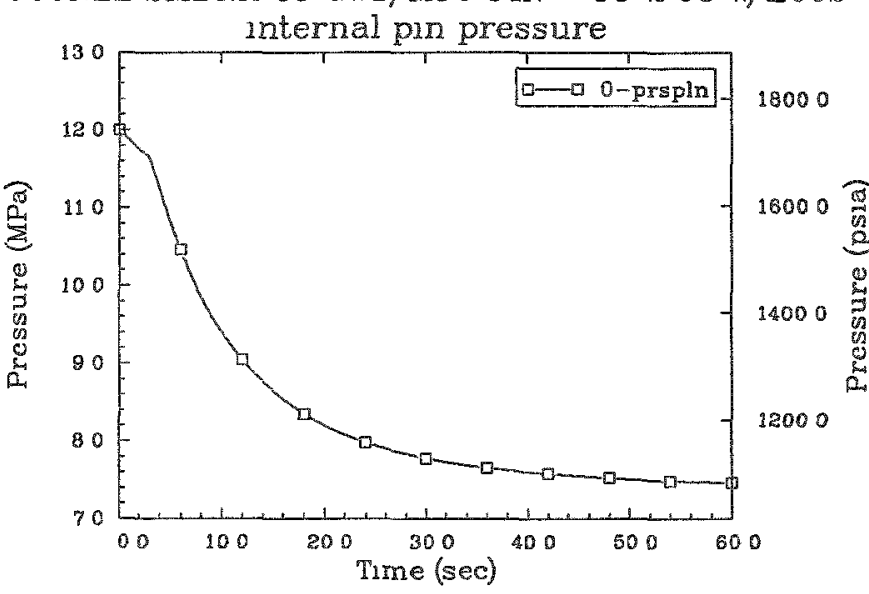

OCONEE SMBRK 35 GWD/MTU PIN--PF 263 W/ECCS

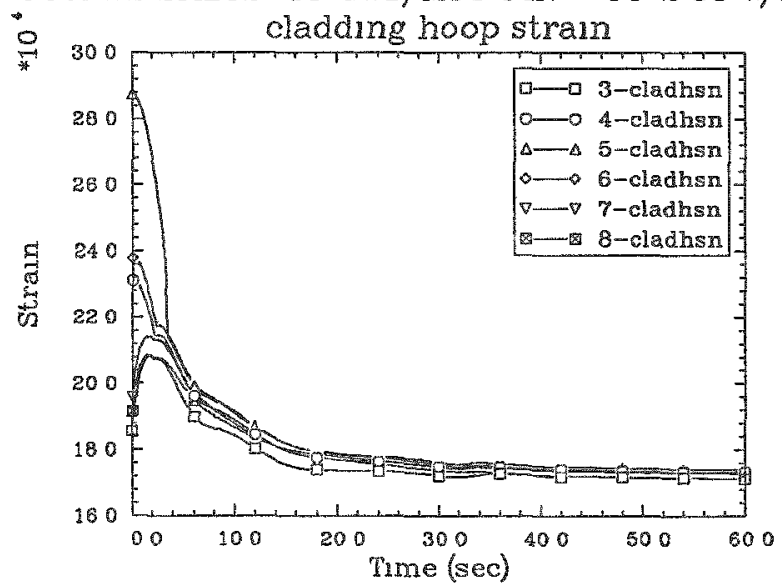

OCONEE SMBRK 35 GWD/MTU PIN--PF 263 W/ECCS fallure probability

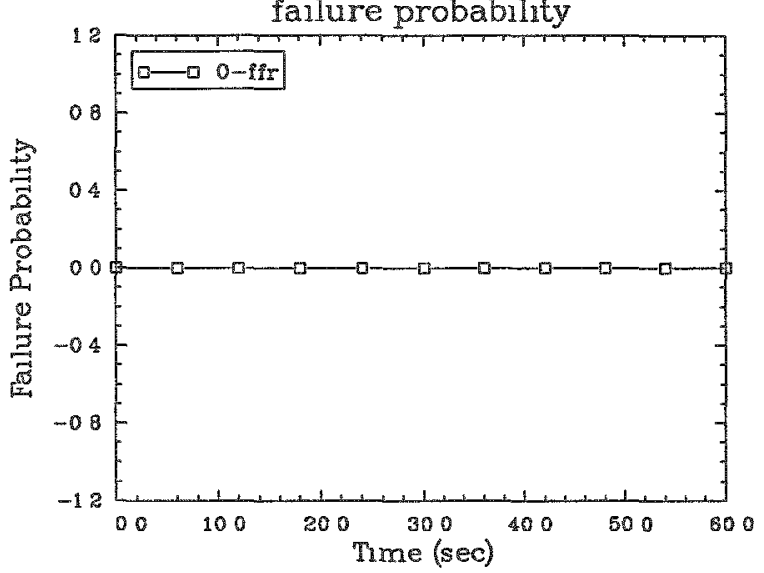

OCONEE SMBRK 35 GWD/MTU PIN--PF 263 W/ECCS

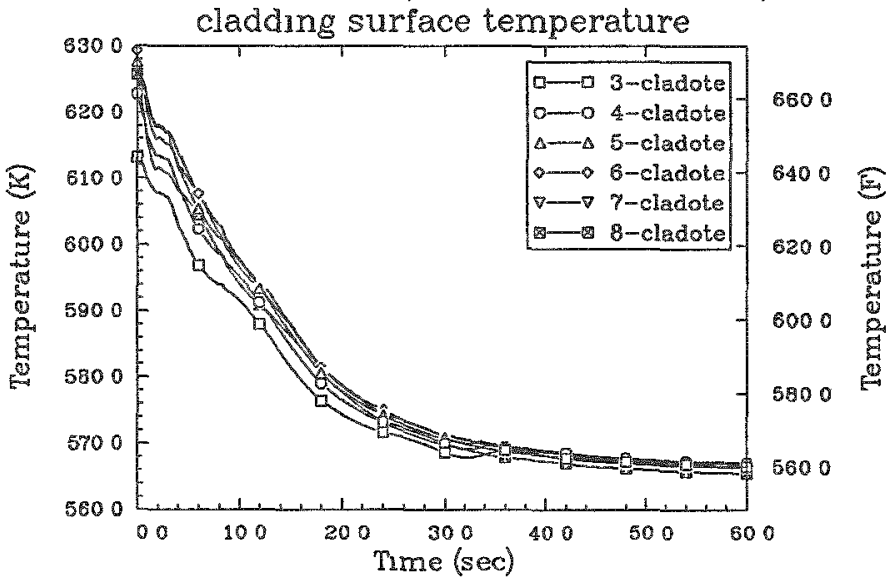

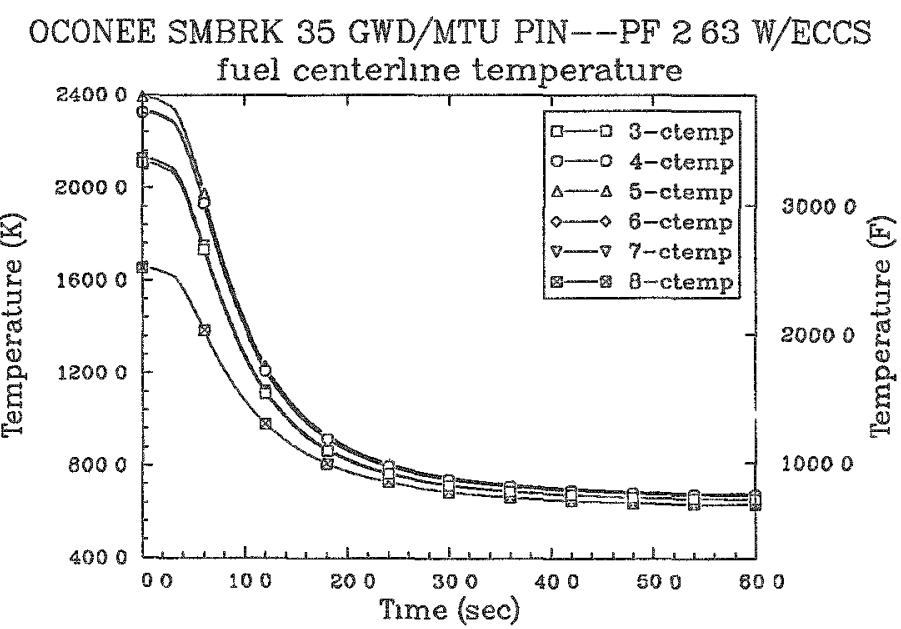

OCONEE SMBRK 35 GWD/MTU PIN--PF 263 W/ECCS

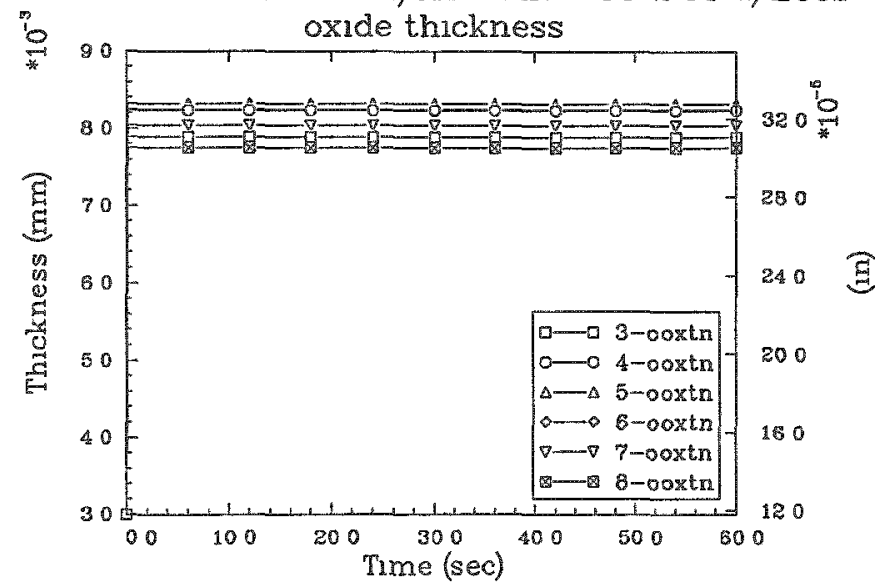


OCONEE SMBRK 20 GWD/MTU PIN-OPF 263 W/ECCS

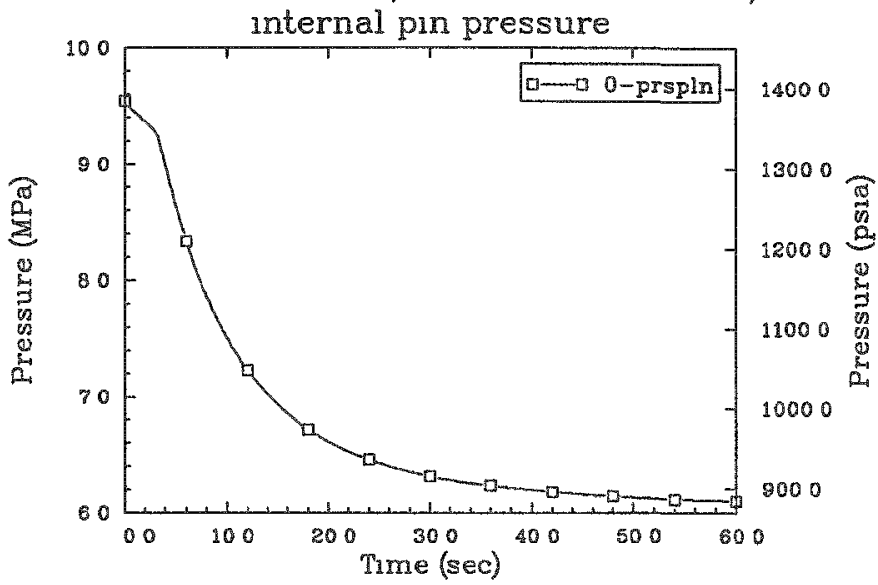

OCONEE SMBRK $20 \mathrm{GWD} / \mathrm{MTU}$ PIN--PF 263 W/ECCS

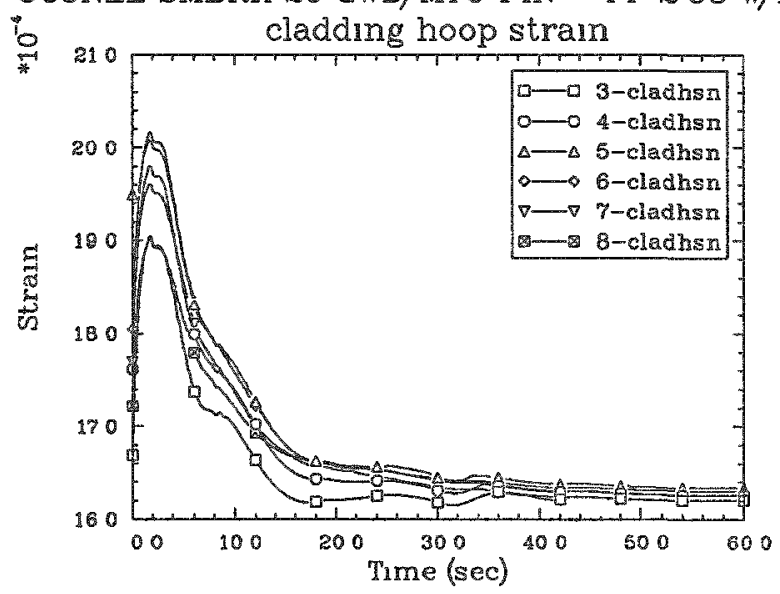

OCONEE SMBRK 20 GWD/MTU PIN--PF 263 W/ECCS

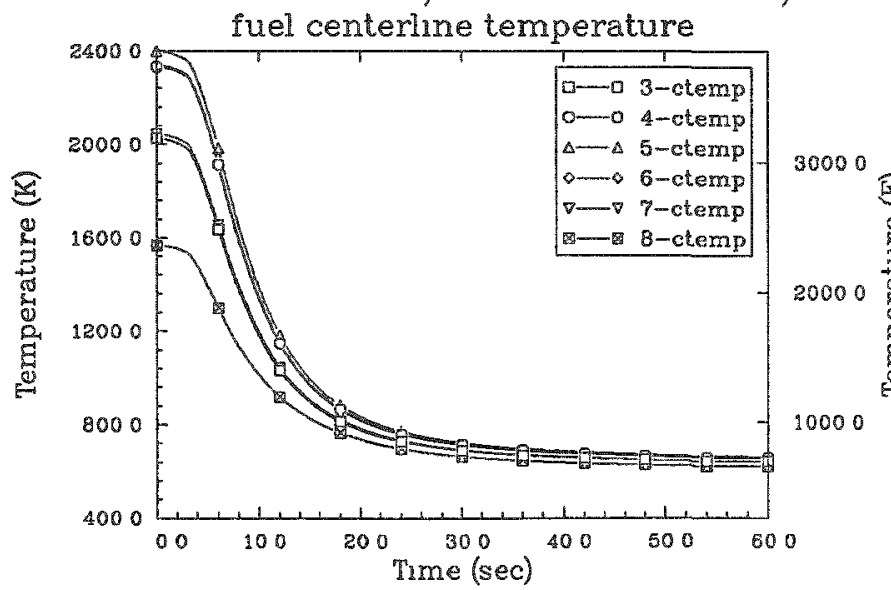

OCONEE SMBRK $20 \mathrm{GWD} / \mathrm{MTU}$ PIN--PF 263 W/ECCS fallure probability

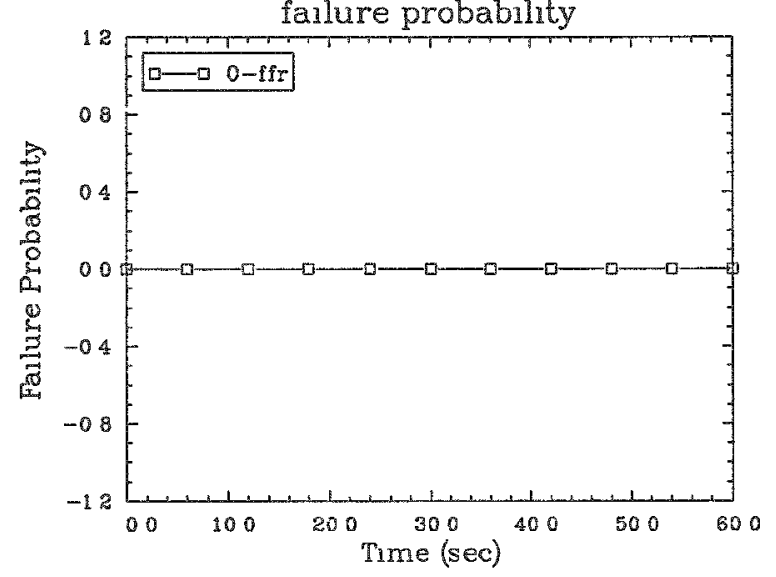

OCONEE SMBRK 20 GWD/MTU PIN--PF 263 W/ECCS

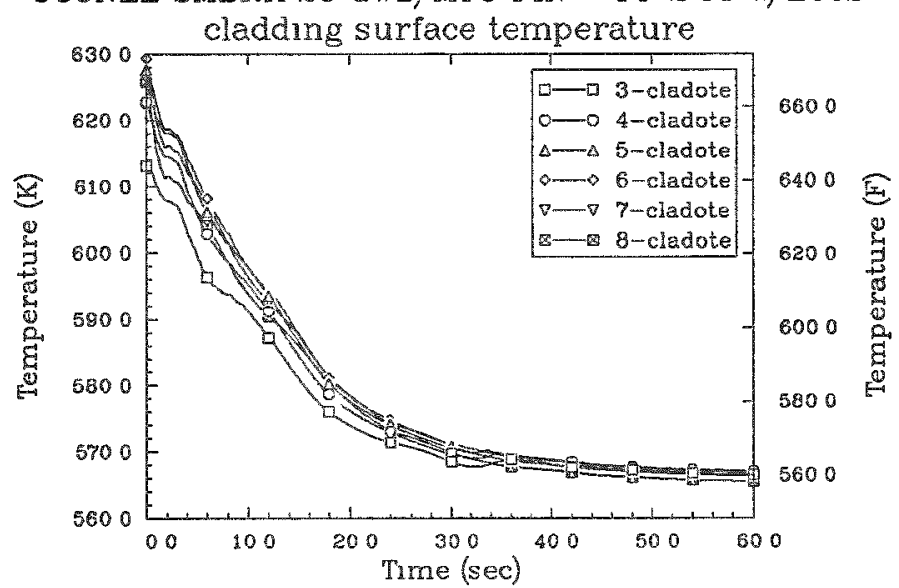

OCONEE SMBRK $20 \mathrm{GWD} / \mathrm{MTU}$ PIN--PF 263 W/ECCS

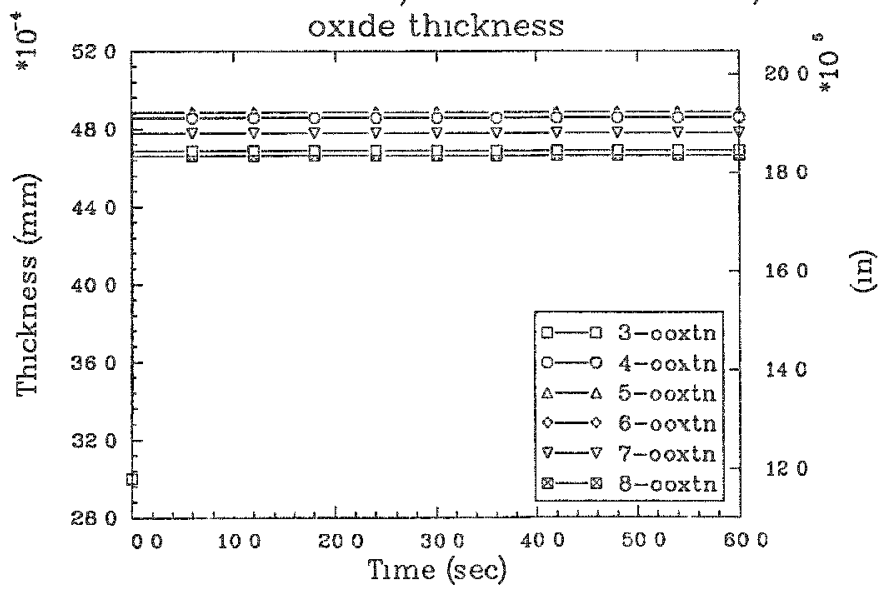


OCONEE SMBRK 5 GWD/MTU PIN--PF 263 W/ECCS

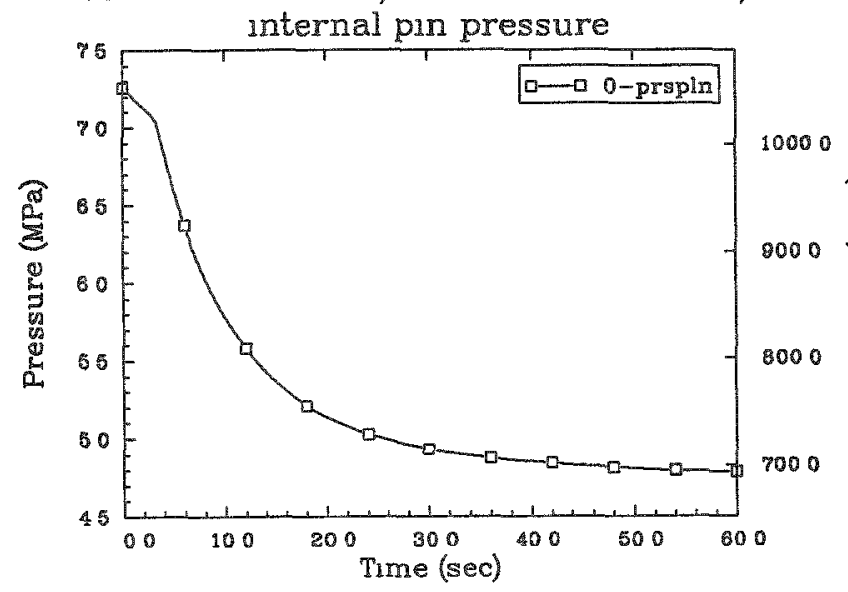

OCONEE SMBRK 5 GWD/MTU PIN--PF 263 W/ECCS

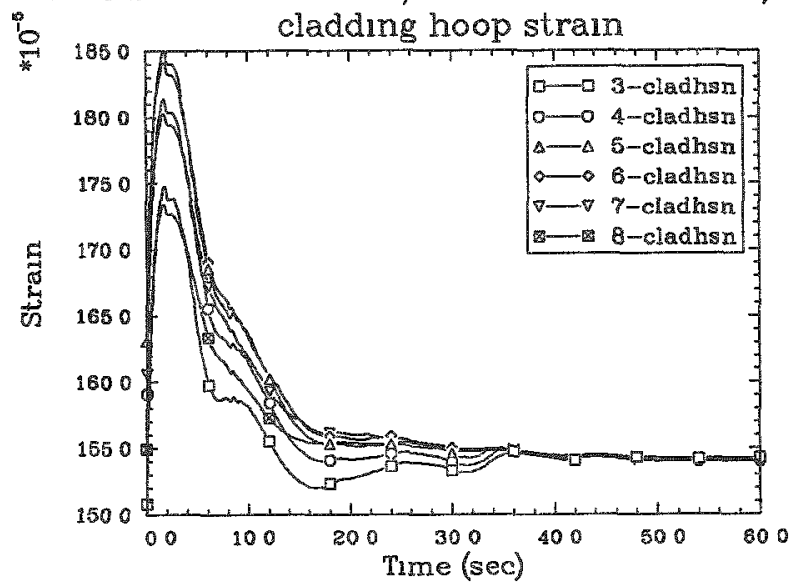

OCONEE SMBRK 5 GWD/MTU PIN--PF 263 W/ECCS

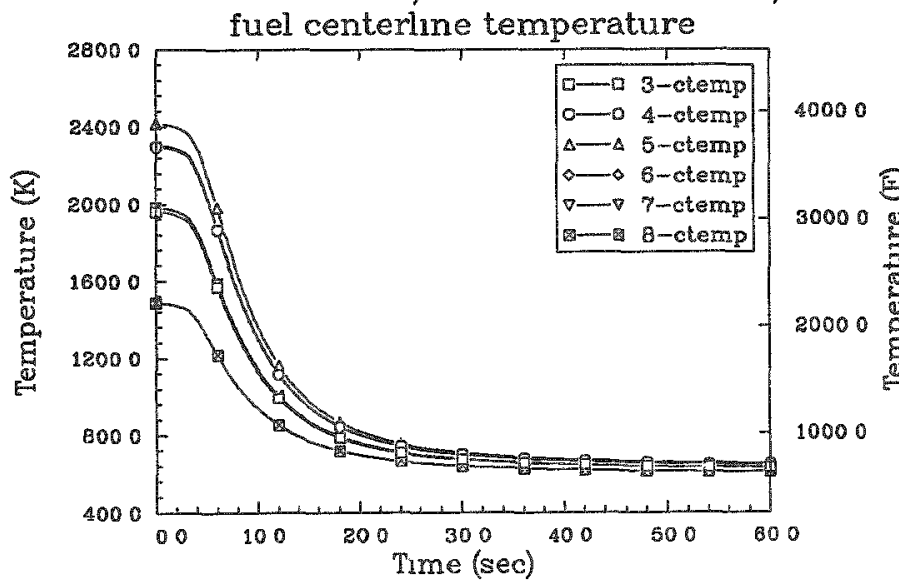

OCONEE SMBRK 5 GWD/MTU PIN--PF 2.63 W/ECCS failure probability

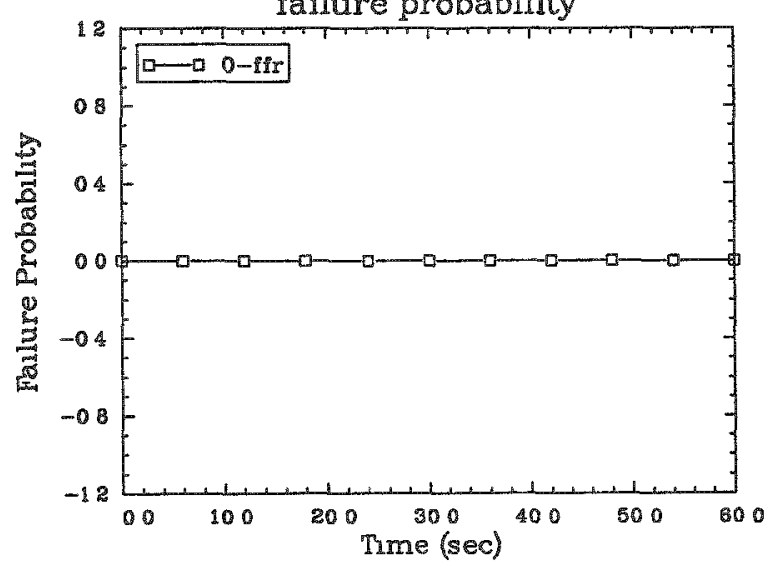

OCONEE SMBRK 5 GWD/MTU PIN--PF 263 W/ECCS

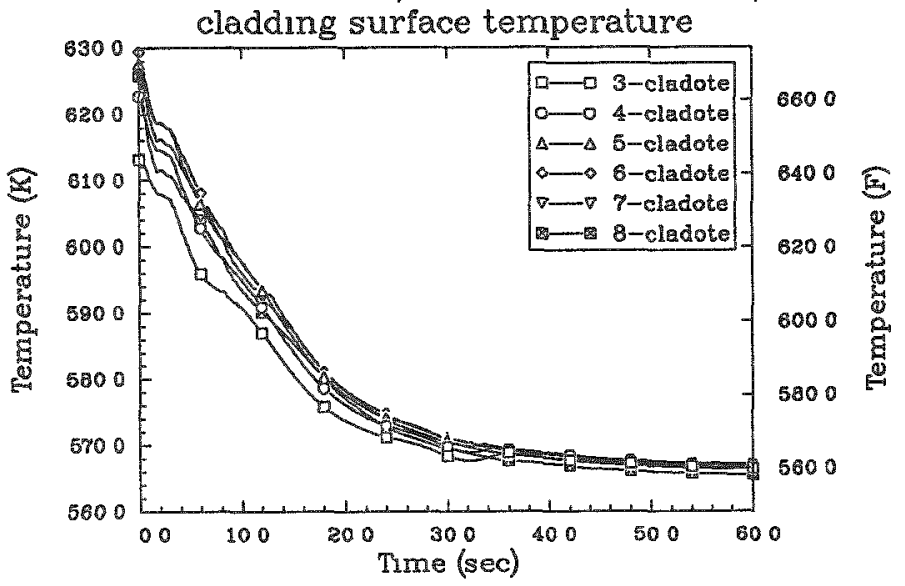

OCONEE SMBRK 5 GWD/MTU PIN--PF 263 W/ECCS

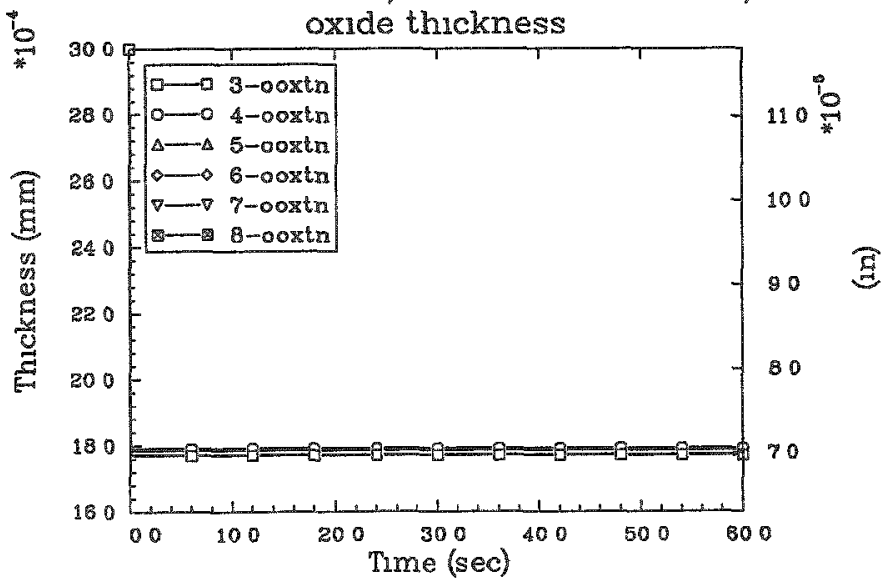


OCONEE 100\%DBA 55 GWD/MTU PIN--PF 2.63 (TRAC)

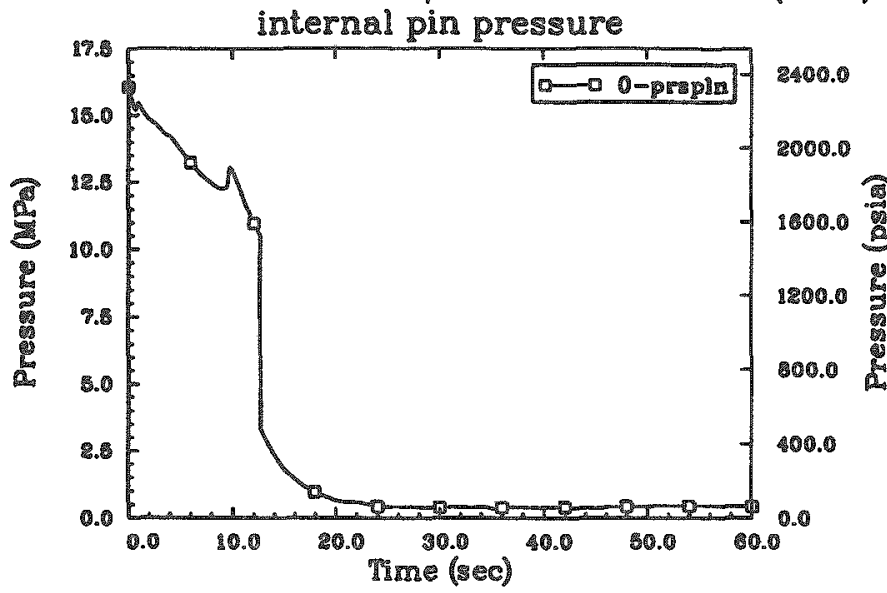

OCONEE 100\%DBA 55 GWD/MTU PIN--PF' 2.63 (TRAC)

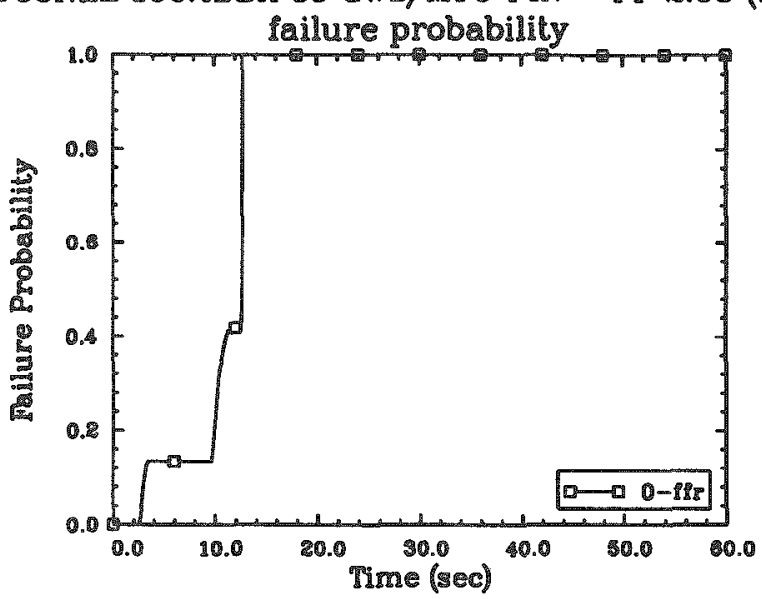

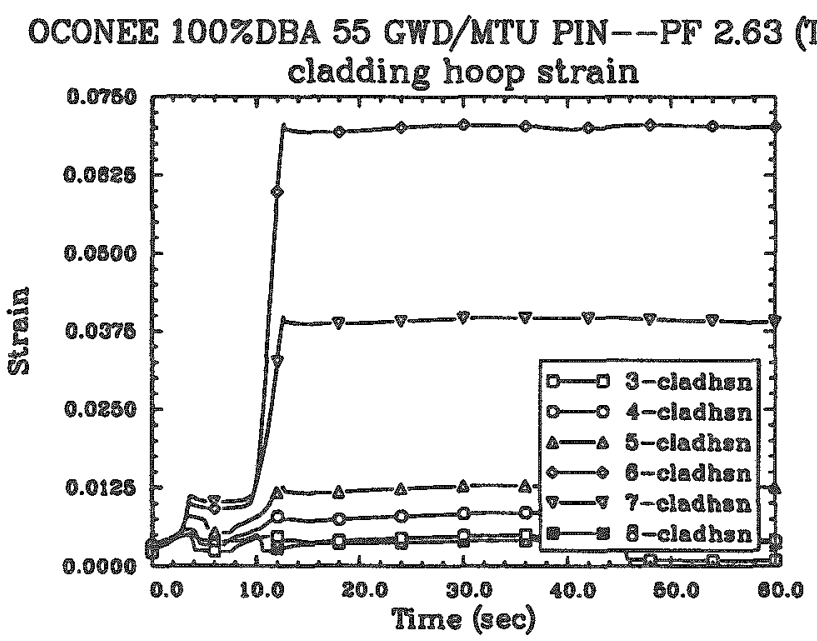

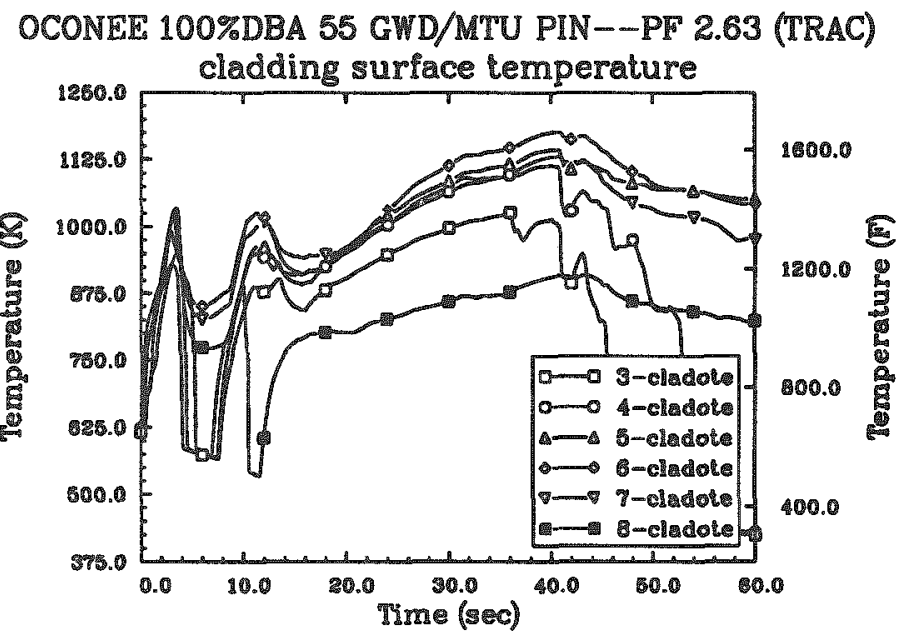

OCONEE 100\%DBA 55 GWD/MTU PIN--PF 2.63 (TRAC)

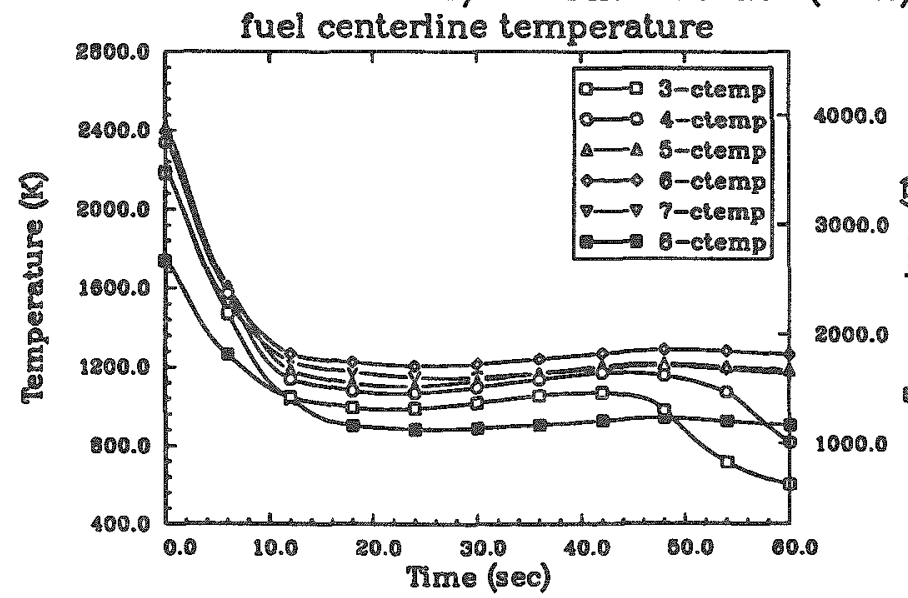

OCONEE 100\%DBA 55 GWD/MTU PIN--PF 2.63 (TRAC)

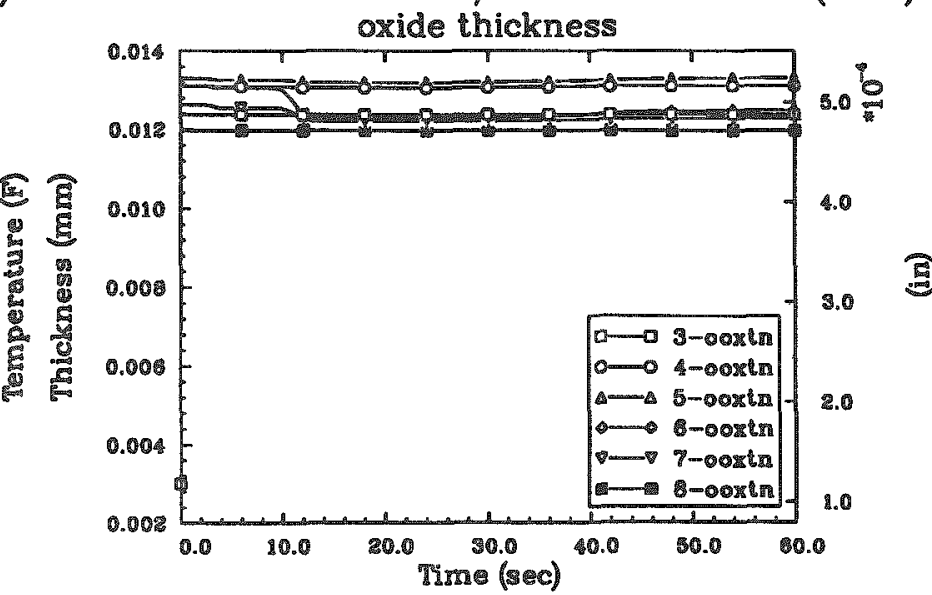


OCONEE 100\%DBA 35 GWD/MTU PIN--PF 2.63 (TRAC)

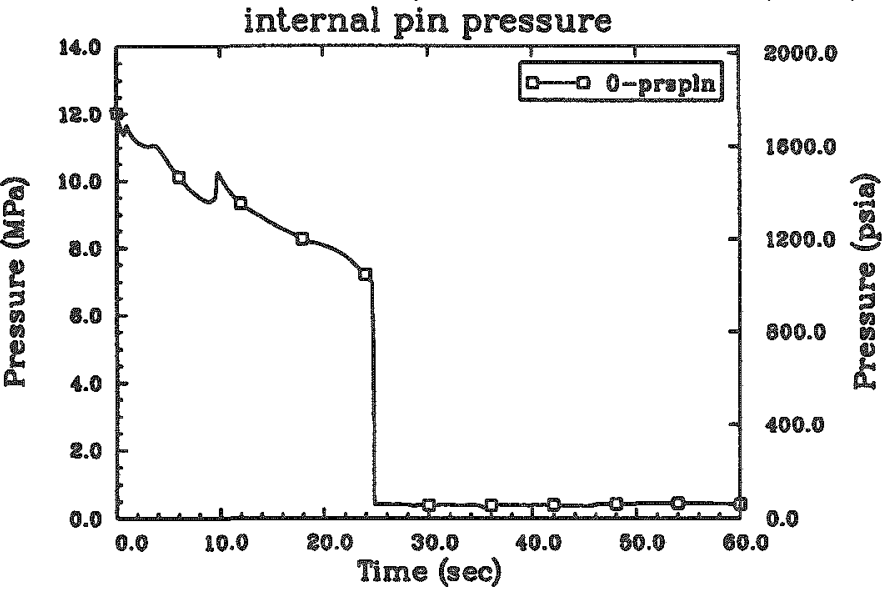

OCONEE 100\%DBA 35 GWD/MTU PIN--PF 2.63 (TRAC)

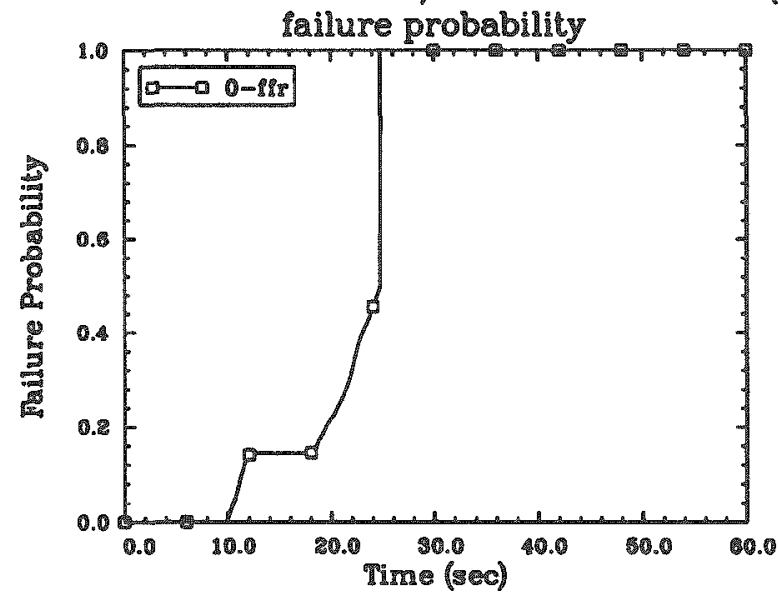

OCONEE 100\%DBA 35 GWD/MTU PIN--PF 2.63 (TRAC) cladding hoop strain

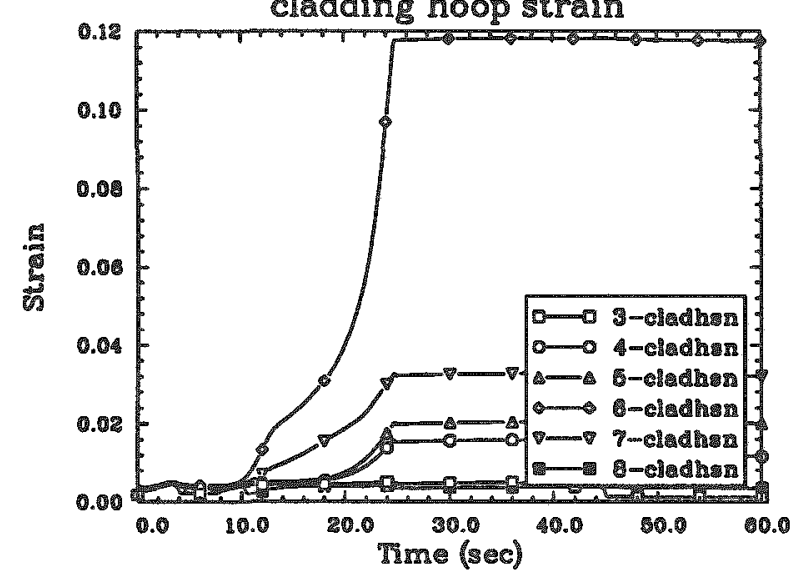

OCONEE 100\%DBA 35 GWD/MTU PIN--PF 2.63 (TRAC)

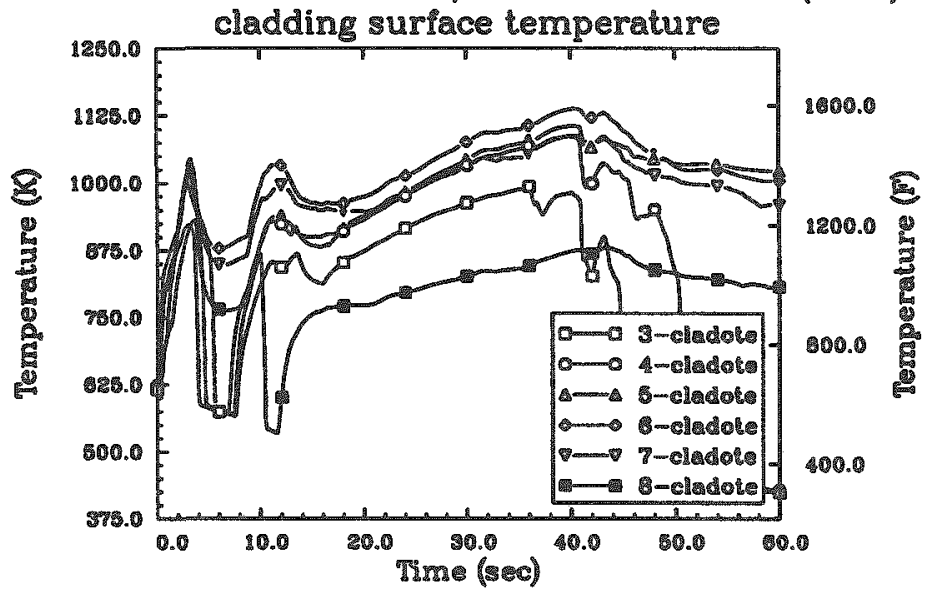

OCONEE 100\%DBA 35 GWD/MTU PIN--PF 2.63 (TRAC)

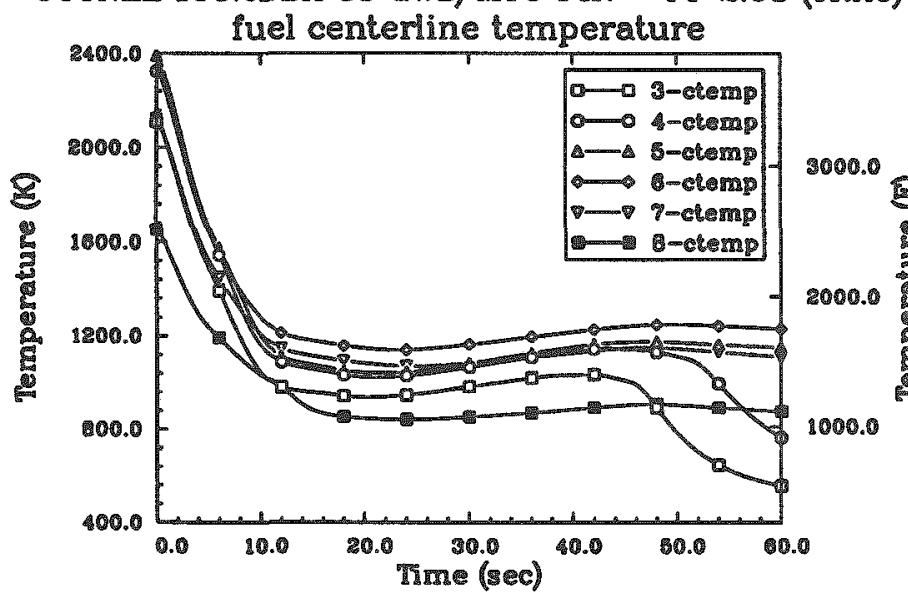

OCONEE 100\%DBA 35 GWD/MTU PIN-DF 2.63 (TRAC)

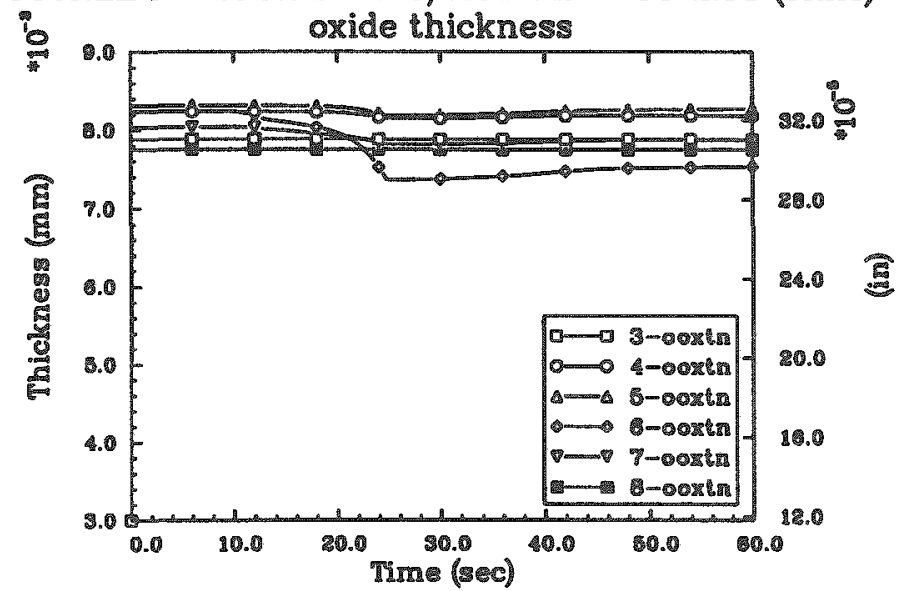


OCONEE 100\%DBA 20 GWD/MTU PIN--PF 2.83 (TRAC)

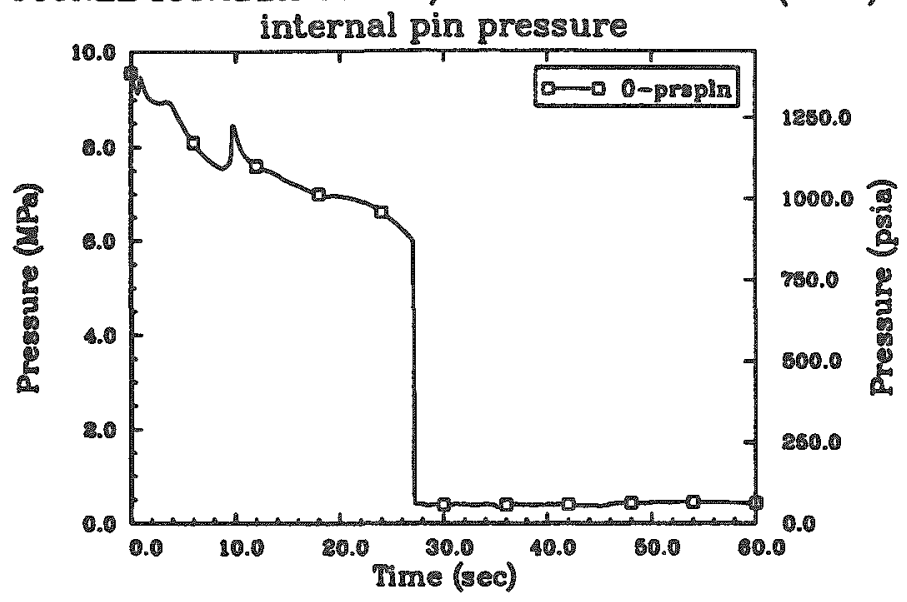

OCONEE 100\%DBA $20 \mathrm{GWD}$ MTU PIN--PF 2.63 (TRAC)

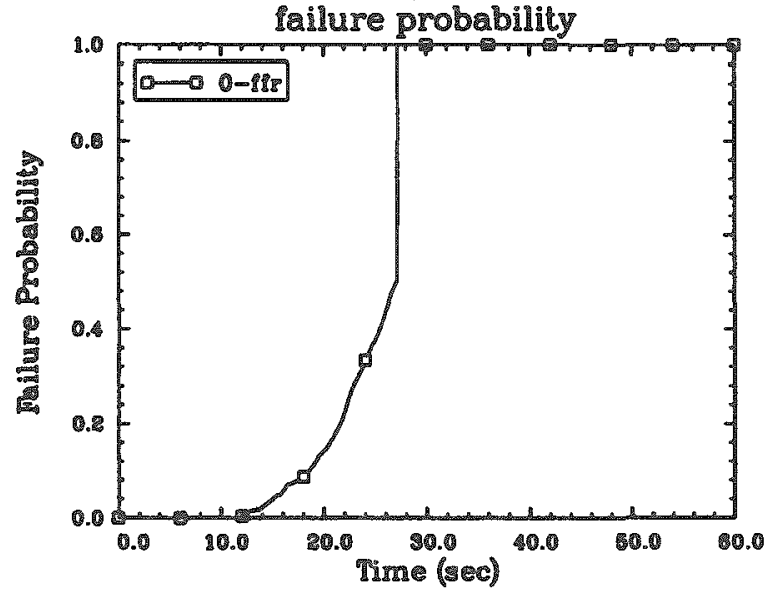

OCONEE 100\%DBA 20 GWD/MTU PIN--PF 2.63 (TRAC)

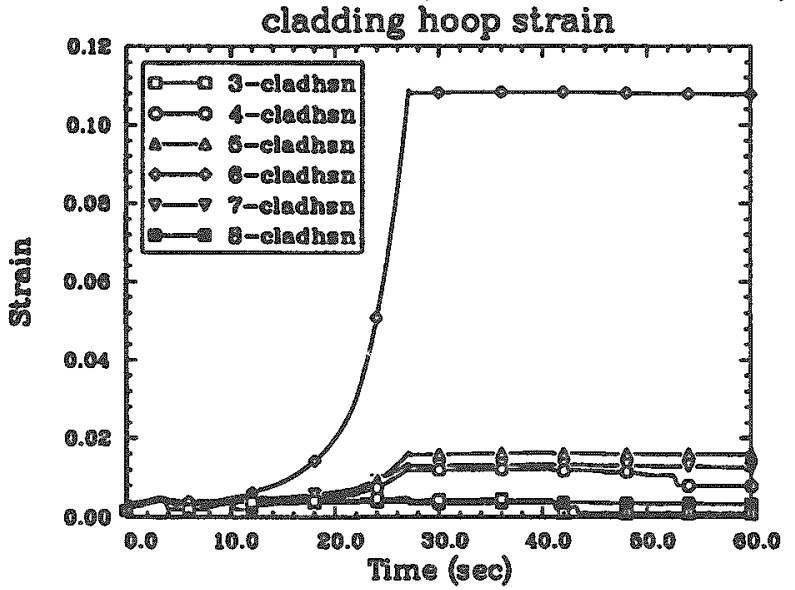

OCONEE 100\%DBA $20 \mathrm{GWD/MTU}$ PIN--PF 2.63 (TRAC)

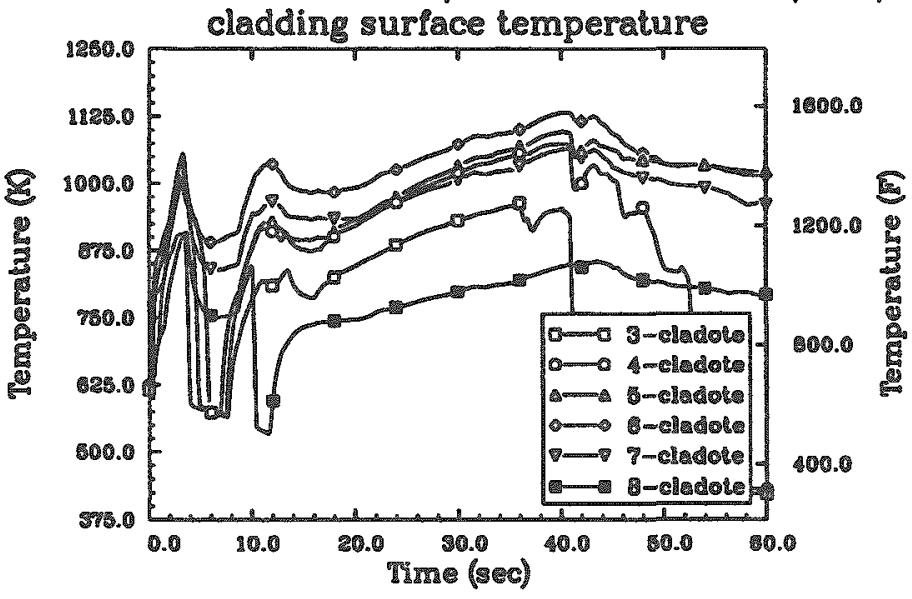

OCONEE 100\%DBA 20 GWD/MTU PIN--PF 2.63 (TRAC)

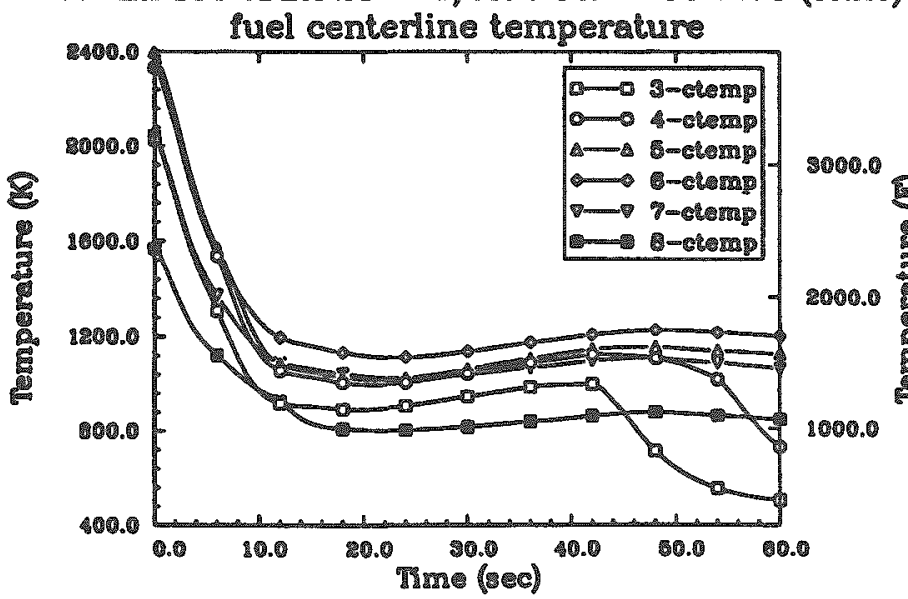

OCONEE 100\%DBA 20 GWD/MTU PIN-DF 2.63 (TRAC) b oxide thickness

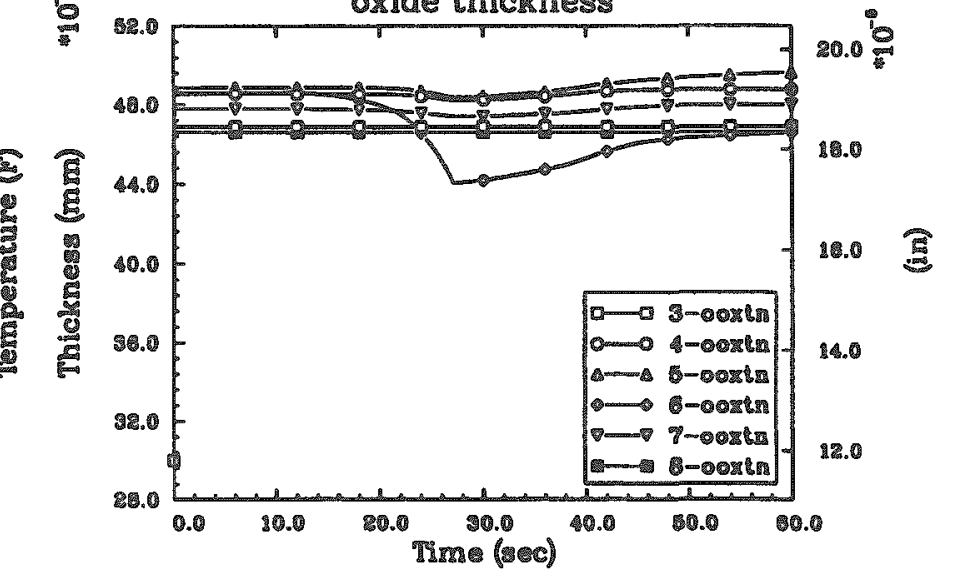


OCONEE 100\%DBA 5 GWD/MTU PIN--PF 2.63 (TRAC)

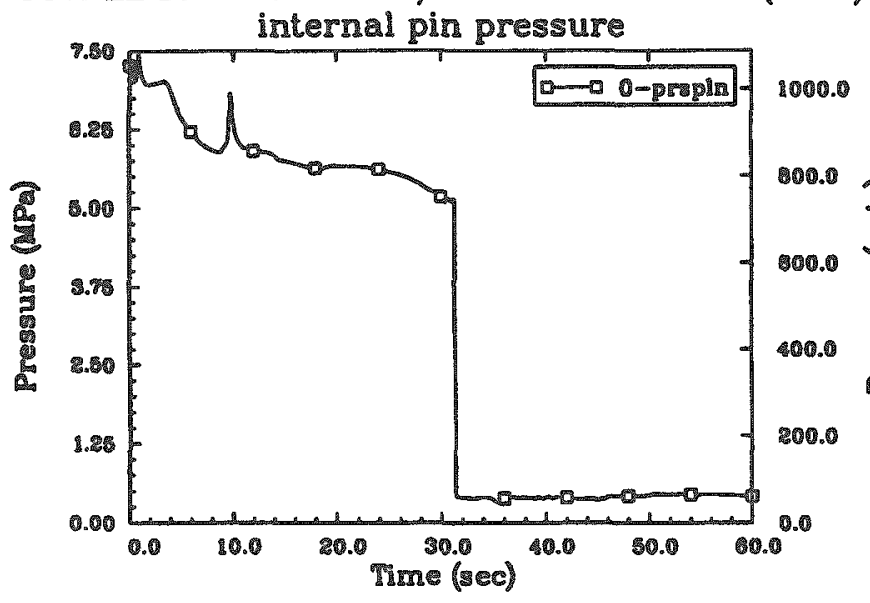

OCONEE 100\%DBA 5 GWD/MTU PIN--PF 2.63 (TRAC)

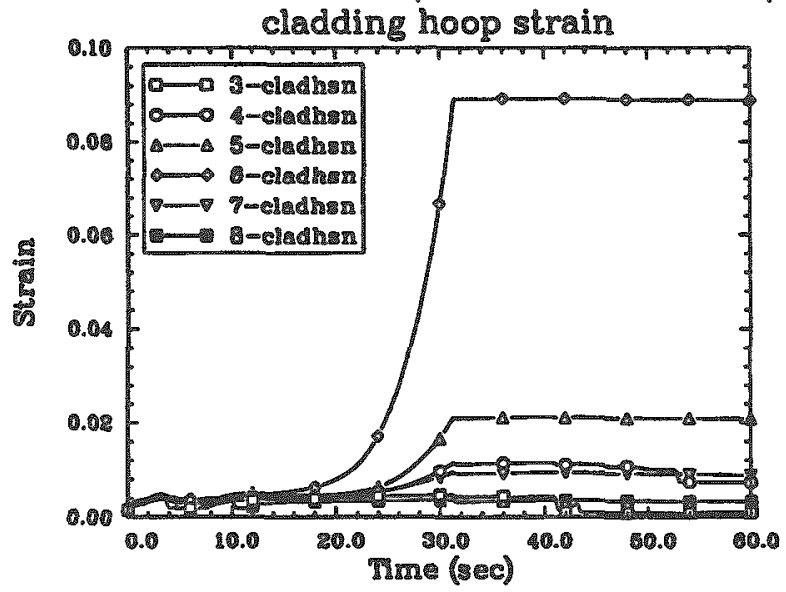

OCONEE 100\%DBA 5 GWD/MTU PIN--PF 2.63 (TRAC)

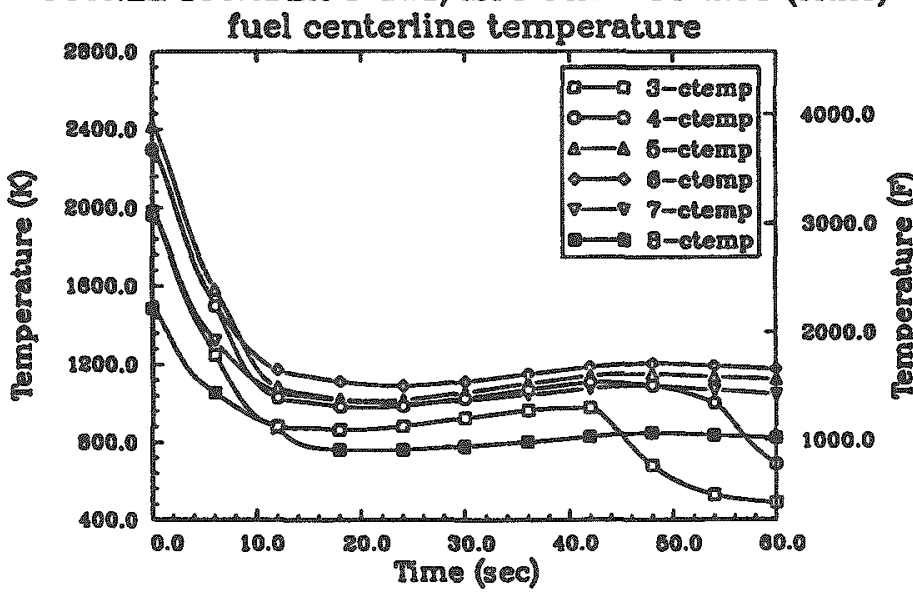

OCONEE 100\%DBA 5 GWD/MTU PIN--PF 2.63 (TRAC) failure probability

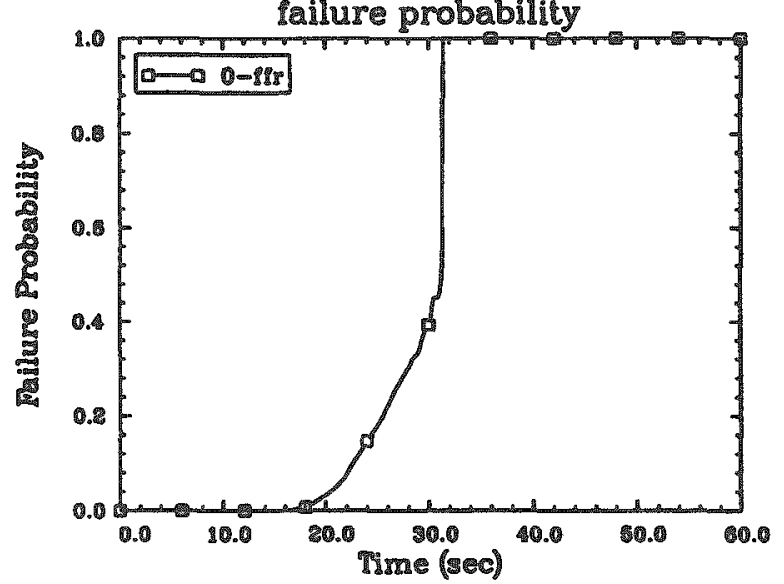

OCONEE 100\%DBA 5 GWD/MTU PIN--PF 2.63 (TRAC)

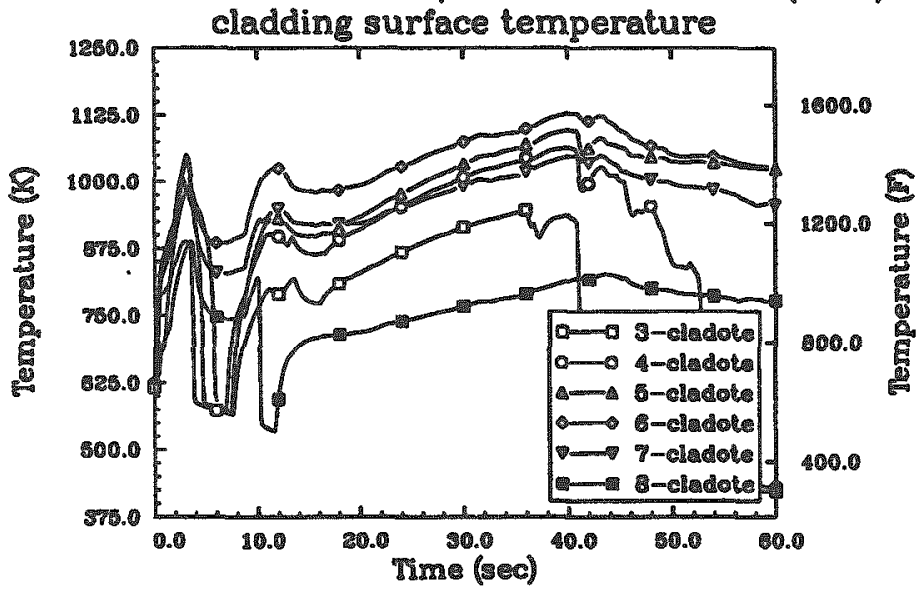

OCONEE 100\%DBA 5 GWD/MTU PIN--PF 2.63 (TRAC)

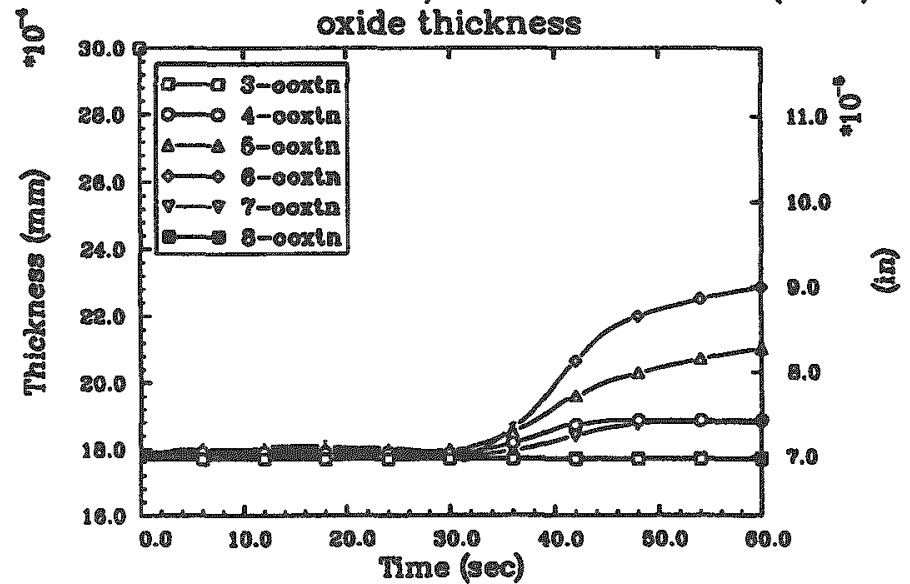


OCONEE 100\%DBA 55 GWD/TU PIN--PF 2.4 (TRAC)

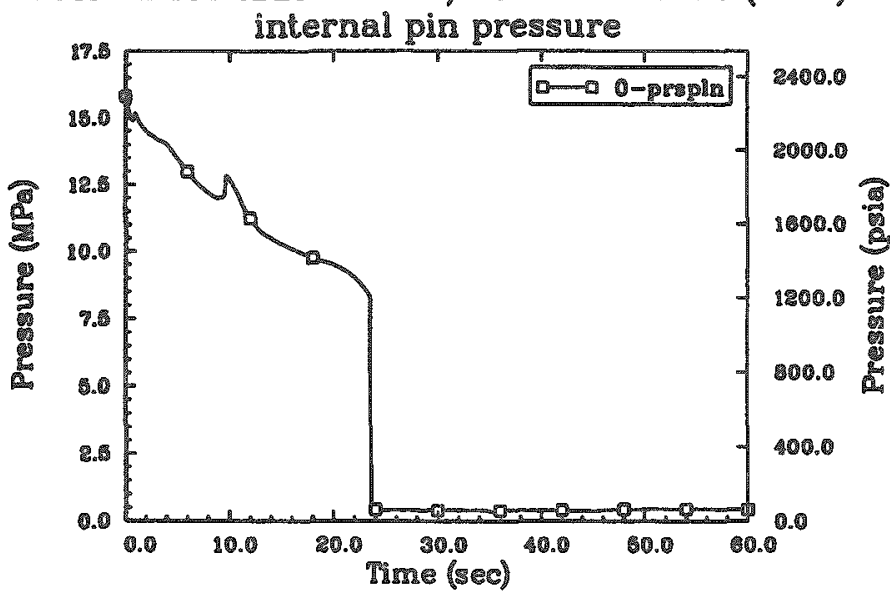

OCONEE 100\%DBA 55 GWD/TU PIN--PF 2.4 (TRAC) cladding hoop strain

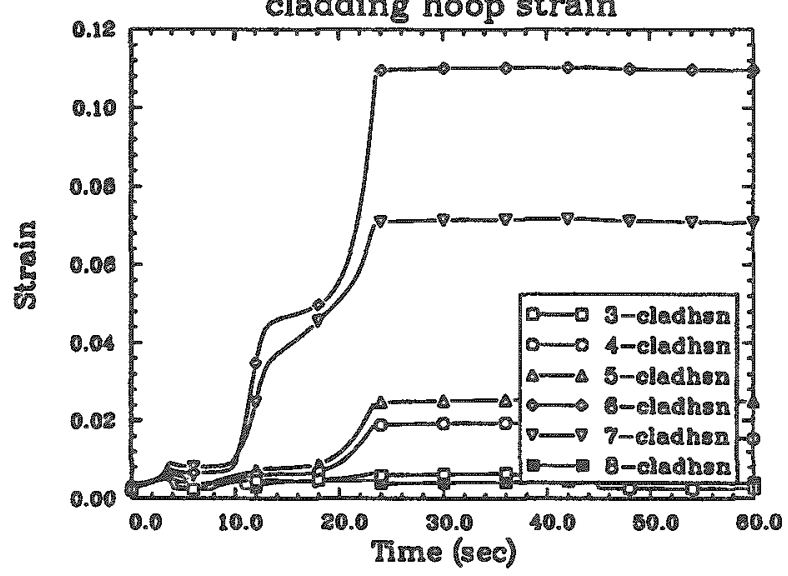

OCONEE 100\%DBA 55 GWD/TU PIN--PF 2.4 (TRAC)

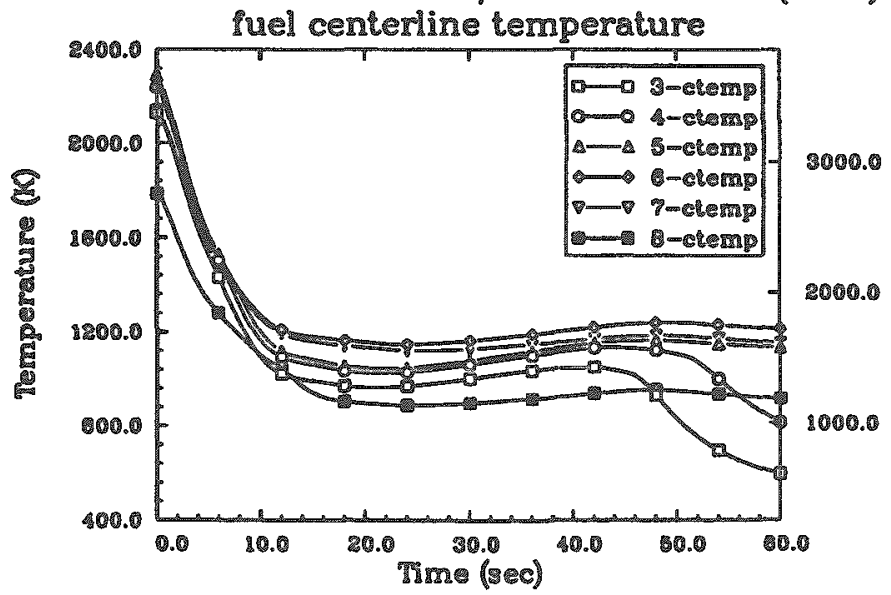

OCONEE 100\%DBA 55 GWD/TU PIN--PF 2.4 (TRAC) failure probability

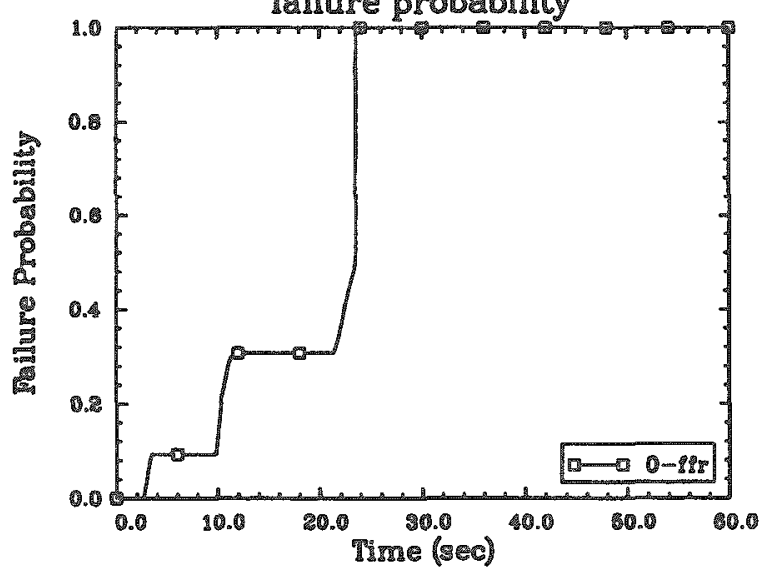

OCONEE 100\%DBA 55 GWD/TU PIN--PF 2.4 (TRAC)

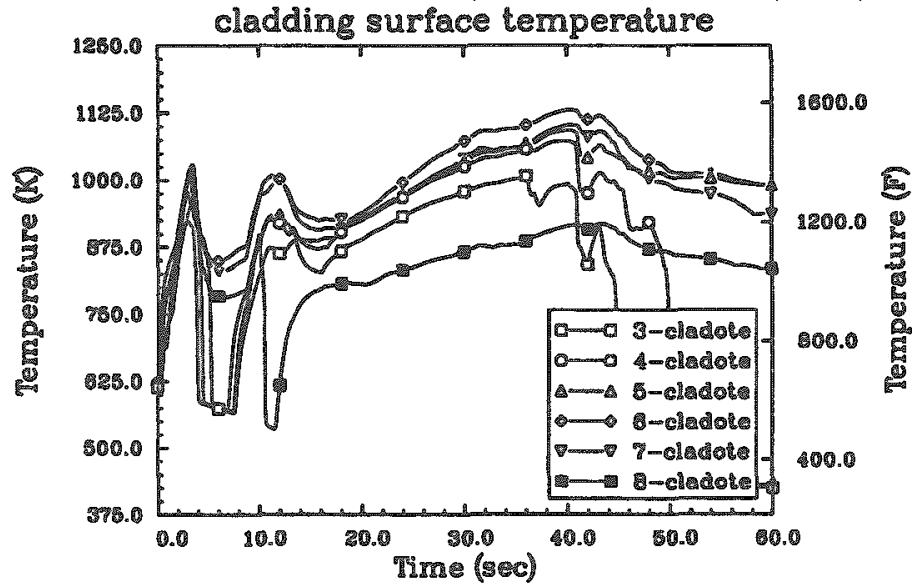

OCONEE 100\%DBA 55 GWD/TU PIN--PF 2.4 (TRAC)

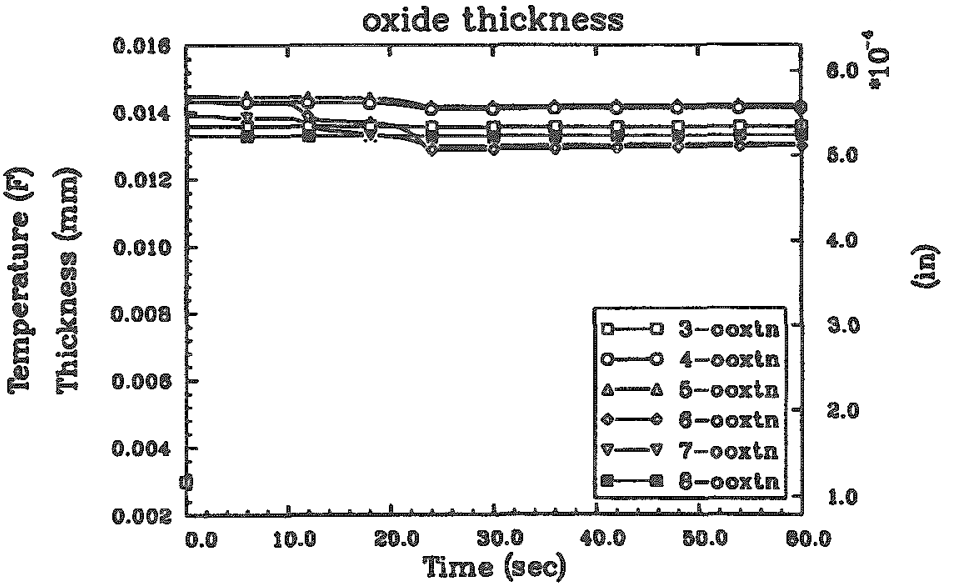


OCONEE 100\%DBA 35 GWD/MTU PIN-DF 2.4 (TRAC)

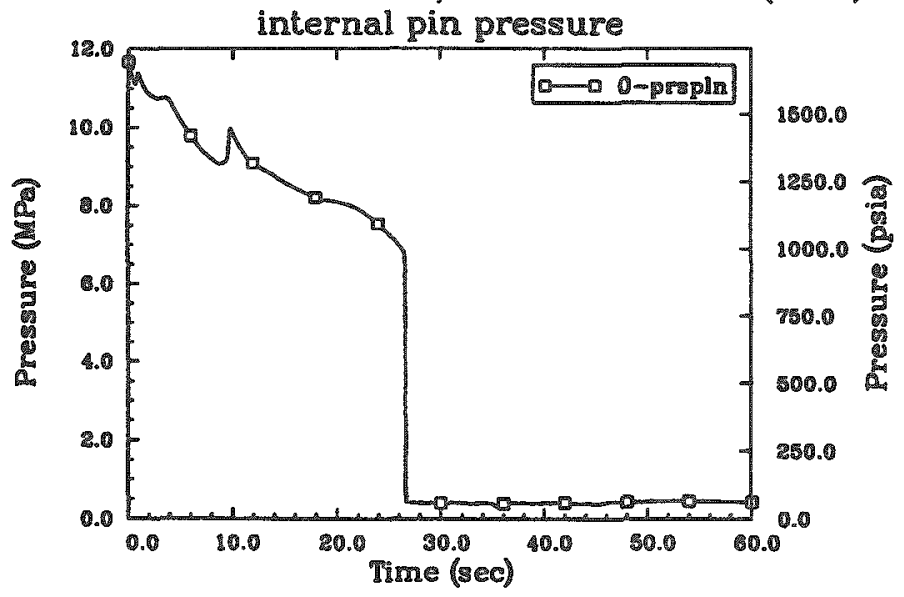

OCONEE 100\%DBA 35 GWD/MTU PIN--PF 2.4 (TRAC)

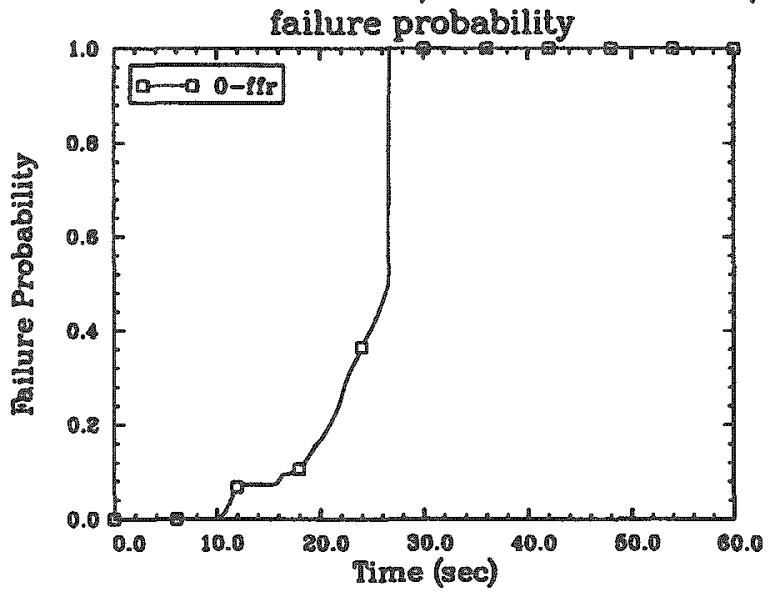

OCONEE 100\%DBA 35 GWD/MTU PIN--PF 2.4 (TRAC)

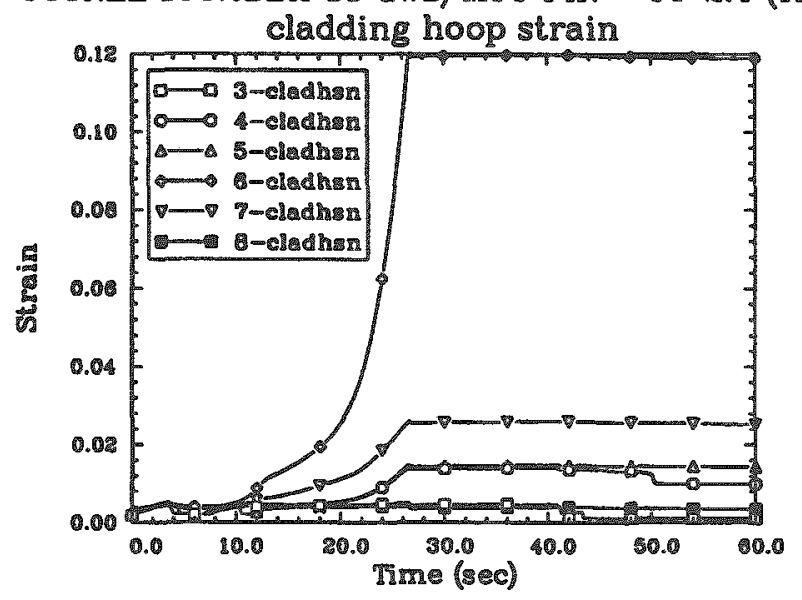

OCONEE 100\%DBA 35 GWD/MTU PIN--PF 2.4 (TRAC)

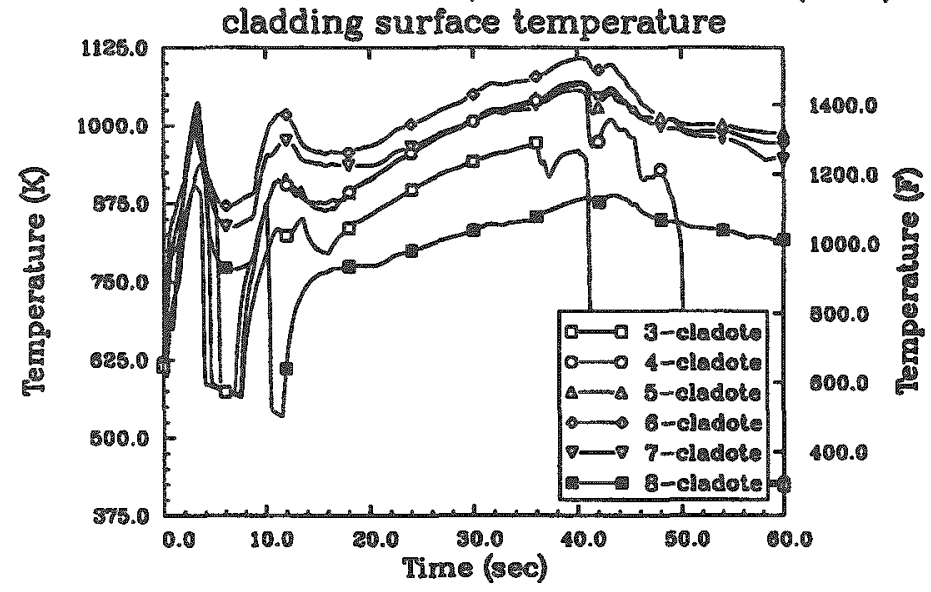

OCONEE 100\%DBA 35 GWD/MTU PIN-DF 2.4 (TRAC)

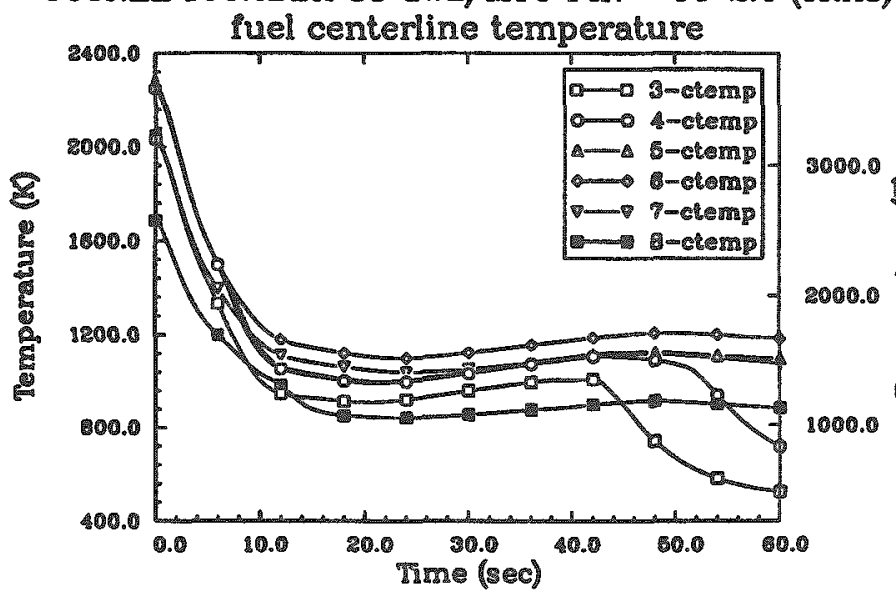

OCONEE 100\%DBA 35 GWD/MTU PIN-DP 2.4 (TRAC)

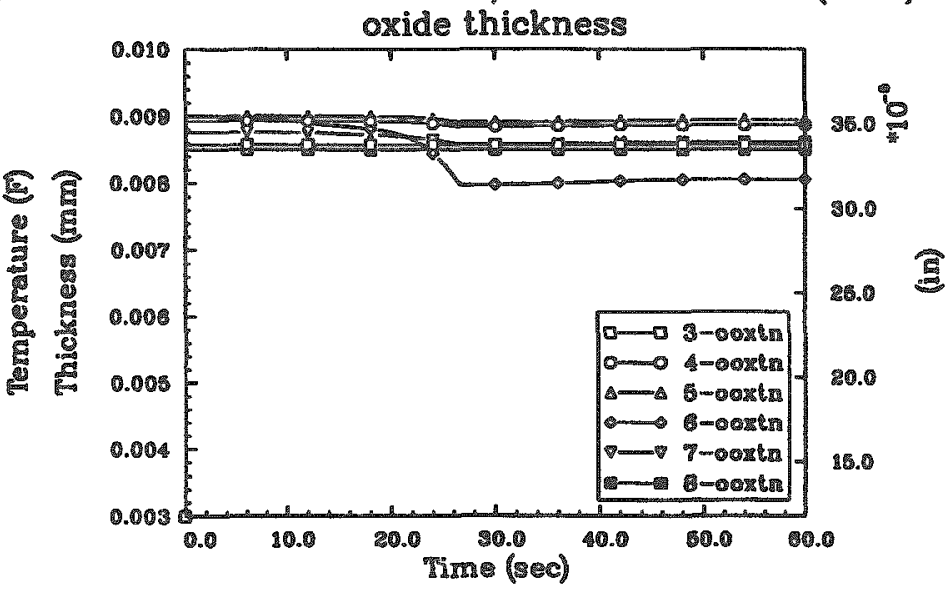


OCONEE 100\%DBA $20 \mathrm{GWD} / \mathrm{MTU}$ PIN--PF 2.4 (TRAC)

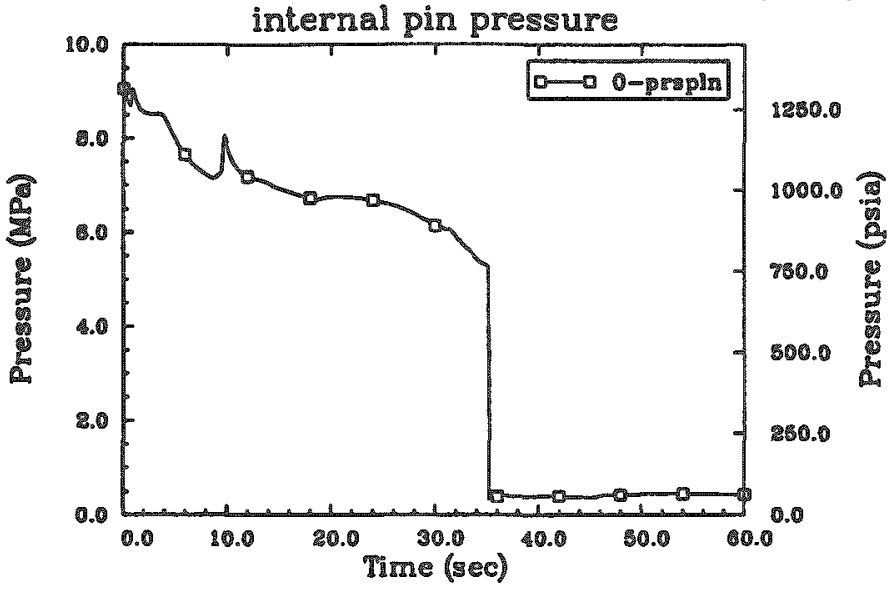

OCONEE 100\%DBA $20 \mathrm{GWD} / \mathrm{MTU}$ PIN-PF 2.4 (TRAC) failure probability

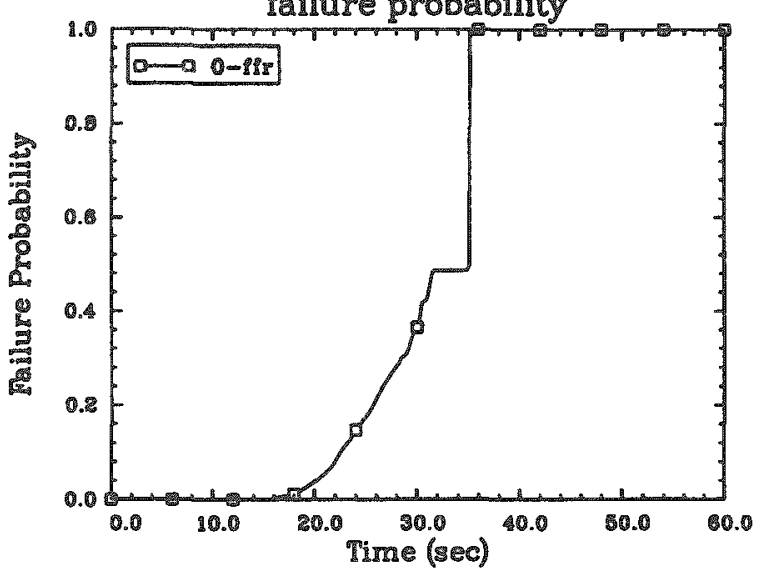

OCONEE 100\%DBA 20 GWD/MTU PIN--PF 2.4 (TRAC)

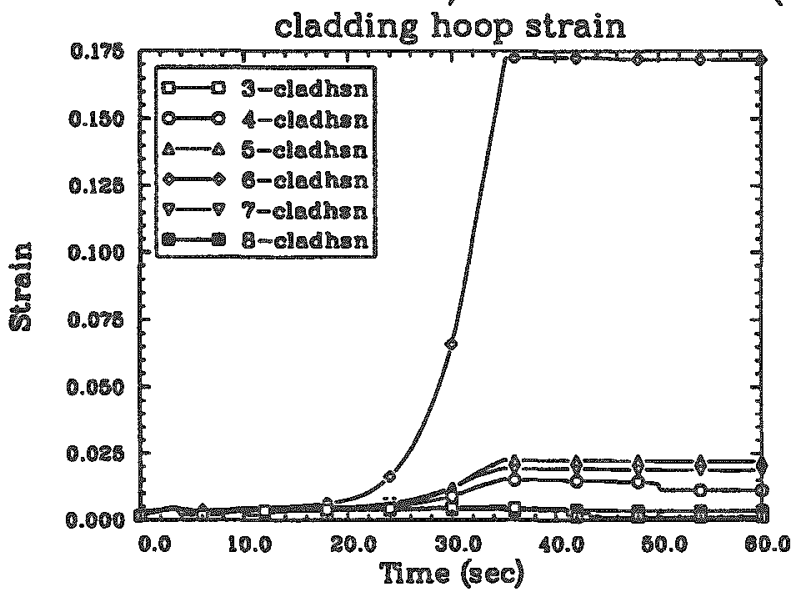

OCONEE 100\%DBA 20 GWD/MTU PIN-DF 2.4 (TRAC)

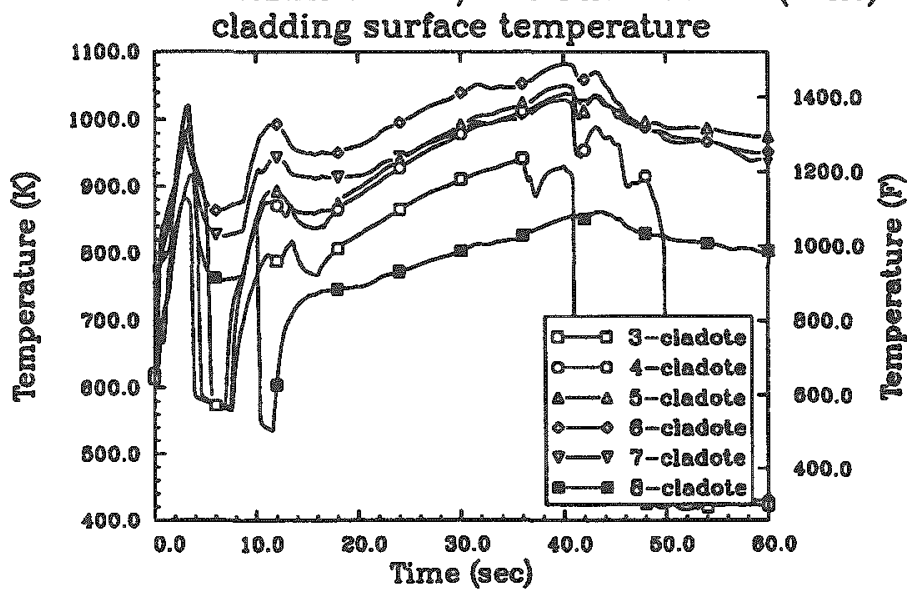

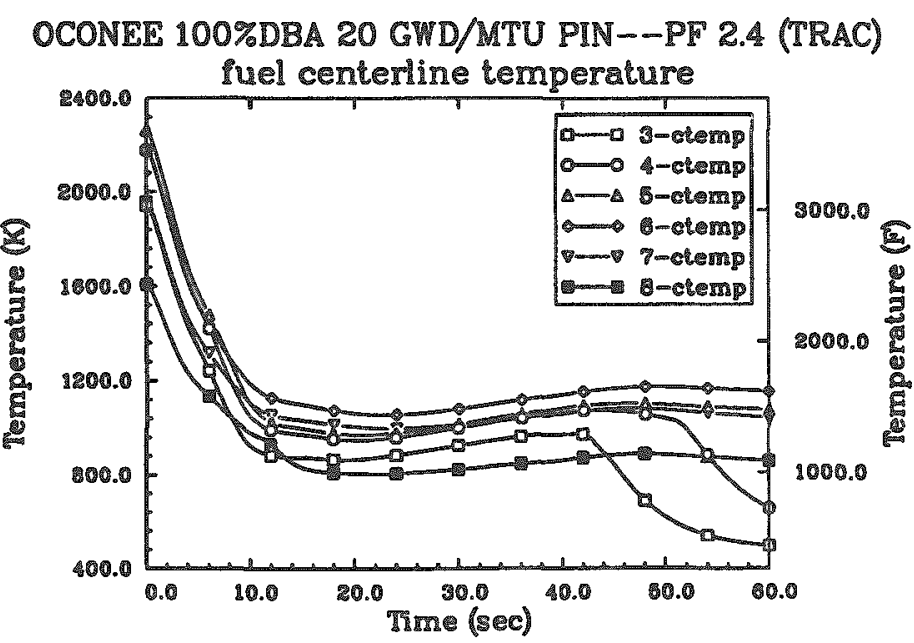

OCONEE 100\%DBA $20 \mathrm{GWD} / \mathrm{MTU}$ PIN--PF 2.4 (TRAC)

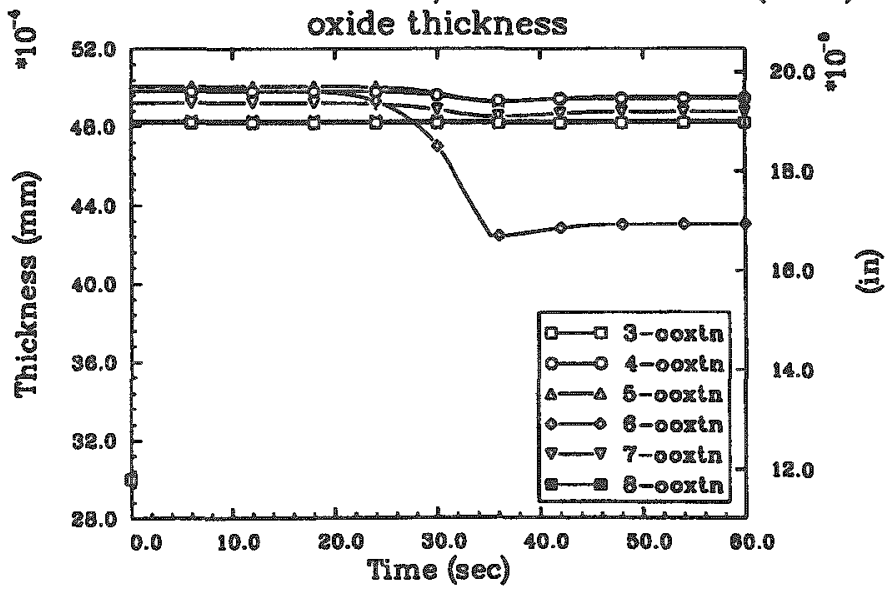



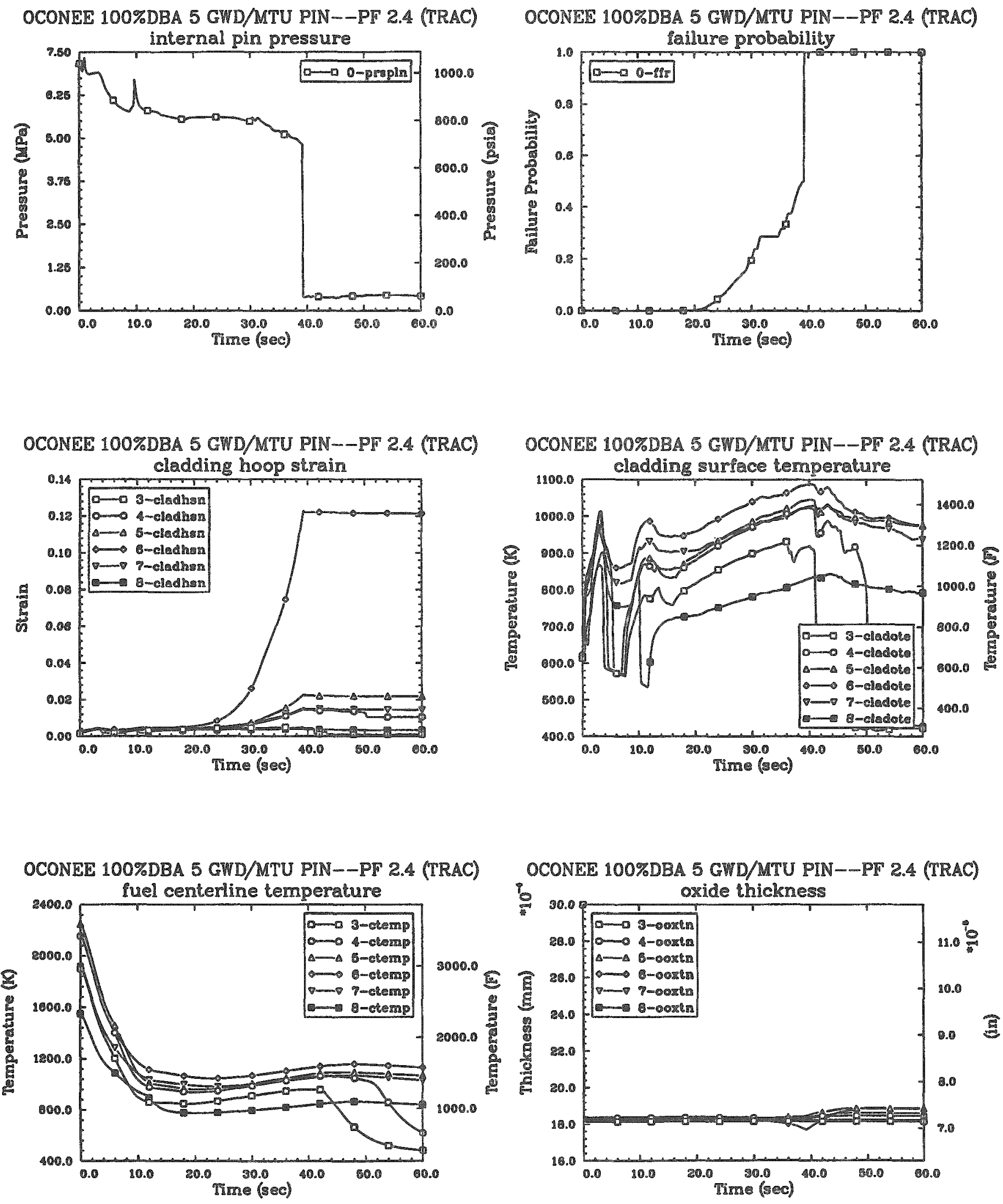

OCONEE 100\%DBA 5 GWD/MTU PIN-DP 2.4 (TRAC)

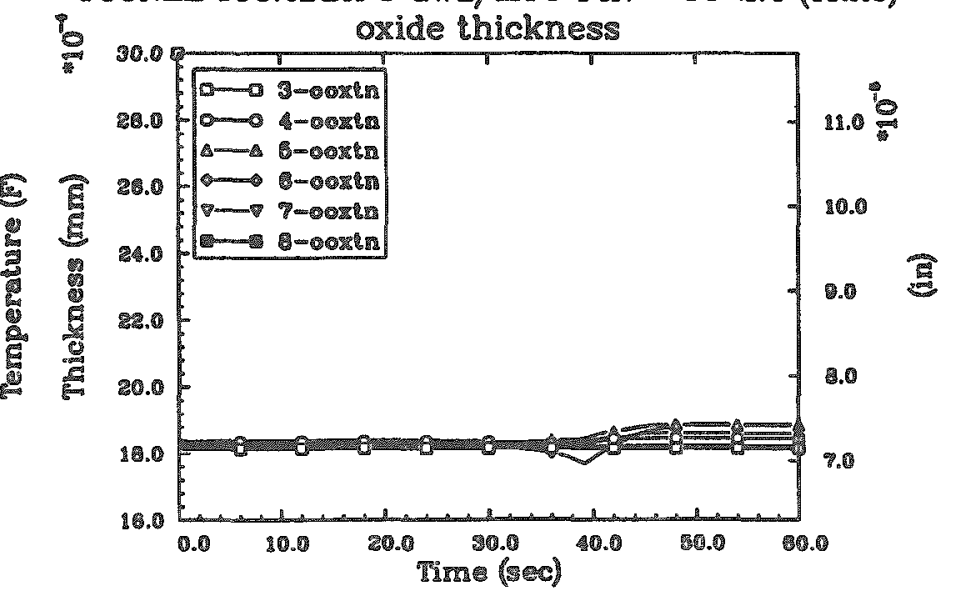


OCONEE 100\%DBA 55 GWD/MTU PIN--PF 2.2 (TRAC)

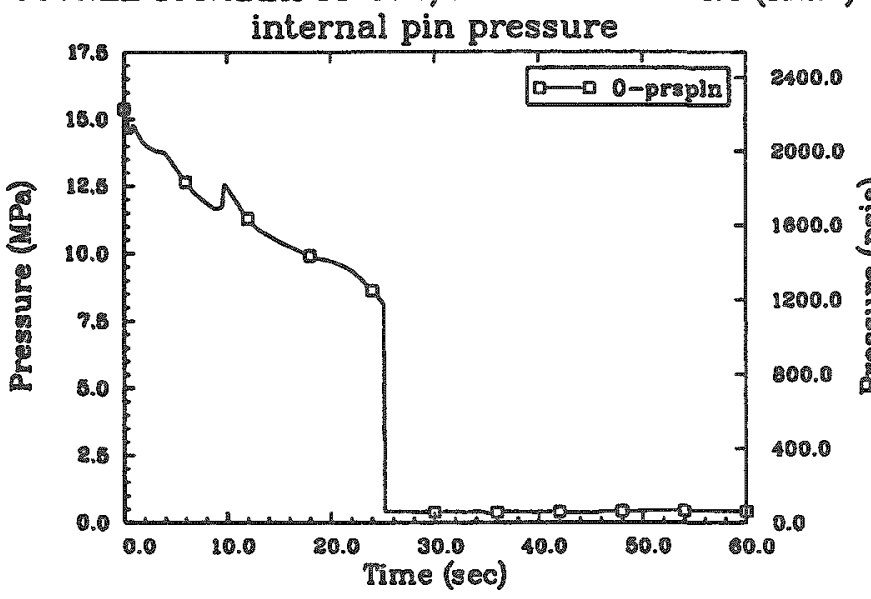

OCONEE 100\%DBA 55 GWD/MTU PIN-PF 2.2 (TRAC)

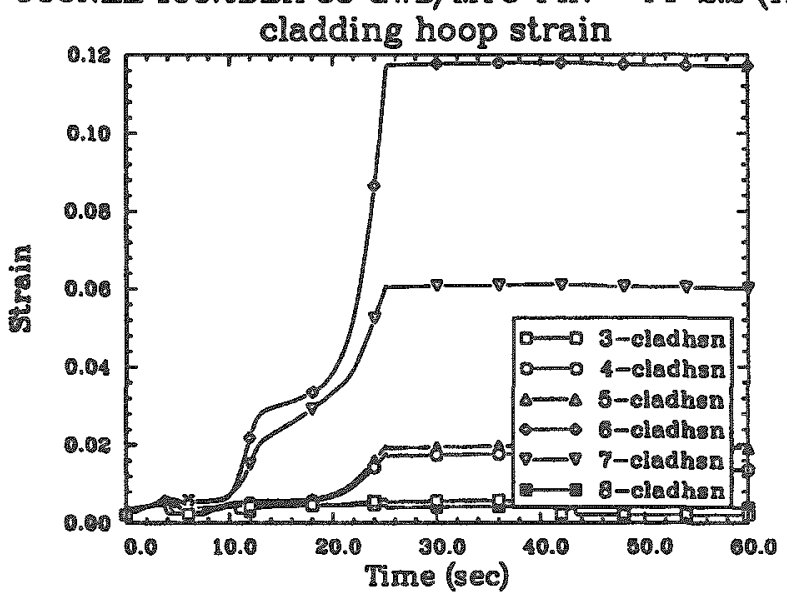

OCONEE 100\%DBA 55 GWD/MTU PIN--PF 2.2 (TRAC) failure probability

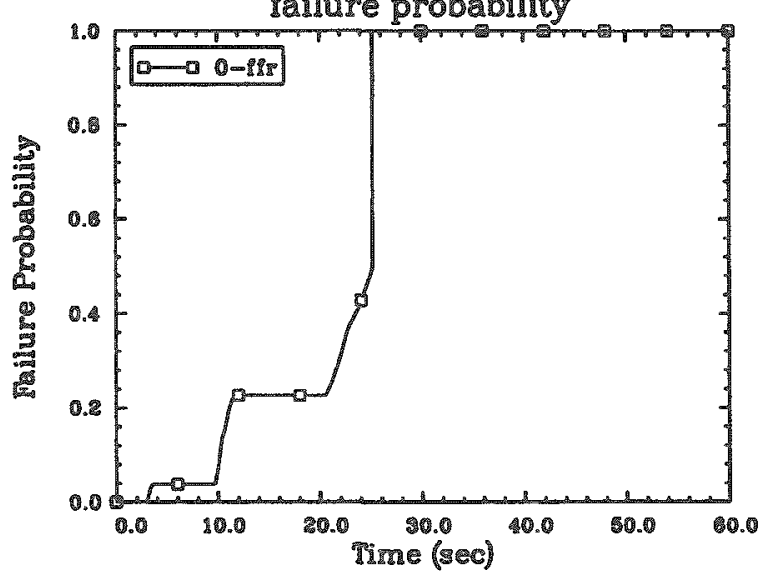

OCONEE 100\%DBA 55 GWD/MTU PIN--PF 2.2 (TRAC)
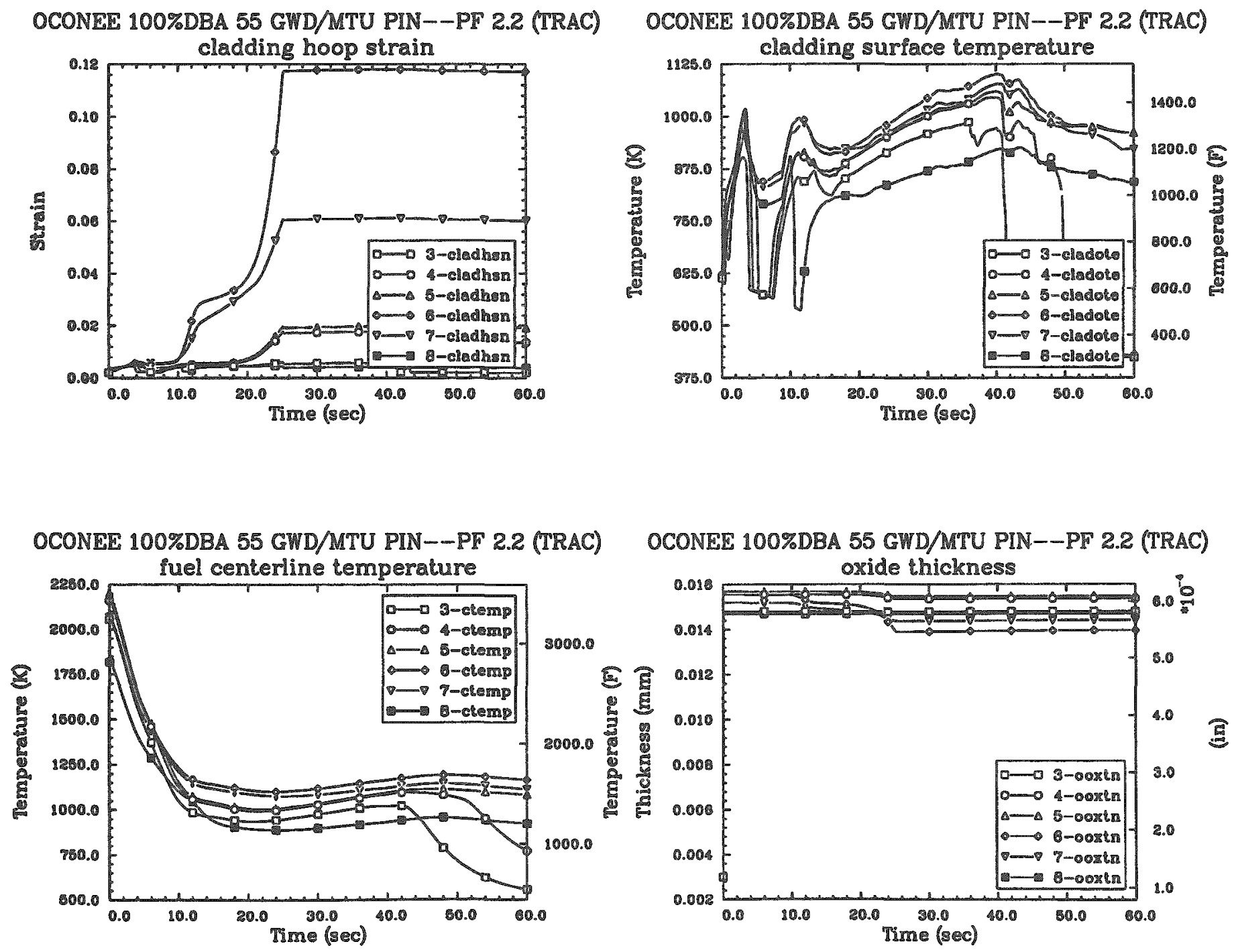

OCONEE 100\%DBA 55 GWD/MTU PIN--PF 2.2 (TRAC)

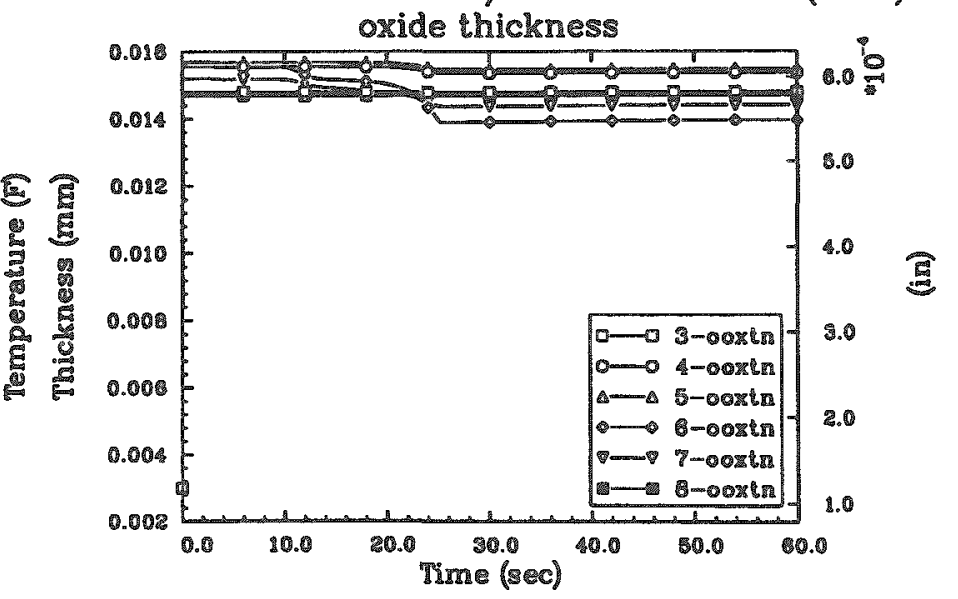


OCONEE 100\%DBA 35 GWD/MTU PIN--PF 2.2 (TRAC)

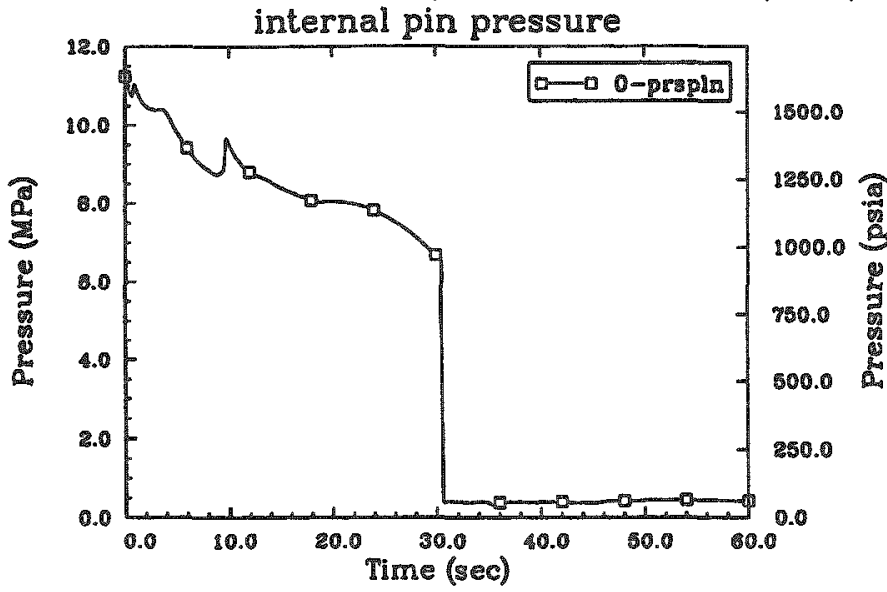

OCONEE 100\%DBA 35 GWD/MTU PIN--PF 2.2 (TRAC) failure probability

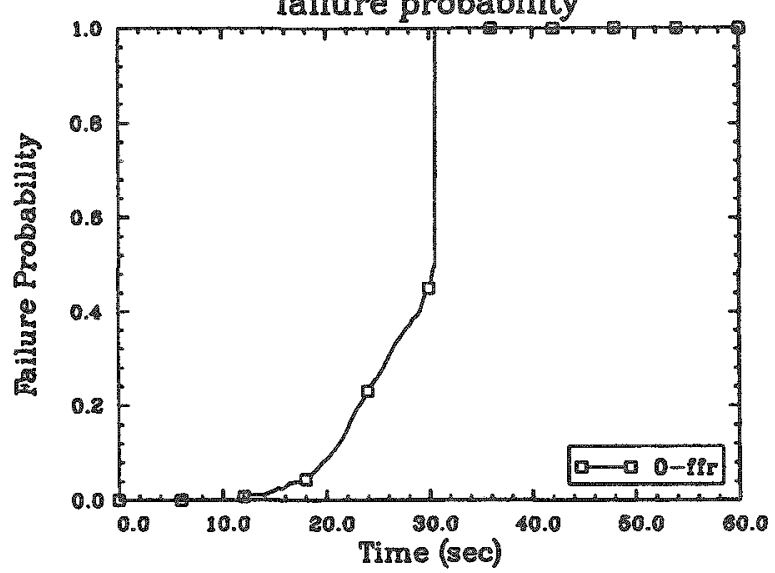

OCONEE 100\%DBA 35 GWD/MTU PIN--PF 2.2 (TRAC) cladding hoop strain

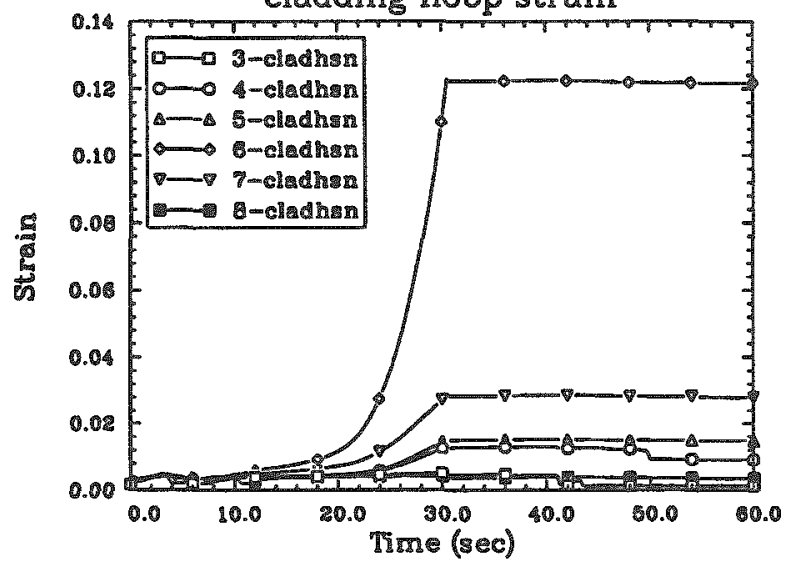

OCONEE 100\%DBA 35 GWD/MTU PIN--PF 2.2 (TRAC)

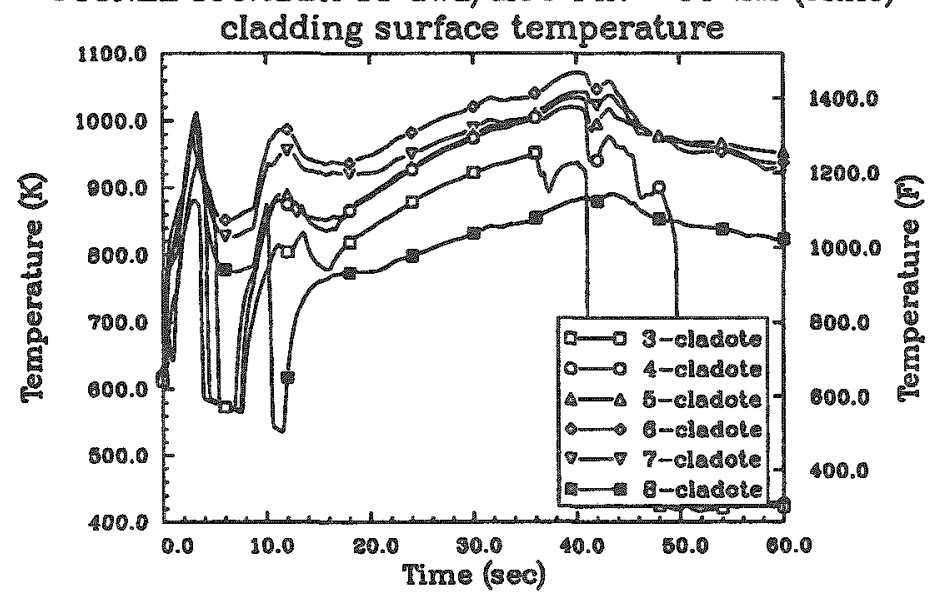

OCONEE 100\%DBA 35 GWD/MTU PIN--PF 2.2 (TRAC)

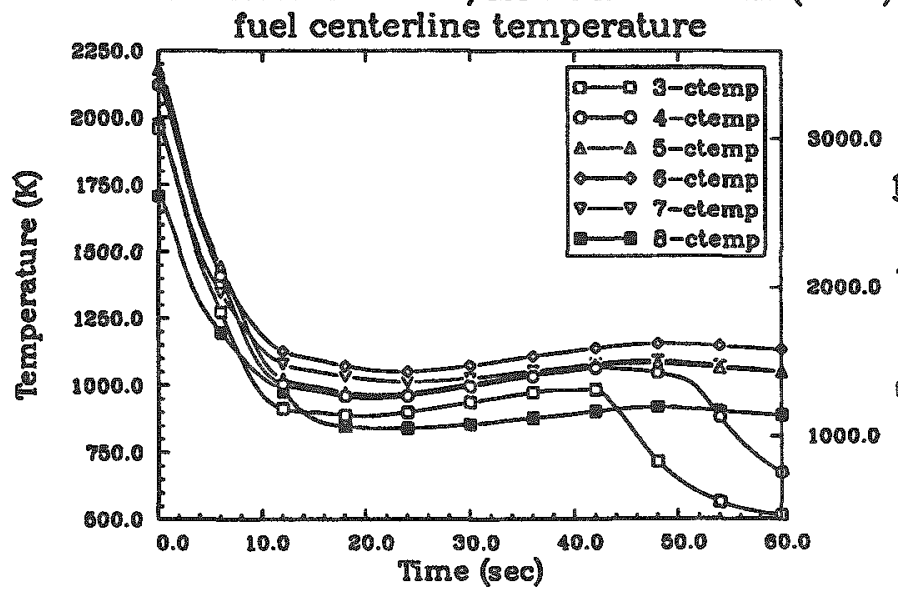

OCONEE 100\%DBA 35 GWD/MTU PIN--PF 2.2 (TRAC)

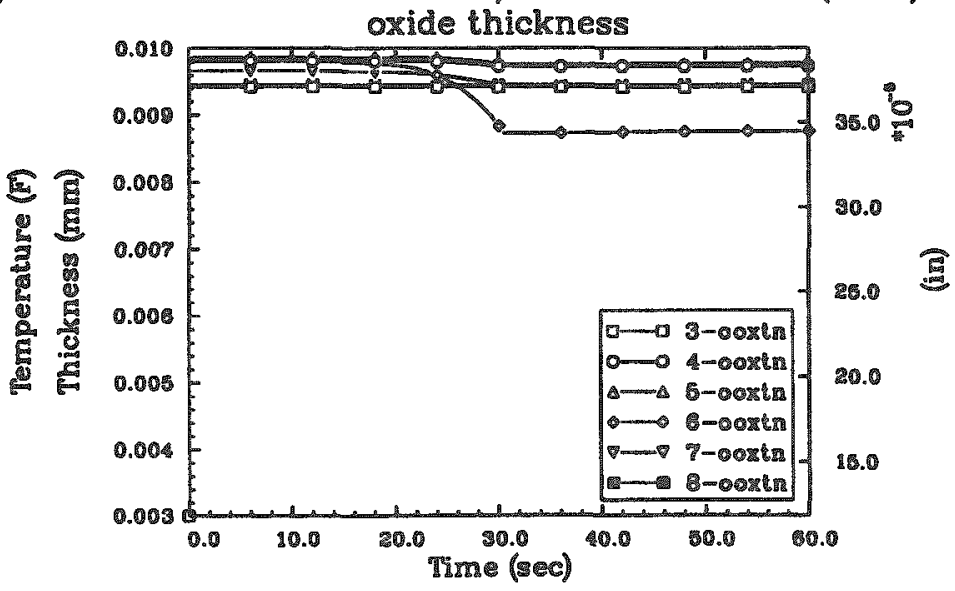


OCONEE 100\%DBA 20 GWD/MTU PIN-DF 2.2 (TRAC) internal pin pressure

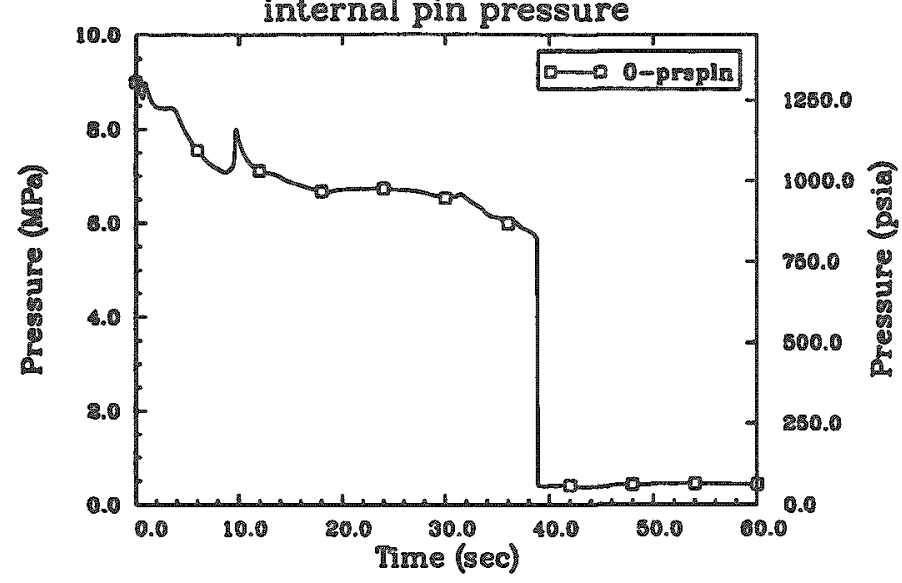

OCONEE 100\%DBA $20 \mathrm{GWD} / \mathrm{MTU}$ PIN-DP 2.2 (TRAC) failure probabilily

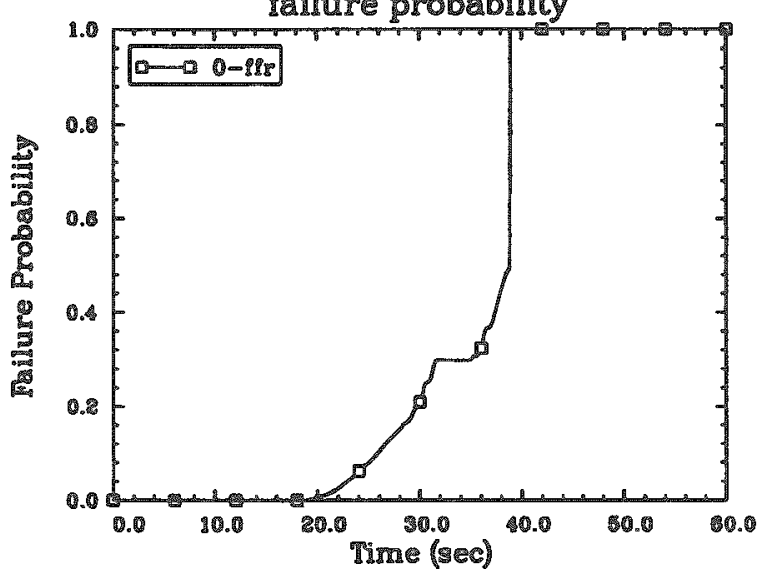

OCONEE 100\%DBA 20 GWD/MTU PIN-DF 2.2 (TRAC)

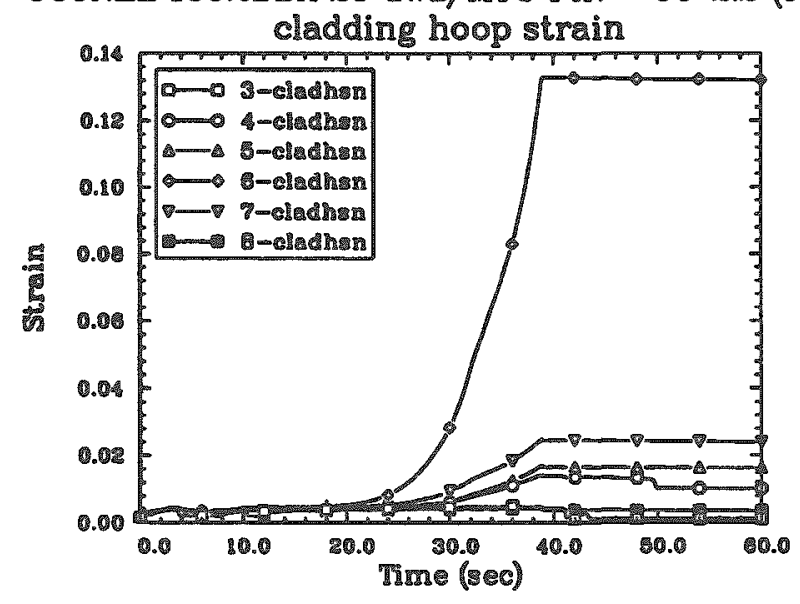

OCONEE 100\%DBA 20 GWD/MTU PIN-DP 2.2 (TRAC)

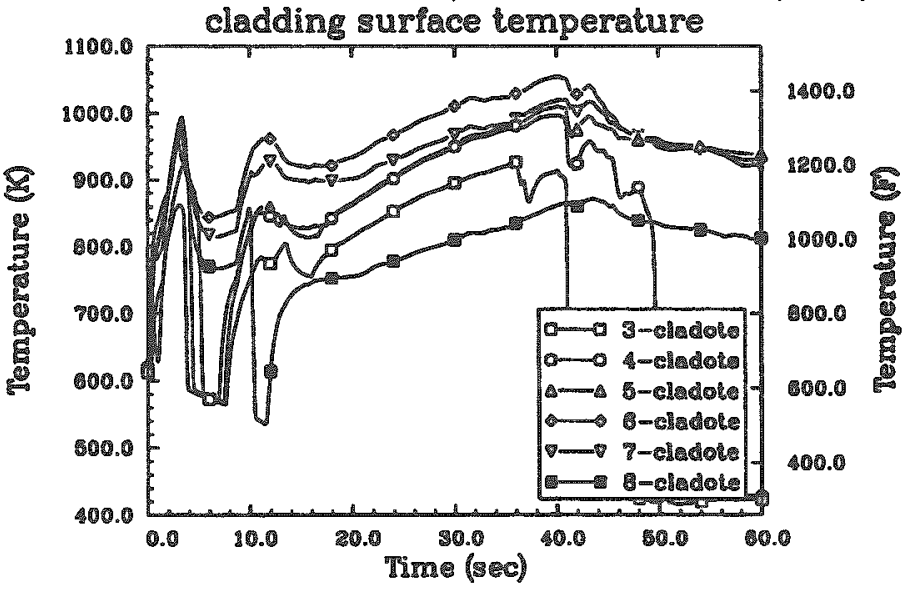

OCONEE 100\%DBA 20 GWD/MTU PIN-DPF 2.2 (TRAC)

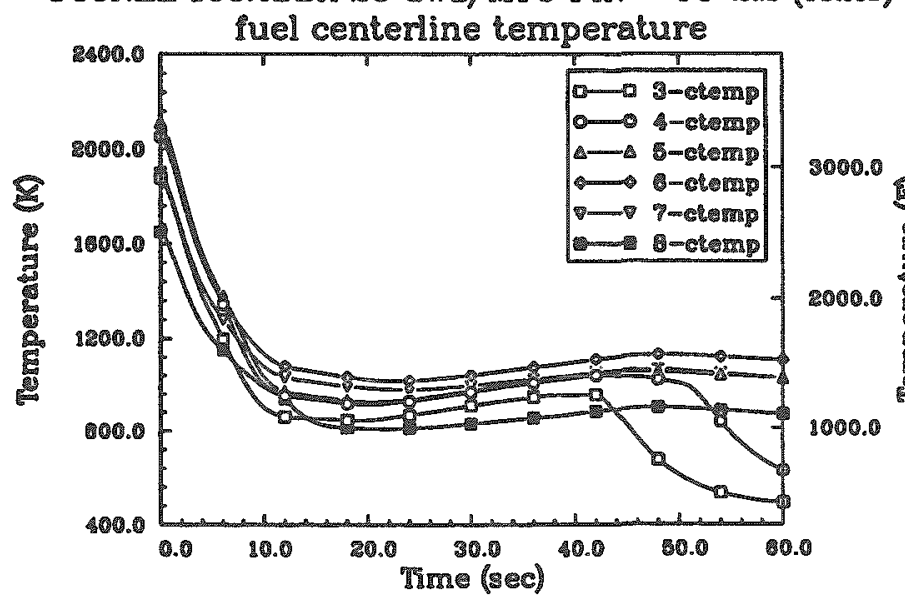

OCONEE 100\%DBA 20 GWD/MTU PIN--PF 2.2 (TRAC)

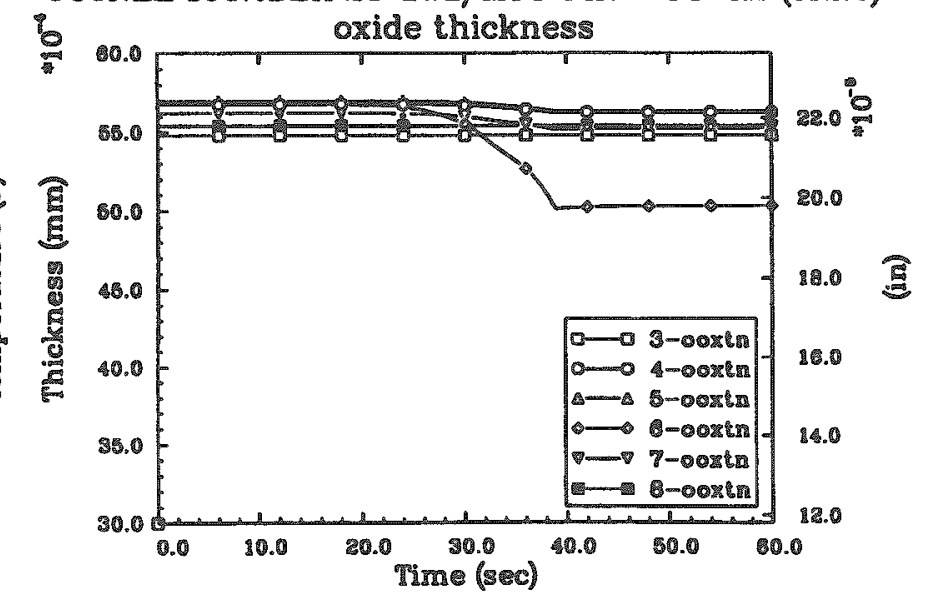


OCONEE 100\%DBA 5 GWD/MTU PIN--PF' 2.2 (TRAC)

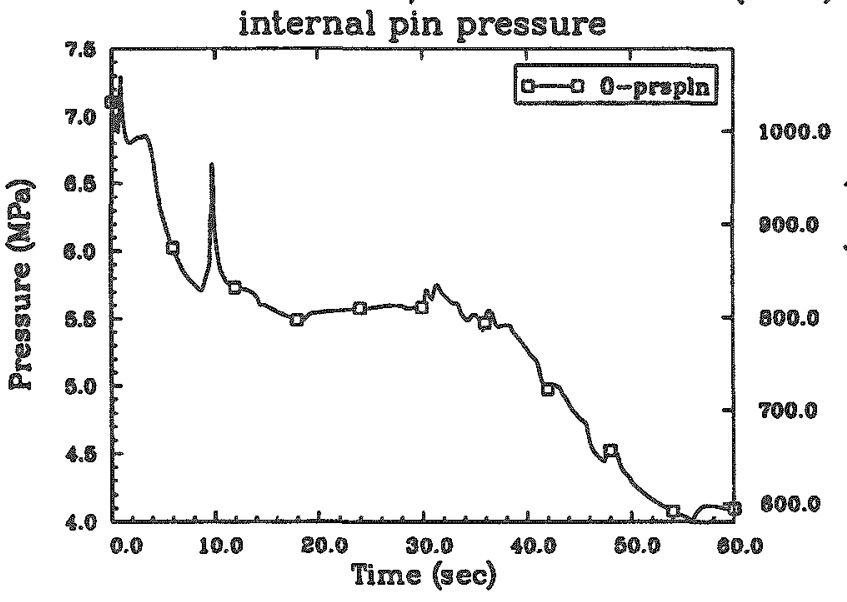

OCONEE 100\%DBA 5 GWD/MTU PIN-PF 2.2 (TRAC)

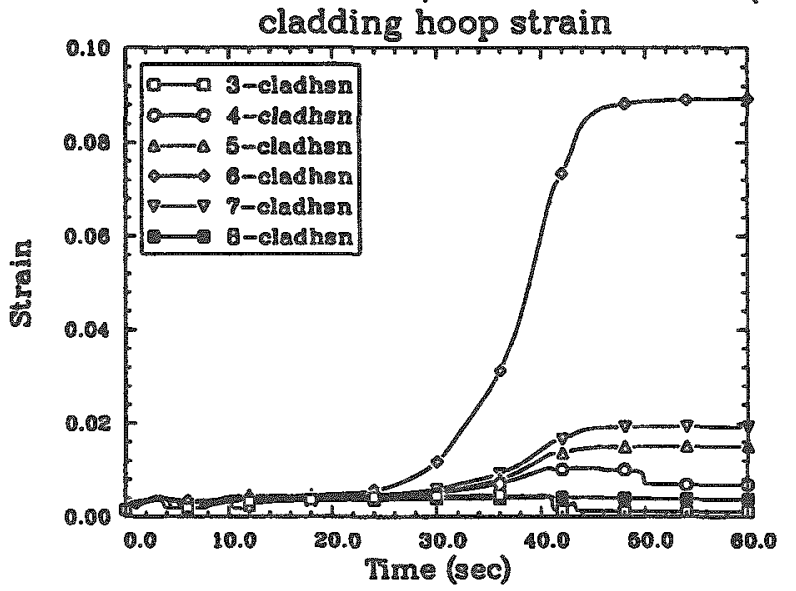

OCONEE 100\%DBA 5 GWD/MTU PIN-PF 2.2 (TRAC) lailure probability

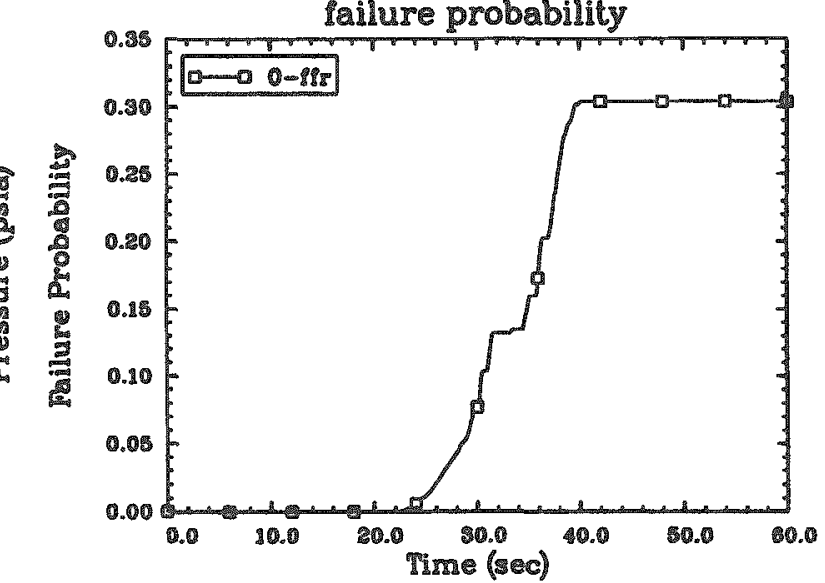

OCONEE 100\%DBA 5 GWD/MTU PIN-DP 2.2 (TRAC)

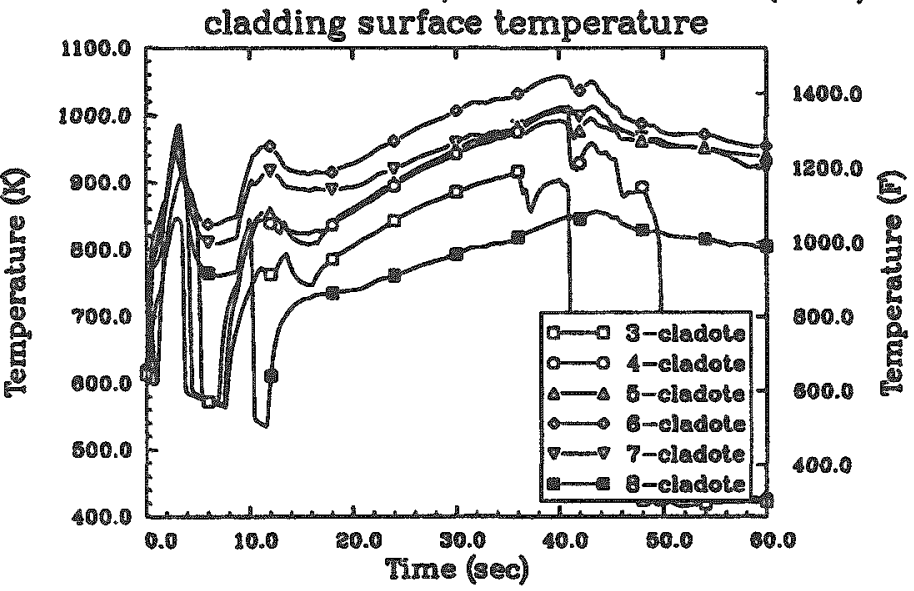

OCONEE 100\%DBA 5 GWD/MTU PIN--PF 2.2 (TRAC)

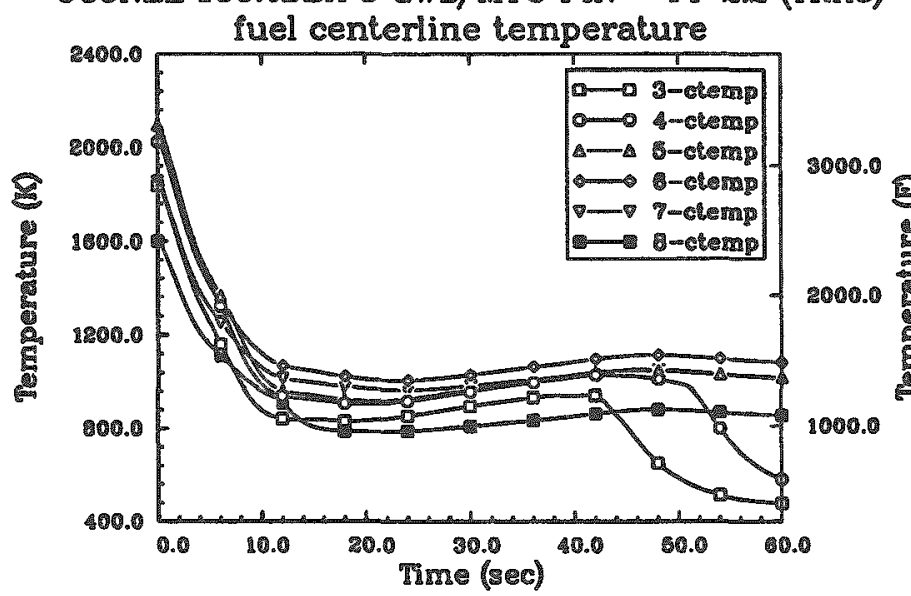

OCONEE 100\%DBA 5 GWD/MTU PIN--PF 2.2 (TRAC)

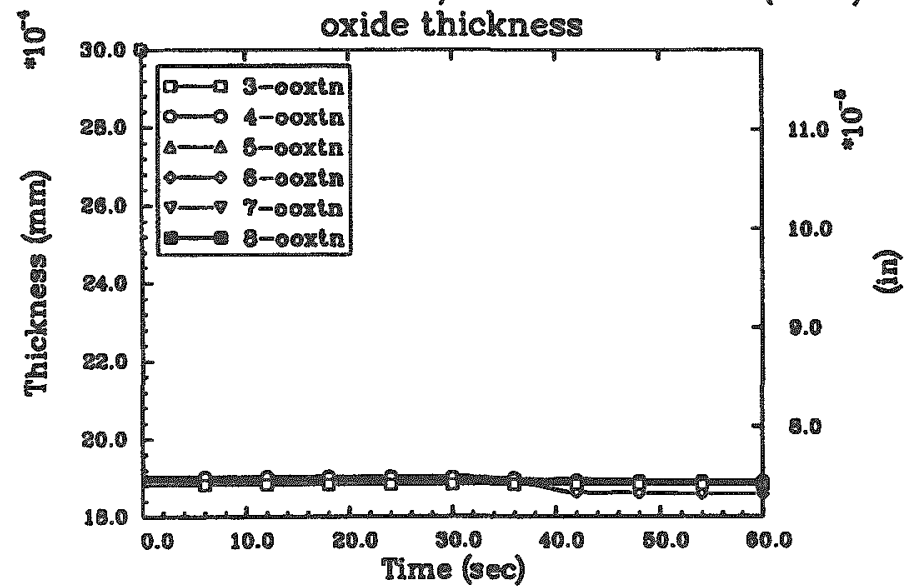


OCONEE 100\%DBA 55 GWD/MTU PIN--PF 2.0 (TRAC)

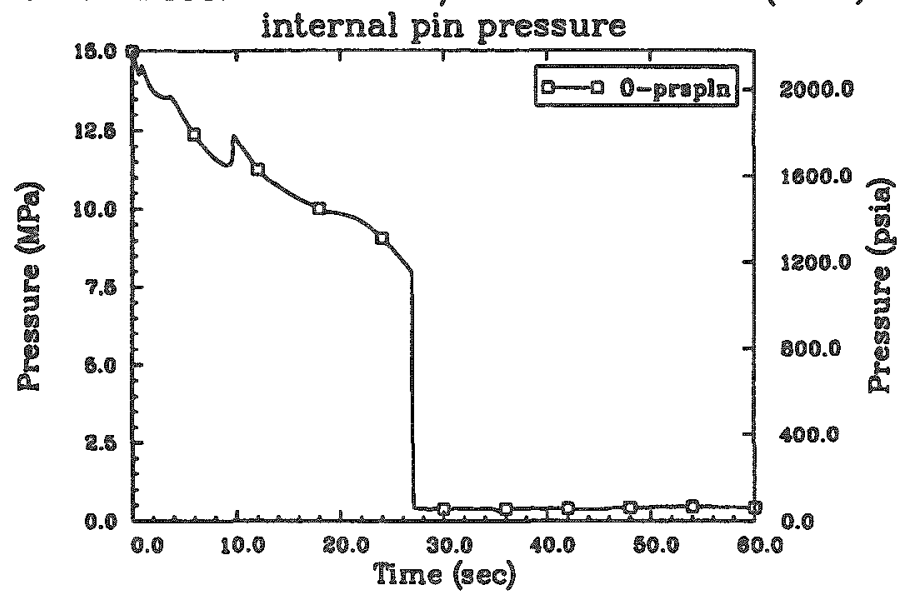

OCONEE 100\%DBA 55 GWD/MTU PIN--PF 2.0 (TRAC) failure probability

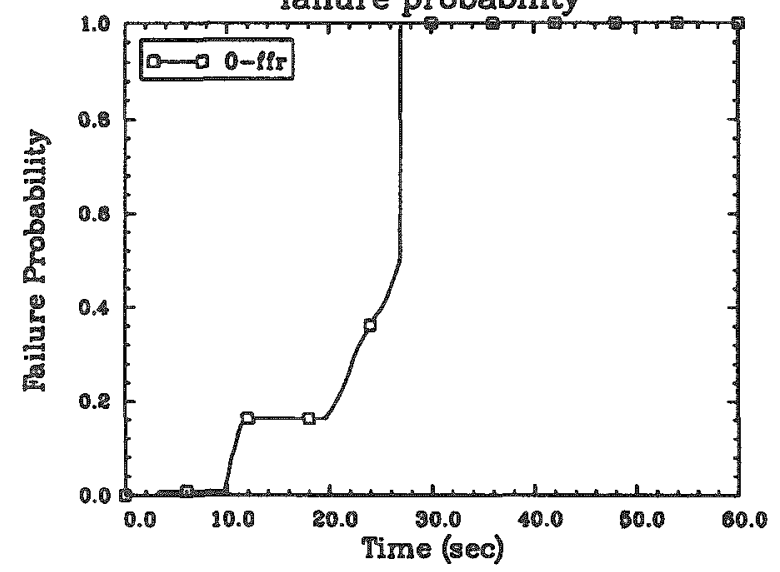

OCONEE 100\%DBA 55 GWD/MTU PIN--PF 2.0 (TRAC) cladding hoop strain

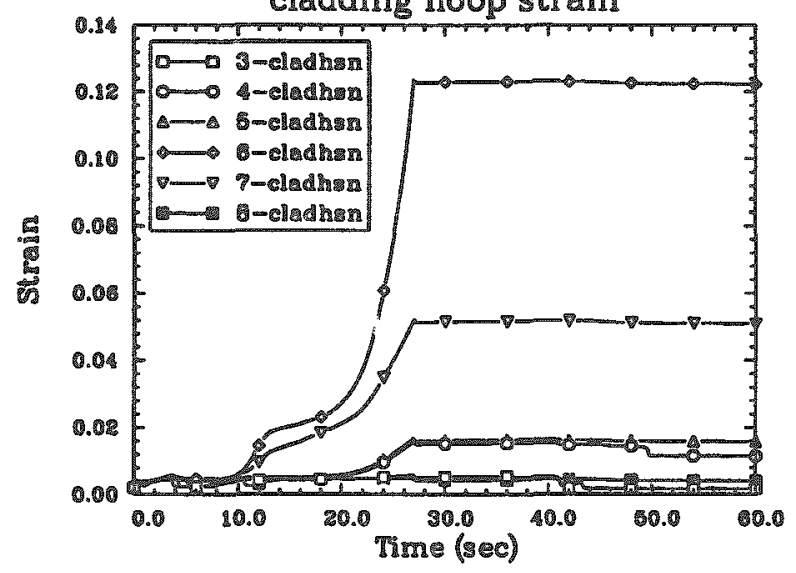

OCONEE 100\%DBA 55 GWD/MTU PIN--PF 2.0 (TRAC)

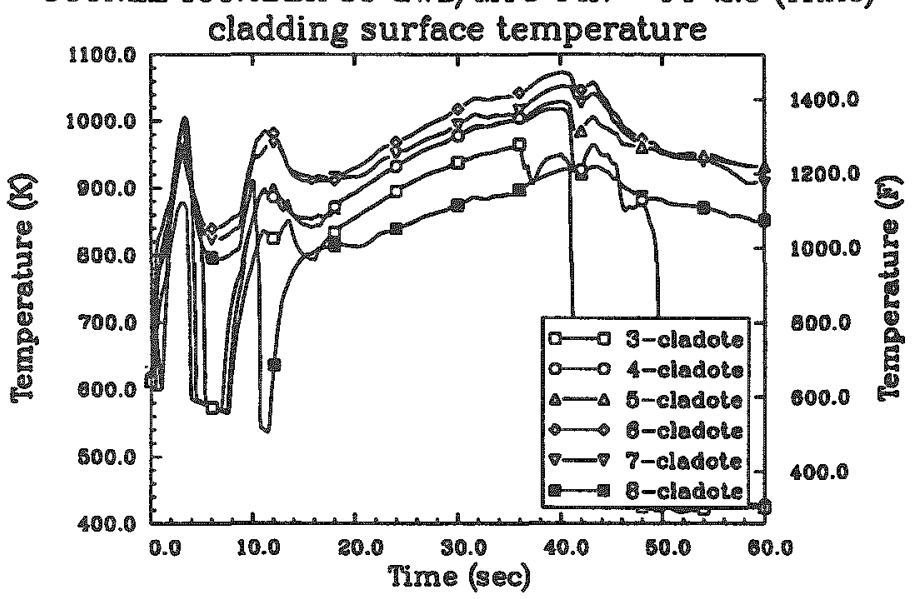

OCONEE 100\%DBA 55 GWD/MTU PIN--PF 2.0 (TRAC)

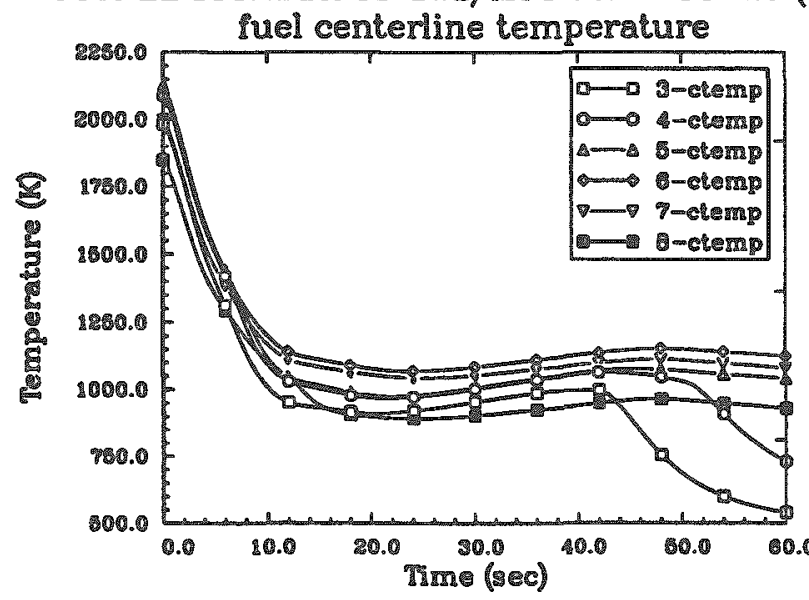

OCONEE 100\%DBA 55 GWD/MTU PIN--PF 2.0 (TRAC) oxide thickness

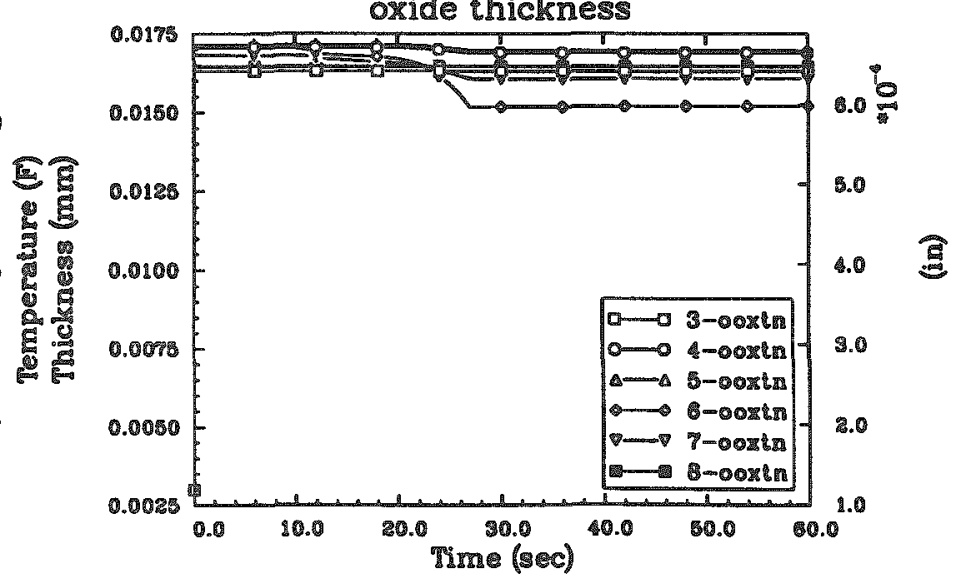


OCONEE 100\%DBA 35 GWD/MTU PIN--PF 2.0 (TRAC) internal pin pressure

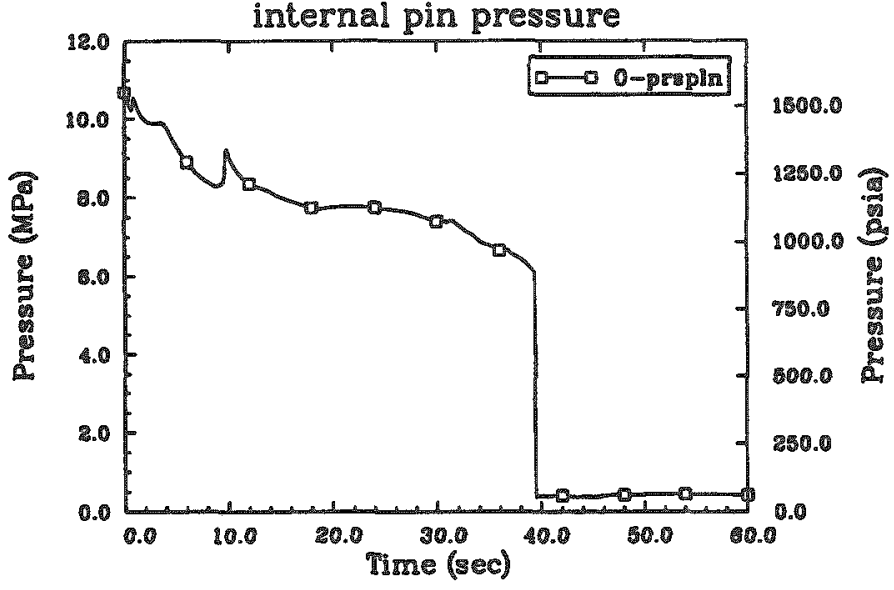

OCONEE 100\%DBA 35 GWD/MTU PIN--PF 2.0 (TRAC) cladding hoop strain
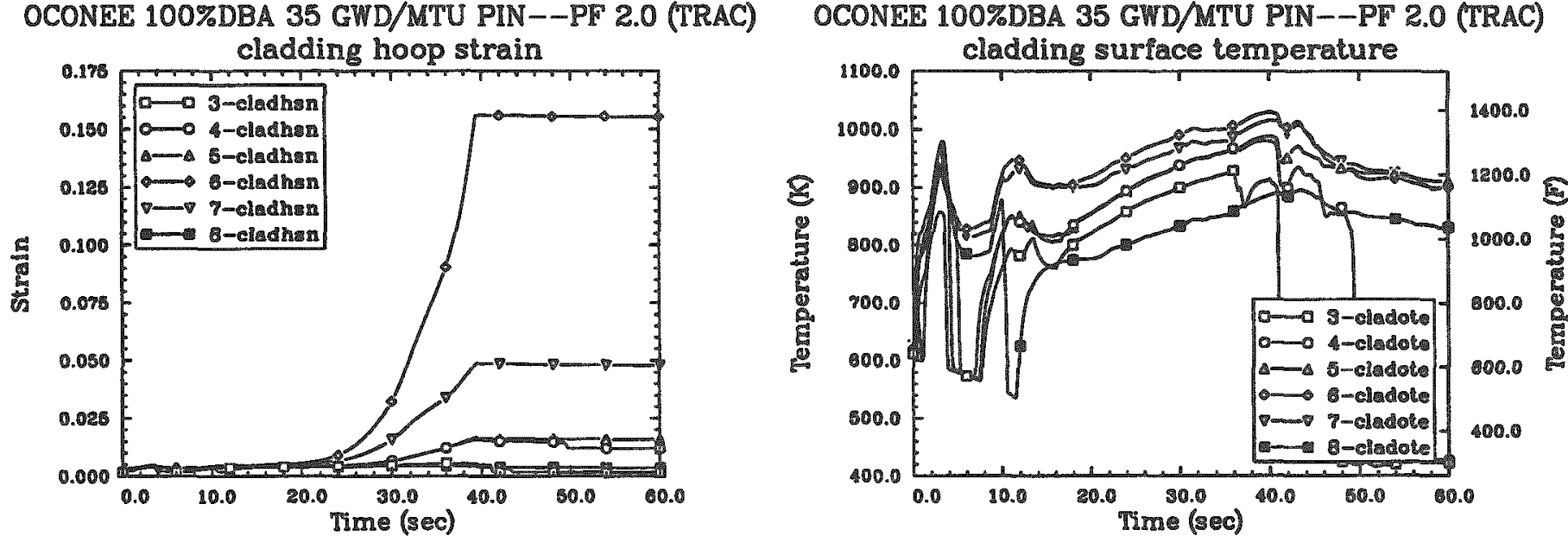

OCONEE 100\%DBA 35 GWD/MTU PIN--PF 2.0 (TRAC)

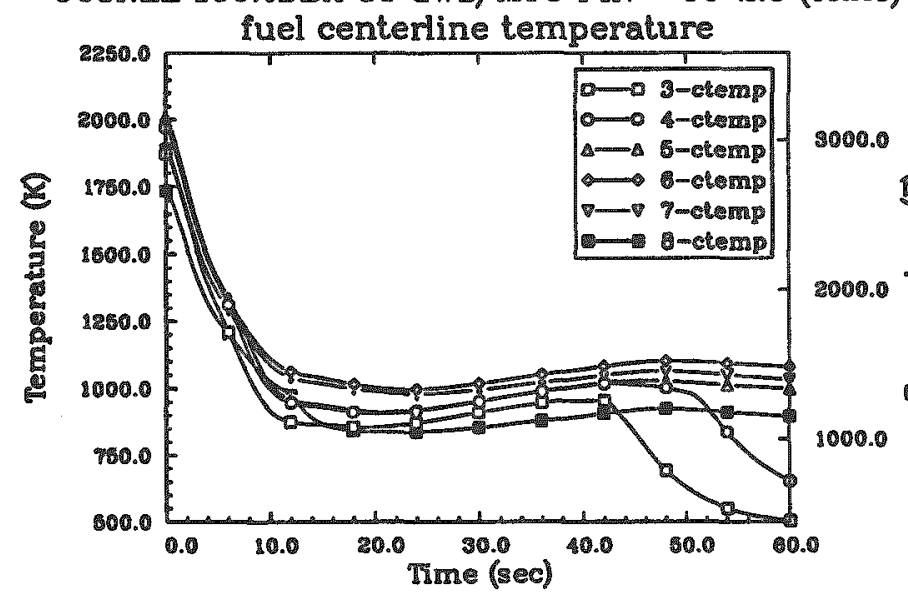

OCONEE 100\%DBA 35 GWD/MTU PIN--PF 2.0 (TRAC)

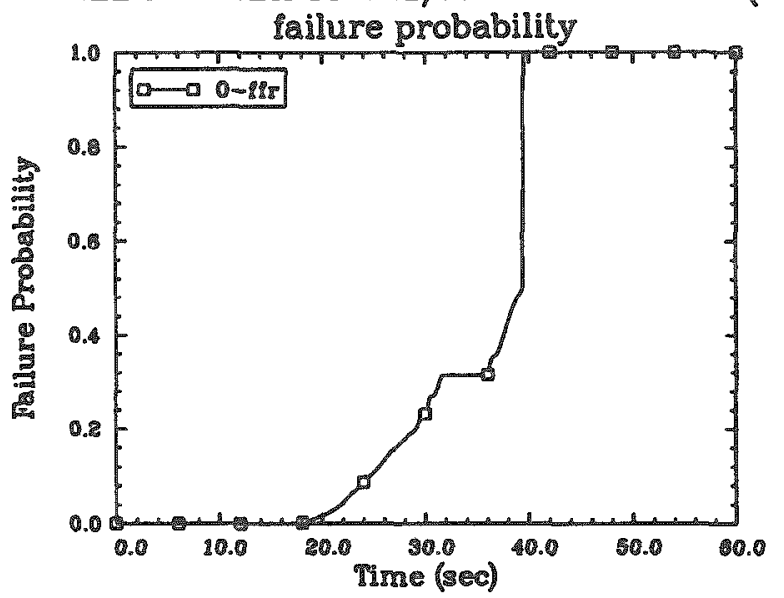


OCONER 100\%DBA 20 GWD/MTU PIN-DF 2.0 (TRAC) internal pin pressure

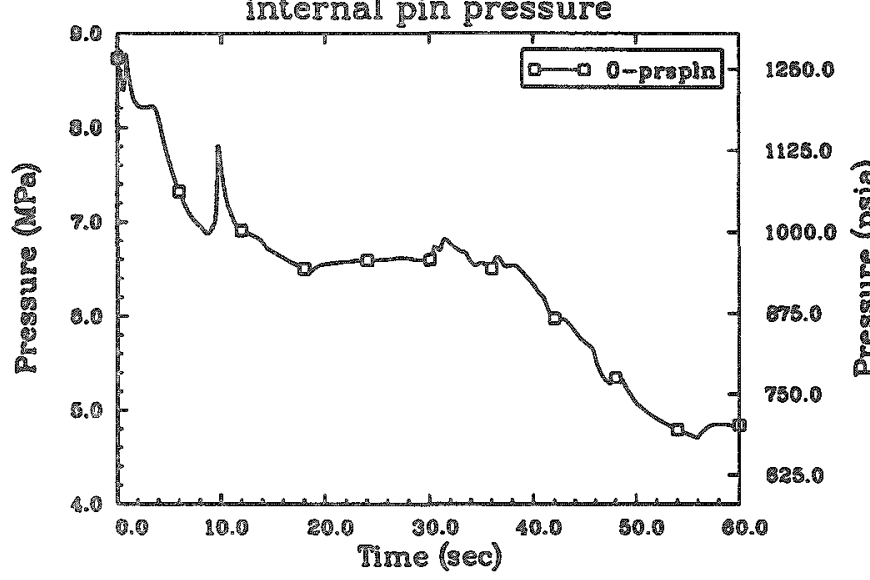

OCONEE 100\%DBA 20 GWD/MTU PIN--PF 2.0 (TRAC) failure probability

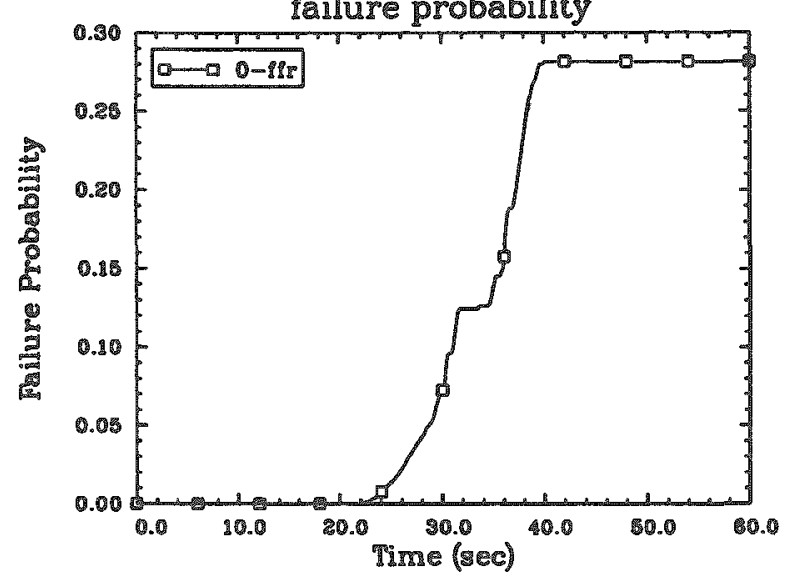

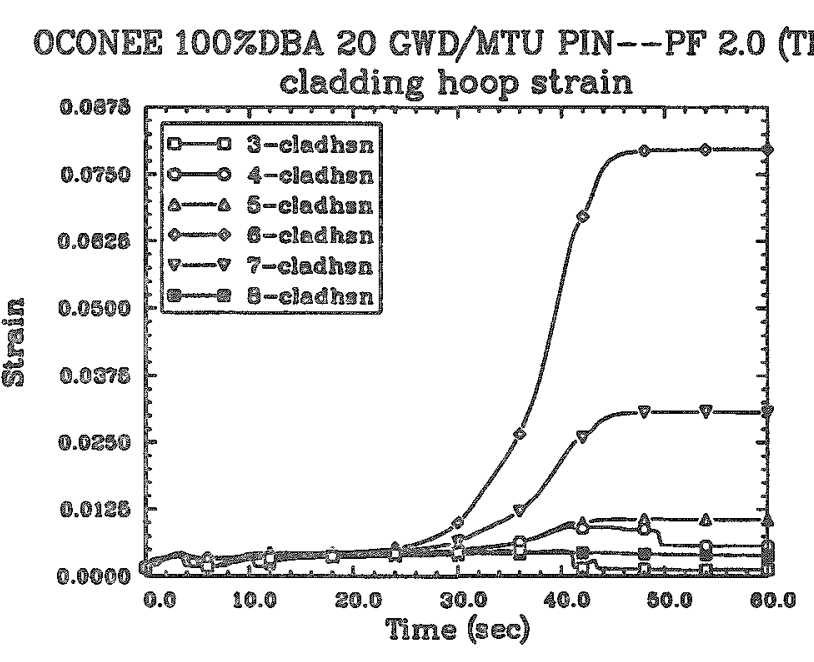

OCONER 100\%DBA 20 GWD/MTU PIN-DF 2.0 (TRAC)

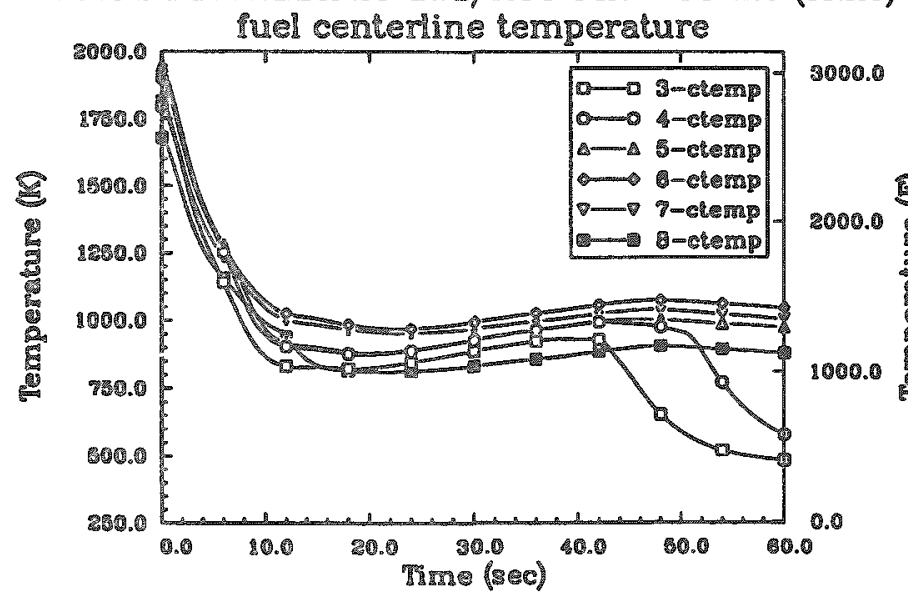

OCONEE 100\%DBA 20 GWD/MTU PIN--PF 2.0 (TRAC)

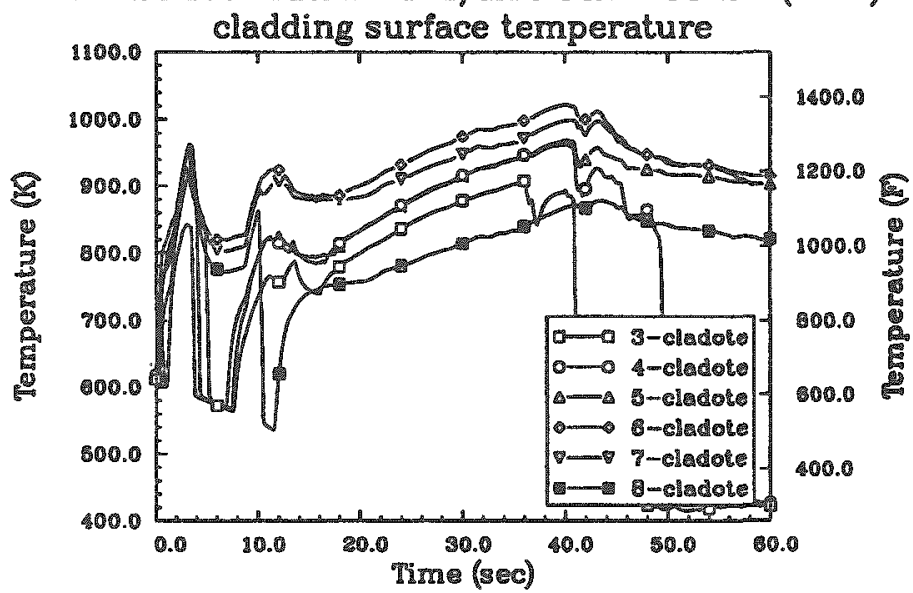

OCONEE 100\%DBA 20 GWD/MTU PIN-DF 2.0 (TRAC)

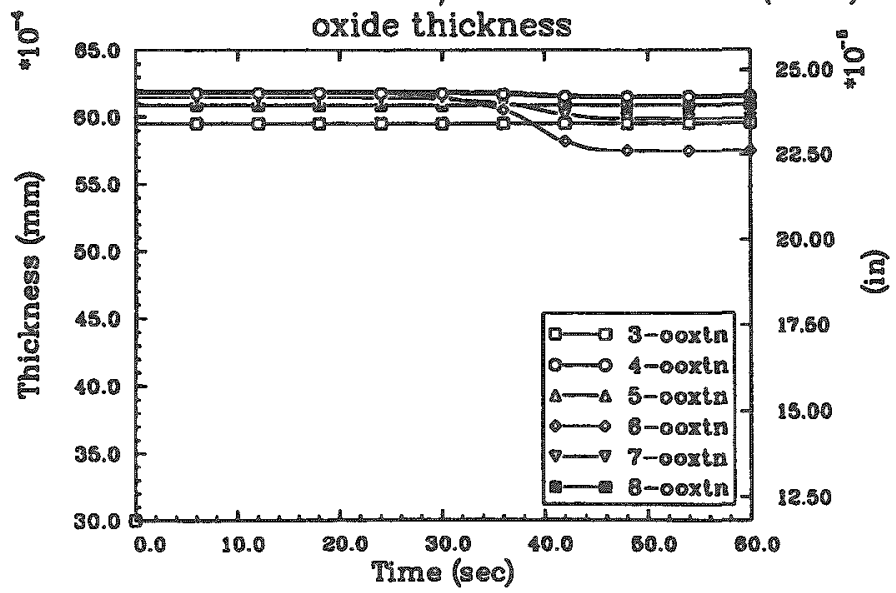


OCONEE 100\%DBA 5 GWD/MTU PIN--PF 2.0 (TRAC)

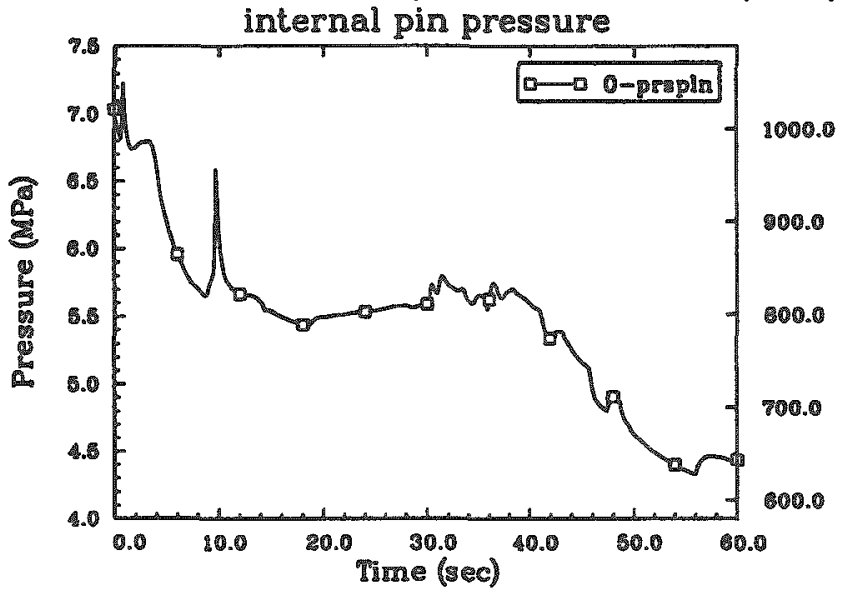

OCONEE 100\%DBA 5 GWD/MTU PIN-DF 2.0 (TRAC)

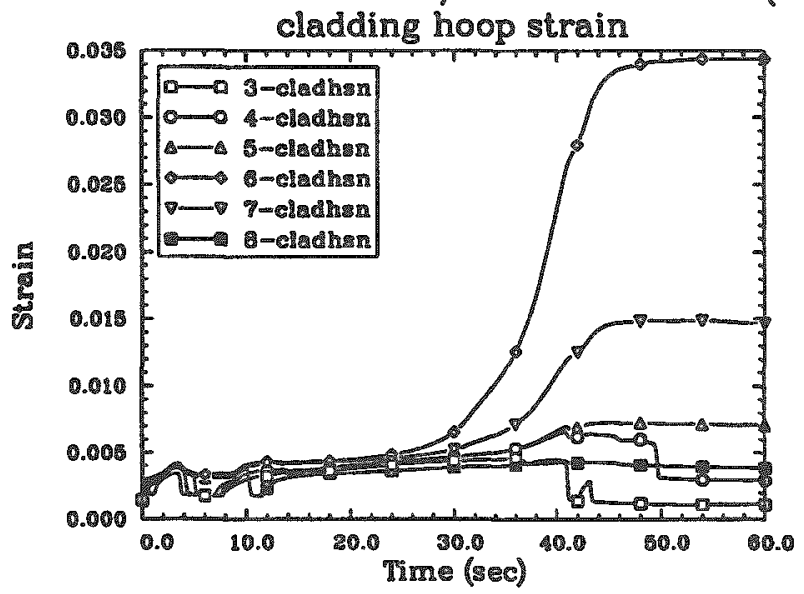

OCONEE 100\%DBA 5 GWD/MTU PIN--PF 2.0 (TRAC)

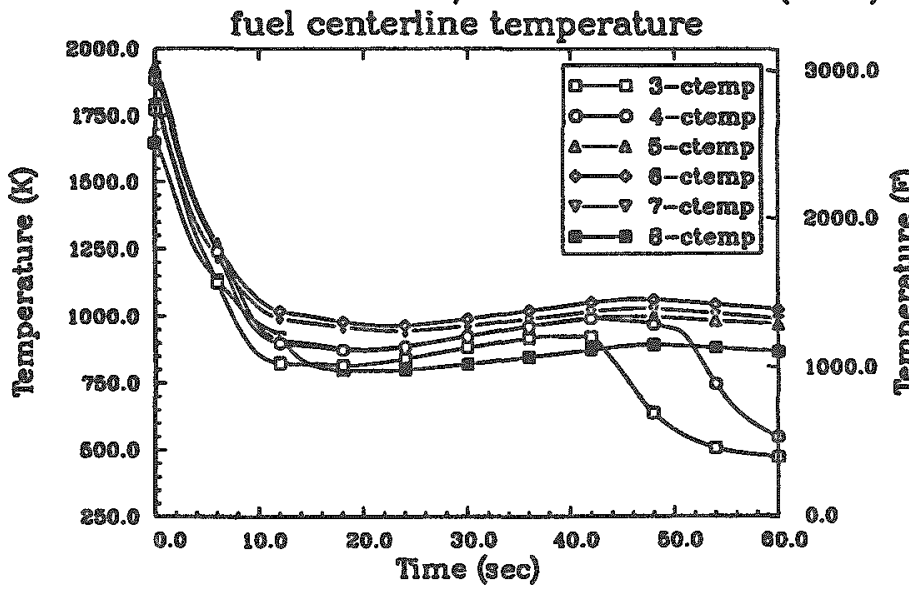

OCONEE 100\%DBA 5 GWD/MTU PIN--PF 2.0 (TRAC)

fallure probability
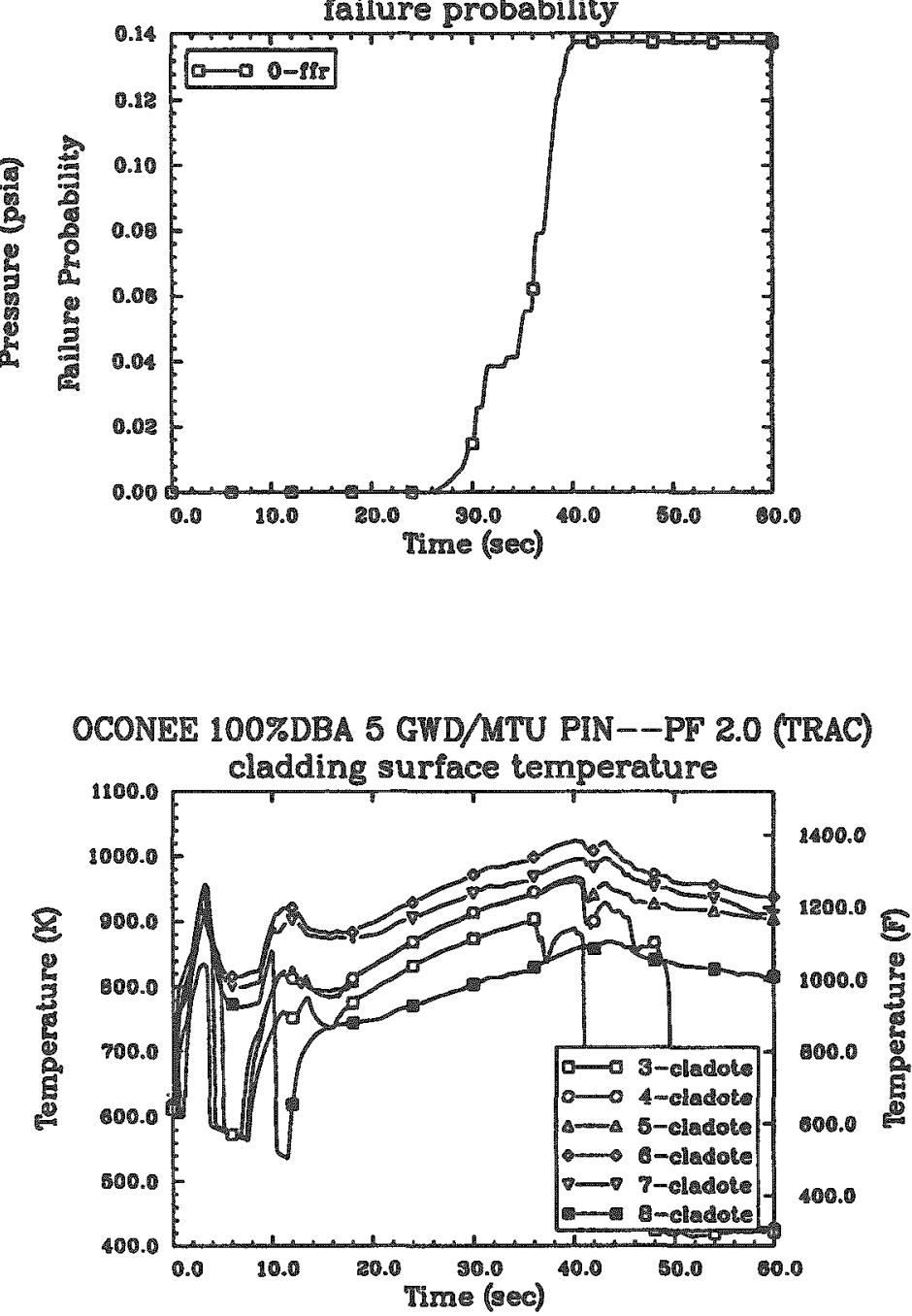

OCONEE 100\%DBA 5 GWD/MTU PIN--PF 2.0 (TRAC)

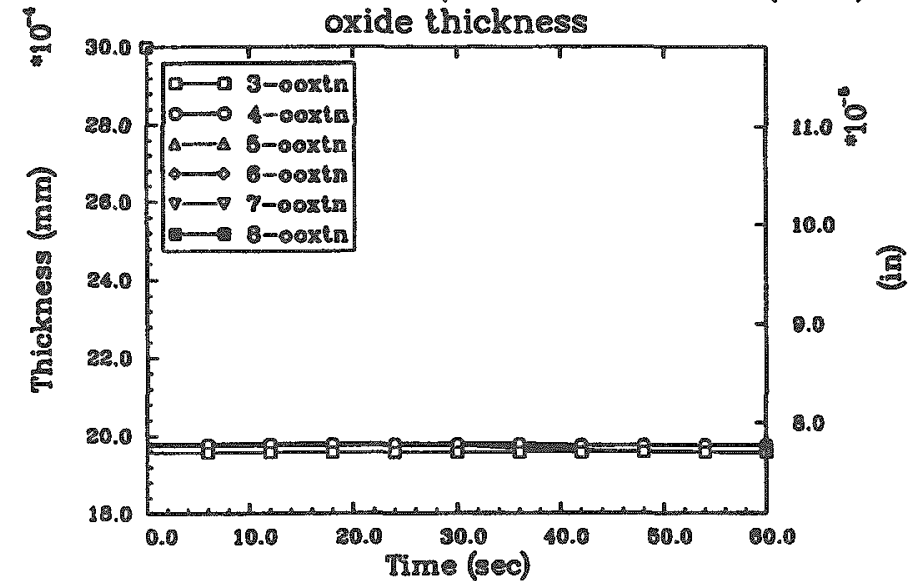




\section{K-3. SCDAP/RELAP5/MOD3 AND TRAC-PF1/MOD1 PLOTTED RESULTS FOR DCONEE}

Table K-3. Description of SCDAP/RELAP5/M003 and TRAC-PFI/MOD1 plot variables for Oconee.

Variable

Description

SCDAP/RELAP5/M003 Variables:

0 -rktpow

0-rkfipow

0-rkgapow

4040-cntrivar

$550010000-p$

$615010000-p$

4100-cntrivar

Total core thermal power (watts)

Total core fission power (watts)

Total core decay heat (watts)

collapsed reactor water level, hot channel (m)

Reactor upper head pressure (pa)

$702000000-m f 7$ owj

700 -acvlig

$100000000-m f 7$ owj

200000000-mflowj

181010000-mflowj

251000000-mflowj

$281000000-m$ m 7 owj

$150010000-m f 7$ owj

$571040000-v 0 i d g$

Pressurizer dome pressure (pa)

Total break flow $(\mathrm{kg} / \mathrm{s})$

Accumulator flow - total flow $(\mathrm{kg} / \mathrm{s})$.

Accumulator liquid volume - total $\left(\mathrm{m}^{3}\right)$

Total intact loop hot leg flow $(\mathrm{kg} / \mathrm{s})$

Total broken loop hot leg flow $(\mathrm{kg} / \mathrm{s})$

Total intact loop cold leg flow $(\mathrm{kg} / \mathrm{s})$

Total broken loop cold leg flow $(\mathrm{kg} / \mathrm{s})$

Total intact 10op cold leg flow $(\mathrm{kg} / \mathrm{s})$

570040000 -voidg

Hot channel flow at the core midplane $(\mathrm{kg} / \mathrm{s})$

$0-d t$

Intact loop downcomer void fraction

Time step size (s)

TRAC-PF1/MODI Plot Variables:

RPOWER0010001 Total core thermal power (watts)

CORELEVEL

P0770001

PUP0010001

MFLOWTOTBRK

MFLOWACCTOT

ACQLIQTOTINT

MFLOWO100001

MFLOW0200001

MFLOW0140001

MFLOW0240001

MFLOW0340001

MFLOWTOTO10701

ALPHA0010719

ALPHA0010722

DELTO000001

collapsed reactor water level, core average (m)

Pressurizer dome pressure (pa)

Reactor upper head pressure (pa)

Total break flow $(\mathrm{kg} / \mathrm{s})$

Total accumulator $(\mathrm{kg} / \mathrm{s})$

Accumulator liquid volume - total intact loop $\left(\mathrm{m}^{3}\right)$

Total intact loop hot leg flow $(\mathrm{kg} / \mathrm{s})$

Total broken loop hot leg flow $(\mathrm{kg} / \mathrm{s})$

Total intact loop cold leg flow $(\mathrm{kg} / \mathrm{s})$

Total broken loop cold leg flow $(\mathrm{kg} / \mathrm{s})$

Total intact 10op cold leg flow $(\mathrm{kg} / \mathrm{s})$

Hot channel flow at the core midplane $(\mathrm{kg} / \mathrm{s})$

Broken loop downcomer void fraction at the core midplane elevation

Intact 100p downcomer void fraction at the core midplane elevation

Time step size (s) 
OCONEE 100\% DBA, TRAC-PF1 VS. RELAP5/MOD3

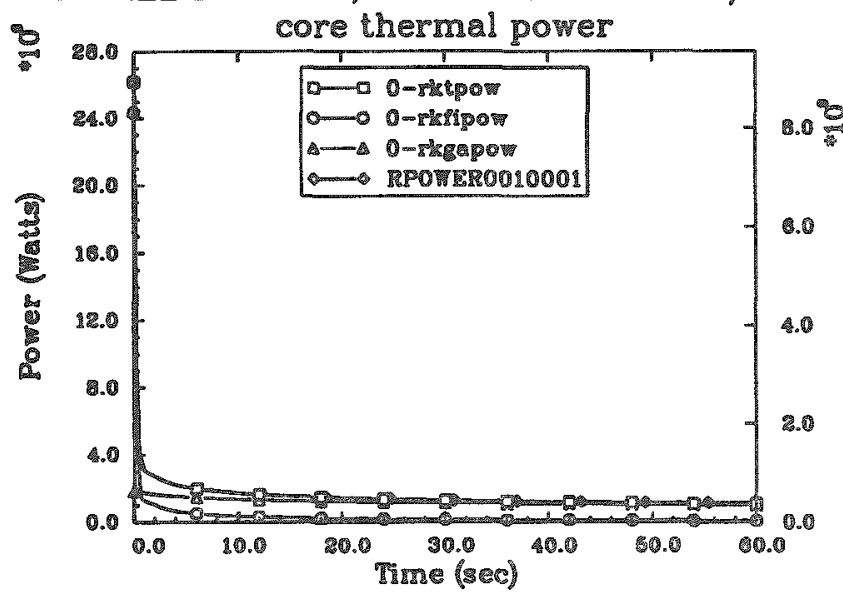

OCONEE 100\% DBA, TRAC-PII VS. RELAP5/MOD3

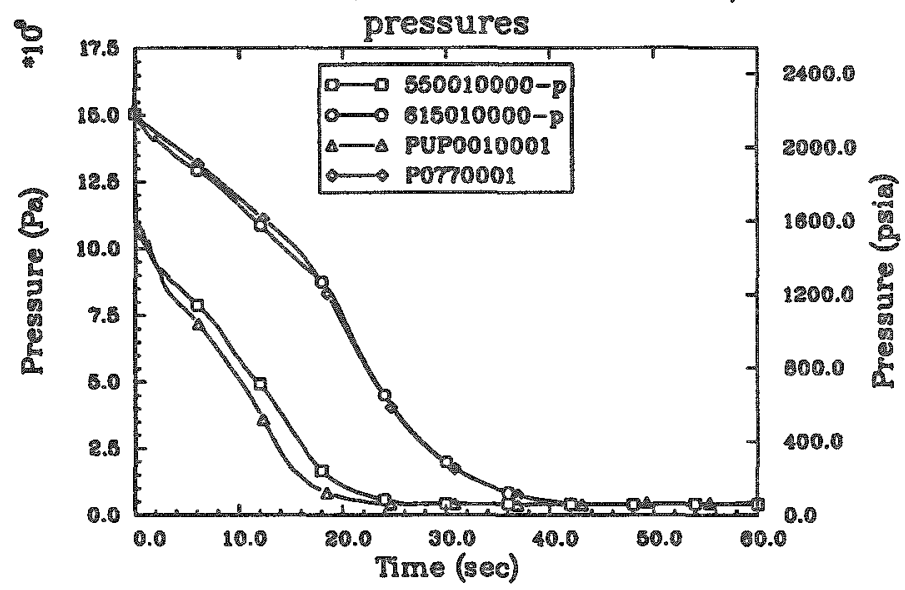

OCONEE 100\% DBA, TRAC-PF1 VS. RELAP5/MOD3

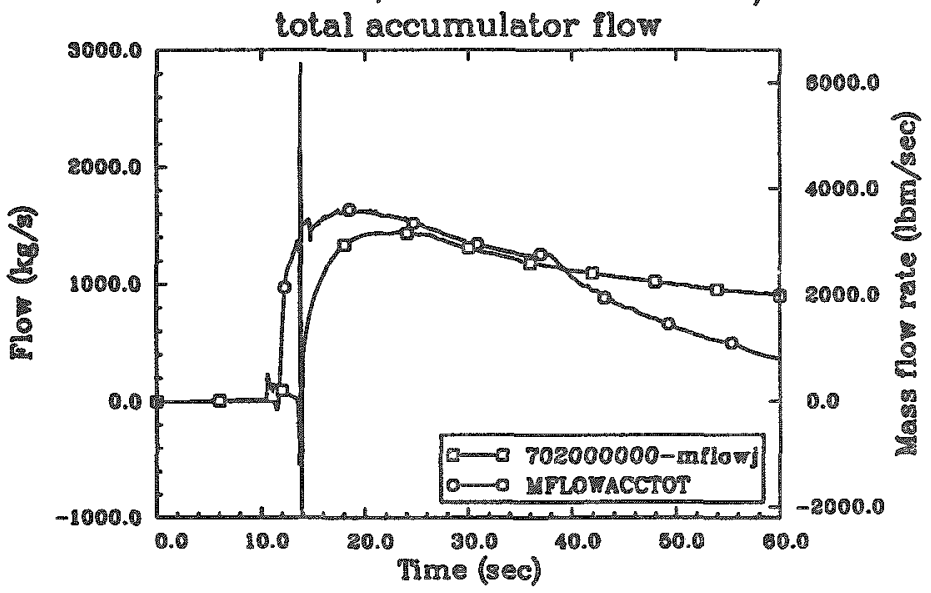

OCONEE 100\% DBA, TRAC-PF1 VS. RELAP5/MOD3 collapsed reactor water level

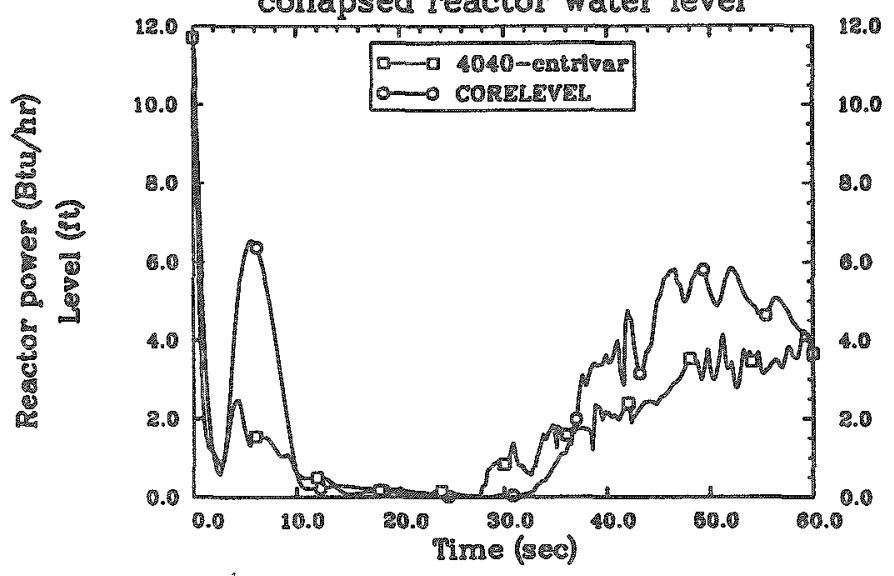

OCONES 100\% DBA TRAC-PFI VS. RELAPS/MOD3

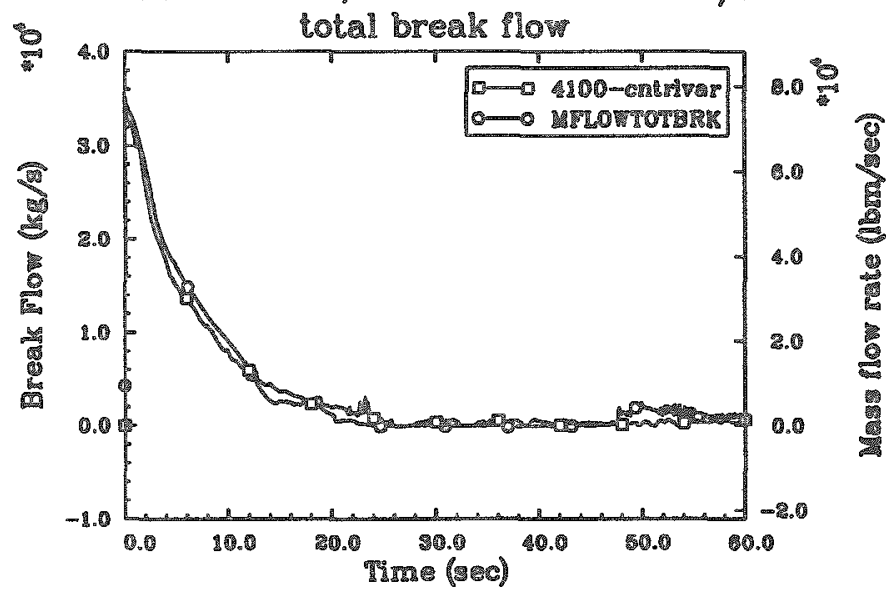

OCONEE 100\% DBA, TRAC-PF1 VS. RELAP5/MOD3

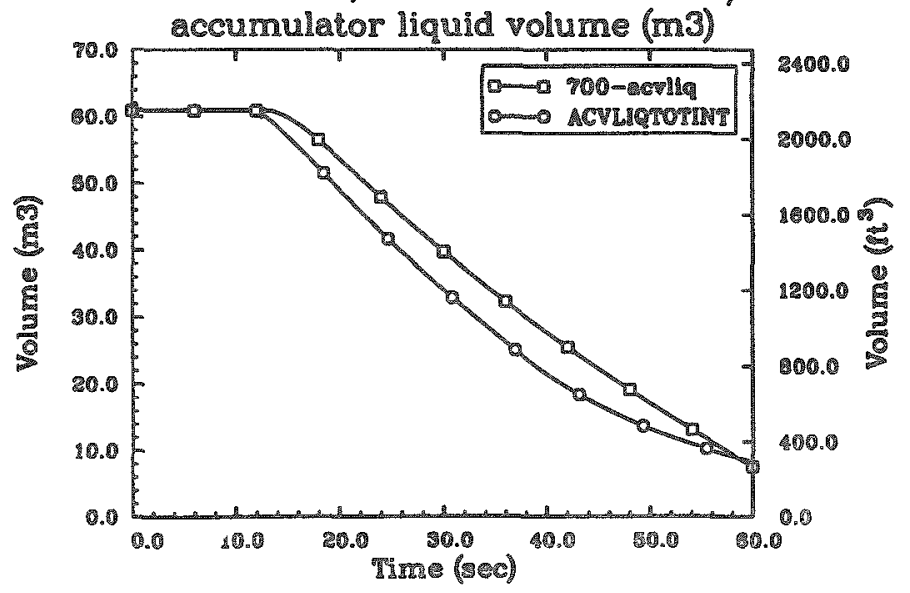


OCONEE 100\% DBA, TRAC-PF1 VS. RELAP5/MOD3

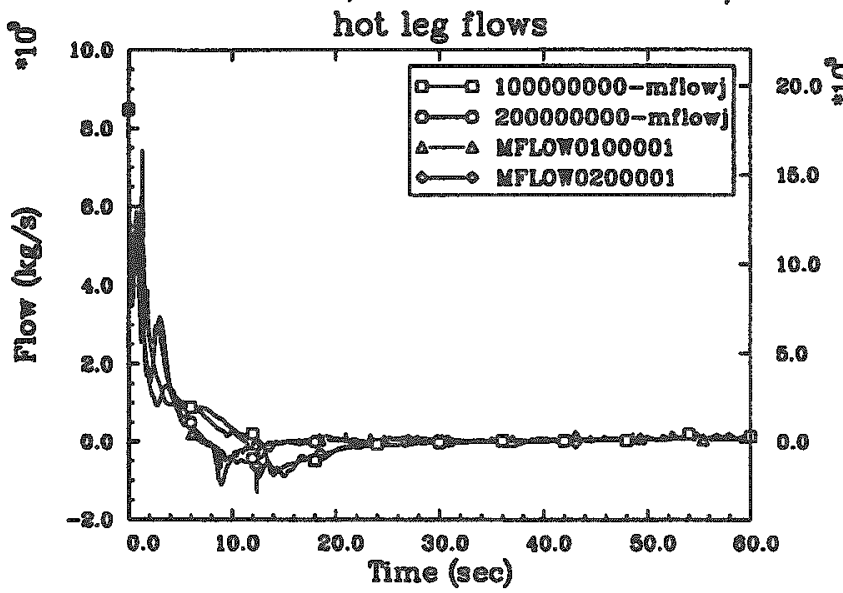

OCONEE 100\% DBA,TRAC-PFI VS. RELAP5/MOD3

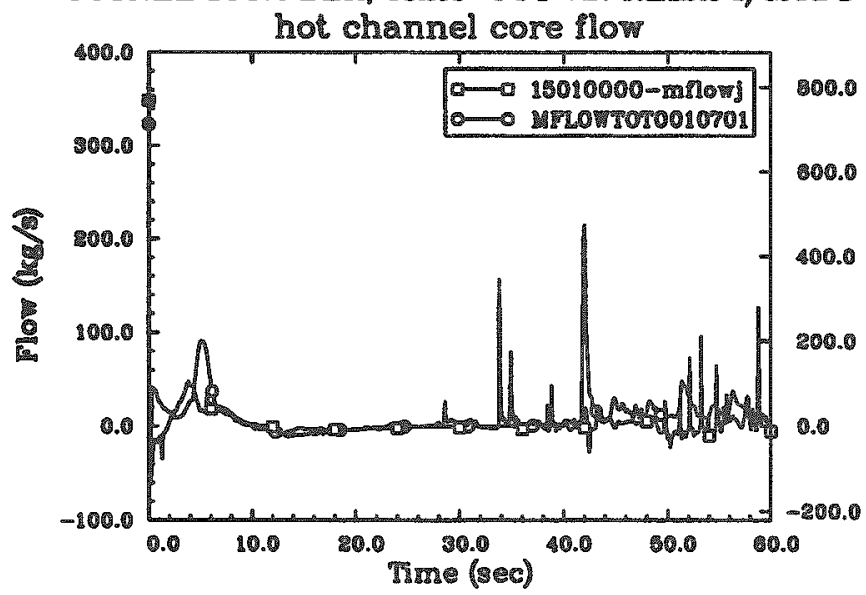

OCONEE 100\% DBA, TRAC-PFI VS. RELAP5/MOD3

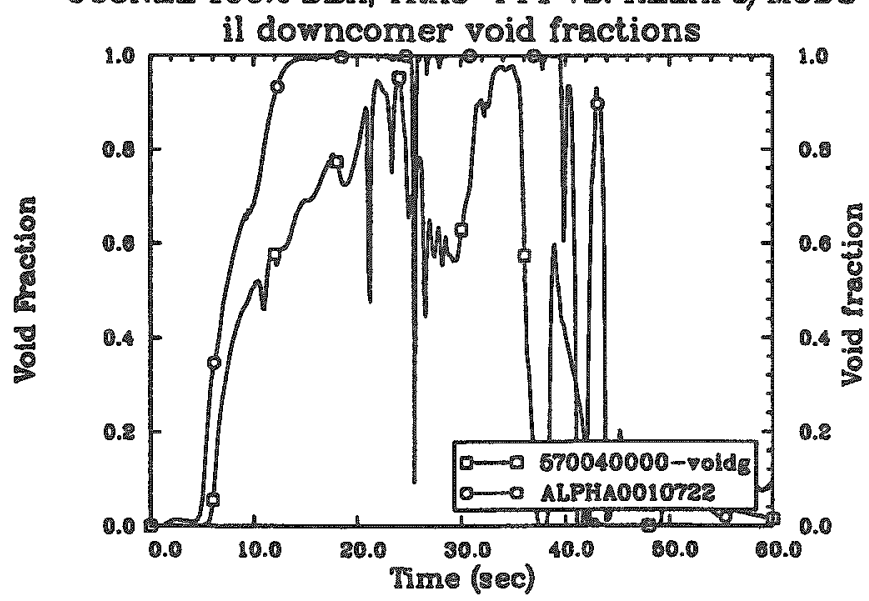

OCONEE 100\% DBA, TRAC-PF1 VS. RELAPS/MOD3

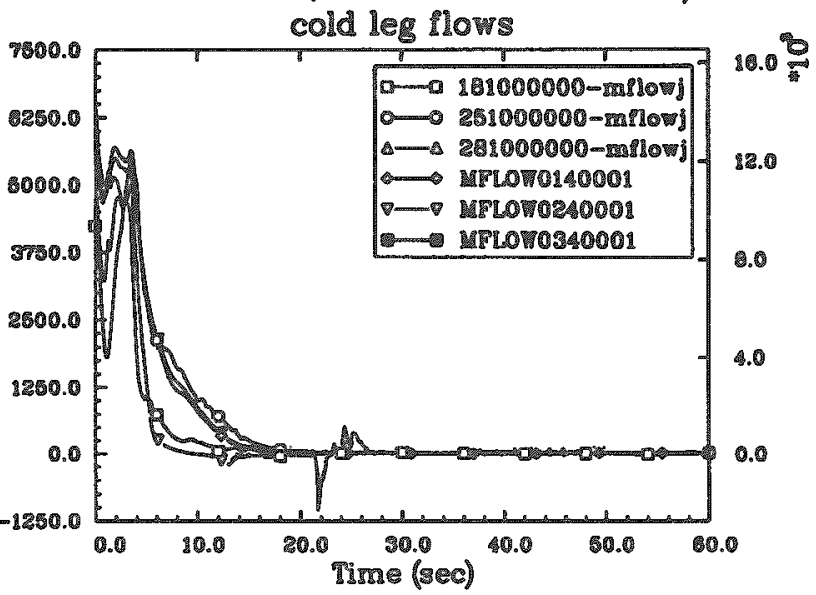

OCONEE 100\% DBA。 TRAC-PF1 VS. RELAP5/MOD3

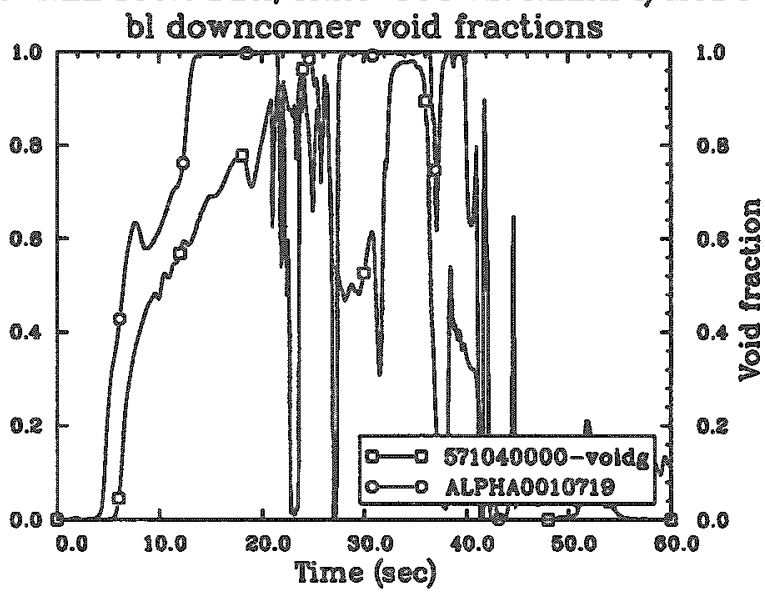

OCONEE 100\% DBA, TRAC-PF1 VS. RELAPS/MOD3

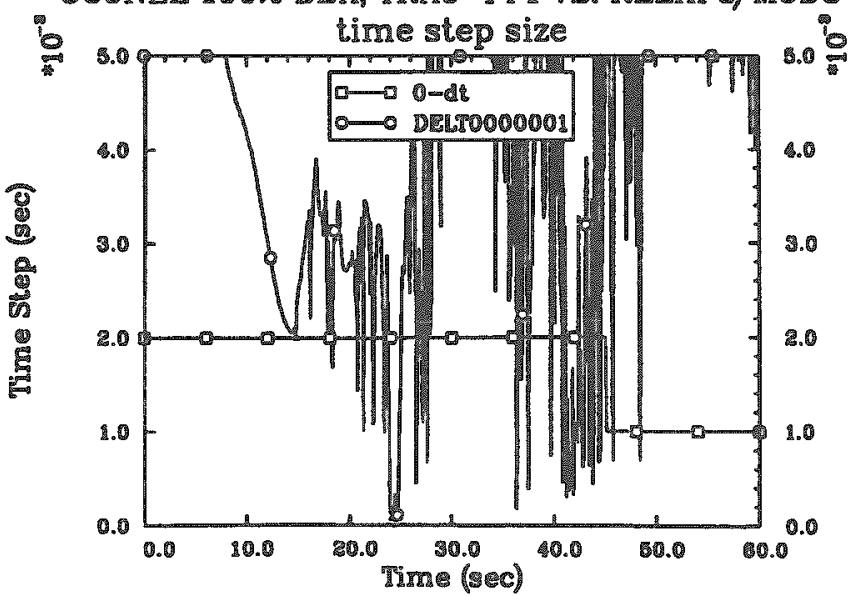




\section{Appendix L}

Plots for the Timing Analysis of PWR Fuel Pin Failures for Seabrook 


\title{
APPENDIX L \\ Plots for the TIMING ANalysis OF PWR \\ Fuel Pin FaIluRes for SEABROOK
}

\begin{abstract}
Appendix $L$ contains the plotted results for the timing analysis of PWR fuel pin failures for the Seabrook reactor. Section L-1 contains the SCDAP/RELAP5/MOD3 plots of total core power, collapsed reactor water level, reactor upper head and pressurizer dome pressures, containment pressure, fission product release, internal pin pressures, fuel centerline temperatures, cladding surface temperatures, hoop strains, total break flow, accumulator flow, accumulator liquid volume, hot leg flows, cold leg flows, hot channel core flow, downcomer void fractions, mass error, time step size, and cpu time for the nine accident scenarios. Section L-2 contains the FRAP-T6 plots of failure probability, internal pin pressure, cladding hoop strain, cladding surface temperature, fuel centerline temperature, and oxide thickness for the nine accident scenarios with peaking factors of $2.232,2.2,2.0$, and 1.8 and burnups of 50,35,20, and 5 GWd/MTU and using SCDAP/RELAP5/MOD3 thermaThydrautic boundary condition data. For the 100\% design basis accident (DBA) case, plots of results obtained using TRAC-PFI/MOD1 thermal-hydraulic boundary condition data are also included. Section L-3 contains plots comparing TRACPF1/MOD1 and SCDAP/RELAP5/M0D3 results for the 100\% DBA case for the following variables: core thermal power, collapsed reactor water level, presurizer dome and reactor upper head pressures, total break flow, accumulator flow, accumulator liquid volume, hot leg flow, cold leg flow, hot channel core flow, downcomer void fractions, and time step size. Tables $L-1, L-2$, and $L-3$ provide a listing of the plot variables.
\end{abstract}




\section{L-1. SCDAP/RELAP5/M0D3 PLOTTEd Results for SEABroOK}

Table L-1. Description of SCDAP/RELAP5/MOD3 plot variables for Seabrook.

\begin{tabular}{|c|c|}
\hline Variable & Description \\
\hline 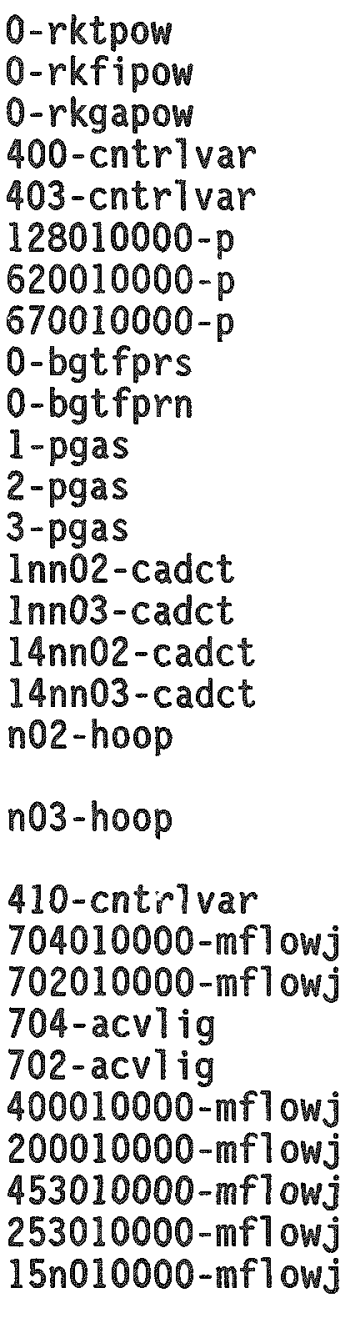 & 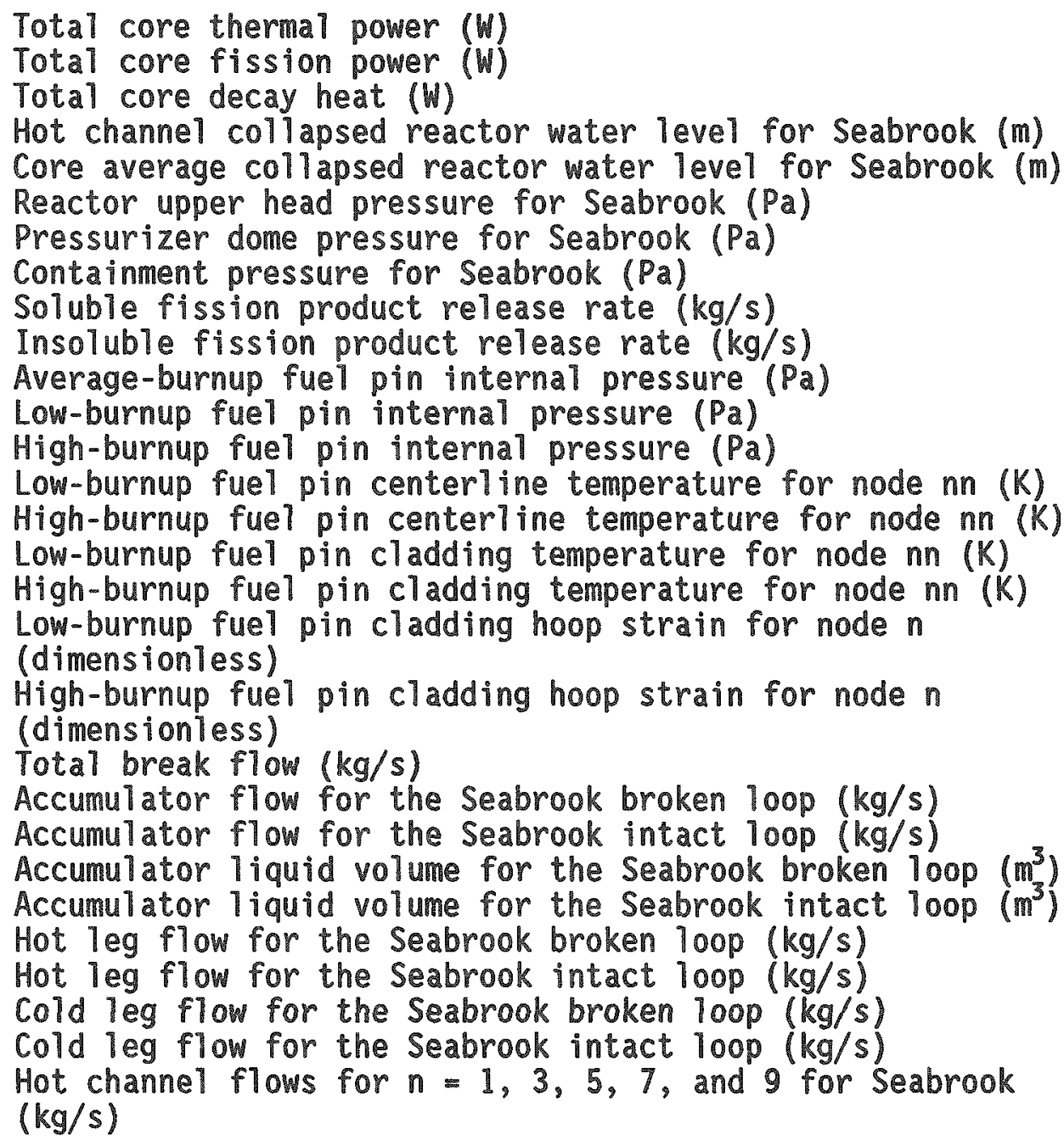 \\
\hline
\end{tabular}




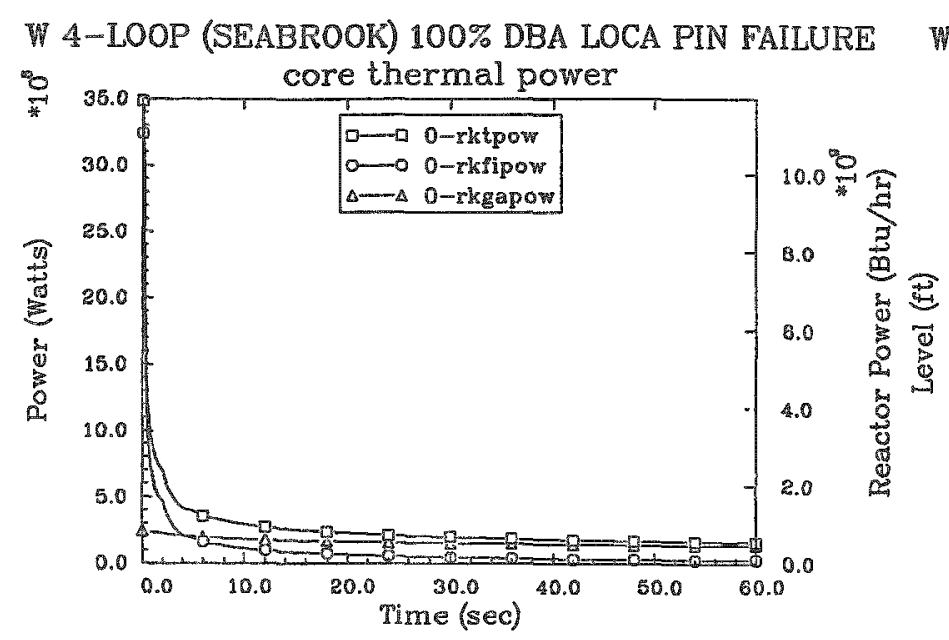

W 4-LOOP (SEABROOK) 100\% DBA LOCA PIN FALURE
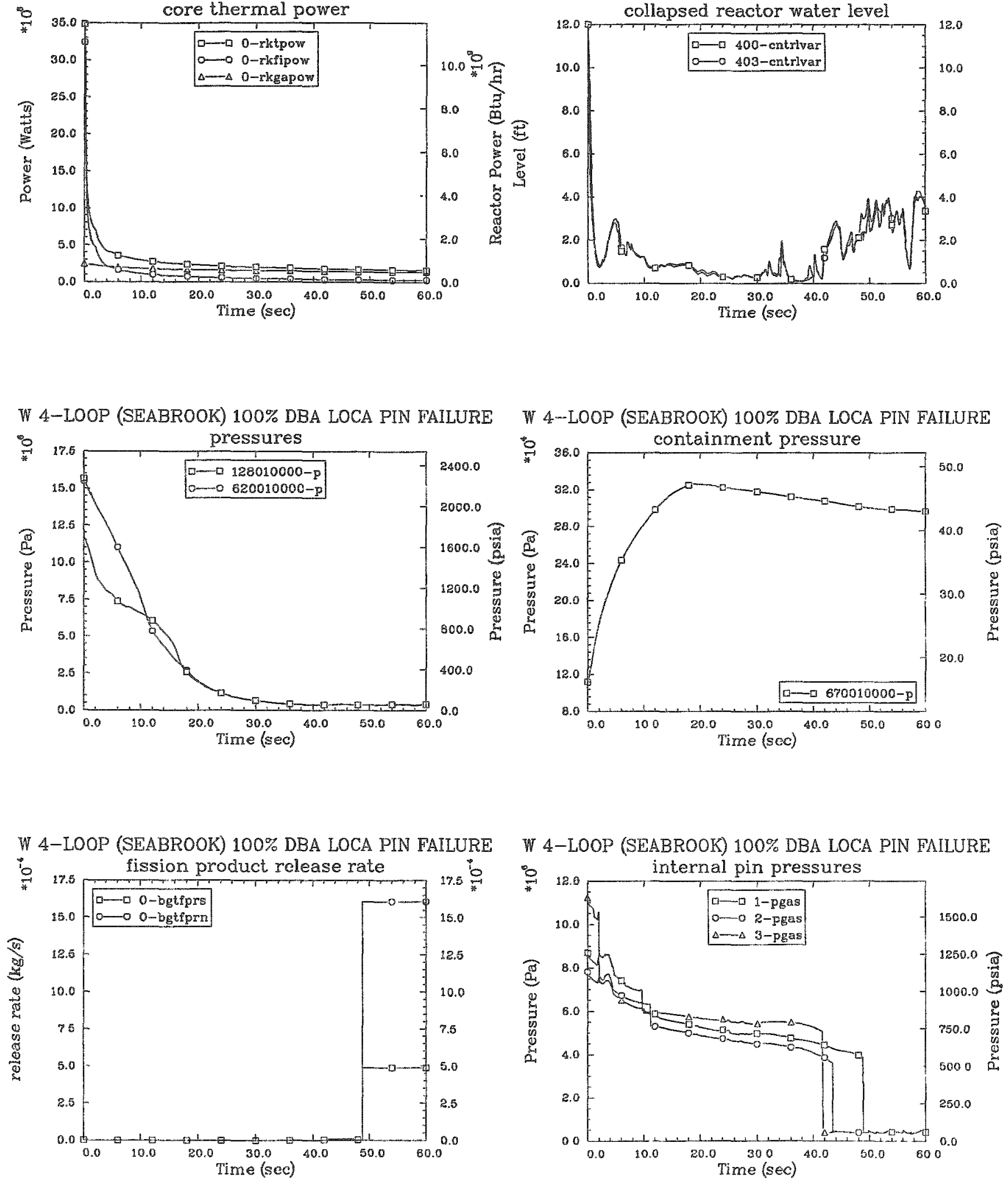

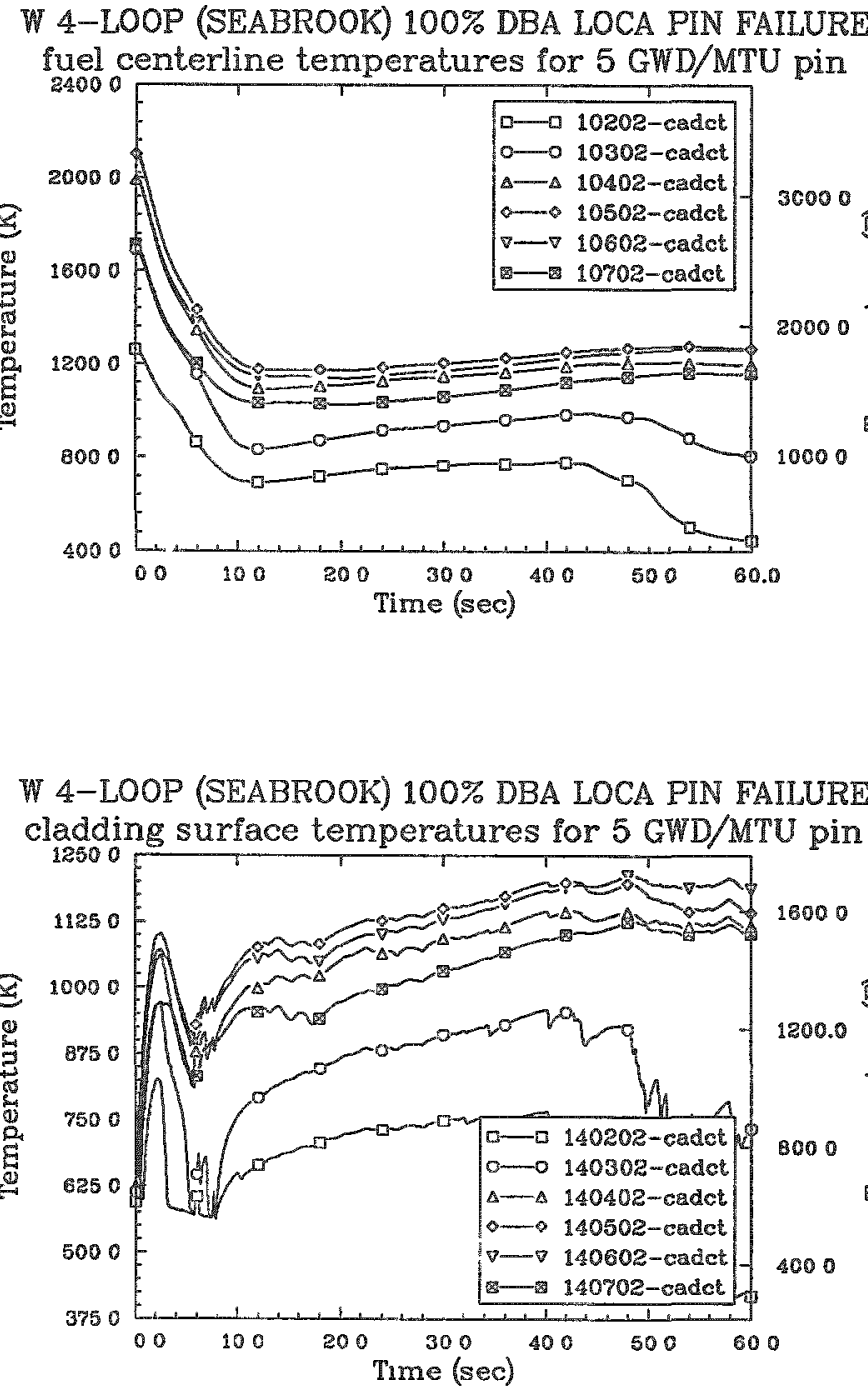

W 4-LOOP (SEABROOK) 100\% DBA LOCA PIN FAILURE

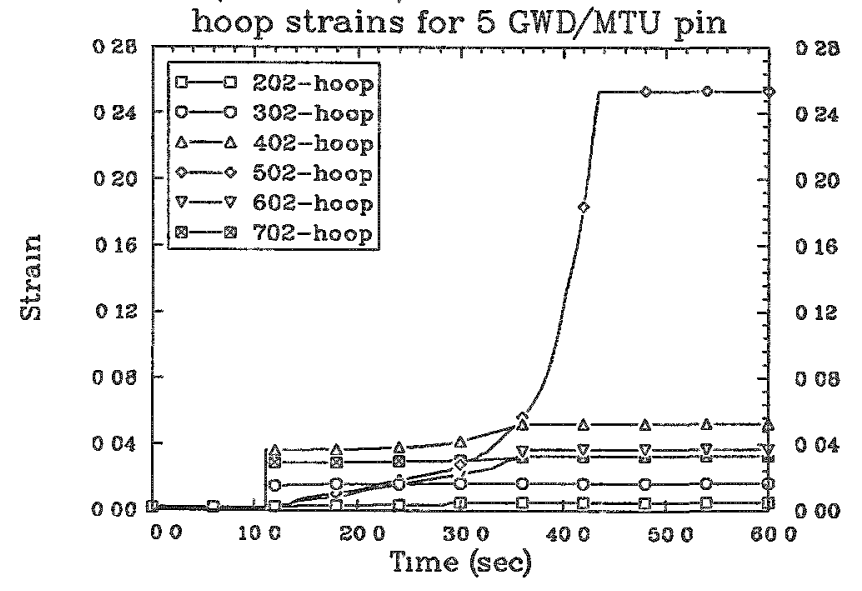

W 4-LOOP (SEABROOK) 100\% DBA LOCA PIN FAILURE fuel centerlne temperatures for $50 \mathrm{GWD} / \mathrm{MTU}$ pin

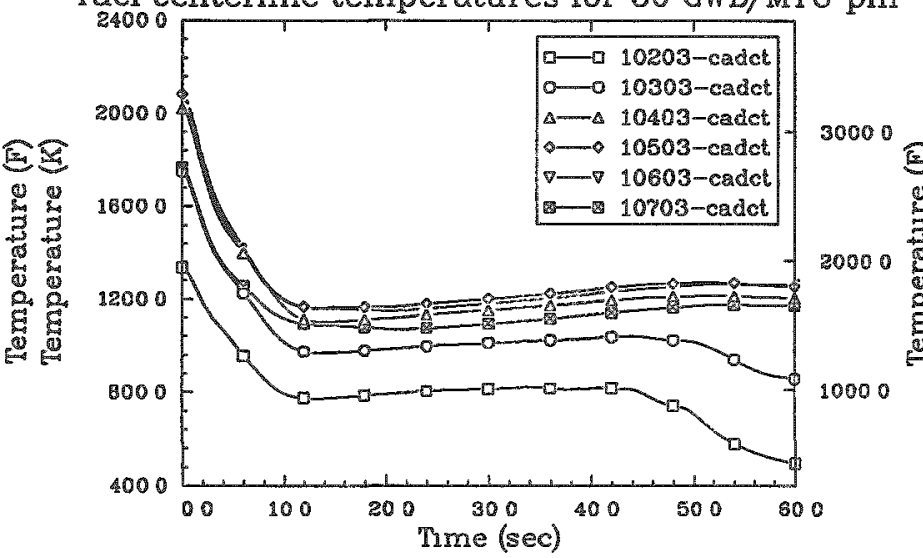

W 4-LOOP (SEABROOK) 100\% DBA LOCA PIN FAILURE cladding surface temperatures for $50 \mathrm{GWD} / \mathrm{MTU}$ pin
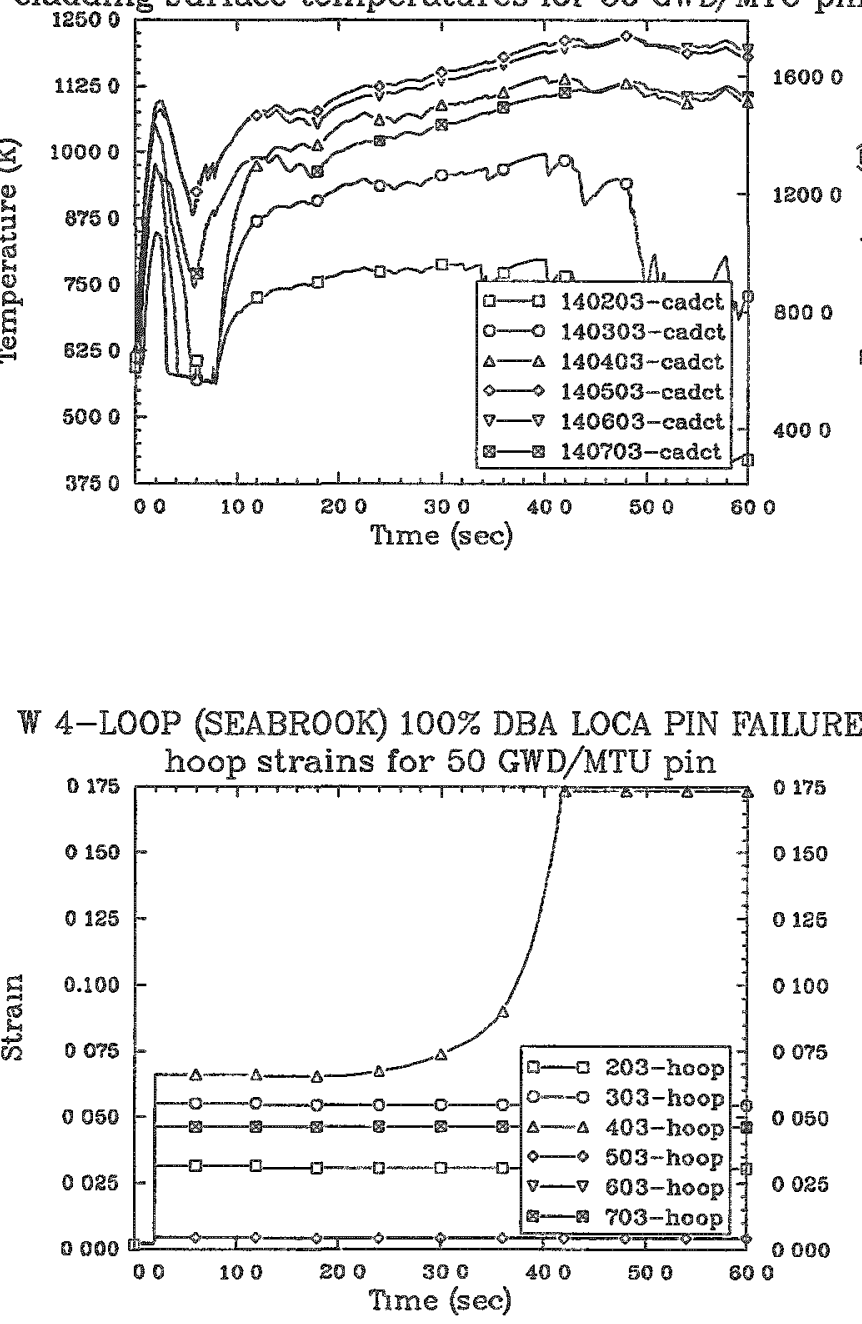

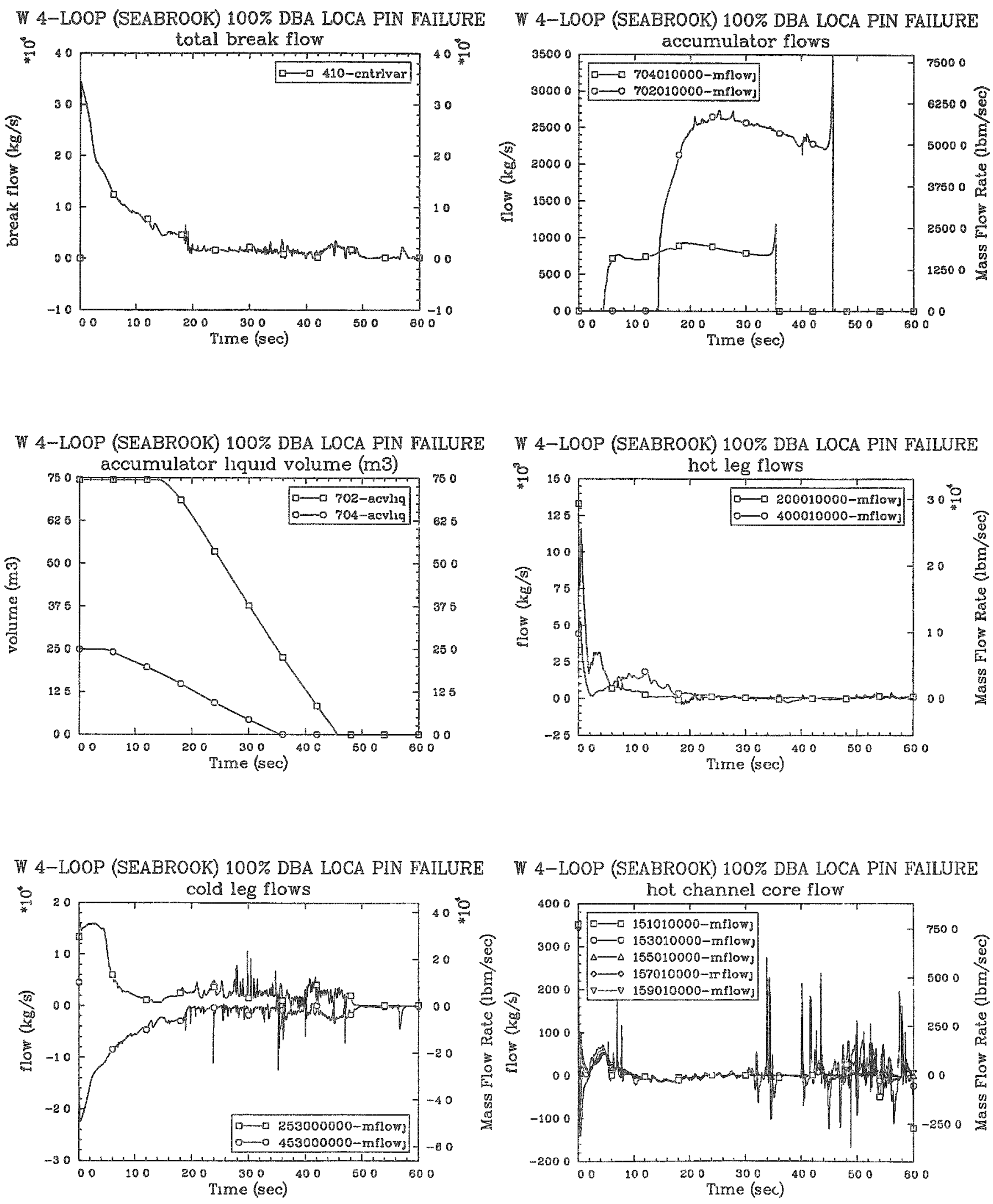
W 4-LOOP (SEABROOK) 100\% DBA LOCA PIN FAILURE bl downcomer void fractions

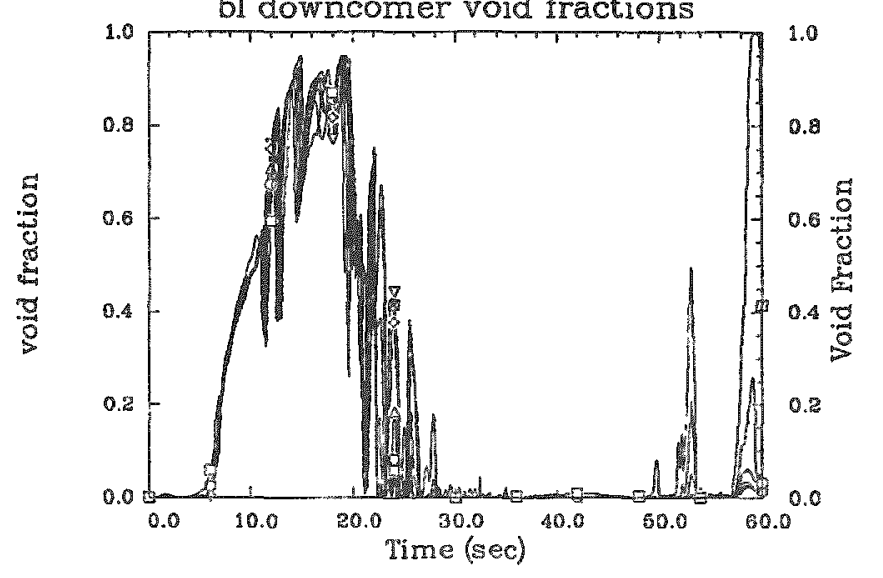

W 4-LOOP (SEABROOK) 100\% DBA LOCA PIN FAILURE

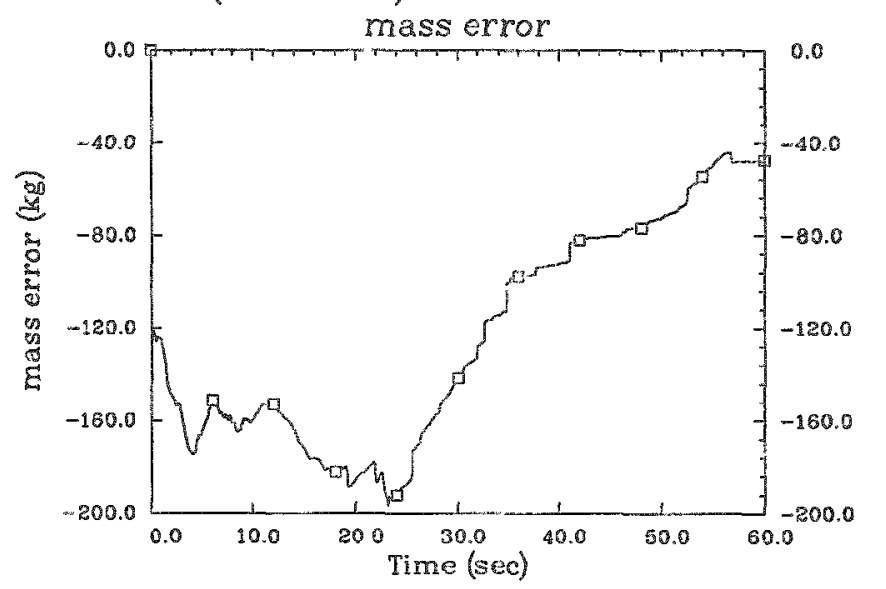

W 4-LOOP (SEABROOK) 100\% DBA LOCA PIN FAILURE

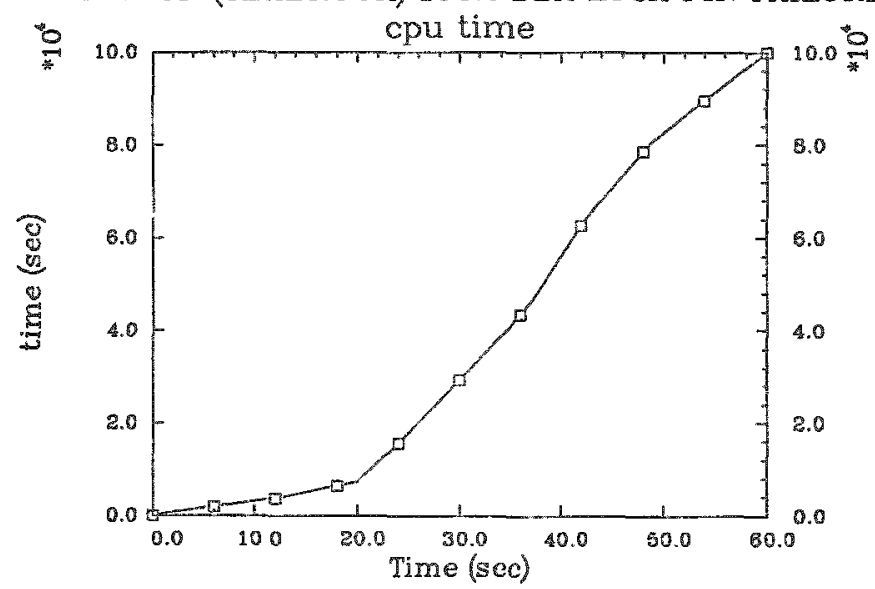

W 4-LOOP (SEABROOK) 100\% DBA LOCA PIN FAILURE
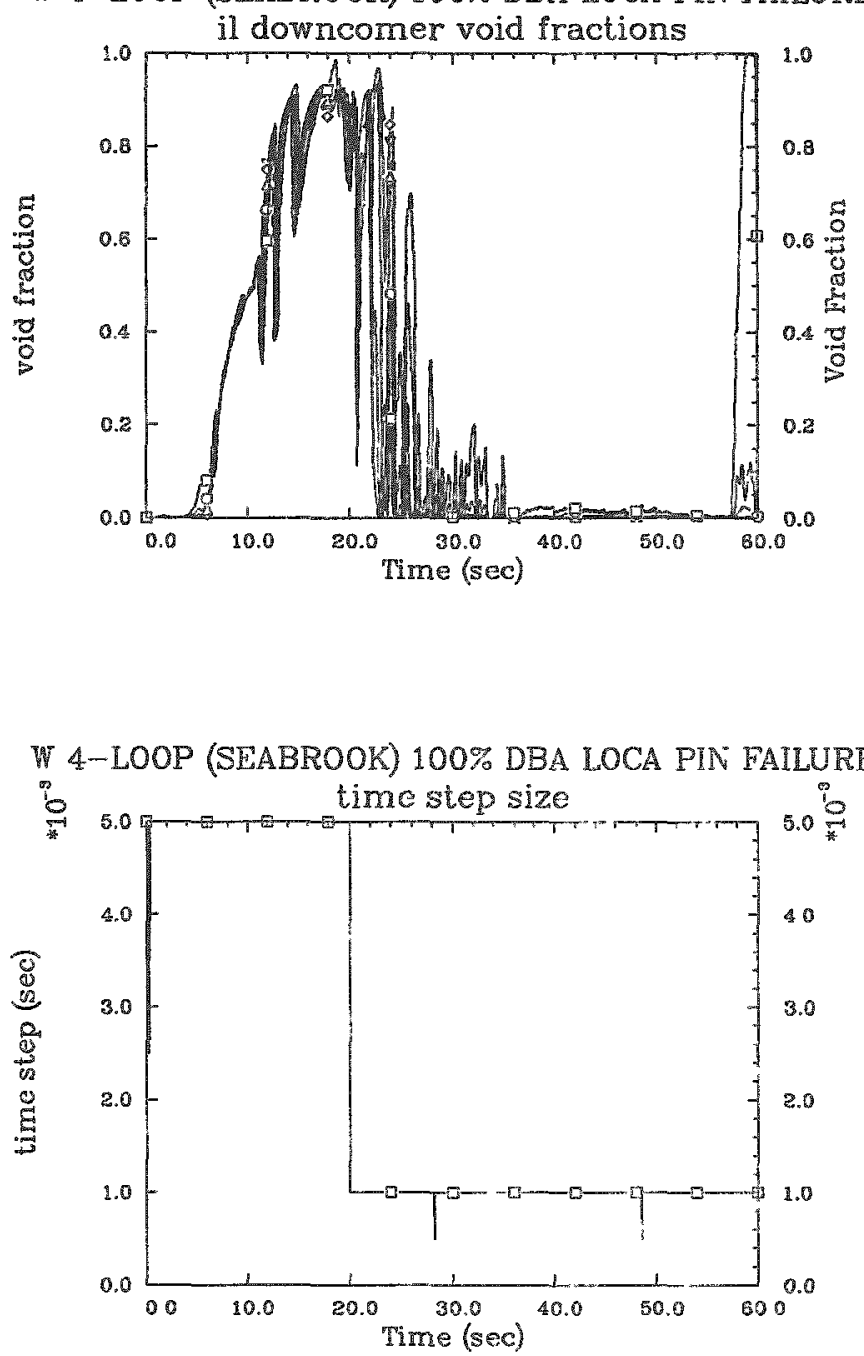


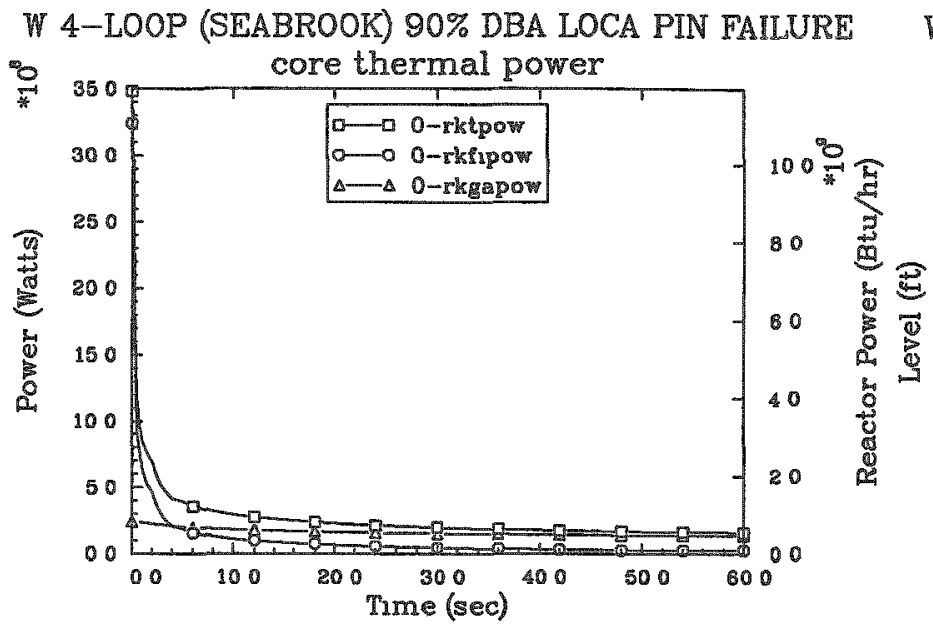

W 4-LOOP (SEABROOK) 90\% DBA LOCA PIN FAILURE collapsed reactor water level
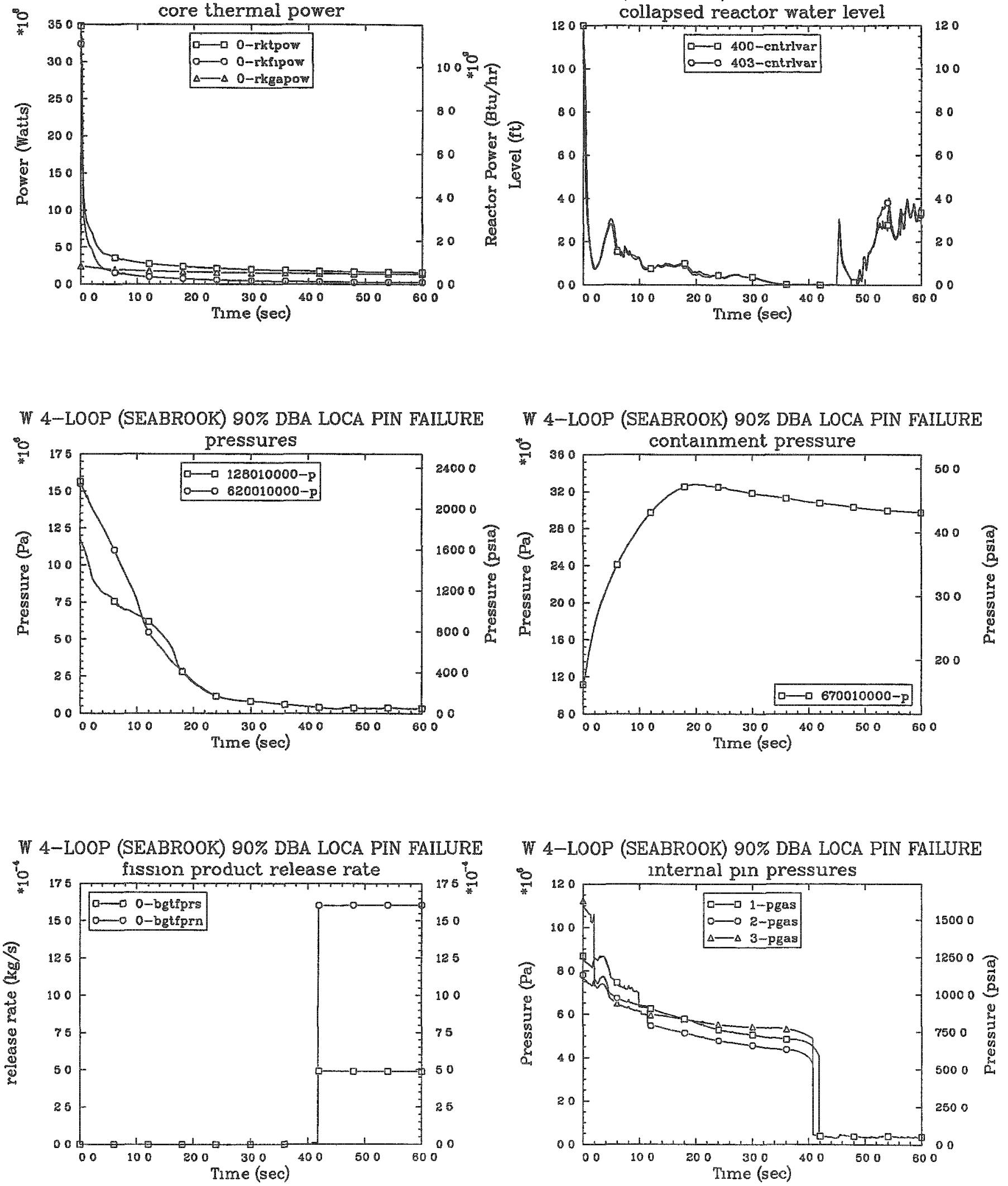
W 4-LOOP (SEABROOK) 90\% DBA LOCA PIN FAILURE fuel centerline temperatures for 5 GWD/MTU pin

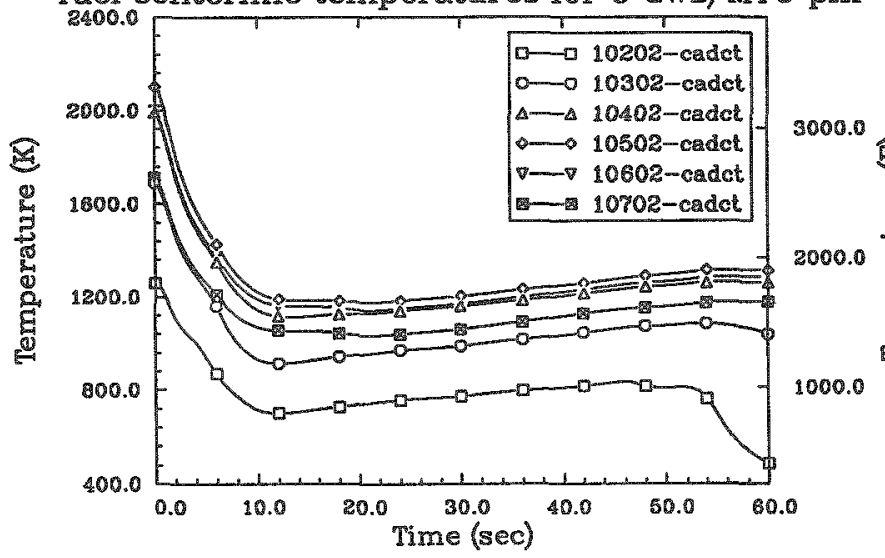

W 4-LOOP (SEABROOK) 90\% DBA LOCA PIN FAILURE cladding surface temperatures for $5 \mathrm{GWD} / \mathrm{MTU}$ pin

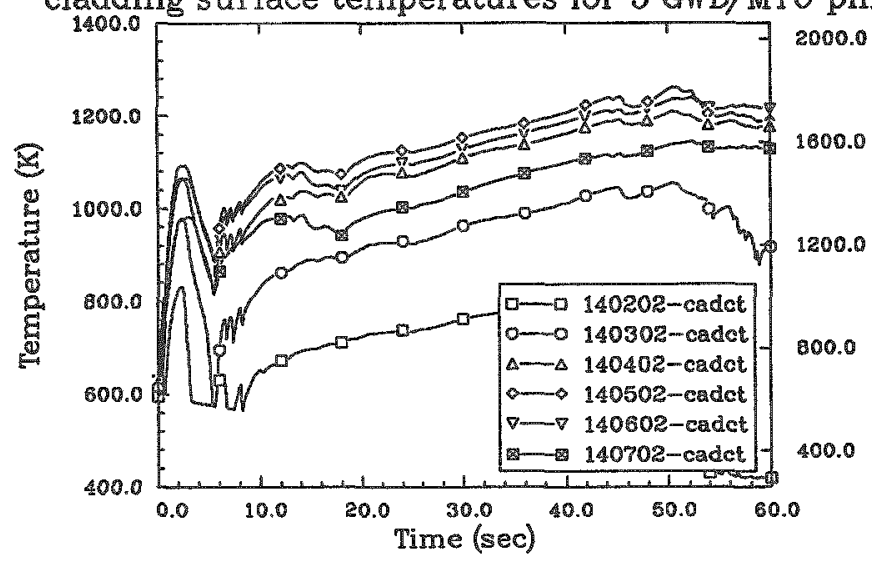

W 4-LOOP (SEABROOK) 90\% DBA LOCA PIN FAILURE

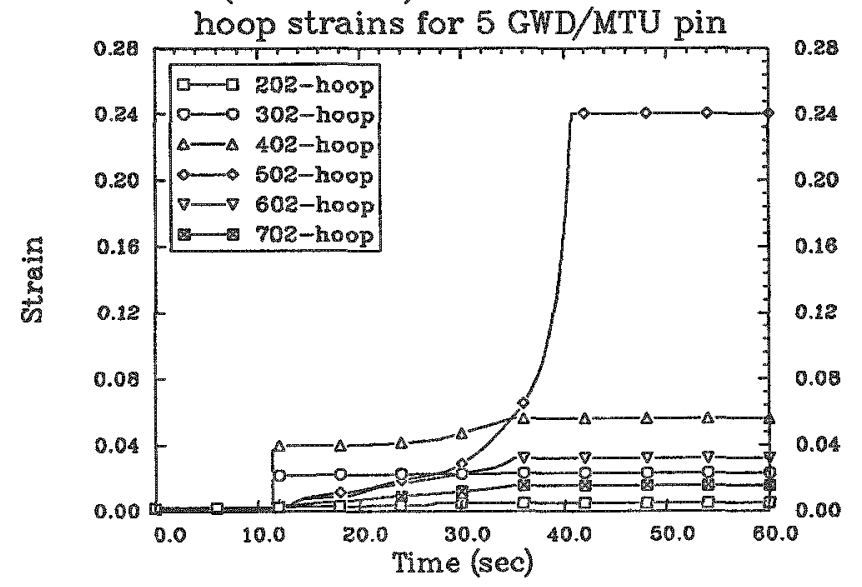

W 4-LOOP (SEABROOK) 90\% DBA LOCA PIN FAILURE fuel centerline temperatures for $50 \mathrm{GWD} / \mathrm{MTU}$ pin

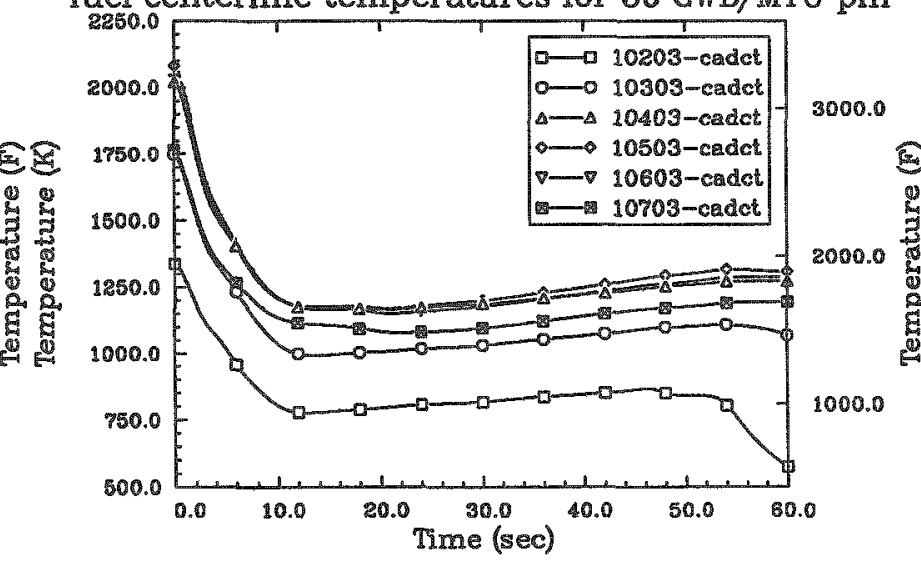

W 4-LOOP (SEABROOK) 90\% DBA LOCA PIN FAILURE cladding surface temperatures for $50 \mathrm{GWD} / \mathrm{MTU}$ pin.

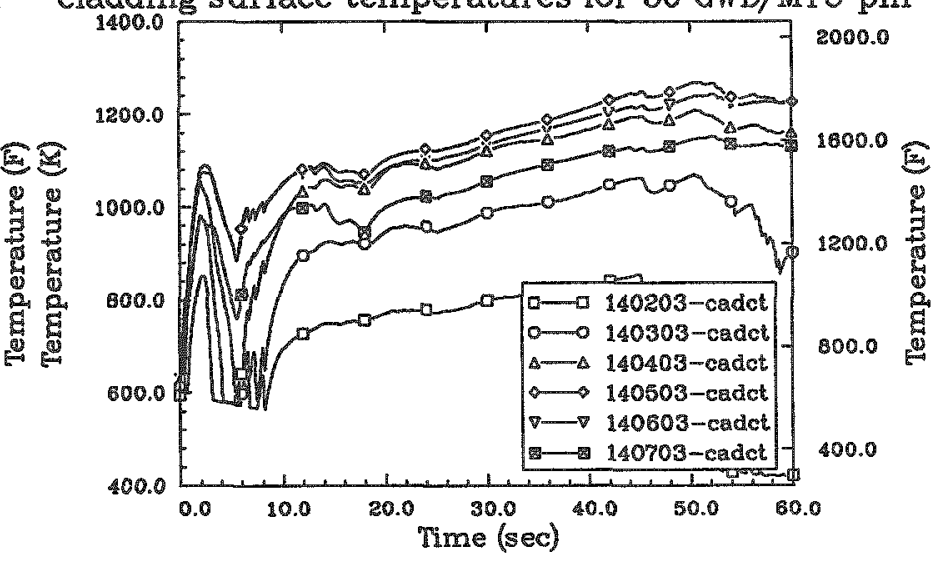

W 4-LOOP (SEABROOK) 90\% DBA LOCA PIN FAILURE

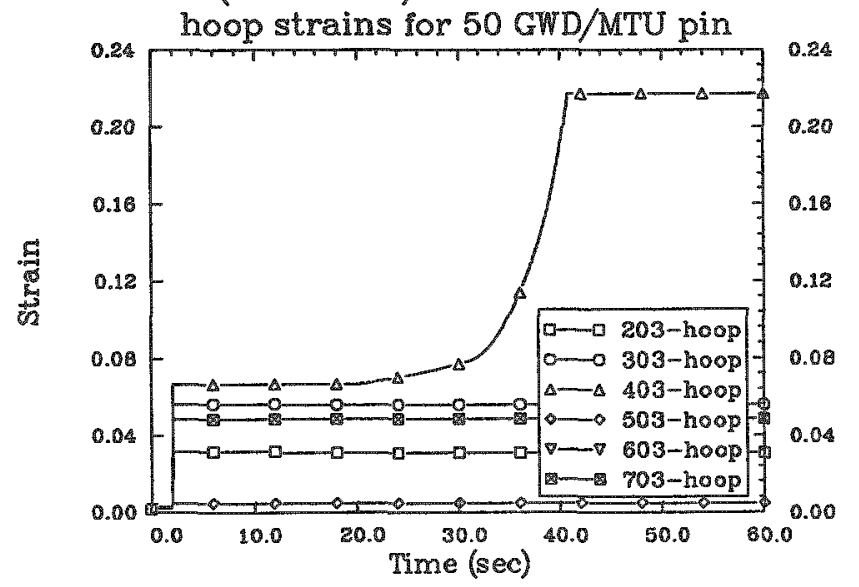



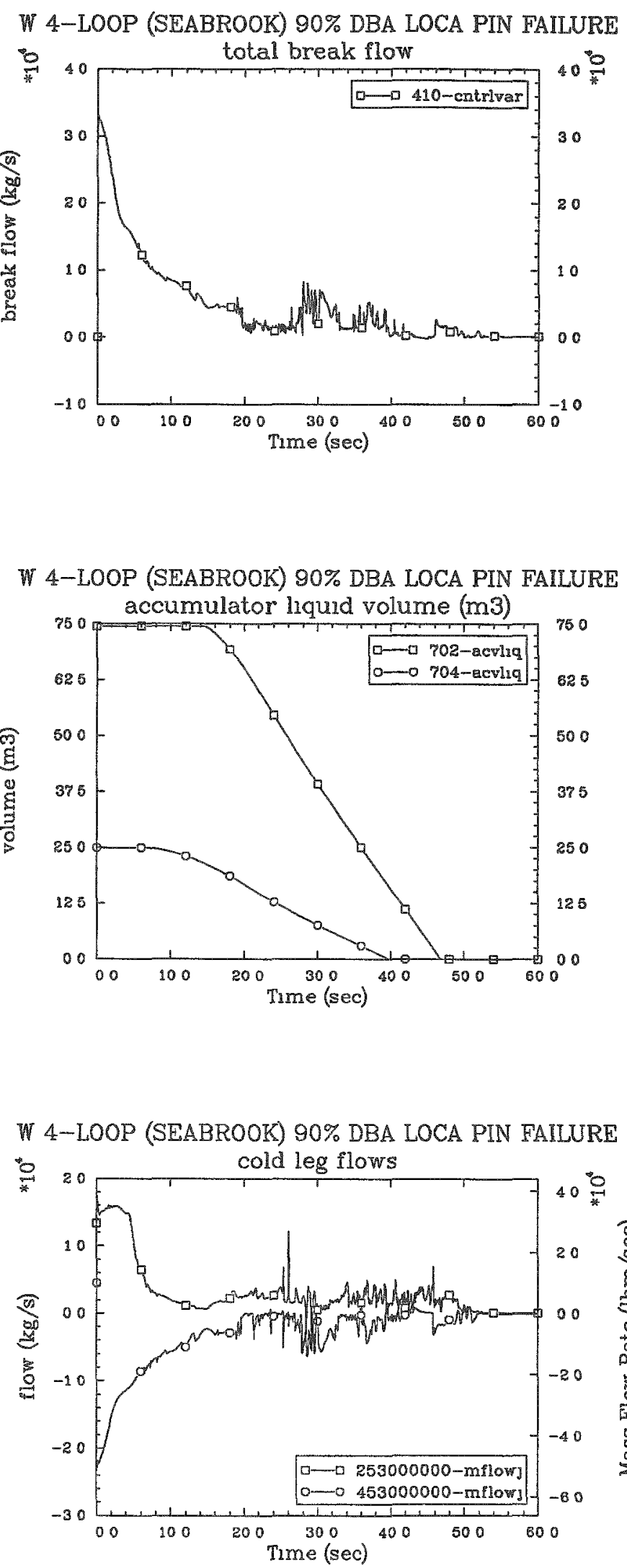
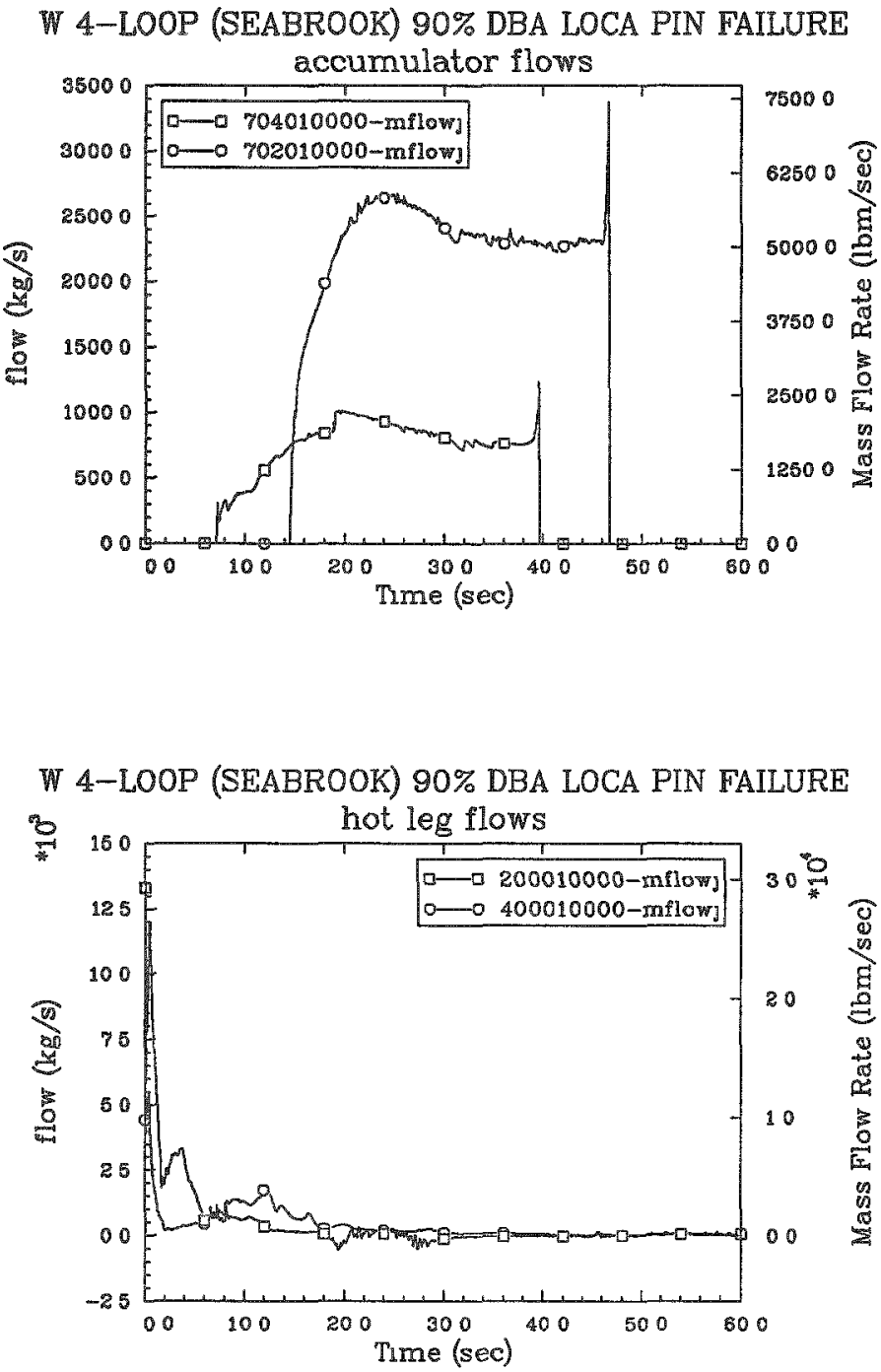

W 4-LOOP (SEABROOK) 90\% DBA LOCA PIN FAILURE

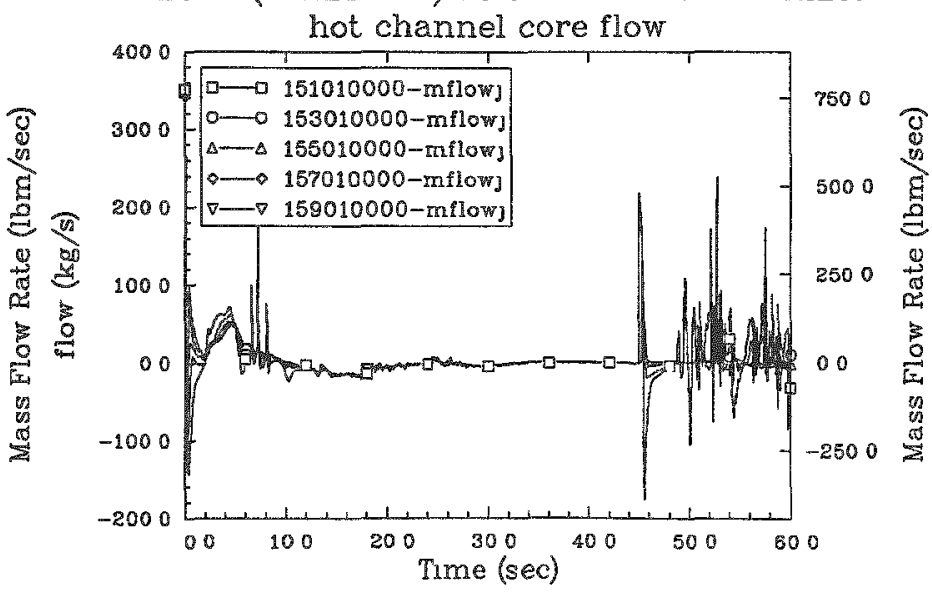




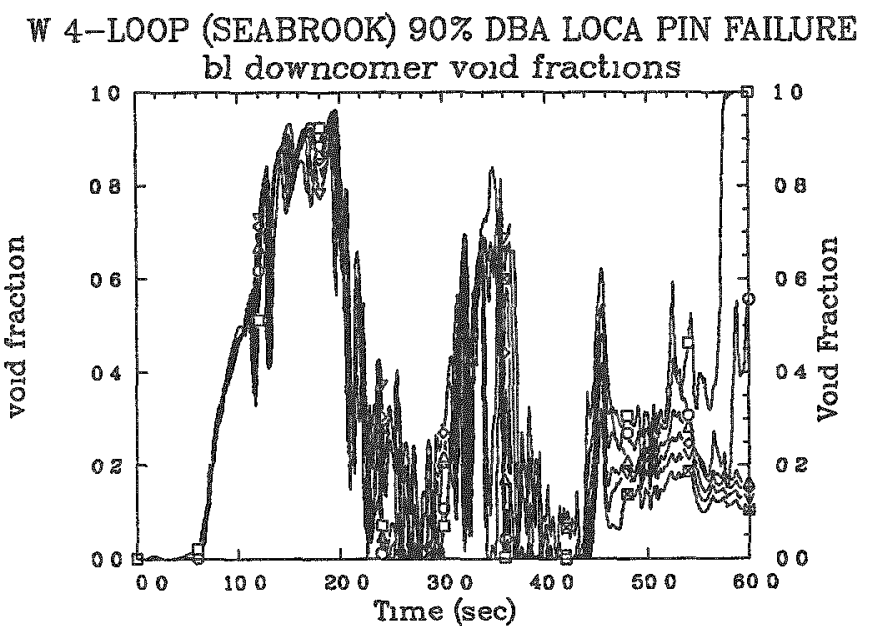

W 4-LOOP (SEABROOK) 90\% DBA LOCA PIN FAILURE

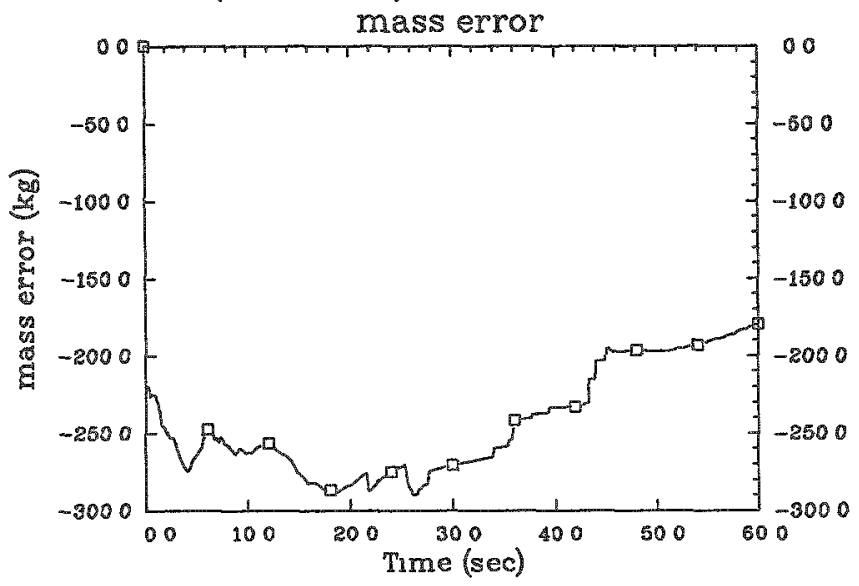

W 4-LOOP (SEABROOK) 90\% DBA LOCA PIN FAILURE

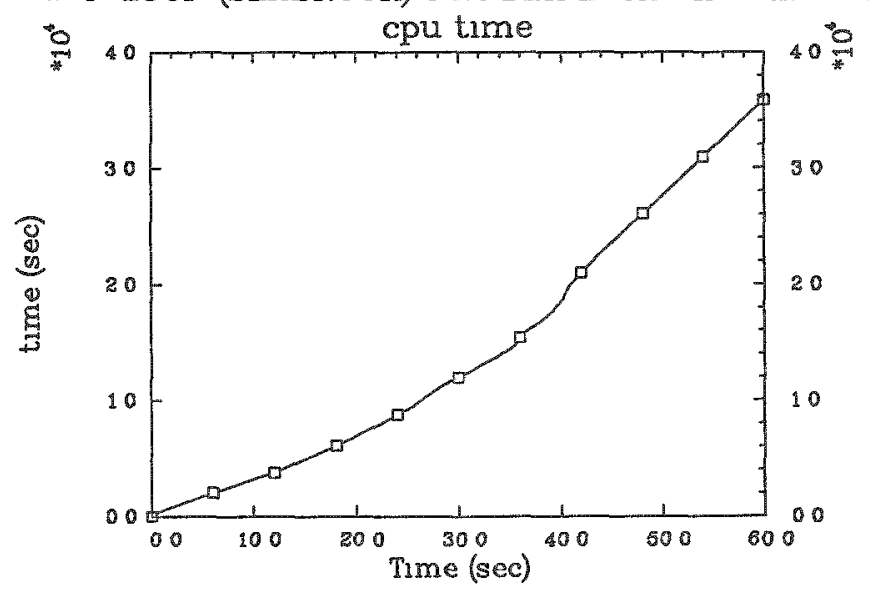

W 4-LOOP (SEABROOK) 90\% DBA LOCA PIN FAILURE
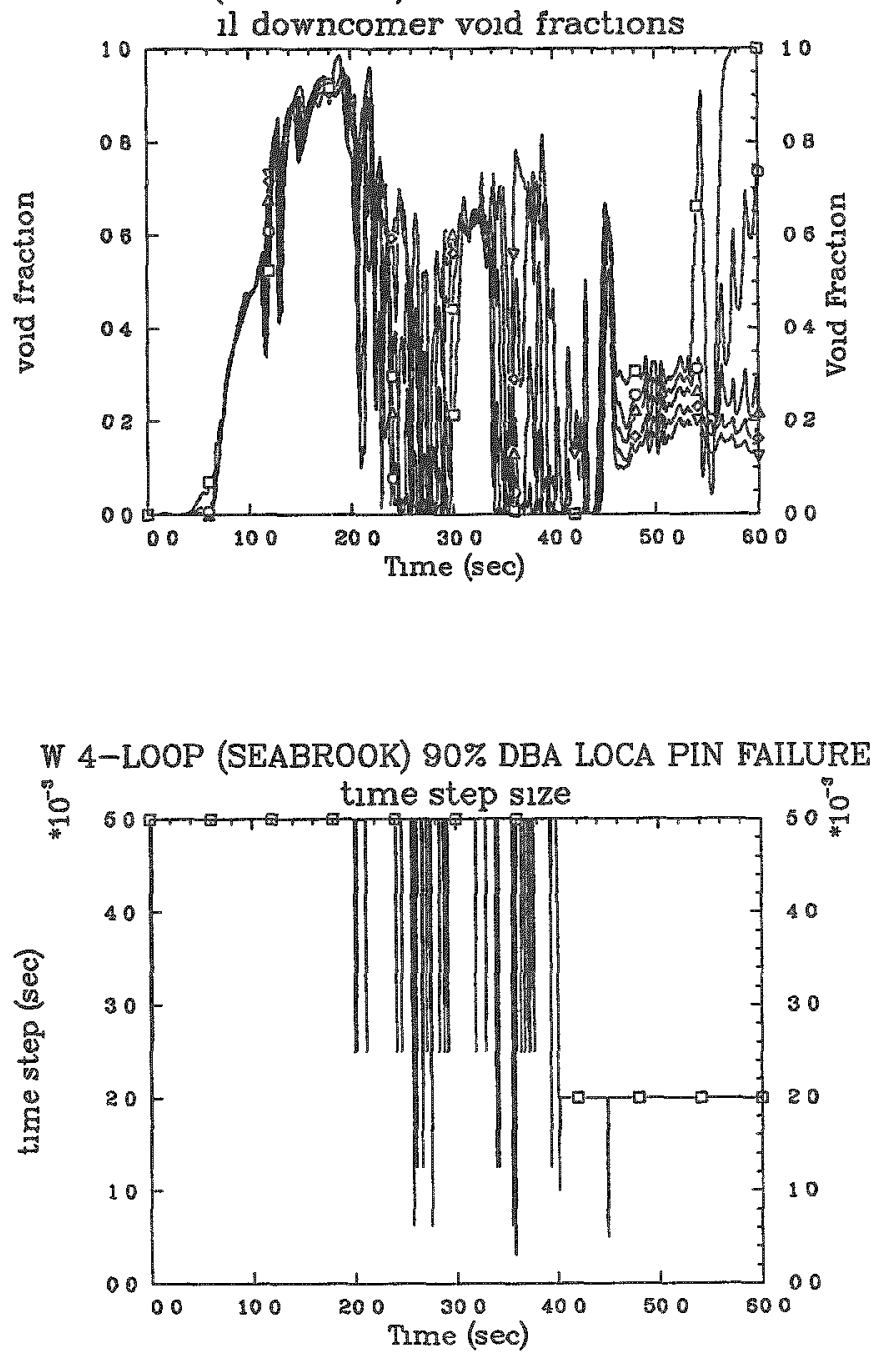

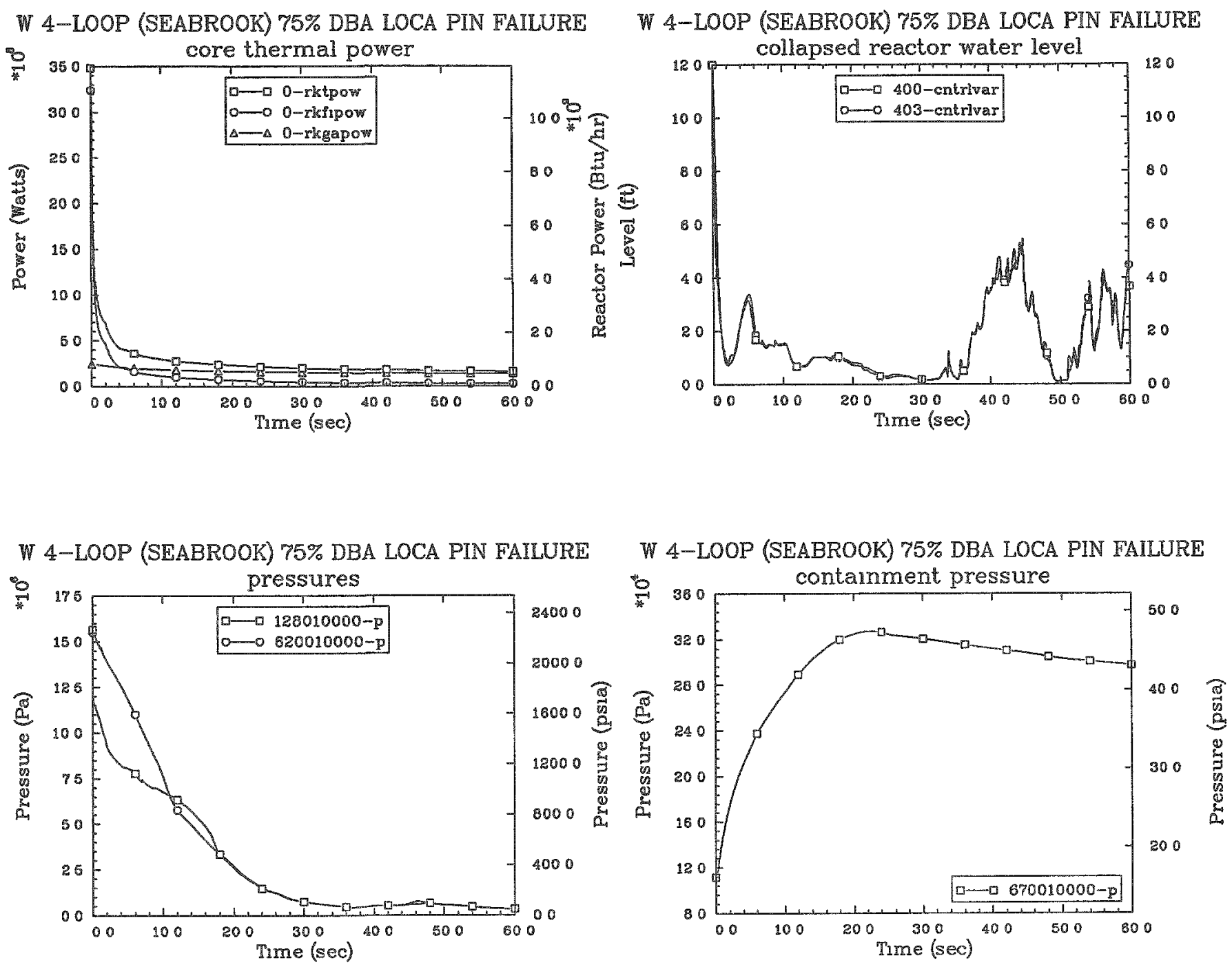

W 4-LOOP (SEABROOK) 75\% DBA LOCA PIN FAILURE

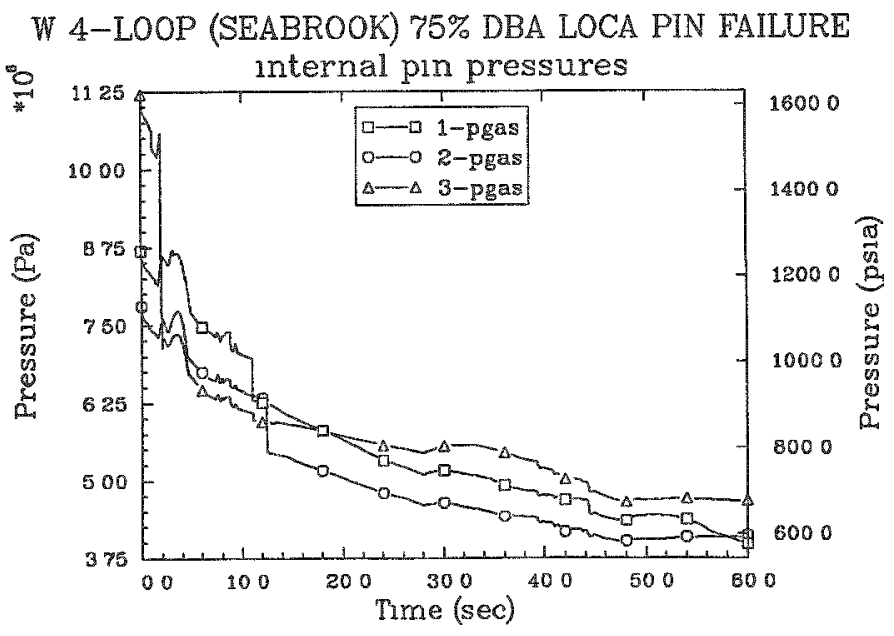


W 4-LOOP (SEABROOK) 75\% DBA LOCA PIN FAILURE fuel centerline temperatures for $5 \mathrm{GWD} / \mathrm{MTU}$ pin

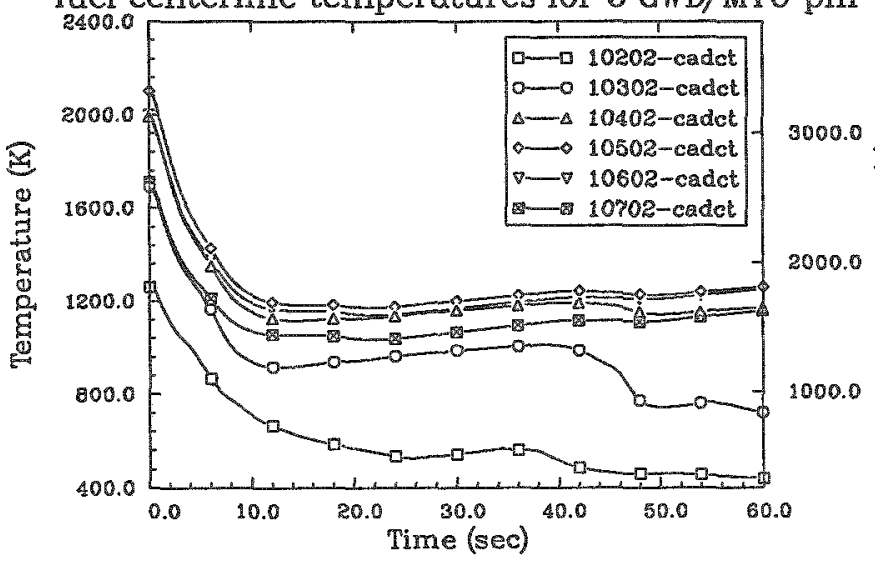

W 4-LOOP (SEABROOK) 75\% DBA LOCA PIN FAILURE cladding surface temperatures for 5 GWD/MTU pin

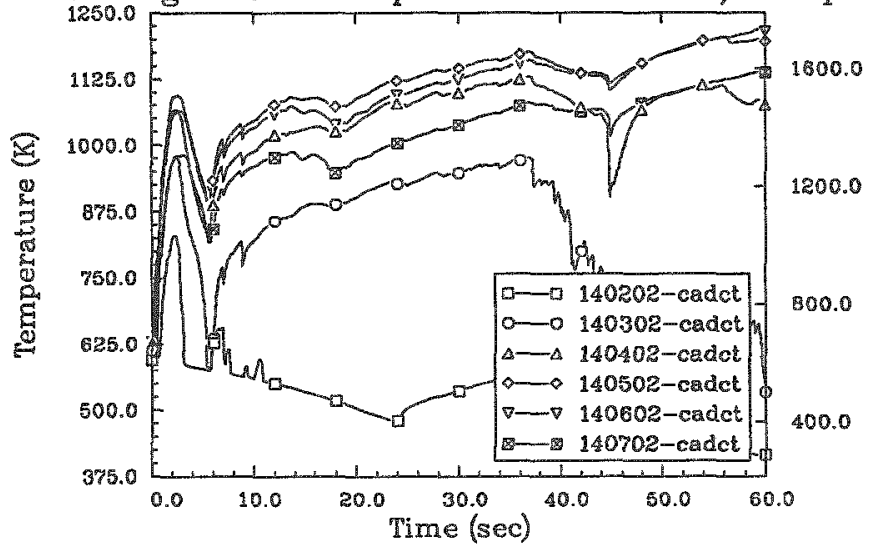

W 4-LOOP (SEABROOK) 75\% DBA LOCA PIN FAILURE

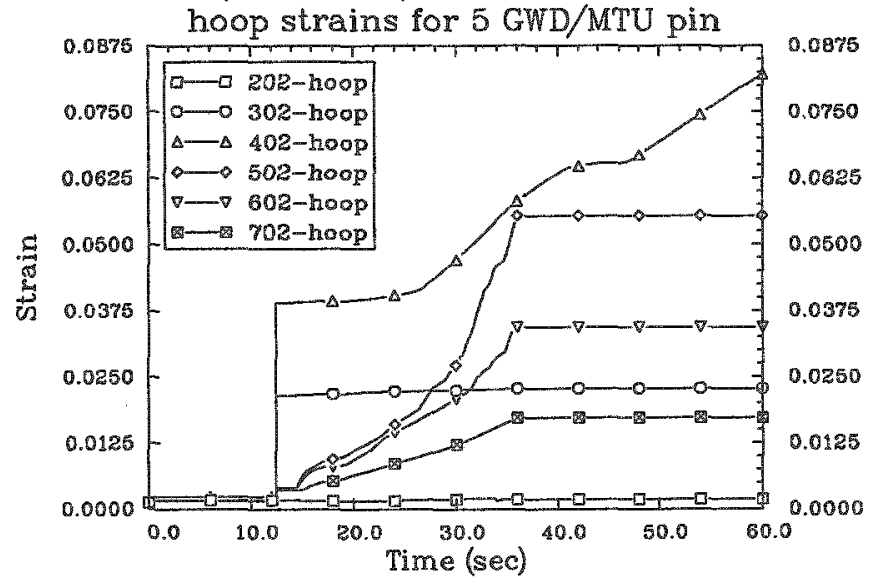

W 4-LOOP (SEABROOK) 75\% DBA LOCA PIN FAILURE fuel centerline temperatures for $50 \mathrm{GWD} / \mathrm{MTU}$ pin

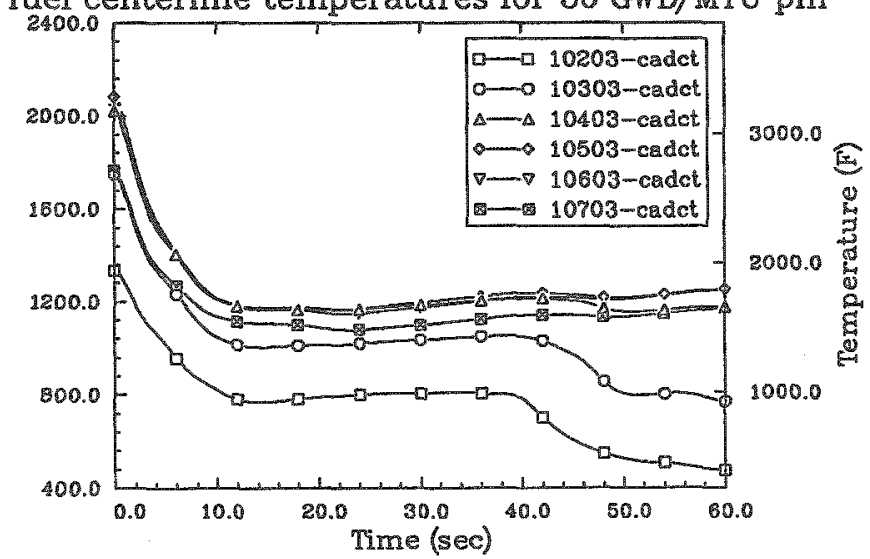

W 4-LOOP (SEABROOK) 75\% DBA LOCA PIN FAILURE cladding surface temperatures for $50 \mathrm{GWD} / \mathrm{MTU}$ pin

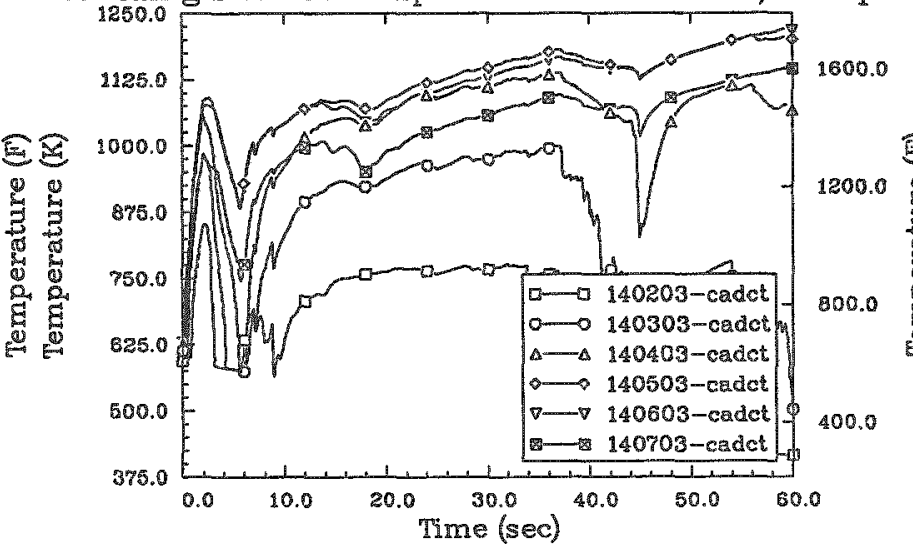

W 4-LOOP (SEABROOK) 75\% DBA LOCA PIN FAILURE

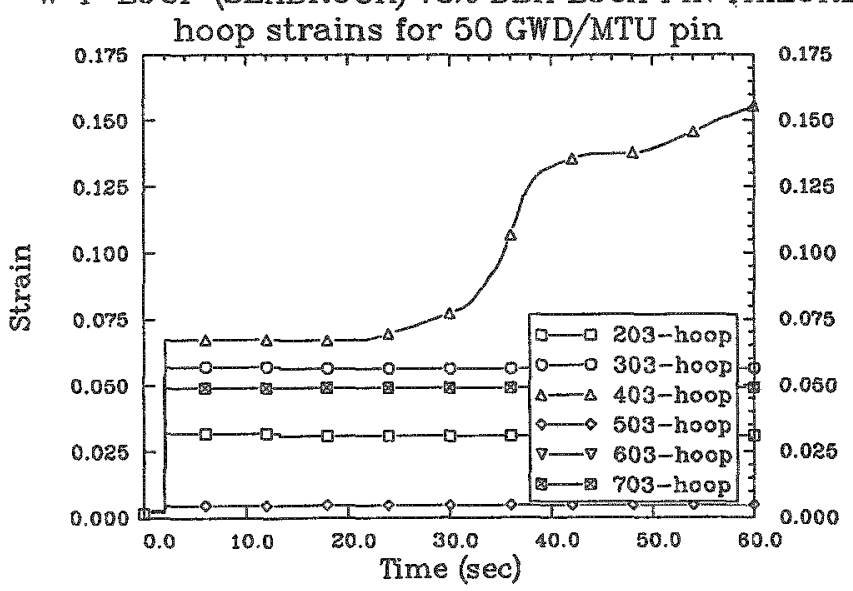



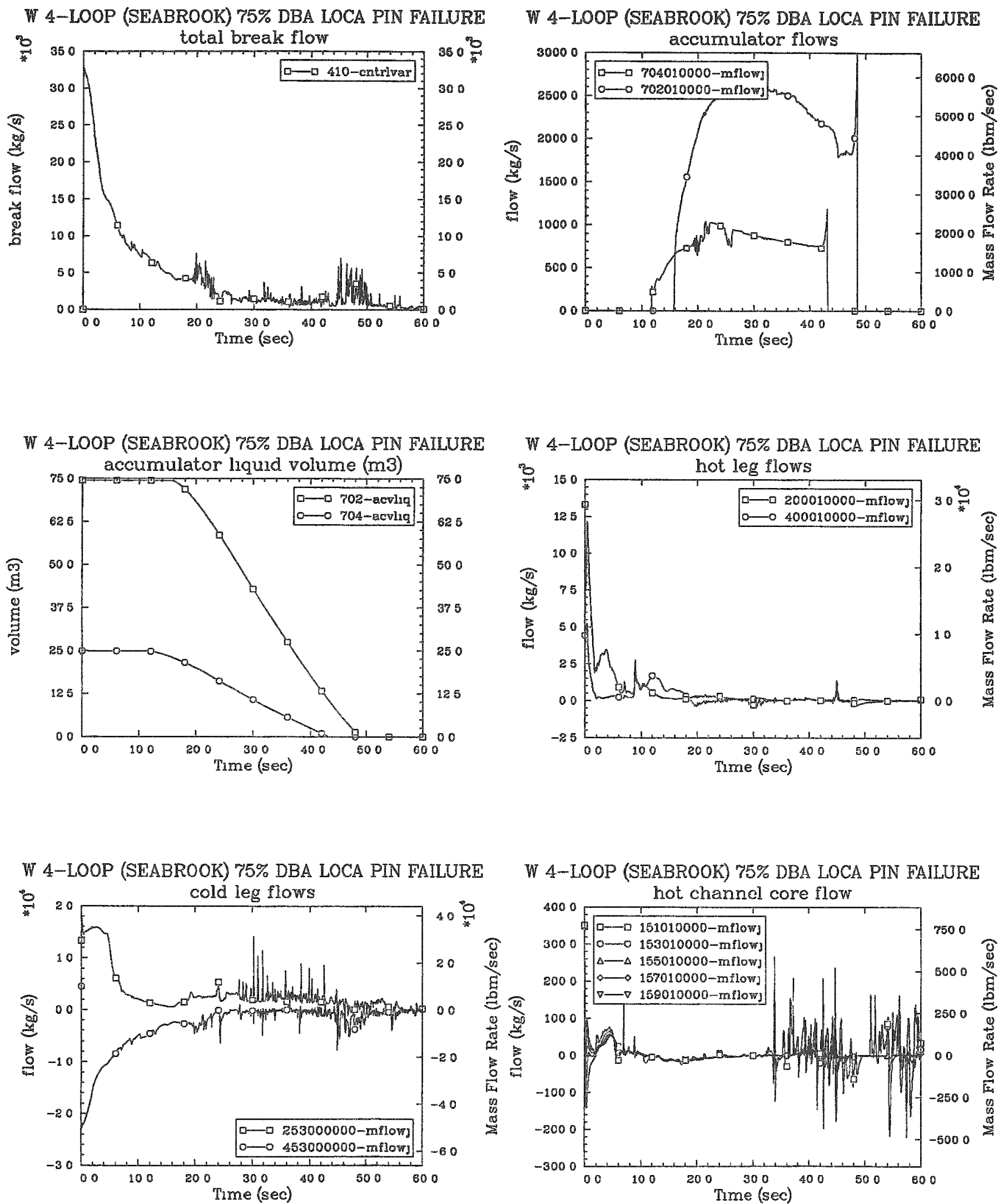

W 4-LOOP (SEABROOK) 75\% DBA LOCA PIN FAILURE

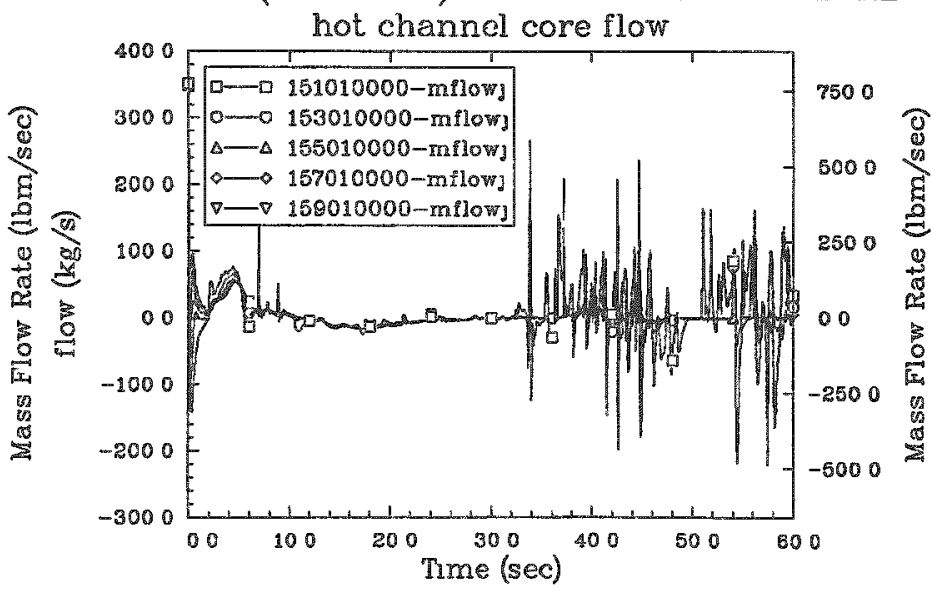


W 4-LOOP (SEABROOK) 75\% DBA LOCA PIN FAILURE

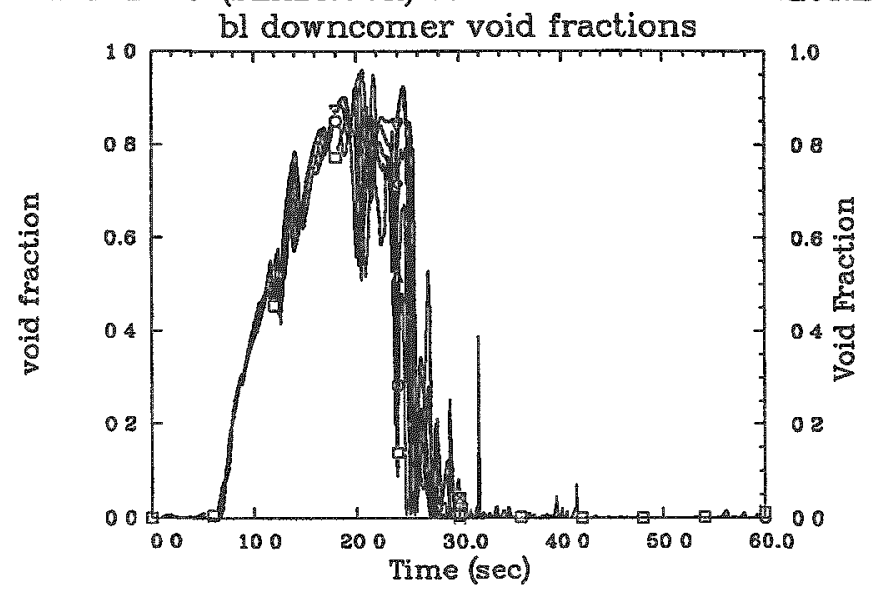

W 4-LOOP (SEABROOK) 75\% DBA LOCA PIN FAILURE

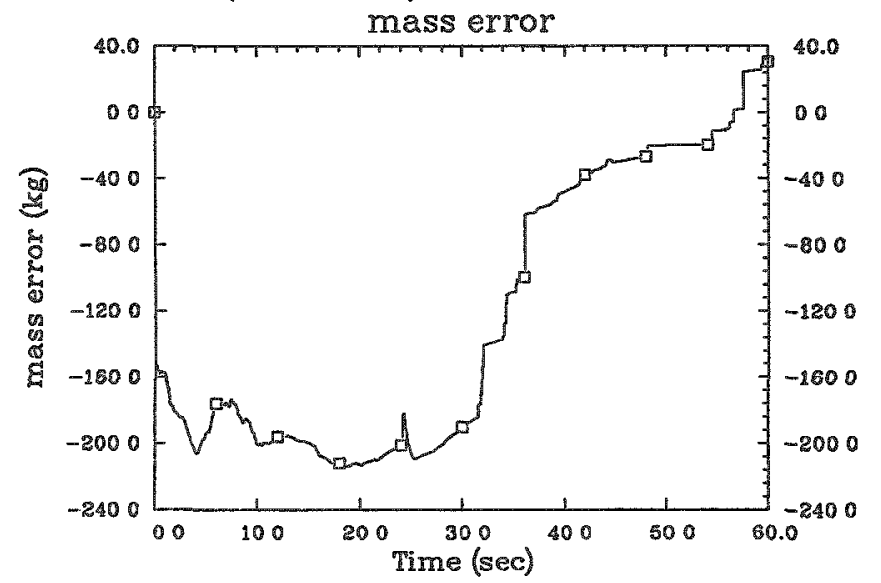

W 4-LOOP (SEABROOK) 75\% DBA LOCA PIN FAILURE

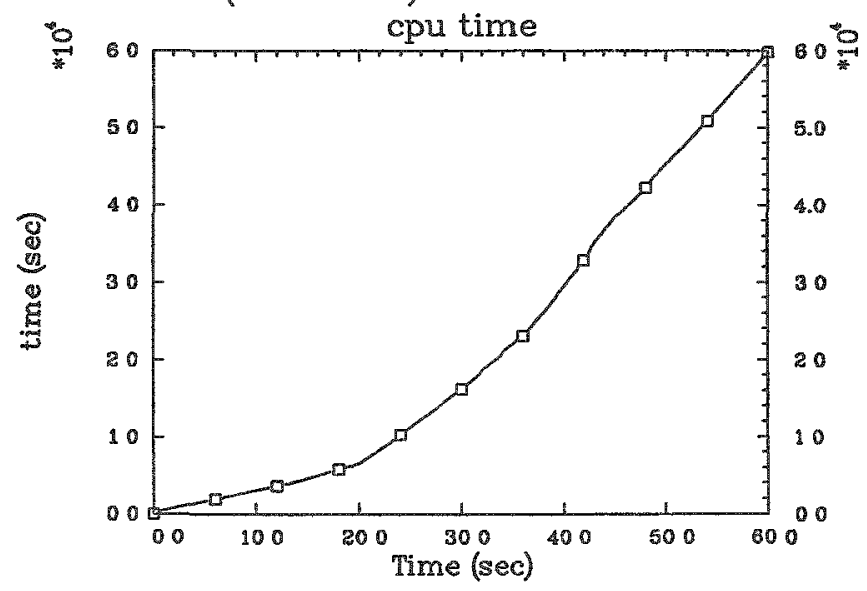

W 4-LOOP (SEABROOK) 75\% DBA LOCA PIN FAILURE

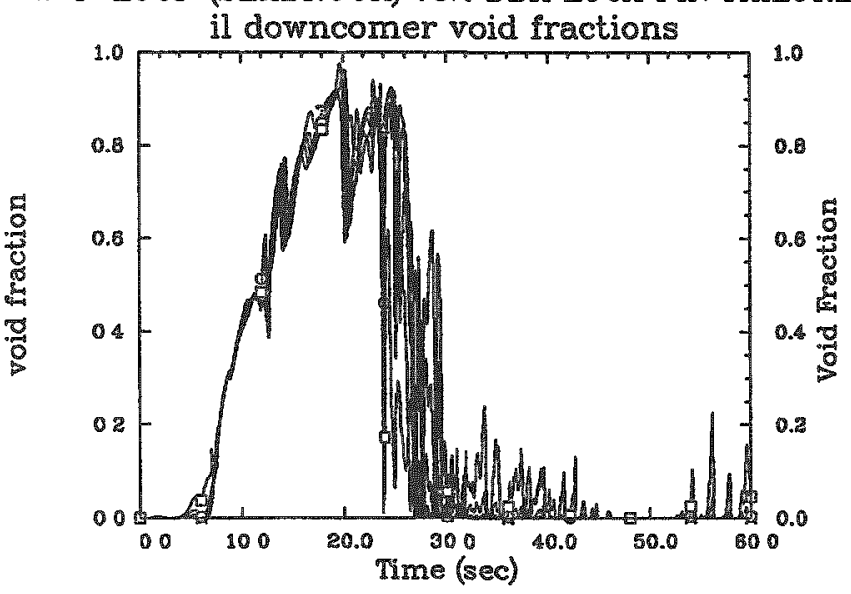

W 4-LOOP (SEABROOK) 75\% DBA LOCA PIN FAILURE

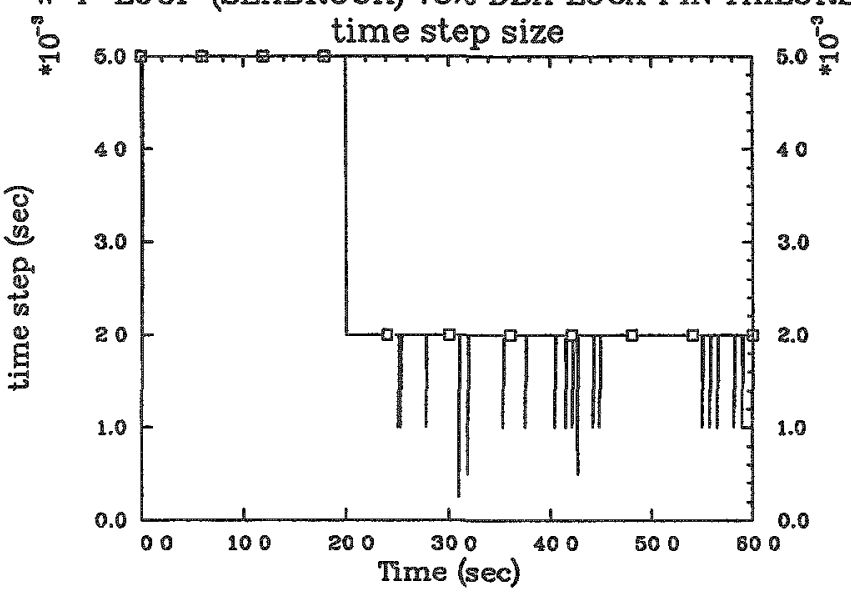




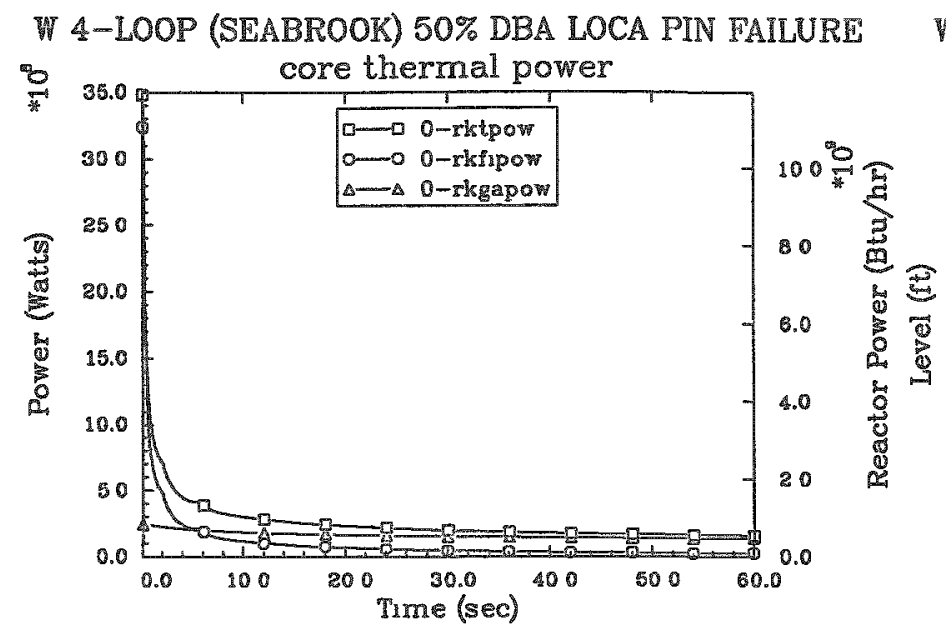

W 4-LOOP (SEABROOK) 50\% DBA LOCA PIN FAILURE
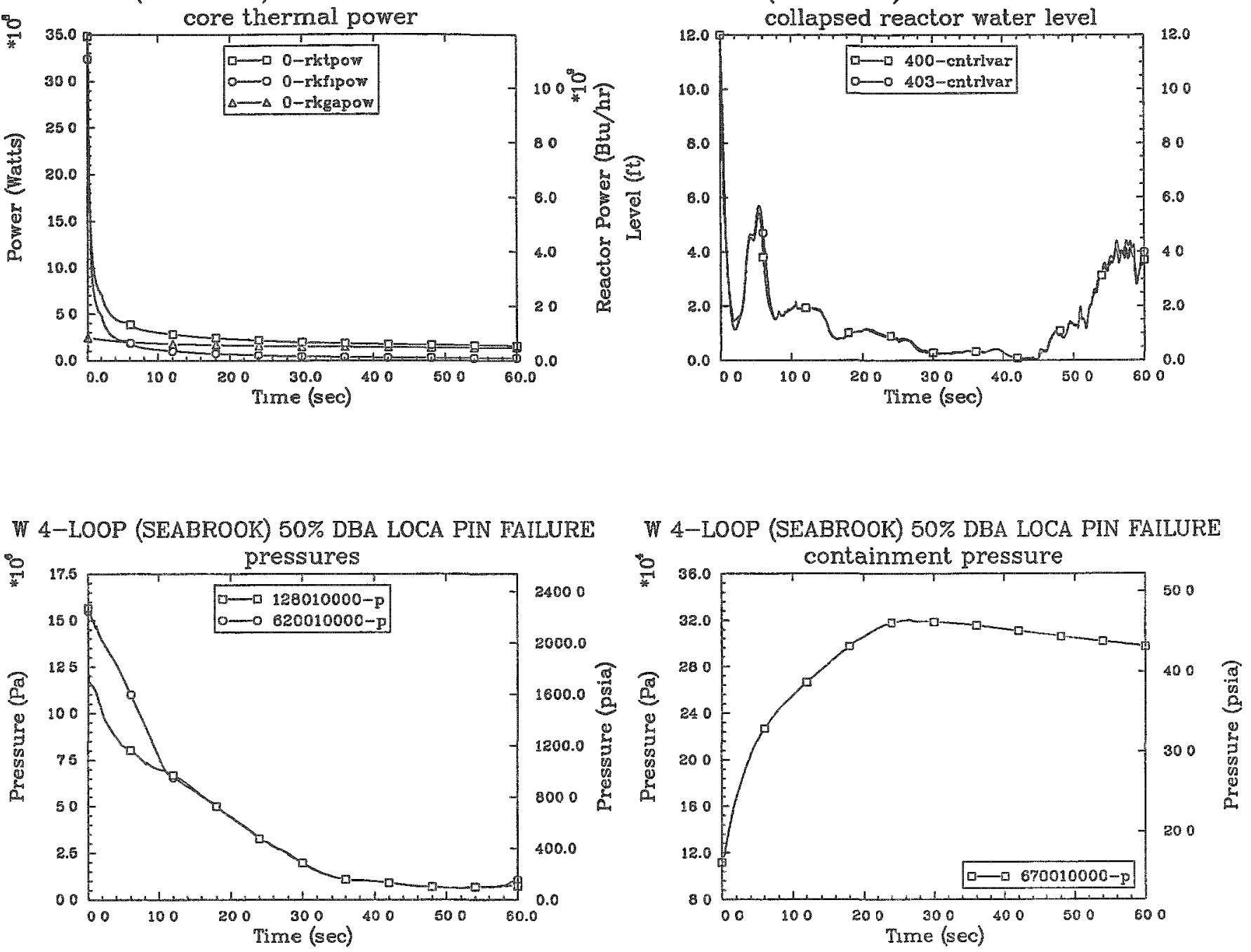

W 4-LOOP (SEABROOK) 50\% DBA LOCA PIN FAILURE
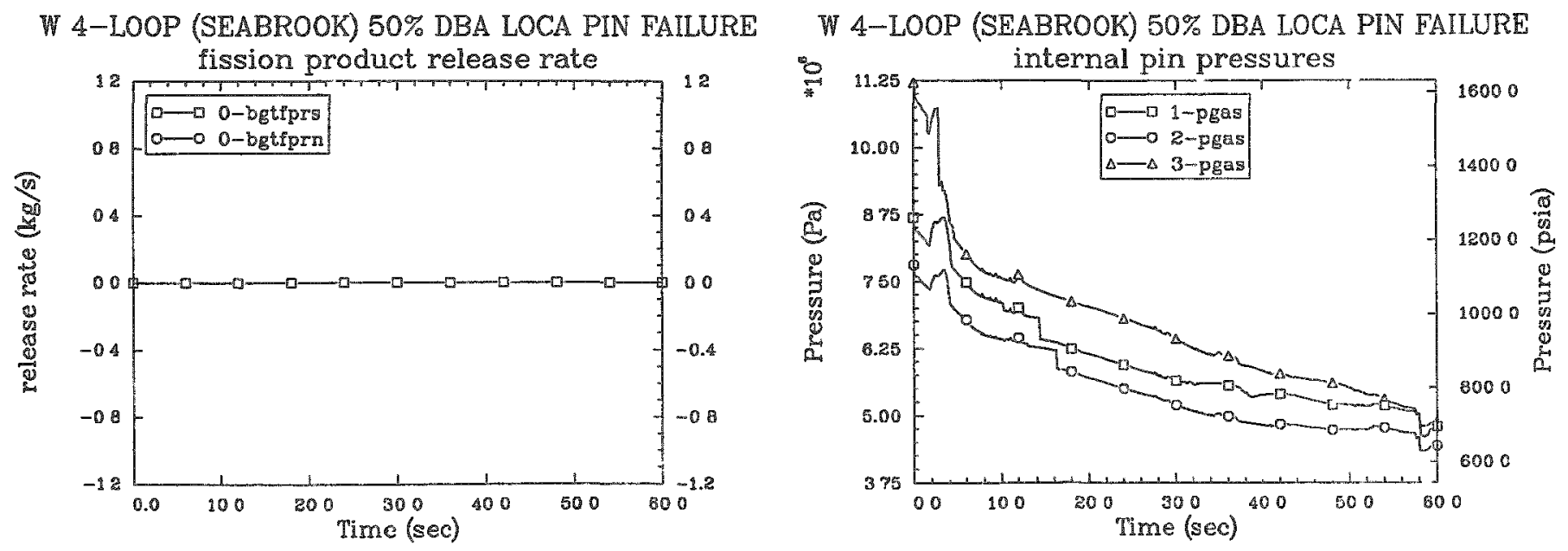


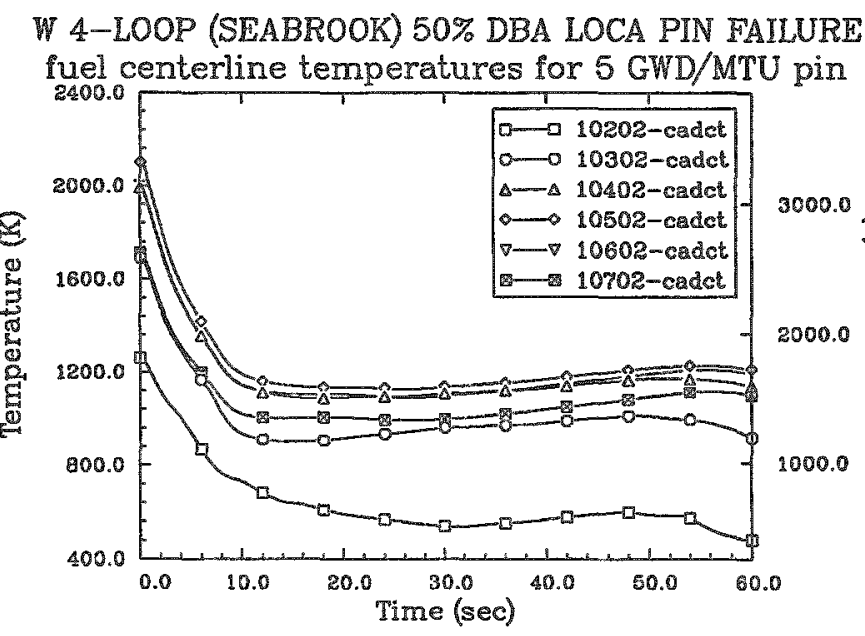

W 4-LOOP (SEABROOK) 50\% DBA LOCA PIN FAILURE cladding surface temperatures for $5 \mathrm{GWD} / \mathrm{MTU}$ pin

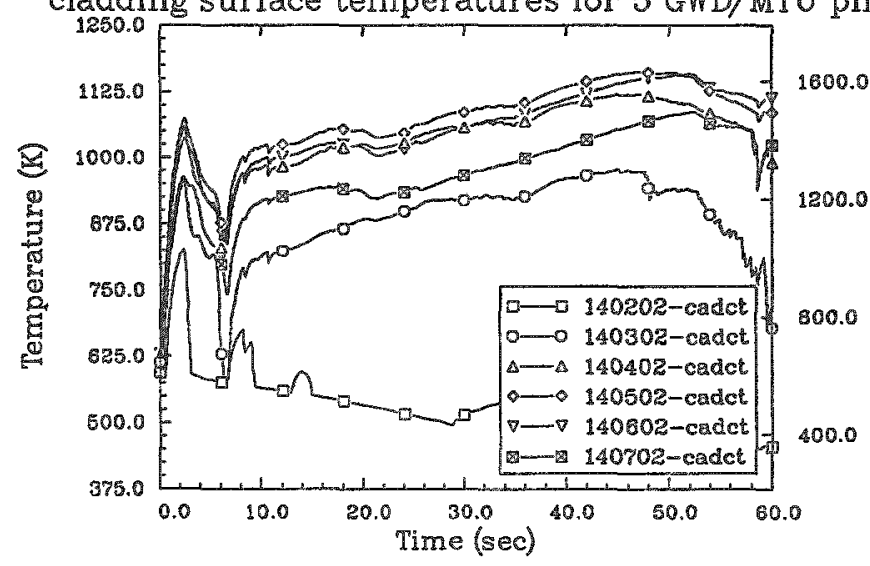

W 4-LOOP (SEABROOK) 50\% DBA LOCA PIN FAILURE

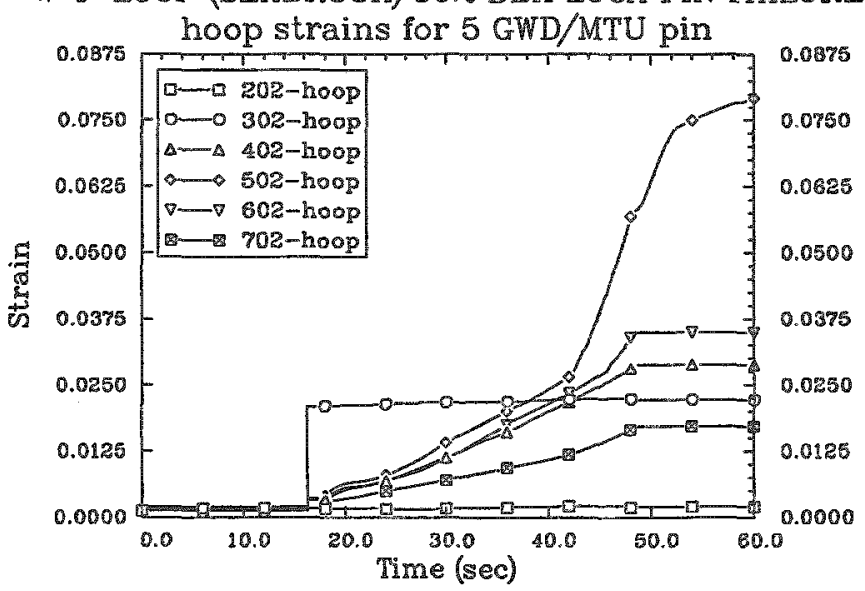

7-LOOP (SEABROOK) 50\% DBA LOCA PIN FAILURE Iuel centerline temperatures for $50 \mathrm{GWD} / \mathrm{MTU}$ pin

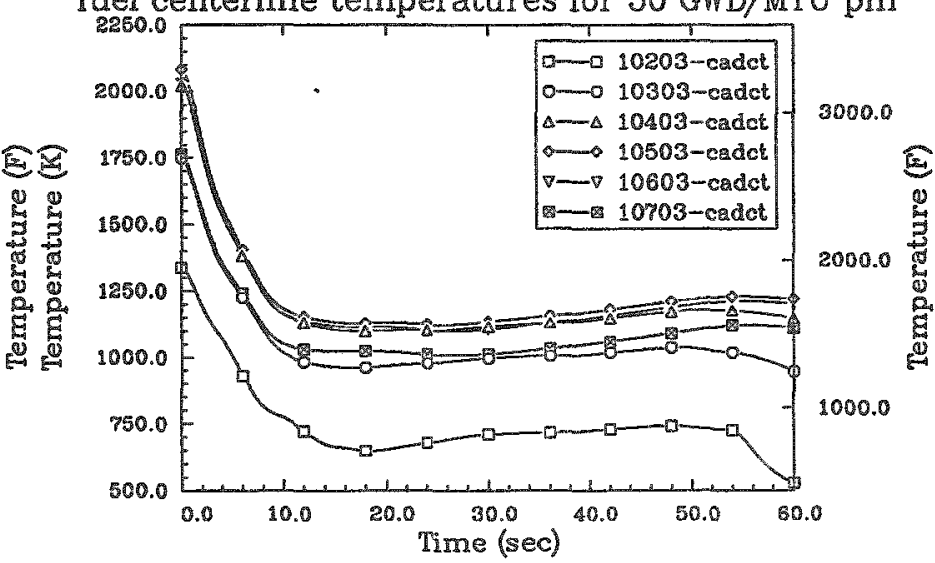

W 4-LOOP (SEABROOK) 50\% DBA LOCA PIN FAILURE cladding surface temperatures for $50 \mathrm{GWD} / \mathrm{MTU}$ pin

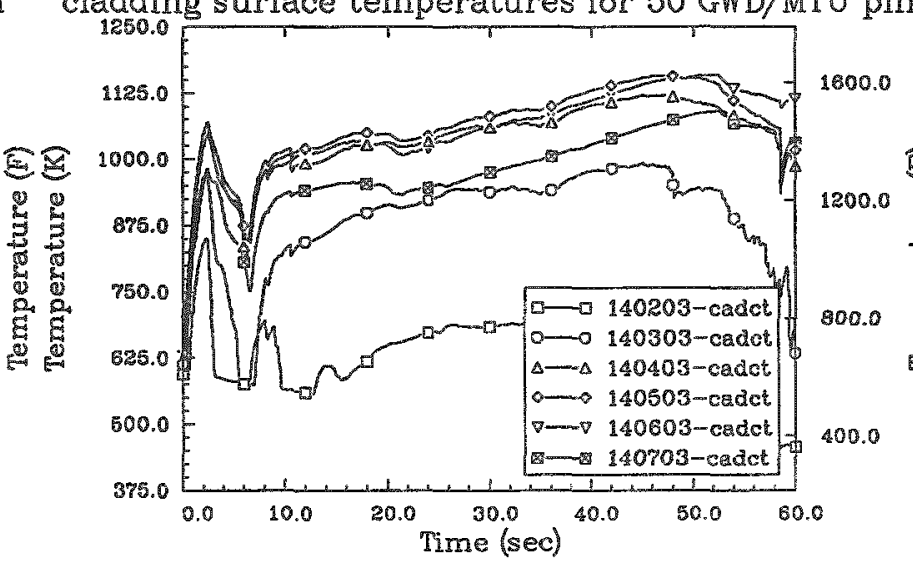

W 4-LOOP (SEABROOK) 50\% DBA LOCA PIN FAILURE

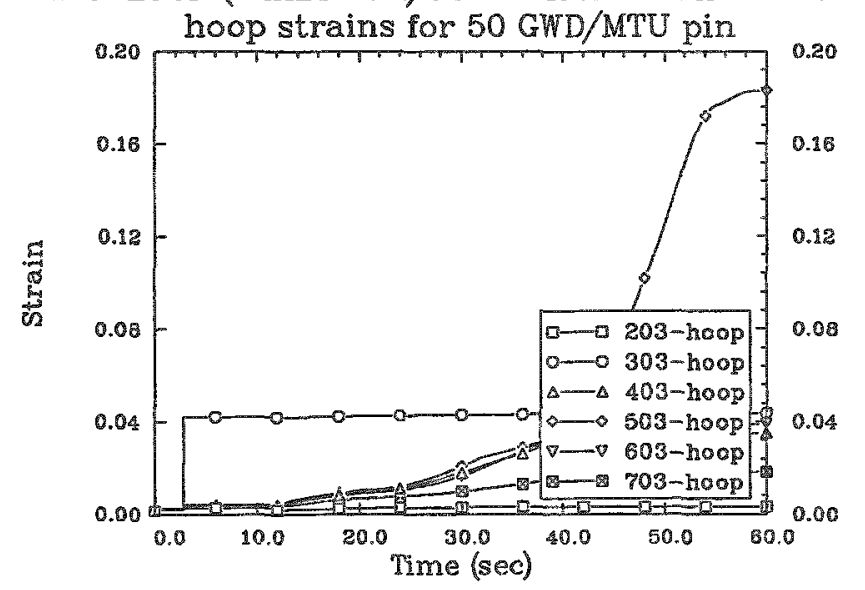



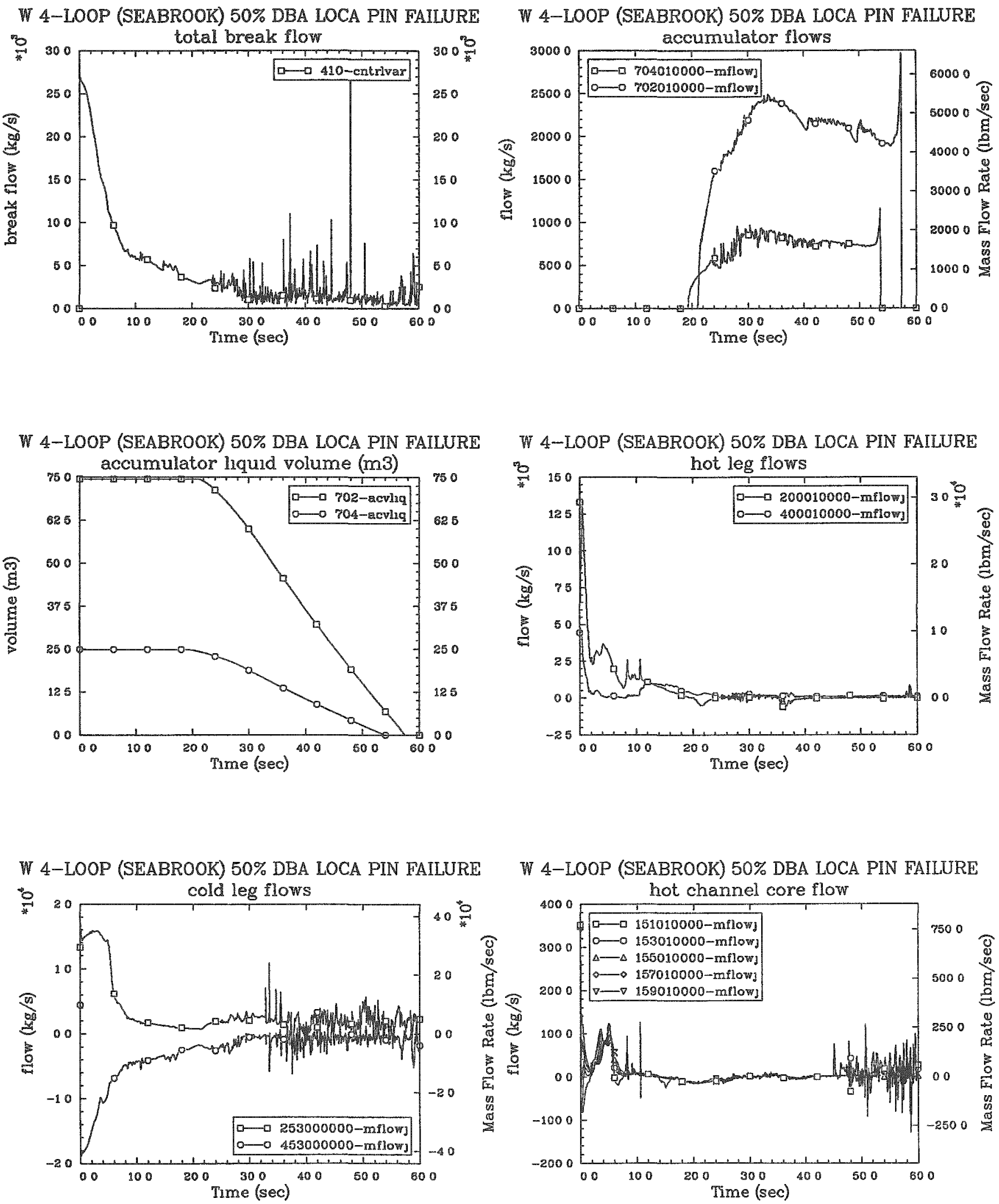
W 4-LOOP (SEABROOK) 50\% DBA LOCA PIN FAILURE

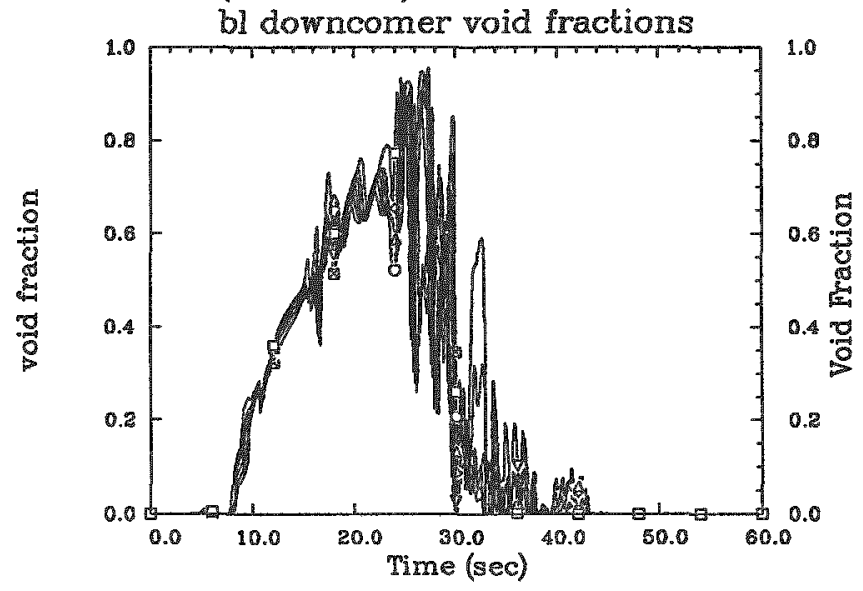

W 4-LOOP (SEABROOK) 50\% DBA LOCA PIN FAILURE

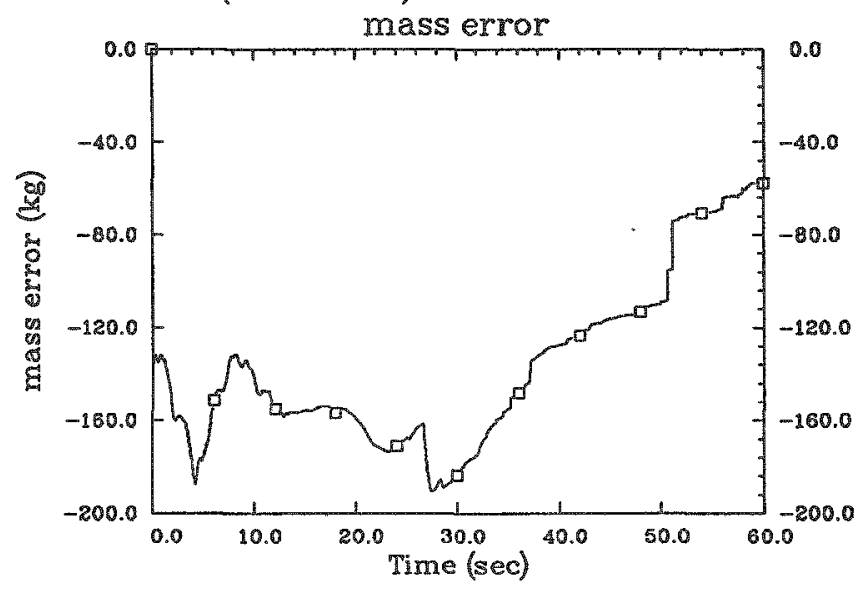

W 4-LOOP (SEABROOK) 50\% DBA LOCA PIN FAILURE

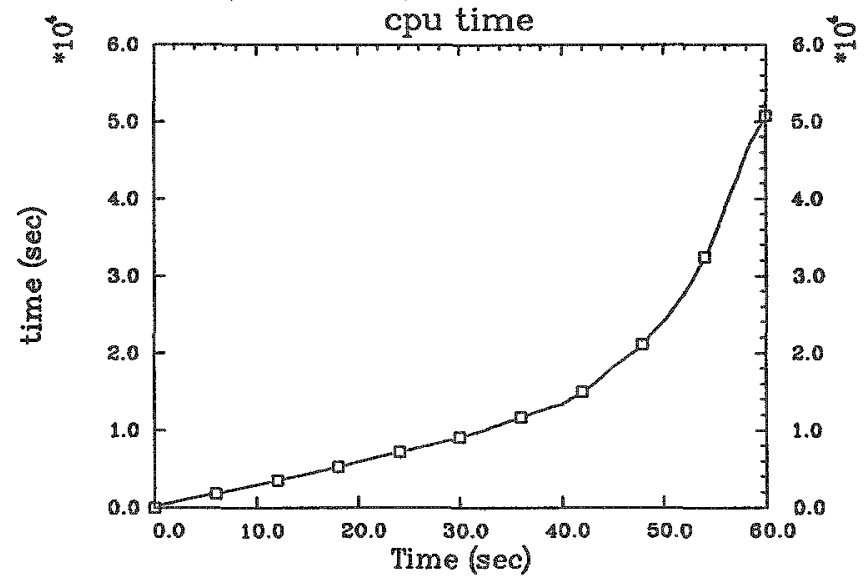

W 4-LOOP (SEABROOK) 50\% DBA LOCA PIN FAILURE

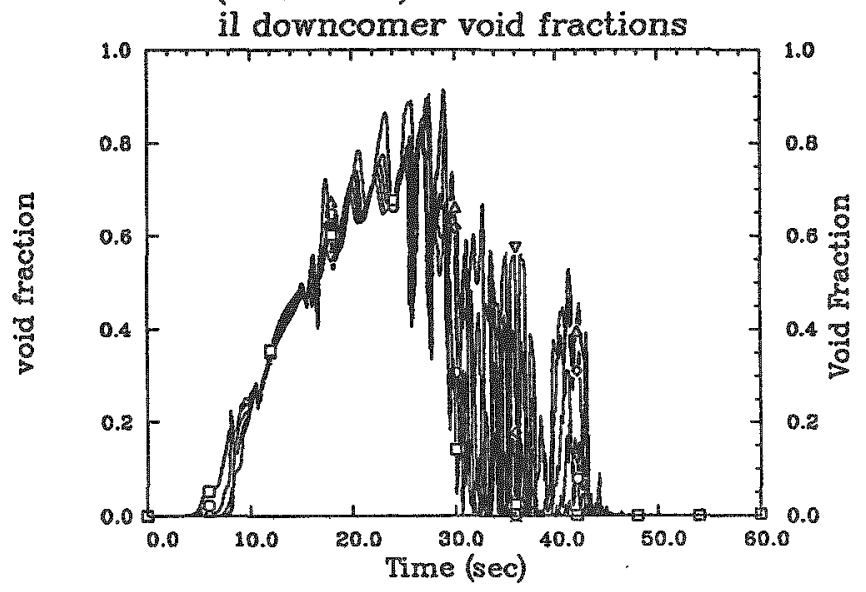

W 4-LOOP (SEABROOK) 50\% DBA LOCA PIN FAILURE

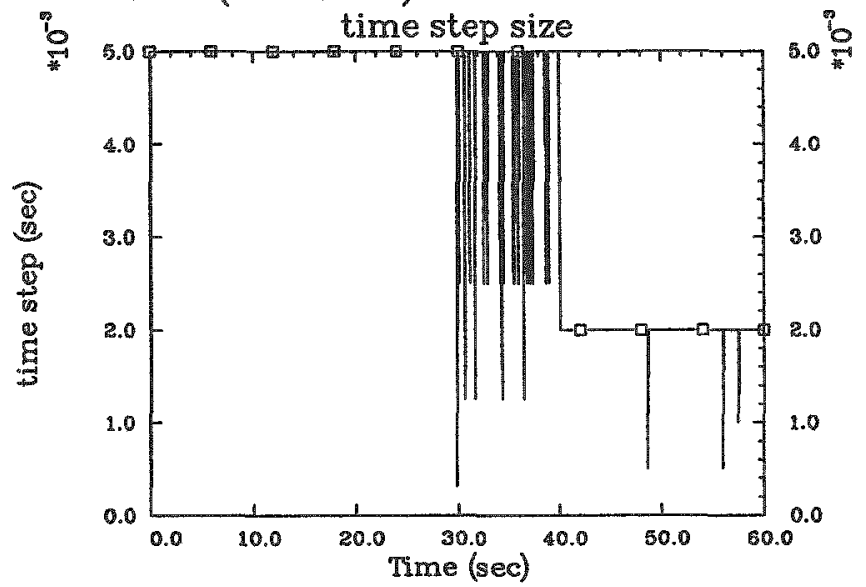




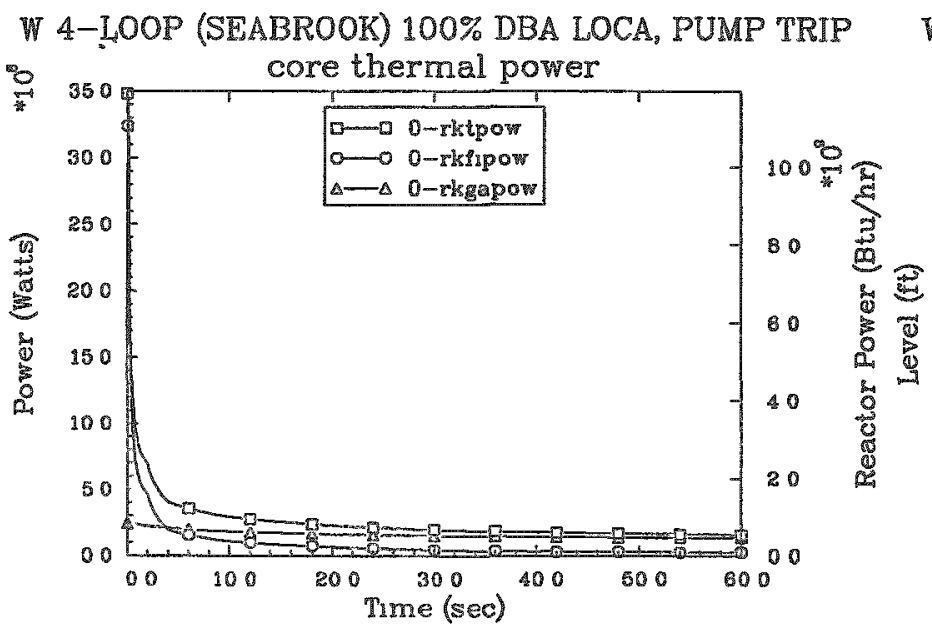

W 4-LOOP (SEABROOK) 100\% DBA LOCA, PUMP TRIP

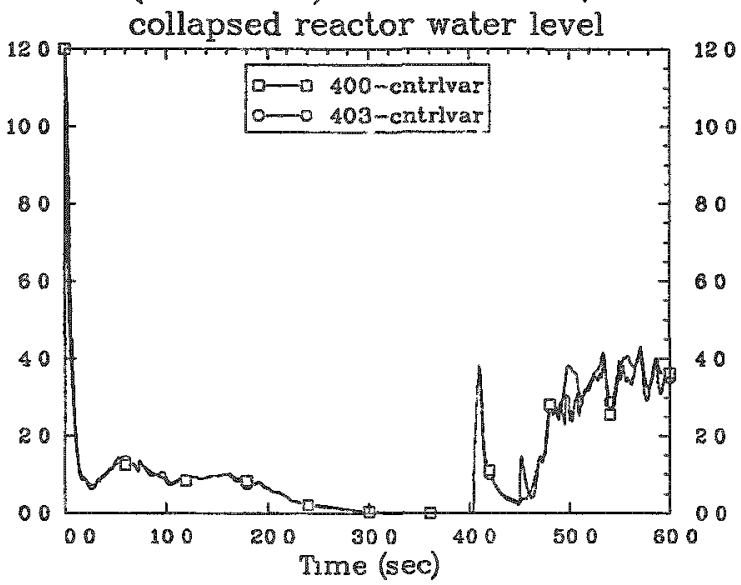

W 4-LOOP (SEABROOK) 100\% DBA LOCA, PUMP TRIP

W 4-LOOP (SEABROOK) 100\% DBA LOCA, PUMP TRIP
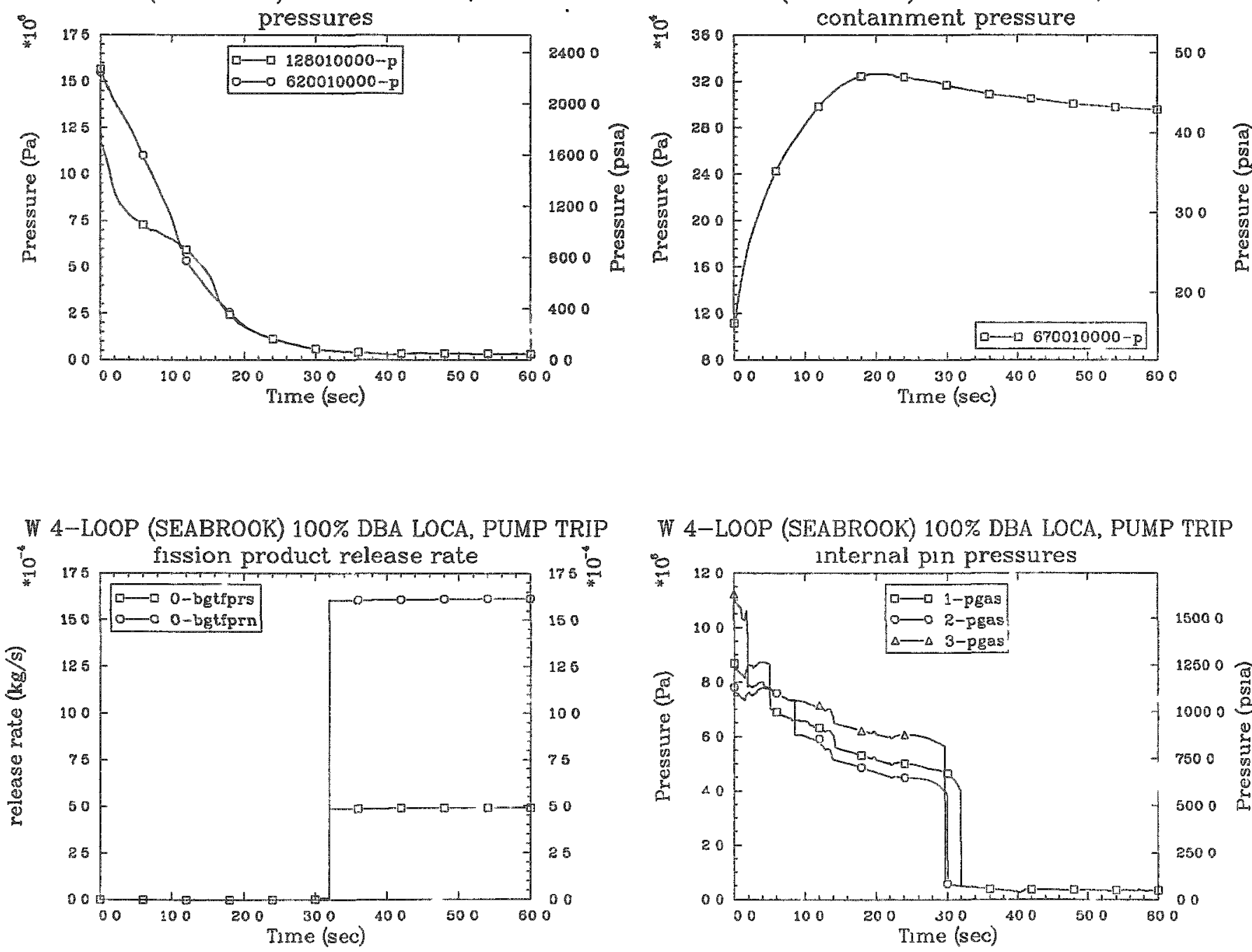

W 4-LOOP (SEABROOK) 100\% DBA LOCA, PUMP TRIP

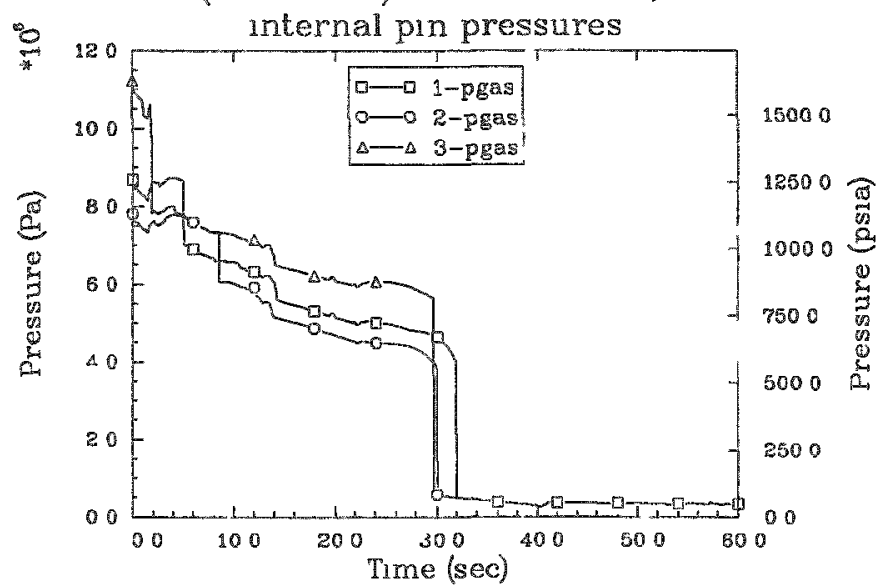


W 4-LOOP (SEABROOK) 100\% DBA LOCA, PUMP TRIP fuel centerline temperatures for 5 GWD/MTU pin

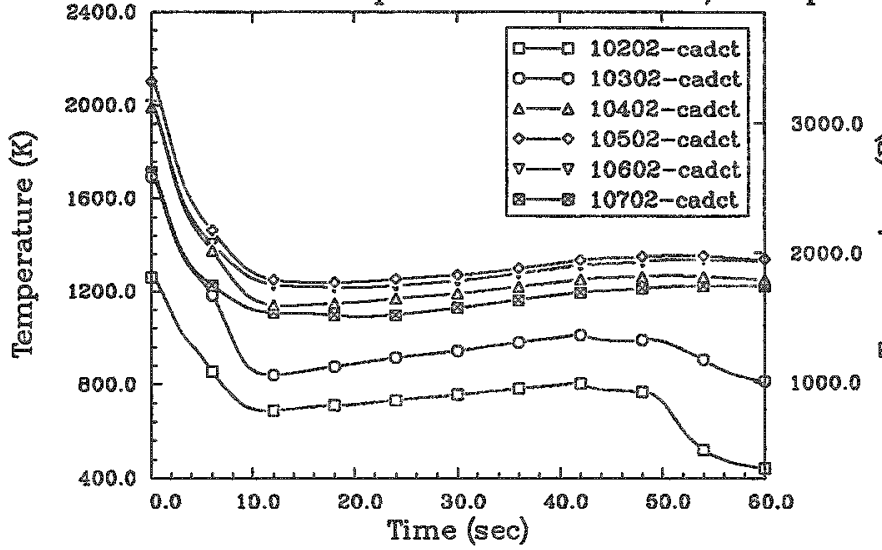

W 4-LOOP (SEABROOK) 100\% DBA LOCA, PUMP TRIP cladding surface temperatures for $5 \mathrm{GWD} / \mathrm{MTU}$ pin

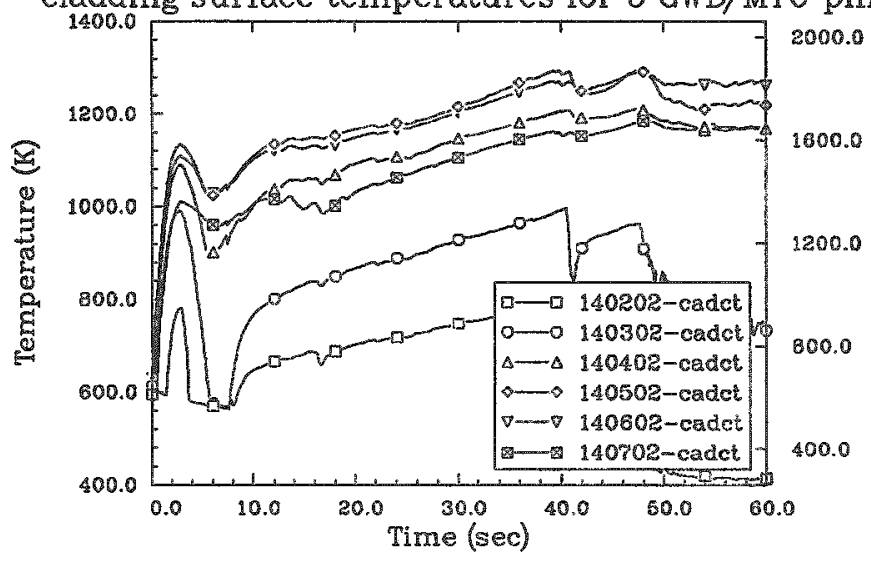

W 4-LOOP (SEABROOK) 100\% DBA LOCA, PUMP TRIP

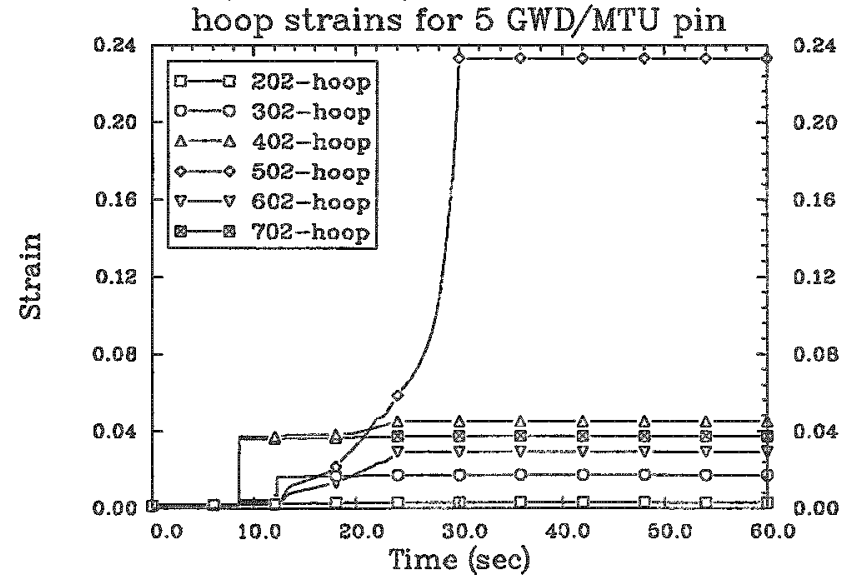

W 4-LOOP (SEABROOK) 100\% DBA LOCA, PUMP TRIP fuel centerline temperatures for $50 \mathrm{GWD} / \mathrm{MTU}$ pin

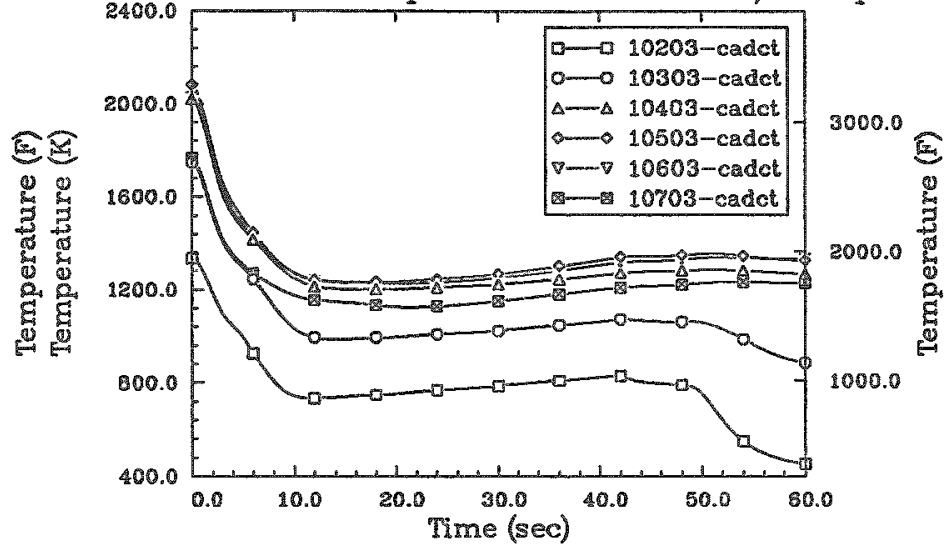

W 4-LOOP (SEABROOK) 100\% DBA LOCA, PUMP TRIP cladding surface temperatures for $50 \mathrm{GWD} / \mathrm{MTU}$ pin
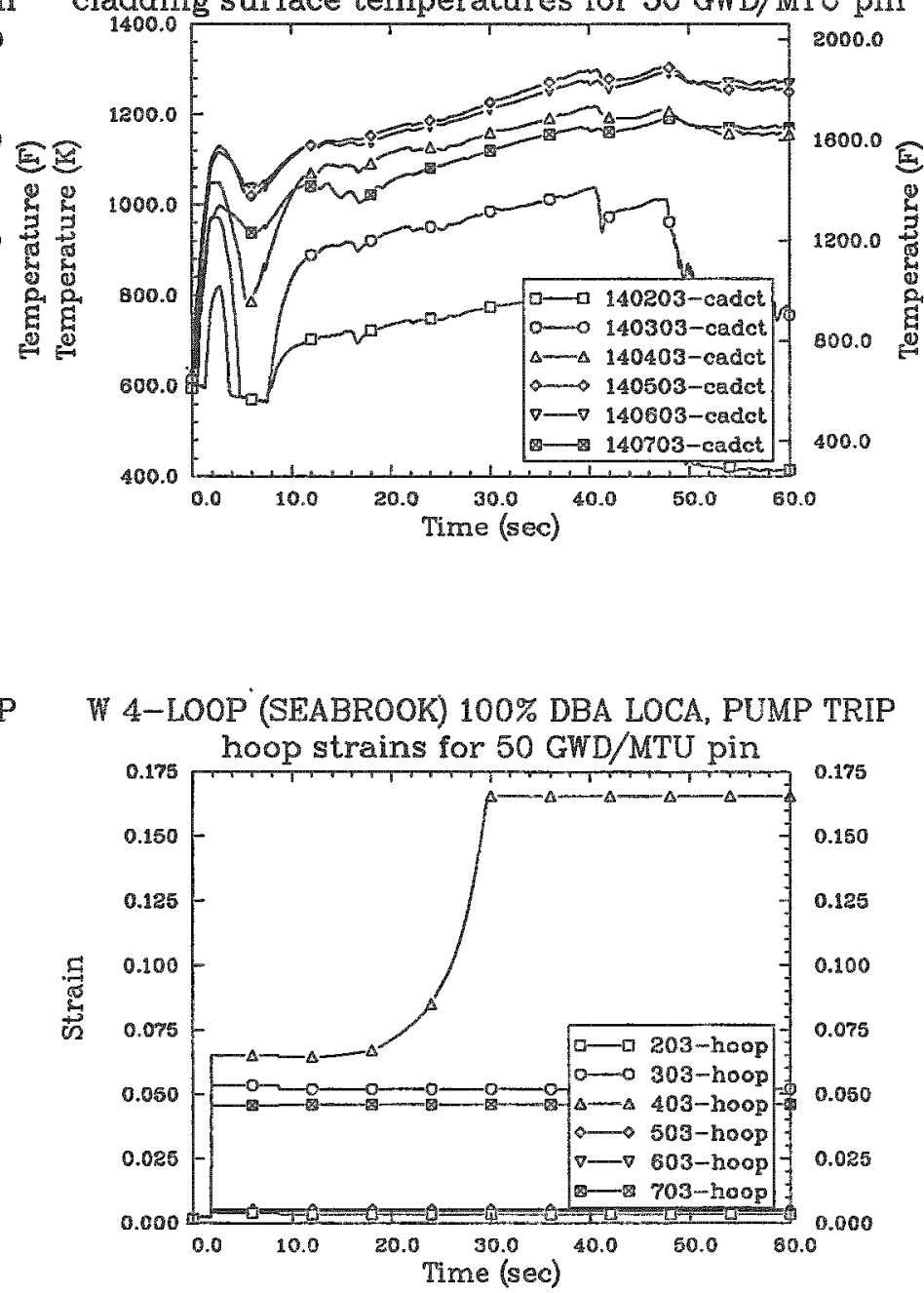

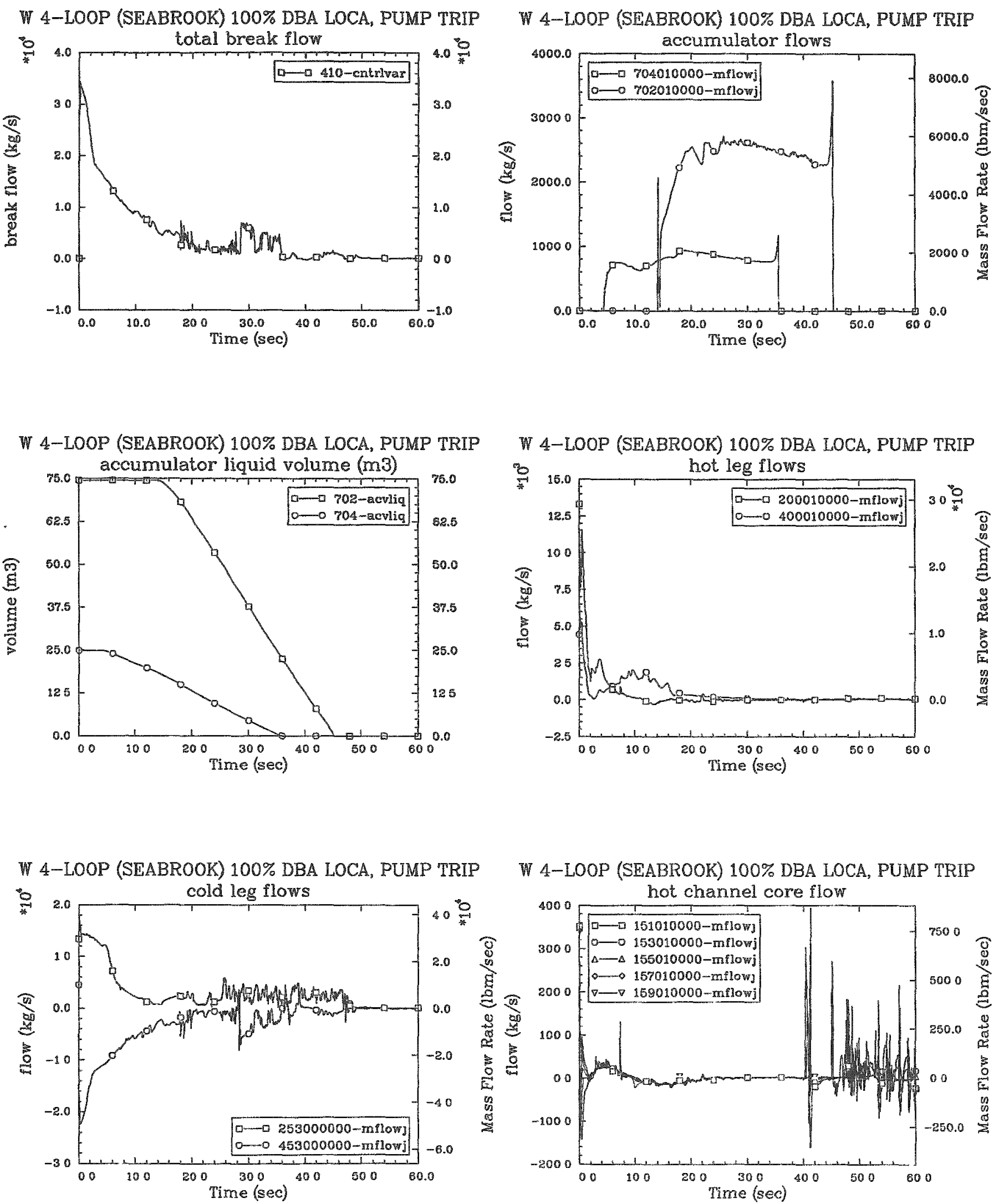

W 4-LOOP (SEABROOK) 100\% DBA LOCA, PUMP TRIP

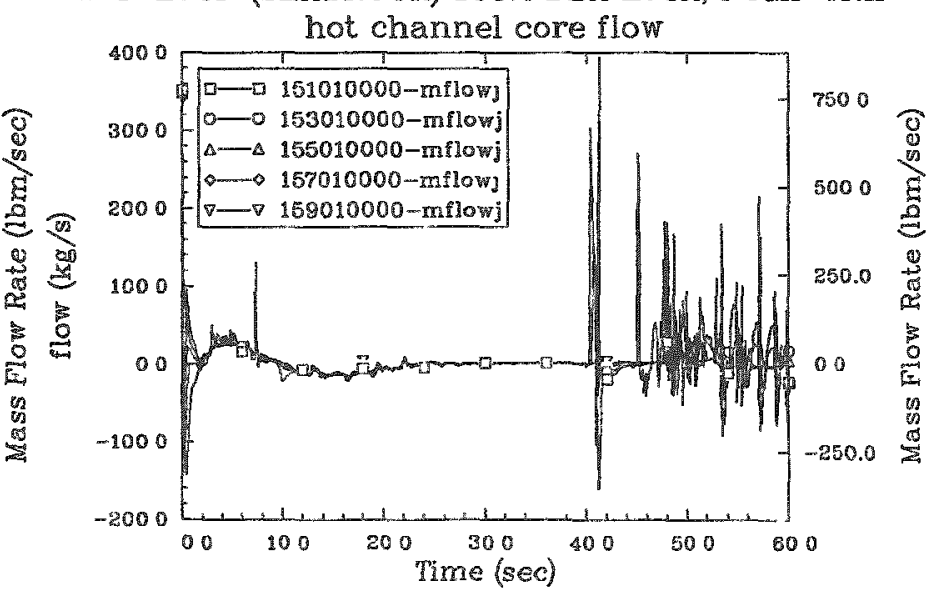



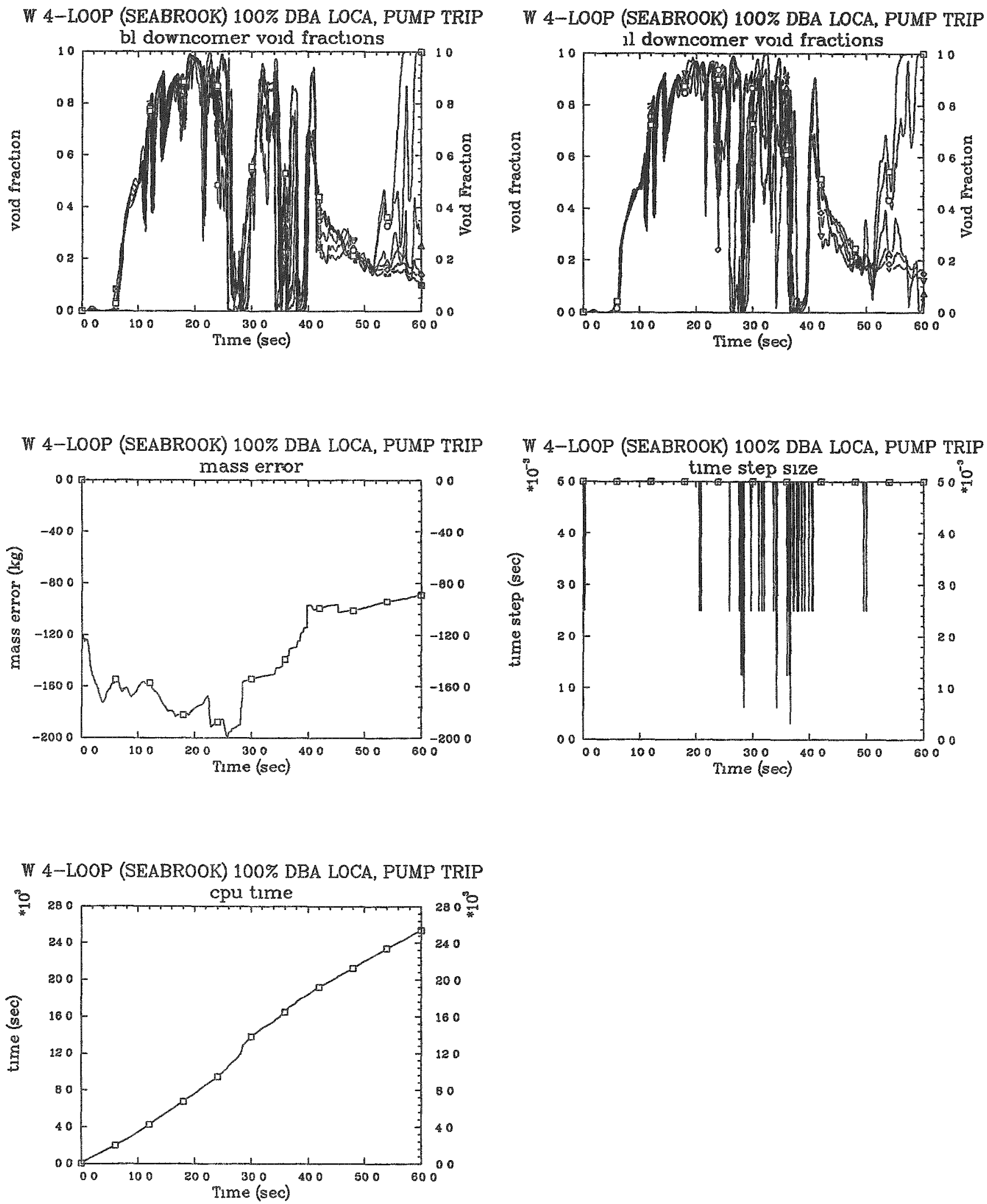

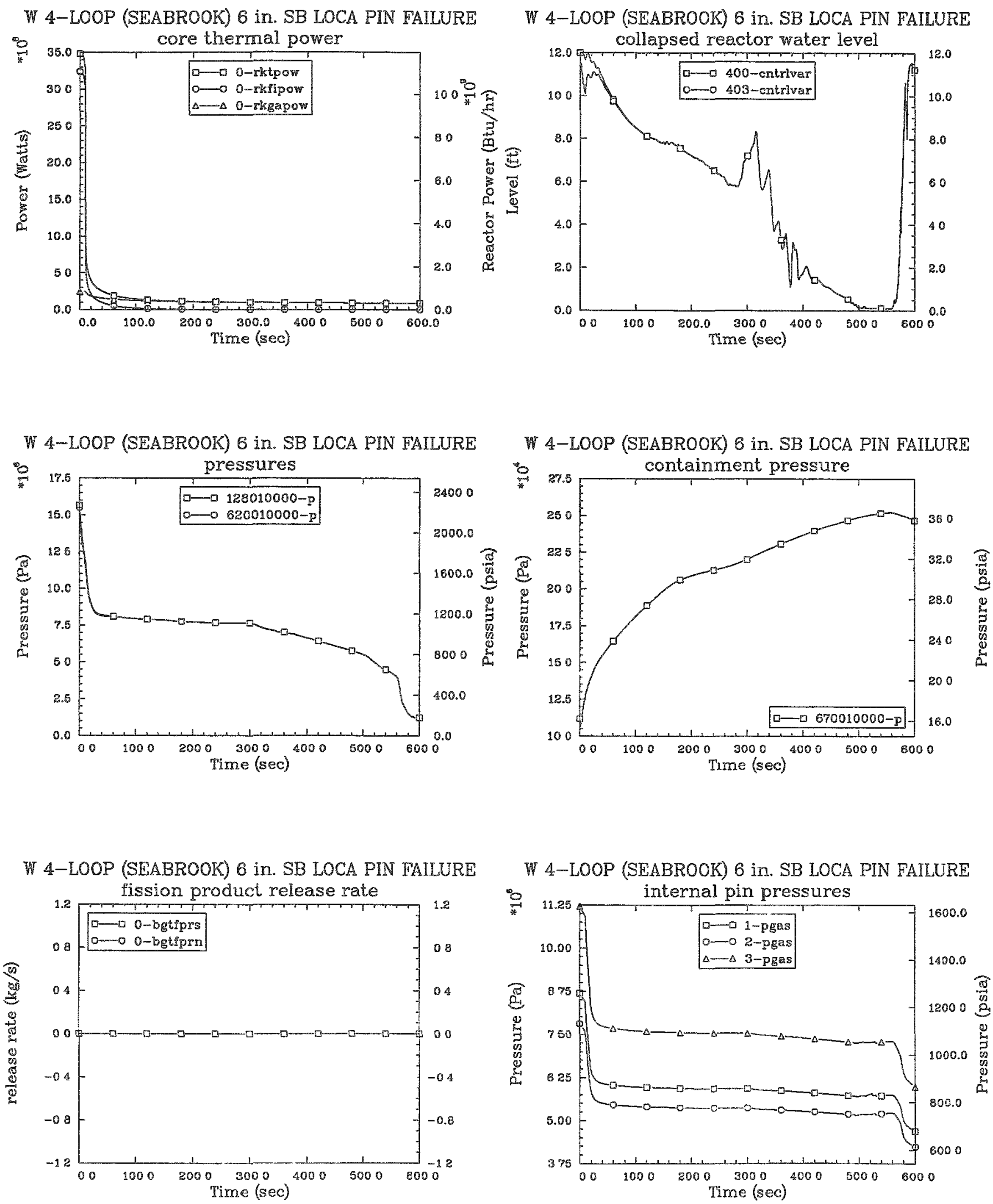
W 4-LOOP (SEABROOK) 6 in. SB LOCA PIN FAILURE fuel centerline temperatures for 5 GWD/MTU pin

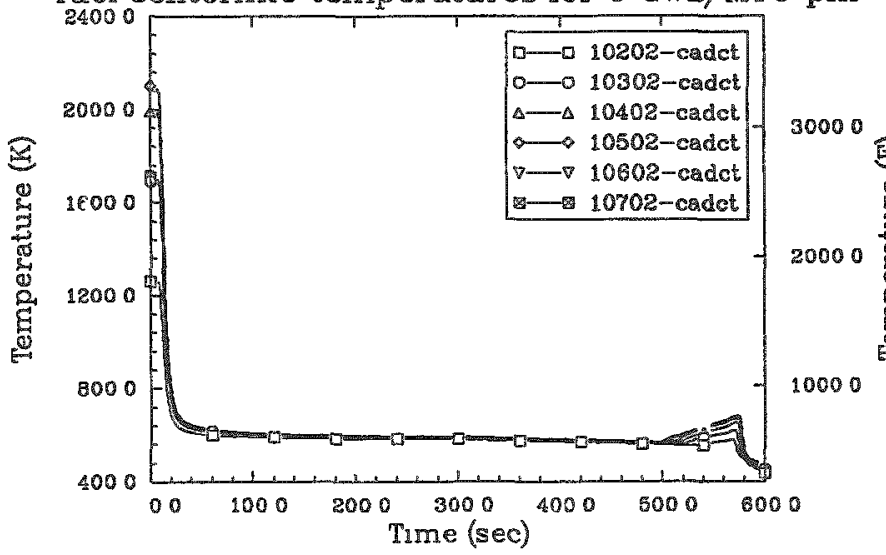

W 4-LOOP (SEABROOK) 6 in SB LOCA PIN FAILURE cladding surface temperatures for 5 GWD/MTU pin

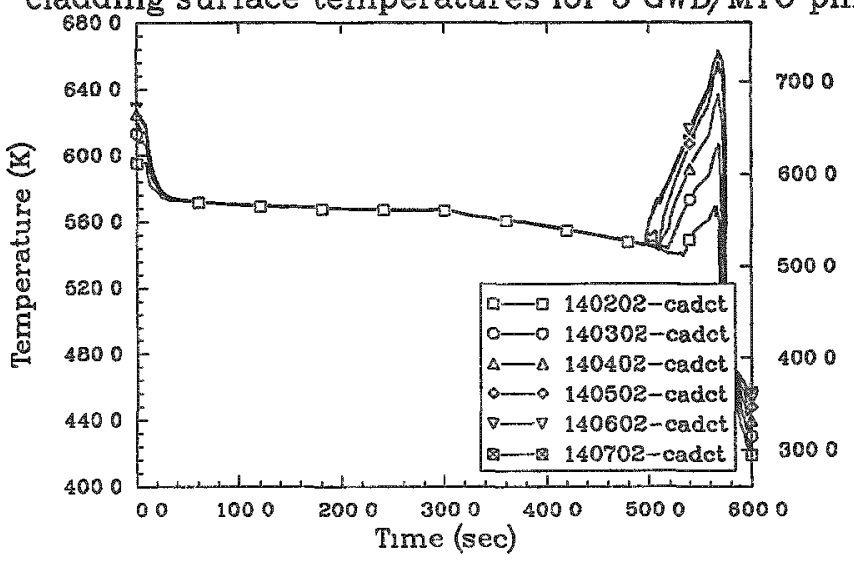

W 4-LOOP (SEABROOK) 6 in SB LOCA PIN FAILURE

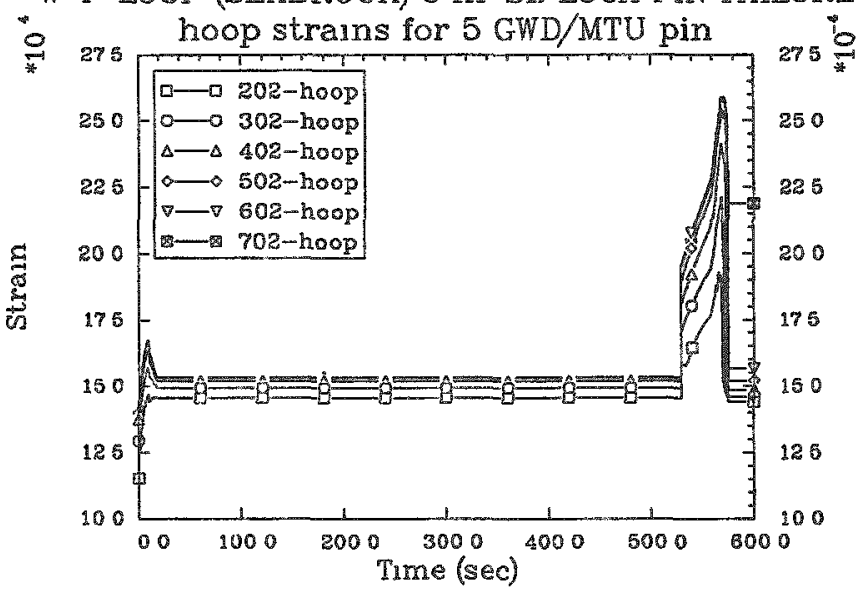

W 4-LOOP (SEABROOK) 6 in SB LOCA PIN FAILURE fuel centerline temperatures for $50 \mathrm{GWD} / \mathrm{MTU}$ pin

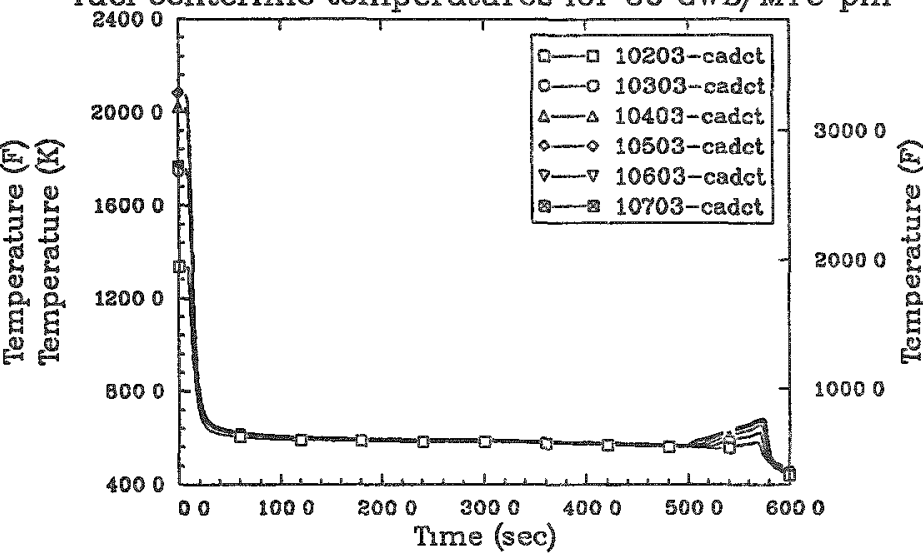

W 4-LOOP (SEABROOK) 6 in SB LOCA PIN FAILURE cladding surface temperatures for $50 \mathrm{GWD} / \mathrm{MTU}$ pin

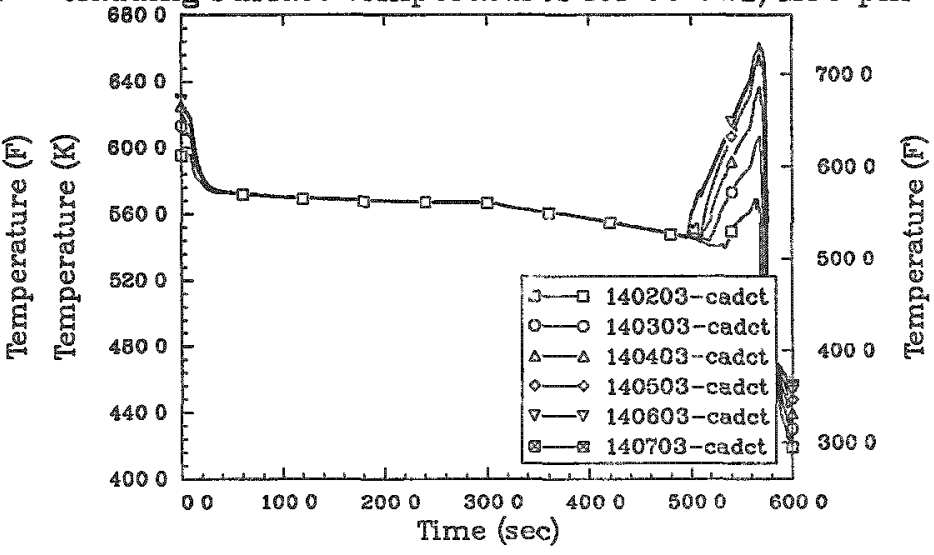

W 4-LOOP (SEABROOK) 6 in SB LOCA PIN FAILURE

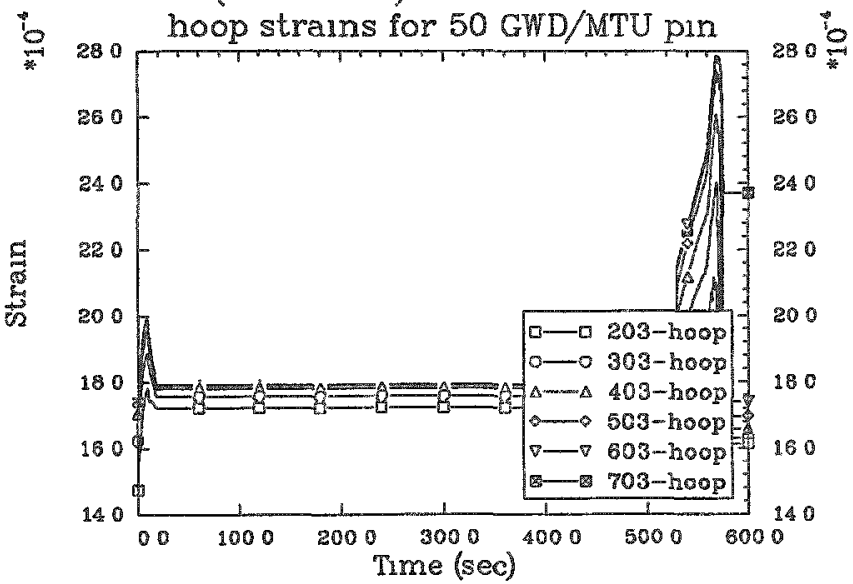


W 4-LOOP (SEABROOK) 6 in. SB LOCA PIN FAILURE

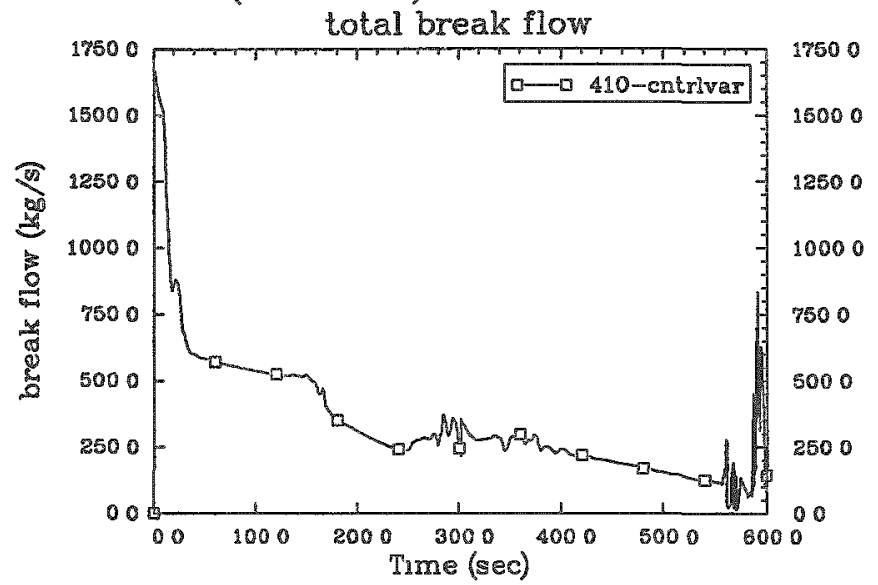

W 4-LOOP (SEABROOK) 6 in SB LOCA PIN FALUURE

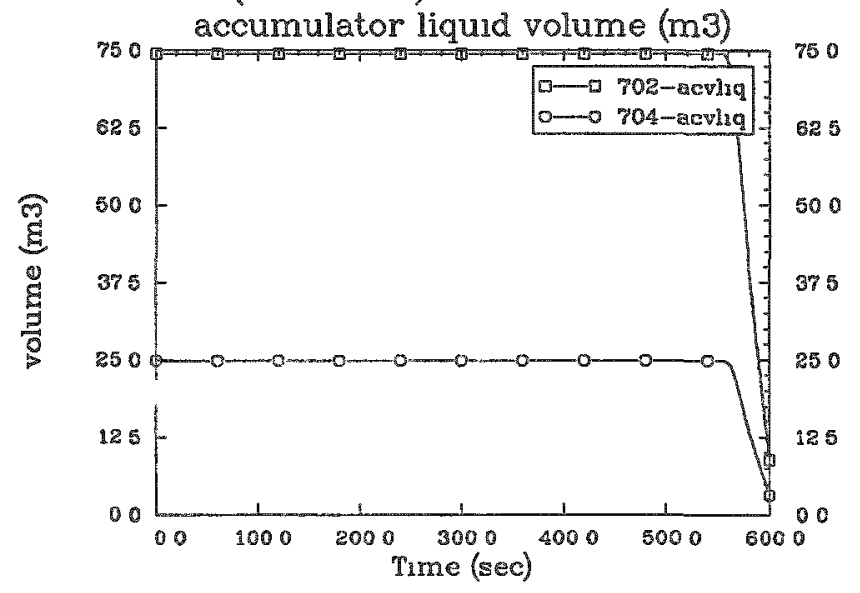

W 4-LOOP (SEABROOK) 6 in SB LOCA PIN FAILURE

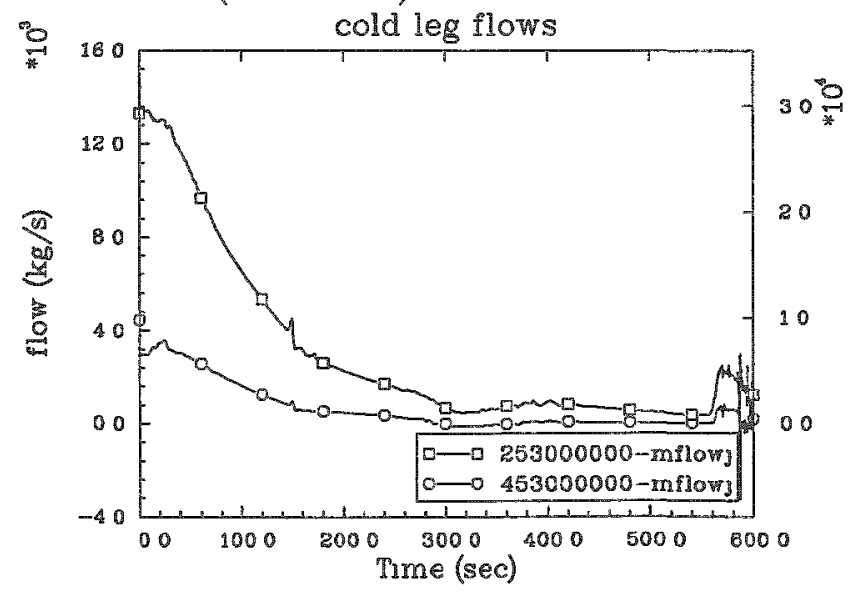

W 4-LOOP (SEABROOK) 6 in. SB LOCA PIN FAILURE accumulator flows
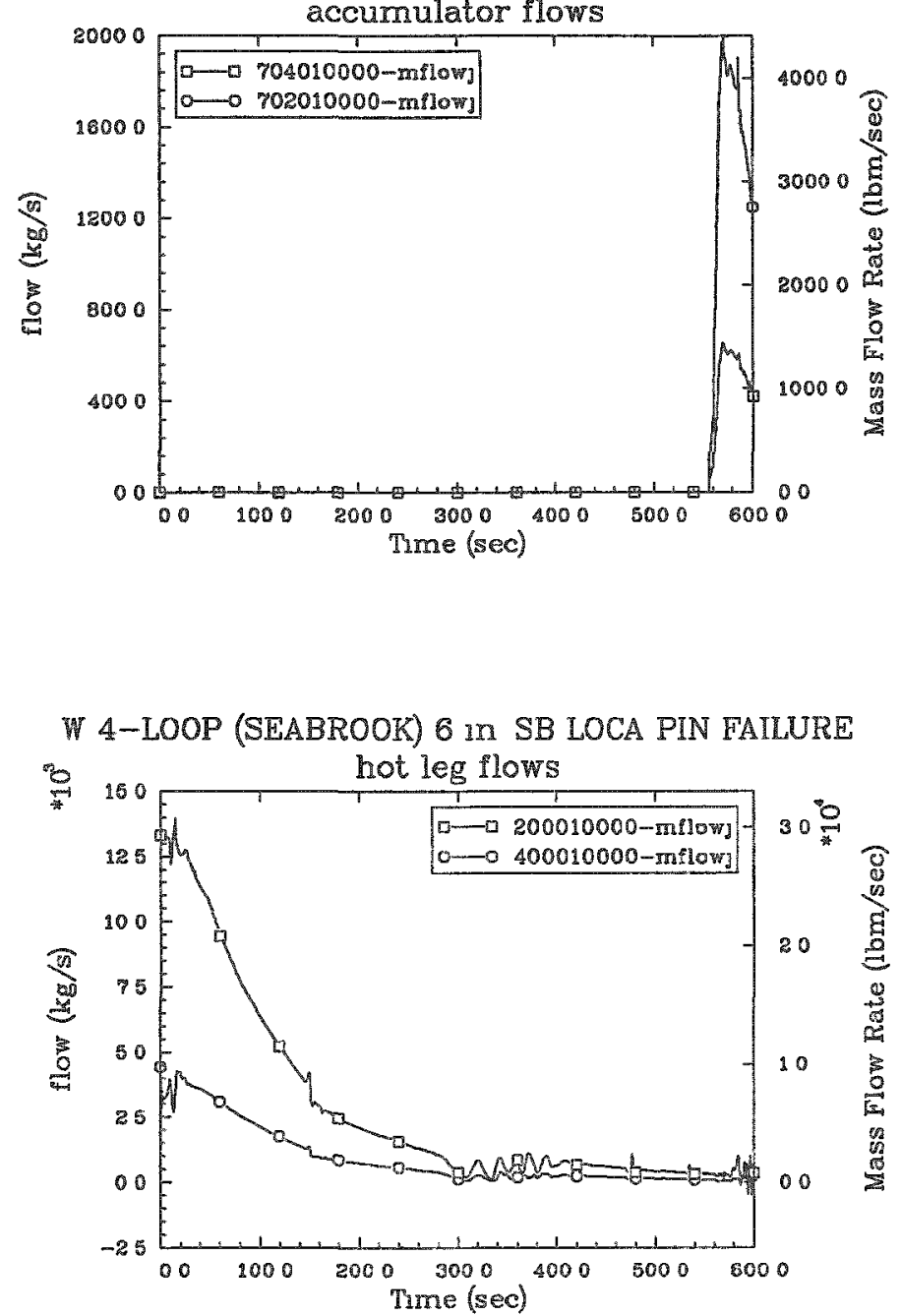

W 4-LOOP (SEABROOK) 6 In SB LOCA PIN FAILURE

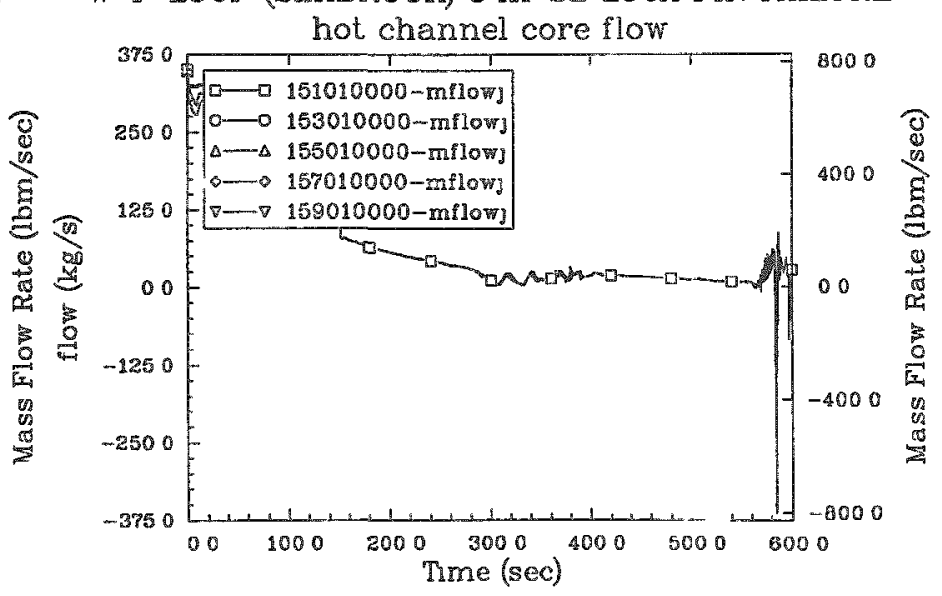



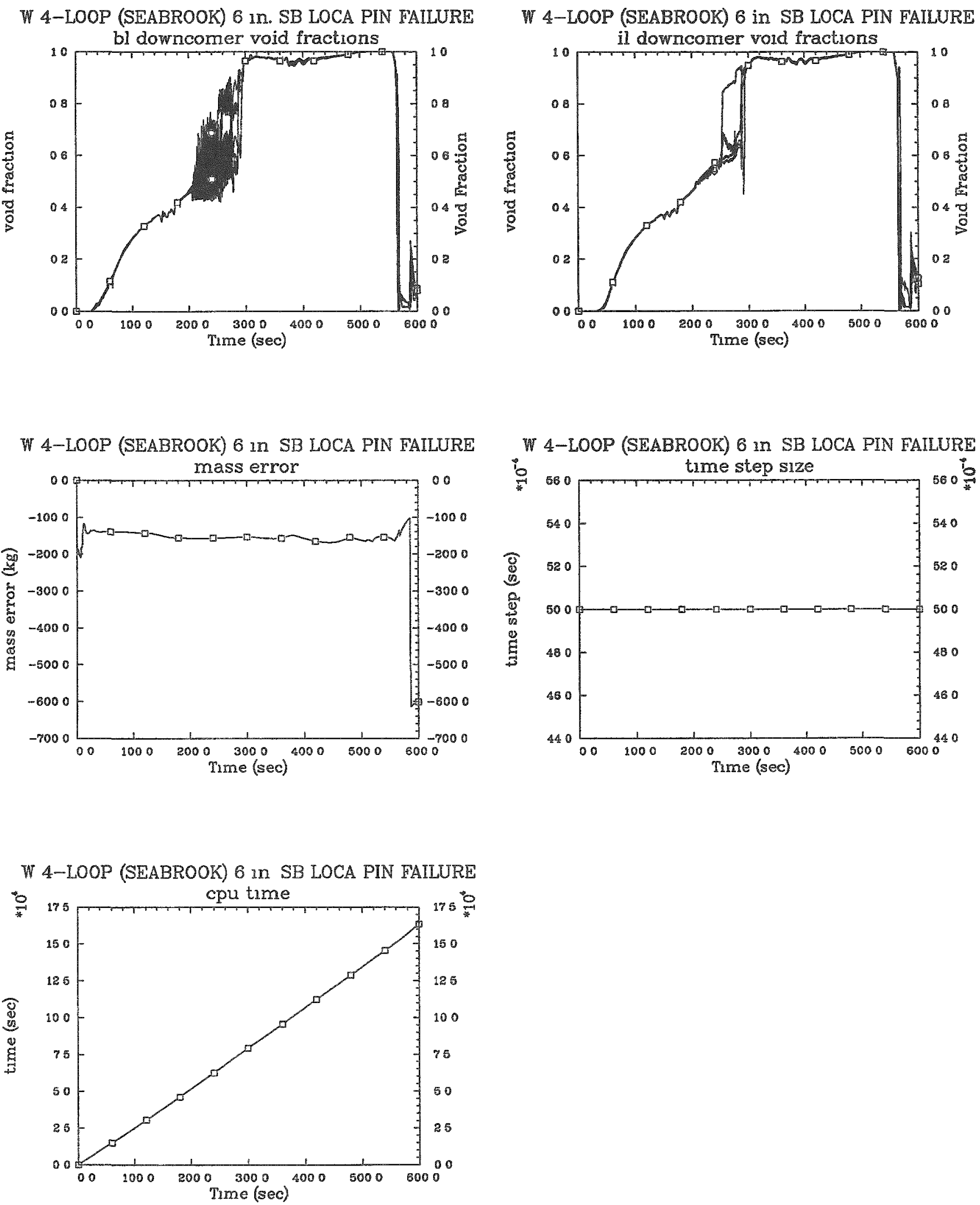

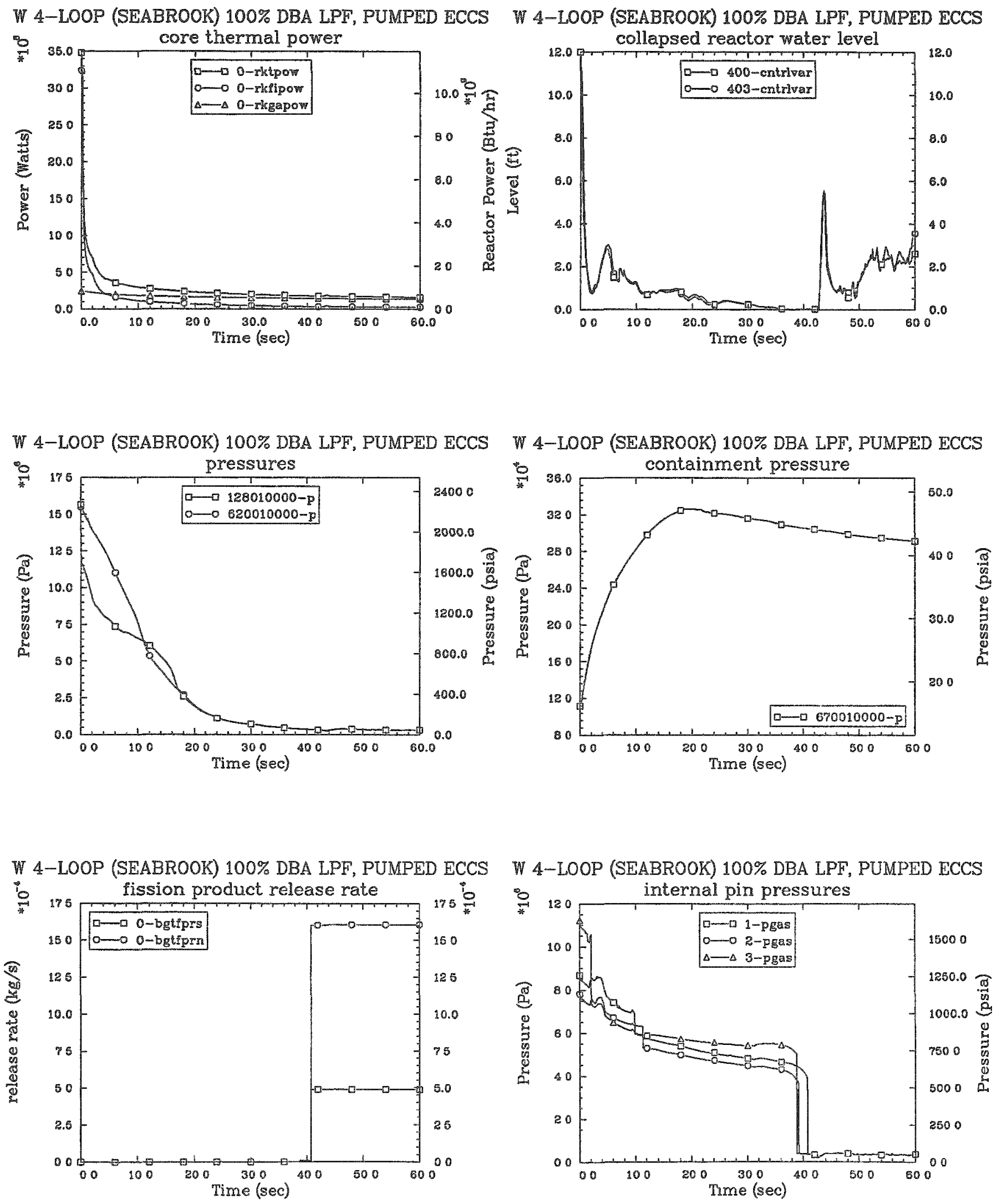
W 4-LOOP (SEABROOK) 100\% DBA LPF, PUMPED ECCS fuel centerline temperatures for 5 GWD/MTU pin

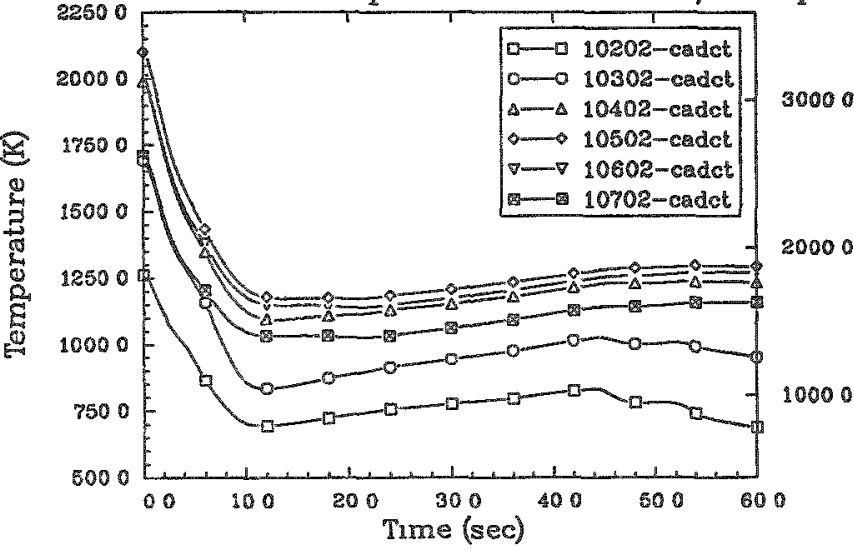

W 4-LOOP (SEABROOK) 100\% DBA LPF, PUMPED ECCS cladding suriace temperatures for $5 \mathrm{GWD} / \mathrm{MTU}$ pin

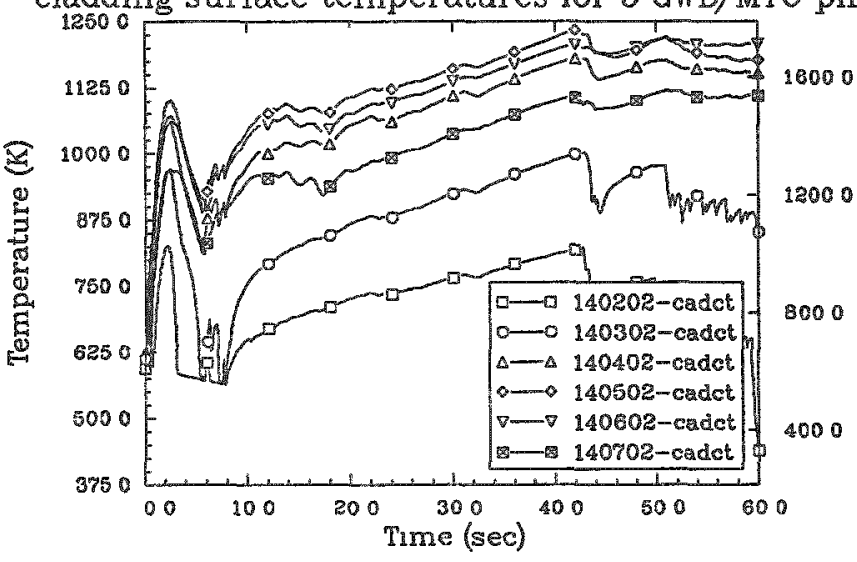

W 4-LOOP (SEABROOK) 100\% DBA LPF, PUMPED ECCS

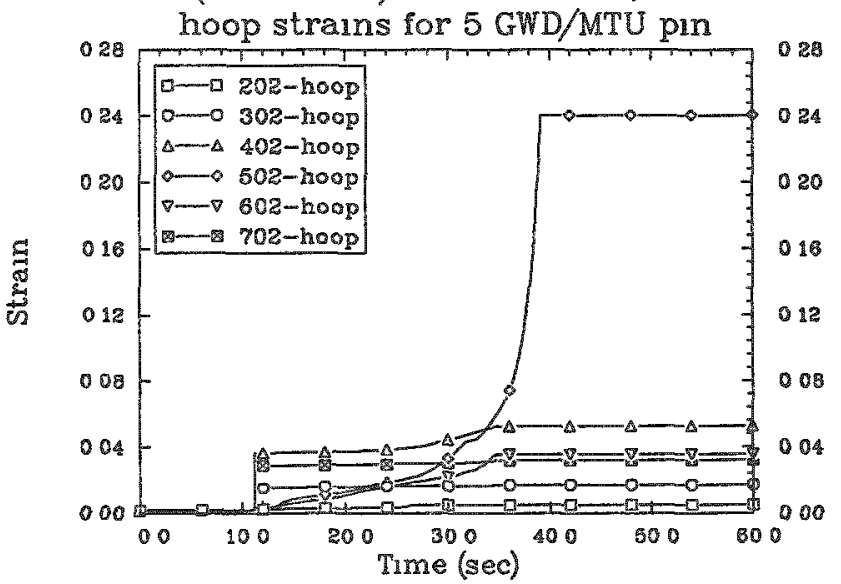

W 4-LOOP (SEABROOK) 100\% DBA LPT, PUMPED ECCS fuel centerline temperatures for $50 \mathrm{GWD} / \mathrm{MTU}$ pin

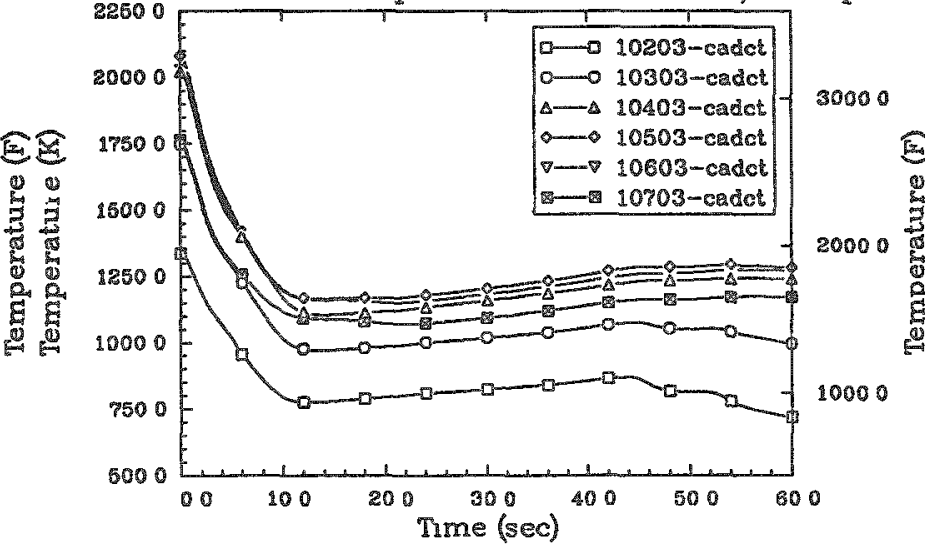

W 4-LOOP (SEABROOK) 100\% DBA LPF, PUMPED ECCS cladding surface temperatures for $50 \mathrm{GWD} / \mathrm{MTU}$ pin

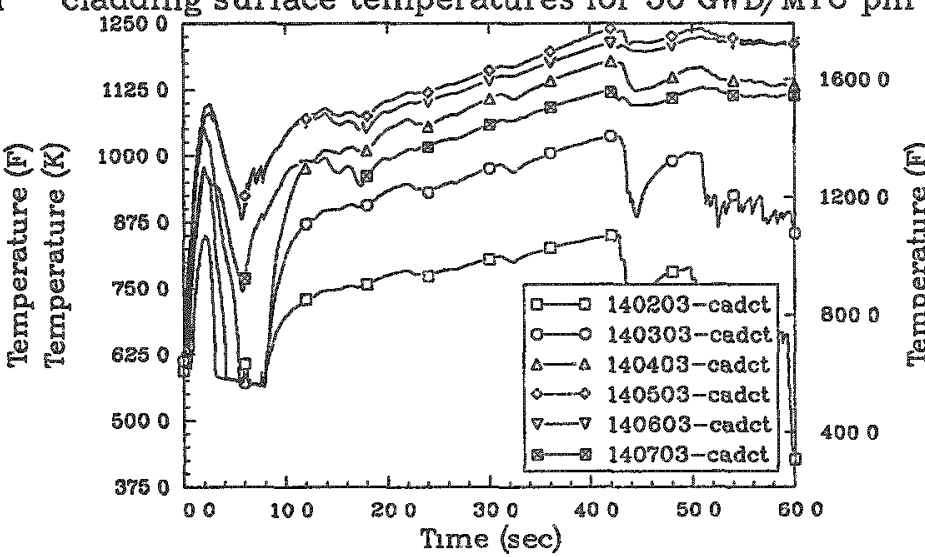

W 4-LOOP (SEABROOK) 100\% DBA LPF, PUMPED ECCS

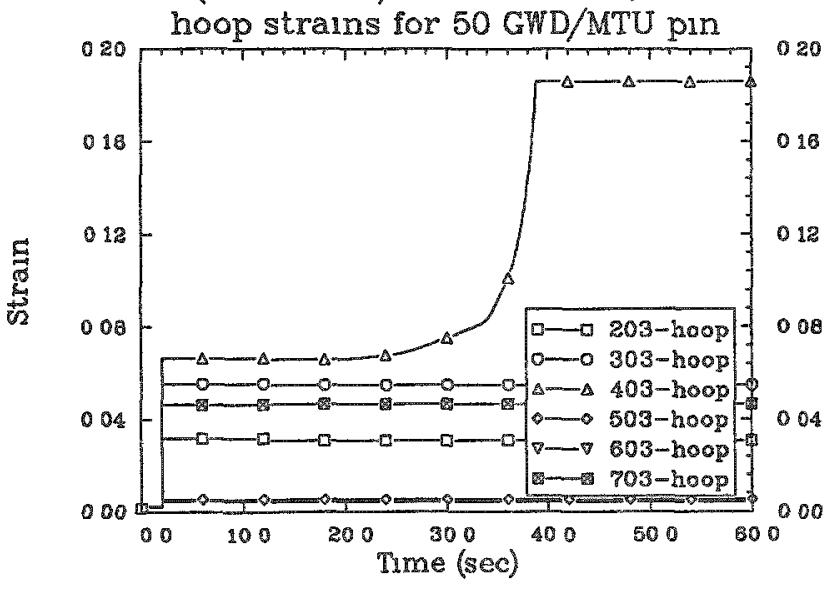


W 4-LOOP (SEABROOK) 100\% DBA LPF, PUMPED ECCS

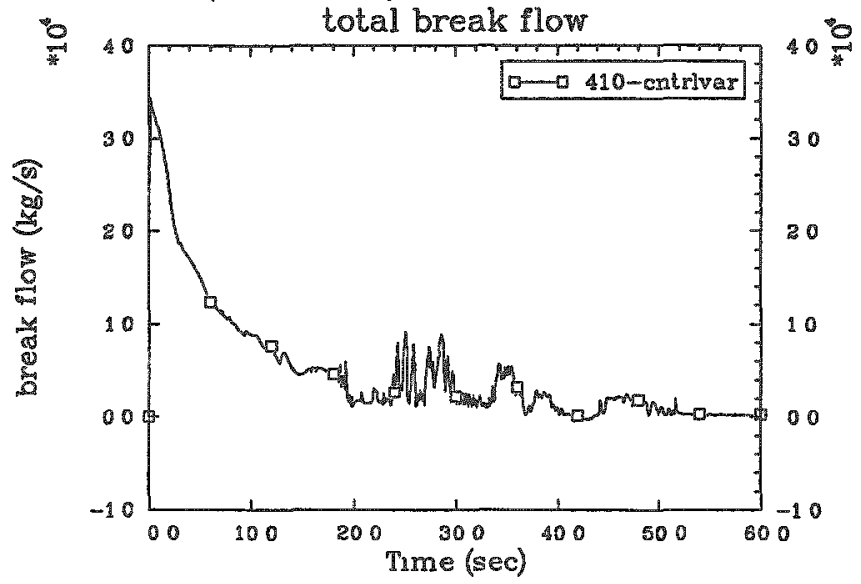

W 4-LOOP (SEABROOK) 100\% DBA LPF, PUMPED ECCS

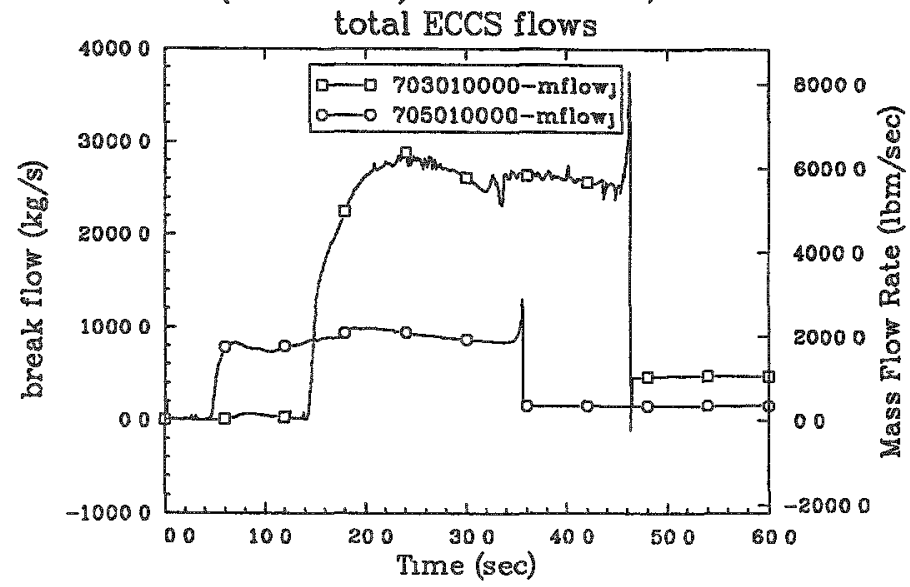

W 4-LOOP (SEABROOK) 100\% DBA LPF, PUMPED ECCS

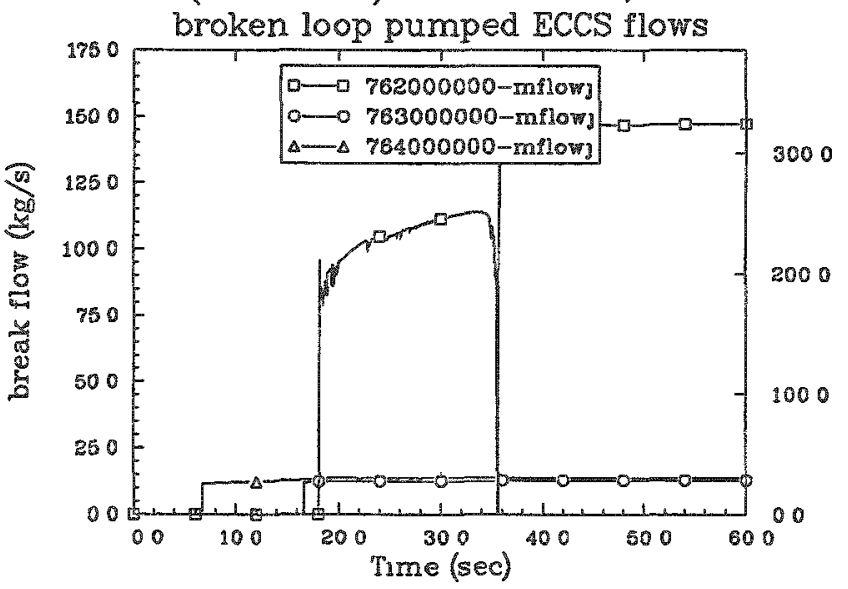

W 4-LOOP (SEABROOK) 100\% DBA LPF, PUMPED ECCS

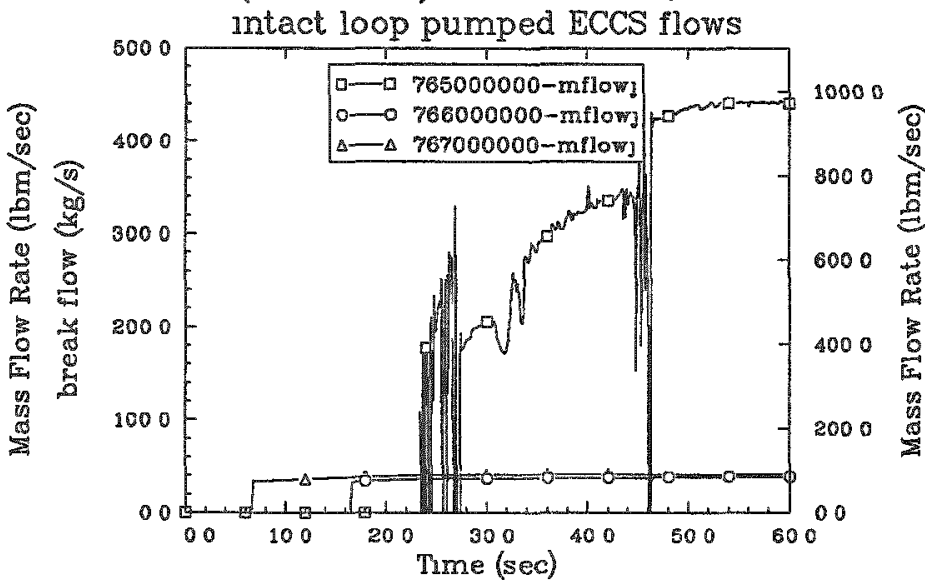

W 4-LOOP (SEABROOK) 100\% DBA LPF, PUMPED ECCS

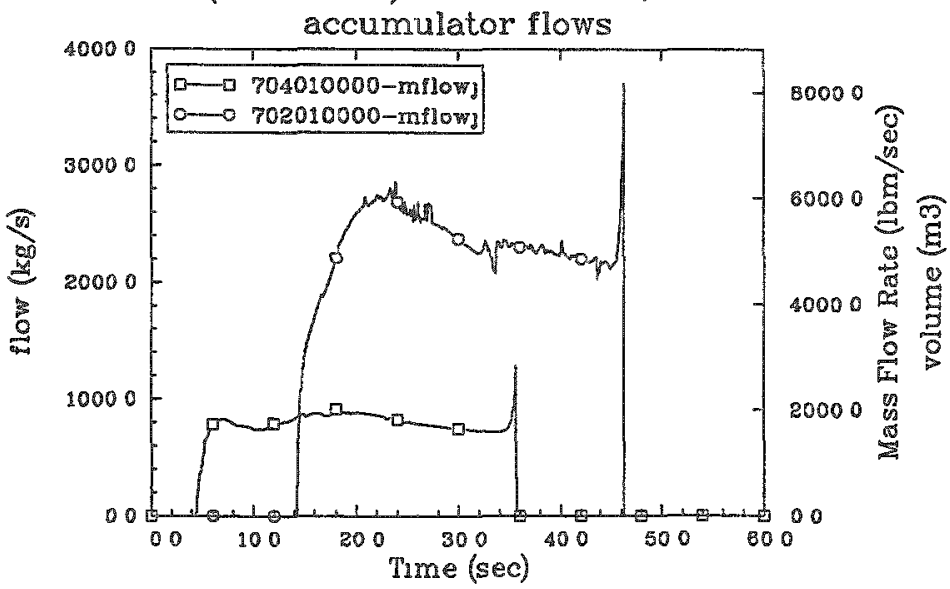

W 4-LOOP (SEABROOK) 100\% DBA LPF, PUMPED ECCS

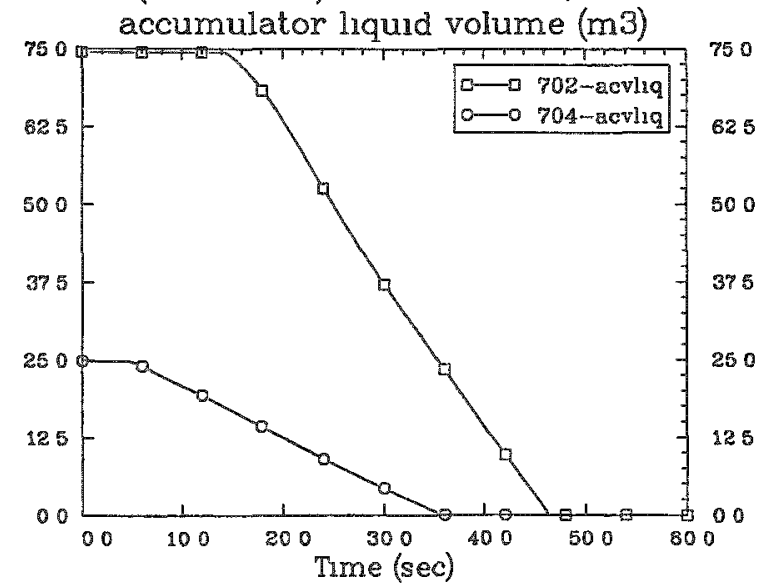


W 4-LOOP (SEABROOK) 100\% DBA LPF, PUMPED ECCS

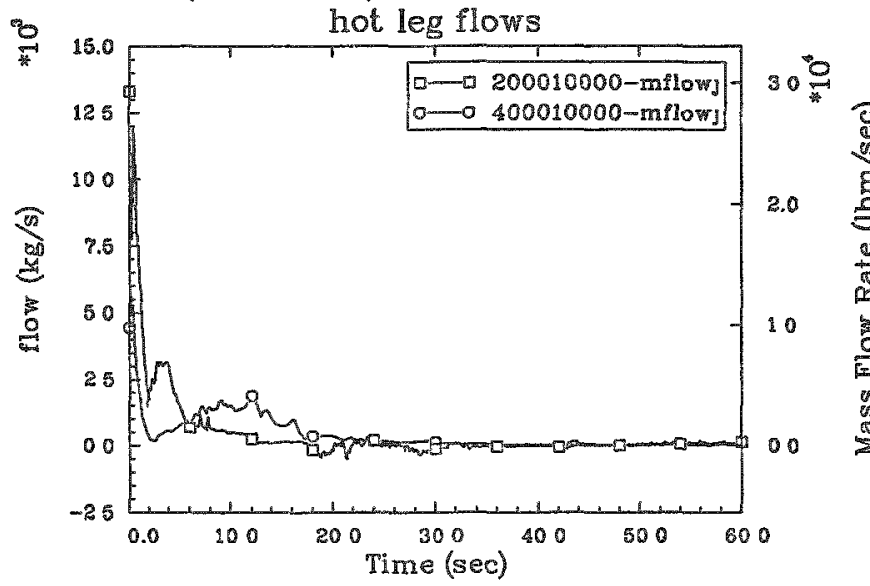

W 4-LOOP (SEABROOK) 100\% DBA LPF, PUMPED ECCS

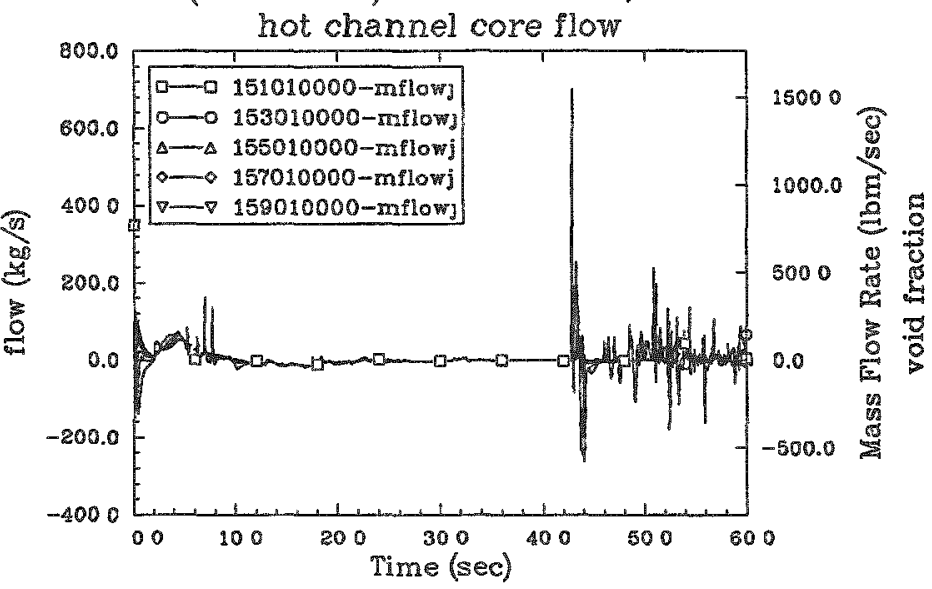

W 4-LOOP (SEABROOK) 100\% DBA LPF, PUMPED ECCS

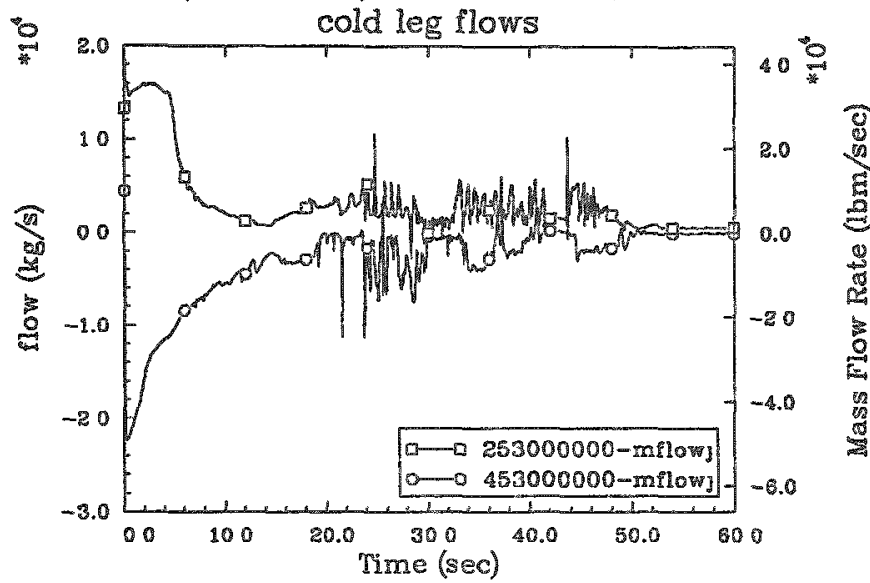

W 4-LOOP (SEABROOK) 100\% DBA LPF, PUMPED ECCS bl downcomer void fractions

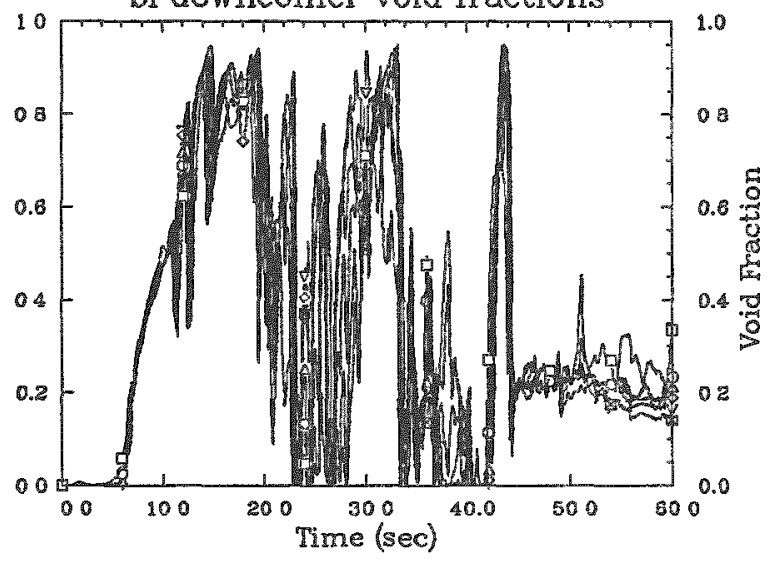

W 4-LOOP (SEABROOK) 100\% DBA LPF, PUMPED ECCS

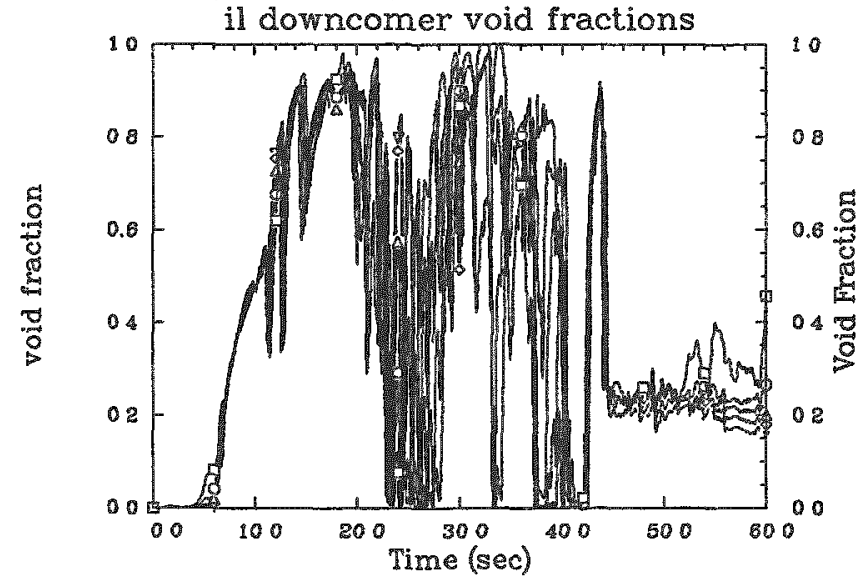

W 4-LOOP (SEABROOK) 100\% DBA LPF, PUMPED ECCS

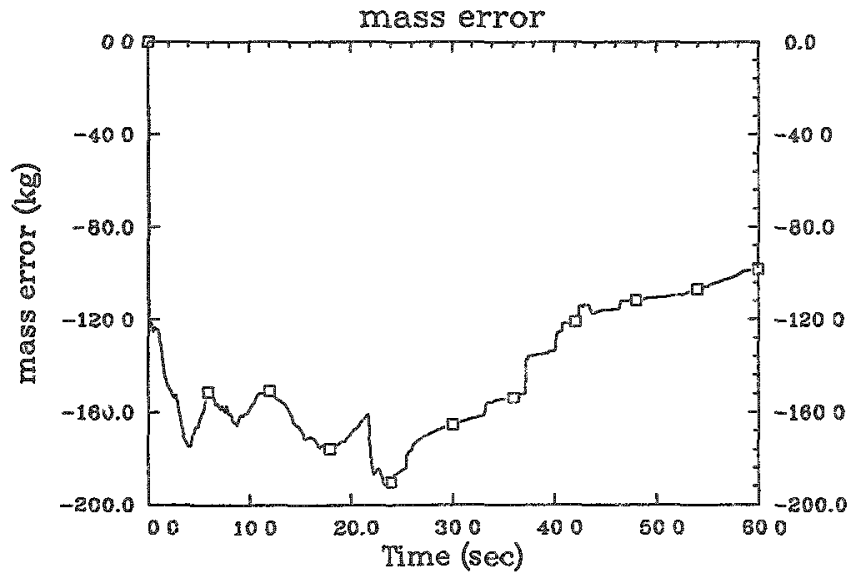



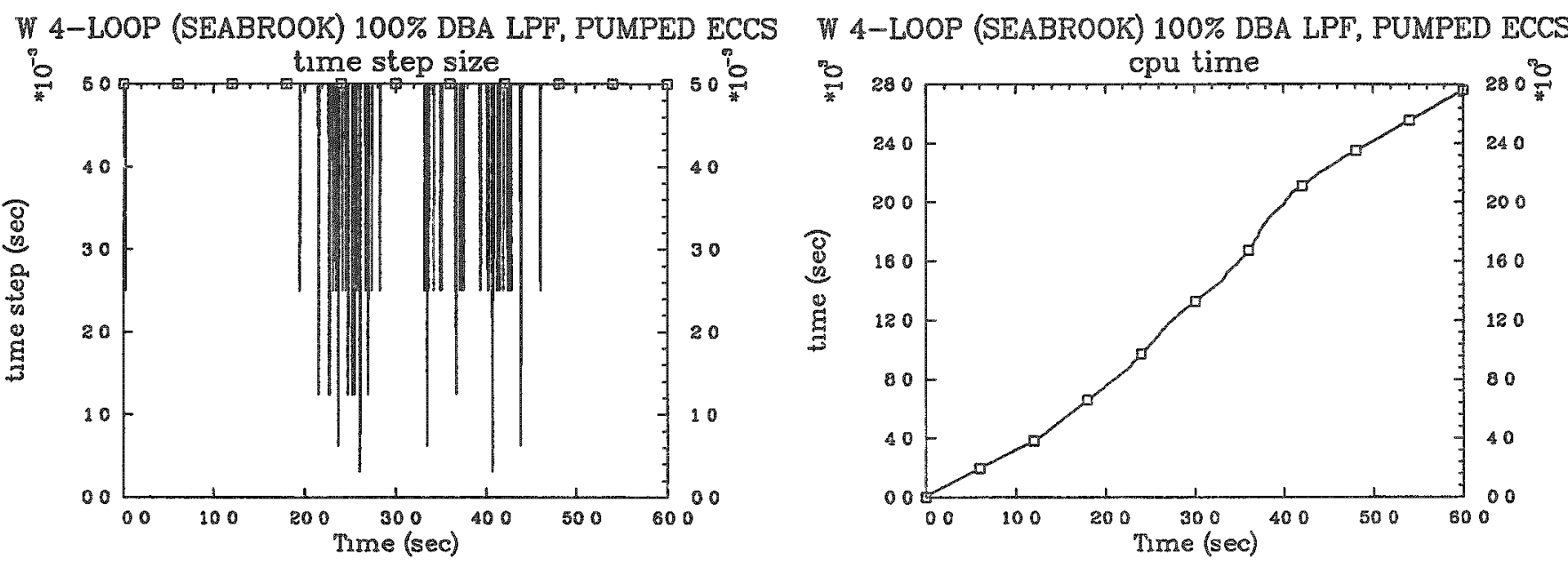


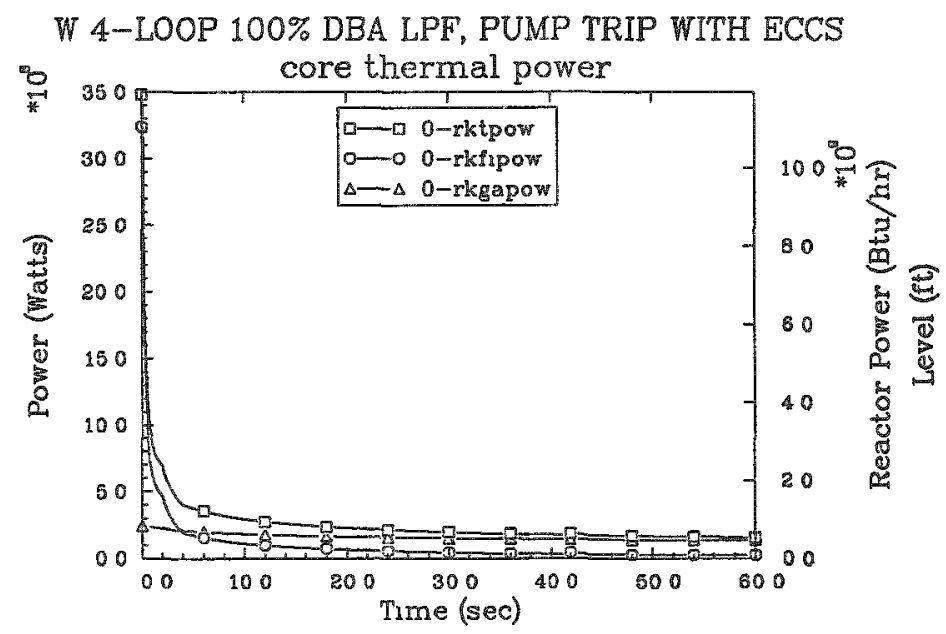

W 4-LOOP 100\% DBA LPF, PUMP TRIP WITH ECCS collapsed reactor water level

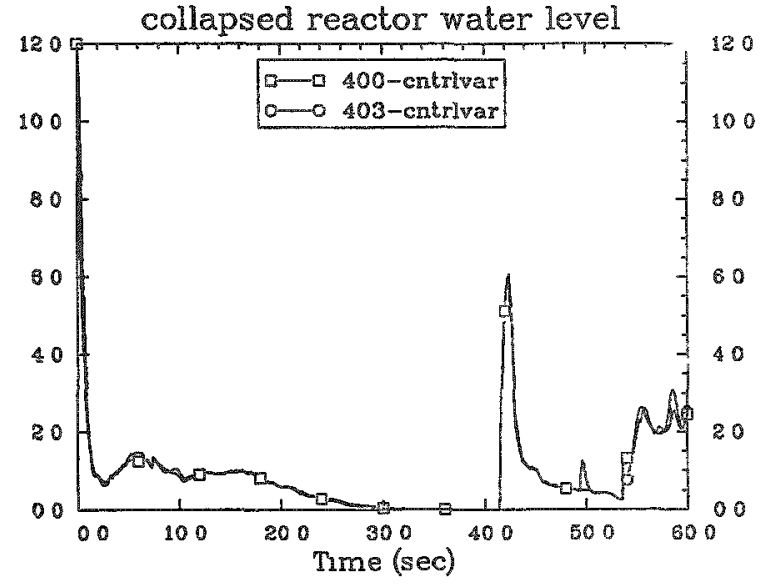

W 4-LOOP 100\% DBA LPF, PUMP TRIP WITH ECCS

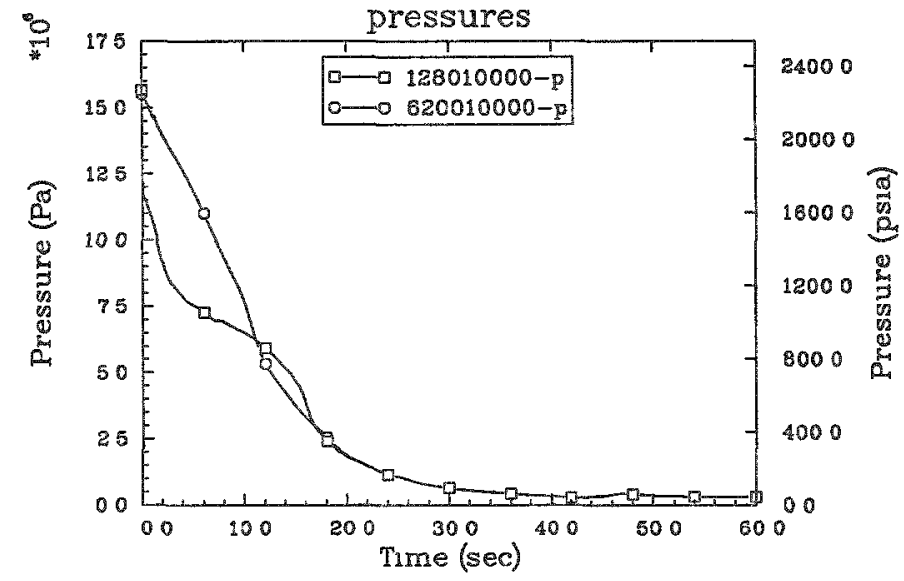

W 4-LOOP 100\% DBA LPF, PUMP TRIP WITH ECCS

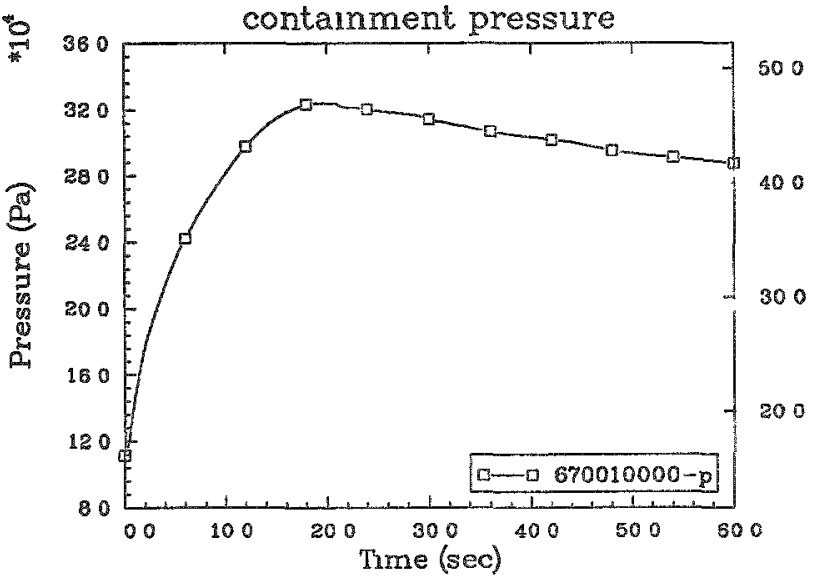

W 4-LOOP 100\% DBA LPF, PUMP TRIP WITH ECCS

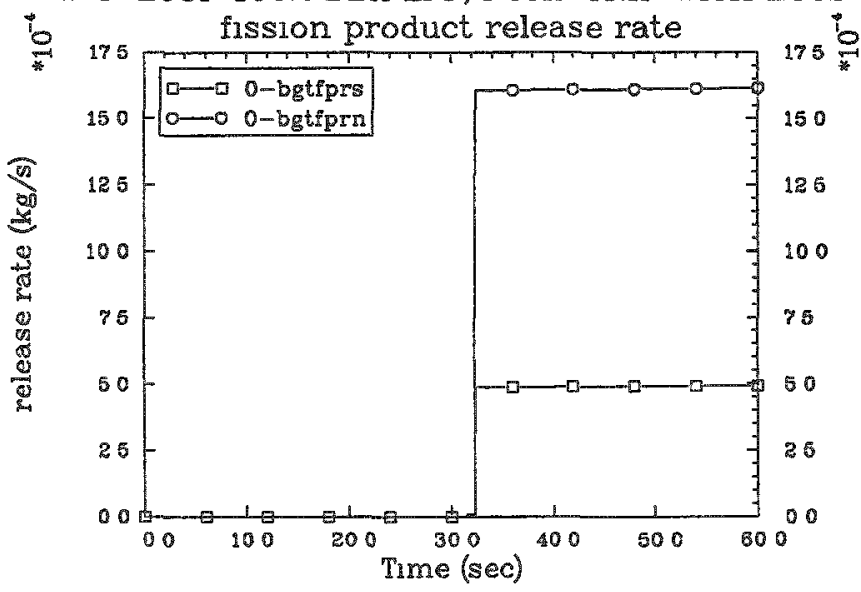

W 4-LOOP $100 \%$ DBA LPF, PUMP TRIP WITH ECCS

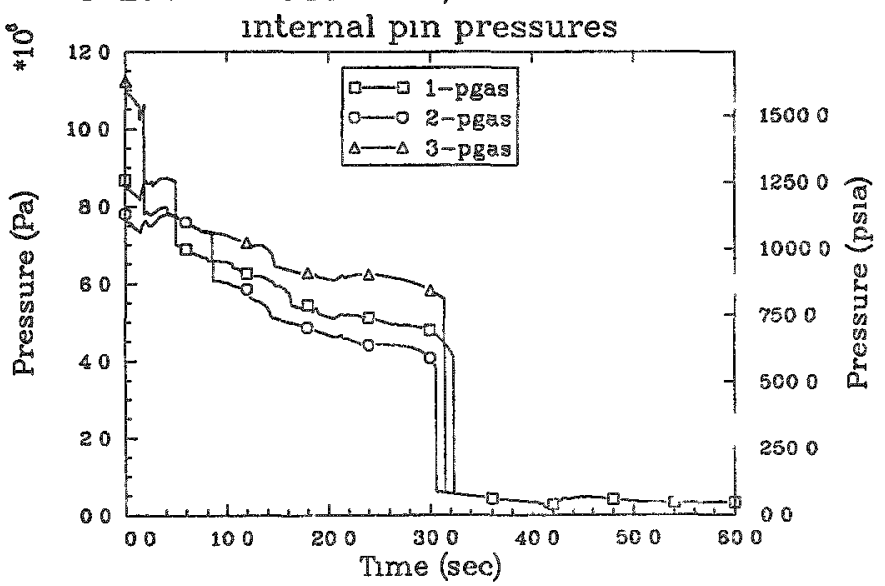


W 4-LOOP $100 \%$ DBA LPF, PUMP TRIP WITH ECCS fuel centerline temperatures for $5 \mathrm{GWD} / \mathrm{MTU}$ pin

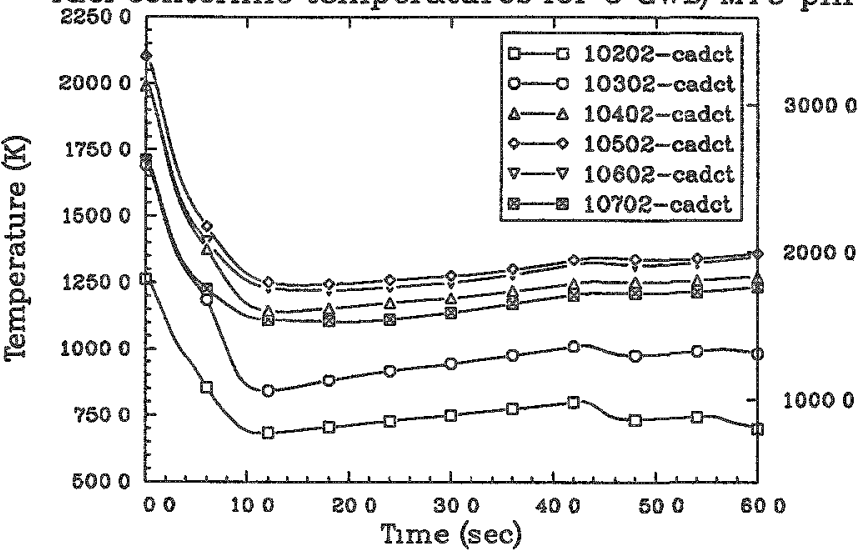

W 4-LOOP 100\% DBA LPF, PUMP TRIP WITH ECCS cladding surface temperatures for 5 GWD/MTU pin

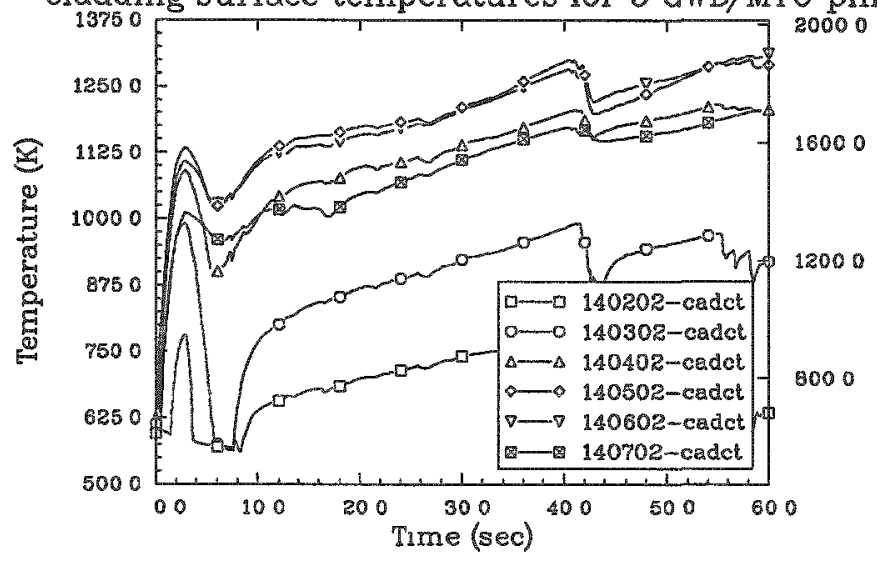

W 4-LOOP 100\% DBA LPF, PUMP TRIP WITH ECCS

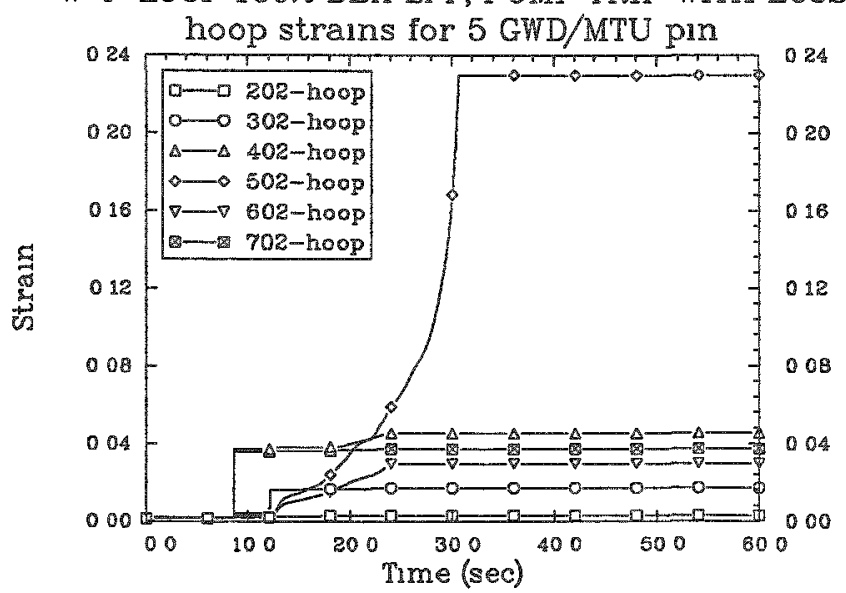

W 4-LOOP 100\% DBA LPF, PUMP TRIP WITH ECCS fuel centerline temperatures for $50 \mathrm{GWD} / \mathrm{MTU}$ pin

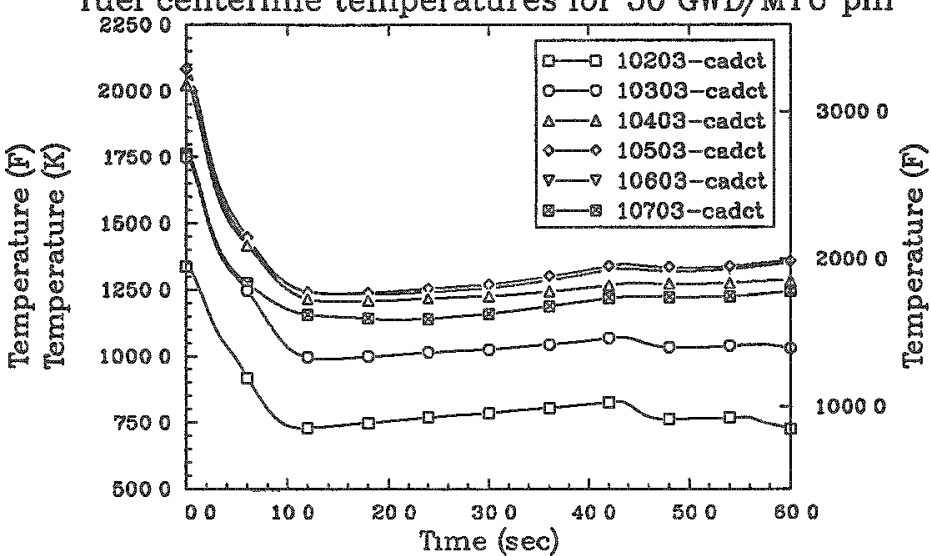

W 4-LOOP 100\% DBA LPF, PUMP TRIP WITH ECCS cladding surface temperatures for $50 \mathrm{GWD} / \mathrm{MTU}$ pin

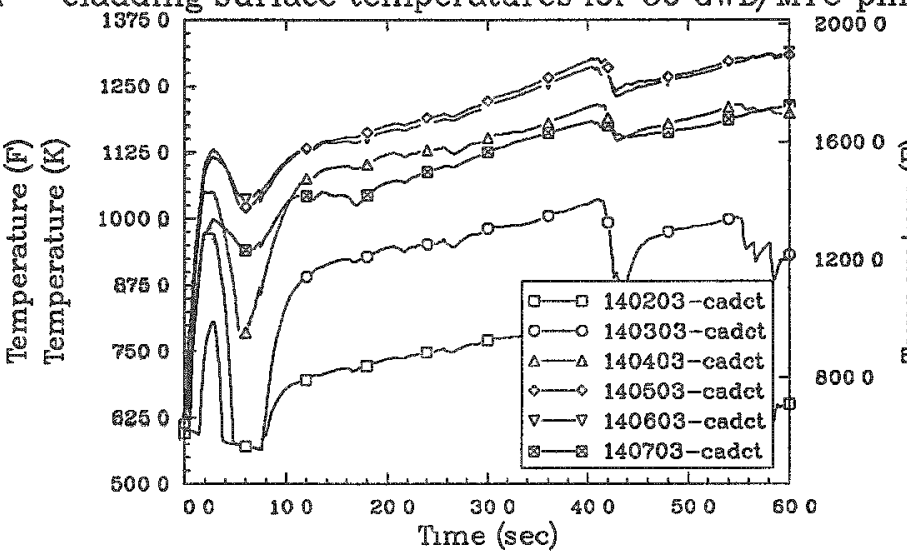

W 4-LOOP 100\% DBA LPF, PUMP TRIP WITH ECCS

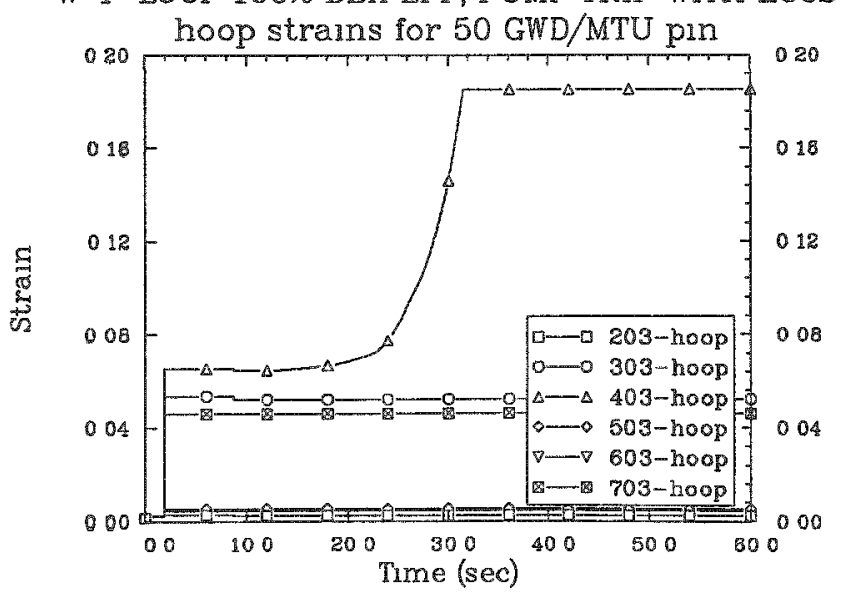



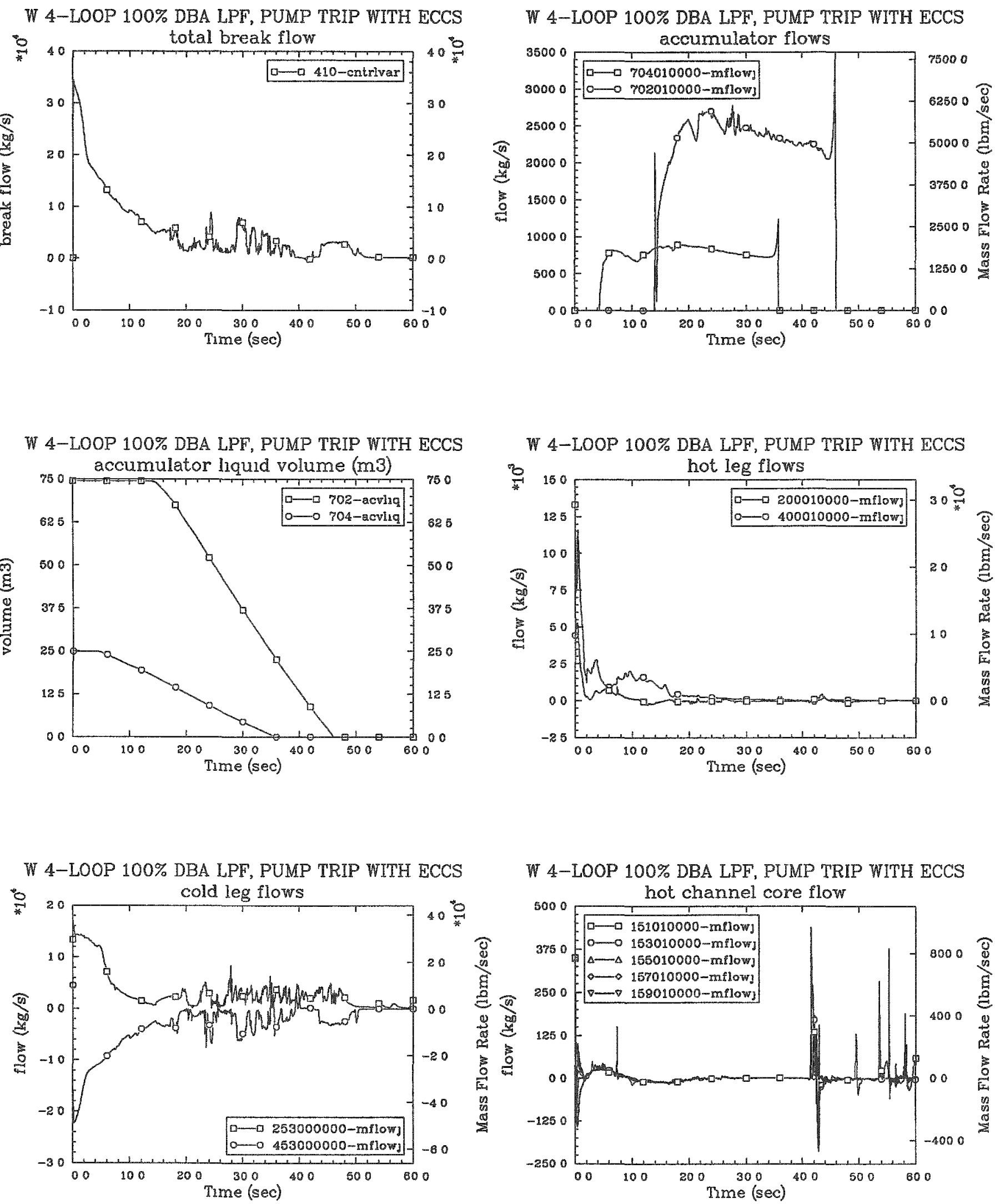

W 4-LOOP 100\% DBA LPF, PUMP TRIP WITH ECCS

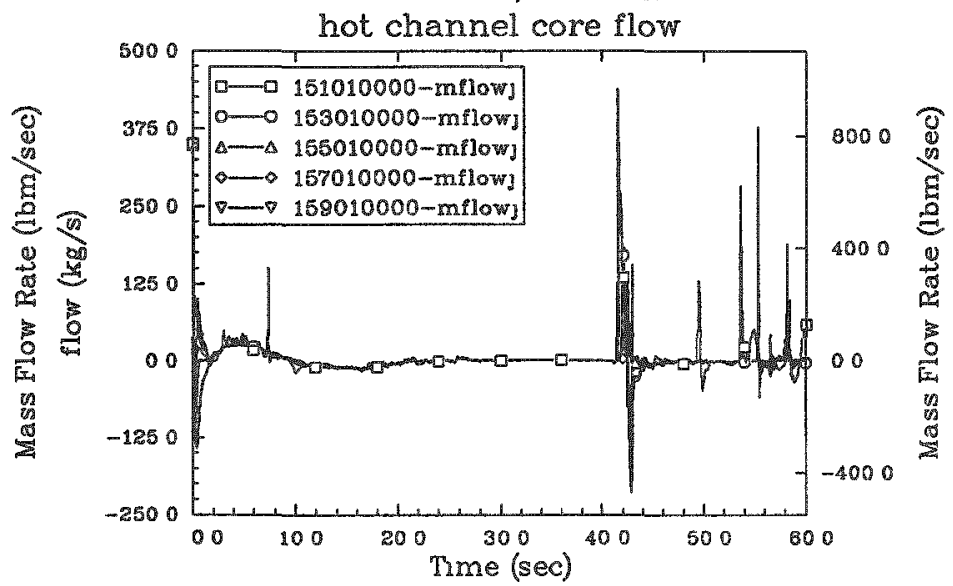


W 4-LOOP 100\% DBA LPF, PUMP TRIP WTTH ECCS

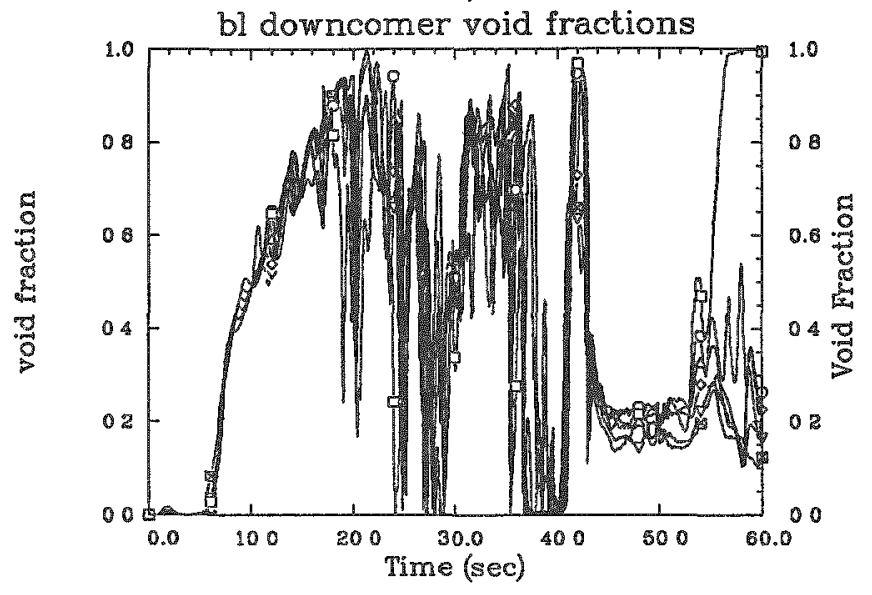

W 4-LOOP 100\% DBA LPF, PUMP TRIP WITH ECCS

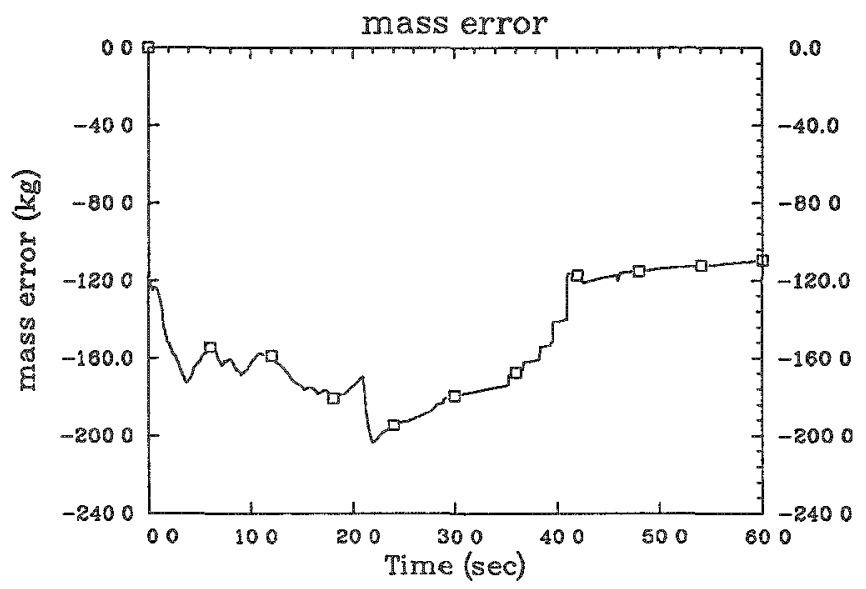

W 4-LOOP 100\% DBA LPF, PUMP TRIP WITH ECCS

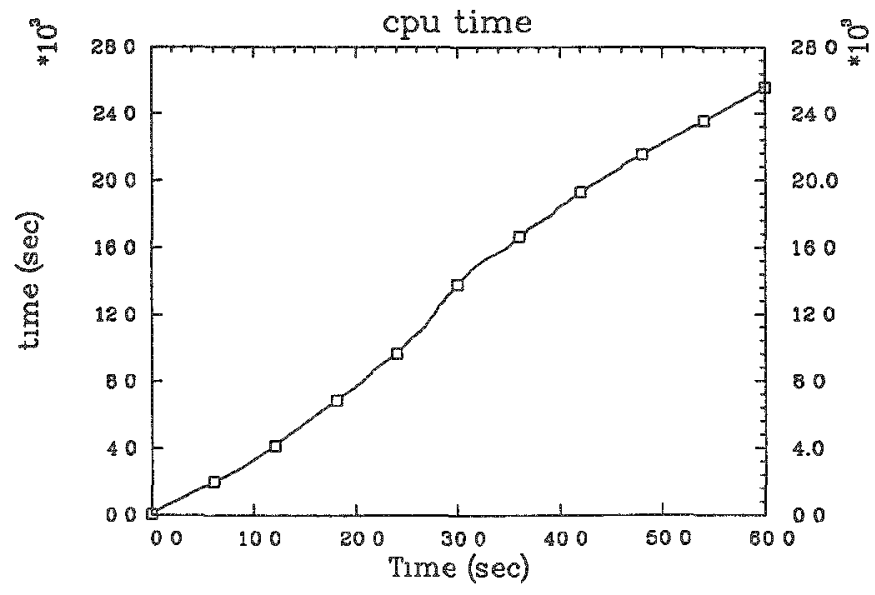

W 4-LOOP $100 \%$ DBA LPF, PUMP TRIP WITH ECCS

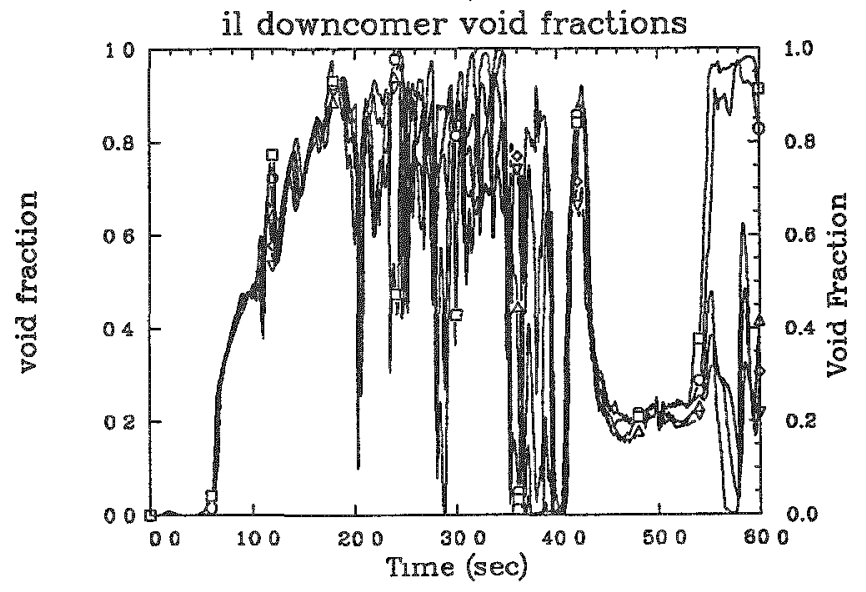

W 4-LOOP 100\% DBA LPF, PUMP TRIP WITH ECCS

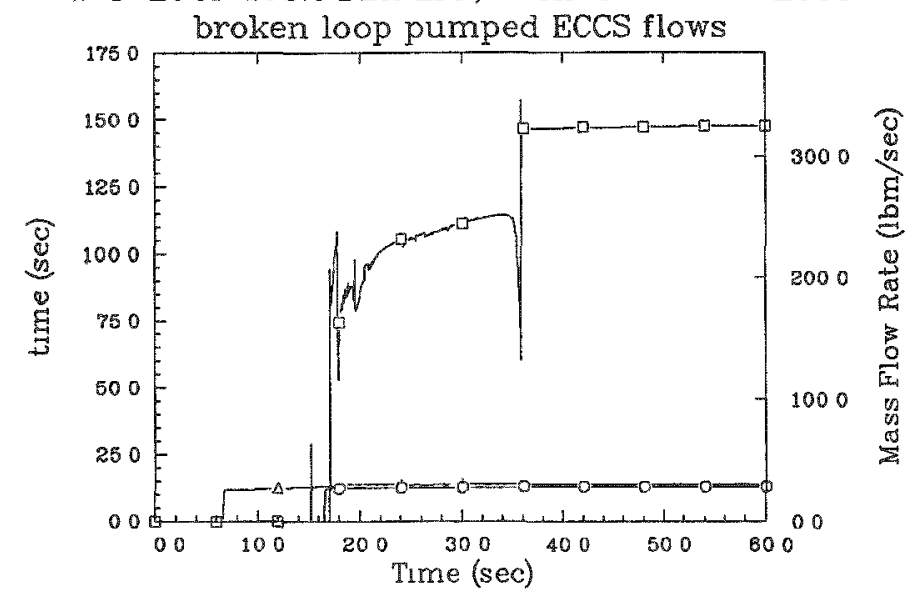


W 4-LOOP 100\% DBA LPF, PUMP TRIP WITH ECCS

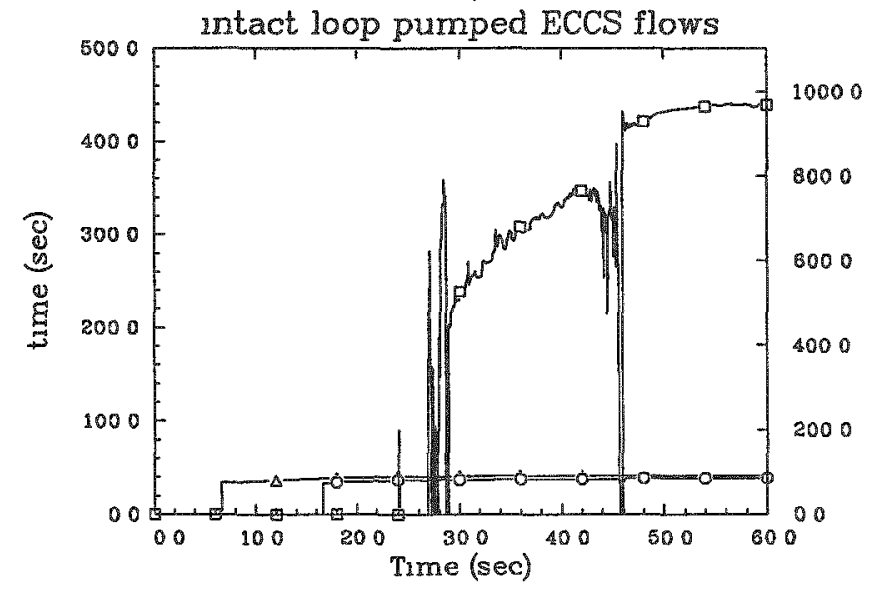

W 4-LOOP 100\% DBA LPF, PUMP TRIP WITH ECCS total ECCS flows

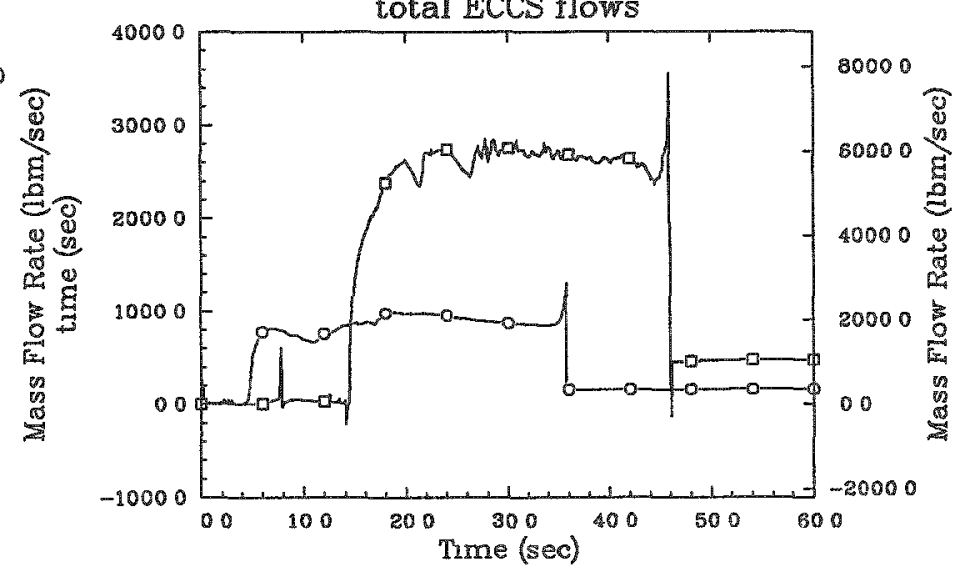




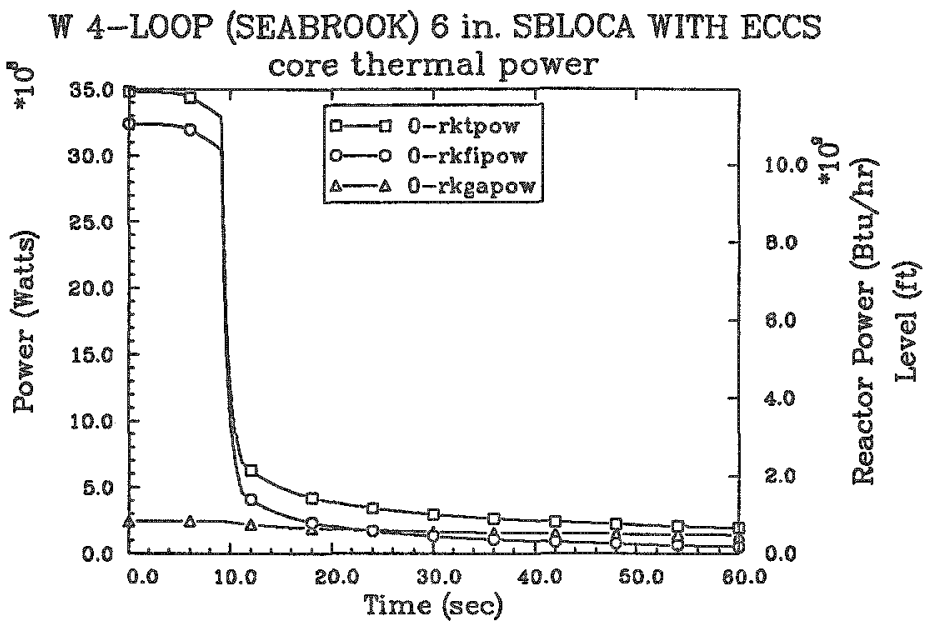

W 4-LOOP (SEABROOK) 6 in. SBLOCA WTTH ECCS

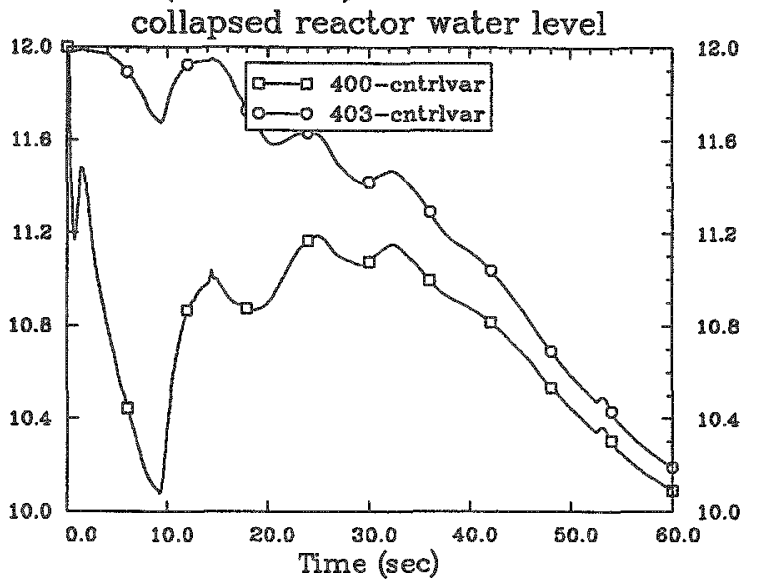

W 4-LOOP (SEABROOK) 6 in. SBLOCA WITH ECCS

$\circ$

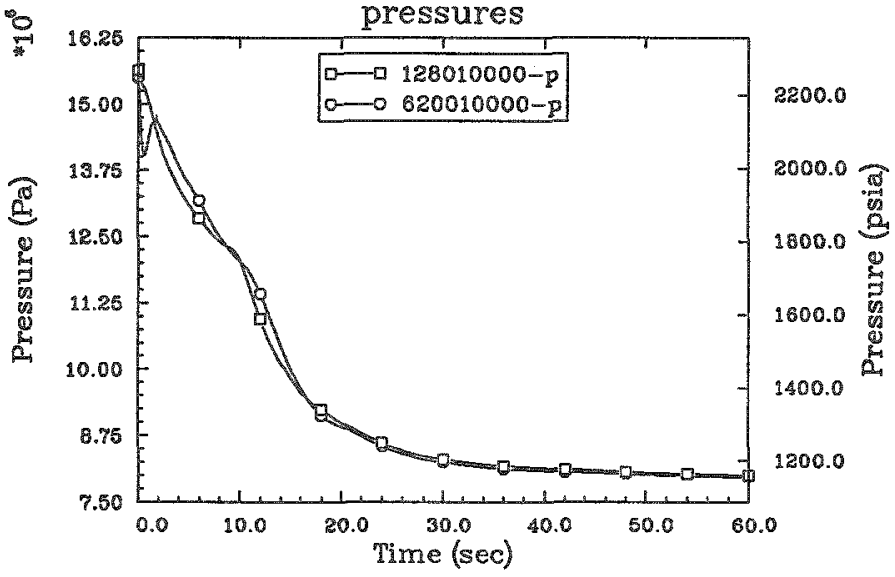

W 4-LOOP (SEABROOK) 6 in. SBLOCA WITH ECCS

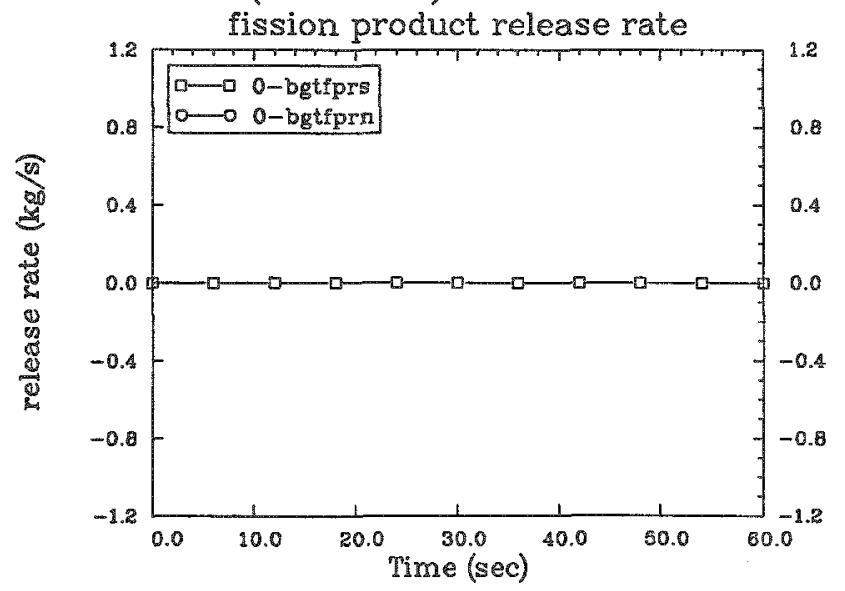

W 4-LOOP (SEABROOK) 6 in. SBLOCA WITH ECCS

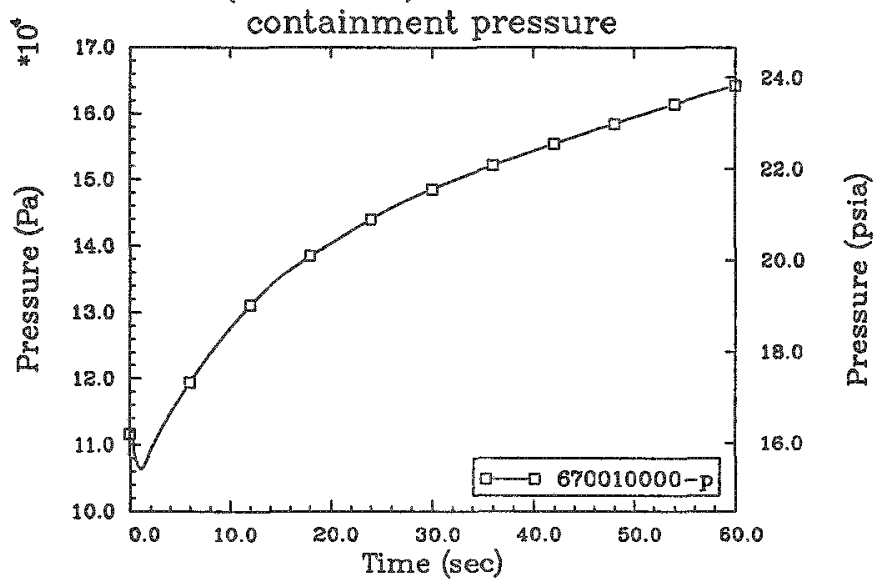

W 4-LOOP (SEABROOK) 6 in. SBLOCA WITH ECCS

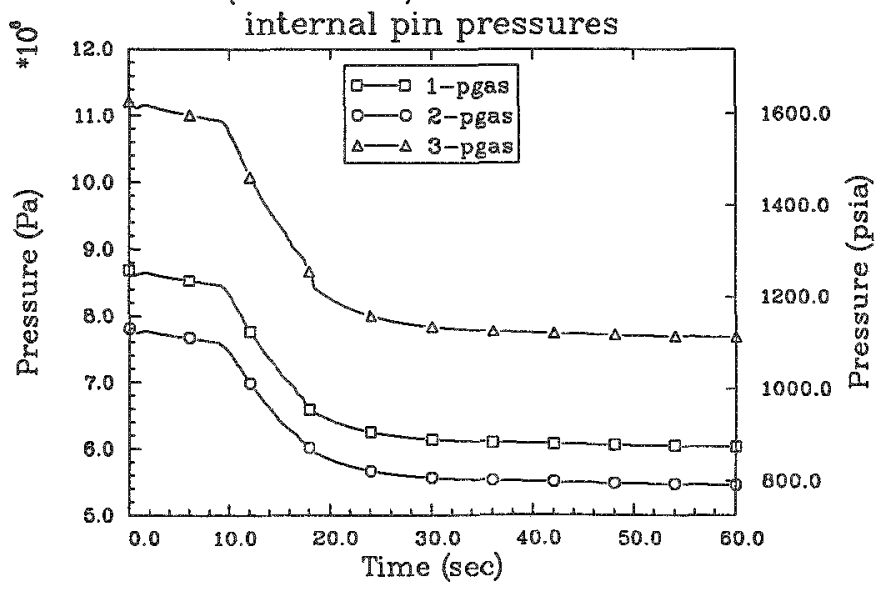


W 4-LOOP (SEABROOK) 6 in. SBLOCA WITH ECCS

fuel centerline temperatures for 5 GWD/MTU pin

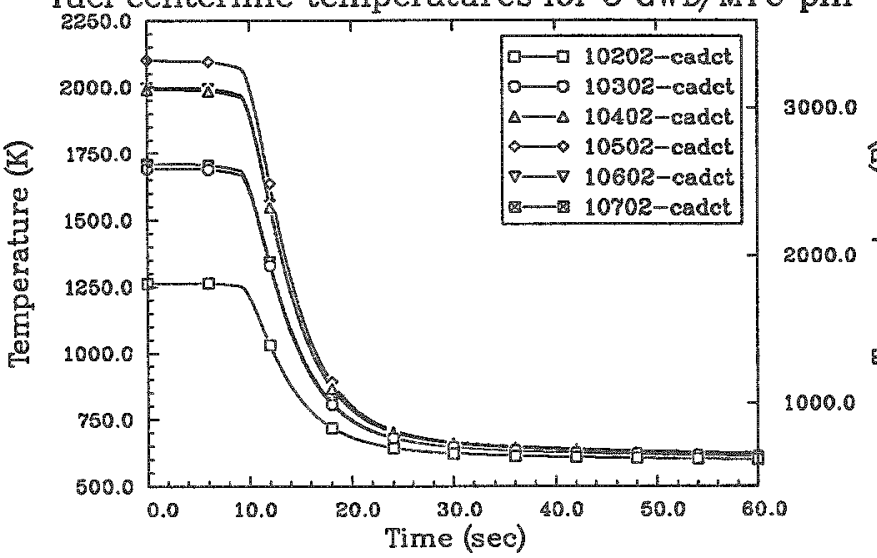

W 4-LOOP (SEABROOK) 6 in. SBLOCA WITH ECCS cladding surface temperatures for 5 GWD/MTU pin

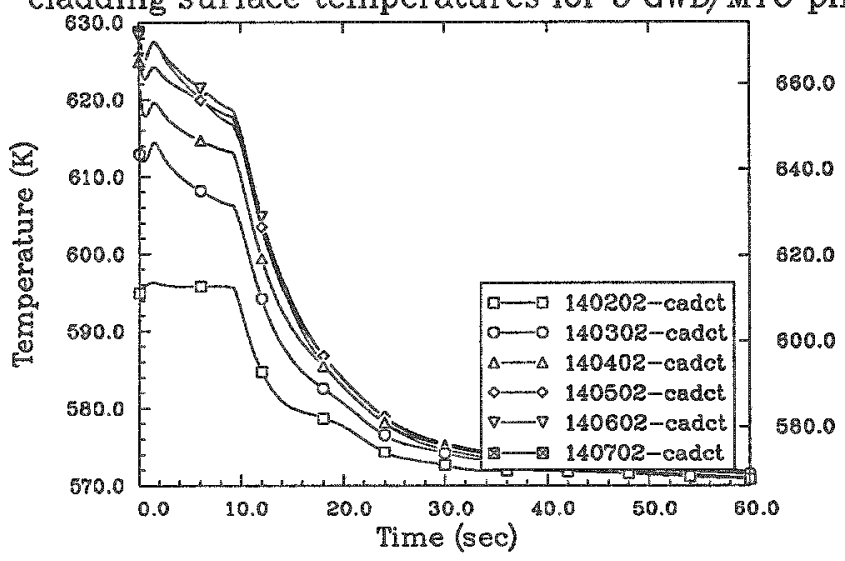

W 4-LOOP (SEABROOK) 6 in. SBLOCA WITH ECCS

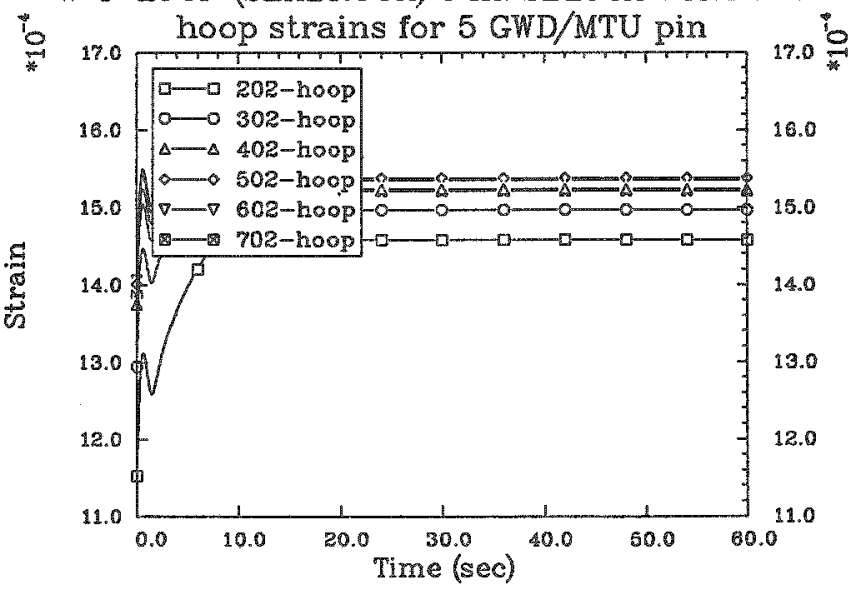

W 4-LOOP (SEABROOK) 6 in. SBLOCA WTTH ECCS iuel centerline temperatures for $50 \mathrm{GWD} / \mathrm{MTU}$ pin

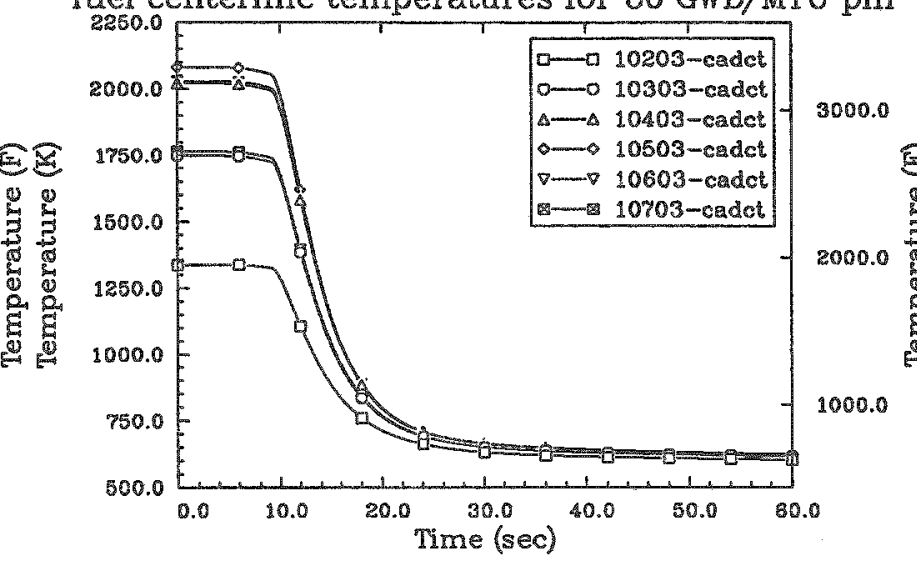

W 4-LOOP (SEABROOK) 6 in. SBLOCA WITH ECCS cladding surface temperatures for $50 \mathrm{GWD} / \mathrm{MTU}$ pin

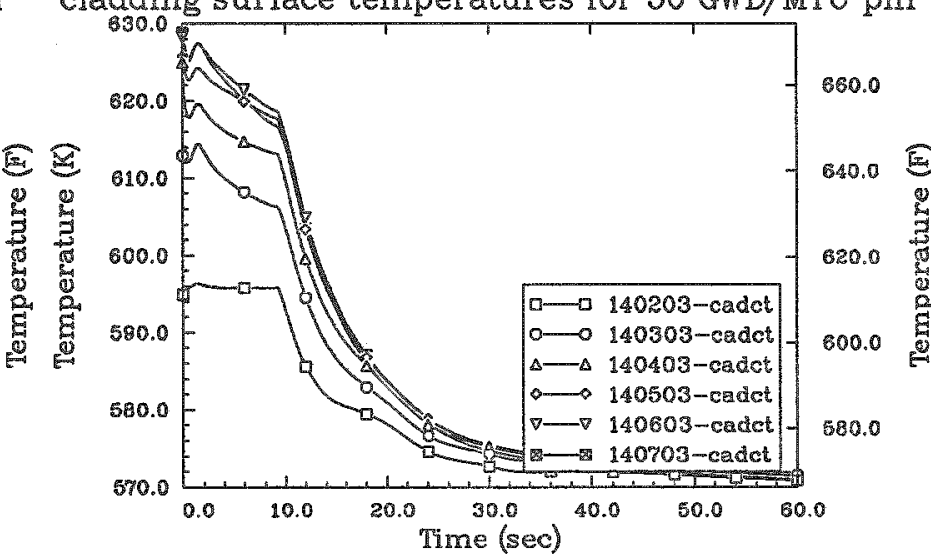



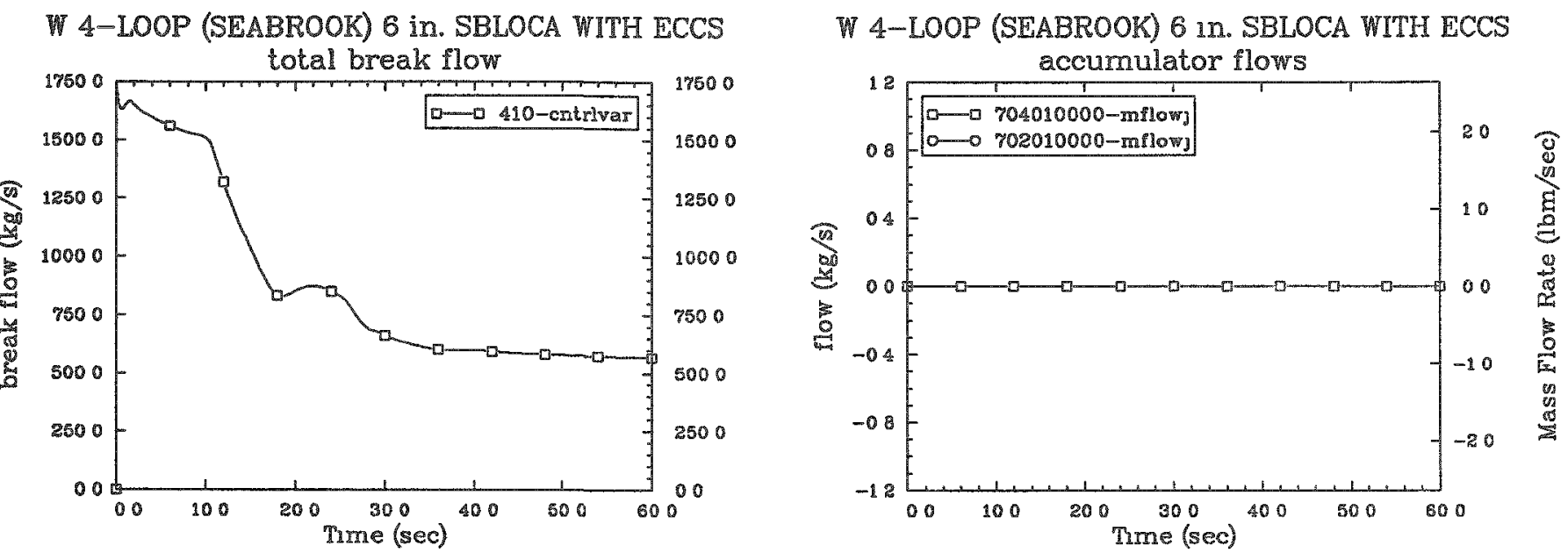

W 4-LOOP (SEABROOK) 6 in SBLOCA WITH ECCS
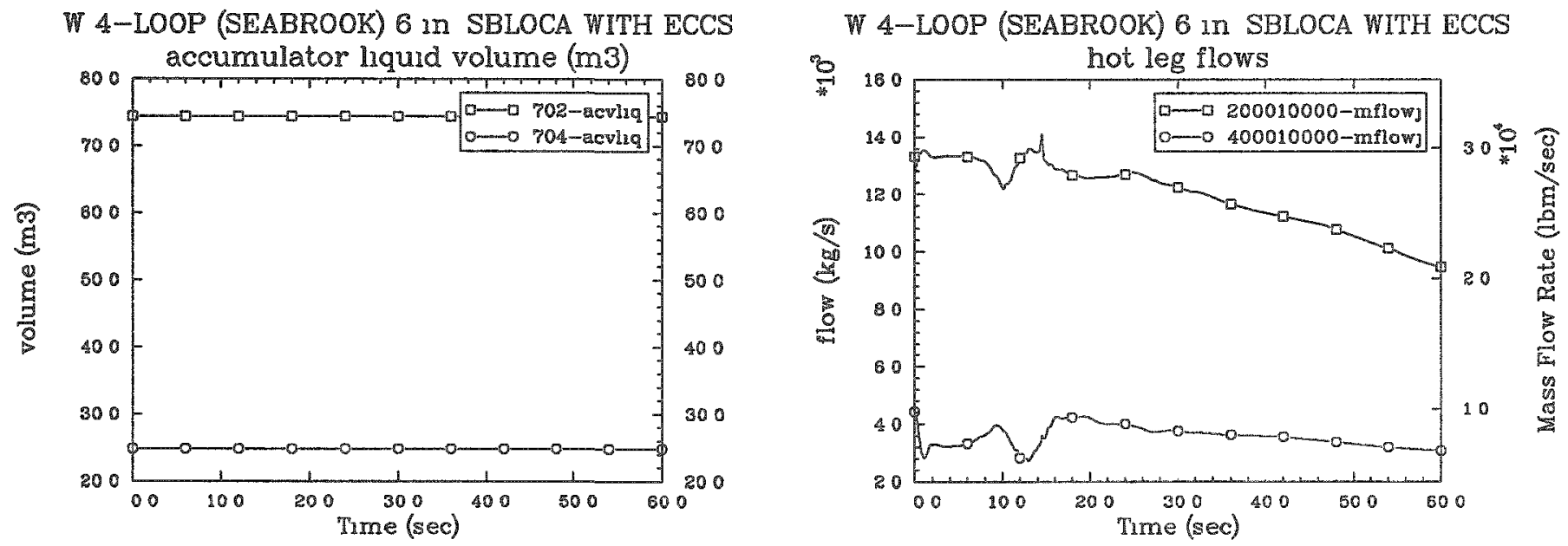

W 4-LOOP (SEABROOK) 6 in SBLOCA WITH ECCS

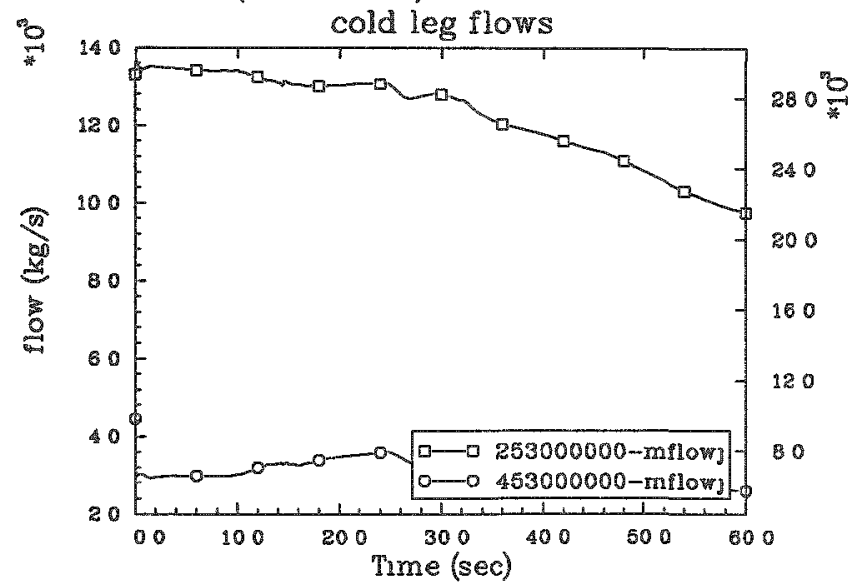

W 4-LOOP (SEABROOK) 6 in SBLOCA WITH ECCS

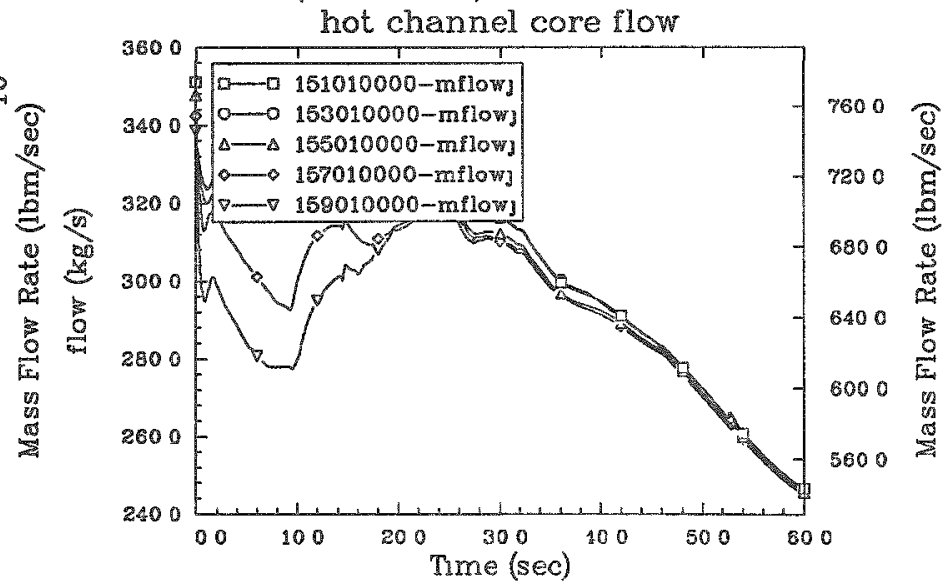




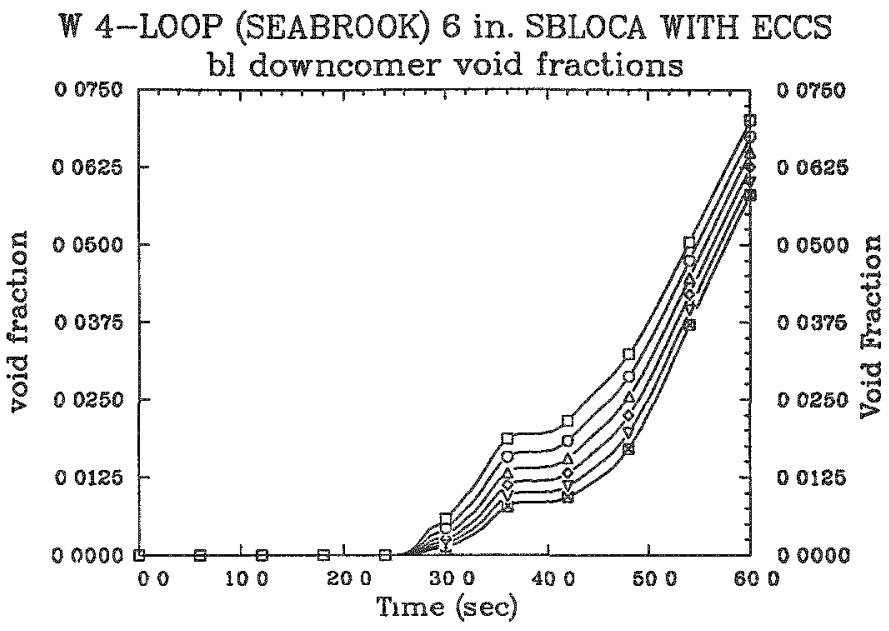

W 4-LOOP (SEABROOK) 6 in. SBLOCA WITH ECCS

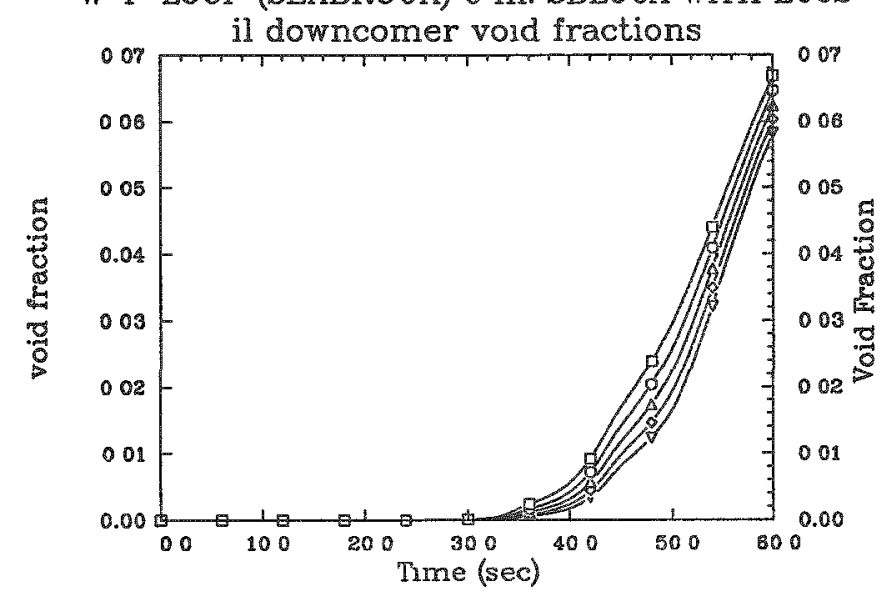

W 4-LOOP (SEABROOK) 6 in SBLOCA WITH ECCS

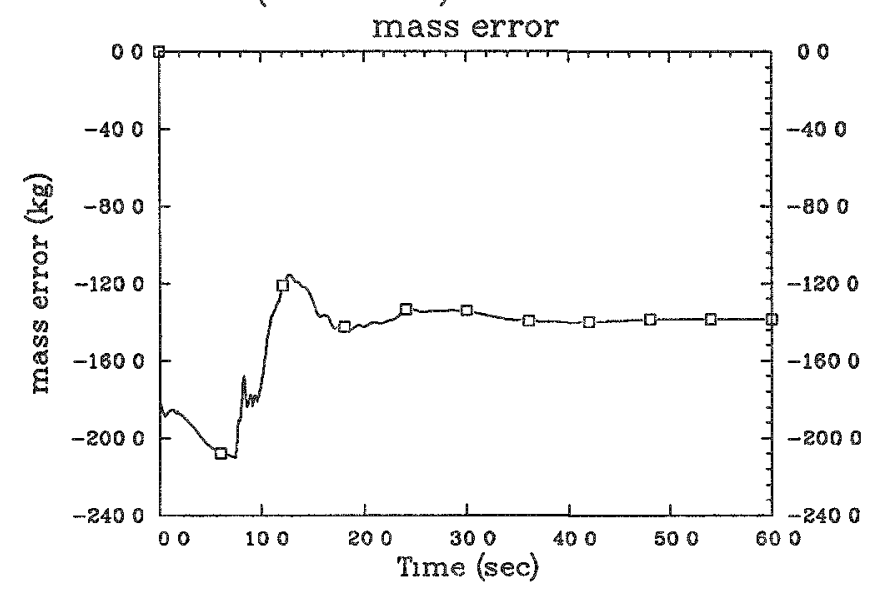

W 4-LOOP (SEABROOK) 6 in SBLOCA WITH ECCS

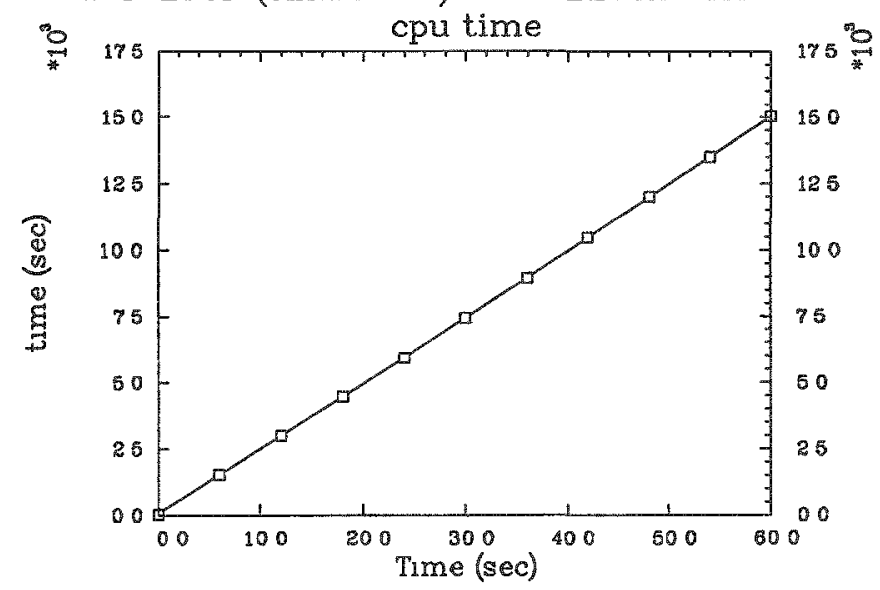

W 4-LOOP (SEABROOK) $6 \mathrm{in.} \mathrm{SBLOCA} \mathrm{WITH} \mathrm{ECCS}$

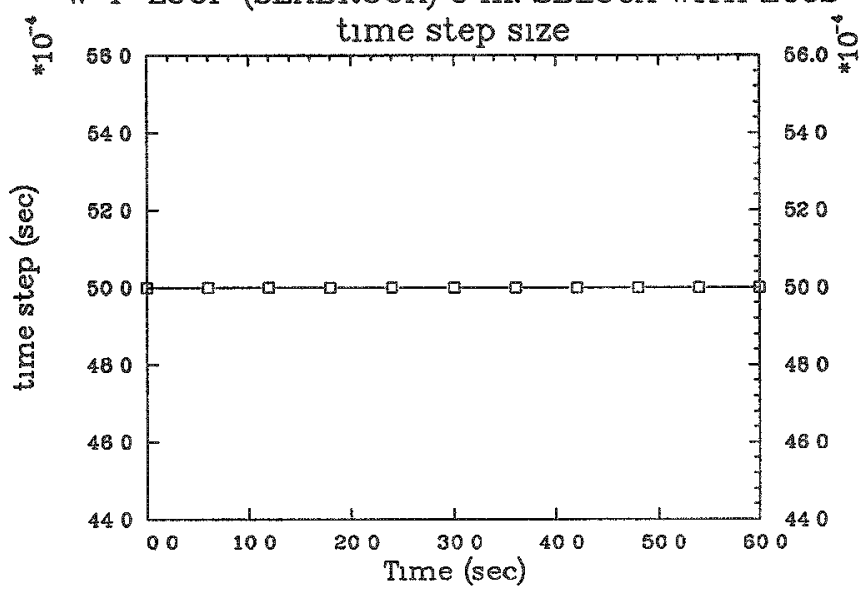

W 4-LOOP (SEABROOK) $6 \mathrm{~m}$. SBLOCA WITH ECCS

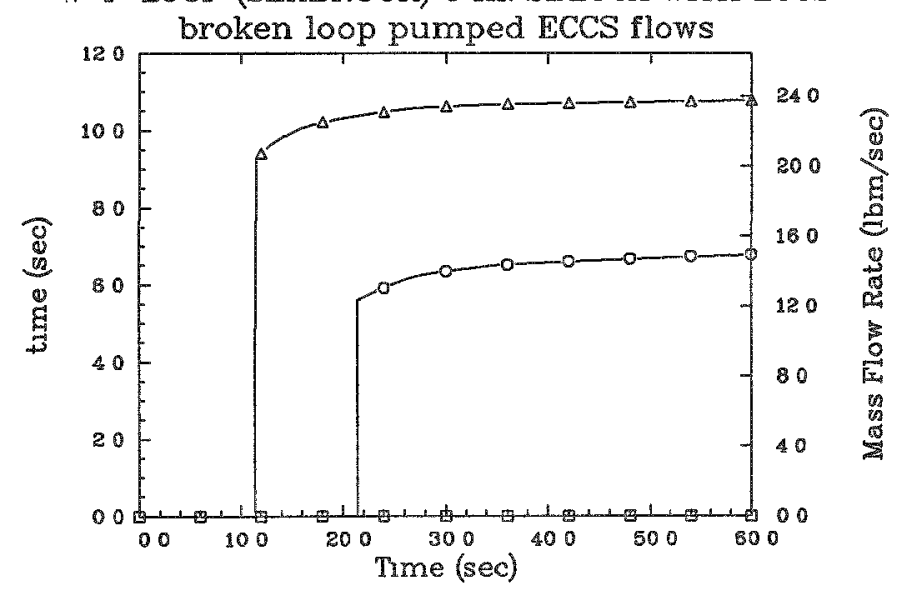


W 4-LOOP (SEABROOK) 6 in SBLOCA WITH ECCS

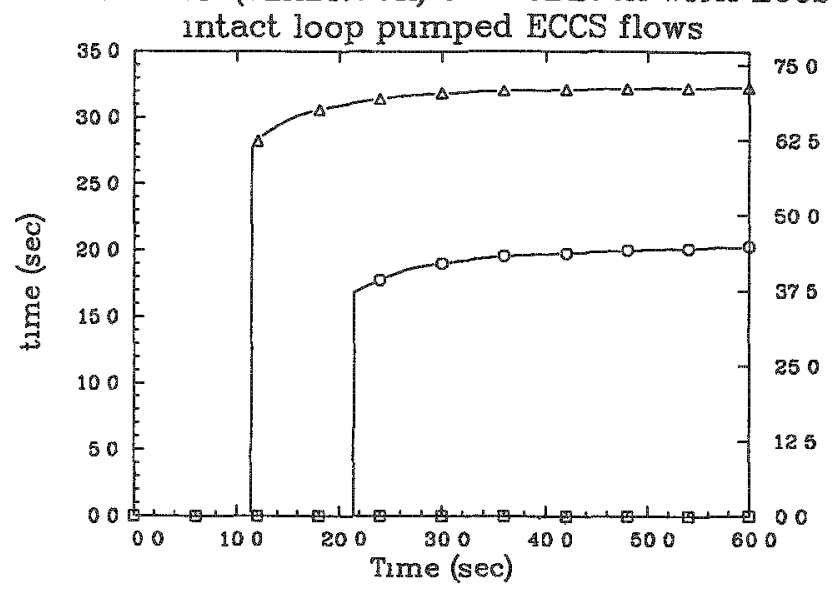

W 4-LOOP (SEABROOK) 6 in SBLOCA WITH ECCS total ECCS flows

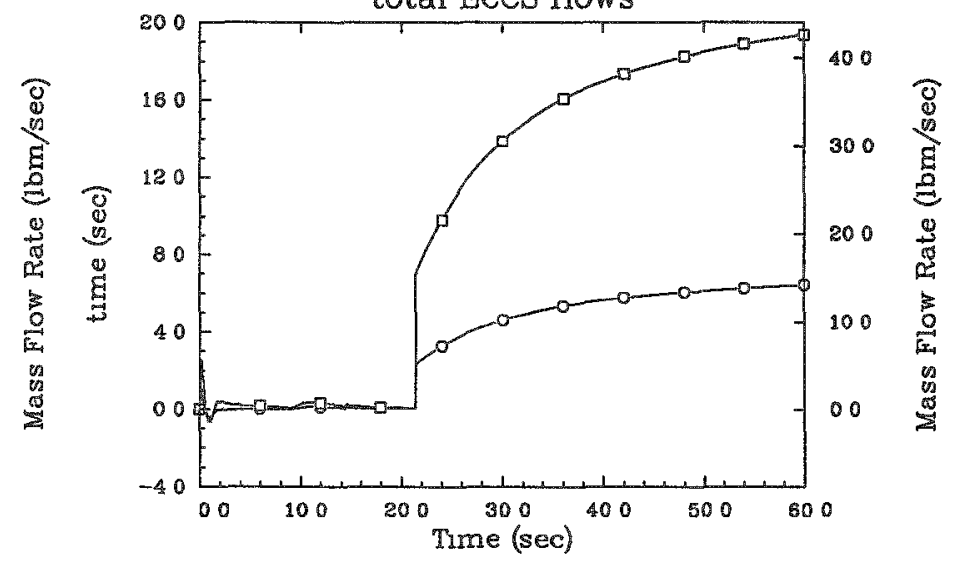




\section{L-2. FRAP-T6 Plotted REsults for SEABrook}

Table L-2. Description of FRAP-T6 plot variables for Seabrook.

\begin{tabular}{ll}
\hline Variable & \multicolumn{1}{c}{ Description } \\
\hline 0-ffr & Failure probability \\
0-prspln & Internal pin pressure (psia, Pa) \\
n-cladhsn & Cladding hoop strain at axial node $n$ \\
$n$-cladote & Cladding surface temperature at axial node $n\left({ }^{\circ} \mathrm{F}, \mathrm{K}\right)$ \\
$n$-ctemp & Fuel centerline temperature at axial node $n\left({ }^{\circ} \mathrm{F}, \mathrm{K}\right)$ \\
$n$-ooxtn & Oxide thickness at axial node $n$ (in., mn) \\
\hline
\end{tabular}



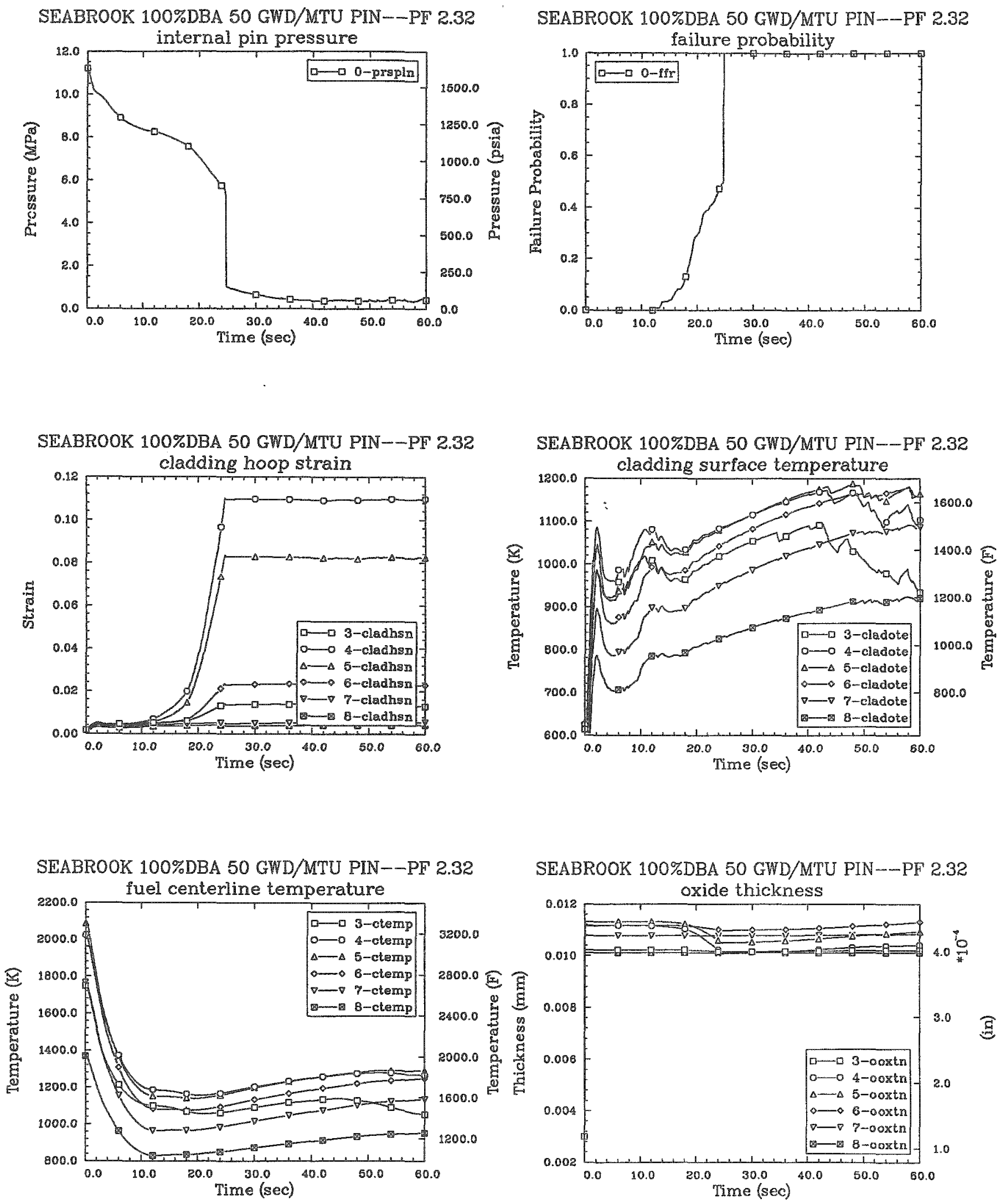

SEABROOK 100\%DBA 50 GWD/MTU PIN--PF 2.32

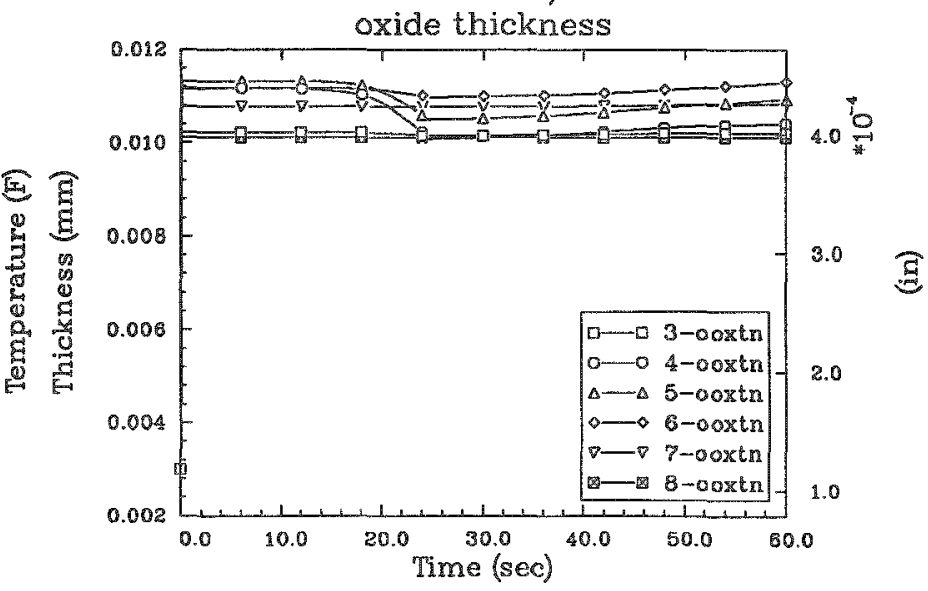


SEABROOK 100\%DBA 35 GWD/MTU PIN--PF 2.32

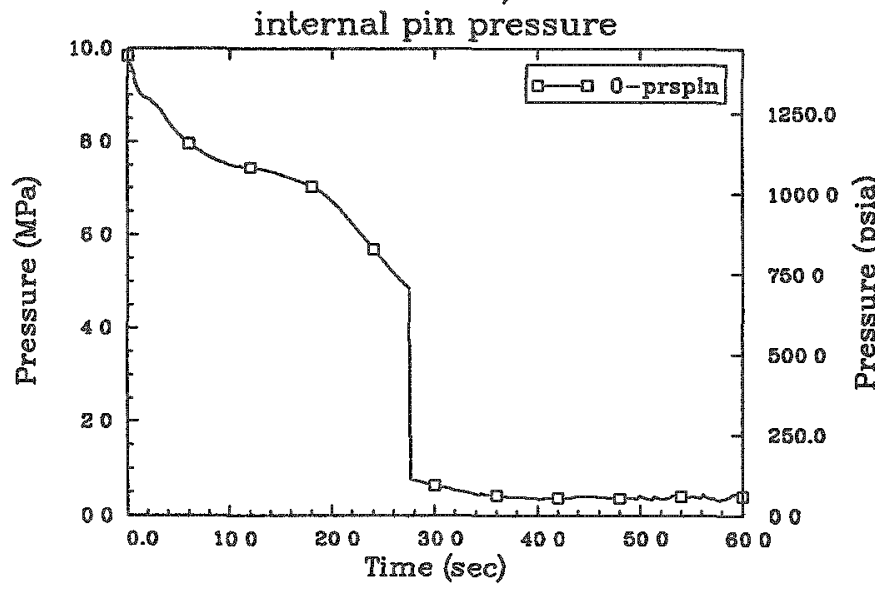

SEABROOK 100\%DBA 35 GWD/MTU PIN--PF 2.32

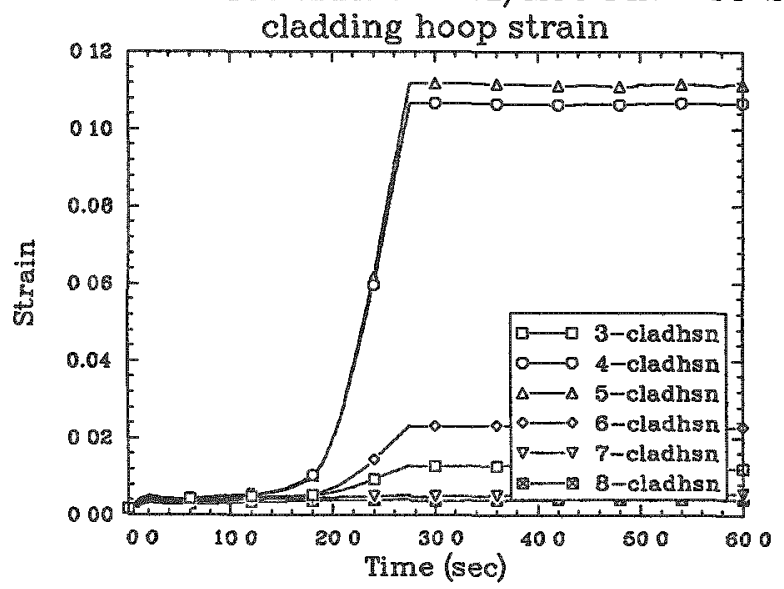

SEABROOK 100\%DBA 35 GWD/MTU PIN--PF 2.32 failure probability

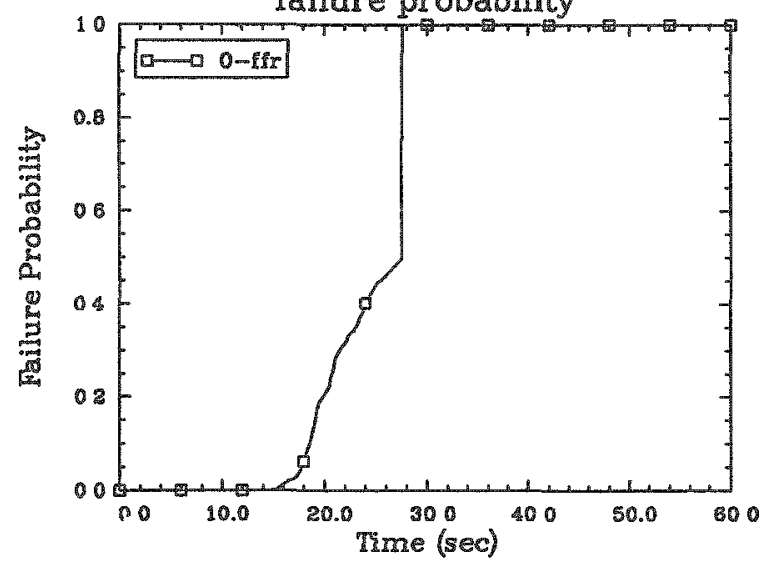

SEABROOK 100\%DBA 35 GWD/MTU PIN--PF 2.32 cladding surface temperature

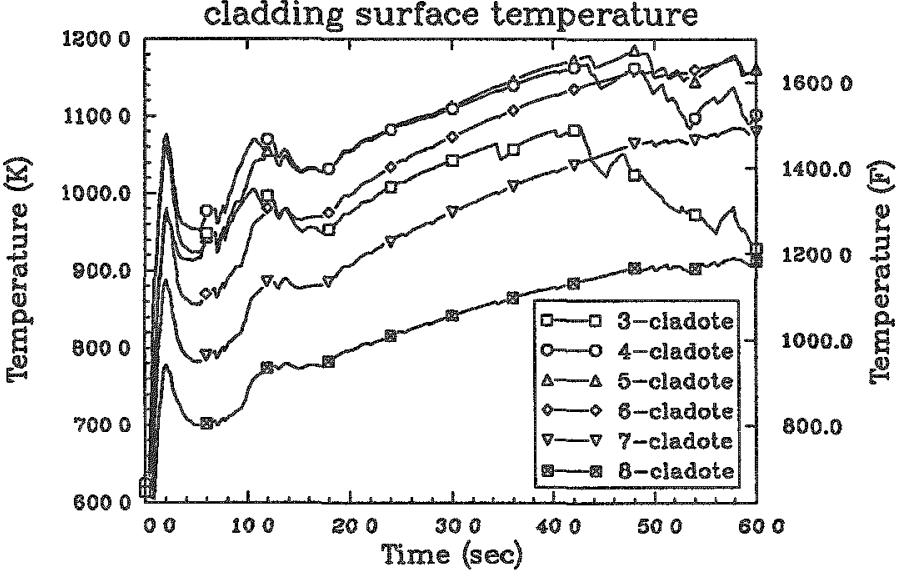

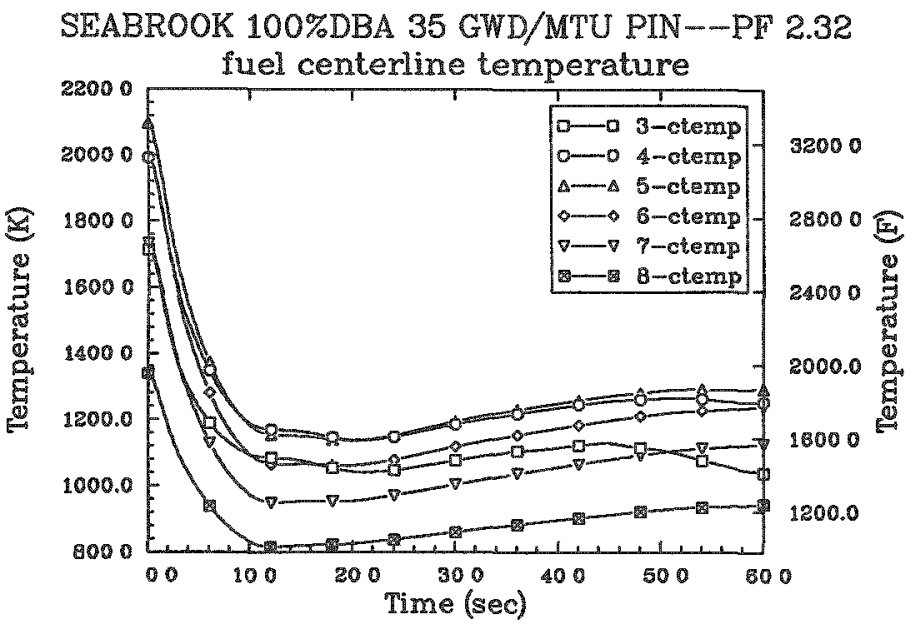

SEABROOK 100\%DBA 35 GWD/MTU PIN--PF 2.32

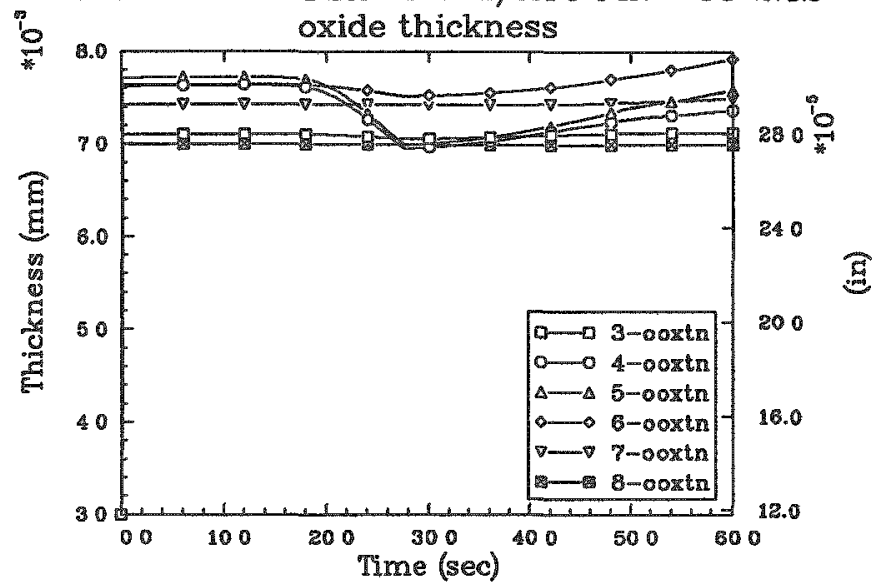


SEABROOK 100\%DBA 20 GWD/MTU PIN--PF 232

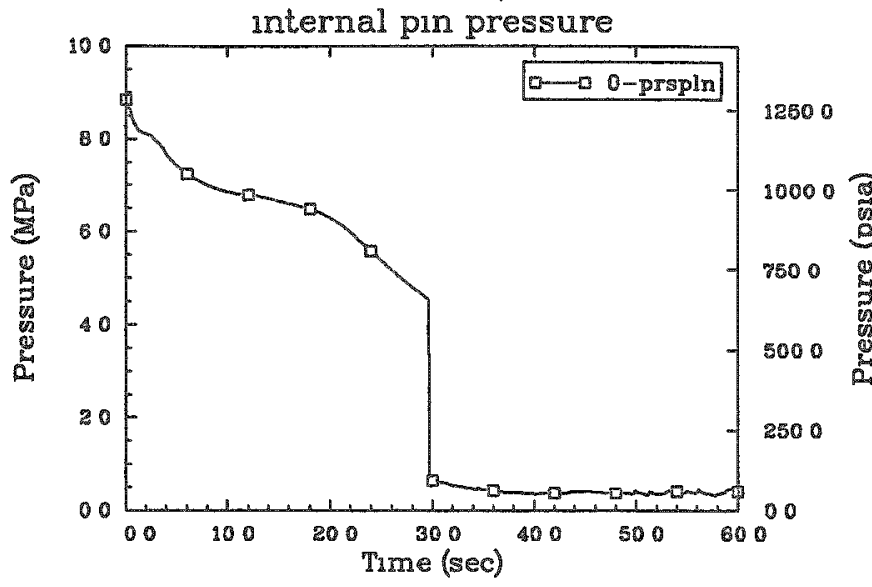

SEABROOK 100\%DBA $20 \mathrm{GWD} / \mathrm{MTU}$ PIN--PF 232

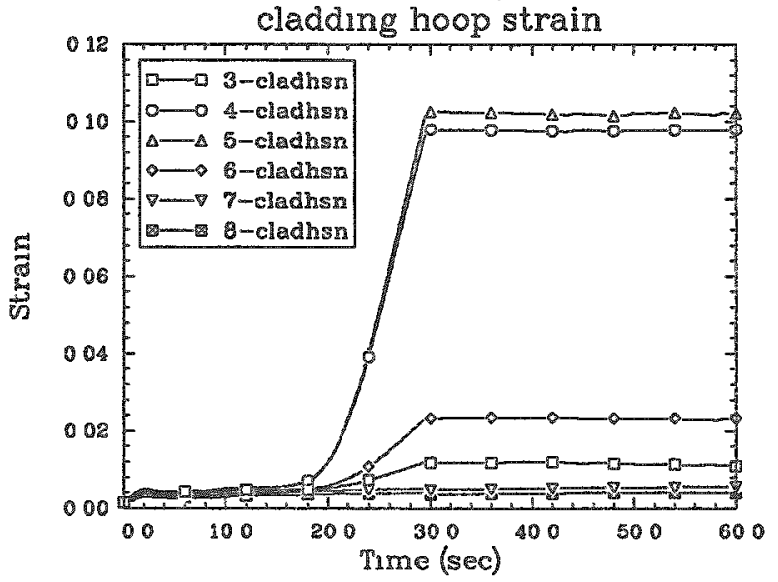

SEABROOK 100\%DBA 20 GWD/MTU PIN--PF 232

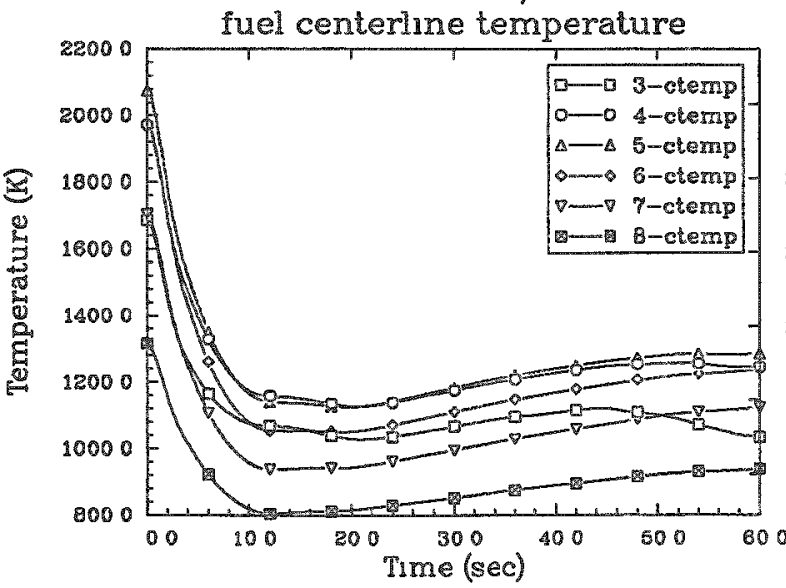

SEABROOK 100\%DBA 20 GWD/MTU PIN--PF 2.32 fallure probability

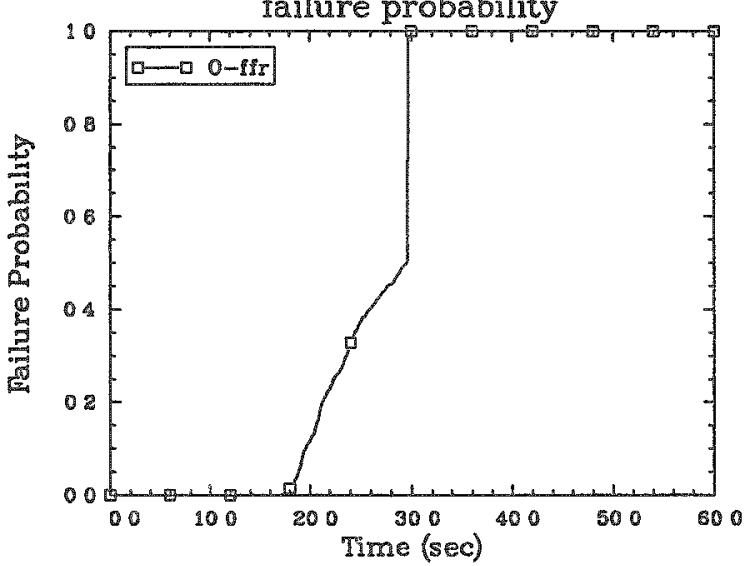

SEABROOK 100\%DBA 20 GWD/MTU PIN--PF 232

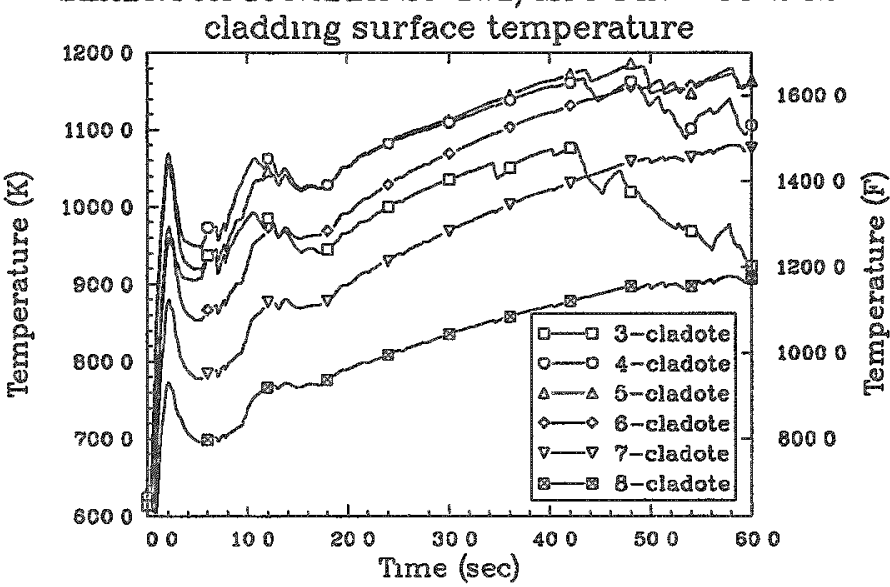

SEABROOK 100\%DBA 20 GWD/MTU PIN--PF 232

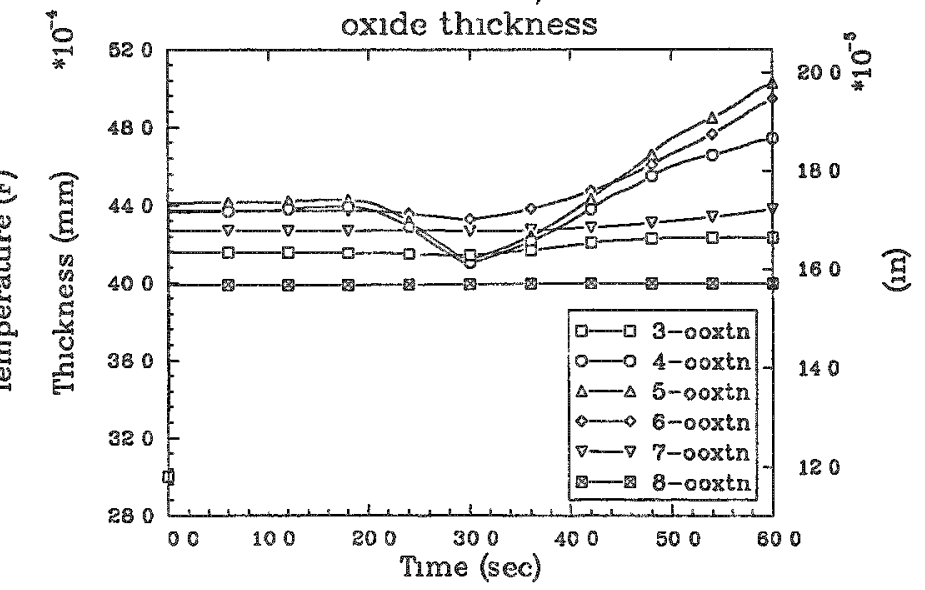




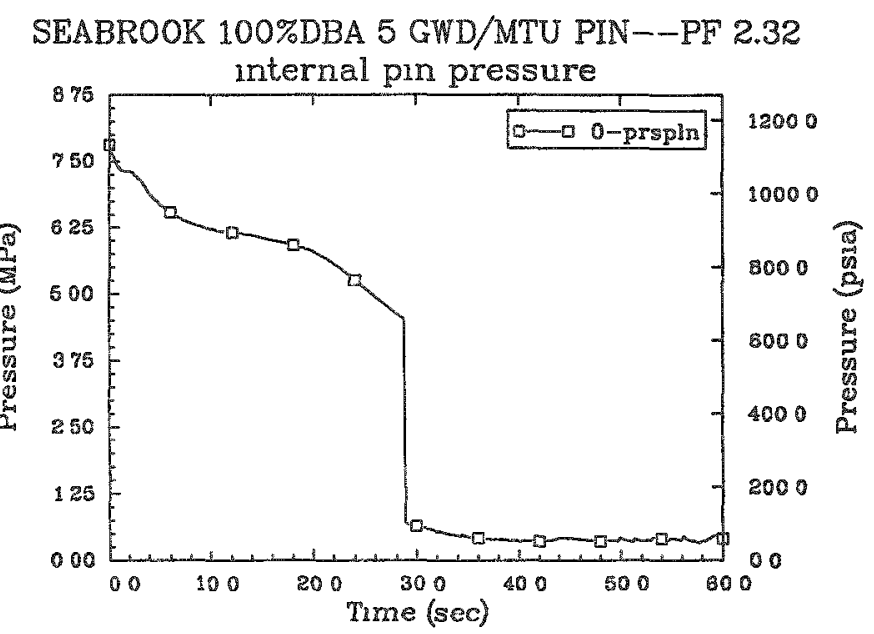

SEABROOK 100\%DBA 5 GWD/MTU PIN--PF 232
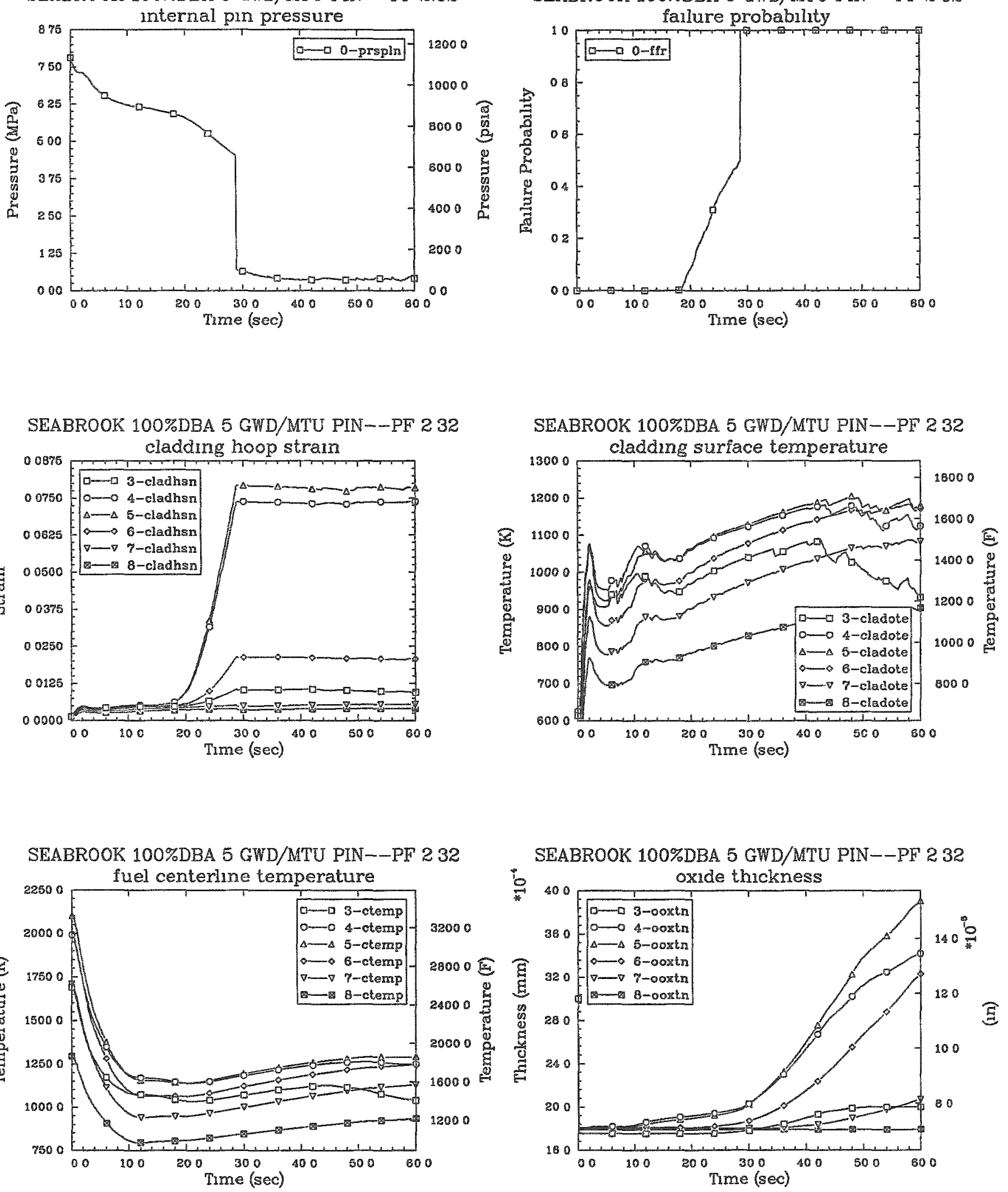
SEABROOK 100\%DBA 50 GWD/MTU PIN--PF 2.2
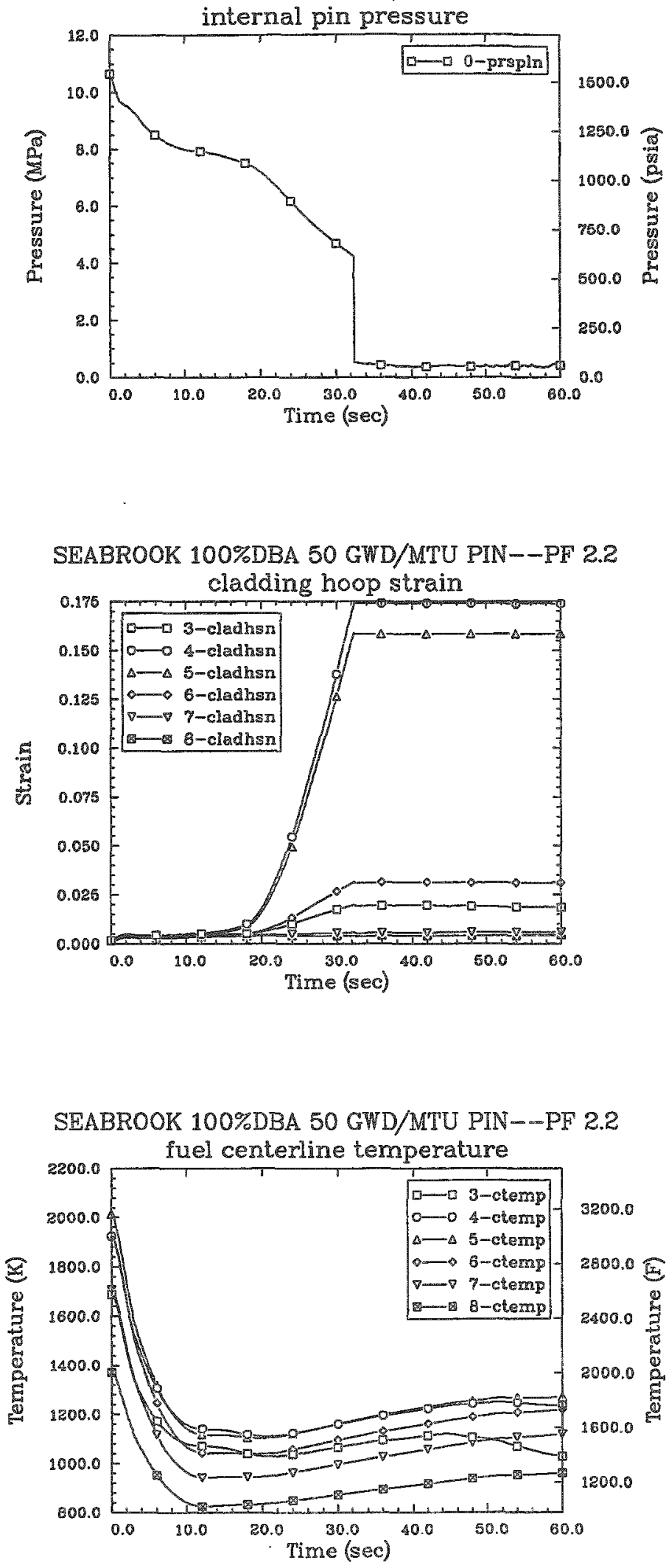

SEABROOK 100\%DBA 50 GWD/MTU PIN--PF 2.2 failure probability

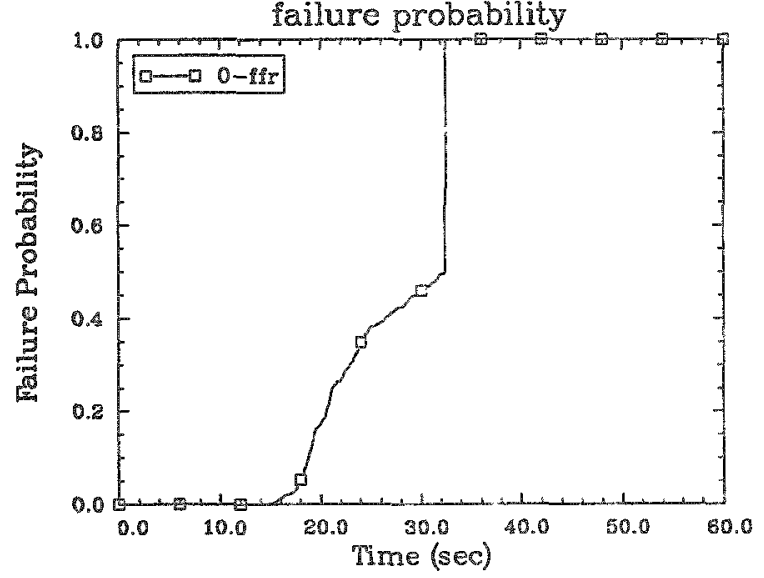

SEABROOK 100\%DBA 50 GWD/MTU PIN--PF 2.2

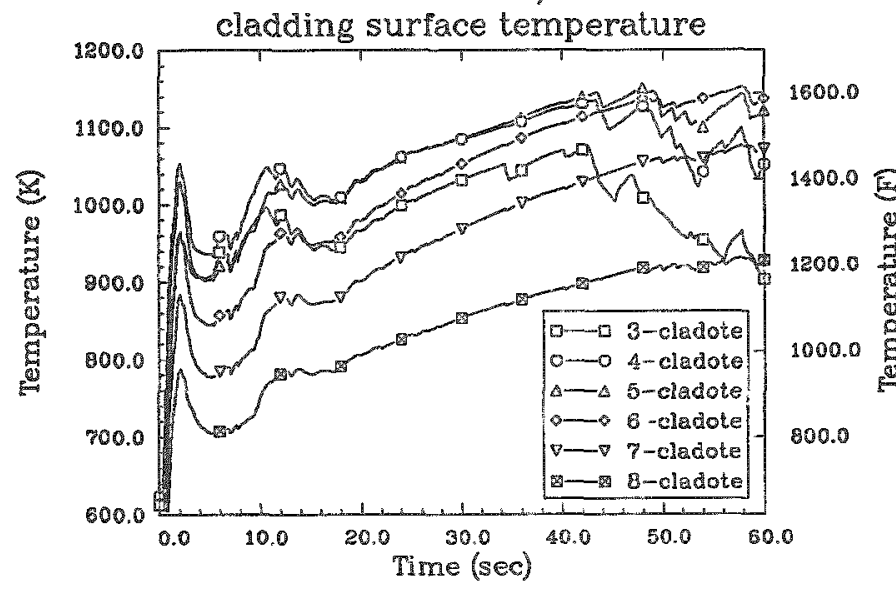

SEABROOK 100\%DBA 50 GWD/MTU PIN--PF 2.2

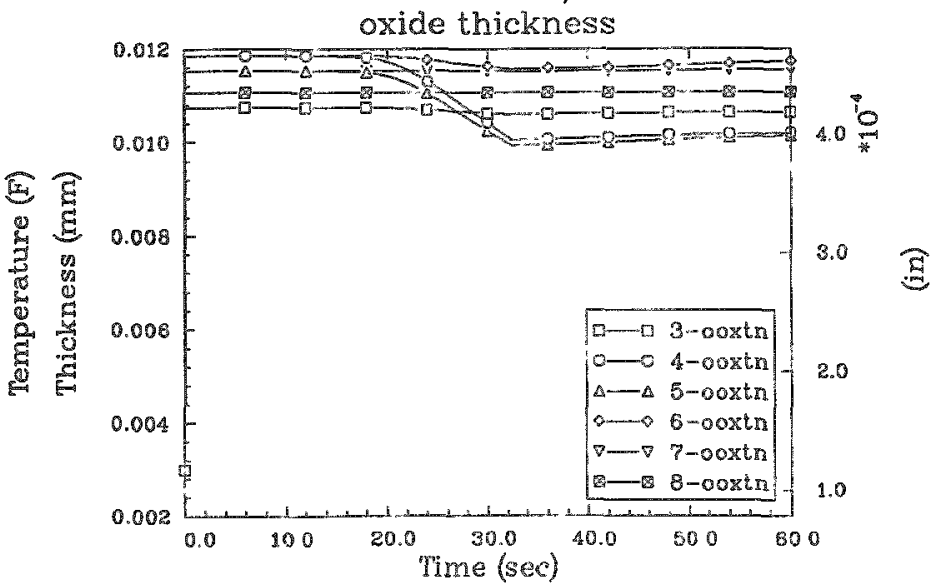


SEABROOK 100\%DBA 35 GWD/MTU PIN--PF 22
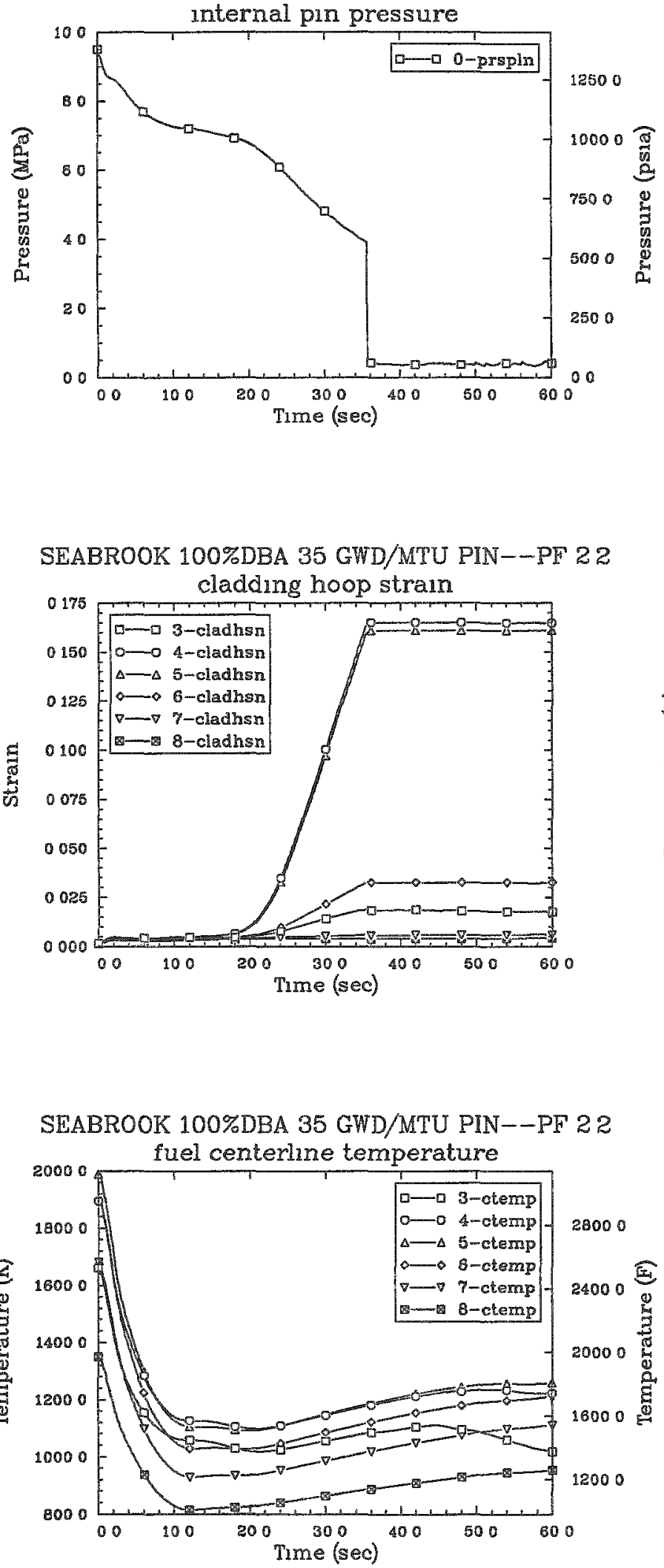

SEABROOK 100\%DBA 35 GWD/MTU PIN--PF 22
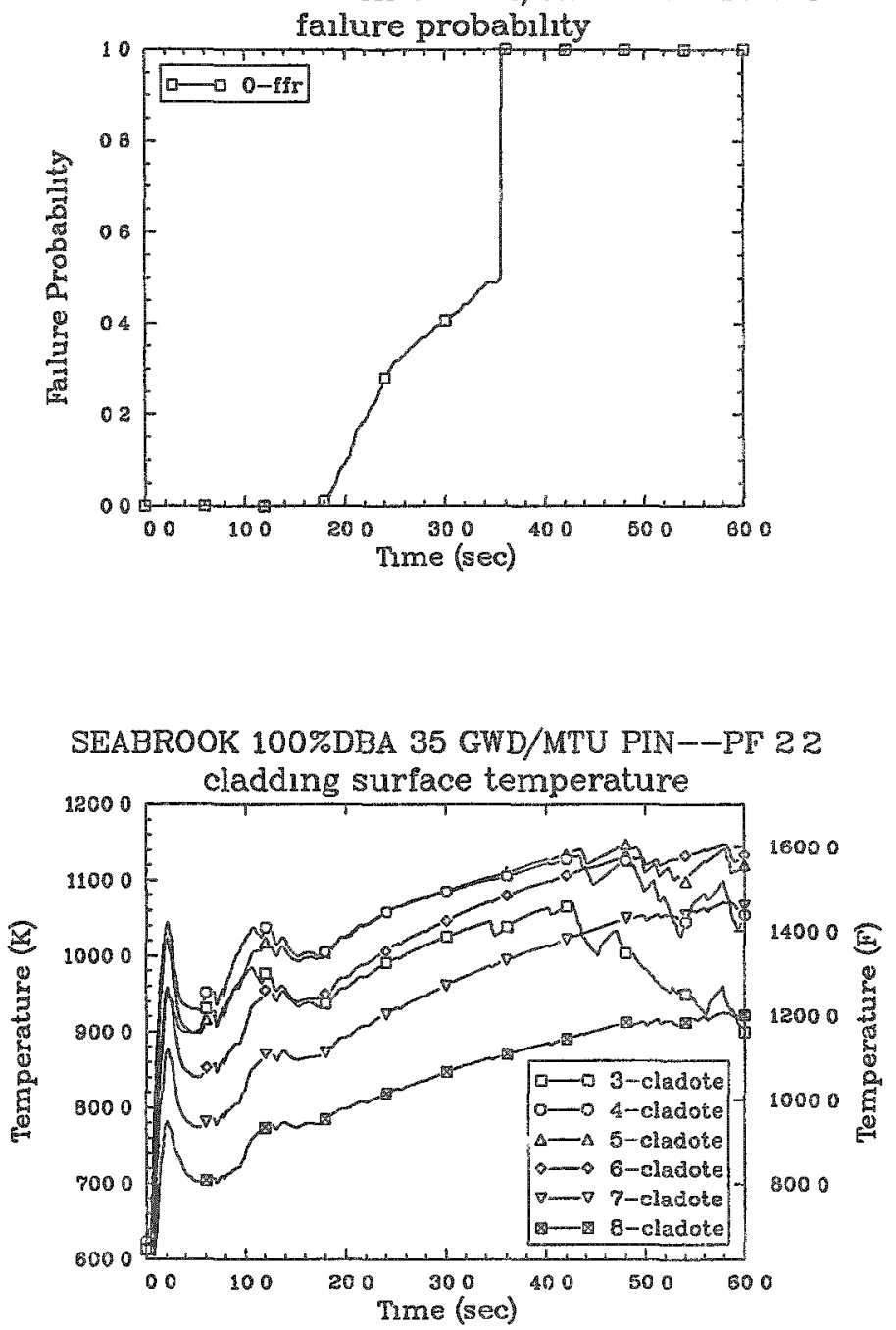

SEABROOK 100\%DBA 35 GWD/MTU PIN--PF 22

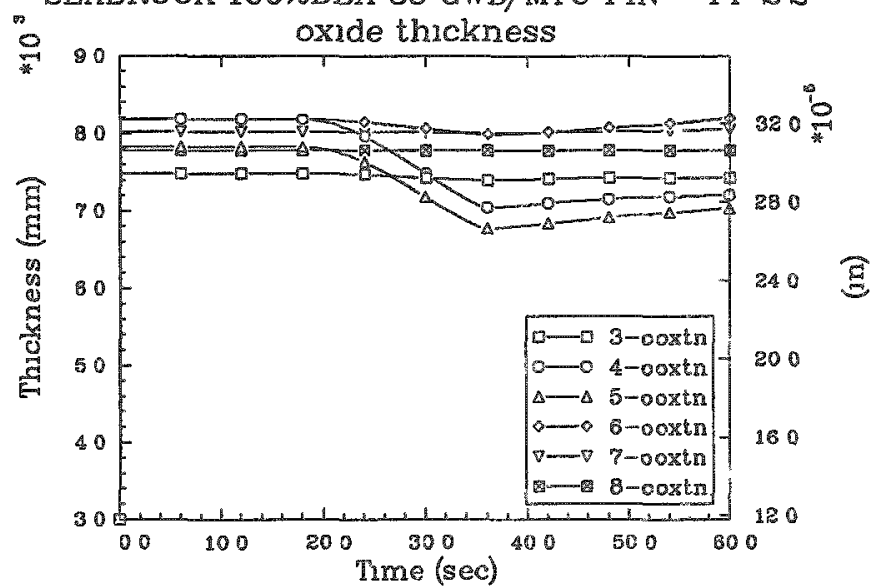


SEABROOK 100\%DBA $20 \mathrm{GWD} / \mathrm{MTU}$ PIN--PF 22
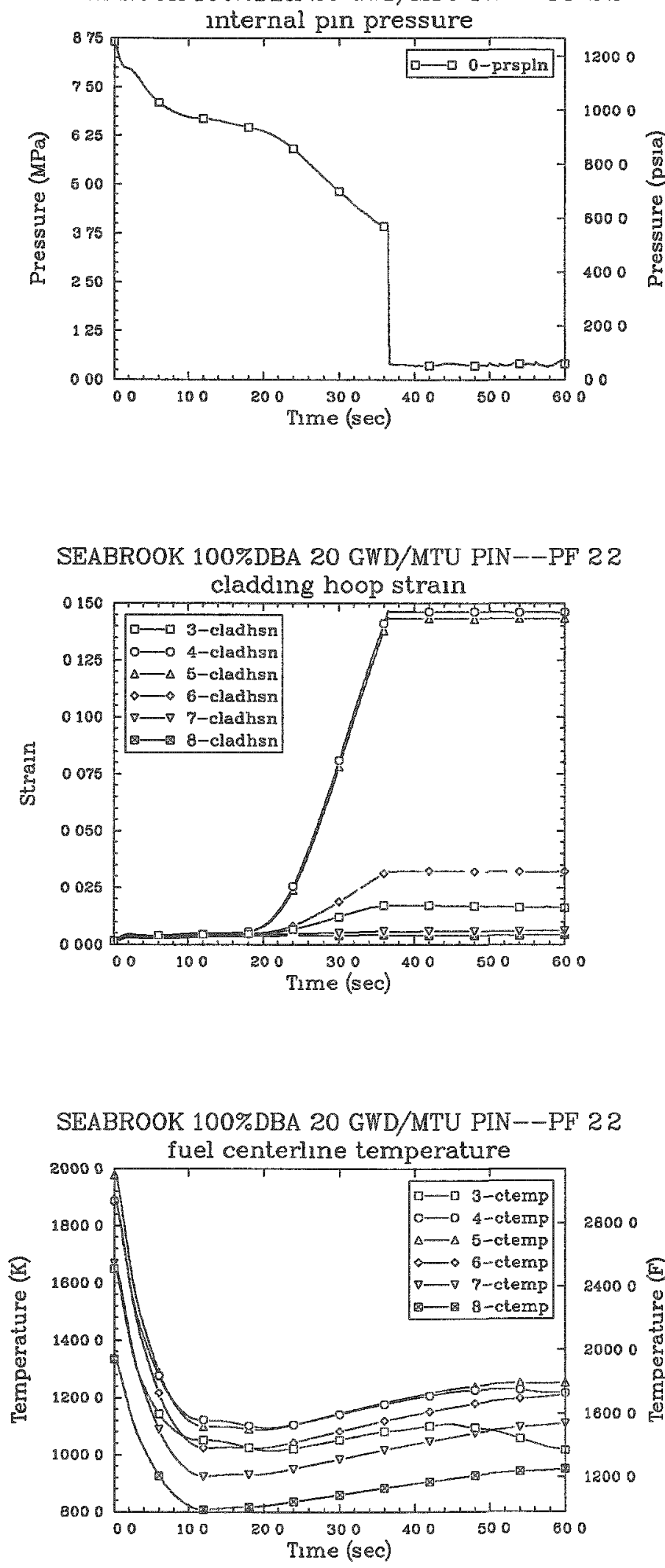

SEABROOK 100\%DBA 20 GWD/MTU PIN--PF 22 fallure probability
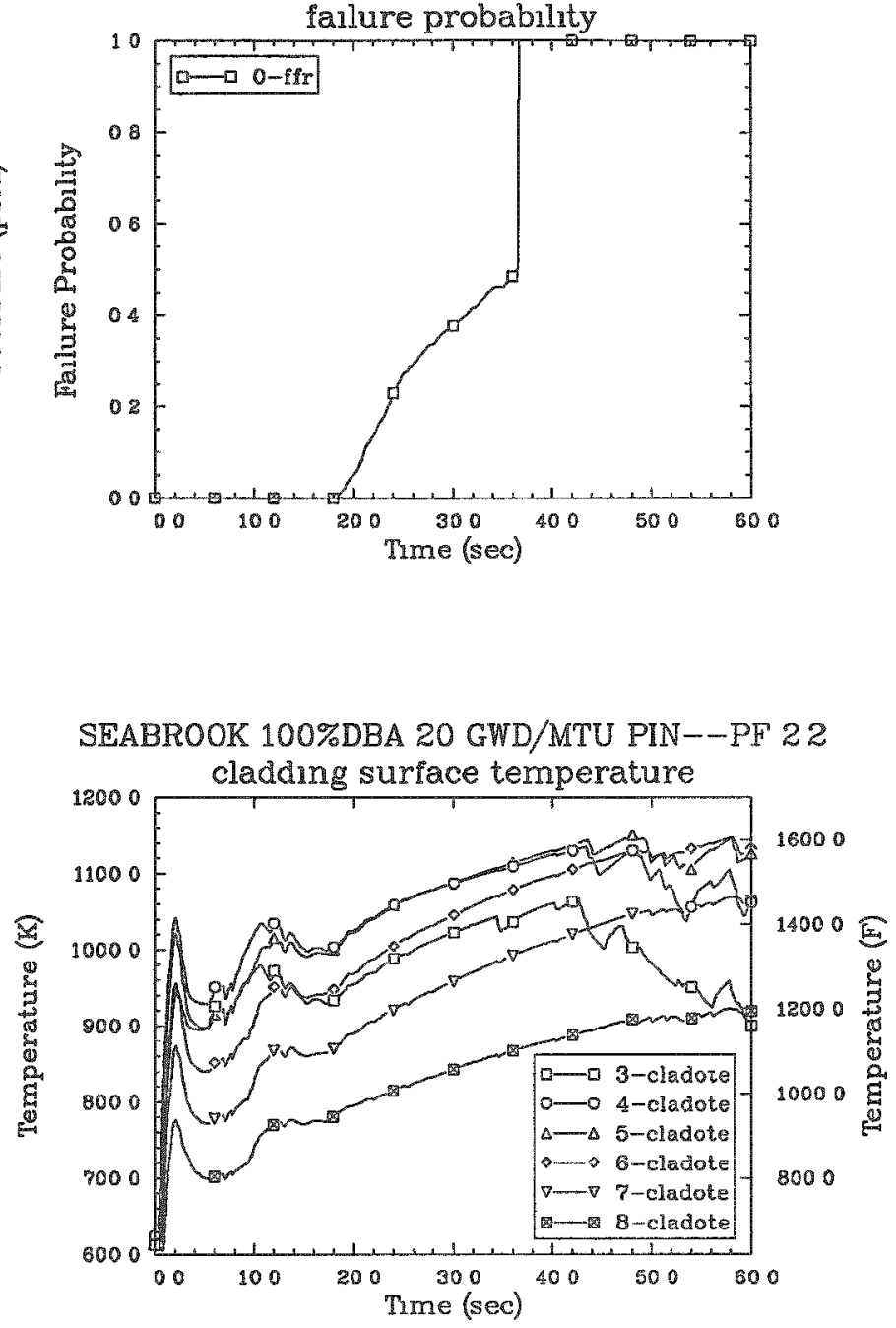

SEABROOK 100\%DBA $20 \mathrm{GWD} / \mathrm{MTU}$ PIN--PF 22 葛

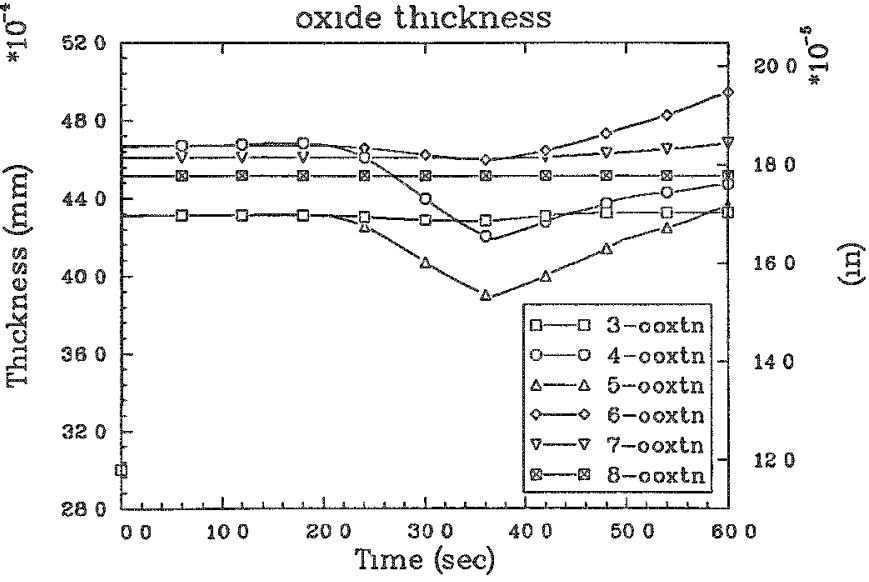



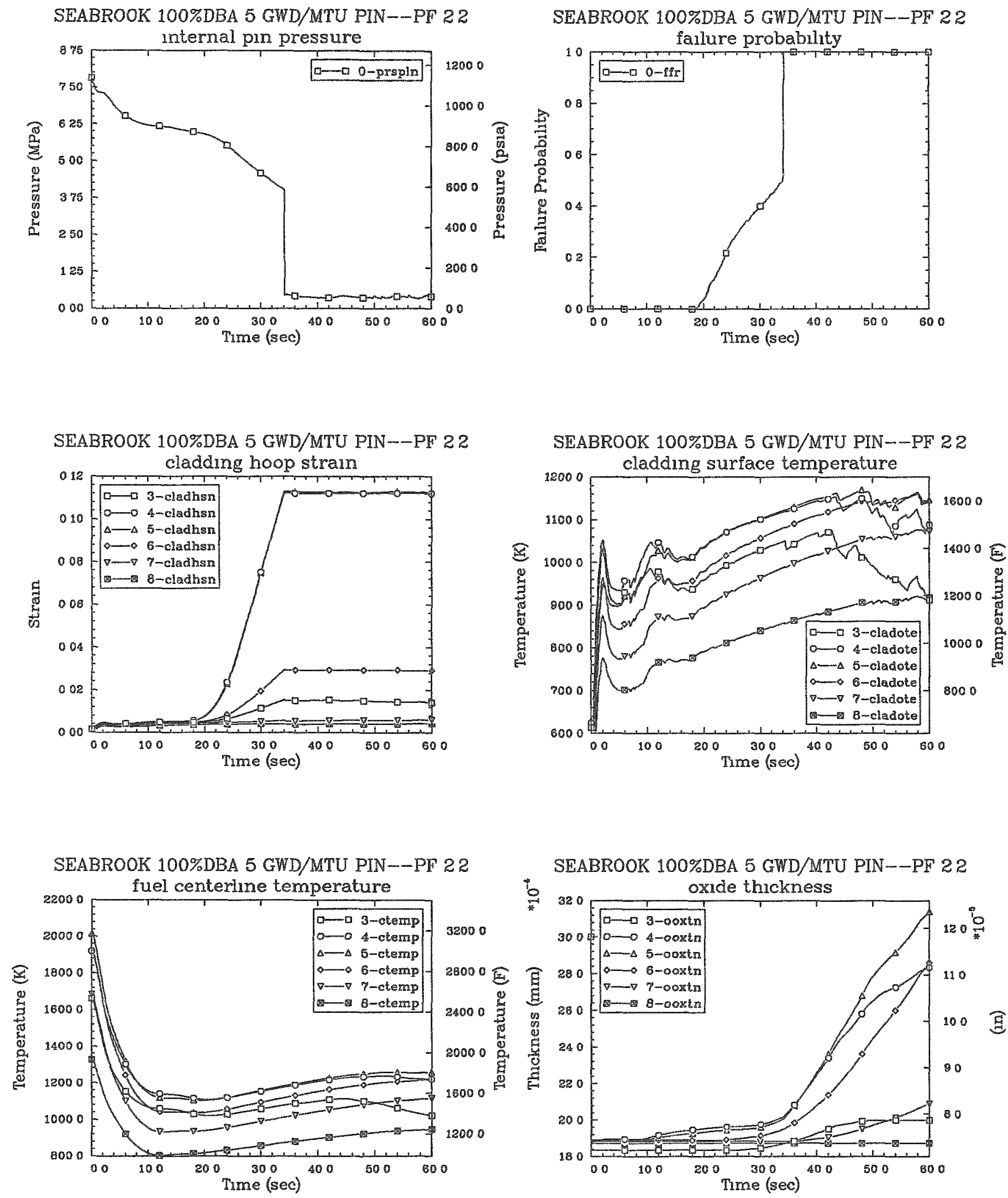


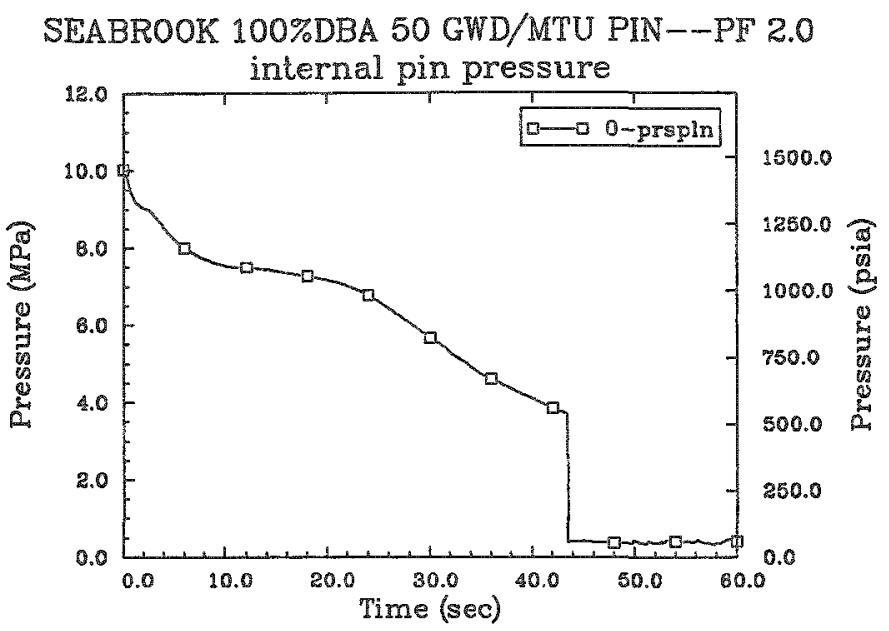

SEABROOK 100\%DBA 50 GWD/MTU PIN--PF 2.0

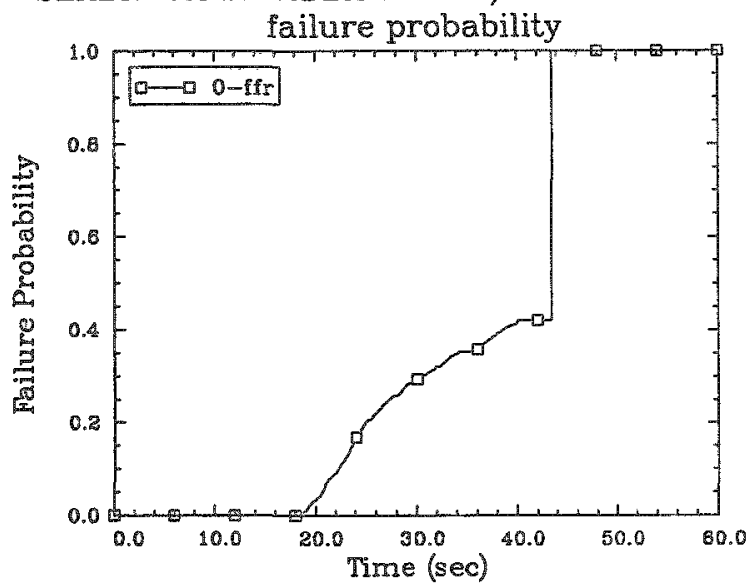

SEABROOK 100\%DBA $50 \mathrm{GWD} / \mathrm{MTU}$ PIN--PF 2.0
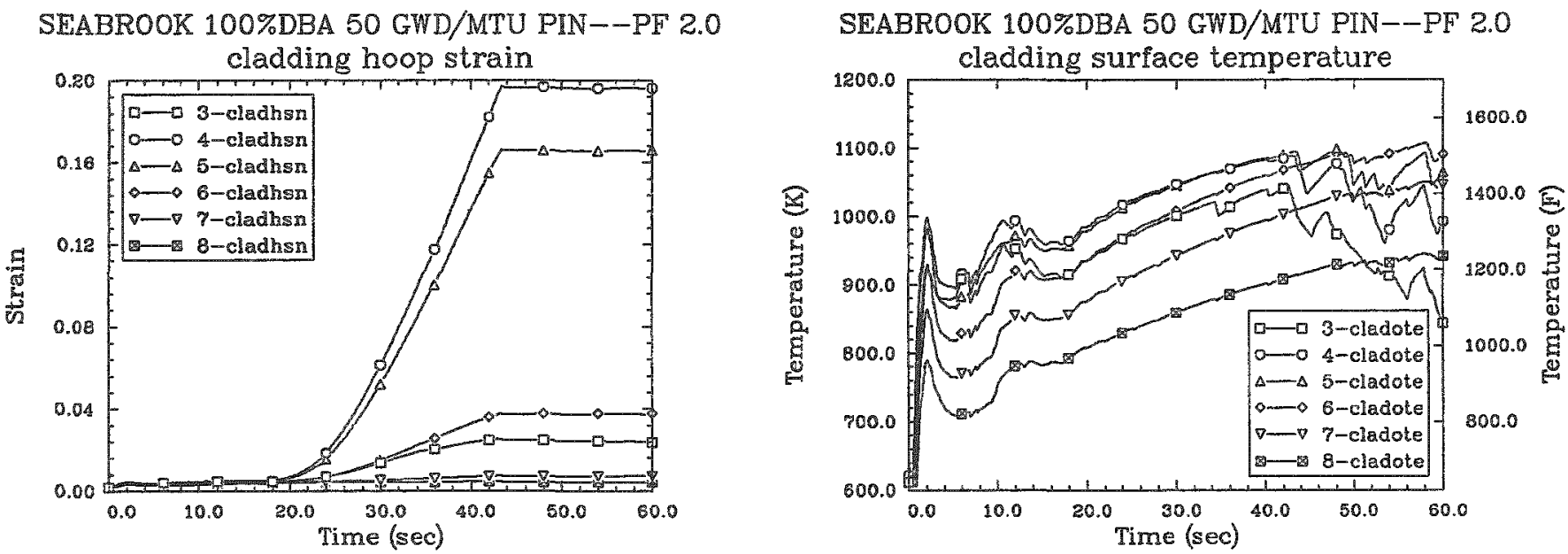

SEABROOK 100\%DBA 50 GWD/MTU PIN--PF 2.0

SEABROOK 100\%DBA $50 \mathrm{GWD} / \mathrm{MTU}$ PIN--PF 2.0 fuel centerline temperature

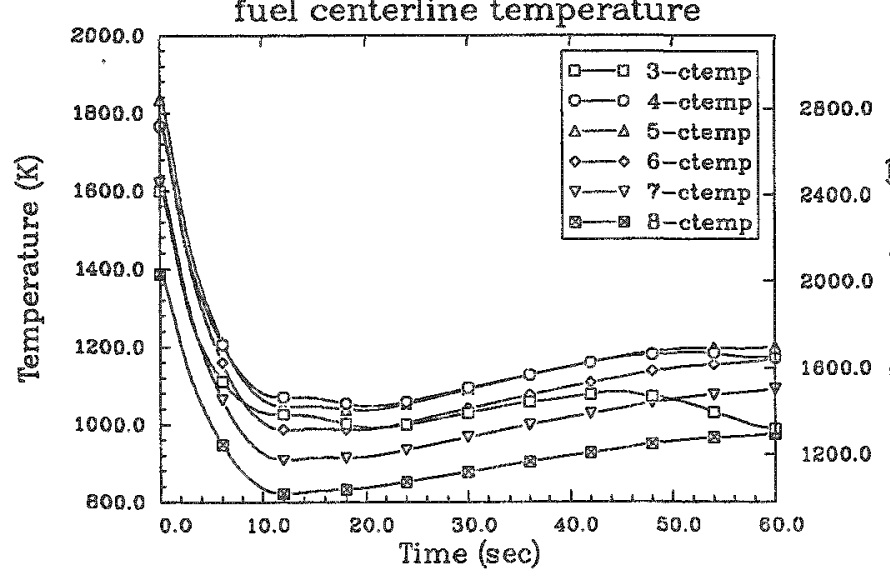
oxide thickness

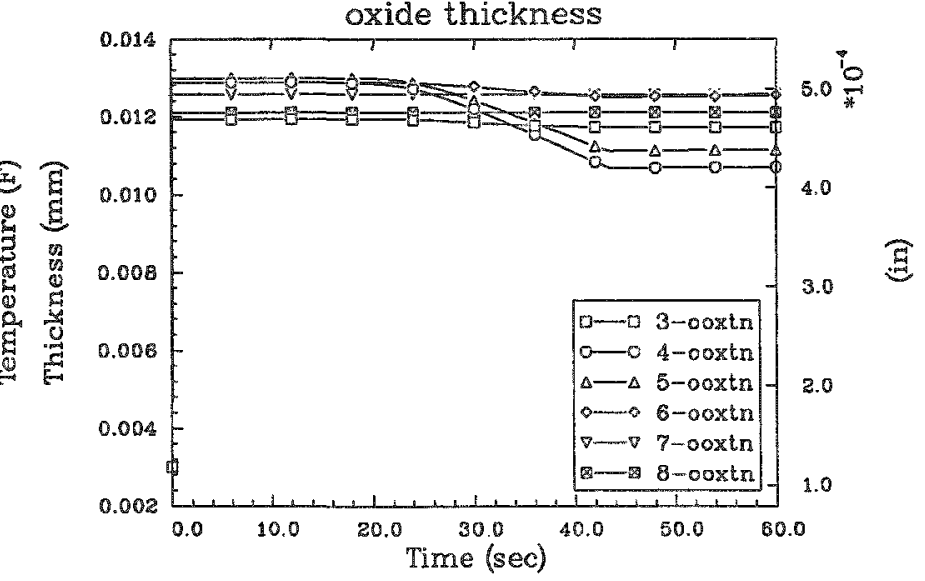




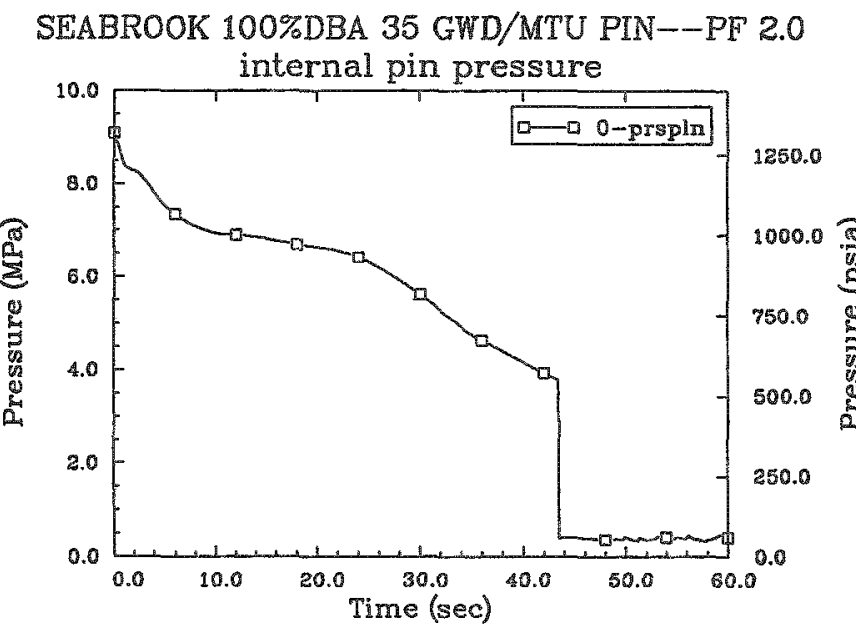

SEABROOK 100\%DBA 35 GWD/MTU PIN--PF 2.0

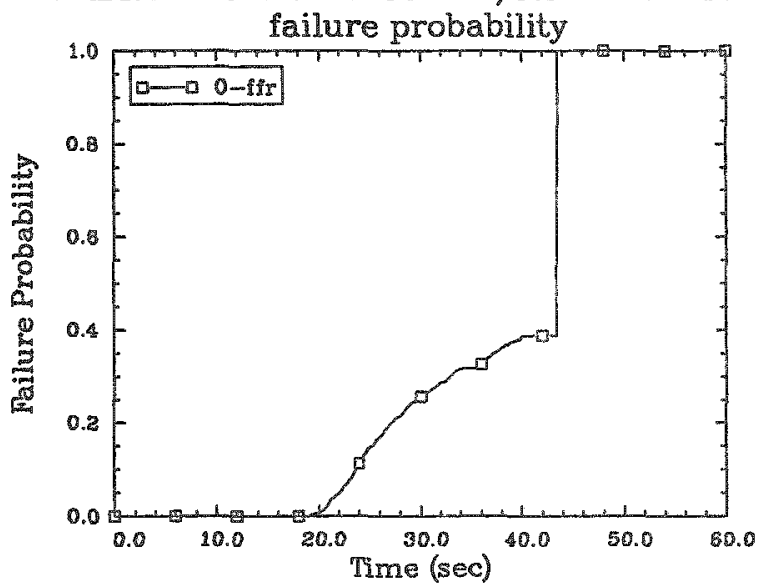

SEABROOK 100\%DBA 35 GWD/MTU PIN--PF 2.0
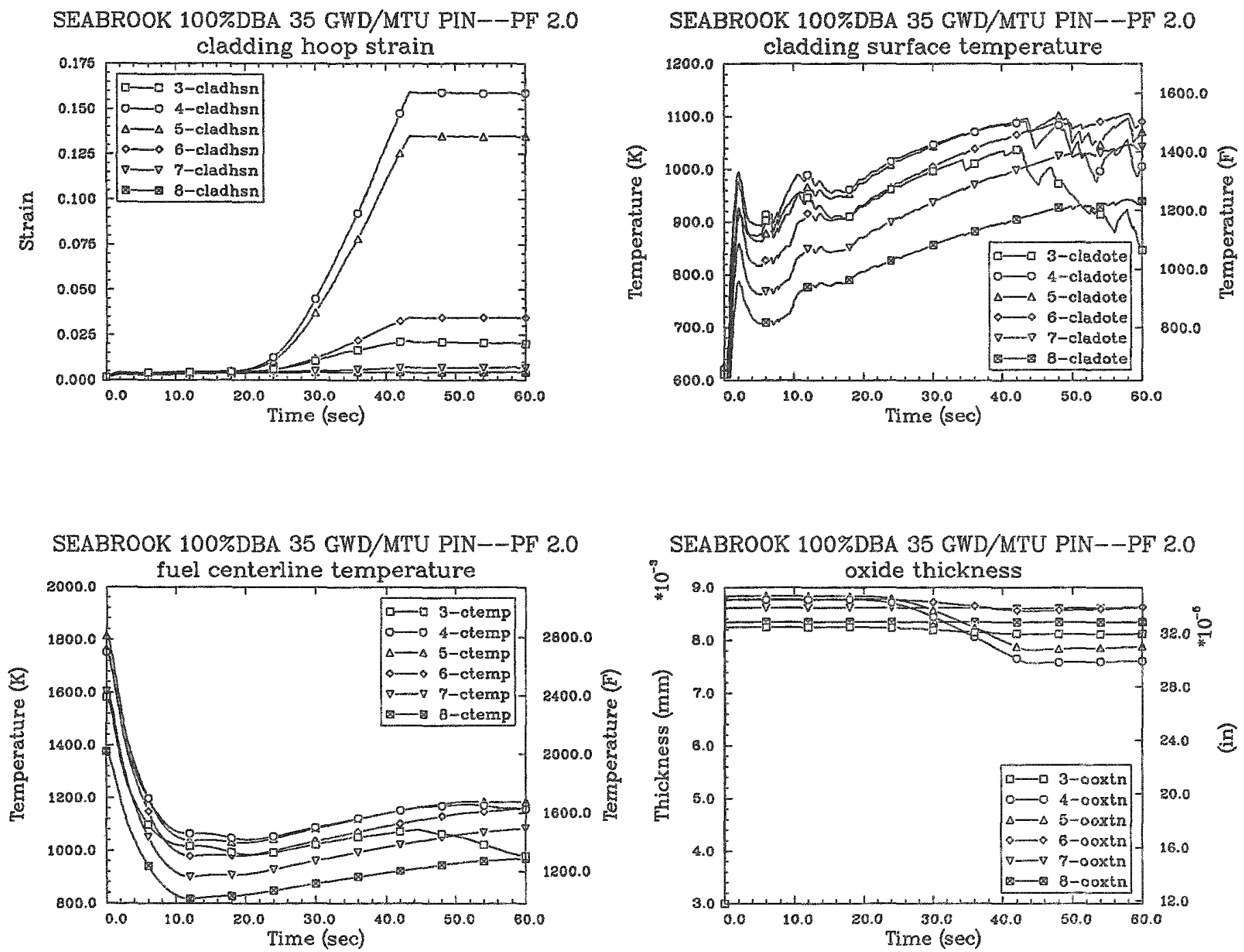


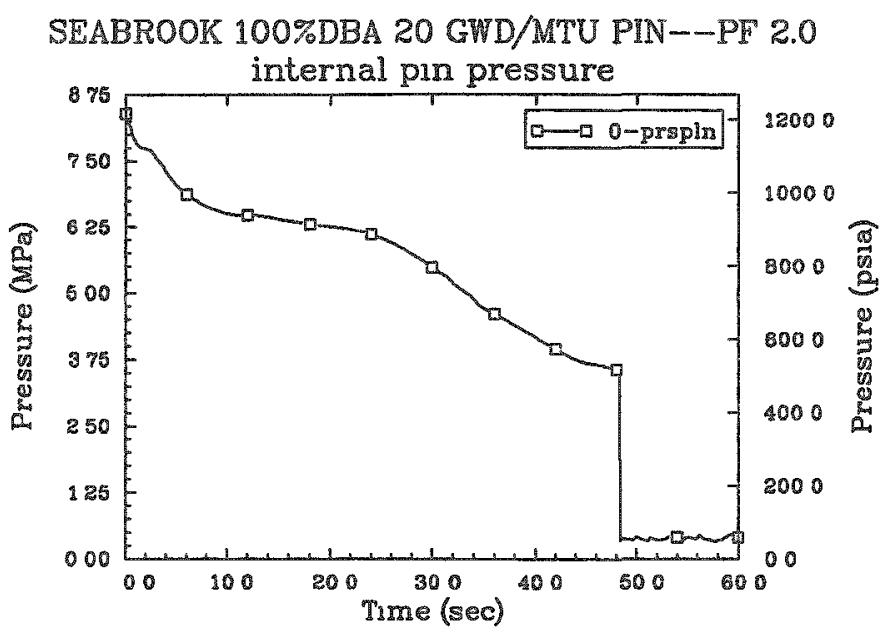

SEABROOK 100\%DBA 20 GWD/MTU PIN--PF 20
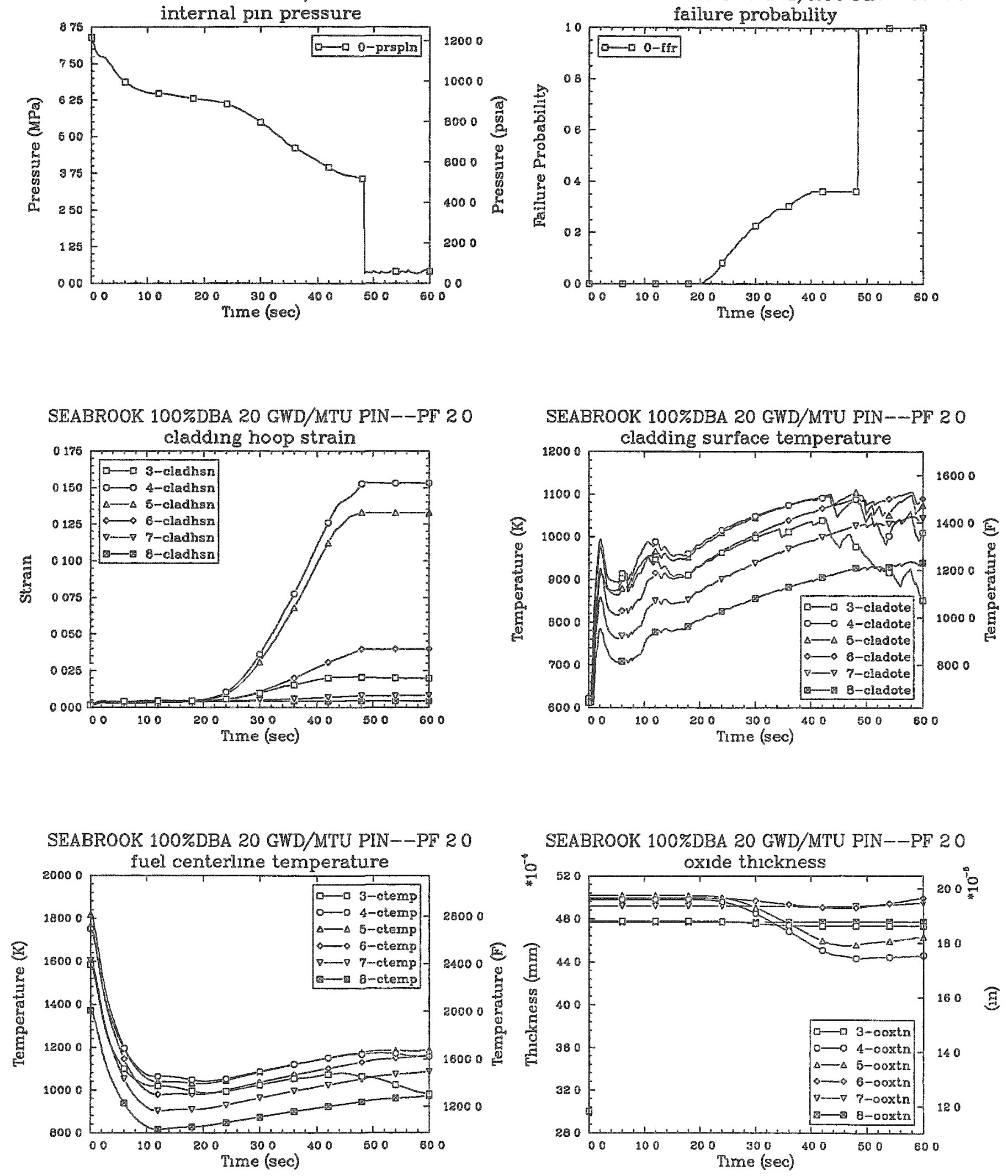
SEABROOK 100\%DBA 5 GWD/MTU PIN--PF 2.0
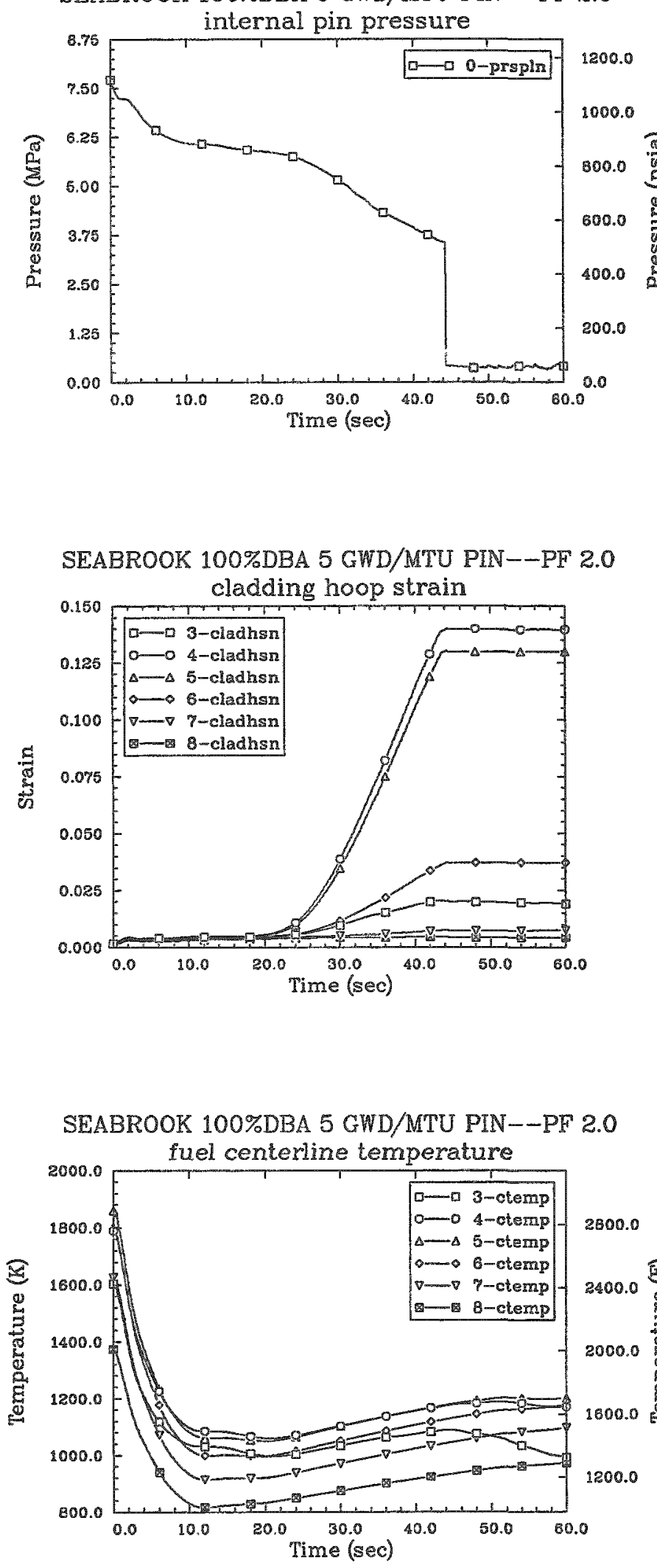

SEABROOK 100\%DBA 5 GWD/MTU PIN--PF 2.0
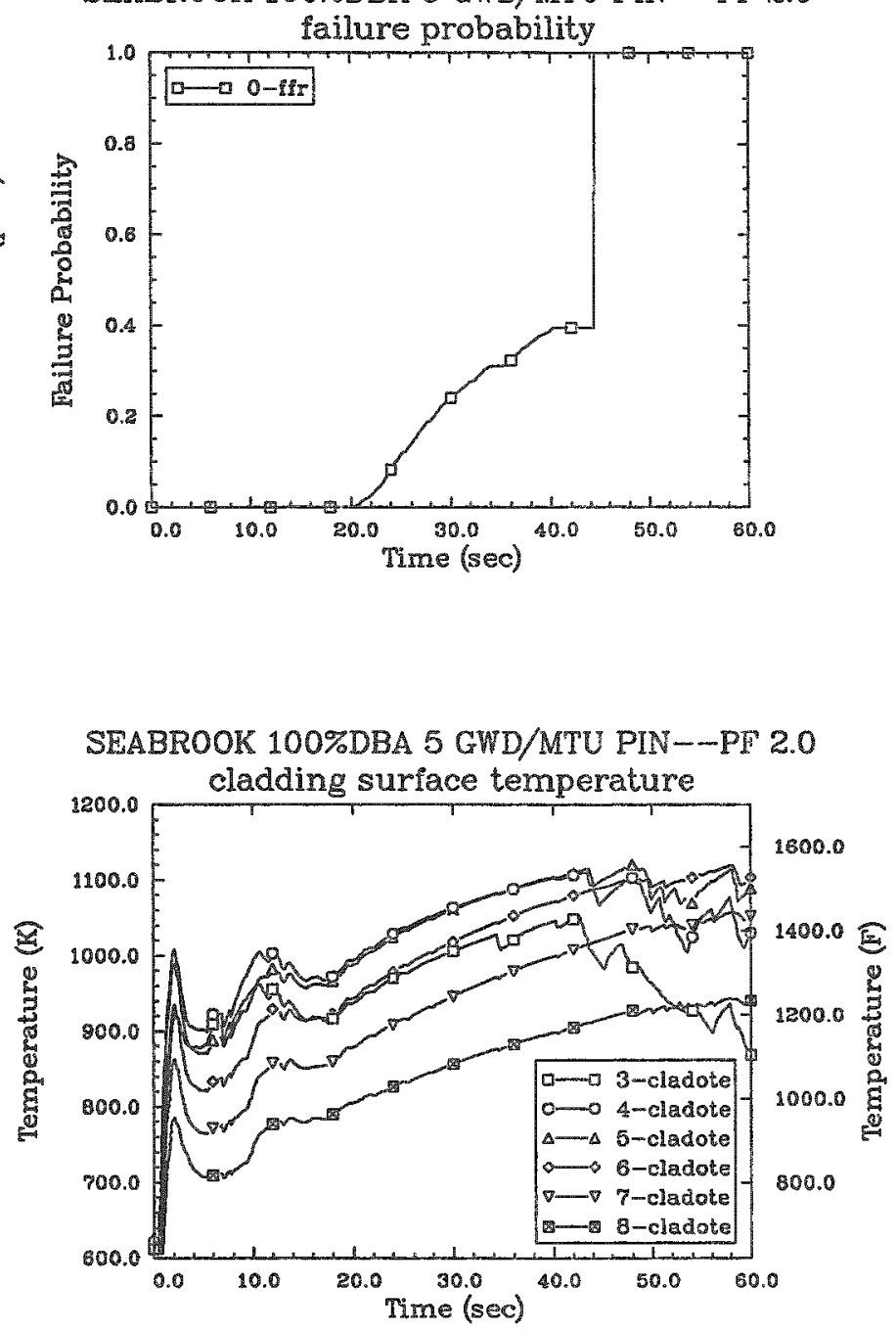

SEABROOK 100\%DBA 5 GWD/MTU PIN--PF 2.0

인

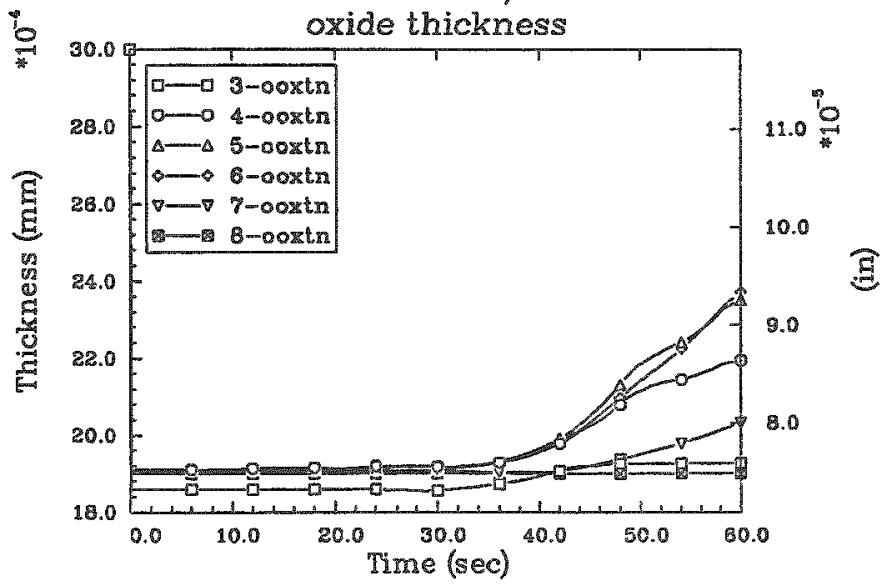



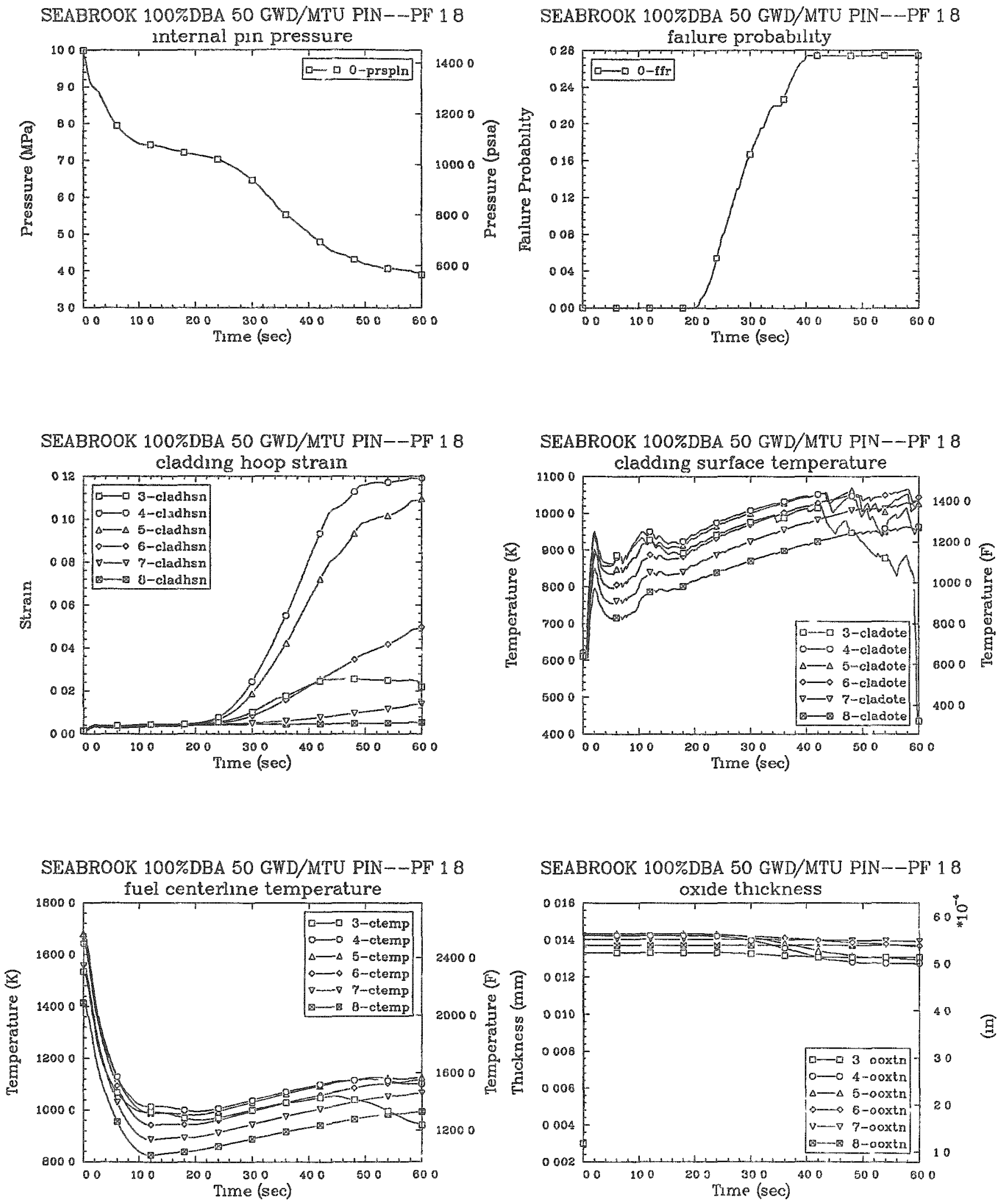


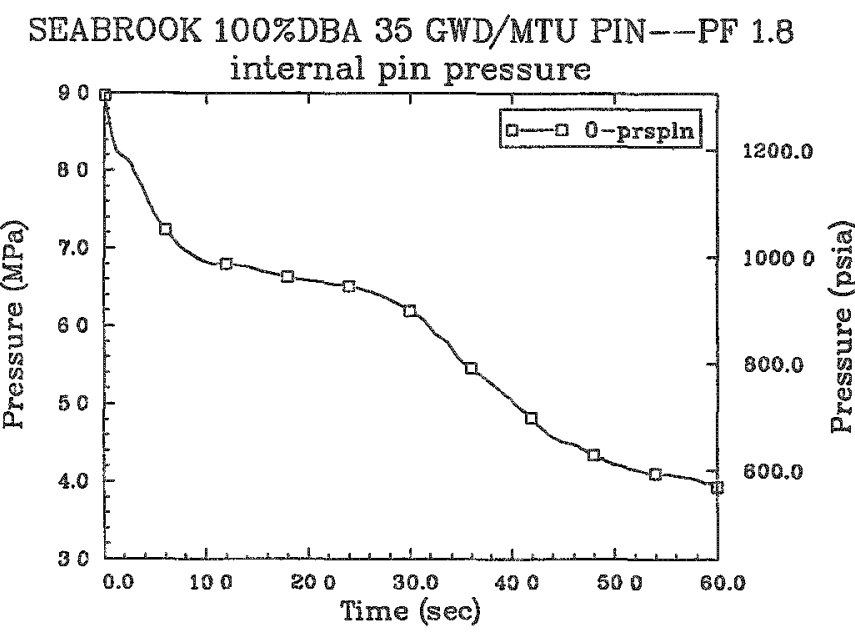

SEABROOK 100\%DBA 35 GWD/MTU PIN--PF 1.8

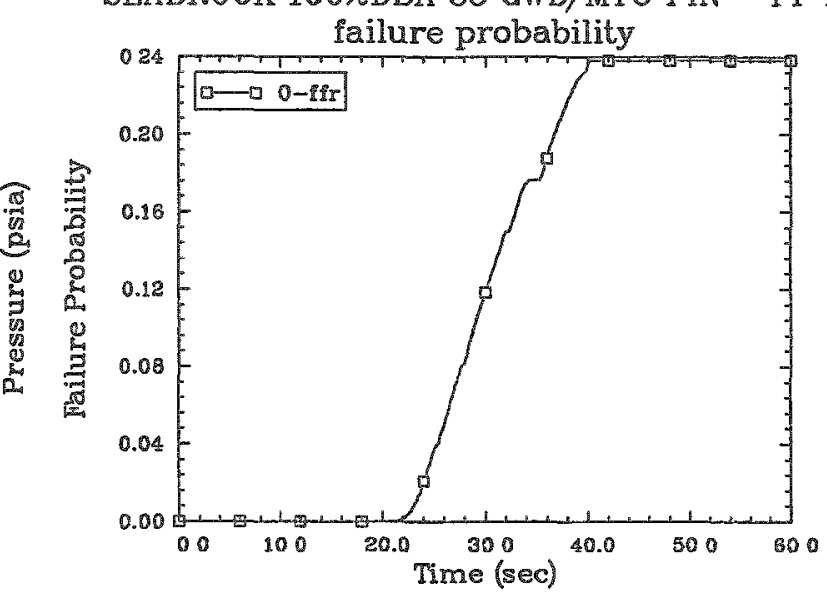

\section{SEABROOK 100\%DBA 35 GWD/MTU PIN--PF 1.8}
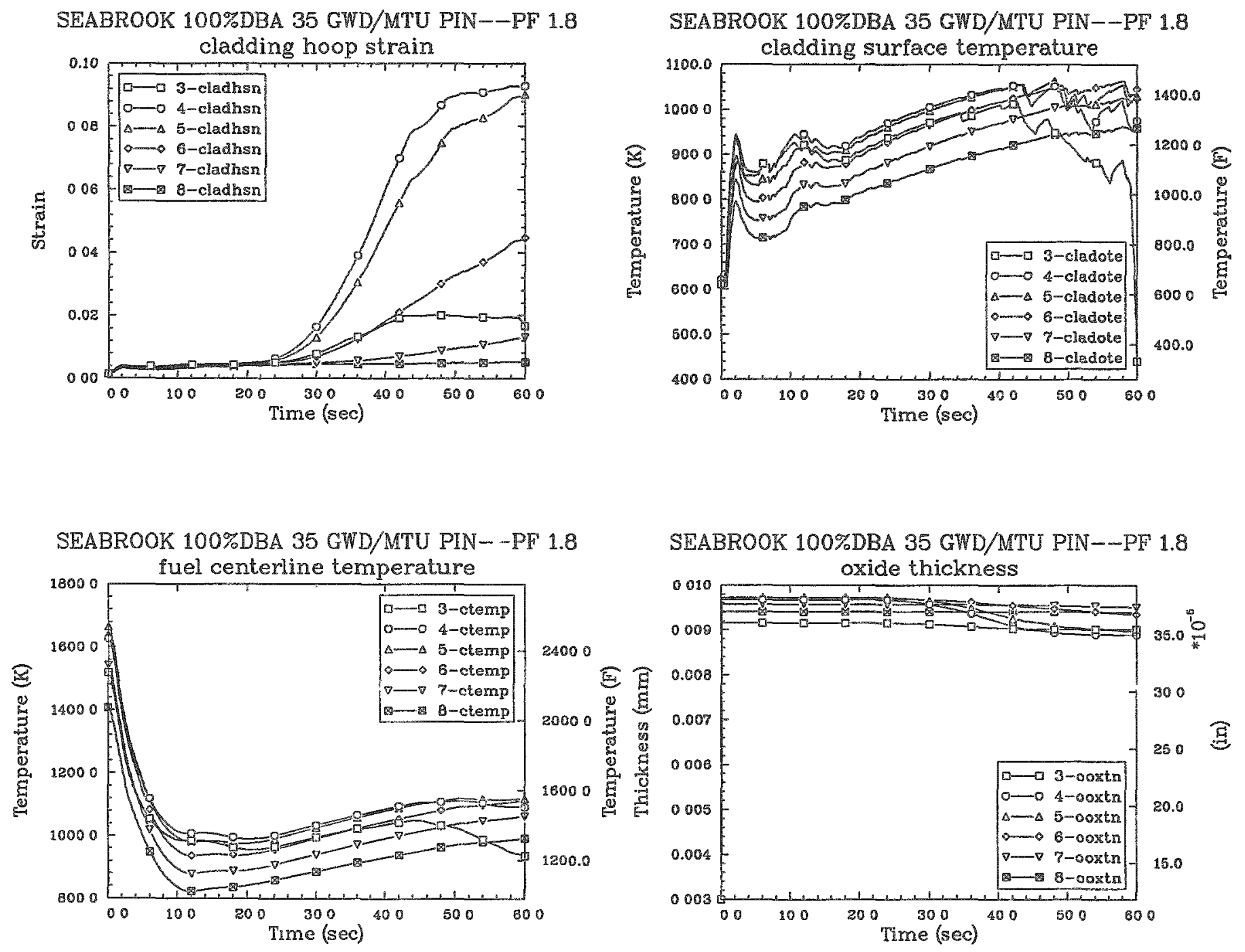


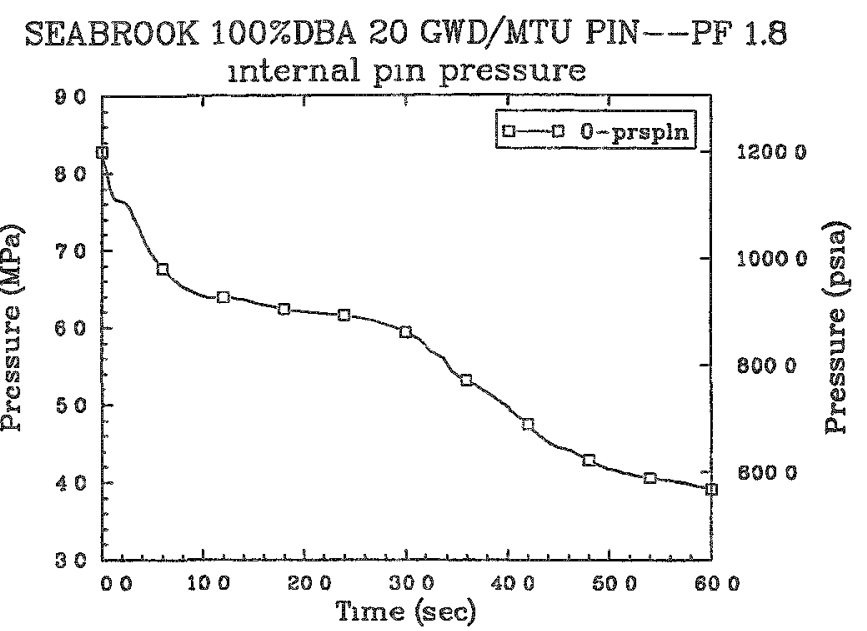

SEABROOK 100\%DBA 20 GWD/MTU PIN--PF 1.8
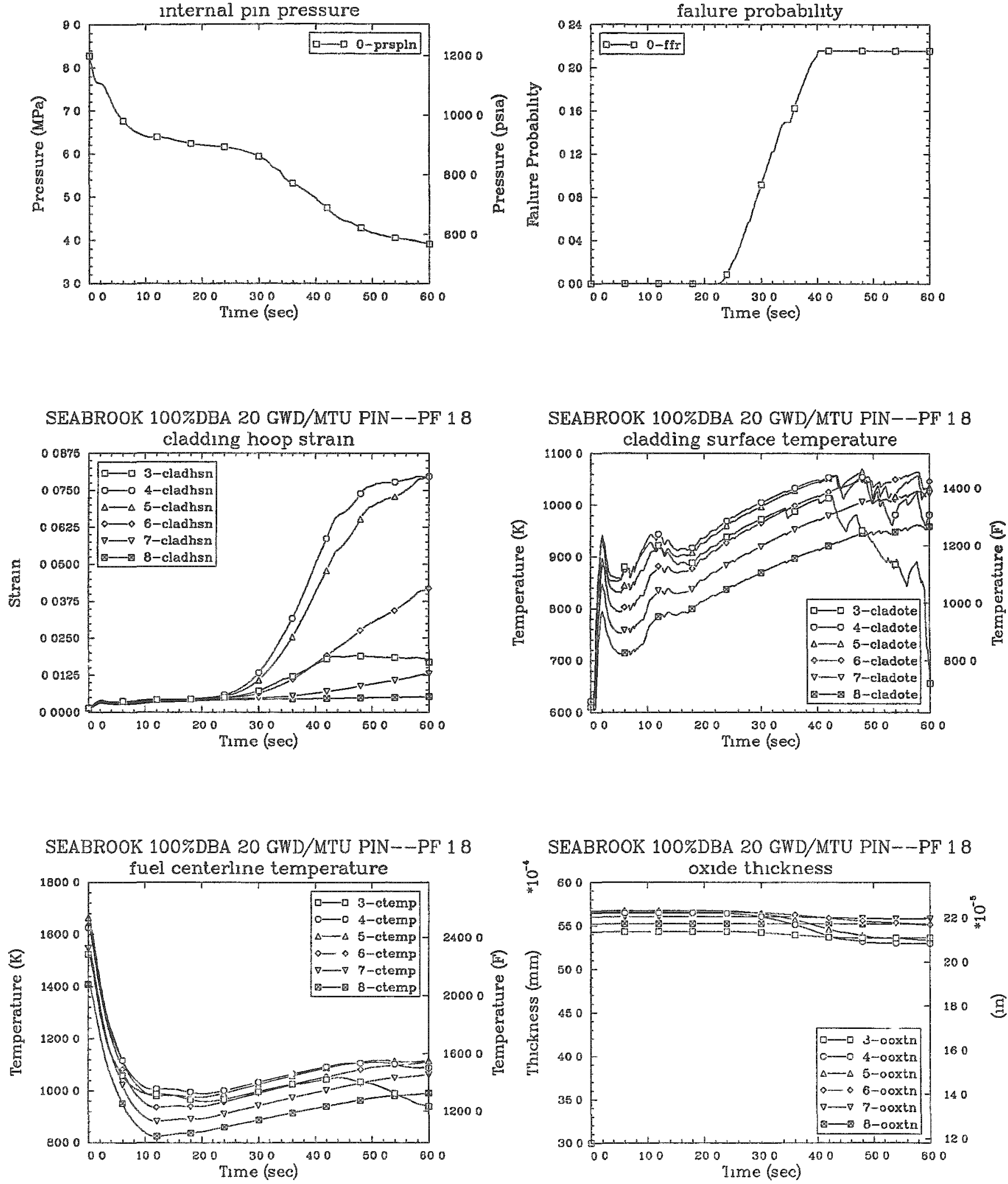

SEABROOK 100\%DBA 20 GWD/MTU PIN--PF 18

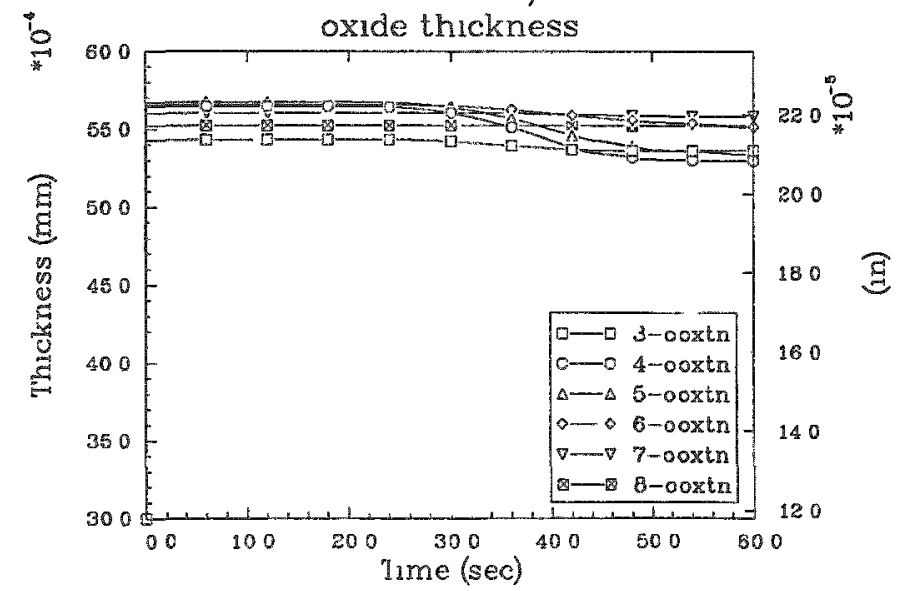



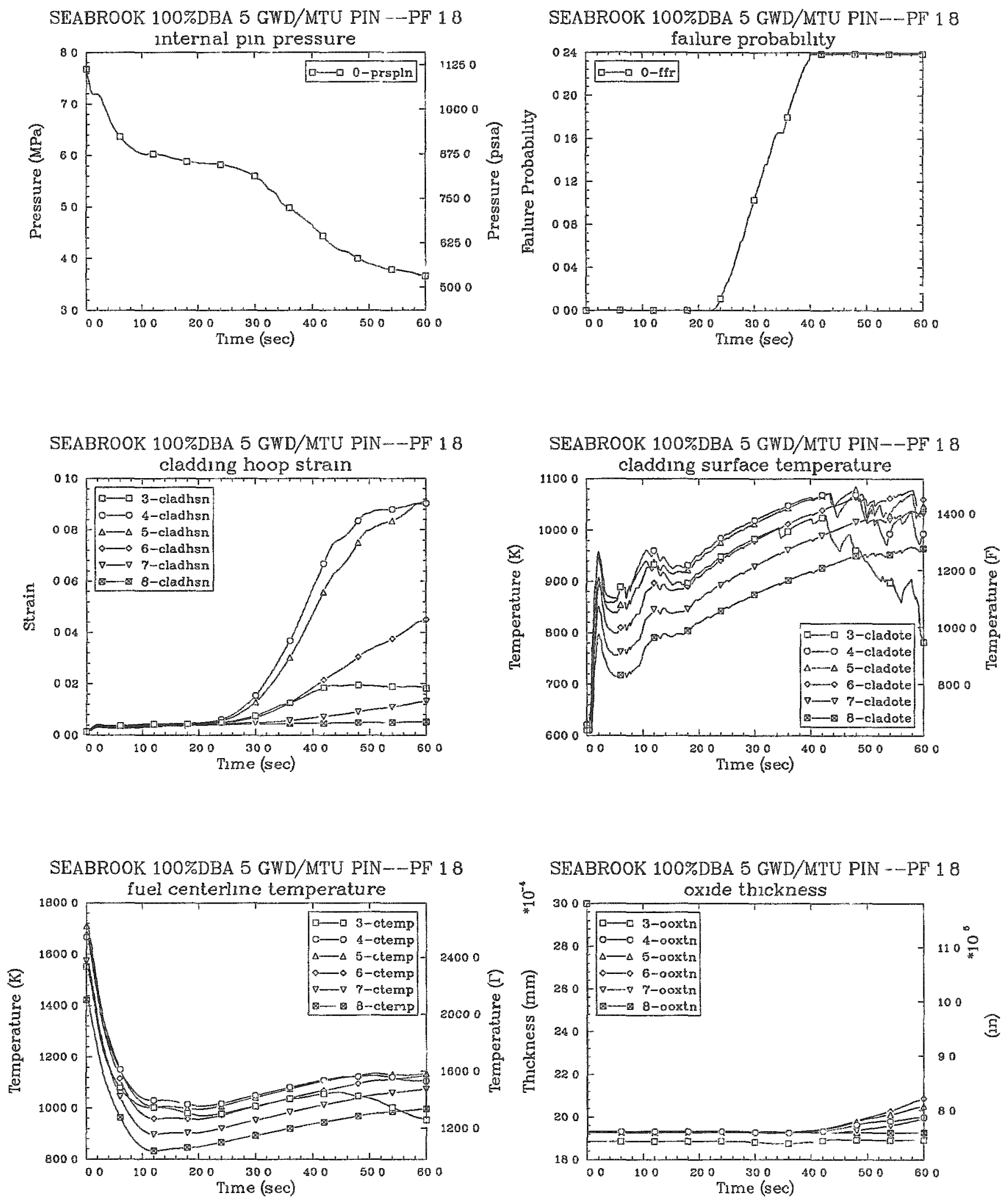

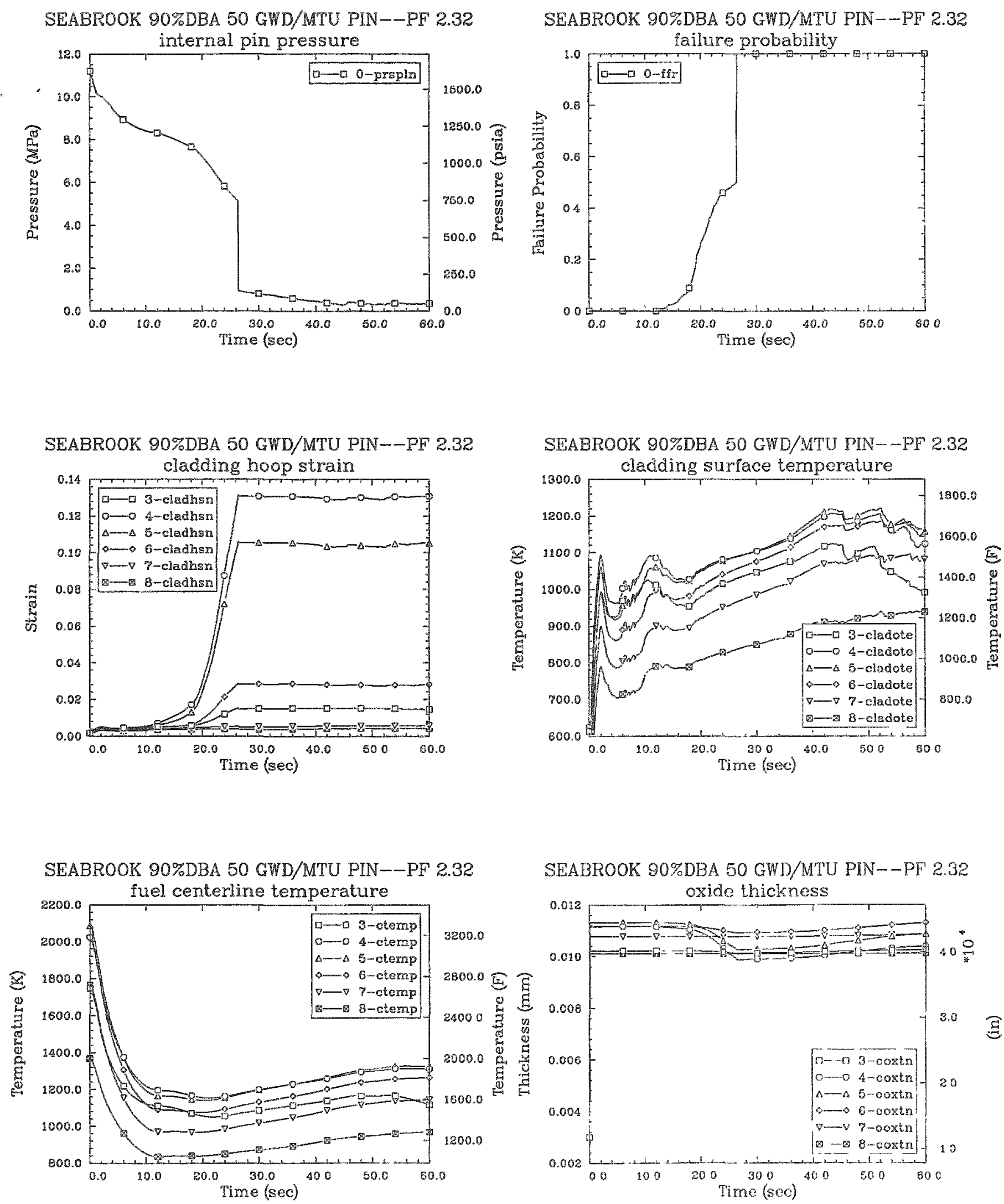

SEABROOK 90\%DBA 50 GWD/MTU PIN--PF 2.32

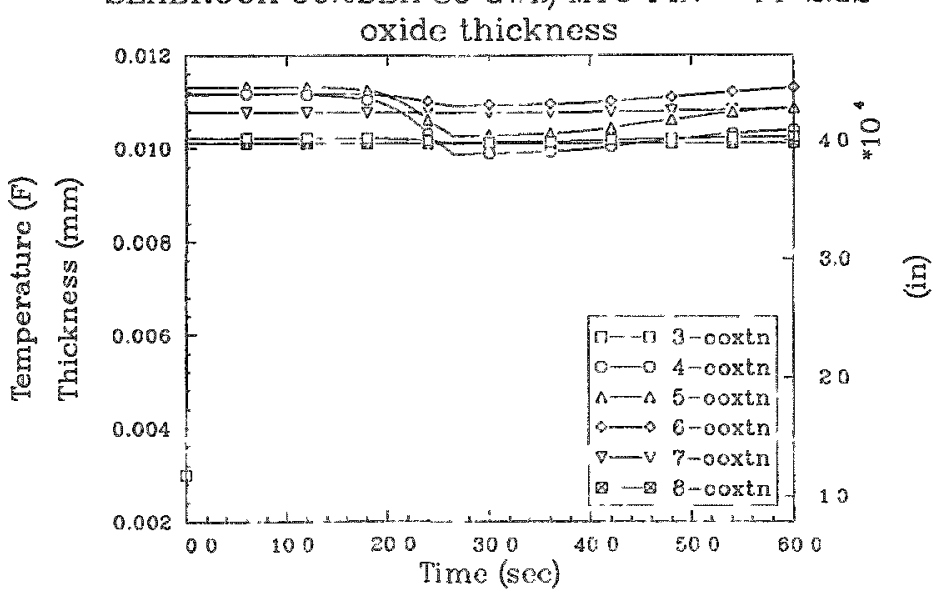



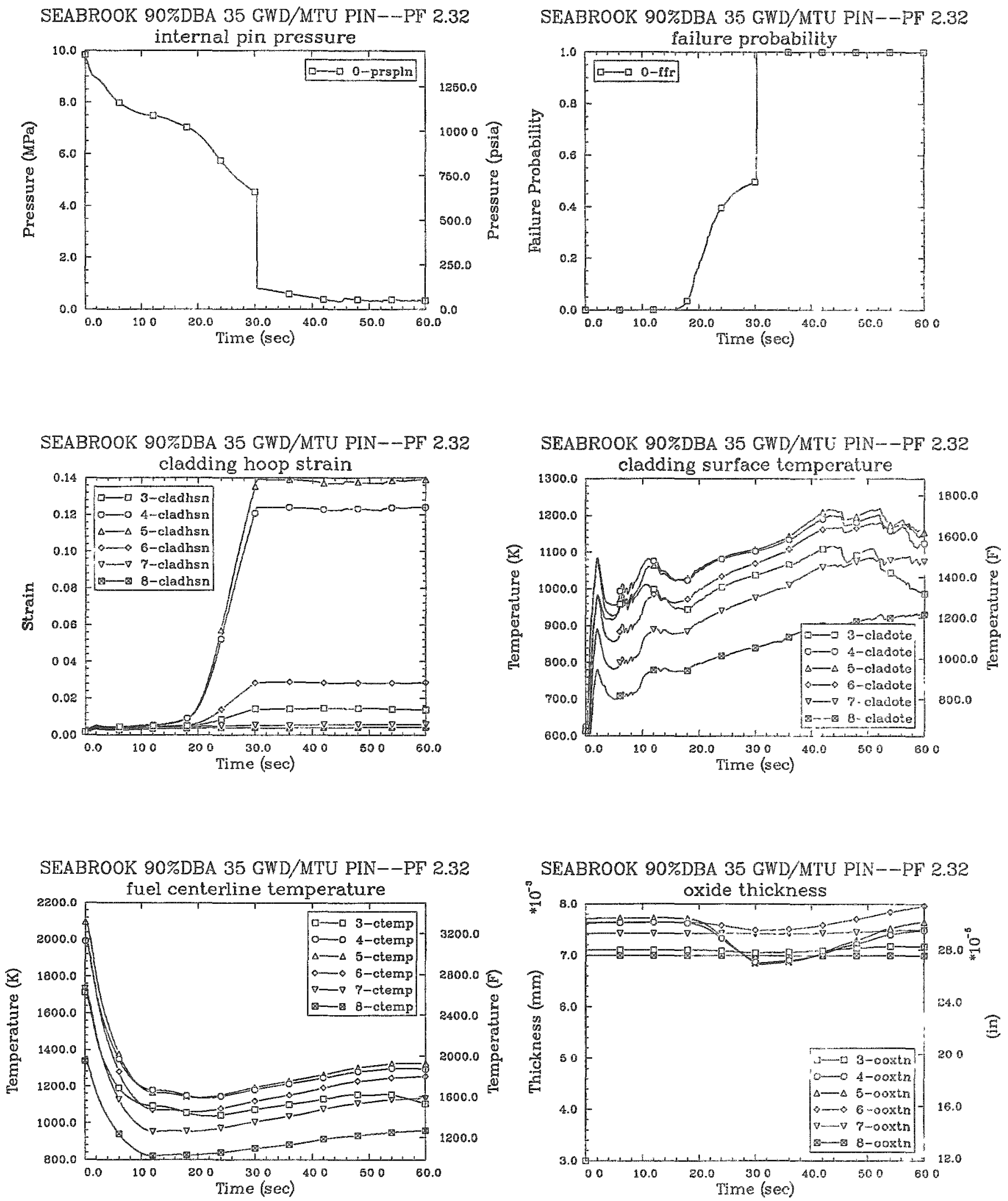
SEABROOK 90\%DBA 20 GWD/MTU PIN--PF 2.32

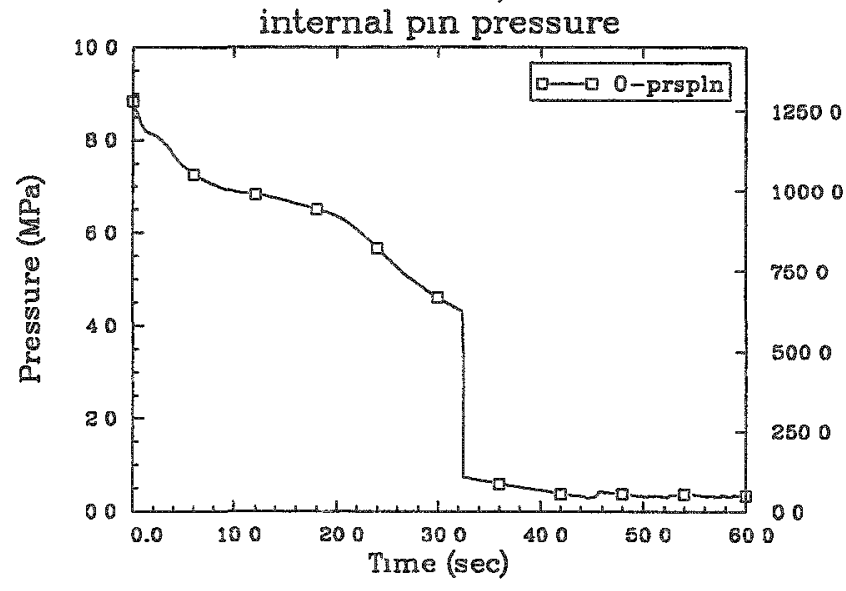

SEABROOK 90\%DBA 20 GWD/MTU PIN--PF 2.32

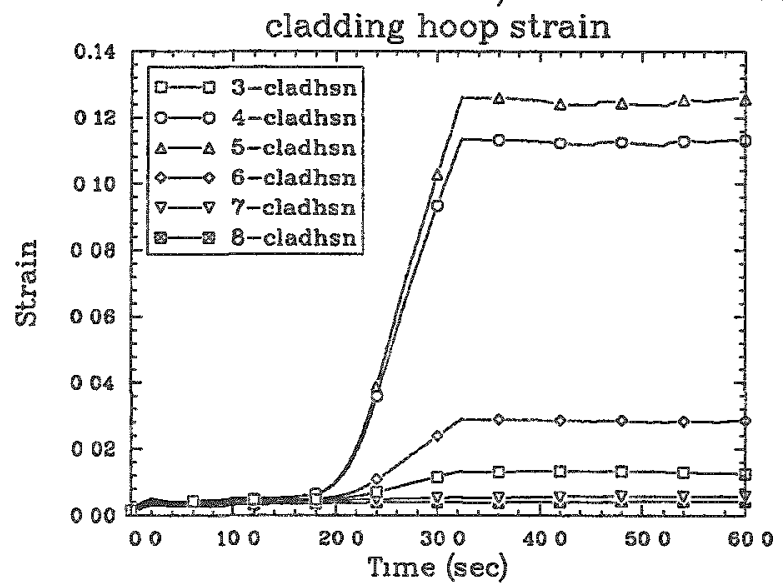

SEABROOK 90\%DBA 20 GWD/MTU PIN--PF 2.32

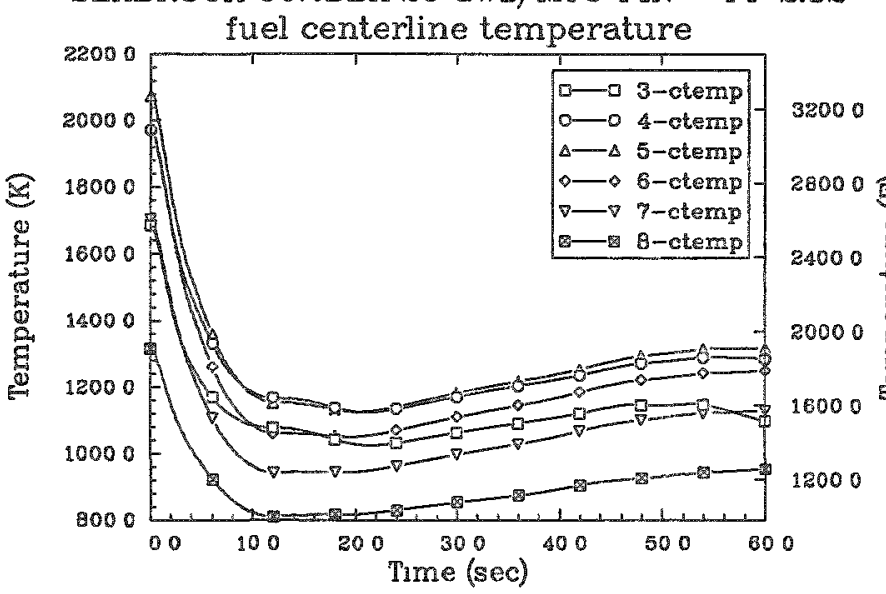

SEABROOK 90\%DBA 20 GWD/MTU PIN--PF 2.32 failure probability

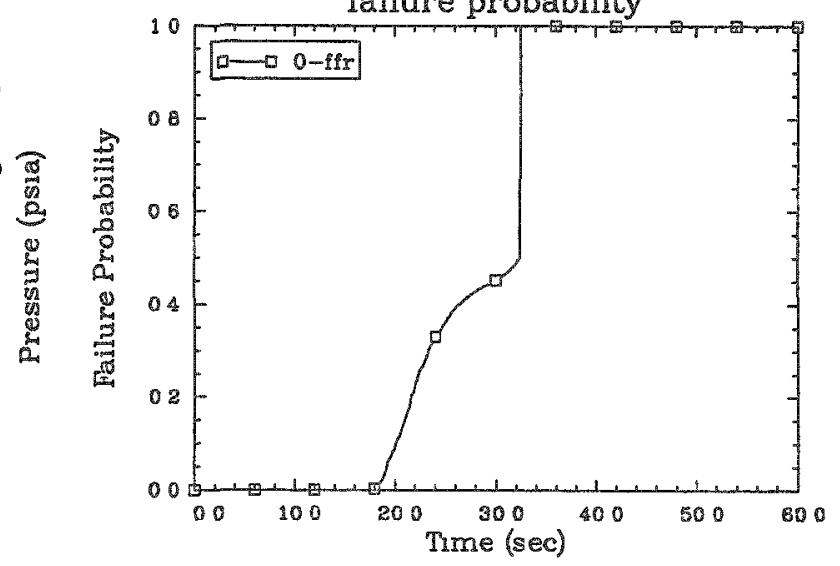

SEABROOK 90\%DBA 20 GWD/MTU PIN--PF 2.32

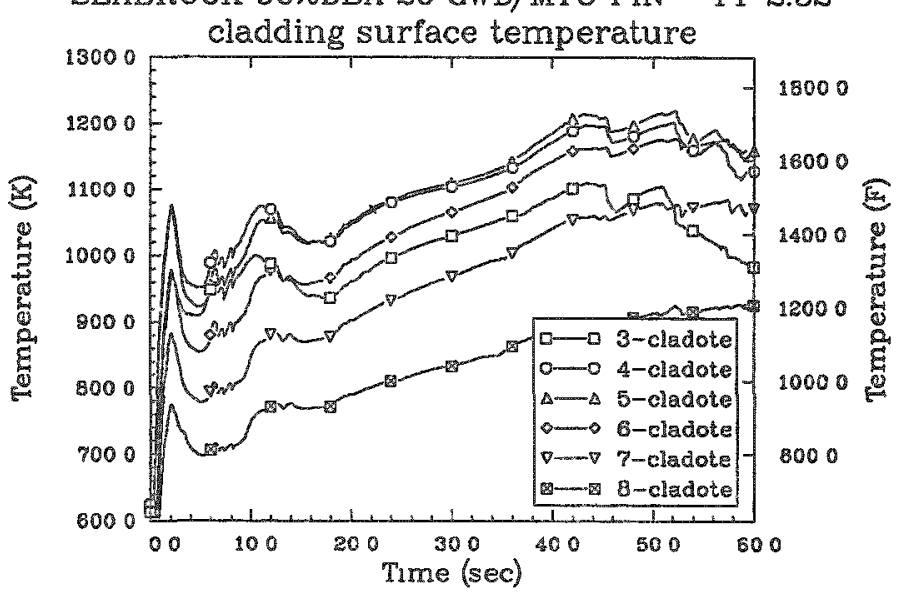

SEABROOK 90\%DBA 20 GWD/MTU PIN--PF 232

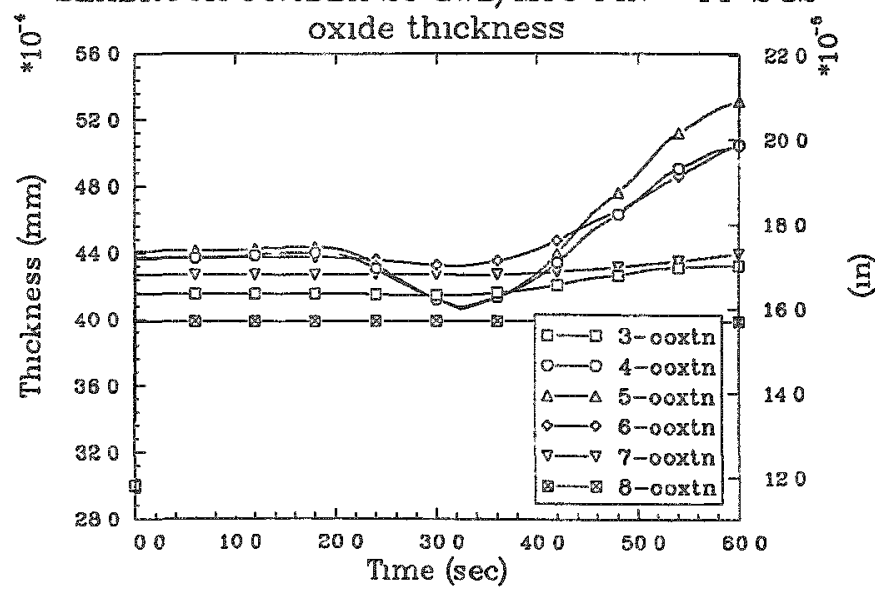


SEABROOK 90\%DBA 5 GWD/MTU PIN--PF 2.32

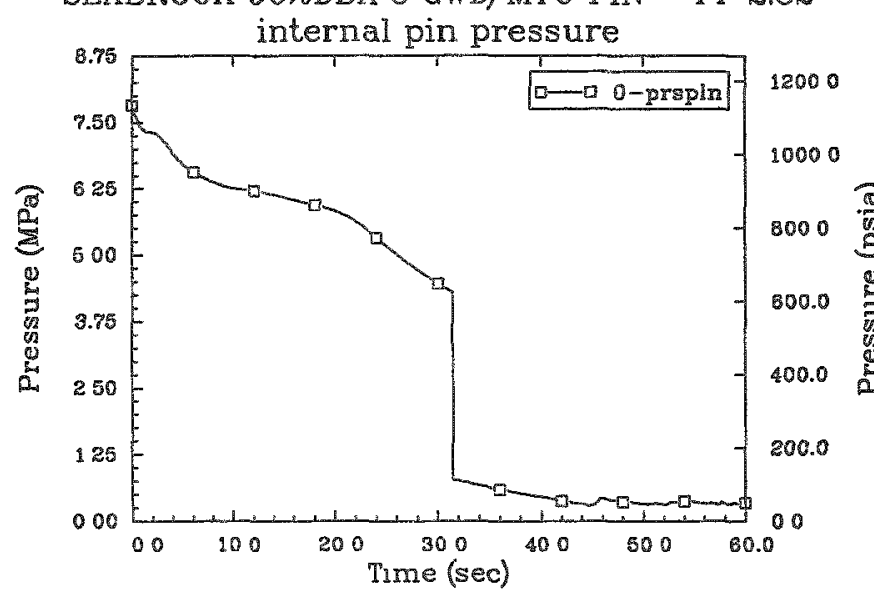

SEABROOK 90\%DBA 5 GWD/MTU PIN--PF 2.32

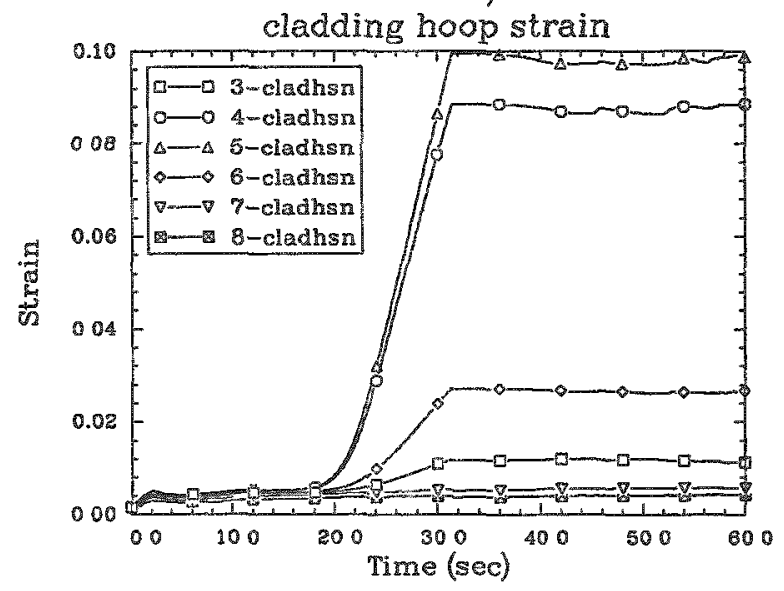

SEABROOK 90\%DBA 5 GWD/MTU PIN--PF 2.32

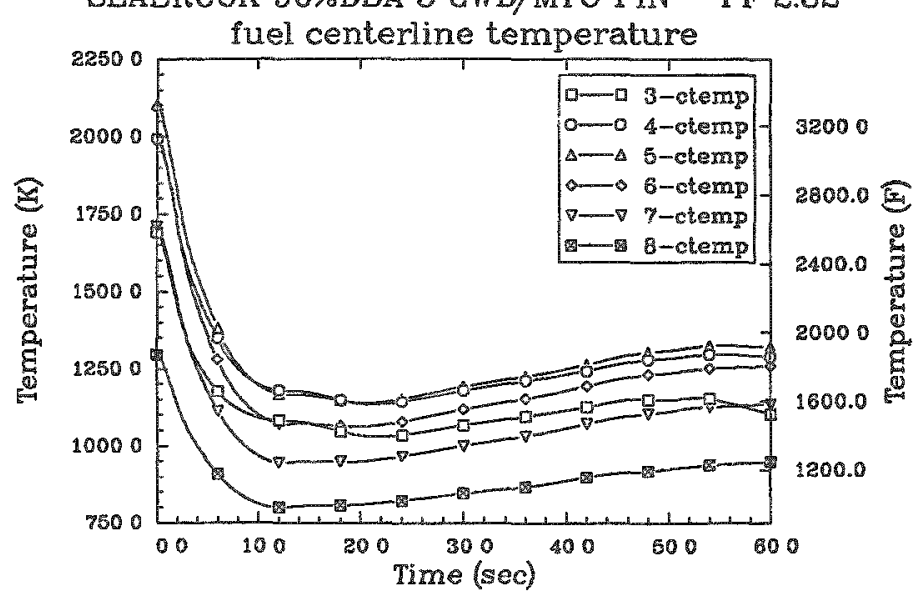

SEABROOK 90\%DBA 5 GWD/MTU PIN--PF 2.32
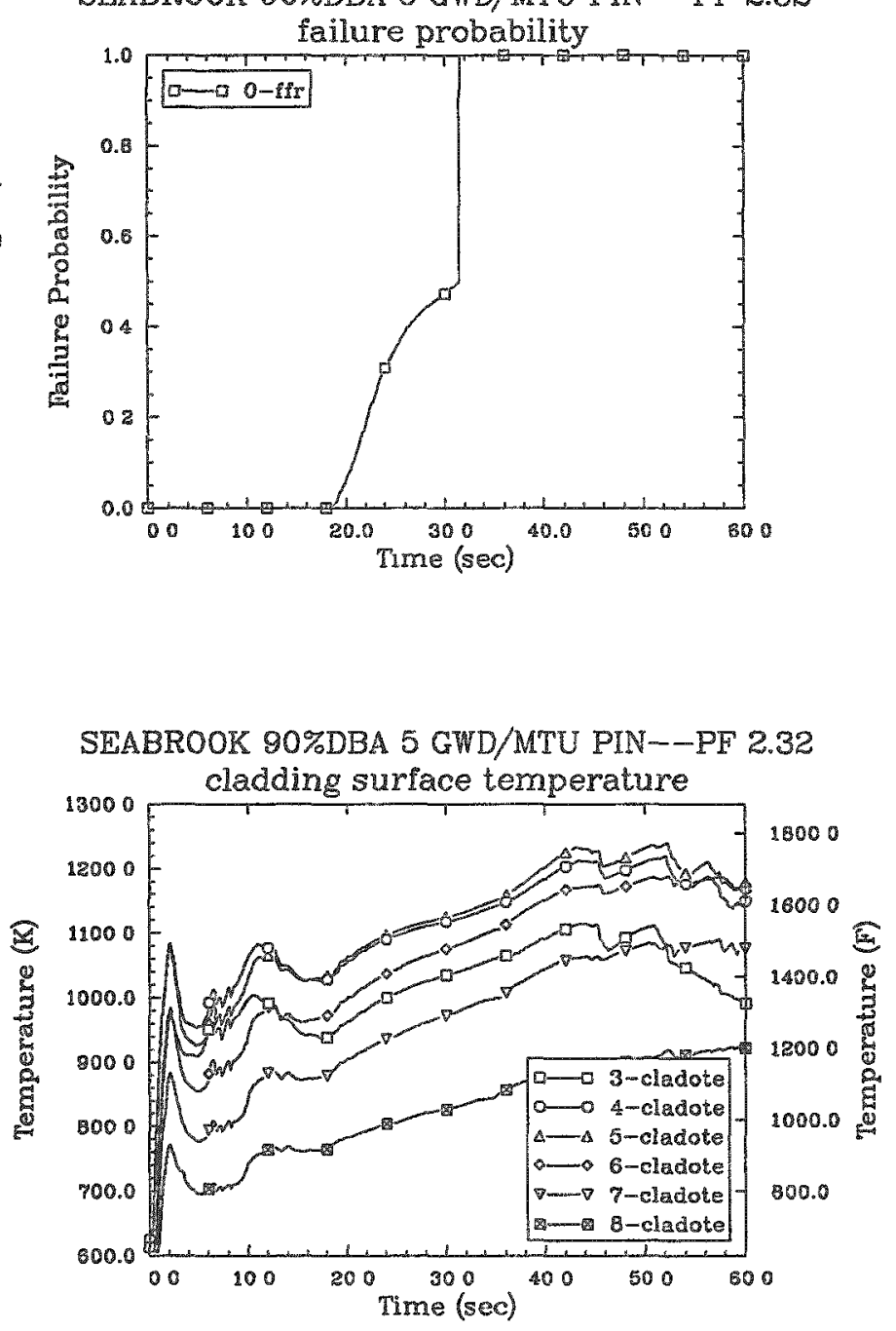

SEABROOK 90\%DBA 5 GWD/MTU PIN--PF 2.32

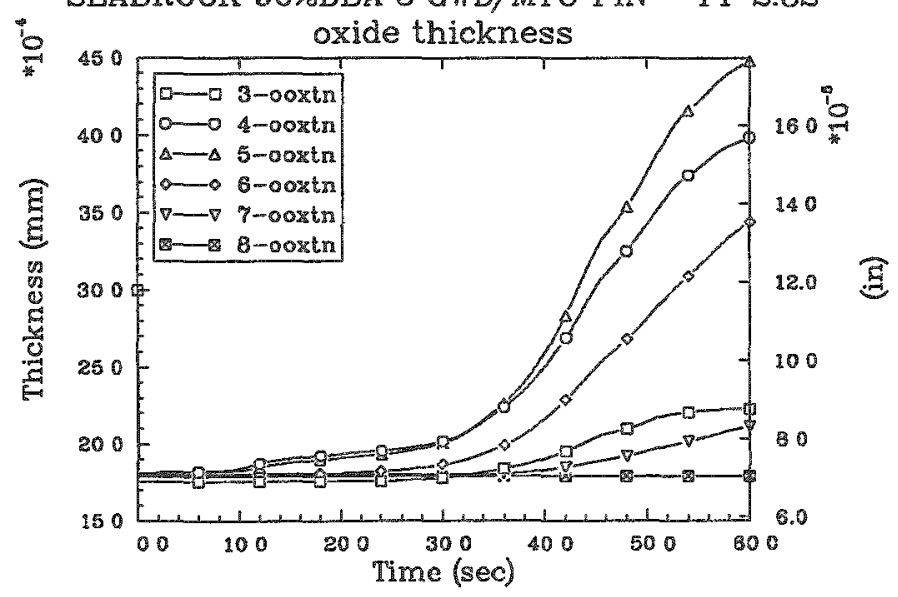




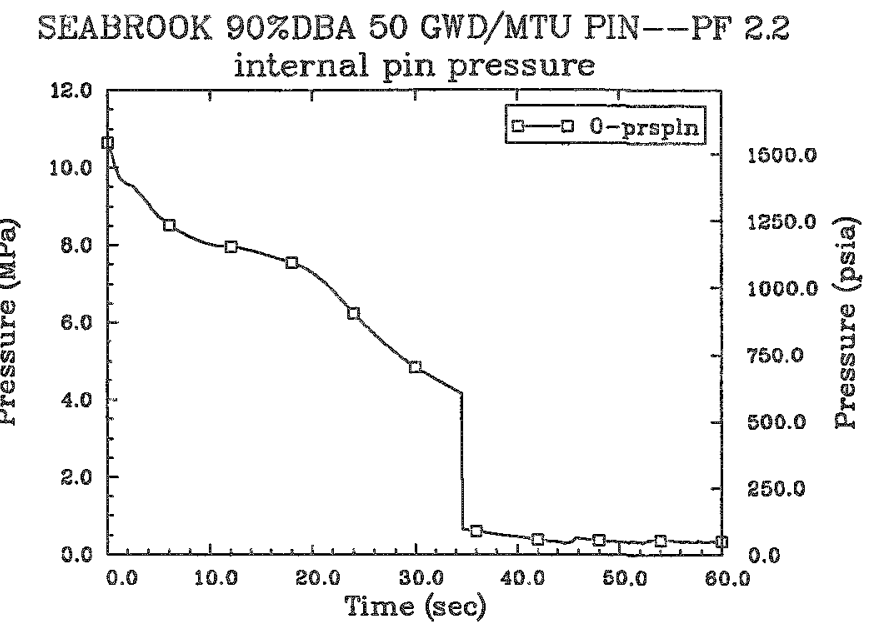

SEABROOK 90\%DBA 50 GWD/MTU PIN-PF 2.2
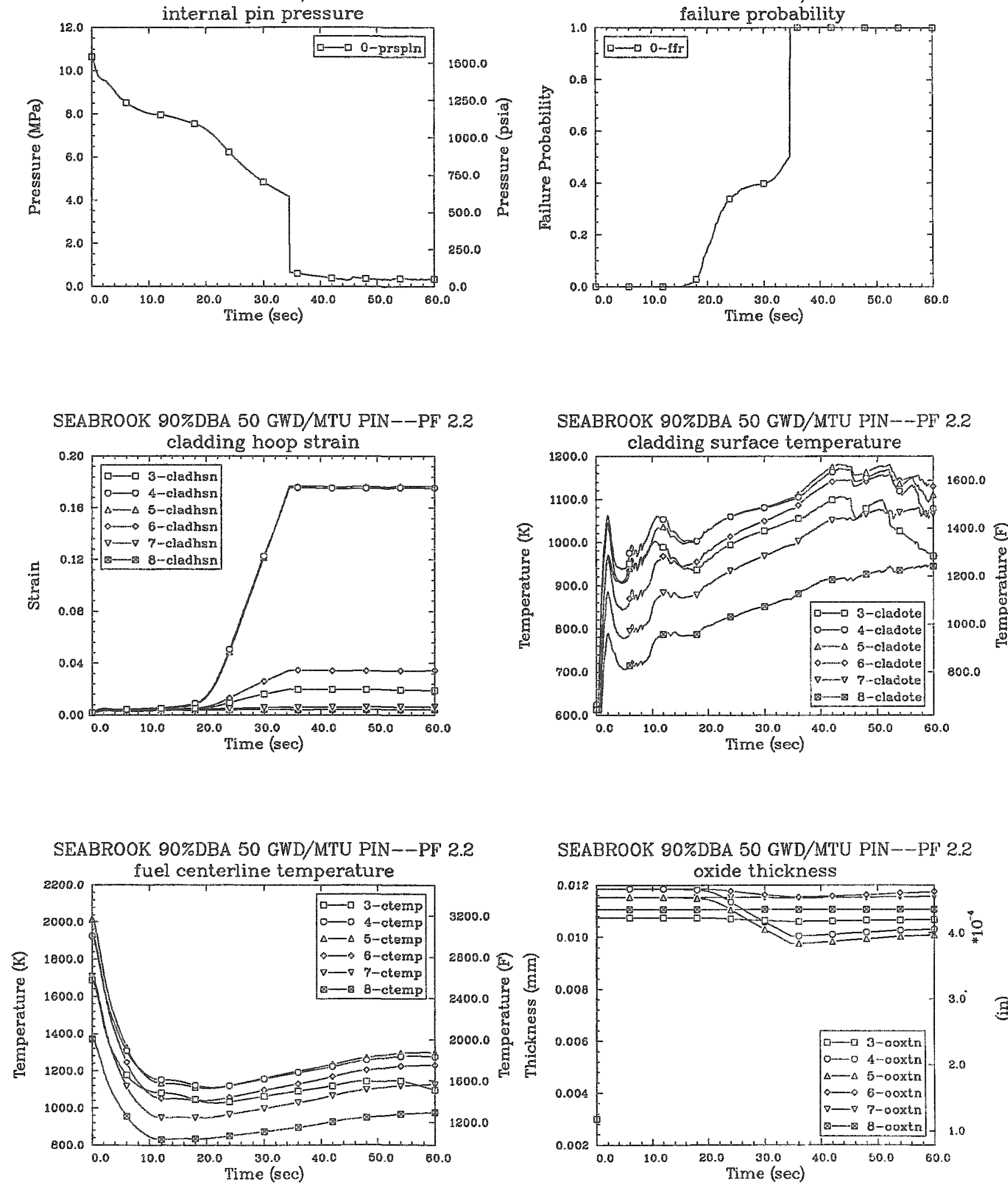

SEABROOK 90\%DBA 50 GWD/MTU PIN--PF 2.2

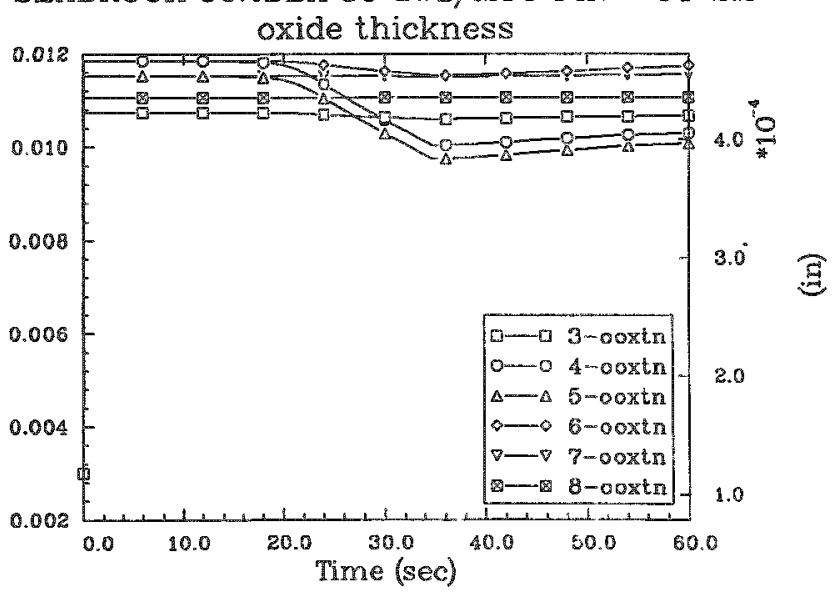


SEABROOK 90\%DBA 35 GWD/MTU PIN--PF 2.2

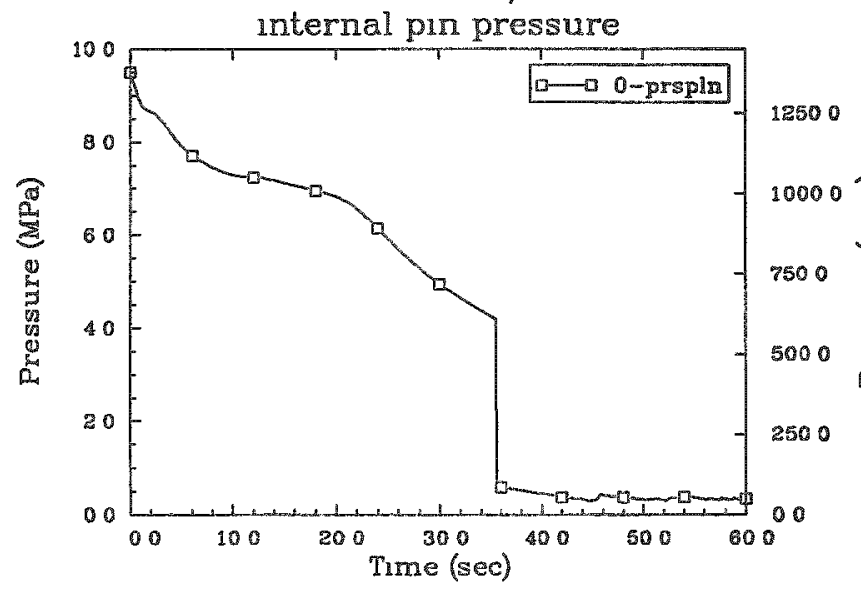

SEABROOK 90\%DBA 35 GWD/MTU PIN--PF 22

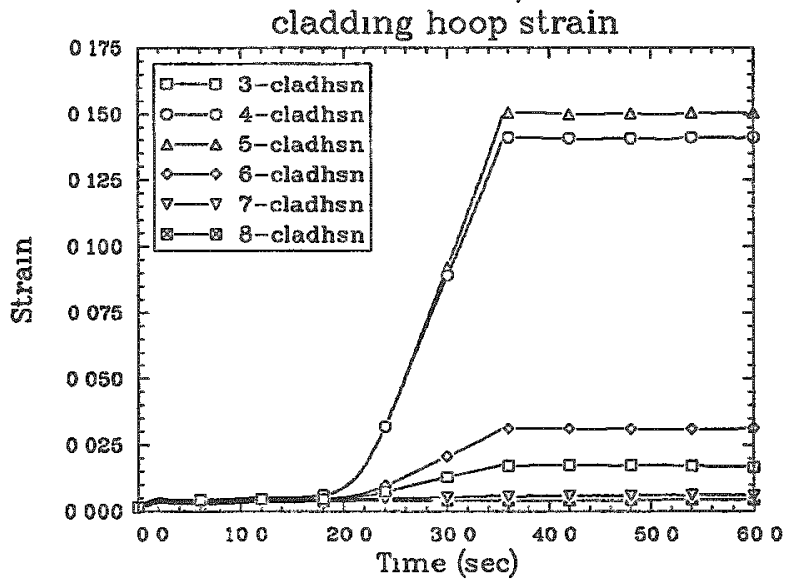

SEABROOK 90\%DBA 35 GWD/MTU PIN-PF 22

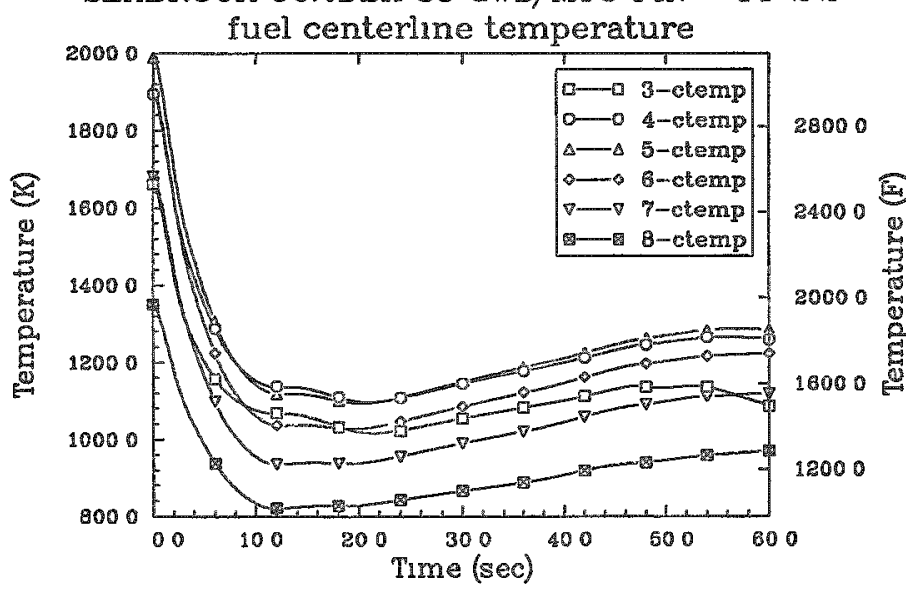

SEABROOK 90\%DBA 35 GWD/MTU PIN--PF 22
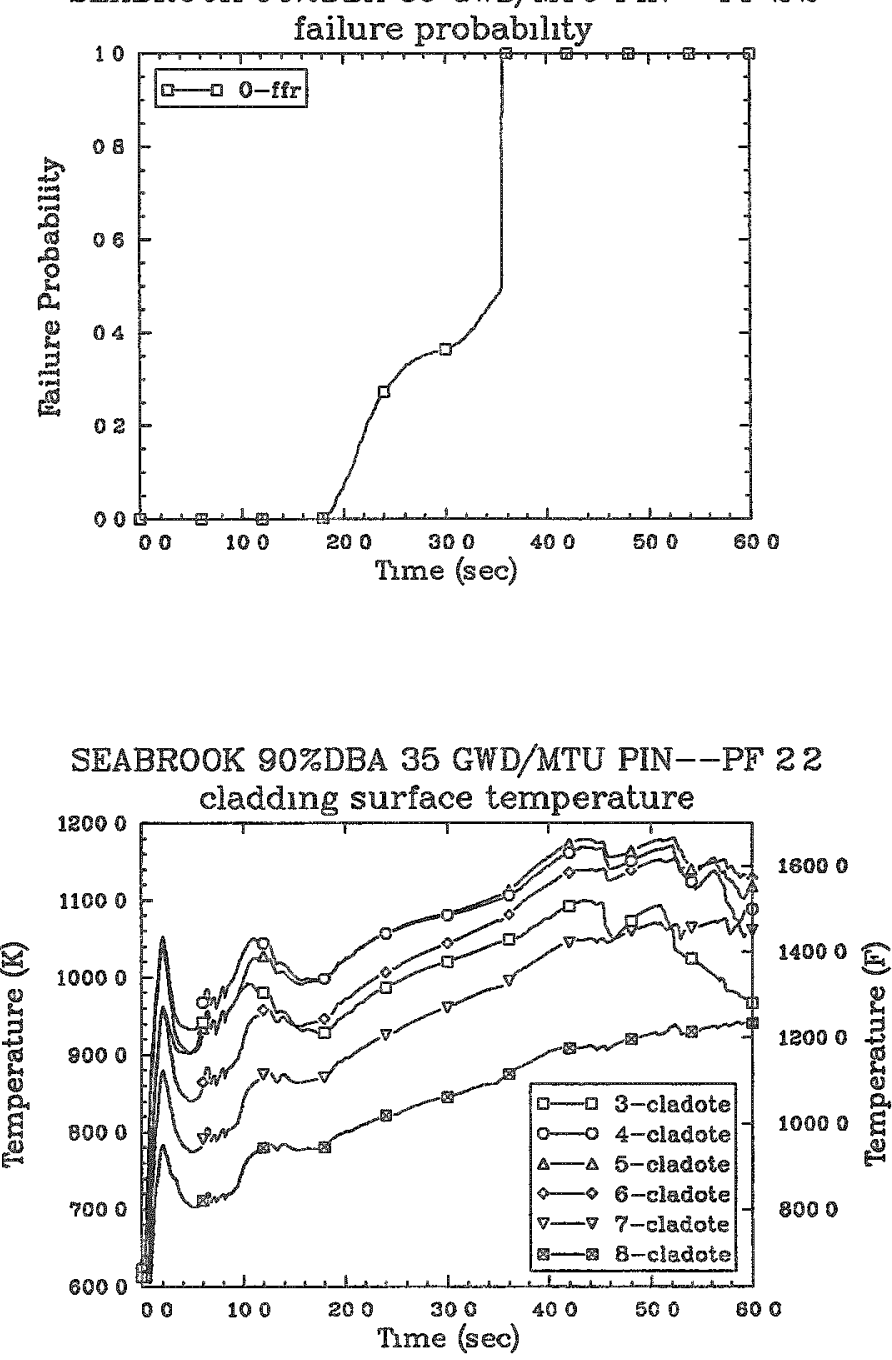

SEABROOK 90\%DBA 35 GWD/MTU PIN--PF 22

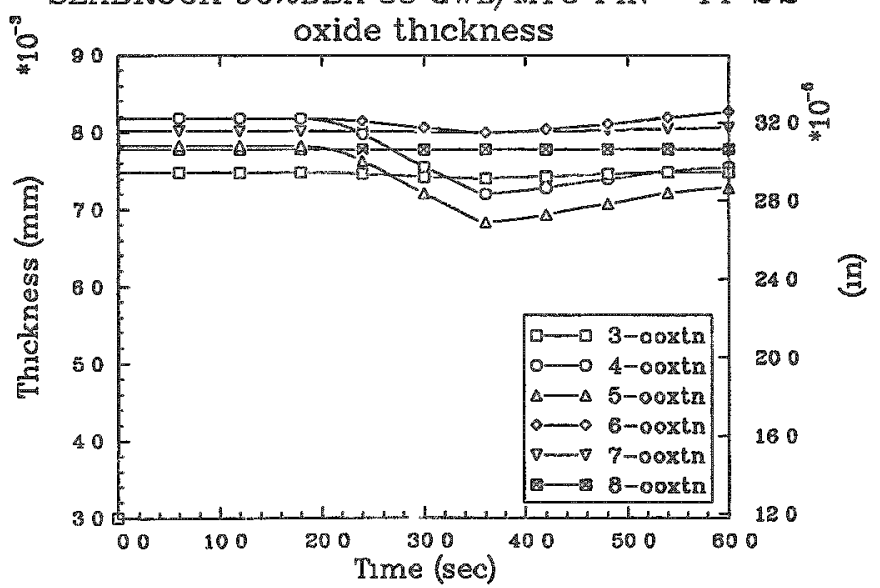



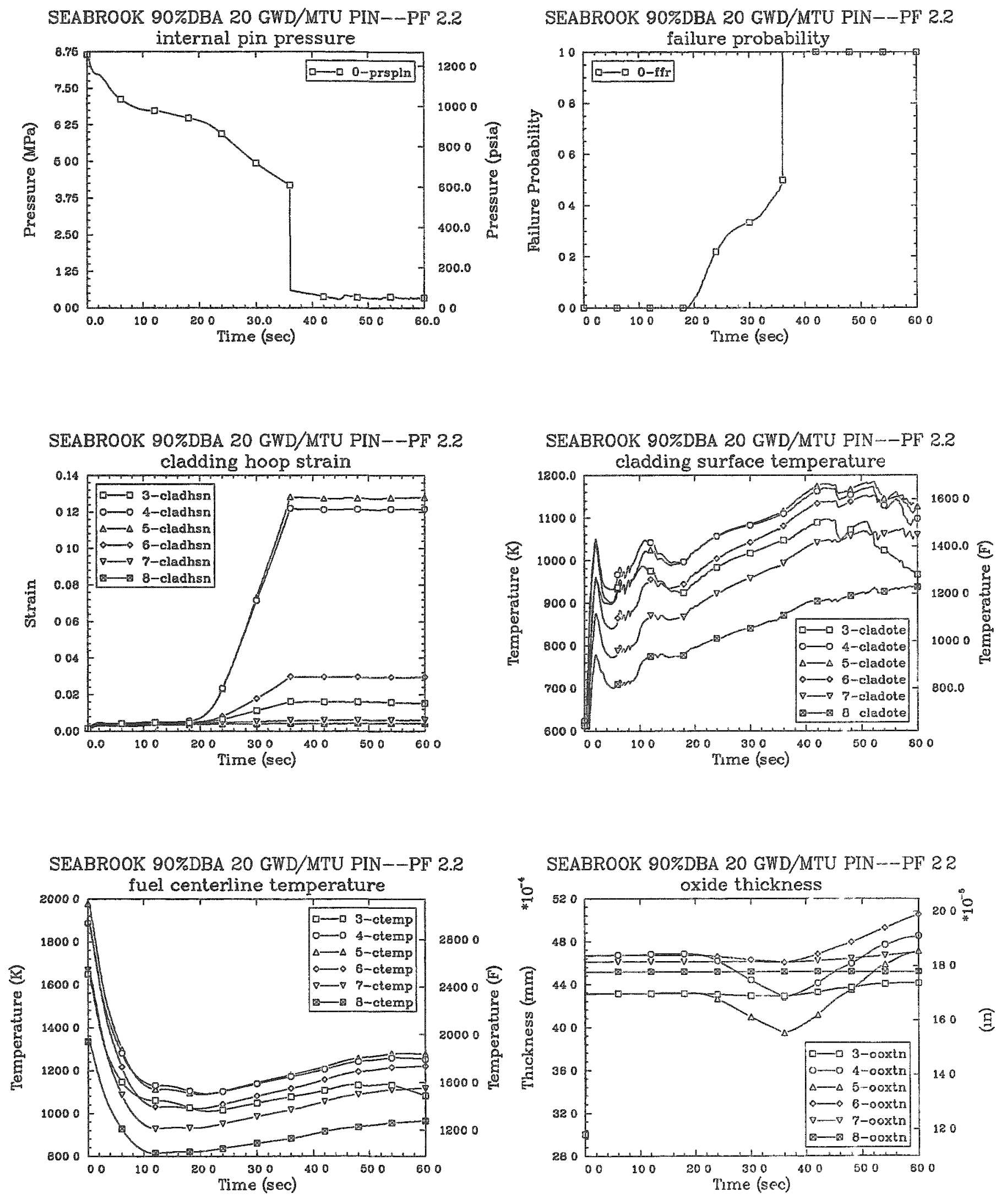


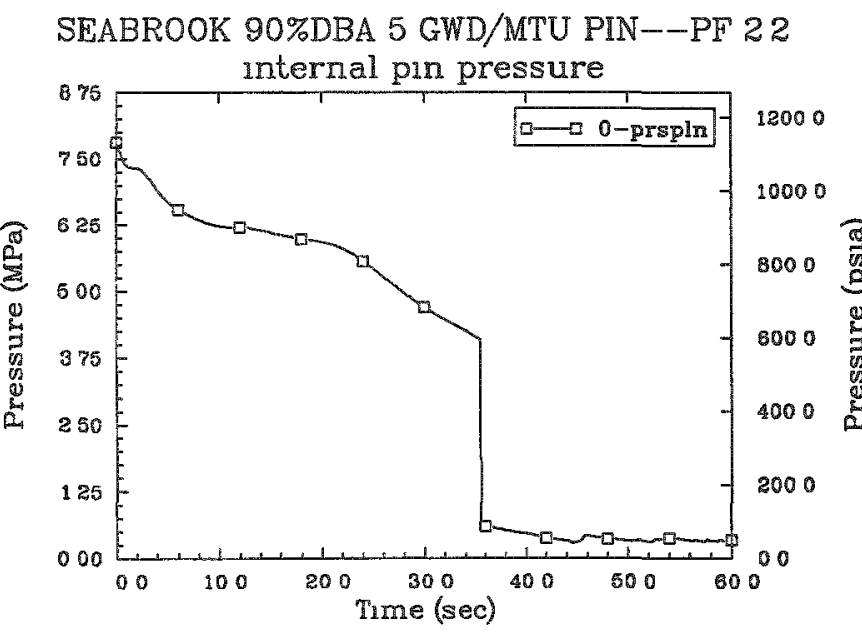

SEAEROOK 90\%DBA 5 GWD/MTU PIN--PF 22
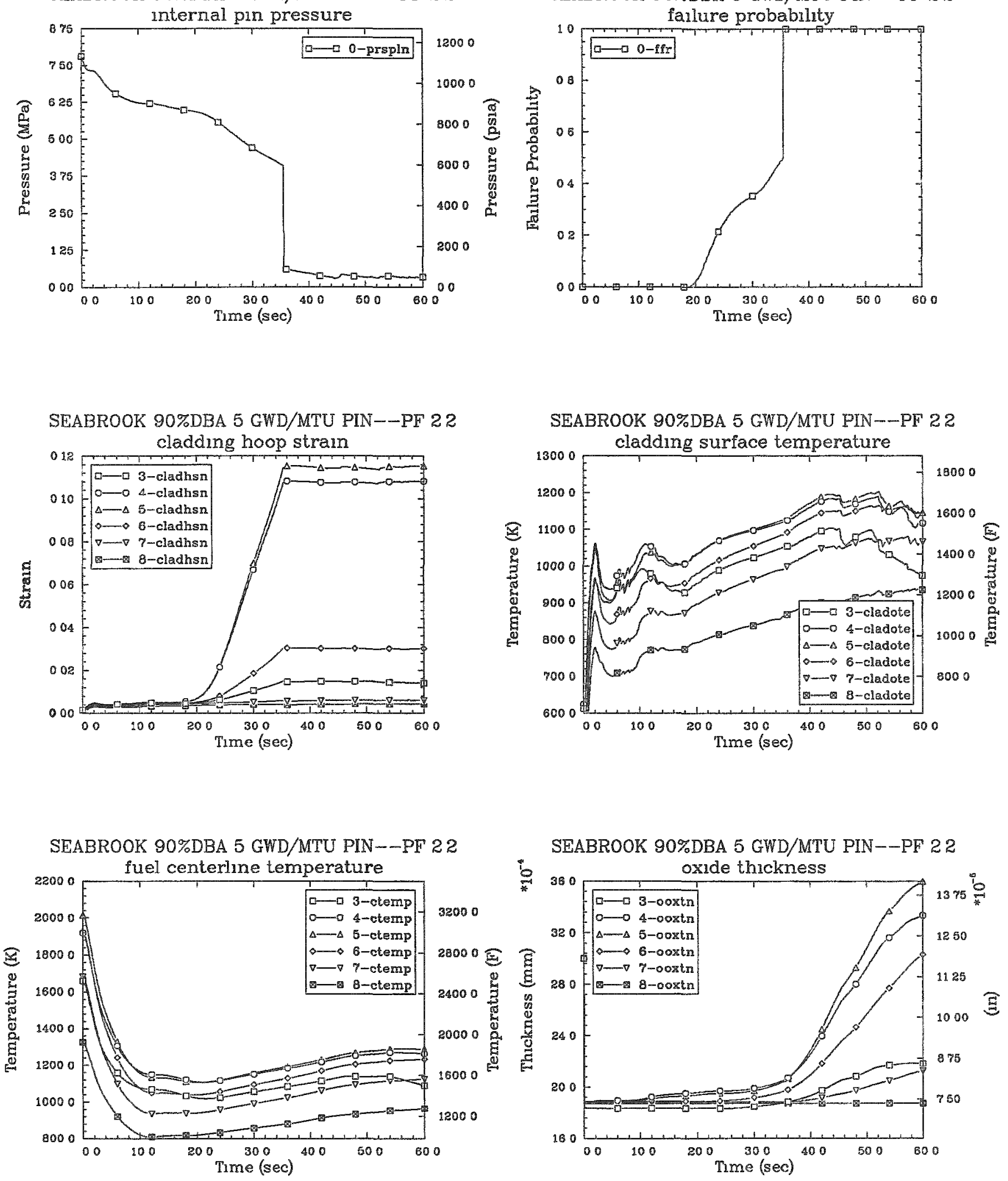

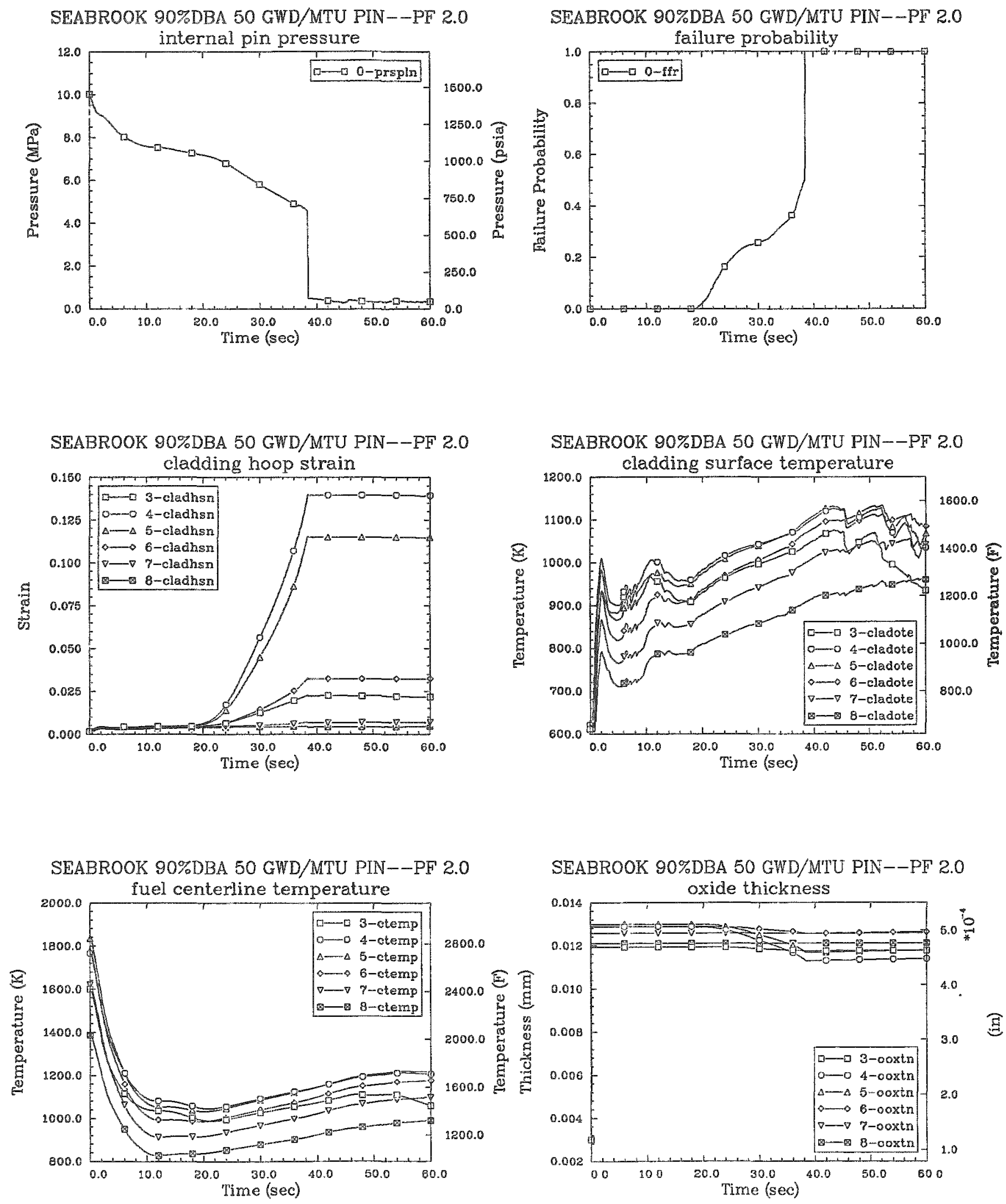

SEABROOK 90\%DBA 50 GWD/MIU PIN--PF 2.0

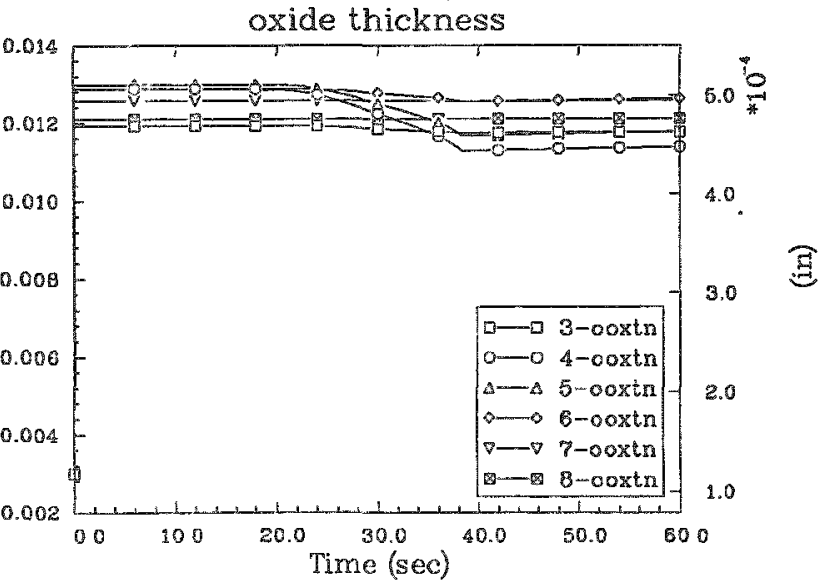


SEABROOK 90\%DBA 35 GWD/MTU PIN--PF 2.0

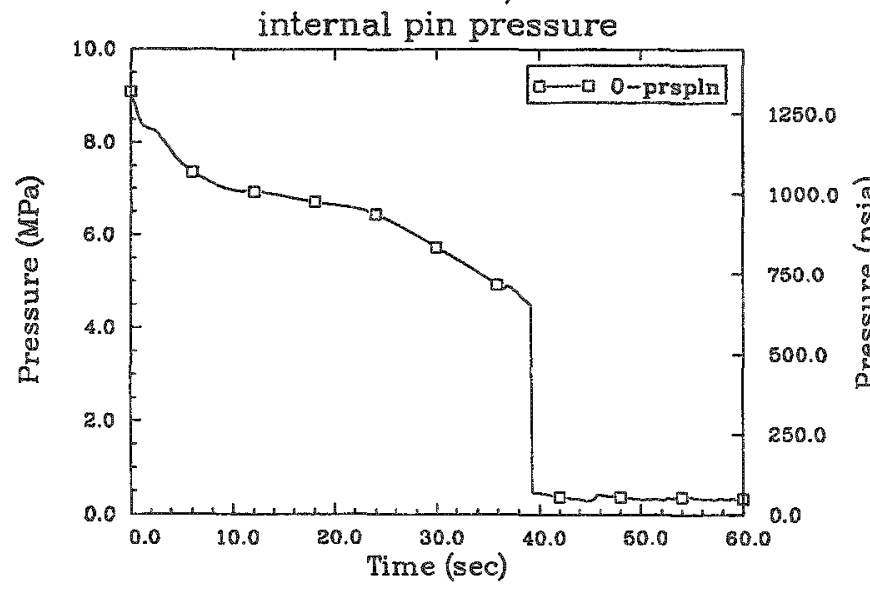

SEABROOK 90\%DBA 35 GWD/MTU PIN--PF 2.0
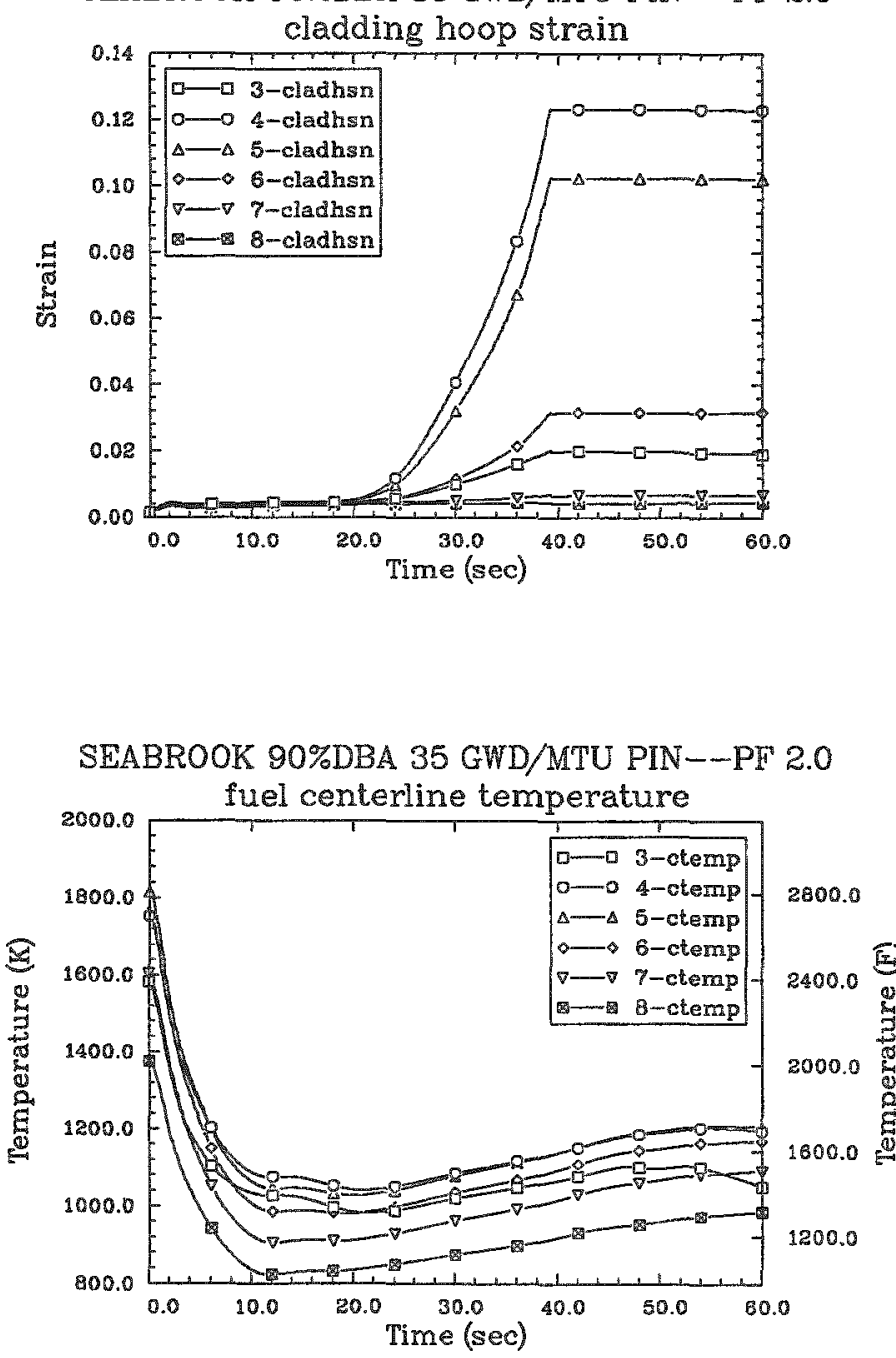

SEABROOK 90\%DBA 35 GWD/MTU PIN-DF 2.0

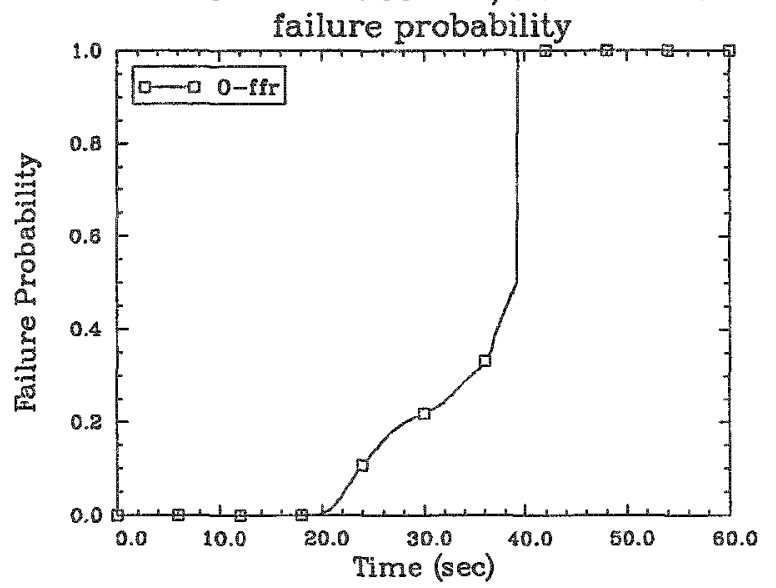

SEABROOK 90\%DBA 35 GWD/MTU PIN--PF 2.0 cladding surface temperature

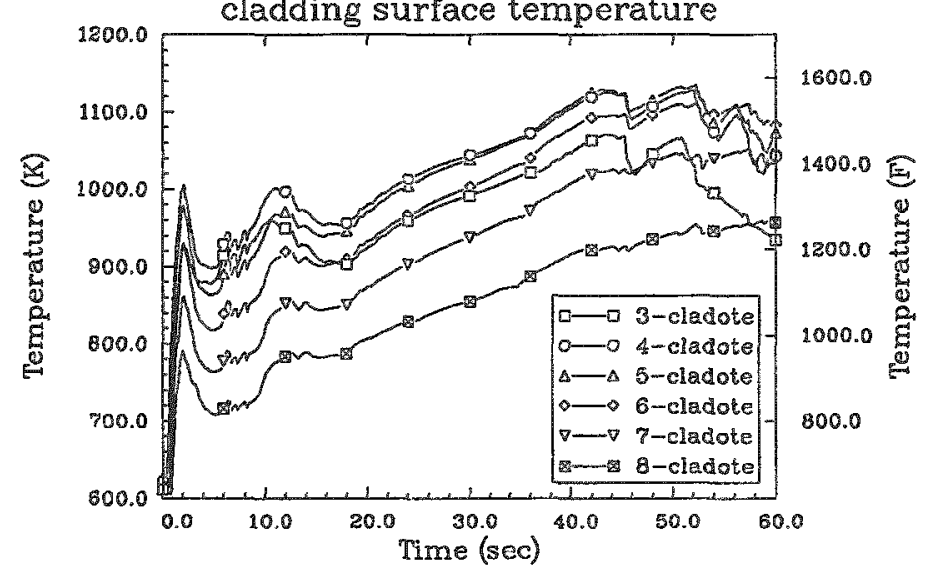

SEABROOK 90\%DBA 35 GWD/MTU PIN--PF 2.0

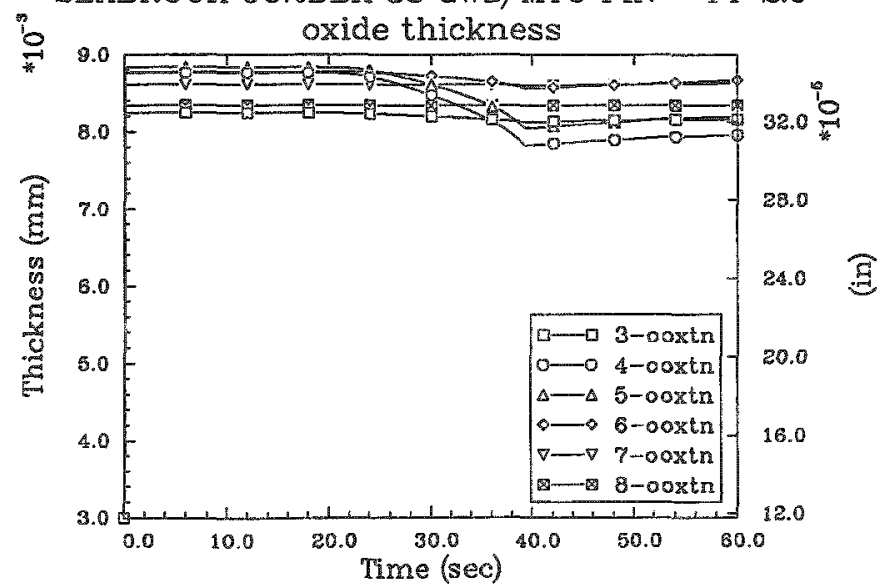


SEABROOK 90\%DBA $20 \mathrm{GWD} / \mathrm{MTU}$ PIN--PF 2.0

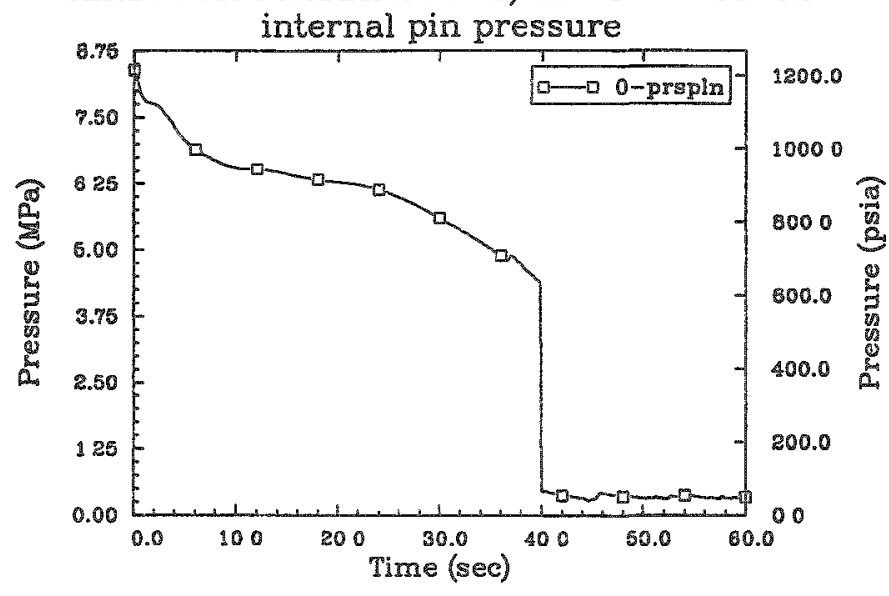

SEABROOK 90\%DBA 20 GWD/MTU PIN--PF 2.0 cladding hoop strain

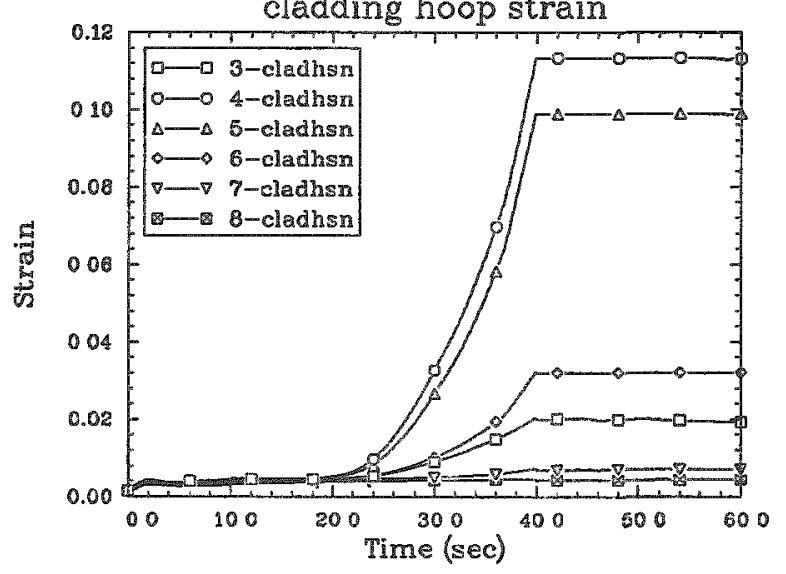

SEABROOK 90\%DBA $20 \mathrm{GWD} / \mathrm{MTU}$ PIN-PF 2.0

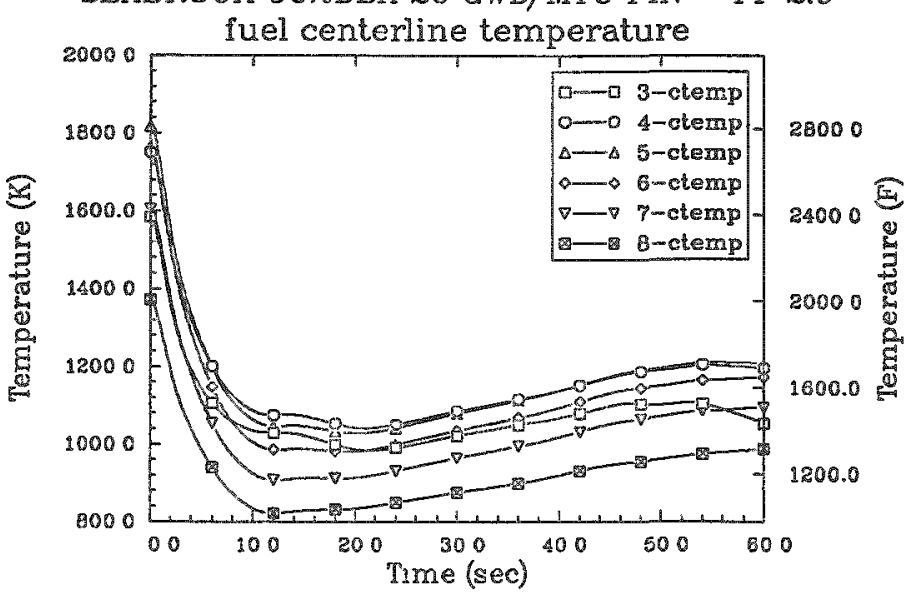

SEABROOK 90\%DBA $20 \mathrm{GWD} / \mathrm{MTU}$ PIN--PF 2.0

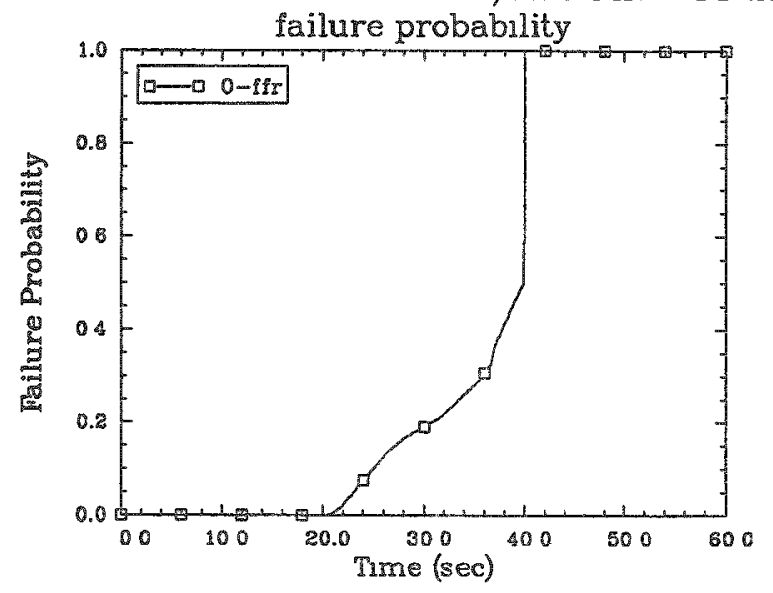

SEABROOK 90\%DBA 20 GWD/MTU PIN--PF 2.0

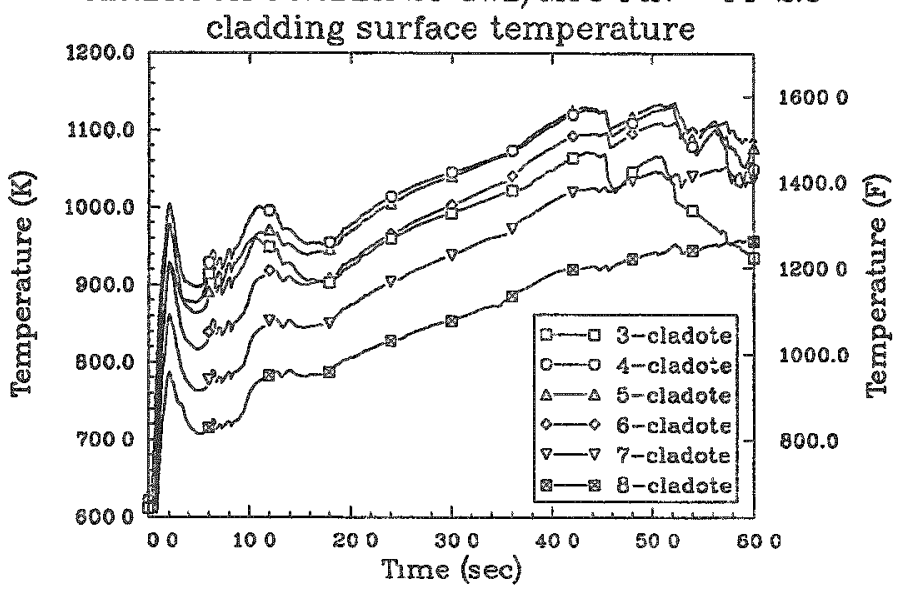

SEABROOK 90\%DBA 20 GWD/MTU PIN--PF 2.0

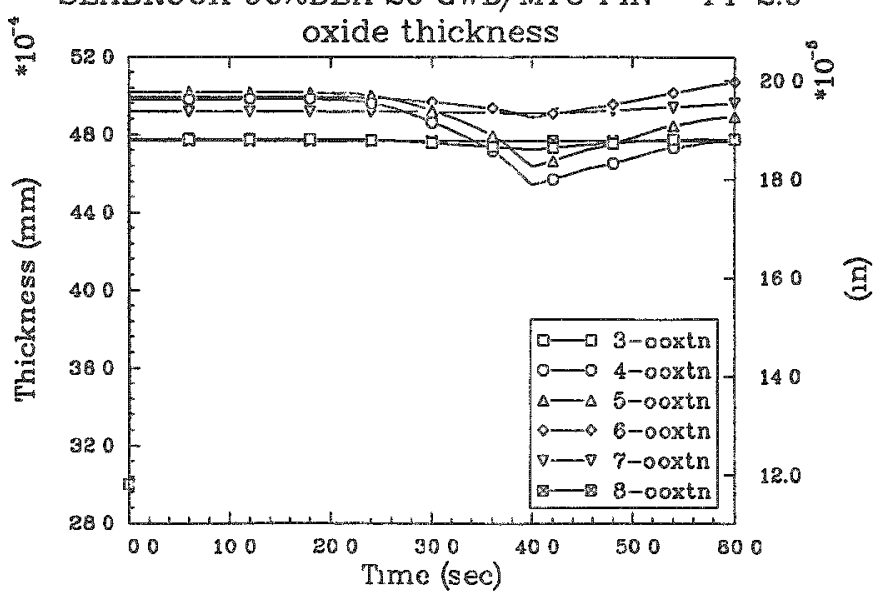



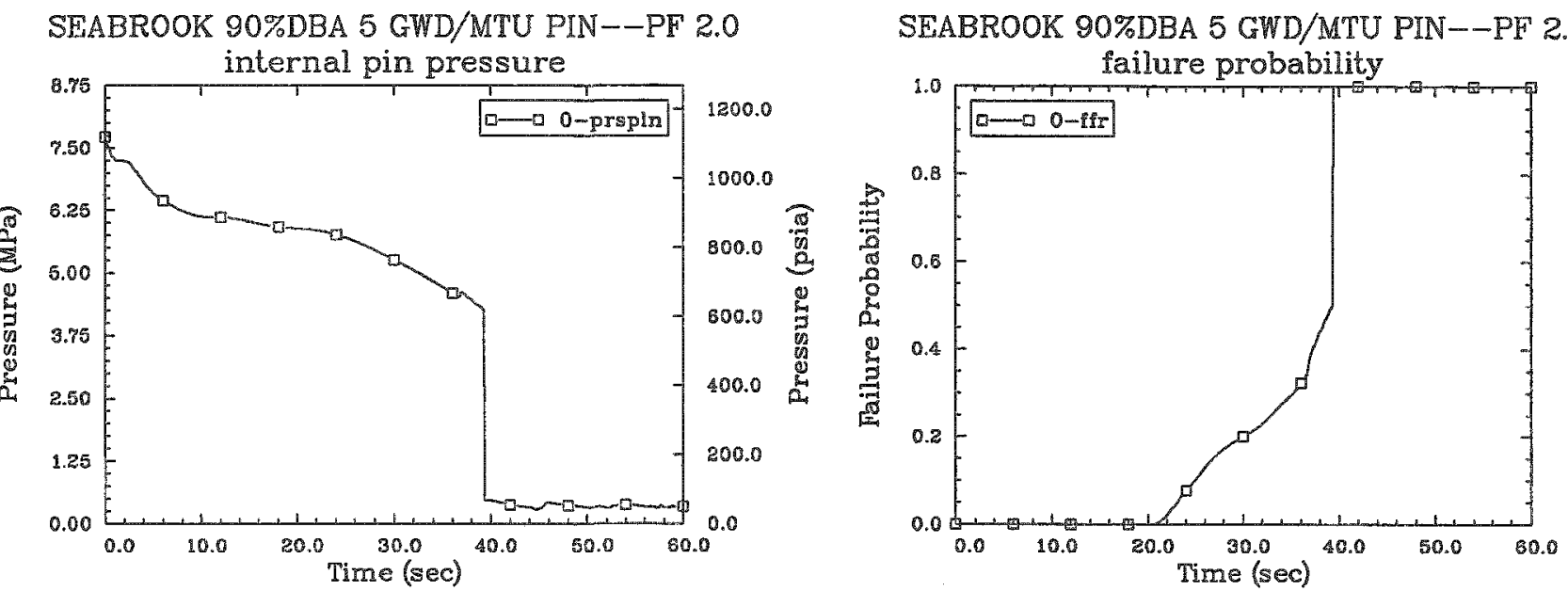

SEABROOK 90\%DBA 5 GWD/MTU PIN--PF 2.0 cladding hoop strain

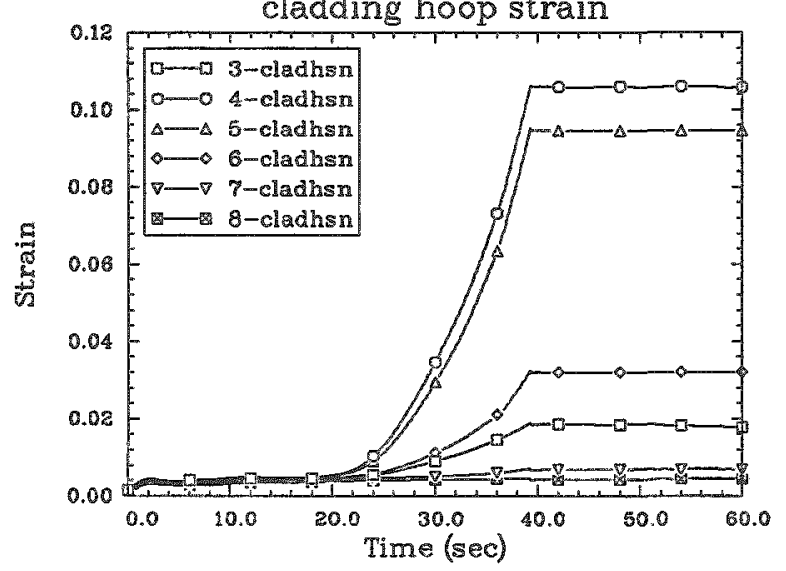

SEABROOK 90\%DBA 5 GWD/MTU PIN--PF 2.0
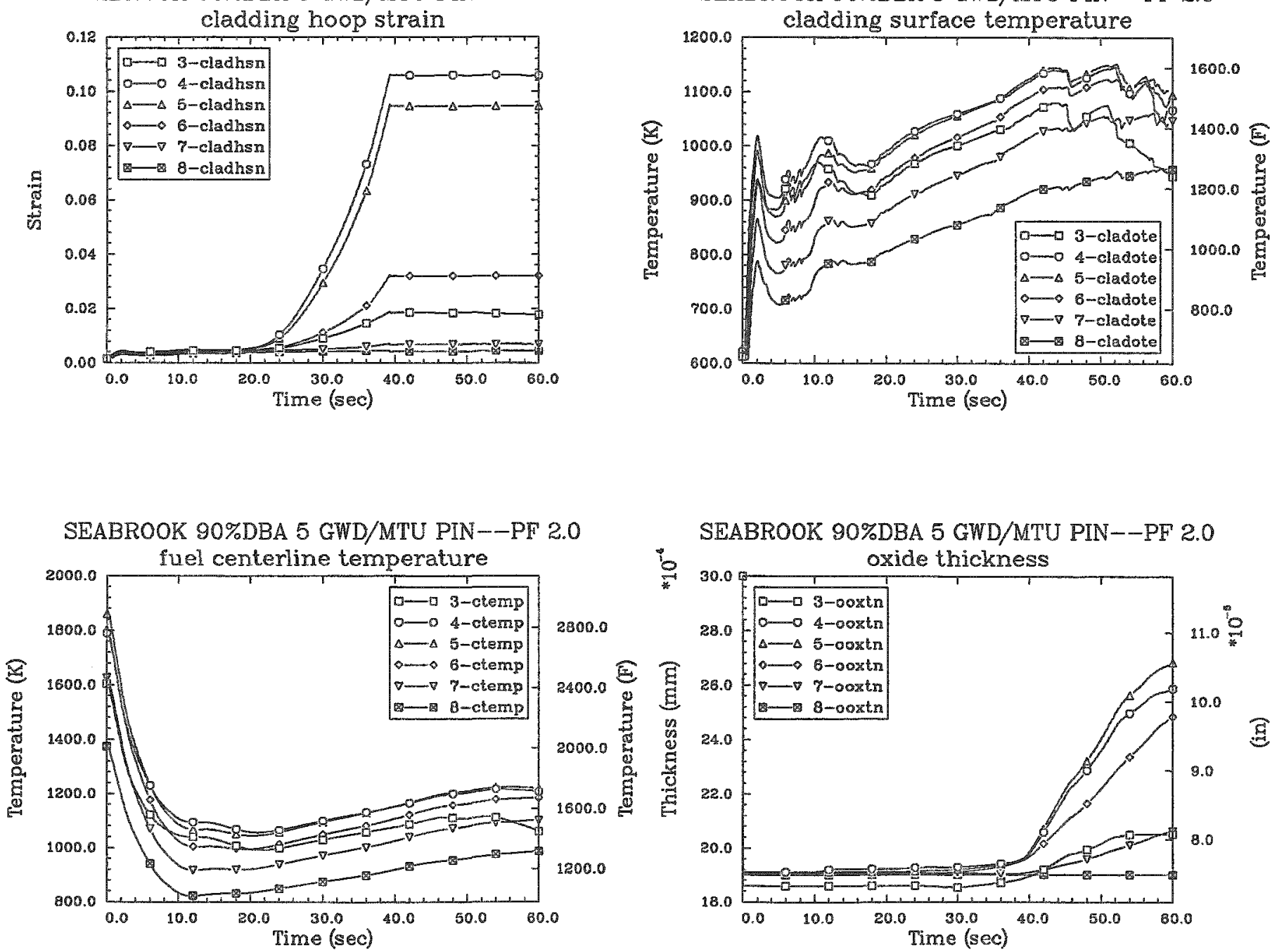
SEABROOK 90\%DBA 50 GWD/MTU PIN-PF' 1.8
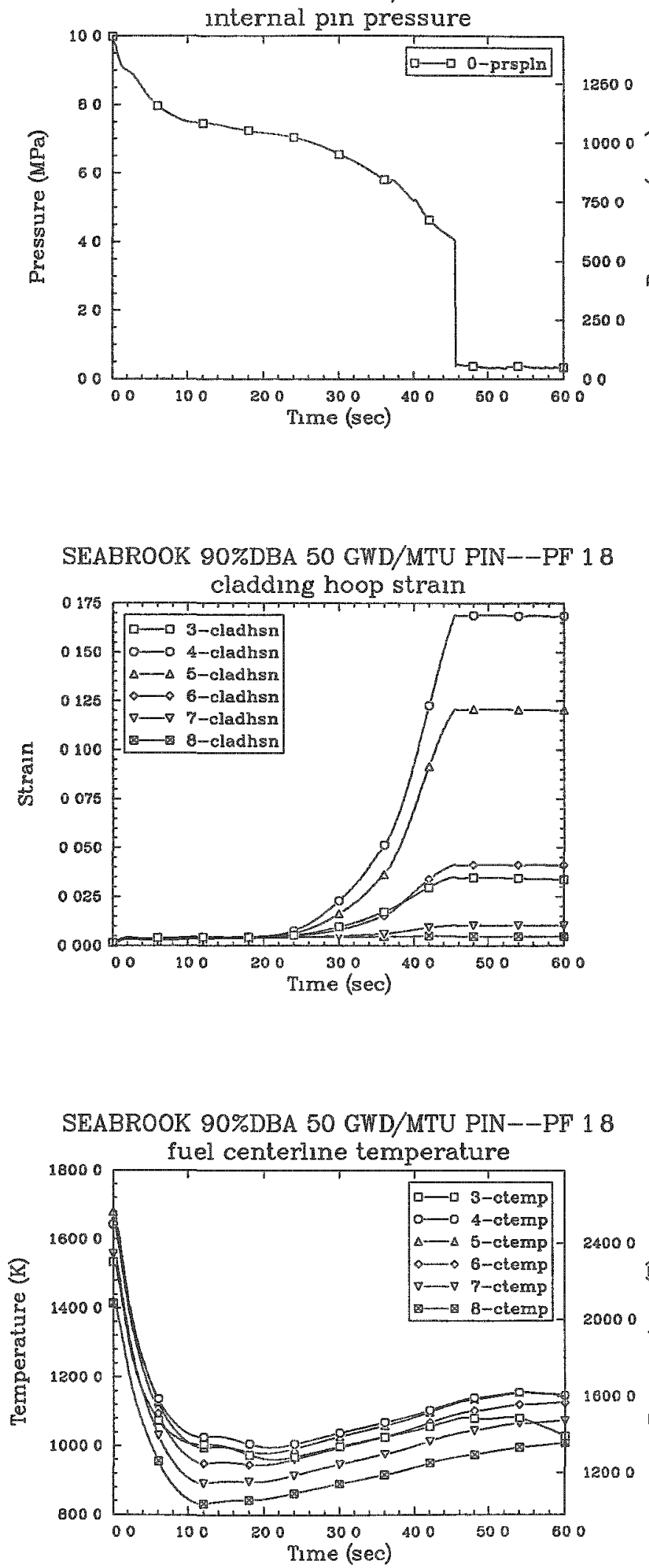

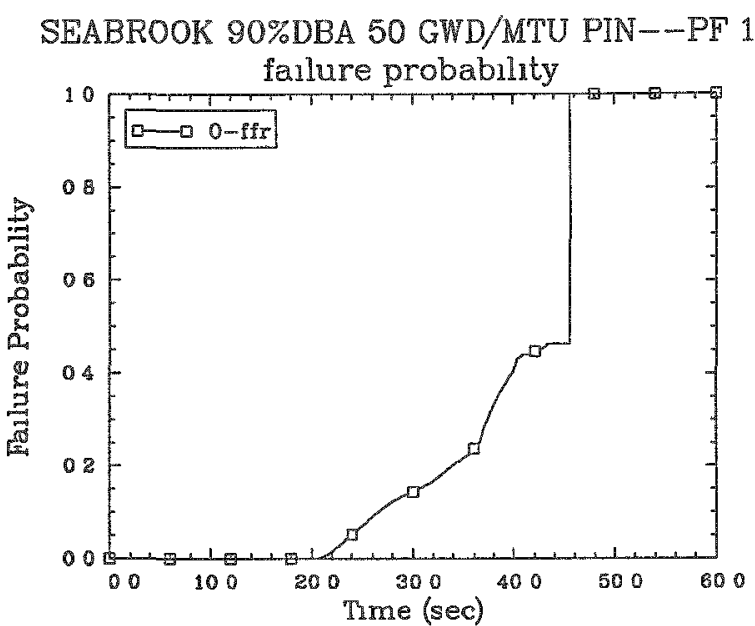

SEABROOK 90\%DBA 50 GWD/MTU PIN--PF 18

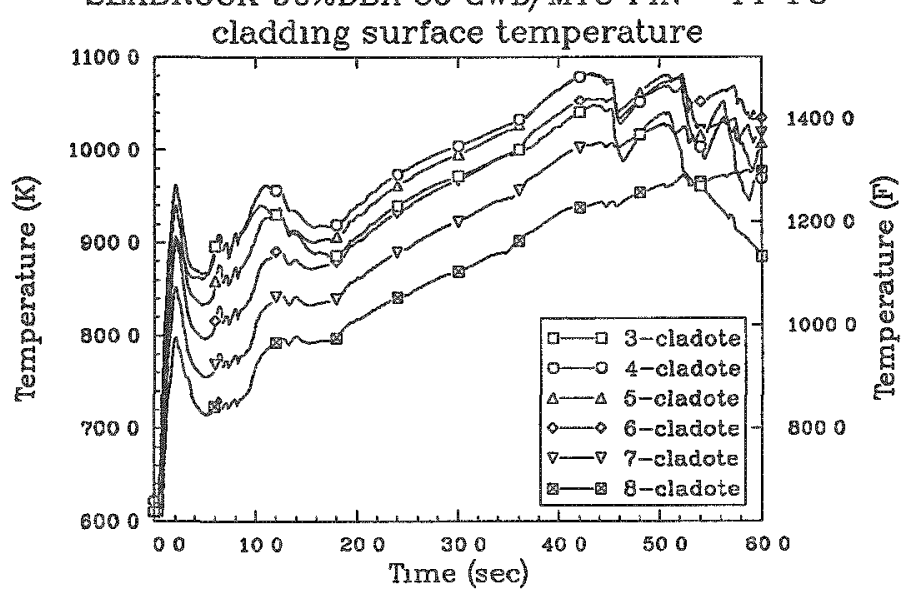

SEABROOK 90\%DBA 50 GWD/MTU PIN--PF 18

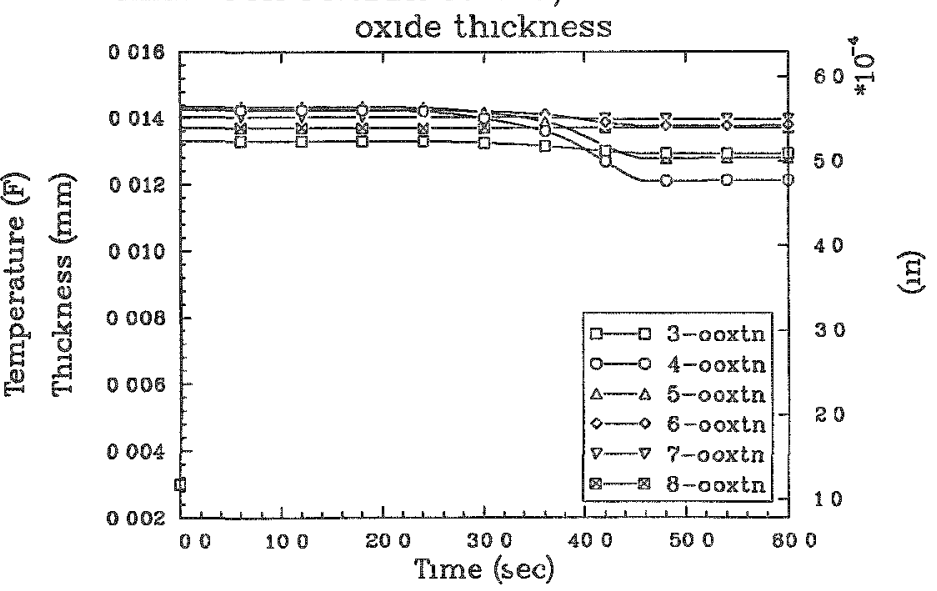




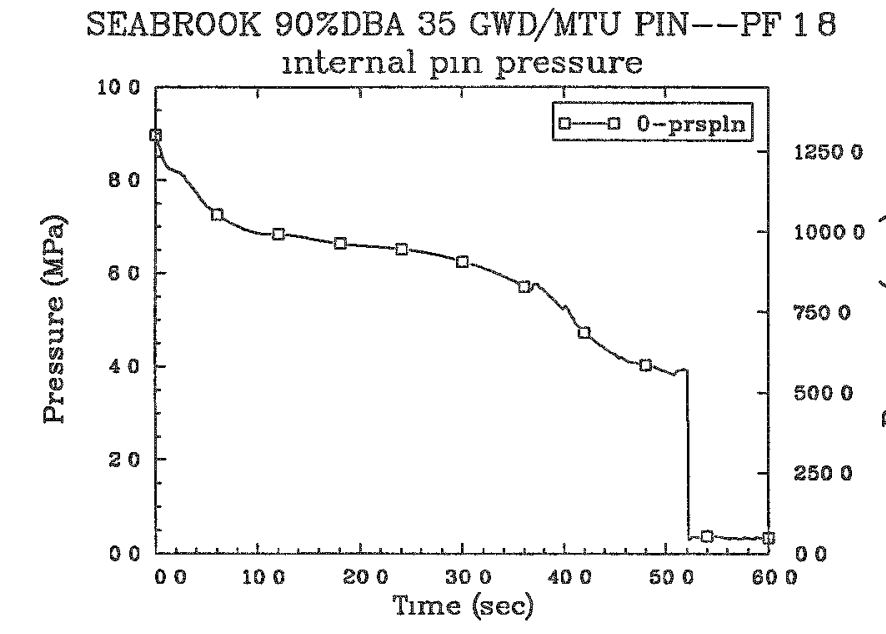

SEABROOK 90\%DBA 35 GWD/MTU PIN--PF 18
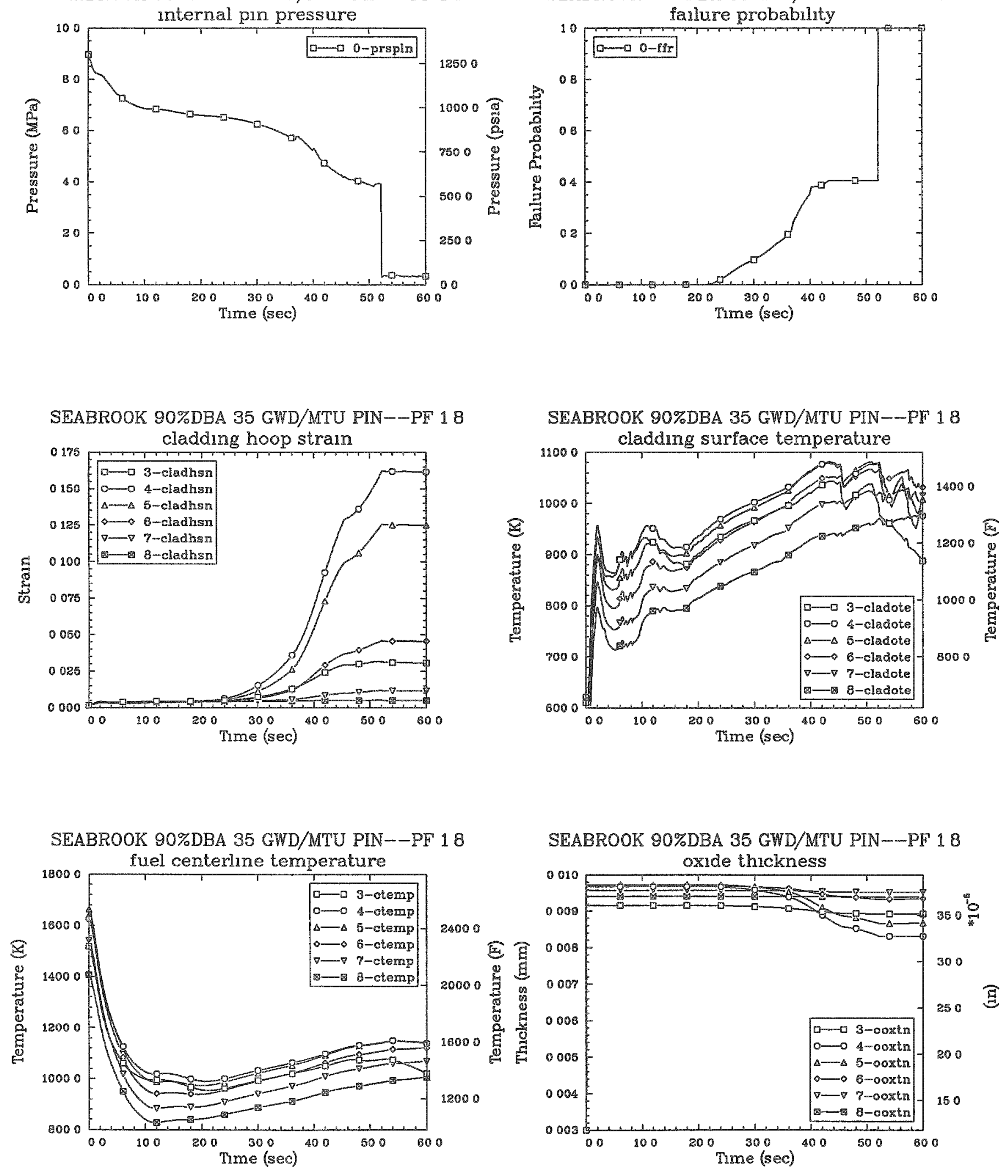

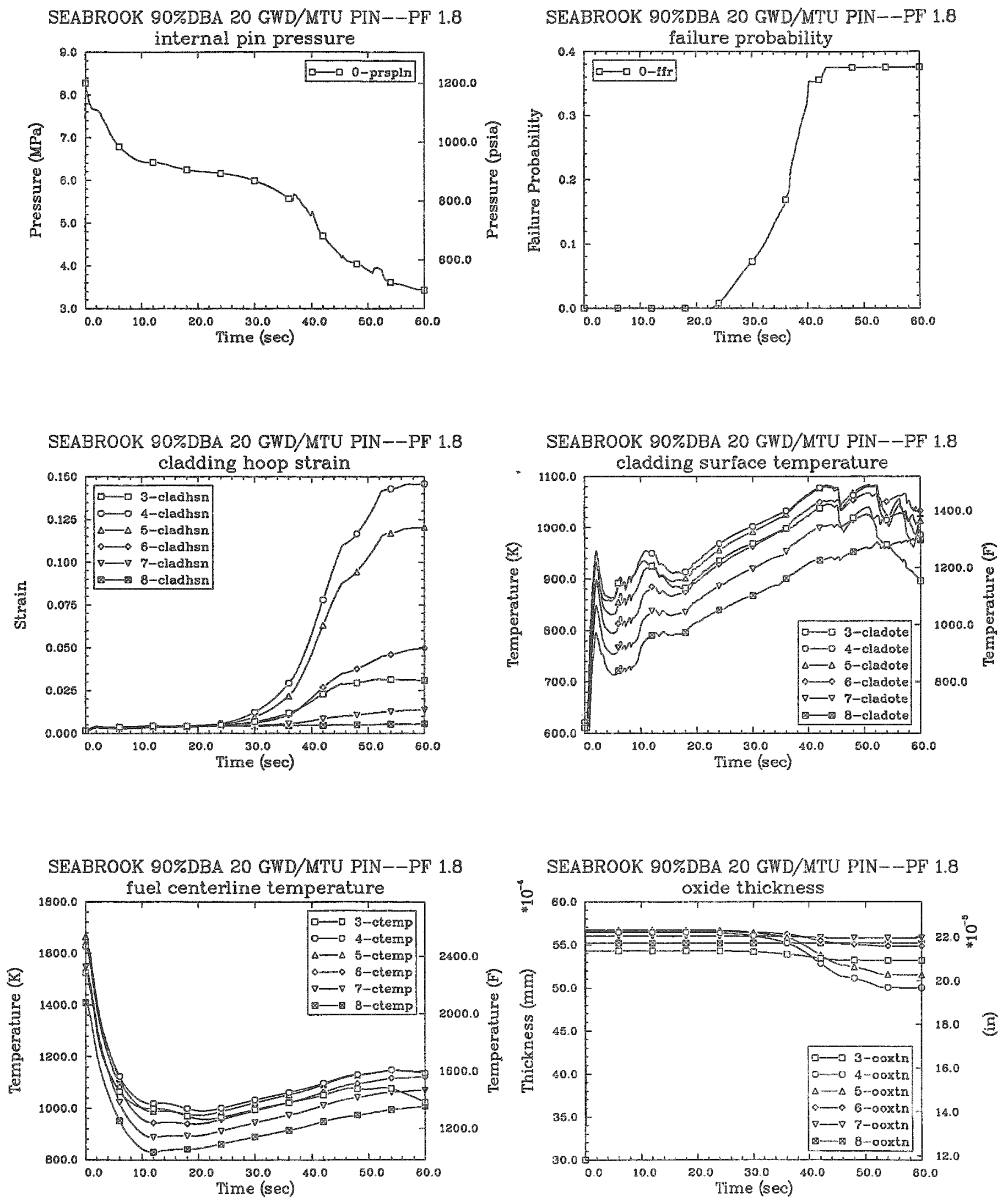

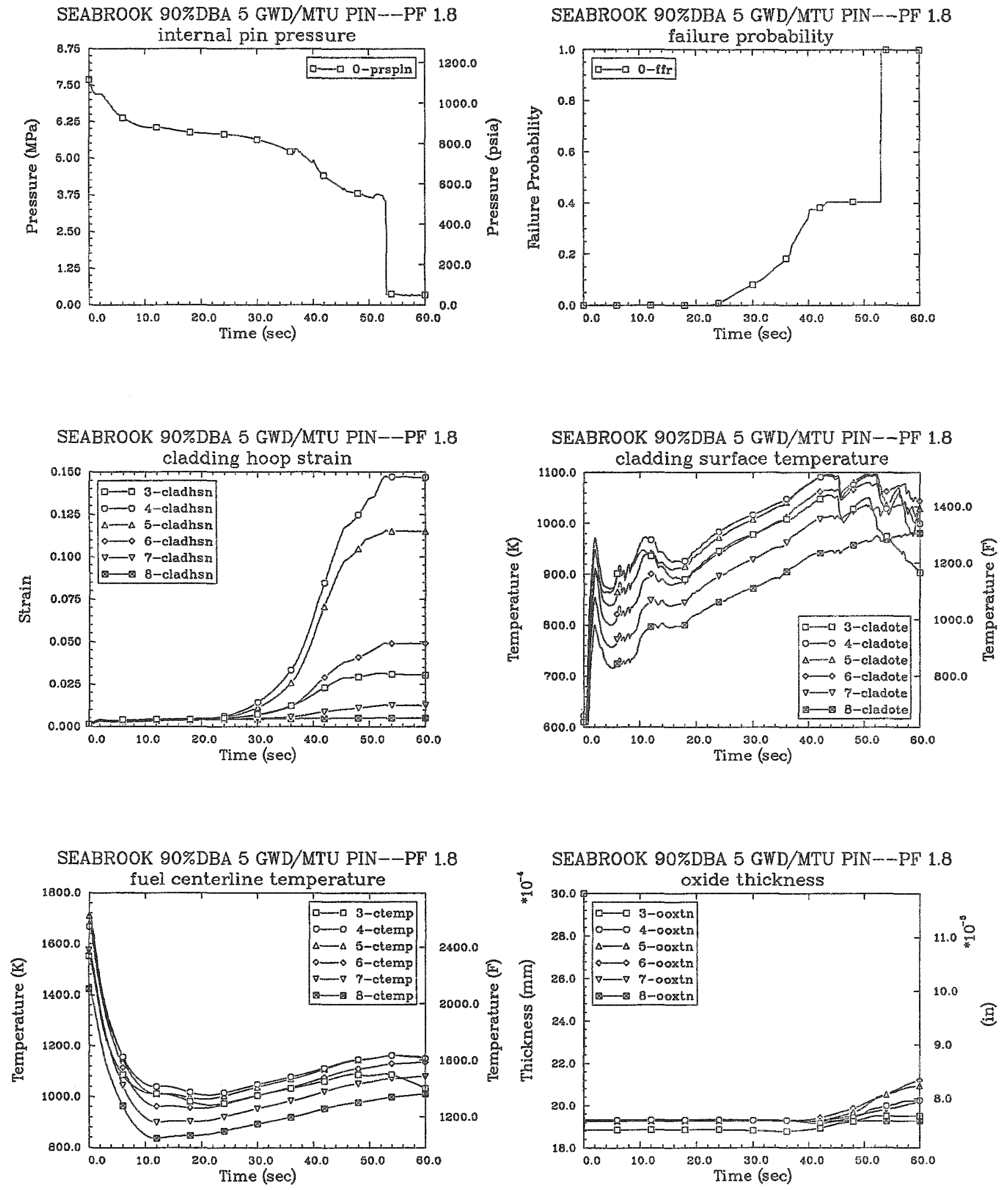
SEABROOK 75\%DBA $50 \mathrm{GWD} / \mathrm{MTU}$ PIN--PF 2.32

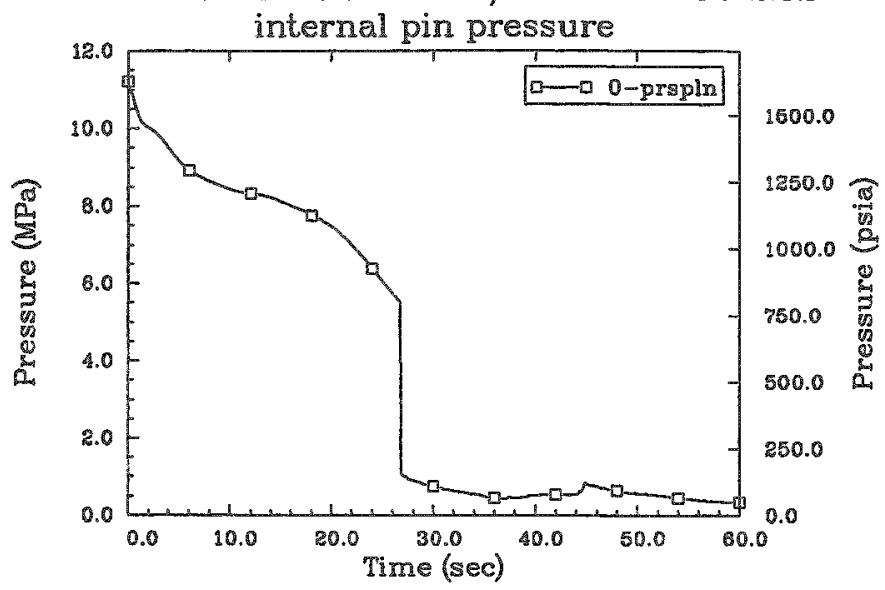

SEABROOK 75\%DBA 50 GWD/MTU PIN--PF 2.32

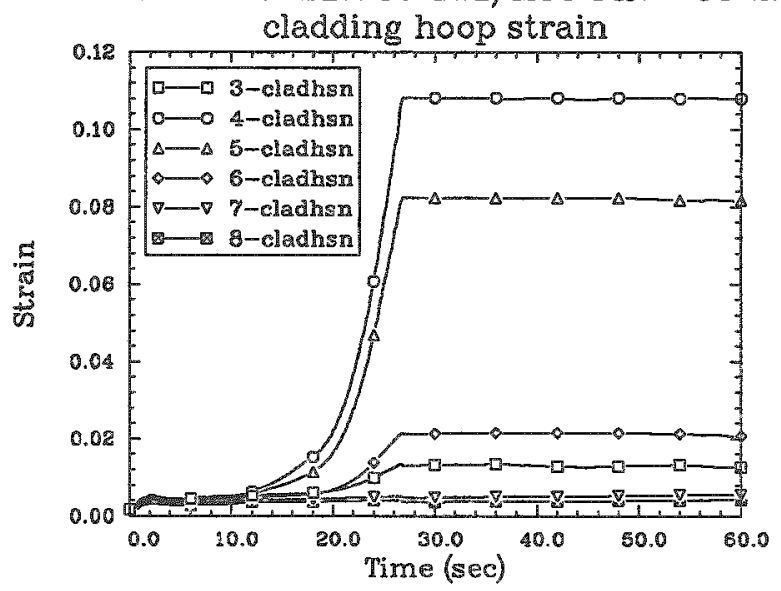

SEABROOK 75\%DBA 50 GWD/MTU PIN--PF 2.32

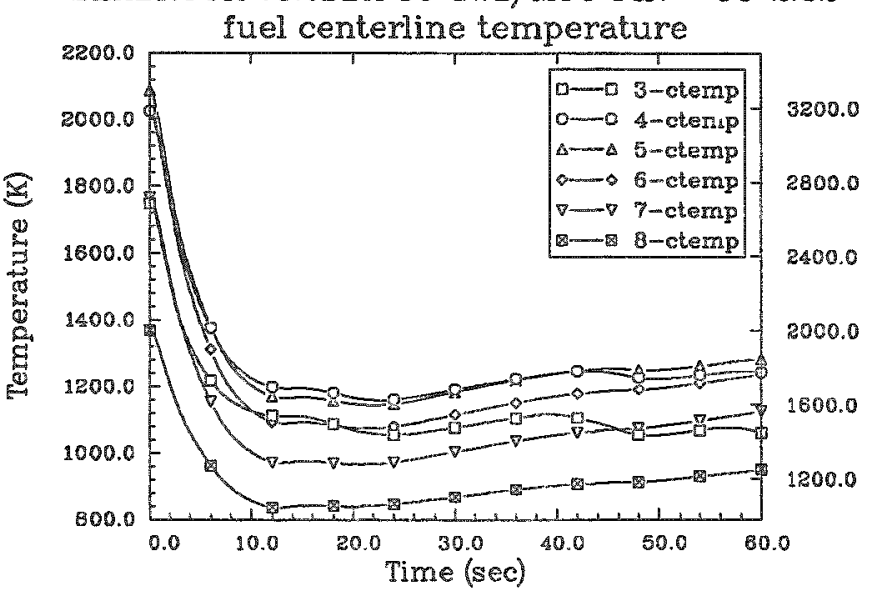

SEABROOK 75\%DBA 50 GWD/MTU PIN--PF 2.32 failure probability

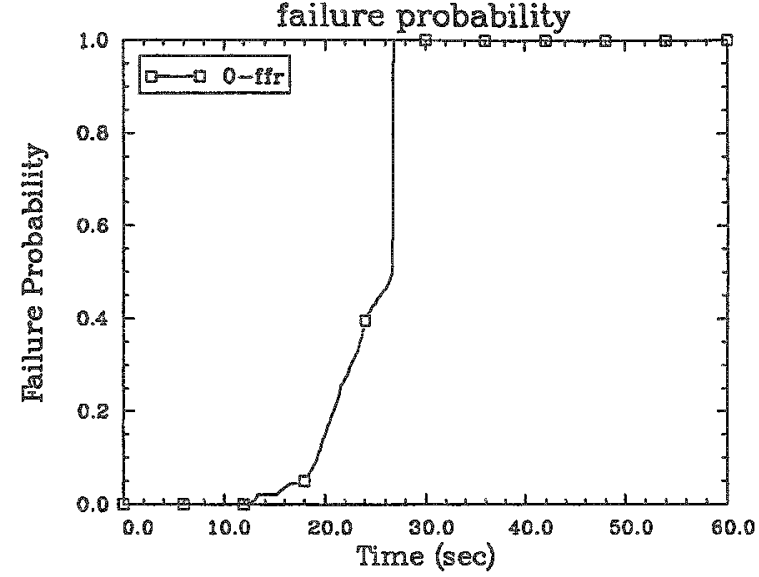

SEABROOK 75\%DBA 50 GWD/MTU PIN--PF 2.32 cladding surface temperature

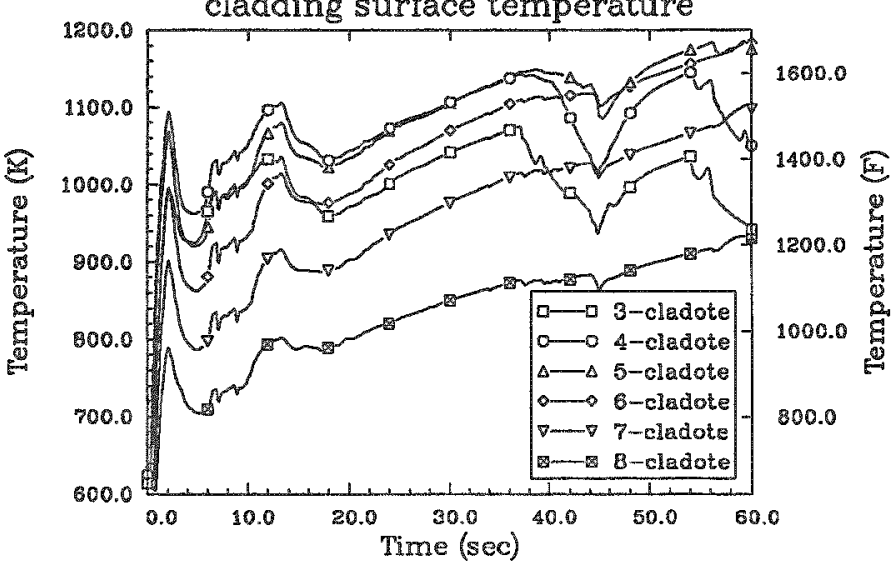

SEABROOK 75\%DBA 50 GWD/MTU PIN--PF 2.32 oxide thickness

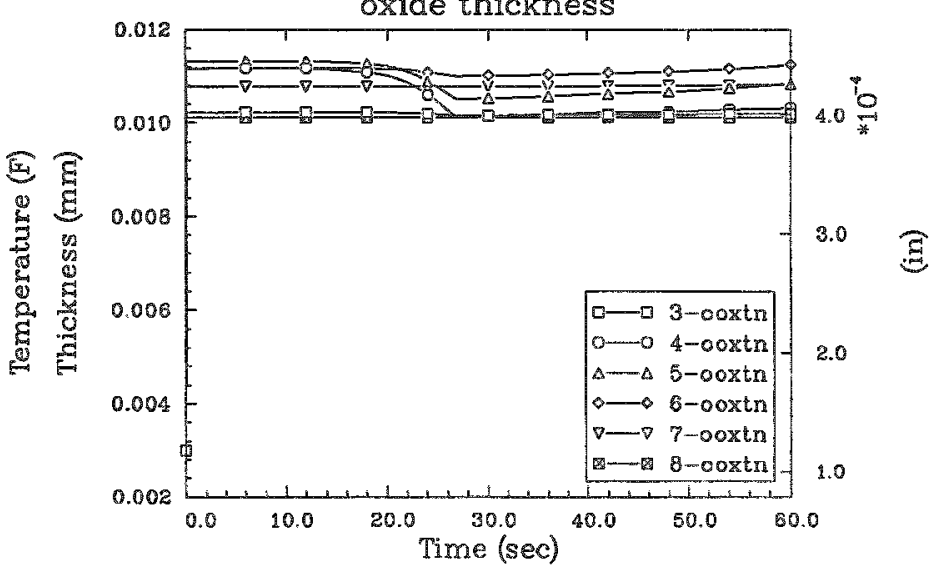



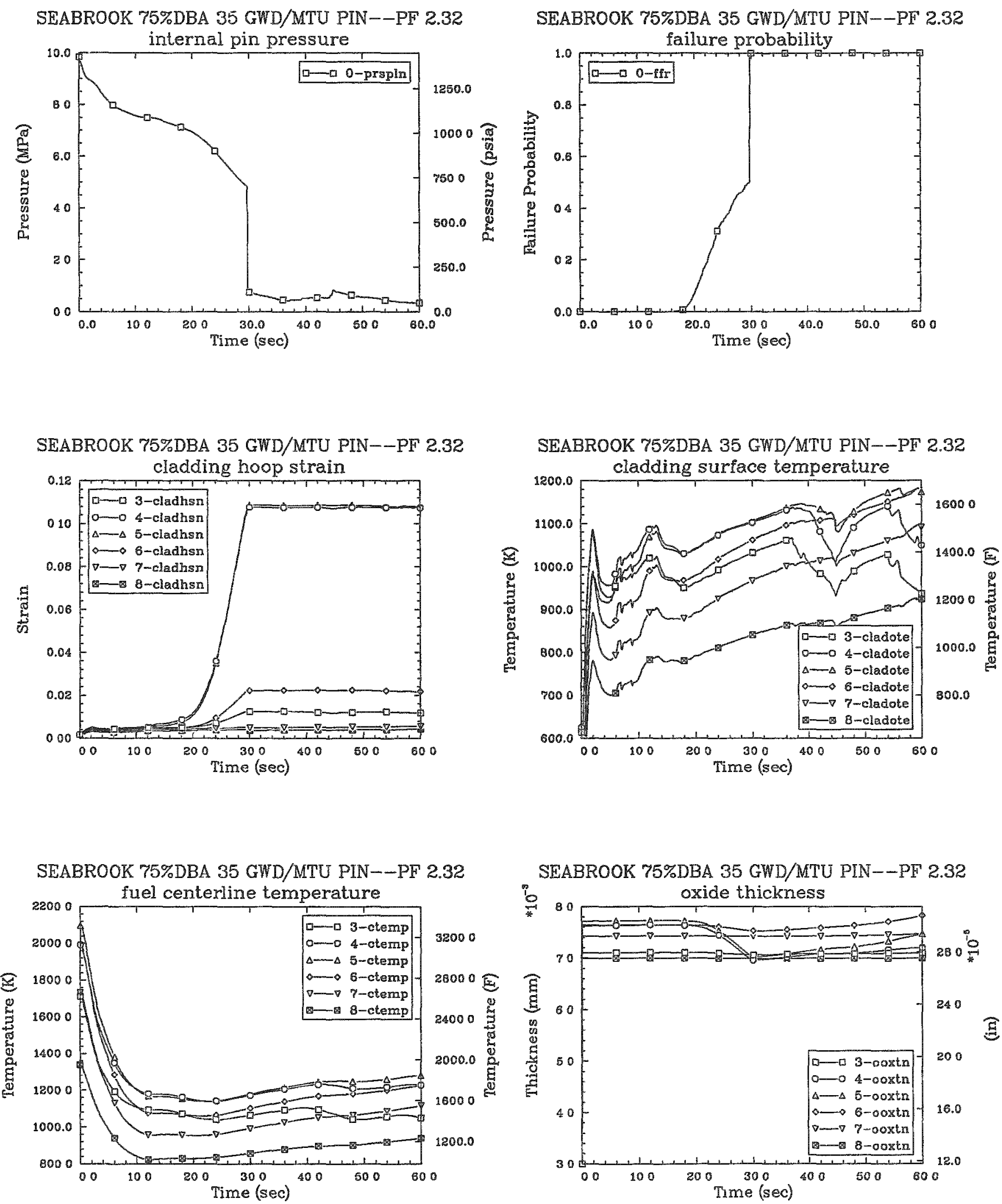

SEABROOK 75\%DBA 35 GWD/MTU PIN--PF 2.32

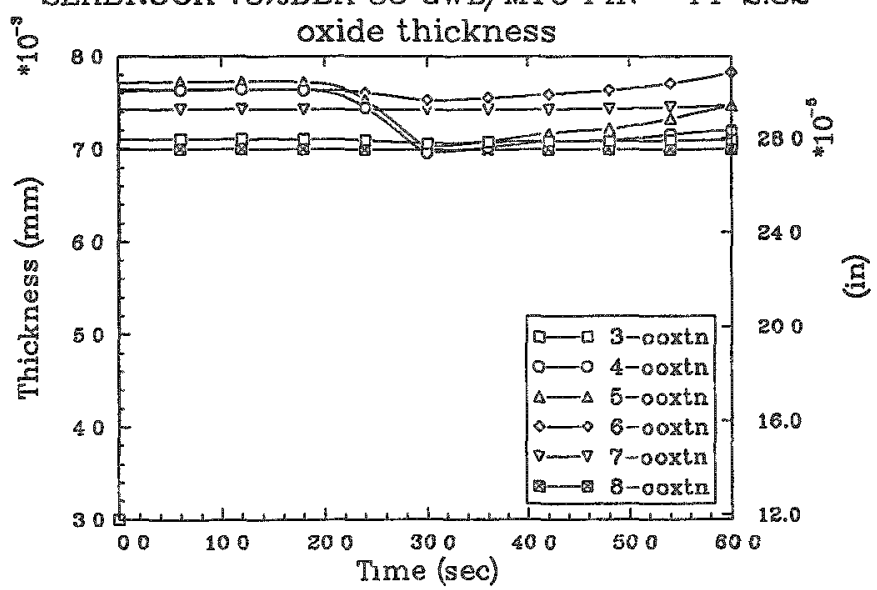




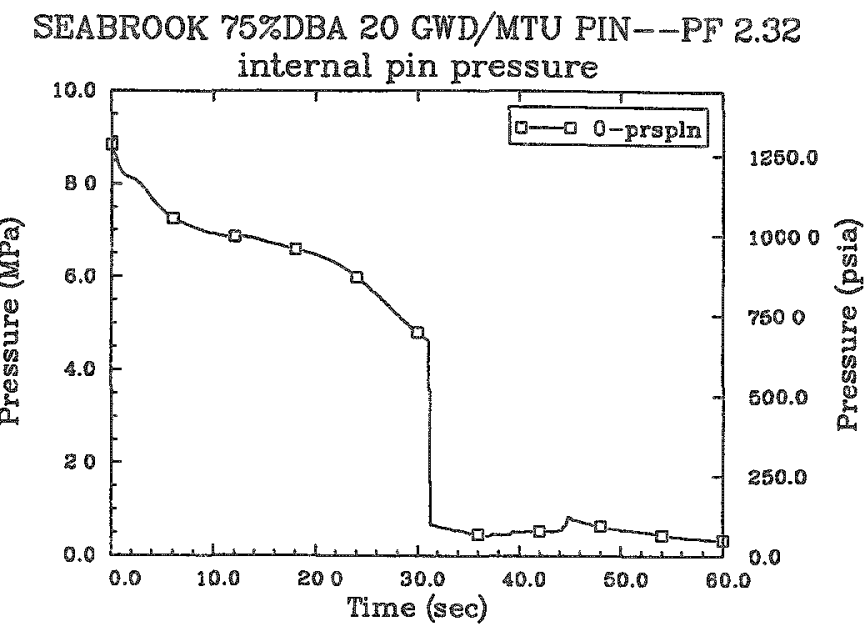

SEABROOK 75\%DBA 20 GWD/MTU PIN--PF 2.32
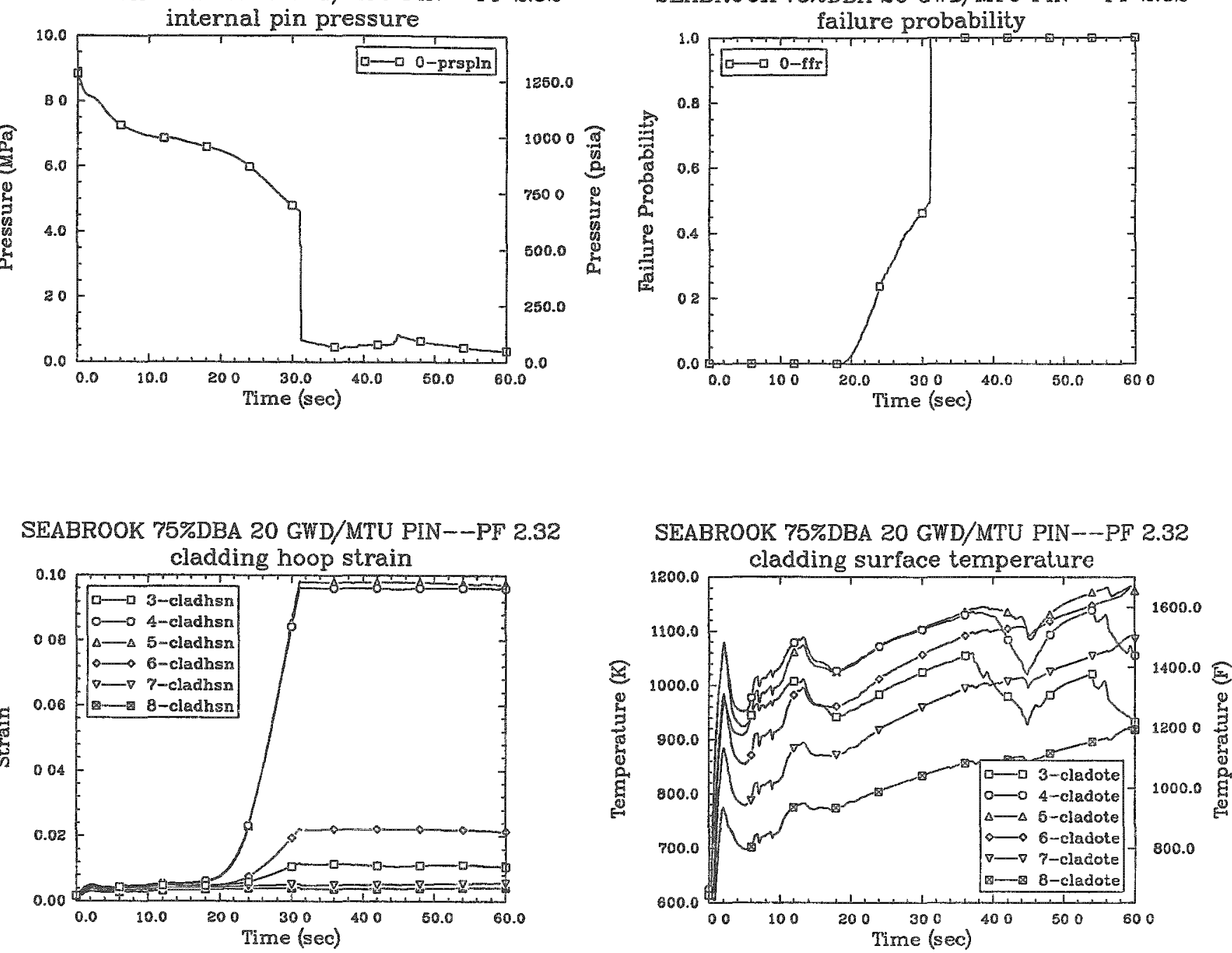

SEABROOK 75\%DBA 20 GWD/MTU PIN--PF 2.32

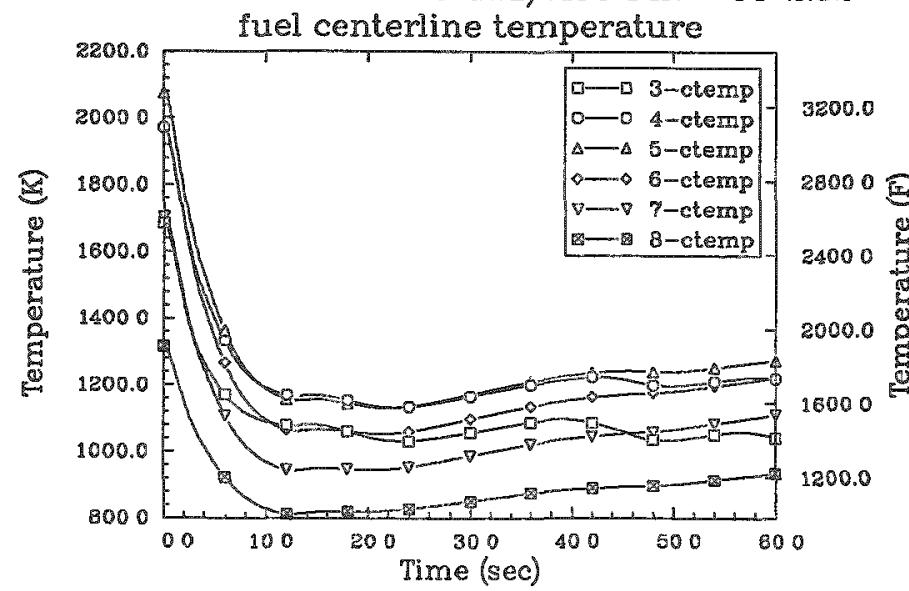

SEABROOK 75\%DBA 20 GWD/MTU PIN--PF 2.32

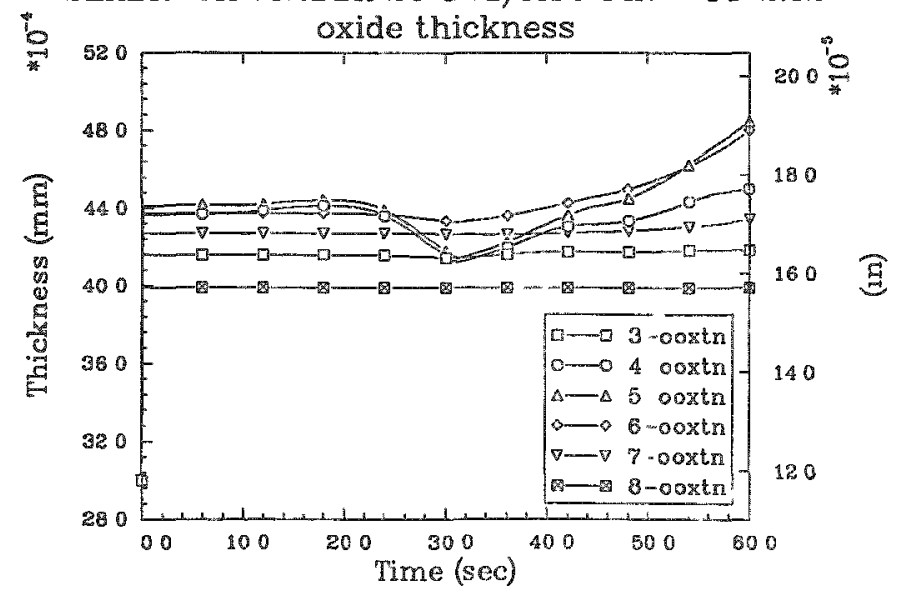




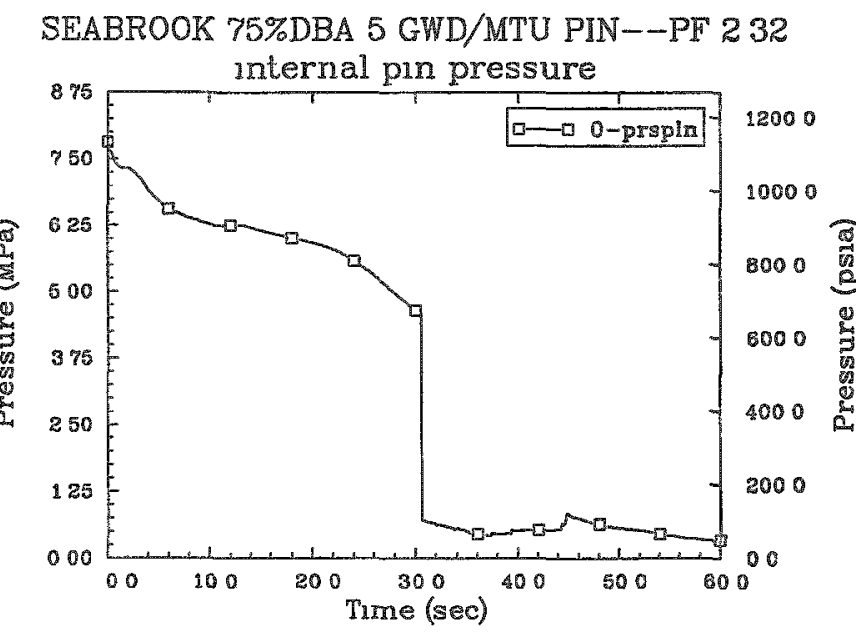

SEABROOK 75\%DBA 5 GWD/MTU PIN--PF 232

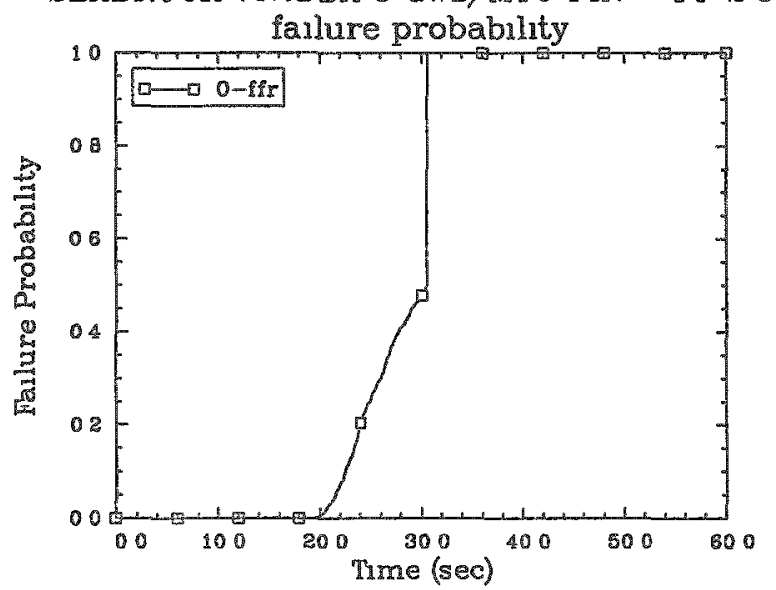

SEABROOK 75\%DBA 5 GWD/MTU PIN--PF 232

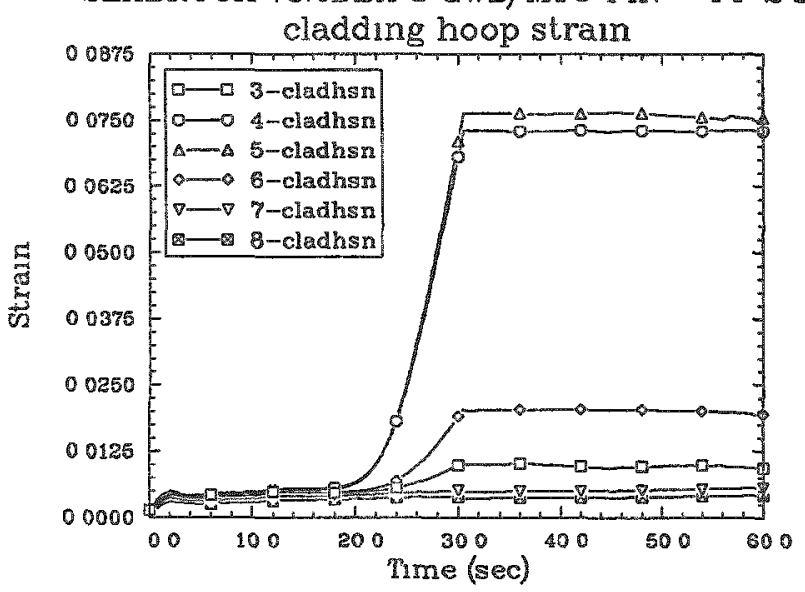

SEABROOK 75\%DBA 5 GWD/MTU PIN--PF' 232

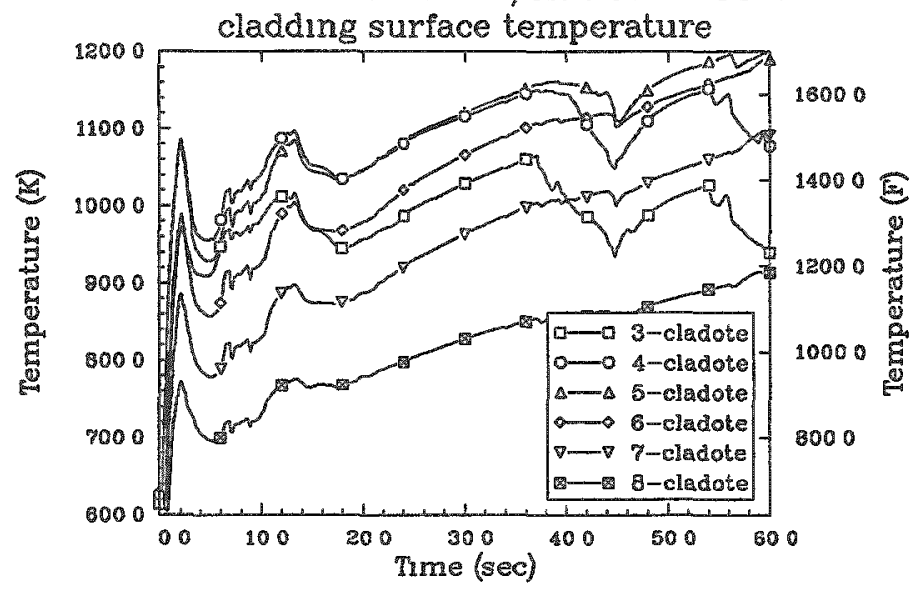

SEABROOK 75\%DBA 5 GWD/MTU PIN--PF 232

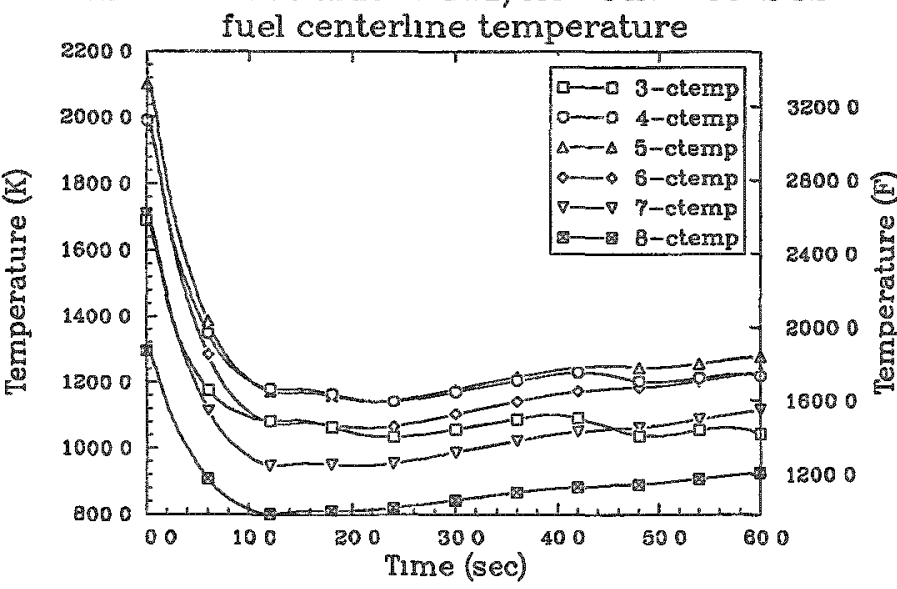

SEABROOK 75\%DBA 5 GWD/MTU PIN--PF 232

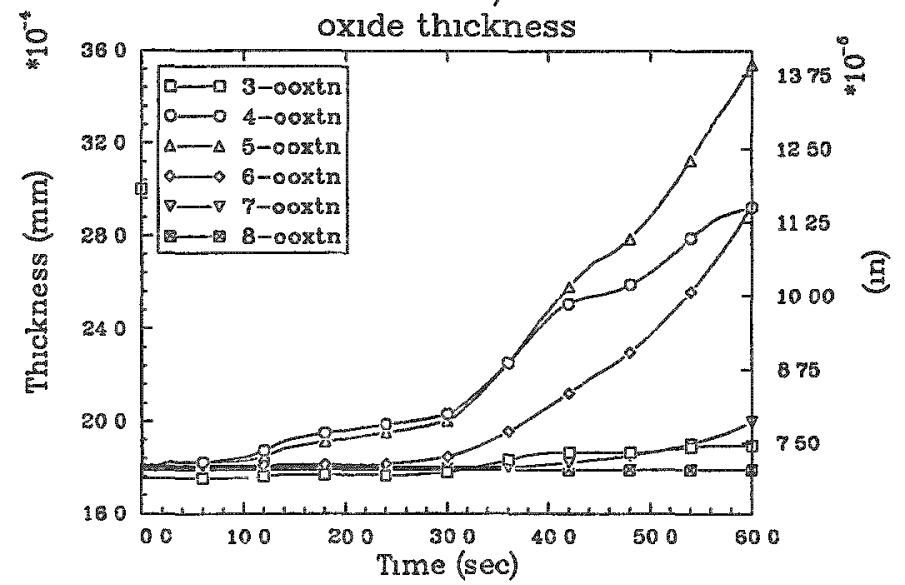



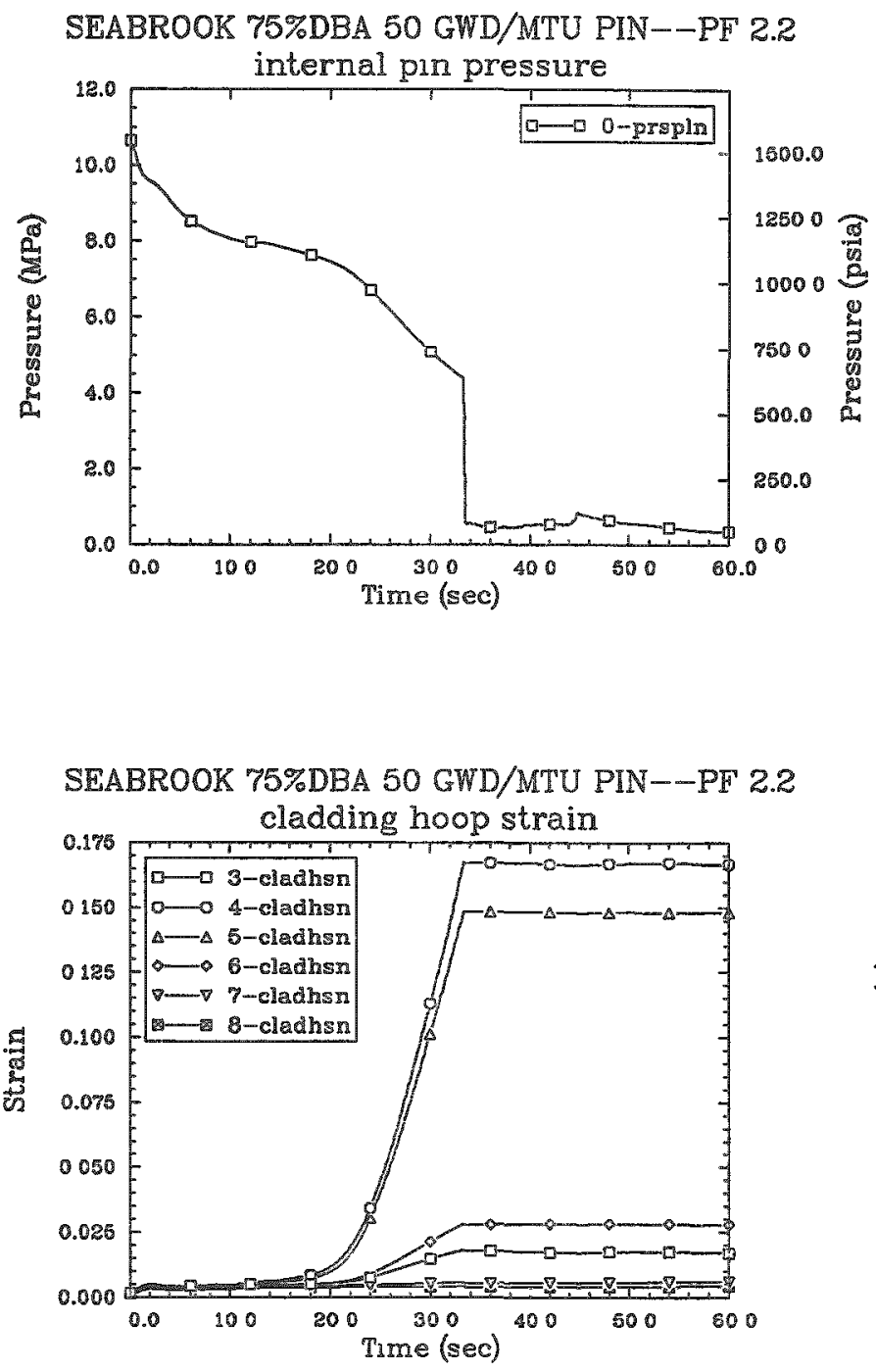

SEABROOK 75\%DBA 50 GWD/MTU PIN--PF 2.2

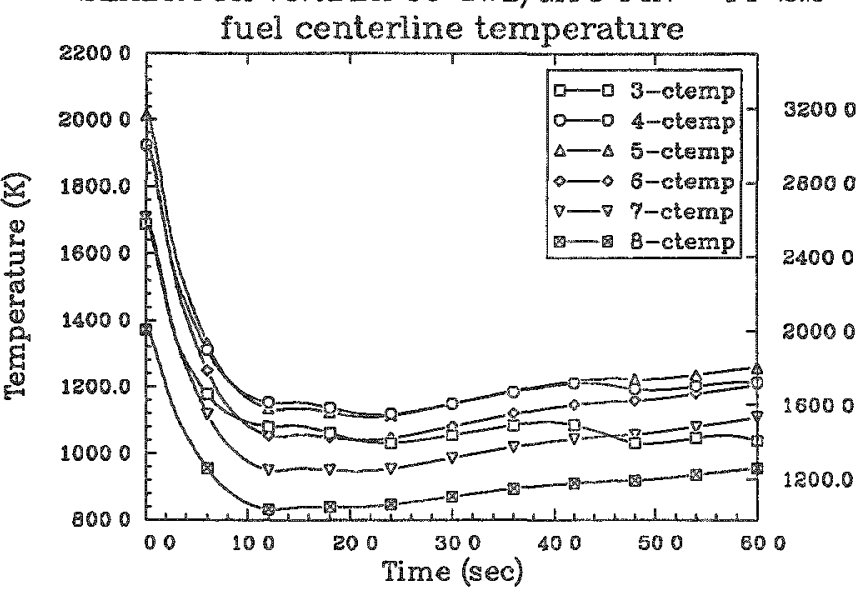

SEABROOK 75\%DBA 50 GWD/MTU PIN--PF 2.2

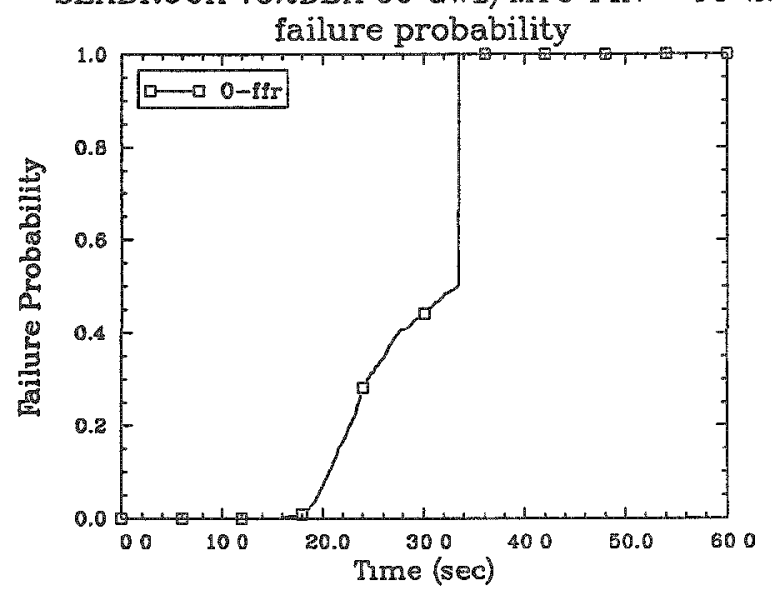

SEABROOK 75\%DBA 50 GWD/MTU PIN-PF 2.2

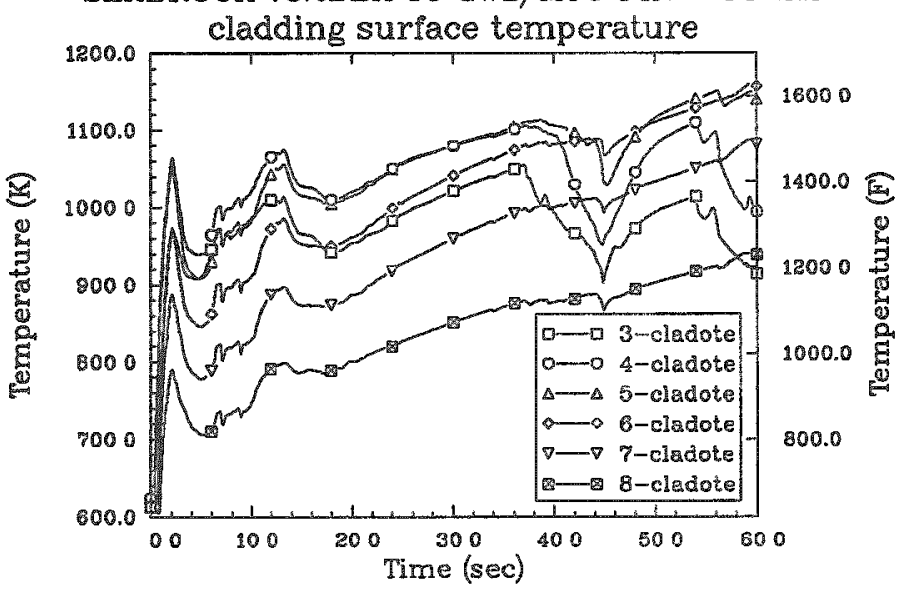

SEABROOK $75 \%$ DBA 50 GWD/MTU PIN--PF 22 oxide thickness

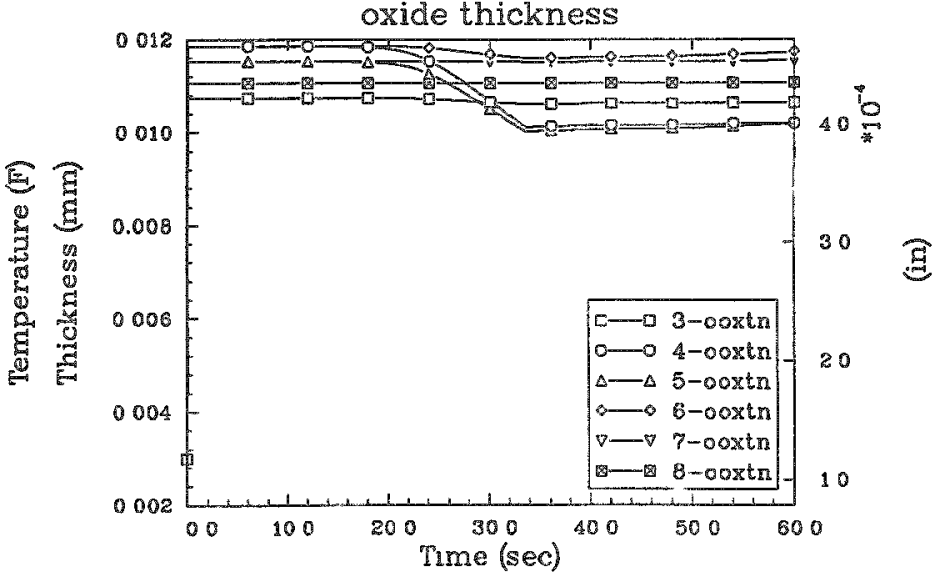



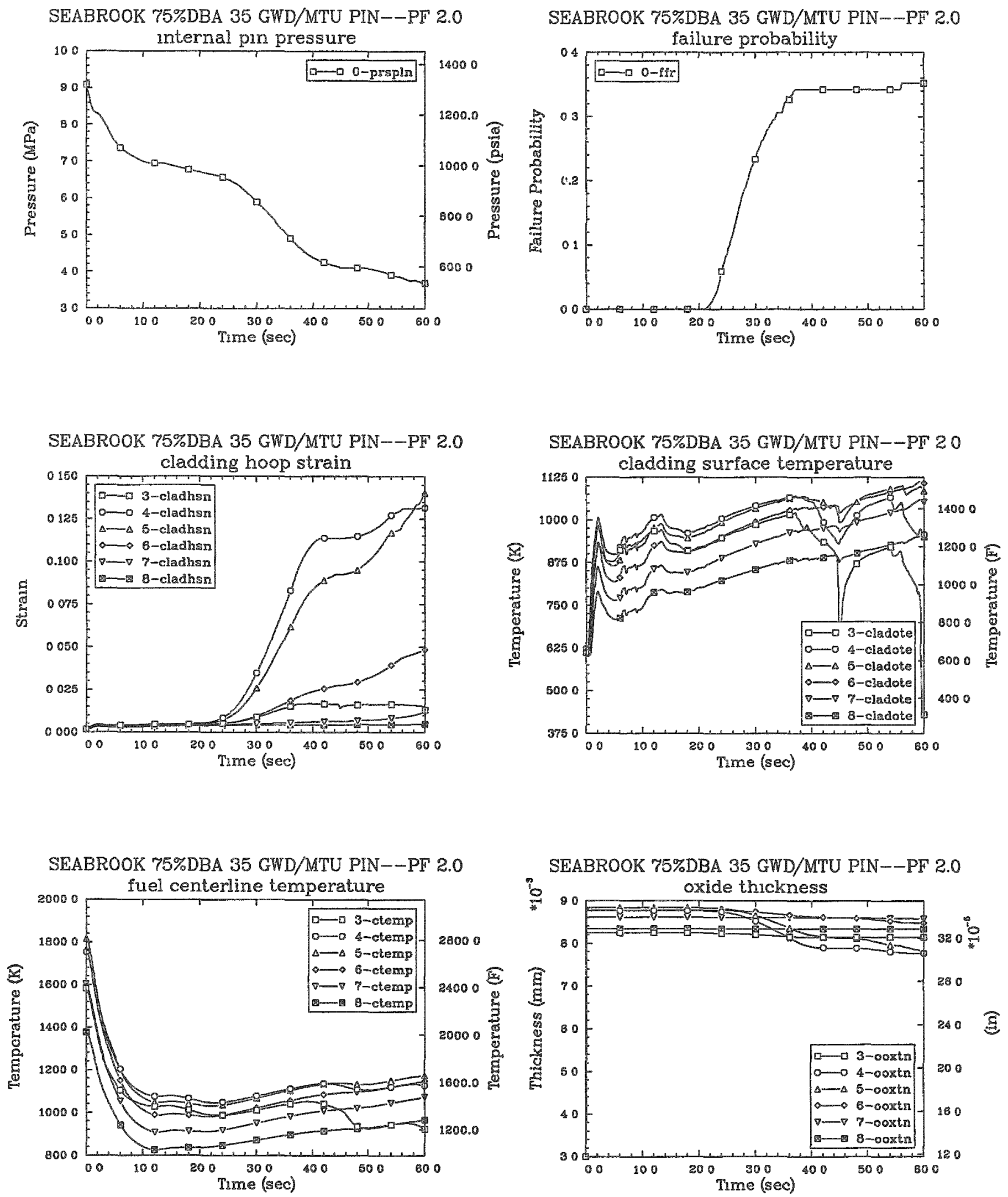


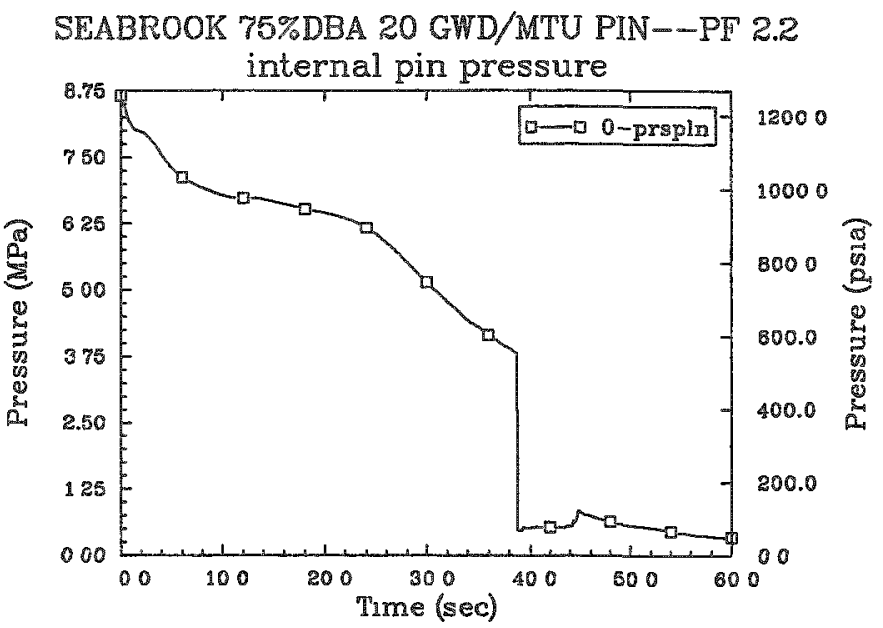

SEABROOK 75\%DBA 20 GWD/MTU PIN--PF 2.2
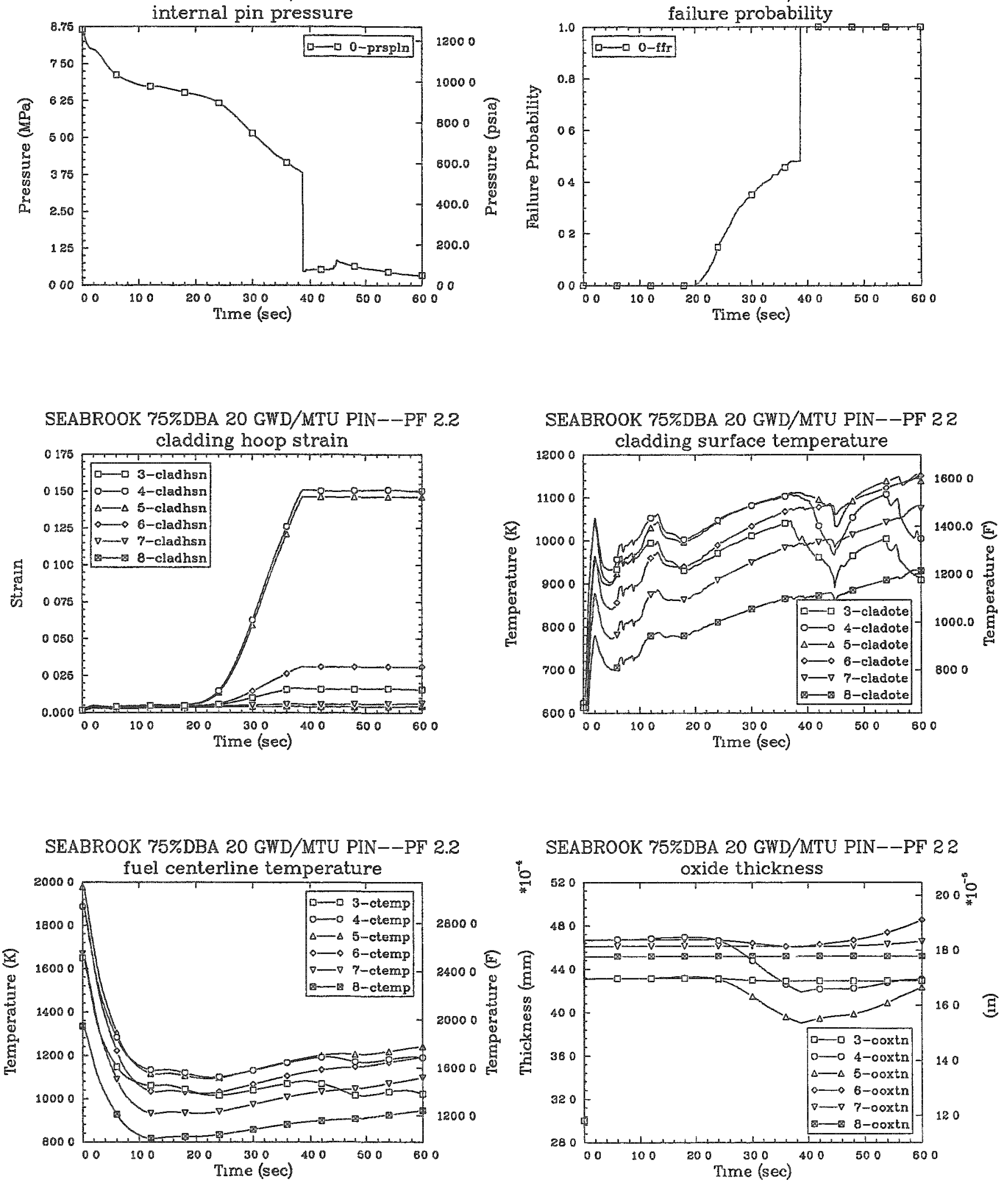


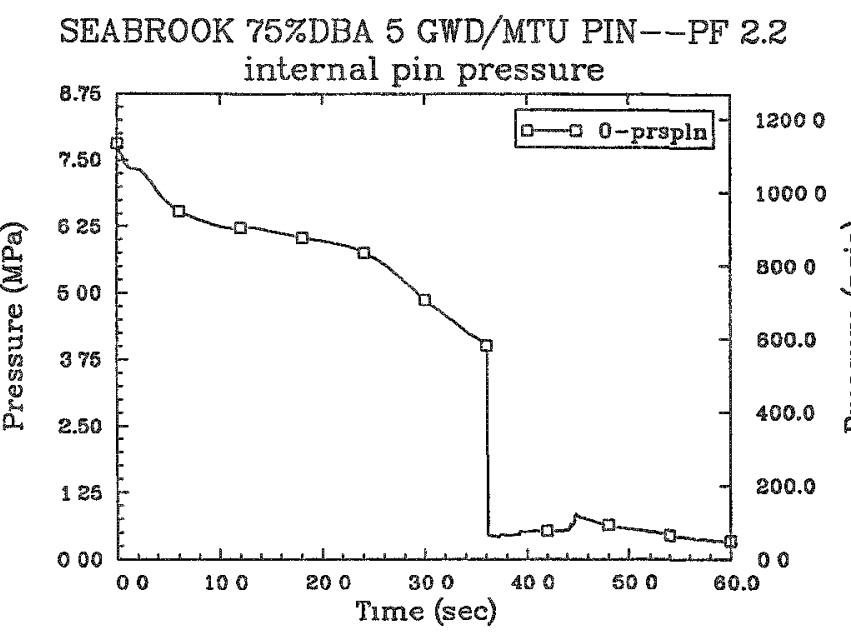

SEABROOK 75\%DBA 5 GWD/MTU PIN--PF 2.2
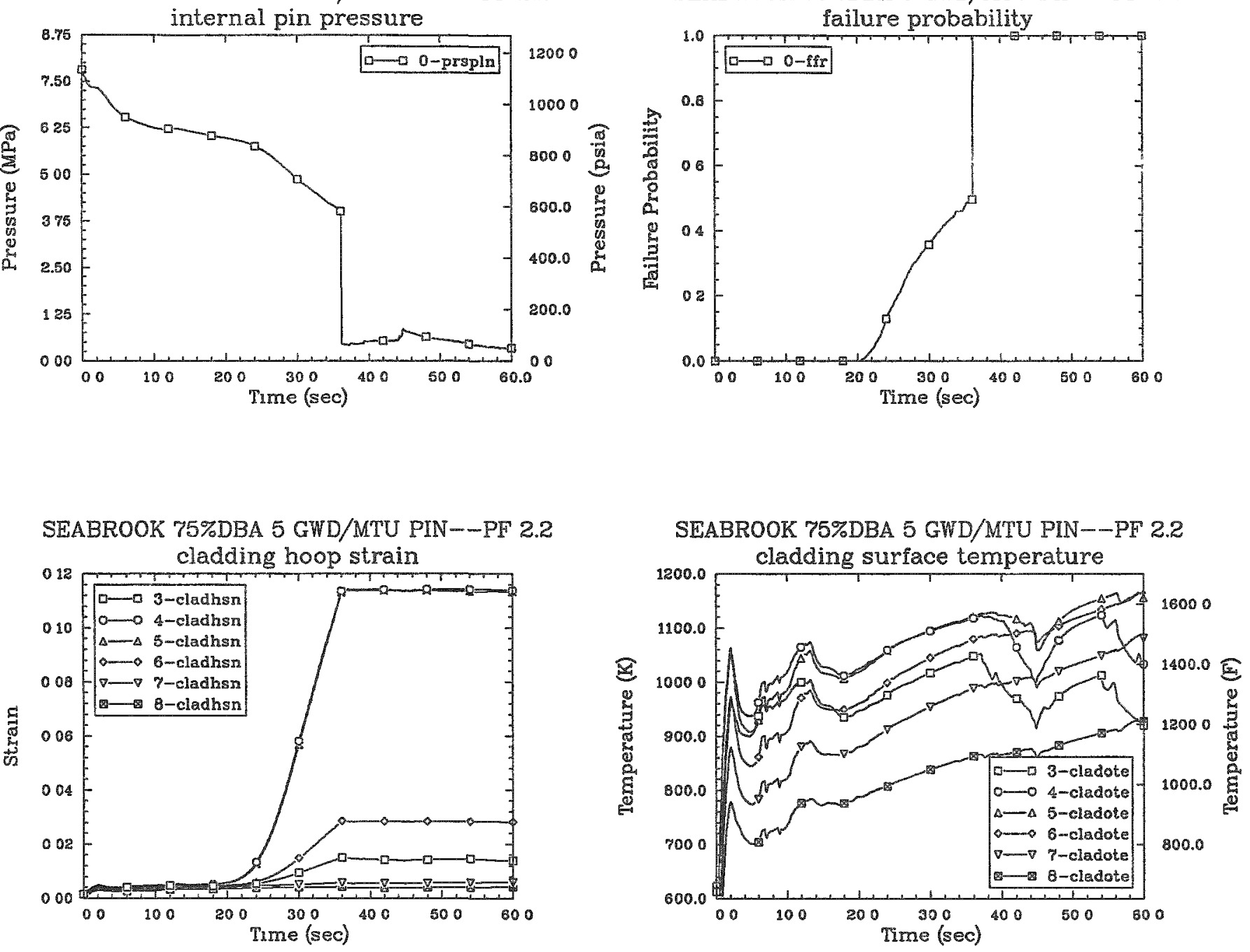

SEABROÒK 75\%DBA 5 GWD/MTU PIN--PF 2.2
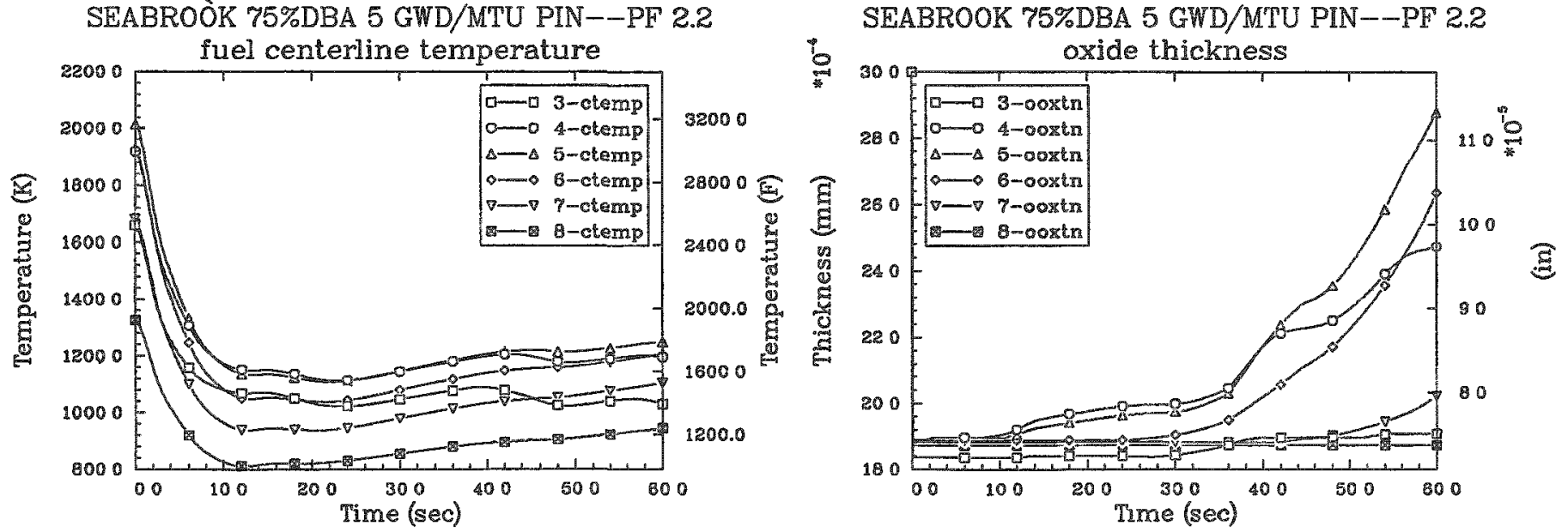


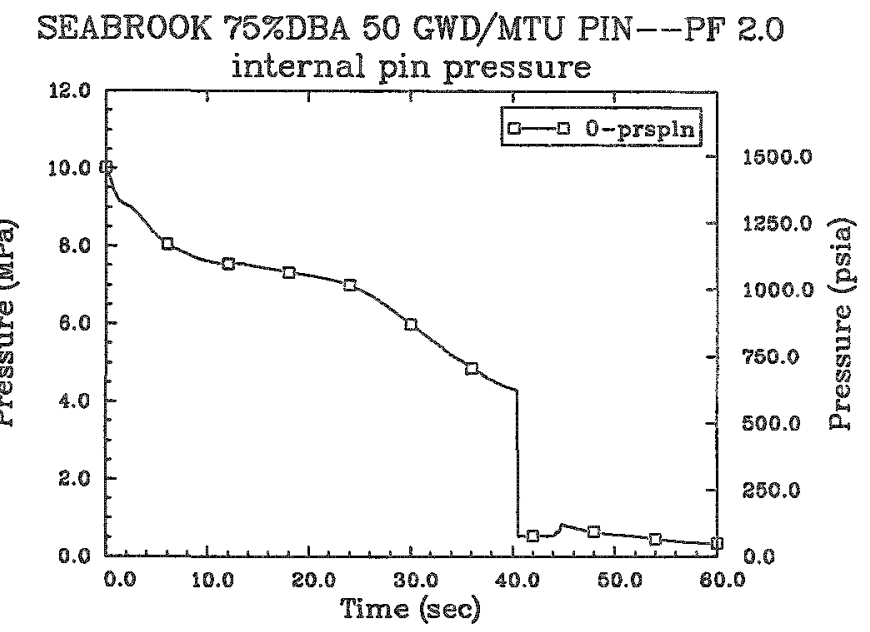

SEABROOK 75\%DBA $50 \mathrm{GWD} / \mathrm{MTU}$ PIN--PF 2.0
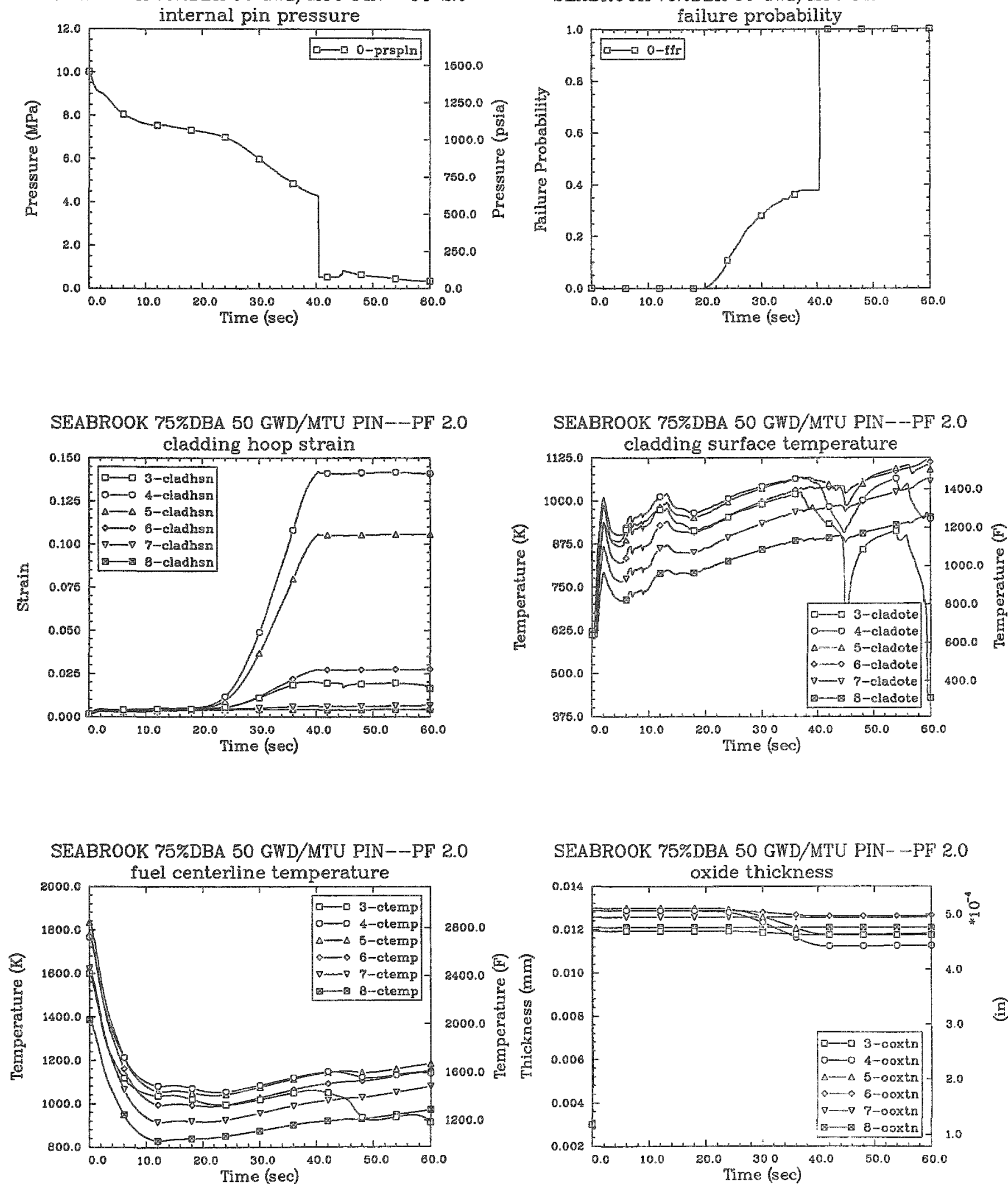

SEABROOK 75\%DBA 50 GWD/MTU PIN--PF 2.0

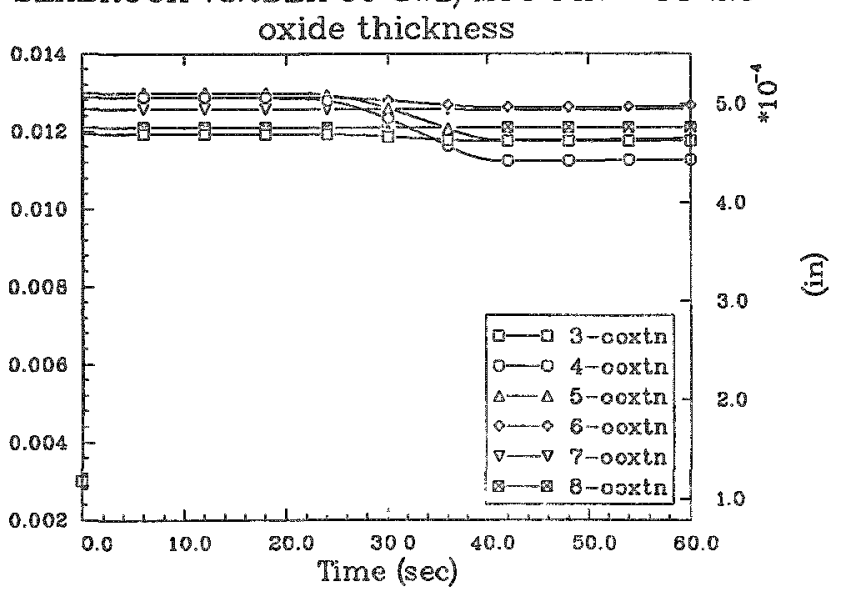


SEABROOK 75\%DBA 35 GWD/MTU PIN--PF 2.0

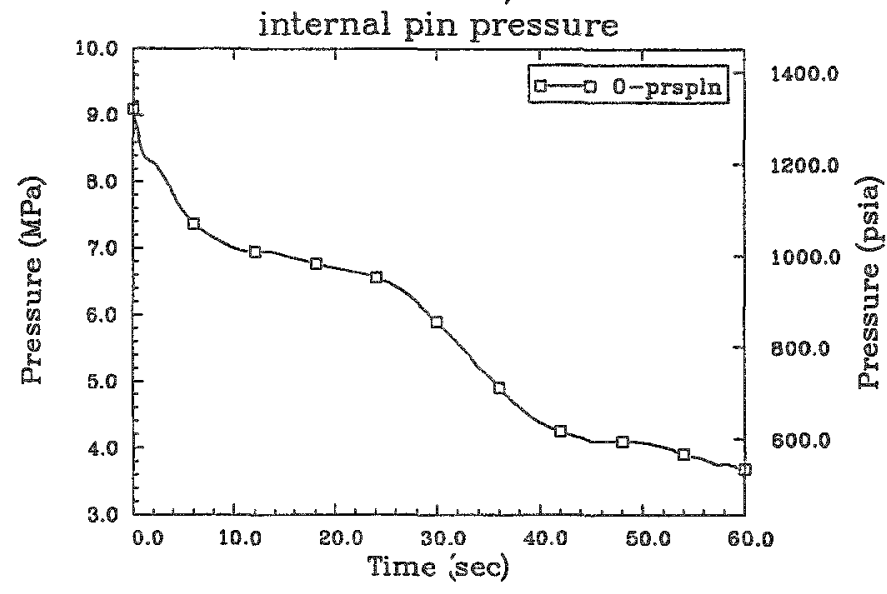

SEABROOK 75\%DBA 35 GWD/MTU PIN--PF 2.0 cladding hoop strain
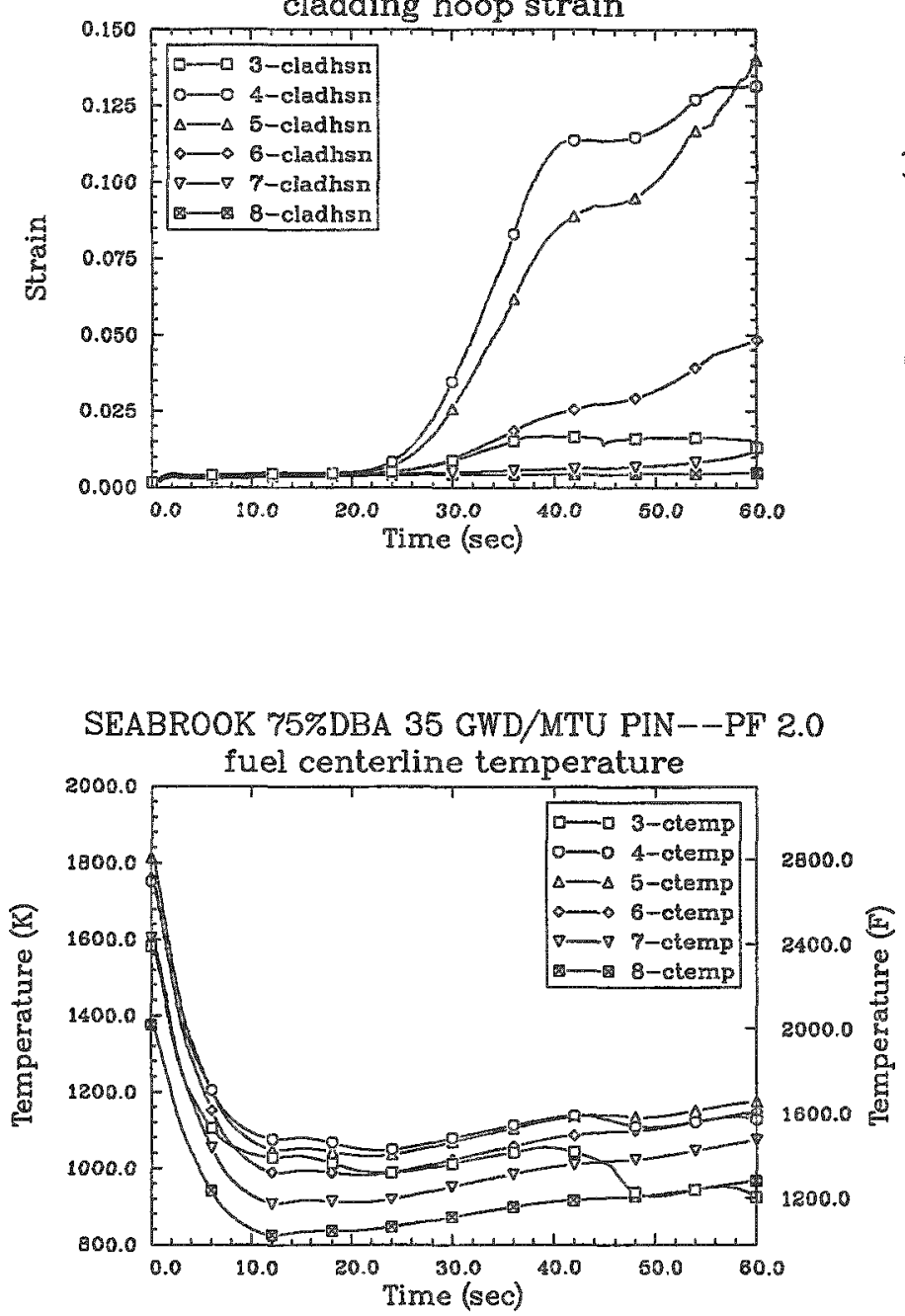

SEABROOK 75\%DBA 35 GWD/MTU PIN--PF 2.0

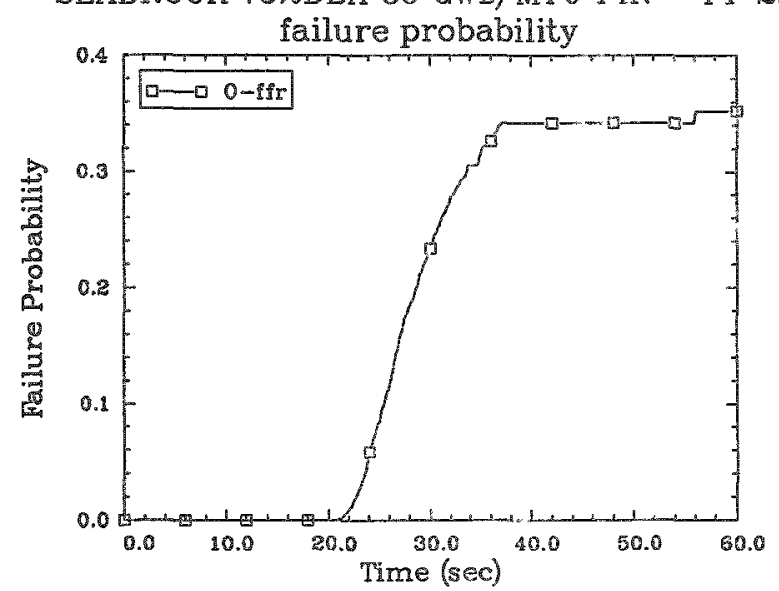

SEABROOK 75\%DBA 35 GWD/MTU PIN--PF 2.0

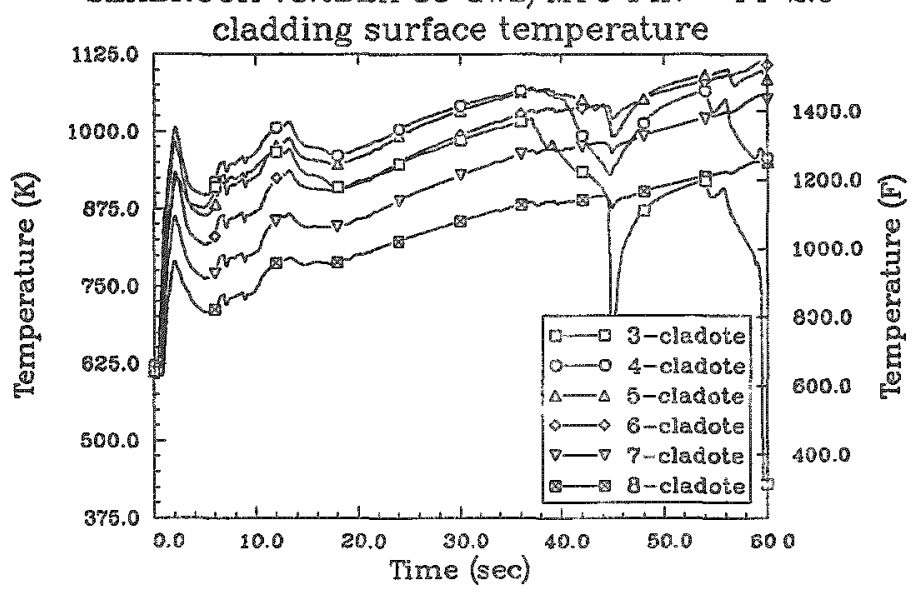

SEABROOK $75 \%$ DBA 35 GWD/MTU PIN--PF 2.0

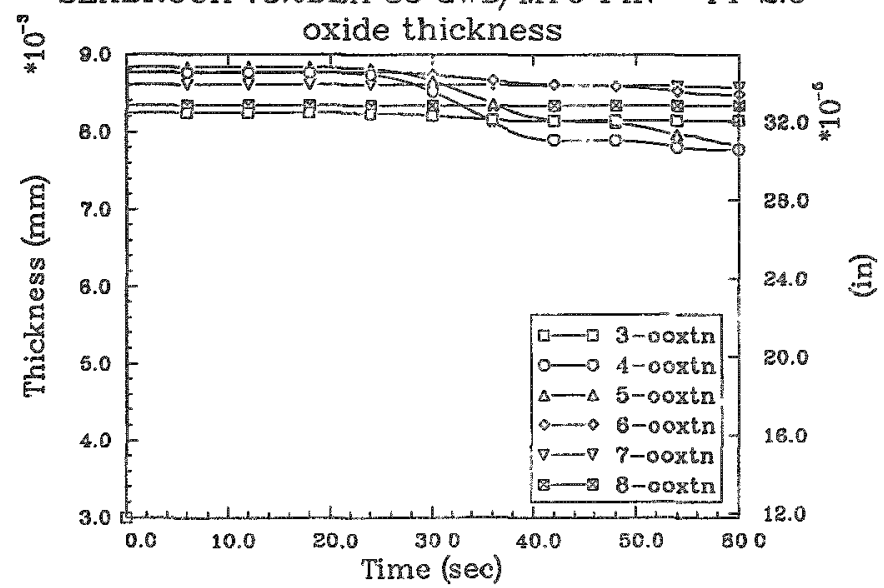



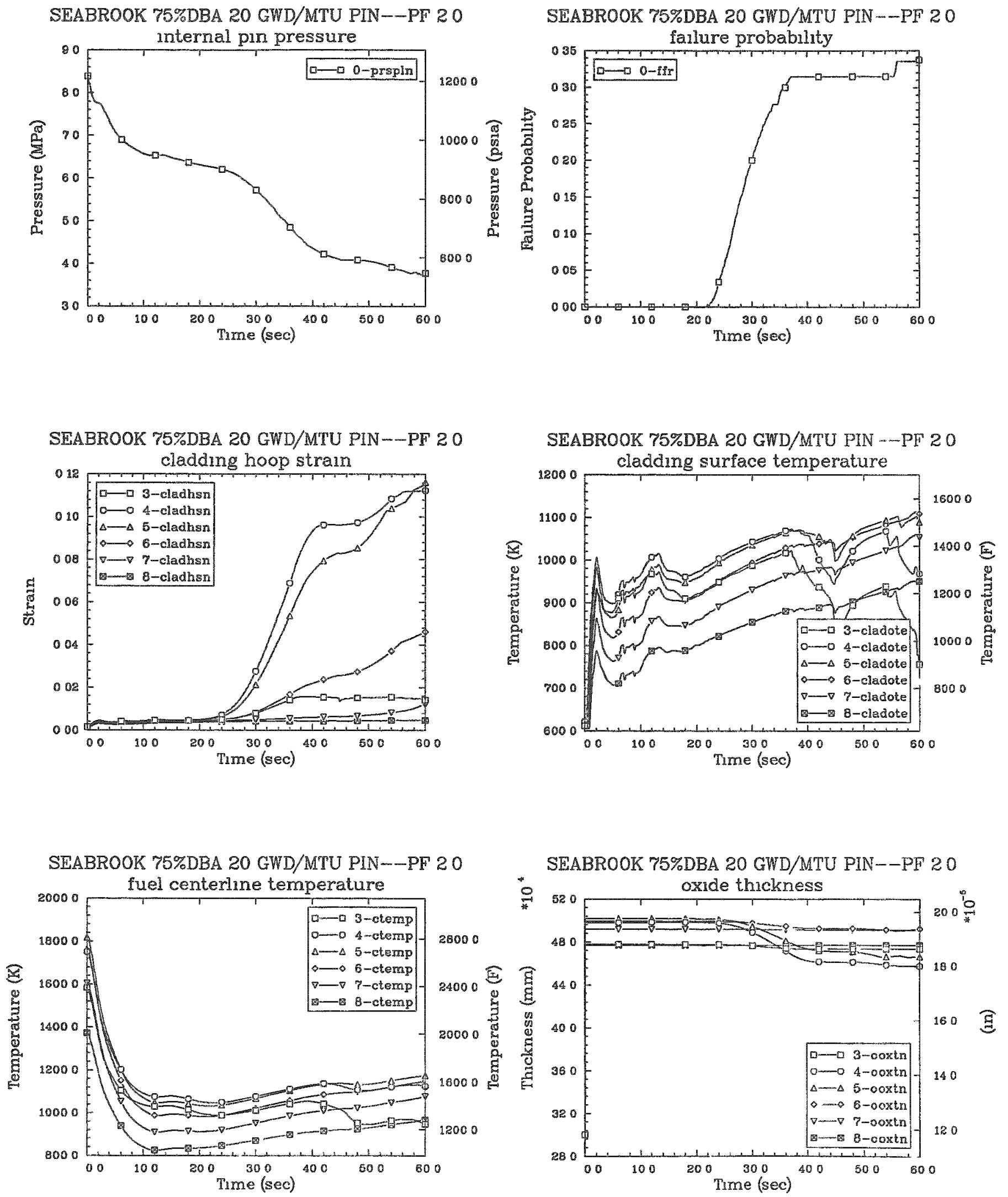


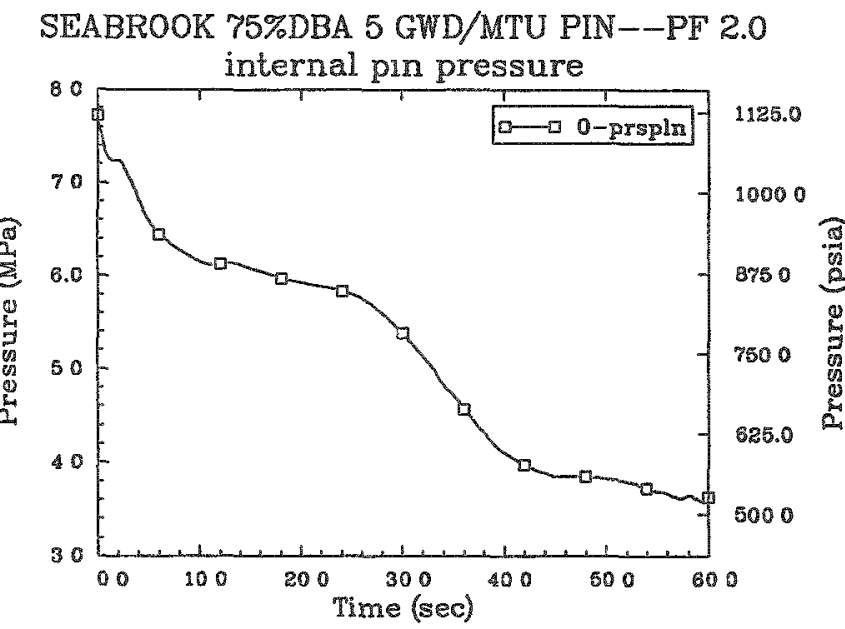

SEABROOK 75\%DBA 5 GWD/MTU PIN--PF 2.0
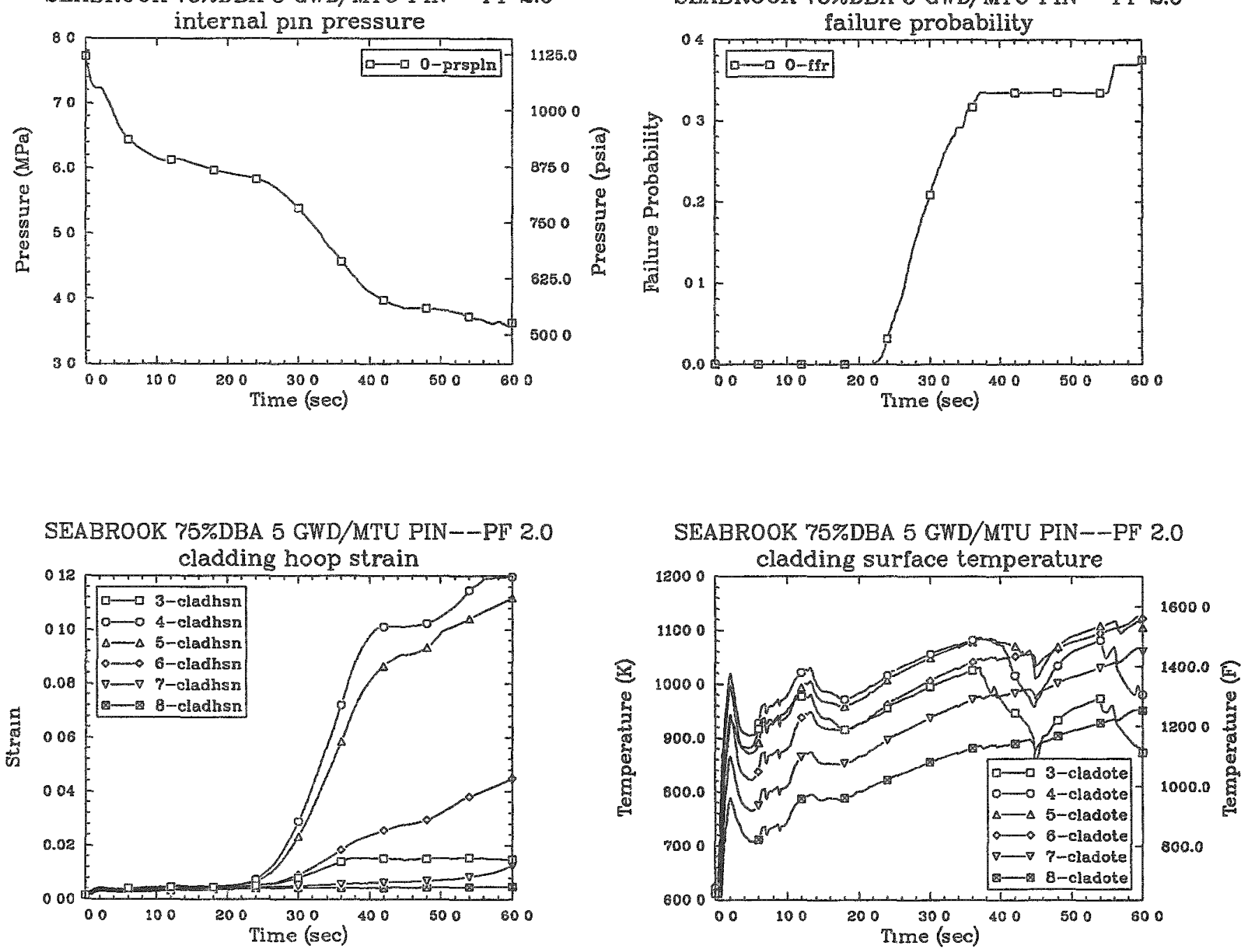

SEABROOK 75\%DBA 5 GWD/MTU PIN--PF 2.0
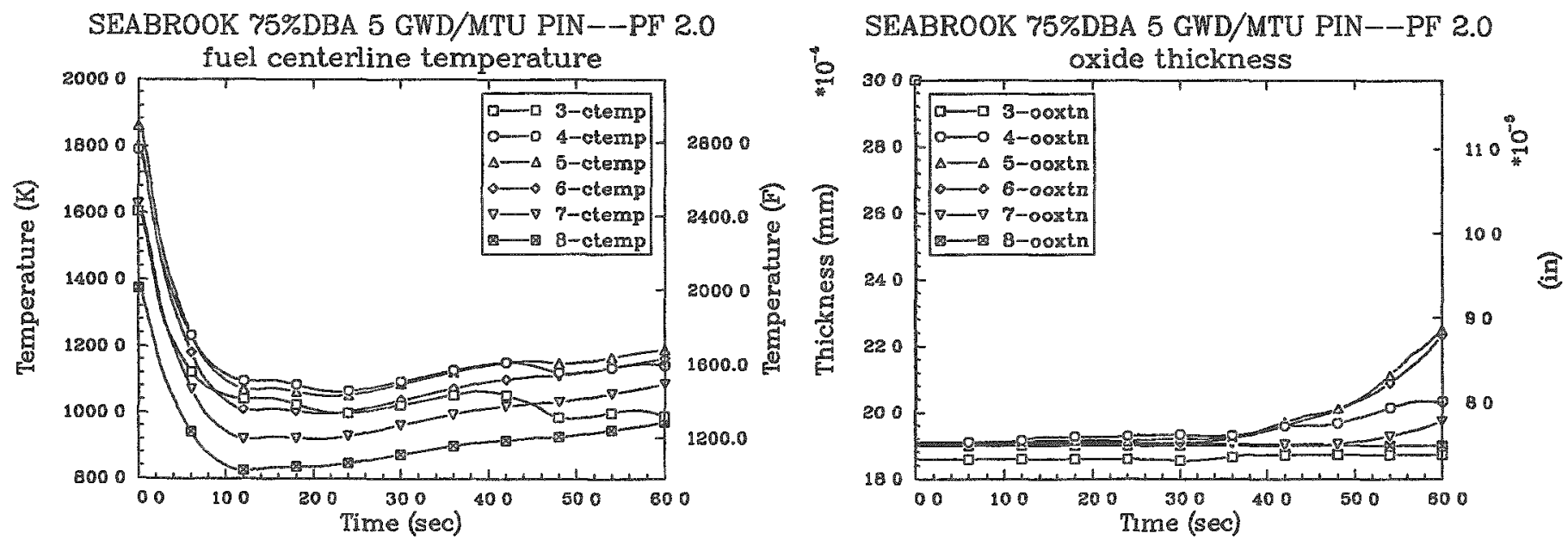

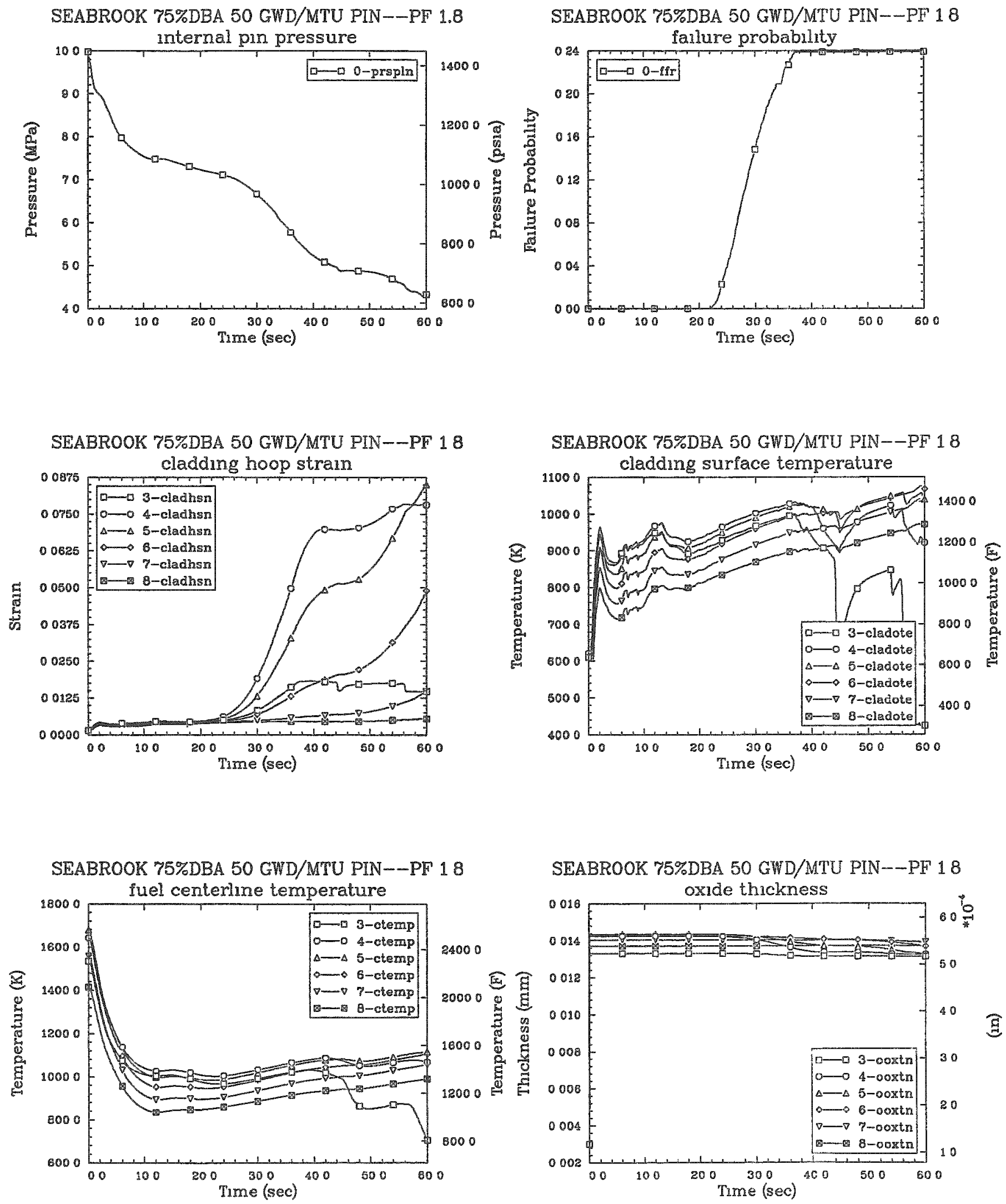

SEABROOK 75\%DBA 50 GWD/MTU PIN--PF 18

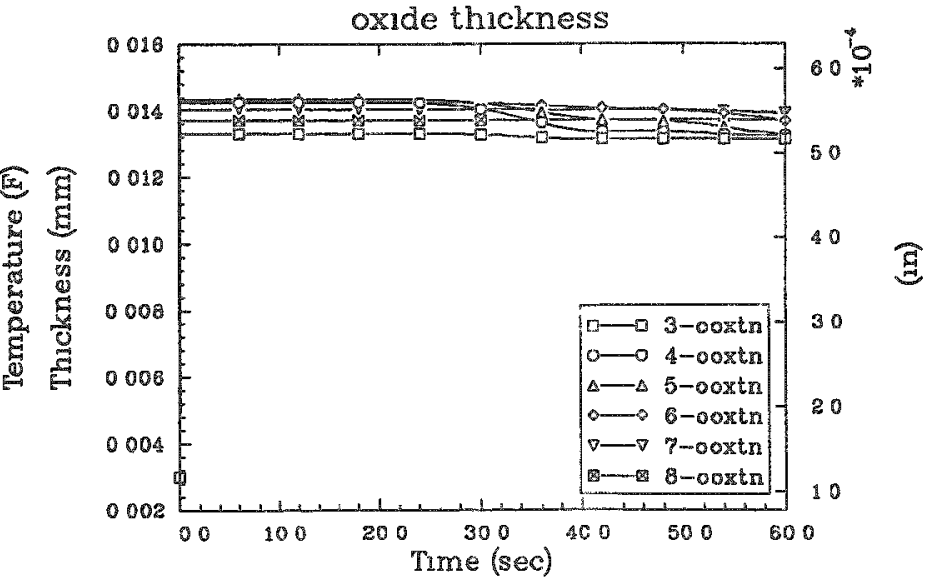



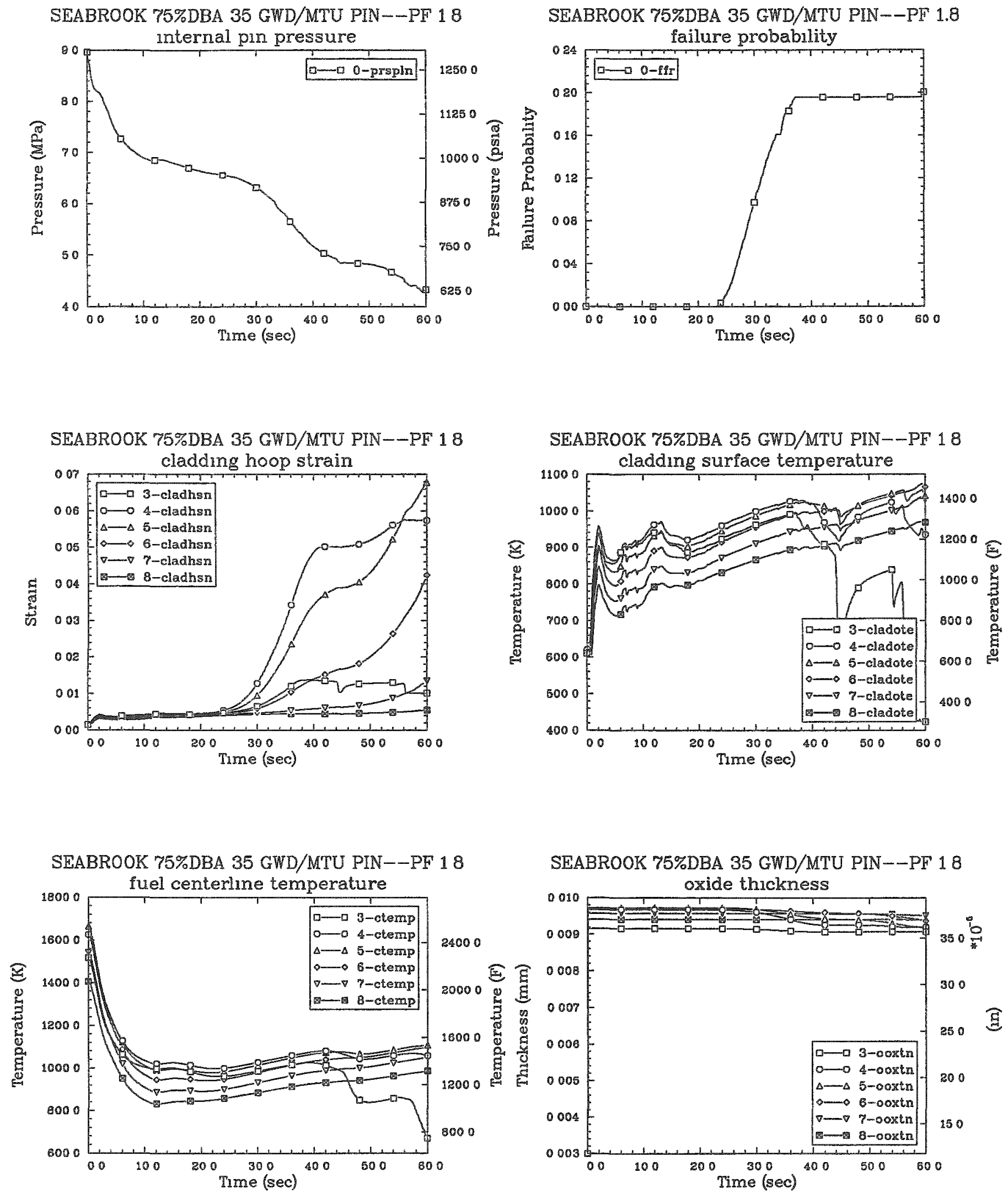

\section{SEABROOK 75\%DBA 35 GWD/MTU PIN--PF 18} oxide thickness

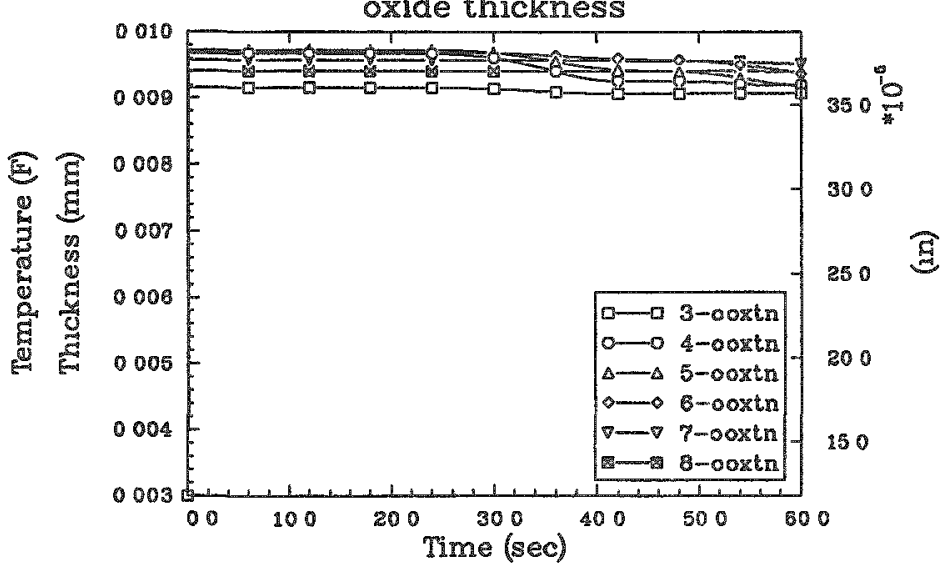



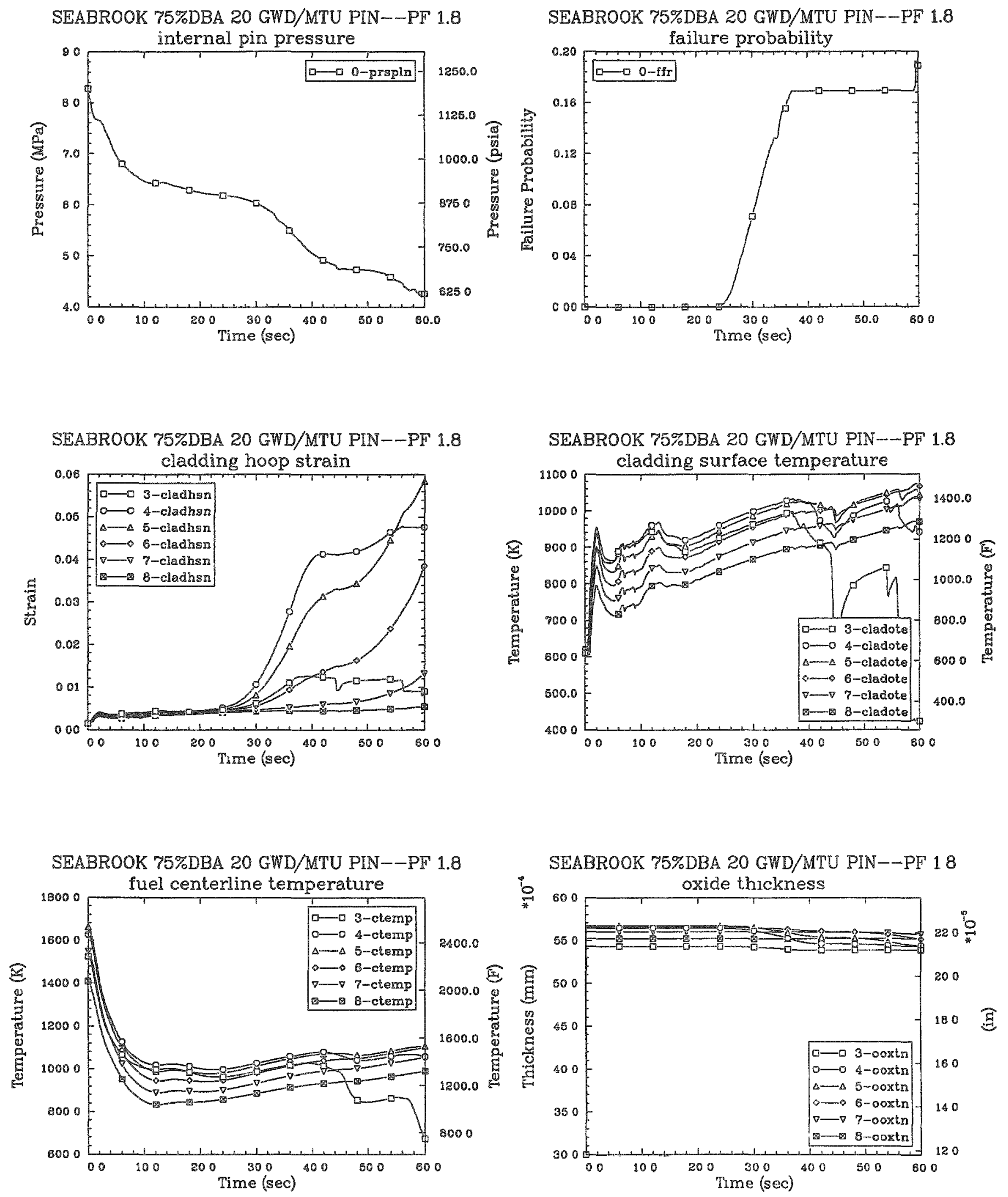

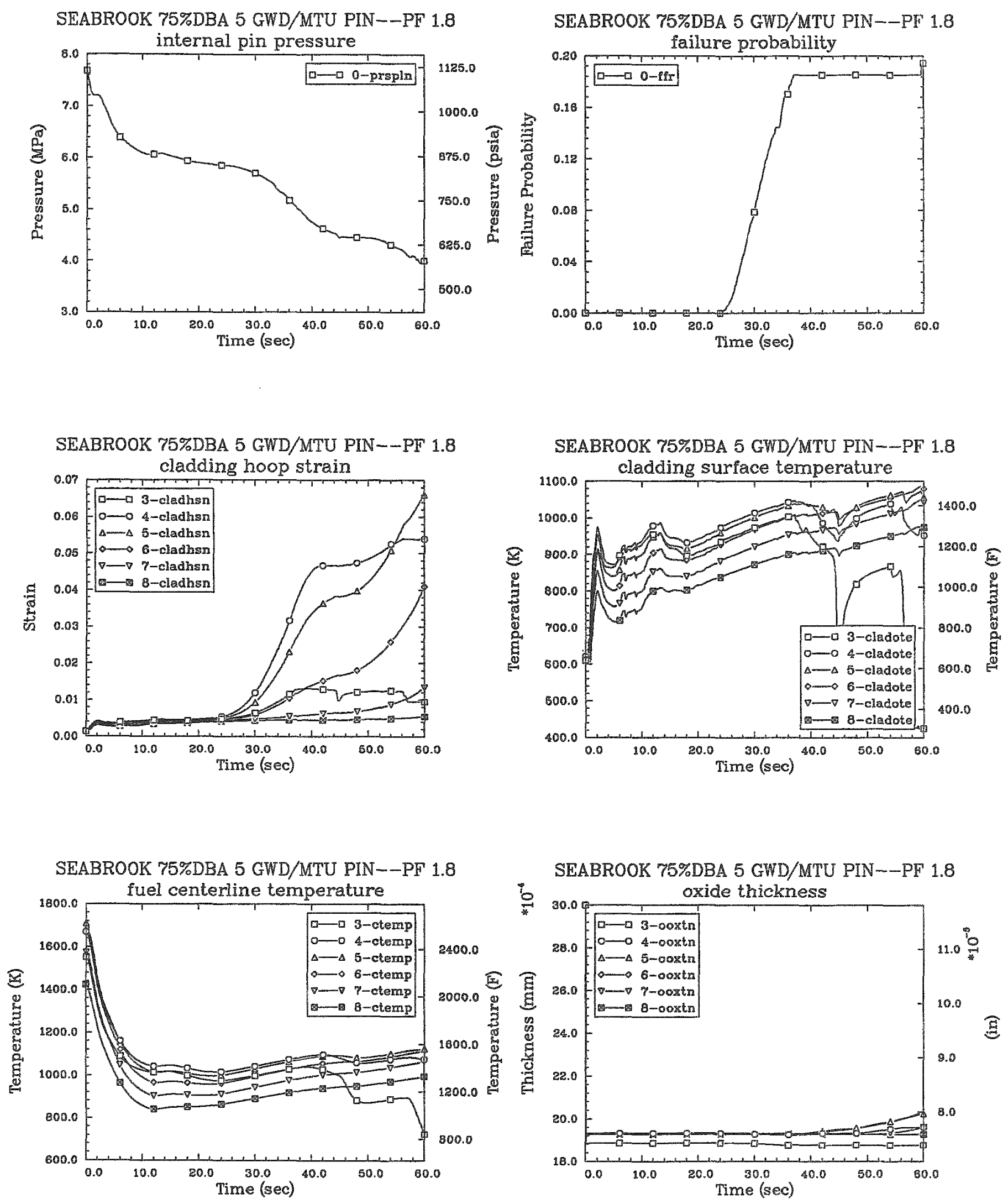

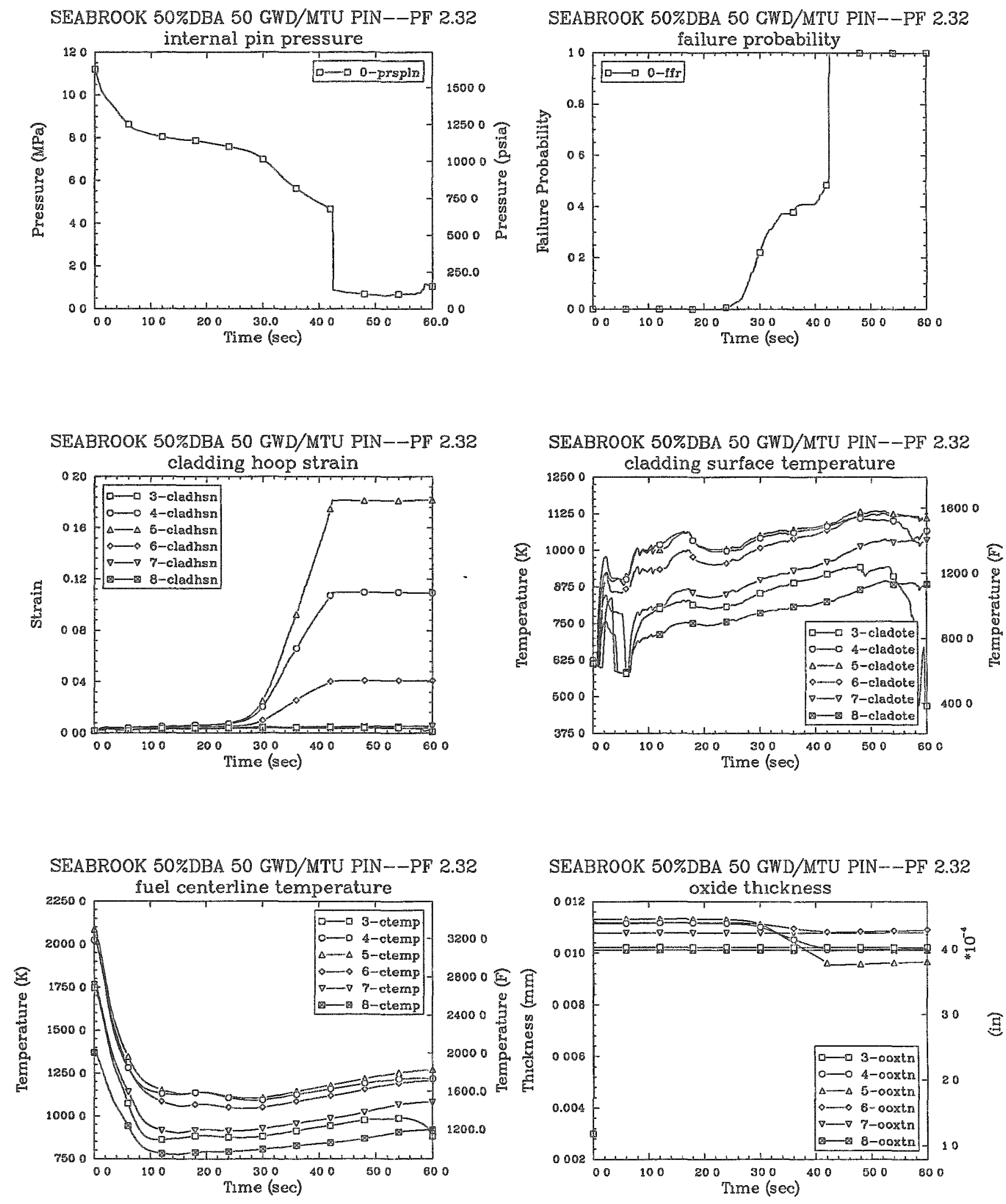

SEABROOK 50\%DBA $50 \mathrm{GWD} / \mathrm{MTU}$ PIN-PF 2.32

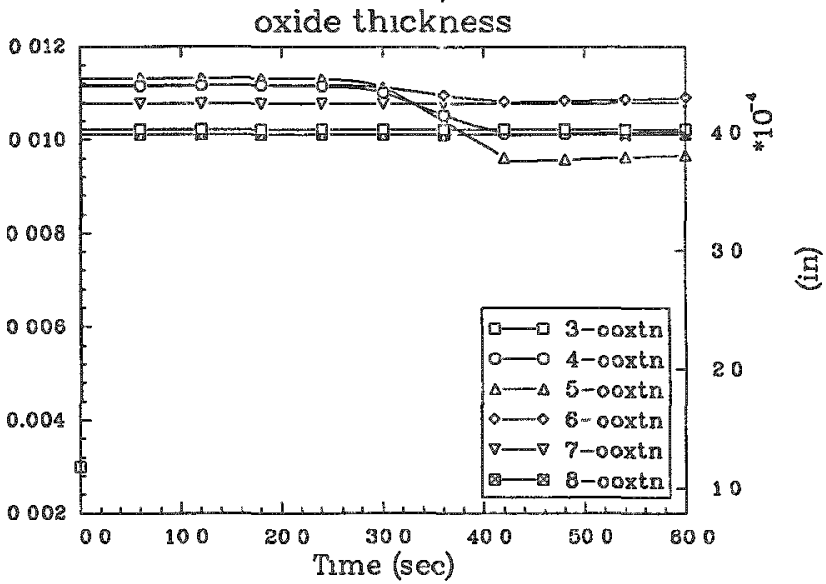


SEABROOK 50\%DBA 35 GWD/MTU PIN--PF 2.32

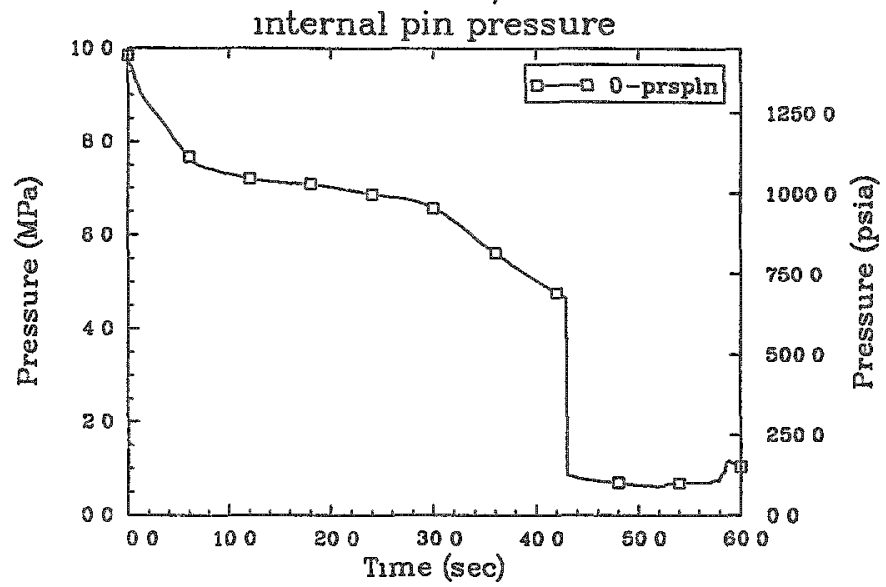

SEABROOK 50\%DBA 35 GWD/MTU PIN--PF 2.32

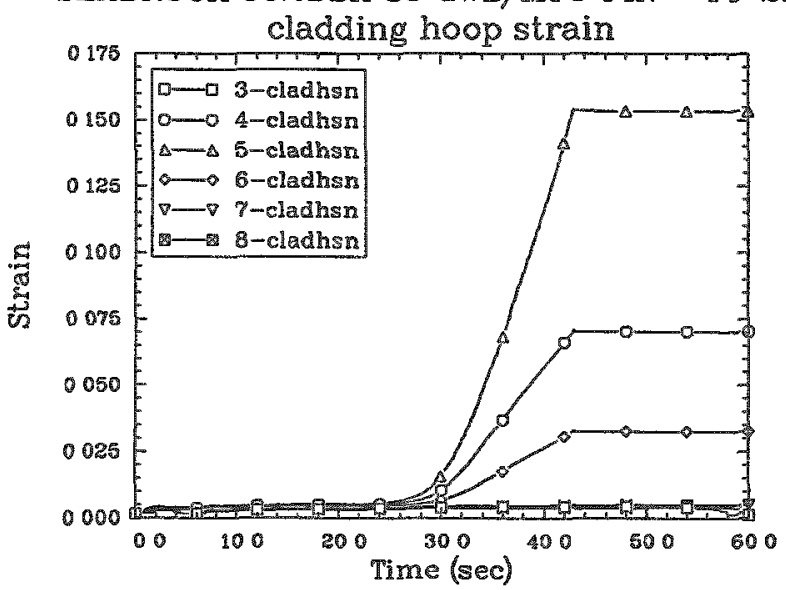

SEABROOK 50\%DBA 35 GWD/MTU PIN--PF 2.32

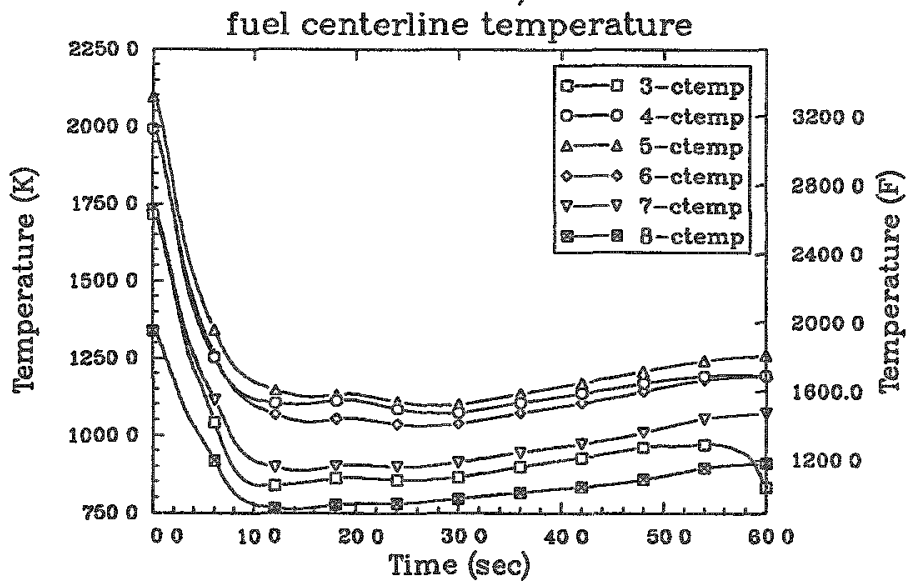

SEABROOK 50\%DBA 35 GWD/MTU PIN--PF 2.32

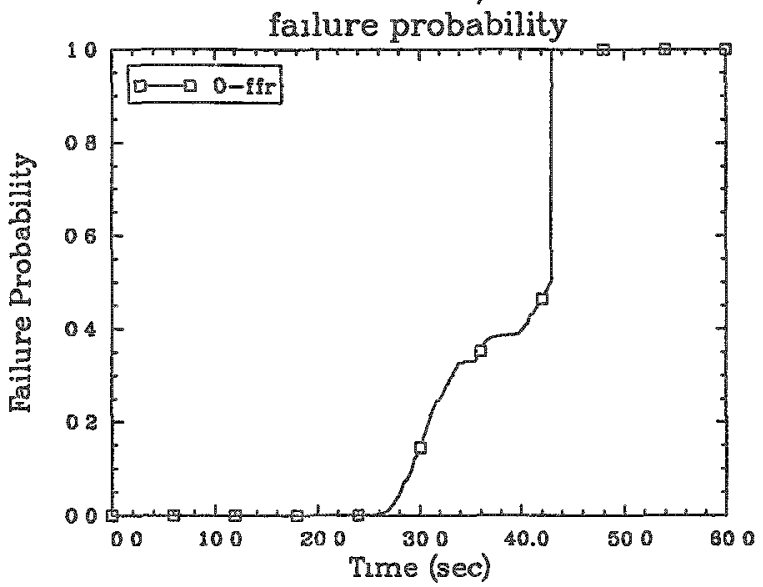

SEABRDOK 50\%DBA 35 GWD/MTU PIN--PF 2.32

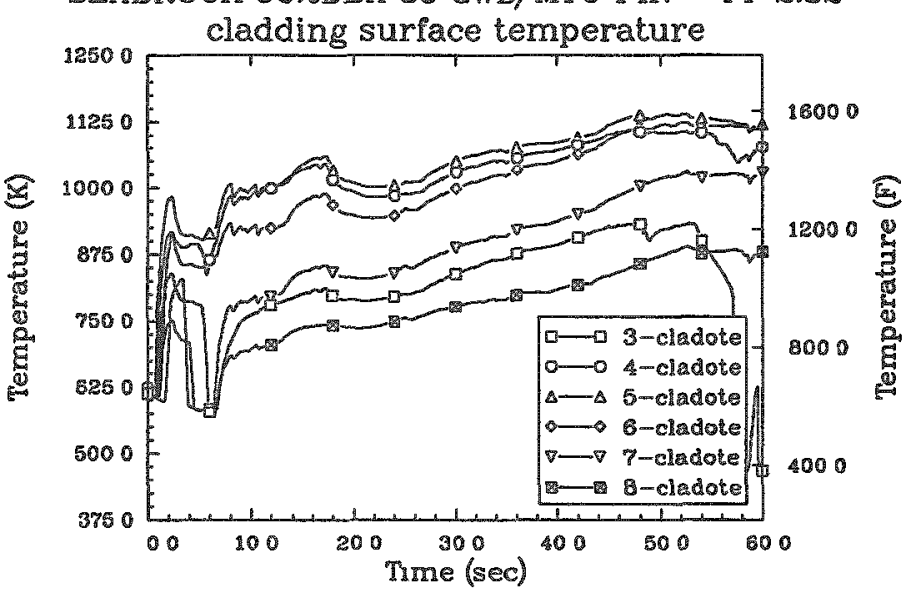

SEABROOK 50\%DBA 35 GWD/MTU PIN--PF 2.32

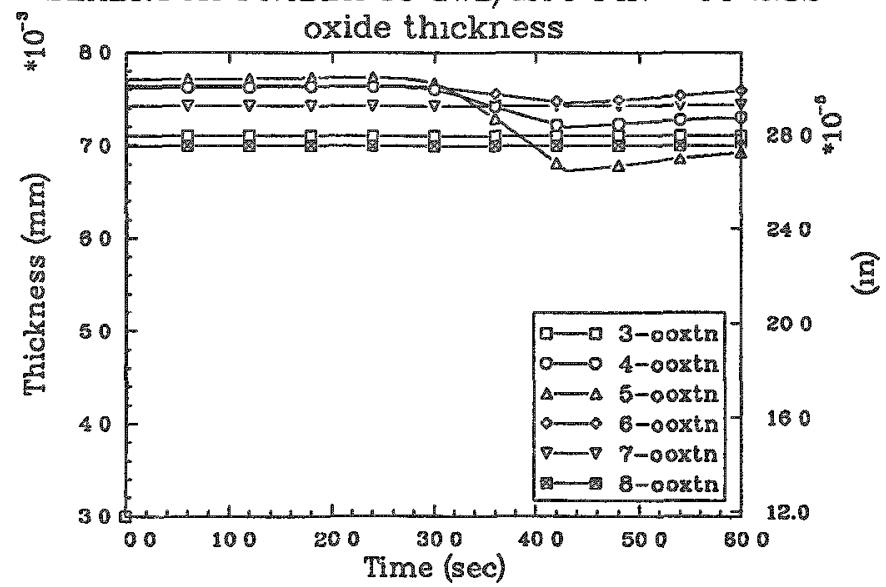


SEABROOK 50\%DBA $20 \mathrm{GWD} / \mathrm{MTU}$ PIN--PF 232

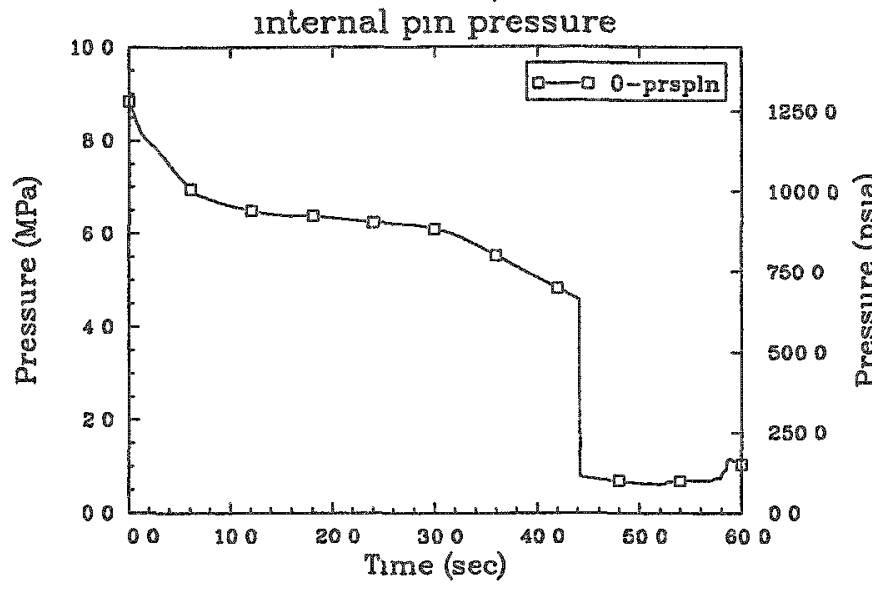

SEABROOK 50\%DBA $20 \mathrm{GWD} / \mathrm{MTU}$ PIN--PF 232

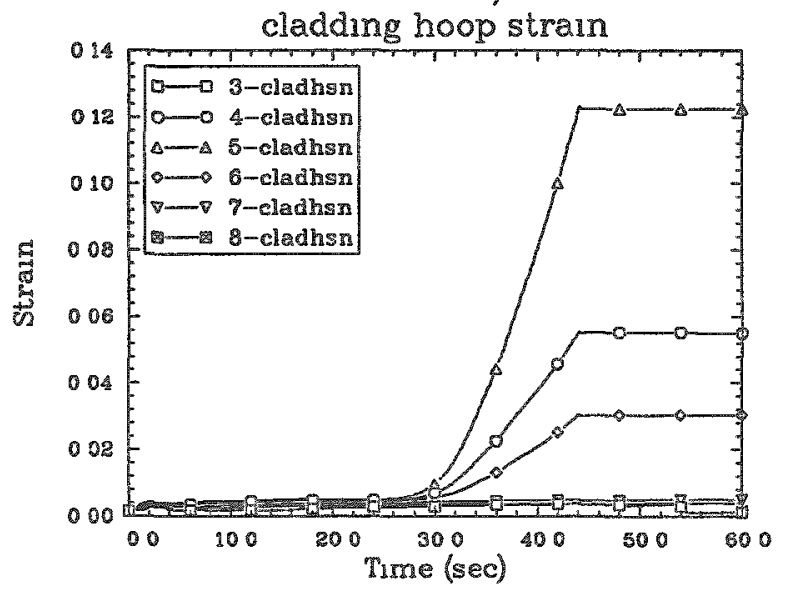

SEABROOK 50\%DBA $20 \mathrm{GWD} / \mathrm{MTU}$ PIN--PF 232

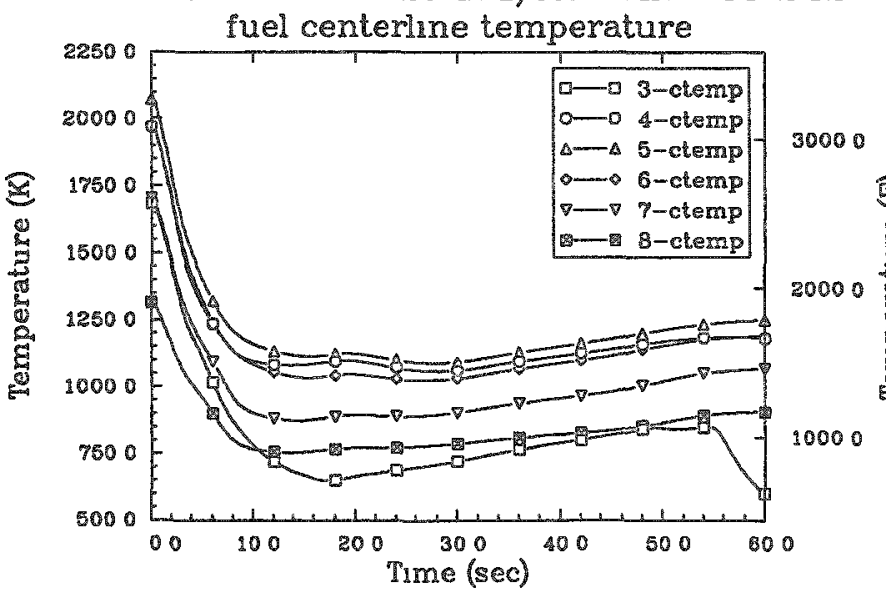

SEABROOK 50\%DBA 20 GWD/MTU PIN-PF 232

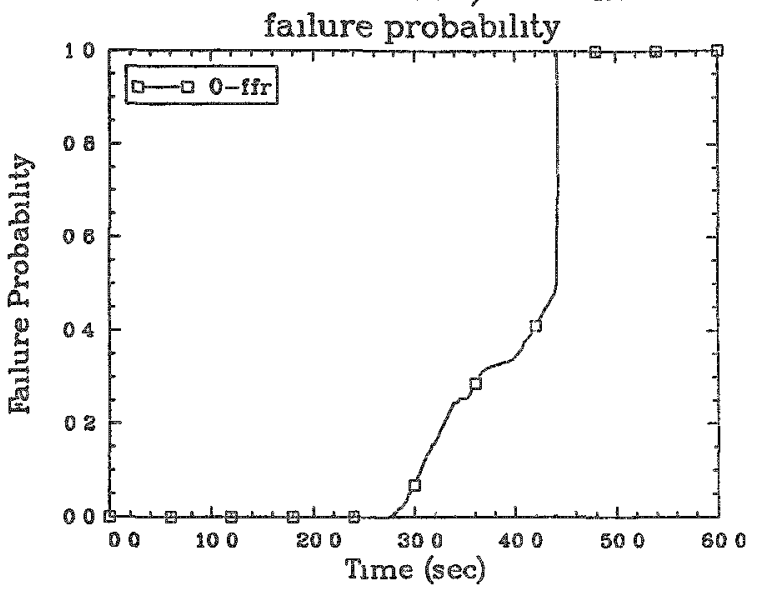

SEABROOK 50\%DBA 20 GWD/MTU PIN--PF 232

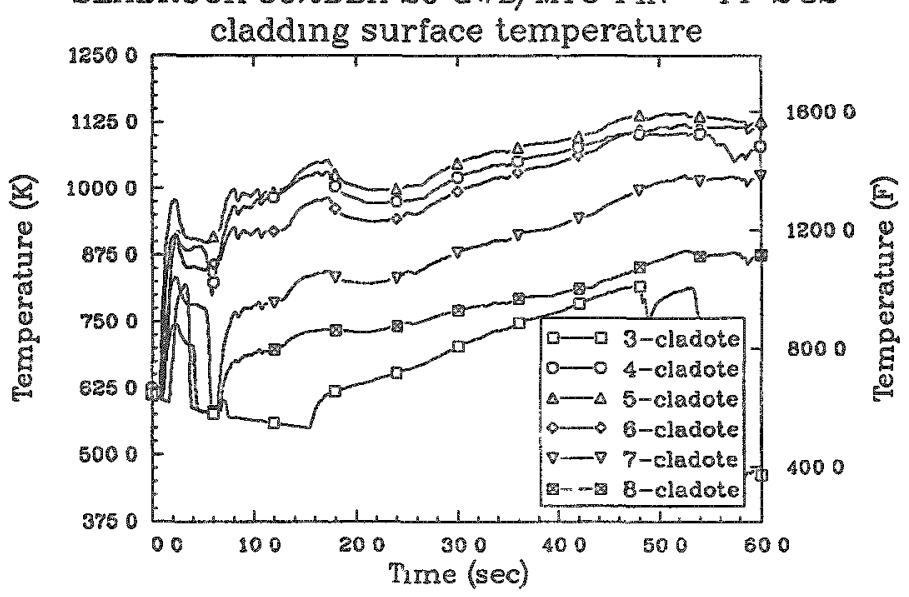

SEABROOK 50\%DBA 20 GWD/MTU PIN--PF 232

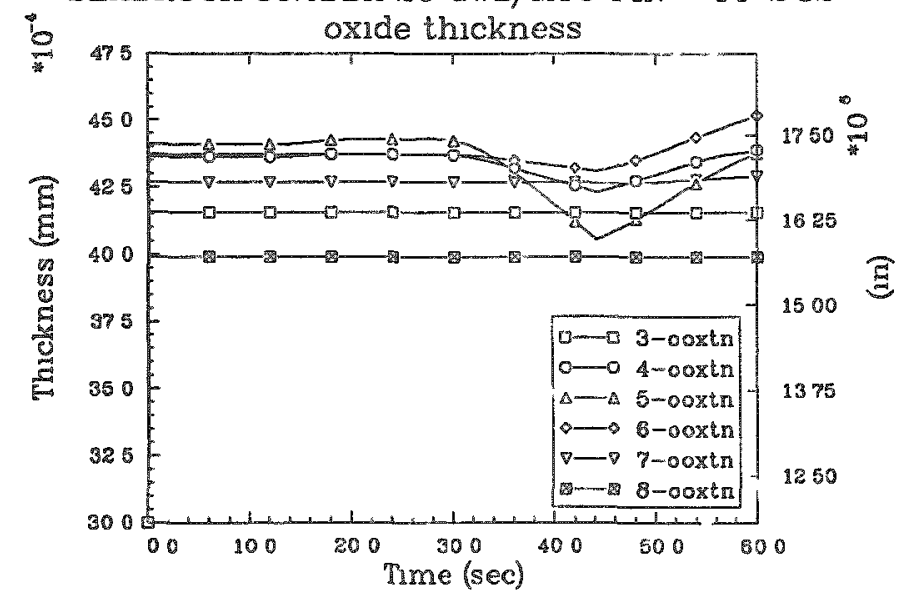


SEABROOK 50\%DBA 5 GWD/MTU PIN--PF 2.32

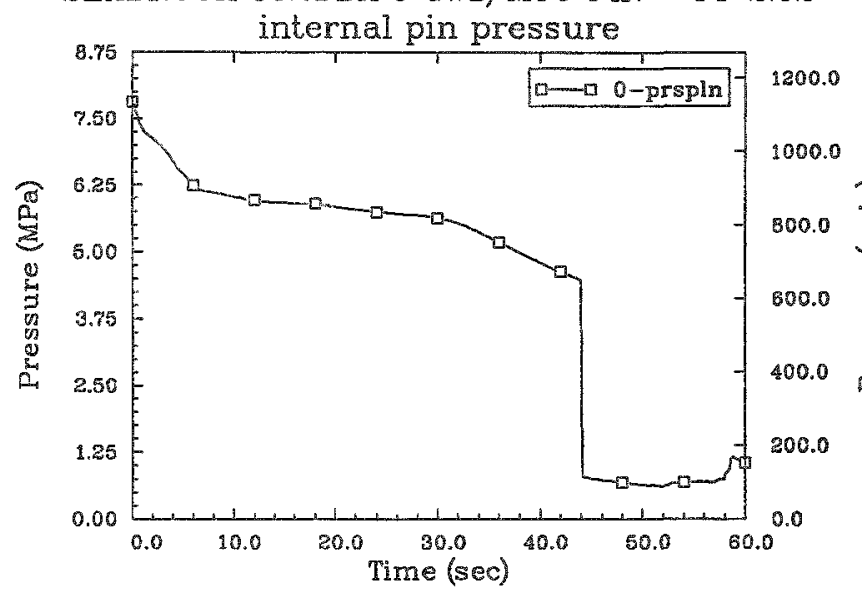

SEABROOK 50\%DBA 5 GWD/MTU PIN--PF 2.32

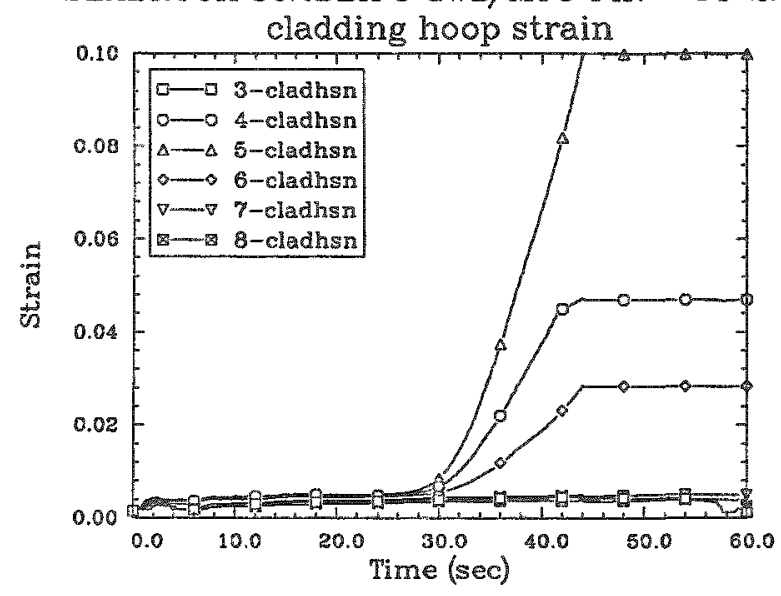

SEABROOK 50\%DBA 5 GWD/MTU PIN-PF 2.32

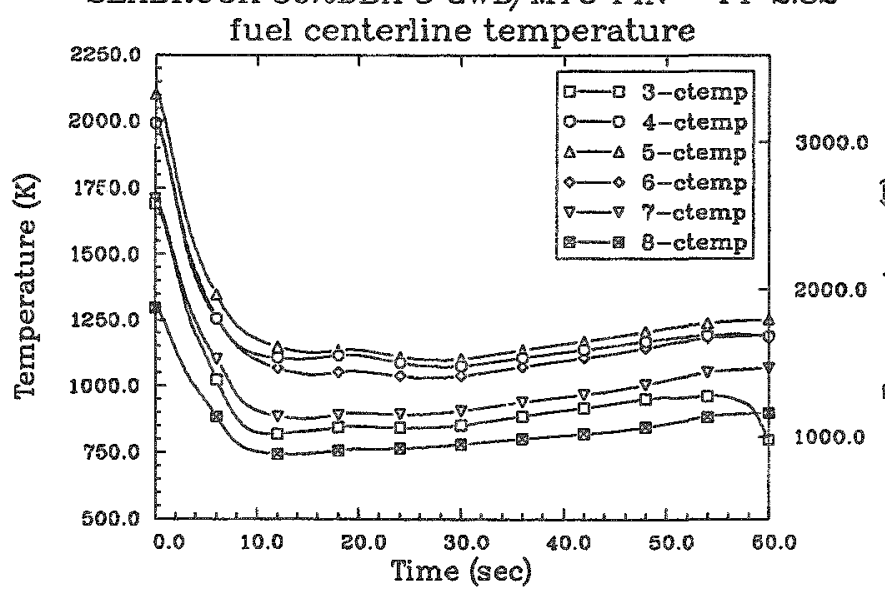

SEABROOK 50\%DBA 5 GWD/MTU PIN--PF 2.32

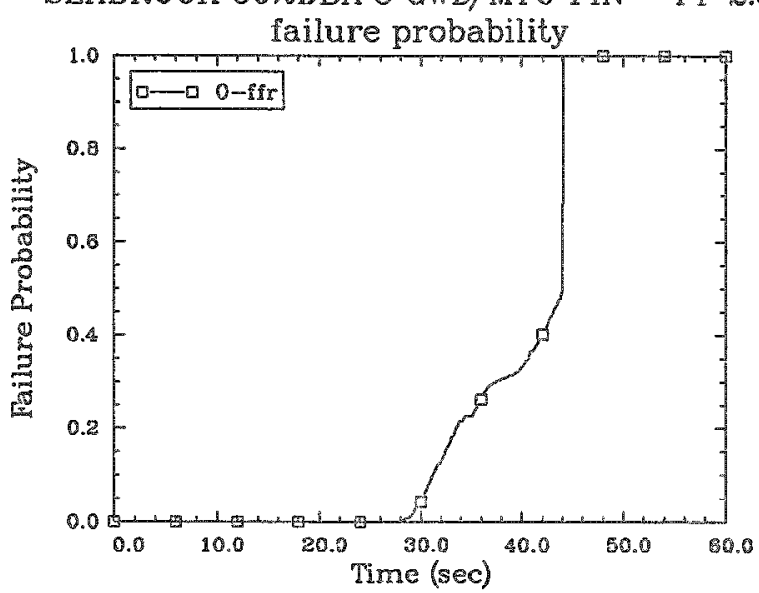

SEABROOK 50\%DBA 5 GWD/MTU PIN--PF 2.32

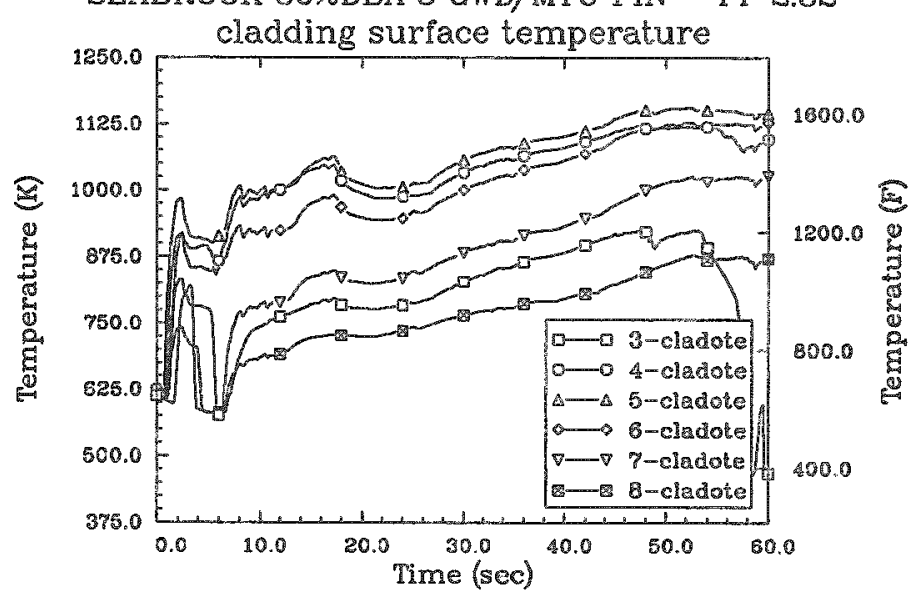

SEABROOK 50\%DBA 5 GWD/MTU PIN--PF 2.32

$i$ oxide thickness

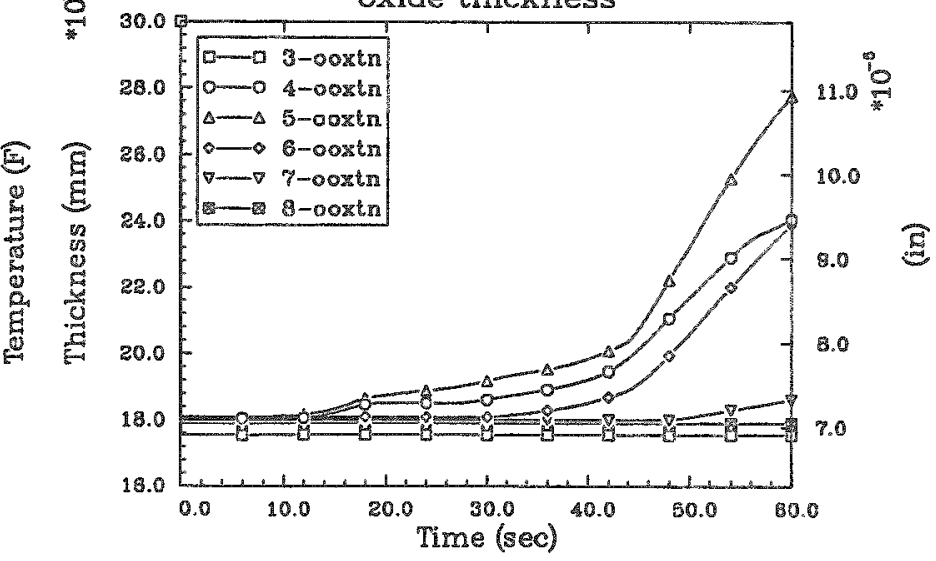



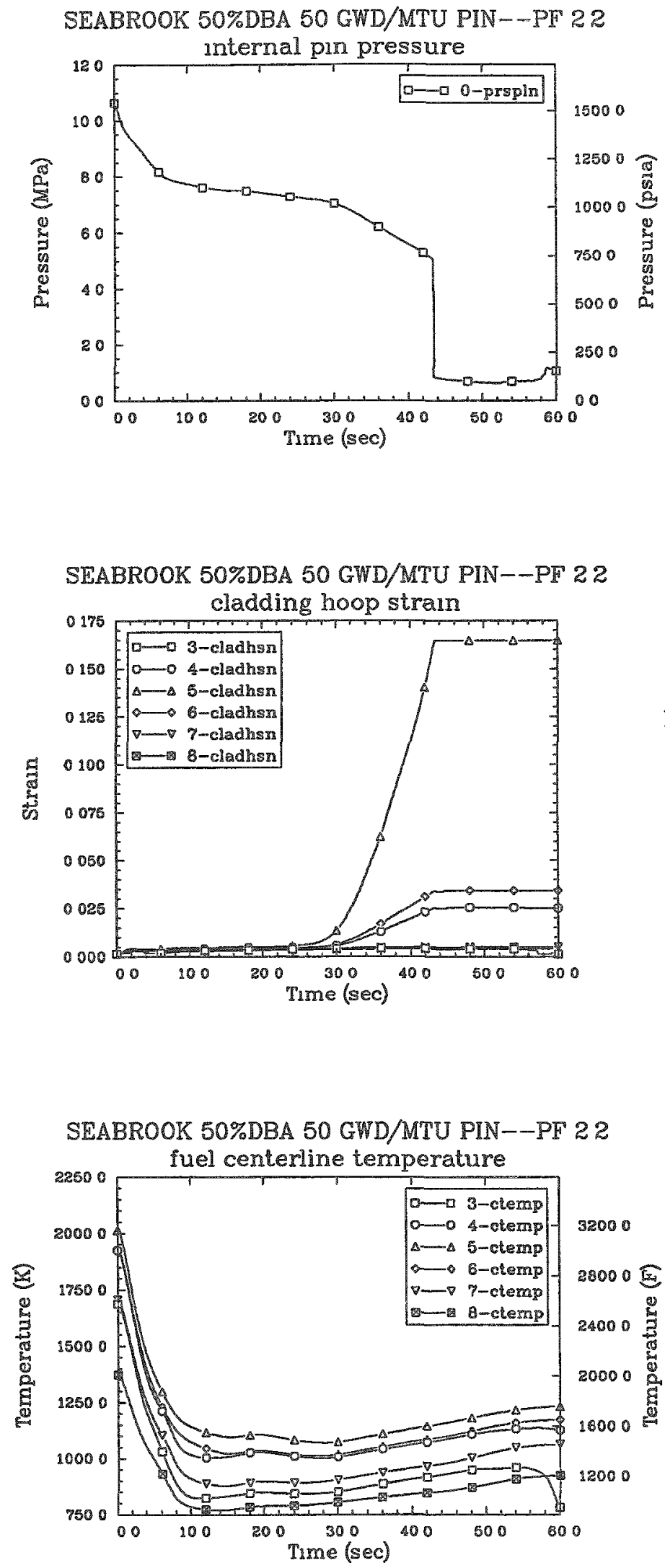

SEABROOK 50\%DBA 50 GWD/MTU PIN--PF 22

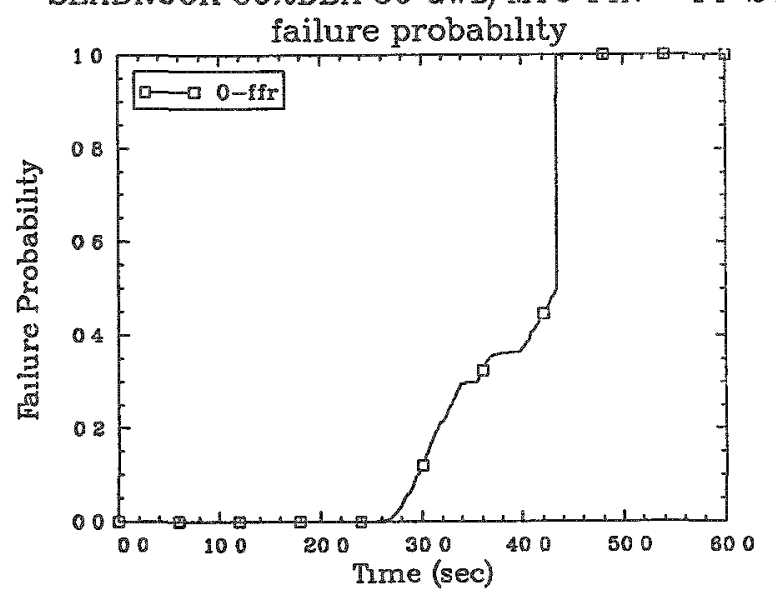

SEABROOK 50\%DBA 50 GWD/MTU PIN--PF 22

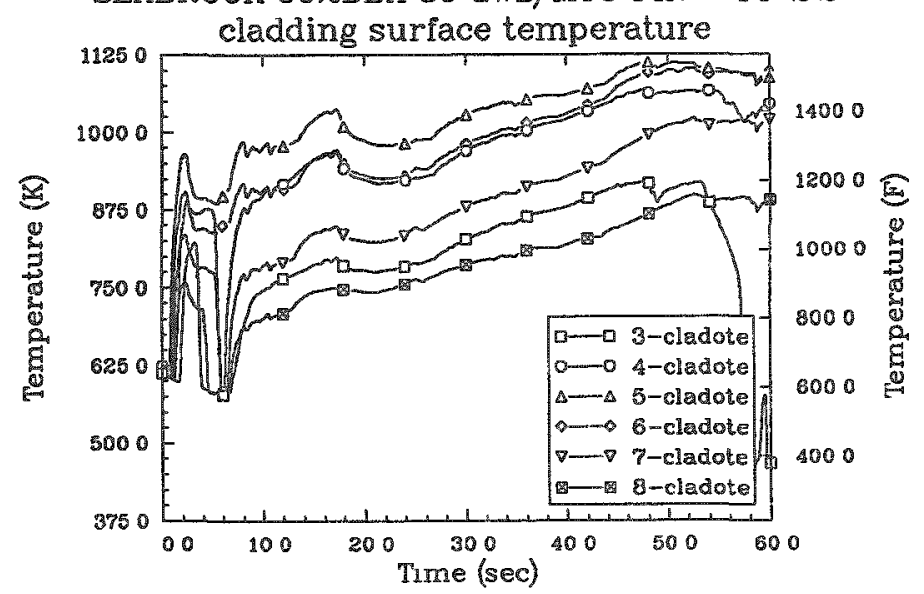

SEABROOK 50\%DBA 50 GWD/MTU PIN--PF 22

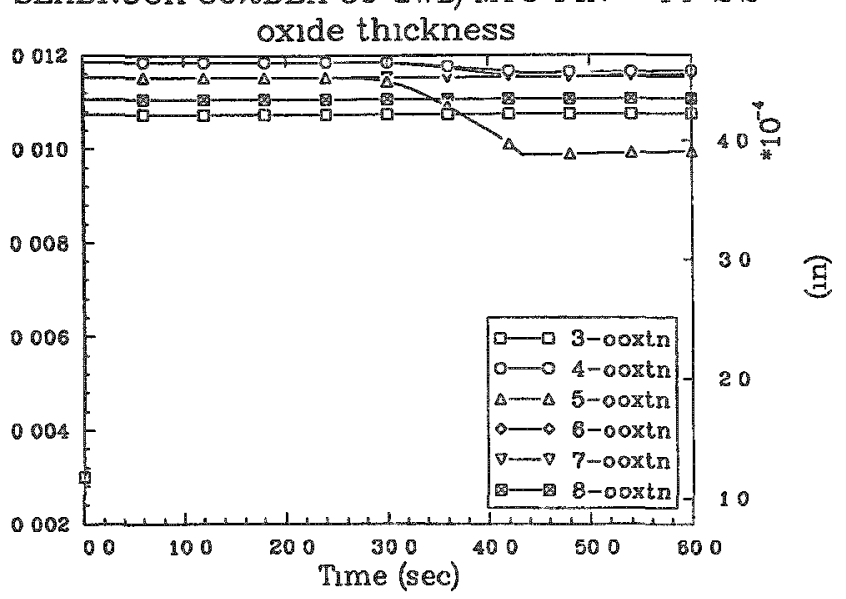


SEABROOK 50\%DBA 35 GWD/MTU PIN--PF 2.2

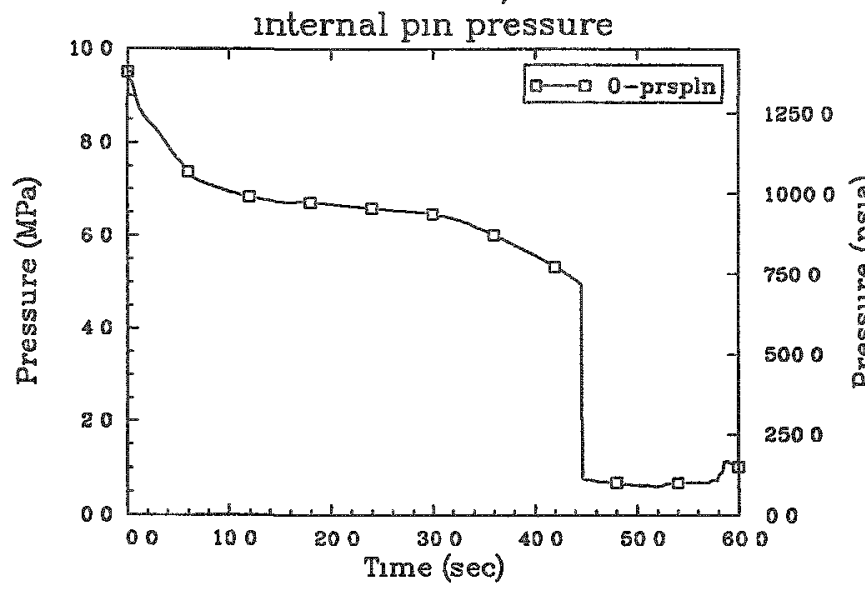

SEABROOK 50\%DBA 35 GWD/MTU PIN--PF 2.2

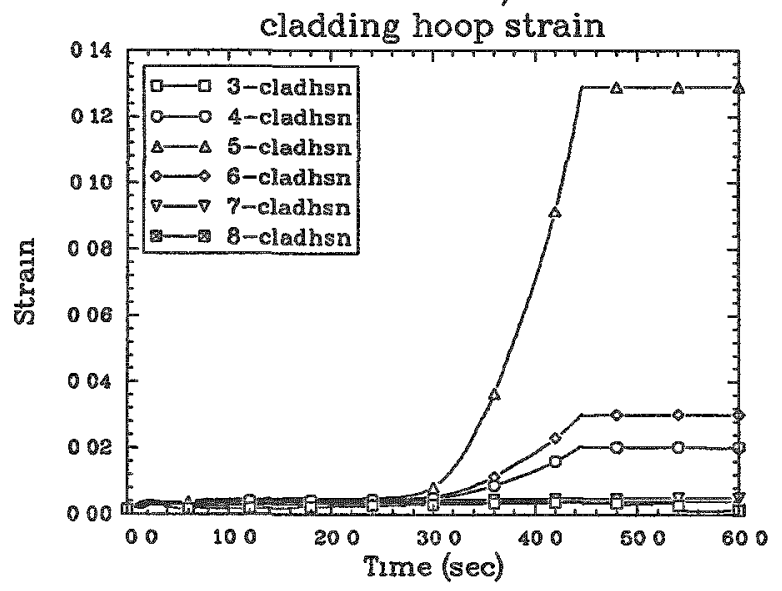

SEABROOK 50\%DBA 35 GWD/MTU PIN--PF 2.2

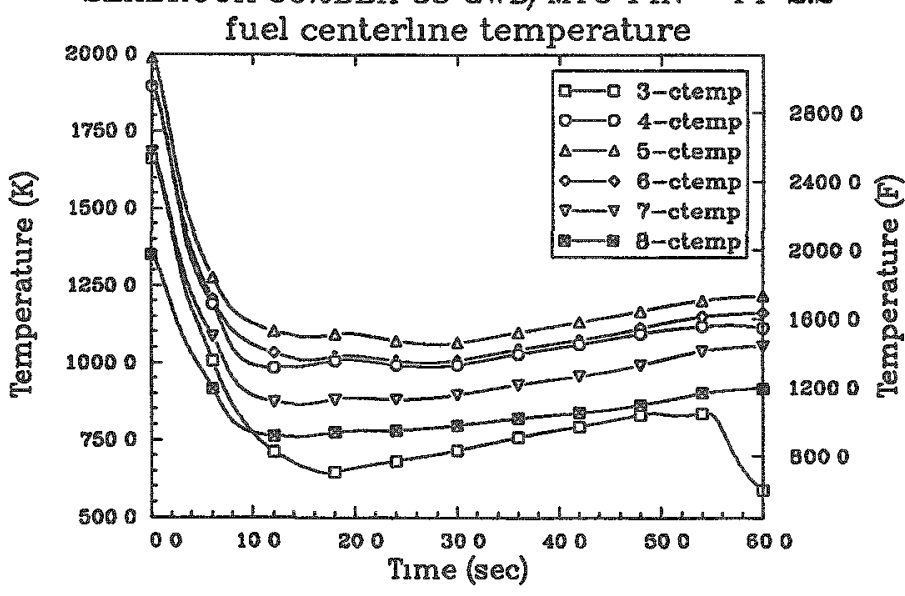

SEABROOK 50\%DBA 35 GWD/MTU PIN--PF 2.2

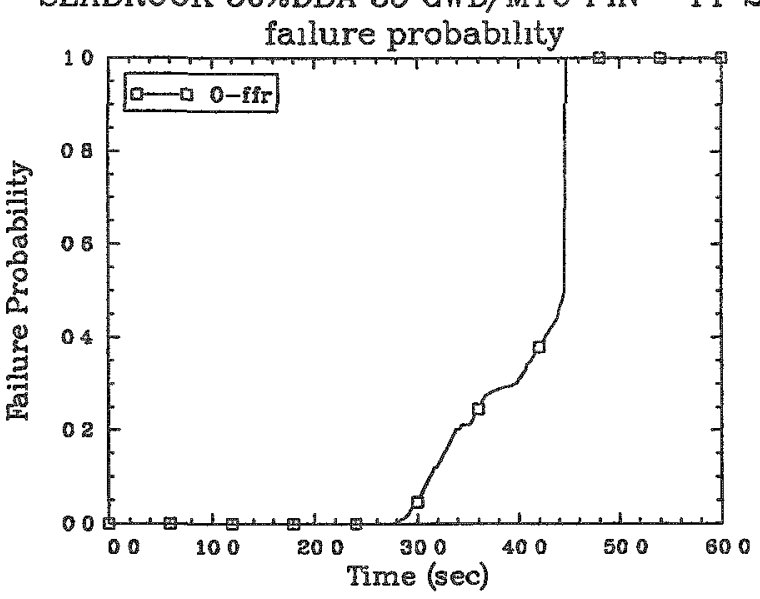

SEABROOK 50\%DBA 35 GWD/MTU PIN-DF 22

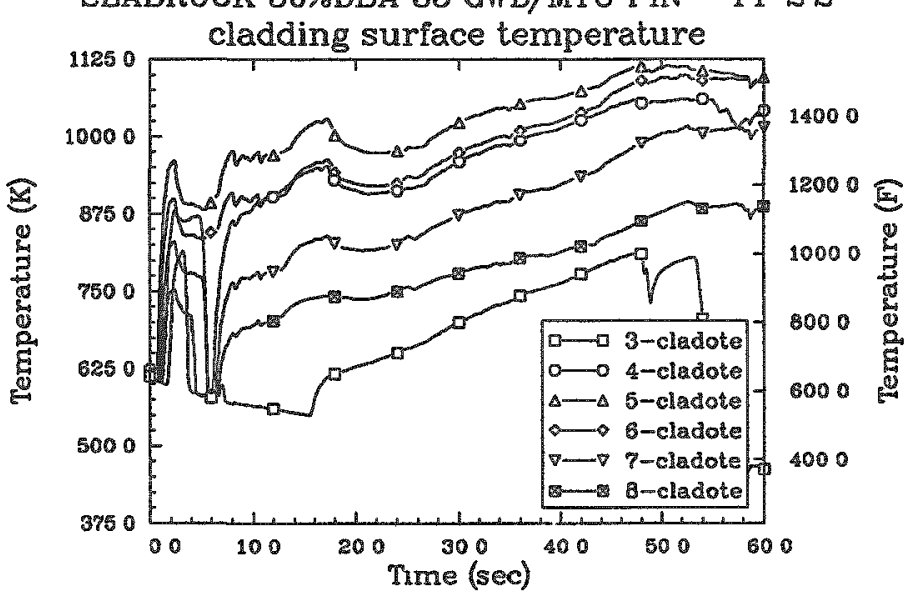

SEABROOK 50\%DBA 35 GWD/MTU PIN--PF 22

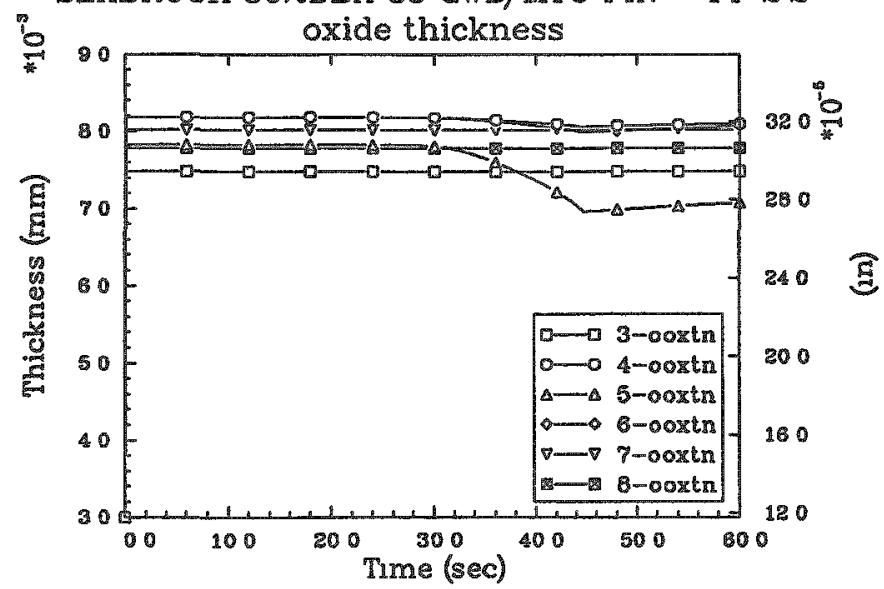




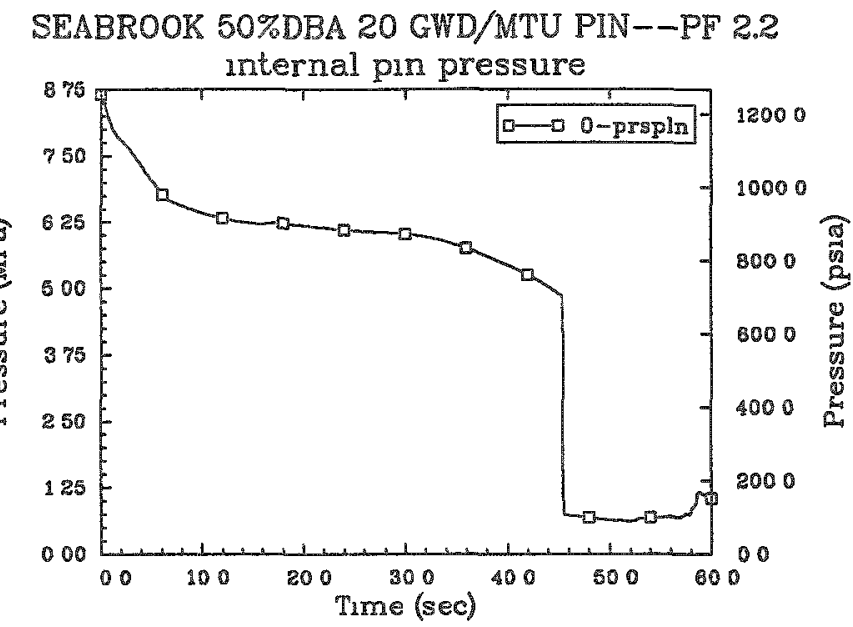

SEABROOK 50\%DBA 20 GWD/MTU PIN--PF 22

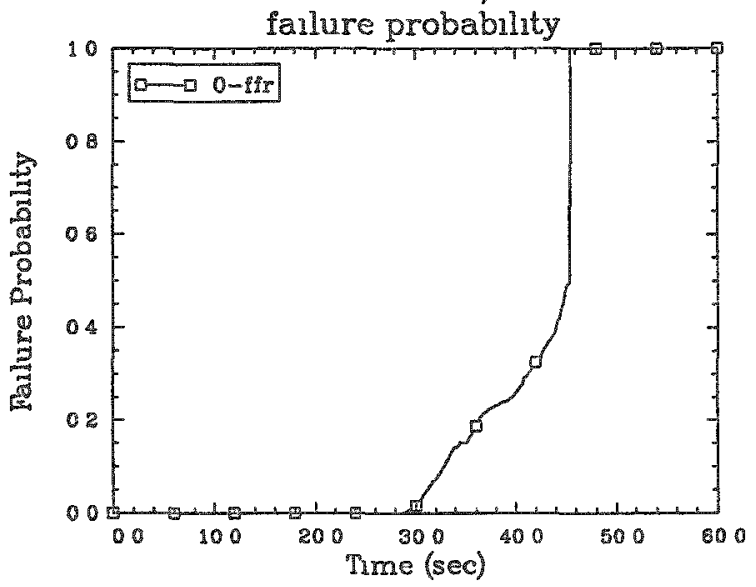

SEABROOK 50\%DBA 20 GWD/MTU PIN--PF' 22

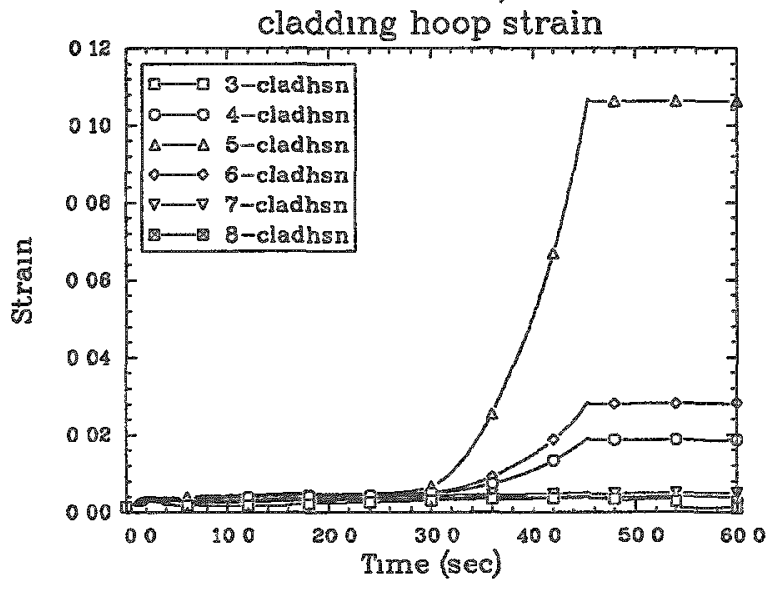

SEABROOK 50\%DBA $20 \mathrm{GWD} / \mathrm{MTU}$ PIN--PF 22

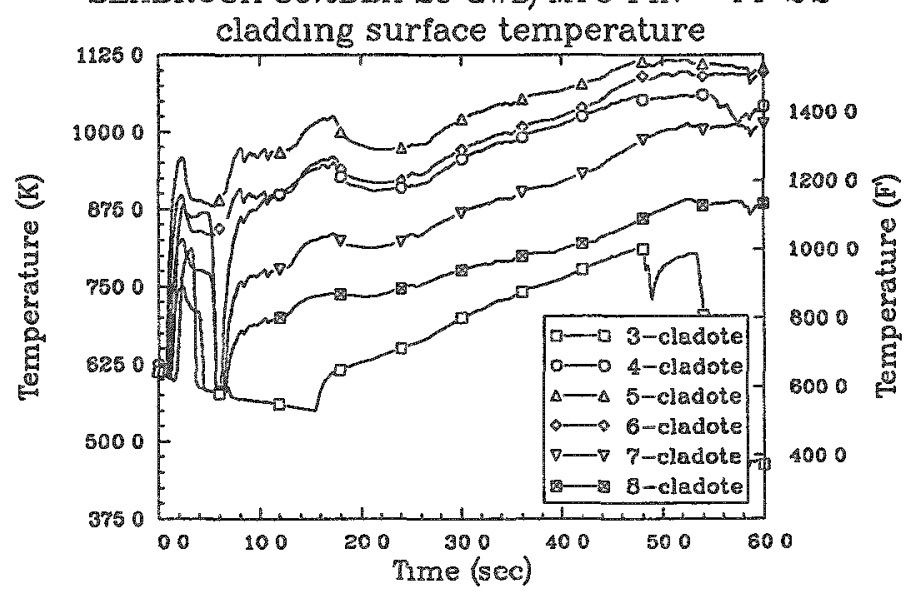

SEABROOK 50\%DBA 20 GWD/MTU PIN--PF 22
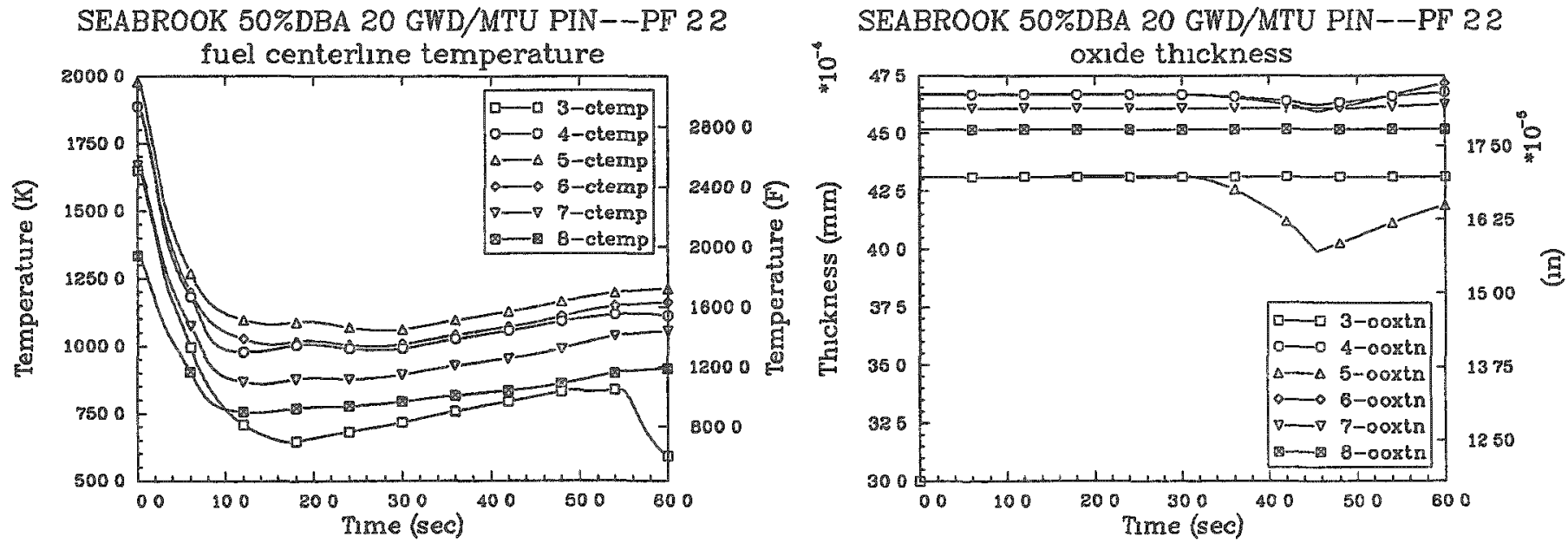


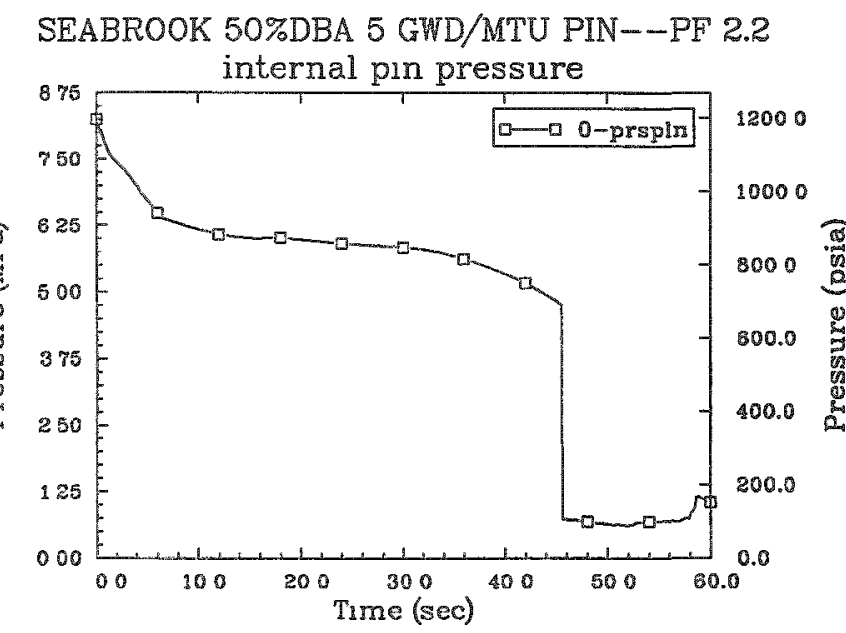

\section{SEABROOK 50\%DBA 5 GWD/MTU PIN--PF 2.2}
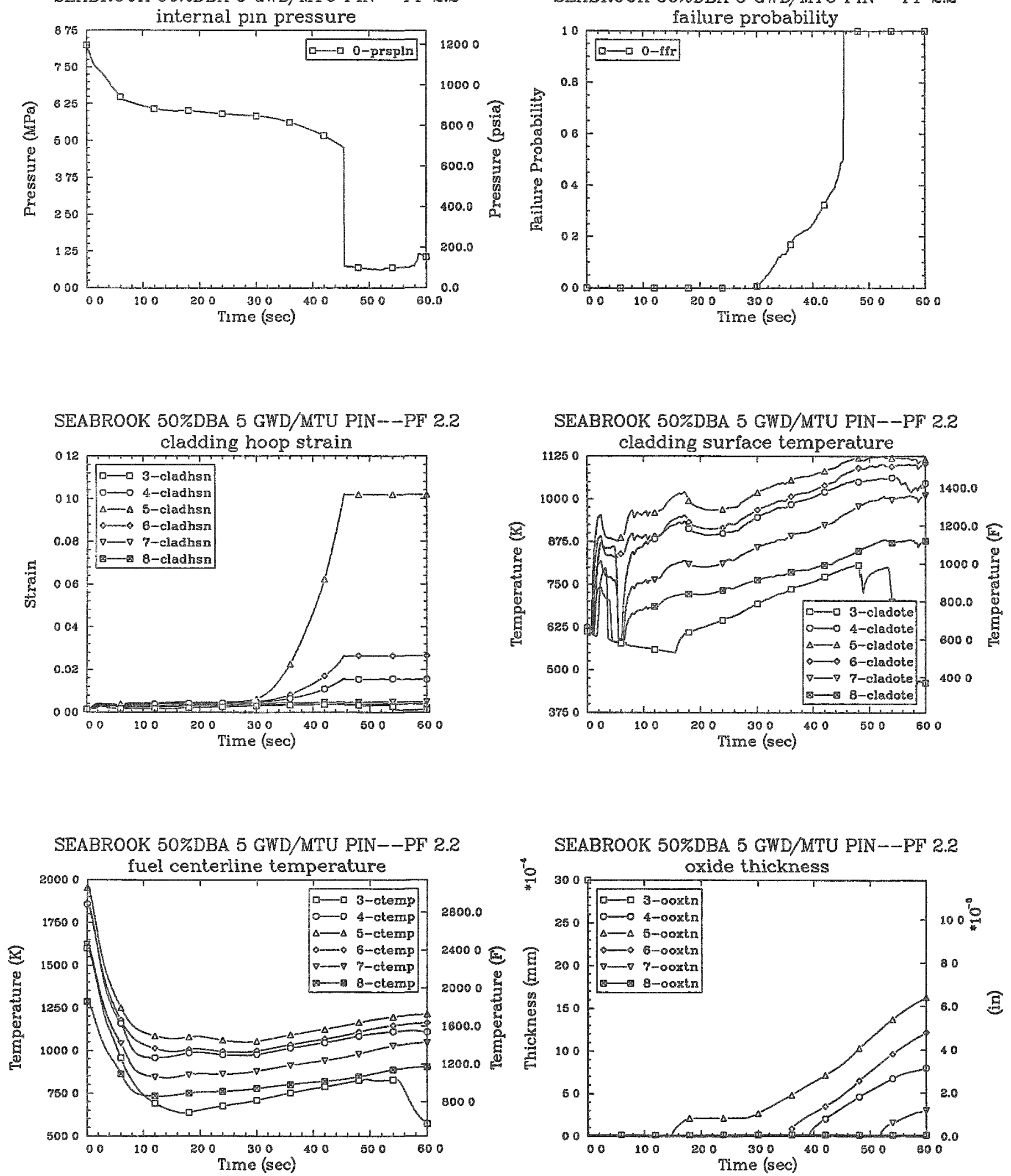
SEABROOK 50\%DBA 50 GWD/MTU PIN-PF 2.0
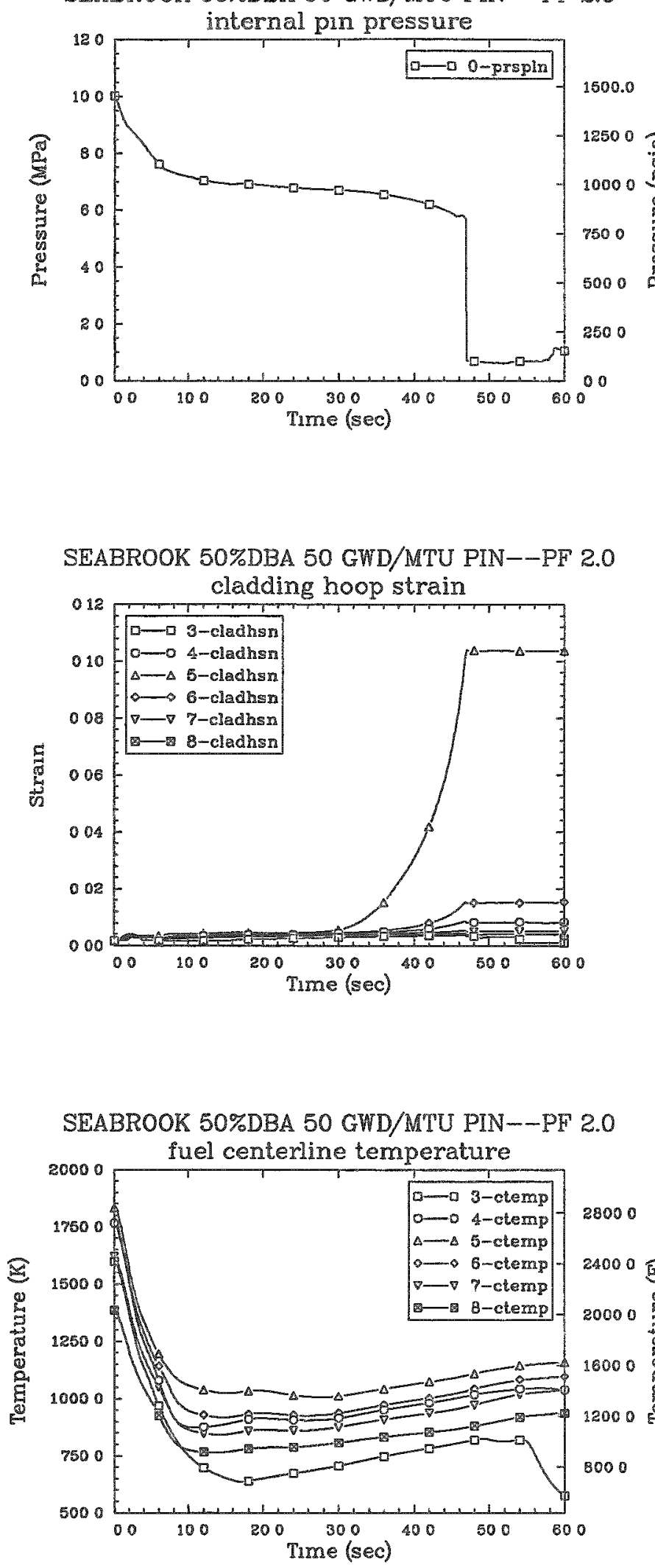

SEABROOK 50\%DBA 50 GWD/MTU PIN--PF 2.0 fallure probability

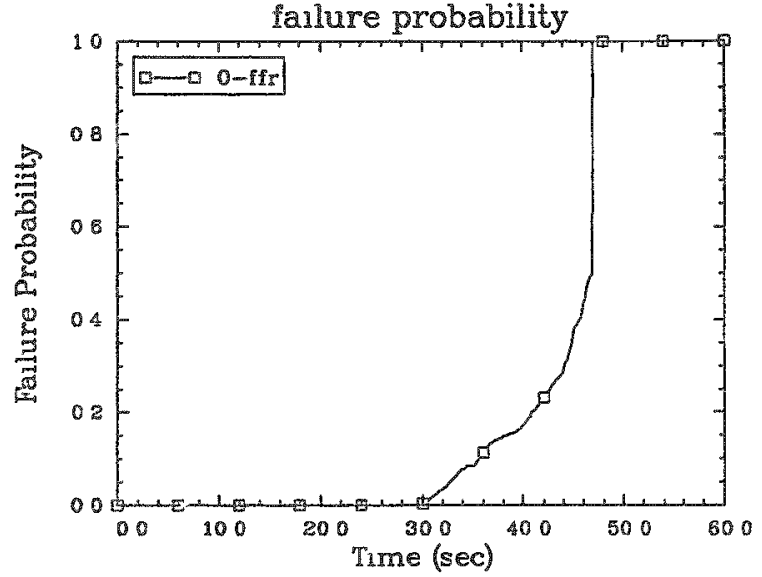

SEABROOK 50\%DBA $50 \mathrm{GWD} / \mathrm{MTU}$ PIN--PF 20 cladding surface temperature

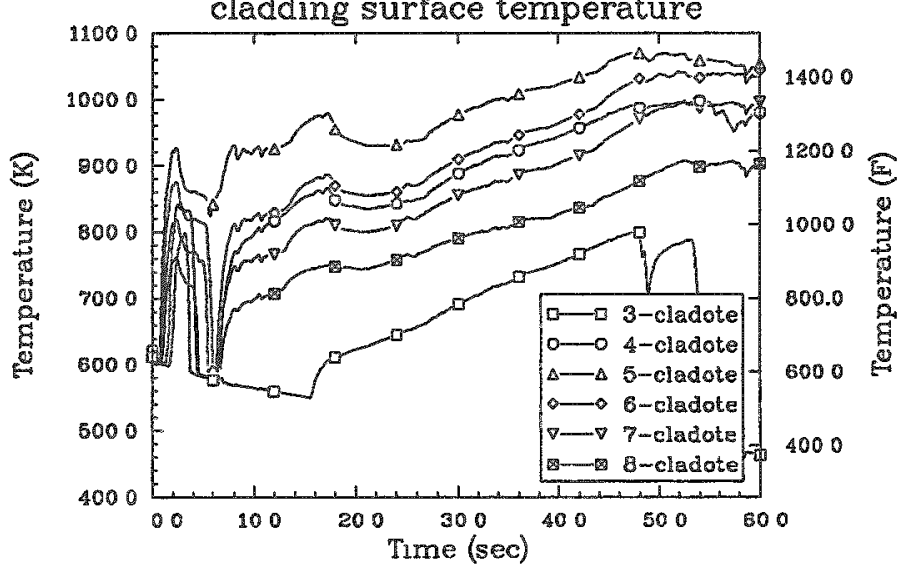

SEABROOK 50\%DBA 50 GWD/MTU PIN--PF 20 oxide thickness

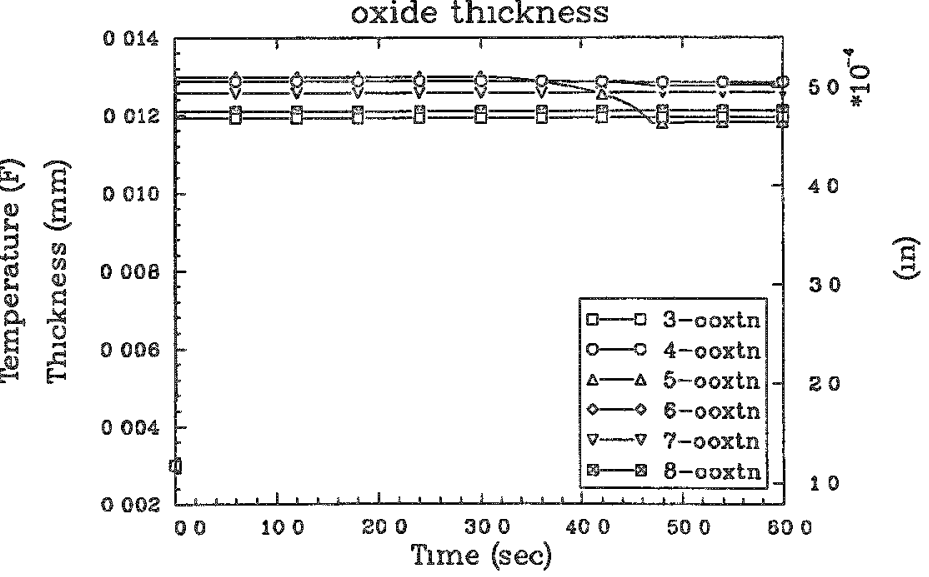


SEABROOK 50\%DBA 35 GWD/MTU PIN--PF 20
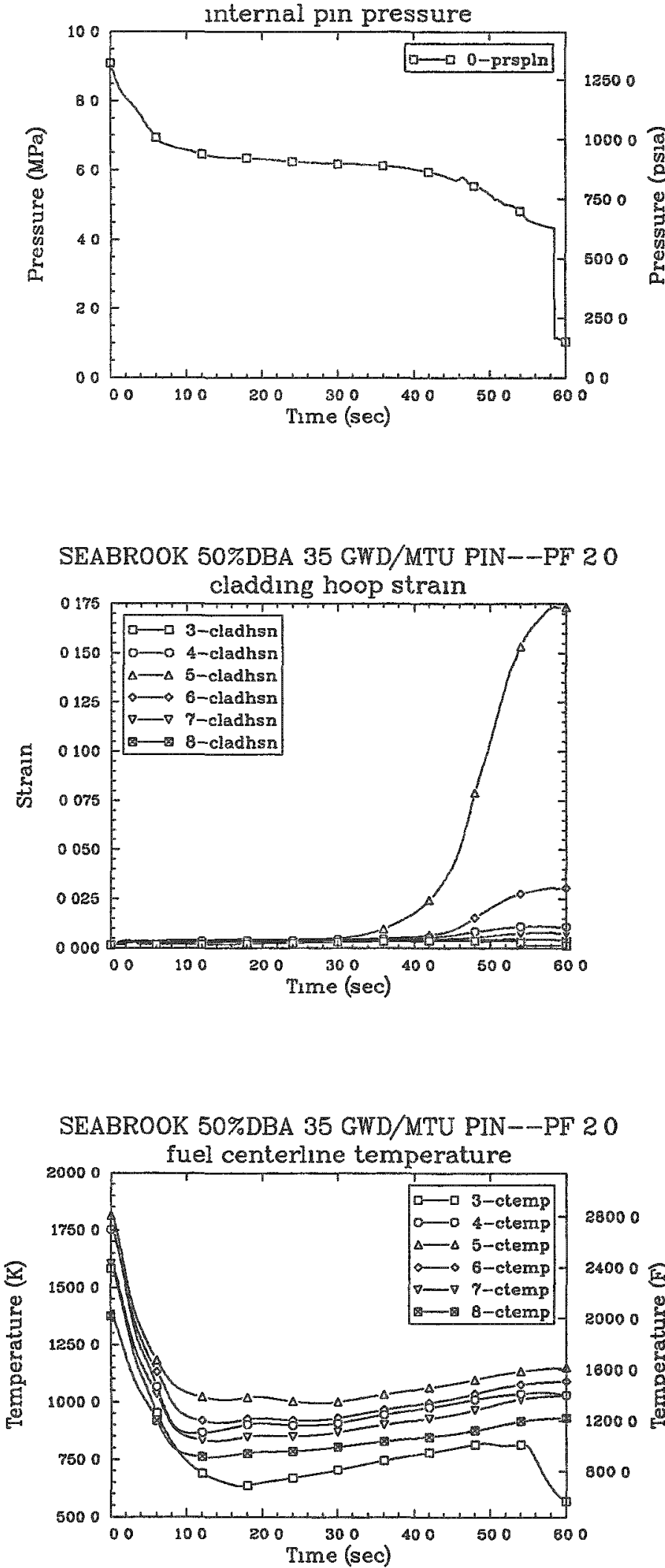

SEABROOK 50\%DBA 35 GWD/MTU PIN--PF 20 fallure probability

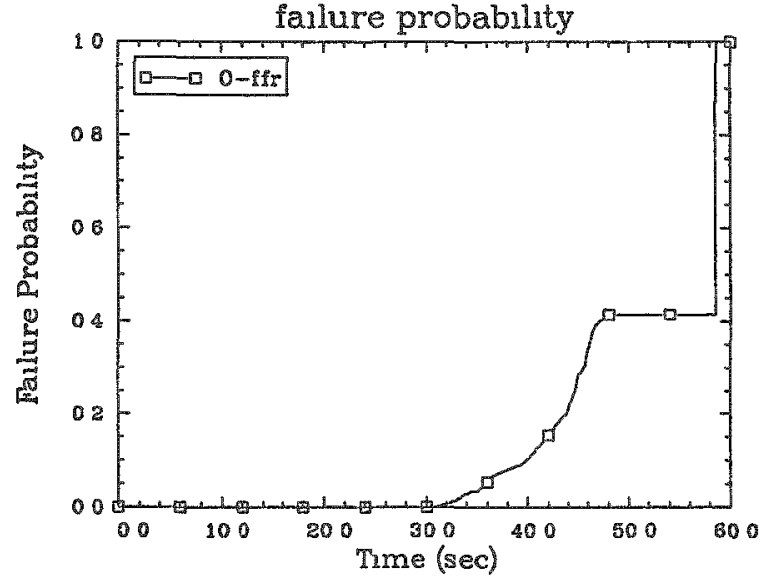

SEABROOK 50\%DBA 35 GWD/MTU PIN--PF 20

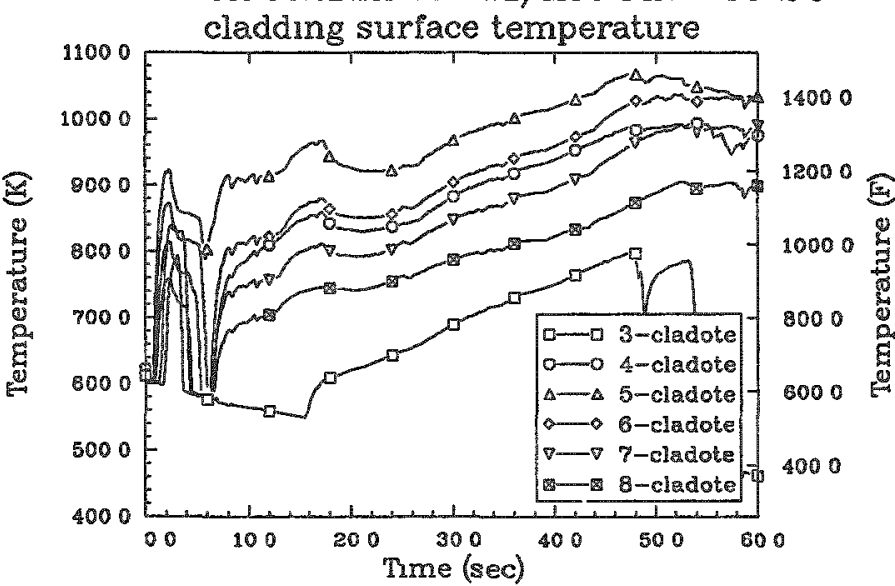

SEABROOK 50\%DBA 35 GWD/MTU PIN--PF 20

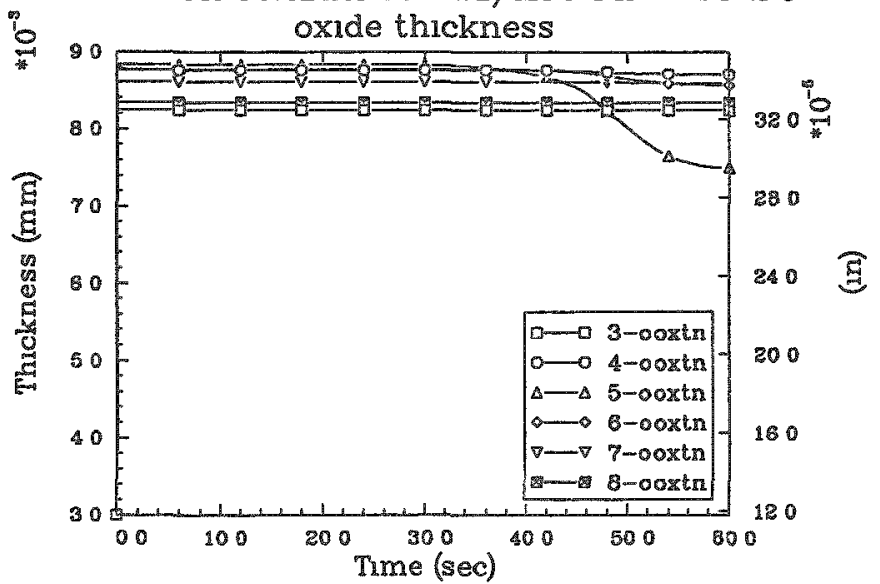


SEABROOK 50\%DBA 20 GWD/MTU PIN--PF 2.0
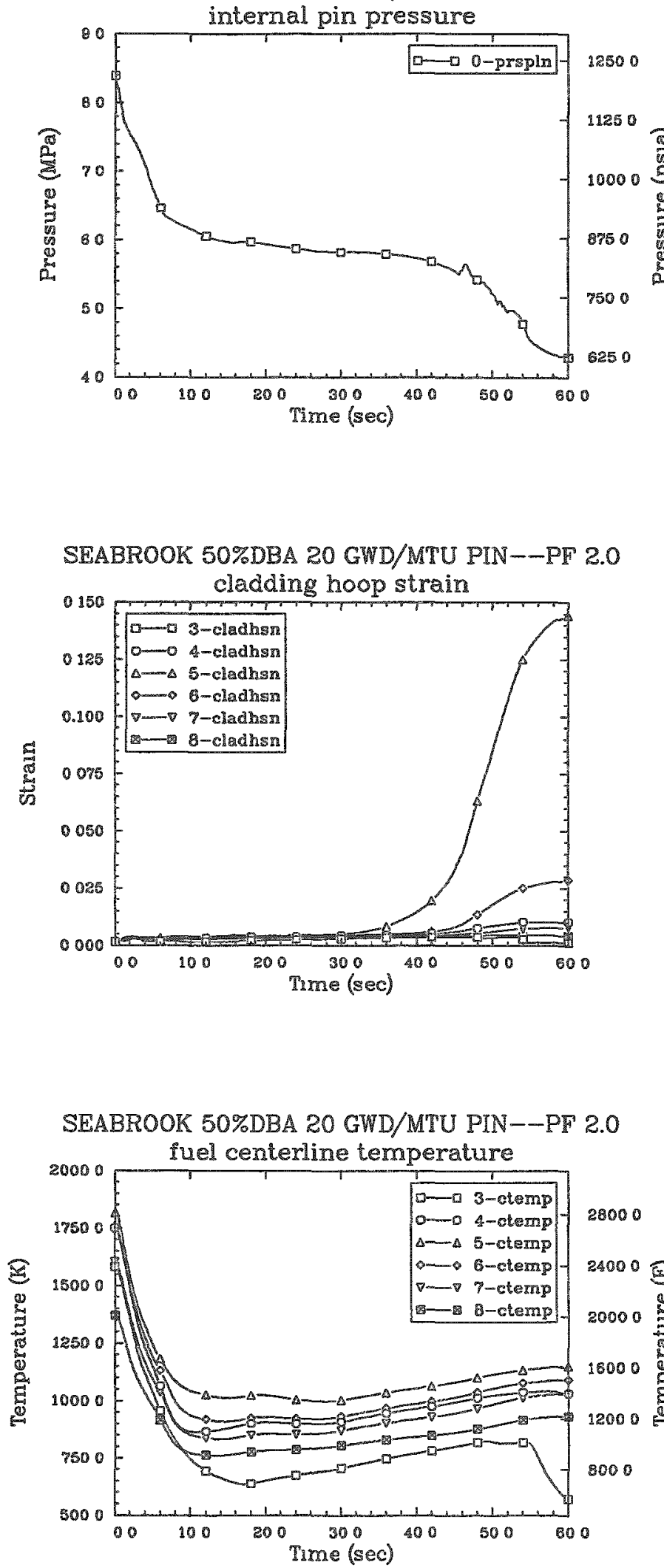

SEABROOK 50\%DBA 20 GWD/MTU PIN--PF 2.0 failure probability

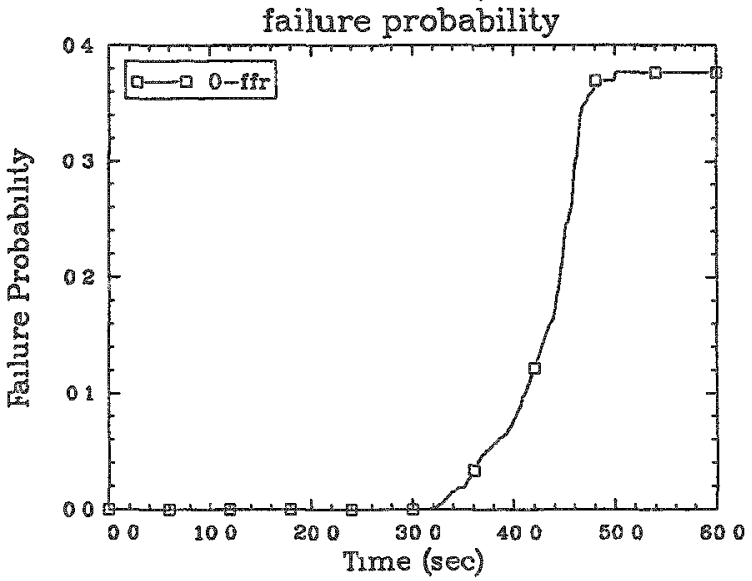

SEABROOK 50\%DBA 20 GWD/MTU PIN--PF 2.0

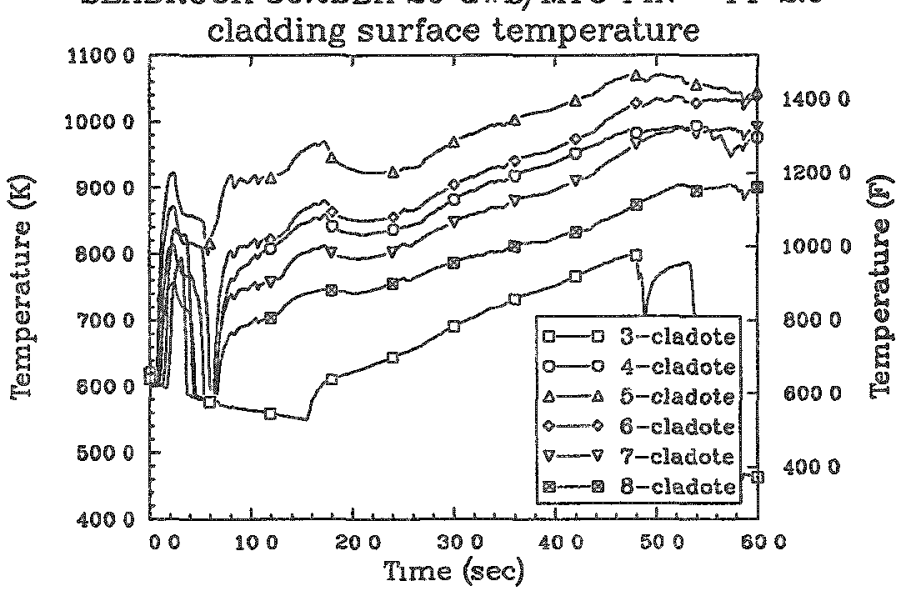

SEABROOK 50\%DBA 20 GWD/MTU PIN--PF 20

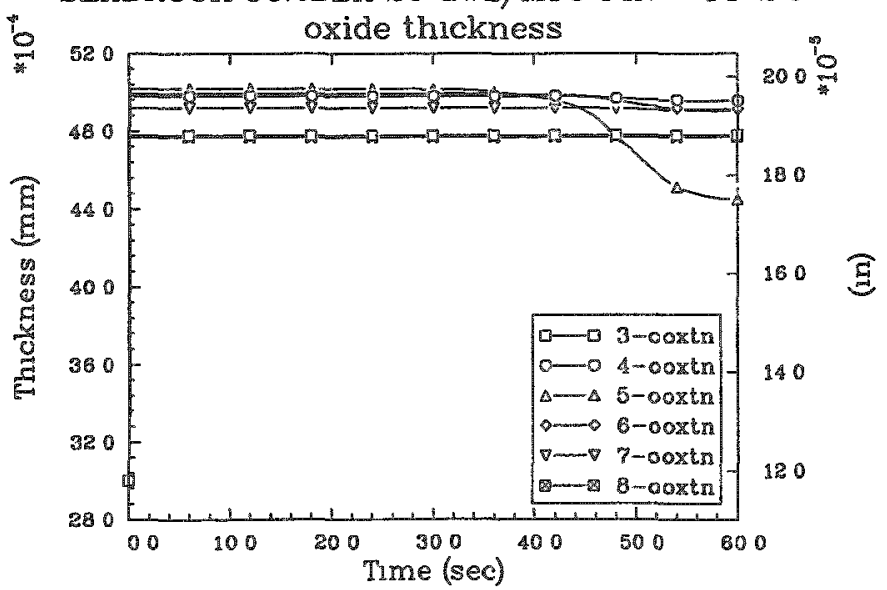


SEABROOK 50\%DBA 5 GWD/MTU PIN--PF 2.0
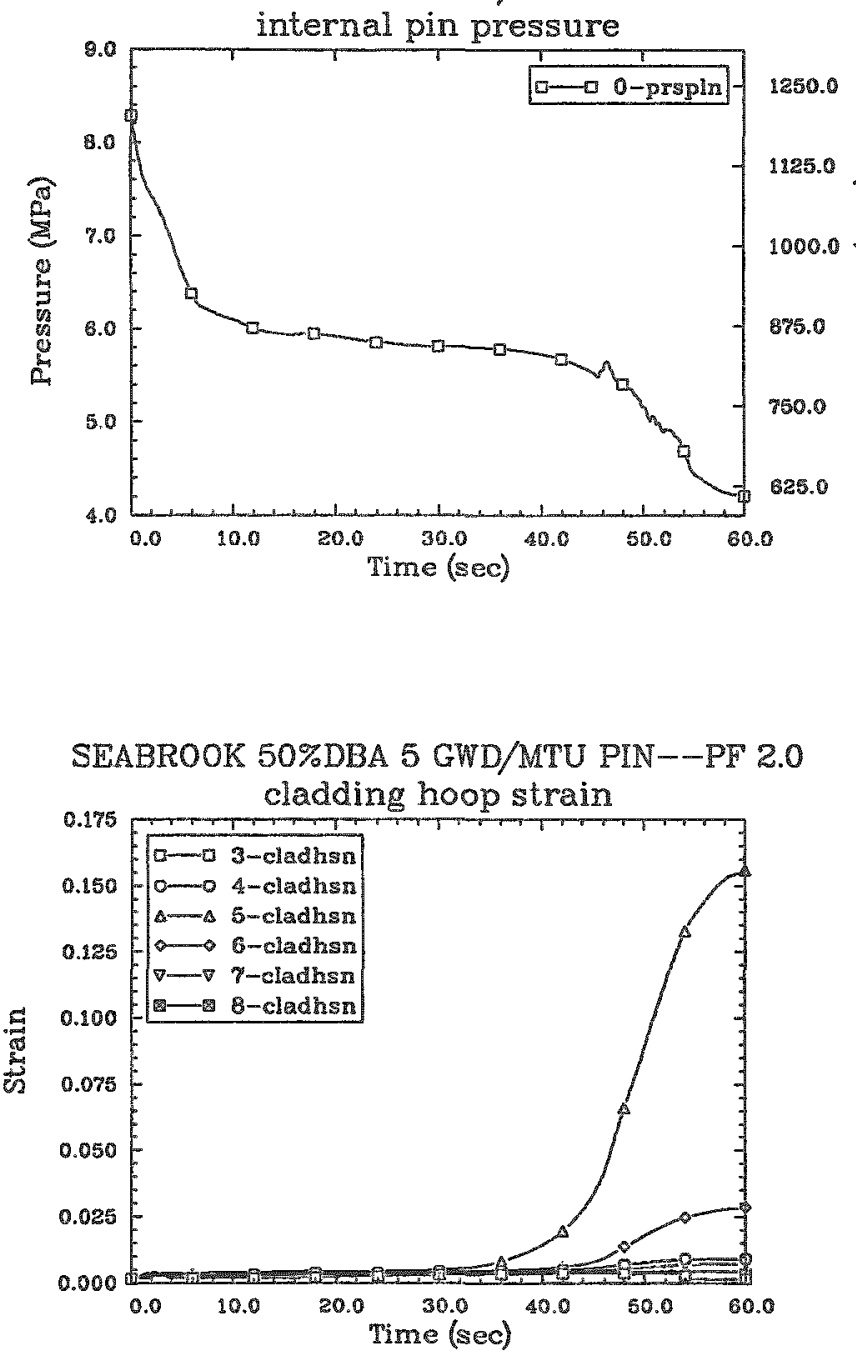

SEABROOK 50\%DBA 5 GWD/MTU PIN--PF 2.0

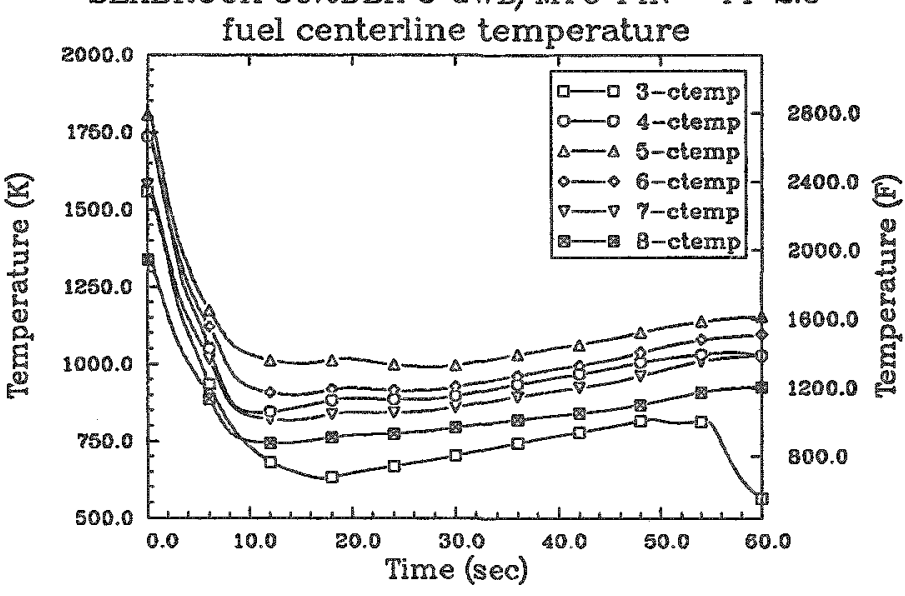

SEABROOK 50\%DBA 5 GWD/MTU PIN--PF 2.0 failure probability

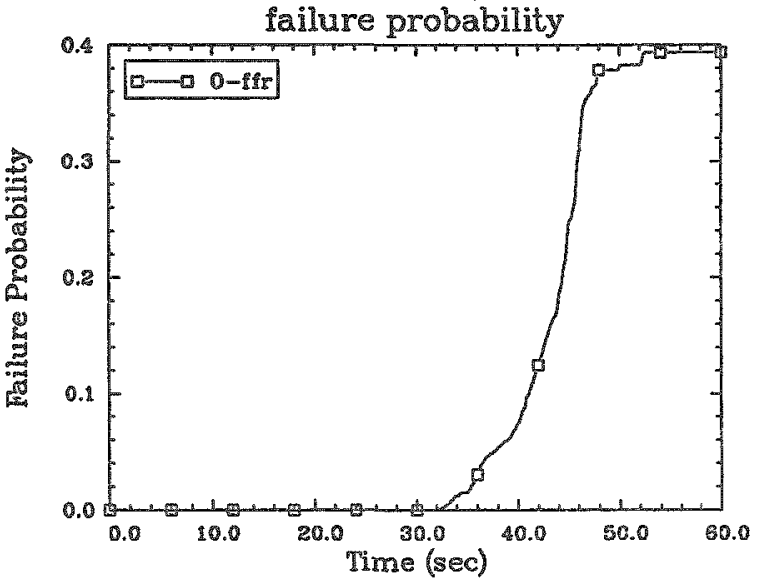

SEABROOK 50\%DBA 5 GWD/MTU PIN--PF 2.0

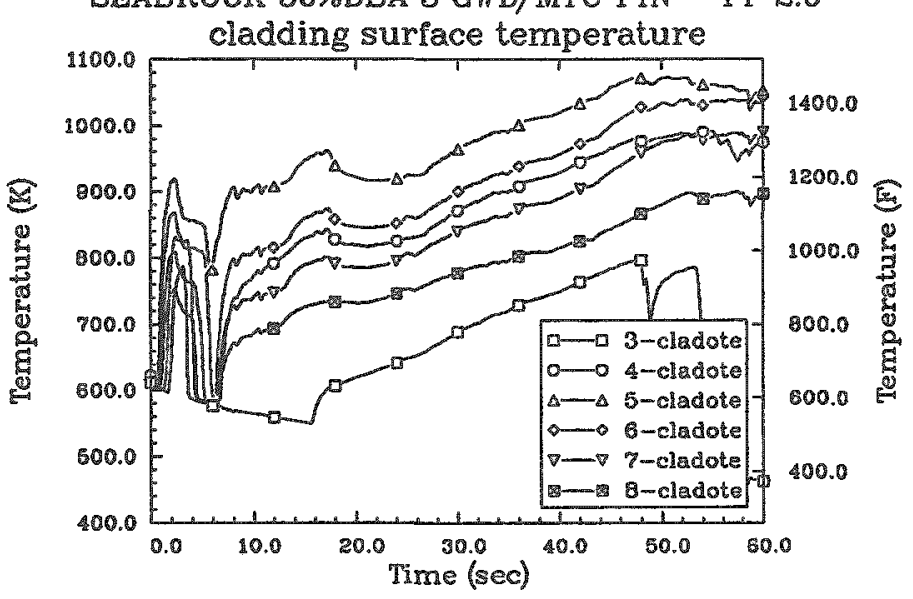

SEABROOK 50\%DBA 5 GWD/MTU PIN--PF 2.0

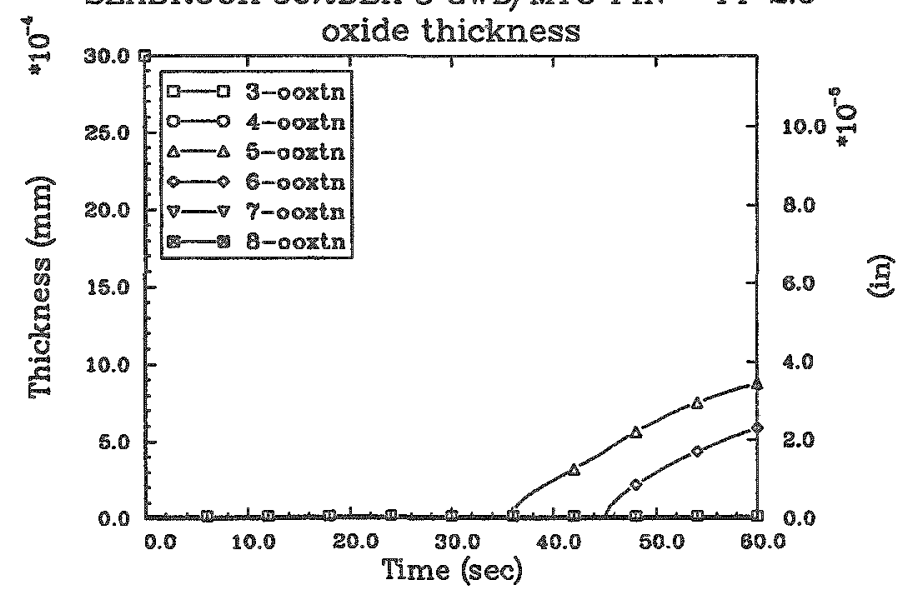


SEABROOK 50\%DBA 50 GWD/MTU PIN--PF 18
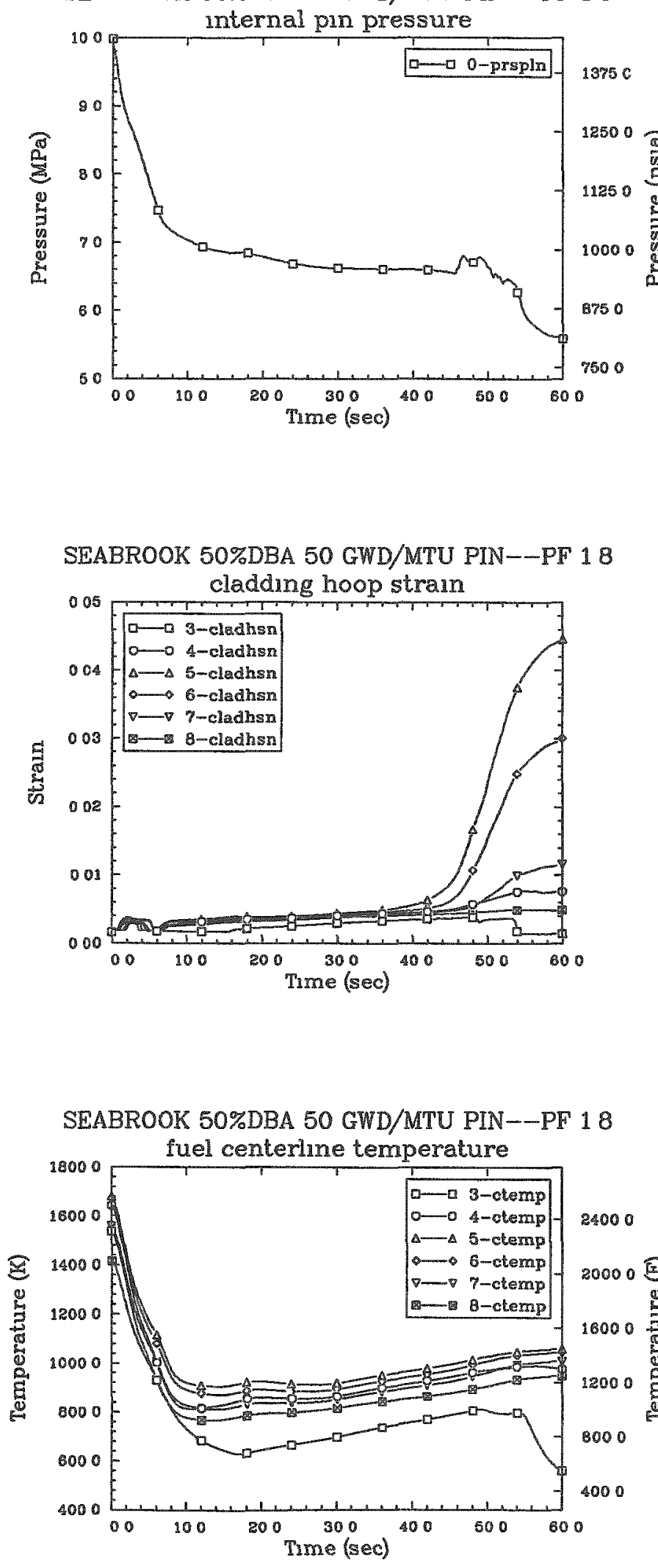

SEABROOK 50\%DBA 50 GWD/MTU PIN--PF 18 fallure probability

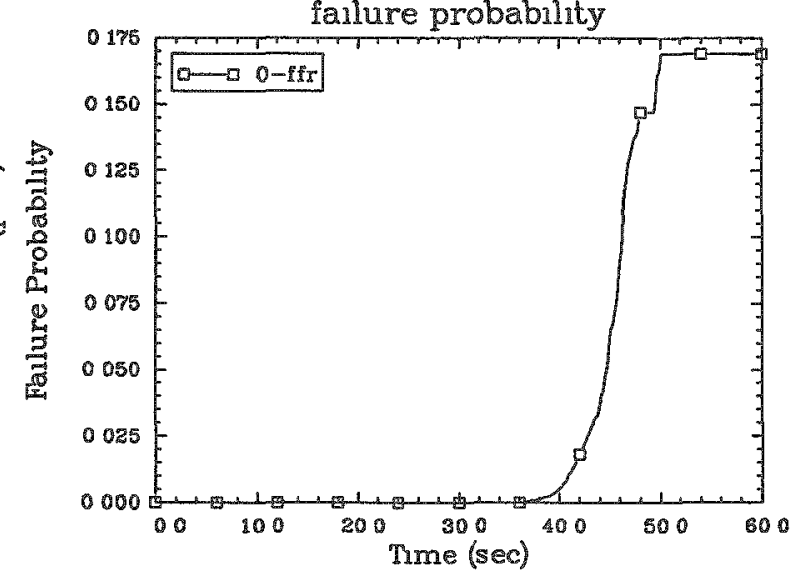

SEABROOK 50\%DBA 50 GWD/MTU PIN--PF 18

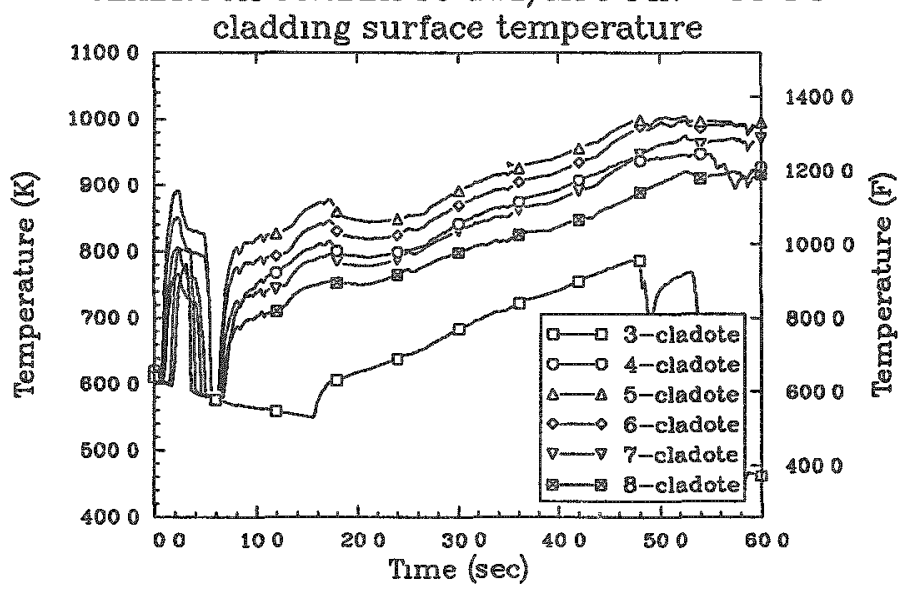

SEABRO0K 50\%DBA 50 GWD/MTU PIN--PF 18

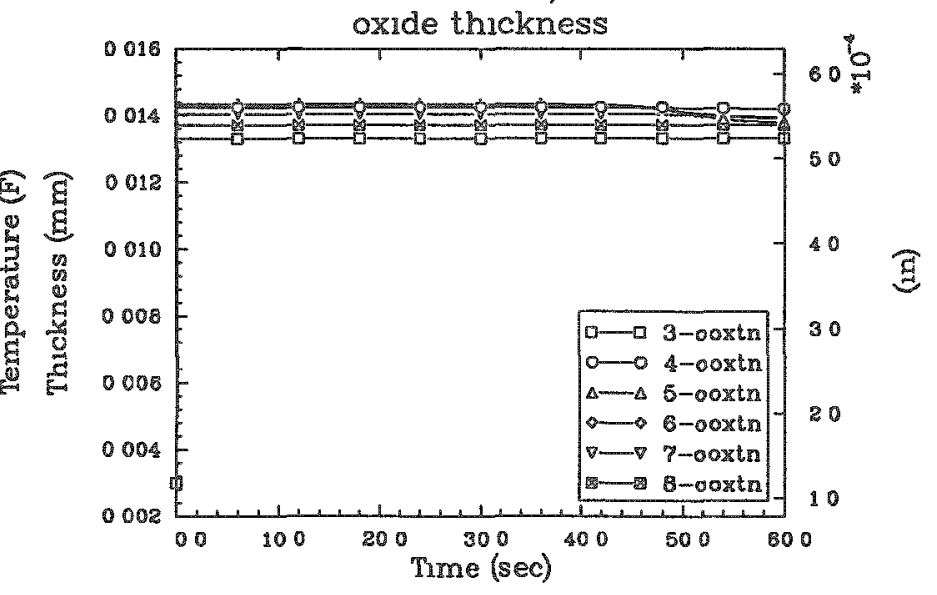



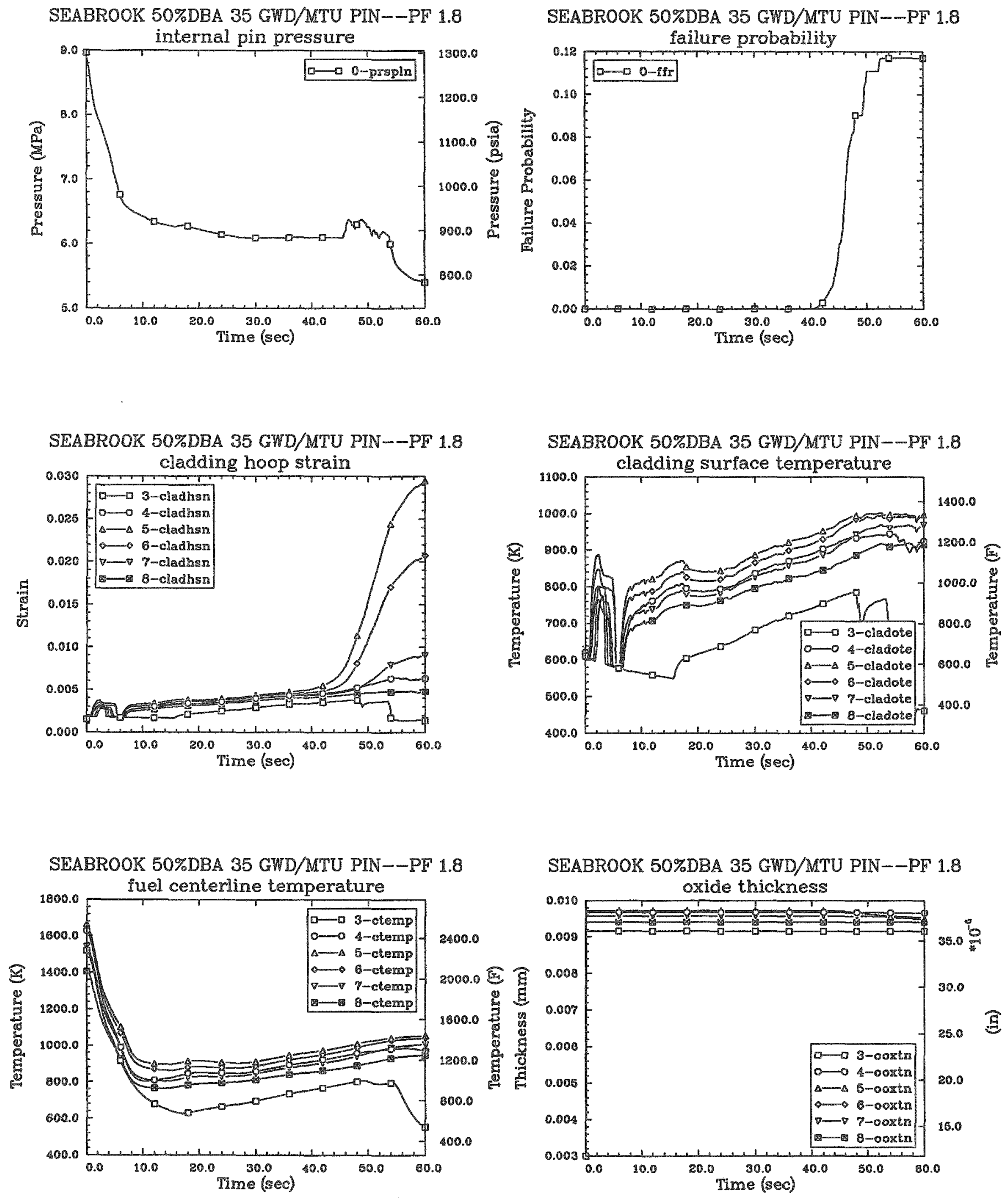

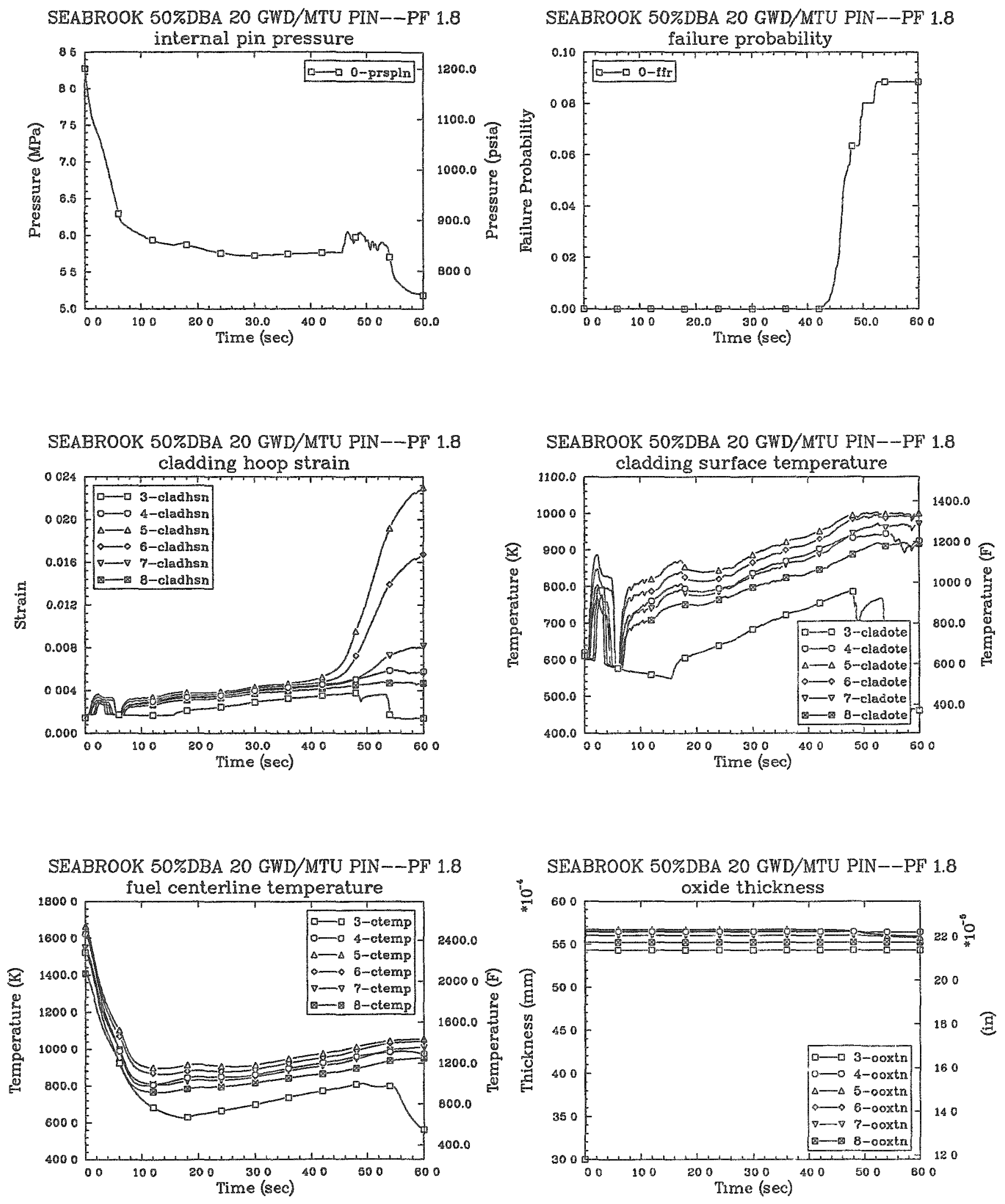

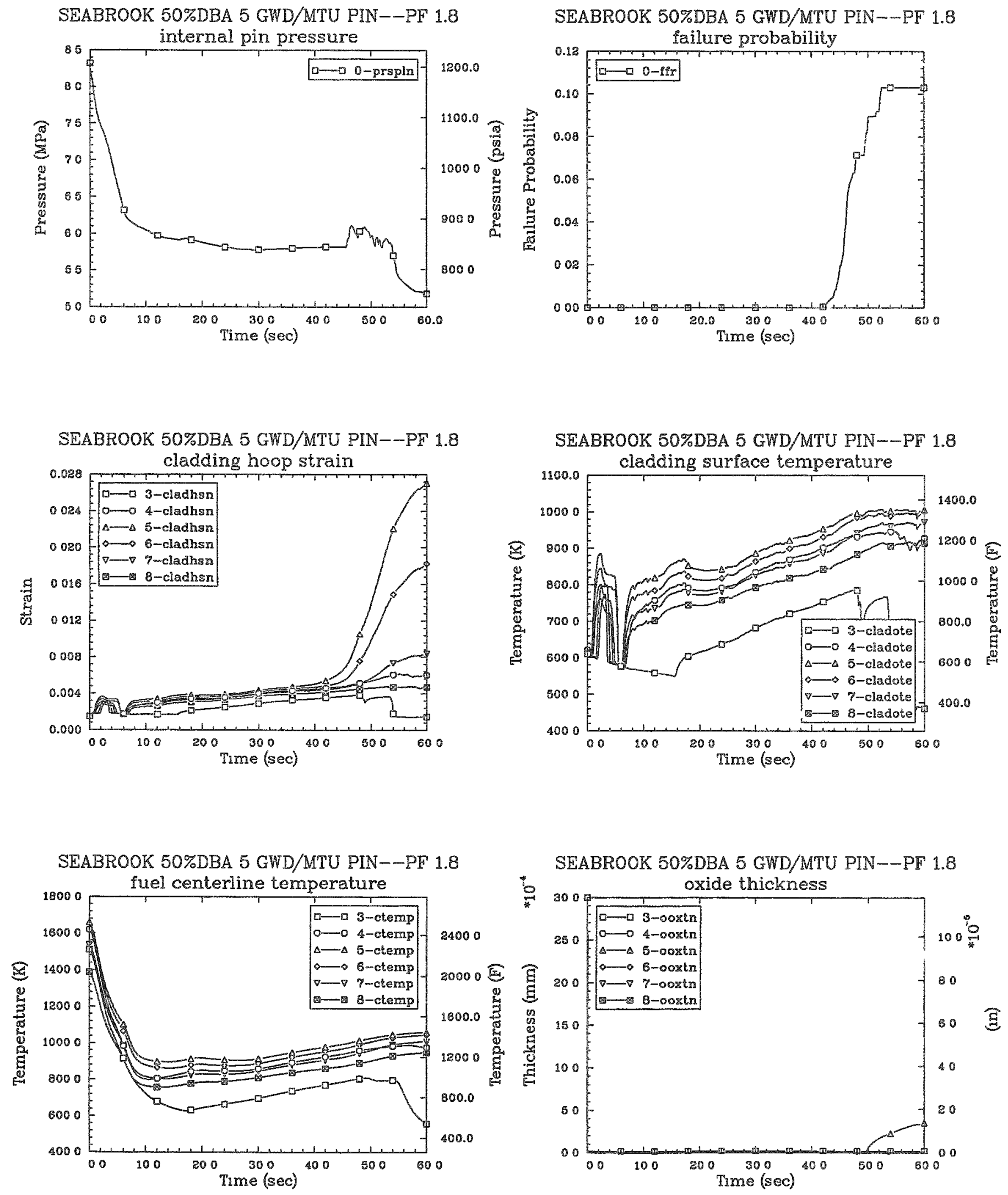
SEABROOK 100\%DBA 50 GWD/MTU PIN--PF2.32 W/TRIPSEABROOK 100\%DBA 50 GWD/MTU PIN--PF2.32 W/TRIP
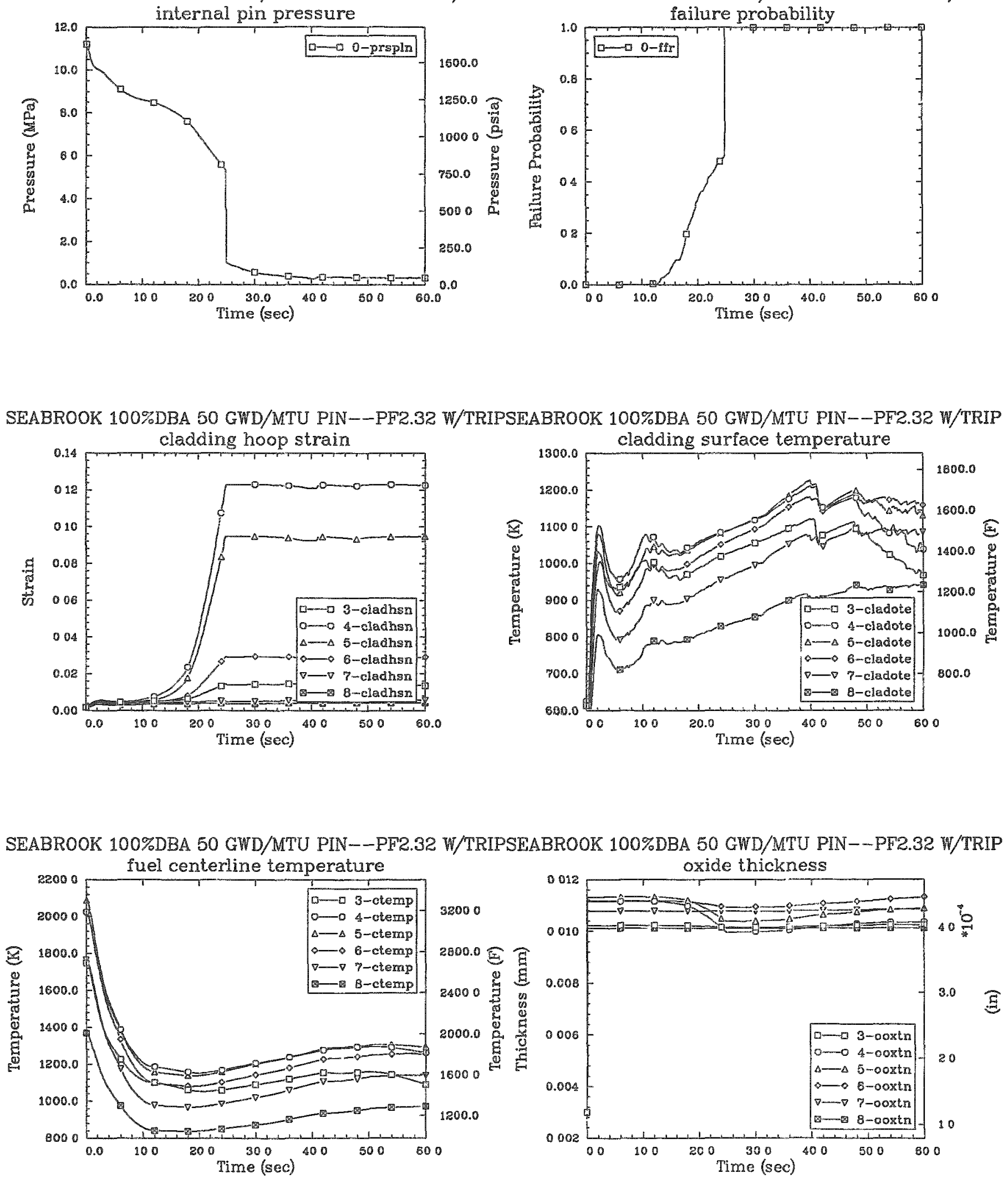
SEABROOK 100\%DBA 35 GWD/MTU PIN---PF2 32 W/TRIPSEABROOK 100\%DBA 35 GWD/MTU PIN--PF2 32 W/TRIP

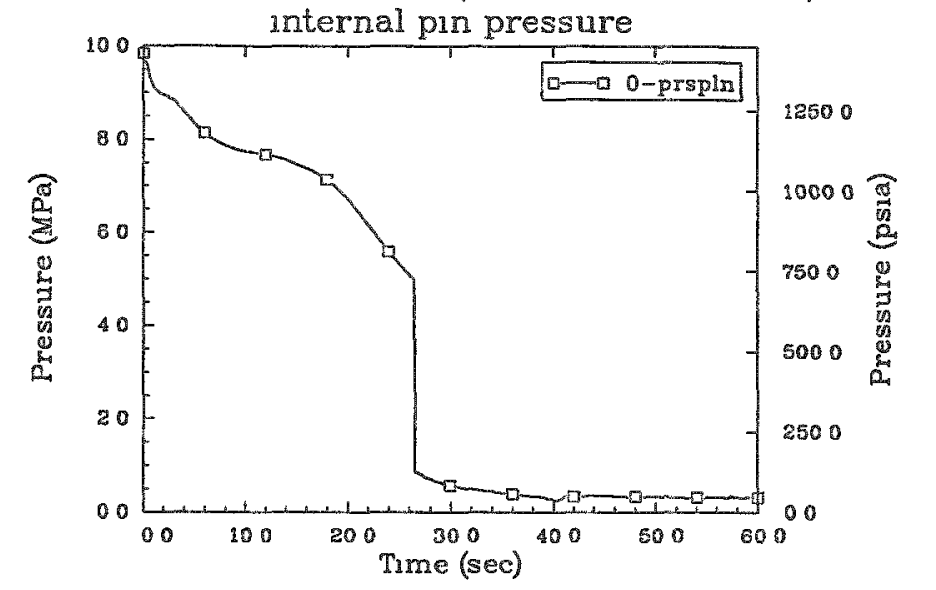

fallure probability
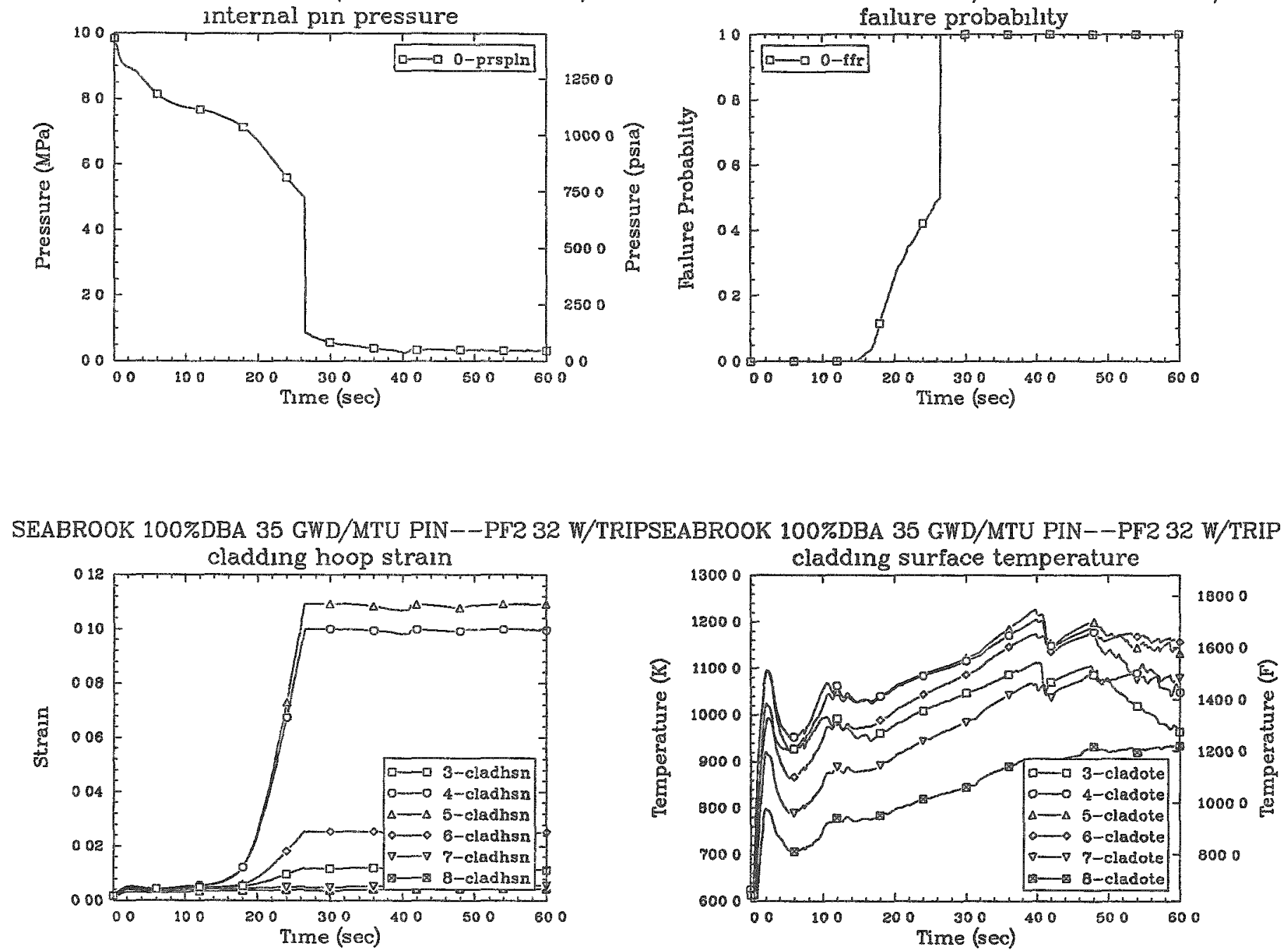

SEABROOK 100\%DBA 35 GWD/MTU PIN--PF2 32 W/TRIPSEABROOK 100\%DBA 35 GWD/MTU PIN--PF2 32 W/TRIP
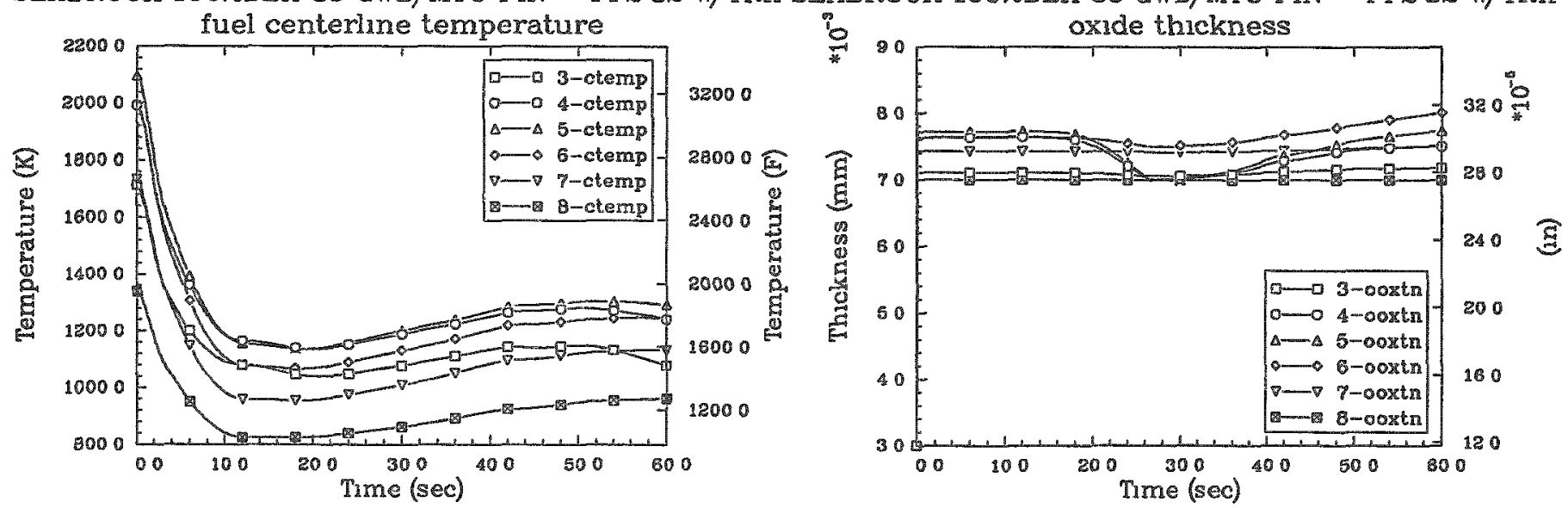

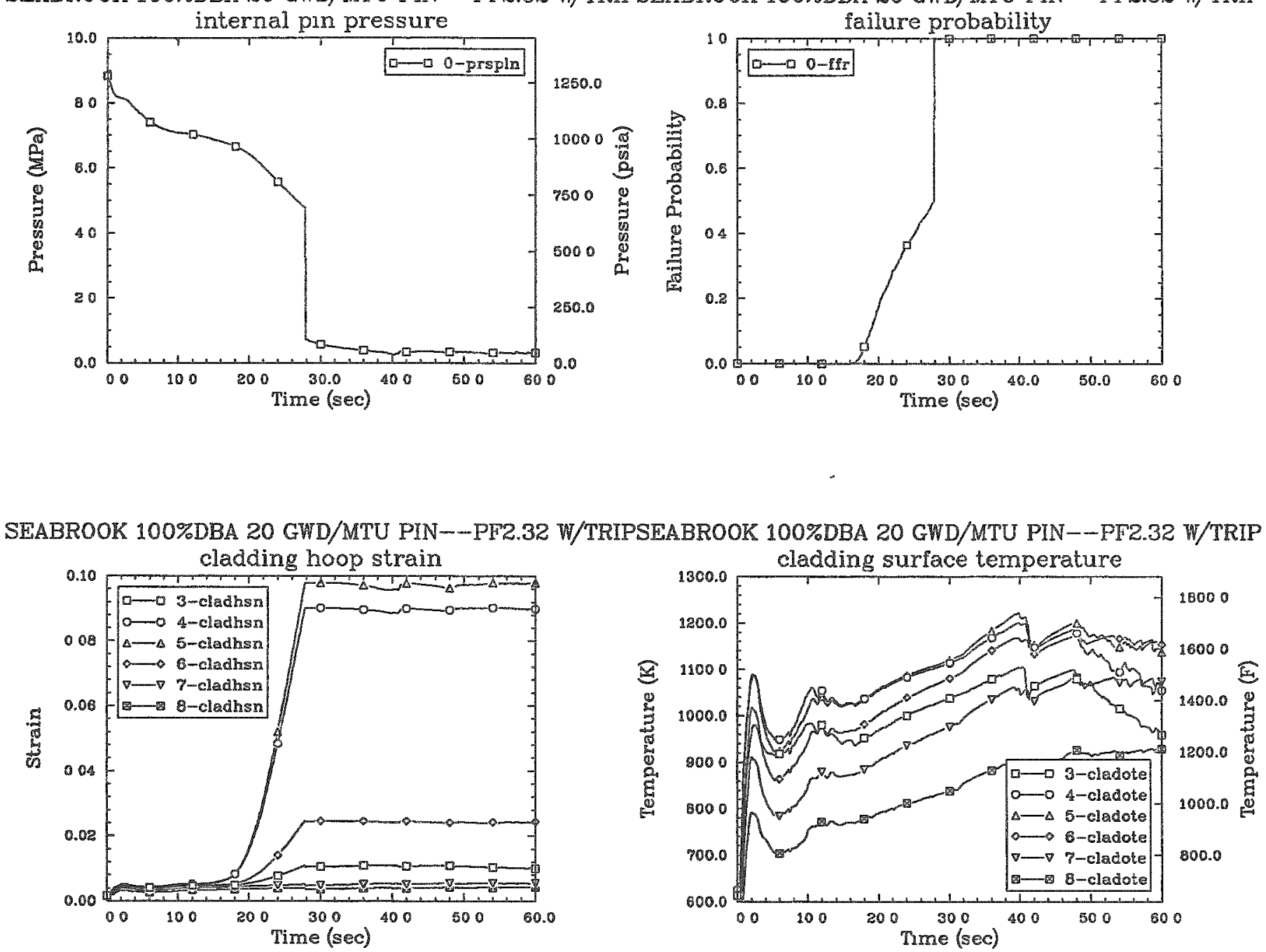

SEABROOK 100\%DBA 20 GWD/MTU PIN--PF2.32 W/TRIPSEABROOK 100\%DBA 20 GWD/MTU PIN--PF2.32 W/TRIP
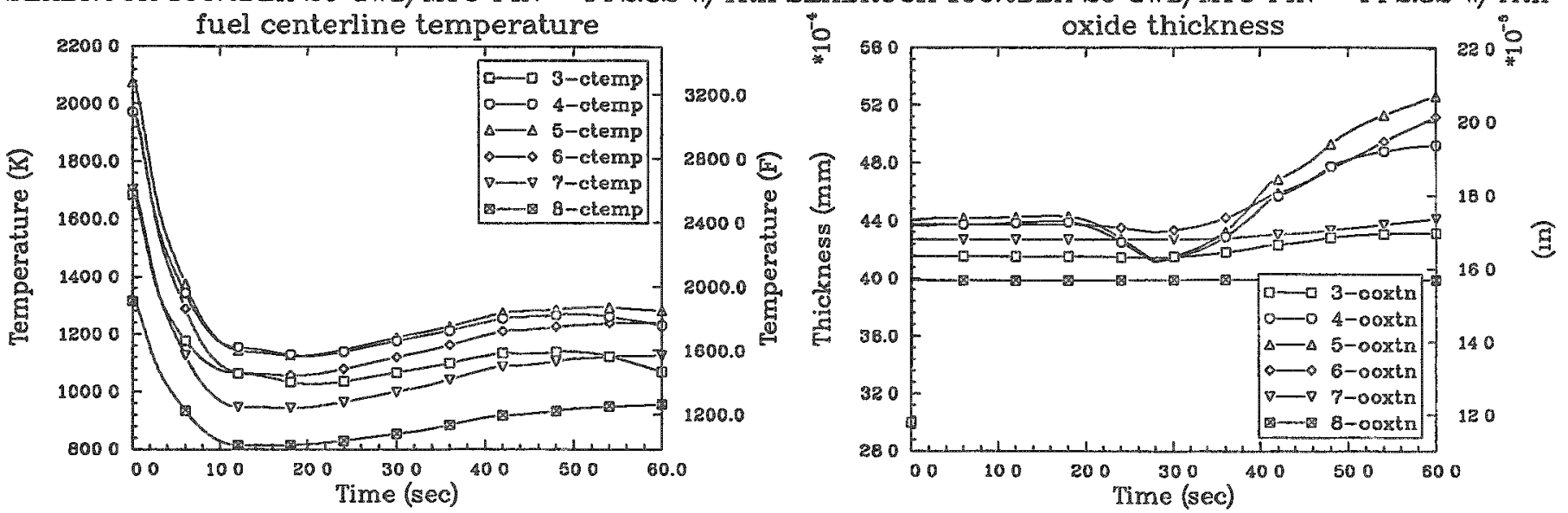

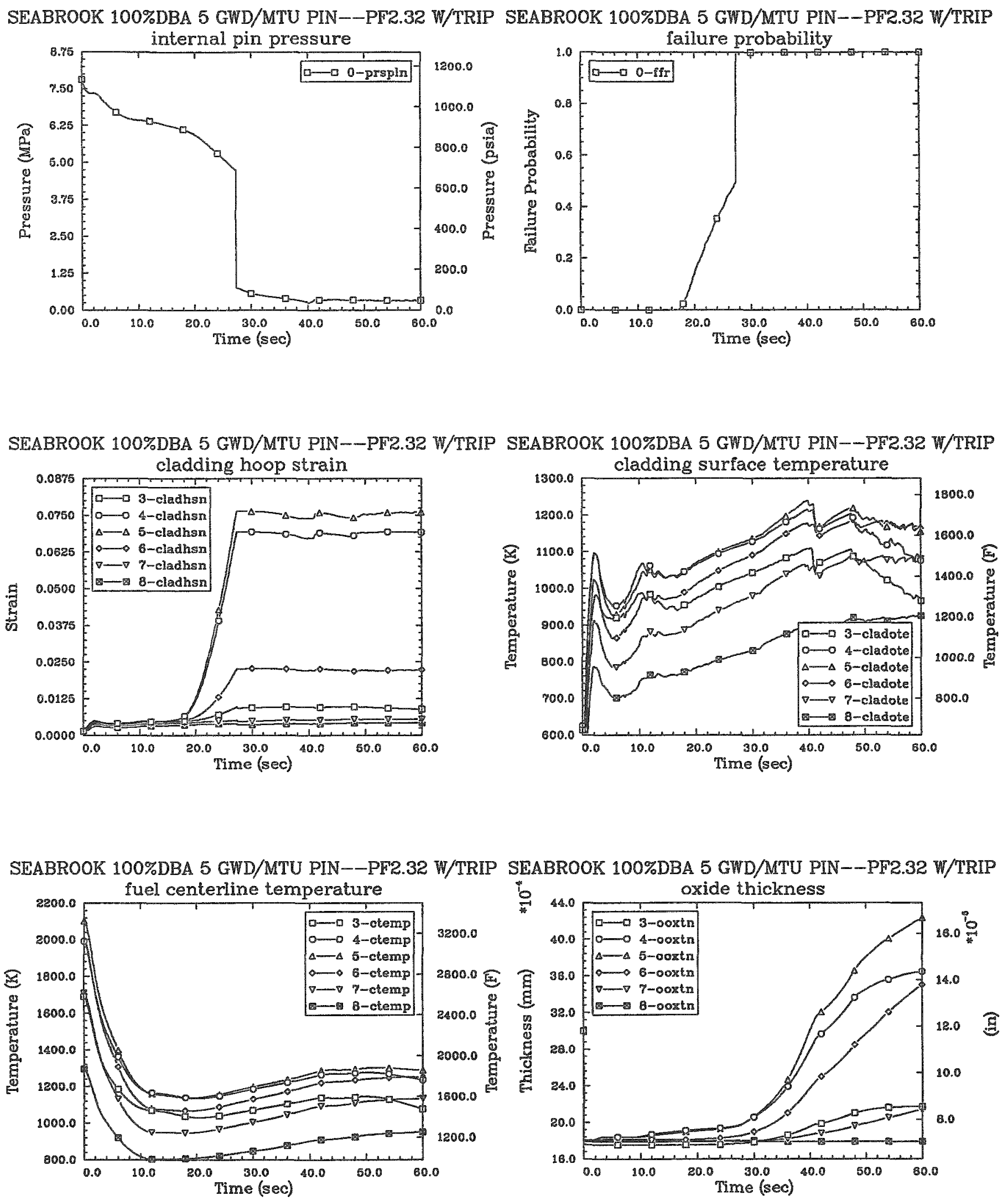
SEABROOK 100\%DBA 50 GWD/MTU PIN--PF2 2 W/TRIP SEABROOK 100\%DBA 50 GWD/MTU PIN--PF2 2 W/TRIP
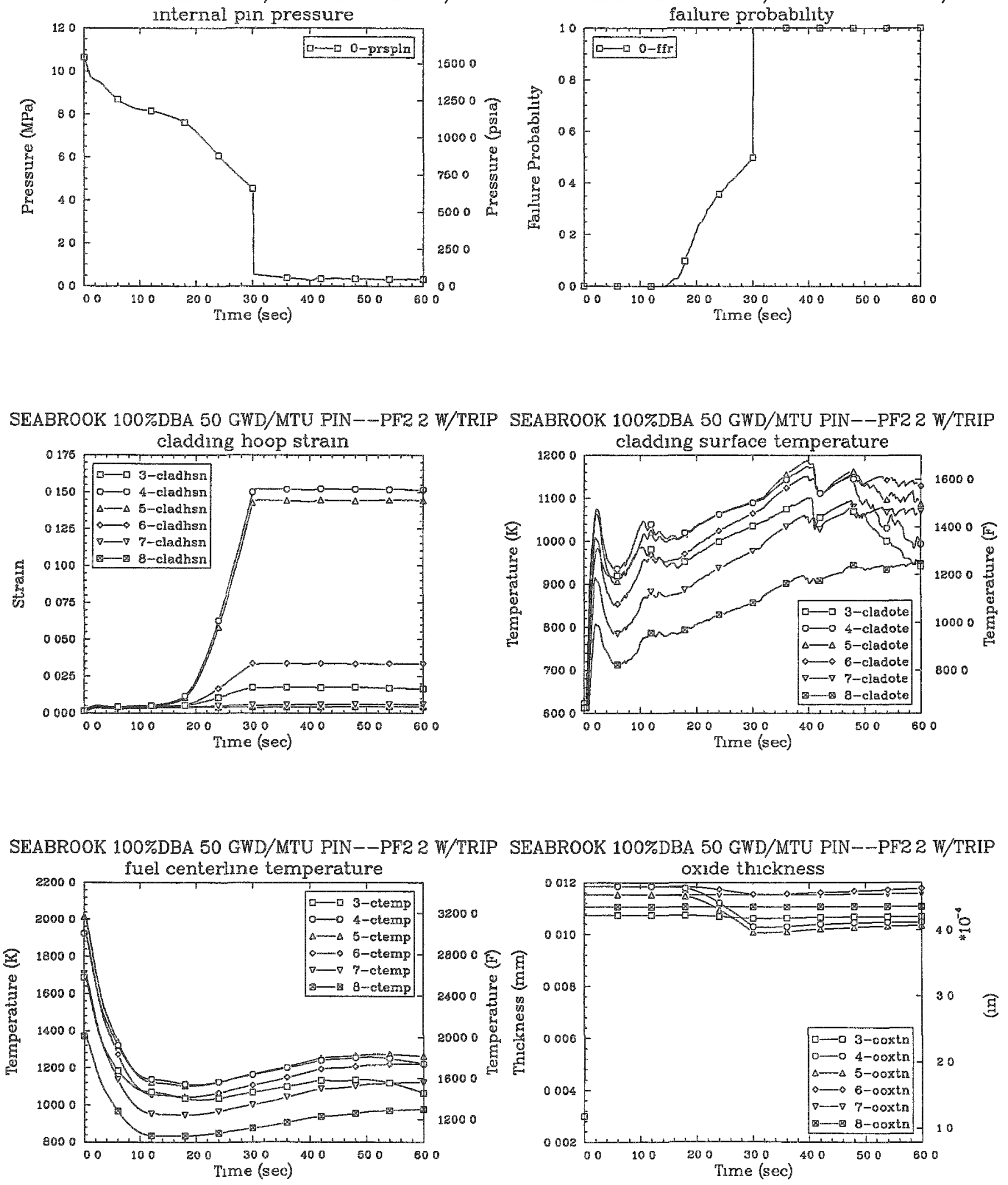
SEABROOK 100\%DBA 35 GWD/MTU PIN--PF2.2 W/TRIP SEABROOK 100\%DBA 35 GWD/MTU PIN--PF2.2 W/TRIP
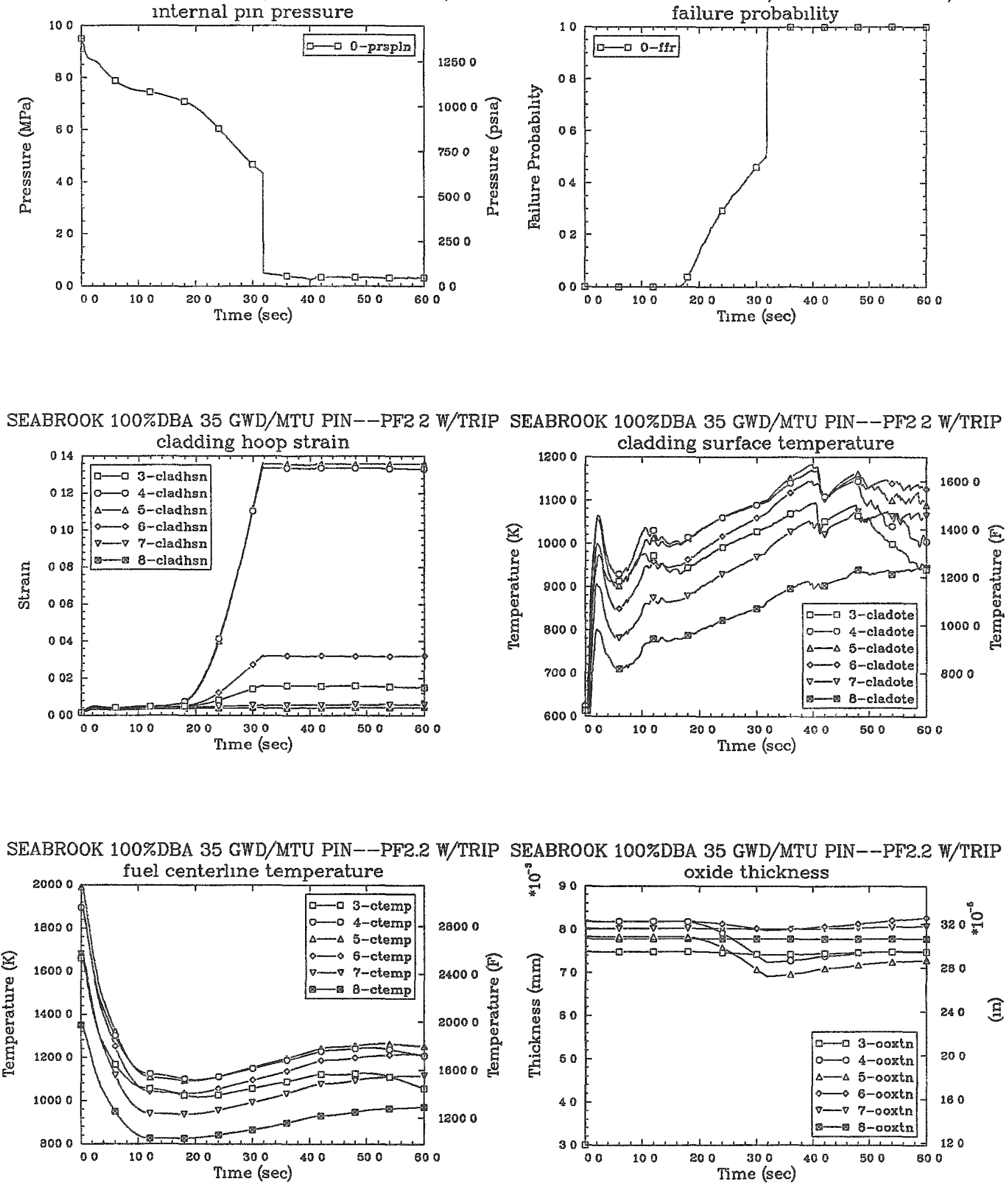
SEABROOK 100\%DBA $20 \mathrm{GWD} / \mathrm{MTU}$ PIN--PF2.2 W/TRIP SEABROOK 100\%DBA 20 GWD/MTU PIN--PF22 W/TRIP
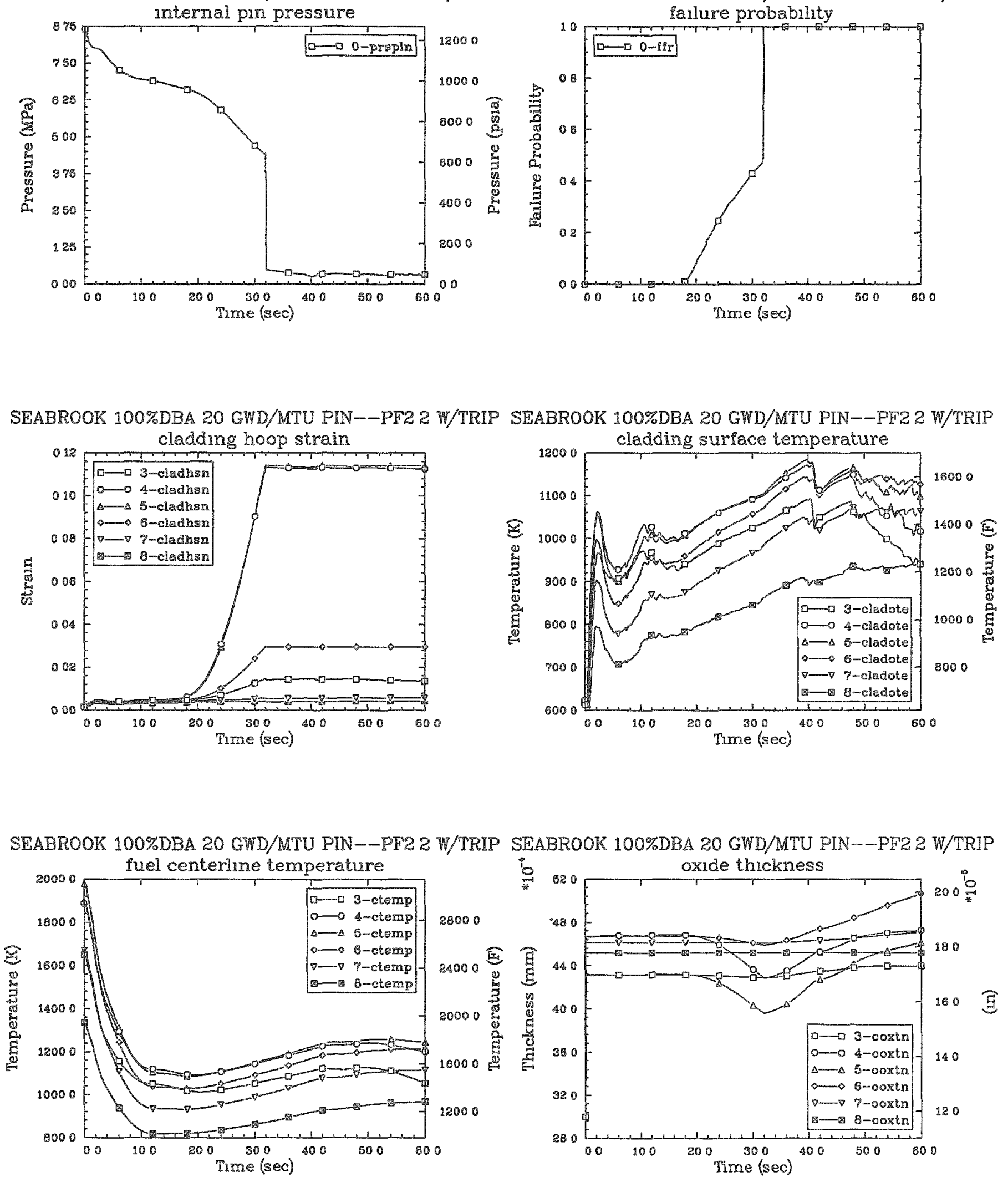
SEABROOK 100\%DBA 5 GWD/MTU PIN--PF2.2 W/TRIP

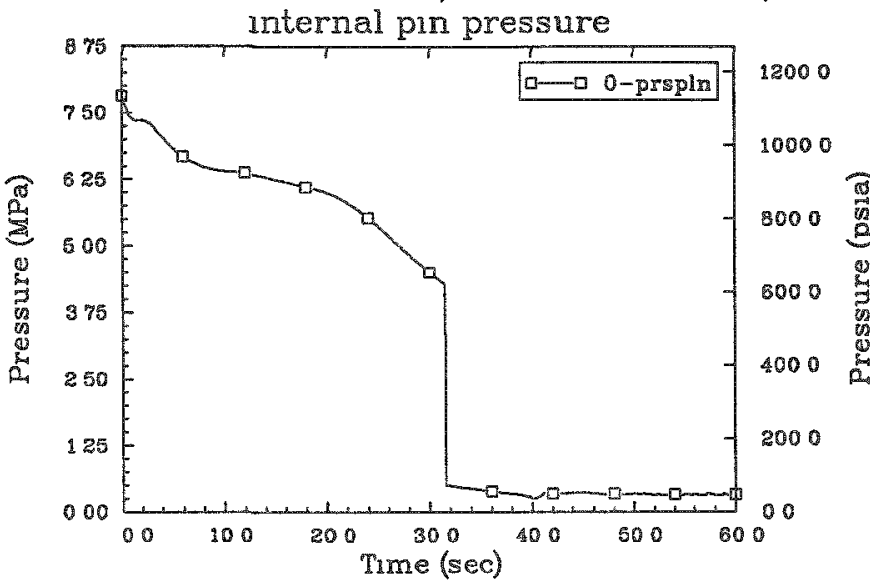

SEABROOK 100\%DBA 5 GWD/MTU PIN--PF2 2 W/TRIP

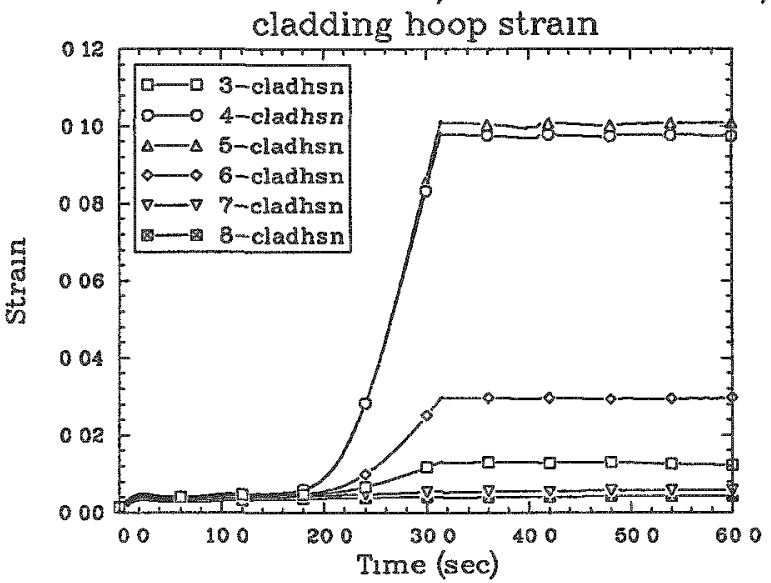

SEABROOK 100\%DBA 5 GWD/MTU PIN--PF2.2 W/TRIP

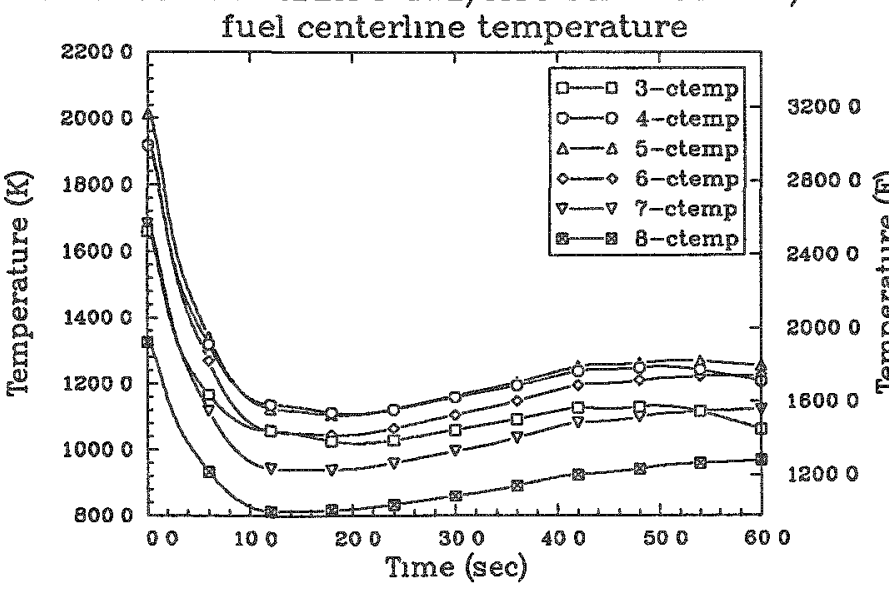

SEABROOK 100\%DBA 5 GWD/MTU PIN--PF2 2 W/TRIP failure probability

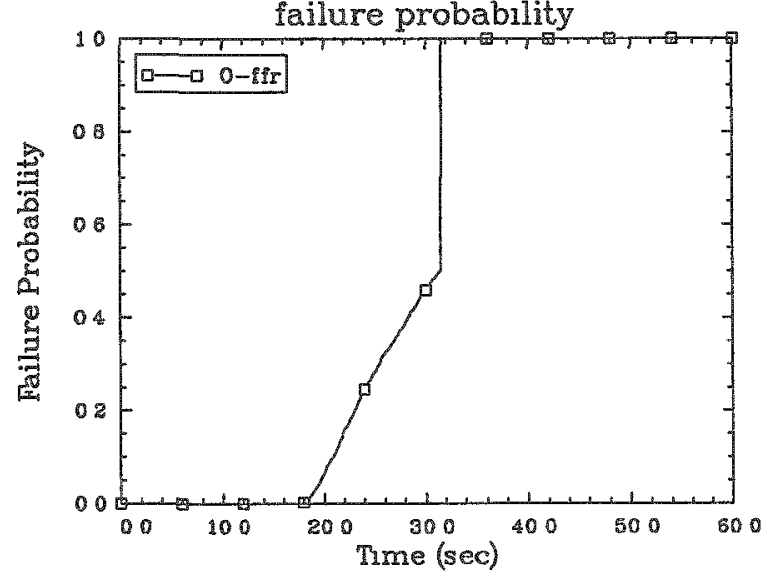

SEABROOK 100\%DBA 5 GWD/MTU PIN--PF2 2 W/TRIP

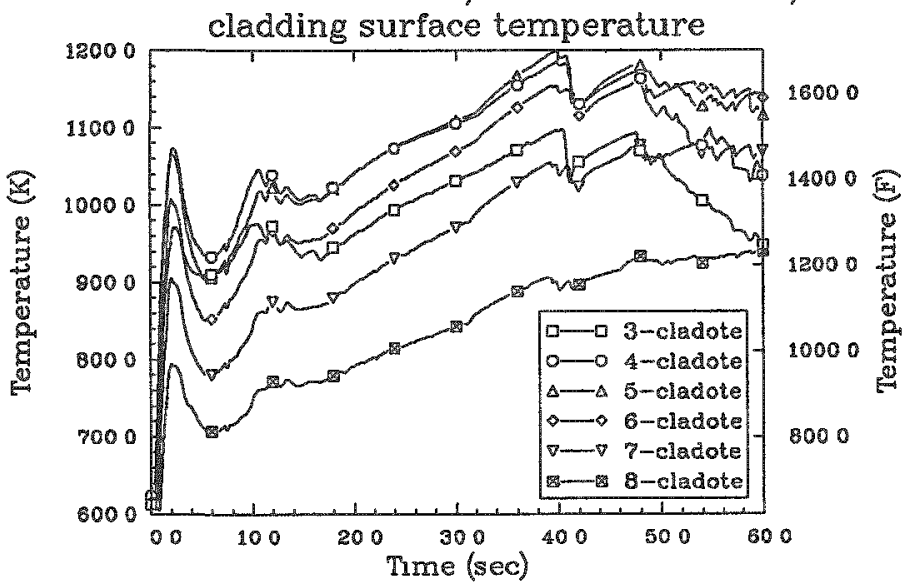

SEABROOK 100\%DBA 5 GWD/MTU PIN--PFZ2 W/TRIP

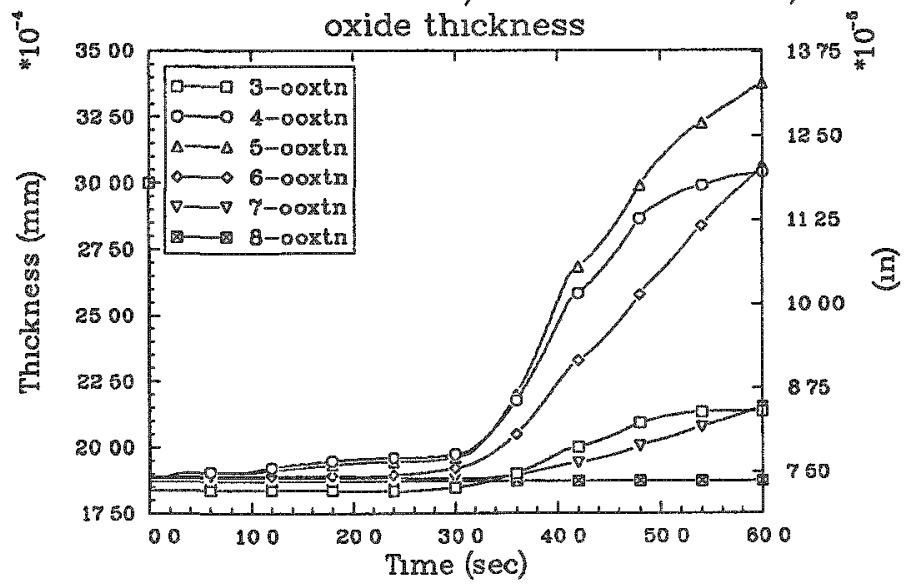



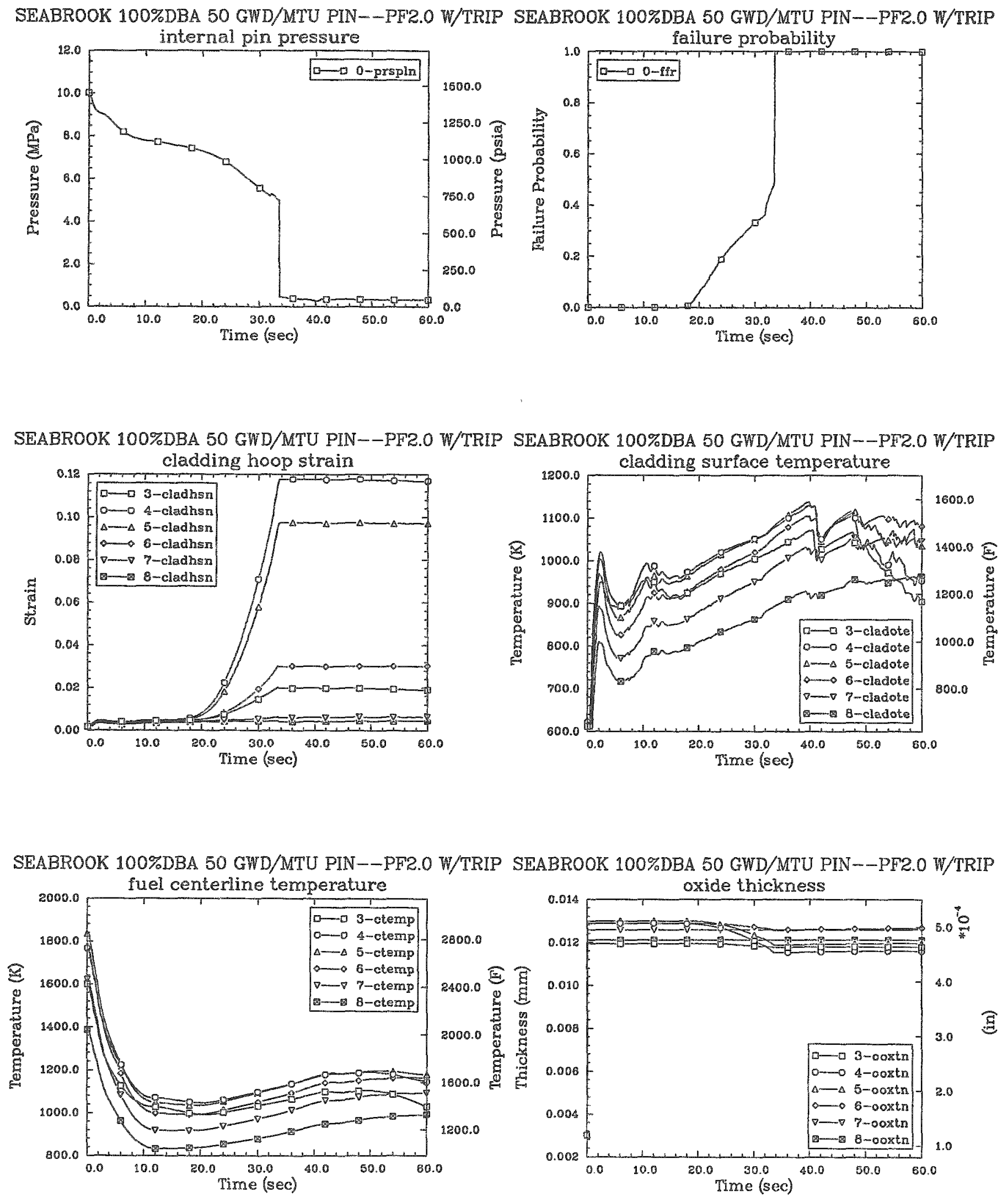
SEABROOK 100\%DBA 35 GWD/MTU PIN--PF2.0 W/TRIP SEABROOK 100\%DBA 35 GWD/MTU PIN--PF2 0 W/TRIP
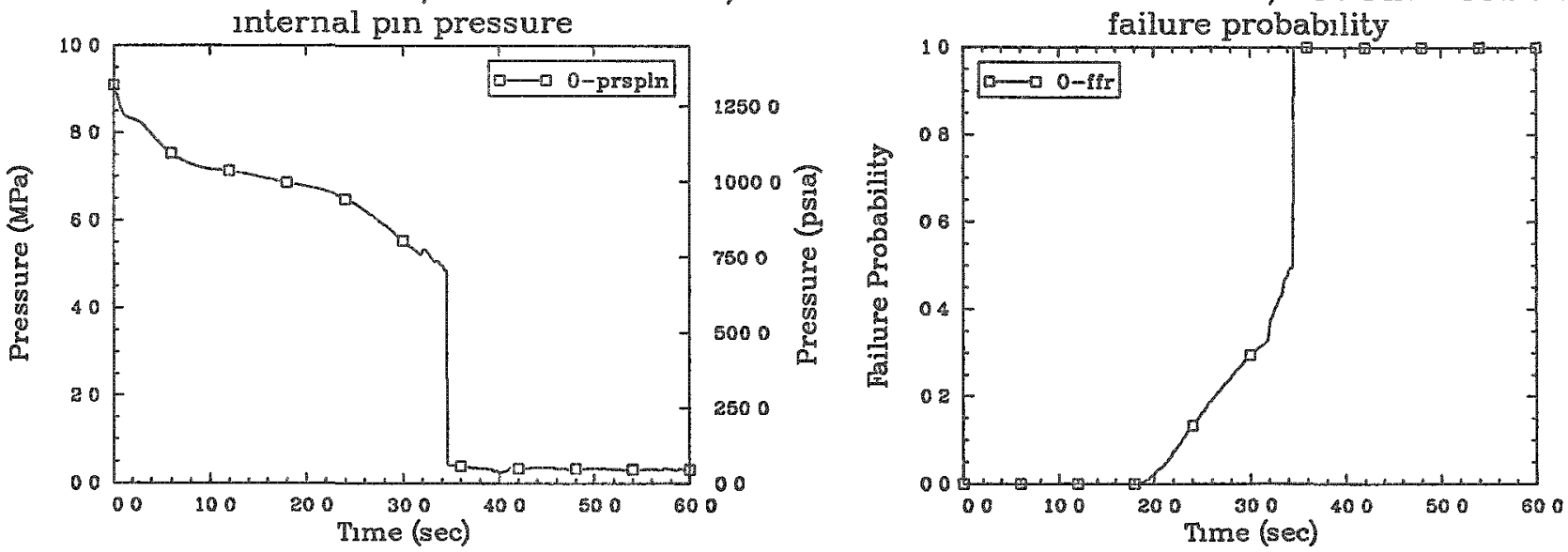

SEABROOK 100\%DBA 35 GWD/MTU PIN--PF2.0 W/TRIP SEABROOK 100\%DBA 35 GWD/MTU PIN--PF20 W/TRIP
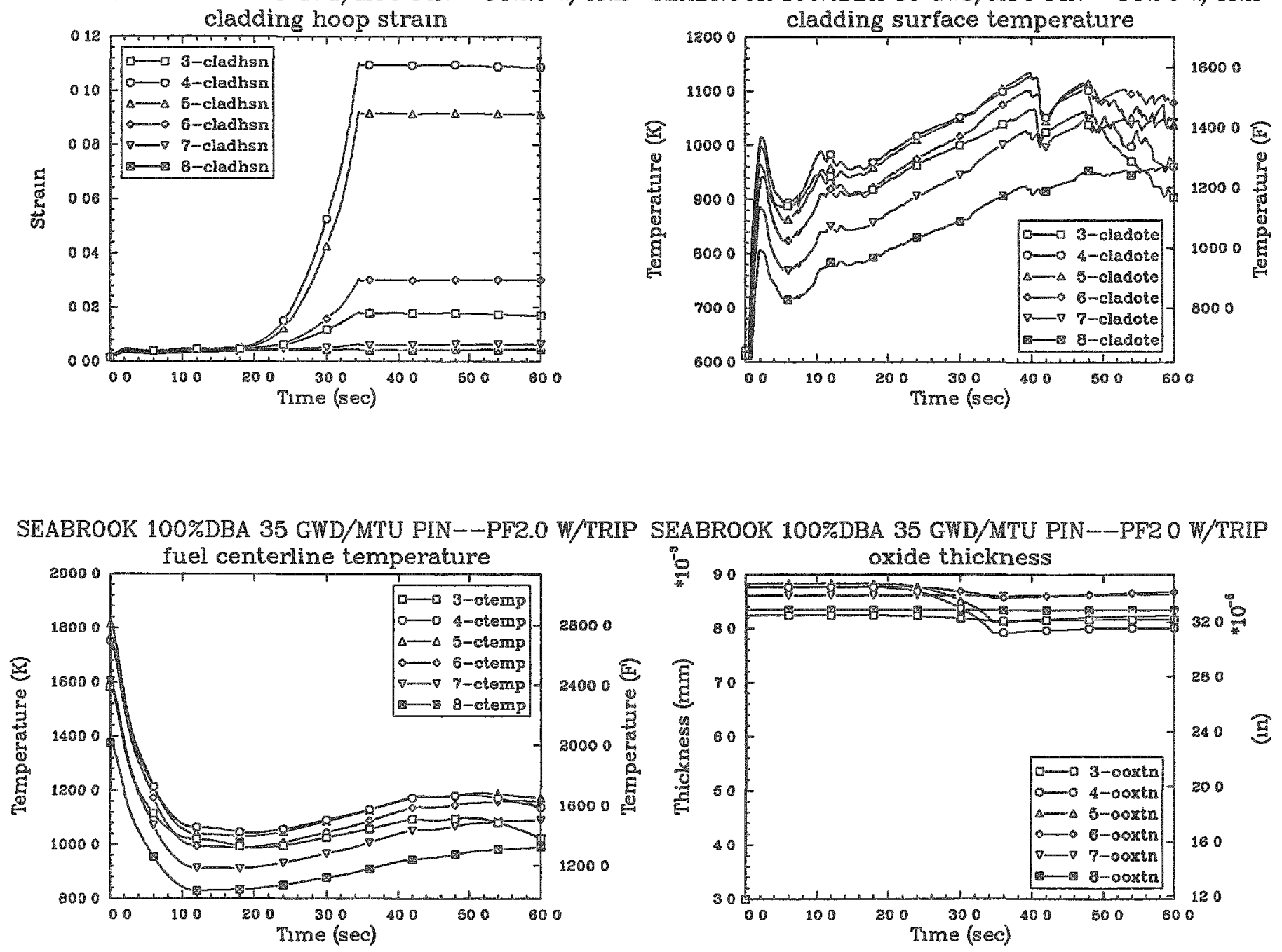
SEABROOK 100\%DBA $20 \mathrm{GWD} / \mathrm{MTU}$ PIN--PF2 0 W/TRIP SEABROOK 100\%DBA $20 \mathrm{GWD} / \mathrm{MTU}$ PIN--PF2 0 W/TRIP
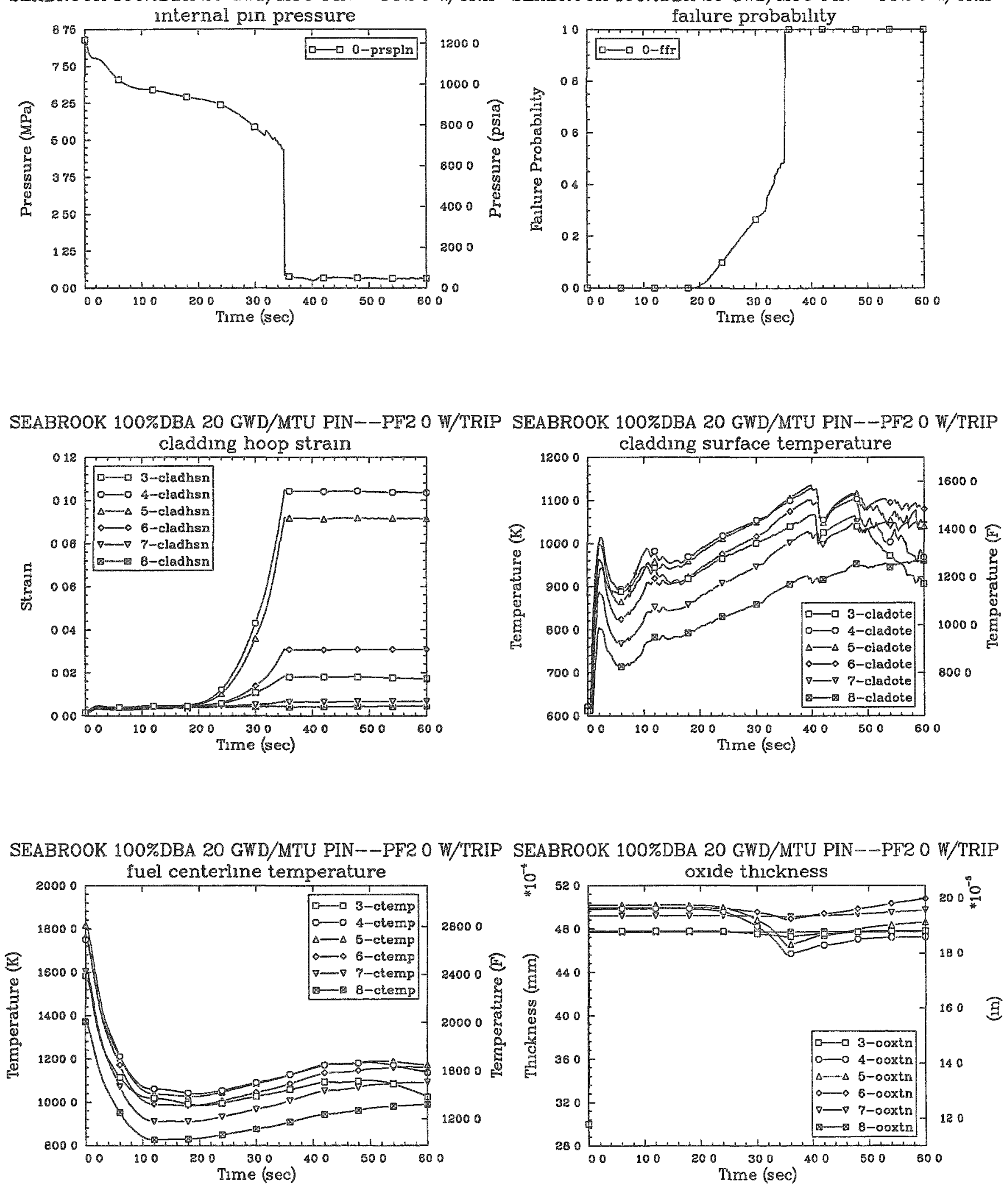
SEABROOK 100\%DBA 5 GWD/MTU PIN--PF2.0 W/TRIP

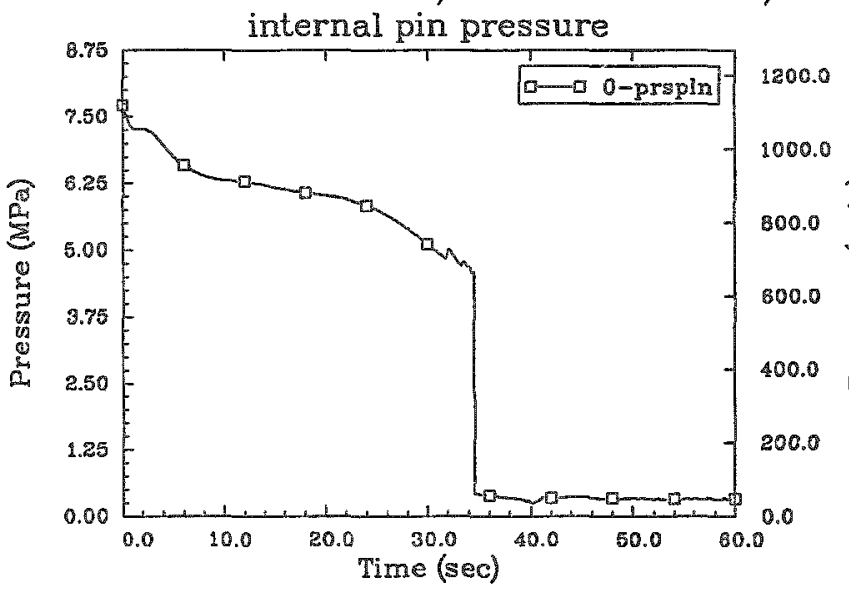

SEABROOK 100\%DBA 5 GWD/MTU PIN--PF2.0 W/TRIP failure probability

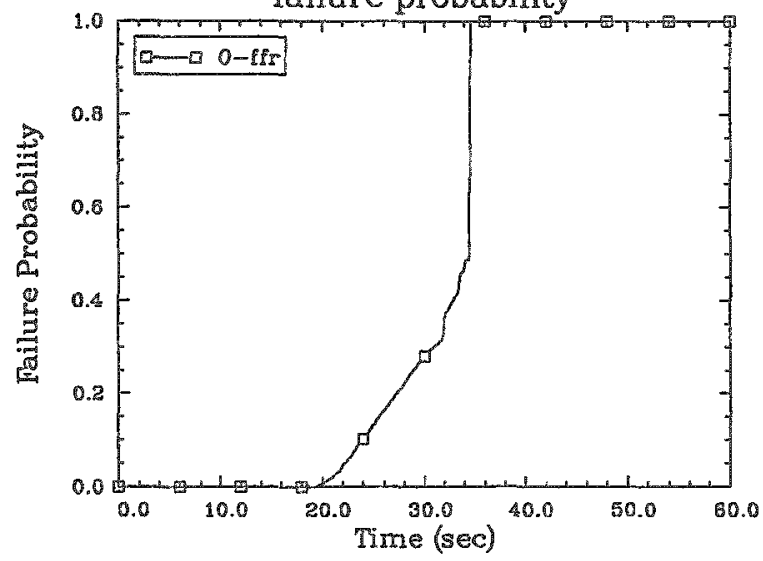

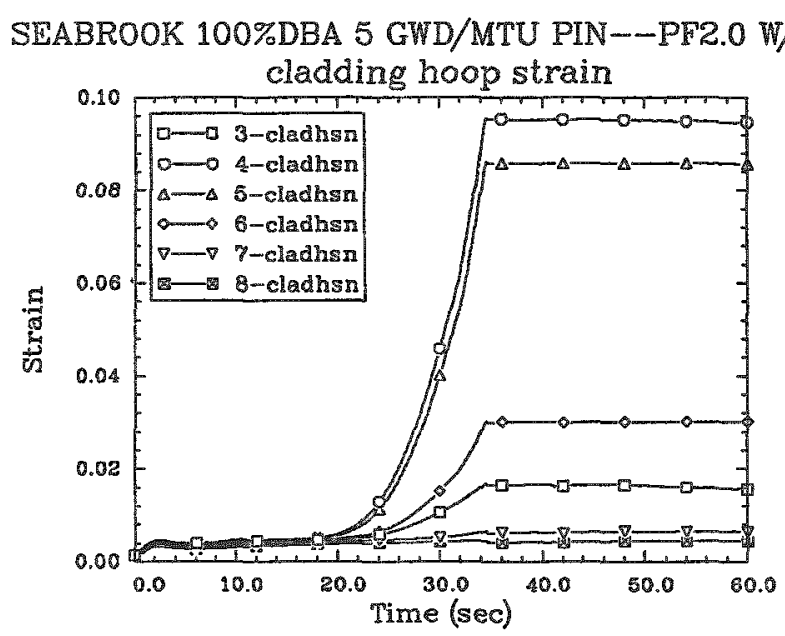
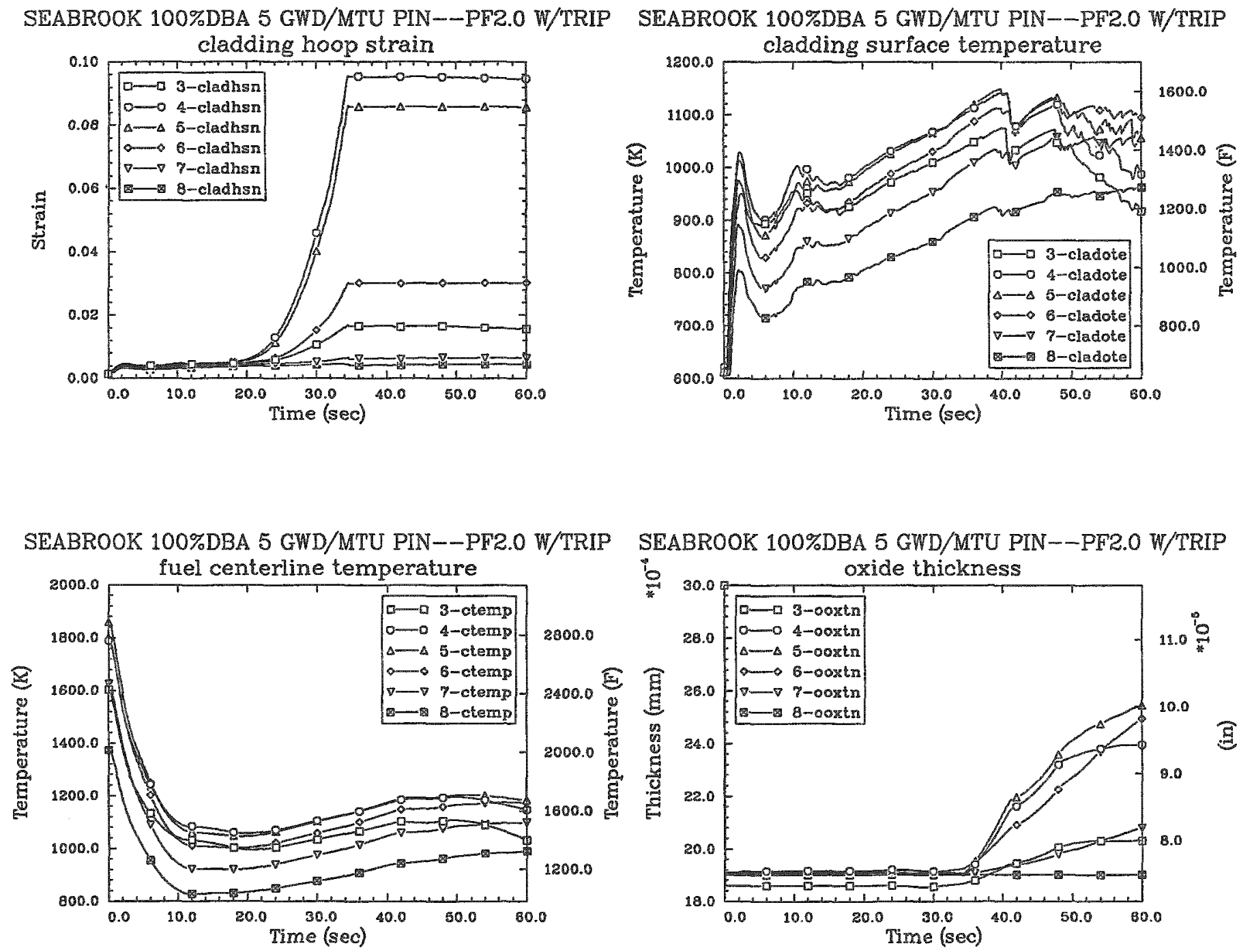

SEABROOK 100\%DBA 5 GWD/MTU PIN--PF2.0 W/TRIP io

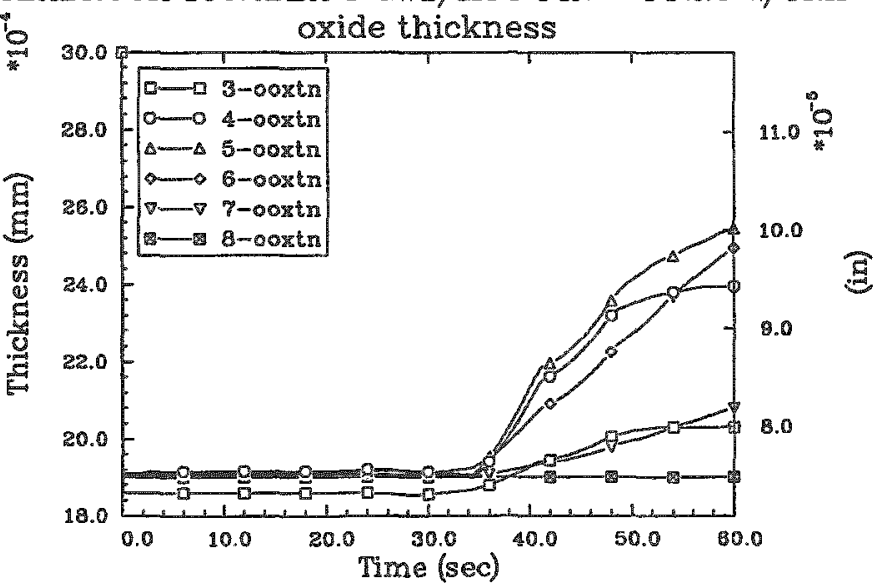


SEABROOK 100\%DBA 50 GWD/MTU PIN--PF1.8 W/TRIP SEABROOK 100\%DBA 50 GWD/MTU PIN--PF1.8 W/TRIP

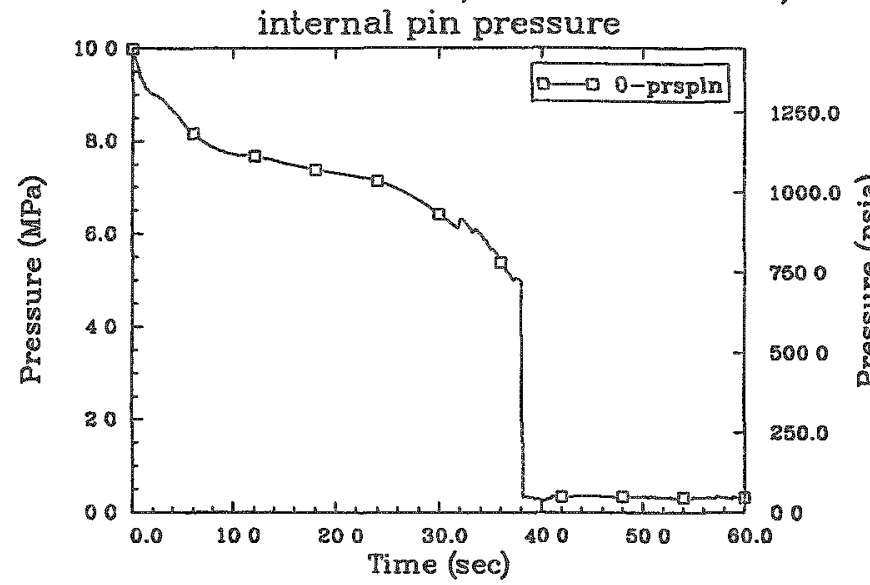

failure probability
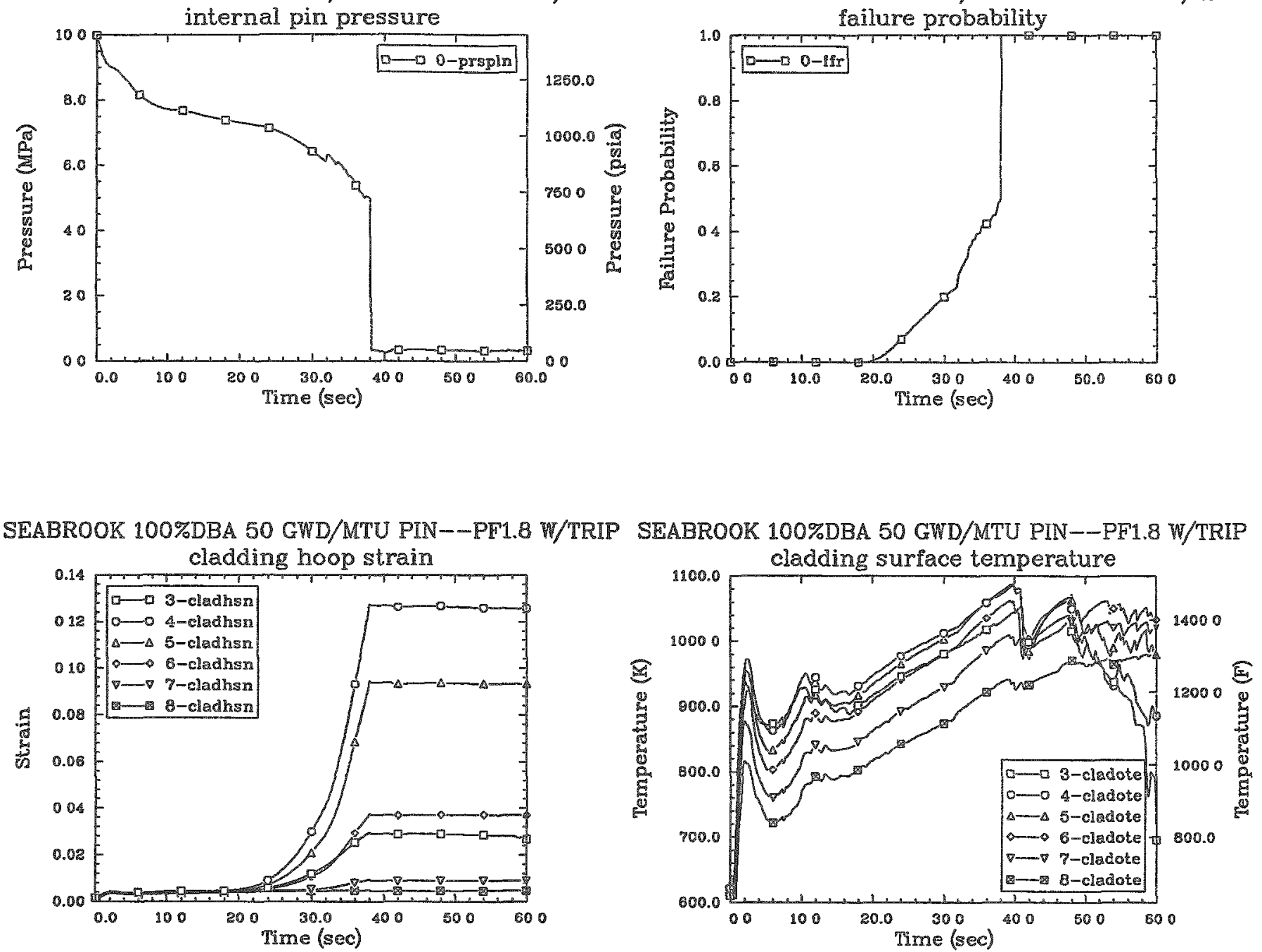

SEABROOK 100\%DBA 50 GWD/MTU PIN--PF1.8 W/TRIP SEABROOK 100\%DBA 50 GWD/MTU PIN--PF1.8 W/TRIP
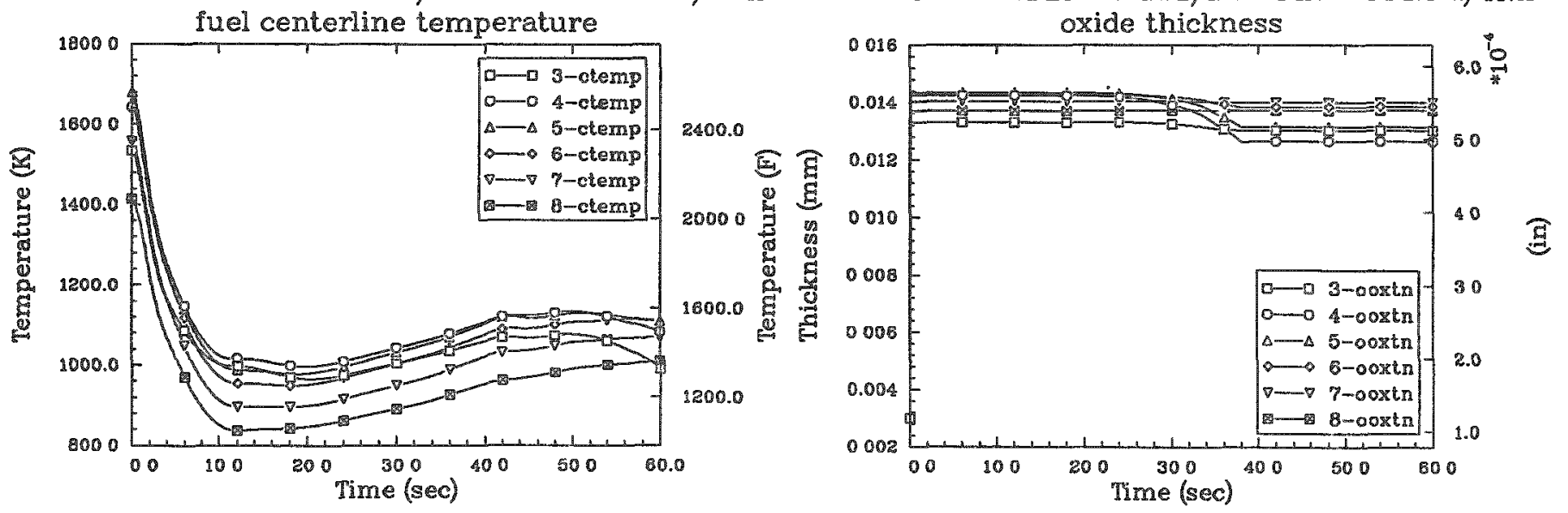

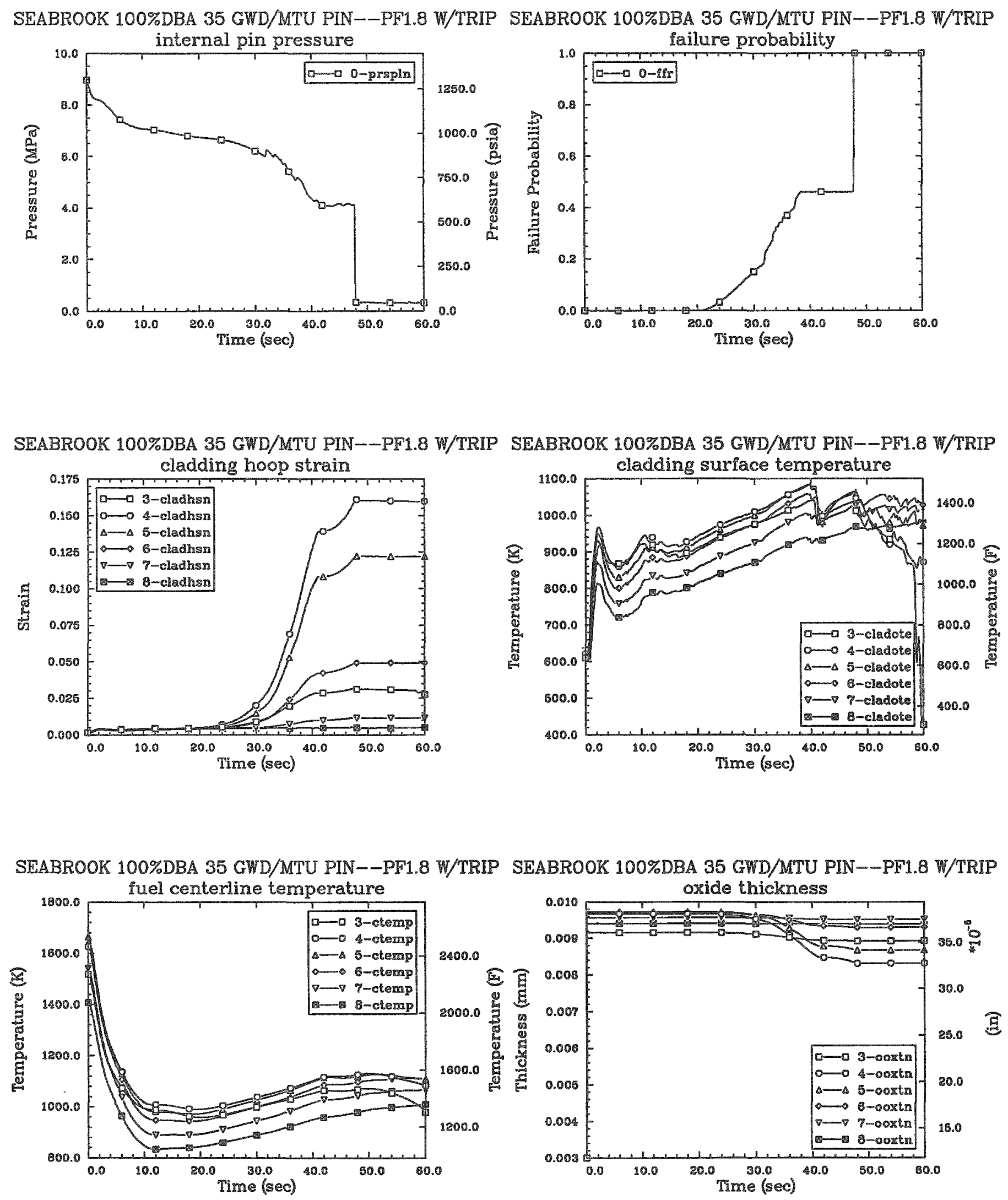
SEABROOK 100\%DBA 20 GWD/MTU PIN--PF1.8 W/TRIP SEABROOK 100\%DBA 20 GWD/MTU PIN--PF1 8 W/TRIP
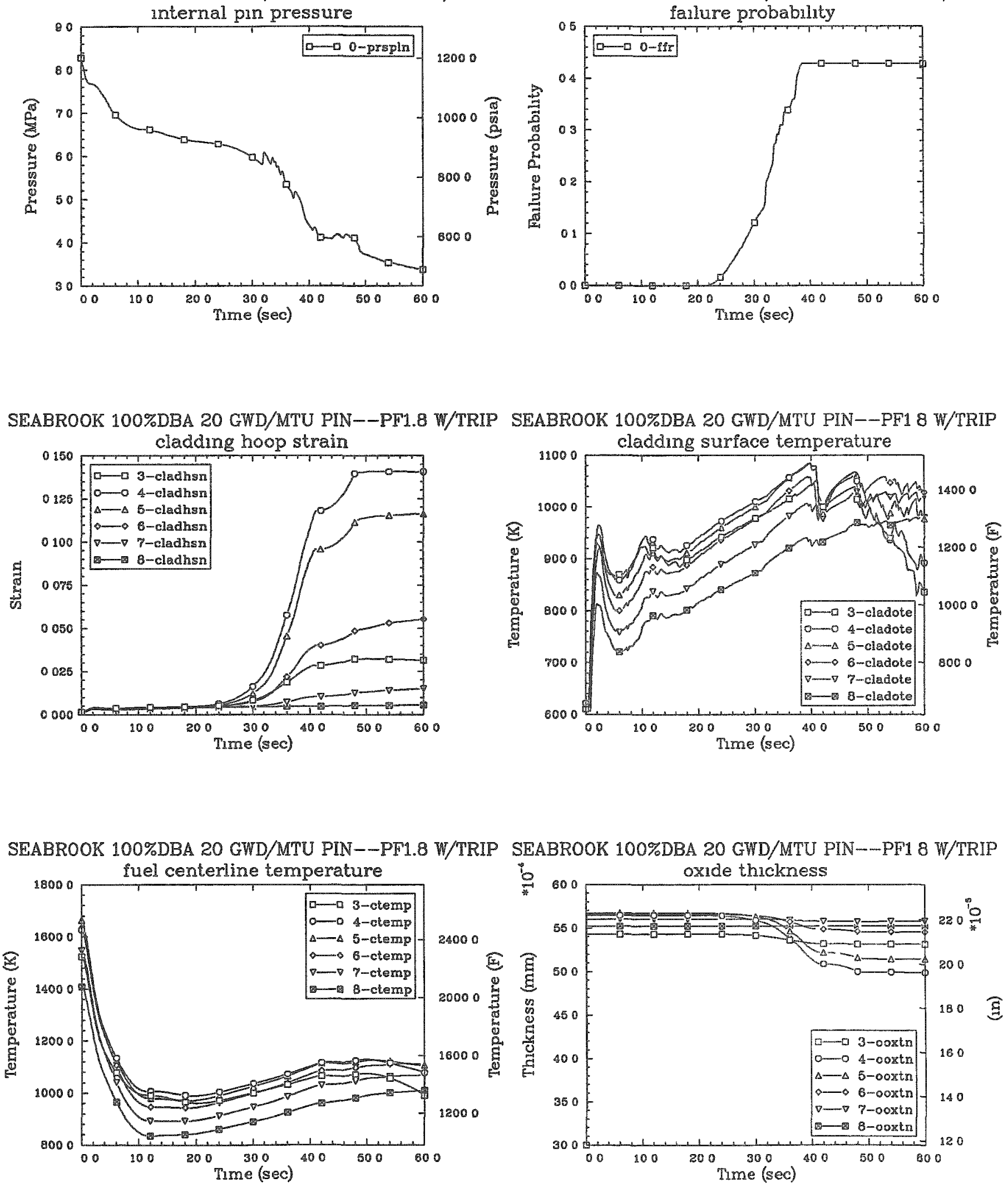
SEABROOK 100\%DBA 5 GWD/MTU PIN--PF1 8 W/TRIP

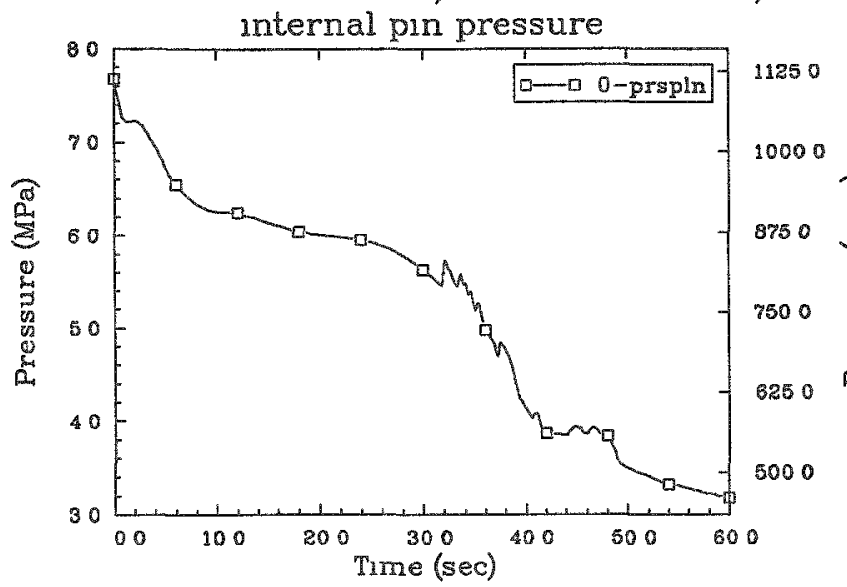

SEABROOK 100\%DBA 5 GWD/MTU PIN--PF1 8 W/TRIP fallure probabiluty

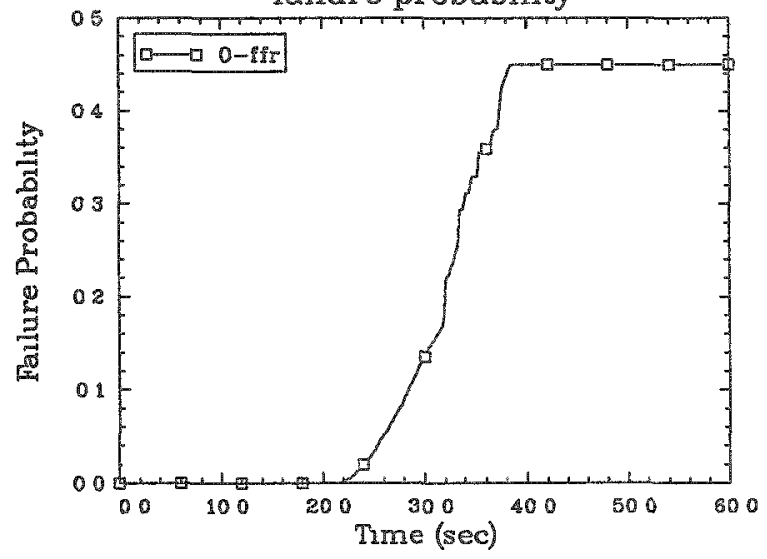

SEABROOK 100\%DBA 5 GWD/MTU PIN--PF1 8 W/TRIP

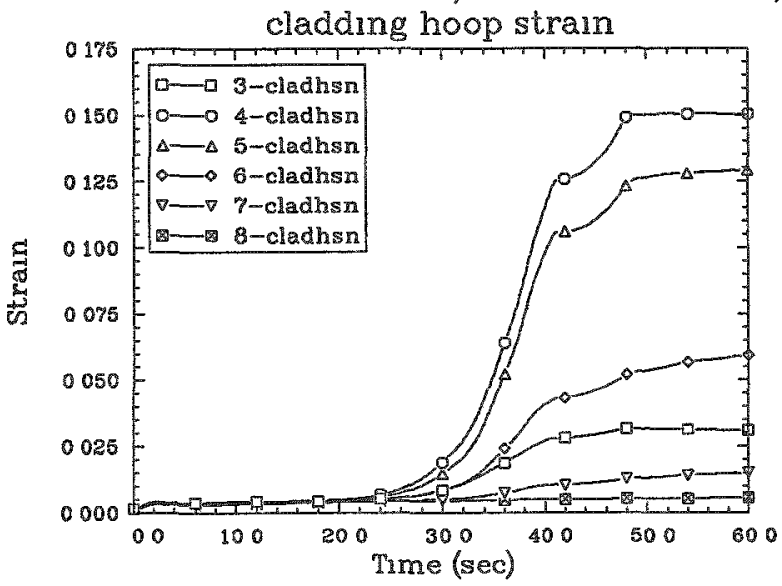

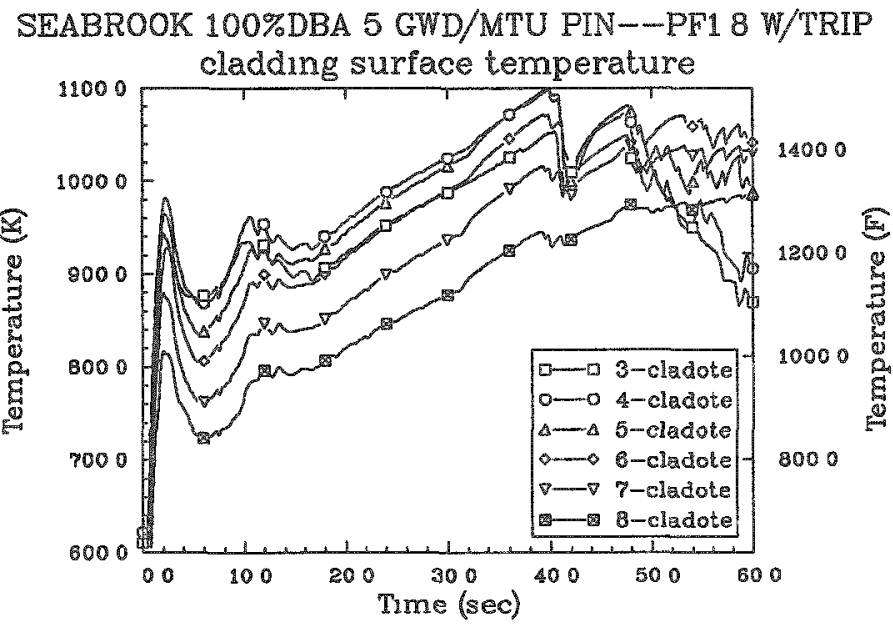

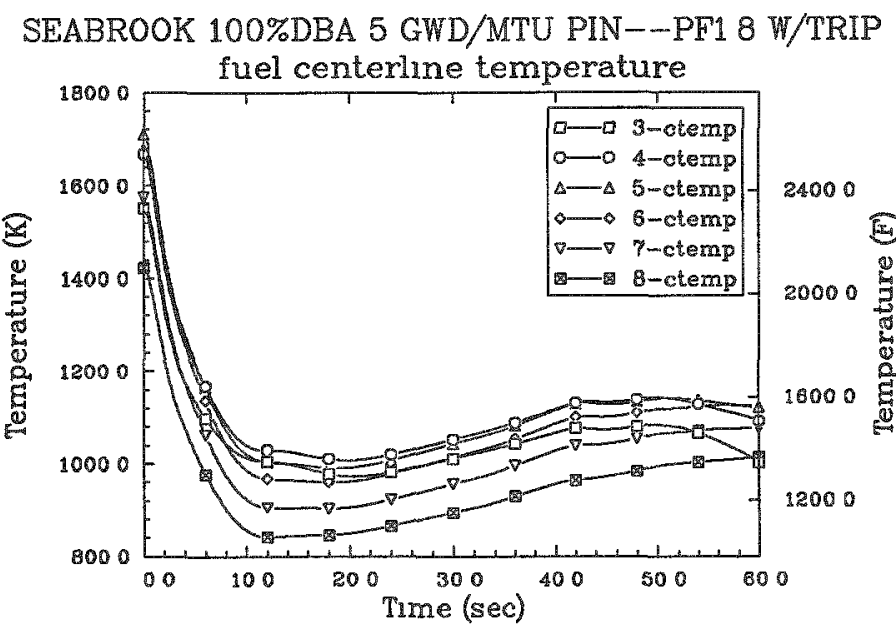

SEABROOK 100\%DBA 5 GWD/MTU PIN--PFI 8 W/TRIP

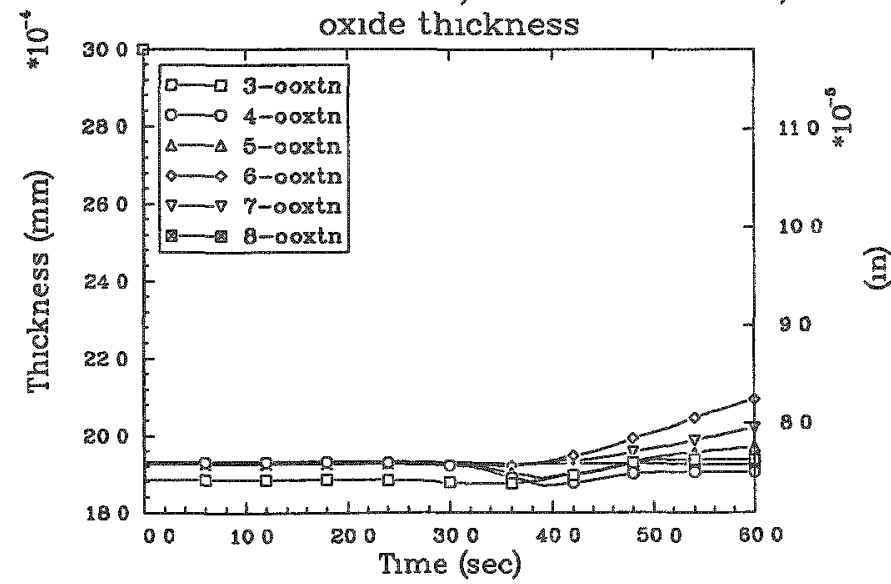



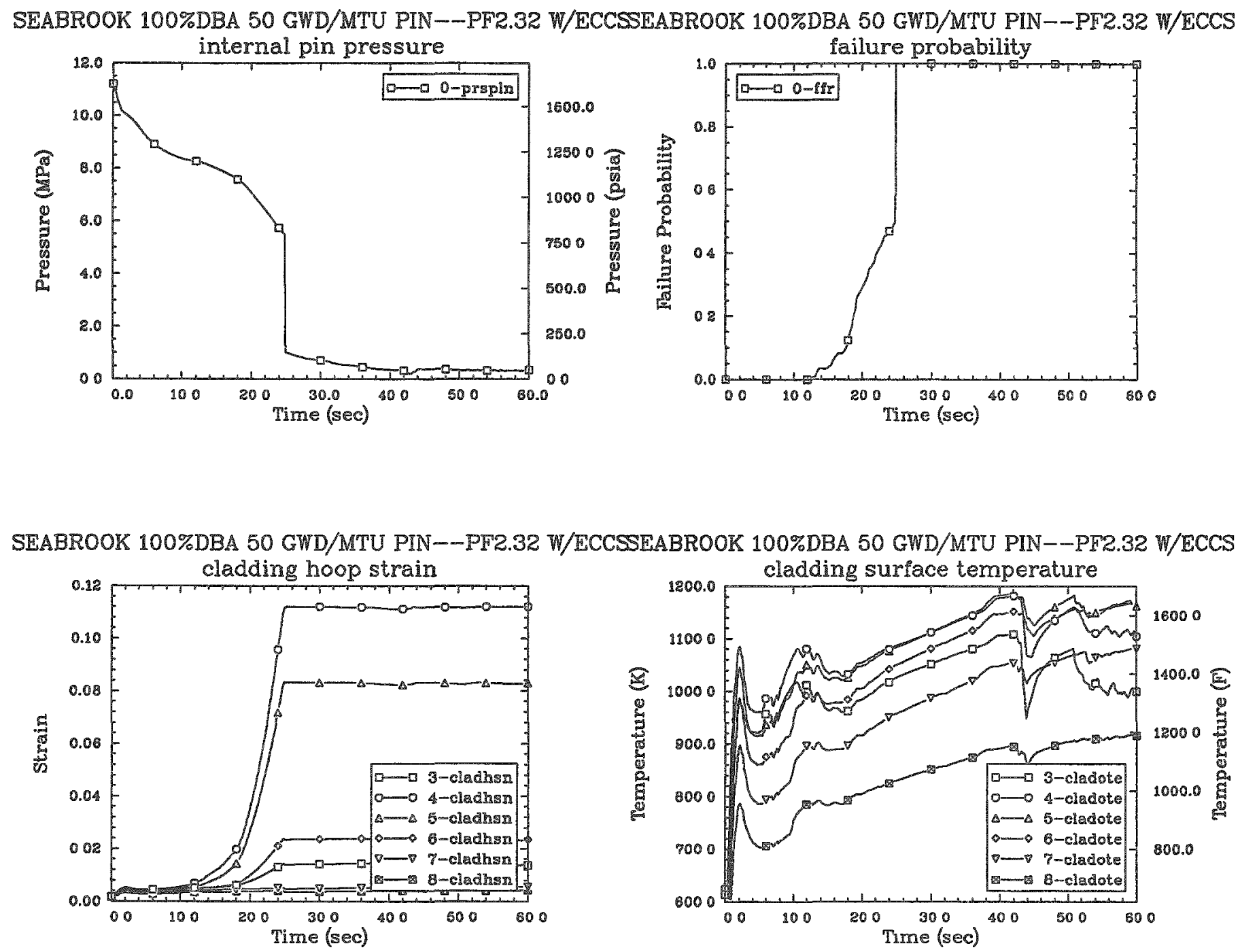

SEABROOK 100\%DBA 50 GWD/MTU PIN--PF2.32 W/ECCSSEABROOK 100\%DBA 50 GWD/MTU PIN--PF2.32 W/ECCS
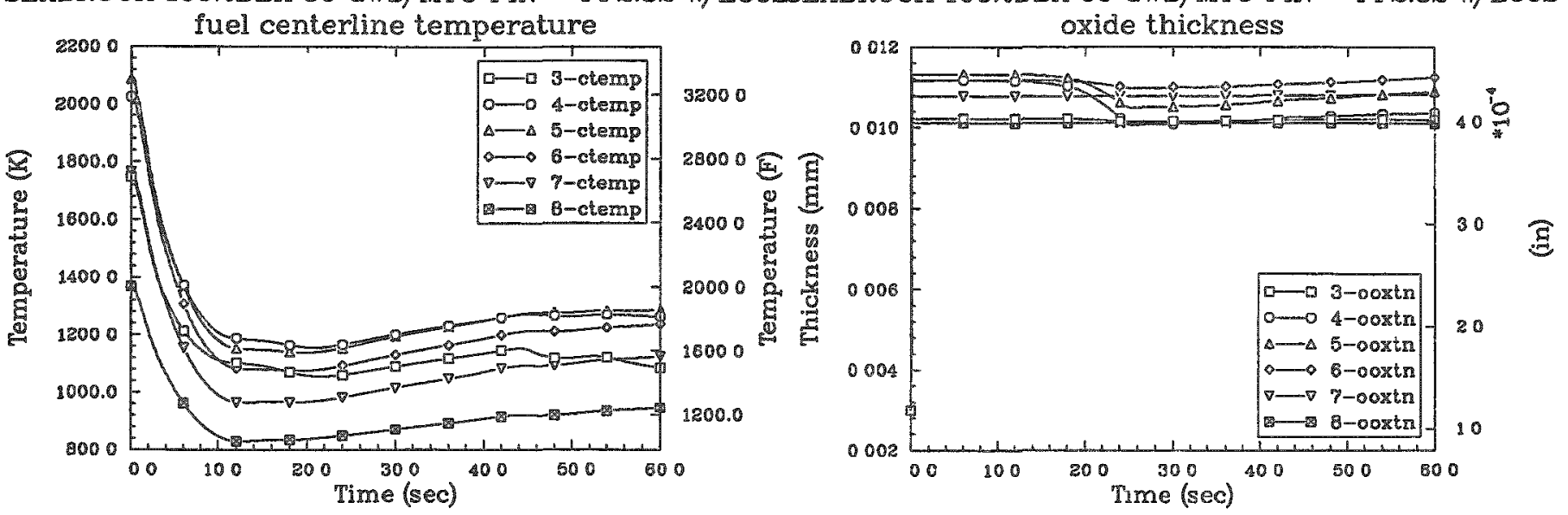
SEABROOK 100\%DBA 35 GWD/MTU PIN--PF2.32 W/ECCSSEABROOK 100\%DBA 35 GWD/MTU PIN--PF2.32 W/ECCS

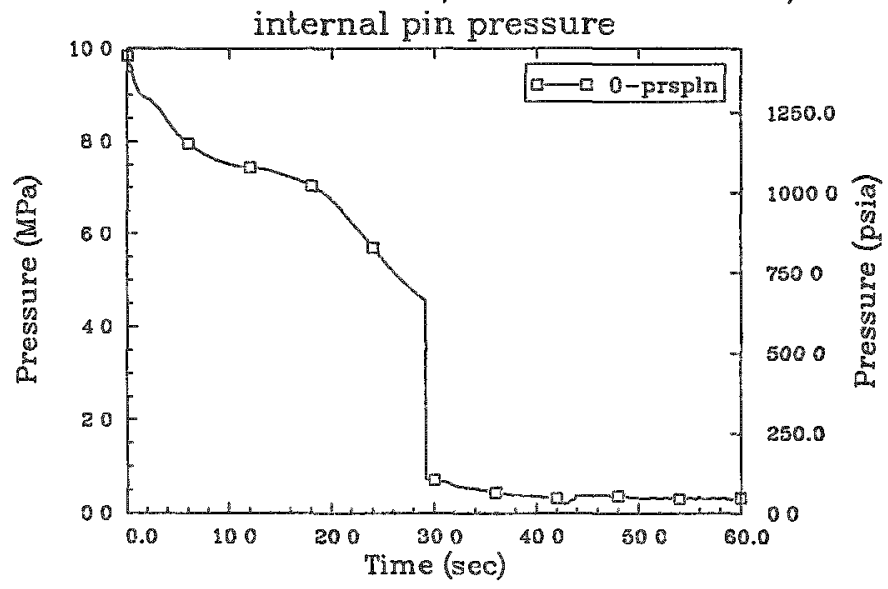

failure probability

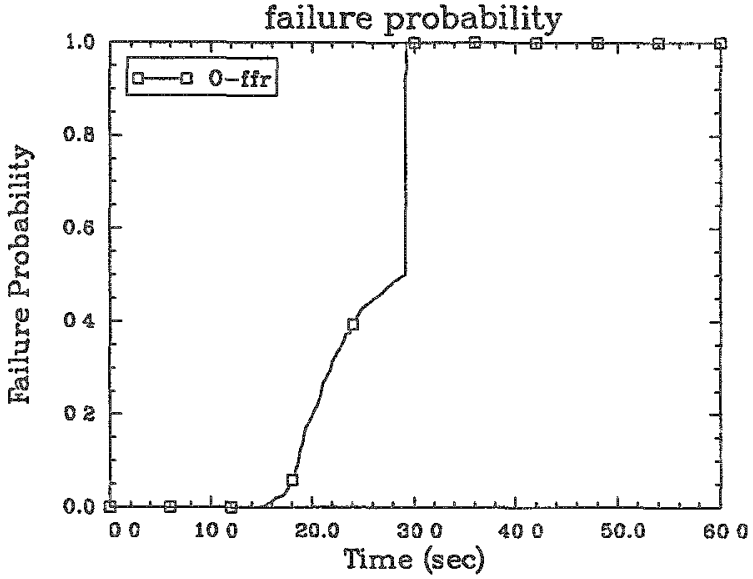

SEABROOK 100\%DBA 35 GWD/MTU PIN--PF2.32 W/ECCSSEABROOK 100\%DBA 35 GWD/MTU PIN--PF2.32 W/ECCS
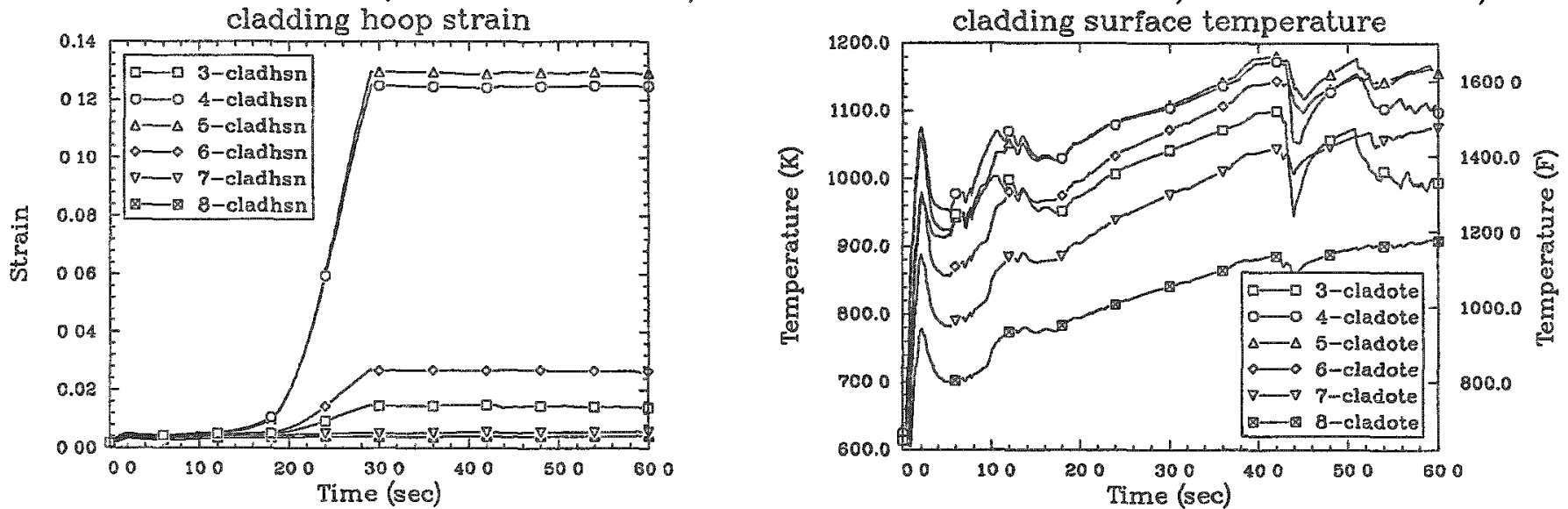

SEABROOK 100\%DBA 35 GWD/MTU PIN--PF2.32 W/ECCSSEABROOK 100\%DBA 35 GWD/MTU PIN--PF2.32 W/ECCS
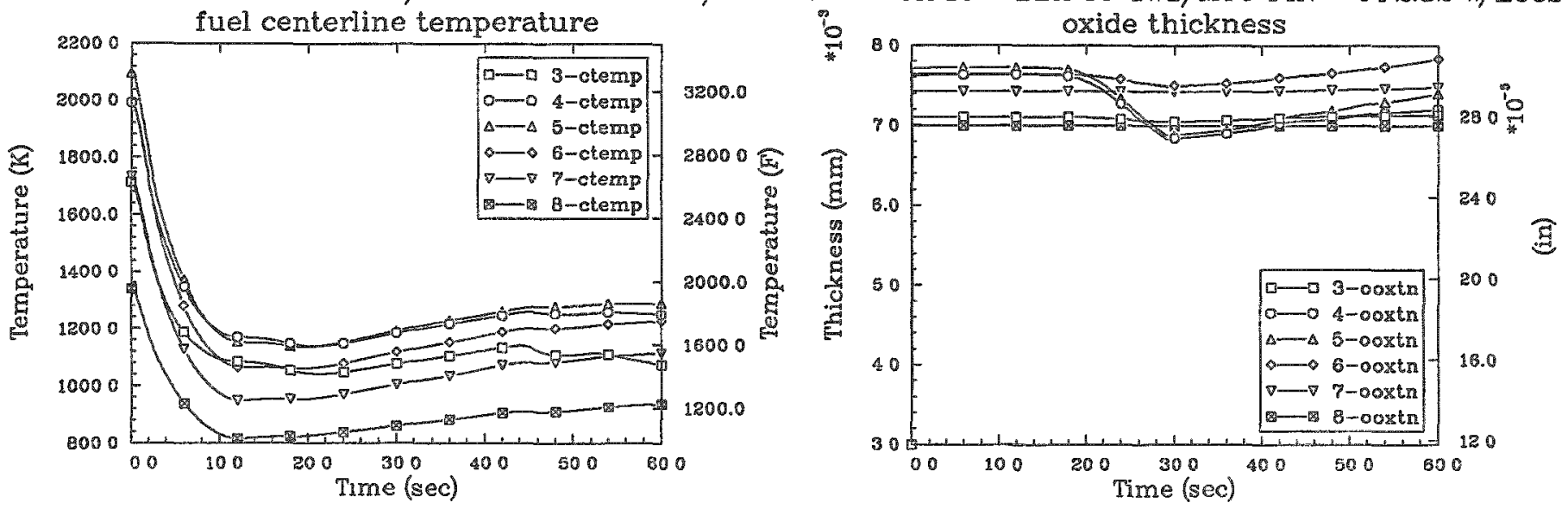
SEABROOK 100\%DBA 20 GWD/MIU PIN- -PF2 32 W/ECCSSEABROOK 100\%DBA 20 GWD/MIU PIN-PF2 32 W/ECCS
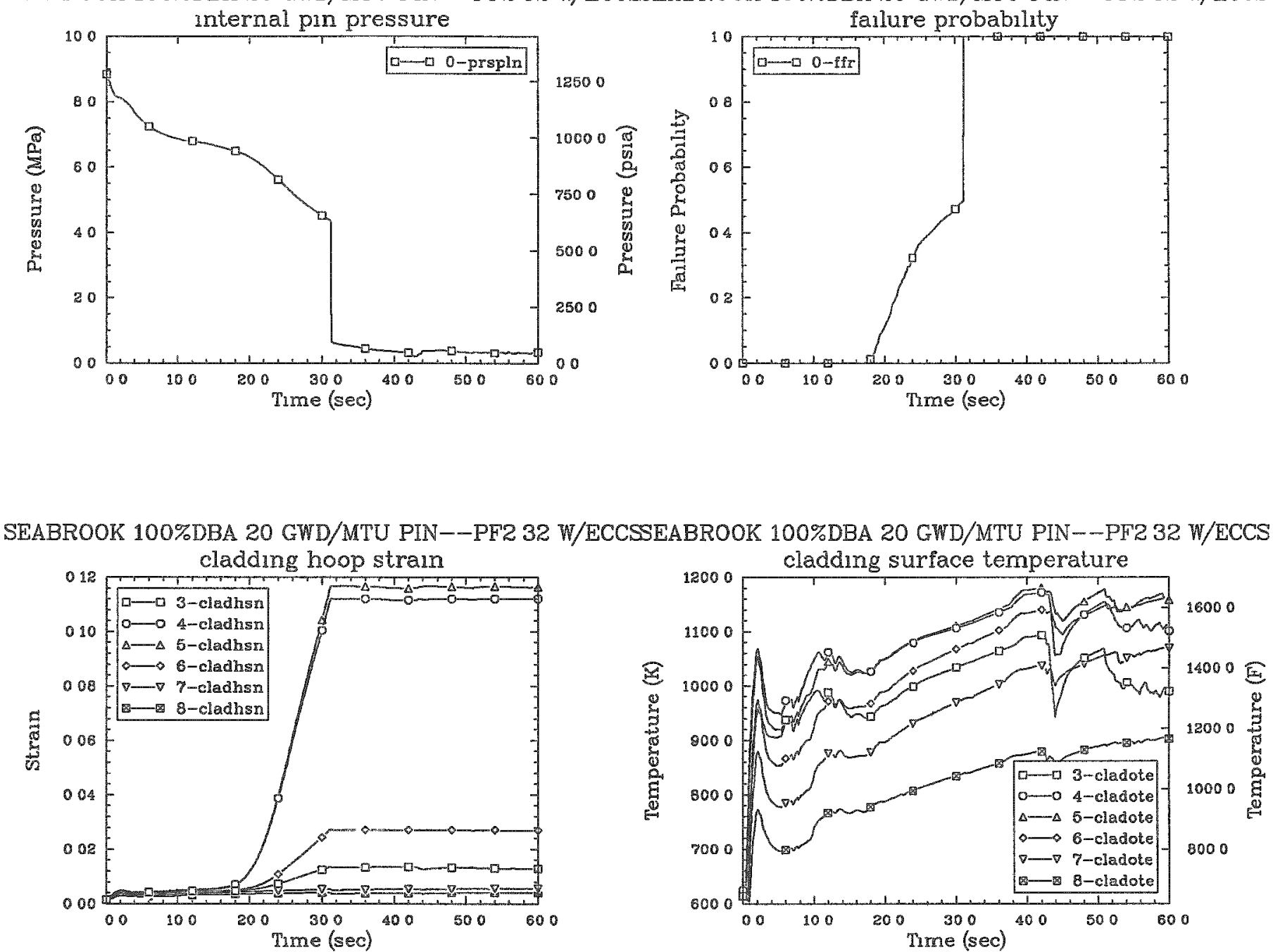

SEABROOK 100\%DBA 20 GWD/MTU PIN--PF2 32 W/ECCSSEABROOK 100\%DBA 20 GWD/MTU PIN--PF2 32 W/ECCS
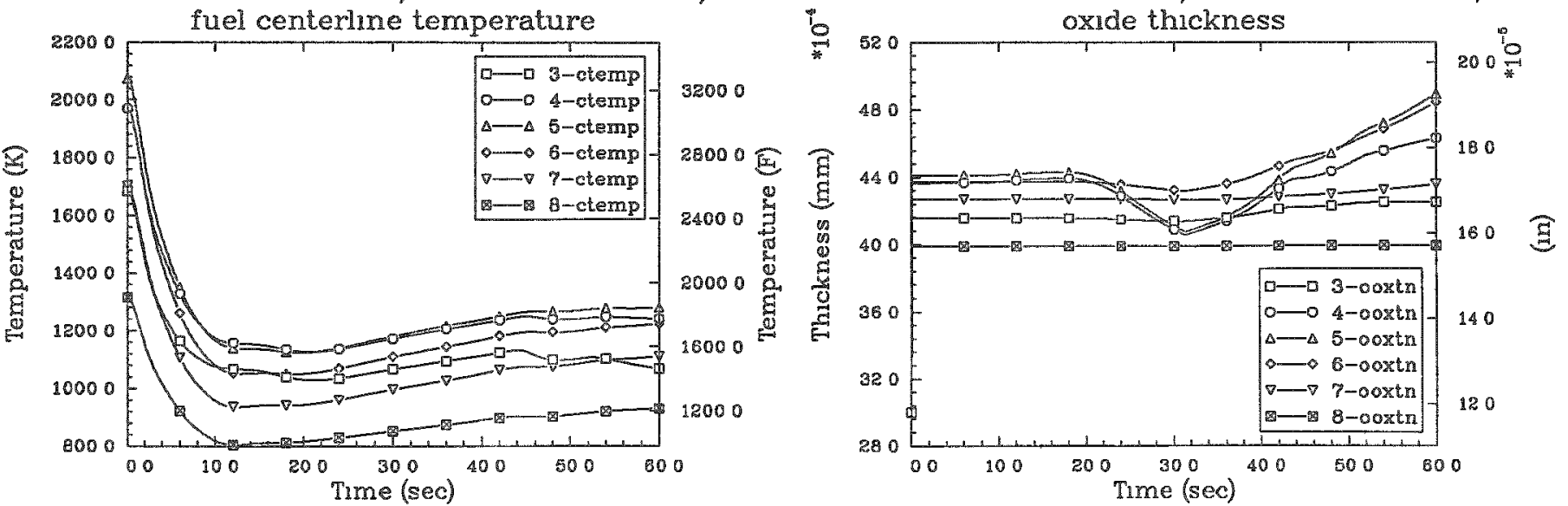
SEABROOK 100\%DBA 5 GWD/MTU PIN--PF2.32 W/ECCS SEABROOK 100\%DBA 5 GWD/MTU PIN--PF2.32 W/ECCS

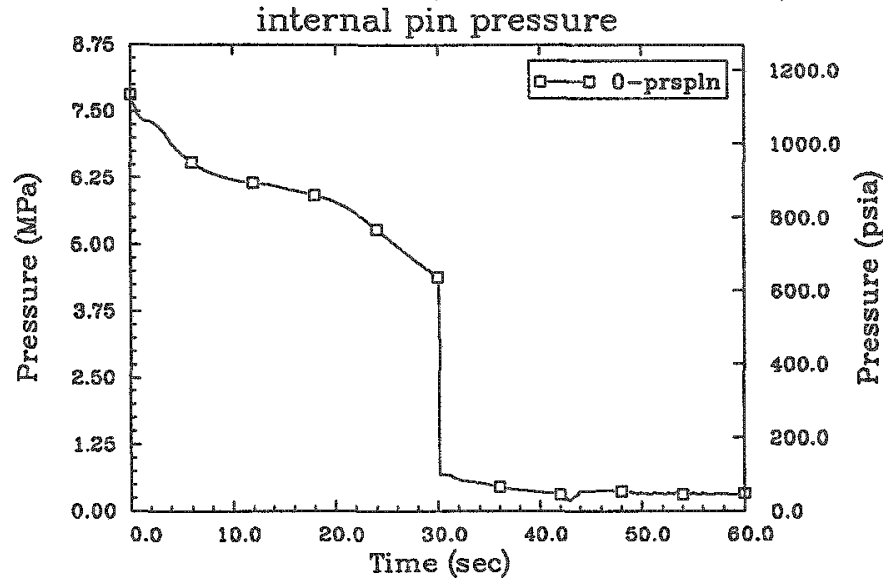

failure probability
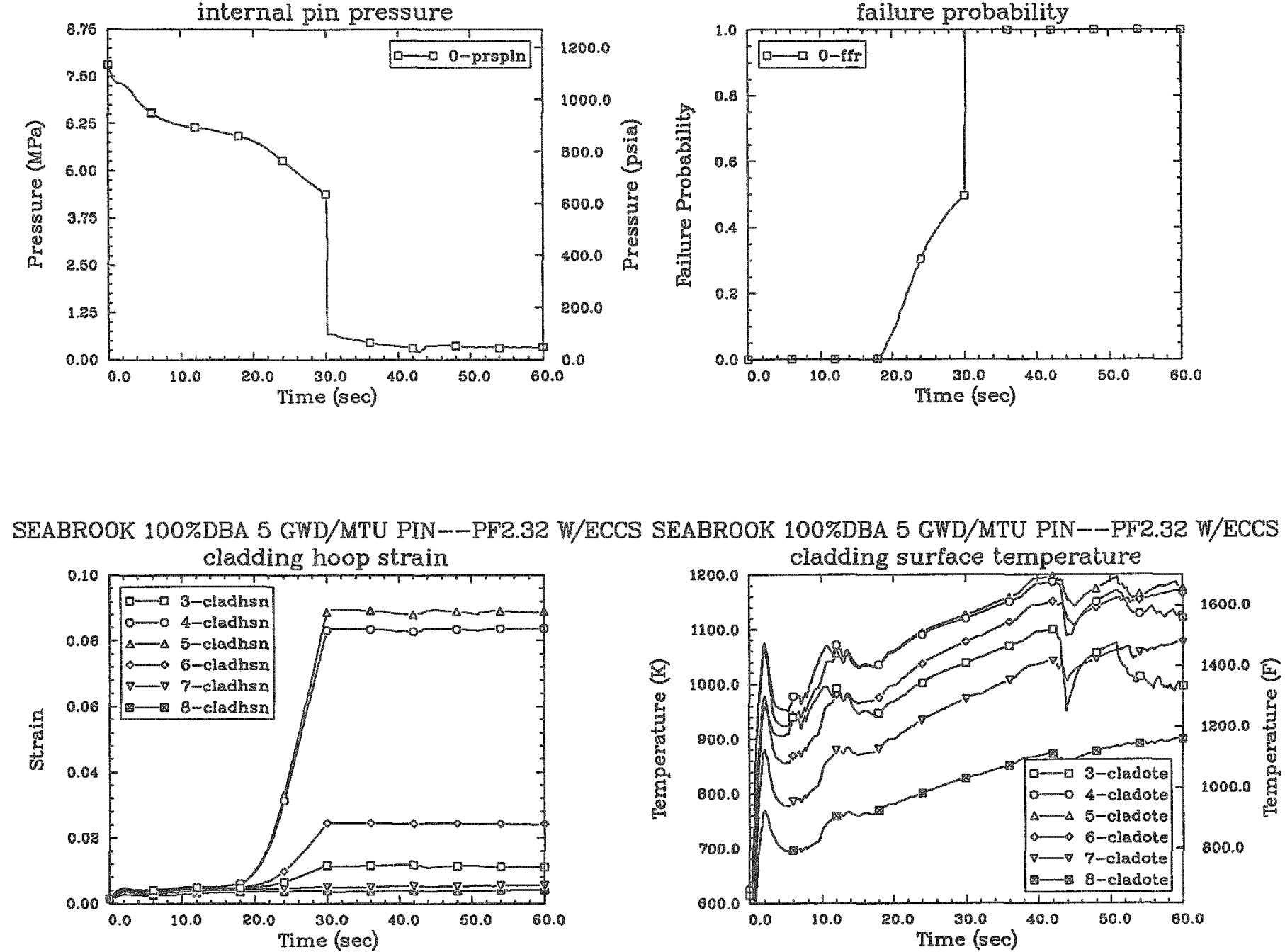

SEABROOK 100\%DBA 5 GWD/MTU PIN--PF2.32 W/ECCS SEABROOK 100\%DBA 5 GWD/MTU PIN--PF2.32 W/ECCS
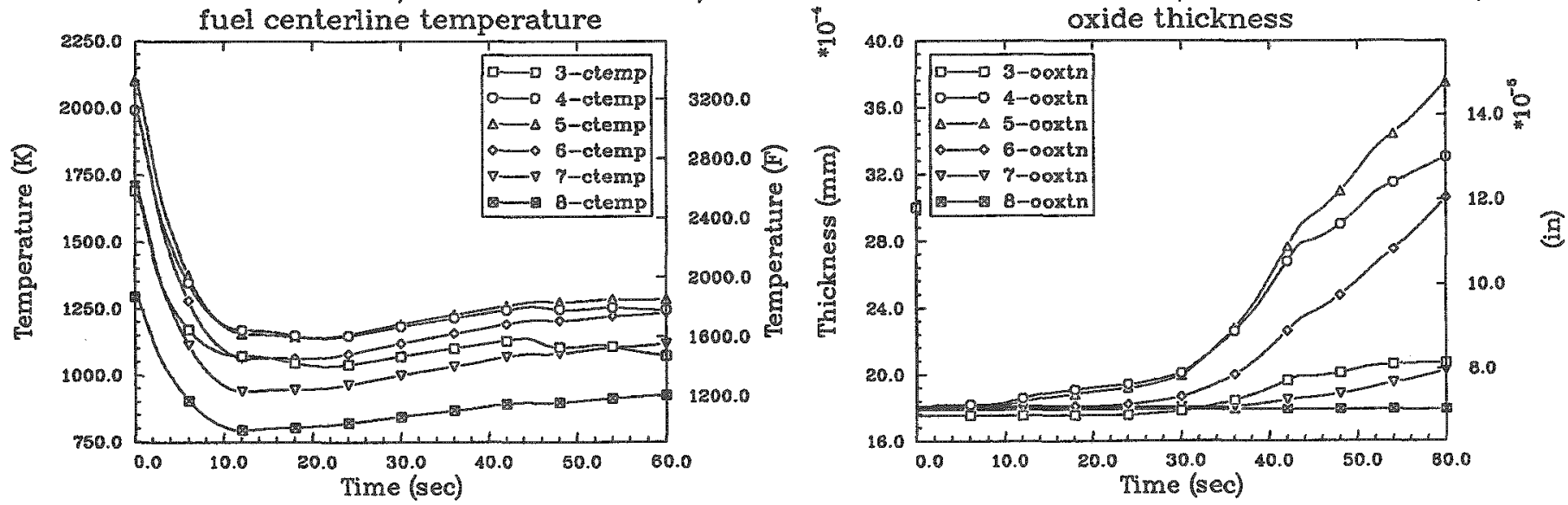
SEABROOK 100\%DBA 50 GWD/MTU PIN-PF22 W/ECCS SEABROOK 100\%DBA 50 GWD/MTU PIN--PF2 2 W/ECCS
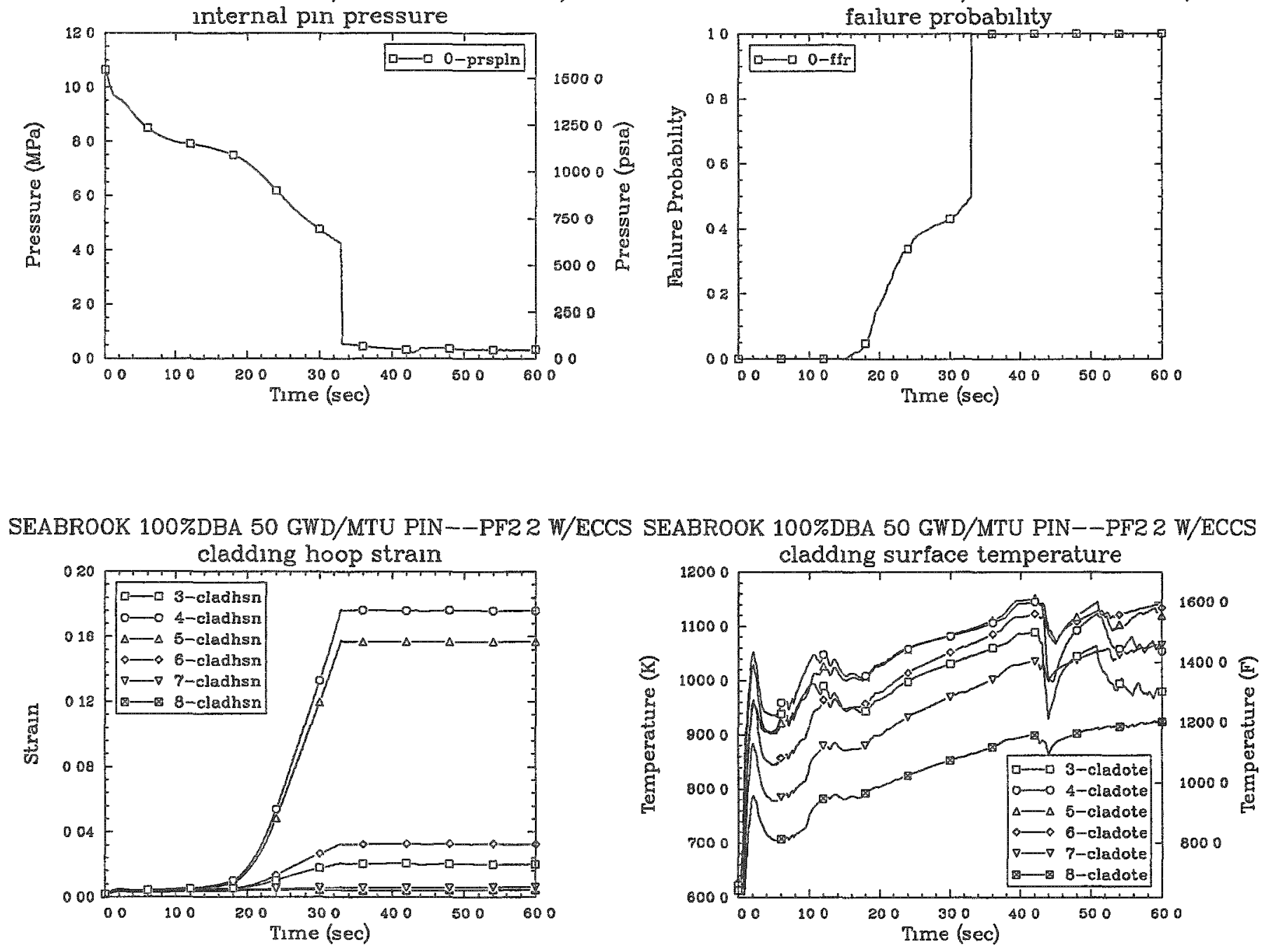

SEABROOK 100\%DBA 50 GWD/MTU PIN--PF2 2 W/ECCS SEABROOK 100\%DBA 50 GWD/MTU PIN--PF2 2 W/ECCS
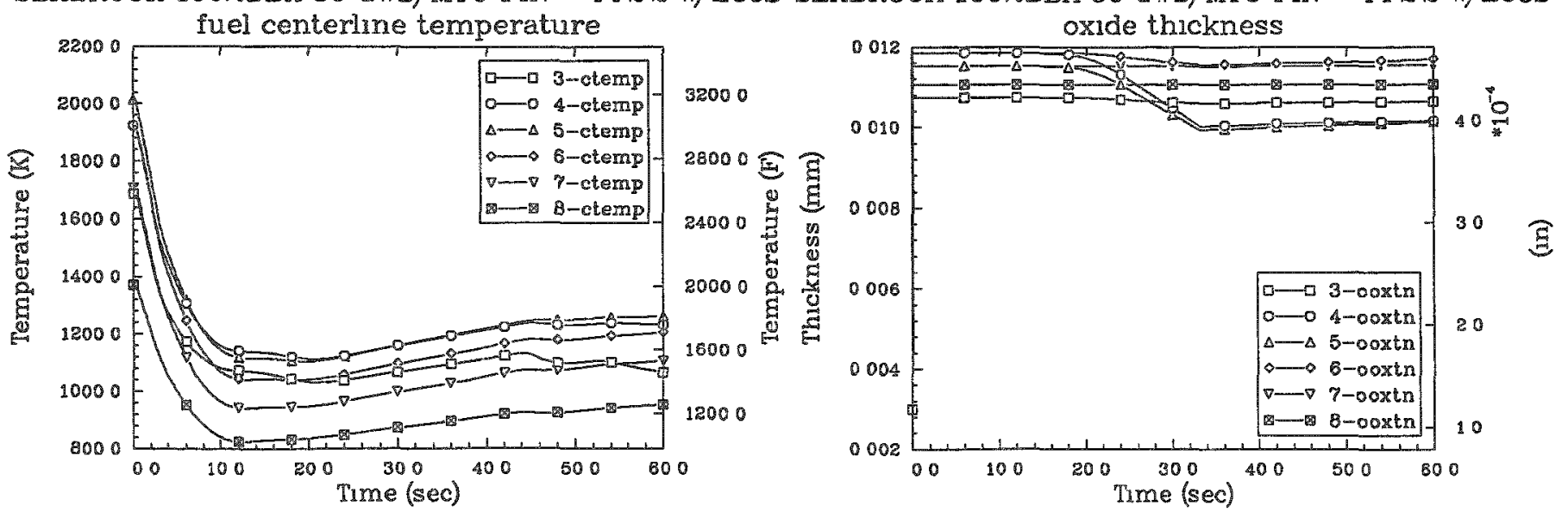
SEABROOK 100\%DBA 35 GWD/MTU PIN--PF2 2 W/ECCS SEABROOK 100\%DBA 35 GWD/MTU PIN--PF2.2 W/ECCS
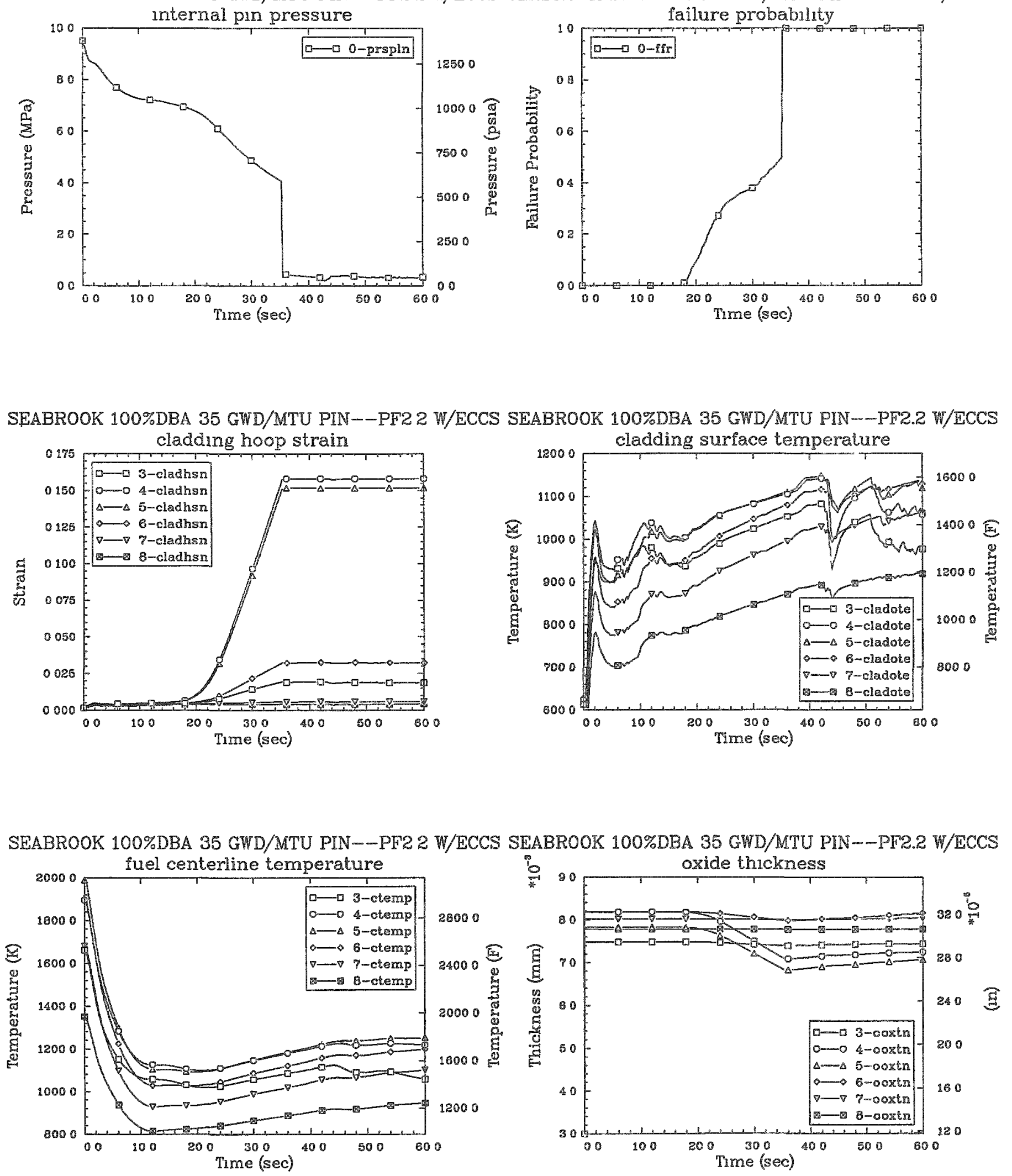
SEABROOK 100\%DBA 20 GWD/MTU PIN--PF2.2 W/ECCS SEABROOK 100\%DBA 20 GWD/MTU PIN--PF22 W/ECCS
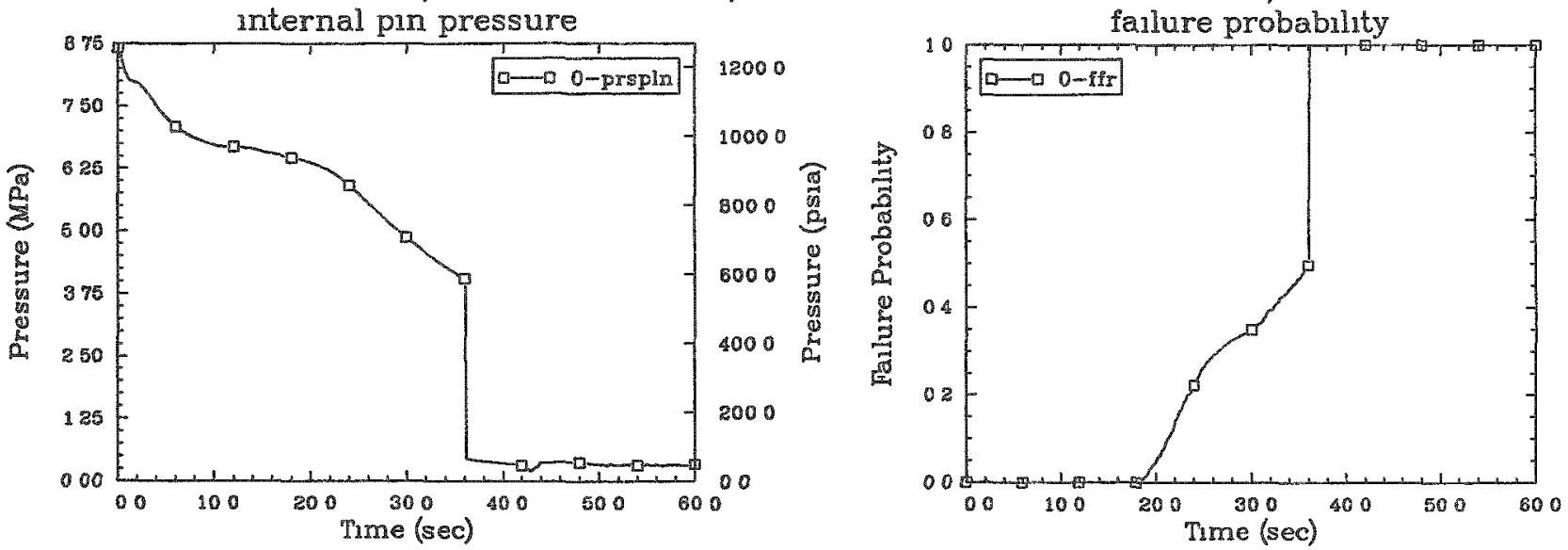

SEABROOK 100\%DBA $20 \mathrm{GWD} / \mathrm{MTU}$ PIN--PF2 2 W/ECCS SEABROOK 100\%DBA $20 \mathrm{GWD} / \mathrm{MTU}$ PIN--PF2 2 W/ECCS
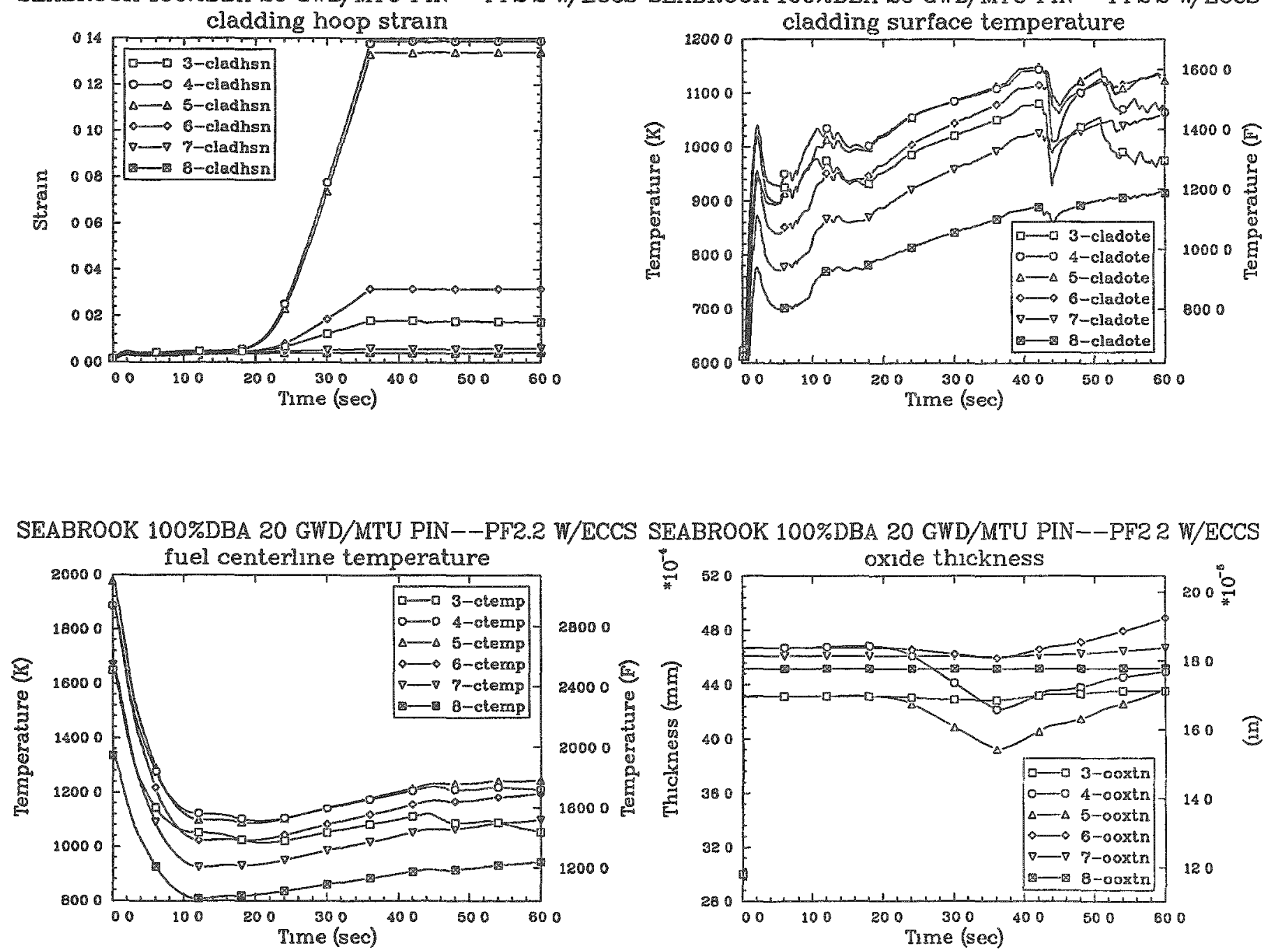
SEABROOK 100\%DBA 5 GWD/MTU PIN--PF2.2 W/ECCS

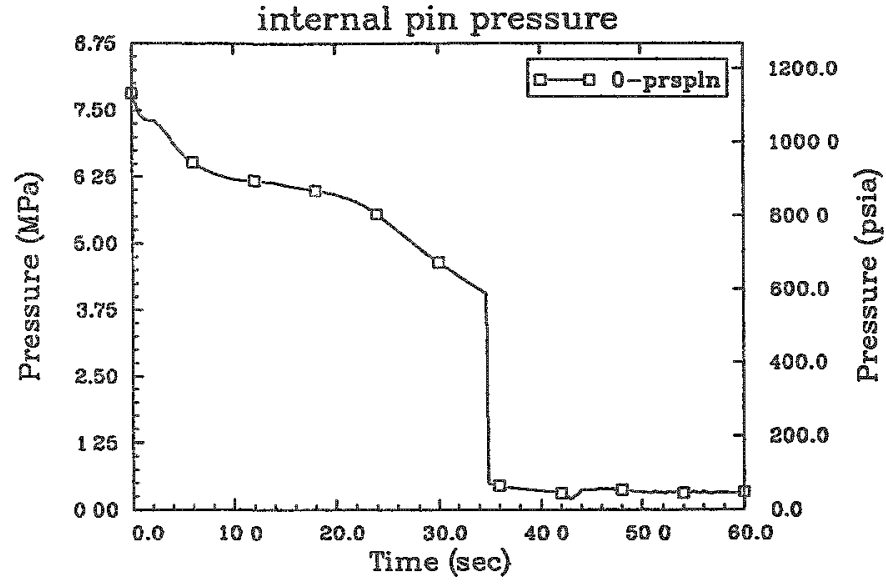

SEABROOK 100\%DBA 5 GWD/MTU PIN--PF2.2 W/ECCS

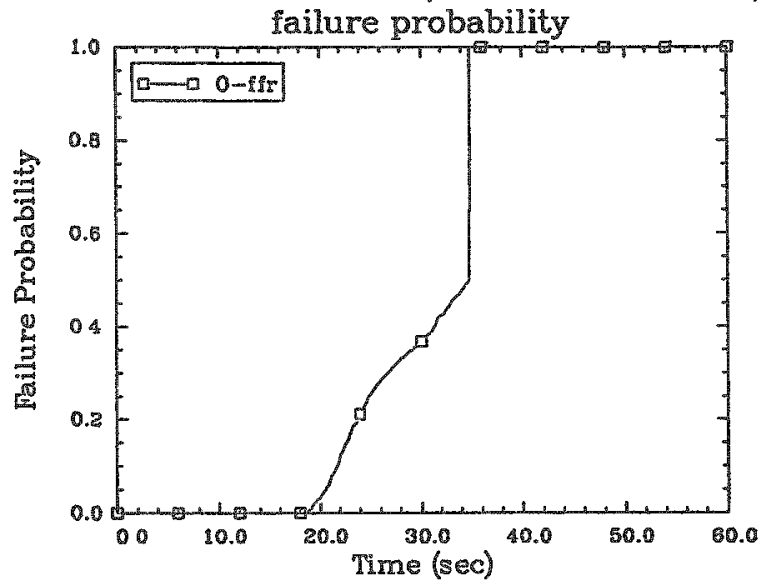

SEABROOK 100\%DBA 5 GWD/MTU PIN--PF2.2 W/ECCS

SEABROOK 100\%DBA 5 GWD/MTU PIN--PF2.2 W/ECCS
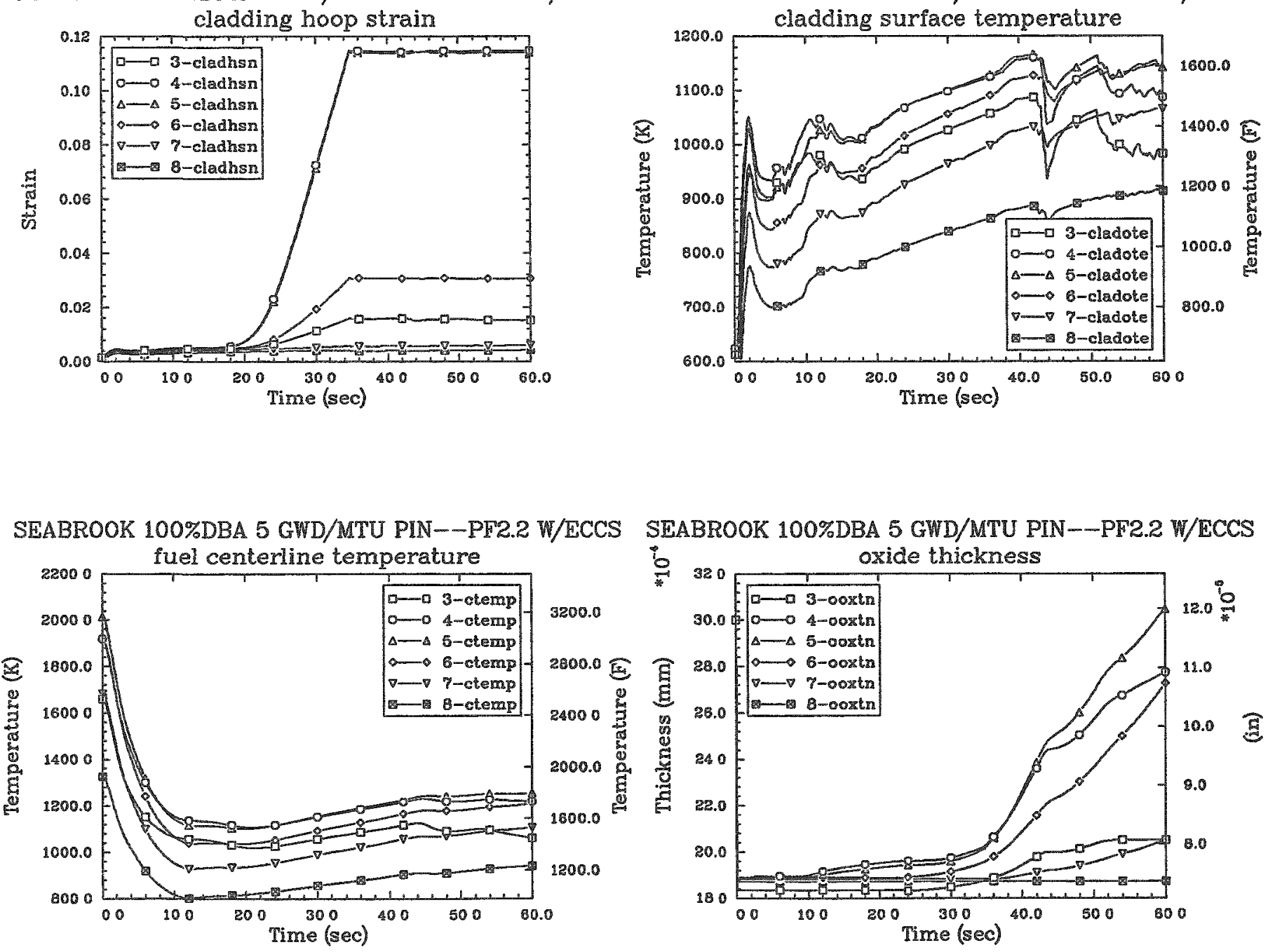
SEABROOK 100\%DBA 50 GWD/MTU PIN-DF2.0 W/ECCS SEABROOK 100\%DBA 50 GWD/MTU PIN--PF2.0 W/ECCS
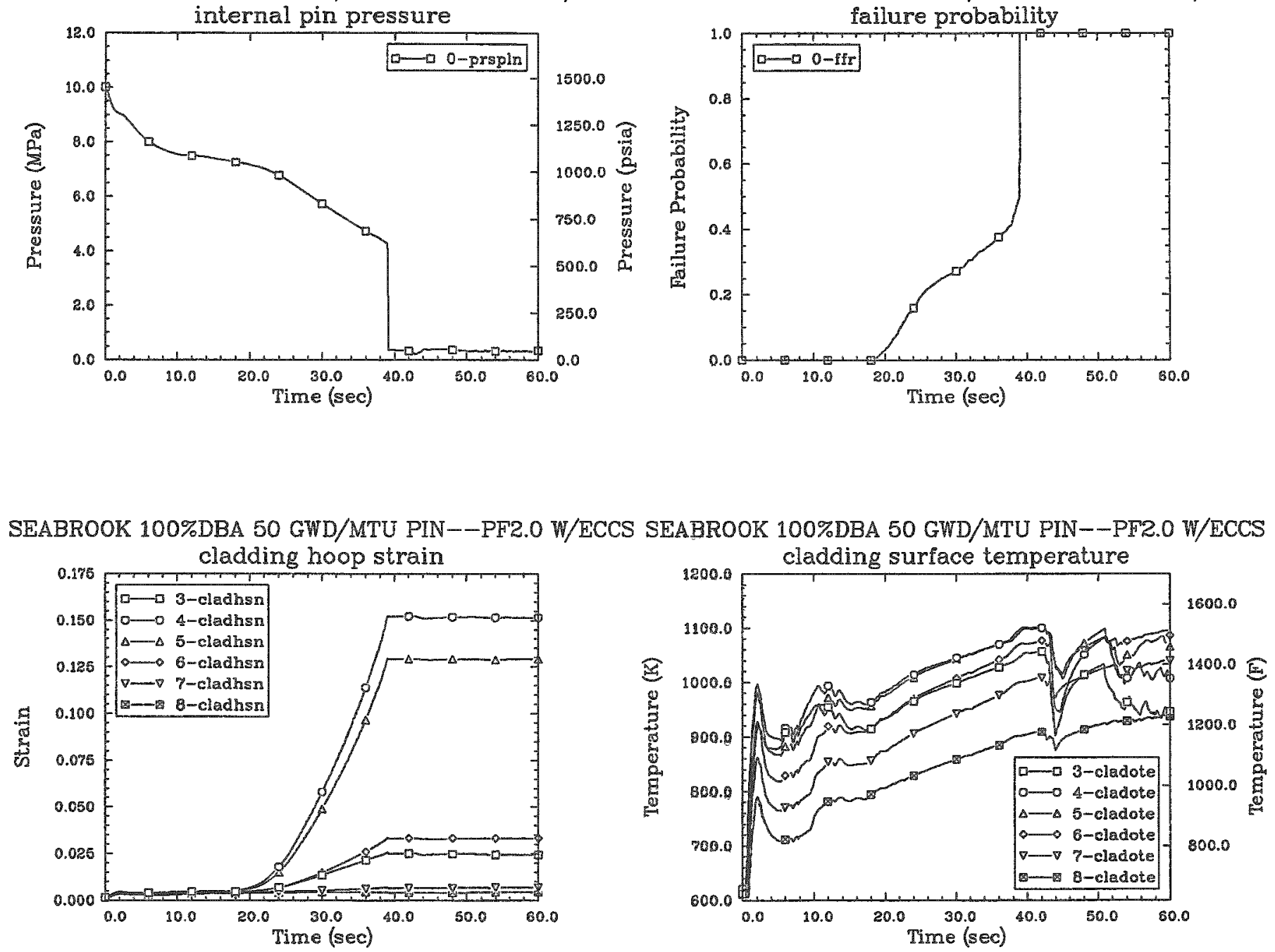

SEABROOK 100\%DBA 50 GWD/MTU PIN--PF2.0 W/ECCS SEABROOK 100\%DBA 50 GWD/MTU PIN--PF2.0 W/ECCS
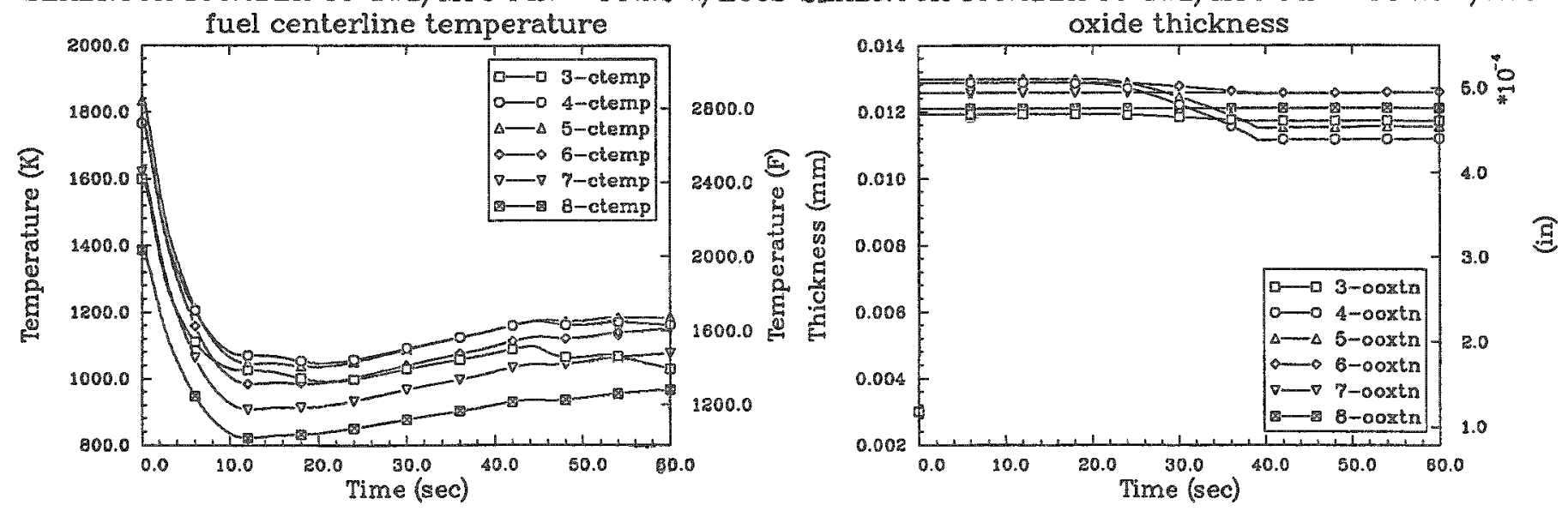
SEABROOK 100\%DBA 35 GWD/MTU PIN--PF2 0 W/ECCS SEABROOK 100\%DBA 35 GWD/MTU PIN--PF2 0 W/ECCS

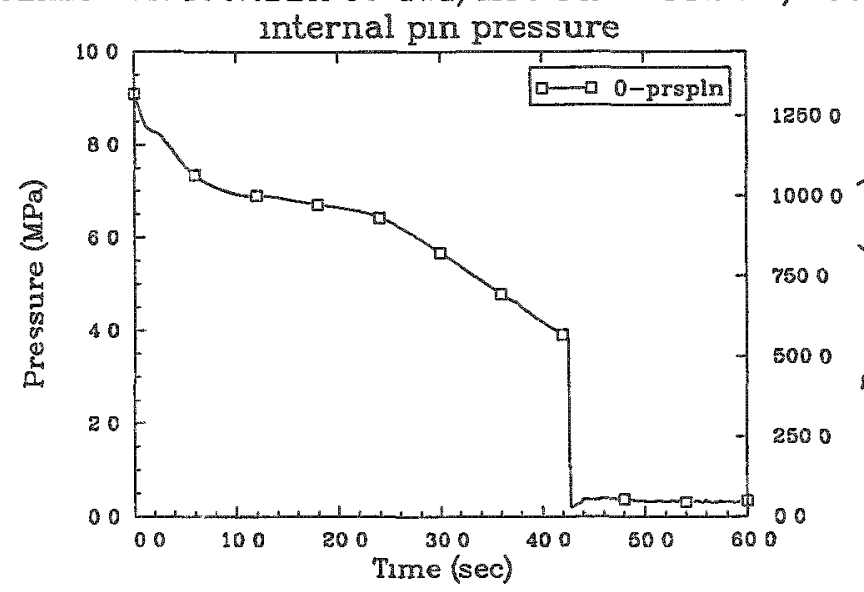

fallure probability
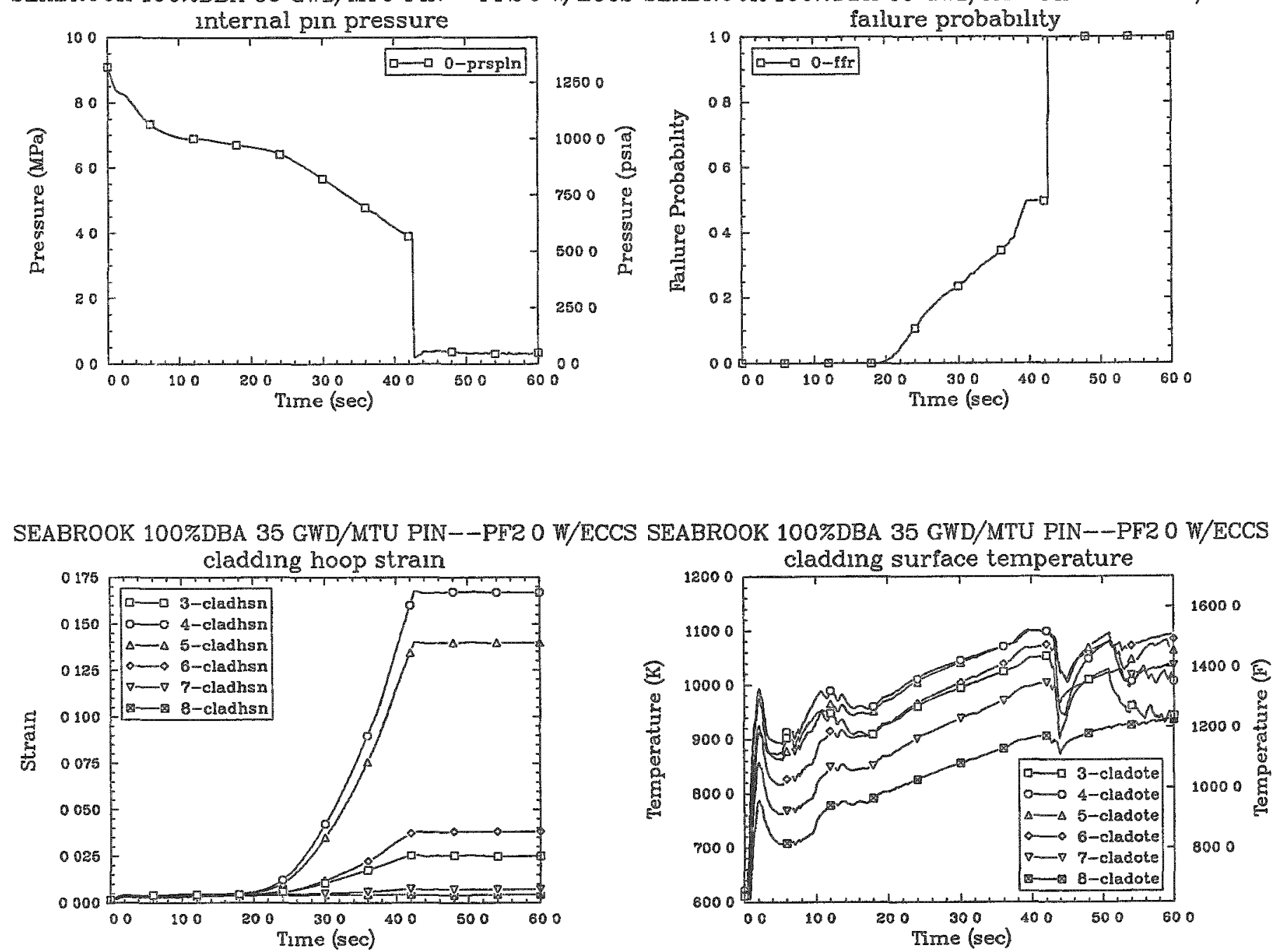

SEABROOK 100\%DBA 35 GWD/MTU PIN--PF2 O W/ECCS SEABROOK 100\%DBA 35 GWD/MTU PIN--PF2 0 W/ECCS
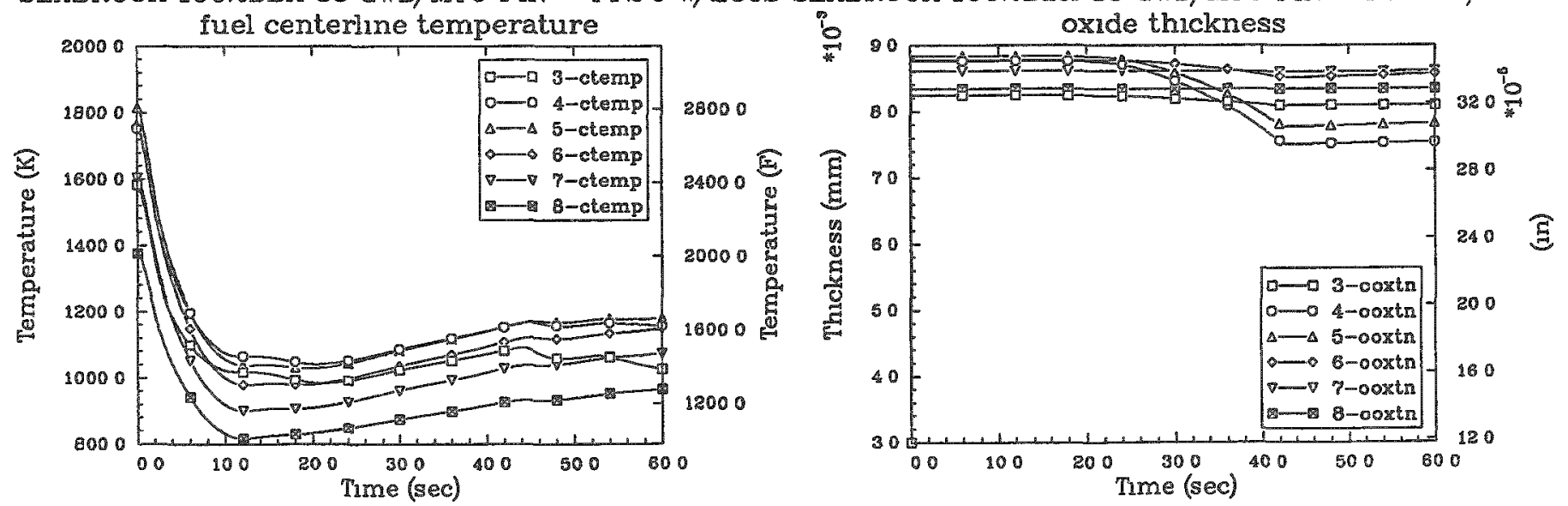
SEABROOK 100\%DBA 20 GWD/MTU PIN--PF20 W/ECCS SEABROOK 100\%DBA 20 GWD/MTU PIN--PF20 W/ECCS
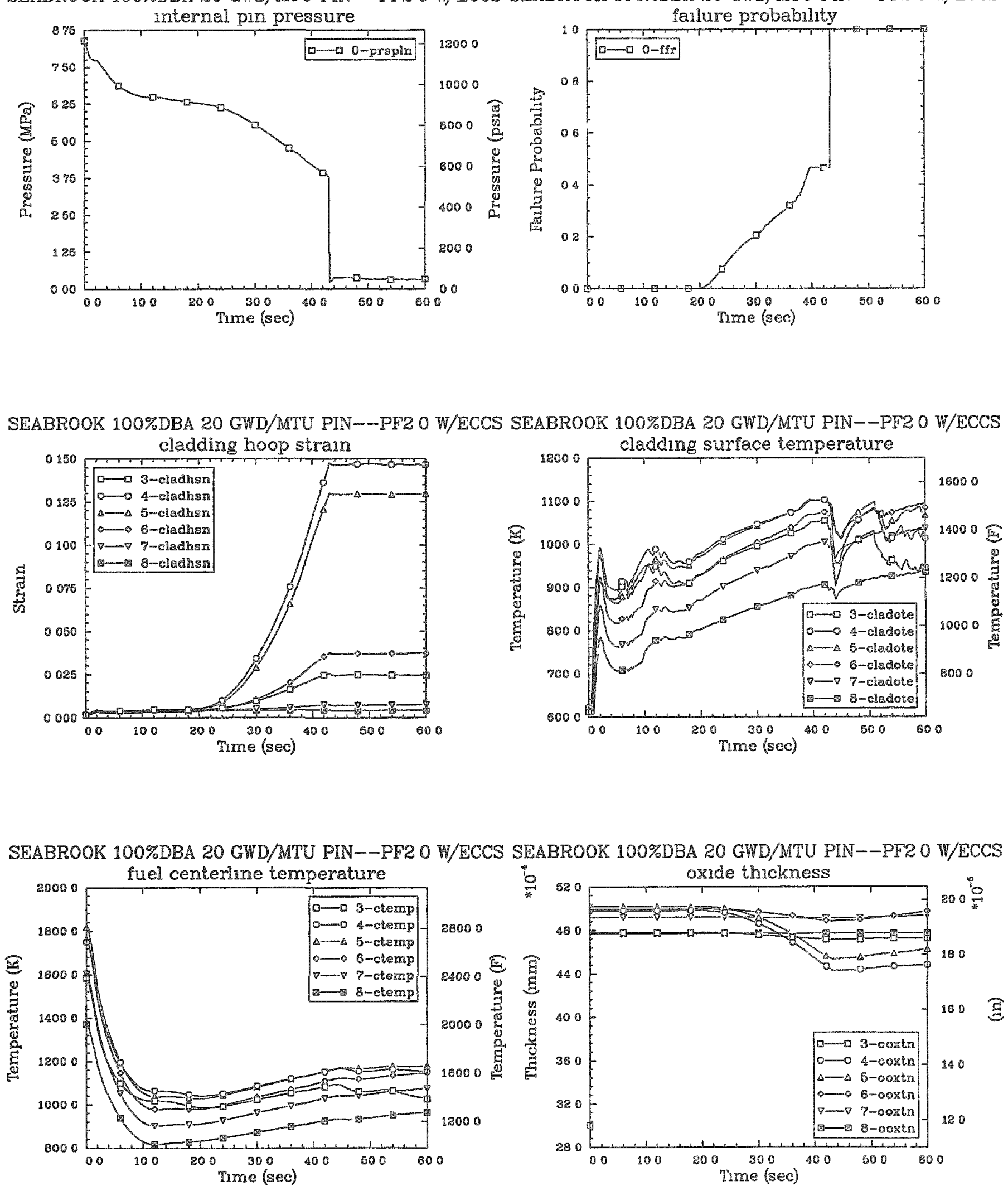
SEABROOK 100\%DBA 5 GWD/MTU PIN--PF2.0 W/ECCS

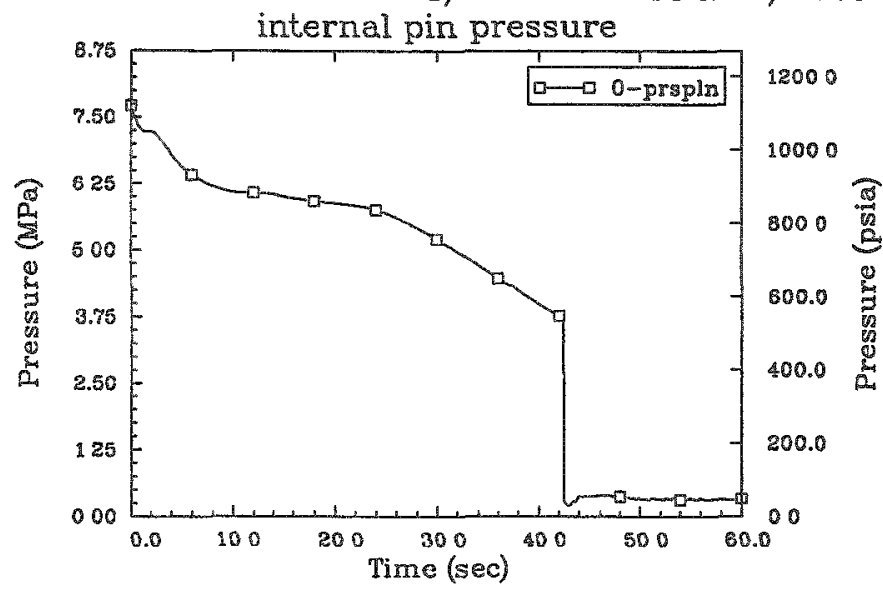

SEABROOK 100\%DBA 5 GWD/MTU PIN--PF2.0 W/ECCS

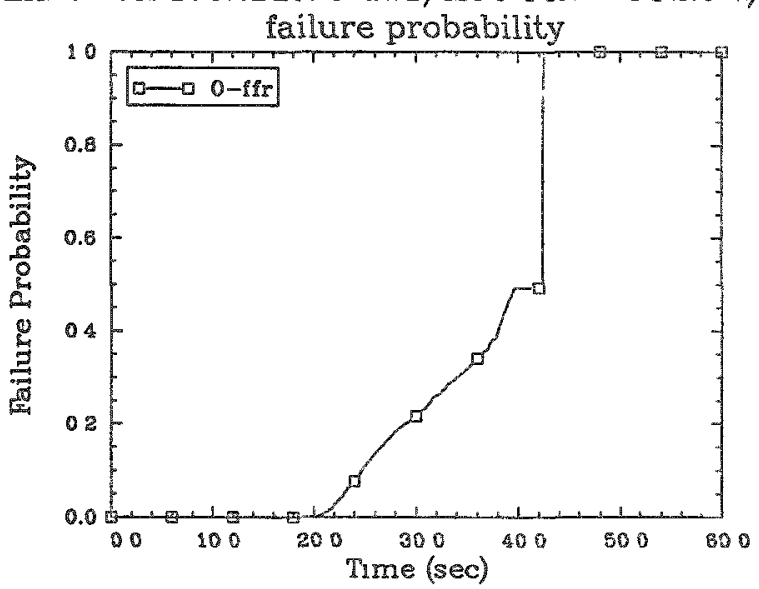

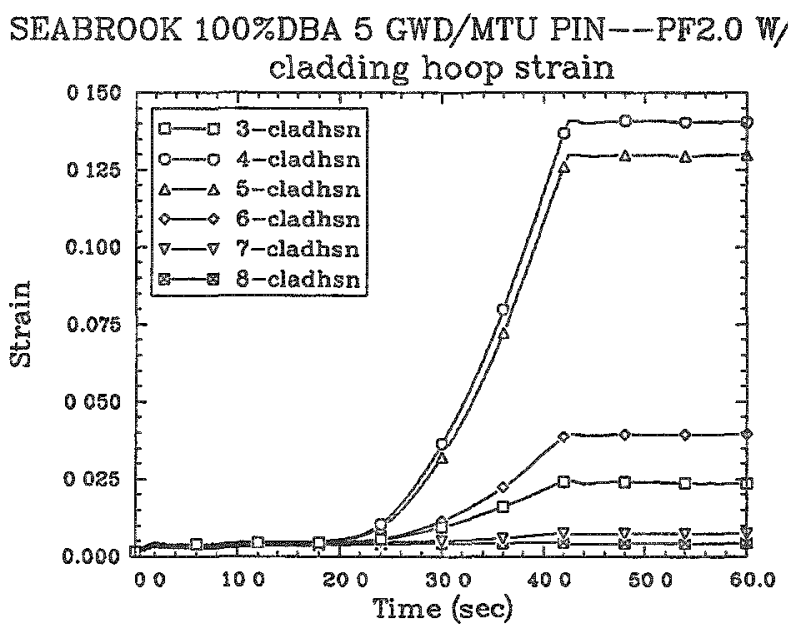

SEABROOK 100\%DBA 5 GWD/MTU PIN--PF2.0 W/ECCS

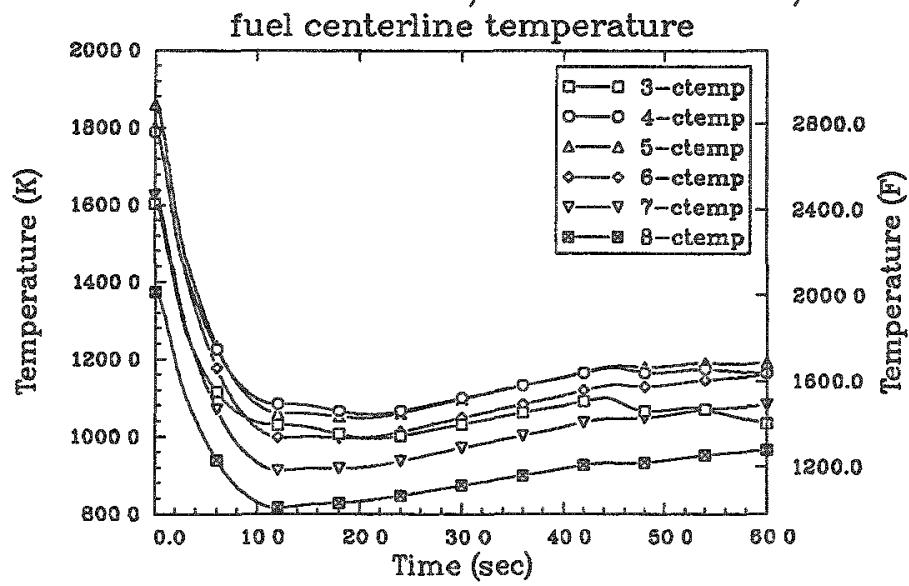

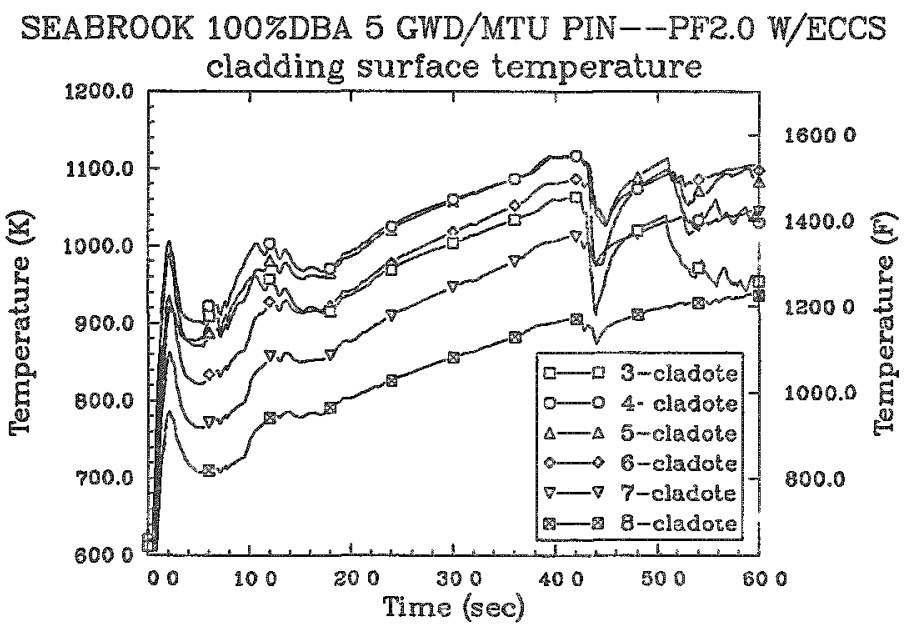

SEABROOK 100\%DBA 5 GWD/MTU PIN--PF2.0 W/ECCS

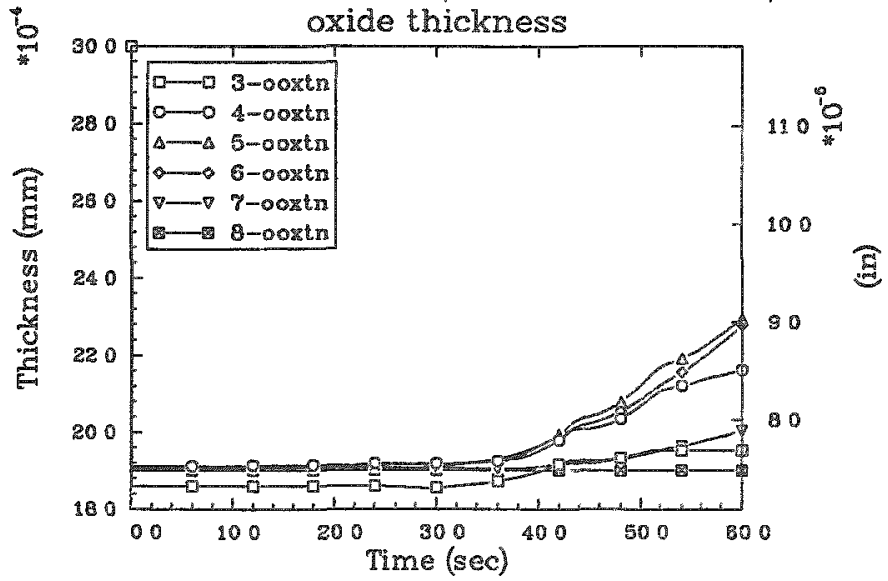


SEABROOK 100\%DBA 50 GWD/MTU PIN--PF1.8 W/ECCS SEABROOK 100\%DBA 50 GWD/MTU PIN--PF1.8 W/ECCS

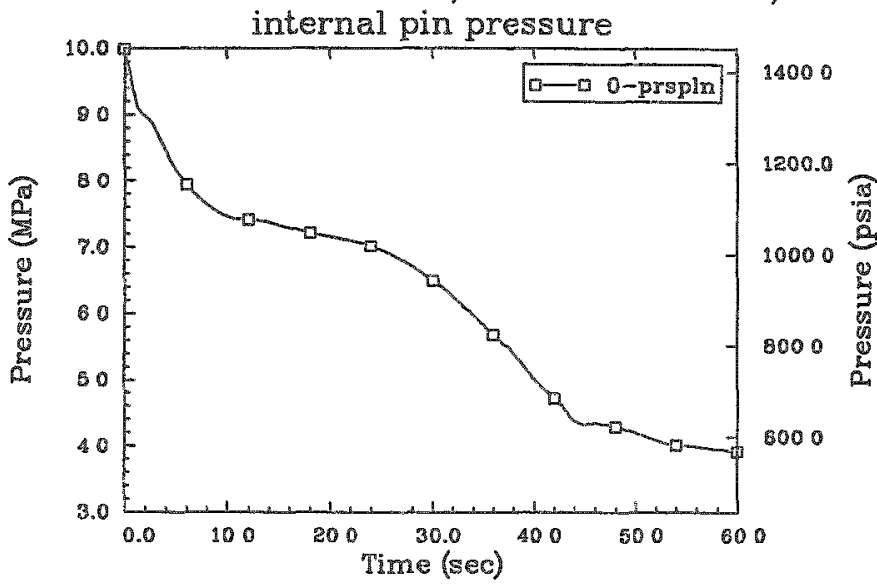
failure probability

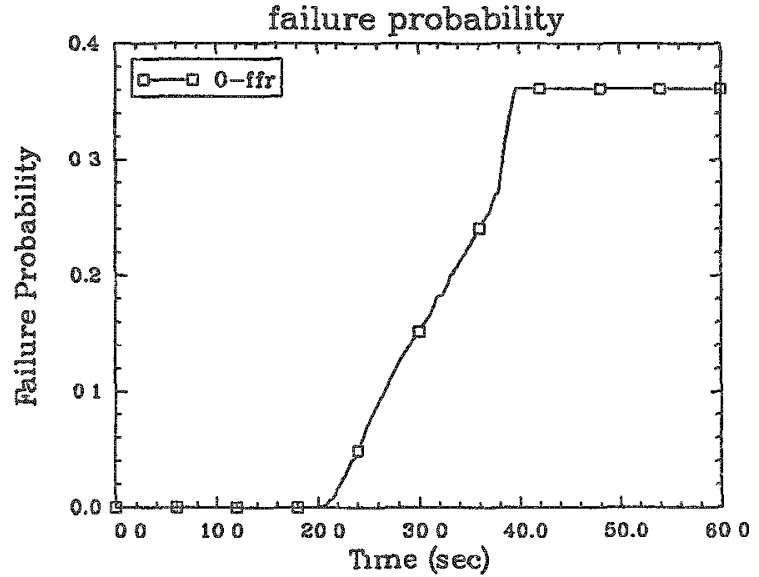

SEABROOK 100\%DBA 50 GWD/MTU PIN-PF1.8 W/ECCS SEABROOK 100\%DBA 50 GWD/MTU PIN--PF1.8 W/ECCS
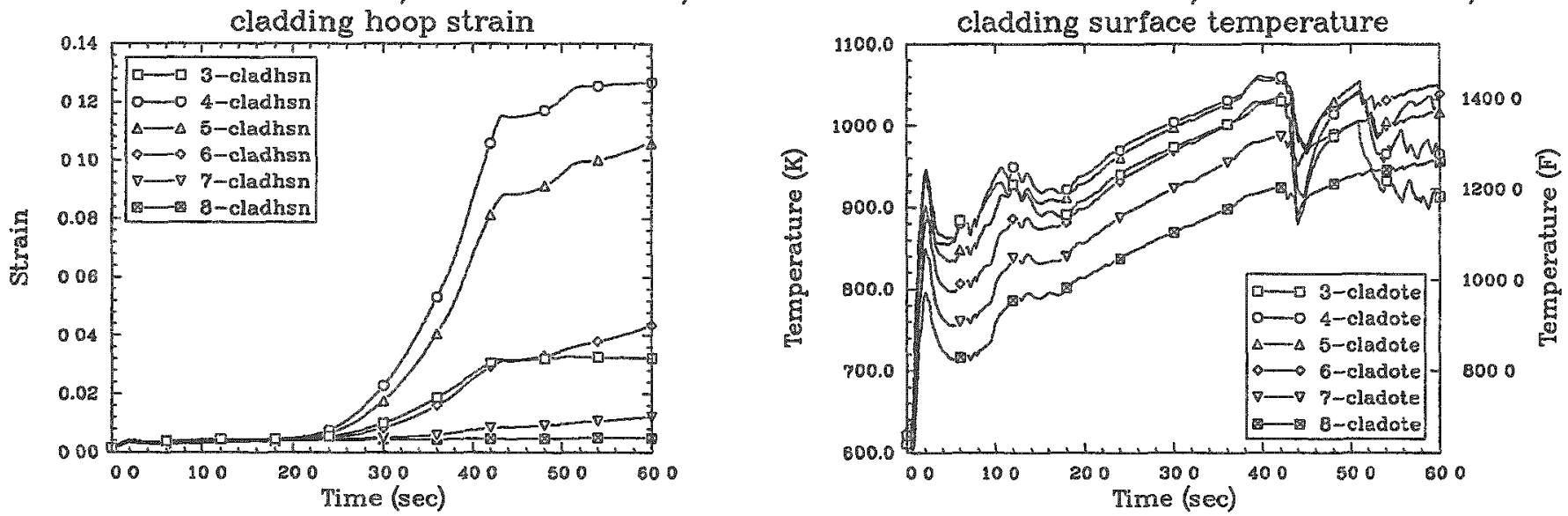

SEABROOK 100\%DBA 50 GWD/MTU PIN--PF1.8 W/ECCS SEABROOK 100\%DBA 50 GWD/MTU PIN--PF1.8 W/ECCS
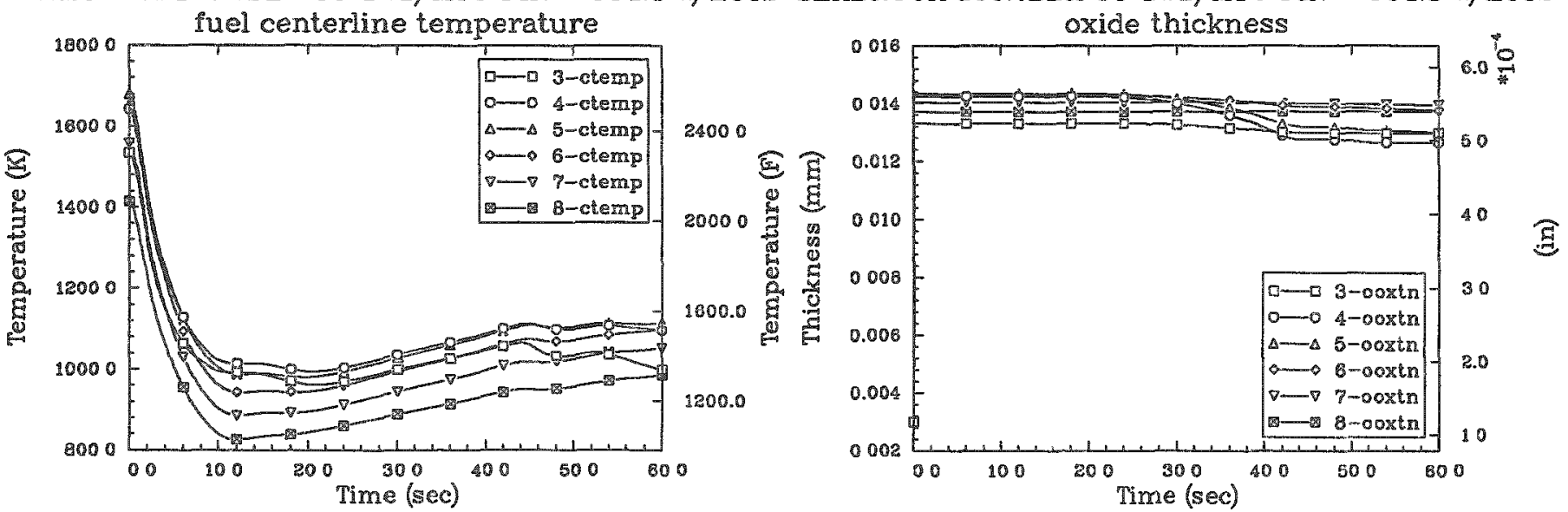
SEABROOK 100\%DBA 35 GWD/MTU PIN--PF1.8 W/ECCS SEABROOK 100\%DBA 35 GWD/MTU PIN--PF1.8 W/ECCS
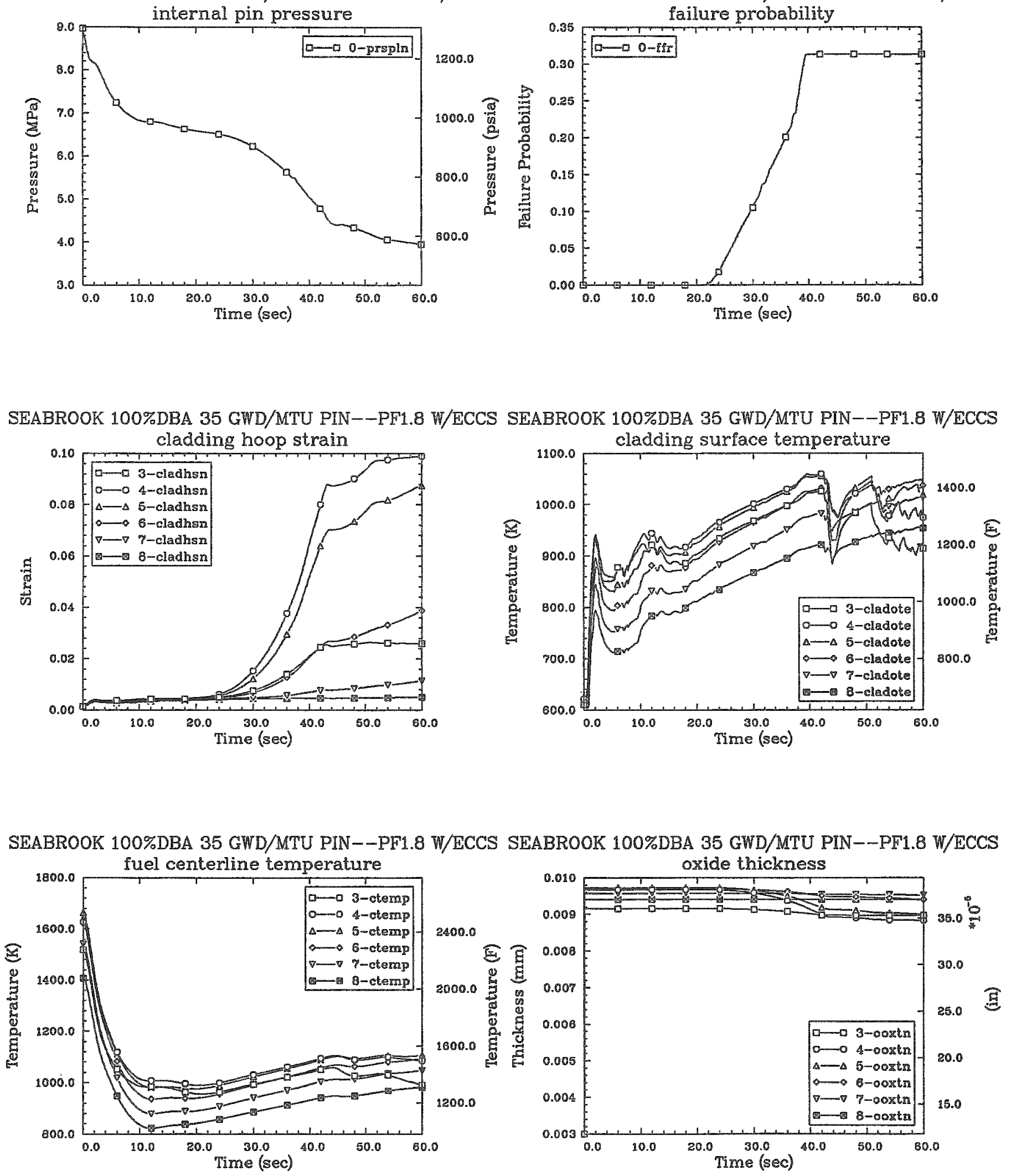

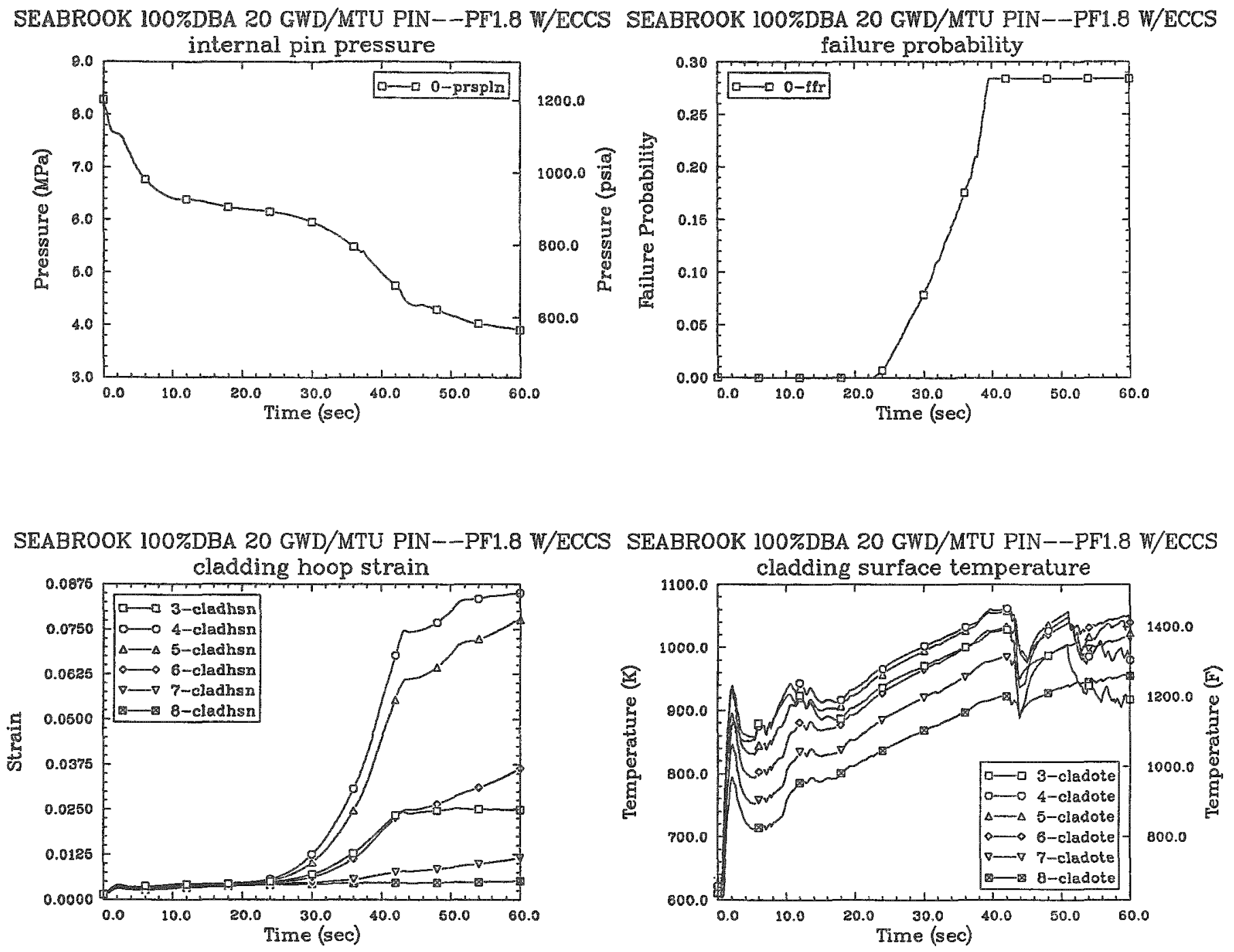

SEABROOK 100\%DBA 20 GWD/MTU PIN--PF1.8 W/ECCS
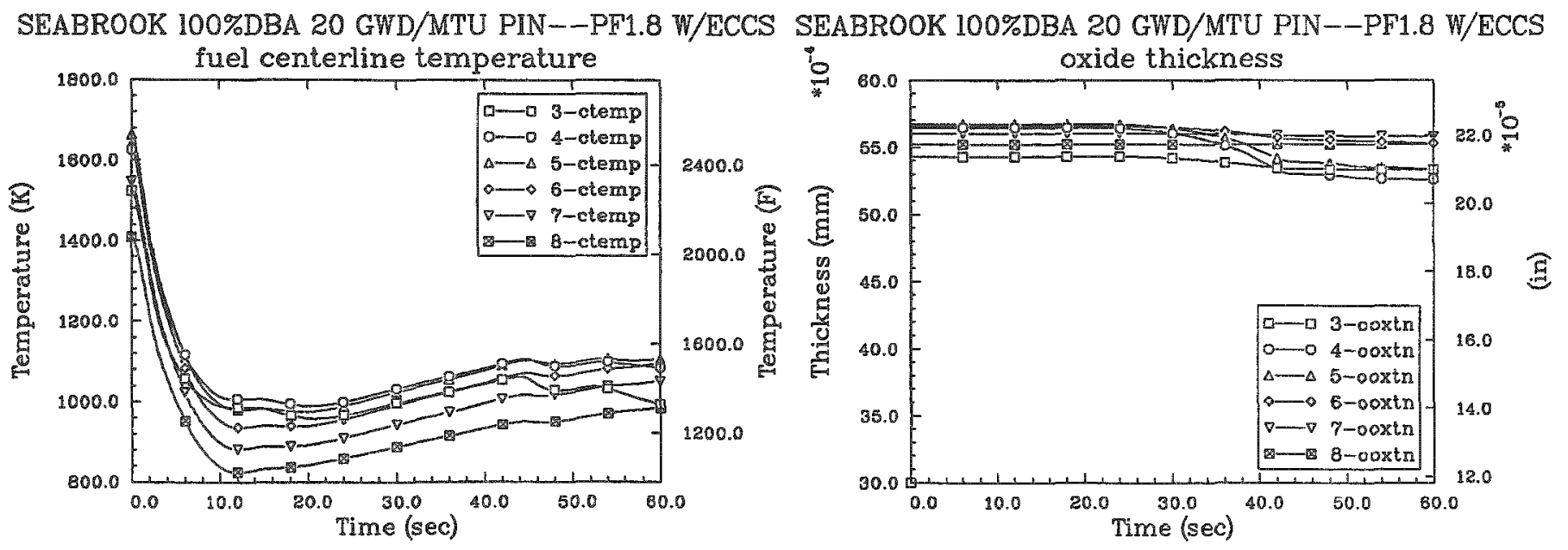
SEABROOK 100\%DBA 5 GWD/MTU PIN--PF1.8 W/ECCS
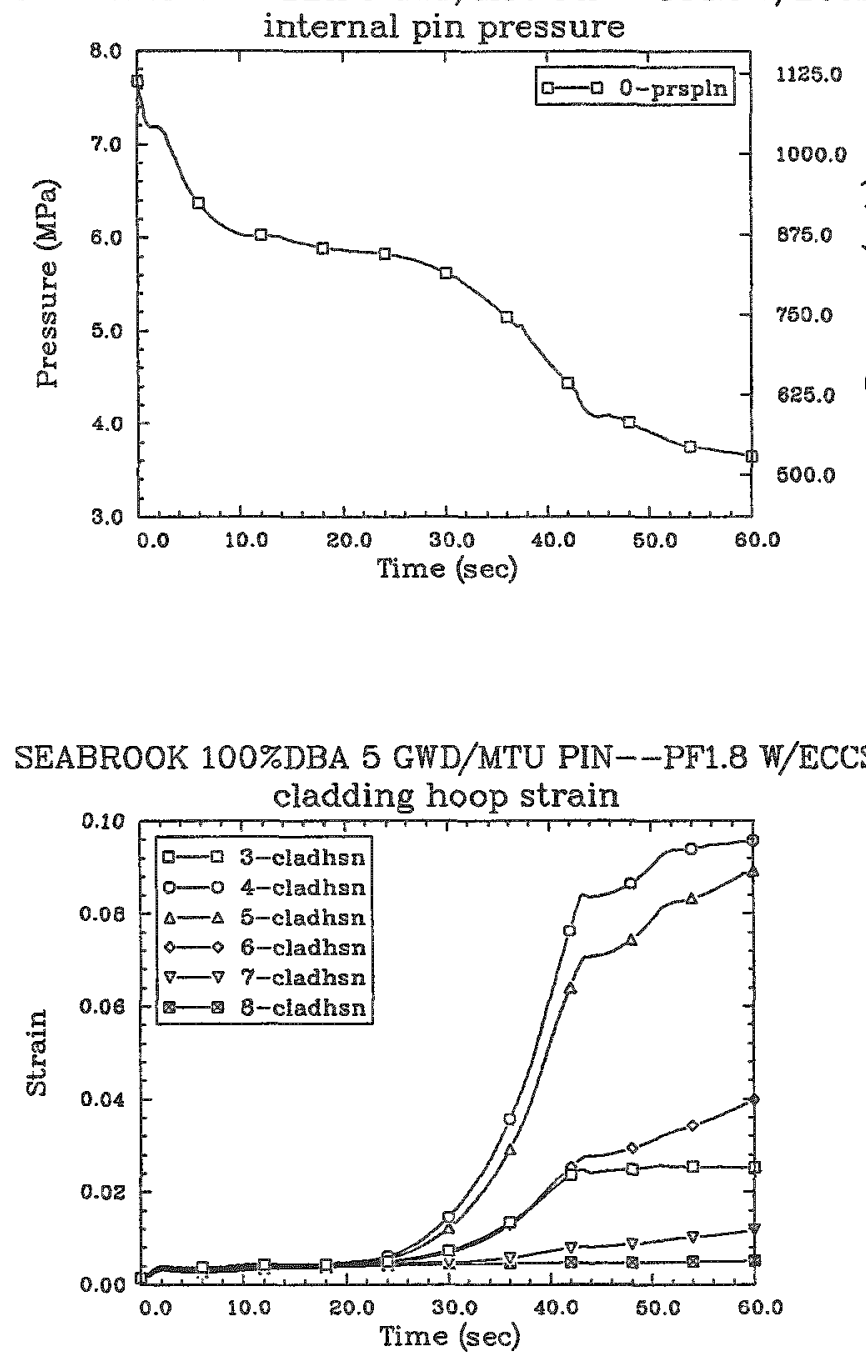

ECCS
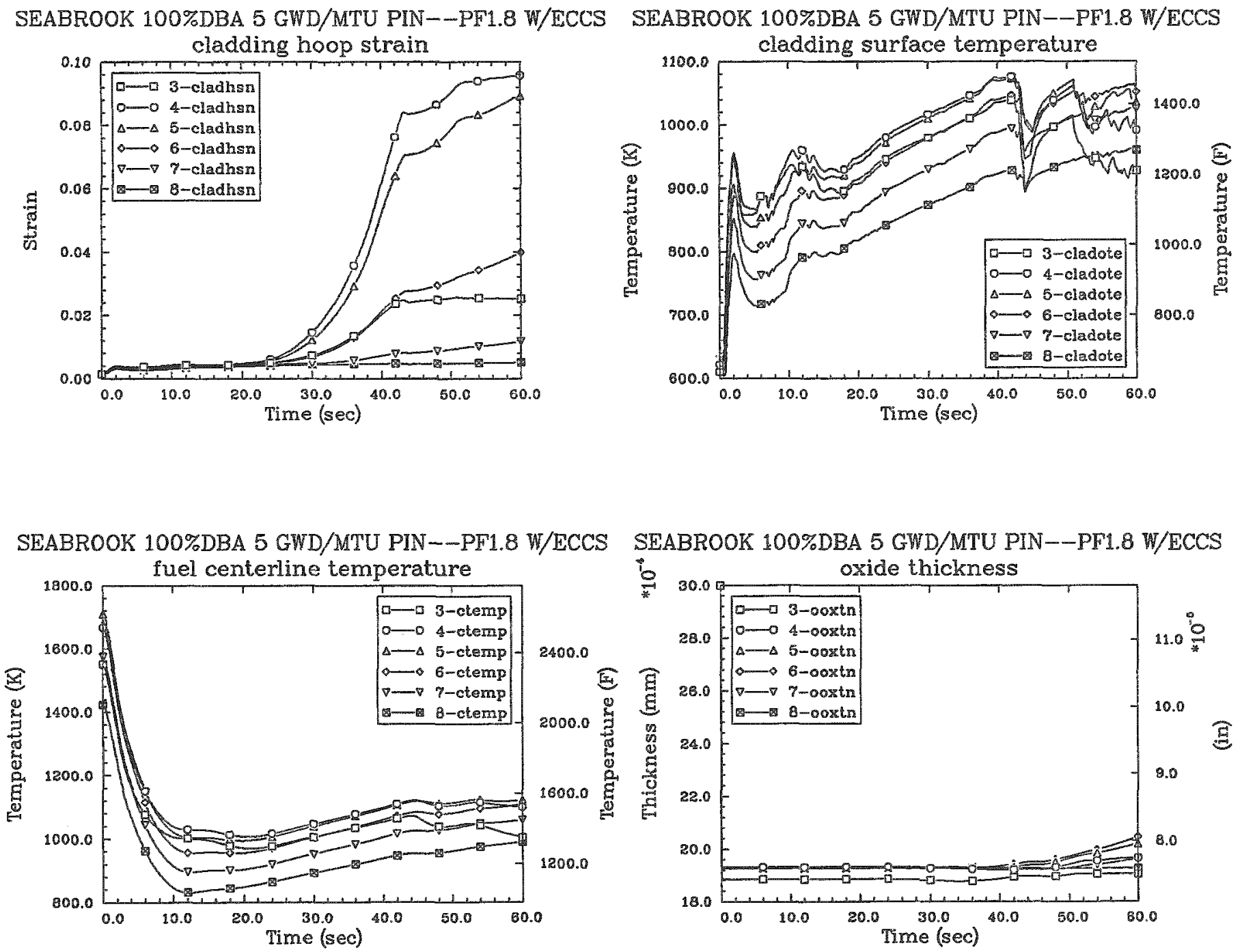

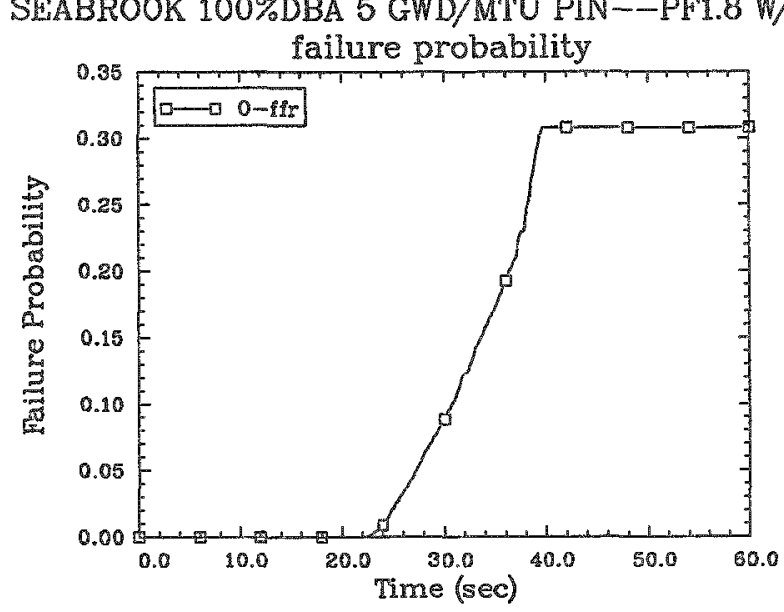


SEABROOK 100\%DBA 50 GWD/MTU PIN--PF2.32 W/E\&PISEABROOK 100\%DBA 50 GWD/MTU PIN--PF2.32 W/E\&PT
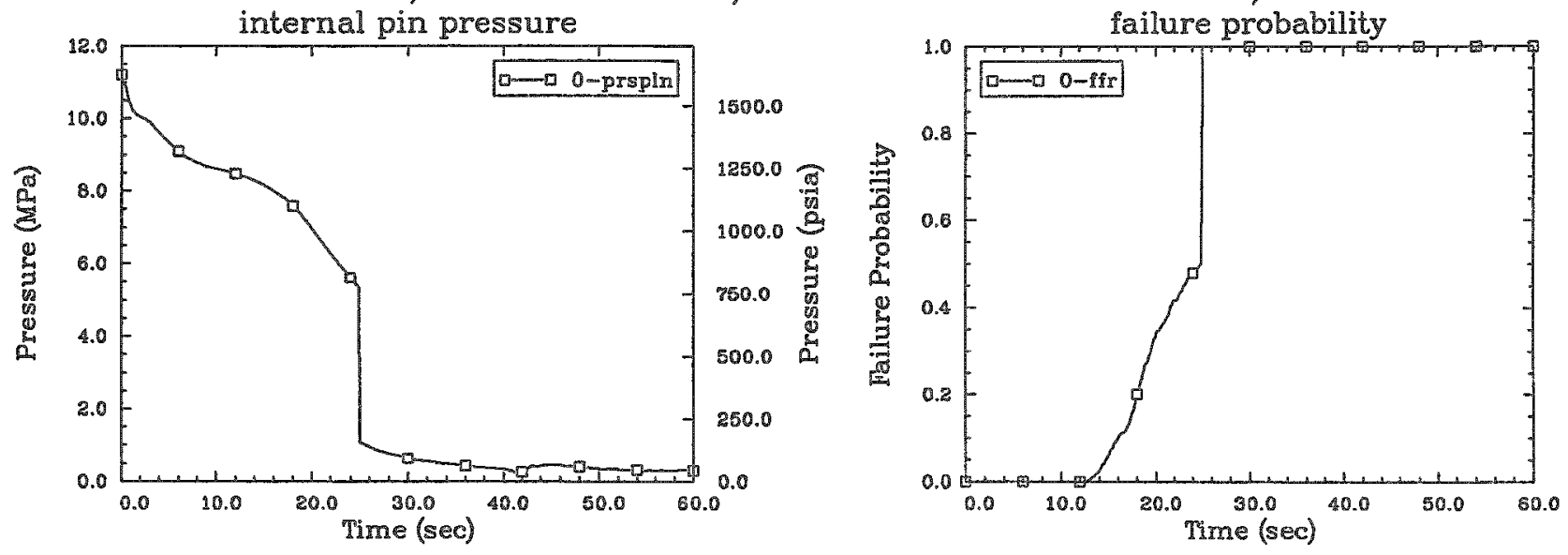

SEABROOK 100\%DBA 50 GWD/MTU PIN--PF2.32 W/E\&CPTSEABROOK 100\%DBA 50 GWD/MTU PIN--PF2.32 W/E\&PT
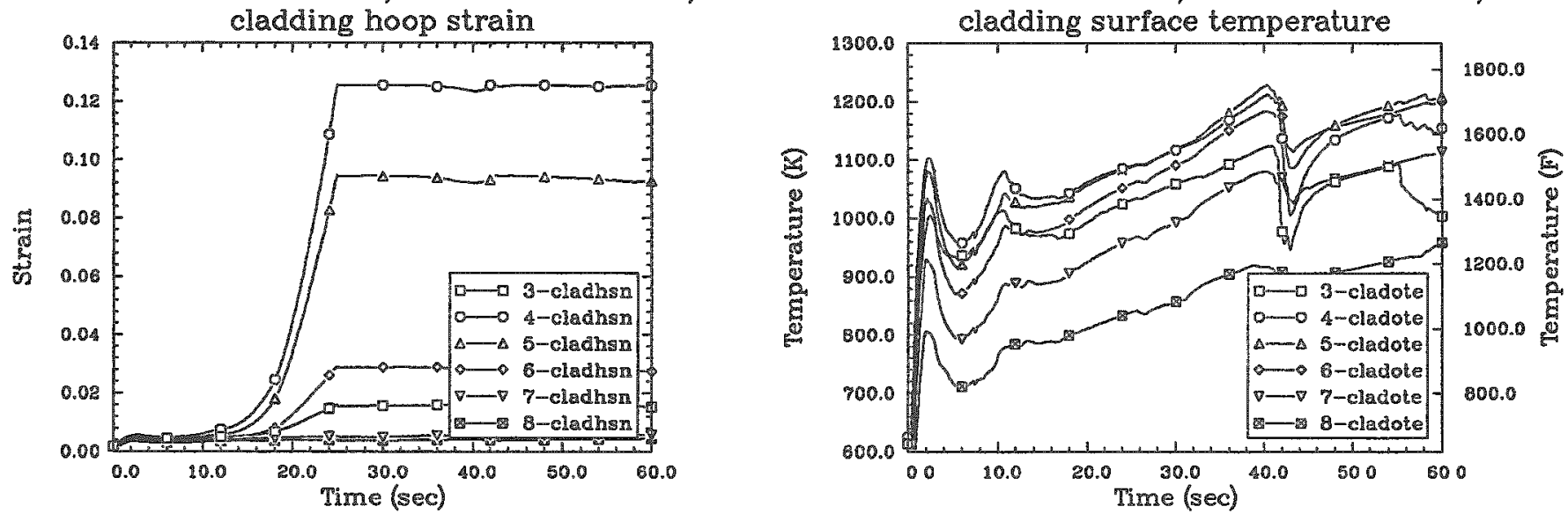

SEABROOK 100\%DBA 50 GWD/MTU PIN--PF2.32 W/E\&PTSEABROOK 100\%DBA 50 GWD/MTU PIN--PF2.32 W/E\&PT
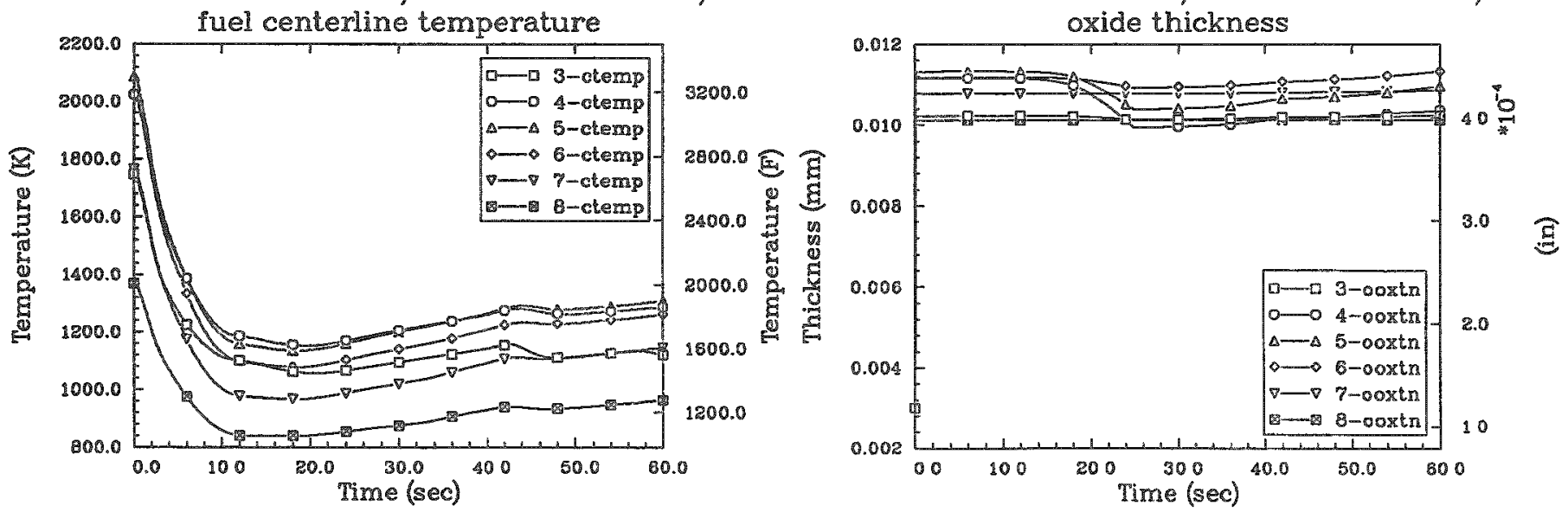
SEABROOK 100\%DBA 35 GWD/MTU PIN--PF2.32 W/E\&PTEABROOK 100\%DBA 35 GWD/MTU PIN--PF2.32 W/E\&PT

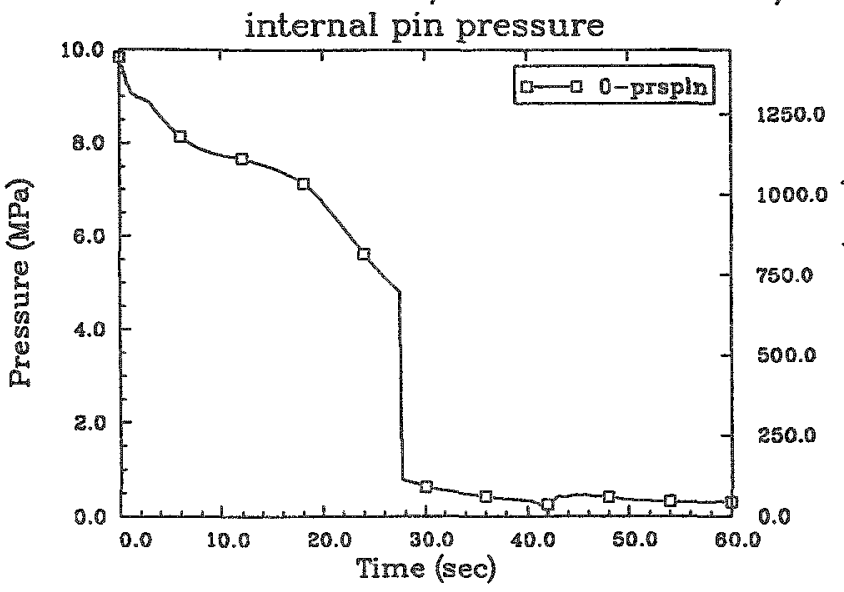

failure probability
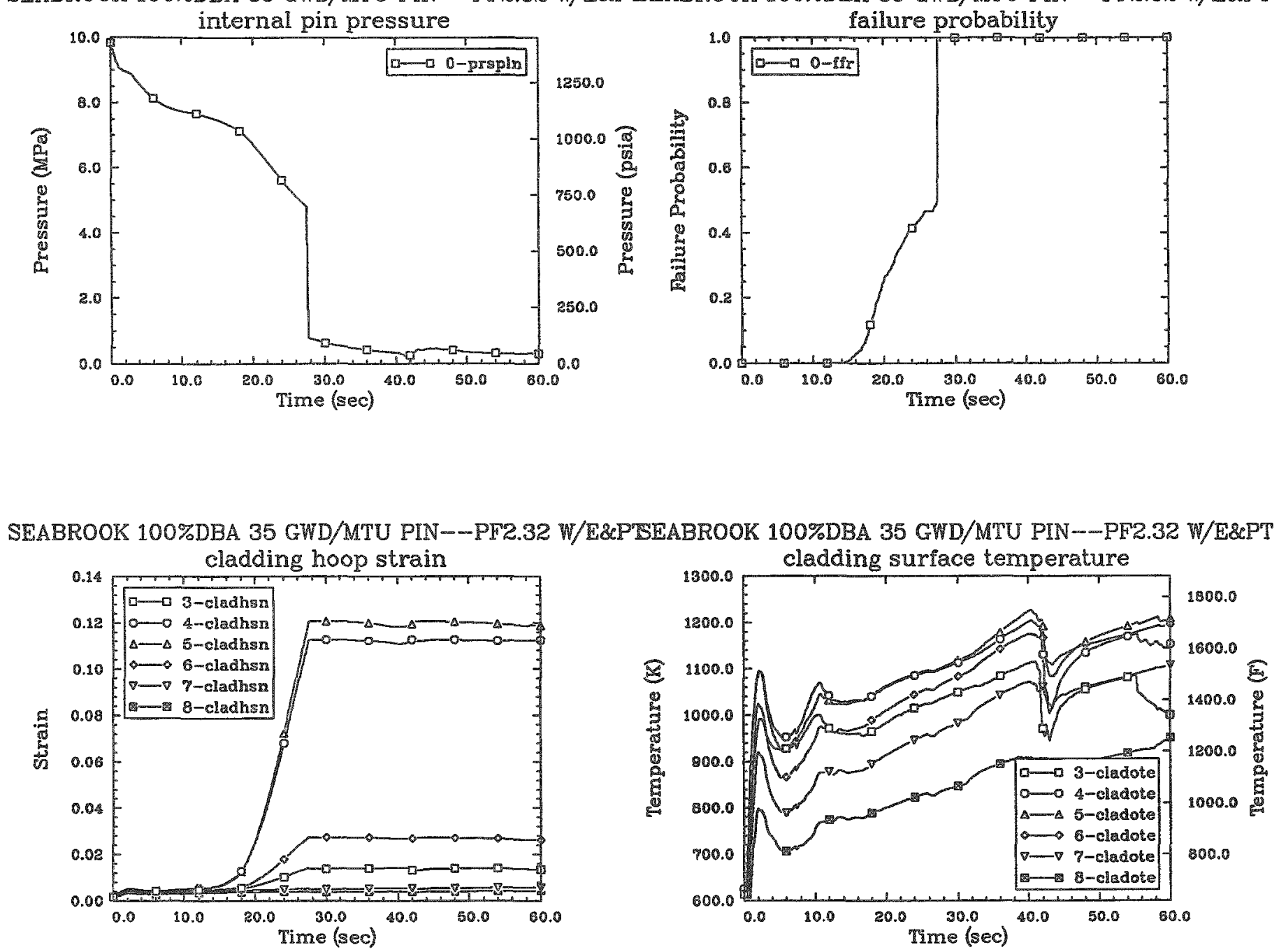

SEABROOK 100\%DBA 35 GWD/MTU PIN--PF2.32 W/E\&PTEABROOK 100\%DBA 35 GWD/MTU PIN--PF2.32 W/E\&PT
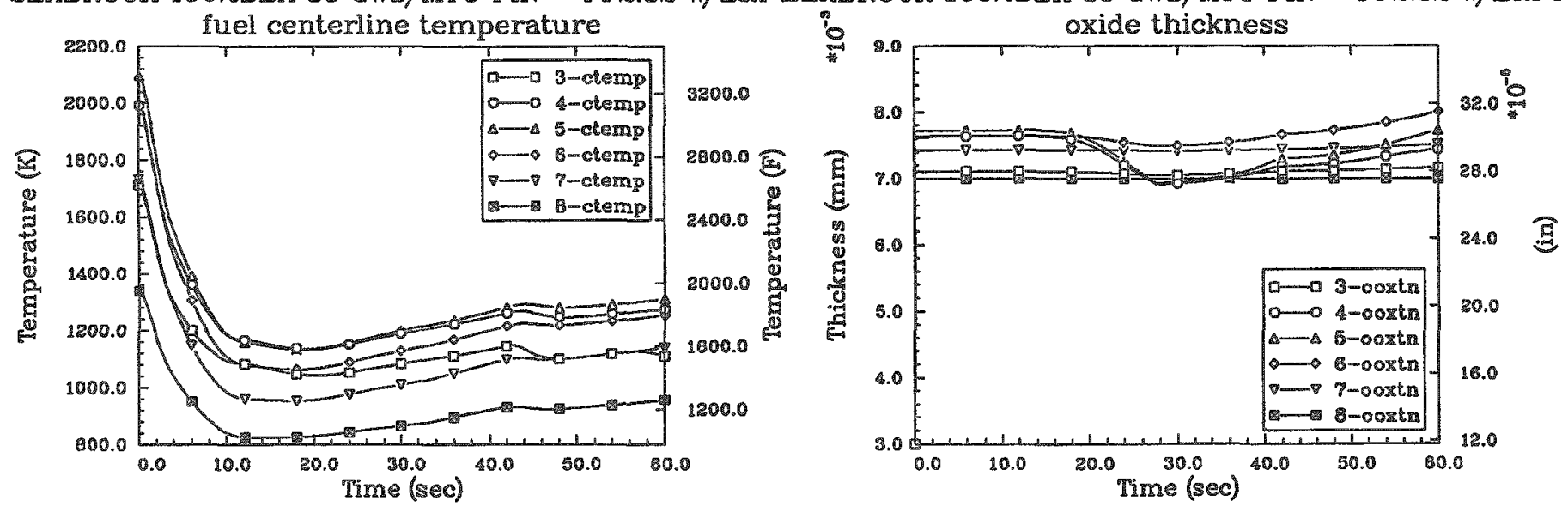
SEABROOK 100\%DBA 20 GWD/MTU PIN--PF2 32 W/E\&PTSEABROOK 100\%DBA 20 GWD/MTU PIN--PF2.32 W/E\&PT
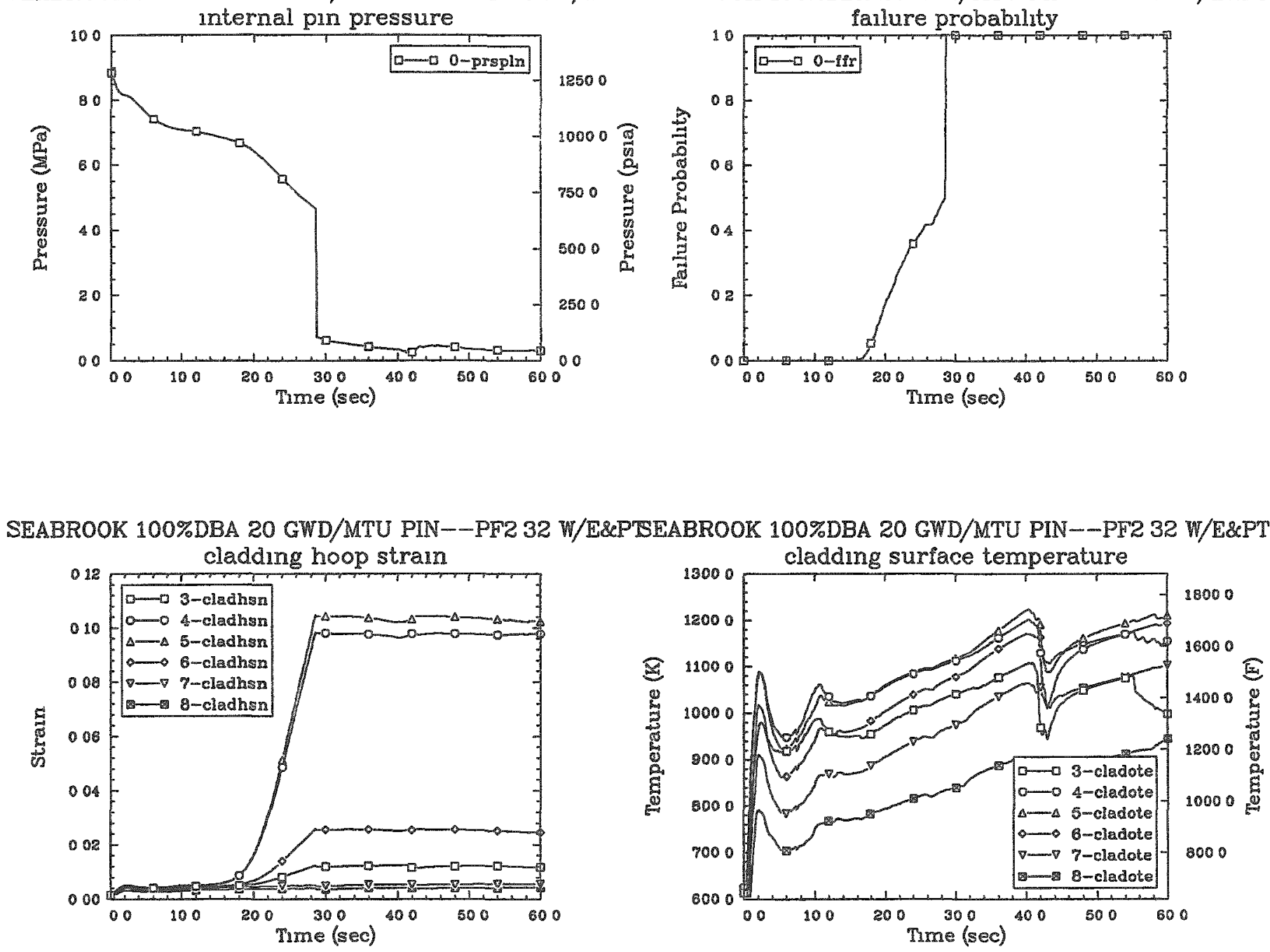

SEABROOK 100\%DBA 20 GWD/MTU PIN--PF2 32 W/E\&PSEABROOK 100\%DBA 20 GWD/MTU PIN--PF2 32 W/E\&PT
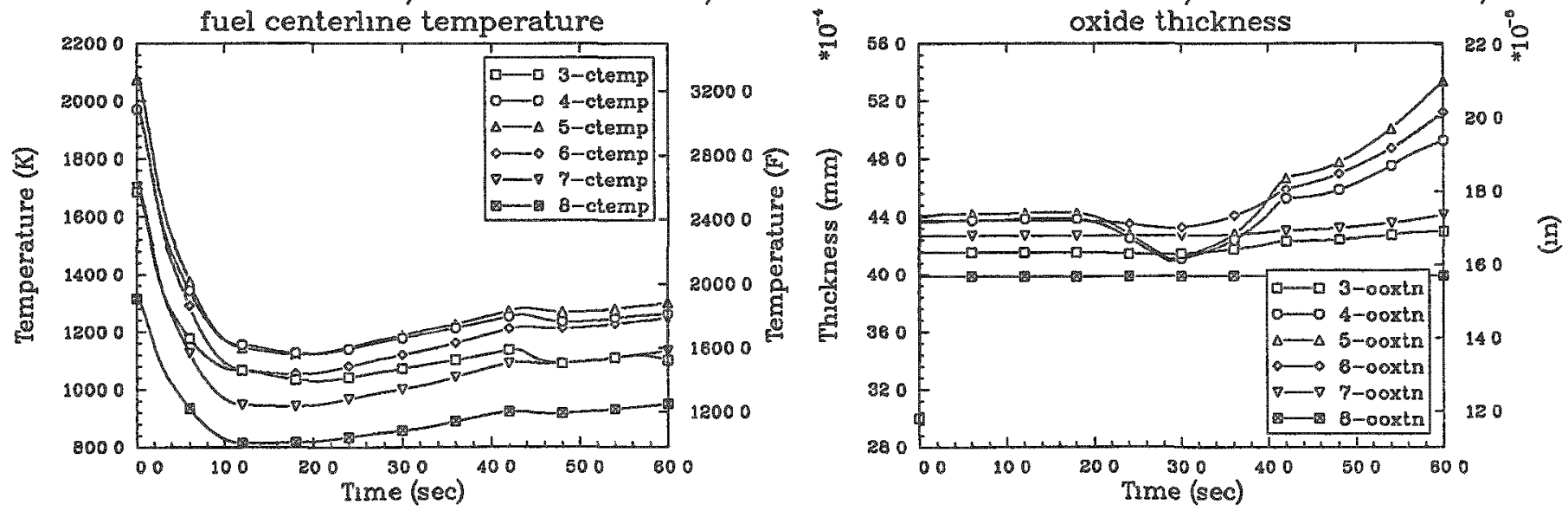
SEABROOK 100\%DBA 5 GWD/MTU PIN--PF2.32 W/E\&PT SEABROOK 100\%DBA 5 GWD/MTU PIN--PF2.32 W/E\&PT
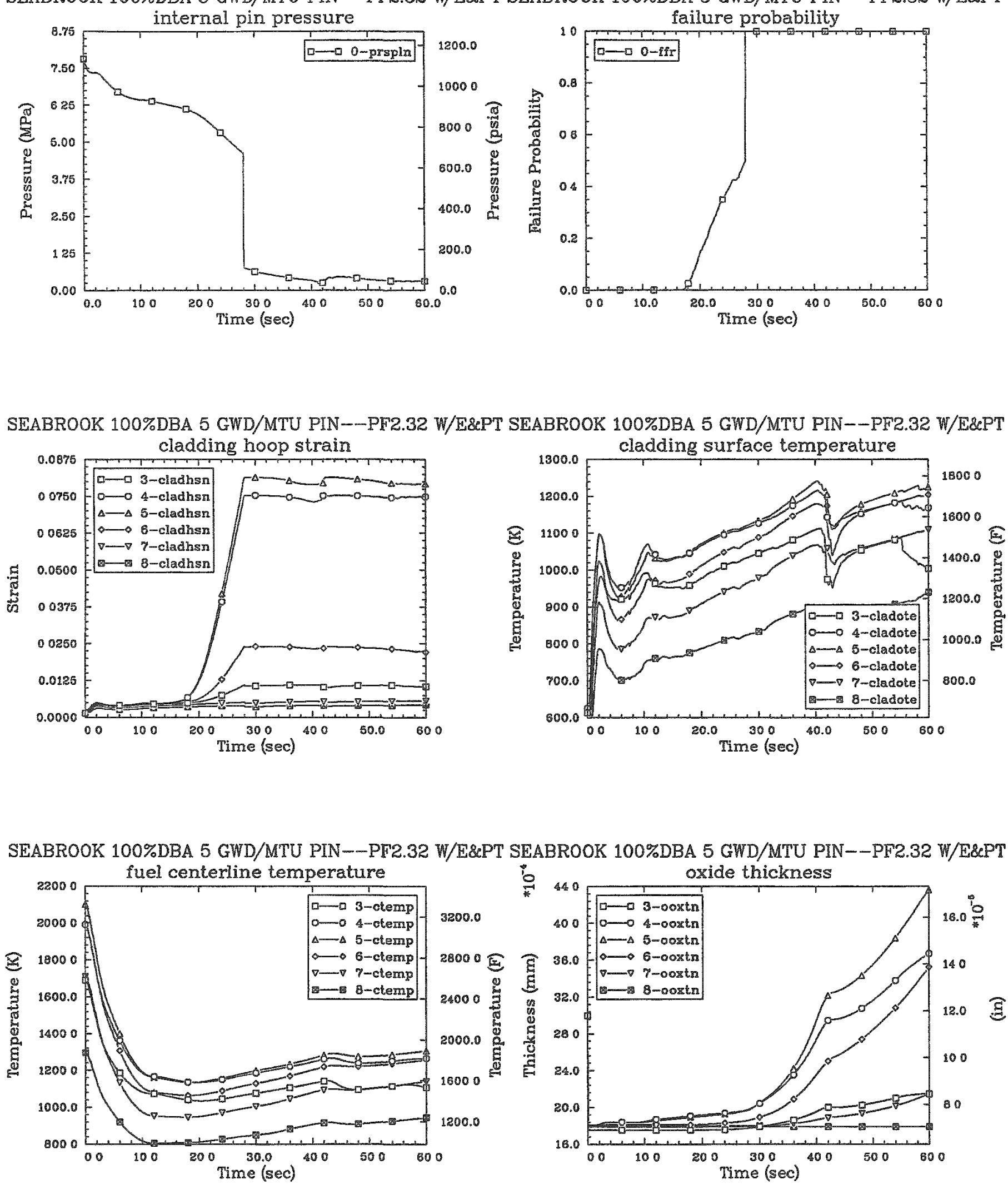

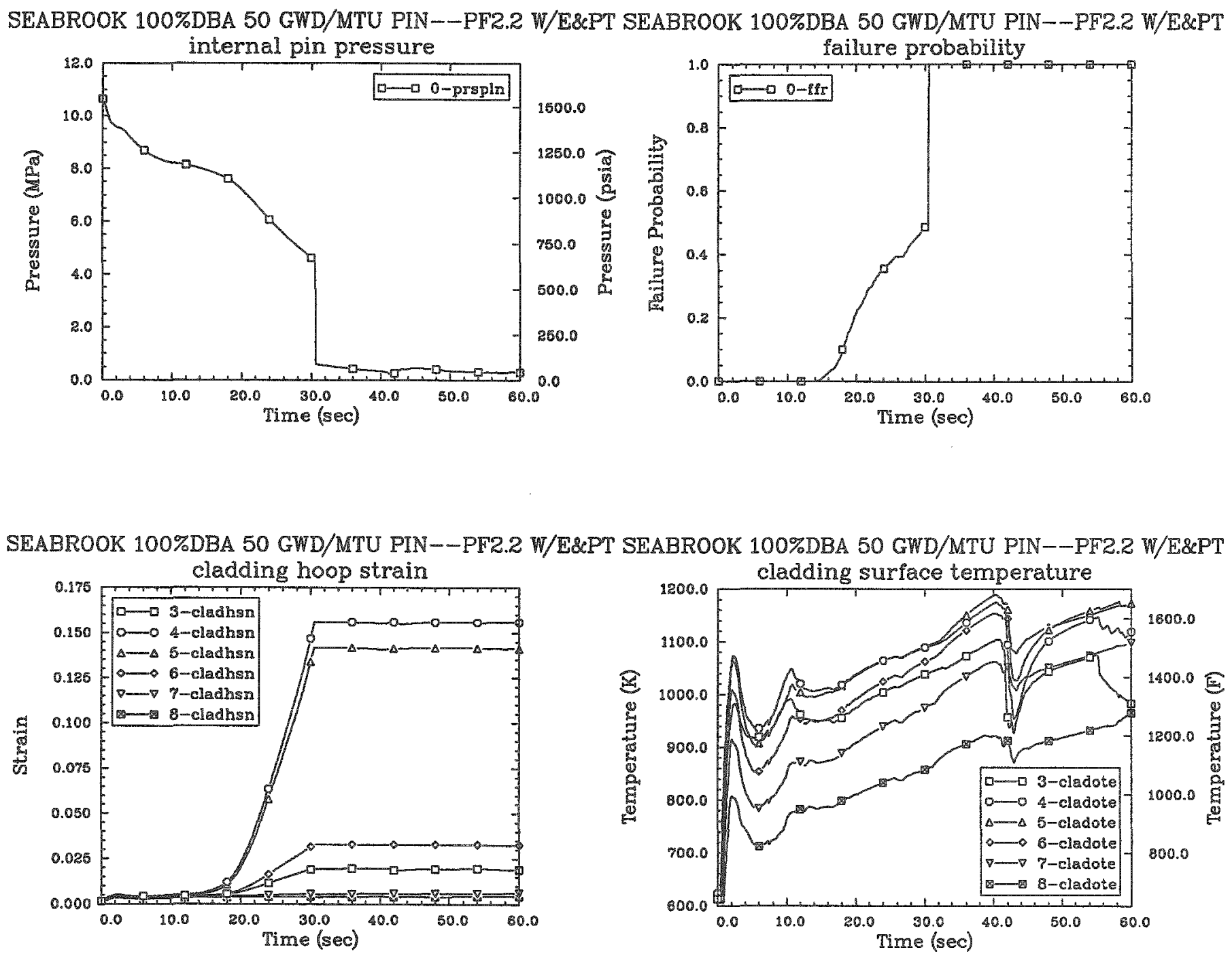

SEABROOK 100\%DBA 50 GWD/MTU PIN--PF2.2 W/E\&PT SEABROOK 100\%DBA 50 GWD/MTU PIN--PF2.2 W/E\&PT
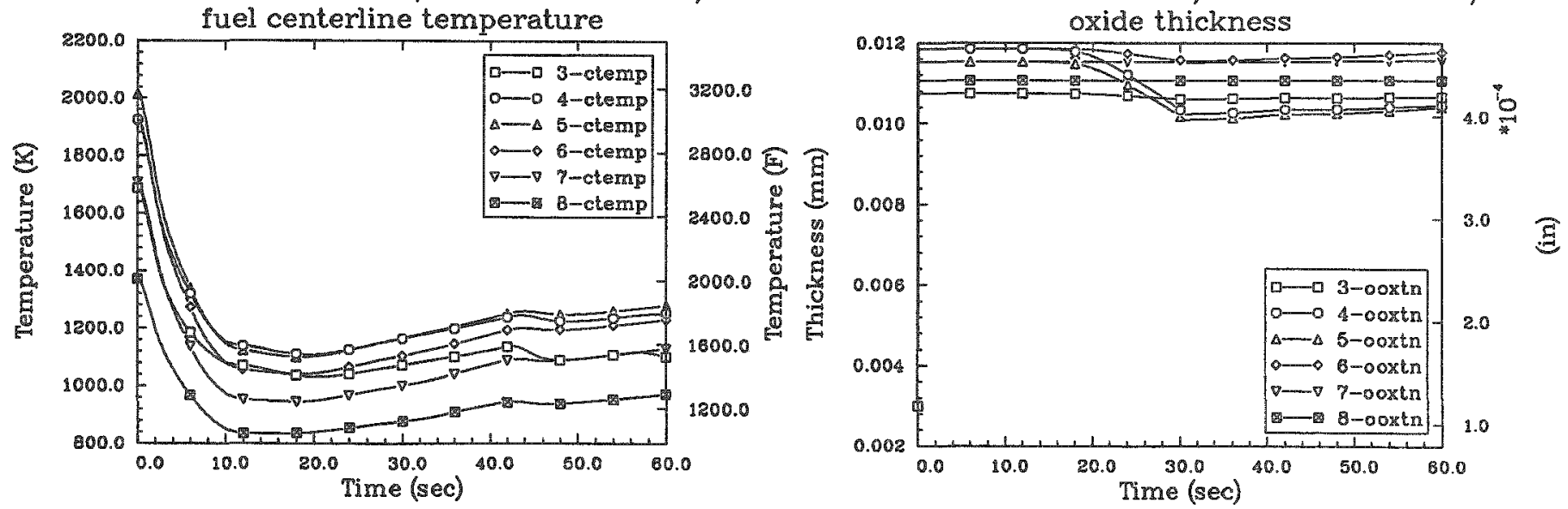
SEABROOK 100\%DBA 35 GWD/MTU PIN--PF2.2 W/E\&PT SEABROOK 100\%DBA 35 GWD/MTU PIN--PF2.2 W/E\&PT
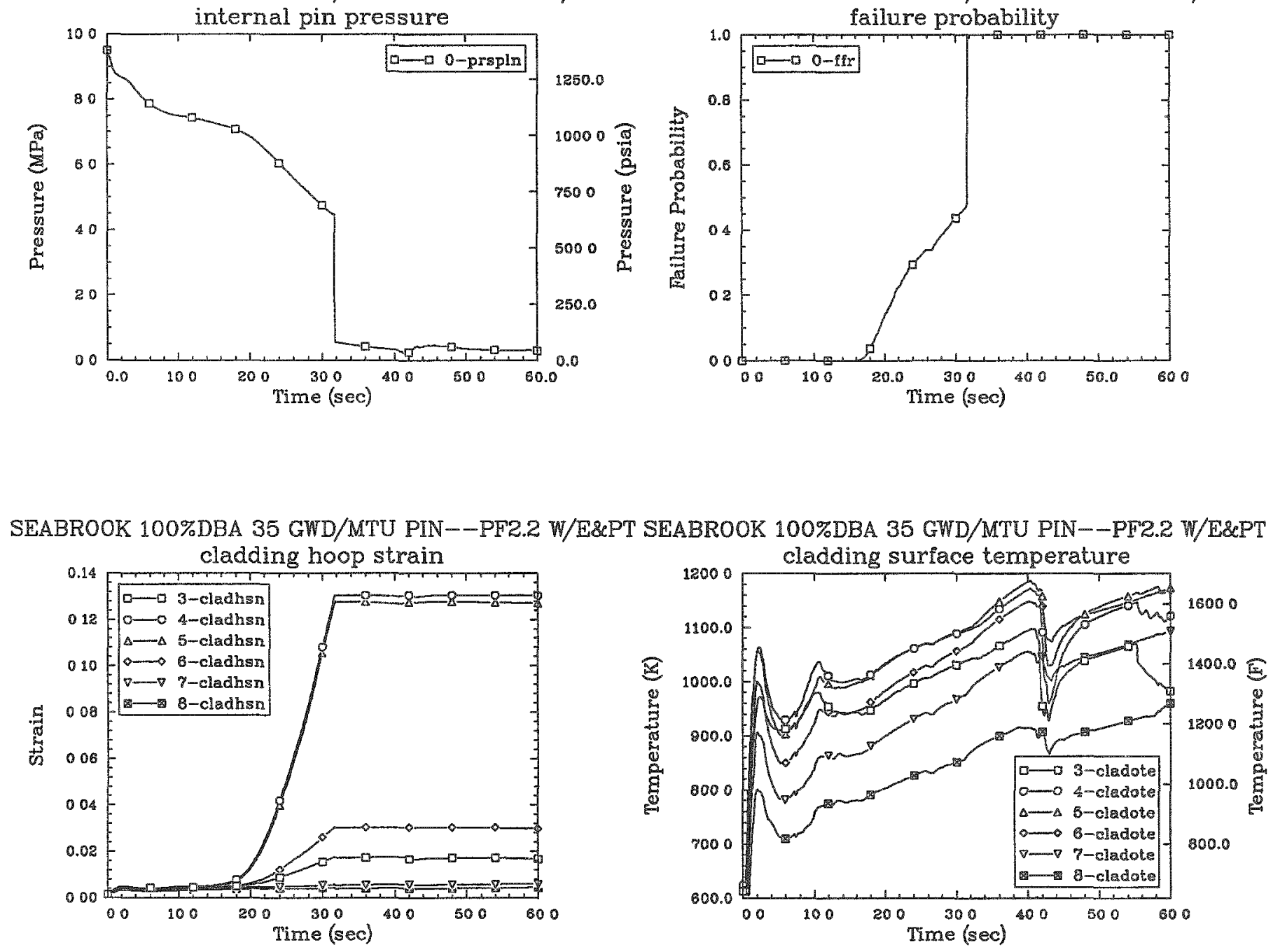

SEABROOK 100\%DBA 35 GWD/MTU PIN--PF2.2 W/E\&PT SEABROOK 100\%DBA 35 GWD/MTU PIN--PF2.2 W/E\&PT
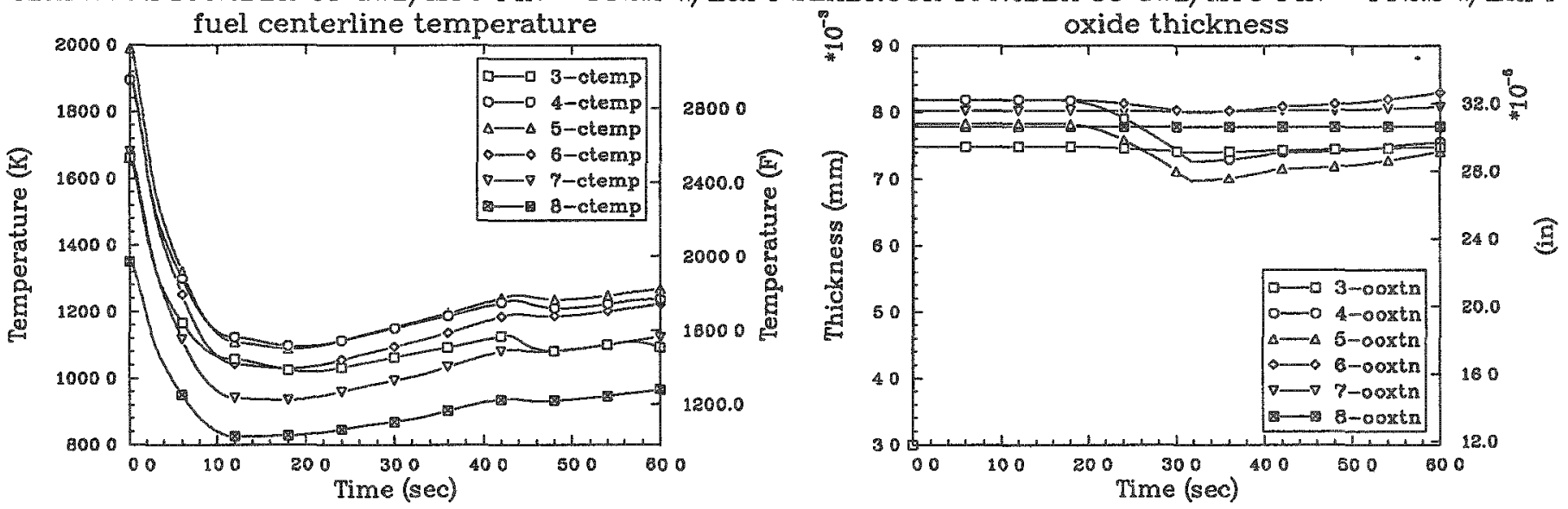
SEABROOK 100\%DBA 20 GWD/MTU PIN--PF2.2 W/E\&PT SEABROOK 100\%DBA 20 GWD/MTU PIN--PF2.2 W/E\&PT

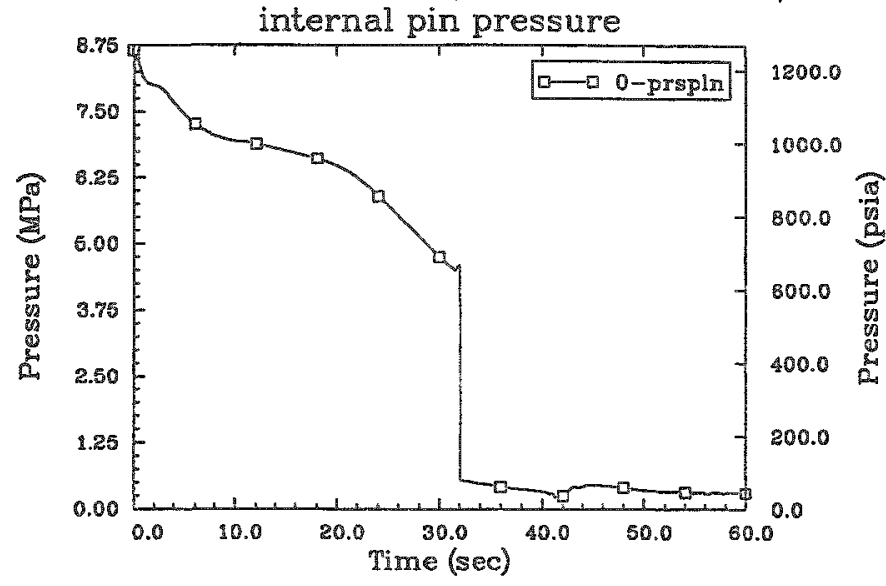

failure probability
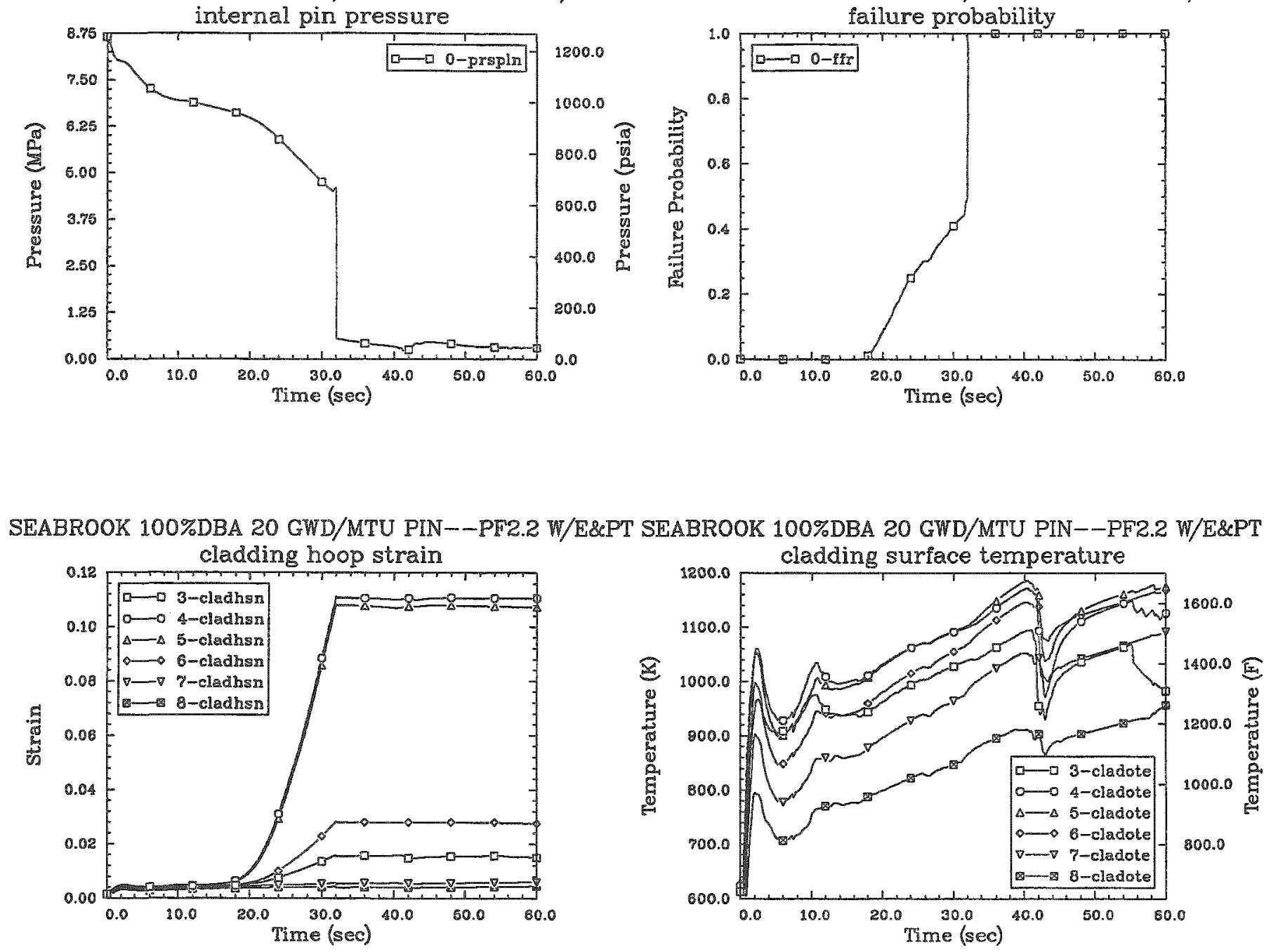

SEABROOK 100\%DBA 20 GWD/MTU PIN--PF2.2 W/E\&PT SEABROOK 100\%DBA 20 GWD/MTU PIN--PF2.2 W/E\&PT
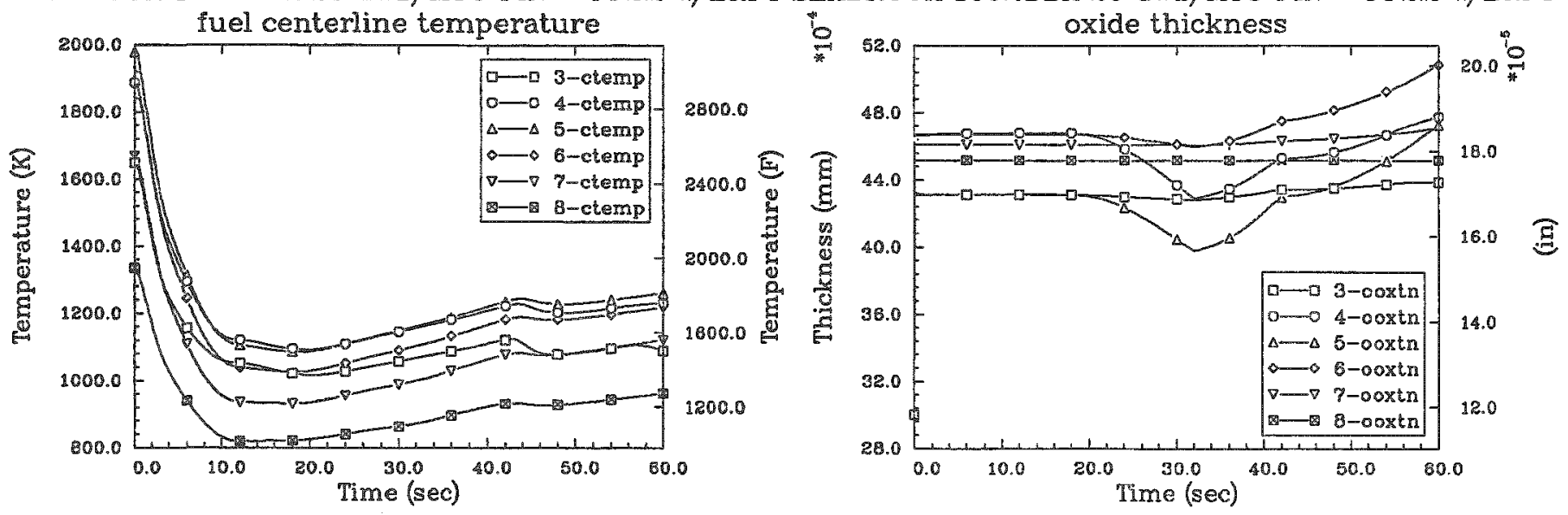

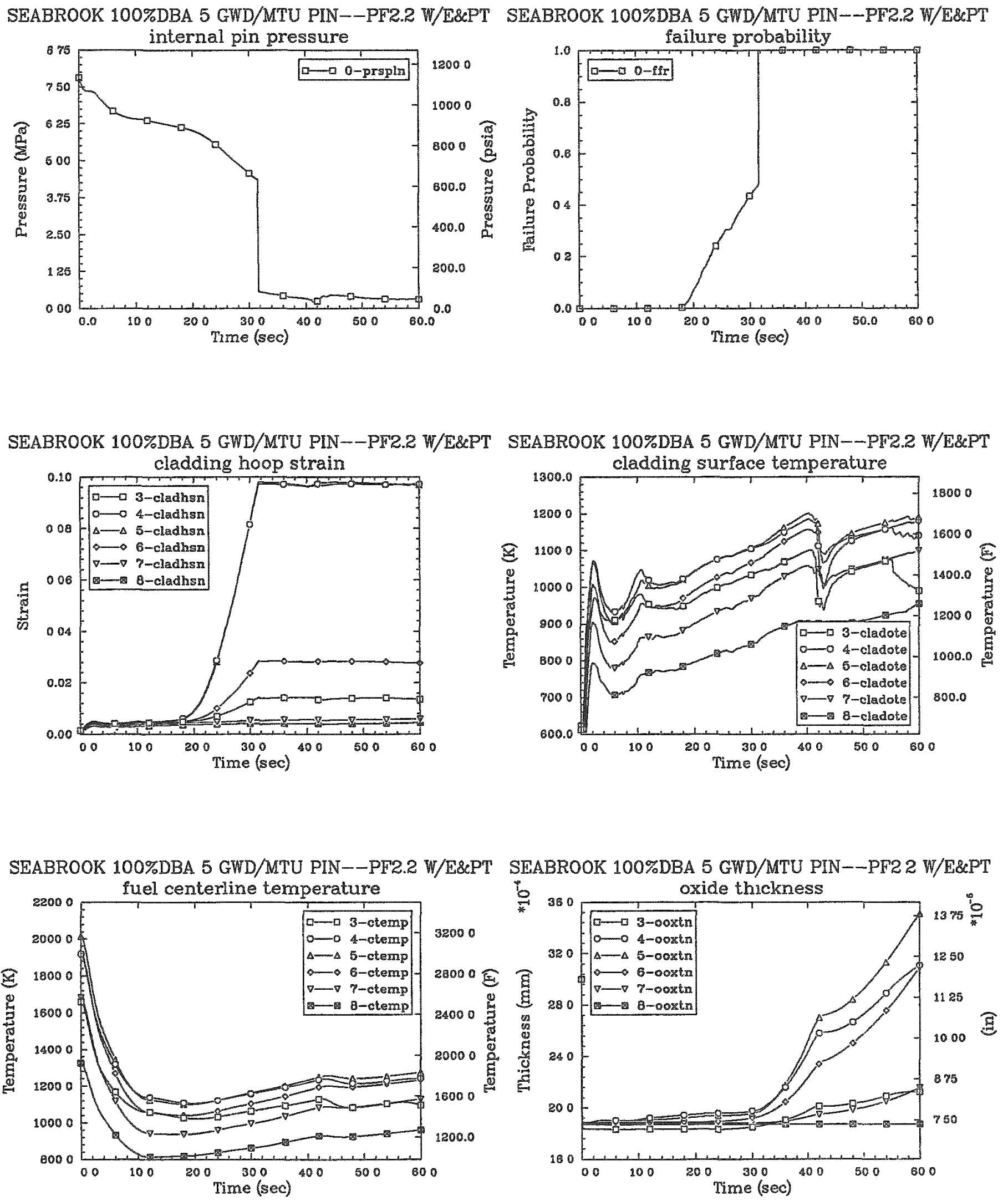
SEABROOK 100\%DBA 50 GWD/MTU PIN--PF2.0 W/E\&PT SEABROOK 100\%DBA 50 GWD/MTU PIN--PF2.0 W/E\&PT
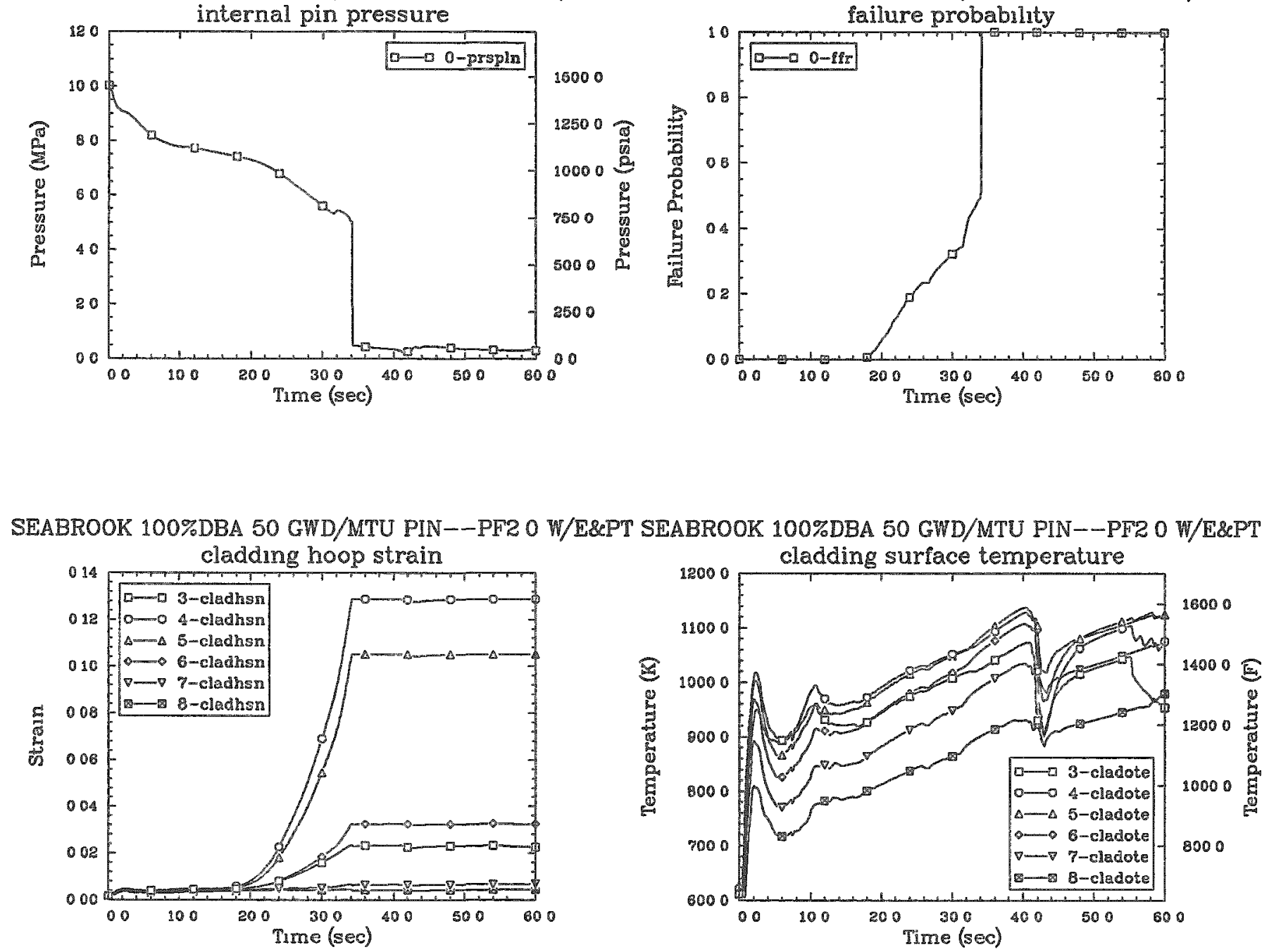

SEABROOK 100\%DBA 50 GWD/MTU PIN--PF2.0 W/E\&PT SEABROOK 100\%DBA 50 GWD/MTU PIN--PF2 0 W/E\&PT
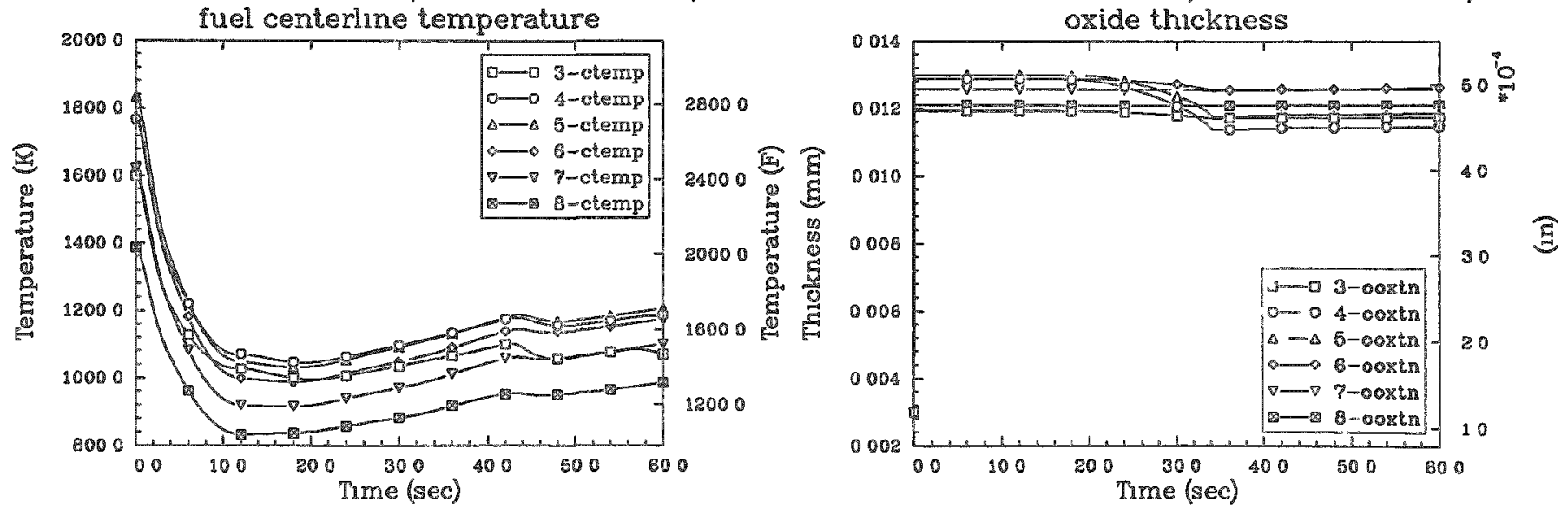

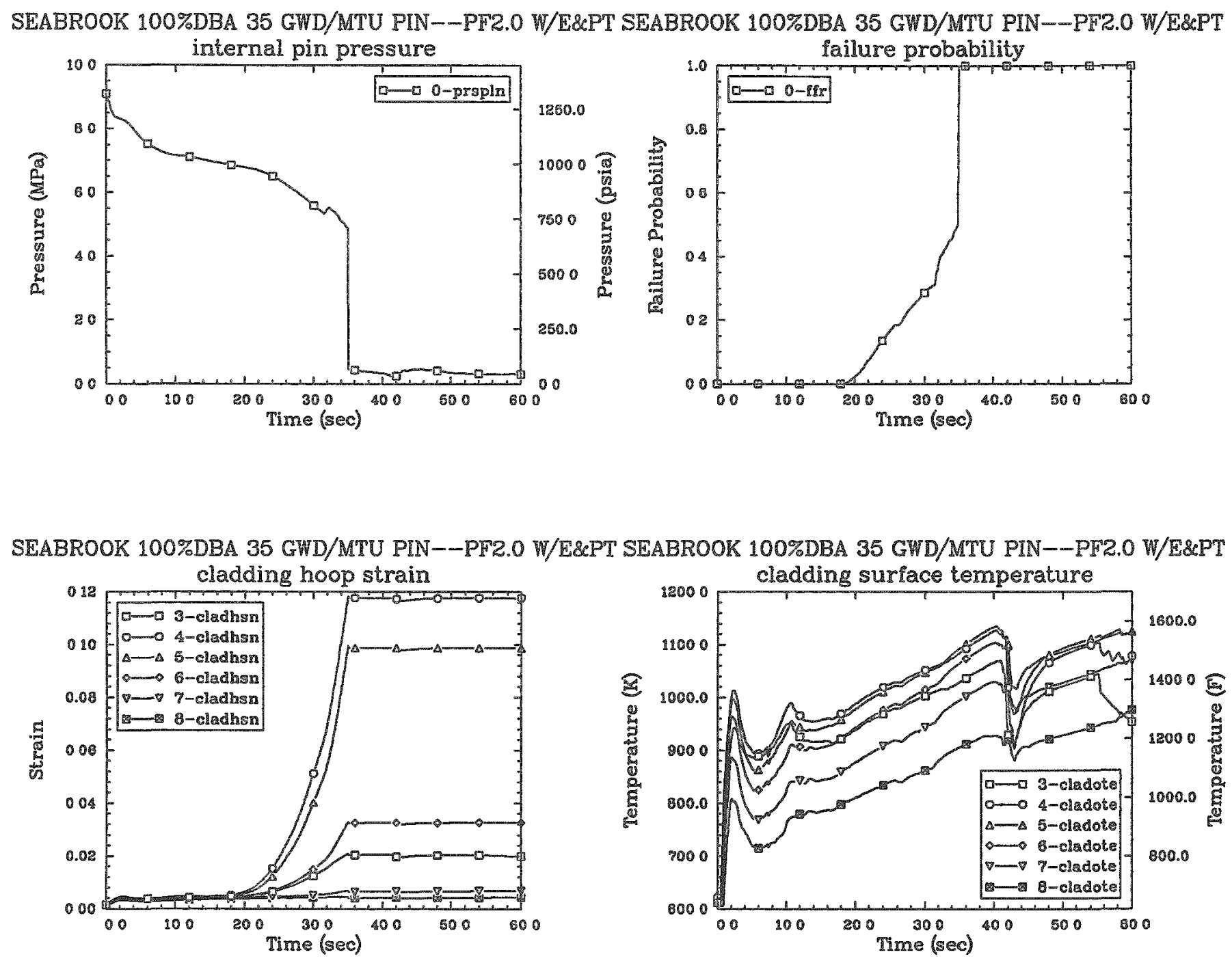

SEABROOK 100\%DBA 35 GWD/MTU PIN--PF2.0 W/E\&PT SEABROOK 100\%DBA 35 GWD/MTU PIN--PF2.0 W/E\&PT
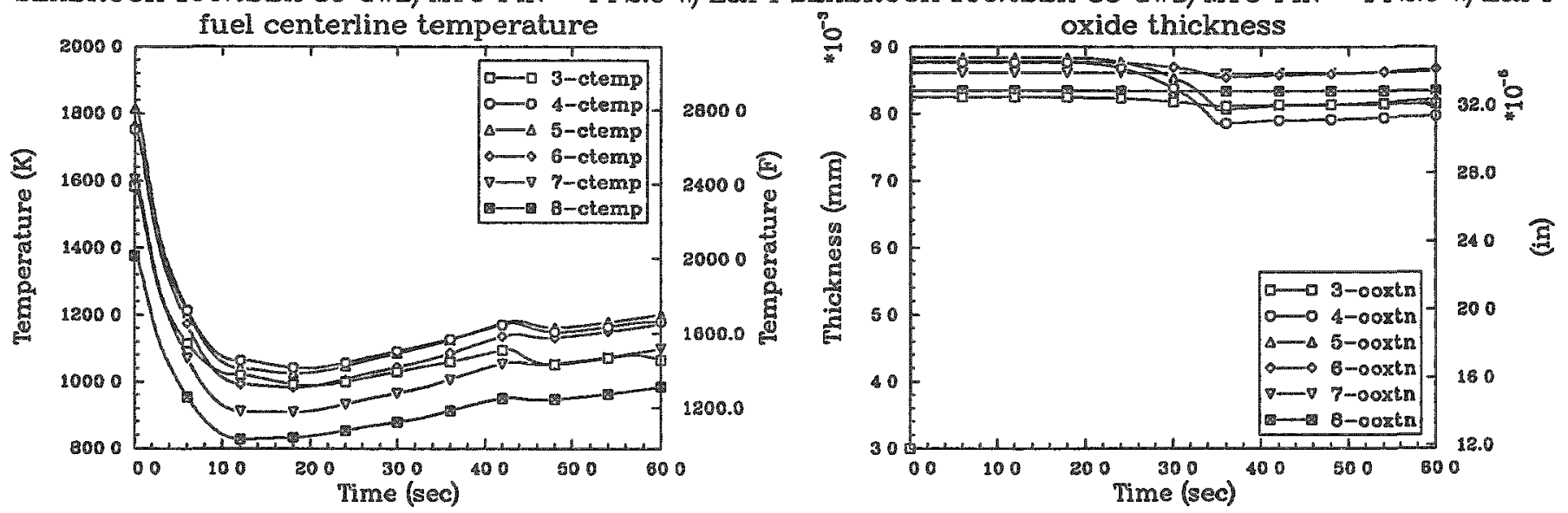

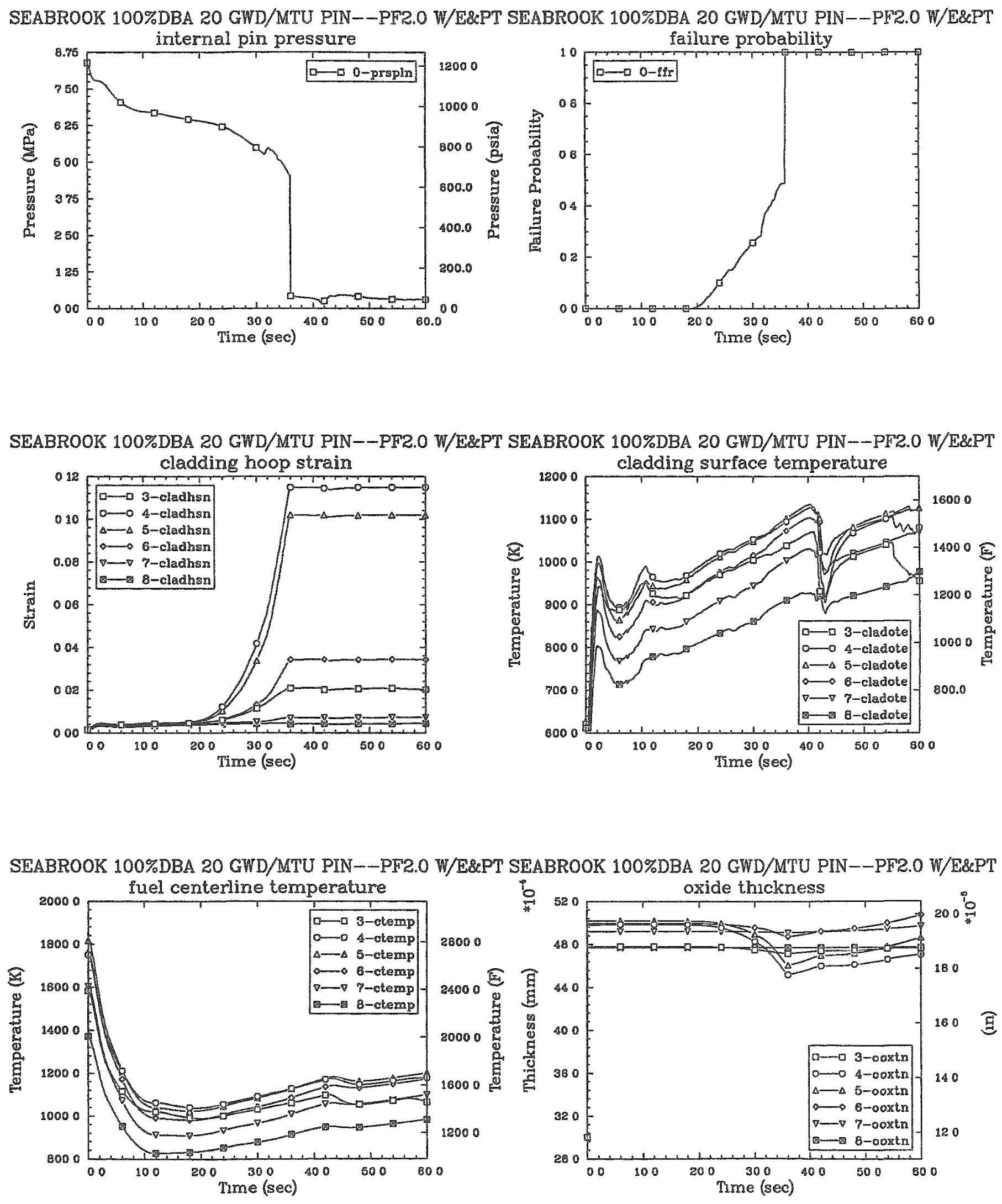
SEABROOK 100\%DBA 5 GWD/MTU PIN--PF2.0 W/E\&PT SEABROOK 100\%DBA 5 GWD/MTU PIN--PF2.0 W/E\&PT
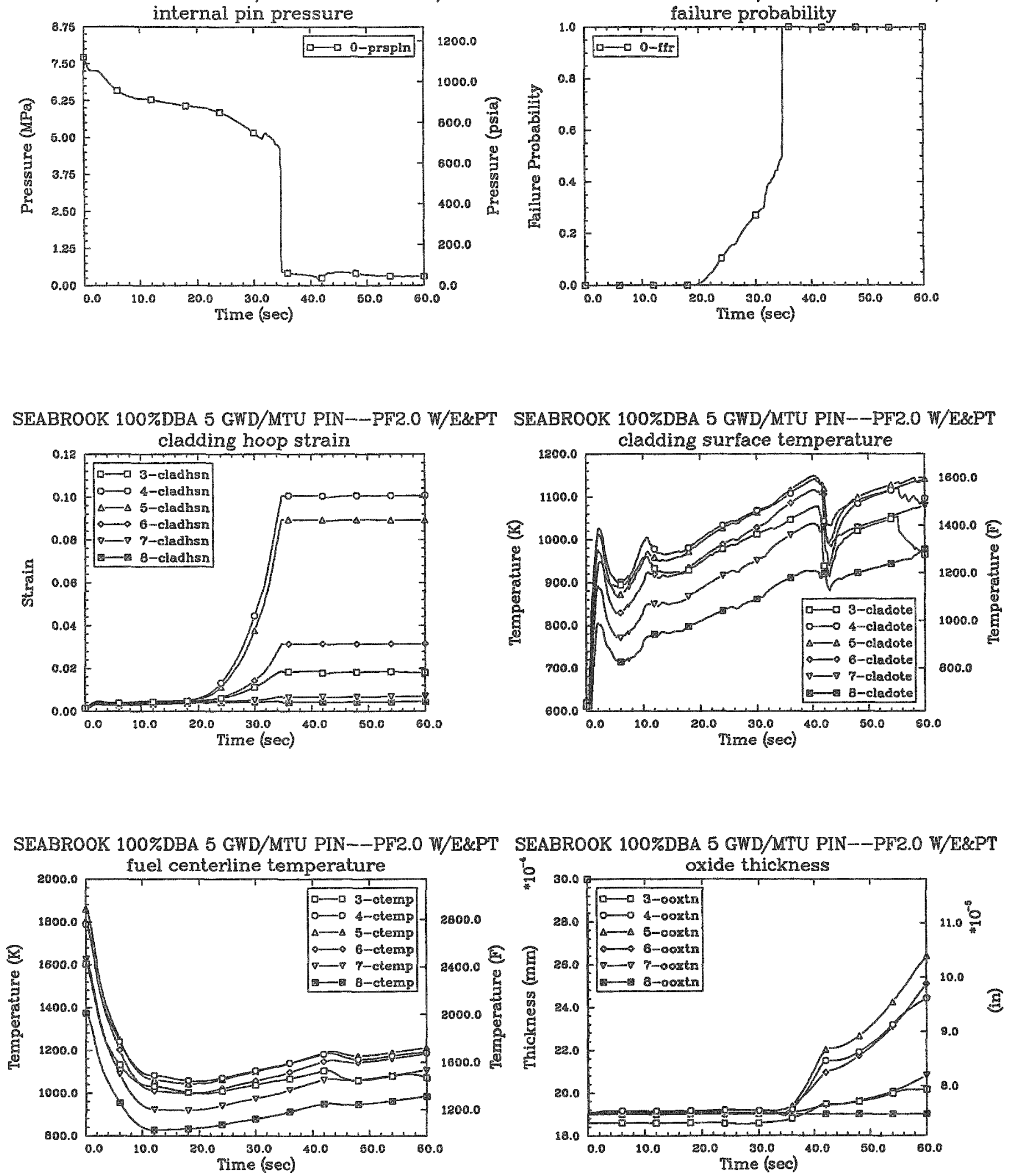

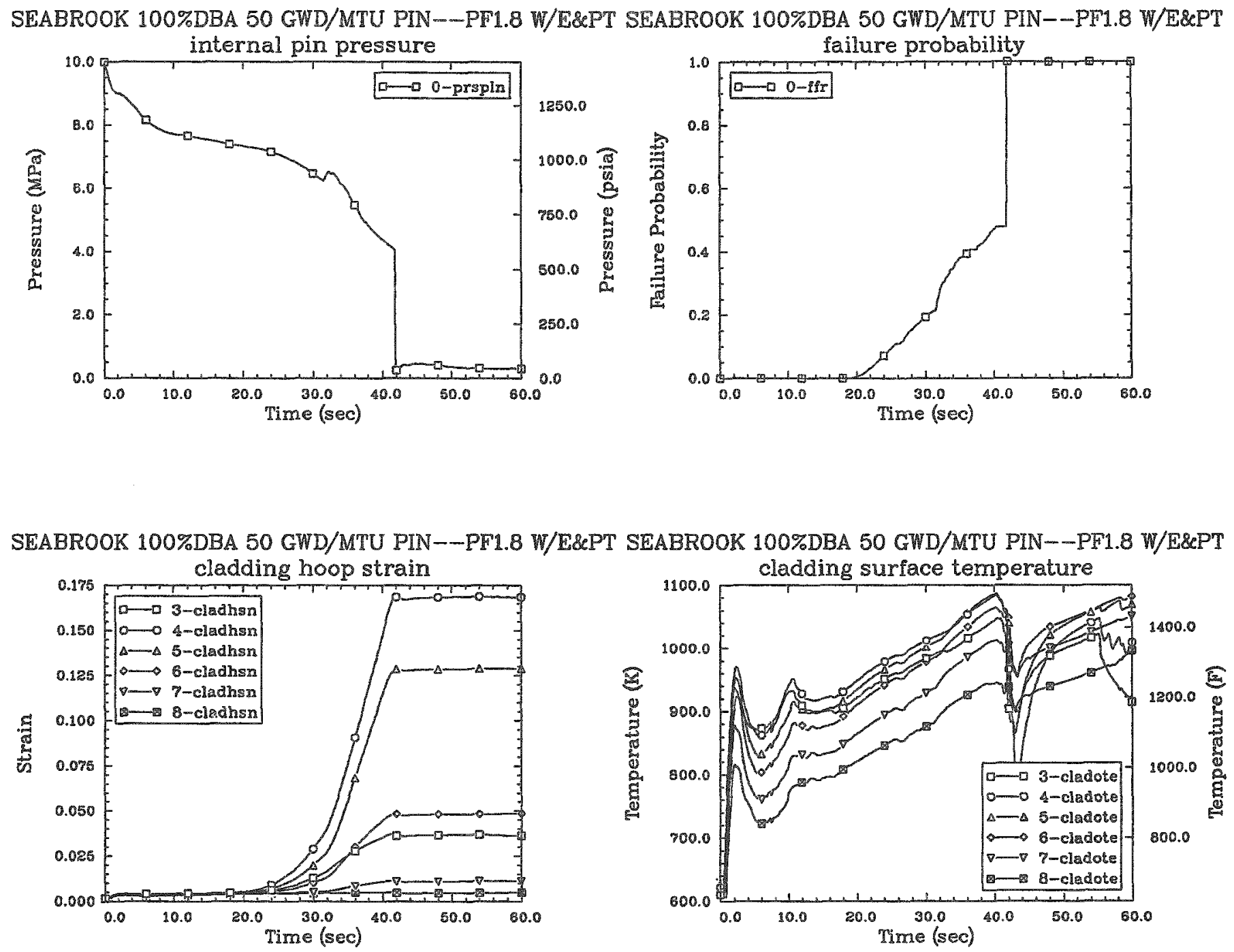

SEABROOK 100\%DBA 50 GWD/MTU PIN--PF1.8 W/E\&PT SEABROOK 100\%DBA 50 GWD/MTU PIN--PF1.8 W/E\&PT
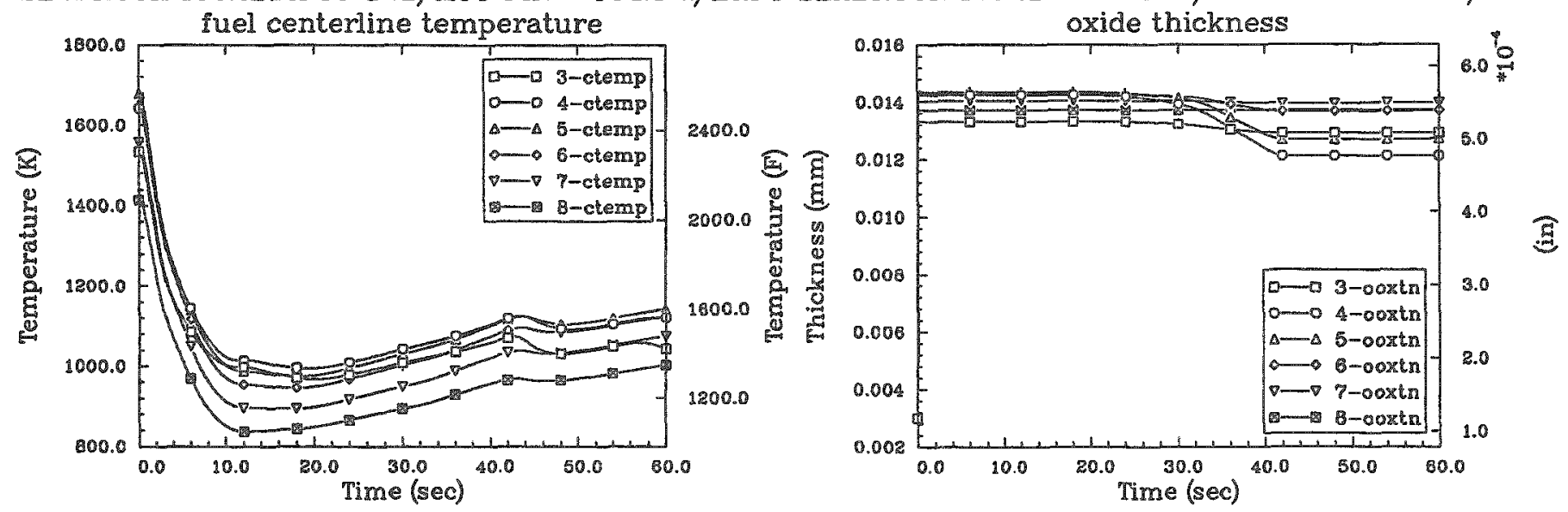
SEABROOK 100\%DBA 35 GWD/MTU PIN--PF1.8 W/E\&PT SEABROOK 100\%DBA 35 GWD/MTU PIN--PF1.8 W/E\&PT
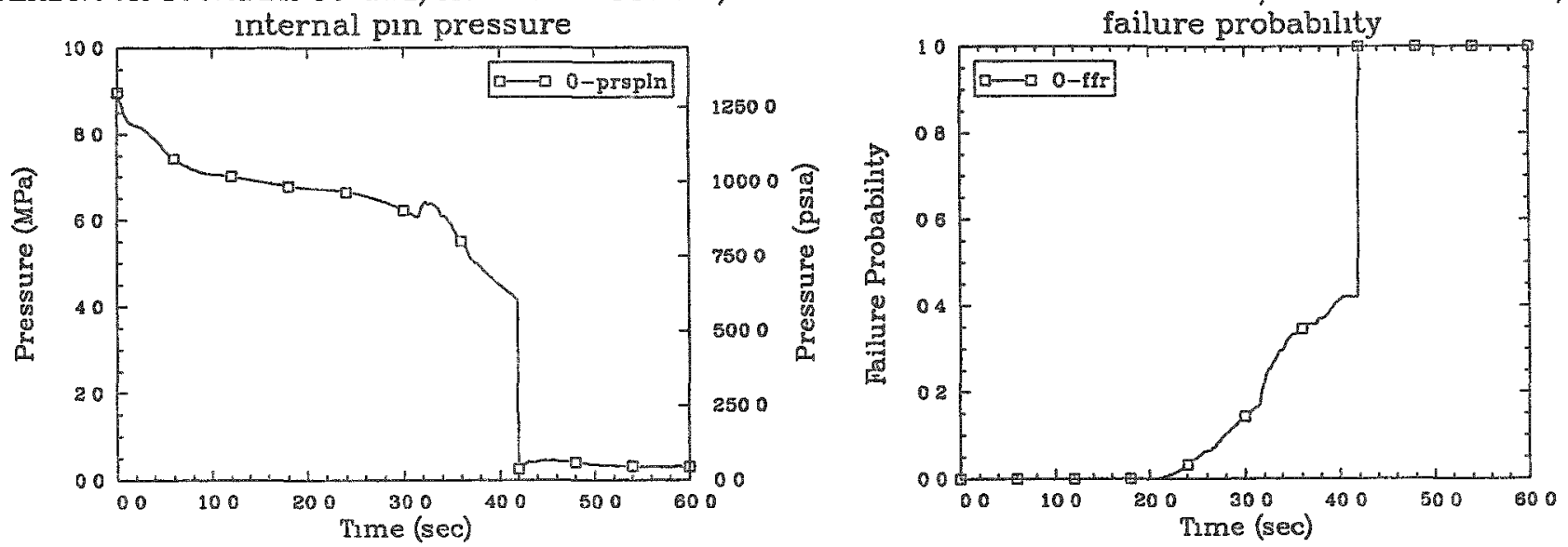

SEABROOK 100\%DBA 35 GWD/MTU PIN--PF1.8 W/E\&PT SEABROOK 100\%DBA 35 GWD/MTU PIN--PF1.8 W/E\&PT
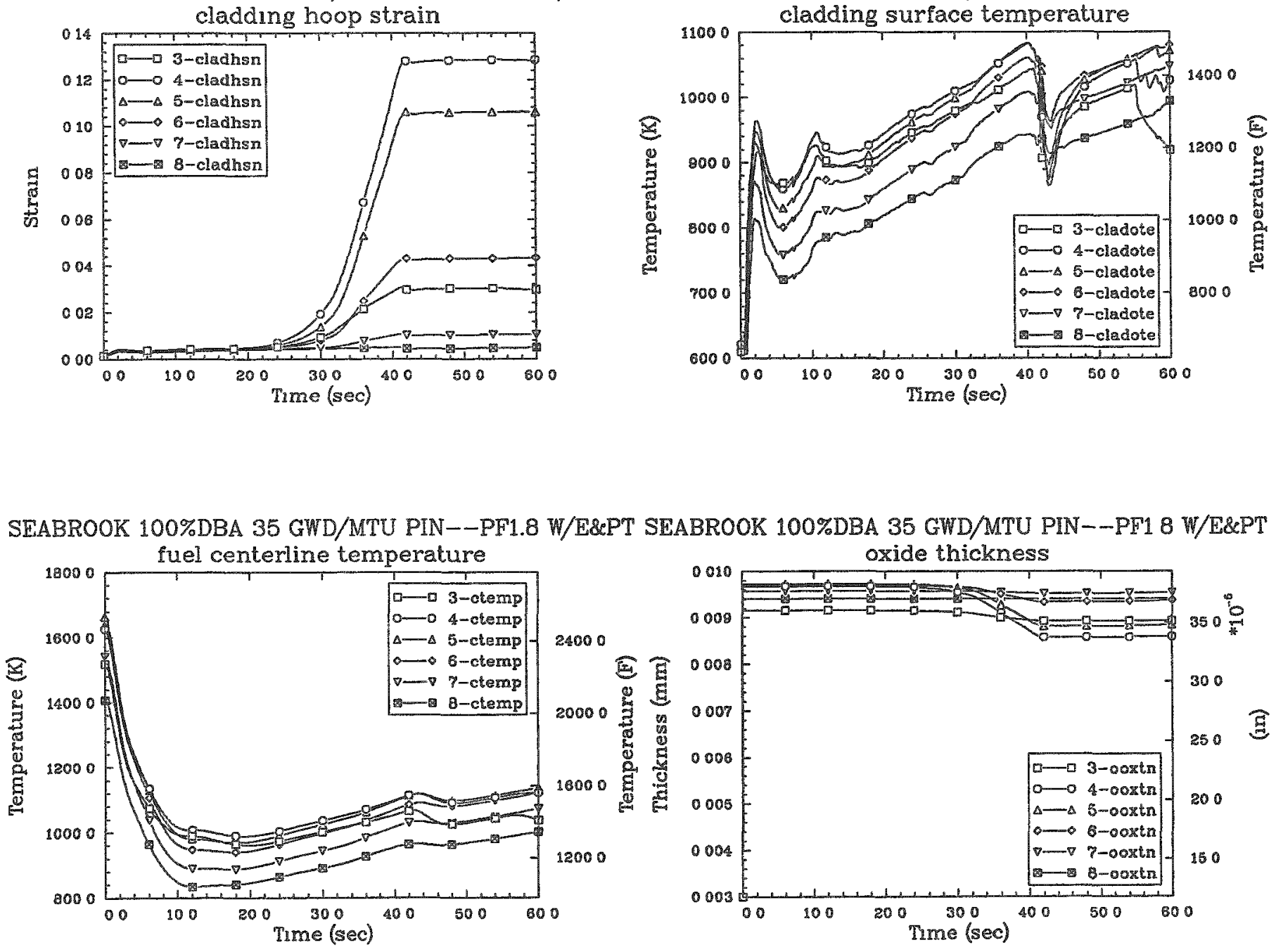

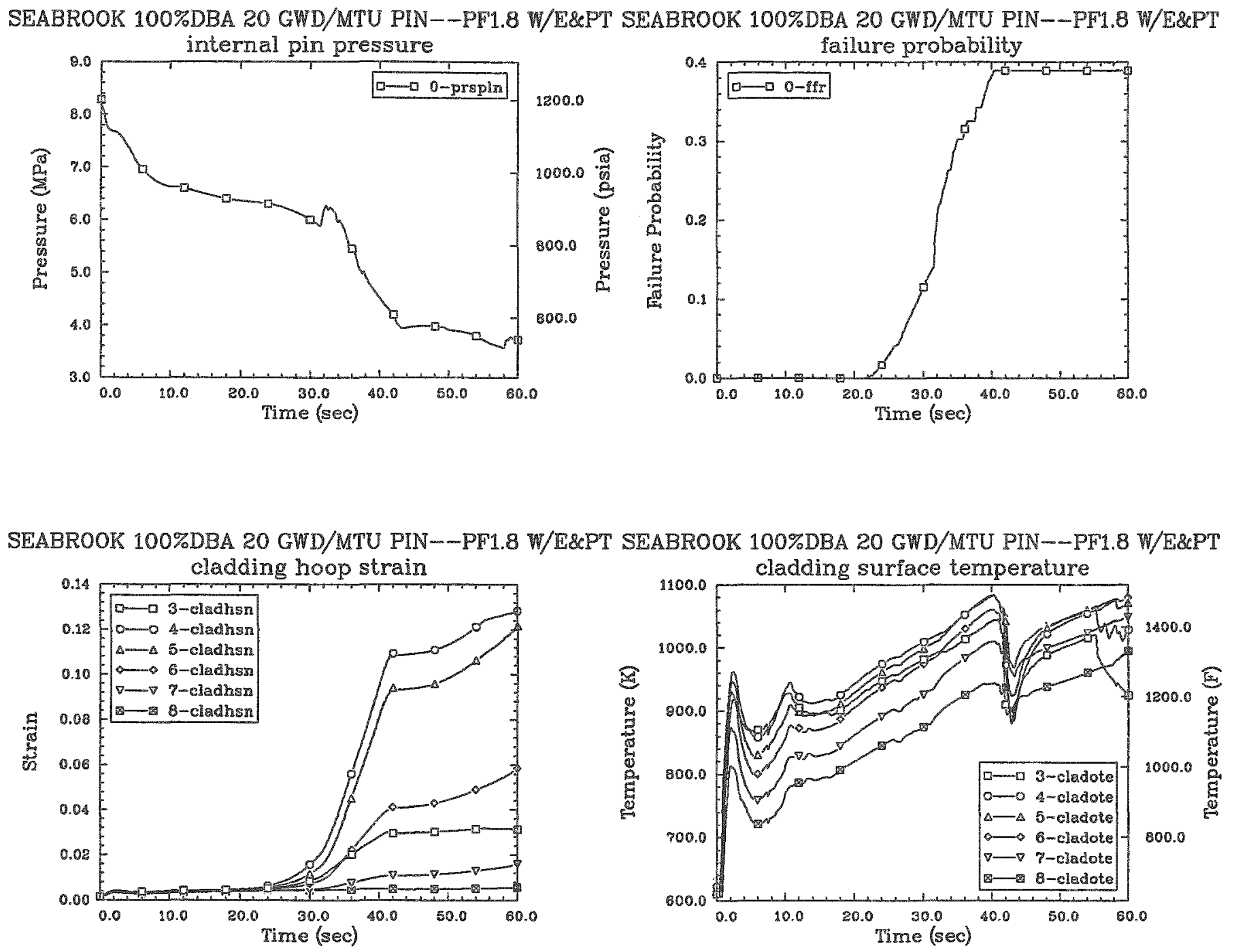

SEABROOK 100\%DBA 20 GWD/MTU PIN--PF1.8 W/E\&PT SEABROOK 100\%DBA 20 GWD/MTU PIN--PF1.8 W/E\&PT
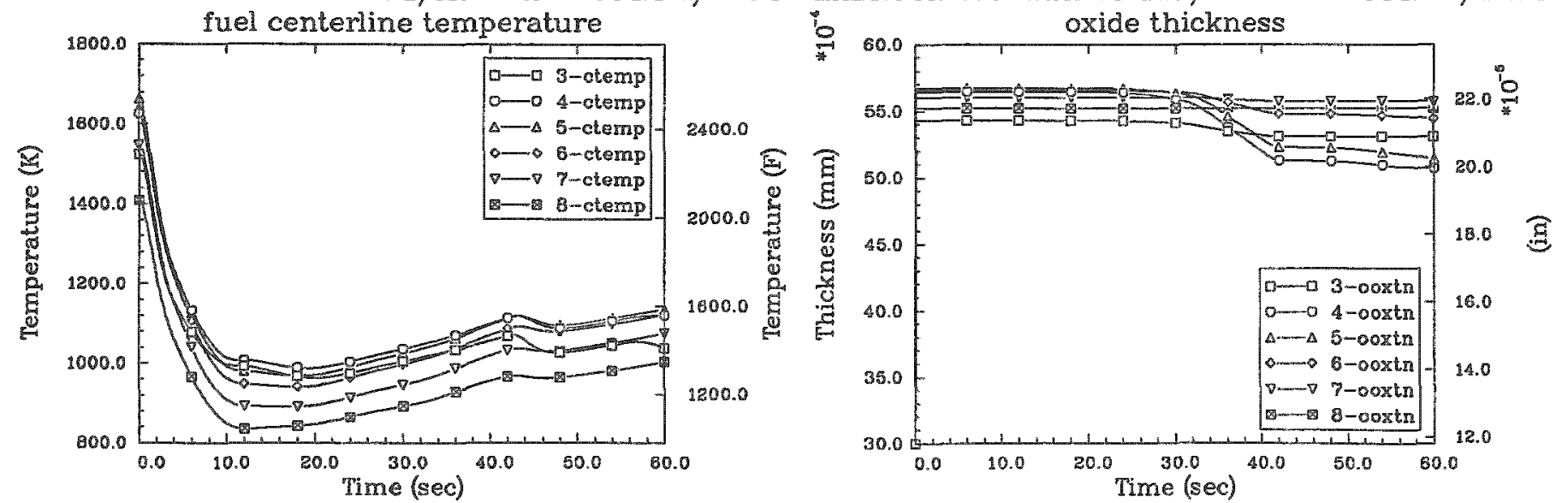
SEABROOK 100\%DBA 5 GWD/MTU PIN--PF1.8 W/ES\&PT SEABROOK 100\%DBA 5 GWD/MTU PIN--PF1.8 W/ES\&PT
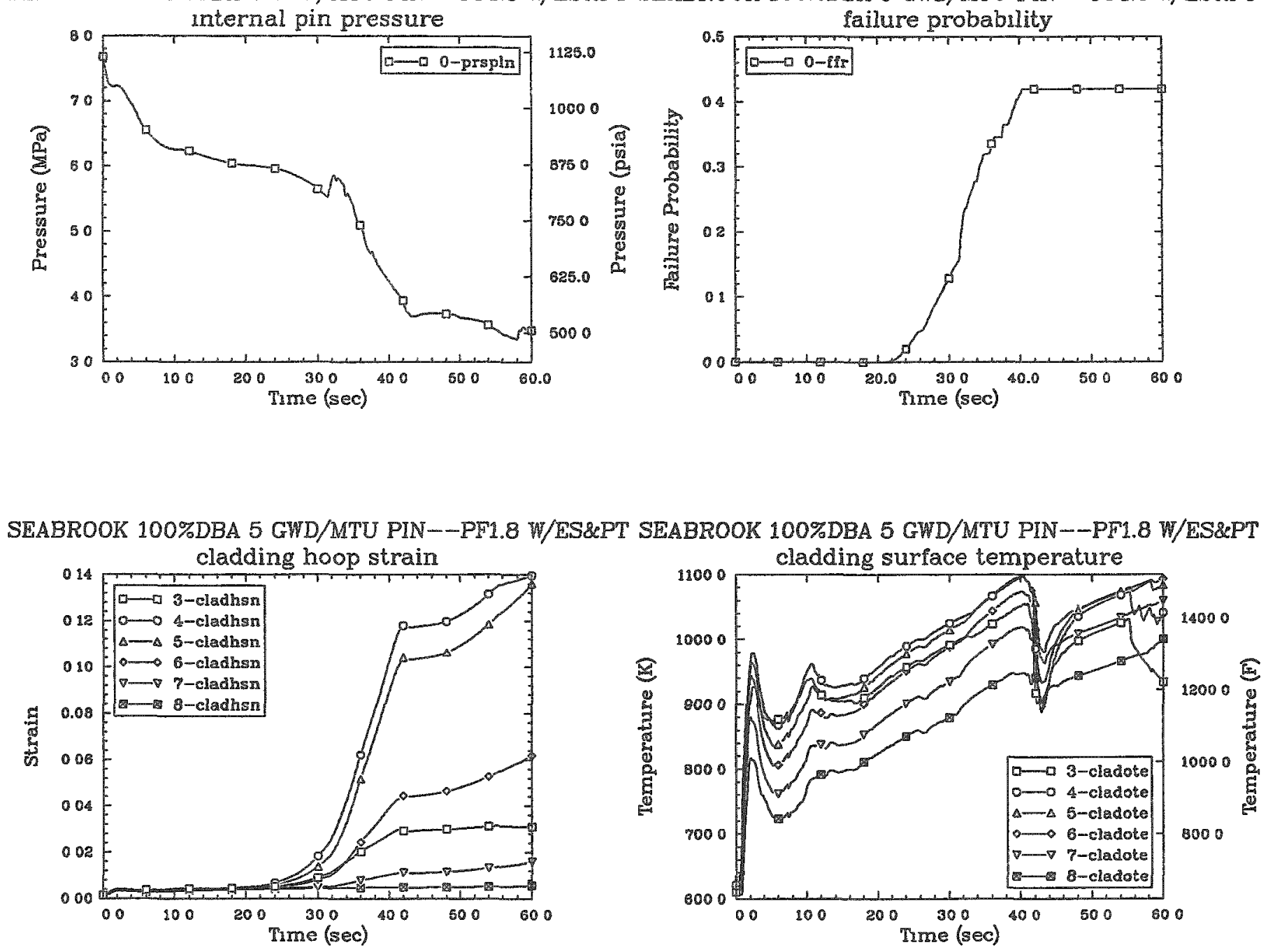

SEABROOK 100\%DBA 5 GWD/MTU PIN--PF1.8 W/ES\&PT SEABROOK 100\%DBA 5 GWD/MTU PIN--PF1.8 W/ES\&PT
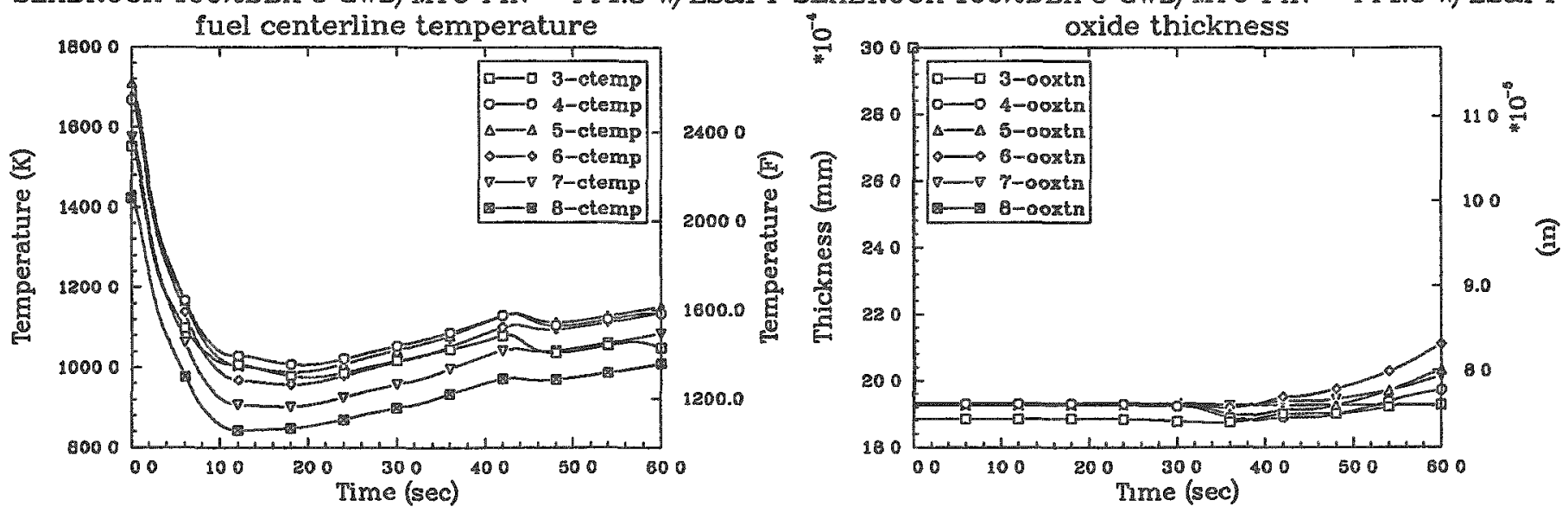

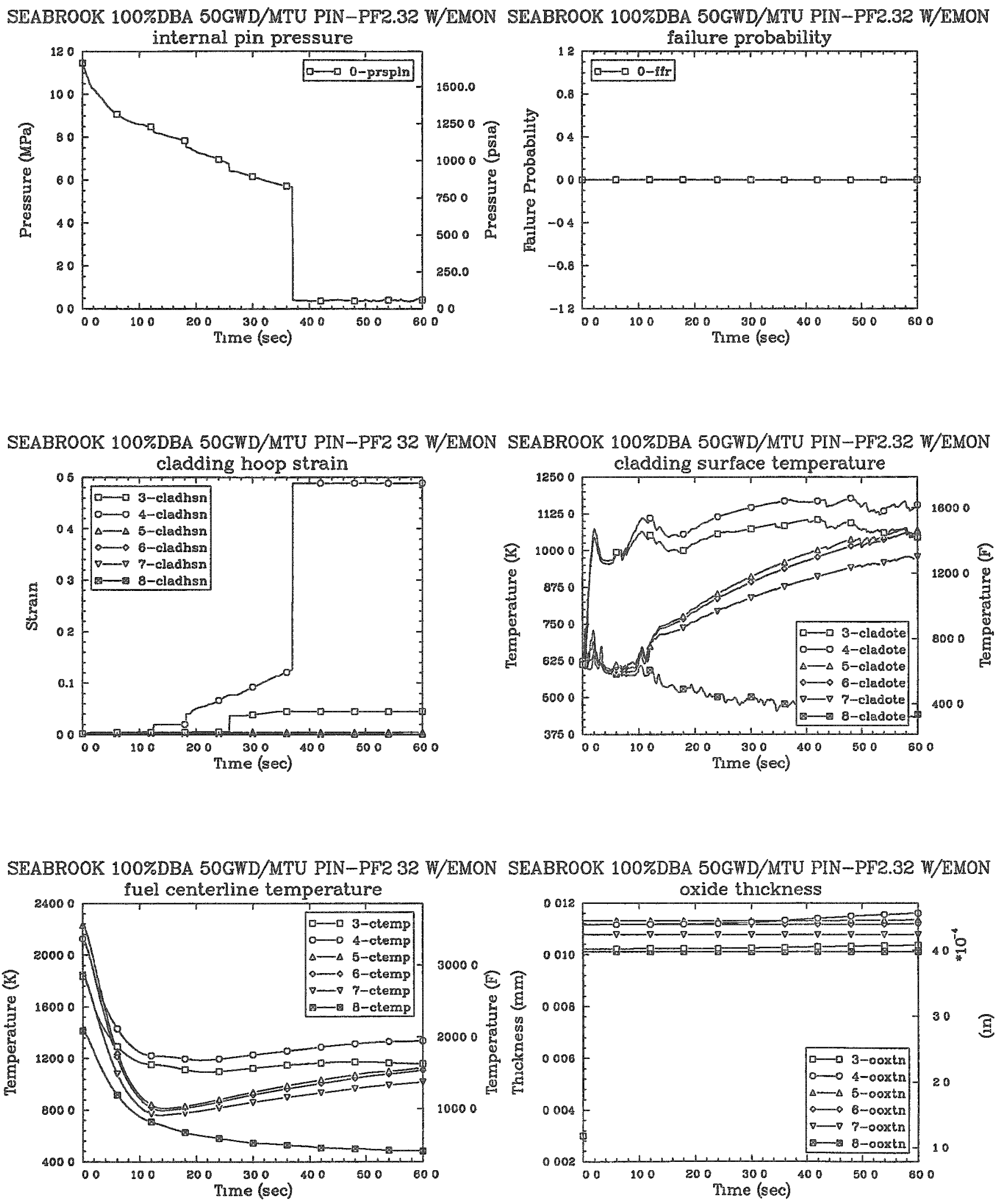
SEABROOK 100\%DBA 35GWD/MTU PIN-PF2.32 W/EMON SEABROOK 100\%DBA 35GWD/MTU PIN-PF2 32 W/EMON
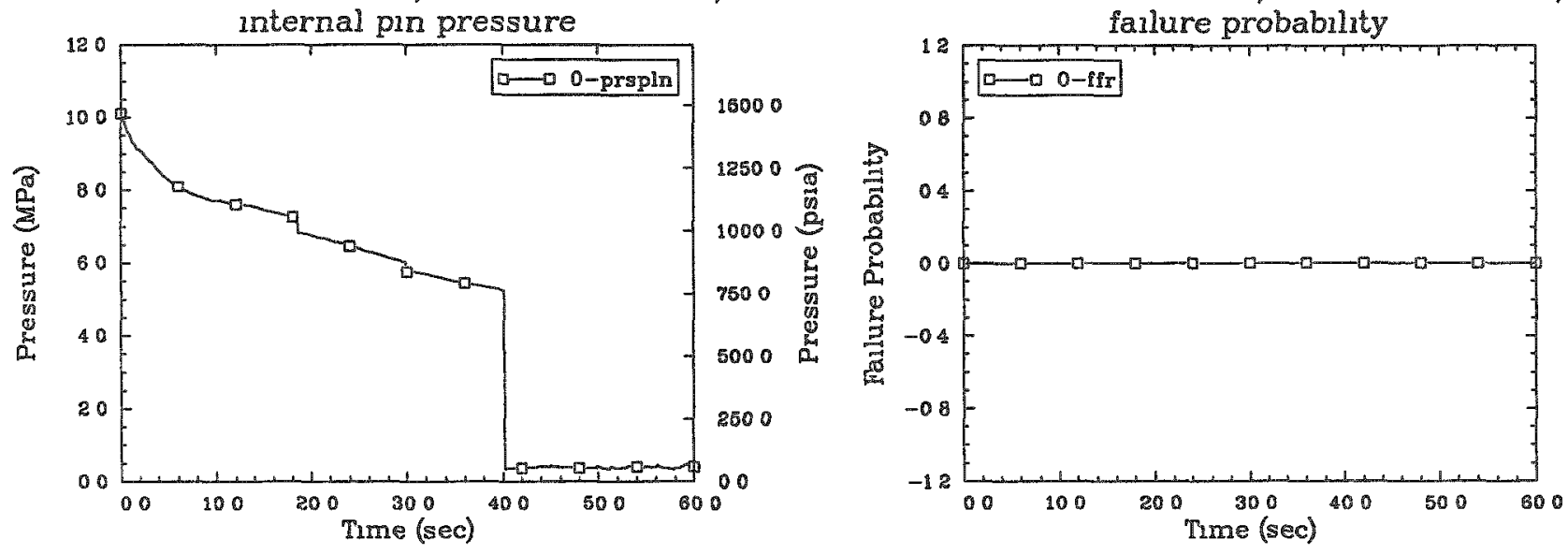

SEABROOK 100\%DBA 35GWD/MTU PIN-PF2 32 W/EMON SEABROOK 100\%DBA 35GWD/MTU PIN-PF2.32 W/EMON
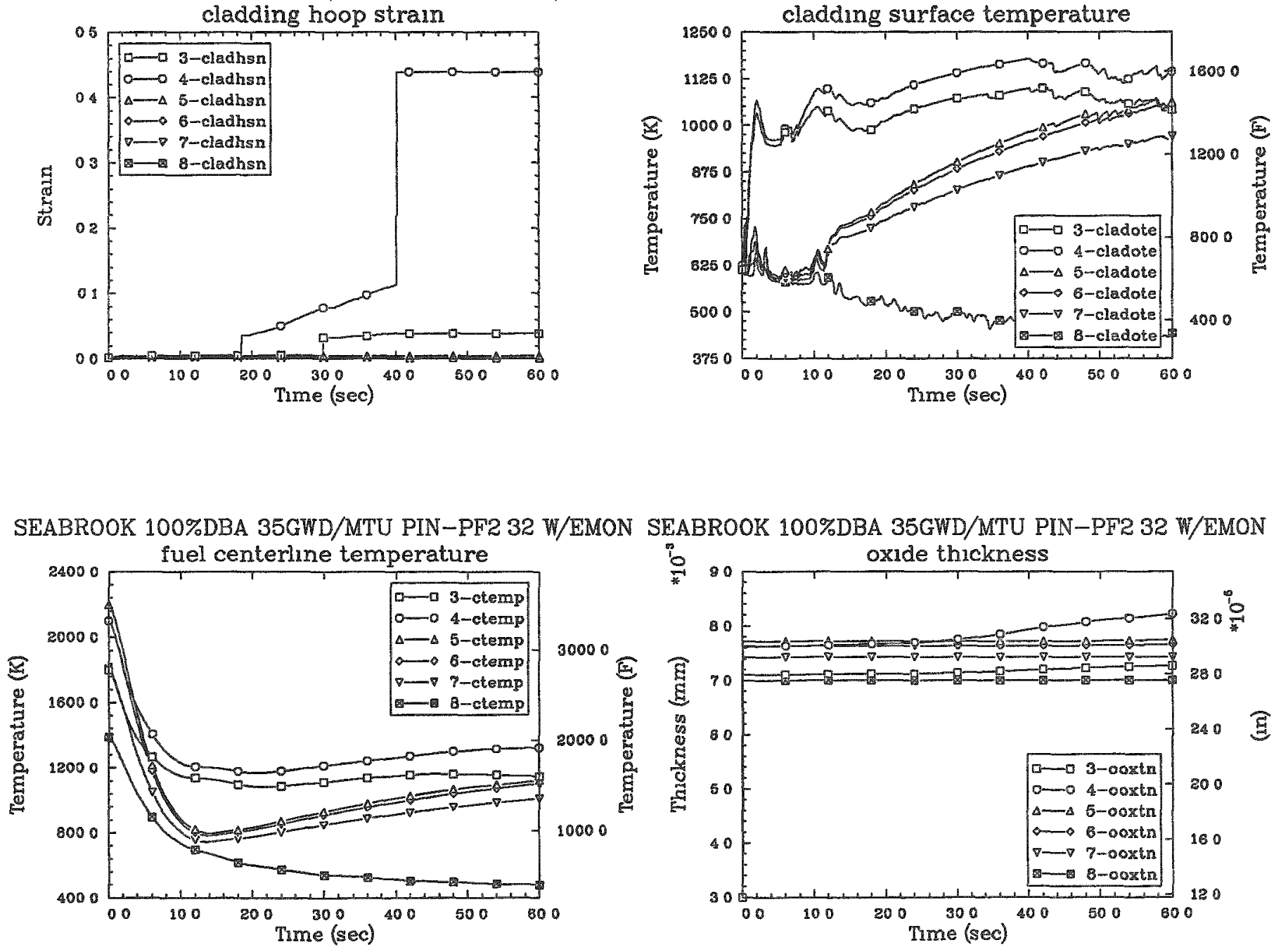
SEABROOK 100\%DBA 20GWD/MTU PIN-PF2 32 W/EMON SEABROOK 100\%DBA 20GWD/MTU PIN-PF2 32 W/EMON
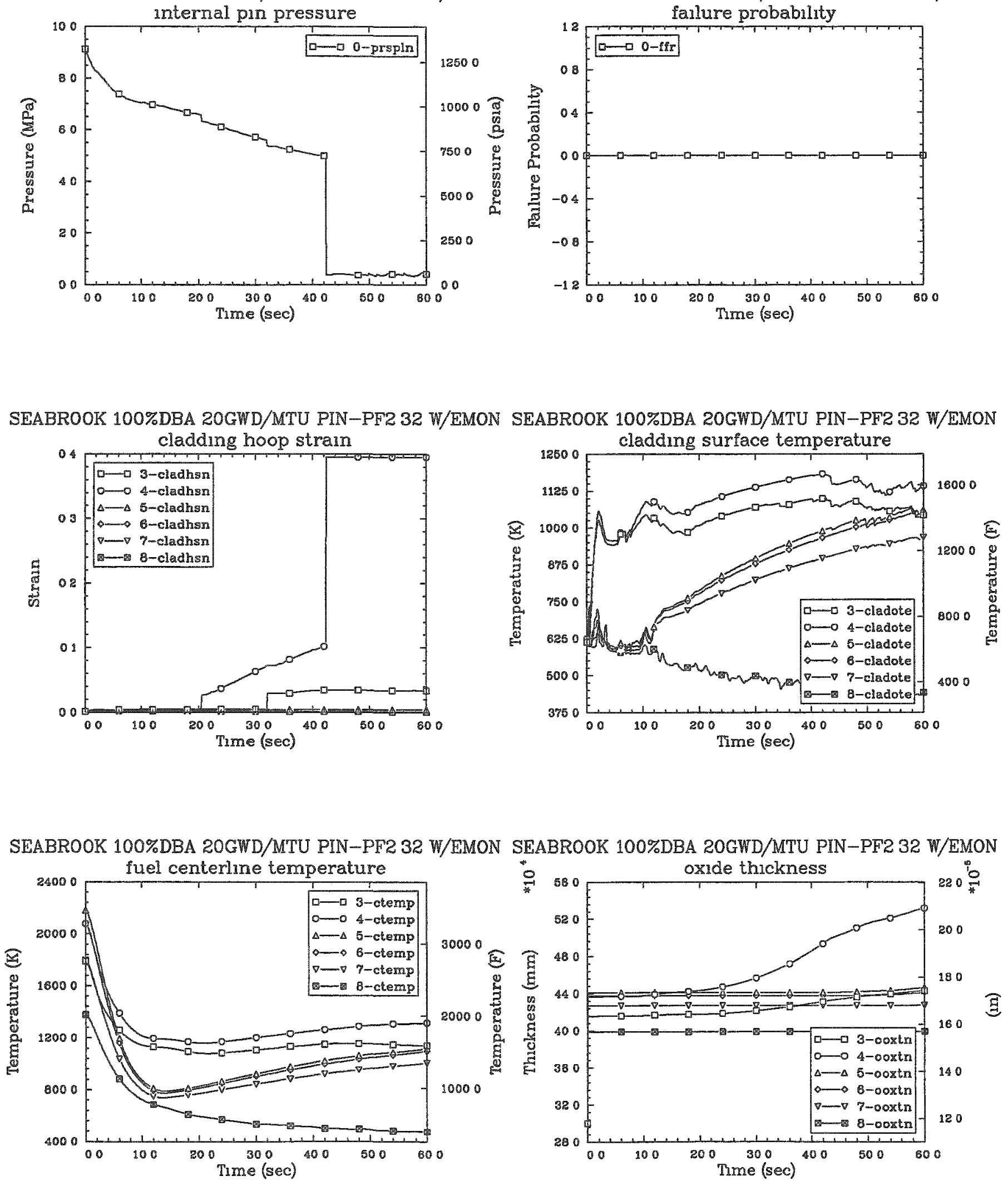
SEABROOK 100\%DBA 5GWD/MTU PIN-PF".32 W/EMON

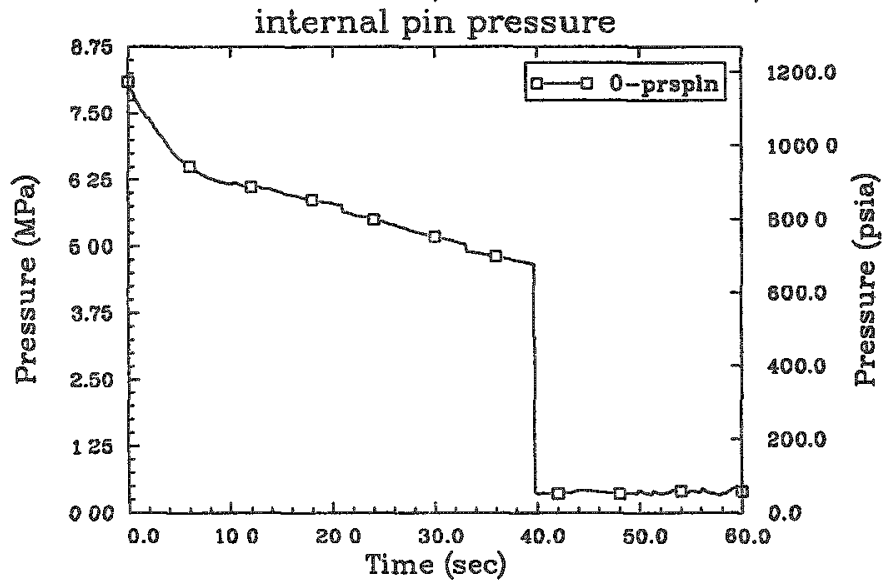

\section{SEABROOK 100\%DBA 5GWD/MTU PIN-PF2.32 W/EMON} cladding hoop strain

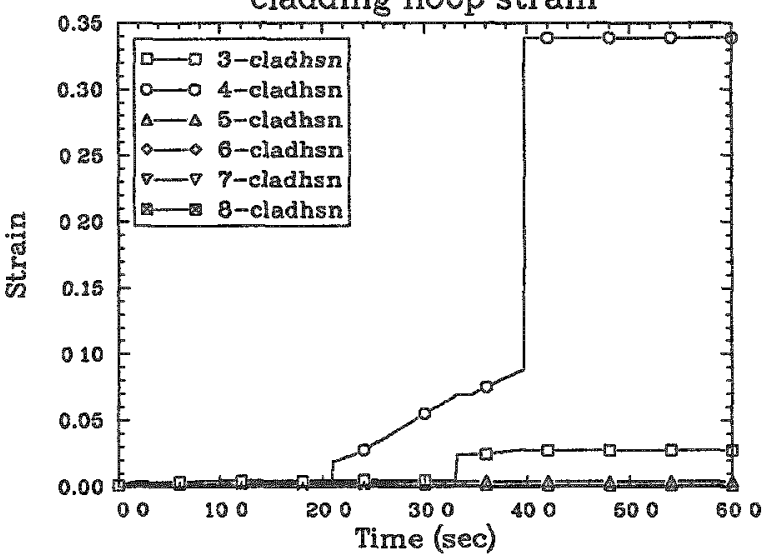

SEABROOK 100\%DBA 5GWD/MTU PIN-PF2.32 W/EMON

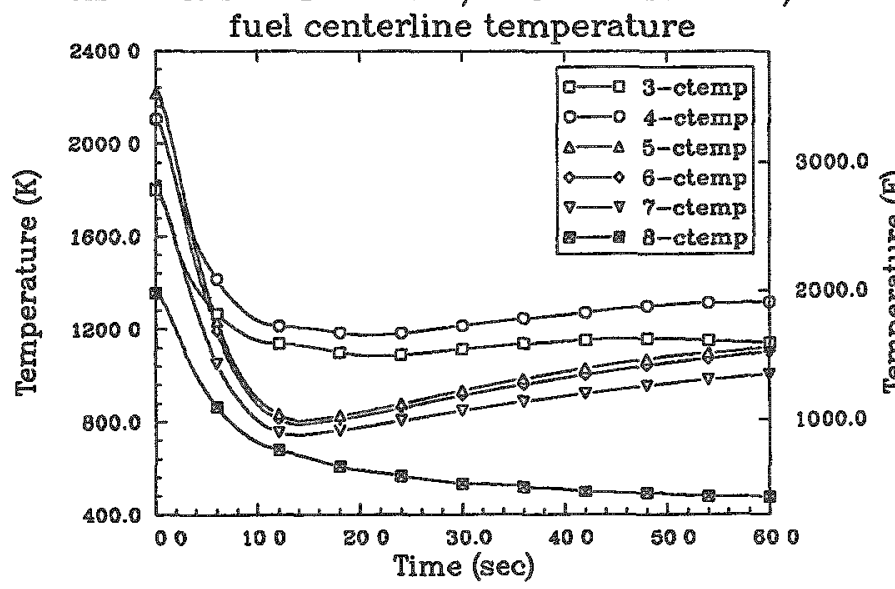

SEABROOK 100\%DBA 5GWD/MTU PIN-PF2.32 W/EMON

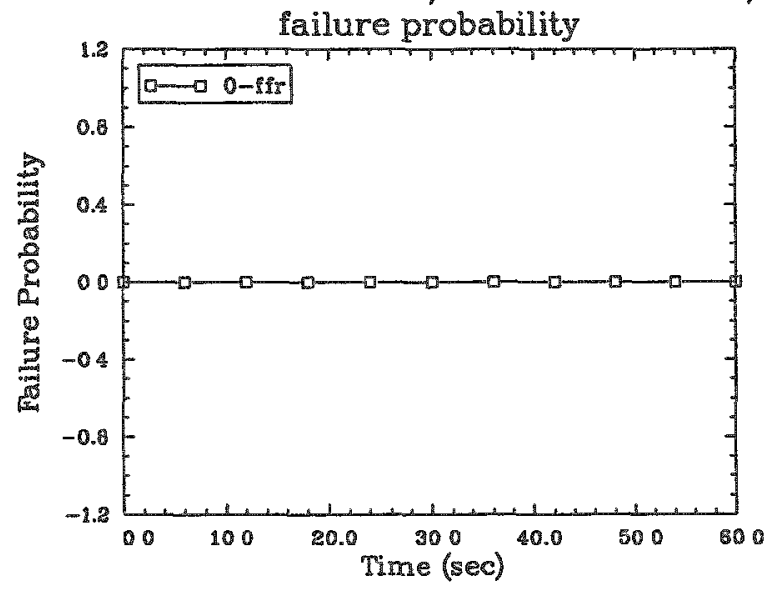

SEABROOK 100\%DBA 5GWD/MTU PIN-PF2.32 W/EMON

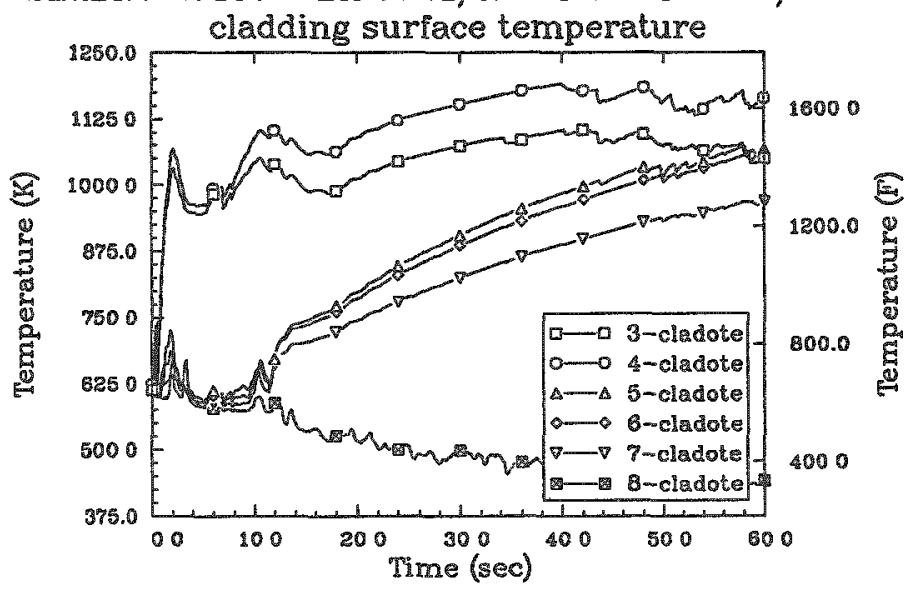

SEABROOK 100\%DBA 5GWD/MTU PIN-PF2.32 W/EMON

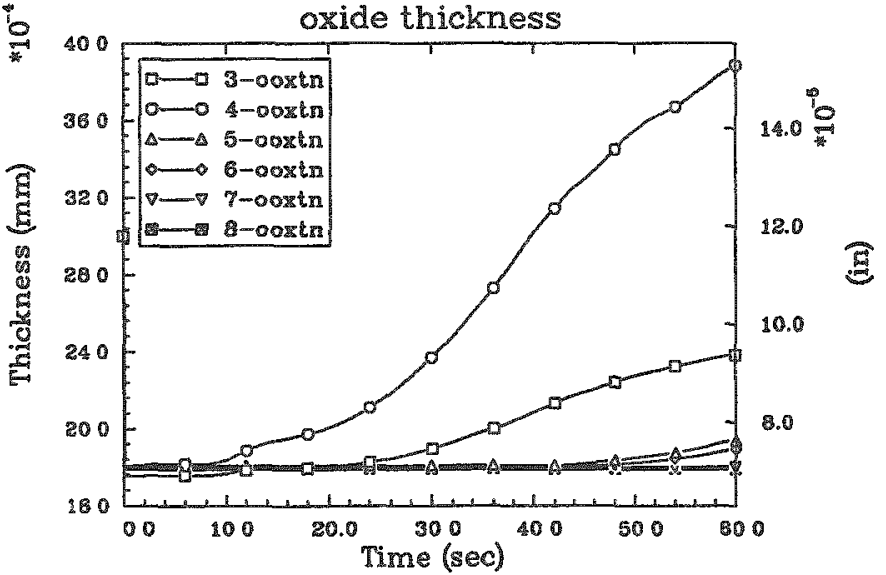


SEABROOK 100\%DBA 50 GWD/MTU PIN--PF2.2 W/EMON SEABROOK 100\%DBA 50 GWD/MTU PIN--PF2 2 W/EMON

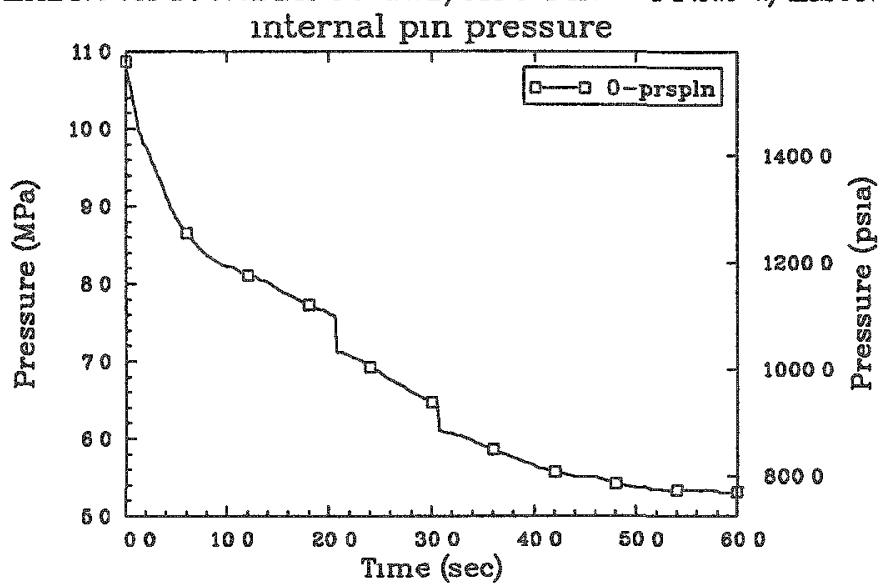

fallure probability
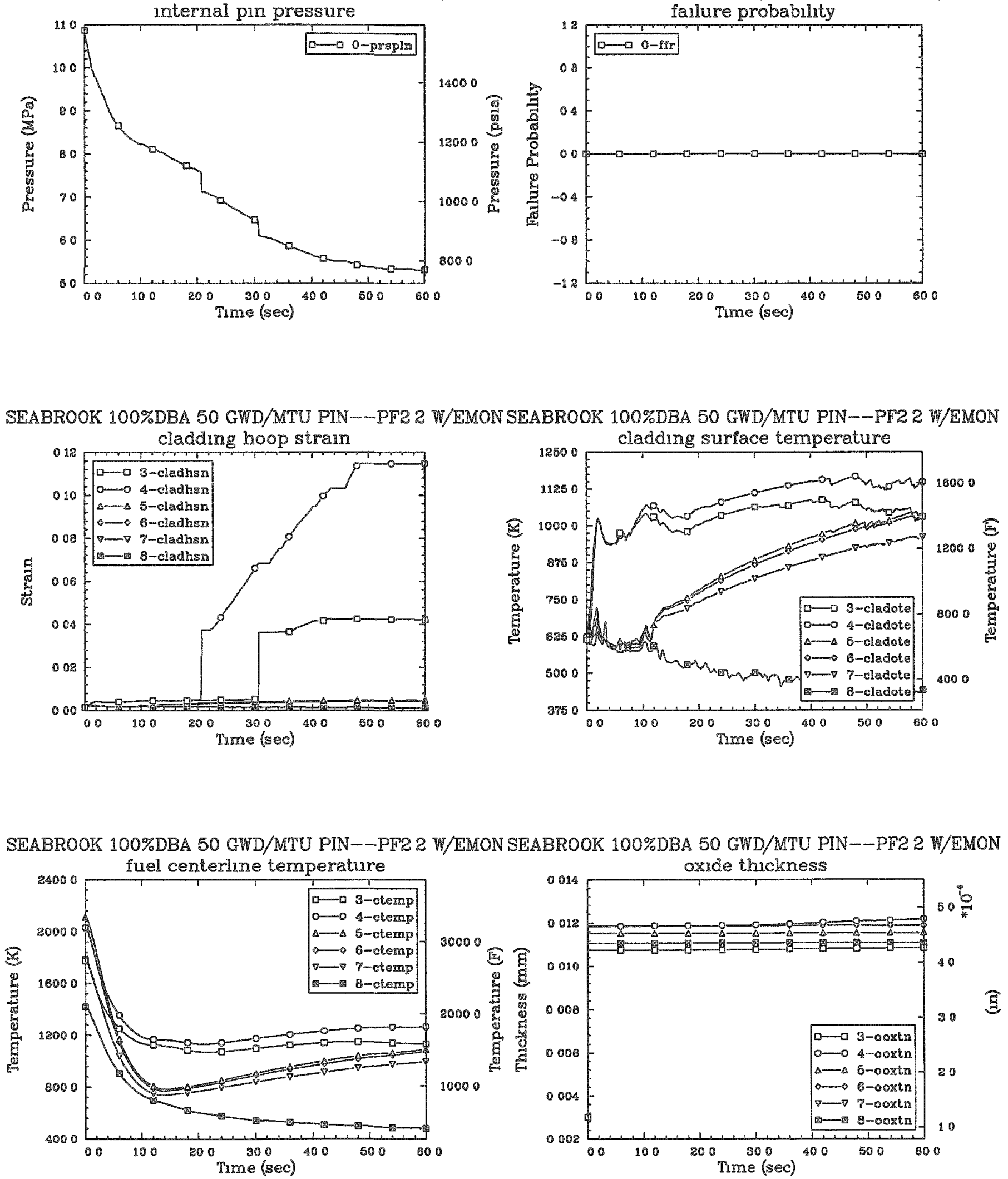
SEABROOK 100\%DBA 35 GWD/MTU PIN--PF2 2 W/EMON SEABROOK 100\%DBA 35 GWD/MTU PIN--PF22 W/EMON
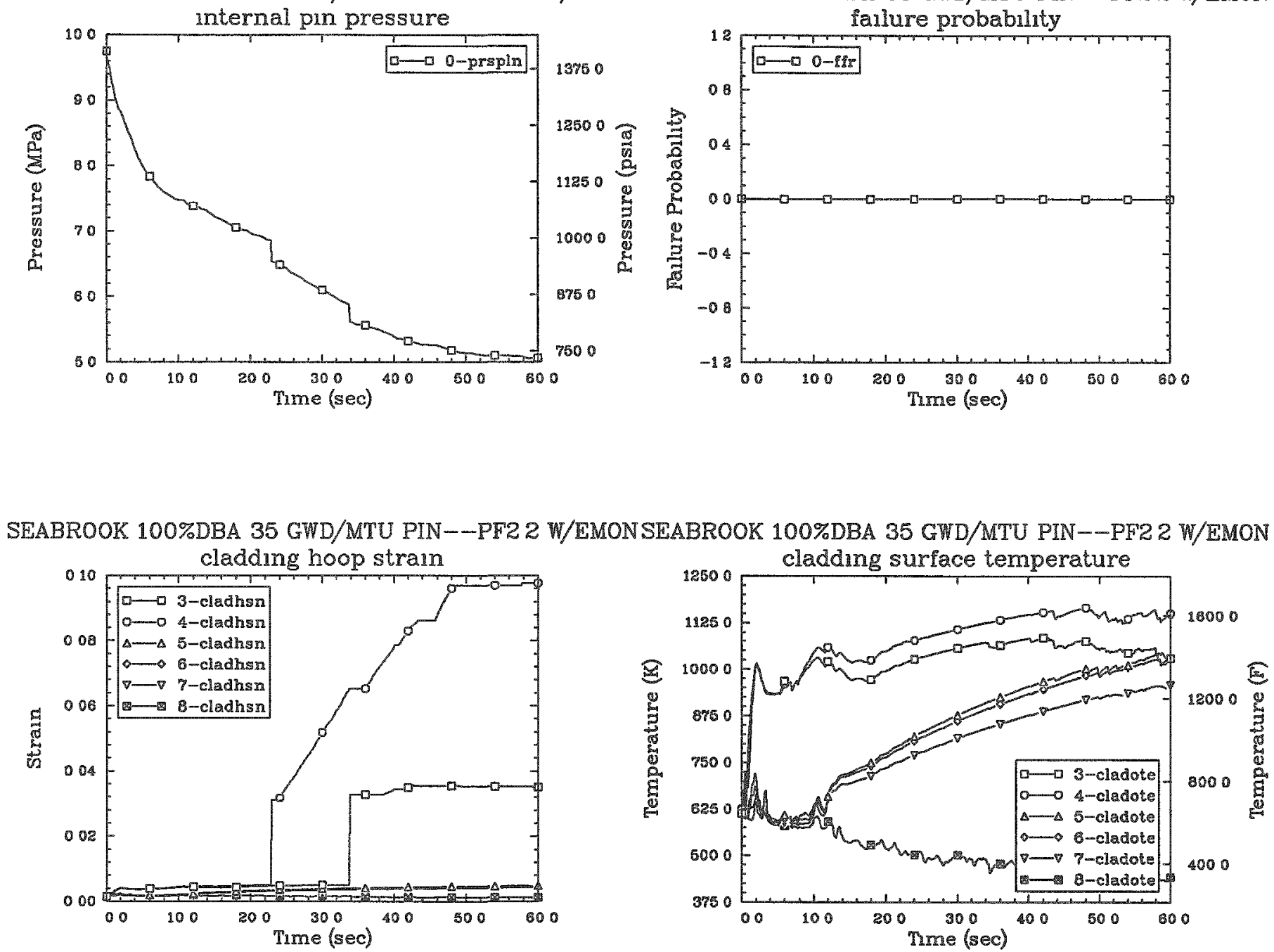

SEABROOK 100\%DBA 35 GWD/MTU PIN--PF2 2 W/EMON SEABROOK 100\%DBA 35 GWD/MTU PIN--PF2 2 W/EMON
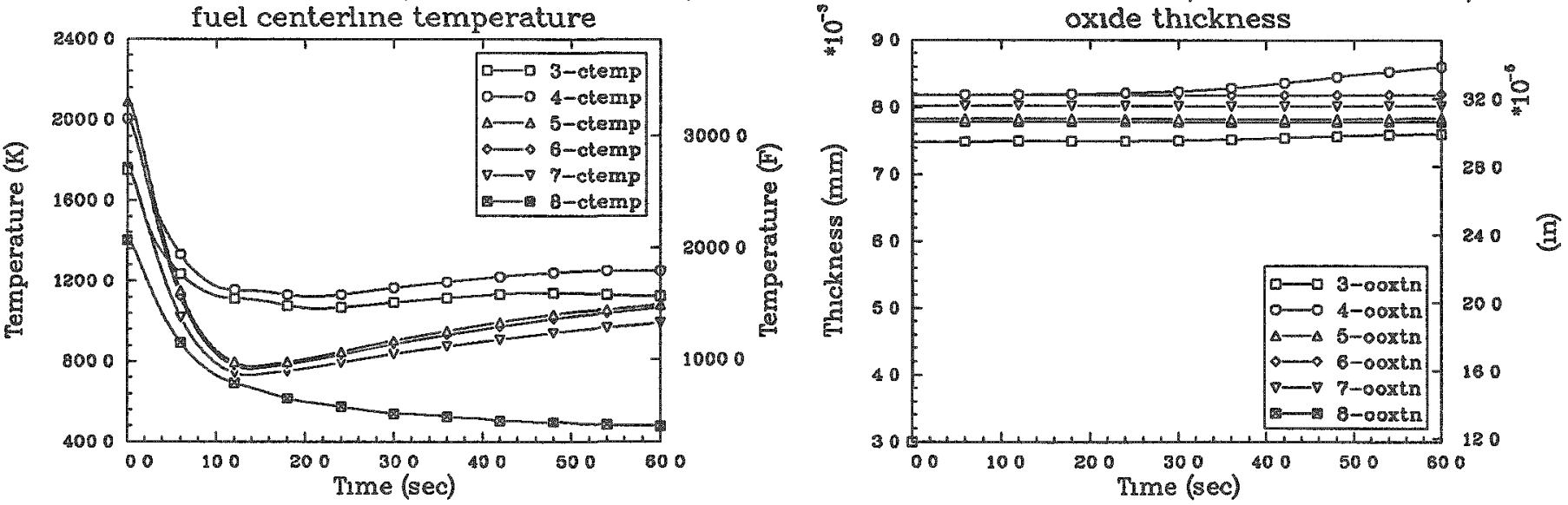

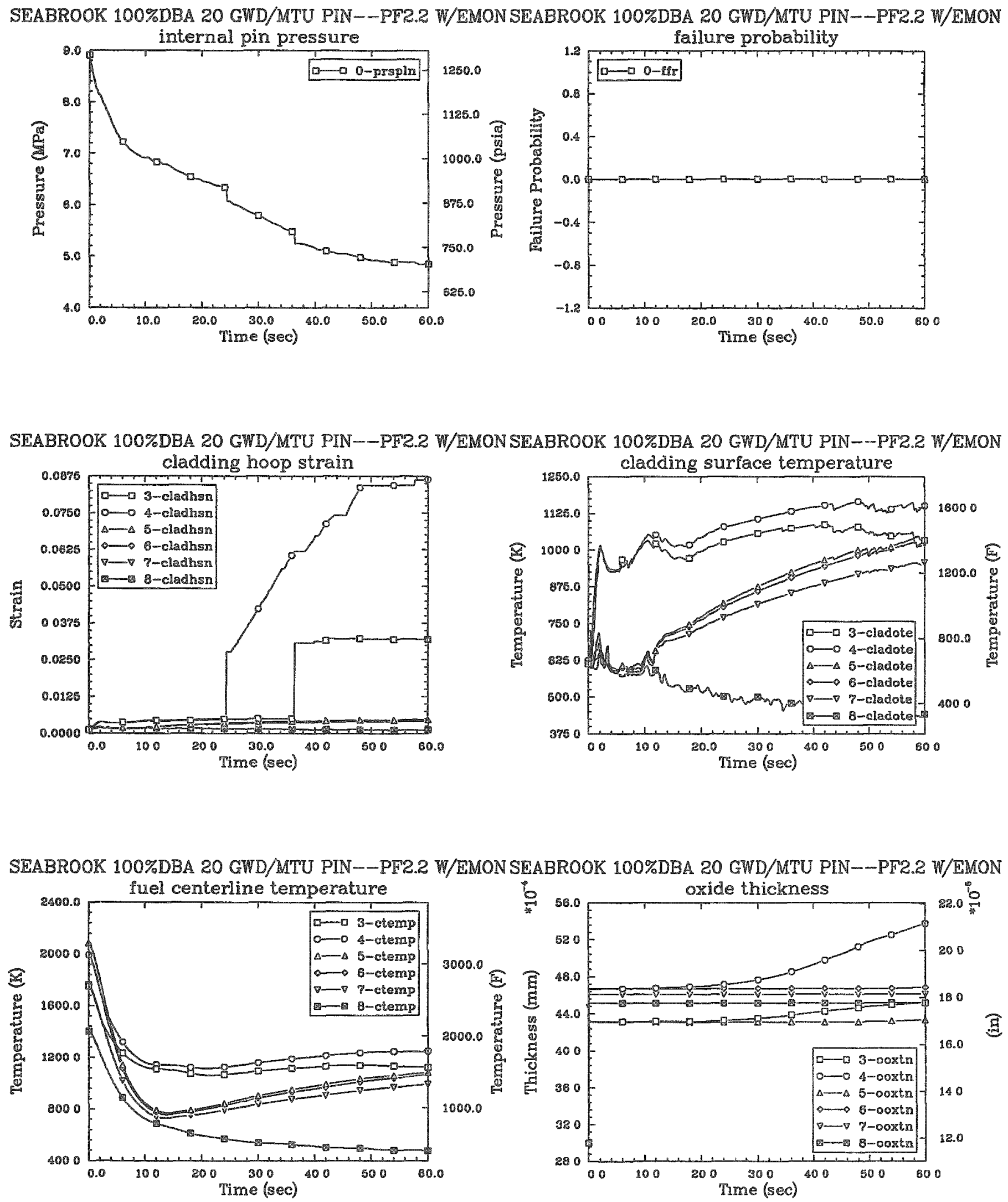

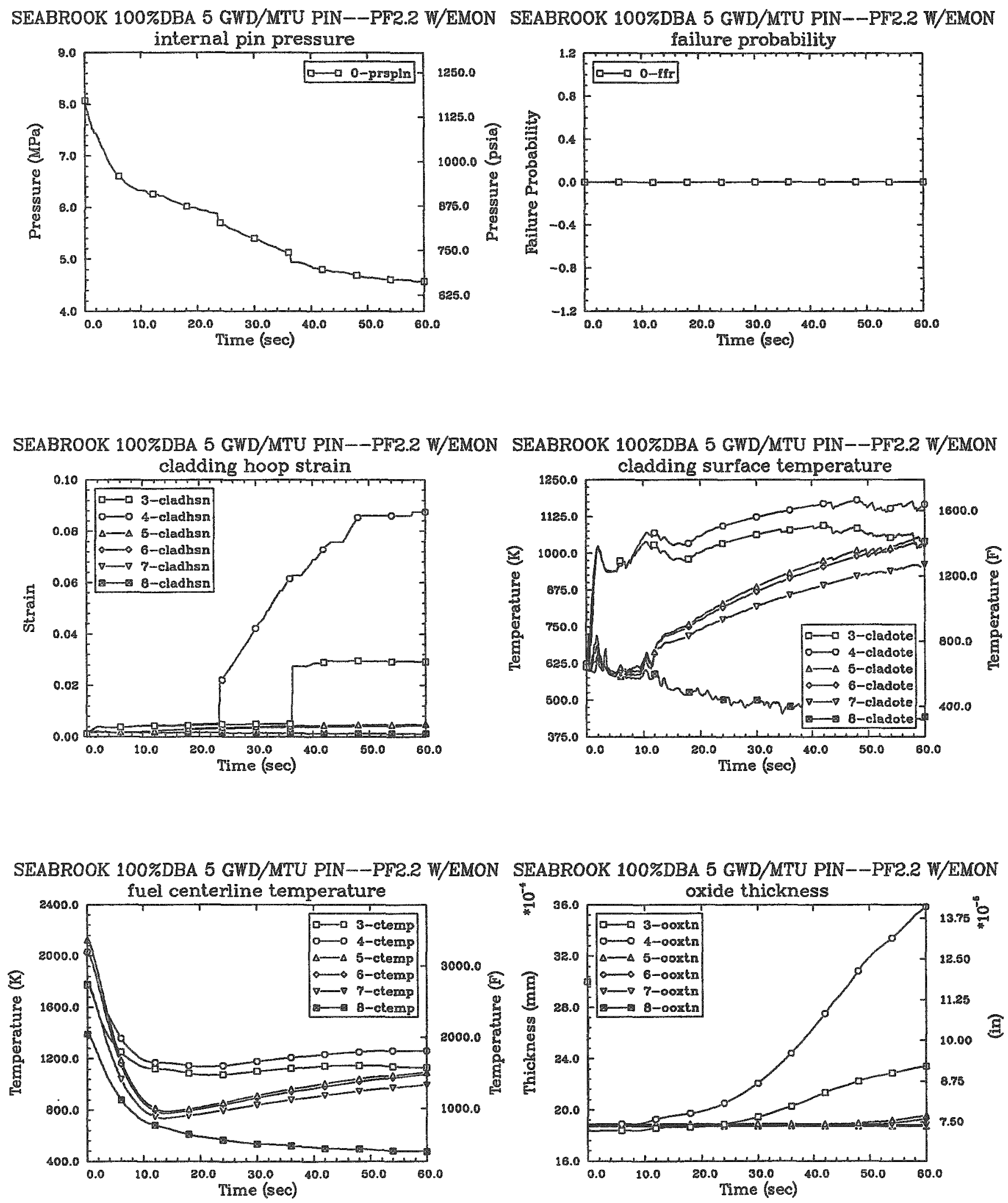

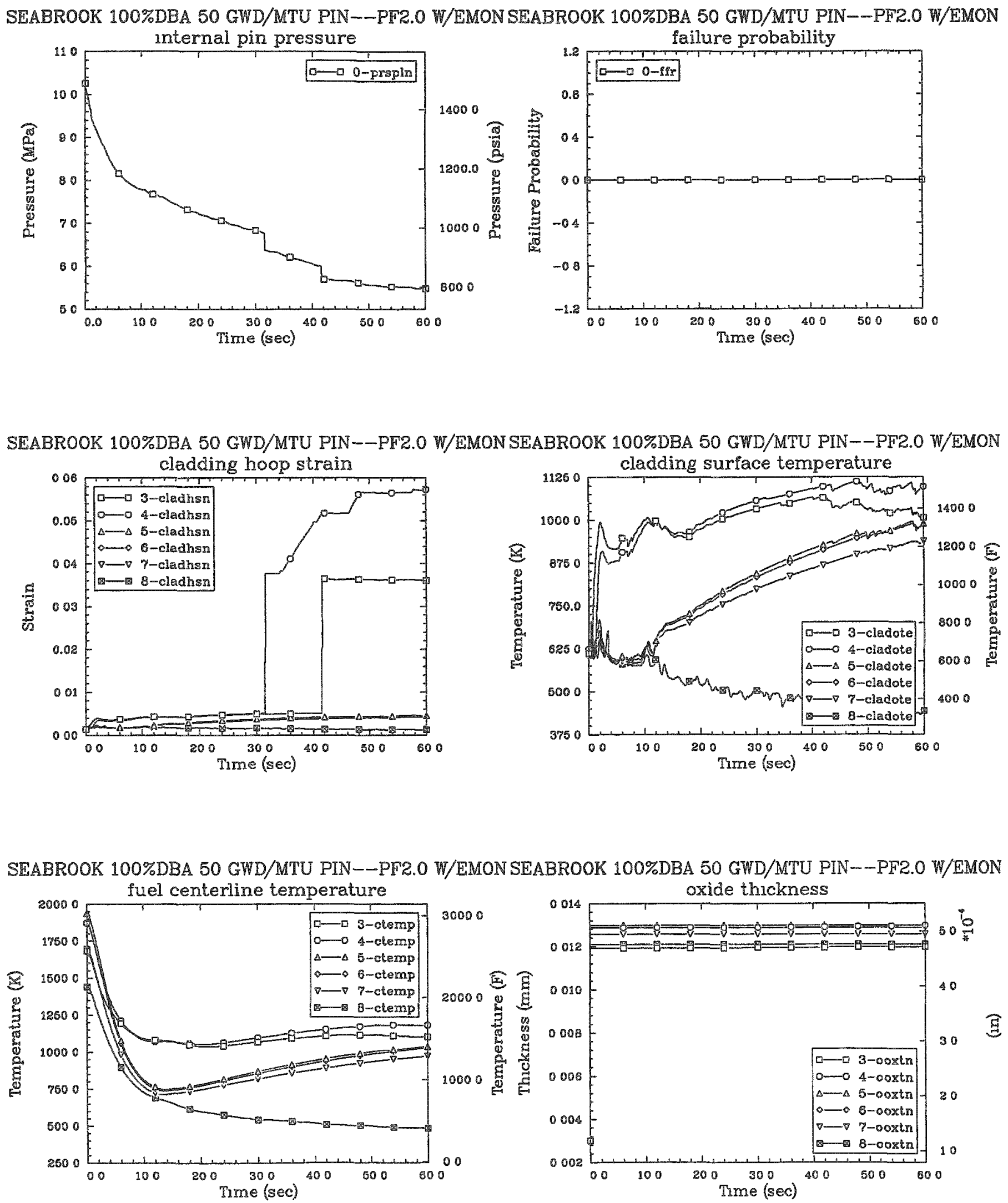

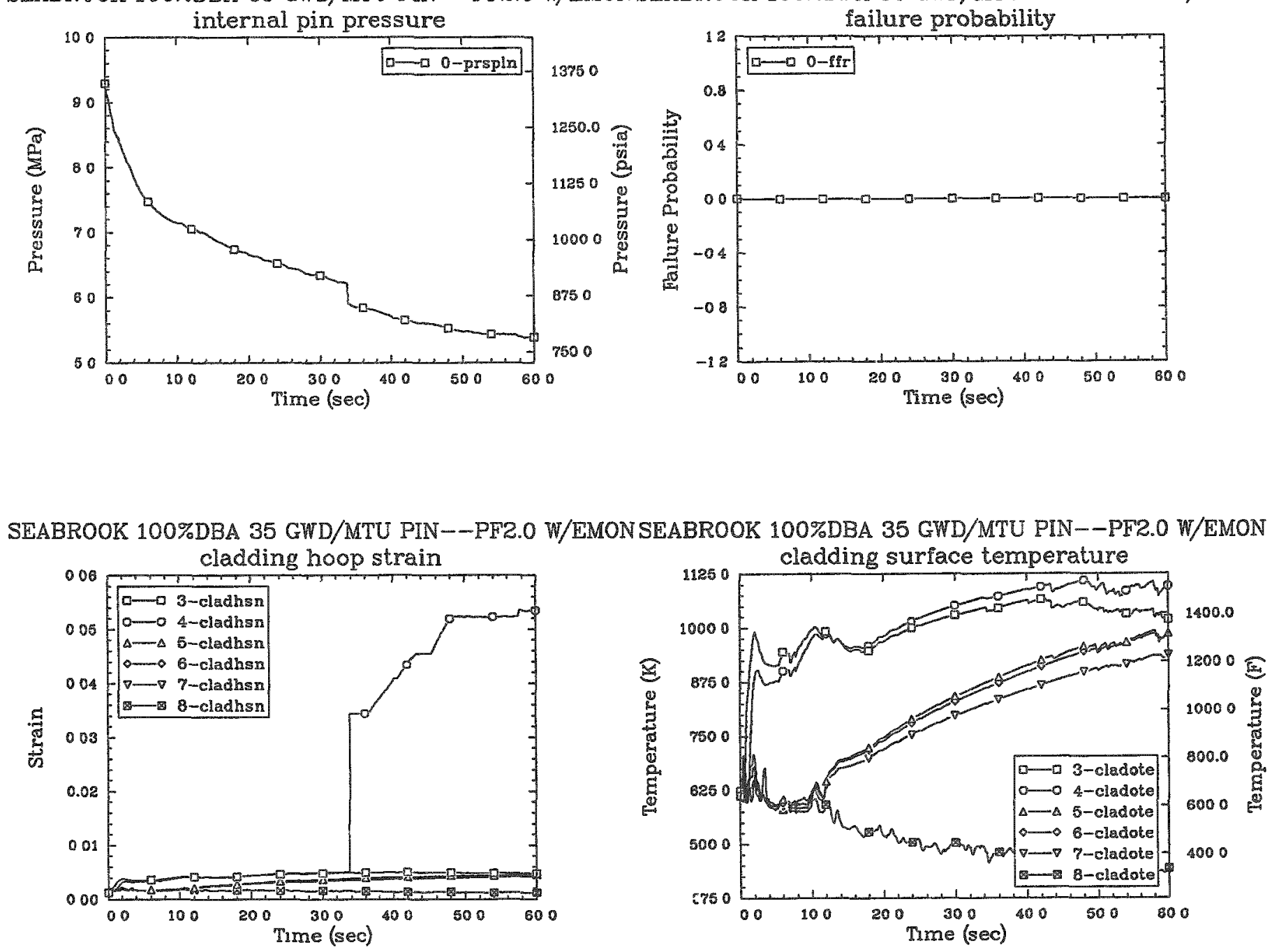

SEABROOK 100\%DBA 35 GWD/MTU PIN--PF2.0 W/EMON SEABROOK 100\%DBA 35 GWD/MTU PIN--PF2.0 W/EMON
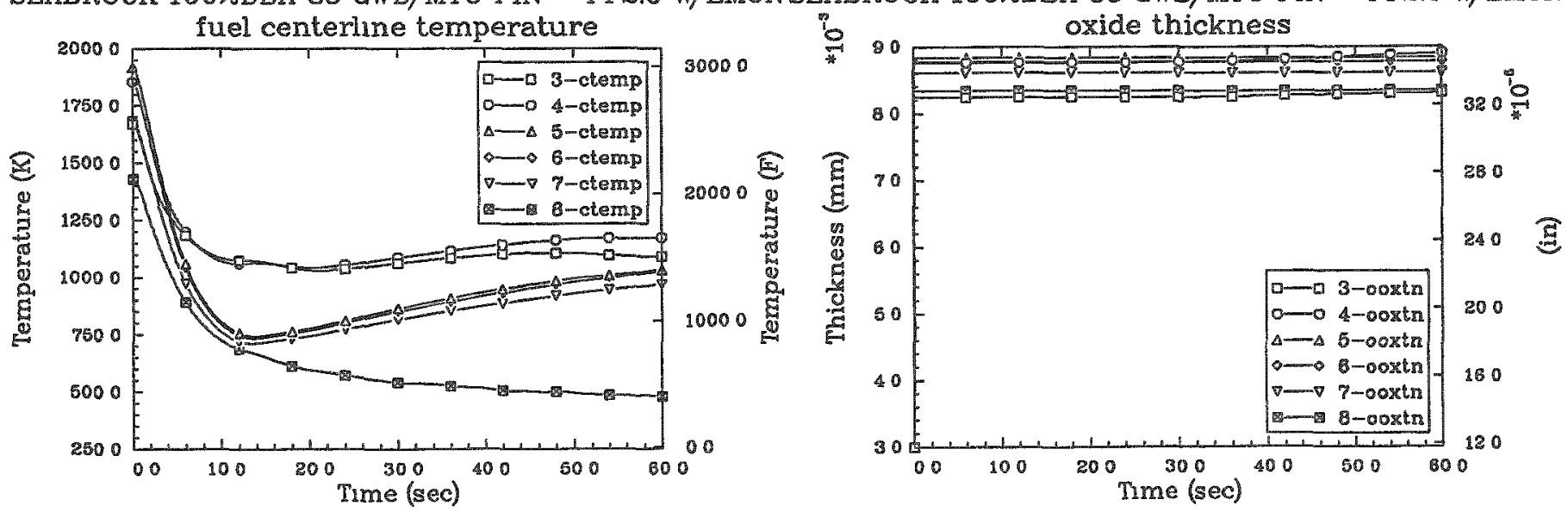

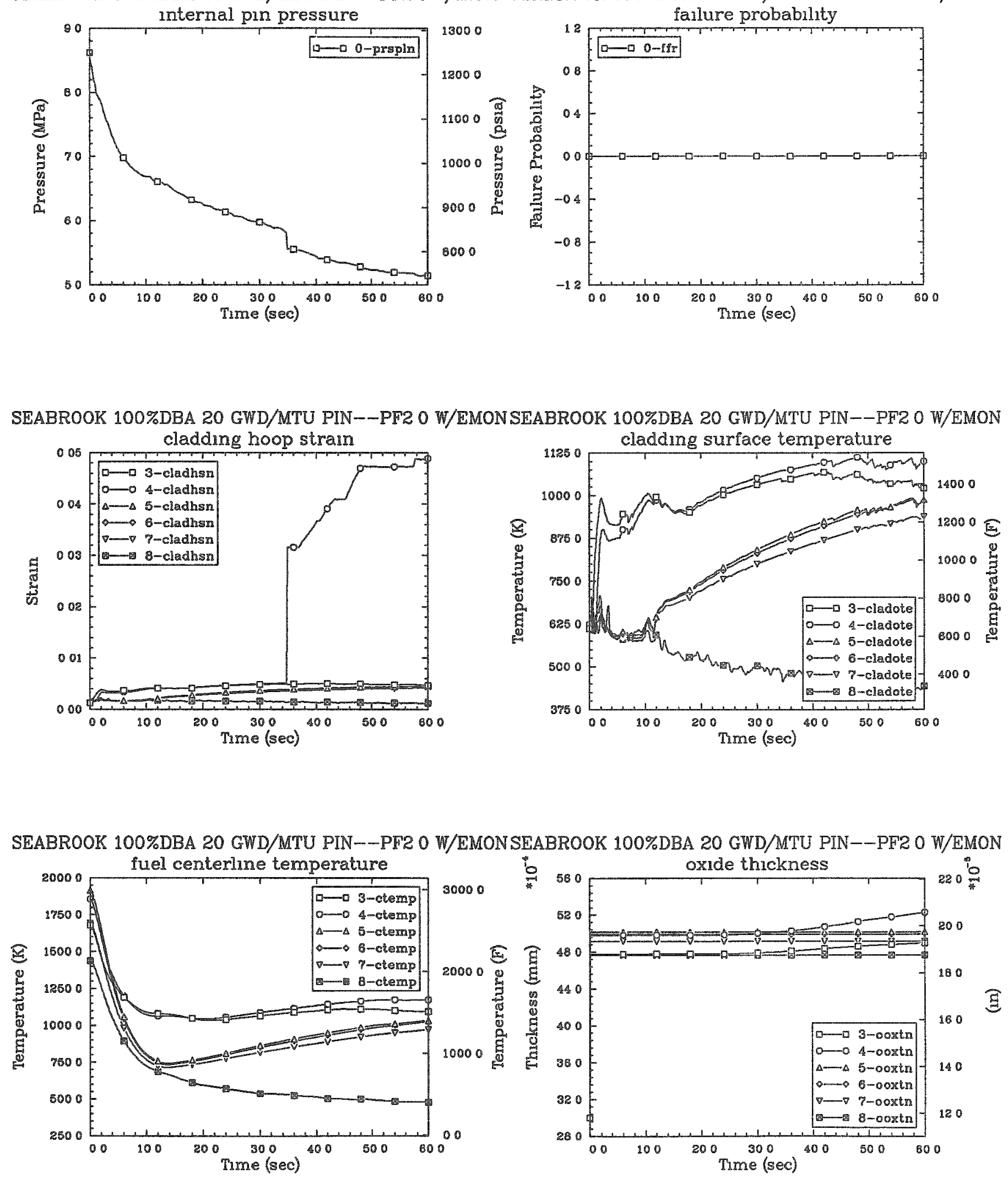
SEABROOK 100\%DBA 5 GWD/MTU PIN--PF2 O W/EMON SEABROOK 100\%DBA 5 GWD/MTU PIN--PF20 W/EMON
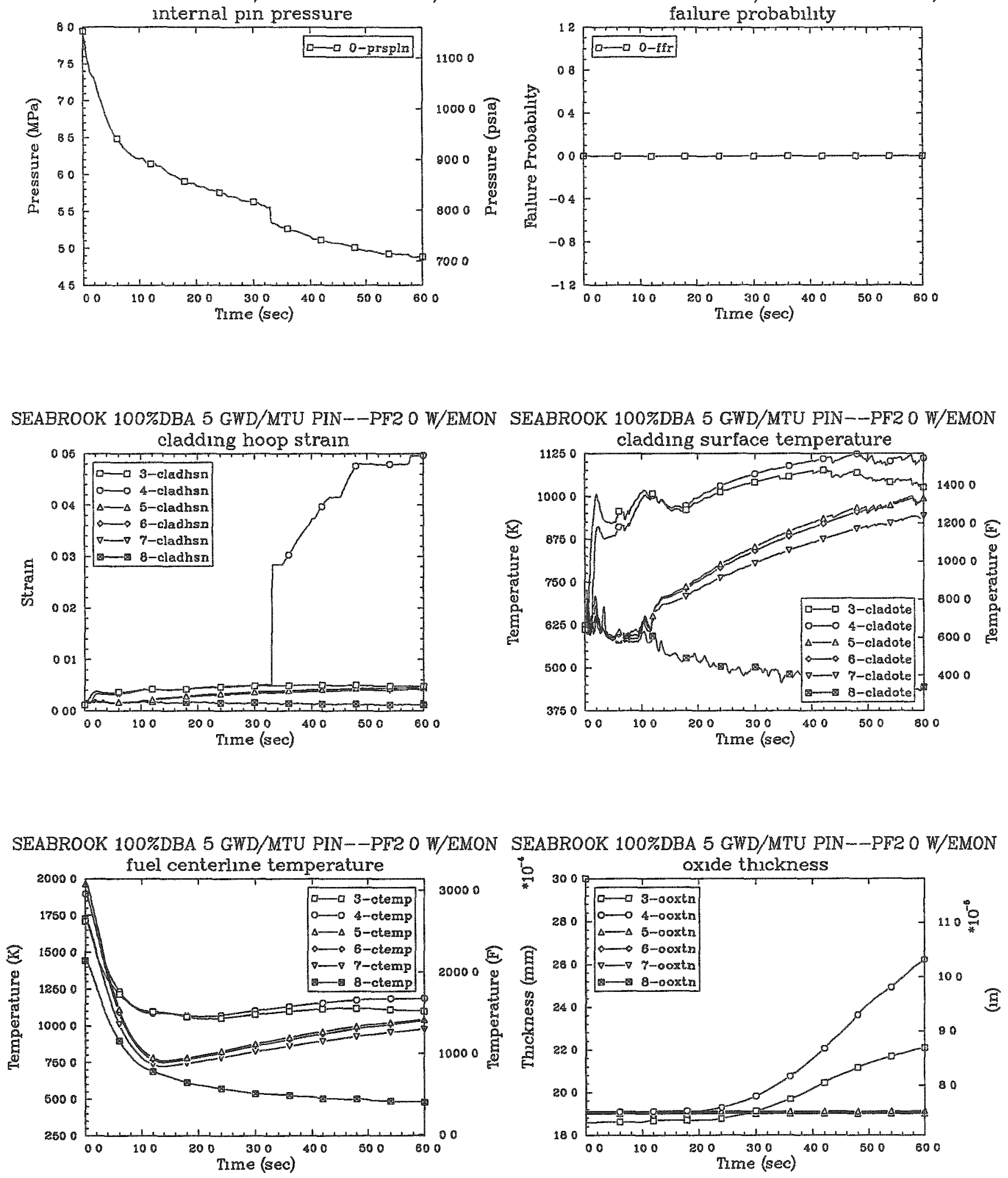
SEABROOK 100\%DBA 50 GWD/MTU PIN--PF1.8 W/EMON SEABROOK 100\%DBA 50 GWD/MTU PIN--PF1.8 W/EMON
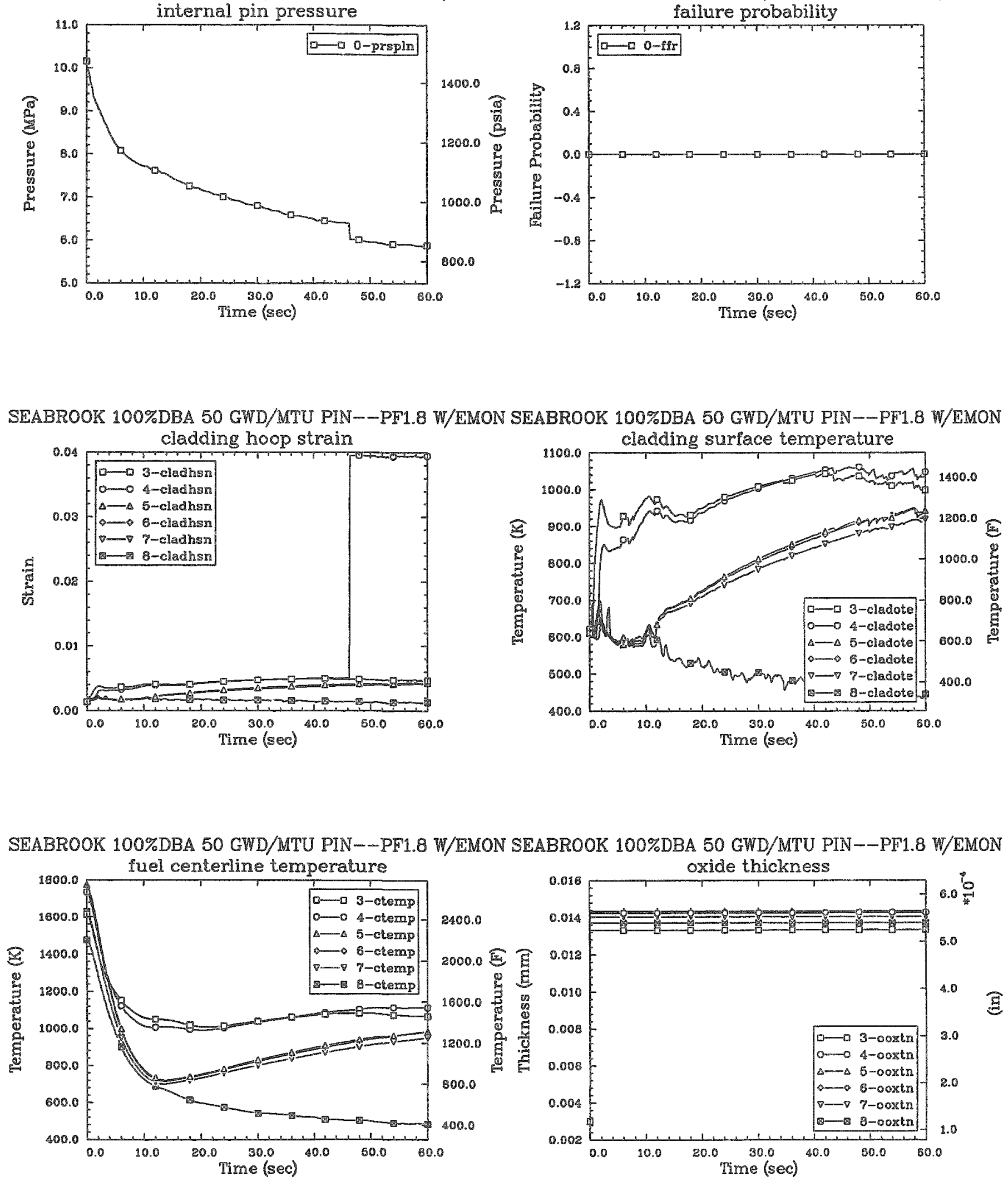
SEABROOK 100\%DBA 35 GWD/MTU PIN--PF1.8 W/EMON SEABROOK 100\%DBA 35 GWD/MTU PIN--PF1.8 W/EMON
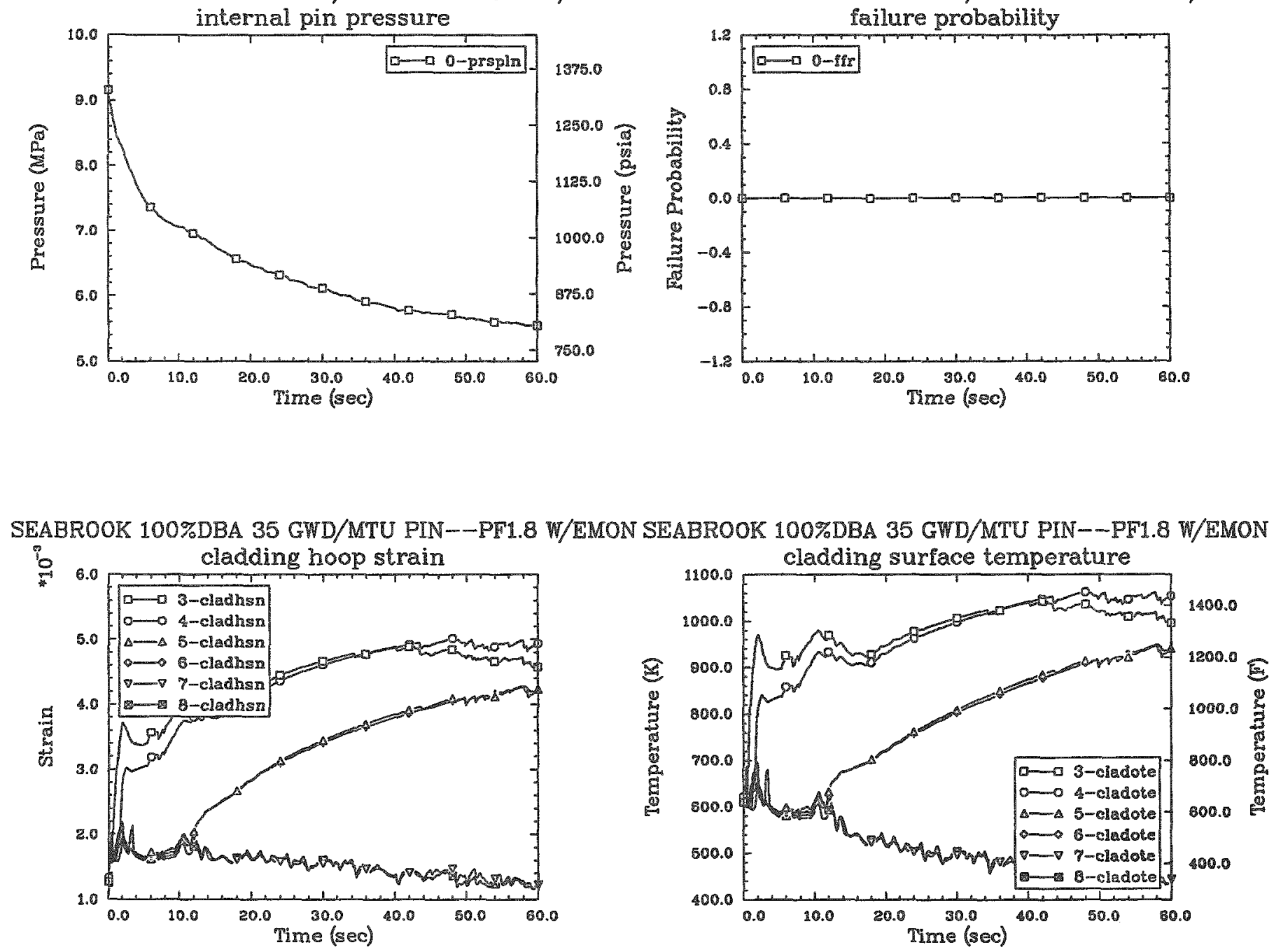

SEABROOK 100\%DBA 35 GWD/MTU PIN--PF1.8 W/EMON SEABROOK 100\%DBA 35 GWD/MTU PIN--PF1.8 W/EMON
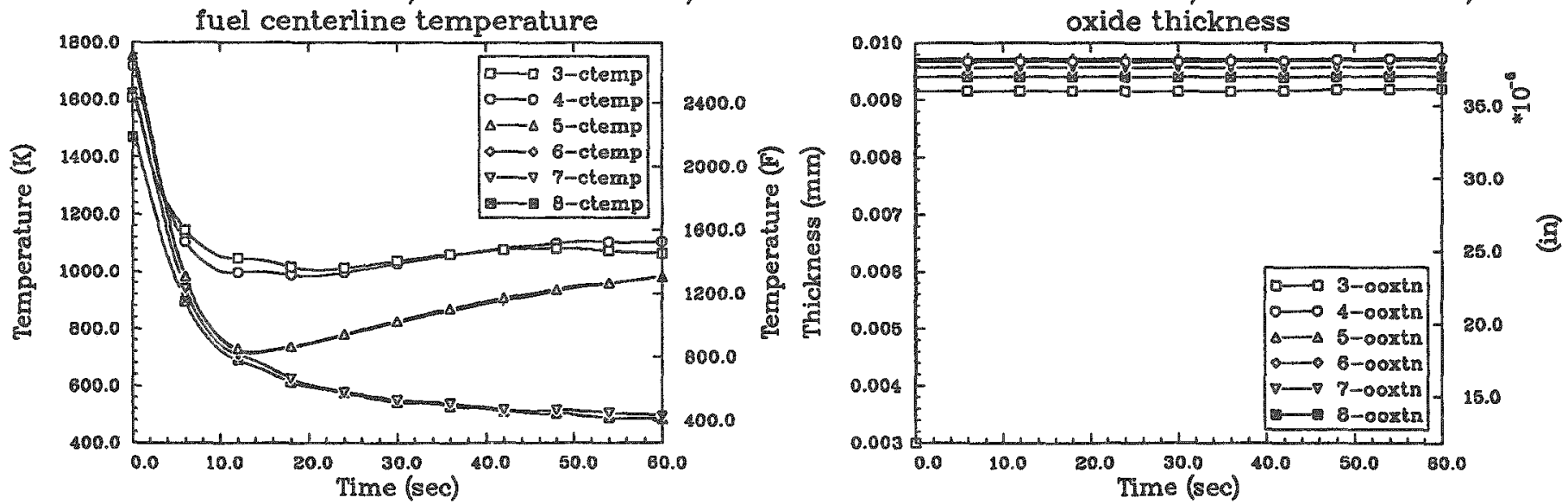

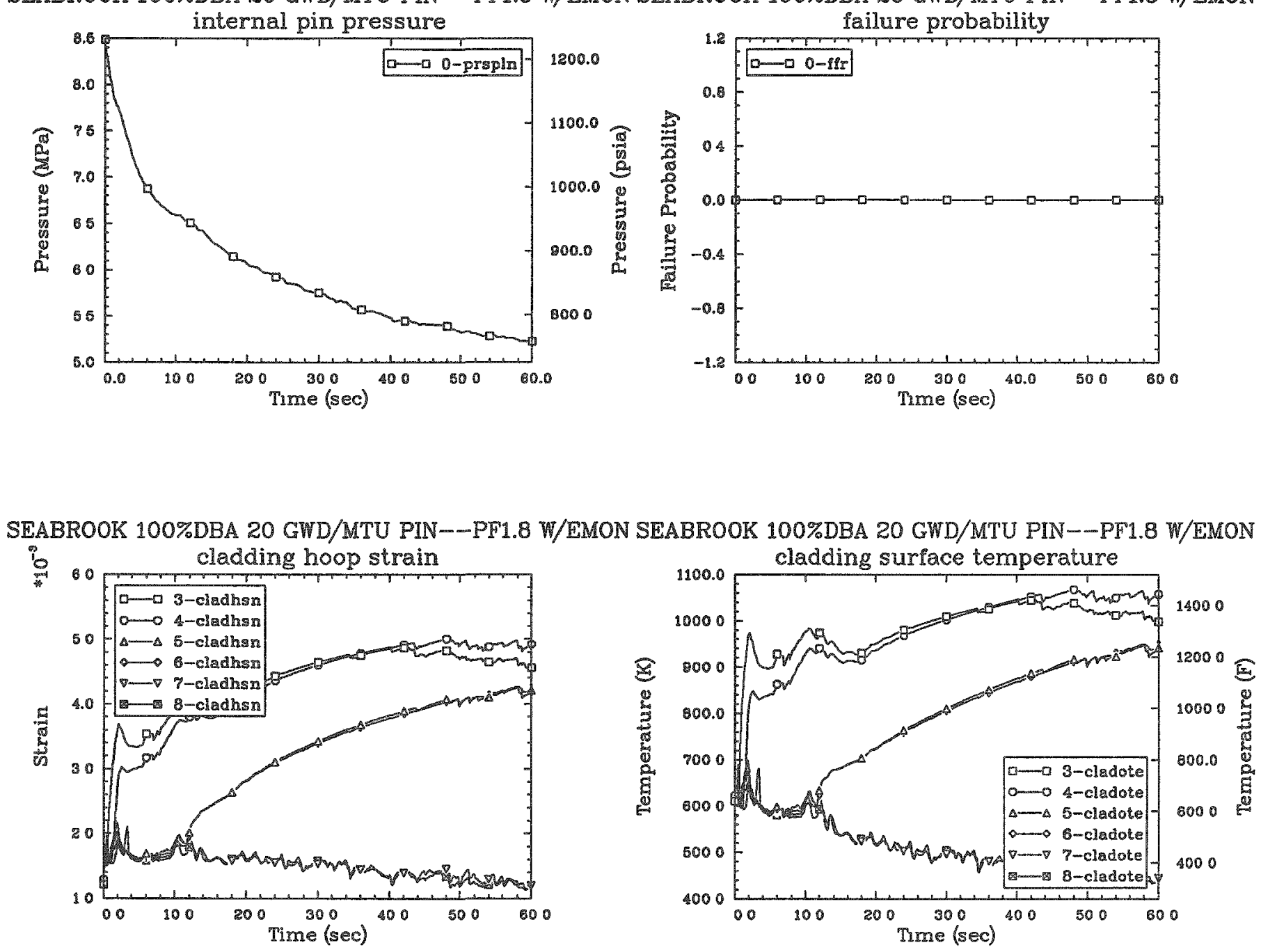

SEABROOK 100\%DBA $20 \mathrm{GWD} / \mathrm{MTU}$ PIN--PF1.8 W/EMON SEABR00K 100\%DBA 20 GWD/MTU PIN--PF1.8 W/EMON
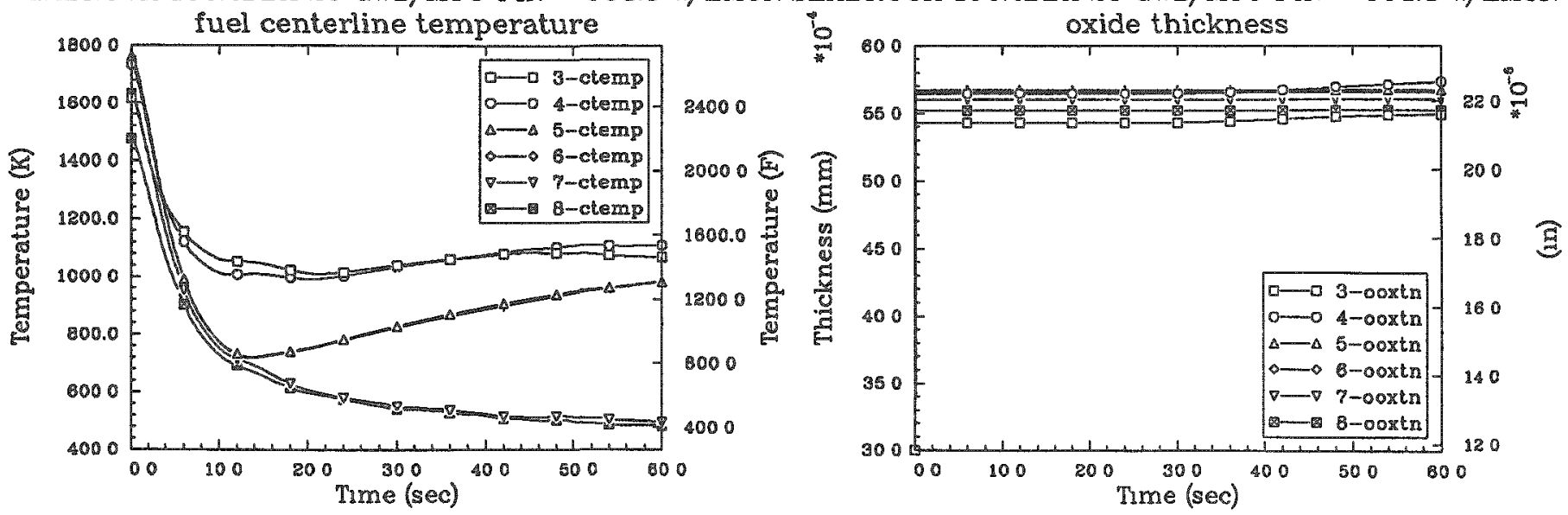

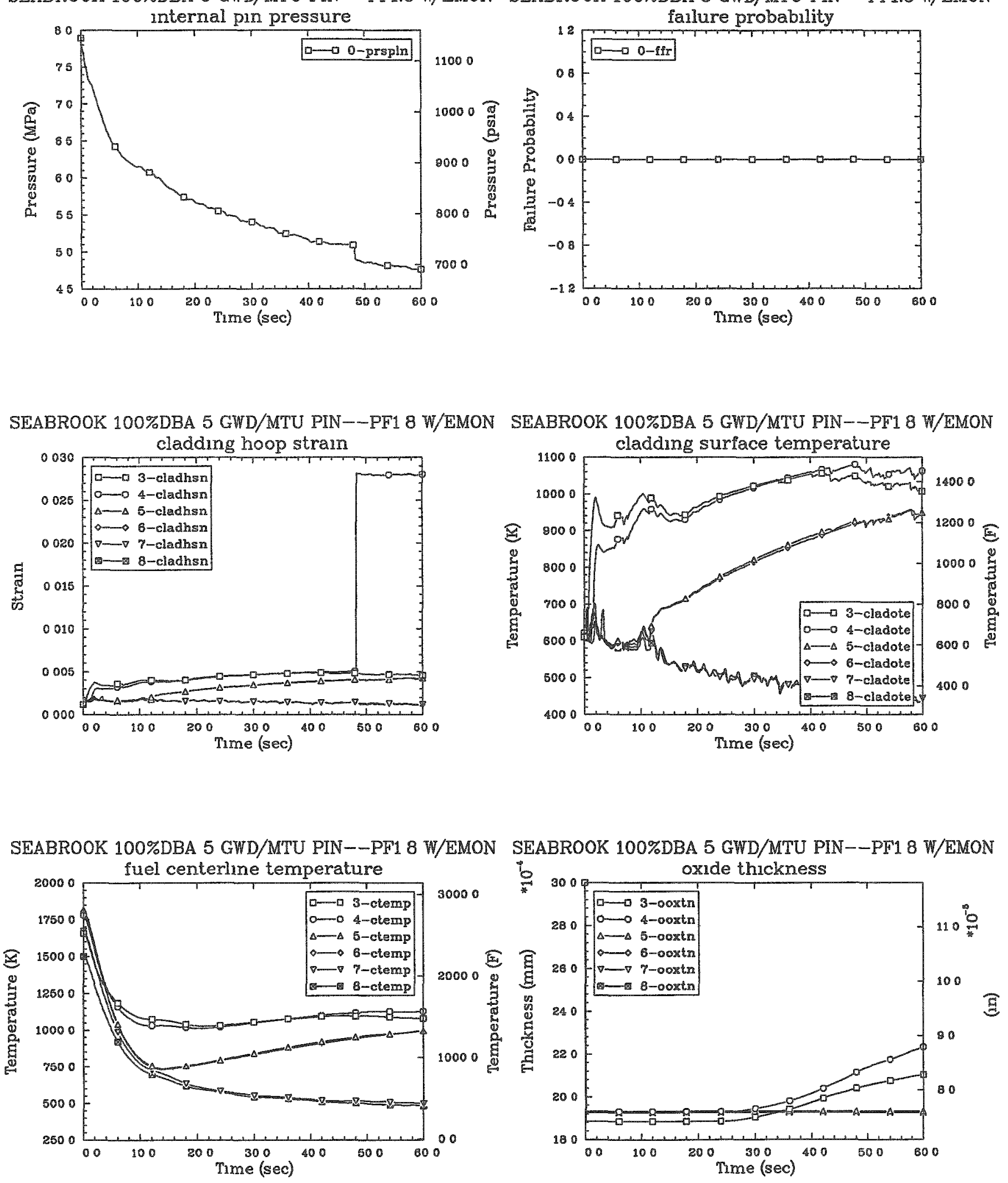


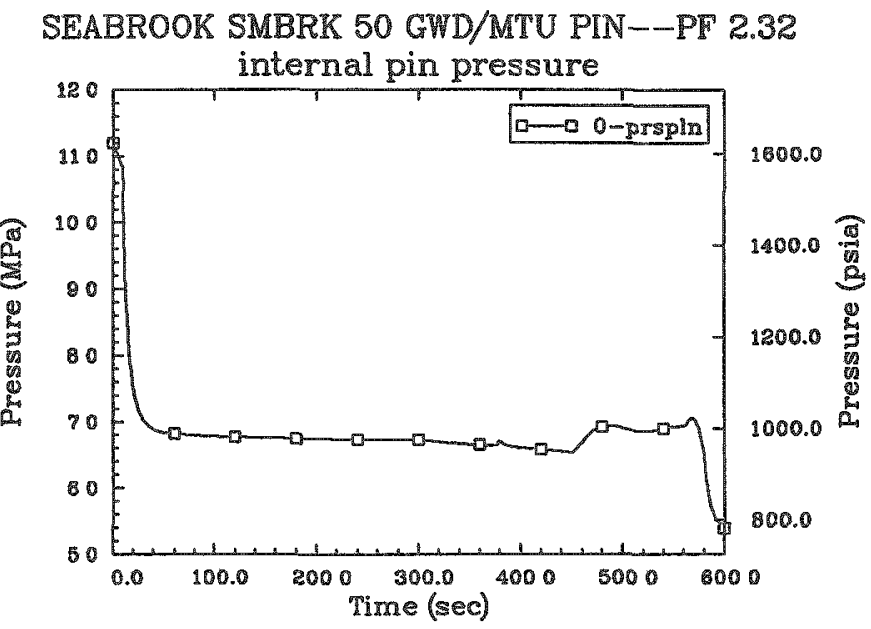

SEABROOK SMBRK 50 GWD/MTU PIN--PF 2.32

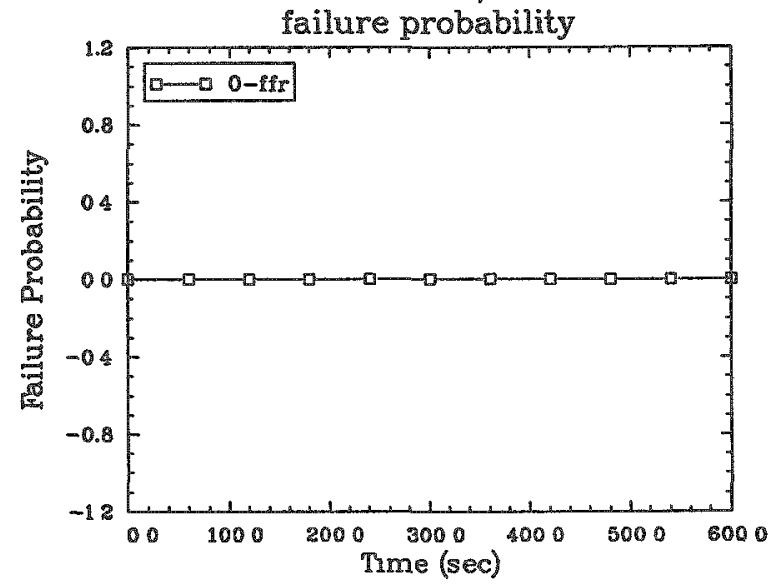

SEABROOK SMBRK $50 \mathrm{GWD} / \mathrm{MTU}$ PIN--PF 2.32
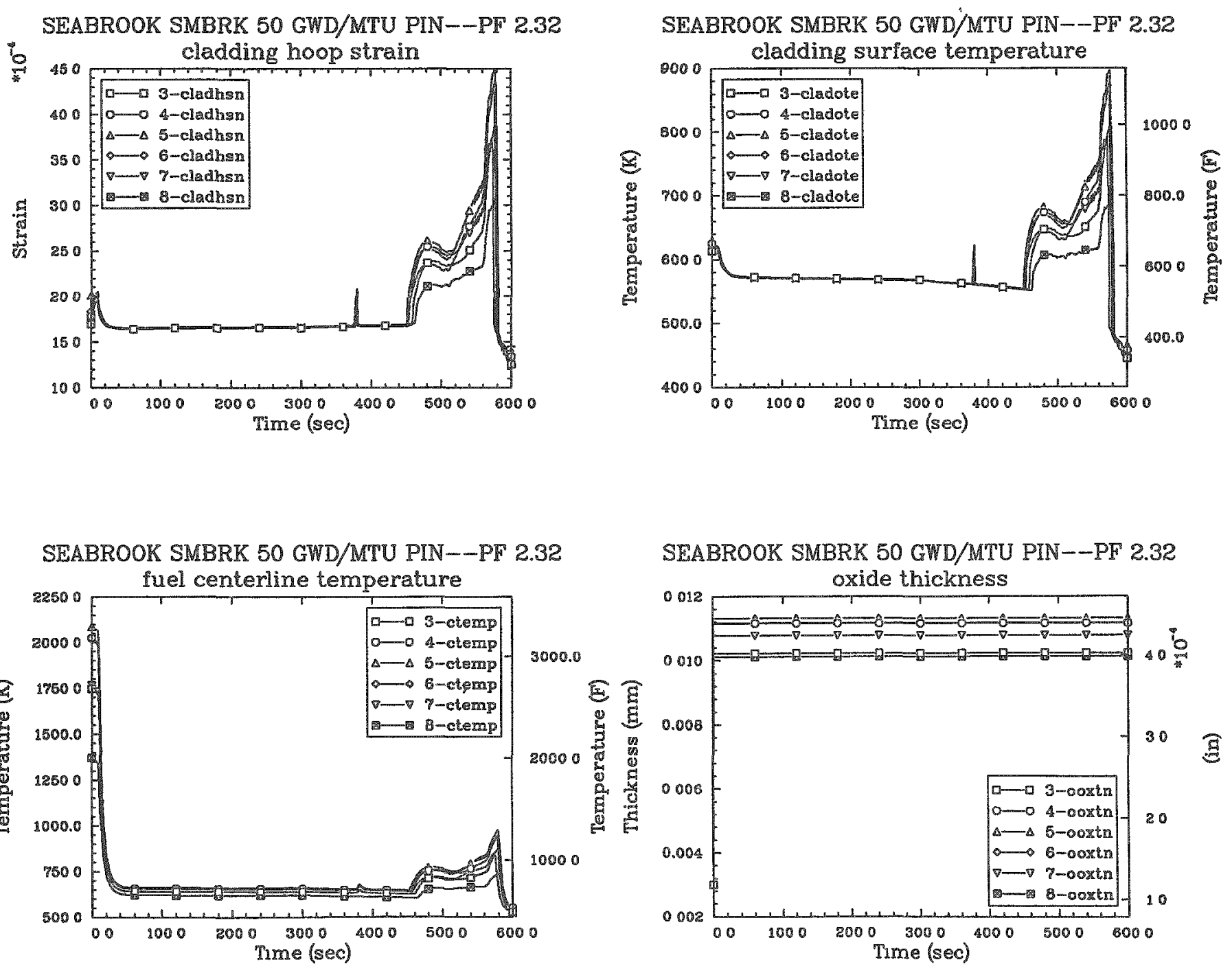

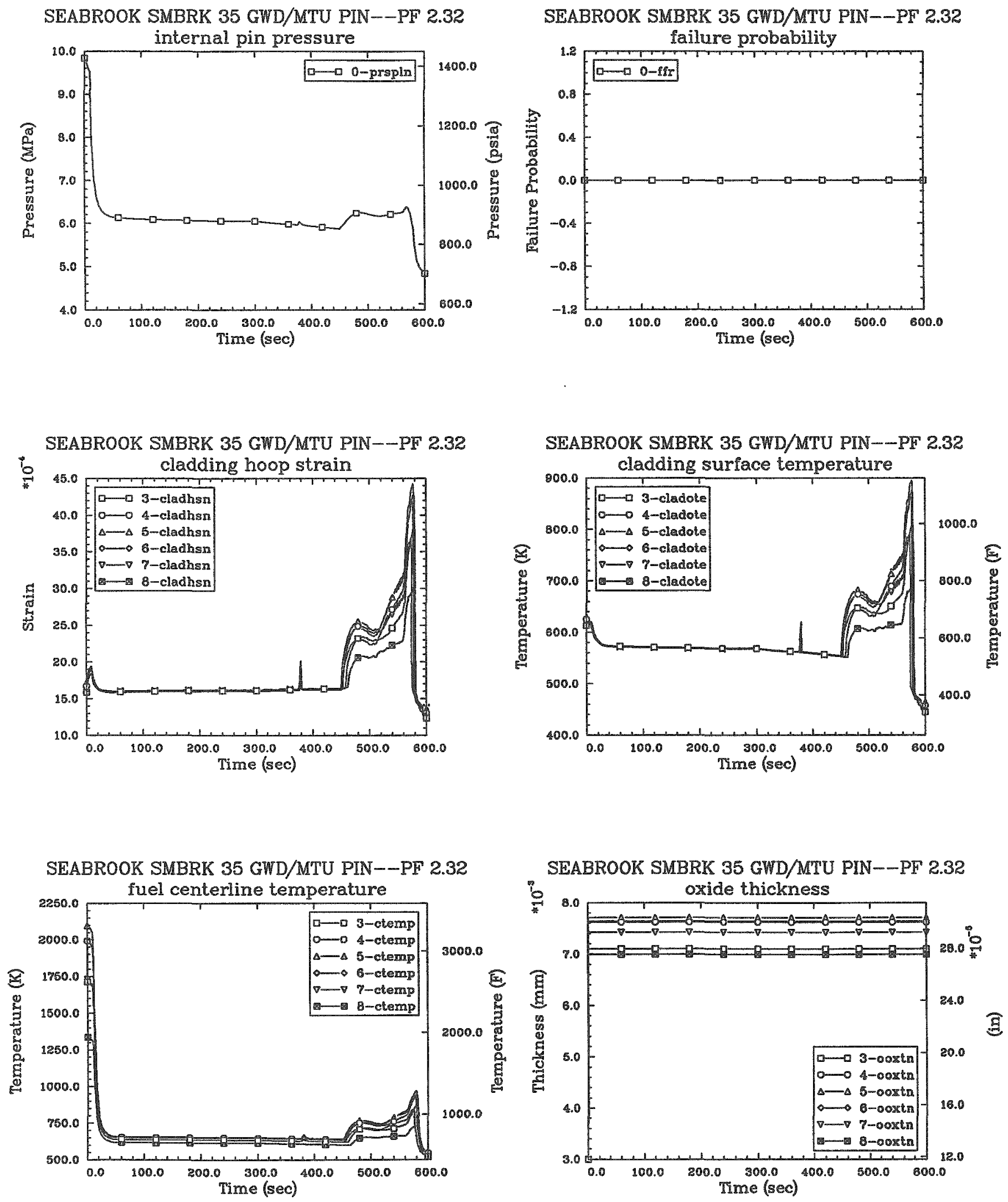

SEABROOK SMBRK 35 GWD/MTU PIN--PF 2.32

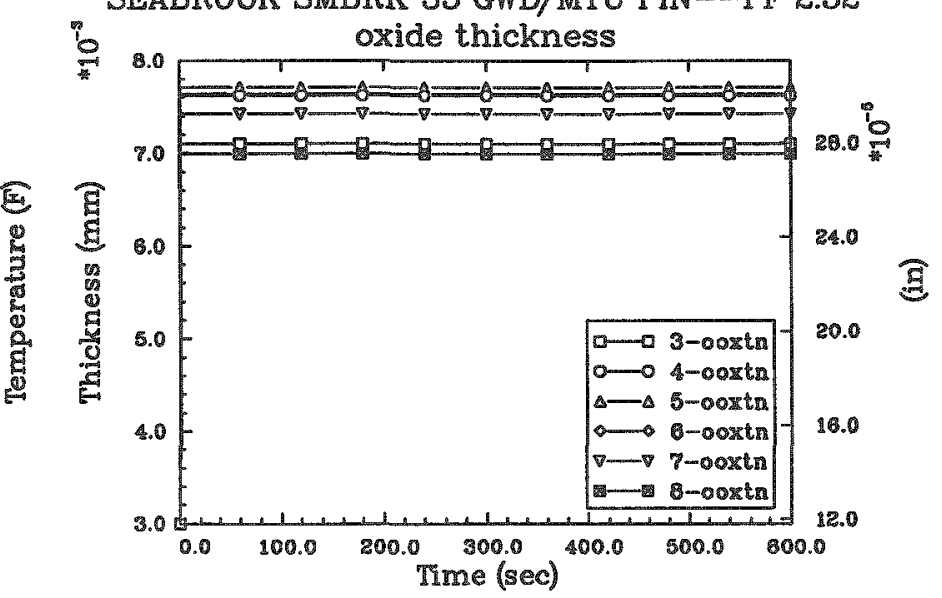



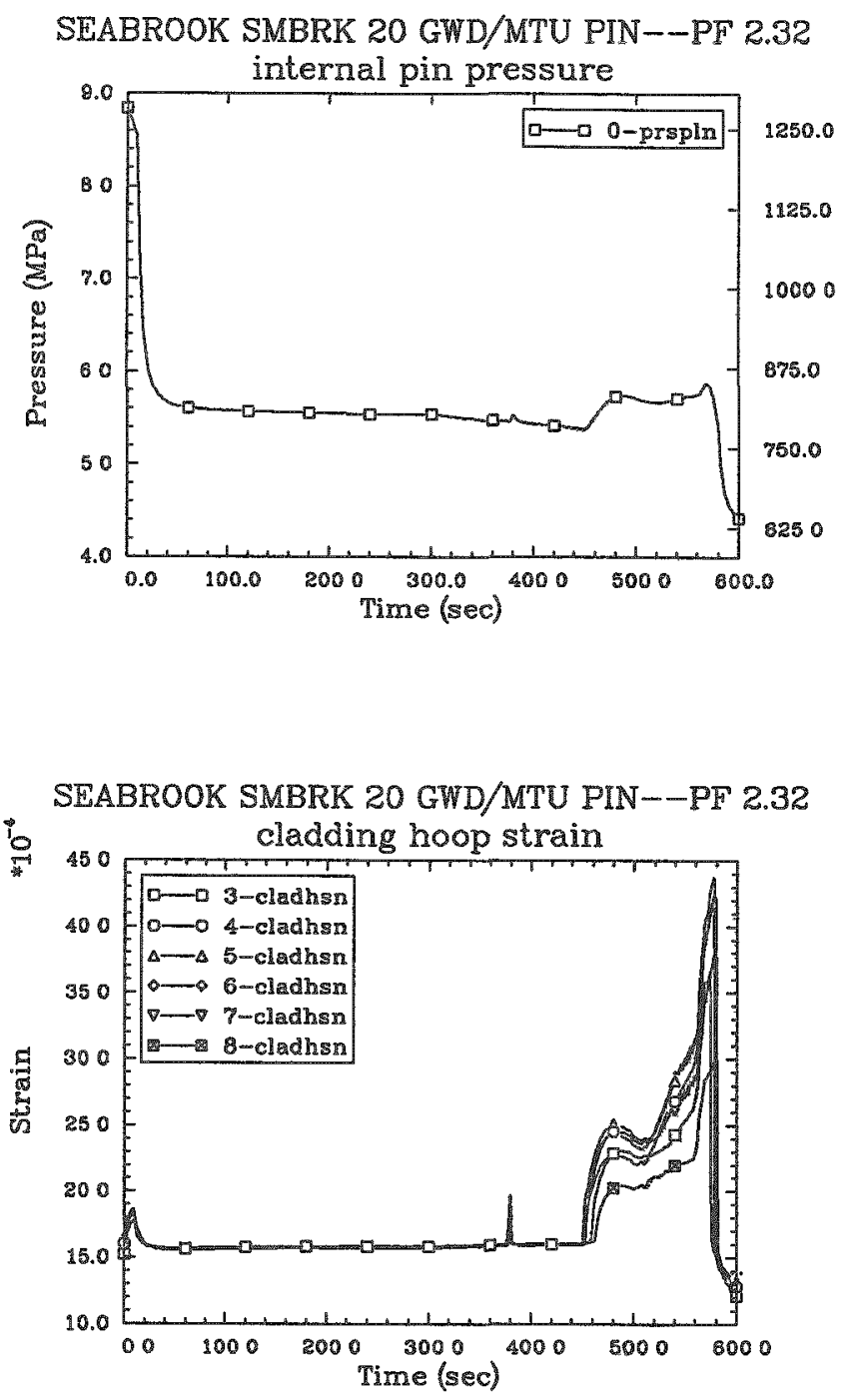

SEABROOK SMBRK 20 GWD/MTU PIN--PF 2.32

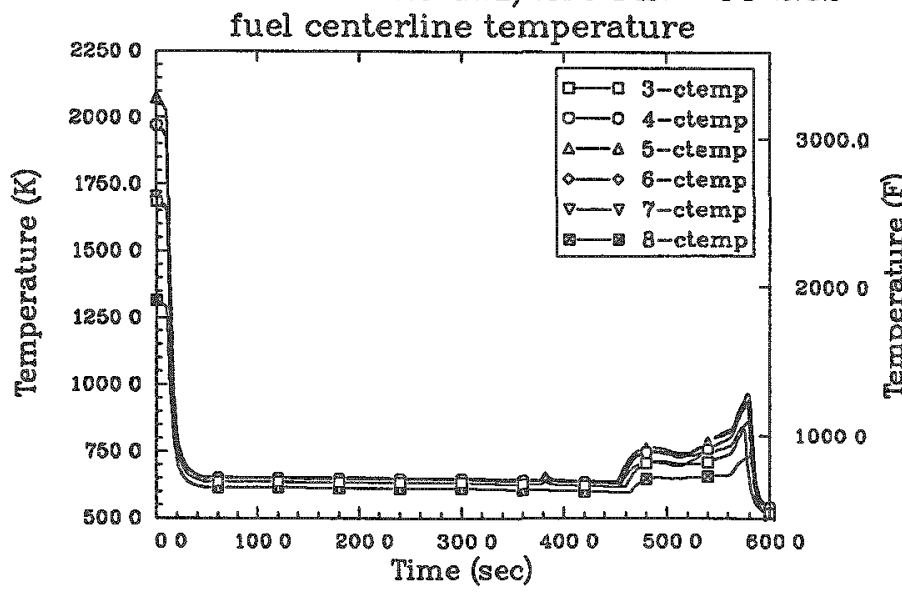

SEABROOK SMBRK $20 \mathrm{GWD} / \mathrm{MTU}$ PIN--PF 2.32 failure probability
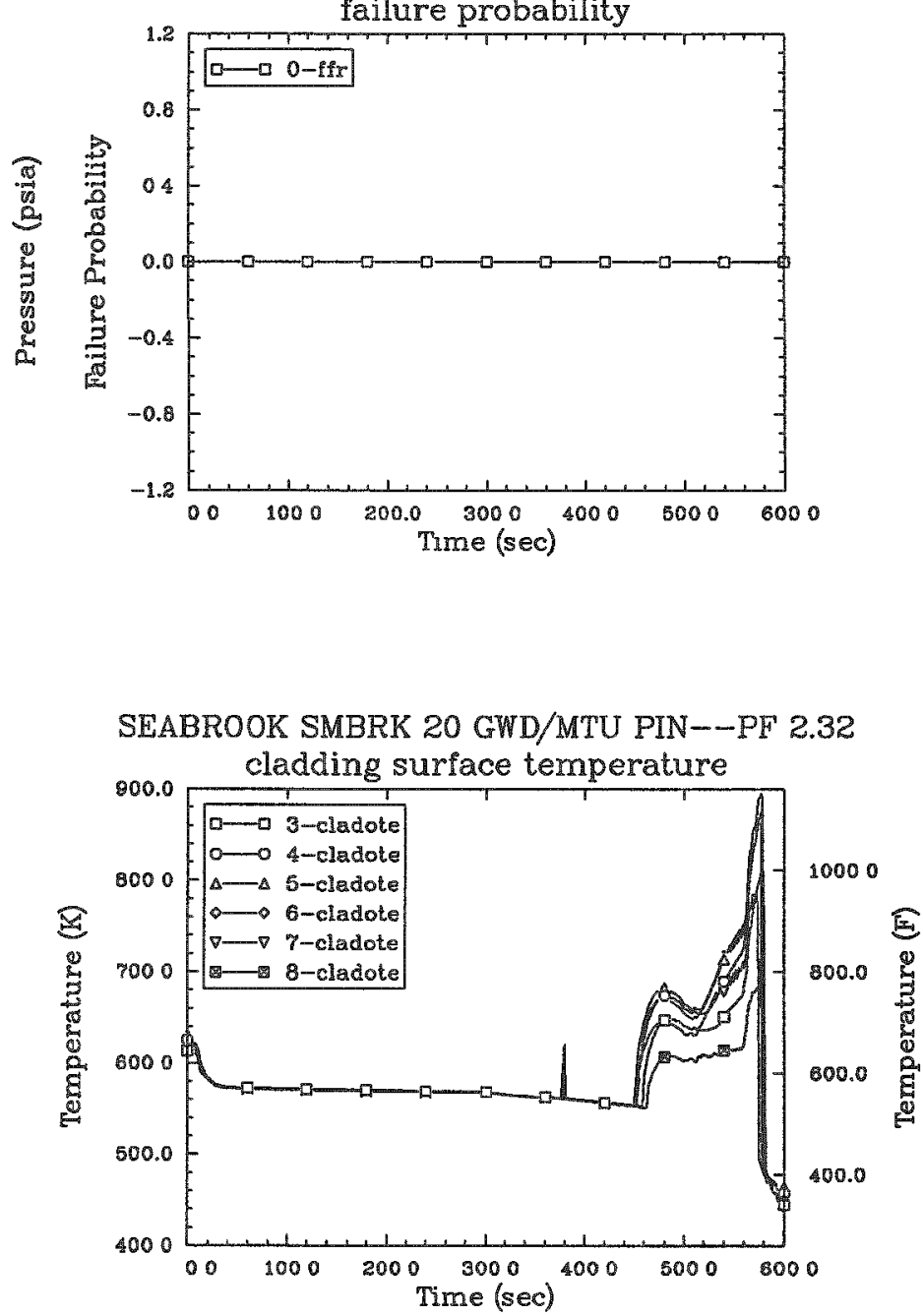

SEABROOK SMBRK 20 GWD/MTU PIN--PF 2.32

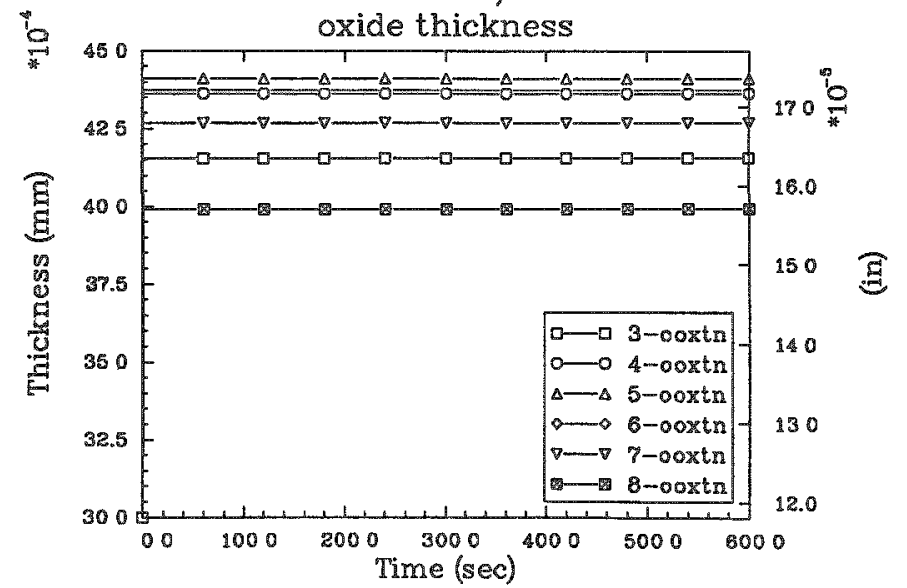


SEABROOK SMBRK 5 GWD/MTU PIN--PF 2.32

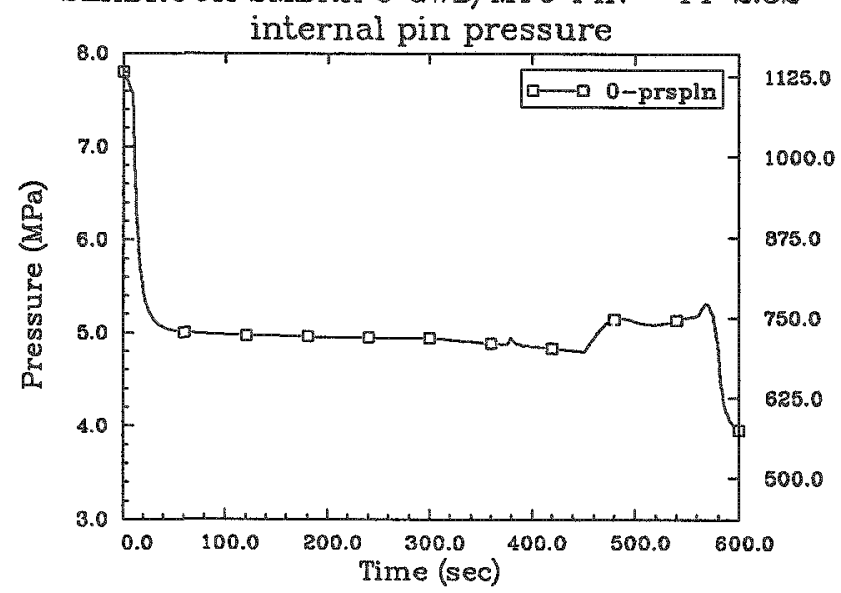

SEABROOK SMBRK 5 GWD/MTU PIN--PF 2.32

io

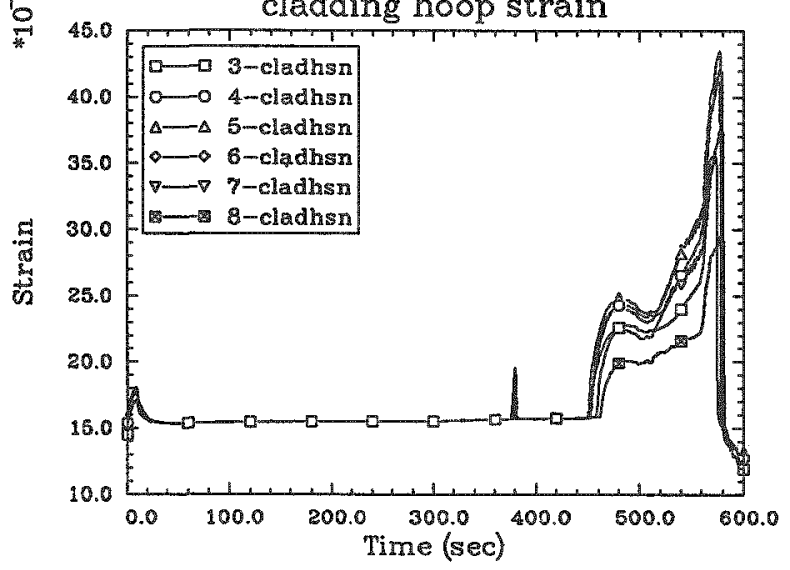

SEABROOK SMBRK 5 GWD/MTU PIN--PF 2.32

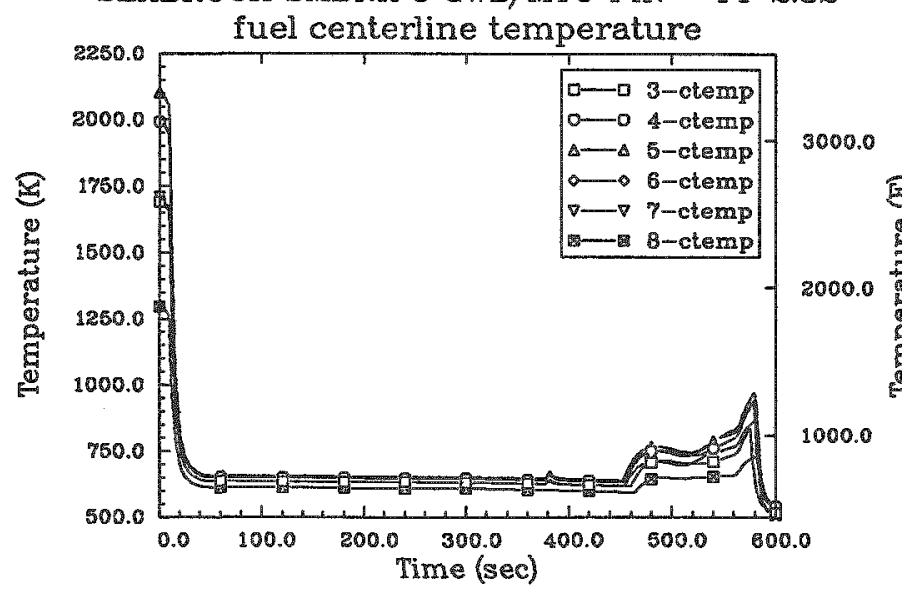

SEABROOK SMBRK 5 GWD/MTU PIN-DF 2.32

failure probability

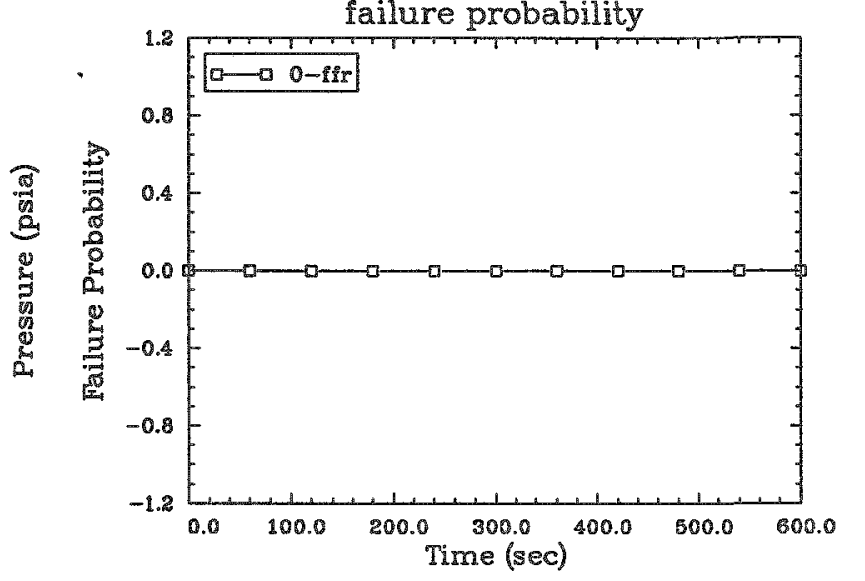

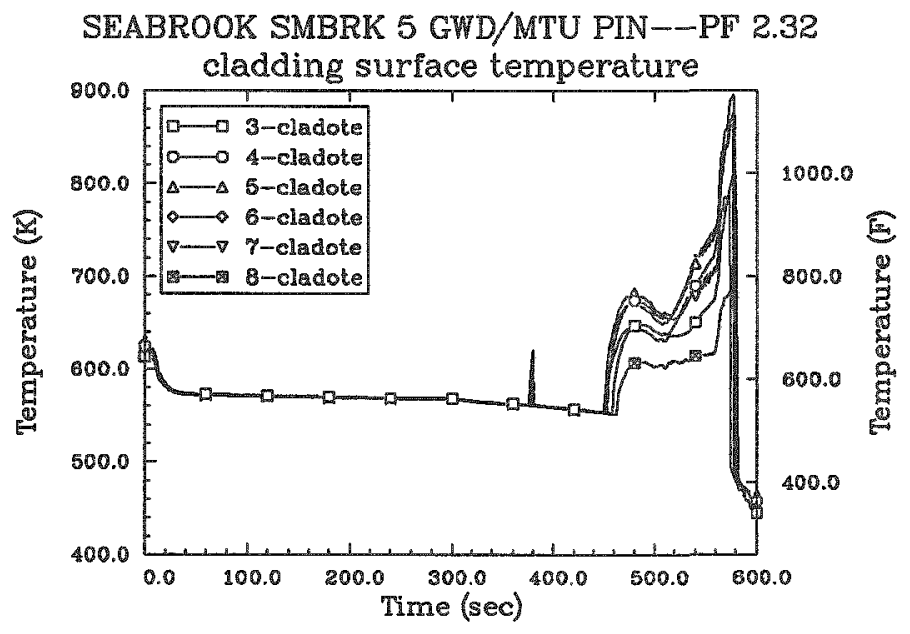


SEABROOK SMBRK 50 GWD/MTU PIN--PF 2.32 W/ECCS SEABROOK SMBRK 50 GWD/MTU PIN--PF 2.32 W/ECCS
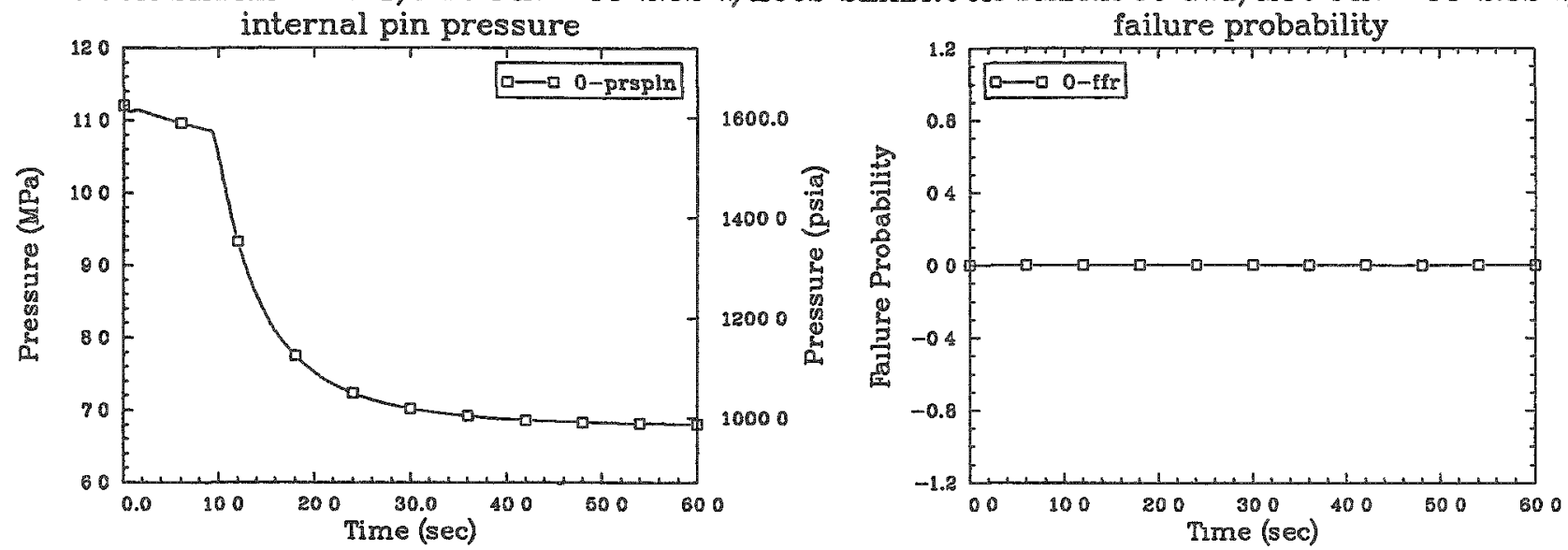

SEABROOK SMBRK 50 GWD/MTU PIN--PF 2.32 W/ECCS SEABROOK SMBRK 50 GWD/MTU PIN--PF 2.32 W/ECCS
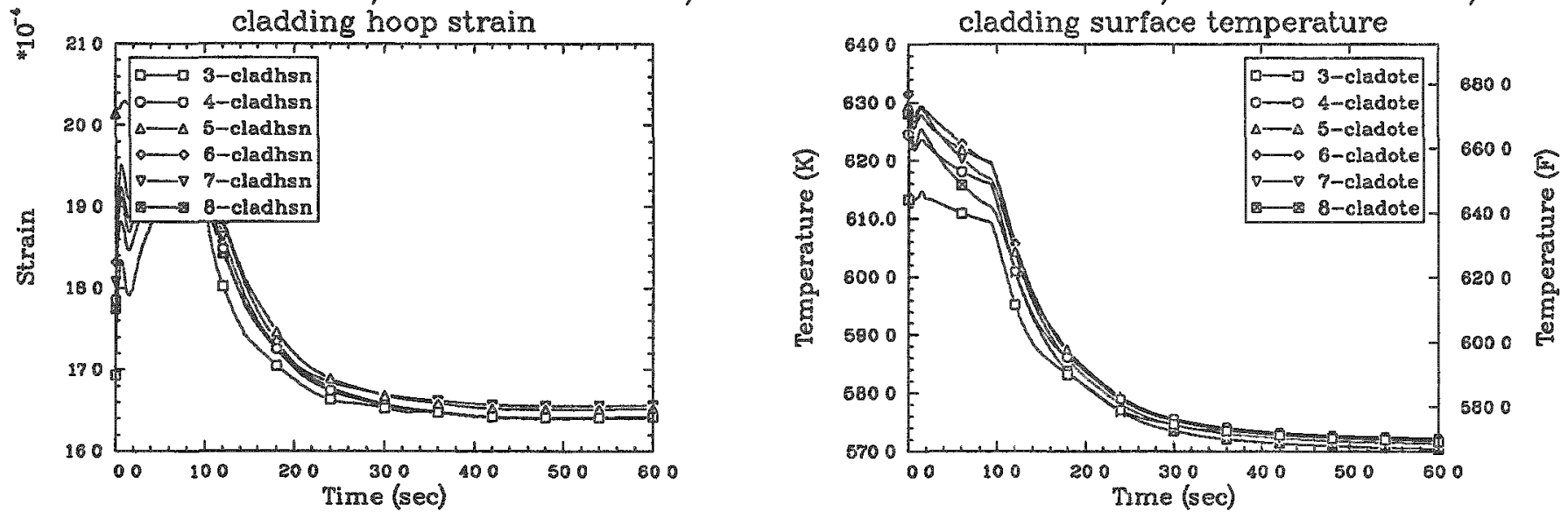

SEABROOK SMBRK 50 GWD/MTU PIN--PF 2.32 W/ECCS SEABROOK SMBRK 50 GWD/MTU PIN--PF 2.32 W/ECCS
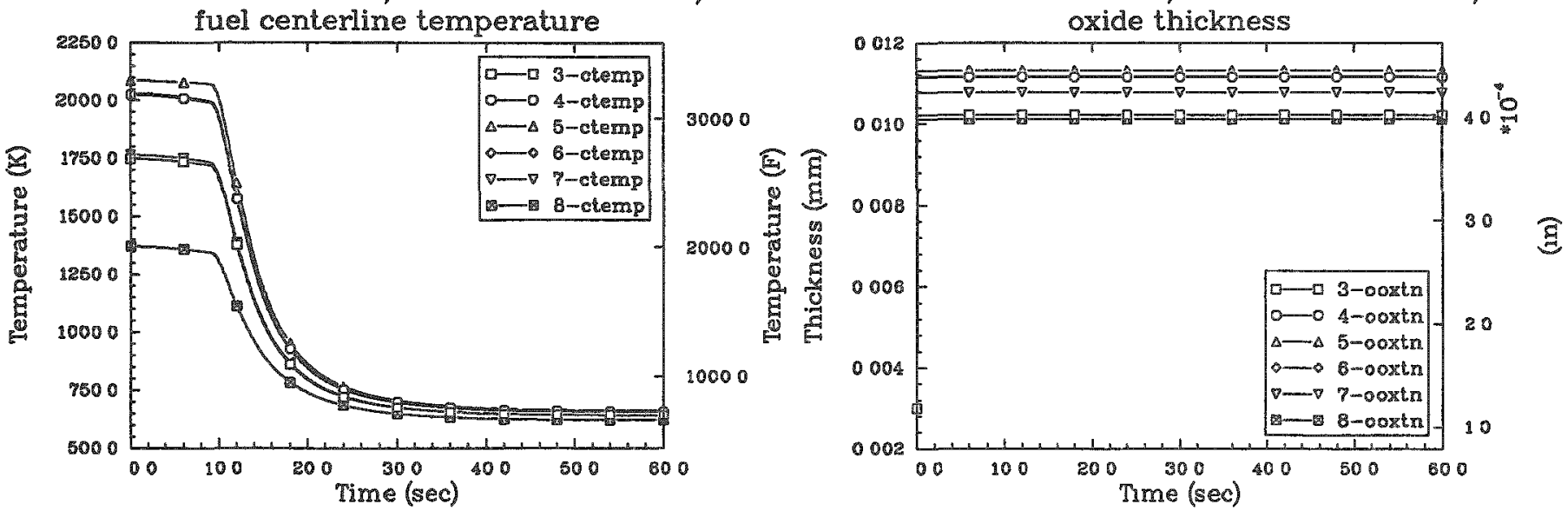
SEABROOK SMBRK 35 GWD/MTU PIN--PF 2.32 W/ECCS SEABROOK SMBRK 35 GWD/MTU PIN--PF 2.32 W/ECCS

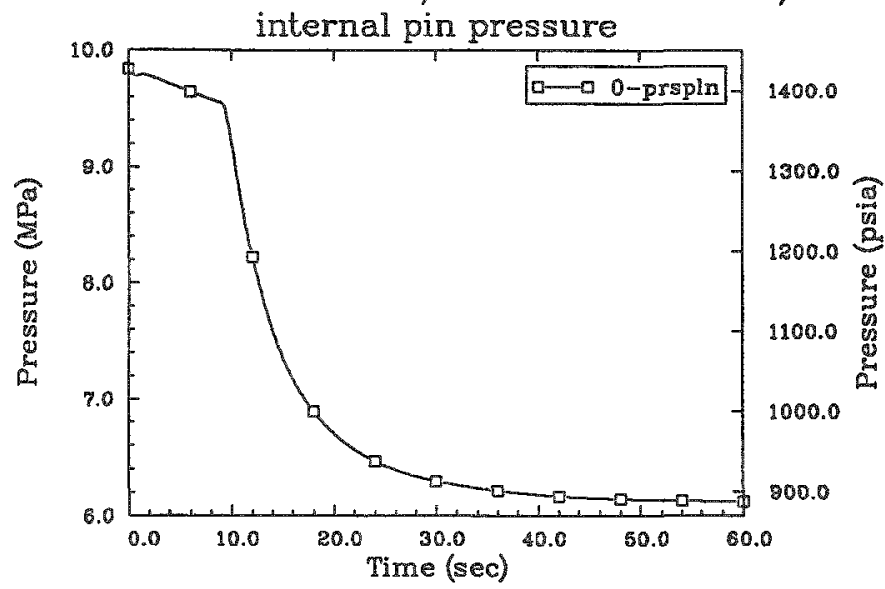
failure probability

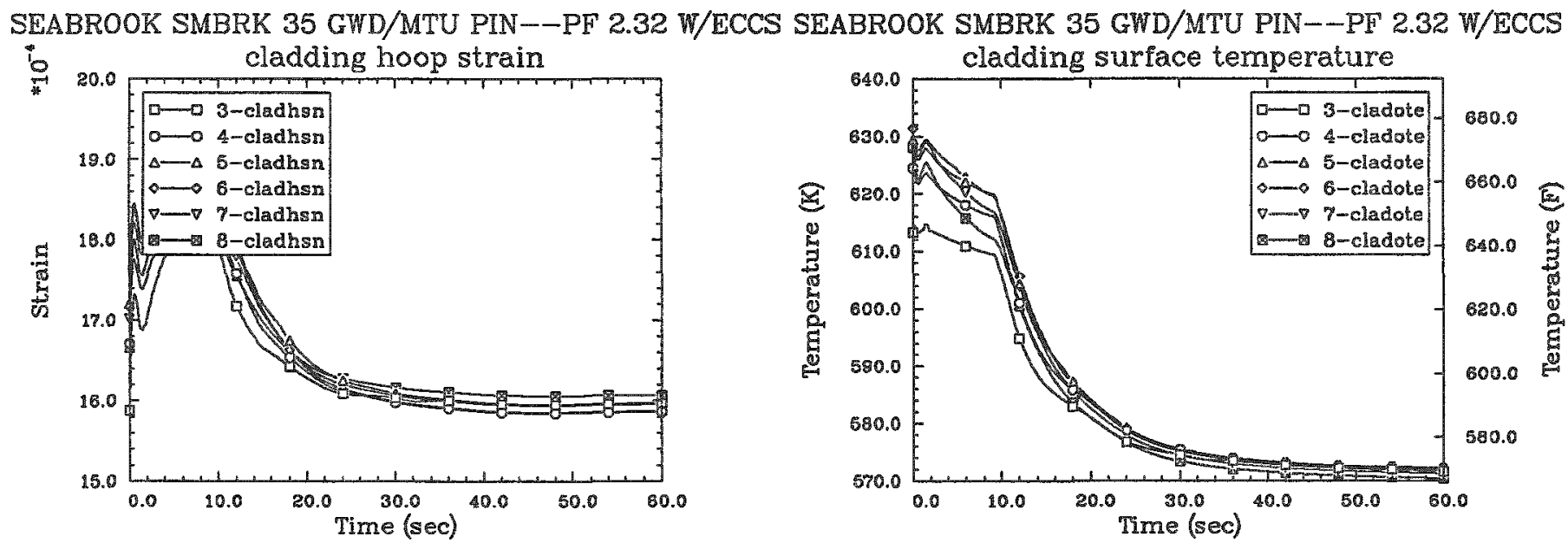

SEABROOK SMBRK 35 GWD/MTU PIN--PF 2.32 W/ECCS SEABROOK SMBRK 35 GWD/MTU PIN--PF 2.32 W/ECCS
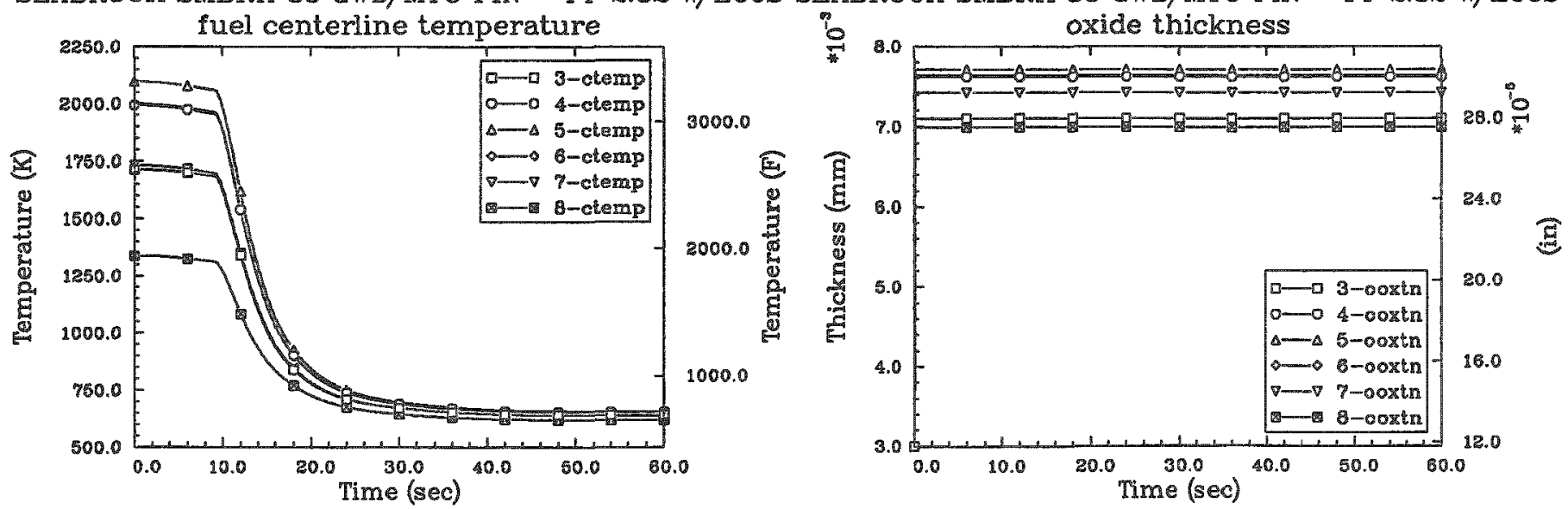
SEABROOK SMBRK 20 GWD/MTU PIN--PF 232 W/ECCS SEABROOK SMBRK 20 GWD/MTU PIN--PF 232 W/ECCS
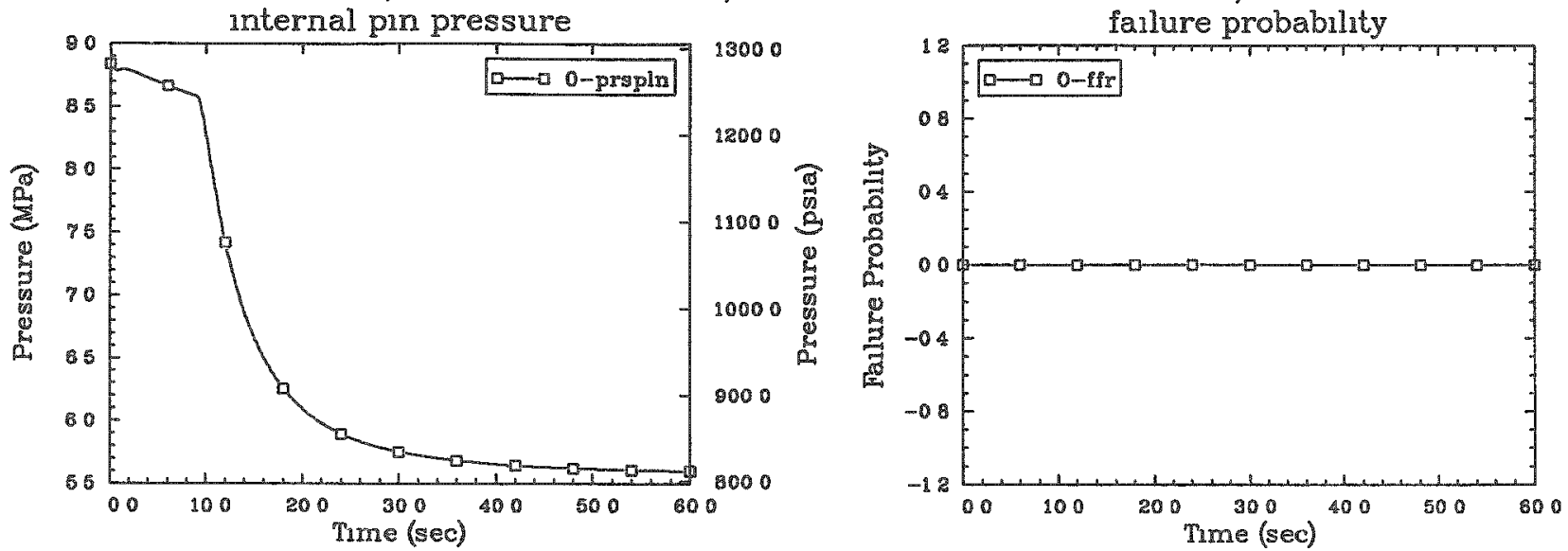

SEABROOK SMBRK 20 GWD/MTU PIN--PF 232 W/ECCS SEABROOK SMBRK 20 GWD/MTU PIN--PF 232 W/ECCS
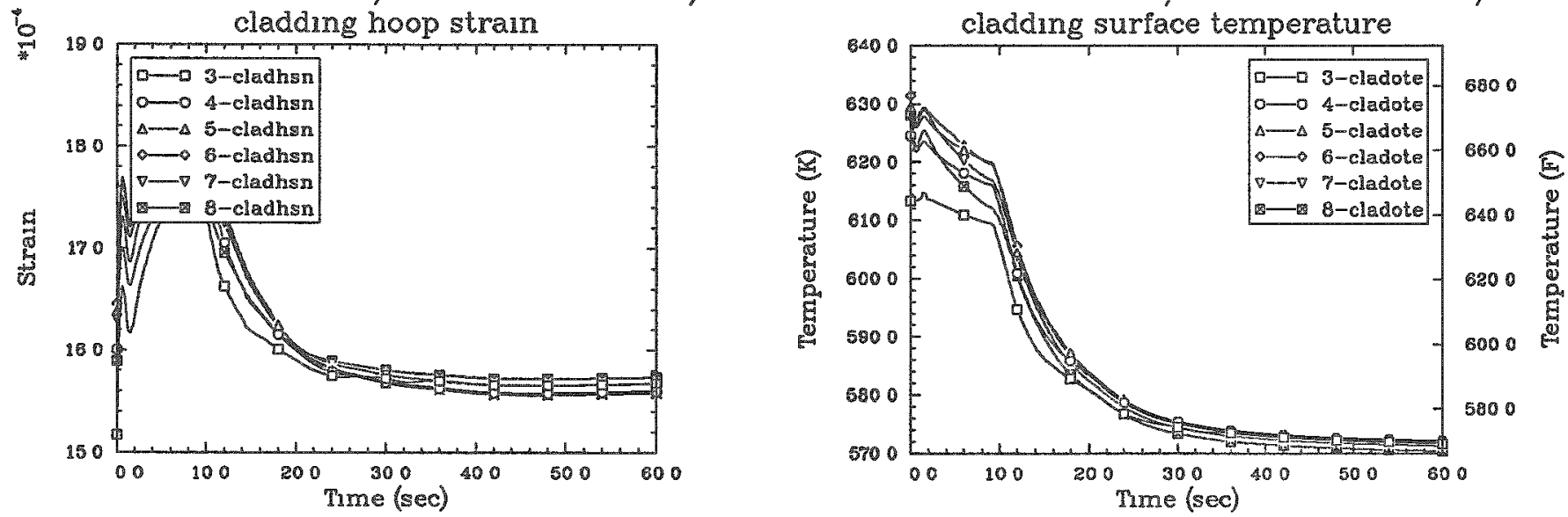

SEABROOK SMBRK 20 GWD/MTU PIN--PF 232 W/ECCS SEABROOK SMBRK 20 GWD/MTU PIN--PF 232 W/ECCS
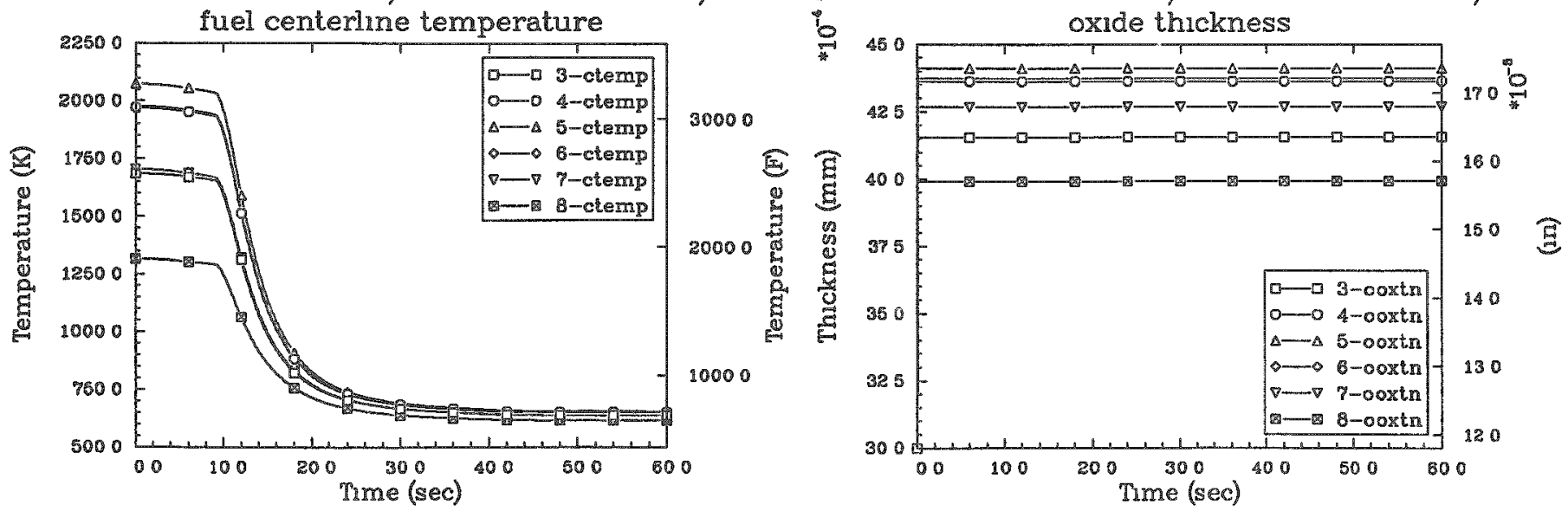
SEABROOK SMBRK 5 GWD/MTU PIN--PF 2.32 W/ECCS

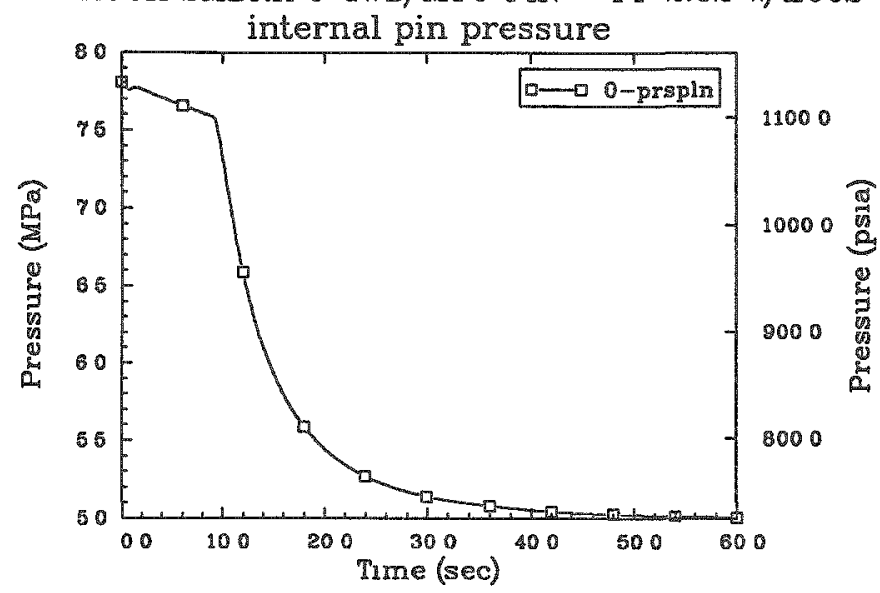

SEABROOK SMBRK 5 GWD/MTU PIN--PF 2.32 W/ECCS

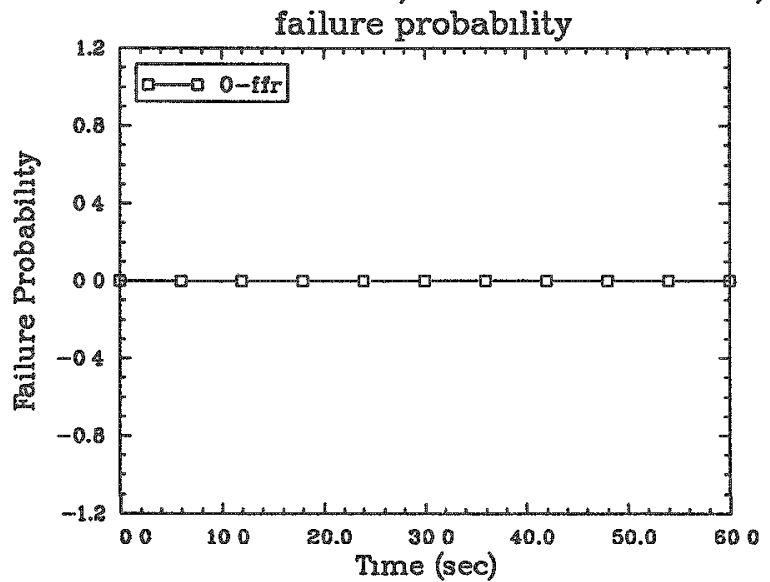

SEABROOK SMBRK 5 GWD/MTU PIN--PF 2.32 W/ECCS

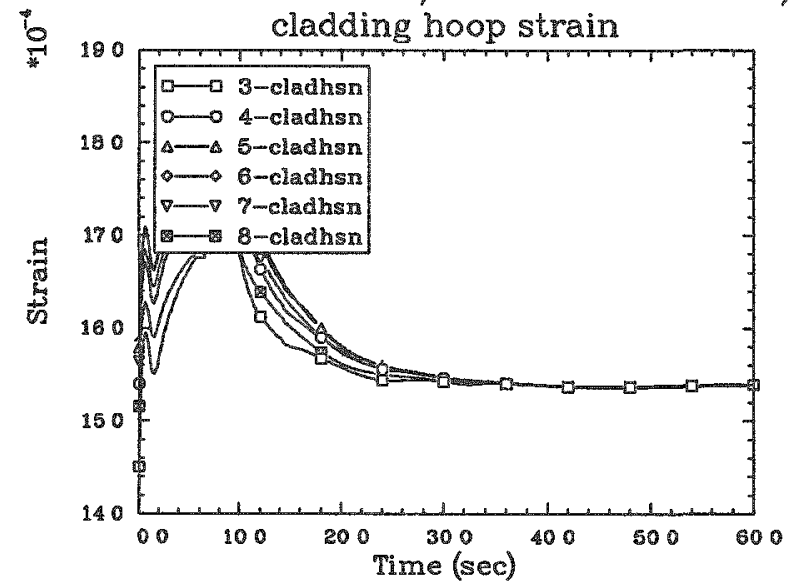

SEABROOK SMBRK 5 GWD/MTU PIN--PF 2.32 W/ECCS

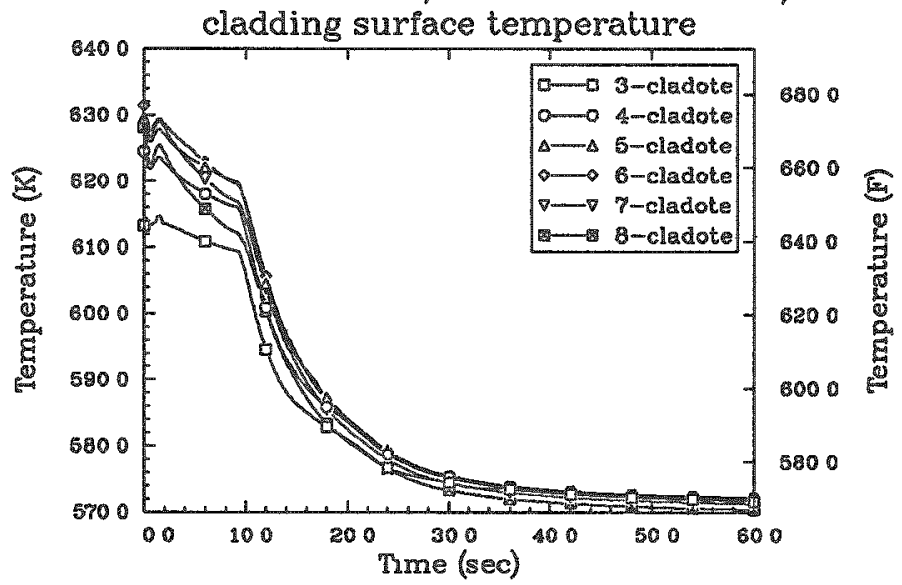

SEABROOK SMBRK 5 GWD/MTU PIN--PF 2.32 W/ECCS

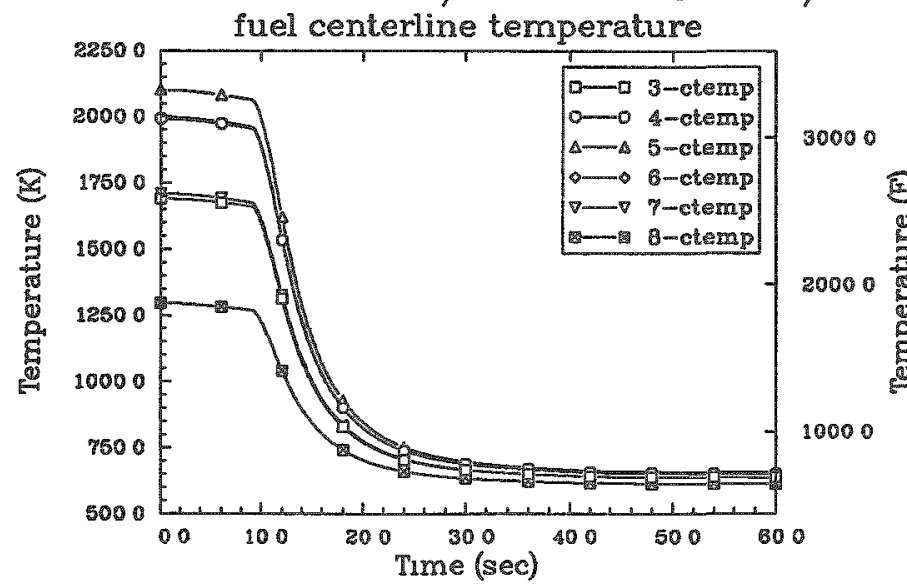

SEABROOK SMBRK 5 GWD/MTU PIN--PF 2.32 W/ECCS

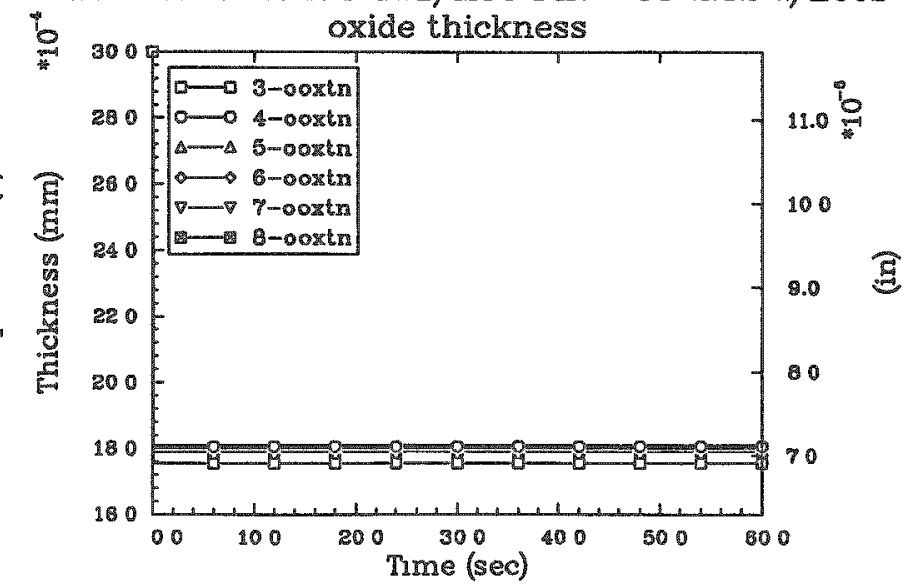


SEABROOK 100\%DBA 50 GWD/MTU PIN--PF 2.32 (TRAC)SEABROOK 100\%DBA 50 GWD/MTU PIN--PF 2.32 (TRAC)
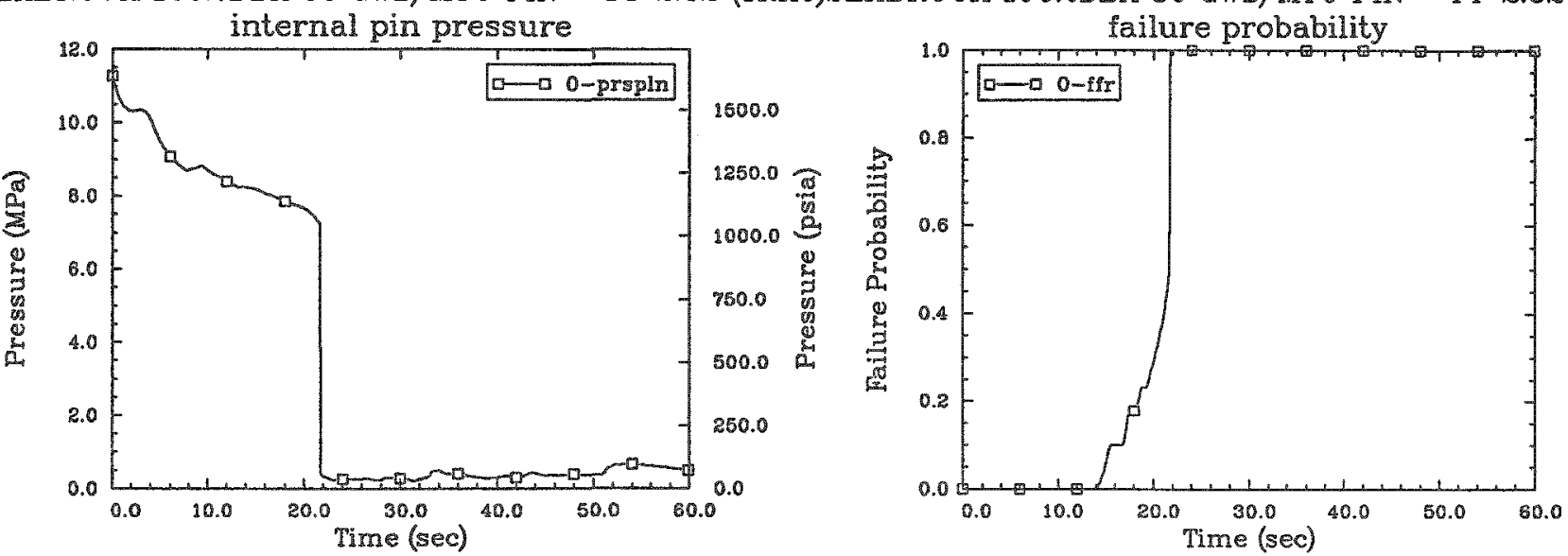

SEABROOK 100\%DBA $50 \mathrm{GWD} / \mathrm{MTU}$ PIN--PF 2.32 (TRAC)SEABROOK 100\%DBA 50 GWD/MTU PIN--PF 2.32 (TRAC)
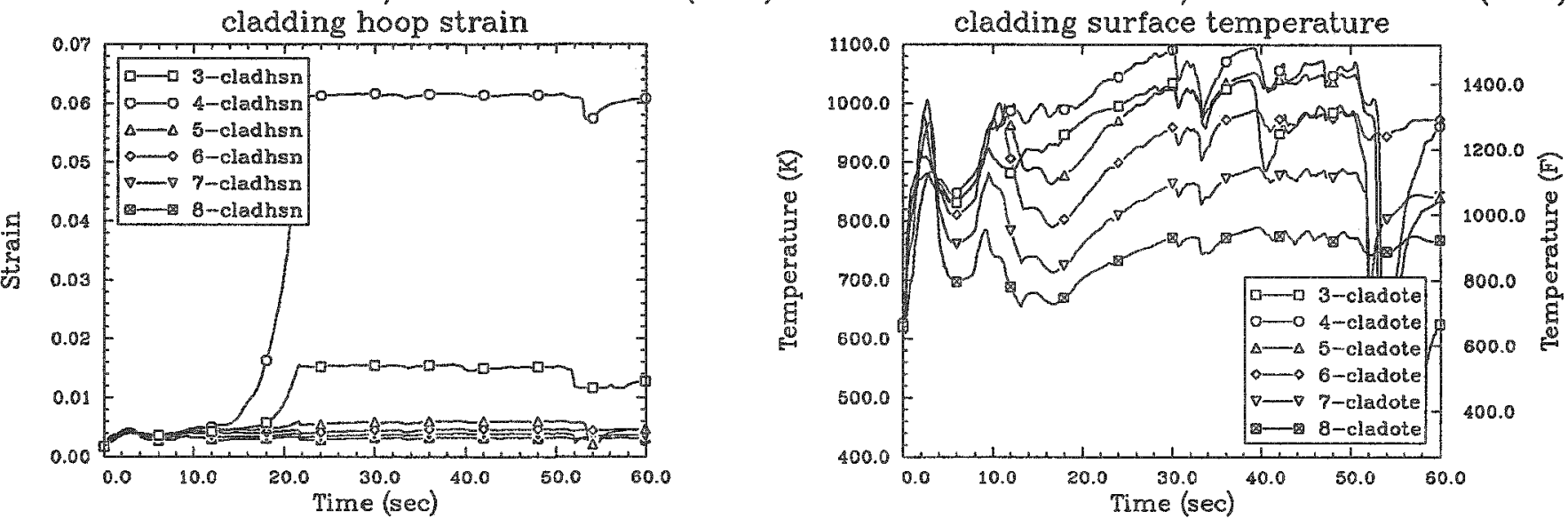

SEABROOK 100\%DBA 50 GWD/MTU PIN--PF' 2.32 (TRAC)SEABROOK 100\%DBA 50 GWD/MTU PIN--PF 2.32 (TRAC)
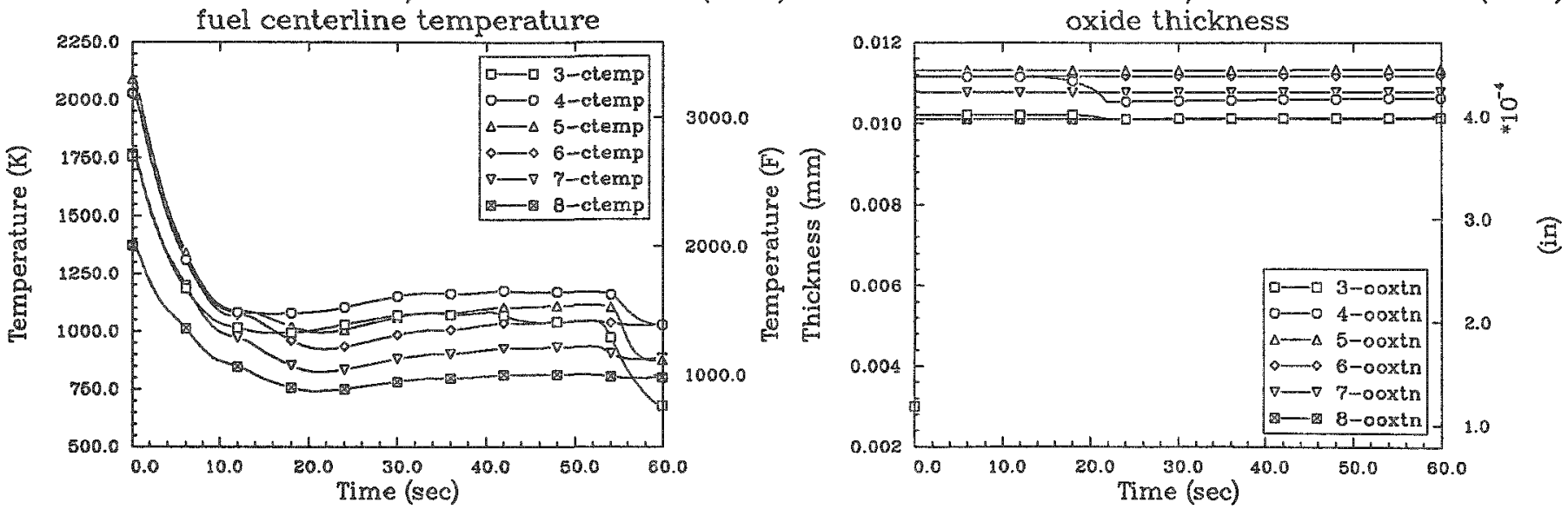
SEABROOK 100\%DBA 35 GWD/MTU PIN--PF 2.32 (TRAC)SEABROOK 100\%DBA 35 GWD/MTU PIN--PF 2.32 (TRAC)
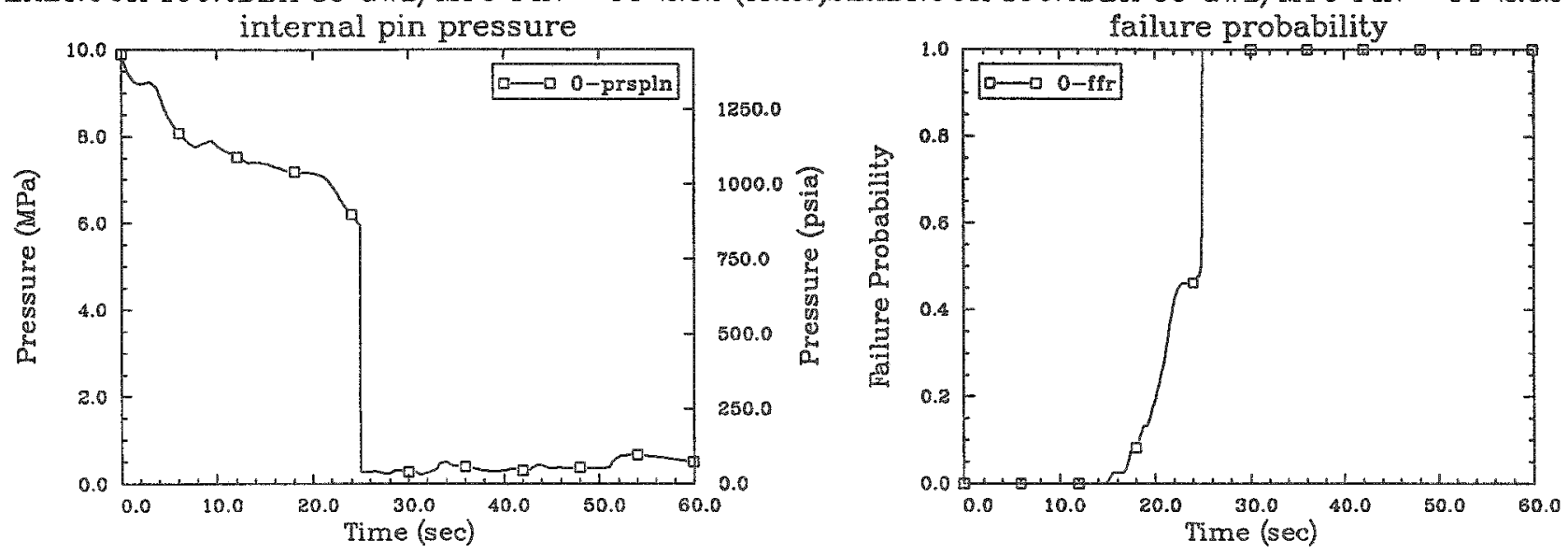

SEABROOK 100\%DBA 35 GWD/MTU PIN--PF 2.32 (TRAC)SEABROOK 100\%DBA 35 GWD/MTU PIN--PF 2.32 (TRAC)
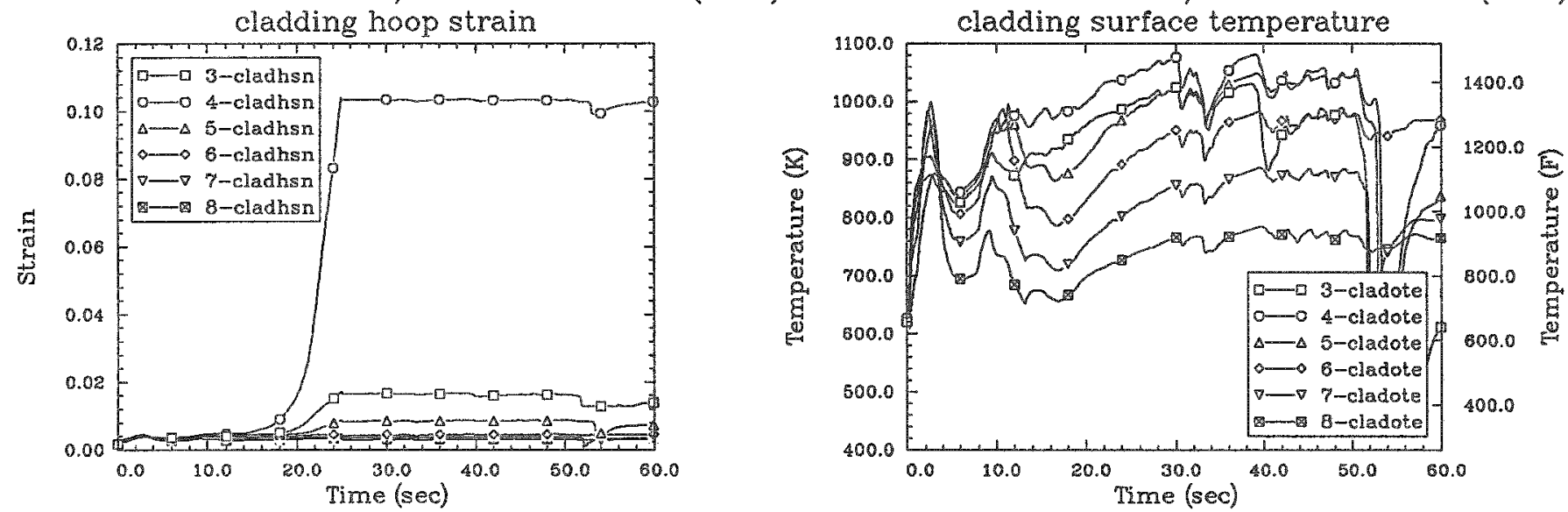

SEABROOK 100\%DBA 35 GWD/MTU PIN--PF 2.32 (TRAC)SEABROOK 100\%DBA 35 GWD/MTU PIN--PF 2.32 (TRAC)
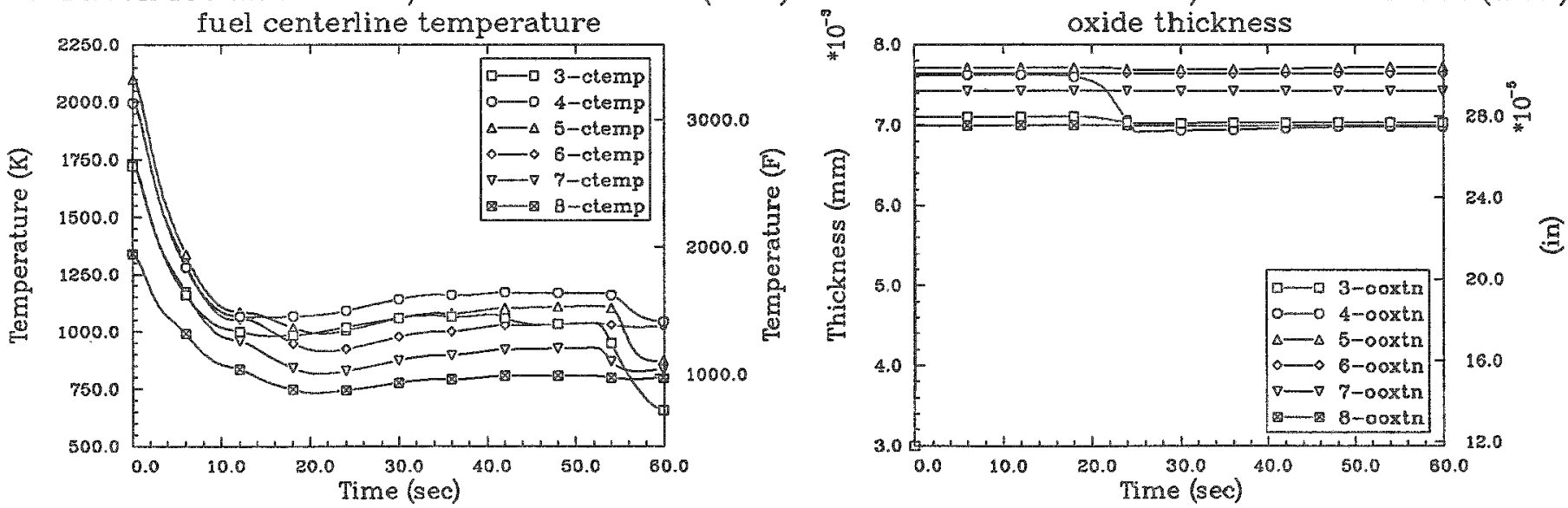
SEABROOK 100\%DBA 20 GWD/MTU PIN--PF 2.32 (TRAC)SEABROOK 100\%DBA 20 GWD/MTU PIN--PF 2.32 (TRAC)
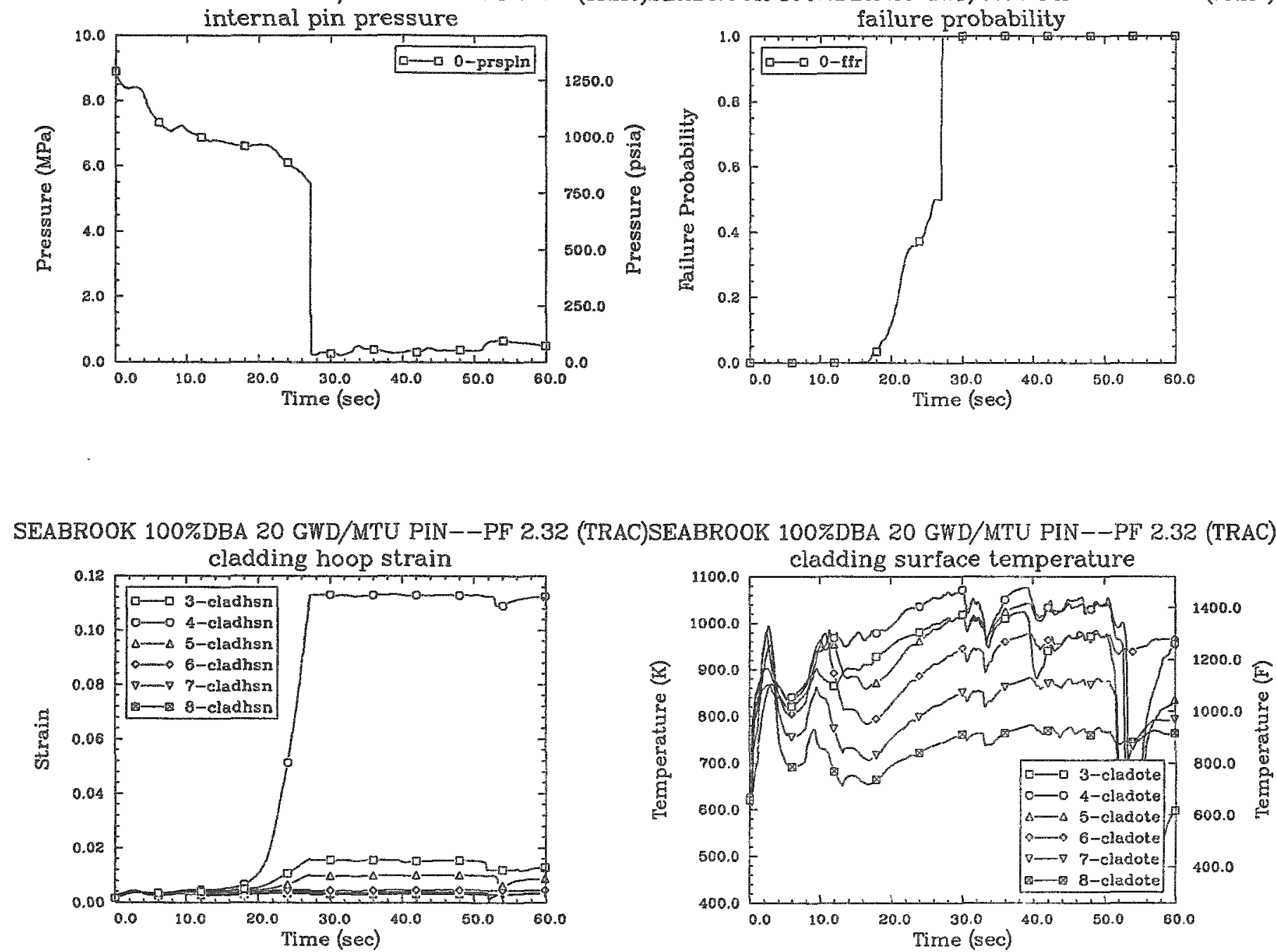

SEABROOK 100\%DBA 20 GWD/MTU PIN--PF 2.32 (TRAC)SEABROOK 100\%DBA 20 GWD/MTU PIN--PF 2.32 (TRAC)
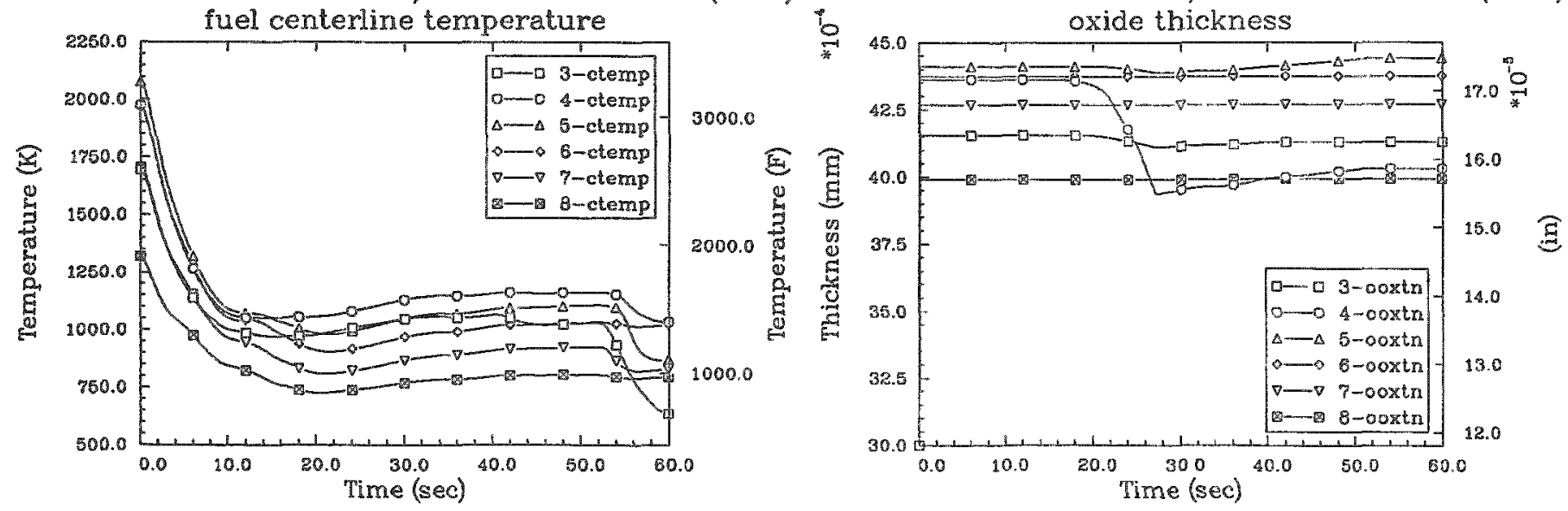
SEABROOK 100\%DBA 5 GWD/MTU PIN--PF 2.32 (TRAC) SEABROOK 100\%DBA 5 GWD/MTU PIN--PF 2.32 (TRAC)
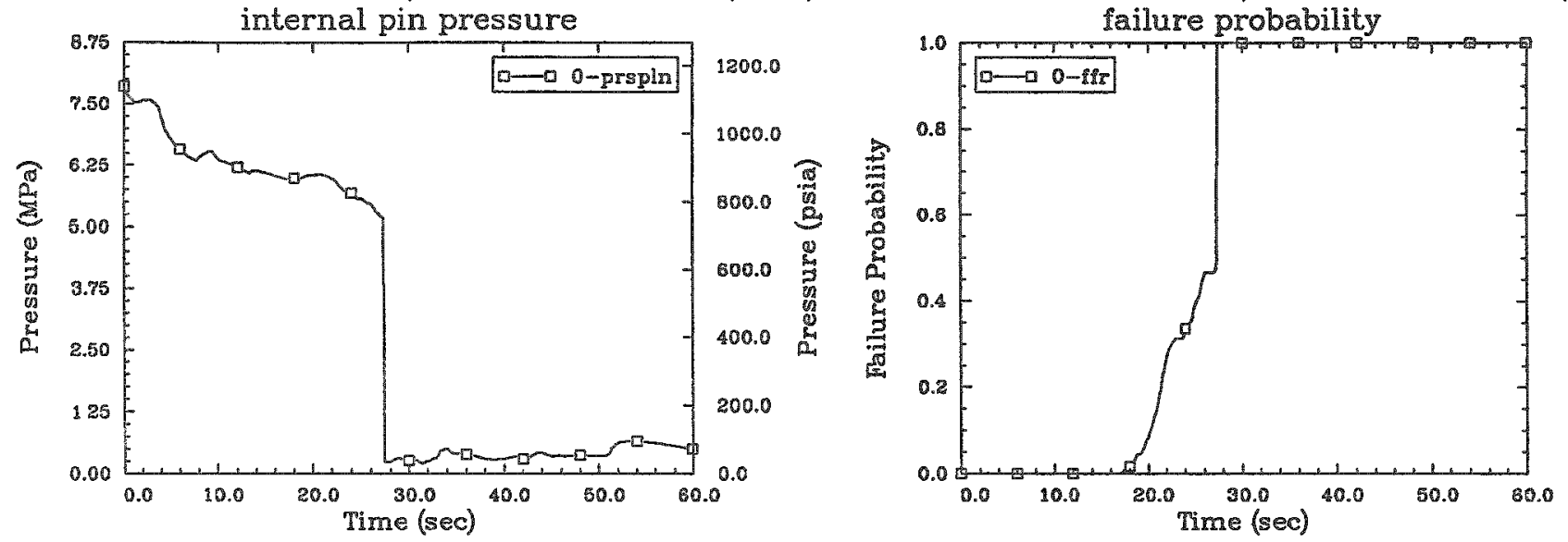

SEABROOK 100\%DBA 5 GWD/MTU PIN--PF 2.32 (TRAC) SEABROOK 100\%DBA 5 GWD/MTU PIN--PF 2.32 (TRAC)
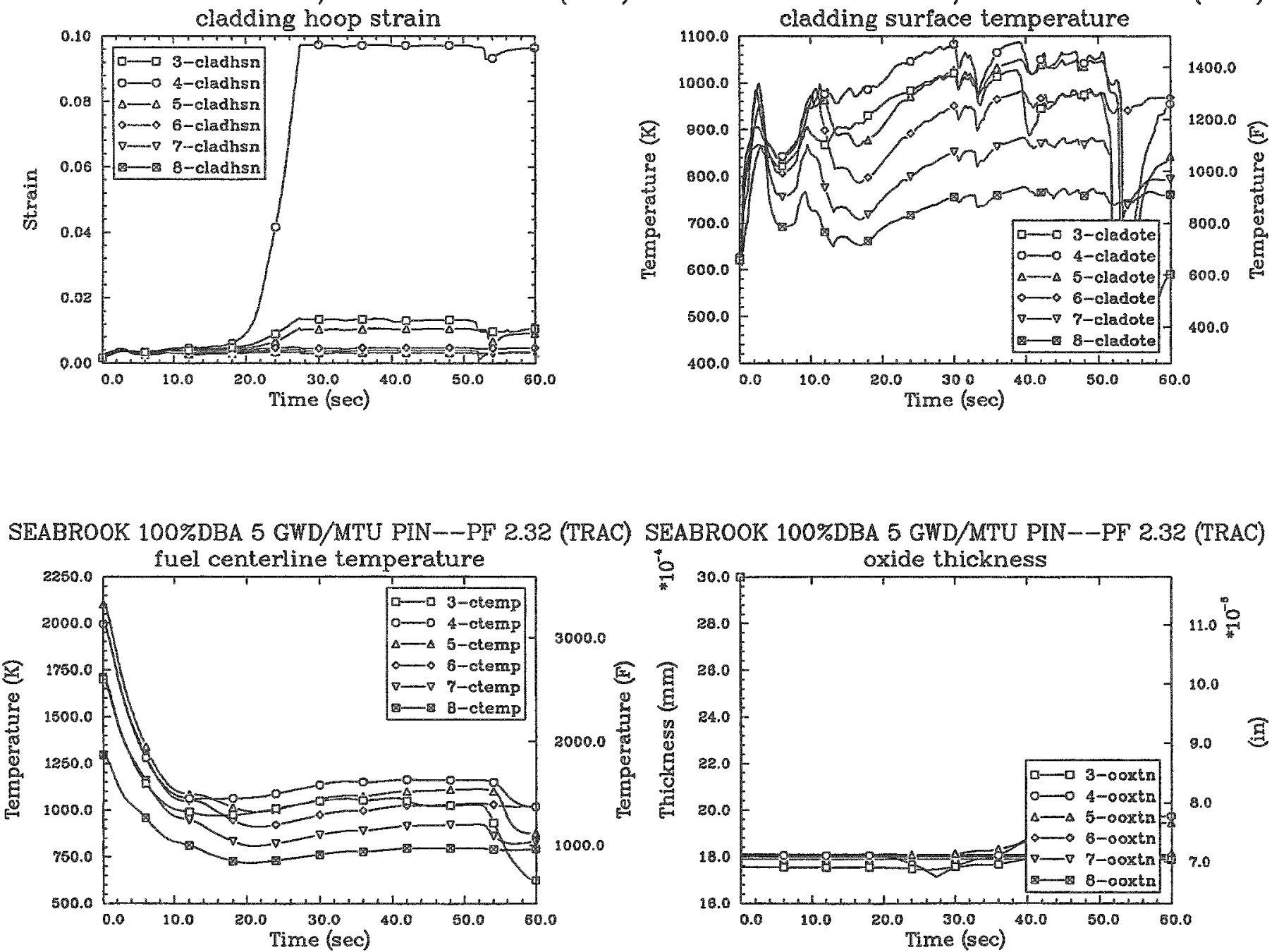
SEABROOK 100\%DBA 50 GWD/MTU PIN--PF 2.2 (TRAC) SEABROOK 100\%DBA 50 GWD/MTU PIN--PF 2.2 (TRAC)

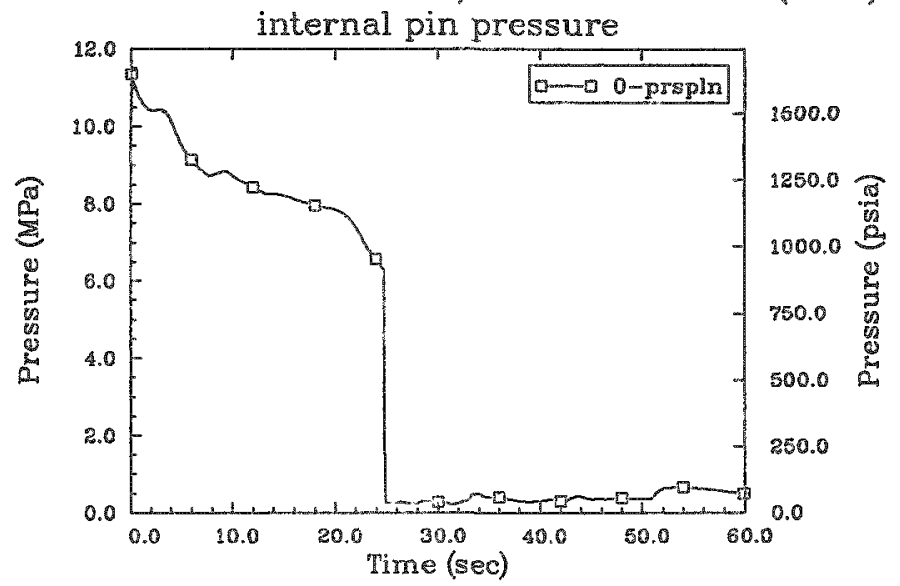

failure probability
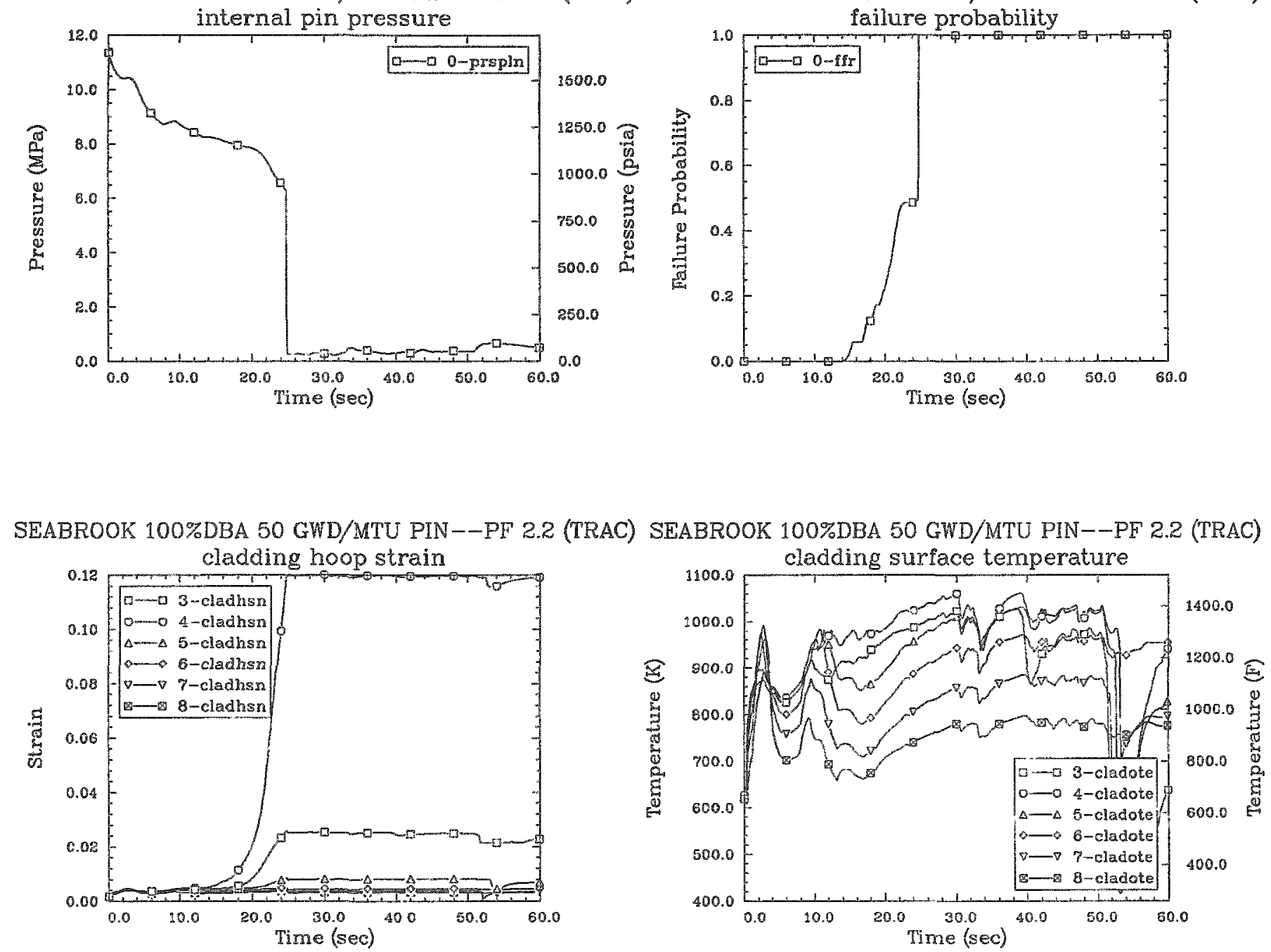

SEABROOK 100\%DBA 50 GWD/MTU PIN--PF 2.2 (TRAC) SFAIBROOK 100\%DBA 50 GWD/MTU PIN--PF 2.2 (TRAC)
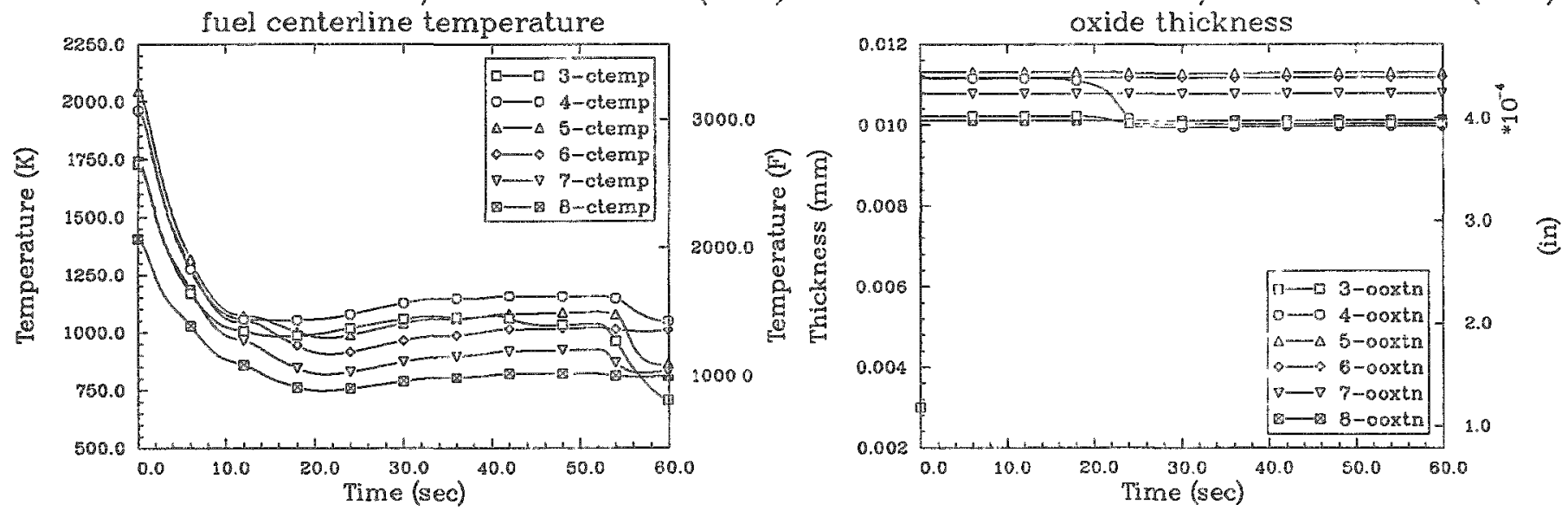
SEABROOK 100\%DBA 35 GWD/MTU PIN--PF 2.2 (TRAC) SEABROOK 100\%DBA 35 GWD/MTU PIN--PF 2.2 (TRAC)
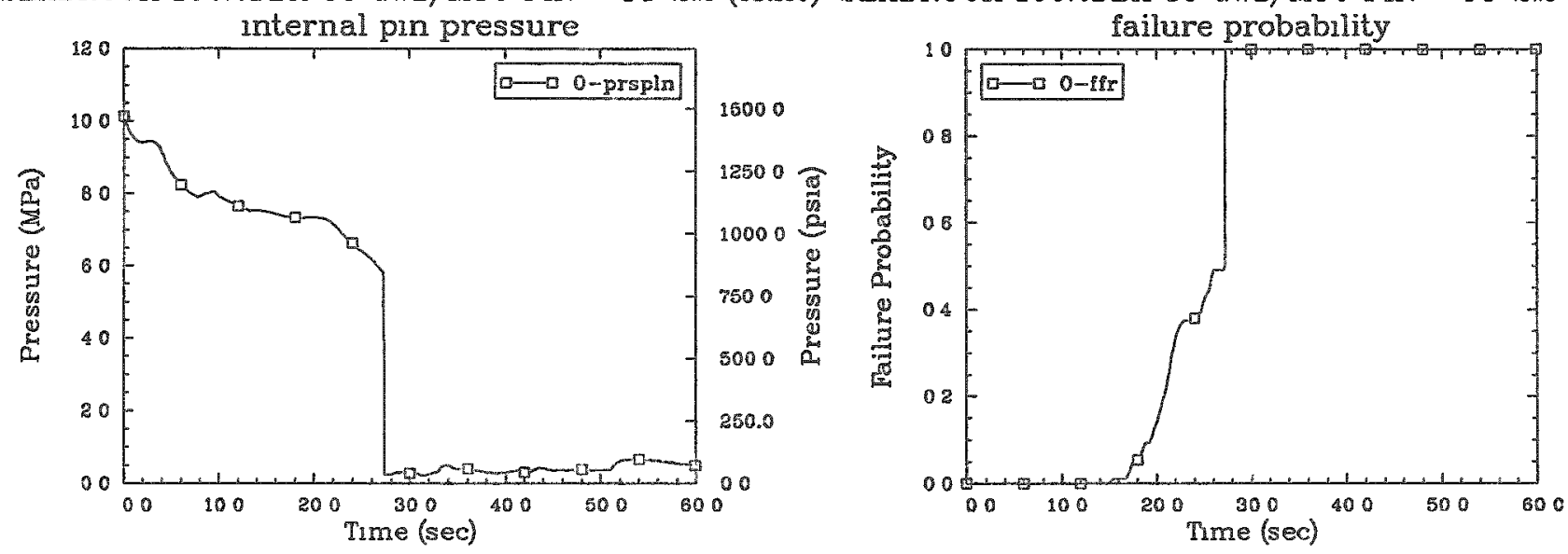

SEABROOK 100\%DBA 35 GWD/MTU PIN--PF 22 (TRAC)

SEABROOK 100\%DBA 35 GWD/MTU PIN--PF 22 (TRAC)
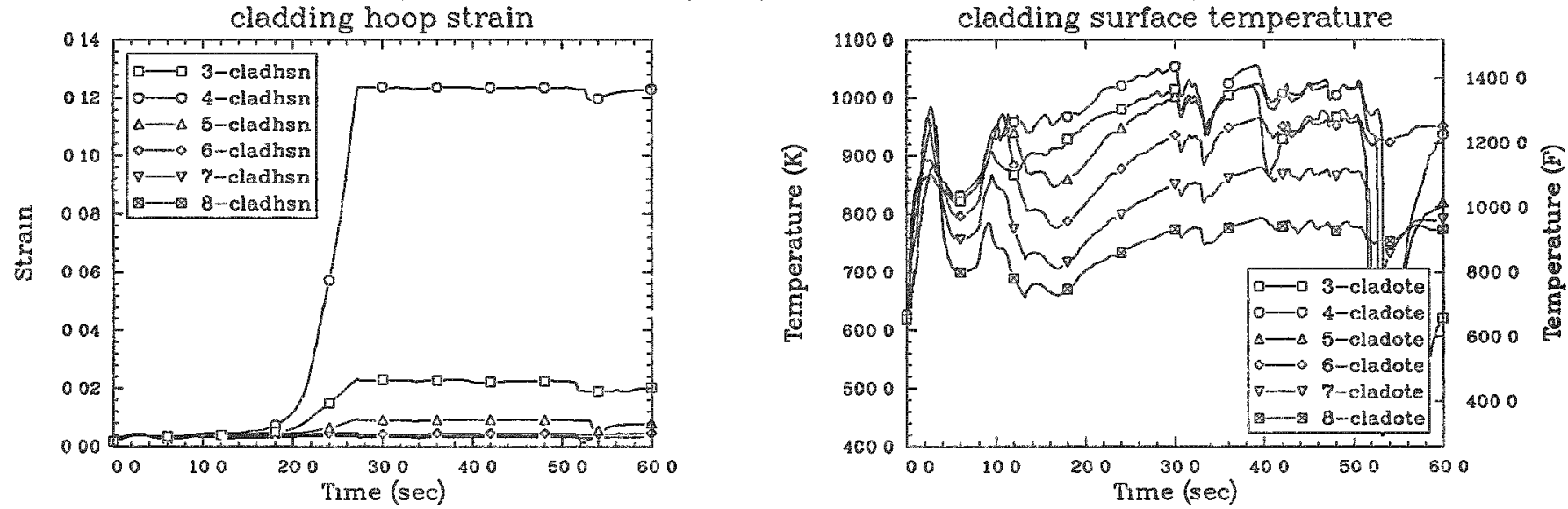

SEABROOK 100\%DBA 35 GWD/MTU PIN--PF 22 (TRAC) SEABROOK 100\%DBA 35 GWD/MTU PIN--PF 22 (TRAC)
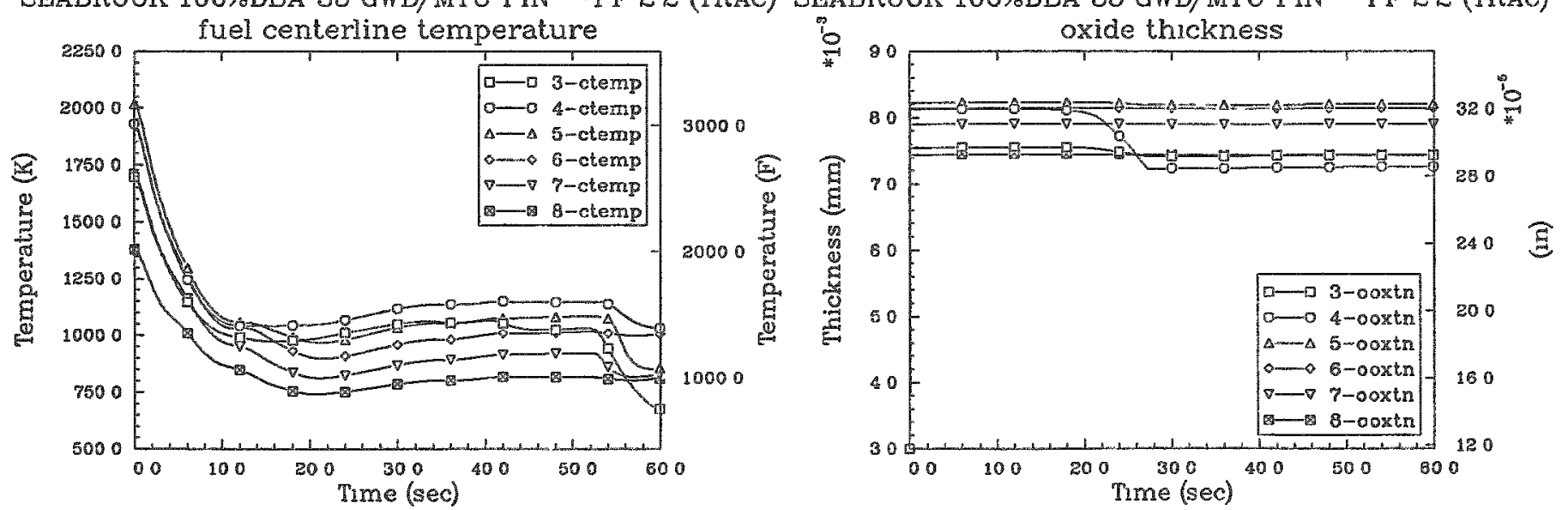
SEABROOK 100\%DBA $20 \mathrm{GWD} / \mathrm{MTU}$ PIN--PF 2.2 (TRAC) SEABROOK 100\%DBA $20 \mathrm{GWD} / \mathrm{MTU}$ PIN--PF 2.2 (TRAC)

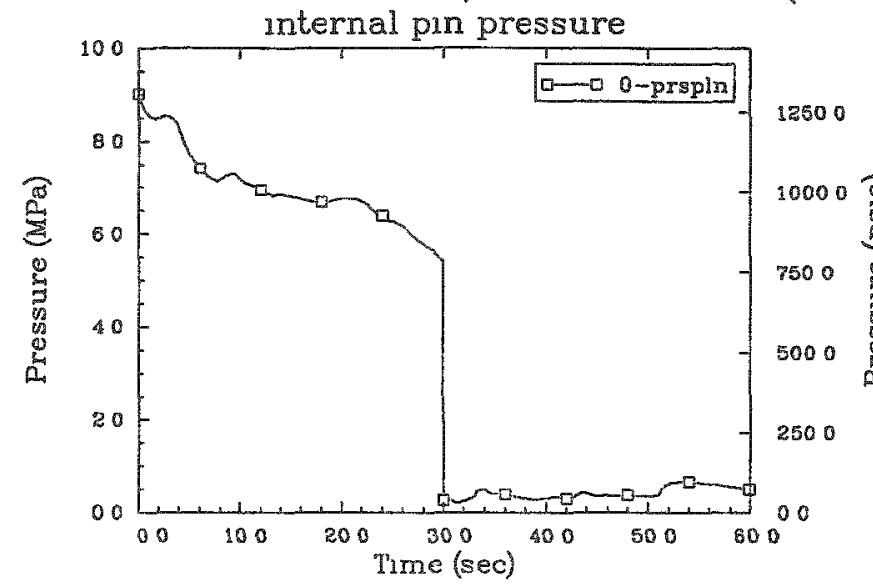

fallure probability

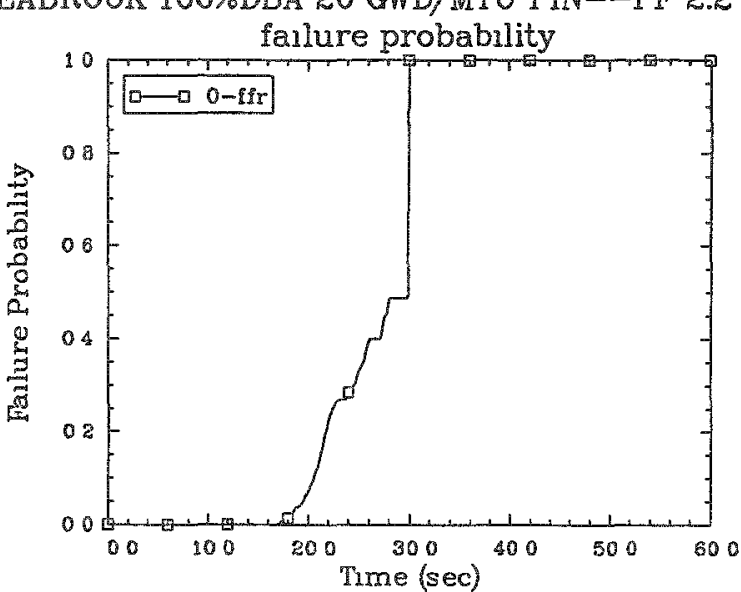

SEABROOK 100\%DBA 20 GWD/MTU PIN--PF 22 (TRAC) SEABROOK 100\%DBA 20 GWD/MTU PIN--PF 22 (TRAC)
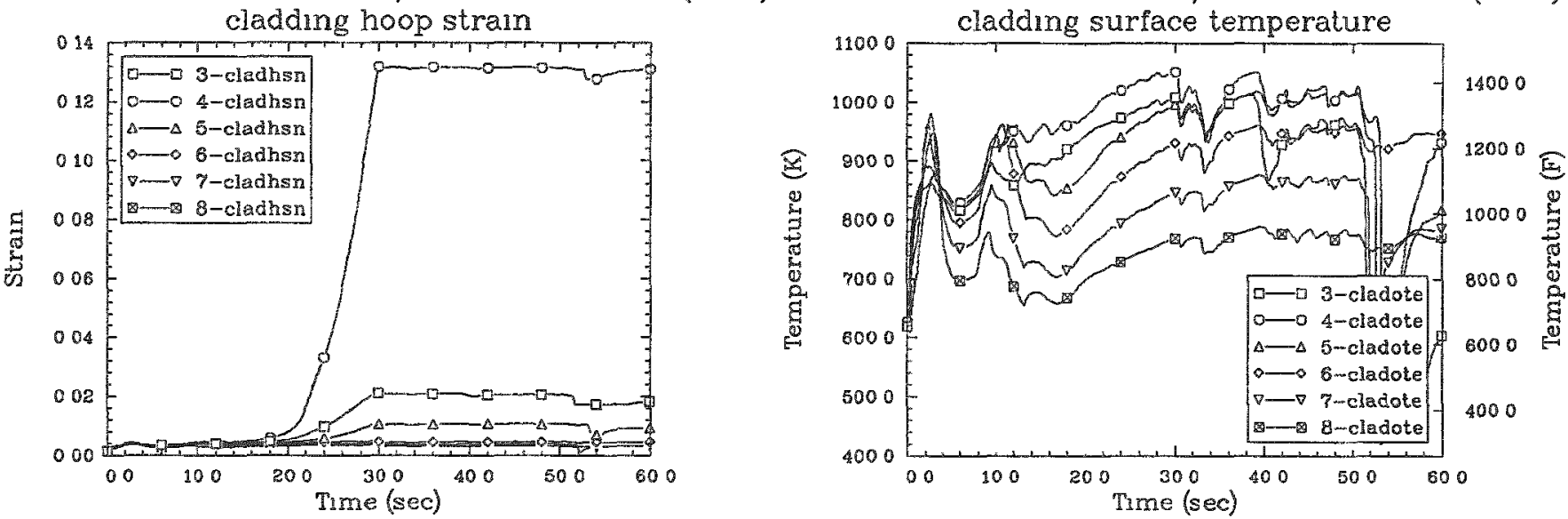

SEABROOK 100\%DBA 20 GWD/MTU PIN--PF 22 (TRAC) SEABROOK 100\%DBA 20 GWD/MTU PIN--PГ 22 (TRAC)
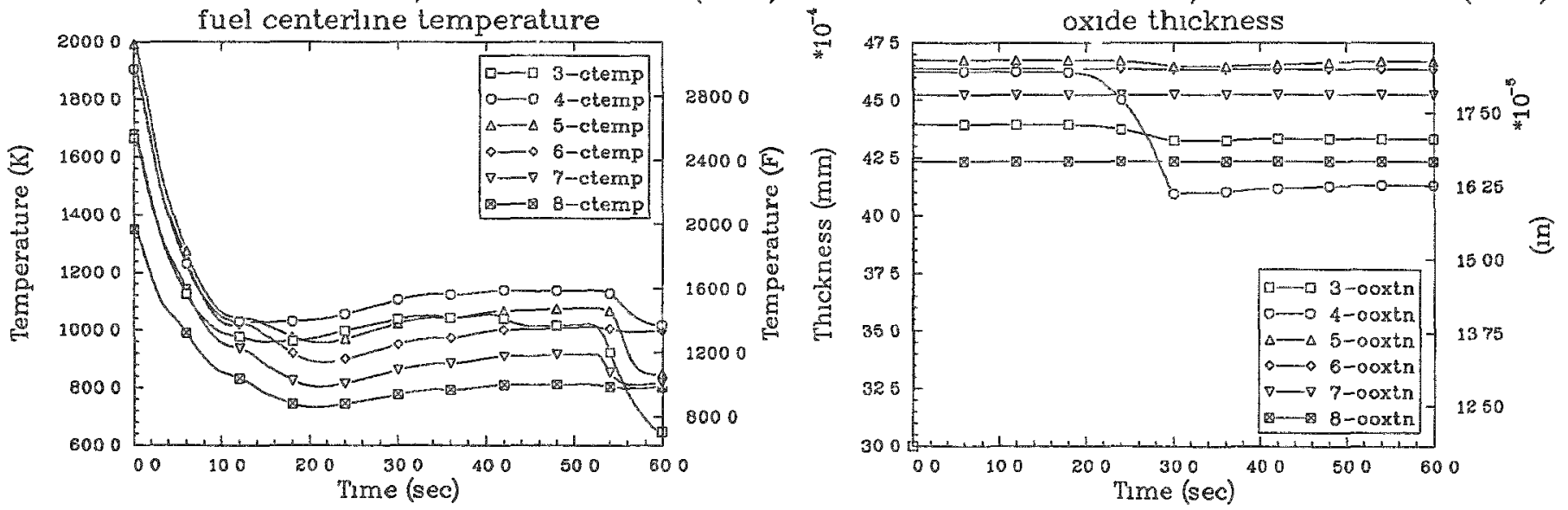
SEABROOK 100\%DBA 5 GWD/MTU PIN--PF 2.2 (TRAC)

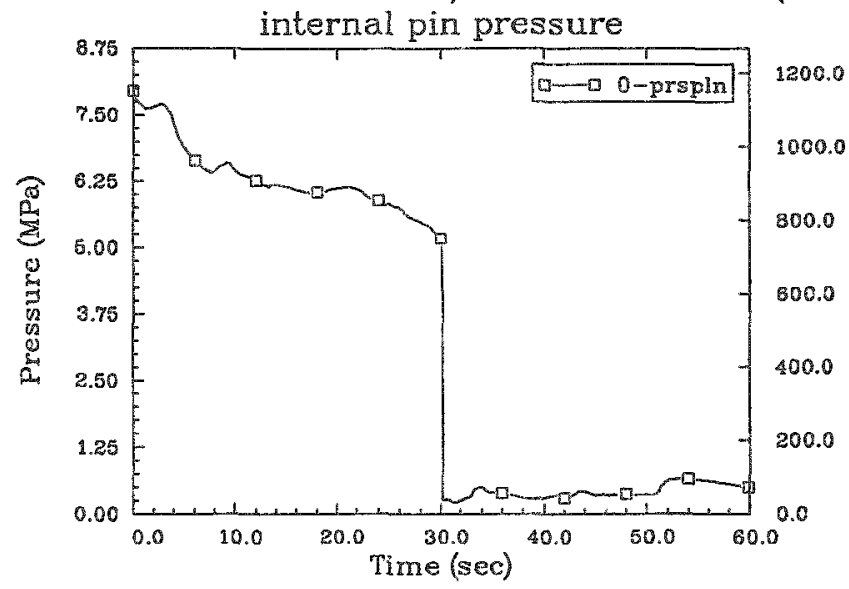

SEABROOK 100\%DBA 5 GWD/MTU PIN--PF 2.2 (TRAC) cladding hoop strain

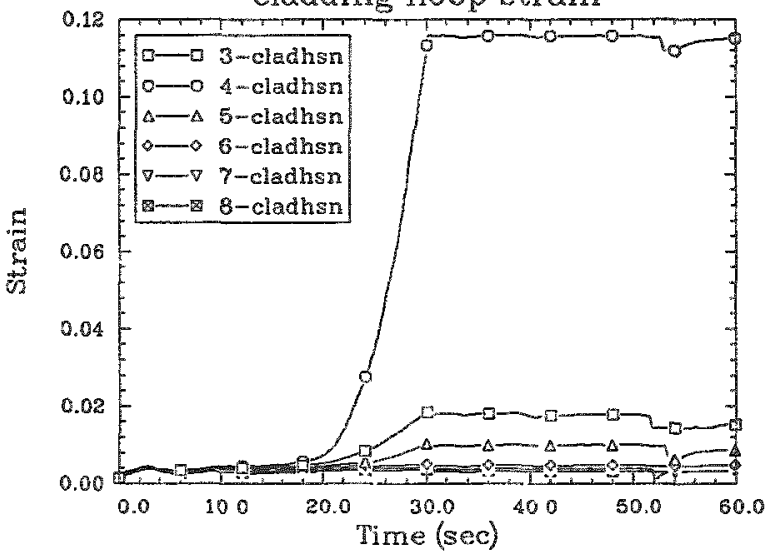

SEABROOK 100\%DBA 5 GWD/MTU PIN--PF 2.2 (TRAC)

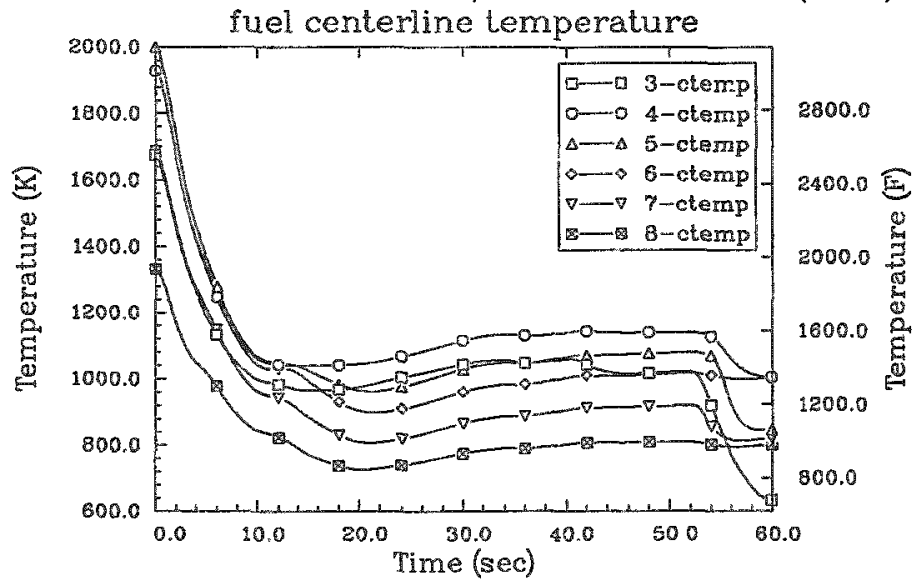

SEABROOK 100\%DBA 5 GWD/MTU PIN--PF 2.2 (TRAC)

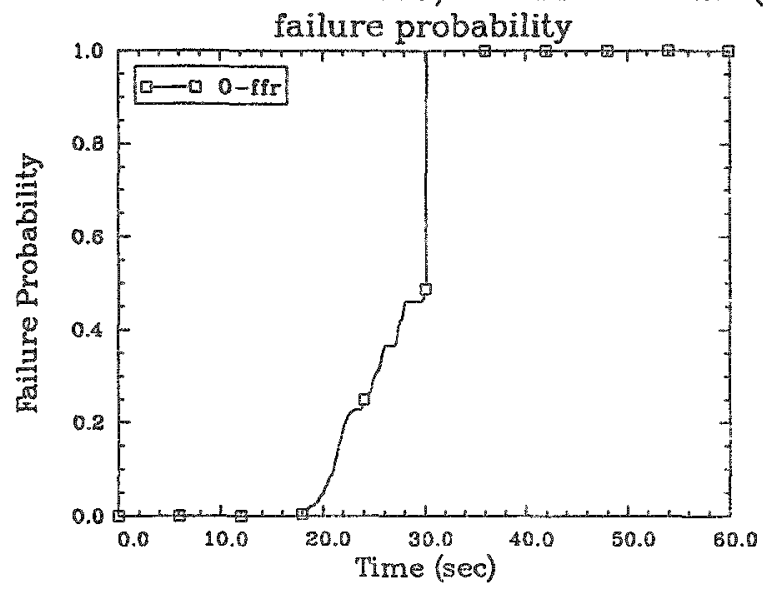

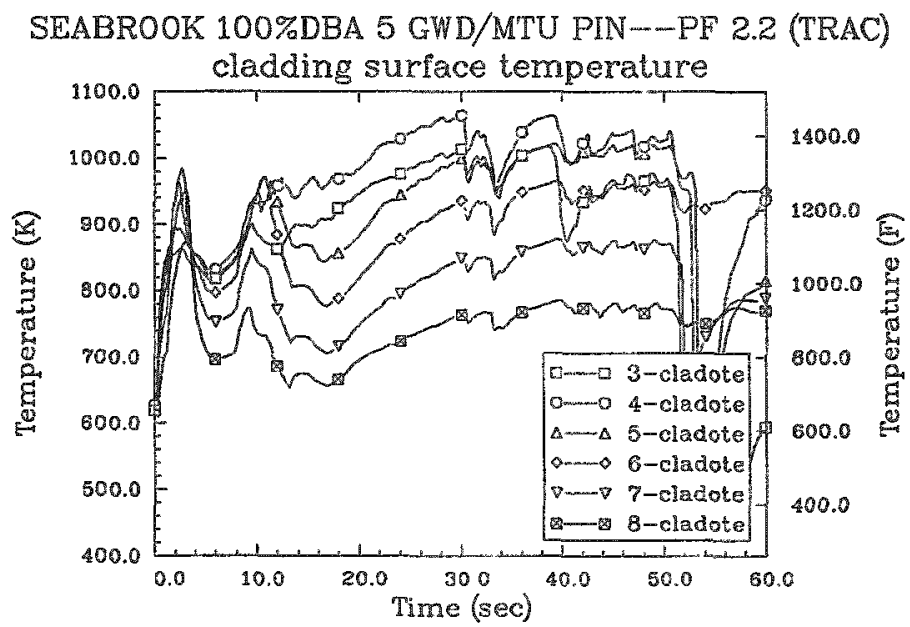

SEABROOK 100\%DBA 5 GWD/MTU PIN-PF 2.2 (TRAC)

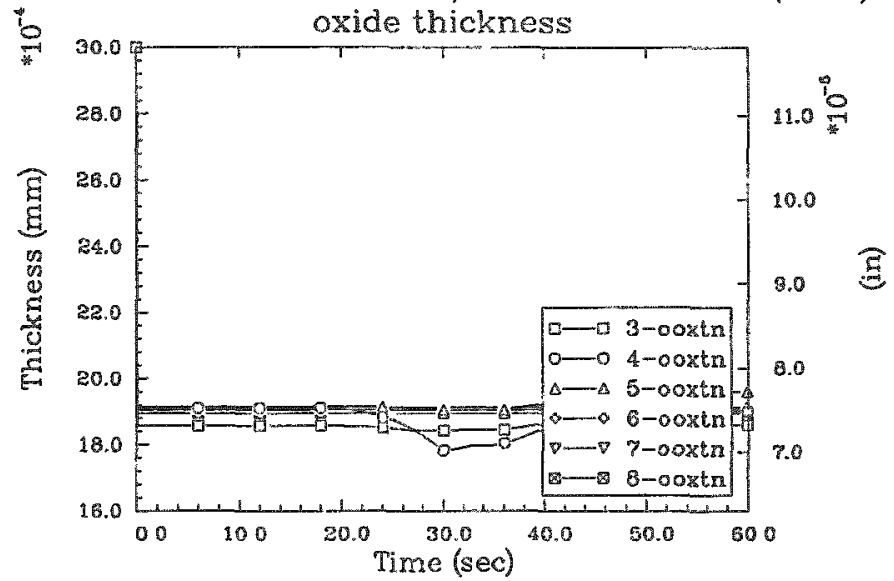


SEABROOK 100\%DBA 50 GWD/MTU PIN--PF 2.0 (TRAC) SEABROOK 100\%DBA 50 GWD/MTU PIN--PF 2.0 (TRAC)
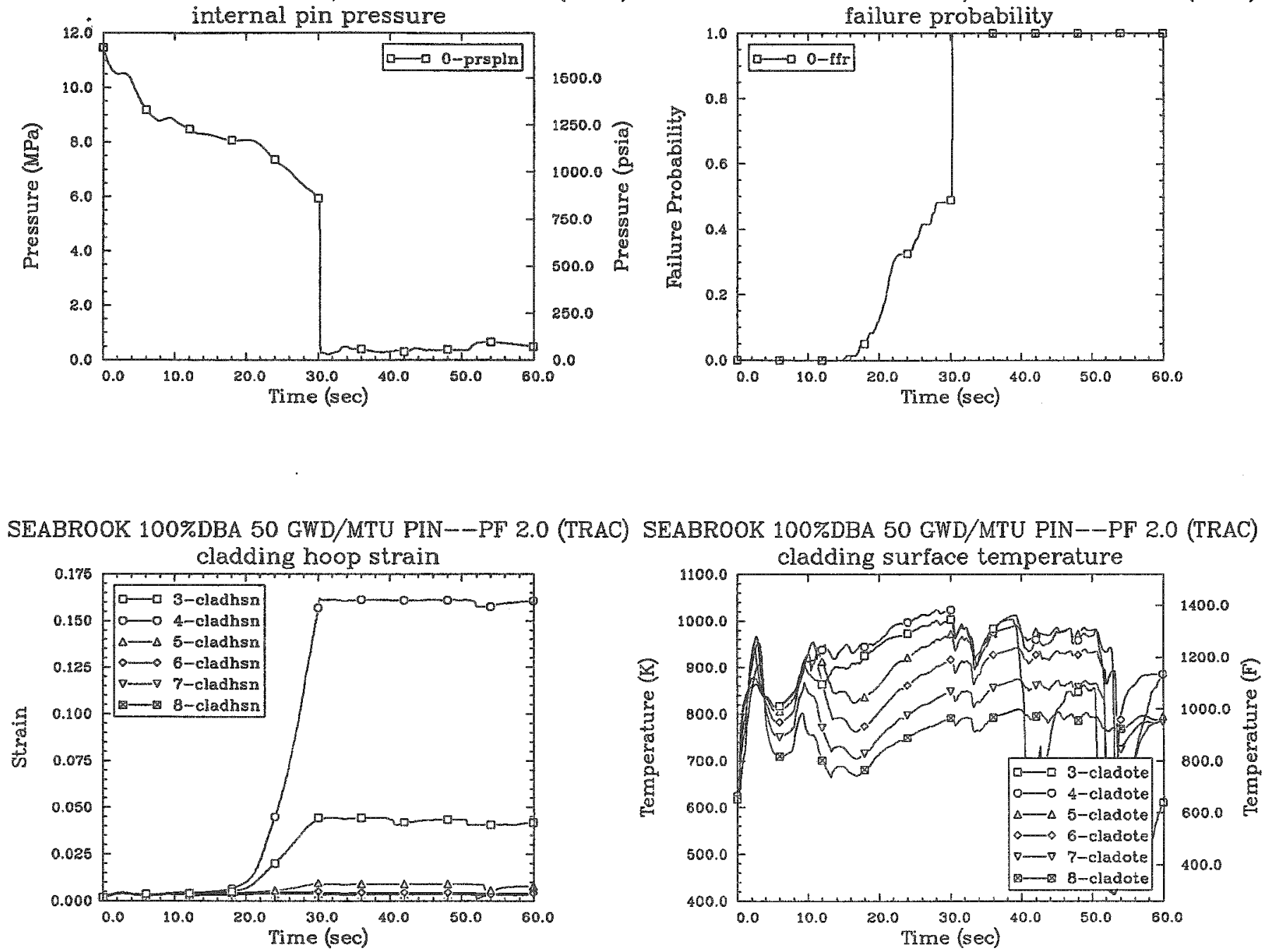

SEABROOK 100\%DBA 50 GWD/MTU PIN--PF 2.0 (TRAC) SEABROOK 100\%DBA 50 GWD/MTU PIN--PF 2.0 (TRAC)
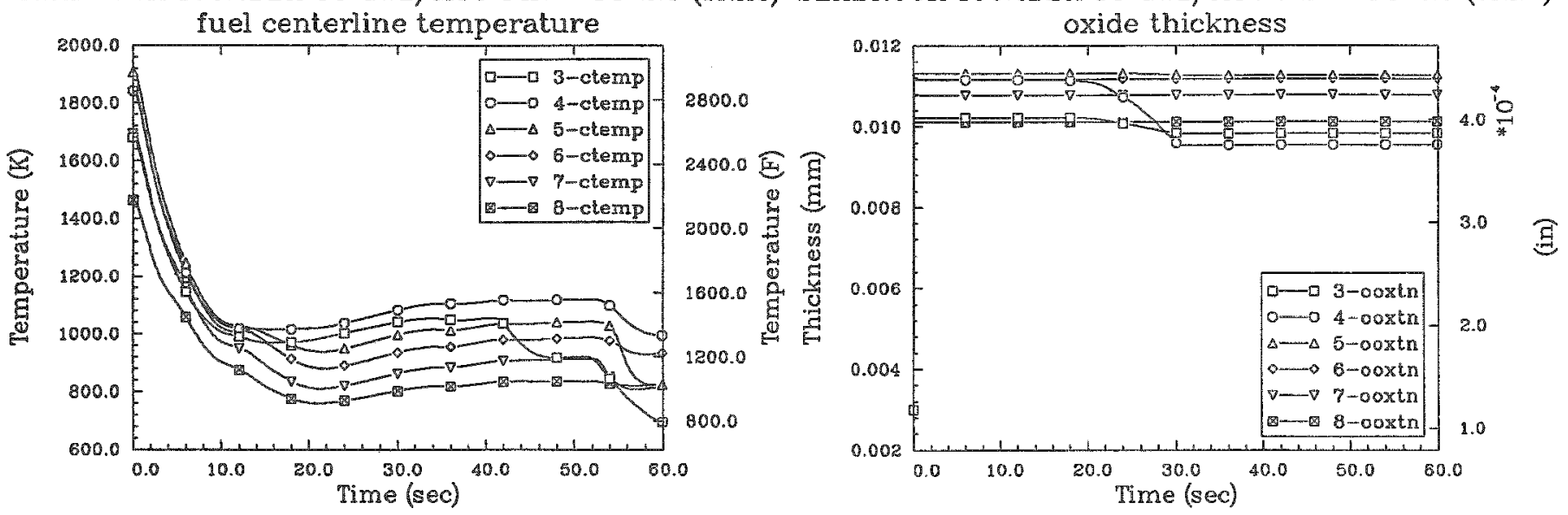
SEABROOK 100\%DBA 35 GWD/MTU PIN--PF 2.0 (TRAC) SEABROOK 100\%DBA 35 GWD/MTU PIN--PF 2.0 (TRAC)
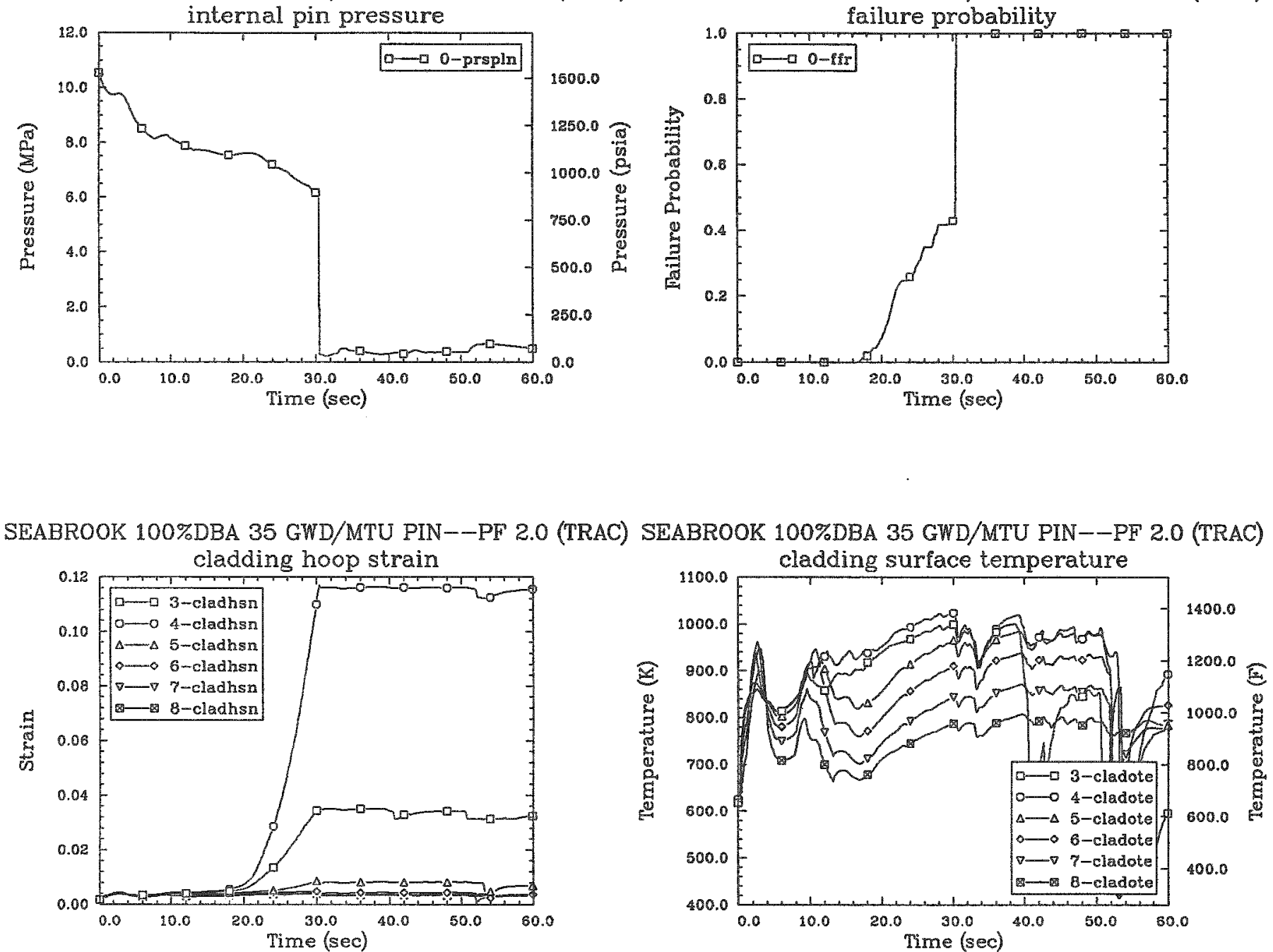

SEABROOK 100\%DBA 35 GWD/MTU PIN--PF 2.0 (TRAC) SEABROOK 100\%DBA 35 GWD/MTU PIN--PF 2.0 (TRAC)

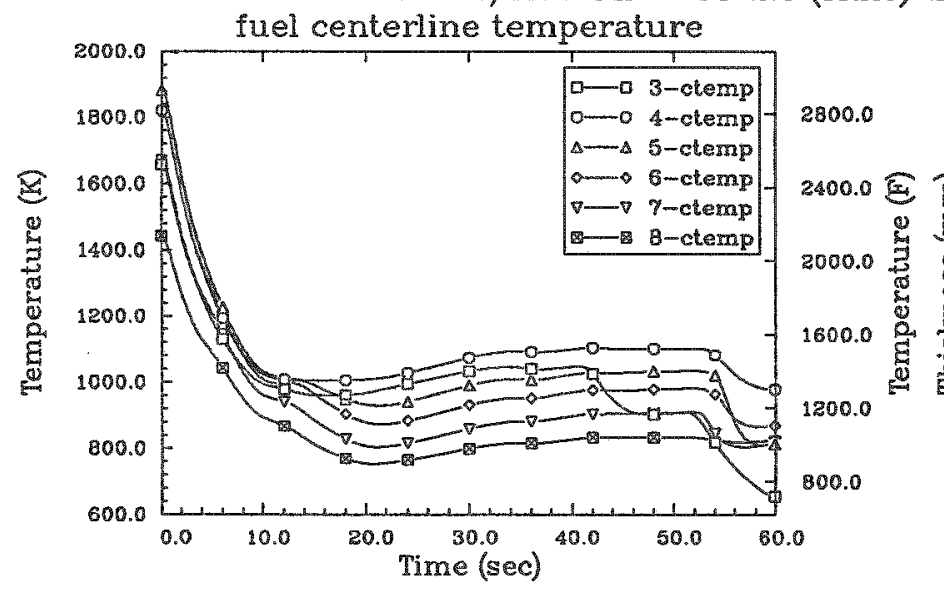
oxide thickness

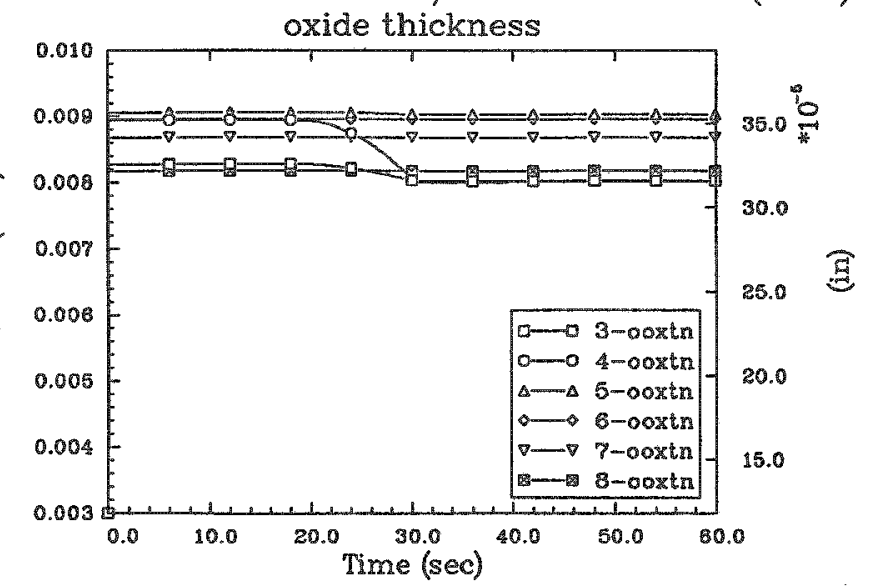


SEABROOK 100\%DBA 20 GWD/MTU PIN--PF 20 (TRAC) SEABROOK 100\%DBA 20 GWD/MTU PIN--PF 2.0 (TRAC)
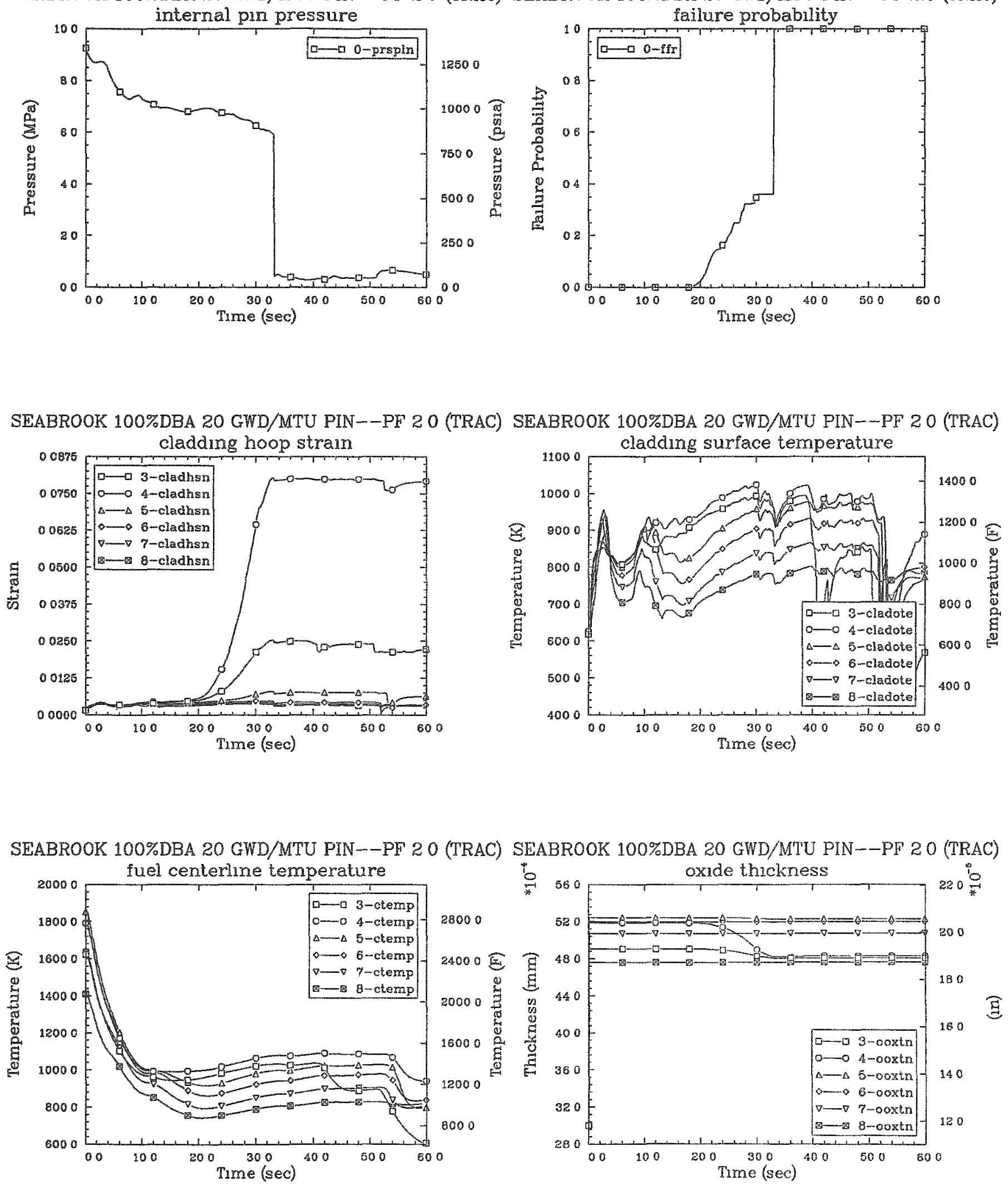
SEABROOK 100\%DBA 5 GWD/MTU PIN--PF 2.0 (TRAC)

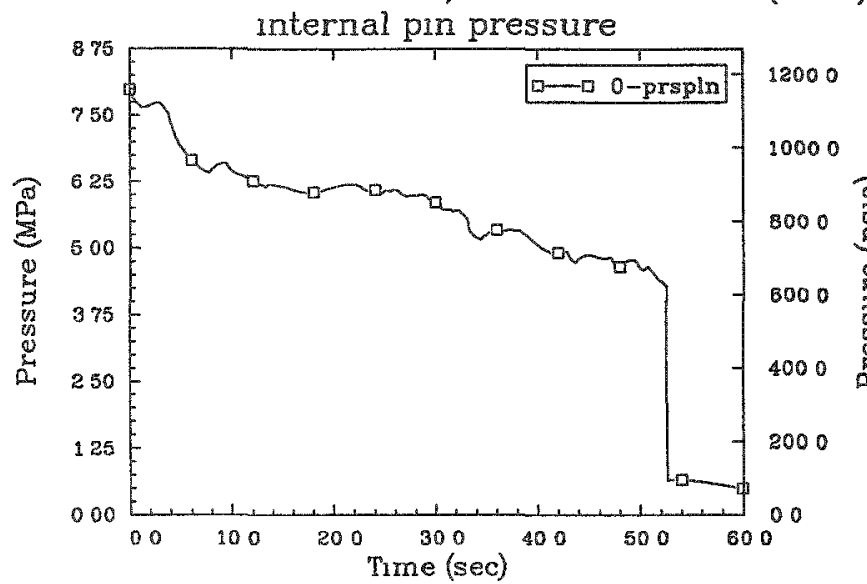

SEABROOK 100\%DBA 5 GWD/MTU PIN--PF 20 (TRAC)

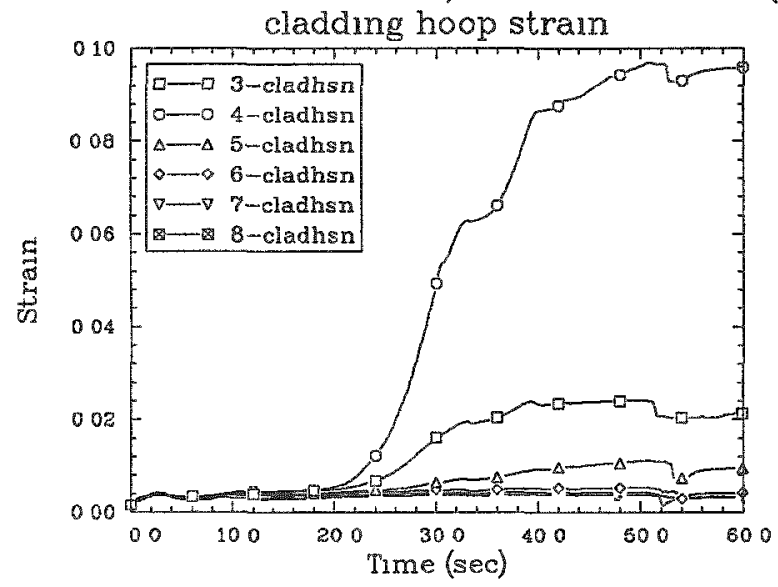

SEABROOK 100\%DBA 5 GWD/MTU PIN--PF 20 (TRAC)

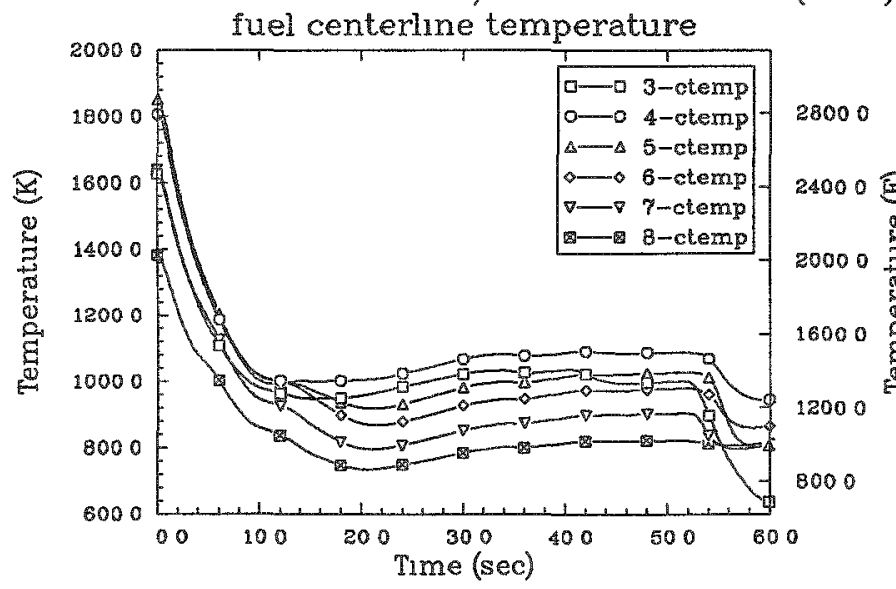

SEABROOK 100\%DBA 5 GWD/MTU PIN--PF 20 (TRAC)

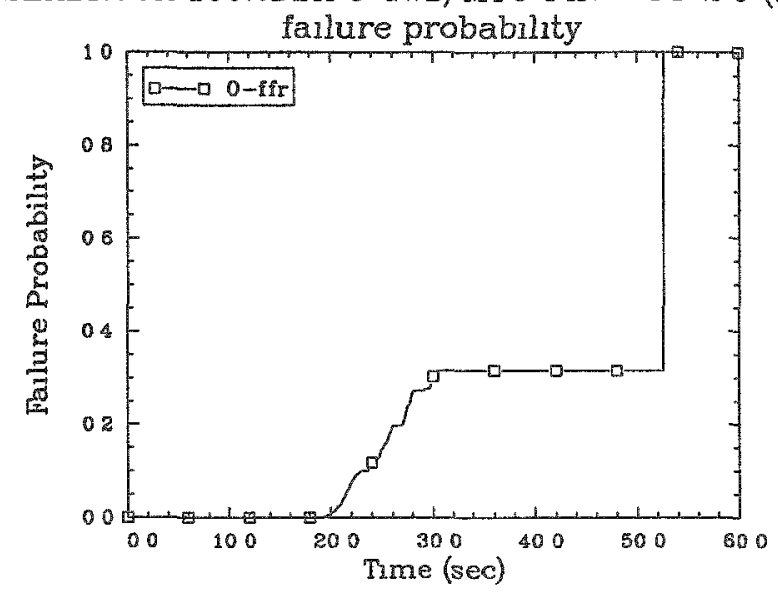

SEABROOK 100\%DBA 5 GWD/MTU PIN--PF 20 (TRAC)

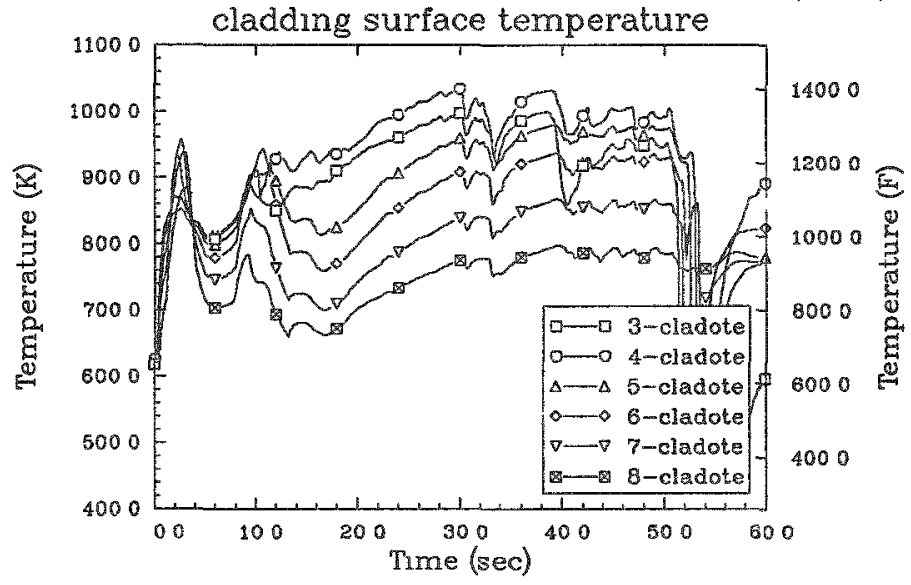

SEABROOK 100\%DBA 5 GWD/MTU PIN--PF 20 (TRAC)

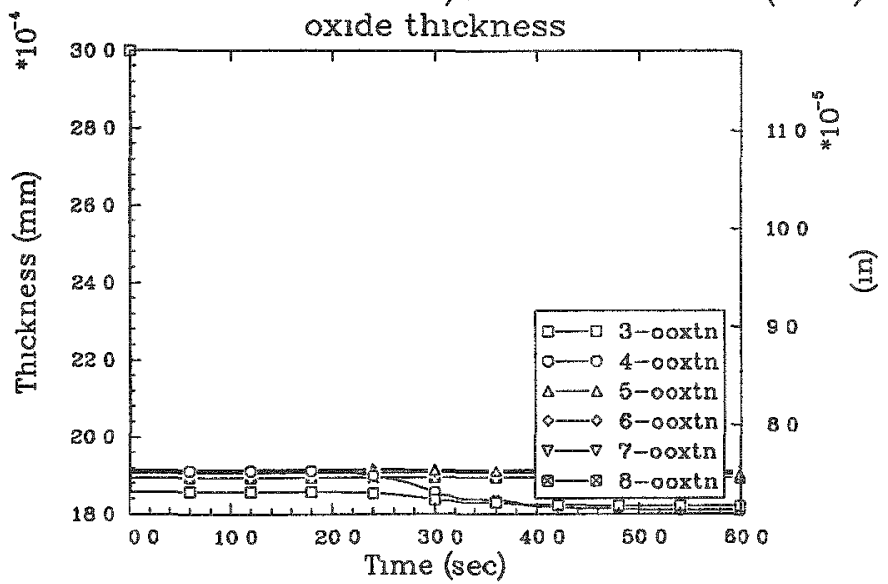


SEABROOK 100\%DBA 50 GWD/MTU PIN--PF 1.8 (TRAC) SEABROOK 100\%DBA 50 GWD/MTU PIN--PF 1.8 (TRAC)
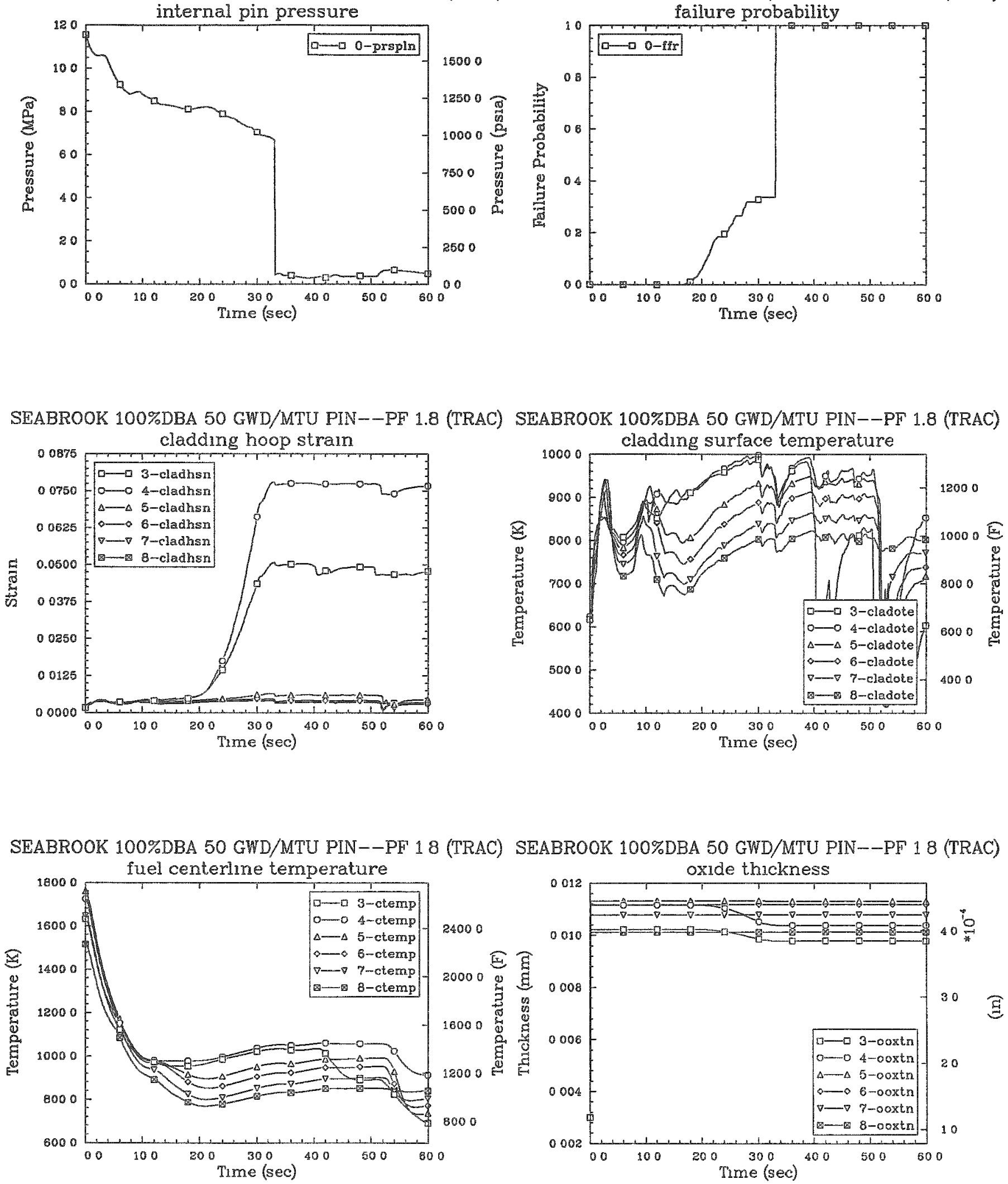
SEABROOK 100\%DBA 35 GWD/MTU PIN--PF 1.8 (TRAC) SEABROOK 100\%DBA 35 GWD/MTU PIN--PF 1.8 (TRAC)
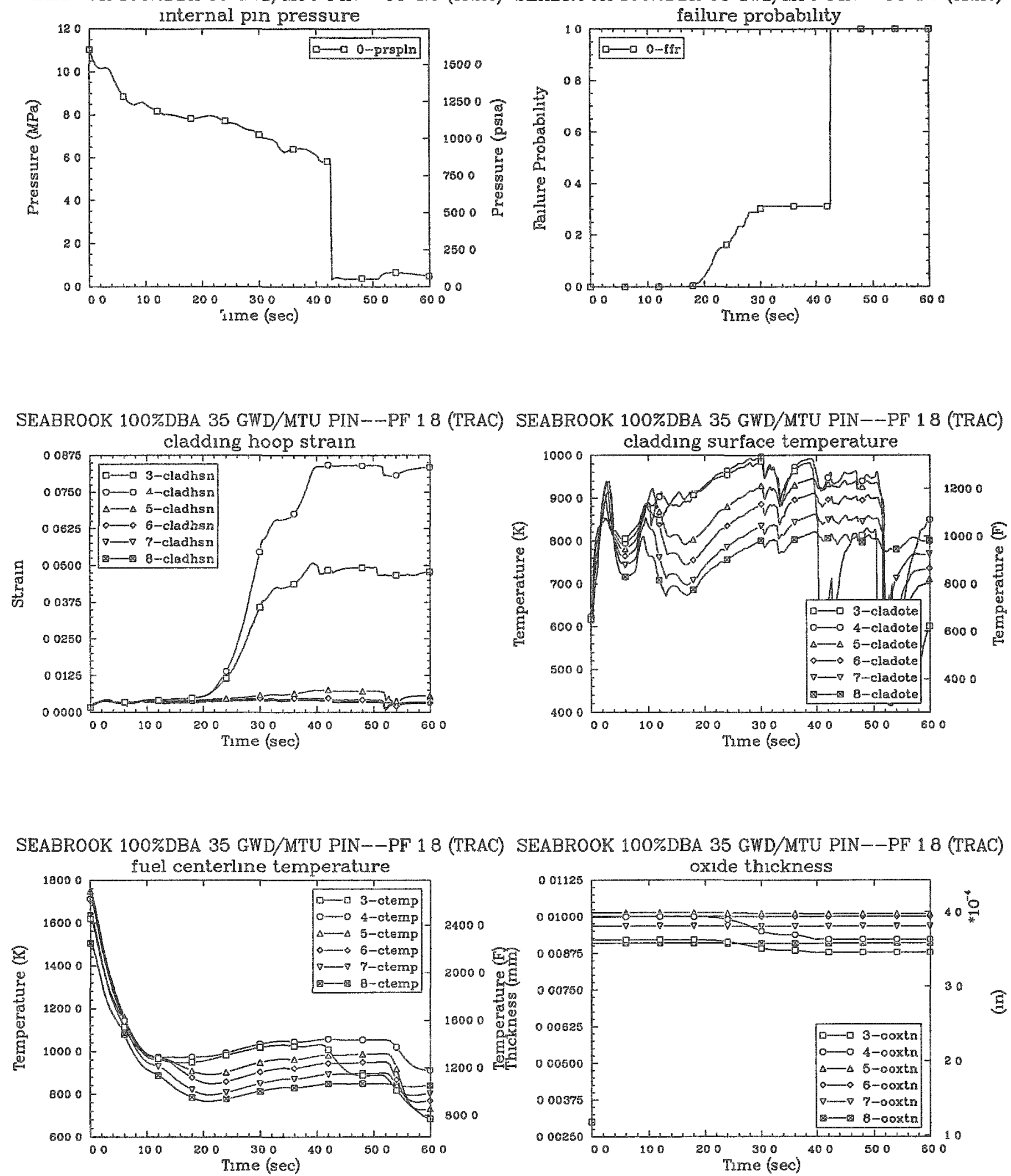

SEABROOK 100\%DBA 35 GWD/MTU PIN--PF 18 (TRAC)

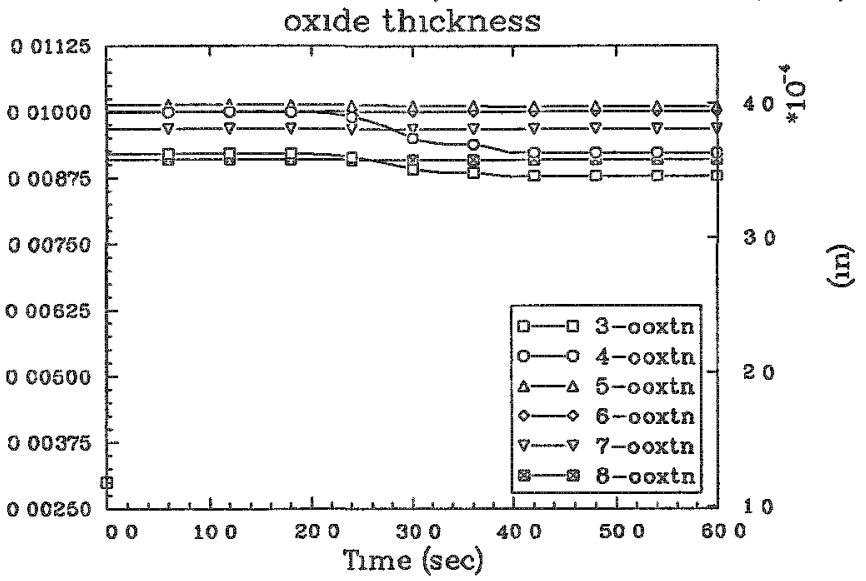



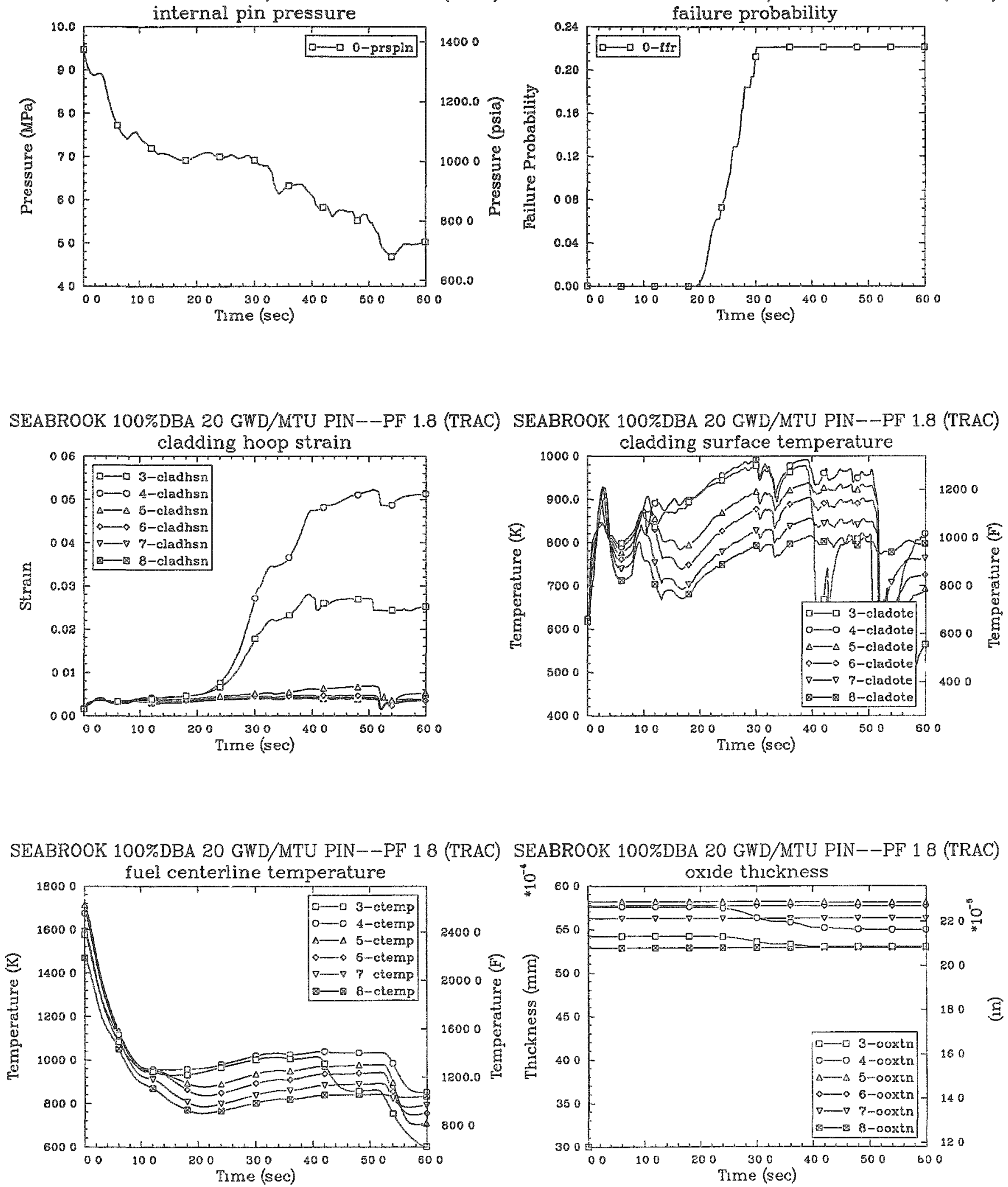
SEABROOK 100\%DBA 5 GWD/MTU PIN--PF 18 (TRAC)

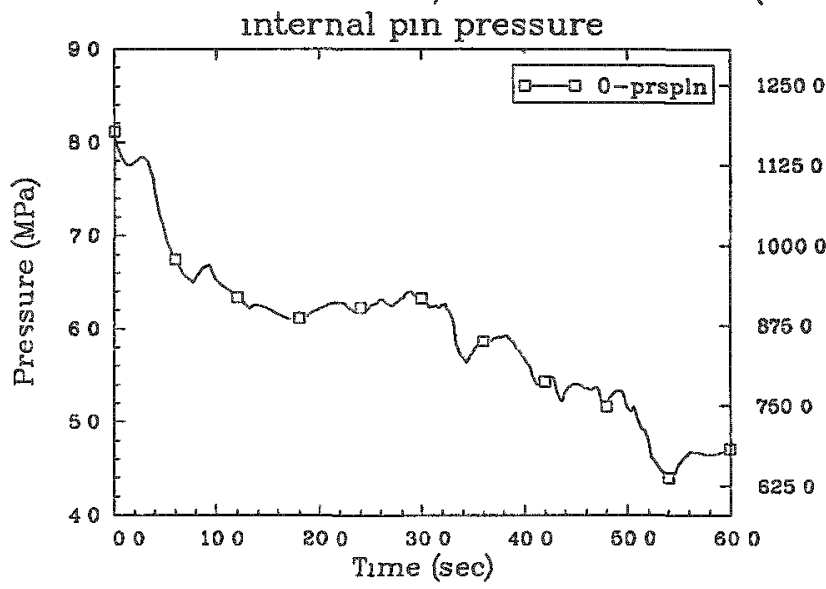

SEABROOK 100\%DBA 5 GWD/MTU PIN--PF 18 (TRAC) cladding hoop strain

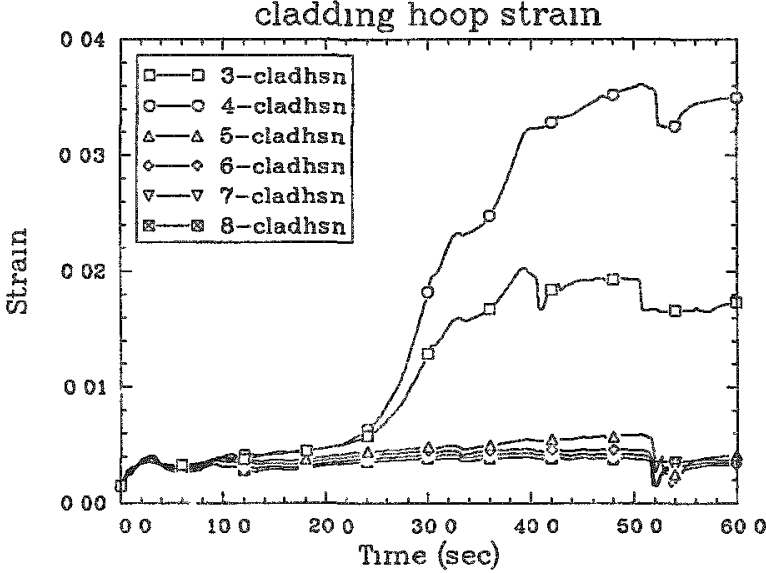

SEABROOK 100\%DBA 5 GWD/MTU PIN--PF 18 (TRAC) fallure probabilnty

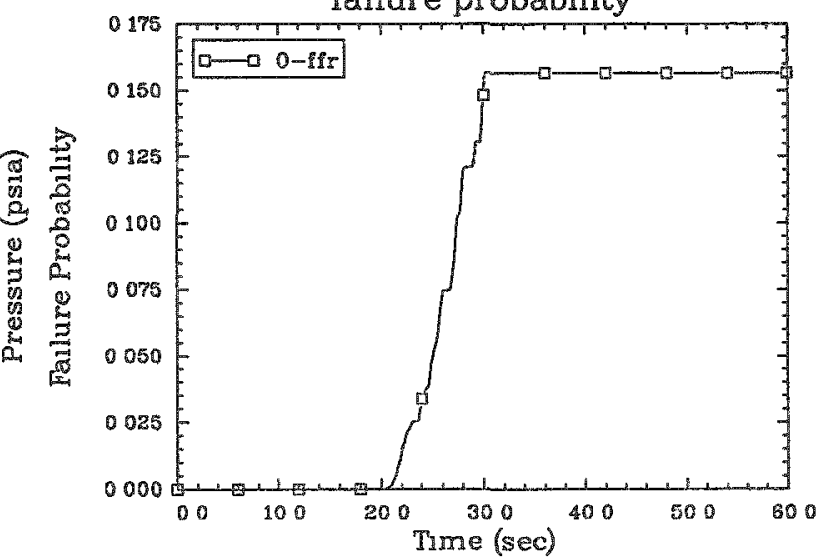

SEABROOK 100\%DBA 5 GWD/MTU PIN--PF 18 (TRAC)

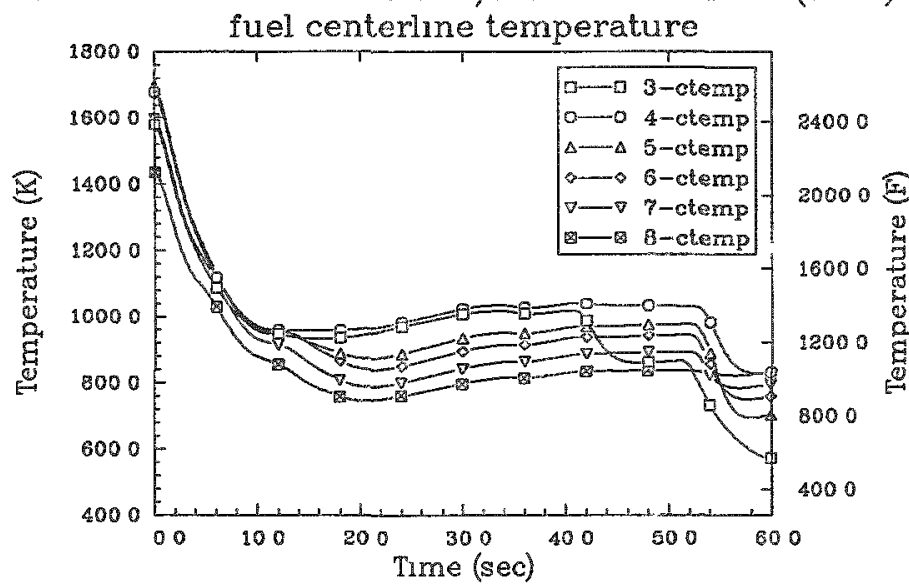

SEABROOK 100\%DBA 5 GWD/MTU PIN--PF 18 (TRAC)

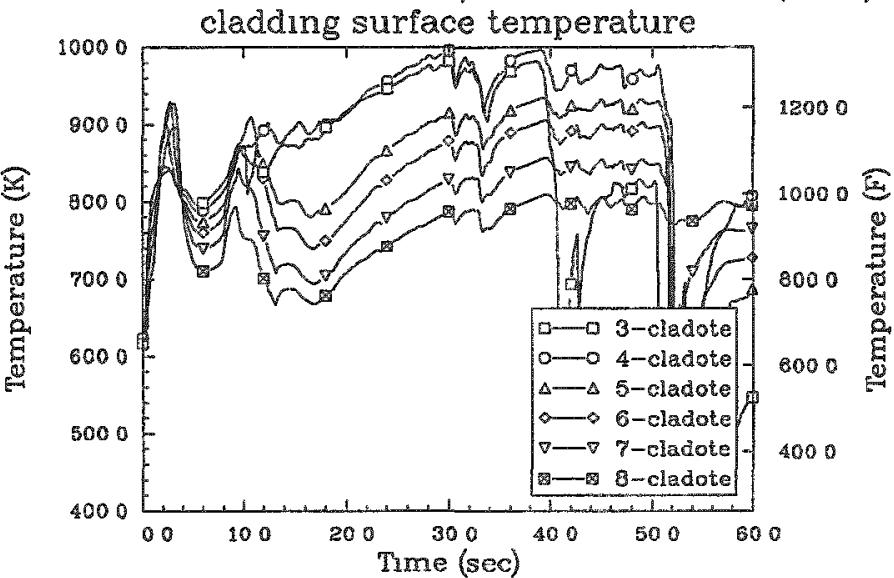

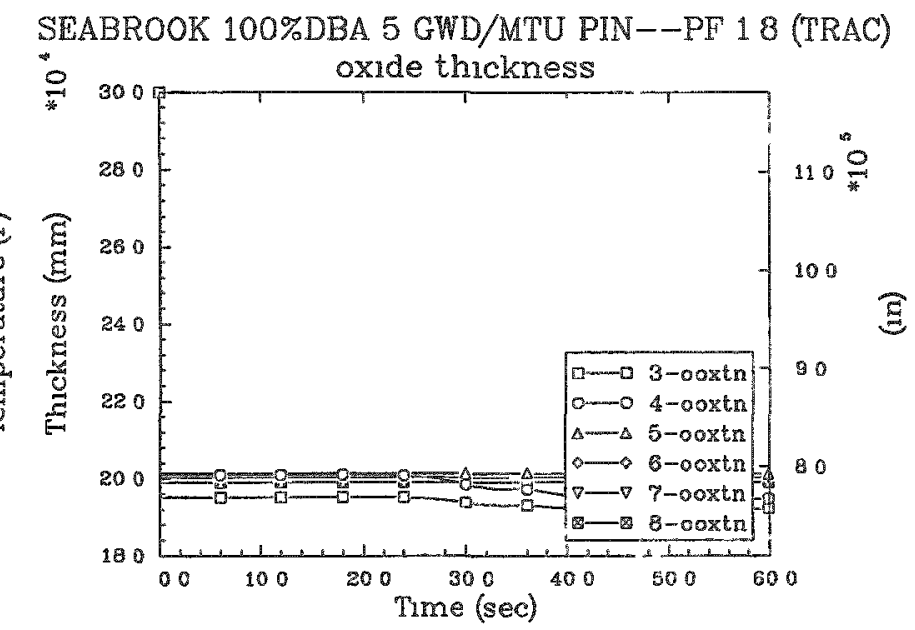




\section{L-3. SCDAP/RELAP5/MOD3 AND TRAC-PF1/MODI PLOTTED RESULTS FOR SEABROOK}

Table L-3. Description of SCDAP/RELAP5/MOD3 and TRAC-PF1/MODI plot variables for Seabrook.

Variable

Description

SCDAP/RELAP5/MOD3 Variables:

0-rktpow

0-rkripow

0-rkgapow

400-cntrivar

403-cntrlvar

128010000-p

$620010000-p$

410-crerlvar

$704010000-$ mflowj

702010000-mf T owj

702 -acvlig

200010000-mf l owj

253010000-mflowj

155010000 -mf 7 owj

1060n0000-voidg

Total core thermal power (W)

Total core fission power (W)

Total core decay heat (W)

Hot channel collapsed reactor water level (m)

Core-average collapsed reactor water level (m)

Reactor upper head pressure $(\mathrm{Pa})$

Pressurizer dome pressure ( $\mathrm{Pa}$ )

Total break flow $(\mathrm{kg} / \mathrm{s})$

Accumulator flow for the broken $100 \mathrm{p}(\mathrm{kg} / \mathrm{s})$

Total accumulator flow for the intact loop $(\mathrm{kg} / \mathrm{s})$

Accumulator liquid volume for the intact loop $\left(\mathrm{m}^{3}\right)$

Total hot leg flow for the intact loop $(\mathrm{kg} / \mathrm{s})$

Total cold leg flow for the intact loop $(\mathrm{kg} / \mathrm{s})$

Hot channel flows at the core midplane $(\mathrm{kg} / \mathrm{s})$

Broken loop downcomer void fraction for node $n$ at the core midplane elevation

1860 n0000-voidg Intact loop downcomer void fraction for node $n$ at the core midplane elevation

$0-d t$

Time step size (s)

TRAC-PFI/MODI Variables:

RPOWER0990001 Total core thermal power (W)

CORELEVEL

PUP0990001

P078001

MFLOWTOTBRK

MFLOW0440002

MFLOWTOTINTAC

ACQLIQTOTINT

MFLOWINTHLEG

MFLOWINTCLEG

MFLOWTOT990801

ALPHA0990814

ALPHA0990813

DELTO000001

Core-average collapsed reactor water level (m)

Reactor upper head pressure $(\mathrm{Pa})$

Pressurizer dome pressure $(\mathrm{PQ})$

Total break flow $(\mathrm{kg} / \mathrm{s})$

Accumulator flow for the broken loop $(\mathrm{kg} / \mathrm{s})$

Total accumulator flow for the intact loop $(\mathrm{kg} / \mathrm{s})$

Accumulator liquid volume for the intact loop $\left(\mathrm{m}^{3}\right)$

Total hot leg flow for the intact loop $(\mathrm{kg} / \mathrm{s})$

Total cold leg flow for the intact $100 p(\mathrm{~kg} / \mathrm{s})$

Hot channel flows at the core midplane $(\mathrm{kg} / \mathrm{s})$

Broken loop downcomer void fraction for node $n$ at the core midplane elevation

Intact loop downcomer void fraction for node $n$ at the core midplane elevation

Time step size ( $s$ ) 
SEABROOK 100\% DBA, TRAC-PF1 VS. RELAP5/MOD3

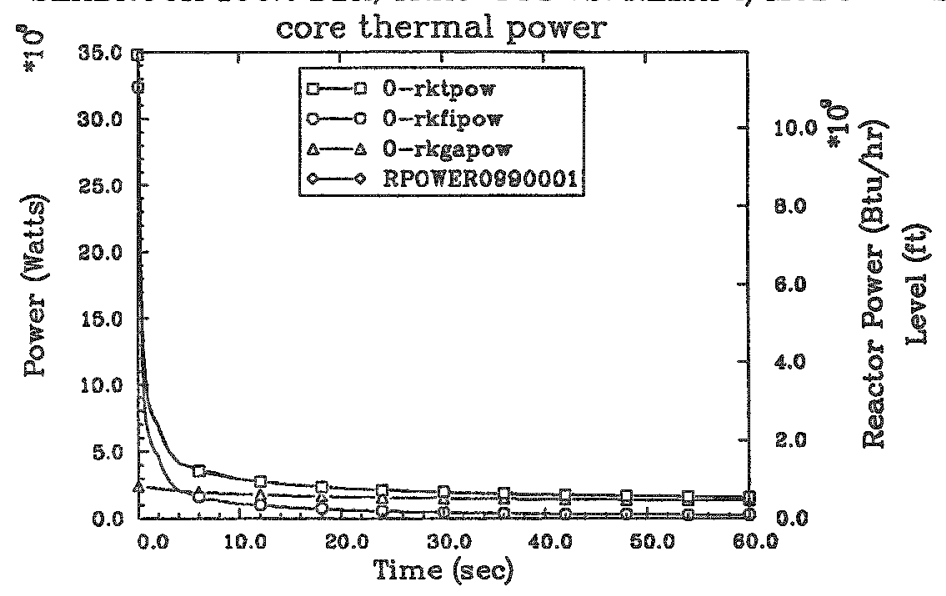

SEABROOK 100\% DBA, TRAC-PF1 VS. RELAP5/MOD3 collapsed reactor water level

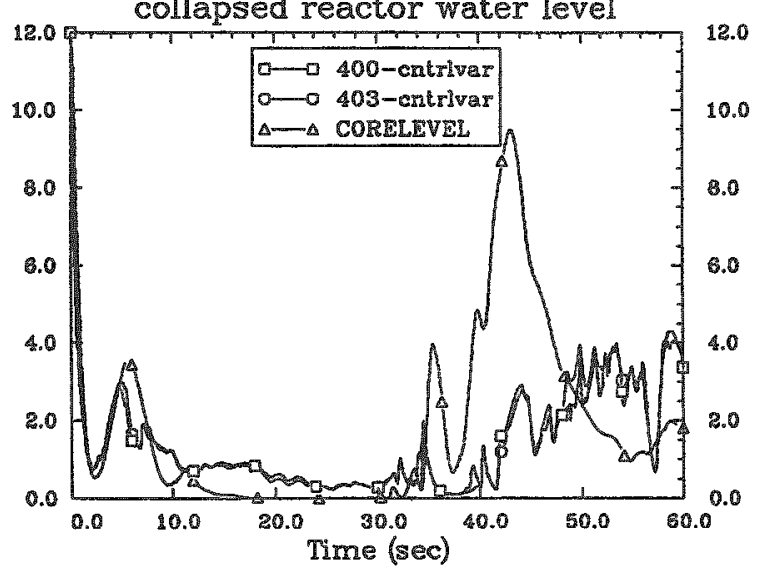

SEABROOK 100\% DBA, TRAC-PF1 VS. RELAP5/MOD3

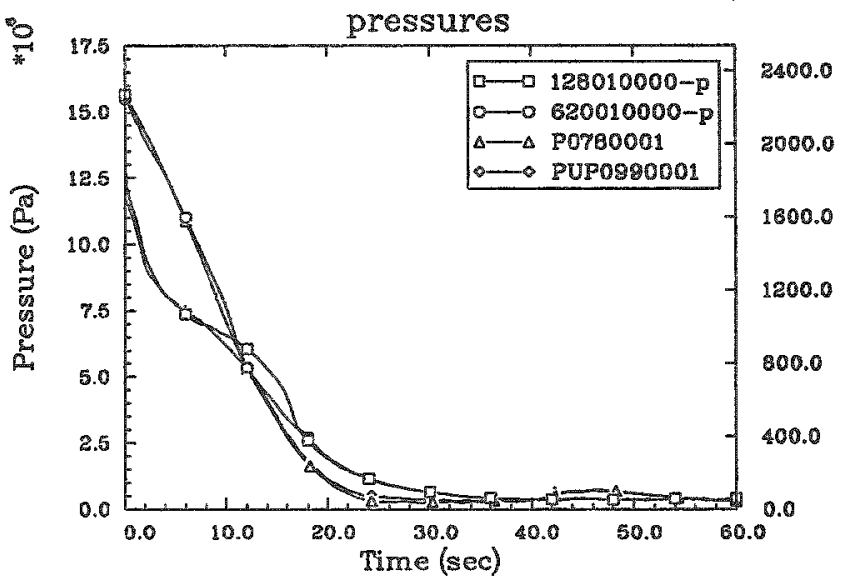

SEABROOK 100\% DBA, TRAC-PF1 VS. RELAP5/MOD3

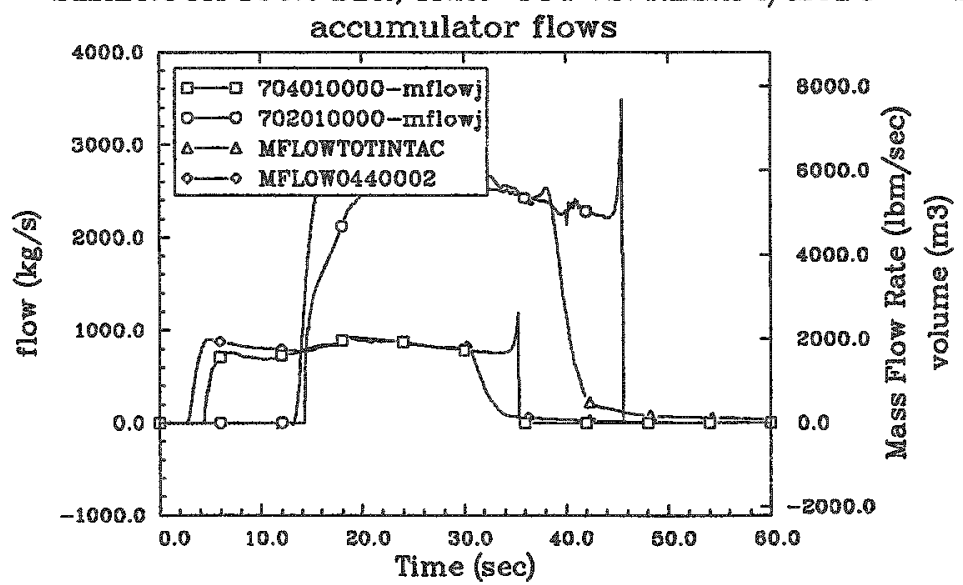

SEABROOK $100 \%$ DBA, TRAC-PF1 VS. RELAP5/MOD3

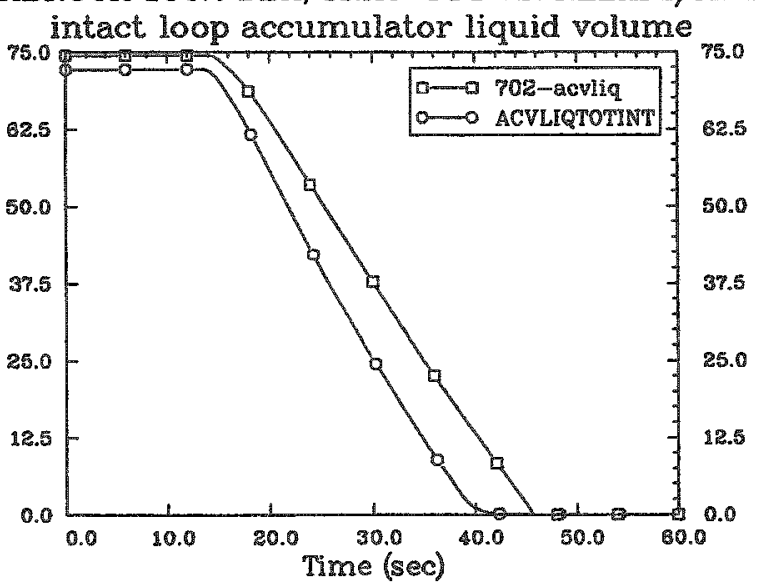


SEABROOK 100\% DBA, TBAC-PF1 VS. RELAP5/MOD3

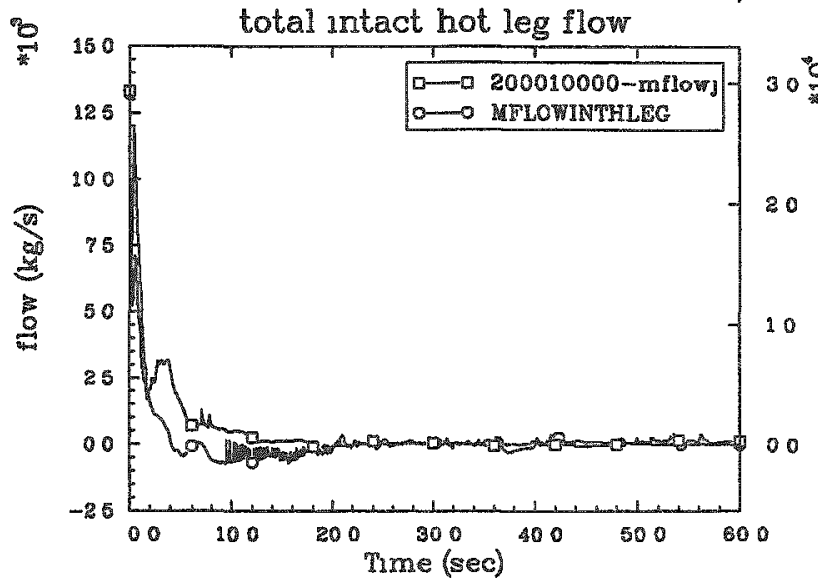

SEABROOK 100\% DBA, TRAC-PF1 VS. RELAP5/MOD3 hot channel core flow

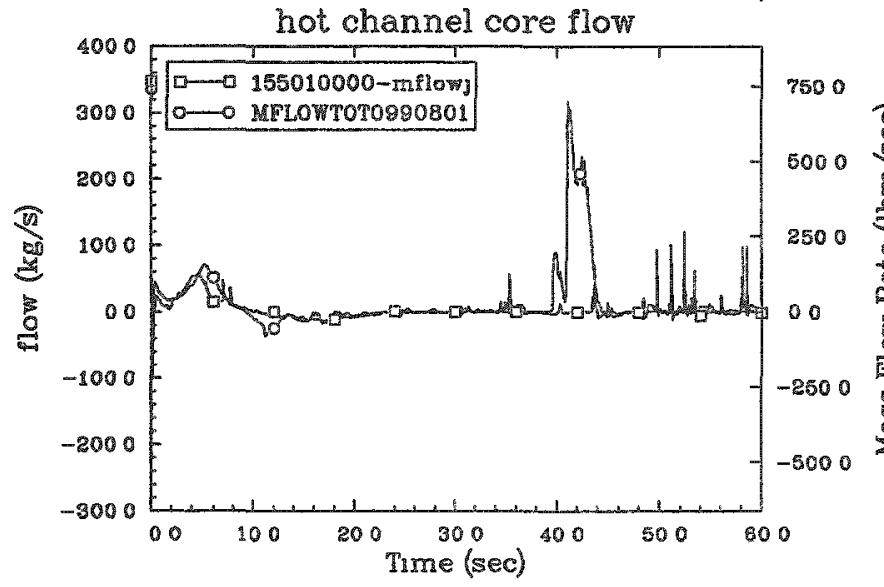

SEABROOK 100\% DBA, TRAC-PF1 VS RELAP5/MOD3

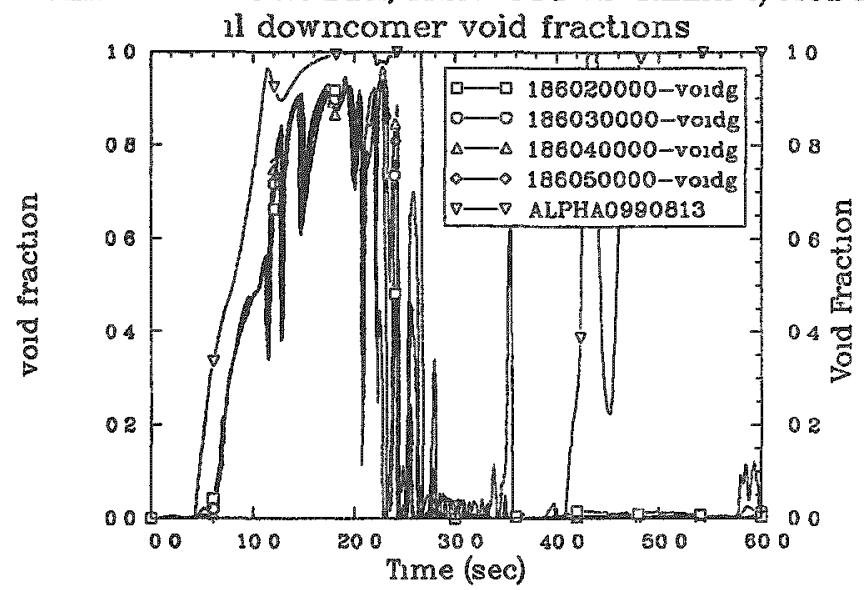

SEABROOK 100\% DBA, TRAC-PF1 VS. RELAP5/MOD3

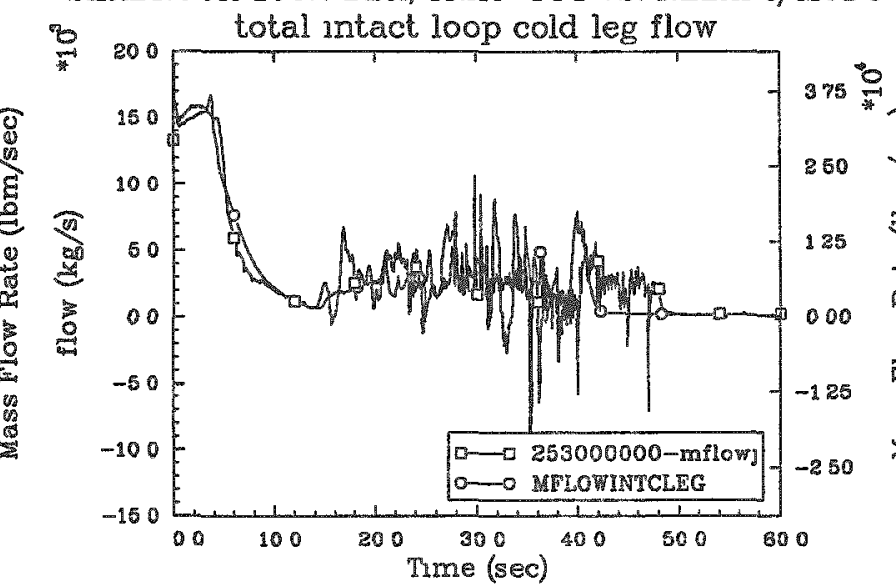

SEABROOK 100\% DBA, TRAC-PFI VS RELAP5/MOD3 bl downcomer vold fractions

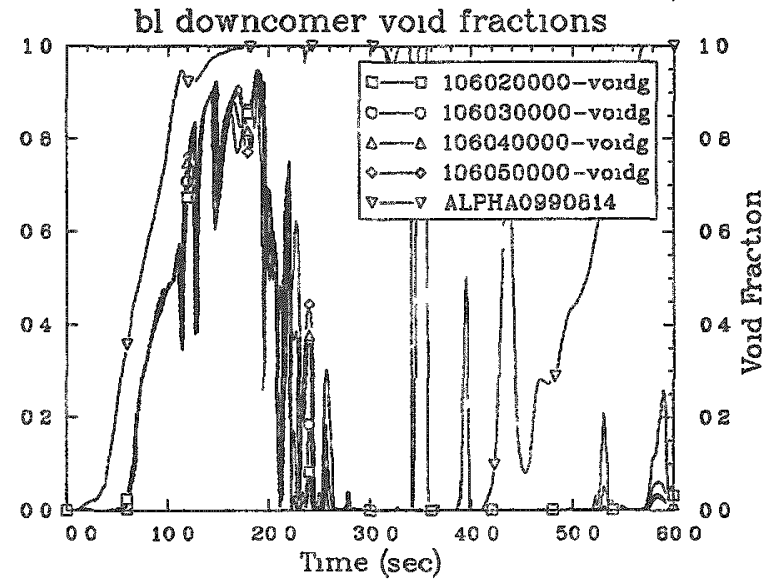

SEABROOK 100\% DBA, TRAC-PF1 VS RELAP5/MOD3

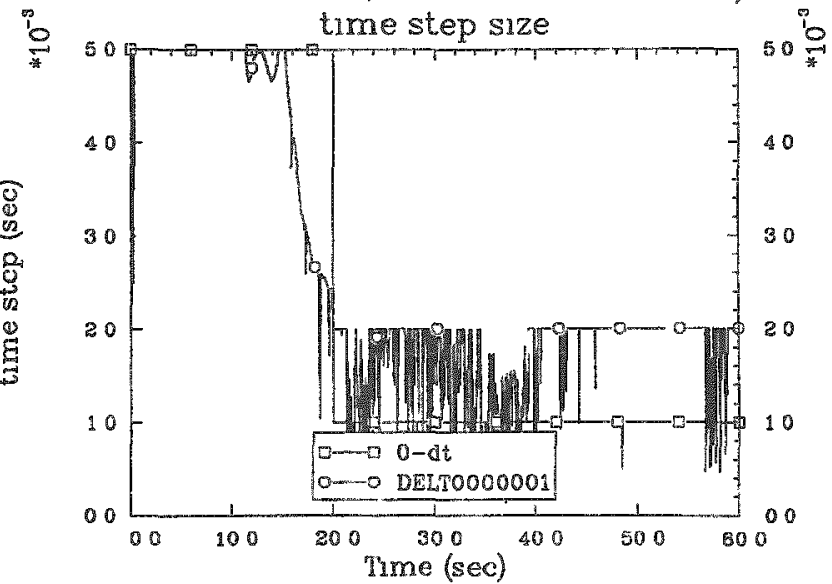

